\title{
Coal Industry Annual 1995
}

\section{October 1996}

\section{Energy Information Administration}

Office of Coal, Nuclear, Electric and Alternate Fuels

U.S. Department of Energy

Washington, DC 20585

This report was prepared by the Energy Information Administration, the independent statistical and analytical agency within the Department of Energy. The information contained herein should not be construed as advocating or reflecting any policy position of the Department of Energy or any other organization. 


\section{Contacts}

This publication was prepared by Frederick L. Freme, Michelle Bowles, Stephen Scott, Thomas Murphy, and Mary K. Paull under the direction of Noel C. Balthasar, Chief, Coal Data Branch, Coal and Electric Data and Renewables Division, Office of Coal, Nuclear, Electric, and Alternate Fuels. Specific infor- mation about the data in this report can be obtained from Frederick L. Freme (202/426-1152), or e-mail FFREME @EIA.DOE.GOV. All other questions on coal statistics should be directed to the National Energy Information Center (202/586-8800), or e-mail INFOCTR@EIA.DOE.GOV. 


\section{DISCLAIMER}

Portions of this document may be illegible in electronic image products. Images are produced from the best available original document. 


\section{DISCLAIMER}

This report was prepared as an account of work sponsored by an agency of the United States Goverament. Neither the United States Government nor any agency thereof, nor any of their employees, makes any wartanty, express or implied, or assumes any legal liability or responsibility for the accuracy, completeness, or usefulness of any information, apparatus, product, or process disclosed, or represents that its use would not infringe privately owned rights. Reference herein to any specific commercial product, process, or service by trade name, trademark, manufacturer, or otherwise does not necessarily constitute or imply its endorsement, recommendation, or favoring by the United States Government or any agency thereof. The views and opinions of authors expressed herein do not necessarily state or reflect those of the United States Government or any agency thereof. 


\section{Preface}

Coal Industry Annual 1995 provides comprehensive information about U.S. coal production, number of mines, prices, productivity, employment, productive capacity, and recoverable reserves. U.S. coal production for 1995 and previous years is based on the annual survey EIA-7A, "Coal Production Report."

This report presents data on coal consumption, coal distribution, coal stocks, coal prices, coal quality, and emissions for Congress, Federal and State agencies, the coal industry, and the general public. Appendix A contains a compilation of coal statistics for the major coal-producing States. This report does not include coal consumption data for nonutility power producers that are not in the manufacturing, agriculture, mining, construction, or commercial sectors. Consumption for nonutility power producers not included in this report is estimated to be 21 million short tons for 1995 .

The data presented in the report were collected and published by the Energy Information Administration (EIA), to fulfill its data collection and dissemination responsibilities, as specified in the Federal Energy Administration Act of 1974 (Public Law 93-275), as amended. Data for the Demonstrated Reserve Base (DRB) are now reported in U.S. Coal Reserves: A
Review and Update (DOE/EIA-0529). However, this report presents data on the recoverable portion of the DRB in Table 105.

The base year for the implicit price deflator, which is used to convert nominal figures to real figures was changed to 1992 from the previous base year of 1987 (Table D3).

This report constitutes the 20th annual report on coal production published by EIA and continues the series formerly included in the Minerals Yearbook published by the Bureau of Mines.

The Office of Coal, Nuclear, Electric and Alternate Fuels gratefully acknowledges the cooperation of the respondents in supplying the information presented in this report and appreciates the valuable assistance of State coal mining agencies; the U.S. Department of the Interior: the Bureau of Land Management, the Minerals Management Service; the U.S. Department of Labor: the Mine Safety and Health Administration, the Bureau of Labor Statistics; the U.S. Department of Commerce: the Bureau of the Census; and the King Publishing Corporation. 



\section{Contents}

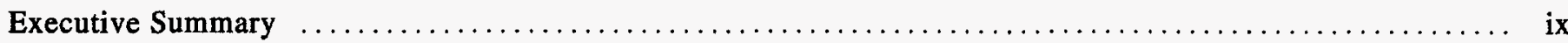

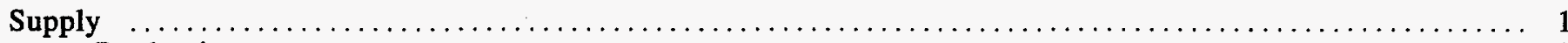

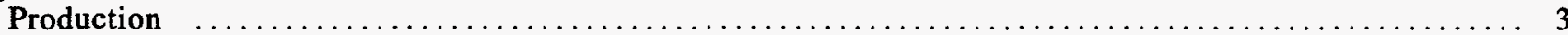

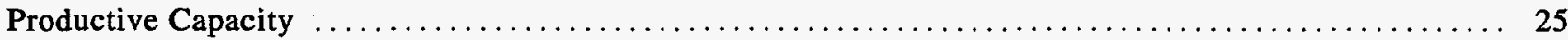

Recoverable Coal Reserves at Producing Mines $\ldots \ldots \ldots \ldots \ldots \ldots \ldots \ldots \ldots \ldots \ldots \ldots \ldots \ldots \ldots \ldots \ldots \ldots$

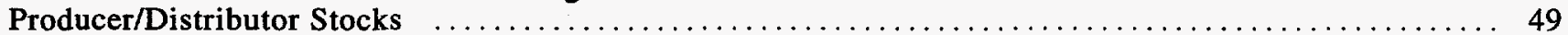

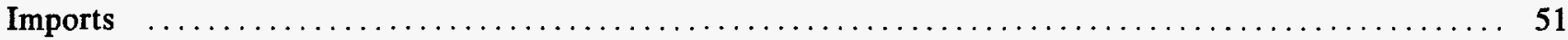

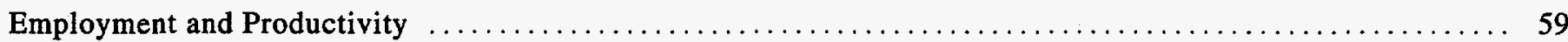

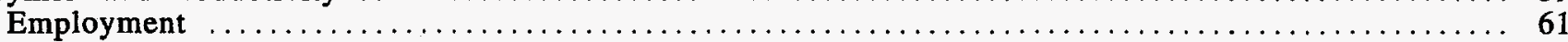

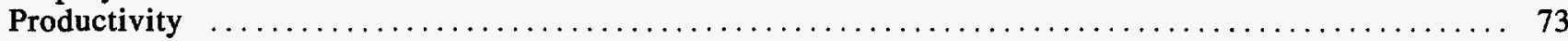

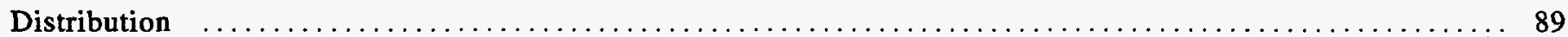

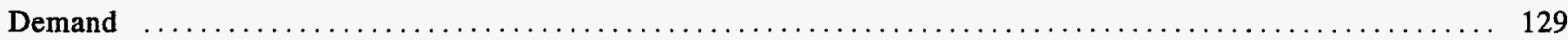

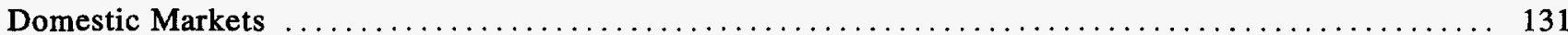

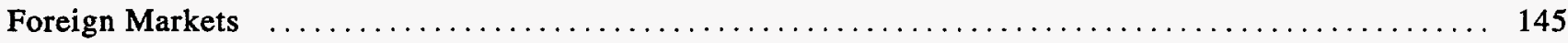

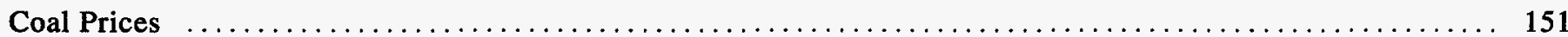

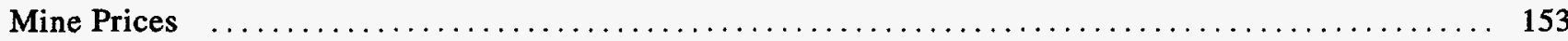

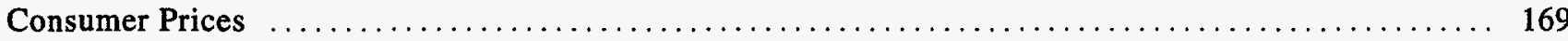

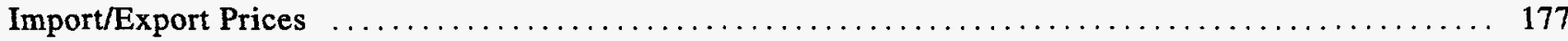

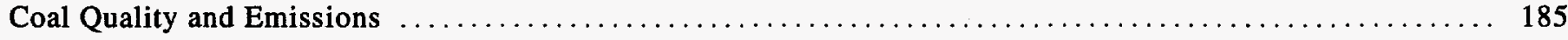

Appendices

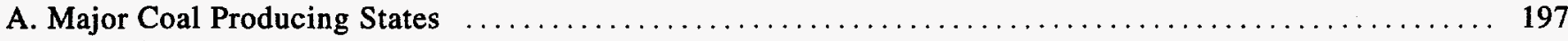

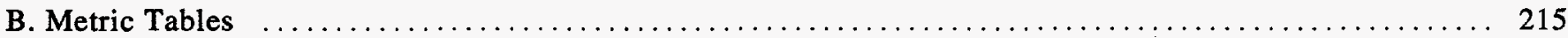

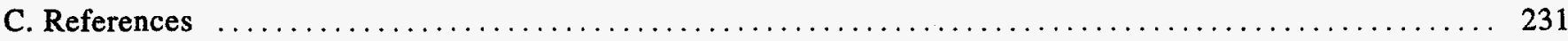

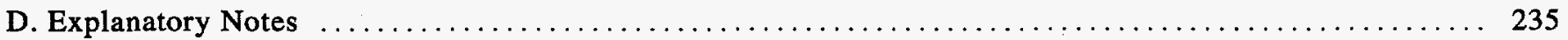

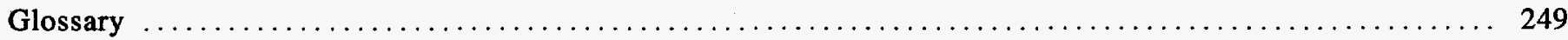




\section{Tables}

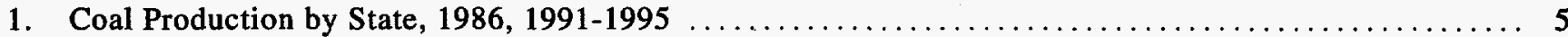

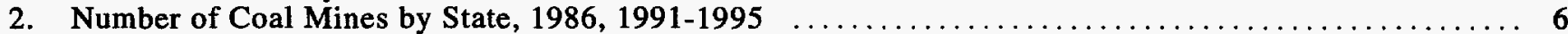

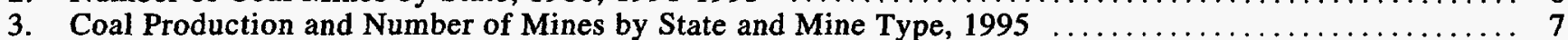

4. Coal Production and Number of Mines by State, County, and Mine Type, $1995 \ldots \ldots \ldots \ldots \ldots \ldots \ldots \ldots$

5. Underground Coal Production by State and Mining Method, $1995 \ldots \ldots \ldots \ldots \ldots \ldots \ldots \ldots \ldots \ldots \ldots$

6. Coal Production and Number of Mines by State, Mine Type, and Mine Production Range, $1995 \ldots \ldots, 13$

7. U.S. Coal Production by Coalbed Thickness and Mine Type, $1995 \ldots \ldots \ldots \ldots \ldots \ldots \ldots \ldots \ldots \ldots \ldots . \ldots 15$

8. U.S. Coal Production and Coalbed Thickness by Major Coalbeds and Mine Type, $1995 \ldots \ldots \ldots \ldots \ldots 16$

9. Coal Production and Number of Mines by State and Coal Rank, $1995 \ldots \ldots \ldots \ldots \ldots \ldots \ldots \ldots \ldots \ldots \ldots$

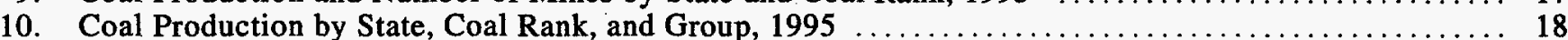

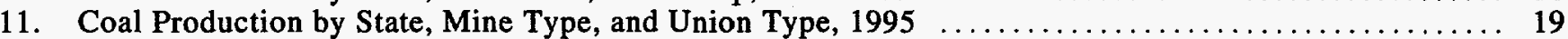

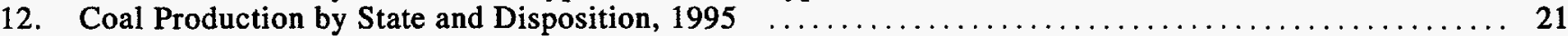

13. Coal Mining Acreage, Production and Royalties from Federal and Indian Leases by State, $1995 \ldots \ldots \ldots 22$

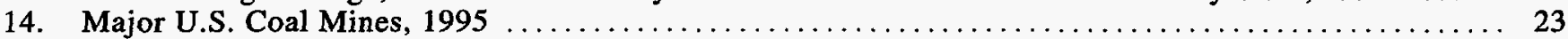

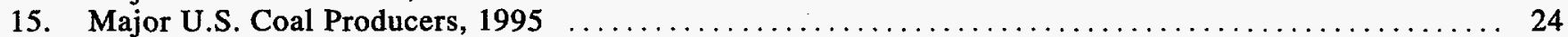

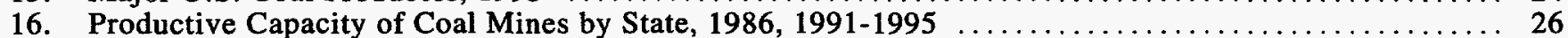

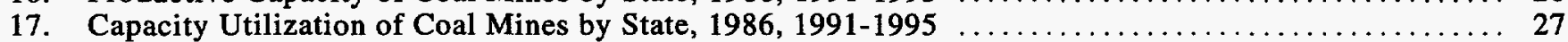

18. Production, Productive Capacity, and Capacity Utilization of Coal Mines by State and Mine Type, 199528

19. Productive Capacity and Capacity Utilization of Underground Coal Mines by State and Mining Method, 1995

20. Productive Capacity and Capacity Utilization of Coal Mines by State and Coal Rank, 1995 ........ 30

21. Productive Capacity and Capacity Utilization of Coal Mines by State and Mine Production Range, 199531

22. Productive Capacity and Productivity of Coal Mines by State and Capacity Utilization Range, 1995 ... 32

23. Productive Capacity and Capacity Utilization of Coal Mines by State and Recoverable Reserves Range, 1995

24. Productive Capacity and Capacity Utilization of Coal Mines by State, Mine Type, and Union Type,

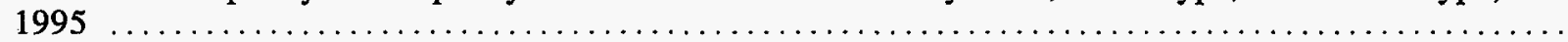

25. Recoverable Coal Reserves at Producing Mines by State, $1986,1991-1995 \ldots \ldots \ldots \ldots \ldots \ldots \ldots$

26. Average Recovery Percentage at Producing Coal Mines by State, 1986, 1991-1995

27. Recoverable Coal Reserves and Average Recovery Percentage at Producing Mines by State and Mine

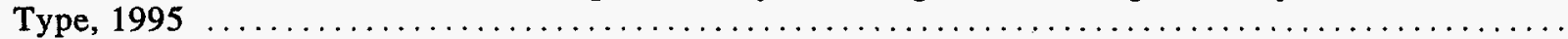

28. Recoverable Coal Reserves at Producing Underground Mines by State and Mining Method, 1995

29. A verage Recovery Percentage at Producing Underground Coal Mines by State and Mining Method, 1995

30. Recoverable Coal Reserves and Average Recovery Percentage at Producing U.S. Mines by Mine Production Range and Mine Type, 1995

31. Recoverable Coal Reserves and Average Recovery Percentage at Producing U.S. Mines by Coalbed Thickness and Mine Type, 1995

32. Recoverable Coal Reserves and Average Recovery Percentage at Producing Mines by State, Mine Type, and Union Type, 1995

33. Status of Recoverable Coal Reserves and Coal Production from Producing Federal Coal Leases by State, Fiscal Year 1995

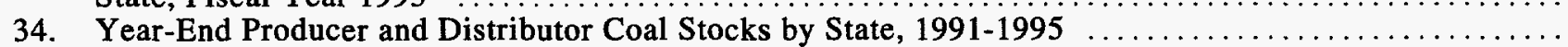

35. U.S. Coal Imports by Continent and Country of Origin, 1986, 1991-1995 $\ldots \ldots \ldots \ldots \ldots \ldots \ldots \ldots$

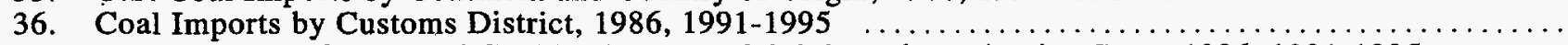

37. U.S. Receipts of Imported Coal by Country of Origin and Destination State, 1986, 1991-1995 ........

38. Imported Coal Received at Electric Utilities by Country of Origin and Destination State, 1986, 1991-1995

39. Imported Coal Received at Manufacturing and Coke Plants by Country of Origin and Destination State, 1993-1995

40. Average Number of Miners by State, 1986, 1991-1995

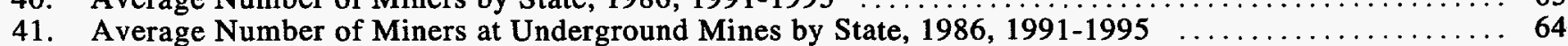

42. Average Number of Miners at Surface Mines by State, 1986, $1991-1995 \ldots \ldots \ldots \ldots \ldots \ldots \ldots \ldots$

43. Average Number of Miners by State and Mine Production Range, $1995 \ldots \ldots \ldots \ldots \ldots \ldots \ldots \ldots .66$

44. Average Number of Miners at Underground Mines by State and Mine Production Range, $1995 \ldots \ldots \ldots 67$

45. Average Number of Miners at Surface Mines by State and Mine Production Range, $1995 \ldots \ldots \ldots 6$ 
46. Average Number of Miners by State, Mine Type, and Union Type, 1995

47. U.S. Coal Mine Injuries, 1986, 1991-1995

48. Coal Mining Productivity by State, 1986, 1991-1995

49. Underground Coal Mining Productivity by State, 1986, 1991-1995

50. Surface Coal Mining Productivity by State, 1986, 1991-1995

51. Coal Mining Productivity by State and Mine Type, 1995

52. Weighted Average Number of Days Worked by State and Mine Type, 1986, 1991-1995

53. Weighted Average Number of Days Worked by Mine Production Range, 1995

54. Underground Coal Mining Productivity by State and Mining Method, 1995

55. U.S. Coal Mining Productivity by Coalbed Thickness and Mining Method, 1995

Coal Mining Productivity by State, Mine Type, and Mine Production Range, 1995

Coal Mining Productivity by State, Mine Type, and Union Type, 1995

58. Distribution of U.S. Coal by State of Origin, 1991-1995

59. Domestic and Foreign Distribution of U.S. Coal by State of Origin, 1991-1995

60. Major U.S. Coal Distributors, 1995

61. Domestic Distribution of U.S. Coal by Coal-Producing Region and State, and Destination Census

Division and State, 1991-1995

62. Foreign Distribution of U.S. Coal by Major Coal-Exporting States and Destination, 1991-1995

63. Foreign Distribution of U.S. Metallurgical Coal by Major Coal-Exporting States and Destination, 1991-1995

64. Foreign Distribution of U.S. Steam Coal by Major Coal-Exporting States and Destination, 1991-1995

65. Distribution of U.S. Coal by Origin, Destination, and Method of Transportation, 1995

66. Major U.S. Coal Consumers, 1995

67. Coal Consumption by Census Division and State, 1986, 1991-1995

68. Year-End Consumer Coal Stocks by Census Division and State, 1986, 1991-1995

69. Coal Consumption at Electric Utility Plants by Census Division and State, 1986, 1991-1995

70. Year-End Coal Stocks at Electric Utility Plants by Census Division and State, 1986, 1991-1995

71. Coal Consumption at Other Industrial Plants by Census Division and State, 1986, 1991-1995

Year-End Coal Stocks at Other Industrial Plants by Census Division and State, 1986, 1991-1995

73. Coal Carbonized at Coke Plants by Census Division and State, 1986, 1991-1995

74. Year-End Coal Stocks at Coke Plants by Census Division and State, 1986, 1991-1995

75. Coal Consumption by Residential and Commercial Sector, by Census Division and State, 1986, 1991-1995

79. Coal Exports by Customs District, 1986, 1991-1995

80. Average Mine Price of Coal by State, 1986, 1991-1995

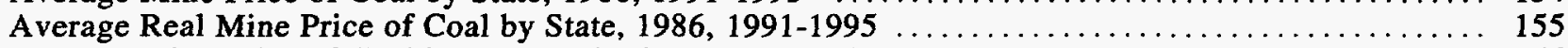

82. Average Mine Price of Coal by State and Mine Type, 1995

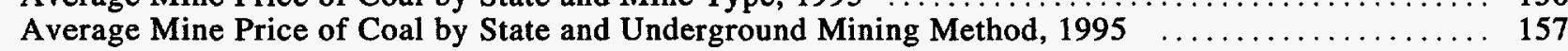

84. Coal Production, Number of Mines, and Average Mine Price, by State and County, $1995 \ldots \ldots \ldots \ldots \ldots$

85. Average Mine Price by State and Coal Rank, 1995

86. Average Mine Price of U.S. Coal by Mine Production Range and Mine Type, 1995

87. Average Mine Price of U.S. Coal by Coalbed Thickness and Mine Type, 1995

88. Average Mine Price of Coal by State and Productivity Range, 1995

89. Average Mine Price of Underground Coal by State and Productivity Range, 1995

90. Average Mine Price of Surface Coal by State and Productivity Range, 1995

91. Average Mine Price by State and Disposition, 1995

92. Average Price of Coal Delivered to Electric Utilities by Census Division and State, 1986, 1991-1995

93. Average Real Price of Coal Delivered to Electric Utilities by Census Division and State, 1986, 1991-1995

94. Average Price of Coal Delivered to Other Industrial Plants by Census Division and State, 1986, 1991-1995

95. Average Real Price of Coal Delivered to Other Industrial Plants by Census Division and State, 1986, 1991-1995

Average Price of Coal Delivered to Coke Plants by Census Division and State, 1986, 1991-1995

97. Average Real Price of Coal Delivered to Coke Plants by Census Division and State, 1986, 1991-1995

98. Average Price of U.S. Coal Imports by Continent and Country of Origin, 1986, 1991-1995

99. Average Price of U.S. Coal Exports by Destination, 1986, 1991-1995

100 .

Average Real Price of U.S. Coal Exports by Destination, 1986, 1991-1995

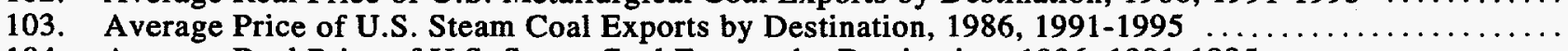

105. Estimate of the Recoverable Reserves of Coal by Sulfur Range, State, and Mine Type

106. Average Quality of Coal Received at Electric Utilities by Census Division and State, 1986, 1991-1995 
107. Average Quality of Coal Received at Manufacturing and Coke Plants by Census Division and State, 1993-1995

A1. Alabama Coal Statistics, 1986, 1991-1995

A2. Arizona Coal Statistics, $1986,1991-1995 \ldots \ldots \ldots \ldots \ldots \ldots \ldots \ldots \ldots \ldots \ldots \ldots \ldots \ldots \ldots \ldots \ldots \ldots$

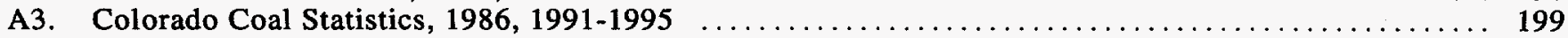

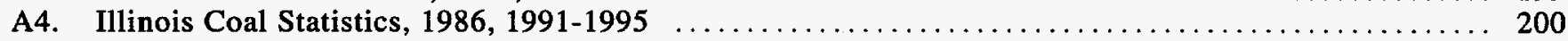

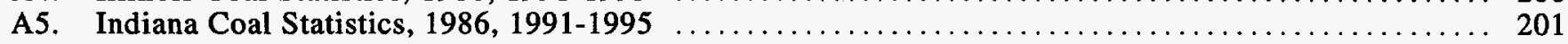

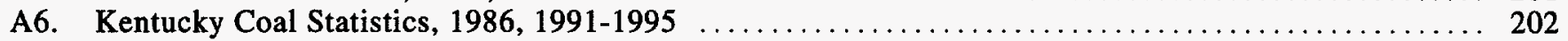

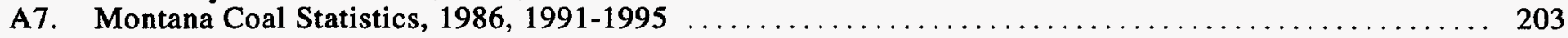

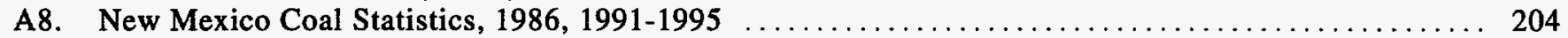

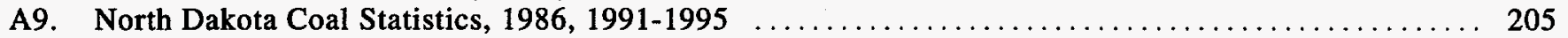

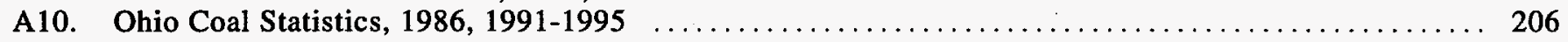

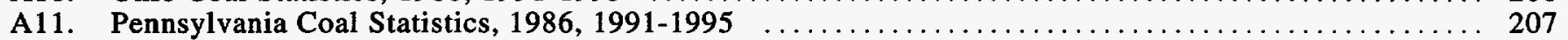

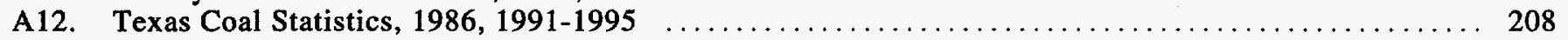

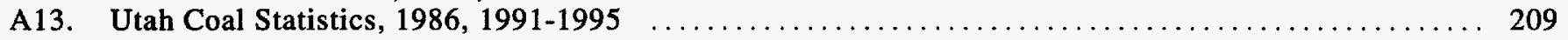

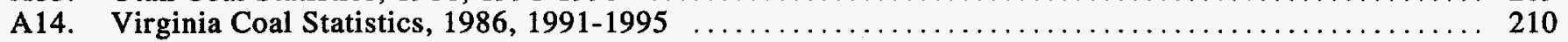

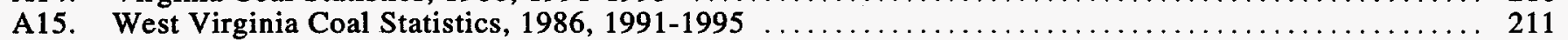

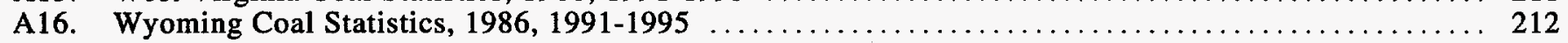

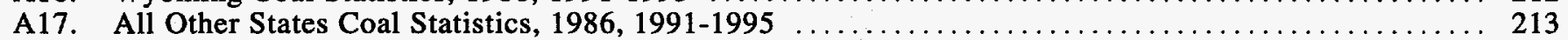

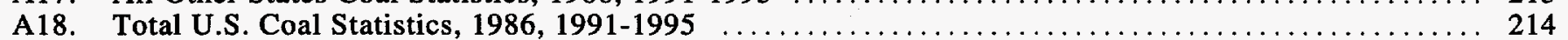

B1. Trends in U.S. Coal Production, Imports, Consumption, Exports, and Stocks, 1986, 1991-1995 ..... 215

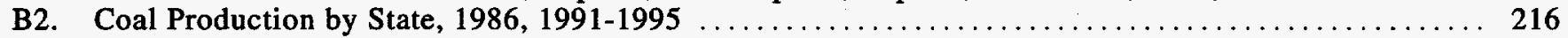

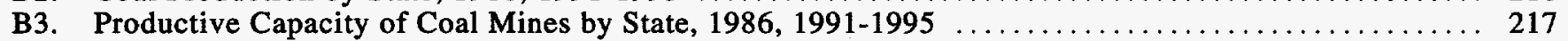

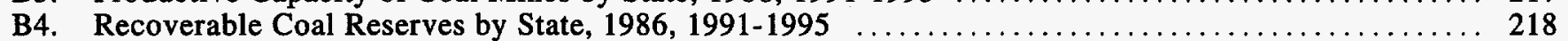

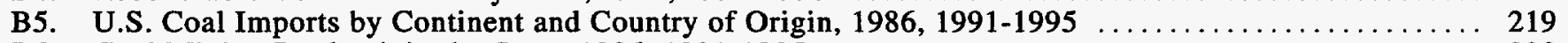

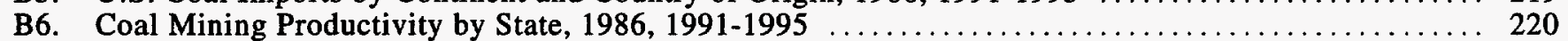

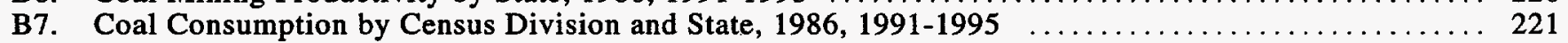

B8. Year-End Consumer Coal Stocks by Census Division and State, 1986, 1991-1995 ............ 222

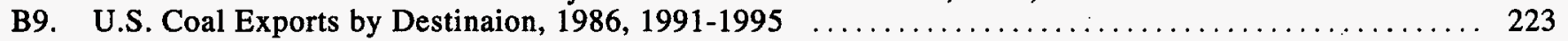

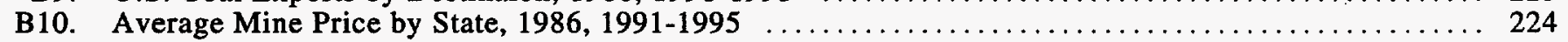

B11. Average Price of Coal Delivered to Electric Utilities by Census Division and State, 1986, 1991-1995 225

B 12. Average Price of Coal Delivered to Other Industrial Plants by Census Division and State, 1986, 1991-1995 ........................................................... 226

B13. Average Price of Coal Delivered to Coke Plants by Census Division and State, 1986, 1991-1995 .... 227

B14. Average Price of U.S. Coal Imports by Continent and Country of Origin, 1986, 1991-1995 _...... 228

B15. Average Price of U.S. Coal Exports by Destination, 1986, 1991-1995 ....................... 229

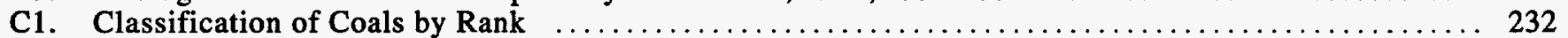

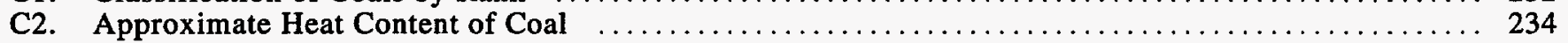

D1. Sources of Data for Total U.S. Coal Production and Number of Mining Operations $\ldots \ldots \ldots \ldots \ldots .236$

D2. Interquartile Range and Average Mine Price by State and Mine Type, $1995 \ldots \ldots \ldots \ldots \ldots \ldots \ldots 239$

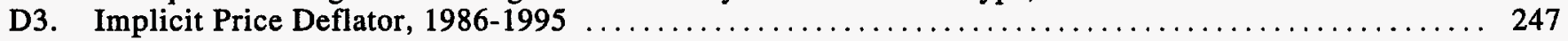

\section{Illustrations}

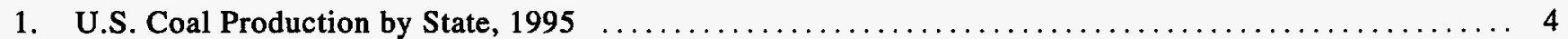

2. Recoverable Coal Reserves at Producing U.S. Mines by Mine Type and by Region, $1986-1995 \ldots \ldots \ldots . \ldots$

3. Average Recovery Percentage at Producing U.S. Coal Mines by Mine Type and by Region, 1986-1995 38

4. U.S. Coal Imports, 1986-1995

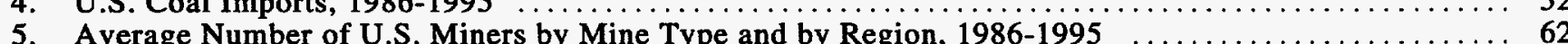

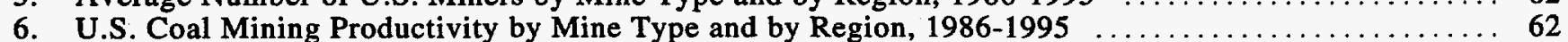

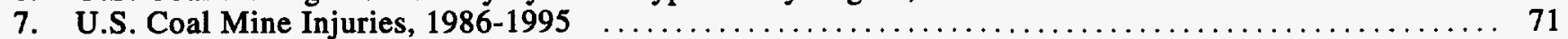

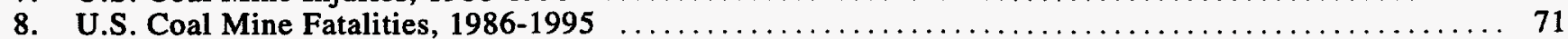

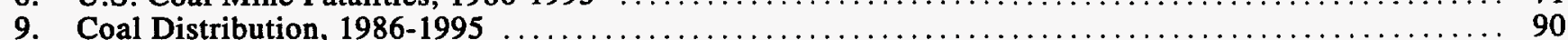

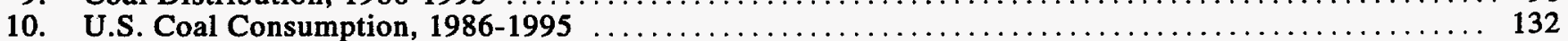

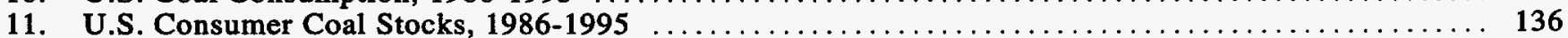

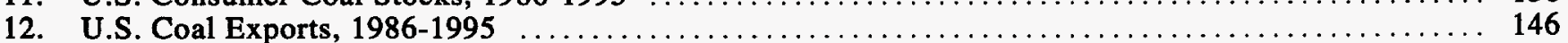

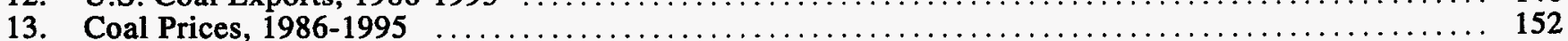

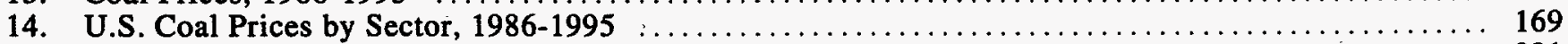

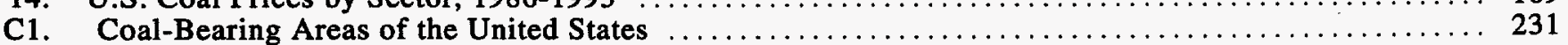




\section{Executive Summary}

\section{Coal's Role in the U.S. Energy Picture}

Total U.S. energy production in 1995 continued the upward swing begun in 1994, rising 1 percent to 71.16 quadrillion Btu. Coal production in Btu terms dropped less than 1 percent to 21.91 quadrillion Btu $(1,033$ million short tons), mainly because the production of low-Btu coal in Wyoming increased by 11 percent. Nuclear electric power and renewable energy sources, in particular conventional hydroelectric power and biofuels, were up from their 1994 levels, while natural gas and oil production declined (Table ES1).

Total U.S. energy consumption reached a record level of 90.62 quadrillion Btu in 1995, the fourth consecutive year of growth. Coal consumption reached 19.62 quadrillion Btu (941 million short tons), a 1-percent increase over the 1994 level. Consumption of natural gas, nuclear electric, hydroelectric power, and biofuels also rose, while consumption of petroleum went down.

The electric utility sector accounted for 88 percent of the total coal consumed in 1995. Coal-fired units represented 55 percent of utility generation of electricity, 1 percent less than in 1994. Petroleum generation paralleled this decline. Electricity generated by hydroelectric and nuclear power, and by natural gas increased (Table ES2).

U.S. coal exports rebounded after 3 years of decline to again become the primary U.S. energy export. In 1995, U.S. coal exports rose to 2.32 (89 million short tons) quadrillion Btu, 23 percent more than in 1994. In comparison, coal imports, accounting for less than 1 percent of U.S. energy imports, declined to 0.18 ( 7 million short tons) quadrillion Btu, 5 percent less than in 1994.

\section{Production}

U.S. coal production totaled 1,033 million short tons, about the same as in 1994. Wyoming's production set a record high; it remained the leading coal-producing State, followed by West Virginia, and Kentucky. On the other hand, Kentucky's production was at the lowest level since 1985; Indiana and Virginia's coal output was the lowest since 1978; and Ohio had the lowest level of production since 1940. (This output does not include about 4 million short tons of low-Btu waste coal, which is recovered and used by independent power producers and reported to their respective State governments.)

Coal production for States east of the Mississippi River declined to 544 million short tons, as demand for low price subbituminous coal from Wyoming increased. The States with the largest decrease in production were Kentucky, Indiana, Illinois, Ohio, and Virginia, which together had 24 million short tons less coal output than in 1994. The eastern States had a combined net loss of 244 mines and 6,982 miners. However, miner productivity in this region rose 5 percent in 1995 to 3.45 short tons per miner per hour, the second consecutive year of substantial gain. This is due in part to the closure of some small, lessproductive mines and to the increased utilization of more productive mining methods (example longwall).

Coal production west of the Mississippi River reached a record 489 million short tons, despite 6 fewer mines in the region and 266 fewer miners. Wyoming's coal output, which was 27 million short tons higher than in 1994 , accounted for most of the growth. Miner productivity in the region rose 7 percent to 14.18 short tons per miner per hour, in part due to the use of new, upgraded equipment at the mines.

\section{Coal Mine Prices}

The average price of U.S. coal in 1995 was $\$ 18.83$ per short ton, 3 percent less than in 1994. The price of coal from mines west of the Mississippi River fell 4 percent from the 1994 level. Coal prices at mines east of the Mississippi River also dipped, but by less than 1 percent.

\section{Coal Imports}

U.S. coal imports in 1995 were 7 million short tons, 5 percent less than in 1994. Colombia accounted for most of the decrease in tonnage, with over 600,000 short tons less coal shipped in 1995 than in 1994. The decline was partially offset by increased shipments from Venezuela and Australia. Colombia exported 19 percent less coal and Venezuela exported 21 percent more to the United States electric utility sector. 
Table ES1. U.S. Energy Overview, Selected Years, 1986, 1991-1995

(Quadrillion Btu)

\begin{tabular}{|c|c|c|c|c|c|c|c|c|c|}
\hline \multirow{2}{*}{$\begin{array}{l}\text { Activity and } \\
\text { Energy Source }\end{array}$} & \multirow{2}{*}{$1995^{P}$} & \multirow{2}{*}{1994} & \multirow{2}{*}{1993} & \multirow{2}{*}{1992} & \multirow{2}{*}{1991 . } & \multirow{2}{*}{1986} & \multirow{2}{*}{$\begin{array}{c}\text { Percent } \\
\text { Change } \\
\text { 1994-1995 }\end{array}$} & \multicolumn{2}{|c|}{$\begin{array}{l}\text { Average Annual } \\
\text { Percent Change }\end{array}$} \\
\hline & & & & & & & & 1991-1995 & 1986-1995 \\
\hline Production & 71.16 & R 70.62 & 68.32 & ${ }^{R} 69.96$ & R 70.41 & 64.35 & 0.8 & $\mathbf{0 . 3}$ & 1.1 \\
\hline 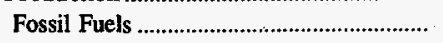 & 57.40 & R 57.83 & $\mathrm{R}^{55.71}$ & 57.55 & 57.83 & 56.58 & -.7 & -.2 & .2 \\
\hline 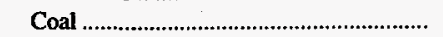 & 21.91 & R 22.07 . & 20.22 & 21.59 & 21.59 & 19.51 & -.7 & .4 & 1.3 \\
\hline 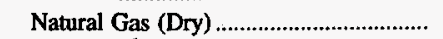 & 19.23 & R 19.27 & R 18.58 & 18.38 & 18.23 & 16.54 & -.2 & 1.3 & 1.7 \\
\hline Crude Oil 1 & 13.82 & R 14.10 & 14.49 & 15.22 & 15.70 & 18.38 & -2.0 & -3.1 & -3.1 \\
\hline Natural Gas Plant Liquids ............................ & 2.44 & 2.39 & 2.41 & 2.36 & 2.31 & 2.15 & 2.1 & 1.4 & 1.4 \\
\hline Nuclear Electric Power............................... & 7.19 & R 6.84 & 6.52 & 6.61 & 6.58 & 4.47 & 5.1 & 2.2 & 5.4 \\
\hline Hydroelectric Pumped Storage 2 & -.03 & R -.03 & -.04 & -.04 & -.05 & (3) & - & - & - \\
\hline 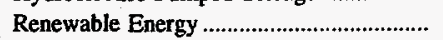 & 6.60 & R 5.99 & ${ }^{R} 6.13$ & $\mathrm{R}_{5.84}$ & $R_{6.05}$ & 3.30 & 10.2 & 2.2 & 8.0 \\
\hline Conventional Hydroelectric Power.......... & 3.20 & 2.67 & 2.88 & 2.61 & 2.98 & 3.07 & 19.6 & 1.8 & .4 \\
\hline 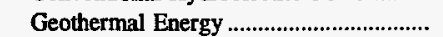 & .36 & $\mathrm{R}^{-38}$ & $\mathrm{R} .38$ & R. .37 & R.35 & .22 & -5.2 & 1.0 & 5.7 \\
\hline Biofuels 4 & 2.94 & R 2.85 & $\mathbf{R}_{2.78}$ & $\mathrm{R}_{2.79}$ & 2.64 & .01 & 3.1 & 2.7 & 83.8 \\
\hline 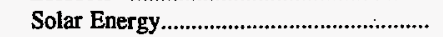 & .07 & .07 & .07 & .07 & .07 & - & - & - & - \\
\hline 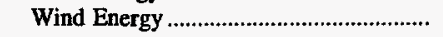 & .04 & .04 & .03 & .03 & .03 & * & 13.6 & 10.3 & 81.5 \\
\hline Imports. & 22.44 & R 22.71 & $\mathbf{R}_{21.54}$ & ${ }^{R} 19.66$ & 18.58 & 14.44 & -1.2 & 4.8 & 5.0 \\
\hline 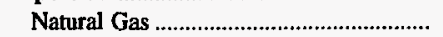 & 2.80 & $R_{2.68}$ & 2.40 & 2.16 & 1.80 & .75 & 4.2 & 11.6 & 15.8 \\
\hline Crude Oil 5 & 15.74 & ${ }^{R} 15.34$ & 14.75 & 13.25 & 12.55 & 9.00 & 2.6 & 5.8 & 6.4 \\
\hline Petroleum Products 6 & 3.20 & R 3.91 & 3.76 & 3.71 & 3.79 & 4.20 & -18.1 & -4.1 & -3.0 \\
\hline 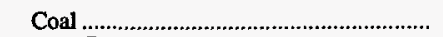 & .18 & R .19 & R. .18 & R. 10 & ${ }^{\mathrm{R}} .08$ & .05 & -5.3 & 22.5 & 15.3 \\
\hline Other 7 & .52 & R .59 & $\mathrm{R}_{.45}$ & R.43 & $\mathbf{R} .36$ & .44 & -11.9 & 9.6 & 1.9 \\
\hline 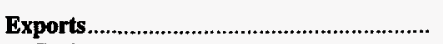 & 4.58 & R 4.12 & 4.35 & 5.02 & 5.22 & 4.06 & 11.0 & -3.2 & 1.4 \\
\hline Coal & 2.32 & 1.88 & 1.96 & 2.68 & 2.85 & 2.25 & 23.5 & -5.0 & .3 \\
\hline Crude Oil & 1.99 & 1.99 & 2.12 & 2.01 & 2.13 & 1.67 & .2 & -1.6 & 2.0 \\
\hline Other ${ }^{8}$ & .27 & $\mathrm{R} .26$ & .27 & .33 & .24 & .14 & 3.4 & 2.8 & 7.7 \\
\hline 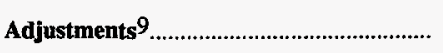 & 1.59 & $\mathrm{R}-.32$ & ${ }^{R} 1.51$ & ${ }^{R} .65$ & ${ }^{\mathrm{R}} .27$ &.- .44 & - & - & - \\
\hline Consumption 10 & 90.62 & R 88.90 & ${ }^{R} 87.03$ & ${ }^{R} \mathbf{8 5 . 2 6}$ & ${ }^{R} 84.05$ & $\mathbf{7 4 . 3 0}$ & 1.9 & 1.9 & 2.2 \\
\hline Fossil Fuels & 76.47 & R 75.64 & R 74.12 & 72.55 & 71.23 & 66.15 & 1.1 & 1.8 & 1.6 \\
\hline 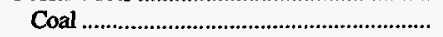 & 19.62 & 19.54 & 19.43 & 18.87 & 18.77 & 17.26 & .4 & 1.1 & 1.4 \\
\hline Coal Coke Net Imports............................... & .03 & .02 & .02 & .03 & .01 & -.02 & - & - & - \\
\hline Natural Gas 11 & 22.20 & R 21.34 & ${ }^{R} 20.83$ & 20.13 & 19.61 & 16.71 & 4.0 & 3.1 & 3.2 \\
\hline Petroleum 12 & 34.62 & $R_{34.73}$ & 33.84 & 33.53 & 32.85 & 32.20 & -.3 & 1.3 & .8 \\
\hline 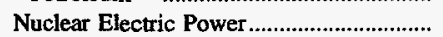 & 7.19 & ${ }^{R} 6.84$ & 6.52 & 6.61 & 6.58 & 4.47 & 5.1 & 2.2 & 5.4 \\
\hline 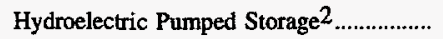 & -.03 & $\mathrm{R}-.03$ & -.04 & -.04 & -.05 & $(3)$ & - & - & - \\
\hline Renewable Energy & 6.88 & ${ }^{R} 6.30$ & ${ }^{R} 6.40$ & ${ }^{R} 6.11$ & R 6.27 & 3.68 & 9.3 & 2.3 & 7.2 \\
\hline Conventional Hydroelectric Power 13 & 3.46 & R 2.96 & $\mathrm{R}_{3.14}$ & $\mathrm{R}_{2.85}$ & $\mathrm{R}_{3.18}$ & 3.45 & 17.0 & 2.1 & * \\
\hline Geothermal Energy 14 & .36 & $\mathrm{R}^{-38}$ & ${ }^{\mathrm{R}} .38$ & ${ }^{R} .37$ & $\mathrm{R} .35$ & .22 & -5.2 & 1.0 & 5.7 \\
\hline Biofuels 4 & 2.94 & R 2.85 & ${ }^{\mathrm{R}} 2.78$ & R 2.79 & 2.64 & .01 & 3.1 & 2.7 & 83.8 \\
\hline 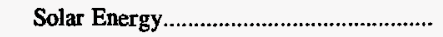 & .07 & .07 & .07 & .07 & .07 & - & - & - & - \\
\hline 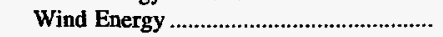 & .04 & .04 & .03 & .03 & .03 & * & 13.6 & 10.3 & 81.5 \\
\hline
\end{tabular}

1 Includes lease condensate.

2 Represents total pumped storage facility production minus energy used for pumping.

3 Pumped storage is included in conventional hydroelectric power.

4 Includes wood, wood waste, peat, wood liquors, railroad ties, pitch, wood sludge, municipal solid waste, agricultural waste, straw, tires, landfill gases, fish oils, and/or other waste.

5 Includes imports of crude oil for the Stategic Petroleum Reserve, which began in 1977.

6 Includes imports of unfinished oils and natural gas plant liquids.

7 "Other" imports are electricity and coal coke.

8 "Other" exports are natural gas, petroleum products, electricity and coal coke.

9 A balancing item. Includes stock changes, losses, gains, miscellaneous blending components, and unaccounted for supply.

10 From 1990, the portion of net imports of electricity that is derived from nonrenewable energy sources is included directly in "Consumption."

11 Includes supplemental gaseous fuels.

12 Petroleum products supplied, including natural gas plant liquids and crude oil burned as fuel.

13 Includes net imports of electricity.

14 Includes electricity imports from Mexico that are derived from geothermal energy.

* Data round to zero.

$\mathbf{R}$ Revised data.

P Preliminary data.

Notes: Coal Consumption does not include coal consumed by independent power producers. Total may not equal sum of components due to independent rounding.

Source: Energy Information Administration, Annual Energy Review 1995, DOE/EIA-0384(95), Table 1.1. 
Table ES2. Share of U.S. Electric Utility Net Generation of Electricity by Source, 1986, 1991-1995 (Percent)

\begin{tabular}{|c|c|c|c|c|c|c|}
\hline Energy Source & 1995 & 1994 & 1993 & 1992 & 1991 & 1986 \\
\hline Coal & 55.2 & 56.2 & 56.9 & 56.3 & 54.9 & 55.7 \\
\hline 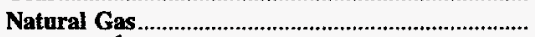 & 10.3 & 10.0 & 9.0 & 9.4 & 9.4 & 10.0 \\
\hline 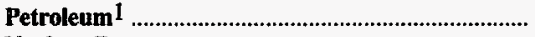 & 2.0 & 3.1 & 3.5 & 3.2 & 3.9 & 5.5 \\
\hline 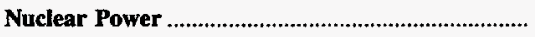 & 22.5 & 22.0 & 21.2 & 22.1 & 21.7 & 16.6 \\
\hline Hydroelectric Power & 9.8 & 8.4 & 9.2 & 8.6 & 9.8 & 11.7 \\
\hline Geothermal and Other ${ }^{2}$ & .2 & .3 & .3 & .4 & .4 & .5 \\
\hline Total & 100.0 & 100.0 & 100.0 & 100.0 & 100.0 & 100.0 \\
\hline
\end{tabular}

1 Distillate fuel oil, residual fuel oil (including crude oil burned as fuel), jet fuel, and petroleum coke.

2 Other is wood, waste, wind, photovoltaic, and solar thermal energy used to generate electricity for distribution.

Note: Total may not equal sum of components due to independent rounding.

Source: Energy Information Administration, Form EIA-759, "Monthly Power Plant Report."

The average price of imported coal in 1995 went up by 13 percent from 1994 to $\$ 34.13$ per short ton. The average price of coal imported from all foreign sources rose in 1995 . Coal imports were valued at $\$ 246$ million, up from $\$ 229$ million in 1994 .

\section{Coal Consumption}

In 1995, domestic coal consumption rose to a record of 941 million short tons, a 1-percent increase over 1994. (This total does not include about 21 million short tons of coal consumed by independent power producers in 1995.) Electric utility coal consumption rose by 1 percent to 829 million short tons, even though net generation at electric utilities rose by 3 percent. Coal consumption at electric utilities was indirectly affected by: a substantial increase in hydro- electric generation due to heavy precipitation, particularly in California and the Pacific Northwest; higher nuclear generation; and abundant supplies of low-cost natural gas which, at some electric utilities, allowed gas to edge out coal as the lowest cost fuel for electric generation.

Industrial plants consumed 73 million short tons of coal, 3 percent less than in 1994. Coal carbonized at coke plants increased by 4 percent to 33 million short tons, while the residential and commercial sectors consumed about 6 million short tons.

The average sulfur content (measured as percent sulfur by weight) of coal received at electric utilities in 1995 was 1.08 percent, down 7 percent from 1994. The quality of coal received at manufacturing and coke plants showed a sulfur content of 1.15 , slightly less than in 1994.

Table ES3. Trends in U.S. Coal Production, Imports, Consumption, Exports, and Stocks, 1986, 1991-1995

(Million Short Tons)

\begin{tabular}{|c|c|c|c|c|c|c|c|c|c|}
\hline \multirow{2}{*}{ Activity } & \multirow{2}{*}{1995} & \multirow{2}{*}{1994} & \multirow{2}{*}{1993} & \multirow{2}{*}{1992} & \multirow{2}{*}{1991} & \multirow{2}{*}{1986} & \multirow{2}{*}{$\begin{array}{l}\text { Percent } \\
\text { Change } \\
\text { 1994-1995 }\end{array}$} & \multicolumn{2}{|c|}{ Average Annual Percent Change } \\
\hline & & & & & & & & 1991-1995 & $1986-1995$ \\
\hline Production & 1,033 & 1,034 & 945 & 998 & 996 & 890 & * & 0.9 & 1.7 \\
\hline 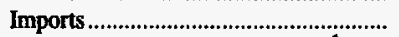 & 7 & 8 & 7 & 4 & 3 & 2 & -5.0 & 20.7 & 14.0 \\
\hline Producer and Distributor Stocks 1 . & 34 & 33 & 25 & 34 & 33 & 34 & 3.7 & 1.1 & .1 \\
\hline Consumption & 941 & 930 & 926 & 892 & 888 & 804 & 1.1 & 1.5 & 1.8 \\
\hline Exports & 89 & 71 & 75 & 103 & 109 & 86 & 24.1 & -5.0 & .4 \\
\hline 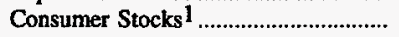 & 135 & 136 & 120 & 164 & 168 & 175 & -1.1 & -5.3 & -2.9 \\
\hline
\end{tabular}

1 Reported as of the last day of the quarter.

* Data round to zero.

Note: Consumption does not include coal consumed by independent power producers

Sources: - Production: Energy Information Administration (EIA), Form EIA-7A, "Coal Production Report"; U.S. Department of Labor, Mine Safety and Health Administration, Form 7000-2, "Quarterly Mine Employment and Coal Production Report"; and State Mining Agency Coal Production Reports. - Imports: U.S. Department of Commerce, Bureau of the Census, "Monthly Report IM 145." ' Producer and Distributor Stocks: ELA, Form EIA-6, "Coal Distribution Report." - Exports: U.S. Department of Commerce, Bureau of the Census, "Monthly Report EM 545."

- Consumption and Consumer Stocks: EIA, Form EIA-759, "Monthly Power Plant Report"; Form EIA-3, Quarterly Coal Consumption Report - Manufacturing Plants"; Form ELA-5, "Coke Plant Report - Quarterly"; and Form EIA-6, "Coal Distribution Report." 
Coal stocks held by consumers on December 31 , 1995 , totaled 135 million short tons, 1.3 million short tons less than in 1994. Compared with 1994, year-end 1995 coal stocks in the electric utility and other industrial sectors declined by 0.3 million short tons and 1.0 million short tons, respectively, while coal stocks at coke plants remained about the same. Producer and distributor stocks at the end of 1995 were 34 million short tons, an increase of 1.2 million short tons.

\section{Delivered Coal Prices}

The price of coal delivered to all consuming sectors except coke plants declined in 1995. The delivered price of coal to the electric utility and other industrial sectors dropped by 4 percent and 1 percent, respectively, to $\$ 27.01$ per short ton and $\$ 32.42$ per short ton. The price of coal delivered to coke plants rose 2 percent to $\$ 47.34$ per short ton.

\section{Coal Exports}

U.S. coal exports rose by 24 percent to 89 million short tons in 1995, reversing the downward trend begun in 1991. A 36-percent increase in shipments to Europe accounted for most of the growth.

Compared with 1994, steam coal exports rose 52 percent in 1995 to 36 million short tons. After declining sharply in 1993, steam coal exports to Europe nearly leveled off in 1994 at 11 million short tons, and then more than doubled in 1995 to 21 million short tons. Steam coal exports to Asia remained about the same while steam coal exports to North America increased 7 percent.

U.S. metallurgical coal exports increased by 10 percent from the 1994 level, to 52 million short tons. Higher shipments to coke plants in Romania, Brazil, the Netherlands, and Japan accounted for most of the increase.

West Virginia continued to dominate coal exports in 1995, shipping over 44 million short tons, a 22-percent increase from 1994. About 78 percent, or 35 million short tons, went to overseas metallurgical coal markets, while exports to overseas steam markets more than doubled reaching 10 million short tons.

The average price of U.S. coal exports in 1995 was $\$ 40.27$ per short ton, a 1-percent increase. U.S. metallurgical coal export prices rose 4 percent to $\$ 44.30$ per short ton. U.S. steam coal export prices went up slightly to $\$ 34.51$ per short ton. Coal exports in 1995 were valued at $\$ 3.6$ billion.
Beacon Group Energy Investment Fund LP paid nearly $\$ 232$ million for 75 percent of Mapco Coal Inc. The two companies formed a limited partnership to operate the coal company. Mapco's production totaled 13.6 million short tons, making it the 18 th ranked coal producing company in the United States.

In February 1995, Kindill Mining purchased three surface mines located in Indiana. The Old Ben 1 and Old Ben 2 surface mines were purchased from Zeigler Coal Holding and the Minnehaha mine was purchased from Cyprus AMAX Coal. The mines will be renamed "Kindill 1", "Kindill 2", and "Kindill 3", respectively. Kindill Mining was formed in 1995 by Wayne Park, formerly of Cyprus AMAX Coal and Old Ben Coal.

Fifty percent of the old Minnehaha (now Kindill 3) mine's production comes from the Addcar highwall mining system, where the miners work both existing highwalls of the mine. The Addcar system will be used as part of a trench mining plan (100-foot-wide trenches will be dug, exposing the coal seam on two sides, worked simultaneously by the highwall miner). The viability of this method in the current low-priced coal market is uncertain. The remaining 50 percent of the mine's coal production will come from a dragline, performing more conventional surface mining in the Indiana No. 6 and No. 7 coal seams. By mid-year 1996, the mine produced nearly 600 thousand short tons. The coal reserves associated with the Minnehaha mine are estimated to be 20 million short tons.

In May 1995, James River Coal purchased Transco Coal from the Williams Companies. The transaction included the Transco subsidiaries Kem Coal, Leeco Inc., Mountain Clay, and Randall Fuel. In return, Williams receives $\$ 65$ million in cash and $\$ 23$ million in preferred stock. The use of preferred stock as partial payment allowed James River to finance debt for future projects, while preserving its equity base. The purchase agreement did not include any Transco properties in Tennessee.

James River Chairman, James Crawford, has stated that Transco's contracts and mines could be consolidated with James Rivers'. He expects the expansion of surface mining operations to depend upon the profitability of sales in the spot and short term markets. Mr. Crawford also stated that James River will concentrate on lower-cost deep mining. James River Coal produced 8 million short tons during 1995, ranking it as the 23rd largest coal producer in the United States. In addition to the acquisition of Transco's assets, James River also sold some of its properties to Leslie Resources. In late December 1995, Leslie Resources completed a purchase of the common stock of James River's Kem Coal (part of the above-mentioned Transco package), Mountain Clay, New Brush Creek Mining, and Aceco Companies. These James River subsidiaries operated surface mines in Leslie, Perry and Knott counties in eastern Kentucky. Other assets in the deal included mining equipment and 88 million tons of combined recoverable reserves. 
In January 1995, Costain Group sold its 80 percent interest in the Dolet Hills Mining Venture to Jones Capital Corporation (Costain's minority partner and a subsidiary of the German-owned construction company Philipp Holzmann AG). In October 1995, Costain agreed to sell its Area 6 surface mine located in Alabama, and 25 to 30 million tons of associated underground reserves.

In 1995, Costain Coal Inc. reported 10.4 million short tons of coal production. They were the 20th ranked coal producing company in the United States.

Completing its exit as a coal producer in West Virginia, the Westmoreland Coal Company sold its remaining assets in the State for $\$ 9$ million in cash, according to a company representative. The assets included a term supply contract with Appalachian Power, which was acquired by Burco Resources of
Big Stone Gap, Virginia, and the Hampton No. 3 prep plant and load-out, which were acquired by Wind River Resources of Bob White, West Virginia. Also included in the transaction was Westmoreland's remaining West Virginia coal lease, which was sold back to the lessor, Penn Virginia Coal.

Addington Resources Inc. (ARI) sold its coal assets to Addington Enterprises, which includes founder Larry Addington and brothers Bruce and Robert, for $\$ 30$ million. Addington Enterprises will assume liabilities as well as future royalty payments. The sale finalized November 1995 included the purchase of the Tennessee Mining Co., Addwest Mining, and four Kentucky mines left after the acquisition by the Pittston Coal Company. Also included in the sale was ARI's mining equipment subsidiary, the developer of the Addcar Highwall mining system. 
Figure ES1. U.S. Coal Production by State, 1995

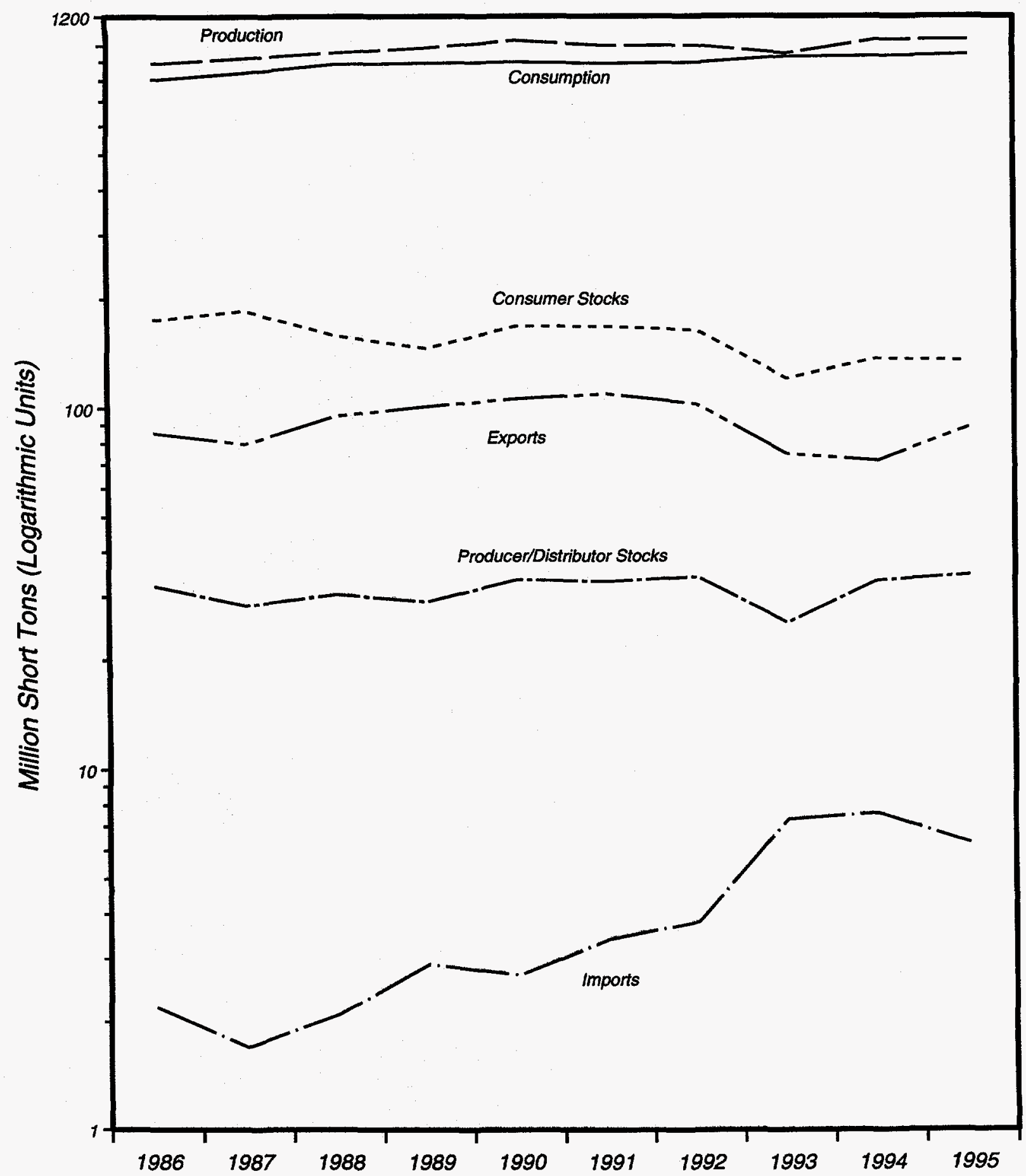

Note: Consumption does not include coal consumed by independent power producers.

Sources: •Production: Energy Information Administration (EIA), Form EIA-7A, "Coal Production "; U.S. Department of Labor, Mine Safety and Health Administration, Form 7000-2, "Quarterly Mine Employment and Coal Production Report"; and State Mining Agency Coal Production Reports. •Imports: U.S. Department of Commerce, Bureau of the Census, "Monthly Report IM-145." •Producer and Distributor Stocks: EIA, Form 6, "Coal Distribution Report." •Exports: U.S. Department of Commerce, Bureau of the Census, "Monthly Report EM545." - Consumption and Consumer Stocks: ElA, Form ElA-759, "Monthly Power Plant Report"; Form ElA-3, "Quarterly Coal Consumption Report - Manufacturing Plants"; Form EIA-5, "Coke Plant Report - Quarterly"; and Form EIA-6, "Coal Distribution Report." 


\begin{tabular}{|c|c|c|c|c|c|c|c|c|c|}
\hline \multirow[t]{2}{*}{ Category } & \multirow[t]{2}{*}{1995} & \multirow[t]{2}{*}{1994} & \multirow{2}{*}{1993} & \multirow{2}{*}{1992} & \multirow{2}{*}{1991} & \multirow{2}{*}{1986} & \multirow{2}{*}{$\begin{array}{c}\text { Percent } \\
\text { Change } \\
\text { 1994-1995 }\end{array}$} & \multicolumn{2}{|c|}{$\begin{array}{l}\text { Average Annual } \\
\text { Percent Change }\end{array}$} \\
\hline & & & & & & & & 1991-1995 & 1986-1995 \\
\hline \multicolumn{10}{|l|}{ Supply (thousand short tons) } \\
\hline Recoverable Reserves & $20,105,197$ & $21,016,526$ & $21,535,283$ & $21,626,971$ & $21,998,540$ & $25,047,698$ & -4.3 & -2.2 & -2.4 \\
\hline Productive Capacity 1 ........................... & $1,299,054$ & $1,320,656$ & $1,261,873$ & $1,241,054$ & NA & 949,182 & -1.6 & NA & 3.5 \\
\hline 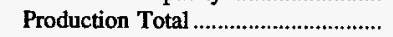 & $1,032,974$ & $1,033,504$ & 945,424 & 997,545 & 995,984 & 890,315 & * & .9 & 1.7 \\
\hline 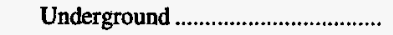 & 396,249 & 399,103 & 351,053 & 407,239 & 407,225 & 360,438 & -.7 & -.7 & 1.0 \\
\hline Surface & 636,725 & 634,401 & 594,371 & 590,306 & 588,759 & 529,877 & .4 & 2.0 & 2.1 \\
\hline 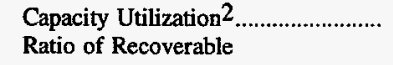 & 79.40 & 78.11 & 74.77 & 80.20 & NA & 93.33 & 1.6 & NA & -1.8 \\
\hline Reserves to Production......................... & 19.5 & 20.3 & 22.8 & 21.7 & 22,1 & 28.1 & -4.3 & -3.1 & -4.0 \\
\hline Number of Miners & 90,252 & 97,500 & 101,322 & 110,196 & 120,602 & 154,645 & -7.4 & -7.0 & -5.8 \\
\hline Productivity Total 2 & 5.38 & 4.98 & 4.70 & 4.36 & 4.09 & 3.01 & 8.0 & 7.1 & 6.6 \\
\hline 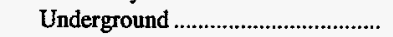 & 3.39 & 3.19 & 2.95 & 2.93 & 2.69 & 2.00 & 6.0 & 5.9 & 6.0 \\
\hline Surface & 8.48 & 7.67 & 7.23 & 6.59 & 6.38 & 4.60 & 10.7 & 7.4 & 7.0 \\
\hline Producer/Distributor Stocks .............. & 34,444 & 33,219 & 25,284 & 33,993 & 32,971 & 34,090 & 3.7 & 1.1 & .1 \\
\hline Imports $^{3}$ & 6,317 & 6,599 & 5,496 & 1,806 & 1,967 & 1,486 & -4.3 & 33.9 & 17.4 \\
\hline \multicolumn{10}{|l|}{ Distribution (thousand short tons) } \\
\hline 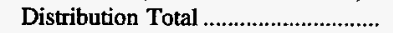 & $1,030,330$ & $1,022,523$ & 959,445 & $998 ; 647$ & 994,146 & NA & .8 & .9 & NA \\
\hline Domestic Distribution Total........... & 940,423 & 949,843 & 883,934 & 897,267 & 885,882 & $\mathrm{NA}$ & -1.0 & 1.5 & NA \\
\hline 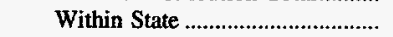 & 336,821 & 353,765 & 339,034 & 355,232 & 345,486 & NA & -4.8 & -.6 & NA \\
\hline To Other States & 603,602 & 596,078 & 544,900 & 542,035 & 540,395 & NA & 1.3 & 2.8 & NA \\
\hline Foreign Distribution Total............... & 89,907 & 72,680 & 75,510 & 101,380 & 108,264 & NA & 23.7 & -4.5 & NA \\
\hline 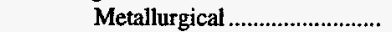 & 54,128 & 51,313 & 52,369 & 62,007 & 62,995 & NA & 5.5 & -3.7 & NA \\
\hline Steam & 35,779 & 21,367 & 23,141 & 39,373 & 45,269 & NA & 67.4 & -5.7 & NA \\
\hline Canada Total & 8,023 & 8,467 & 7,751 & 13,919 & 12,828 & NA & -5.2 & -11.1 & NA \\
\hline 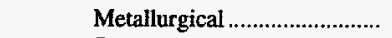 & 6,985 & 7,464 & 6,666 & 9,394 & 8,483 & NA & -6.4 & -4.7 & NA \\
\hline Steam & 1,037 & 1,003 & 1,085 & 4,525 & 4,345 & NA & 3.4 & -30.1 & NA \\
\hline Overseas Total 4 & 81,884 & 64,214 & 67,759 & 87,461 & 95,436 & NA & 27.5 & -3.8 & NA \\
\hline Metallurgical .............................. & 47,143 & 43,849 & 45,703 & 52,614 & 54,511 & NA & 7.5 & -3.6 & NA \\
\hline 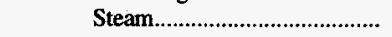 & 34,742 & 20,364 & 22,057 & 34,848 & 40,925 & NA & 70.6 & -4.0 & NA \\
\hline \multicolumn{10}{|l|}{ Demand (thousand short tons) } \\
\hline 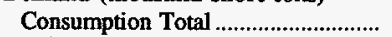 & 940,638 & 930,201 & 925,944 & 892,421 & 887,621 & 804,169 & 1.1 & 1.5 & 1.8 \\
\hline 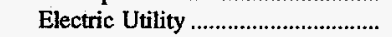 & 829,007 & 817,270 & 813,508 & 779,860 & 772,268 & 685,056 & 1.4 & 1.8 & 2.1 \\
\hline 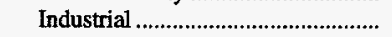 & 72,796 & 75,179 & 74,892 & 74,042 & 75,405 & 75,583 & -3.2 & -.9 & -.4 \\
\hline Coke & 33,011 & 31,740 & 31,323 & 32,366 & 33,854 & 35,862 & 4.0 & -.6 & -.9 \\
\hline Residential/Commercial................... & 5,824 & $6 ; 013$ & 6,221 & 6,153 & 6,094 & 7,667 & -3.1 & -1.1 & -3.0 \\
\hline Consumer Stocks Total ..................... & 134,639 & 136,139 & 120,458 & 163,692 & 167,711 & 175,226 & -1.1 & -5.3 & -2.9 \\
\hline 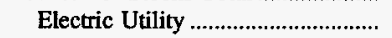 & 126,304 & 126,897 & 111,341 & 154,130 & 157,876 & 161,806 & -.5 & -5.4 & -2.7 \\
\hline 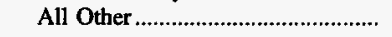 & 8,334 & 9,243 & 9,117 & 9,562 & 9,835 & 13,420 & -9.8 & -4.0 & -5.1 \\
\hline \multicolumn{10}{|c|}{ Coal Prices (nominal dollars per short ton) } \\
\hline 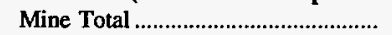 & $\$ 18.83$ & $\$ 19.41$ & $\$ 19.85$ & $\$ 21.03$ & $\$ 21.49$ & $\$ 23.79$ & -3.0 & -3.3 & -2.6 \\
\hline 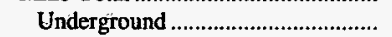 & 26.18 & 26.39 & 26.92 & 27.83 & 28.56 & 30.33 & -.8 & -2.1 & -1.6 \\
\hline 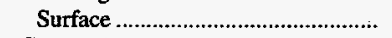 & 14.25 & 15.02 & 15.67 & 16.34 & 16.60 & 19.34 & -5.1 & -3.7 & -3.3 \\
\hline \multicolumn{10}{|l|}{ Consumer } \\
\hline 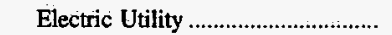 & 27.01 & 28.03 & 28.58 & 29.36 & 30.02 & 33.30 & -3.6 & -2.6 & -2.3 \\
\hline 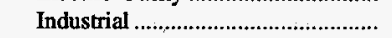 & 32.42 & 32.55 & 32.23 & 32.78 & 33.54 & 35.84 & -.4 & -.8 & -1.1 \\
\hline Coke & 47.34 & 46.56 & 47.44 & 47.92 & 48.88 & 50.85 & 1.7 & -.8 & -.8 \\
\hline
\end{tabular}

1 For 1986, the Form EIA-7A solicited data on "Daily Productive Capacity." To obtain annual productive capacity for a mine in 1986, each mine's daily productive capacity was multiplied by the number of days worked during the year.

2 Capacity utilization (percent) is the ratio of total production to annual productive capacity as reported by mining companies on Form EIA-7A. Productivity (short tons per miner per hour) is calculated by dividing total coal production by the total direct labor hours worked by all employees engaged in production, preparation, processing, development, maintenance, repair, and shop or yard work at mining operations.

3 Imports for 1992 through 1995 include imports to electric utilities, manufacturing plants and coke plants. Imports for 1986 through 1991 include only imports to electric utilities.

4 Includes Mexico.

* Data round to zero.

NA Not available.

Notes: Excludes silt, culm, refuse bank, slurry dam, and dredge operations except for Pennsylvania anthracite. Consumption Total does not include coal consumed by independent power producers. Totals may not equal sum of components due to independent rounding.

Sources: Energy Information Administration, Form EIA-3, "Quarterly Coal Consumption Report - Manufacturing Plants"; Form EIA-5, "Coke Plant Report - Quarterly"; Form EIA-6, "Coal Distribution Report"; Form EIA-7A, "Coal Production Report"; Form EIA-759, "Monthly Power Plant Report"; and U.S. Department of Commerce, Bureau of the Census, "Monthly Report EM 545" and "Monthly Report IM 145." 


\section{Supply}

\section{Production}

U.S. coal production during 1995 totaled 1,033 million short tons, about the same as last year's record level of 1,034 million short tons (Table 1). Although total U.S. coal production was essentially unchanged from 1994, coal production at the State levels changed dramatically. As in 1994, the largest coal-producing State was Wyoming, followed by West Virginia and Kentucky. Wyoming's coal production in 1995 increased 11 percent over its 1994 level to reach a record of 264 million short tons. West Virginia increased its coal production by 1 percent to a level of 163 million short tons, 7 percent less than the record level of 176 million short tons in 1947. Kentucky, the third largest coal-producing State, declined by 5 percent in 1995, to a level of 154 million short tons. Only 11 of the 25 States showed an increase in coal production in 1995.

Regionally, coal production in both the Appalachian and Interior Regions declined in 1995, while the Western Region increased for the ninth year in a row. In Appalachia, even though increases in production in 1995 occurred in Alabama, Maryland, Tennessee, and West Virginia, coal production in the region declined 10.5 million short tons (2.3 percent). The Interior Region registered increases in production in Kansas, Louisiana, and Texas, with only Louisiana obtaining a record level. Overall, the Interior Region declined in 1995 by 11.3 million short tons ( 6.3 percent). In the Western Region, four of the nine States (Alaska, Colorado, Utah and Wyoming) increased their coal production in 1995, with only Alaska failing to reach a record level. Coal production in the Western Region increased 21.3 million short tons, an increase of 5.2 percent. Over the last decade, the Appalachian Region has only increased at an average annual rate of 0.2 percent, while the Western Region has increased 5.5 percent. During the same time period, the Interior Region has declined at an average annual rate of 1.7 percent.

U.S. coal producers operated 2,104 mines during 1995, including 977 underground mines and 1,127 surface mines (Table 3 ). Underground mines produced 396 million short tons and represented 38 percent of total coal production. Underground mines east of the Mississippi accounted for 88.6 percent of all underground coal production in 1995. Surface mines west of the Mississippi represented 69.7 percent of total surface coal production in 1995 . Of the top 20 coal mines in the United States, 19 are surface mines, all located west of the Mississippi; they accounted for 28.9 percent of total U.S. coal production. The top nine mines in the United States are in Wyoming and represent 18.8 percent of total U.S. coal production.

\section{Productive Capacity}

The total estimated productive capacity of U.S. coal mines in 1995 was 1,299 million short tons, a decrease of 1.6 percent compared with the total estimated productive capacity of 1,321 million short tons in 1994 (Table 16). Following the same pattern as coal production, the total estimated productive capacity declined in both the Appalachian and Interior Regions (4.1 and 8.3 percent, respectively), while increasing in the Western Region (3.9 percent). Although total estimated productive capacity declined, coal mine capacity utilization, (defined as the ratio of actual production to productive capacity) rose to 79 percent in 1995 (Table 17). Capacity utilization increased for all three regions in 1995, with the Interior Region showing the largest increase.

\section{Recoverable Reserves}

Estimated recoverable U.S. coal reserves at producing mines in 1995 totaled 20 billion short tons. This 4.3-percent decrease continued a decline that started in 1986 (Table 25). Recoverable reserves at producing mines in the Western Region accounted for 63.3 percent of total reserves, with reserves at producing mines in the Appalachian and Interior Regions representing 22.6 percent and 14.1 percent, respectively.

The average recovery percentage for all U.S. producing mines in 1995 decreased slightly to 80.1 percent from the 80.4 percent in 1994 (Table 26). The estimated recovery percent for all underground mines in 1995 was 54.5 percent, a decline from the $\mathbf{5 6 . 5}$ percent in 1994. The estimated recovery percent at surface mines remained unchanged in 1995 at 90 percent. 


\section{Producers and Distributors Stocks}

Coal stocks held by U.S. coal producers and distributors at the end of 1995 totaled 34 million short tons, an increase of 4 percent compared with stocks at year-end 1994 and the highest year-end stock level since 1982 (Table 34).

\section{Coal Imports}

U.S. coal imports during 1995 totaled 7.2 million short tons, 5 percent less than the 7.6 million short tons imported during 1994 (Table 35). Since 1986, U.S. coal imports have increased at an average annual rate of 14 percent.

Colombia, Venezuela, Canada, and Indonesia were the major sources of imported coal during 1995. They contributed 6.9 million short tons and accounted for 96 percent of total coal imports. 


\section{Production}

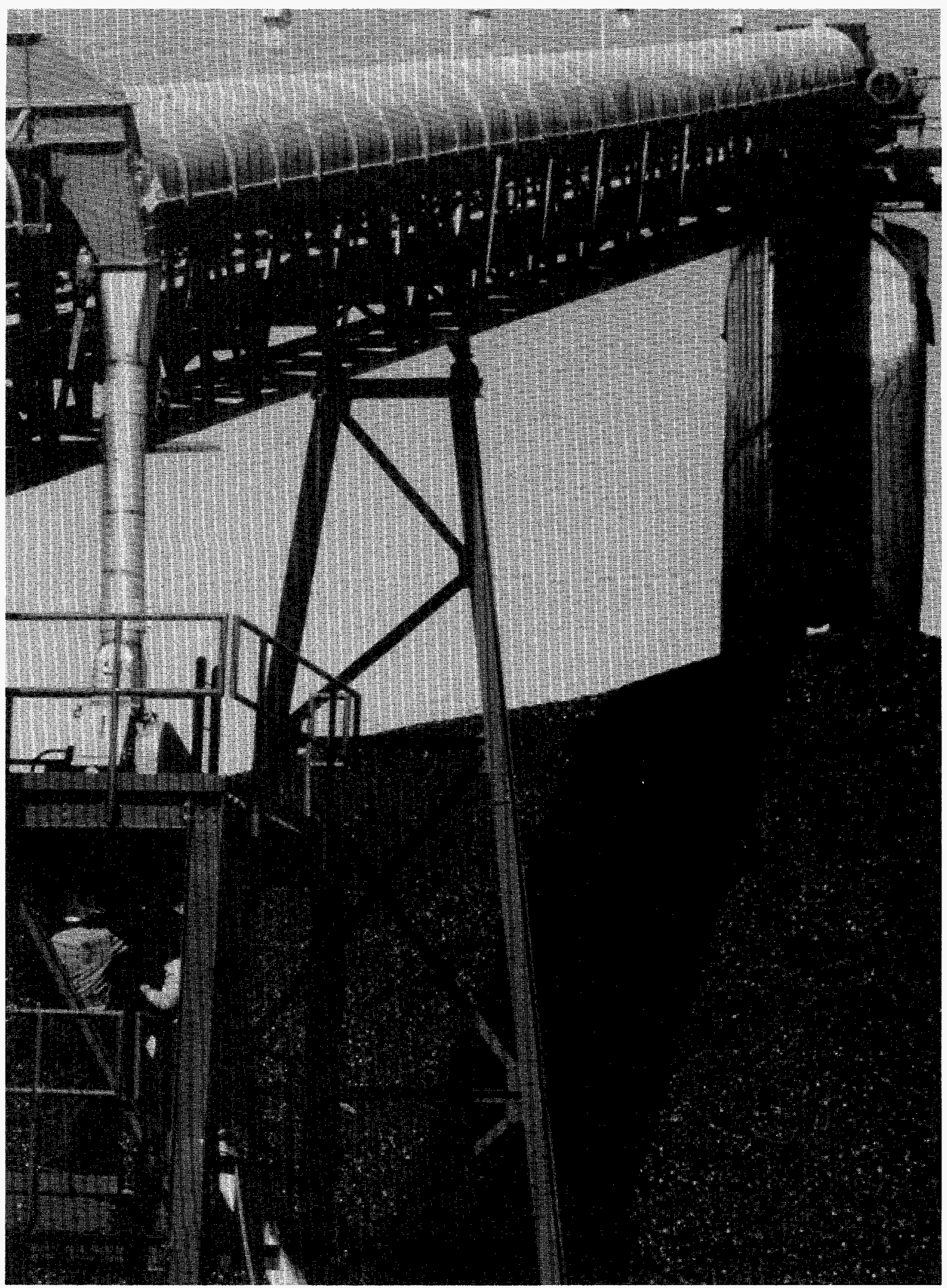

Coal production in 1995 was almost the same as a year ago, totaling 1,033 million short tons. 
Figure 1. U.S. Coal Production by State, 1995

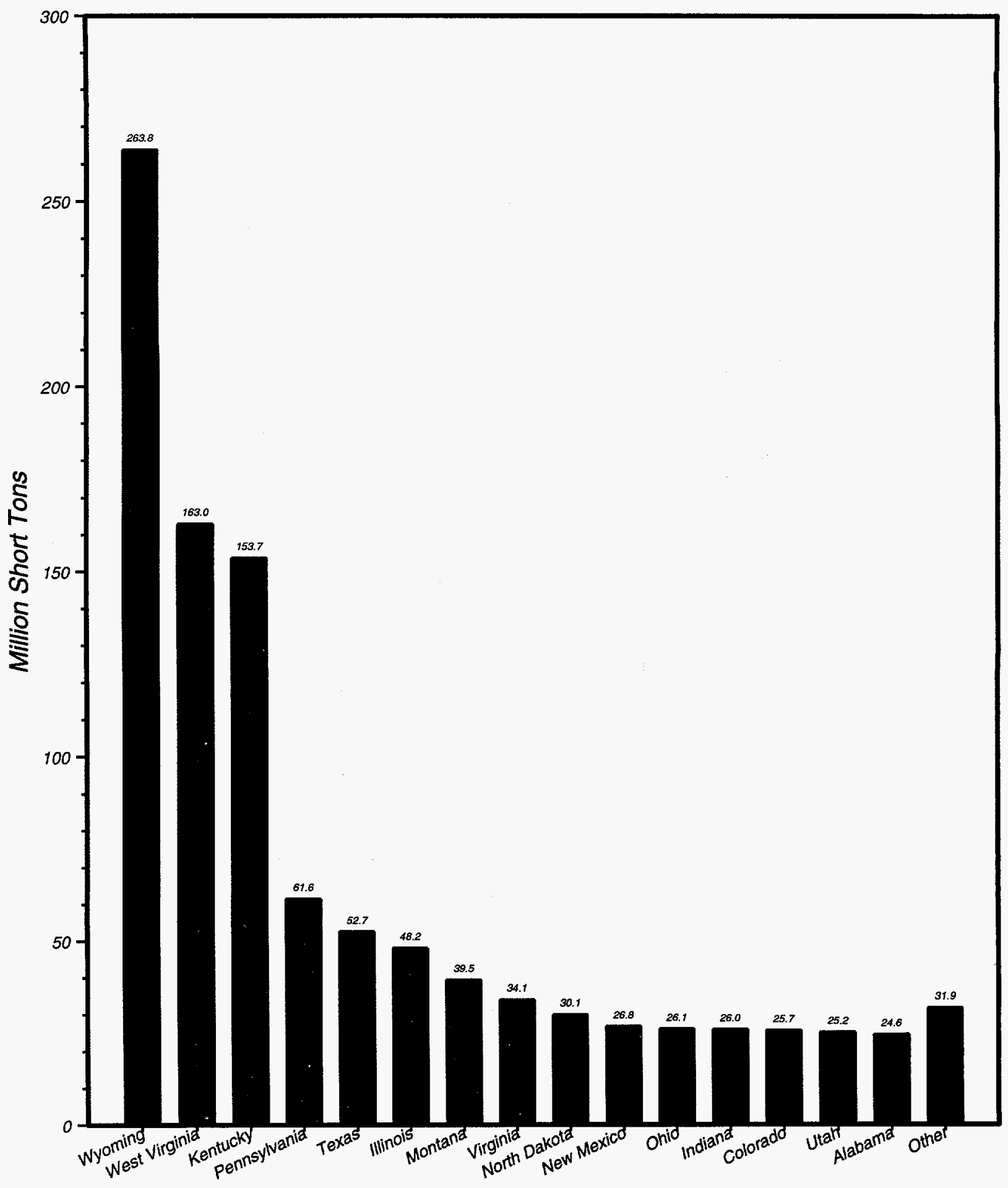

Notes: Other represents States which produced less than 12 million short tons of coal and includes Alaska, Arkansas, Arizona, Kansas, Louisiana, Maryland, Missouri, Oklahoma, Tennessee, and Washington. Coal production excludes silt, culm, refuse bank, slurry dam, and dredge operations except for Pennsylvania anthracite.

Sources: Energy Information Administration, Form EIA-7A, "Coal Production Report"; State Mining Agency Coal Production Reports; and/or U.S. Department of Labor, Mine Safety and Health Administration, Form 7000-2, "Quarterly Mine Employment and Coal Production Report." 
Table 1. Coal Production by State, 1986, 1991-1995 (Thousand Short Tons)

\begin{tabular}{|c|c|c|c|c|c|c|c|c|c|}
\hline \multirow{2}{*}{$\begin{array}{l}\text { Coal-Producing } \\
\text { State and Region }\end{array}$} & \multirow{2}{*}{1995} & \multirow{2}{*}{1994} & \multirow{2}{*}{1993} & \multirow{2}{*}{1992} & \multirow{2}{*}{1991} & \multirow{2}{*}{1986} & \multirow{2}{*}{$\begin{array}{c}\text { Percent } \\
\text { Change } \\
\text { 1994-1995 }\end{array}$} & \multicolumn{2}{|c|}{ Average Annual Percent Chang } \\
\hline & & & & & & & & 1991-1995 & 1986-1995 \\
\hline 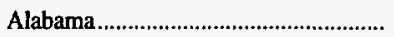 & 24,640 & 23,266 & 24,768 & 25,796 & 27,269 & 25,826 & 5.9 & -2.5 & -0.5 \\
\hline 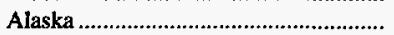 & 1,698 & 1,567 & 1,601 & 1,534 & 1,436 & 1,570 & 8.4 & 4.3 & .9 \\
\hline Arizona & 11,947 & 13,056 & 12,173 & 12,512 & 13,203 & 11,556 & -8.5 & -2.5 & .4 \\
\hline Arkansas & 29 & 51 & 44 & 58 & 52 & 167 & -42.8 & -13.5 & -17.6 \\
\hline 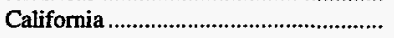 & - & - & - & 103 & 57 & - & - & - & - \\
\hline Colorado & 25,710 & 25,304 & 21,886 & 19,226 & 17,834 & 15,237 & 1.6 & 9.6 & 6.0 \\
\hline 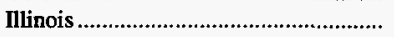 & 48,180 & 52,797 & 41,098 & 59,857 & 60,258 & 61,866 & -8.7 & -5.4 & -2.7 \\
\hline Indiana & 26,007 & 30,927 & 29,295 & 30,466 & 31,468 & 32,852 & -15.9 & -4.6 & -2.6 \\
\hline 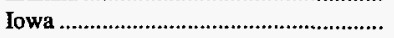 & - & 46 & 175 & 289 & 344 & 484 & -100.0 & - & - \\
\hline 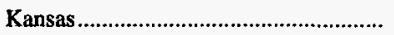 & 285 & 284 & 341 & 363 & 416 & 1,486 & .1 & -9.0 & -16.8 \\
\hline Kentucky Total & 153,739 & 161,642 & 156,299 & 161,068 & 158,980 & 153,933 & -4.9 & -.8 & * \\
\hline Eastern & 118,541 & 124,447 & 120,191 & 119,382 & 117,220 & 112,732 & -4.7 & .3 & .5 \\
\hline Western & 35,198 & 37,195 & 36,108 & 41,686 & 41,760 & 41,201 & -5.4 & -4.2 & -1.7 \\
\hline 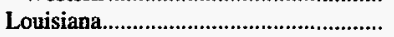 & 3,719 & 3,463 & 3,134 & 3,240 & 3,151 & 2,254 & 7.4 & 4.2 & 5.7 \\
\hline 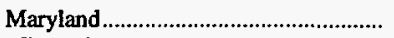 & 3,667 & 3,632 & 3,355 & 3,341 & 3,773 & 3,906 & 1.0 & -.7 & -.7 \\
\hline 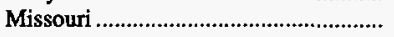 & 548 & 838 & 653 & 2,886 & 2,304 & 4,687 & -34.7 & -30.2 & -21.2 \\
\hline 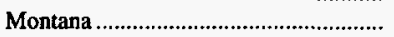 & 39,451 & 41,640 & 35,917 & 38,889 & 38,237 & 33,978 & -5.3 & .8 & 1.7 \\
\hline 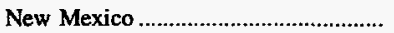 & 26,813 & 28,041 & 28,268 & 24,549 & 21,518 & 21,496 & -4.4 & 5.6 & 2.5 \\
\hline 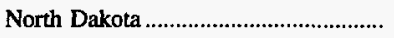 & 30,112 & 32,286 & 31,973 & 31,744 & 29,530 & 25,640 & -6.7 & .5 & 1.8 \\
\hline Ohio & 26,118 & 29,897 & 28,816 & 30,403 & 30,569 & 36,441 & -12.6 & -3.8 & -3.6 \\
\hline Oklahoma & 1,876 & 1,911 & 1,758 & 1,741 & 1,841 & 3,048 & -1.8 & .5 & -5.2 \\
\hline Pennsylvania Total .................................. & 61,576 & 62,237 & 59,700 & 68,981 & 65,381 & 71,648 & -1.1 & -1.5 & -1.7 \\
\hline Anthracite & 4,682 & 4,621 & 4,306 & 3,483 & 3,445 & 4,292 & 1.3 & 8.0 & 1.0 \\
\hline 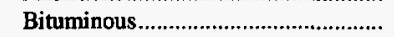 & 56,893 & 57,616 & 55,394 & 65,498 & 61,936 & 67,356 & -1.3 & -2.1 & -1.8 \\
\hline 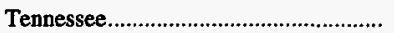 & 3,221 & 2,987 & 3,047 & 3,476 & 4,290 & 6,870 & 7.8 & -6.9 & -8.1 \\
\hline 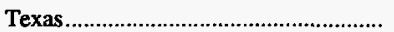 & 52,684 & 52,346 & 54,567 & 55,071 & 53,825 & 48,590 & .6 & -.5 & .9 \\
\hline 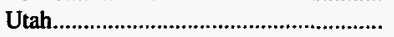 & 25,167 & 24,399 & 21,847 & 21,339 & 21,945 & 14,269 & 3.1 & 3.5 & 6.5 \\
\hline Virginia & 34,099 & 37,129 & 39,317 & 43,024 & 41,954 & 41,178 & -8.2 & -5.0 & -2.1 \\
\hline 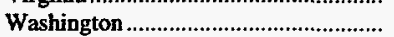 & 4,868 & 4,893 & 4,739 & 5,251 & 5,143 & 4,601 & -.5 & -1.4 & .6 \\
\hline 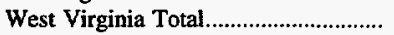 & 162,997 & 161,776 & 130,525 & 162,164 & 167,352 & 129,907 & .8 & -6 & 2.5 \\
\hline Northern & 46,114 & 49,316 & 33,802 & 50,022 & 52,155 & 48,355 & -6.5 & -3.0 & -.5 \\
\hline Southern & 116,883 & 112,460 & 96,723 & 112,142 & 115,196 & 81,552 & 3.9 & .4 & 4.1 \\
\hline Wyoming & 263,822 & 237,092 & 210,129 & 190,172 & 193,854 & 136,826 & 11.3 & 8.0 & 7.6 \\
\hline Appalachian Total ${ }^{1}$ & 434,861 & 445,370 & 409,718 & 456,565 & 457,808 & 428,508 & -2.3 & -1.3 & .2 \\
\hline Interior Total ${ }^{1} \ldots \ldots \ldots \ldots \ldots \ldots \ldots \ldots \ldots \ldots$ & 168,526 & 179,858 & 167,174 & 195,659 & 195,418 & 196,634 & -6.3 & -3.6 & -1.7 \\
\hline Western Total ${ }^{1}$ & 429,587 & 408,276 & 368,532 & 345,321 & 342,758 & 265,172 & 5.2 & 5.8 & 5.5 \\
\hline East of Miss. River................................. & 544,246 & 566,289 & 516,219 & 588,575 & 591,294 & 564,427 & -3.9 & -2.0 & -.4 \\
\hline West of Miss. River................................ & 488,728 & 467,216 & 429,205 & 408,970 & 404,690 & 325,887 & 4.6 & 4.8 & 4.6 \\
\hline U.S. Total & $1,032,974$ & $1,033,504$ & 945,424 & $\mathbf{9 9 7 , 5 4 5}$ & 995,984 & 890,315 & * & .9 & 1.7 \\
\hline
\end{tabular}

1 For a definition of coal-producing regions, see Appendix C.

* Data round to zero.

Notes: Coal production excludes silt, culm, refuse bank, slurry dam, and dredge operations except for Pennsylvania anthracite. Totals may not equal sum of components due to independent rounding.

Sources: Energy Information Administration, Form EIA-7A, “Coal Production Report”; State Mining Agency Coal Production Reports; and/or U.S. Department of Labor, Mine Safety and Health Administration, Form 7000-2, "Quarterly Mine Employment and Coal Production Report." 
Table 2. Number of Coal Mines by State, 1986, 1991-1995

\begin{tabular}{|c|c|c|c|c|c|c|c|c|c|}
\hline \multirow{2}{*}{$\begin{array}{l}\text { Coal-Producing } \\
\text { State and Region }\end{array}$} & \multirow{2}{*}{1995} & \multirow{2}{*}{1994} & \multirow{2}{*}{1993} & \multirow{2}{*}{1992} & \multirow{2}{*}{1991} & \multirow{2}{*}{1986} & \multirow{2}{*}{$\begin{array}{c}\text { Percent } \\
\text { Change } \\
\text { 1994-1995 }\end{array}$} & \multicolumn{2}{|c|}{ Average Annual Percent Change } \\
\hline & & & & & & & & 1991-1995 & 1986-1995 \\
\hline Alabama & 73 & 85 & 85 & 88 & 96 & 113 & -14.1 & -6.6 & -4.7 \\
\hline 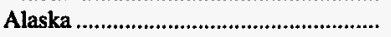 & 1 & 1 & 1 & 2 & 1 & 1 & - & - & - \\
\hline Arizona & 2 & 2 & 2 & 2 & 2 & 2 & - & - & - \\
\hline Arkansas & 3 & 6 & 6 & 6 & 7 & 8 & -50.0 & -19.1 & -10.3 \\
\hline 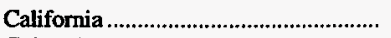 & - & - & - & 1 & 1 & - & - & - & - \\
\hline Colorado & 17 & 18 & 20 & 21 & 21 & 28 & -5.5 & -5.1 & -5.4 \\
\hline 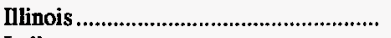 & 31 & 34 & 39 & 43 & 51 & 53 & -8.8 & -11.7 & -5.8 \\
\hline Indiana & 42 & 55 & 56 & 51 & 58 & 71 & -23.6 & -7.8 & -5.7 \\
\hline Iowa & - & 1 & 2 & 2 & 3 & 6 & -100.0 & - & - \\
\hline 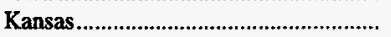 & 1 & 1 & 2 & 2 & 3 & 6 & - & -24.0 & -18.0 \\
\hline Kentucky Total & 598 & 673 & 696 & 752 & 838 & 1,553 & -11.1 & -8.1 & -10.1 \\
\hline 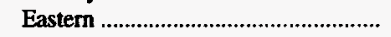 & 540 & 607 & 622 & 684 & 756 & 1,435 & -11.0 & -8.1 & -10.3 \\
\hline Western & 58 & 66 & 74 & 68 & 82 & 118 & -12.1 & -8.3 & -7.6 \\
\hline 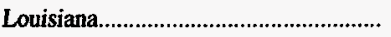 & 2 & 2 & 2 & 2 & 2 & 1 & - & - & 8.0 \\
\hline 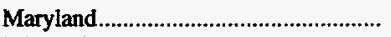 & 20 & 20 & 21 & 24 & 26 & 38 & - & -6.3 & -6.9 \\
\hline 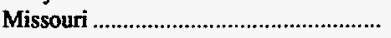 & 6 & 6 & 7 & 5 & 5 & 14 & - & 4.7 & -9.0 \\
\hline 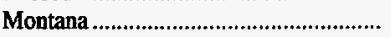 & 8 & 8 & 8 & 8 & 9 & 9 & - & -2.9 & -1.3 \\
\hline 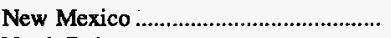 & 7 & 7 & 7 & 7 & 7 & 11 & - & - & -4.9 \\
\hline North Dakota & 6 & 6 & 8 & 8 & 8 & 14 & - & -6.9 & -9.0 \\
\hline Ohio & 113 & 134 & 135 & 149 & 159 & 221 & -15.7 & -8.2 & -7.2 \\
\hline 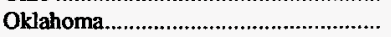 & 13 & 14 & 17 & 20 & 22 & 21 & -7.1 & -12.3 & -5.2 \\
\hline 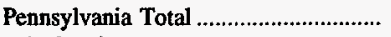 & 459 & 505 & 524 & 578 & 608 & 775 & -9.1 & -6.8 & -5.6 \\
\hline 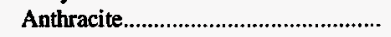 & 134 & 143 & 148 & 166 & 176 & 221 & -6.3 & -6.6 & -5.4 \\
\hline 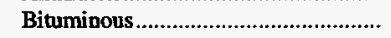 & 325 & 362 & 376 & 412 & 432 & 554 & -10.2 & -6.9 & -5.8 \\
\hline 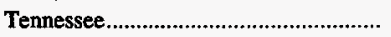 & 25 & 24 & 37 & 50 & 72 & 110 & 4.2 & -23.2 & -15.2 \\
\hline 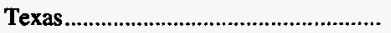 & 14 & 13 & 14 & 15 & 15 & 15 & 7.7 & -1.7 & -.8 \\
\hline 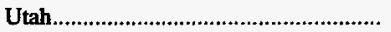 & 13 & 14 & 15 & 15 & 15 & 23 & -7.1 & -3.5 & -6.1 \\
\hline 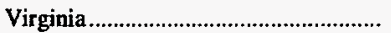 & 194 & 231 & 237 & 258 & 294 & 500 & -16.0 & -9.9 & -10.0 \\
\hline 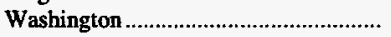 & 3 & 3 & 3 & 3 & 3 & 4 & - & - & -3.1 \\
\hline 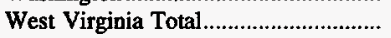 & 424 & 462 & 502 & 604 & 665 & 798 & -8.2 & -10.6 & -6.8 \\
\hline 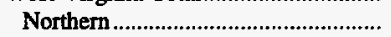 & 98 & 116 & 137 & 166 & 174 & 269 & -15.5 & -13.4 & -10.6 \\
\hline Southern & 326 & 346 & 365 & 438 & 491 & 529 & -5.8 & -9.7 & -5.2 \\
\hline Wyoming & 29 & 29 & 29 & 30 & 31 & 29 & - & -1.6 & - \\
\hline Appalachian Total 1 .............................. & 1,848 & 2,068 & 2,163 & 2,435 & 2,676 & 3,990 & -10.6 & -8.8 & -8.2 \\
\hline Interior Total 1 & 170 & 198 & 219 & 214 & 248 & 313 & -14.1 & -9.0 & -6.5 \\
\hline Western Total ${ }^{1}$ & 86 & 88 & 93 & 97 & 98 & 121 & $-\mathbf{2 . 3}$ & $-\mathbf{3 . 2}$ & -3.7 \\
\hline 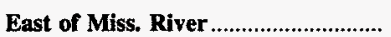 & 1,979 & 2,223 & 2,332 & 2,597 & 2,867 & 4,232 & -11.0 & -8.8 & -8.1 \\
\hline 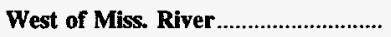 & 125 & 131 & 143 & 149 & 155 & 192 & -4.6 & -5.2 & -4.6 \\
\hline 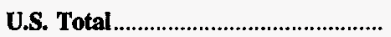 & 2,104 & 2,354 & 2,475 & 2,746 & 3,022 & 4,424 & -10.6 & -8.6 & -7.9 \\
\hline
\end{tabular}

1 For a definition of coal-producing regions, see Appendix $\mathrm{C}$

Note: Excludes silt, culm, refuse bank, slurry dam, and dredge operations except for Pennsylvania anthracite.

Sources: Energy Information Administration, Form EIA-7A, "Coal Production Report"; State Mining Agency Coal Production Reports; and/or U.S. Department of Labor, Mine Safety and Health Administration, Form 7000-2, "Quarterly Mine Employment and Coal Production Report." 
Table 3. Coal Production and Number of Mines by State and Mine Type, 1995 (Thousand Short Tons)

\begin{tabular}{|c|c|c|c|c|c|c|}
\hline \multirow{2}{*}{$\begin{array}{l}\text { Coal-Producing } \\
\text { State and Region }\end{array}$} & \multicolumn{2}{|c|}{ Underground } & \multicolumn{2}{|c|}{ Surface } & \multicolumn{2}{|c|}{ Total } \\
\hline & Number of Mines & Production & Number of Mines & Production & Number of Mines & Production \\
\hline 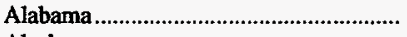 & 11 & 17,605 & 62 & 7,036 & 73 & 24,640 \\
\hline Alaska & - & - & 1 & 1,698 & 1 & 1,698 \\
\hline 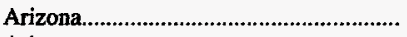 & - & - & 2 & 11,947 & 2 & 11,947 \\
\hline 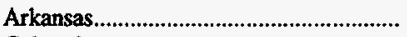 & - & - & 3 & 29 & 3 & 29 \\
\hline 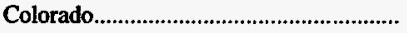 & 12 & 17,187 & 5 & 8,523 & 17 & 25,710 \\
\hline Illinois & 20 & 41,118 & 11 & 7,062 & 31 & 48,180 \\
\hline Indiana & 4 & 3,540 & 38 & 22,467 & 42 & 26,007 \\
\hline Kansas & - & - & 1 & 285 & 1 & 285 \\
\hline 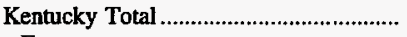 & 361 & 94,207 & 237 & 59,532 & 598 & 153,739 \\
\hline 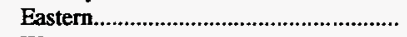 & 339 & 69,856 & 201 & 48,685 & 540 & 118,541 \\
\hline 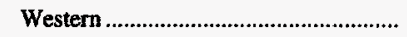 & 22 & 24,351 & 36 & 10,847 & 58 & 35,198 \\
\hline 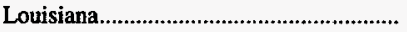 & - & - & 2 & 3,719 & 2 & 3,719 \\
\hline 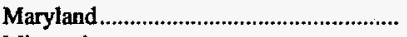 & 3 & 2,891 & 17 & 776 & 20 & 3,667 \\
\hline 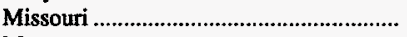 & - & - & 6 & 548 & 6 & 548 \\
\hline 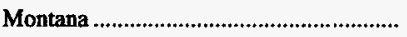 & 1 & 10 & 7 & 39,441 & 8 & 39,451 \\
\hline New Mexico & 1 & 640 & 6 & 26,173 & 7 & 26,813 \\
\hline North Dakota & - & - & 6 & 30,112 & 6 & 30,112 \\
\hline Ohio & 8 & 13,077 & 105 & 13,041 & 113 & 26,118 \\
\hline 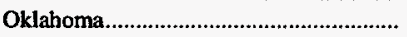 & 1 & 25 & 12 & 1,851 & 13 & 1,876 \\
\hline Pennsylvania Total & 96 & 41,409 & 363 & 20,167 & 459 & 61,576 \\
\hline 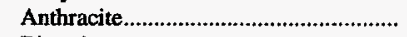 & 45 & 428 & 89 & 4,254 & 134 & 4,682 \\
\hline 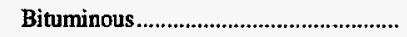 & 51 & 40,981 & 274 & 15,912 & 325 & 56,893 \\
\hline 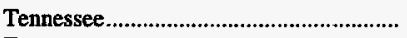 & 14 & 1,964 & 11 & 1,258 & 25 & 3,221 \\
\hline 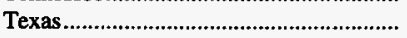 & - & - & 14 & 52,684 & 14 & 52,684 \\
\hline 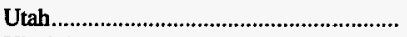 & 13 & 25,167 & - & - & 13 & 25,167 \\
\hline 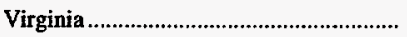 & 151 & 25,372 & 43 & 8,727 & 194 & 34,099 \\
\hline 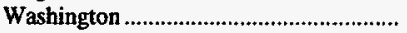 & - & - & 3 & 4,868 & 3 & 4,868 \\
\hline 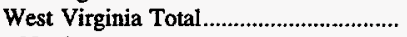 & 278 & 110,029 & 146 & 52,968 & 424 & 162,997 \\
\hline Northern & 53 & 40,726 & 45 & 5,388 & 98 & 46,114 \\
\hline 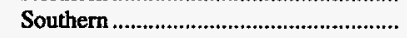 & 225 & 69,303 & 101 & 47,580 & 326 & 116,883 \\
\hline Wyoming & 3 & 2,008 & 26 & 261,814 & 29 & 263,822 \\
\hline Appalachian Total ${ }^{1} \ldots \ldots \ldots \ldots \ldots \ldots \ldots \ldots \ldots$ & 900 & 282,203 & 948 & 152,658 & 1,848 & 434,861 \\
\hline Interior Total 1 & 47 & 69,035 & 123 & 99,491 & 170 & 168,526 \\
\hline Western Total ${ }^{1} \ldots \ldots \ldots \ldots \ldots \ldots \ldots \ldots \ldots \ldots \ldots \ldots \ldots \ldots$ & $\mathbf{3 0}$ & 45,011 & 56 & 384,576 & 86 & 429,587 \\
\hline 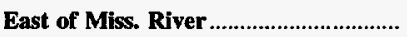 & 946 & 351,212 & 1,033 & 193,033 & 1,979 & 544,246 \\
\hline West of Miss. River................................... & 31 & 45,037 & 94 & 443,691 & 125 & 488,728 \\
\hline 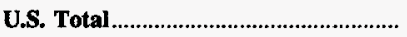 & 977 & 396,249 & 1,127 & 636,725 & 2,104 & $1,032,974$ \\
\hline
\end{tabular}

1 For a definition of coal-producing regions, see Appendix $\mathbf{C}$.

Notes: Coal production excludes silt, culm, refuse bank, slurry dam, and dredge operations except for Pennsylvania anthracite. Totals may not equal sum of components due to independent rounding.

Sources: Energy Information Administration, Form ElA-7A, “Coal Production Report”; State Mining Agency Coal Production Reports; and/or U.S. Department of Labor, Mine Safety and Health Administration, Form 7000-2, "Quarterly Mine Employment and Coal Production Report." 
Table 4. Coal Production and Number of Mines by State, County, and Mine Type, 1995 (Thousand Short Tons)

\begin{tabular}{|c|c|c|c|c|c|c|}
\hline \multirow{2}{*}{$\begin{array}{l}\text { Coal-Producing } \\
\text { State and County }\end{array}$} & \multicolumn{2}{|c|}{ Underground } & \multicolumn{2}{|c|}{ Surface } & \multicolumn{2}{|c|}{ Total } \\
\hline & Number of Mines & Production & Number of Mines & Production & Number of Mines & Production \\
\hline Alabama & 11 & 17,605 & 62 & $\mathbf{7 , 0 3 6}$ & 73 & 24,640 \\
\hline 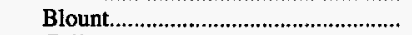 & - & - & 2 & 20 & 2 & 20 \\
\hline 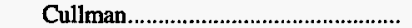 & - & - & 3 & 90 & 3 & 90 \\
\hline Fayette $\ldots \ldots \ldots \ldots \ldots \ldots$ & 1 & 1,984 & - & - & 1 & 1,984 \\
\hline Jackson & - & - & 1 & 46 & 1 & 46 \\
\hline 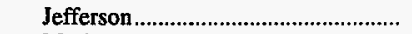 & 5 & 7,138 & 5 & 1,087 & 10 & 8,225 \\
\hline 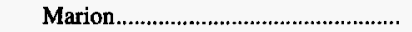 & - & - & 6 & 214 & 6 & 214 \\
\hline 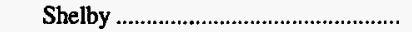 & 1 & 857 & - & - & 1 & 857 \\
\hline 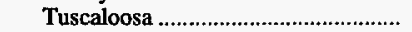 & 3 & 6,390 & 7 & 1,875 & 10 & 8,265 \\
\hline 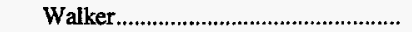 & 1 & 1,237 & 34 & 3,394 & 35 & 4,631 \\
\hline Winston & - & - & 4 & 310 & 4 & 310 \\
\hline 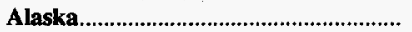 & - & - & 1 & 1,698 & 1 & 1,698 \\
\hline 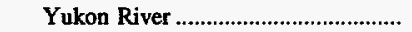 & - & - & 1 & 1,698 & 1 & 1,698 \\
\hline Arizona & - & - & 2 & 11,947 & 2 & 11,947 \\
\hline Navajo & - & - & 2 & 11,947 & 2 & 11,947 \\
\hline 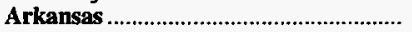 & - & - & 3 & 29 & 3 & 29 \\
\hline Johnson & - & - & 2 & 24 & 2 & 24 \\
\hline 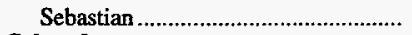 & - & - & 1 & 5 & 1 & 5 \\
\hline 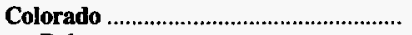 & 12 & 17,187 & $\mathbf{5}$ & $\mathbf{8 , 5 2 3}$ & 17 & 25,710 \\
\hline Delta & 1 & 405 & - & - & 1 & 405 \\
\hline 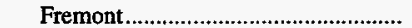 & 1 & 302 & - & - & 1 & 302 \\
\hline 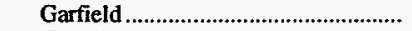 & 1 & * & - & - & 1 & $*$ \\
\hline 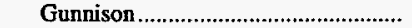 & 3 & 6,870 & - & - & 3 & 6,870 \\
\hline La Plata & 1 & 209 & - & - & 1 & 209 \\
\hline 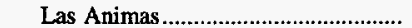 & 1 & 1,156 & - & - & 1 & 1,156 \\
\hline Mesa & 1 & 607 & - & - & 1 & 607 \\
\hline Moffat & 1 & 745 & 2 & 6,388 & 3 & 7,133 \\
\hline Montrose & - & - & 1 & 374 & 1 & 374 \\
\hline 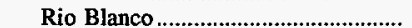 & 1 & 1,047 & - & - & 1 & 1,047 \\
\hline 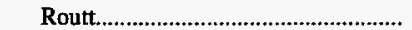 & 1 & 5,847 & 2 & 1,761 & 3 & 7,608 \\
\hline 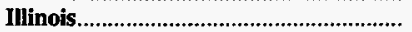 & 20 & 41,118 & $1 \overline{11}$ & $\mathbf{7 , 0 6 2}$ & 31 & 48,180 \\
\hline Clinton & 1 & 2,998 & - & - & 1 & 2,998 \\
\hline Franklin & 2 & 5,298 & - & - & 2 & 5,298 \\
\hline Fulton & - & - & 1 & 469 & 1 & 469 \\
\hline 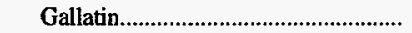 & 1 & 1,086 & - & - & 1 & 1,086 \\
\hline 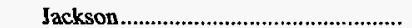 & - & - & 1 & 19 & 1 & 19 \\
\hline Jefferson & 2 & 4,705 & - & - & 2 & 4,705 \\
\hline Logan & 1 & 1,745 & - & - & 1 & 1,745 \\
\hline 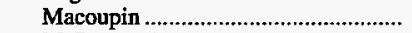 & 3 & 4,815 & - & - & 3 & 4,815 \\
\hline 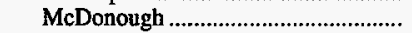 & - & - & 1 & 278 & 1 & 278 \\
\hline 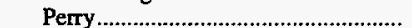 & 2 & 2,494 & 4 & 4,153 & 6 & 6,647 \\
\hline 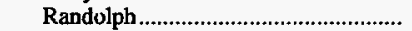 & 2 & 2,891 & - & - & 2 & 2,891 \\
\hline Saline & 3 & 7,463 & 1 & 346 & 4 & 7,809 \\
\hline 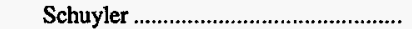 & - & - & 1 & 504 & 1 & 504 \\
\hline 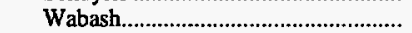 & 1 & 2,557 & - & - & 1 & 2,557 \\
\hline 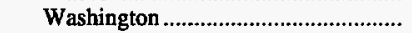 & 1 & 3,259 & - & - & 1 & 3,259 \\
\hline White & 1 & 1,808 & - & - & 1 & 1,808 \\
\hline 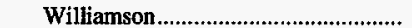 & - & - & 2 & 1,293 & 2 & 1,293 \\
\hline Indiana & 4 & 3,540 & 38 & 22,467 & 42 & 26,007 \\
\hline 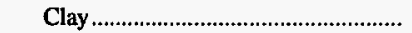 & - & - & 5 & 1,178 & 5 & 1,178 \\
\hline 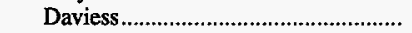 & - & - & 6 & 3,341 & 6 & 3,341 \\
\hline 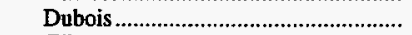 & - & - & 1 & 458 & 1 & 458 \\
\hline 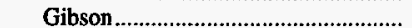 & 1 & 1,541 & 1 & 10 & 2 & 1,550 \\
\hline 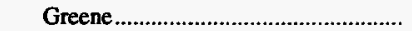 & - & - & 5 & 2,741 & 5 & 2,741 \\
\hline 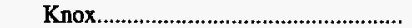 & 2 & 1,714 & 2 & 1,114 & 4 & 2,828 \\
\hline Owen & - & - & 1 & 354 & 1 & 354 \\
\hline 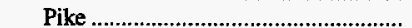 & - & - & 5 & 2,492 & 5 & 2,492 \\
\hline 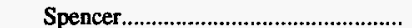 & - & - & 2 & 196 & 2 & 196 \\
\hline Sullivan & 1 & 285 & 2 & 3,538 & 3 & 3,823 \\
\hline 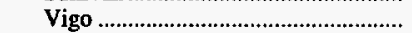 & - & - & 1 & 1,204 & 1 & 1,204 \\
\hline Warrick & - & - & 7 & 5,841 & 7 & 5,841 \\
\hline 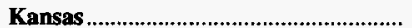 & - & - & 1 & 285 & $\mathbf{1}$ & 285 \\
\hline Crawford & - & - & 1 & 285 & 1 & 285 \\
\hline 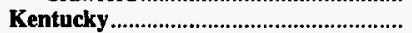 & 361 & 94,207 & 237 & 59,532 & 598 & 153,739 \\
\hline 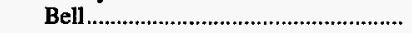 & 14 & 3,319 & 10 & 1,658 & 24 & 4,977 \\
\hline 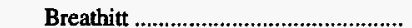 & - & - & 8 & 2,505 & 8 & 2,505 \\
\hline 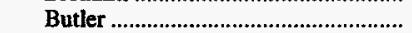 & - & - & 1 & 170 & 1 & 170 \\
\hline 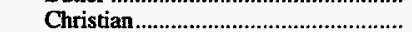 & - & - & $\mathrm{i}$ & 3 & 1 & 3 \\
\hline 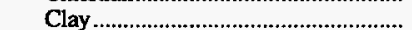 & 1 & 3 & 7 & 254 & 8 & 257 \\
\hline 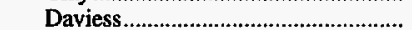 & - & - & 6 & 742 & 6 & 742 \\
\hline Floyd & 39 & 2,902 & 13 & 3,549 & 52 & 6,451 \\
\hline
\end{tabular}

See footnotes at end of table. 
Table 4. Coal Production and Number of Mines by State, County, and Mine Type, 1995 (Continued) (Thousand Short Tons)

\begin{tabular}{|c|c|c|c|c|c|c|}
\hline \multirow{2}{*}{$\begin{array}{l}\text { Coal-Producing } \\
\text { State and County }\end{array}$} & \multicolumn{2}{|c|}{ Underground } & \multicolumn{2}{|c|}{ Surface } & \multicolumn{2}{|c|}{ Total } \\
\hline & Number of Mines & Production & Number of Mines & Production & Number of Mines & Production \\
\hline \multicolumn{7}{|l|}{ Kentucky (Continued) } \\
\hline 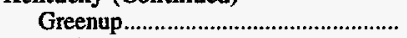 & - & - & 1 & 1 & 1 & 1 \\
\hline 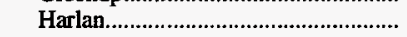 & 47 & 9,917 & 9 & 1,009 & 56 & 10,926 \\
\hline 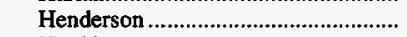 & 3 & 1,728 & 3 & 974 & 6 & 2,702 \\
\hline 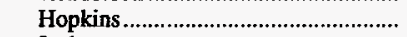 & 6 & 3,520 & 8 & 4,946 & 14 & 8,466 \\
\hline 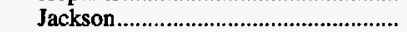 & - & - & 1 & 31 & 1 & 31 \\
\hline Johnson & 6 & 1,222 & 6 & 36 & 12 & 1,258 \\
\hline Knott & 32 & 5,914 & 16 & 6,081 & 48 & 11,994 \\
\hline 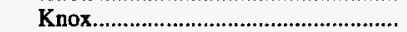 & 18 & 651 & 4 & 73 & 22 & 725 \\
\hline 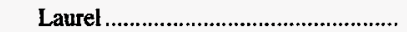 & - & - & 1 & 11 & 1 & 11 \\
\hline 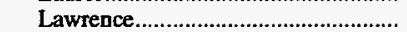 & - & - & 3 & 242 & 3 & 242 \\
\hline Leslie & 8 & 7,428 & 4 & 1,324 & 12 & 8,752 \\
\hline Letcher & 17 & 4,412 & 25 & 3,746 & 42 & 8,159 \\
\hline Magoffin & 1 & 46 & 4 & 959 & 5 & 1,005 \\
\hline 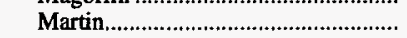 & 23 & 6,670 & 11 & 5,438 & 34 & 12,108 \\
\hline McLean & - & - & 2 & 448 & 2 & 448 \\
\hline 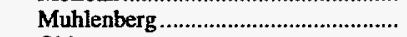 & 2 & 2,137 & 3 & 846 & 5 & 2,983 \\
\hline Ohio & $\overrightarrow{1}$ & 209 & 11 & 2,554 & 12 & 2,763 \\
\hline 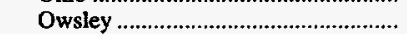 & - & - & 2 & 161 & 2 & 161 \\
\hline Perry & 14 & 4,045 & 15 & 8,060 & 29 & 12,105 \\
\hline Pike & 108 & 22,513 & 53 & 13,012 & 161 & 35,525 \\
\hline Union & 4 & 6,398 & - & - & 4 & 6,398 \\
\hline 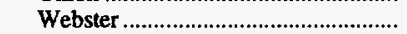 & 6 & 10,359 & 1 & 165 & 7 & 10,524 \\
\hline 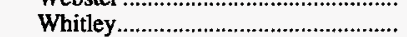 & 11 & 814 & 7 & 522 & 18 & 1,337 \\
\hline Wolfe & - & - & 1 & 12 & 1 & 12 \\
\hline 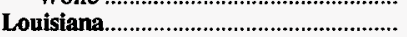 & - & - & 2 & 3,719 & 2 & 3,719 \\
\hline De Soto & - & - & 1 & 2,881 & 1 & 2,881 \\
\hline Red River & - & - & 1 & 838 & 1 & 838 \\
\hline 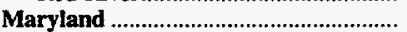 & 3 & 2,891 & 17 & 776 & 20 & 3,667 \\
\hline 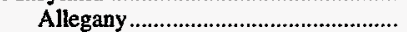 & + & - & 12 & 623 & 12 & 623 \\
\hline Garrett & 3 & 2,891 & 5 & 153 & 8 & 3,045 \\
\hline 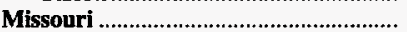 & - & - & 6 & 548 & 6 & 548 \\
\hline Barton. & - & - & 1 & 198 & 1 & 198 \\
\hline 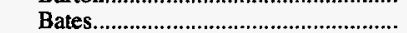 & - & - & 2 & 102 & 2 & 102 \\
\hline 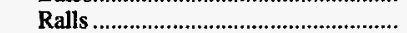 & - & - & 1 & 57 & 1 & 57 \\
\hline Randolph & - & - & 1 & 141 & 1 & 141 \\
\hline Vernon & - & - & 1 & 49 & 1 & 49 \\
\hline Montana & 1 & 10 & 7 & 39,441 & 8 & 39,451 \\
\hline Big Horn & - & - & 4 & 23,179 & 4 & 23,179 \\
\hline 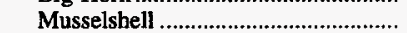 & 1 & 10 & - & - & 1 & 10 \\
\hline Richland & - & - & 1 & 297 & 1 & 297 \\
\hline Rosebud & - & - & 2 & 15,965 & 2 & 15,965 \\
\hline 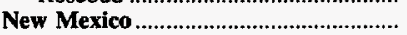 & 1 & 640 & 6 & 26,173 & $\overline{7}$ & $\mathbf{2 6 , 8 1 3}$ \\
\hline 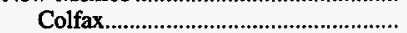 & $\overline{1}$ & 640 & 1 & 1,216 & 2 & 1,855 \\
\hline 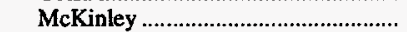 & - & - & 2 & 10,596 & 2 & 10,596 \\
\hline San Juan & - & - & 3 & 14,361 & 3 & 14,361 \\
\hline North Dakota & - & - & 6 & 30,112 & 6 & 30,112 \\
\hline Bowman & - & - & 1 & 1,267 & 1 & 1,267 \\
\hline 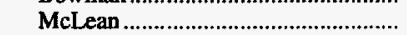 & - & - & 1 & 7,071 & 1 & 7,071 \\
\hline 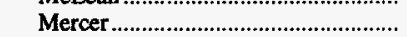 & - & - & 2 & 16,502 & 2 & 16,502 \\
\hline Oliver & - & - & 2 & 5,271 & 2 & 5,271 \\
\hline Ohio & 8 & 13,077 & 105 & 13,041 & $11 \overrightarrow{3}$ & 26,118 \\
\hline 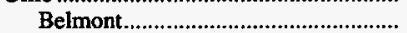 & 1 & 3,946 & 9 & 1,218 & 10 & 5,164 \\
\hline Carroll & - & - & 6 & 203 & 6 & 203 \\
\hline 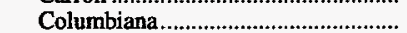 & 1 & 341 & 9 & 615 & 10 & 956 \\
\hline Coshocton & - & - & 6 & 884 & 6 & 884 \\
\hline 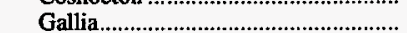 & - & - & 1 & 189 & 1 & 189 \\
\hline 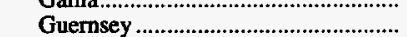 & - & - & 6 & 272 & 6 & 272 \\
\hline 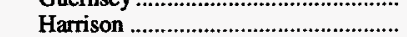 & 1 & 955 & 11 & 1,217 & 12 & 2,172 \\
\hline 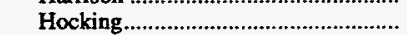 & - & - & 1 & 1 & 1 & 1 \\
\hline Holmes & - & - & 3 & 206 & 3 & 206 \\
\hline 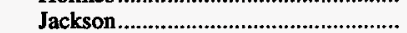 & - & - & 4 & 1,084 & 4 & 1,084 \\
\hline 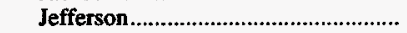 & 2 & 392 & 10 & 373 & 12 & 766 \\
\hline 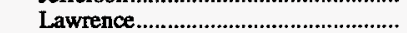 & - & - & 1 & 3 & 1 & 3 \\
\hline 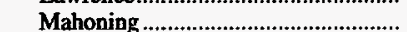 & - & - & 2 & 12 & 2 & 12 \\
\hline Meigs & 2 & 4,721 & - & - & 2 & 4,721 \\
\hline Monroe & 1 & 2,722 & - & - & 1 & 2,722 \\
\hline 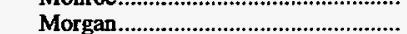 & - & - & 1 & 1,058 & 1 & 1,058 \\
\hline Muskingum & - & - & 2 & 180 & 2 & 180 \\
\hline Noble & - & - & $\overline{1}$ & 823 & 1 & 823 \\
\hline 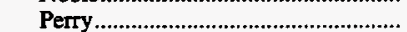 & - & - & 6 & 763 & 6 & 763 \\
\hline
\end{tabular}

See footnotes at end of table. 
Table 4. Coal Production and Number of Mines by State, County, and Mine Type, 1995 (Continued) (Thousand Short Tons)

\begin{tabular}{|c|c|c|c|c|c|c|}
\hline \multirow{2}{*}{$\begin{array}{l}\text { Coal-Producing } \\
\text { State and County }\end{array}$} & \multicolumn{2}{|c|}{ Underground } & \multicolumn{2}{|c|}{ Surface } & \multicolumn{2}{|c|}{ Total } \\
\hline & Number of Mines & Production & Number of Mines & Production & Number of Mines & Production \\
\hline \multicolumn{7}{|l|}{ Ohio (Continued) } \\
\hline Stark & - & - & 6 & 312 & 6 & 312 \\
\hline 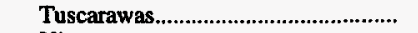 & - & - & 15 & 1,518 & 15 & 1,518 \\
\hline 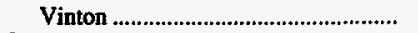 & - & - & 5 & 2,110 & 5 & 2,110 \\
\hline 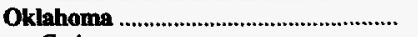 & 1 & 25 & 12 & 1,851 & 13 & 1,876 \\
\hline Craig & - & - & 1 & 139 & 1 & 139 \\
\hline Haskell & - & - & 1 & 16 & 1 & 16 \\
\hline 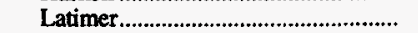 & - & - & 1 & 222 & 1 & 222 \\
\hline Le Flore & - & - & 6 & 1,309 & 6 & 1,309 \\
\hline 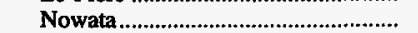 & - & - & 2 & 159 & 2 & 159 \\
\hline 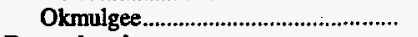 & 1 & 25 & 1 & 6 & 2 & 31 \\
\hline 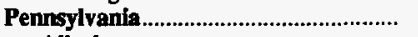 & 96 & 41,409 & 363 & 20,167 & 459 & 61,576 \\
\hline Allegheny & 1 & * & 4 & 27 & 5 & 27 \\
\hline Armstrong & 14 & 4,296 & 27 & 1,078 & 41 & 5,374 \\
\hline Beaver & - & - & 2 & 125 & 2 & 125 \\
\hline Bedford & - & - & 1 & 2 & 1 & 2 \\
\hline 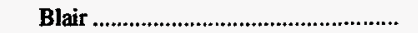 & - & - & 1 & 69 & 1 & 69 \\
\hline 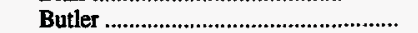 & - & - & 7 & 135 & 7 & 135 \\
\hline 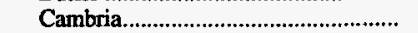 & 3 & 218 & 11 & 1,266 & 14 & 1,484 \\
\hline Carbon & - & - & 1 & 432 & 1 & 432 \\
\hline 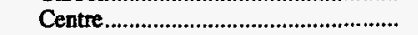 & - & - & 3 & 142 & 3 & 142 \\
\hline 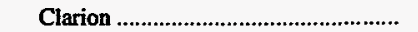 & - & - & 6 & 624 & 6 & 624 \\
\hline 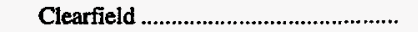 & 2 & 97 & 54 & 4,403 & 56 & 4,499 \\
\hline Clinton & - & - & 1 & 1 & 1 & 1 \\
\hline 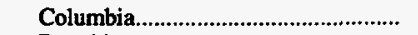 & 1 & 158 & 3 & 45 & 4 & 203 \\
\hline 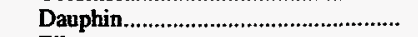 & 1 & 2 & - & - & 1 & 2 \\
\hline Elk & - & - & 5 & 369 & 5 & 369 \\
\hline Fayette & - & - & 27 & 375 & 27 & 375 \\
\hline Greene & 10 & 28,183 & 4 & 207 & 14 & 28,390 \\
\hline Indiana & 9 & 3,491 & 23 & 752 & 32 & 4,243 \\
\hline 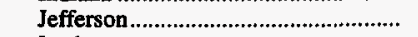 & 2 & 514 & 31 & 947 & 33 & 1,461 \\
\hline 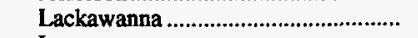 & - & - & 7 & 414 & 7 & 414 \\
\hline 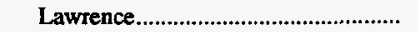 & - & - & 5 & 165 & 5 & 165 \\
\hline 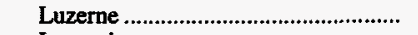 & - & - & 16 & 879 & 16 & 879 \\
\hline 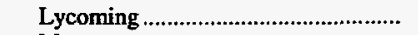 & - & - & 1 & 290 & 1 & 290 \\
\hline Mercer & - & - & 2 & 8 & 2 & 8 \\
\hline 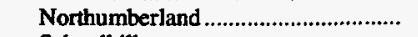 & 12 & 46 & 9 & 288 & 21 & 334 \\
\hline 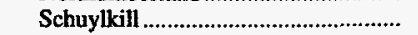 & 31 & 223 & 51 & 2,169 & 82 & 2,392 \\
\hline Snyder & - & - & I & 1 & 1 & 1 \\
\hline 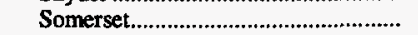 & 8 & 2,034 & 34 & 3,577 & 42 & 5,611 \\
\hline 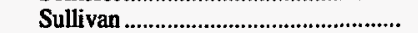 & - & - & 1 & 27 & 1 & 27 \\
\hline 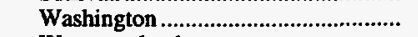 & 2 & 2,148 & 8 & 600 & 10 & 2,749 \\
\hline 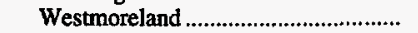 & - & - & 17 & 751 & 17 & 751 \\
\hline 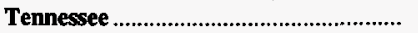 & 14 & 1,964 & 11 & 1,258 & 25 & 3,221 \\
\hline 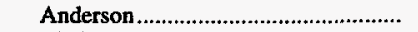 & 1 & 157 & 2 & 59 & 3 & 216 \\
\hline 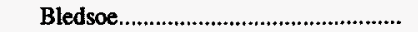 & - & - & 1 & 8 & 1 & 8 \\
\hline 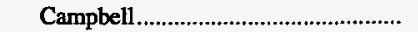 & 6 & 888 & 4 & 365 & 10 & 1,252 \\
\hline 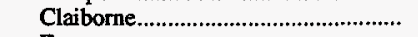 & 1 & 218 & 1 & 147 & 2 & 364 \\
\hline 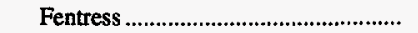 & - & - & 1 & 95 & 1 & 95 \\
\hline Marion & 1 & 43 & 1 & 68 & 2 & 111 \\
\hline 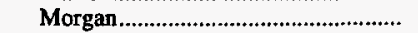 & 1 & 25 & - & - & $\overline{1}$ & 25 \\
\hline 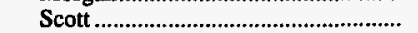 & 1 & 215 & - & - & 1 & 215 \\
\hline 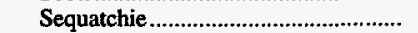 & 3 & 419 & 1 & 517 & 4 & 936 \\
\hline 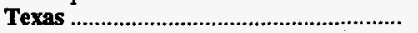 & - & - & 14 & 52,684 & 14 & 52,684 \\
\hline Atascosa & - & - & 1 & 2,924 & 1 & 2,924 \\
\hline 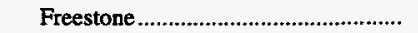 & - & - & 1 & 4,759 & 1 & 4,759 \\
\hline 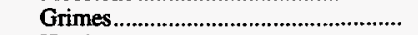 & - & - & 1 & 3,312 & 1 & 3,312 \\
\hline 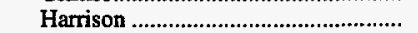 & - & - & 2 & 4,015 & 2 & 4,015 \\
\hline 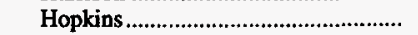 & - & - & 1 & 1,325 & 1 & 1,325 \\
\hline Leon & - & - & 1 & 8,304 & 1 & 8,304 \\
\hline 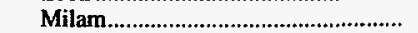 & - & - & 1 & 5,828 & 1 & 5,828 \\
\hline 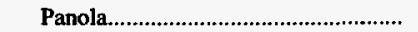 & - & - & 1 & 7,004 & 1 & 7,004 \\
\hline 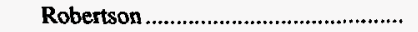 & - & - & 1 & 1,980 & 1 & 1,980 \\
\hline 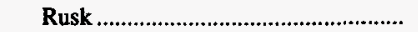 & - & - & 1 & 5,537 & 1 & 5,537 \\
\hline 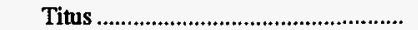 & - & - & 1 & 7,385 & 1 & 7,385 \\
\hline 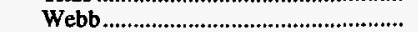 & - & - & 2 & 312 & 2 & 312 \\
\hline Utah & 13 & 25,167 & - & - & 13 & 25,167 \\
\hline 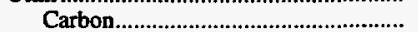 & 6 & 11,227 & - & - & 6 & 11,227 \\
\hline 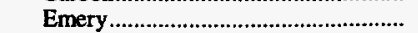 & 6 & 10,066 & - & - & 6 & 10,066 \\
\hline 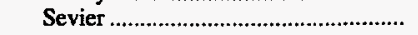 & 1 & 3,874 & - & - & 1 & 3,874 \\
\hline
\end{tabular}

See footnotes at end of table. 
Table 4. Coal Production and Number of Mines by State, County, and Mine Type, 1995 (Continued) (Thousand Short Tons)

\begin{tabular}{|c|c|c|c|c|c|c|}
\hline \multirow{2}{*}{$\begin{array}{l}\text { Coal-Producing } \\
\text { State and County }\end{array}$} & \multicolumn{2}{|c|}{ Underground } & \multicolumn{2}{|c|}{ Surface } & \multicolumn{2}{|c|}{ Total } \\
\hline & Number of Mines & Production & Number of Mines & Production & Number of Mines & Production \\
\hline Virginia & 151 & 25,372 & 43 & 8,727 & 194 & 34,099 \\
\hline 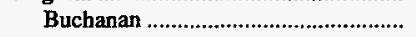 & 69 & 12,572 & 4 & 1,219 & 73 & 13,792 \\
\hline Dickenson & 14 & 1,562 & 10 & 1,142 & 24 & 2,704 \\
\hline Lee & 10 & 1,469 & 3 & 515 & 13 & 1,984 \\
\hline Russell & 8 & 928 & 4 & 801 & 12 & 1,729 \\
\hline Scott & 1 & 21 & - & - & 1 & 21 \\
\hline 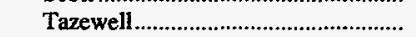 & 13 & 1,936 & 3 & 221 & 16 & 2,156 \\
\hline Wise & 36 & 6,884 & 19 & 4,830 & 55 & 11,713 \\
\hline 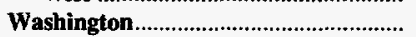 & - & - & 3 & 4,868 & 3 & 4,868 \\
\hline King & - & - & 1 & 241 & 1 & 241 \\
\hline 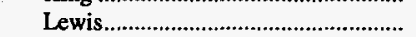 & - & - & 1 & 2,617 & i & 2,617 \\
\hline Thurston & - & - & $i$ & 2,009 & 1 & 2,009 \\
\hline West Virginia & 278 & 110,029 & 146 & 52,968 & 424 & 162,997 \\
\hline 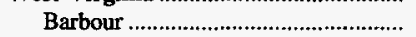 & 4 & 1,508 & 4 & 62 & 8 & 1,570 \\
\hline Boone & 33 & 19,745 & 10 & 7,056 & 43 & 26,801 \\
\hline Braxton & 1 & 201 & - & 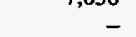 & 1 & 201 \\
\hline 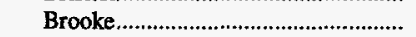 & 1 & 1,051 & - & - & 1 & 1,051 \\
\hline 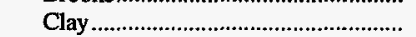 & 1 & 1 & 7 & 4,392 & 8 & 4,392 \\
\hline 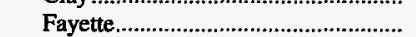 & 6 & 1,894 & 6 & 2,371 & 12 & 4,265 \\
\hline 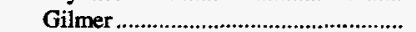 & 1 & 32 & - & - & 1 & 32 \\
\hline Grant & 2 & 2,849 & 3 & 661 & 5 & 3,510 \\
\hline Greenbrier & 3 & 276 & 5 & 61 & 8 & 338 \\
\hline 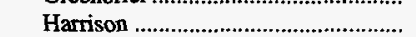 & 6 & 3,956 & 8 & 122 & 14 & 4,078 \\
\hline Kanawha & 9 & 3,090 & 6 & 7,519 & 15 & 10,609 \\
\hline Lincoln & 1 & 3 & - & - & 1 & 3 \\
\hline Logan & 15 & 3,207 & 20 & 15,437 & 35 & 18,644 \\
\hline 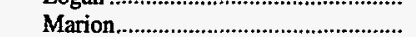 & 5 & 3,594 & 2 & 29 & 7 & 3,623 \\
\hline Marshall & 2 & 7,892 & - & - & 2 & 7,892 \\
\hline 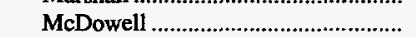 & 62 & 4,089 & 17 & 1,399 & 79 & 5,489 \\
\hline Mineral & - & - & 2 & 121 & 2 & 121 \\
\hline 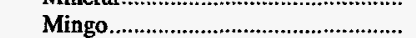 & 38 & 18,961 & 16 & 4,927 & 54 & 23,888 \\
\hline 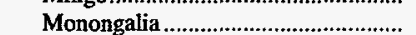 & 9 & 14,026 & 5 & 855 & 14 & 14,880 \\
\hline 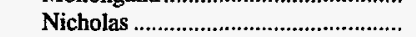 & 12 & 1,797 & 8 & 2,133 & 20 & 3,930 \\
\hline Preston & 6 & 1,672 & 10 & 234 & 16 & 1,906 \\
\hline Raleigh & 20 & 6,948 & 2 & 745 & 22 & 7,693 \\
\hline 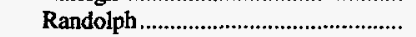 & 4 & 676 & - & - & 4 & 676 \\
\hline 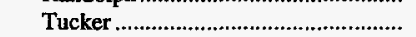 & - & - & 1 & 128 & 1 & 128 \\
\hline 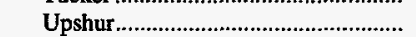 & 5 & 1,141 & 7 & 466 & 12 & 1,607 \\
\hline 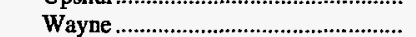 & 5 & 1,426 & 2 & 1,291 & 7 & 2,717 \\
\hline Webster & 7 & 2,128 & 3 & 2,712 & 10 & 4,840 \\
\hline Wyoming & 20 & 7,866 & 2 & 249 & 22 & 8,114 \\
\hline Wyoming & 3 & 2,008 & 26 & 261,814 & 29 & 263,822 \\
\hline 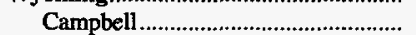 & - & - & 17 & 232,255 & 17 & 232,255 \\
\hline 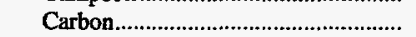 & 2 & 1,990 & 2 & 1,733 & 4 & 3,723 \\
\hline 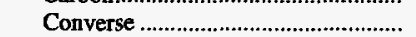 & - & - & 2 & 14,120 & 2 & 14,120 \\
\hline Lincoln & - & - & 2 & 4,444 & 2 & 4,444 \\
\hline 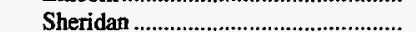 & - & - & 1 & 38 & 1 & 38 \\
\hline 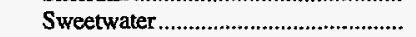 & 1 & 18 & 2 & 9,224 & 3 & 9,242 \\
\hline 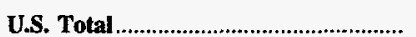 & 977 & 396,249 & 1,127 & 636,725 & 2,104 & $1,032,974$ \\
\hline
\end{tabular}

* Data round to zero.

Notes: Coal production excludes silt, culm, refuse bank, slurry dam, and dredge operations except for Pennsylvania anthracite. Totals may not equal sum of components due to independent rounding.

Sources: Energy Information Administration, Form EIA-7A, "Coal Production Report"; State Mining Agency Coal Production Reports; and/or U.S. Department of Labor, Mine Safety and Health Administration, Form 7000-2, "Quarterly Mine Employment and Coal Production Report." 
Table 5. Underground Coal Production by State and Mining Method, 1995 (Thousand Short Tons)

\begin{tabular}{|c|c|c|c|c|c|}
\hline $\begin{array}{l}\text { Coal-Producing } \\
\text { State and Region }\end{array}$ & Continuous 1 & Conventional 2 & Longwall ${ }^{3}$ & Other 4 & Total \\
\hline 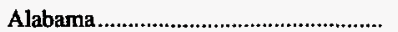 & 1,492 & 1 & 16,112 & - & 17,605 \\
\hline Colorado & 2,650 & 405 & 14,133 & - & 17,187 \\
\hline 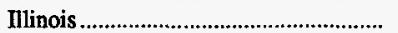 & 23,504 & - & 17,614 & - & 41,118 \\
\hline Indiana & 3,540 & - & - & - & 3,540 \\
\hline 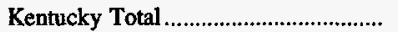 & 63,919 & 13,573 & 16,155 & 559 & 94,207 \\
\hline 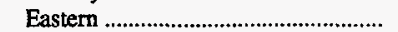 & 47,624 & 13,496 & 8,177 & 559 & 69,856 \\
\hline Western & 16,295 & 77 & 7,978 & - & 24,351 \\
\hline 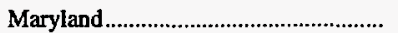 & 296 & - & 2,595 & - & 2,891 \\
\hline 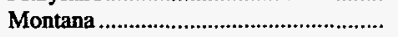 & 10 & - & - & - & 10 \\
\hline New Mexico & - & - & 640 & - & 640 \\
\hline Ohio & 1,688 & - & 11,389 & - & 13,077 \\
\hline Oklahoma. & 25 & - & - & - & 25 \\
\hline 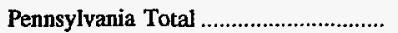 & 11,368 & 592 & 29,364 & 84 & 41,409 \\
\hline Anthracite & 158 & 186 & - & 84 & 428 \\
\hline 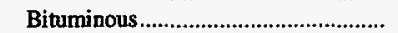 & 11,211 & 406 & 29,364 & - & 40,981 \\
\hline 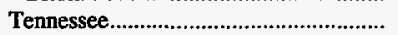 & 1,959 & 5 & - & - & 1,964 \\
\hline 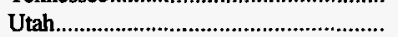 & 1,557 & 324 & 23,285 & - & 25,167 \\
\hline Virginia & 12,197 & 5,318 & 7,797 & 61 & 25,372 \\
\hline 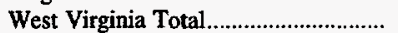 & 47,701 & 13,982 & 48,347 & - & 110,029 \\
\hline 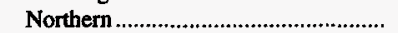 & 5,110 & 3,096 & 32,521 & - & 40,726 \\
\hline Southern & 42,591 & 10,886 & 15,826 & - & 69,303 \\
\hline 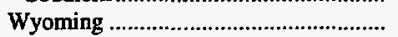 & - & 18 & 1,990 & - & 2,008 \\
\hline Appalachian Total $5 \ldots \ldots \ldots \ldots \ldots \ldots \ldots \ldots \ldots$ & 124,325 & 33,393 & 123,780 & 704 & 282,203 \\
\hline Interior Total 5 & 43,365 & 77 & 25,593 & - & 69,035 \\
\hline Western Total 5 & 4,217 & 747 & 40,048 & - & 45,011 \\
\hline East of Miss. River ................................. & 167,665 & 33,470 & 149,373 & 704 & $\mathbf{3 5 1 , 2 1 2}$ \\
\hline West of Miss. River & 4,242 & 747 & 40,048 & - & $\mathbf{4 5 , 0 3 7}$ \\
\hline U.S. Total & 171,907 & 34,217 & 189,421 & 704 & 396,249 \\
\hline
\end{tabular}

1 Mines that produce greater than 50 percent of coal by continuous mining method.

2 Mines that produce greater than 50 percent of coal by conventional mining method.

3 Mines that have any production from longwall mining method. A typical longwall mining operation uses 80 percent longwall mining and 20 percent continuous mining.

4 Mines that produce coal using shortwall, scoop loading, hand loading, or other mining methods or a 50/50 percent continuous/conventional split in mining method.

5 For a definition of coal-producing regions, see Appendix C.

Note: Totals may not equal sum of components due to independent rounding.

Source: Energy Information Administration, Form EIA-7A, "Coal Production Report." 
Table 6. Coal Production and Number of Mines by State, Mine Type, and Mine Production Range, 1995

(Thousand Short Tons)

\begin{tabular}{|c|c|c|c|c|c|c|c|c|c|c|c|c|}
\hline \multirow{3}{*}{$\begin{array}{l}\text { Coal-Producing State, Region } \\
\text { and Type of Mining }\end{array}$} & \multicolumn{6}{|c|}{ Number of Mines } & \multicolumn{6}{|c|}{ Production } \\
\hline & \multicolumn{12}{|c|}{$\begin{array}{l}\text { Mine Production Range } \\
\text { (thousand short tons) }\end{array}$} \\
\hline & $\begin{array}{l}1,000 \\
\text { and } \\
\text { over }\end{array}$ & $\begin{array}{c}500 \\
\text { to } \\
1,000\end{array}$ & $\begin{array}{c}200 \\
\text { to } \\
500\end{array}$ & $\begin{array}{c}100 \\
\text { to } \\
200\end{array}$ & $\begin{array}{c}10 \\
\text { to } \\
100\end{array}$ & $\begin{array}{c}\text { Less } \\
\text { than } \\
10\end{array}$ & $\begin{array}{c}1,000 \\
\text { and } \\
\text { over }\end{array}$ & $\begin{array}{c}500 \\
\text { to } \\
1,000\end{array}$ & $\begin{array}{c}200 \\
\text { to } \\
500\end{array}$ & $\begin{array}{c}100 \\
\text { to } \\
200\end{array}$ & $\begin{array}{c}10 \\
\text { to } \\
100\end{array}$ & $\begin{array}{c}\text { Less } \\
\text { than } \\
10\end{array}$ \\
\hline Alabama & 9 & $\mathbf{5}$ & 5 & 4 & 35 & 15 & 17,381 & 3,704 & 1,333 & 589 & 1,572 & 61 \\
\hline 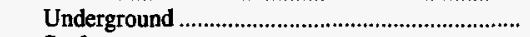 & 8 & 2 & - & - & - & 1 & 16,112 & 1,492 & - & - & - & 1 \\
\hline 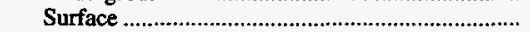 & 1 & 3 & 5 & 4 & 35 & 14 & 1,270 & 2,212 & 1,333 & 589 & 1,572 & 60 \\
\hline Alaska & 1 & - & - & - & - & - & 1,698 & - & - & - & - & - \\
\hline 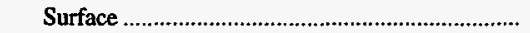 & 1 & - & - & - & - & - & 1,698 & - & - & - & - & - \\
\hline 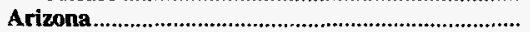 & 2 & - & - & - & - & - & 11,947 & - & - & - & - & - \\
\hline Surface & 2 & - & - & - & - & - & 11,947 & - & - & - & - & - \\
\hline Arkansas & - & - & - & - & 1 & 2 & - & - & - & - & 15 & 14 \\
\hline Surface & - & - & - & - & 1 & $\overline{2}$ & - & - & - & - & 15 & 14 \\
\hline 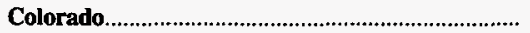 & 8 & 2 & 5 & - & 1 & $\mathbf{1}$ & 22,577 & 1,352 & 1,763 & - & 17 & * \\
\hline Underground & 5 & 2 & 4 & - & - & $\overline{1}$ & 14,446 & 1,352 & 1,389 & - & - & * \\
\hline Surface & 3 & - & 1 & - & 1 & - & 8,131 & - & 374 & - & 17 & - \\
\hline 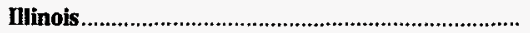 & 21 & 3 & 3 & 1 & 3 & - & 45,079 & 1,743 & 1,093 & 118 & 147 & - \\
\hline Underground & 18 & 2 & - & - & - & - & 39,880 & 1,239 & - & - & - & - \\
\hline Surface & 3 & 1 & 3 & 1 & 3 & - & 5,200 & 504 & 1,093 & 118 & 147 & - \\
\hline 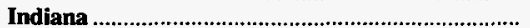 & 9 & 6 & 13 & 6 & 4 & 4 & 15,939 & $\mathbf{4 , 3 2 7}$ & 4,697 & 899 & 121 & 24 \\
\hline 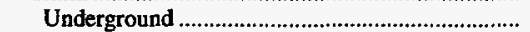 & 2 & - & 2 & - & - & - & 3,045 & - & 495 & - & - & - \\
\hline 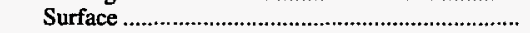 & $\overline{7}$ & 6 & 11 & 6 & 4 & 4 & 12,894 & 4,327 & 4,202 & 899 & 121 & 24 \\
\hline Kansas & - & - & 1 & - & - & - & - & - & 285 & - & - & - \\
\hline Surface & - & - & 1 & - & - & - & - & - & 285 & _ & - & - \\
\hline 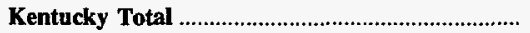 & 27 & 71 & 104 & 94 & 213 & 89 & 47,700 & 48,528 & 33,196 & 13,758 & $\mathbf{1 0 , 2 0 2}$ & 355 \\
\hline Underground & 18 & 40 & 58 & 60 & 134 & 51 & 34,159 & 26,300 & 17,861 & 8,675 & 7,022 & 190 \\
\hline 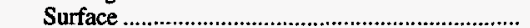 & 9 & 31 & 46 & 34 & 79 & 38 & 13,541 & 22,228 & 15,336 & 5,083 & 3,180 & 165 \\
\hline Eastern & 18 & 56 & 93 & 90 & 203 & 80 & 28,663 & $\mathbf{3 7 , 3 4 7}$ & 29,244 & 13,127 & 9,828 & 331 \\
\hline 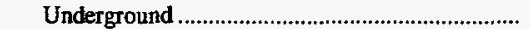 & 10 & 32 & 55 & 60 & 132 & 50 & 16,505 & 20,702 & 16,897 & 8,675 & 6,888 & 189 \\
\hline 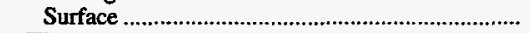 & 8 & 24 & 38 & 30 & 71 & 30 & 12,157 & 16,646 & 12,347 & 4,452 & 2,940 & 143 \\
\hline 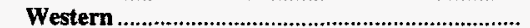 & 9 & 15 & 11 & 4 & 10 & 9 & 19,037 & $\mathbf{1 1 , 1 8 1}$ & 3,952 & 630 & 374 & 24 \\
\hline 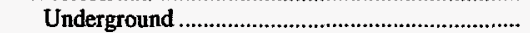 & 8 & 8 & 3 & - & 2 & 1 & 17,653 & 5,598 & 963 & - & 134 & 2 \\
\hline 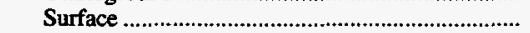 & 1 & 7 & 8 & 4 & 8 & 8 & 1,383 & 5,582 & 2,988 & 630 & 240 & 22 \\
\hline 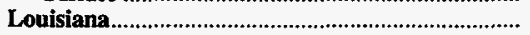 & 1 & 1 & - & - & - & - & 2,881 & 838 & - & - & - & - \\
\hline 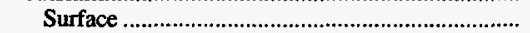 & 1 & 1 & - & - & - & - & 2,881 & 838 & - & - & - & - \\
\hline 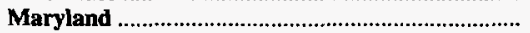 & 1 & - & 2 & 1 & 11 & 5 & 2,595 & - & 540 & 103 & 406 & 24 \\
\hline 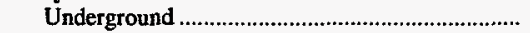 & 1 & - & 1 & - & - & 1 & 2,595 & - & 293 & - & - & 4 \\
\hline 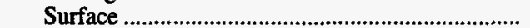 & - & - & 1 & 1 & 11 & 4 & - & - & 248 & 103 & 406 & 20 \\
\hline 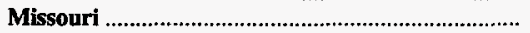 & - & - & - & 2 & 3 & 1 & - & - & - & 340 & 199 & 9 \\
\hline 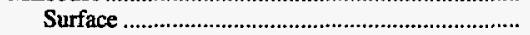 & - & - & - & 2 & 3 & 1 & - & - & - & 340 & 199 & 9 \\
\hline 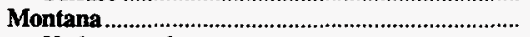 & 6 & - & 1 & - & - & 1 & 39,144 & - & 297 & - & - & 10 \\
\hline Underground & - & - & - & - & - & $\overline{1}$ & - & - & - & - & - & 10 \\
\hline Surface & 6 & - & 1 & - & - & - & 39,144 & - & 297 & - & - & - \\
\hline New Mexico & 6 & 1 & - & - & - & - & 26,173 & 640 & - & - & - & - \\
\hline Underground & - & 1 & - & - & - & - & - & 640 & - & - & - & - \\
\hline Surface & 6 & - & - & - & - & - & 26,173 & - & - & - & - & - \\
\hline 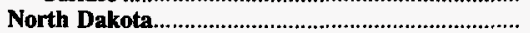 & 6 & - & - & - & - & - & $\mathbf{3 0 , 1 1 2}$ & - & - & - & - & - \\
\hline 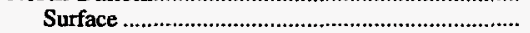 & 6 & - & - & - & - & - & 30,112 & - & - & - & - & - \\
\hline Ohio & 5 & 7 & 13 & 14 & $\mathbf{5 1}$ & 23 & 12,447 & 5,452 & 4,010 & 2,044 & 2,082 & 83 \\
\hline 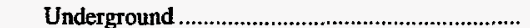 & 4 & 1 & 2 & - & 1 & - & 11,389 & 955 & 661 & - & 73 & - \\
\hline Surface & 1 & 6 & 11 & 14 & 50 & 23 & 1,058 & 4,497 & 3,349 & 2,044 & 2,009 & 83 \\
\hline 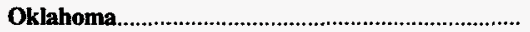 & - & - & 5 & 2 & $\mathbf{5}$ & 1 & - & - & 1,457 & 284 & 129 & 6 \\
\hline Underground & - & - & - & - & 1 & - & - & - & - & - & 25 & - \\
\hline Surface & - & - & 5 & 2 & 4 & 1 & - & - & 1,457 & 284 & 103 & 6 \\
\hline Pennsylvania Total & 9 & 9 & 31 & 37 & 189 & 184 & 32,088 & 6,347 & 10,515 & 5,036 & 6,842 & 747 \\
\hline 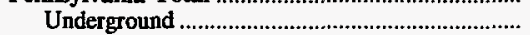 & 8 & 6 & 14 & 6 & 21 & 41 & 30,593 & 4,449 & 4,502 & 917 & 780 & 168 \\
\hline Surface & 1 & 3 & 17 & 31 & 168 & 143 & 1,494 & 1,898 & 6,014 & 4,120 & 6,062 & 579 \\
\hline 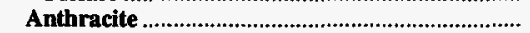 & - & - & 6 & 7 & 40 & 81 & - & - & 2,056 & 977 & 1,361 & 288 \\
\hline 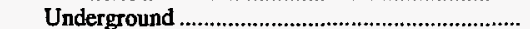 & - & - & - & 1 & 5 & 39 & - & - & - & 158 & 112 & 158 \\
\hline 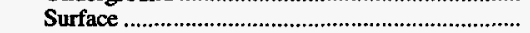 & - & - & 6 & 6 & 35 & 42 & - & - & 2,056 & 819 & 1,249 & 130 \\
\hline 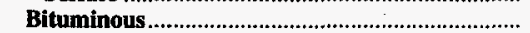 & 9 & 9 & 25 & 30 & 149 & 103 & 32,088 & 6,347 & 8,459 & 4,059 & 5,481 & 459 \\
\hline 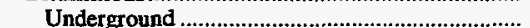 & 8 & 6 & 14 & 5 & 16 & 2 & 30,593 & 4,449 & 4,502 & 759 & 668 & 10 \\
\hline Surface & 1 & 3 & 11 & 25 & 133 & 101 & 1,494 & 1,898 & 3,958 & 3,300 & 4,813 & 449 \\
\hline 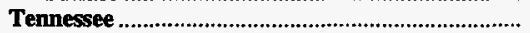 & - & $\mathbf{1}$ & 6 & $\mathbf{5}$ & 8 & 5 & - & 517 & 1,508 & 749 & 433 & 14 \\
\hline Underground & - & - & 5 & 4 & 3 & 2 & - & - & 1,200 & 602 & 156 & 5 \\
\hline 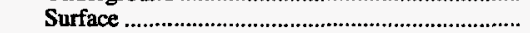 & - & 1 & 1 & 1 & 5 & 3 & - & 517 & 308 & 147 & 277 & 10 \\
\hline 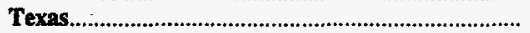 & 11 & - & 2 & - & 1 & - & 52,105 & - & 527 & - & 51 & - \\
\hline Surface & 11 & - & 2 & - & 1 & - & 52,105 & - & 527 & - & 51 & \\
\hline
\end{tabular}

See footnotes at end of table. 
Table 6. Coal Production and Number of Mines by State, Mine Type, and Mine Production Range, 1995 (Continued)

(Thousand Short Tons)

\begin{tabular}{|c|c|c|c|c|c|c|c|c|c|c|c|c|}
\hline \multirow{3}{*}{$\begin{array}{c}\text { Coal-Producing State, Region } \\
\text { and Type of Mining }\end{array}$} & \multicolumn{6}{|c|}{ Number of Mines } & \multicolumn{6}{|c|}{ Production } \\
\hline & \multicolumn{12}{|c|}{$\begin{array}{l}\text { Mine Production Range } \\
\text { (thousand short tons) }\end{array}$} \\
\hline & $\begin{array}{c}1,000 \\
\text { and } \\
\text { over }\end{array}$ & $\begin{array}{c}500 \\
\text { to } \\
1,000\end{array}$ & $\begin{array}{c}200 \\
\text { to } \\
500\end{array}$ & $\begin{array}{c}100 \\
\text { to } \\
200\end{array}$ & $\begin{array}{c}10 \\
\text { to } \\
100\end{array}$ & $\begin{array}{c}\text { Less } \\
\text { than } \\
\mathbf{1 0}\end{array}$ & $\begin{array}{l}1,000 \\
\text { and } \\
\text { over }\end{array}$ & $\begin{array}{c}500 \\
\text { to } \\
1,000\end{array}$ & $\begin{array}{c}200 \\
\text { to } \\
500\end{array}$ & $\begin{array}{c}100 \\
\text { to } \\
200\end{array}$ & $\begin{array}{c}10 \\
\text { to } \\
100\end{array}$ & $\begin{array}{c}\text { Less } \\
\text { than } \\
10\end{array}$ \\
\hline Utah & 9 & 1 & 2 & 1 & - & - & $\mathbf{2 3 , 8 3 3}$ & 529 & 702 & 103 & - & - \\
\hline Underground & 9 & 1 & 2 & 1 & - & - & 23,833 & 529 & 702 & 103 & - & - \\
\hline Virginia & 3 & 7 & 36 & 39 & 91 & 18 & $\mathbf{7 , 3 1 0}$ & 4,047 & 12,352 & $\mathbf{5 , 4 3 4}$ & 4,886 & 71 \\
\hline 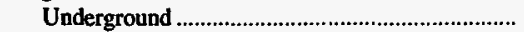 & 3 & 3 & 22 & 32 & 77 & 14 & 7,310 & 1,727 & 7,555 & 4,377 & 4,339 & 64 \\
\hline Surface & - & 4 & 14 & 7 & 14 & 4 & - & 2,320 & 4,797 & 1,057 & 547 & 6 \\
\hline Washington & 2 & - & $\mathbf{1}$ & - & - & - & 4,627 & - & 241 & - & - & - \\
\hline 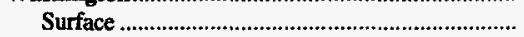 & 2 & - & 1 & - & - & - & 4,627 & - & 241 & - & - & - \\
\hline 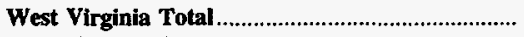 & 41 & 36 & 86 & 57 & 167 & 37 & 94,576 & 25,320 & 27,814 & 7,630 & 7,515 & 142 \\
\hline Underground & 25 & 23 & 68 & 41 & 106 & 15 & 61,362 & 16,049 & 21,798 & 5,495 & 5,263 & 62 \\
\hline Surface & 16 & 13 & 18 & 16 & 61 & 22 & 33,214 & 9,272 & 6,016 & 2,135 & 2,252 & 80 \\
\hline 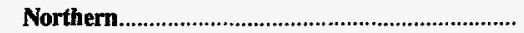 & 13 & 6 & 11 & 11 & 39 & 18 & 34,669 & 4,833 & 3,562 & 1,400 & 1,579 & 72 \\
\hline 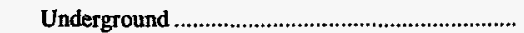 & 11 & 5 & 9 & 6 & 17 & 5 & 32,069 & 4,024 & 2,914 & 765 & 932 & 22 \\
\hline 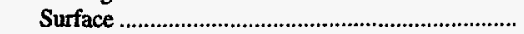 & 2 & 1 & 2 & 5 & 22 & 13 & 2,599 & 809 & 648 & 635 & 647 & 50 \\
\hline Southern & 28 & 30 & $\mathbf{7 5}$ & 46 & 128 & 19 & 59,907 & 20,487 & 24,252 & 6,230 & 5,936 & 70 \\
\hline 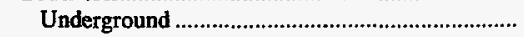 & 14 & 18 & 59 & 35 & 89 & 10 & 29,292 & 12,025 & 18,884 & 4,731 & 4,331 & 40 \\
\hline 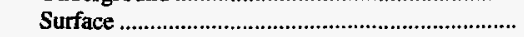 & 14 & 12 & 16 & 11 & 39 & 9 & 30,614 & 8,462 & 5,368 & 1,499 & 1,605 & 30 \\
\hline Wyoming & 21 & 2 & 2 & - & 2 & 2 & 261,643 & 1,498 & 625 & - & 56 & 1 \\
\hline Underground & 1 & - & - & - & 1 & 1 & 1,990 & - & - & - & 18 & * \\
\hline Surface & 20 & 2 & 2 & - & 1 & 1 & 259,653 & 1,498 & 625 & - & 38 & * \\
\hline Appalachian Total ${ }^{1}$ & 86 & 121 & 272 & 247 & 755 & 367 & 195,059 & 82,735 & 87,316 & $\mathbf{3 4 , 7 1 3}$ & 33,564 & 1,474 \\
\hline Underground & 59 & 67 & 167 & 143 & 340 & 124 & 145,866 & 45,373 & 52,906 & 20,066 & 17,500 & 492 \\
\hline 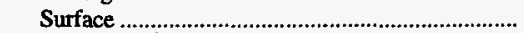 & 27 & 54 & 105 & 104 & 415 & 243 & 49,194 & 37,361 & 34,411 & 14,647 & 16,064 & 981 \\
\hline Interior Total 1 & 51 & 25 & 35 & 15 & 27 & 17 & 135,042 & 18,089 & 12,011 & $\mathbf{2 , 2 7 2}$ & 1,036 & 76 \\
\hline Underground & 28 & 10 & 5 & - & 3 & 1 & 60,578 & 6,837 & 1,458 & - & 160 & 2 \\
\hline Surface & 23 & 15 & 30 & 15 & 24 & 16 & 74,463 & 11,252 & 10,553 & 2,272 & 876 & 75 \\
\hline Western Total ${ }^{1}$ & 61 & 6 & 11 & 1 & 3 & 4 & $\mathbf{4 2 1 , 7 5 3}$ & 4,019 & 3,629 & 103 & 73 & 11 \\
\hline Underground & 15 & 4 & 6 & 1 & 1 & 3 & 40,269 & 2,521 & 2,091 & 103 & 18 & 10 \\
\hline Surface & 46 & 2 & 5 & - & 2 & 1 & 381,484 & 1,498 & 1,538 & - & 56 & * \\
\hline 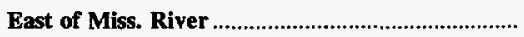 & 125 & 145 & 299 & 258 & 772 & 380 & 275,114 & 99,985 & 97,058 & 36,361 & 34,206 & $\mathbf{1 , 5 2 1}$ \\
\hline Underground & 87 & 77 & 172 & 143 & 342 & 125 & 206,444 & 52,210 & 54,364 & 20,066 & 17,634 & 494 \\
\hline Surface & 38 & 68 & 127 & 115 & 430 & 255 & 68,671 & 47,775 & 42,694 & 16,295 & 16,572 & 1,027 \\
\hline 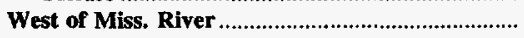 & 73 & 7 & 19 & 5 & 13 & 8 & 476,739 & 4,857 & 5,899 & 726 & 468 & 39 \\
\hline 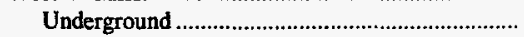 & 15 & 4 & 6 & 1 & 2 & 3 & 40,269 & 2,521 & 2,091 & 103 & 43 & 10 \\
\hline Surface & 58 & 3 & 13 & 4 & 11 & 5 & 436,471 & 2,336 & 3,808 & 624 & 424 & 29 \\
\hline U.S. Total & 198 & 152 & 318 & 263 & 785 & 388 & 751,854 & 104,842 & 102,957 & 37,087 & 34,673 & $\mathbf{1 , 5 6 0}$ \\
\hline Underground & 102 & 81 & 178 & 144 & 344 & 128 & 246,713 & 54,731 & 56,455 & 20,169 & 17,677 & 504 \\
\hline Surface & 96 & 71 & 140 & 119 & 441 & 260 & 505,141 & 50,111 & 46,502 & 16,918 & 16,996 & 1,056 \\
\hline
\end{tabular}

1 For a definition of coal-producing regions, see Appendix C.

* Data round to zero.

Notes: Coal production excludes silt, culm, refuse bank, slurry dam, and dredge operations except for Pennsylvania anthracite. Totals may not equal sum of components due to independent rounding.

Sources: Energy Information Administration, Form EIA-7A, “Coal Production Report"; State Mining Agency Coal Production Reports; and/or U.S. Department of Labor, Mine Safety and Health Administration, Form 7000-2, "Quarterly Mine Employment and Coal Production Report." 
Table 7. U.S. Coal Production by Coalbed Thickness and Mine Type, 1995 (Thousand Short Tons)

\begin{tabular}{|c|c|c|c|}
\hline $\begin{array}{c}\text { Coalbed Thickness } \\
\text { (inches) }\end{array}$ & Underground & Surface & Total \\
\hline$<7$ & - & 356 & 356 \\
\hline $7-12$ & - & 1,737 & 1,737 \\
\hline 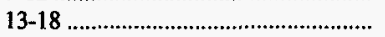 & - & 6,626 & 6,626 \\
\hline $19-24$ & 624 & 15,941 & 16,565 \\
\hline $25-30$ & 5,548 & 22,843 & 28,391 \\
\hline 31-36 & 34,718 & 40,309 & 75,027 \\
\hline $37-42$ & 28,198 & 21,122 & 49,319 \\
\hline 43-48 & 36,970 & 18,819 & 55,789 \\
\hline 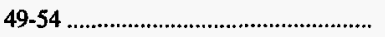 & 33,166 & 18,405 & 51,571 \\
\hline 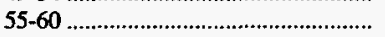 & 43,332 & 15,325 & 58,657 \\
\hline $61-66$ & 39,874 & 14,277 & 54,151 \\
\hline $67-72$ & 35,867 & 16,144 & 52,011 \\
\hline $73-78$ & 17,202 & 10,438 & 27,639 \\
\hline 79-84 & 41,225 & 19,265 & 60,490 \\
\hline $85-90$ & 6,776 & 13,582 & 20,358 \\
\hline $91-96$ & 26,415 & 6,451 & 32,867 \\
\hline $97-102$ & 4,985 & 6,483 & 11,468 \\
\hline 103-108 & 3,825 & 1,382 & 5,207 \\
\hline $109-114$ & 6,082 & 2,500 & 8,582 \\
\hline $115-120$ & 8,241 & 2,241 & 10,483 \\
\hline$>\quad 120$ & 22,696 & 381,423 & 404,119 \\
\hline 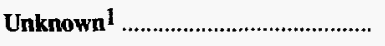 & 504 & 1,056 & 1,560 \\
\hline 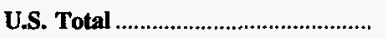 & 396,249 & 636,725 & $1,032,974$ \\
\hline
\end{tabular}

1 Includes mines with production of less than 10,000 short tons, which are required to provide only production data. Notes: Coal production excludes silt, culm, refuse bank, slurry dam, and dredge operations except for Pennsylvania anthracite. Totals may not equal sum of components due to independent rounding.

Sources: Energy Information Administration, Form EIA-7A, "Coal Production Report"; State Mining Agency Coal Production Reports; and/or U.S. Department of Labor, Mine Safety and Health Administration, Form 7000-2, "Quarterly Mine Employment and Coal Production Report." 
Table 8. U.S. Coal Production and Coalbed Thickness by Major Coalbeds and Mine Type, 1995

\begin{tabular}{|c|c|c|c|c|c|c|}
\hline \multirow{2}{*}{$\begin{array}{l}\text { Coalbed ID Number } 1 \\
\text { Coalbed Name } 2\end{array}$} & \multicolumn{3}{|c|}{$\begin{array}{l}\text { Production } \\
\text { (thousand short tons) }\end{array}$} & \multicolumn{3}{|c|}{$\begin{array}{l}\text { Thickness } \\
\text { (inches) }\end{array}$} \\
\hline & Underground & Surface & Total & Average 3 & Low & High \\
\hline 1699 Wyodak & - & 231,457 & 231,457 & 858 & 300 & 1,296 \\
\hline 0036 Pittsburgh & 64,750 & 2,515 & 67,265 & 75 & 30 & 108 \\
\hline 0489 No. 9 & 30,851 & 7,828 & 38,680 & 62 & 42 & 84 \\
\hline 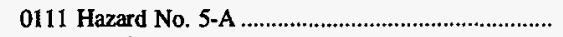 & 12,915 & 23,553 & 36,468 & 76 & 18 & 226 \\
\hline 0484 No. 6 & 30,643 & 4,644 & 35,287 & 79 & 5 & 100 \\
\hline 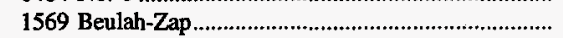 & - & 27,699 & 27,699 & 150 & 132 & 180 \\
\hline 0135 Hazard No. 4 & 18,355 & 2,878 & 21,234 & 45 & 26 & 106 \\
\hline 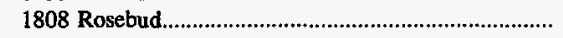 & - & 18,421 & 18,421 & 261 & 216 & 276 \\
\hline 0168 Lower Elkhorn & 16,743 & 1,620 & 18,363 & 60 & 18 & 96 \\
\hline 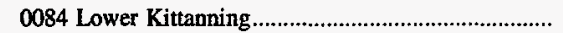 & 5,050 & 12,454 & 17,505 & 59 & 12 & 150 \\
\hline 0103 Stockton-Lewiston & 3,357 & 13,506 & 16,863 & 70 & 12 & 102 \\
\hline 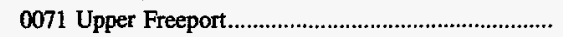 & 12,141 & 3,577 & 15,717 & 62 & 6 & 96 \\
\hline 0344 Pocahontas No. 3 & 14,879 & 237 & 15,116 & 63 & 36 & 83 \\
\hline 0157 Elkhorn No. 3 & 11,411 & 2,768 & 14,178 & 63 & 15 & 120 \\
\hline 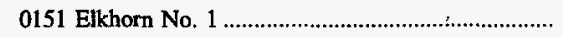 & 10,885 & 2,990 & 13,875 & 46 & 12 & 96 \\
\hline 0154 Elkhorn No. 2 & 11,273 & 2,382 & 13,655 & 52 & 14 & 72 \\
\hline 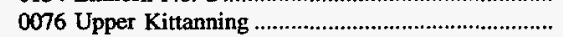 & 6,144 & 4,402 & 10,547 & 50 & 12 & 102 \\
\hline Major Coalbeds Total & 249,398 & 362,930 & 612,329 & 375 & $\mathbf{5}$ & 1,296 \\
\hline Other Coalbeds. & 146,346 & 272,738 & 419,085 & 130 & $\mathbf{5}$ & 960 \\
\hline Unknown4 4 & 504 & 1,056 & 1,560 & NA & NA & NA \\
\hline U.S. Total & 396,249 & 636,725 & $1,032,974$ & 275 & 5 & 1,296 \\
\hline
\end{tabular}

1 The coalbed ID number is a unique code assigned by ElA to each correlated coalbed or to coal-bearing geologic formations, coal groups, or coal zones.

2 The coalbed name given is the name most commonly used in the State having the greatest production from that coalbed. The States having the greatest production for each coalbed are: Eastern Kentucky (coalbeds 0111, 0135, 0151, 0154, 0157, 0168); West Virginia (0036, 0084, 0103, 0344); Pennsylvania (0071,0076); Western Kentucky (0489); Illinois (0484); North Dakota (1569); Montana (1808); Wyoming (1699). In some other States where these are major producing beds, the following alternate coalbed names are also used: 0084, No. 5 Block (Eastern Kentucky); 0111, Coalburg (West Virginia); 0135, Chilton (West Virginia); 0151, Jellico (Tennesee), Taggert (Virginia), Cedar Grove (West Virginia); 0154, Lower Cedar Grove (West Virginia); 0157, Upper Standiford (Virginia), Alma (West Virginia); 0168, No. 2 Gas (West Virginia); 0483, No. 12 (Western Kentucky); 0484, No. 11 (Western Kentucky); 0489, No. 5 (Illinois and Indiana).

3 Average thickness is the bed thickness weighted by bed production.

4 Includes mines with production of less than 10,000 short tons, which are required to provide only production data.

NA Not available.

Notes: Coal production excludes silt, culm, refuse bank, slurry dam, and dredge operations except for Pennsylvania anthracite. A major coalbed is defined here as a coalbed from which 10 million or more short tons were produced during the year. The category "Other Coalbeds" includes all coalbeds from which less than 10 million short tons were produced during the year. In some regions, coalbeds are characteristically discontinuous or uncorrelatable from one location to another, and production is identified by the geological formations, coal groups, or coal zones of the native rock where the coalbeds occur. These types of coalbeds are found primarily in the Rocky Mountain States and even in the Gulf Coast lignite belt. Coalbeds of these types are also included in "Other Coalbeds," even though production may exceed 10 million short tons. Totals may not equal sum of components due to independent rounding.

Sources: Energy Information Administration, Form EIA-7A, "Coal Production Report"; State Mining Agency Coal Production Reports; and/or U.S. Department of Labor, Mine Safety and Health Administration, Form 7000-2, "Quarterly Mine Employment and Coal Production Report." 
Table 9. Coal Production and Number of Mines by State and Coal Rank, 1995 (Thousand Short Tons)

\begin{tabular}{|c|c|c|c|c|c|c|c|c|c|c|}
\hline \multirow[b]{2}{*}{$\begin{array}{l}\text { Coal-Producing } \\
\text { State and Region }\end{array}$} & \multicolumn{2}{|c|}{ Bituminous } & \multicolumn{2}{|c|}{ Subbituminous } & \multicolumn{2}{|c|}{ Lignite } & \multicolumn{2}{|c|}{ Anthracite } & \multicolumn{2}{|c|}{ Total } \\
\hline & $\begin{array}{c}\text { Number } \\
\text { of } \\
\text { Mines }\end{array}$ & Production & $\begin{array}{c}\text { Number } \\
\text { of } \\
\text { Mines }\end{array}$ & Production & $\begin{array}{c}\text { Number } \\
\text { of } \\
\text { Mines }\end{array}$ & Production & $\begin{array}{c}\text { Number } \\
\text { of } \\
\text { Mines }\end{array}$ & Production & $\begin{array}{c}\text { Number } \\
\text { of } \\
\text { Mines }\end{array}$ & Production \\
\hline Alabama & 73 & 24,640 & - & - & - & - & - & - & 73 & 24,640 \\
\hline Alaska & - & - & 1 & 1,698 & - & - & - & - & 1 & 1,698 \\
\hline 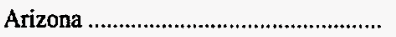 & 2 & 11,947 & - & - & - & - & - & - & 2 & 11,947 \\
\hline 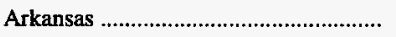 & - & - & - & - & - & - & 3 & 29 & 3 & 29 \\
\hline 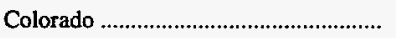 & 13 & 17,174 & 4 & 8,536 & - & - & - & - & 17 & 25,710 \\
\hline Illinois & 31 & 48,180 & - & - & - & - & - & - & 31 & 48,180 \\
\hline Indiana & 42 & 26,007 & - & - & - & - & - & - & 42 & 26,007 \\
\hline Kansas & 1 & 285 & - & - & - & - & - & - & 1 & 285 \\
\hline 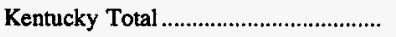 & 598 & 153,739 & - & - & - & - & - & - & 598 & 153,739 \\
\hline 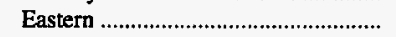 & 540 & 118,541 & - & - & - & - & - & - & 540 & 118,541 \\
\hline 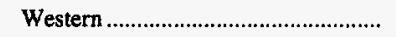 & 58 & 35,198 & - & - & - & - & - & - & 58 & 35,198 \\
\hline 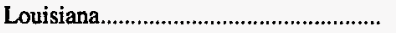 & - & - & - & - & 2 & 3,719 & - & - & 2 & 3,719 \\
\hline Maryland & 20 & 3,667 & - & - & - & - & - & - & 20 & 3,667 \\
\hline 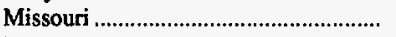 & 6 & 548 & - & - & - & - & - & - & 6 & 548 \\
\hline 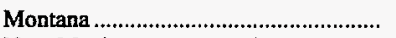 & - & - & 7 & 39,153 & 1 & 297 & - & - & 8 & 39,451 \\
\hline New Mexico & 5 & 14,428 & 2 & 12,385 & - & - & - & - & 7 & 26,813 \\
\hline North Dakota & - & - & - & - & 6 & 30,112 & - & - & 6 & 30,112 \\
\hline 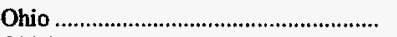 & 113 & 26,118 & - & - & - & - & - & - & 113 & 26,118 \\
\hline 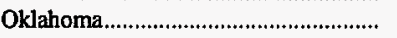 & 13 & 1,876 & - & - & - & - & - & - & 13 & 1,876 \\
\hline 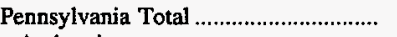 & 325 & 56,893 & - & - & - & - & 134 & 4,682 & 459 & 61,576 \\
\hline 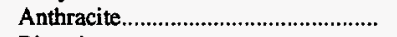 & - & - & - & - & - & - & 134 & 4,682 & 134 & 4,682 \\
\hline 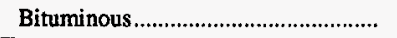 & 325 & 56,893 & - & - & - & - & - & - & 325 & 56,893 \\
\hline 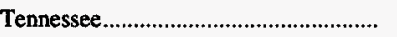 & 25 & 3,221 & - & - & - & - & - & - & 25 & 3,221 \\
\hline 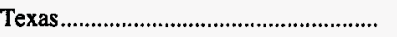 & 2 & 312 & - & - & 12 & 52,372 & - & - & 14 & 52,684 \\
\hline Utah & 13 & 25,167 & - & - & - & - & - & - & 13 & 25,167 \\
\hline 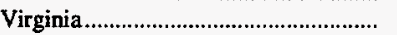 & 194 & 34,099 & - & - & - & - & - & - & 194 & 34,099 \\
\hline 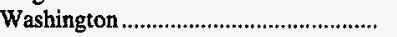 & 1 & 241 & 2 & 4,627 & - & - & - & - & 3 & 4,868 \\
\hline 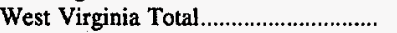 & 424 & 162,997 & - & - & - & - & - & - & 424 & 162,997 \\
\hline 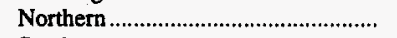 & 98 & 46,114 & - & - & - & - & - & - & 98 & 46,114 \\
\hline 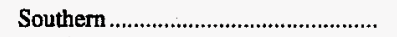 & 326 & 116,883 & - & - & - & - & - & - & 326 & 116,883 \\
\hline 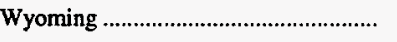 & 4 & 2,224 & 25 & 261,599 & - & - & - & - & 29 & 263,822 \\
\hline 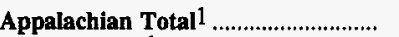 & 1,714 & 430,178 & - & - & - & - & 134 & 4,682 & 1,848 & 434,861 \\
\hline Interior Total 1 & 153 & 112,406 & - & - & 14 & 56,091 & 3 & 29 & 170 & 168,526 \\
\hline 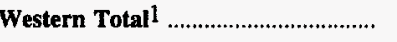 & 38 & 71,181 & 41 & 327,998 & 7 & 30,409 & - & - & 86 & 429,587 \\
\hline East of Miss. River & 1,845 & 539,563 & - & - & - & - & 134 & 4,682 & 1,979 & 544,246 \\
\hline West of Miss. River............................... & 60 & 74,201 & 41 & 327,998 & 21 & 86,500 & 3 & 29 & 125 & 488,728 \\
\hline U.S. Total & 1,905 & 613,765 & 41 & 327,998 & 21 & 86,500 & 137 & 4,711 & 2,104 & $1,032,974$ \\
\hline
\end{tabular}

1 For a definition of coal-producing regions, see Appendix $\mathrm{C}$.

Notes: Coal production excludes silt, culm, refuse bank, slurry dam, and dredge operations except for Pennsylvania anthracite. Totals may not equal sum of components due to independent rounding.

Sources: Energy Information Administration, Form EIA-7A, "Coal Production Report"; State Mining Agency Coal Production Reports; and/or U.S. Department of Labor, Mine Safety and Health Administration, Form 7000-2, "Quarterly Mine Employment and Coal Production Report." 
Table 10. Coal Production by State, Coal Rank, and Group, 1995 (Thousand Short Tons)

\begin{tabular}{|c|c|c|c|c|c|c|c|c|}
\hline $\begin{array}{l}\text { Coal-Producing } \\
\text { State and Region }\end{array}$ & $\begin{array}{l}\text { Bituminous } \\
\text { Low Volatile }\end{array}$ & $\begin{array}{c}\text { Bituminous } \\
\text { Medium } \\
\text { Volatile }\end{array}$ & $\begin{array}{l}\text { Bituminous } \\
\text { High Volatile }\end{array}$ & $\begin{array}{l}\text { Bituminous } \\
\text { Total } 1\end{array}$ & $\begin{array}{l}\text { Subbitumi- } \\
\text { nous }\end{array}$ & Lignite & Anthracite & Total \\
\hline Alabama & 6,734 & 5,036 & 12,567 & 24,640 & - & - & - & 24,640 \\
\hline Alaska & - & - & - & - & 1,698 & - & - & 1,698 \\
\hline 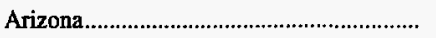 & - & - & 11,947 & 11,947 & - & - & - & 11,947 \\
\hline 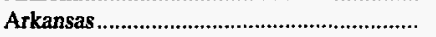 & - & - & - & - & - & - & 29 & 29 \\
\hline Colorado & - & 1,156 & 16,019 & 17,174 & 8,536 & - & - & 25,710 \\
\hline 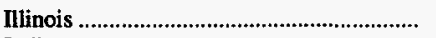 & - & - & 48,180 & 48,180 & - & - & - & 48,180 \\
\hline 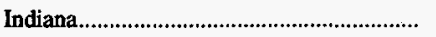 & - & - & 26,007 & 26,007 & - & - & - & 26,007 \\
\hline 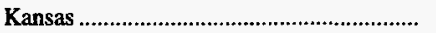 & - & - & 285 & 285 & - & - & - & 285 \\
\hline 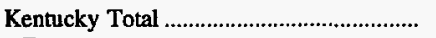 & 10,427 & 3,618 & 139,684 & 153,739 & - & - & - & 153,739 \\
\hline Eastern & 371 & 3,293 & 114,867 & 118,541 & - & - & - & 118,541 \\
\hline Western & 10,056 & 326 & 24,817 & 35,198 & - & - & - & 35,198 \\
\hline 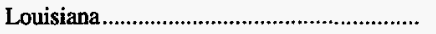 & - & - & - & - & - & 3,719 & - & 3,719 \\
\hline Maryland & 3,644 & - & 23 & 3,667 & - & - & - & 3,667 \\
\hline 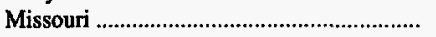 & - & - & 548 & 548 & - & - & - & 548 \\
\hline 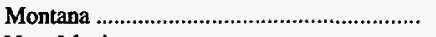 & - & - & - & - & 39,153 & 297 & - & 39,451 \\
\hline 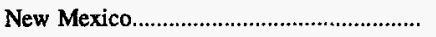 & - & 640 & 13,788 & 14,428 & 12,385 & - & - & 26,813 \\
\hline 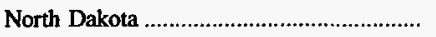 & - & - & - & - & - & 30,112 & - & 30,112 \\
\hline 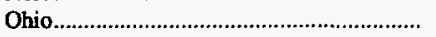 & 18 & 747 & 24,849 & 26,118 & - & - & - & 26,118 \\
\hline Oklahoma & 586 & 584 & 706 & 1,876 & - & - & - & 1,876 \\
\hline Pennsylvania Total & 5,141 & 11,972 & 39,709 & 56,893 & - & - & 4,682 & 61,576 \\
\hline Anthracite & - & - & - & - & - & - & 4,682 & 4,682 \\
\hline Bituminous & 5,141 & 11,972 & 39,709 & 56,893 & - & - & - & 56,893 \\
\hline 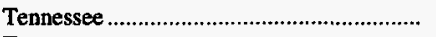 & - & 850 & 2,372 & 3,221 & - & - & - & 3,221 \\
\hline 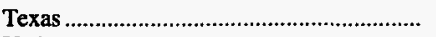 & - & 312 & - & 312 & - & 52,372 & - & 52,684 \\
\hline Utah & - & - & 25,167 & 25,167 & - & - & - & 25,167 \\
\hline 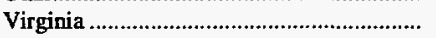 & 7,310 & 7,554 & 19,235 & 34,099 & - & - & - & 34,099 \\
\hline Washington & - & - & 241 & 241 & 4,627 & - & - & 4,868 \\
\hline 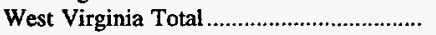 & 18,431 & 16,608 & 127,921 & 162,997 & - & - & - & 162,997 \\
\hline Northern & 4,094 & 2,098 & 39,899 & 46,114 & - & - & - & 46,114 \\
\hline 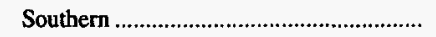 & 14,337 & 14,510 & 88,022 & 116,883 & - & - & - & 116,883 \\
\hline 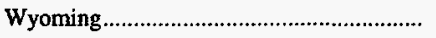 & - & * & 2,224 & 2,224 & 261,599 & - & - & 263,822 \\
\hline 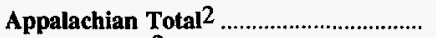 & 41,649 & 46,061 & 341,544 & 430,178 & - & - & 4,682 & 434,861 \\
\hline Interior Total 2 & 10,642 & 1,222 & 100,542 & 112,406 & - & 56,091 & 29 & 168,526 \\
\hline Western Total ${ }^{2} \ldots \ldots \ldots \ldots \ldots \ldots$ & - & 1,796 & 69,385 & 71,181 & 327,998 & 30,409 & - & 429,587 \\
\hline East of Miss. River & $\mathbf{5 1 , 7 0 5}$ & 46,386 & 440,548 & 539,563 & - & - & 4,682 & 544,246 \\
\hline West of Miss. River & 586 & 2,692 & 70,923 & 74,201 & 327,998 & 86,500 & 29 & 488,728 \\
\hline U.S. Total & 52,291 & 49,078 & $\mathbf{5 1 1}, \mathbf{4 7 1}$ & 613,765 & 327,998 & 86,500 & 4,711 & $1,032,974$ \\
\hline
\end{tabular}

1 Includes bituminous production with volatile content not reported.

2 For a definition of coal-producing regions, see Appendix C.

* Data round to zero.

Notes: Refer to the Classification of Coals by Rank table in Appendix $\mathrm{C}$ for coal group definitions. Coal production excludes silt, culm, refuse bank, slurry dam, and dredge operations except for Pennsylvania anthracite. Totals may not equal sum of components due to independent rounding.

Sources: Energy Information Administration, Form EIA-7A, "Coal Production Report"; State Mining Agency Coal Production Reports; and/or U.S. Department of Labor, Mine Safety and Health Administration, Form 7000-2, "Quarterly Mine Employment and Coal Production Report." 
Table 11. Coal Production by State, Mine Type, and Union Type, 1995 (Thousand Short Tons)

\begin{tabular}{|c|c|c|c|c|c|}
\hline $\begin{array}{l}\text { Coal-Producing } \\
\text { State and Region }\end{array}$ & UMWA & Other Unions & Union Totạl & Nonunion & Total \\
\hline 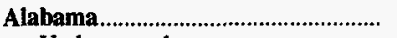 & 17,901 & - & 17,901 & 6,678 & 24,579 \\
\hline 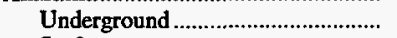 & 14,259 & - & 14,259 & 3,344 & 17,604 \\
\hline 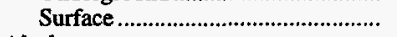 & 3,642 & - & 3,642 & 3,333 & 6,976 \\
\hline 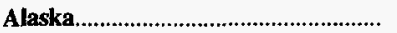 & - & 1,698 & 1,698 & - & 1,698 \\
\hline 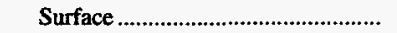 & - & 1,698 & 1,698 & - & 1,698 \\
\hline 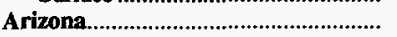 & 11,947 & - & 11,947 & - & 11,947 \\
\hline 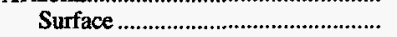 & 11,947 & - & 11,947 & - & 11,947 \\
\hline 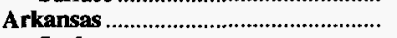 & - & - & - & 15 & 15 \\
\hline Surface & - & - & - & 15 & 15 \\
\hline 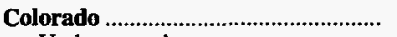 & 5,556 & 2,024 & 7,580 & 18,130 & 25,710 \\
\hline 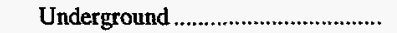 & 3,420 & - & 3,420 & 13,766 & 17,187 \\
\hline 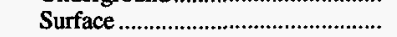 & 2,135 & 2,024 & 4,160 & 4,363 & 8,523 \\
\hline Illinois. & 35,557 & 2,878 & 38,434 & 9,746 & 48,180 \\
\hline 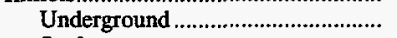 & 29,524 & 2,532 & 32,055 & 9,063 & 41,118 \\
\hline Surface & 6,033 & 346 & 6,379 & 683 & 7,062 \\
\hline Indiana & 12,619 & - & 12,619 & 13,364 & 25,983 \\
\hline 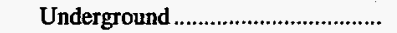 & 1,825 & - & 1,825 & 1,714 & 3,540 \\
\hline Surface & 10,793 & - & 10,793 & 11,650 & 22,443 \\
\hline 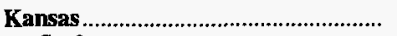 & 285 & - & 285 & - & 285 \\
\hline Surface & 285 & - & 285 & - & 285 \\
\hline 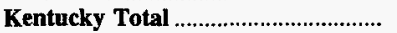 & 22,760 & 1,284 & 24,043 & 129,341 & 153,384 \\
\hline 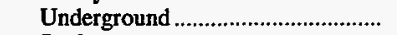 & 16,431 & 315 & 16,746 & 77,270 & 94,017 \\
\hline 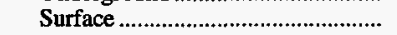 & 6,328 & 968 & 7,297 & 52,071 & 59,367 \\
\hline 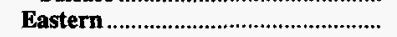 & 13,069 & 1,284 & 14,353 & 103,857 & 118,210 \\
\hline Underground & 8,584 & 315 & 8,899 & 60,768 & 69,667 \\
\hline 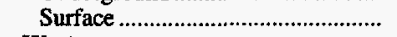 & 4,485 & 968 & 5,454 & 43,089 & 48,543 \\
\hline Western & 9,690 & - & 9,690 & 25,484 & 35,174 \\
\hline 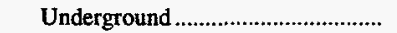 & 7,847 & - & 7,847 & 16,502 & 24,350 \\
\hline 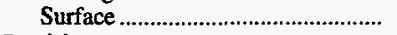 & 1,843 & - & 1,843 & 8,982 & 10,825 \\
\hline Louisiana & - & - & - & 3,719 & 3,719 \\
\hline Surface & - & - & - & 3,719 & 3,719 \\
\hline 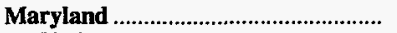 & - & - & - & 3,643 & 3,643 \\
\hline 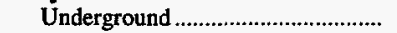 & - & - & - & 2,887 & 2,887 \\
\hline Surface & - & - & - & 756 & 756 \\
\hline 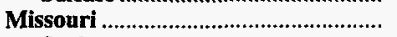 & - & - & - & 539 & 539 \\
\hline 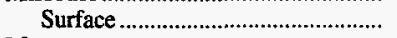 & - & - & - & 539 & 539 \\
\hline 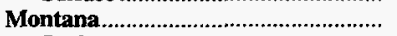 & 15,284 & 15,643 & 30,926 & 8,515 & 39,441 \\
\hline Surface & 15,284 & 15,643 & 30,926 & 8,515 & 39,441 \\
\hline New Mexico & $\mathbf{8 , 4 7 8}$ & 14,361 & 22,840 & 3,973 & 26,813 \\
\hline 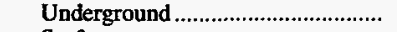 & 640 & - & 640 & - & 640 \\
\hline 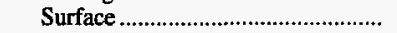 & 7,839 & 14,361 & 22,200 & 3,973 & 26,173 \\
\hline 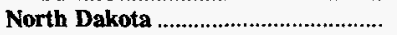 & 3,889 & 4,039 & 7,928 & 22,184 & 30,112 \\
\hline Surface & 3,889 & 4,039 & 7,928 & 22,184 & 30,112 \\
\hline Ohio & 13,260 & 60 & 13,320 & 12,715 & 26,035 \\
\hline 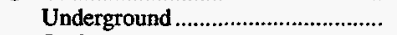 & 11,389 & - & 11,389 & 1,688 & 13,077 \\
\hline Surface & 1,871 & 60 & 1,931 & 11,027 & 12,958 \\
\hline 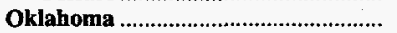 & - & - & - & 1,870 & 1,870 \\
\hline Underground & - & - & - & 25 & 25 \\
\hline 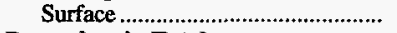 & - & - & - & 1,845 & 1,845 \\
\hline Pennsylvania Total ................................ & 21,734 & 63 & 21,796 & 39,032 & 60,828 \\
\hline 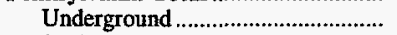 & 19,502 & - & 19,502 & 21,739 & 41,241 \\
\hline 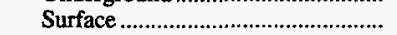 & 2,232 & 63 & 2,294 & 17,293 & 19,587 \\
\hline Anthracite & 1,214 & 63 & 1,277 & $\mathbf{3 , 1 1 8}$ & 4,394 \\
\hline 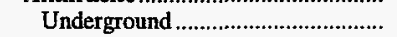 & - & - & - & 270 & 270 \\
\hline 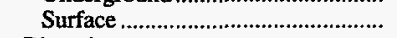 & 1,214 & 63 & 1,277 & 2,848 & 4,124 \\
\hline 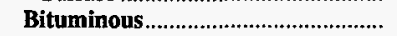 & 20,519 & - & 20,519 & 35,915 & 56,434 \\
\hline 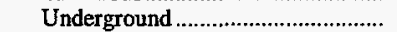 & 19,502 & - & 19,502 & 21,469 & 40,971 \\
\hline Surface & 1,017 & - & 1,017 & 14,445 & 15,463 \\
\hline 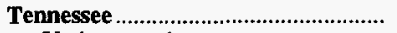 & - & - & - & $\mathbf{3 , 2 0 7}$ & $\mathbf{3 , 2 0 7}$ \\
\hline 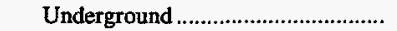 & - & - & - & 1,959 & 1,959 \\
\hline 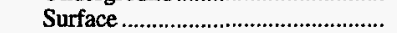 & - & - & - & 1,248 & 1,248 \\
\hline Texas & - & 32,103 & 32,103 & 20,581 & 52,684 \\
\hline Surface & - & 32,103 & 32,103 & 20,581 & 52,684 \\
\hline Utah & 8,737 & - & 8,737 & 16,430 & 25,167 \\
\hline 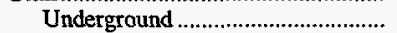 & 8,737 & - & 8,737 & 16,430 & 25,167 \\
\hline 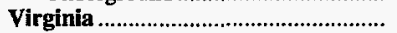 & 8,514 & 491 & 9,005 & 25,024 & 34,029 \\
\hline 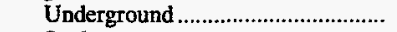 & 8,174 & - & 8,174 & 17,133 & 25,308 \\
\hline 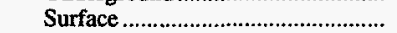 & 340 & 491 & 831 & 7,891 & 8,721 \\
\hline 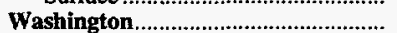 & - & 4,627 & 4,627 & 241 & 4,868 \\
\hline Surface & - & 4,627 & 4,627 & 241 & 4,868 \\
\hline West Virginia Total ............................... & 94,616 & - & 94,616 & 68,239 & 162,855 \\
\hline 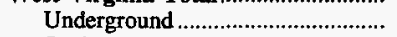 & 67,613 & - & 67,613 & 42,354 & 109,967 \\
\hline Surface & 27,003 & - & 27,003 & 25,885 & 52,888 \\
\hline
\end{tabular}

See footnotes at end of table 
Table 11. Coal Production by State, Mine Type, and Union Type, 1995 (Continued)

(Thousand Short Tons)

\begin{tabular}{|c|c|c|c|c|c|}
\hline $\begin{array}{l}\text { Coal-Producing } \\
\text { State and Region }\end{array}$ & UMWA & Other Unions & Union Total & Nonunion & Total \\
\hline 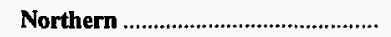 & 32,669 & - & 32,669 & 13,374 & 46,043 \\
\hline 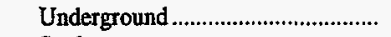 & 32,669 & - & 32,669 & 8,036 & 40,704 \\
\hline 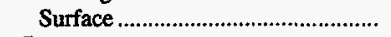 & - & - & - & 5,339 & 5,339 \\
\hline Southern & 61,947 & - & 61,947 & 54,865 & 116,812 \\
\hline 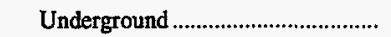 & 34,944 & - & 34,944 & 34,319 & 69,263 \\
\hline Surface & 27,003 & - & 27,003 & 20,546 & 47,550 \\
\hline 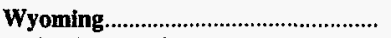 & 4,483 & 10,252 & 14,735 & 249,087 & 263,822 \\
\hline 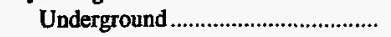 & - & - & - & 2,008 & 2,008 \\
\hline Surface & 4,483 & 10,252 & 14,735 & 247,079 & 261,814 \\
\hline Appalachian Total ${ }^{1}$............................... & 169,095 & 1,897 & 170,991 & 262,396 & 433,387 \\
\hline 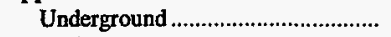 & 129,522 & 315 & 129,837 & 151,874 & 281,710 \\
\hline 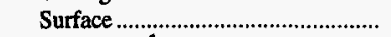 & 39,573 & 1,582 & 41,154 & 110,522 & 151,676 \\
\hline 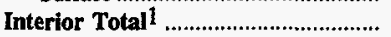 & $\mathbf{5 8 , 1 5 1}$ & 34,981 & 93,131 & 75,318 & 168,450 \\
\hline 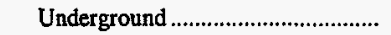 & 39,196 & 2,532 & 41,728 & 27,305 & 69,033 \\
\hline 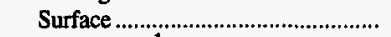 & 18,954 & 32,449 & 51,403 & 48,013 & 99,416 \\
\hline Western Total ${ }^{1}$ & $\mathbf{5 8 , 3 7 3}$ & 52,644 & 111,017 & 318,560 & 429,577 \\
\hline 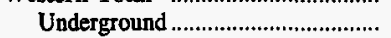 & 12,797 & - & 12,797 & 32,204 & 45,001 \\
\hline 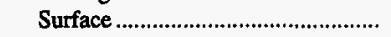 & 45,577 & 52,644 & 98,220 & 286,356 & 384,576 \\
\hline 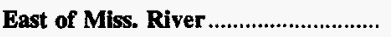 & 226,961 & 4,774 & 231,735 & 310,990 & 542,724 \\
\hline 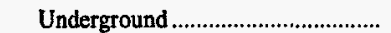 & 168,718 & 2,847 & 171,565 & 179,154 & 350,718 \\
\hline 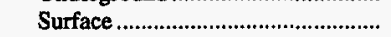 & 58,242 & 1,927 & 60,170 & 131,836 & 192,006 \\
\hline West of Miss. River ........................... & 58,658 & 84,747 & 143,405 & 345,284 & 488,689 \\
\hline 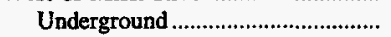 & 12,797 & - & 12,797 & 32,230 & 45,026 \\
\hline Surface & 45,861 & 84,747 & 130,608 & 313,054 & 443,663 \\
\hline 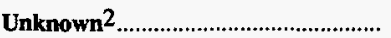 & NA & NA & $\mathbf{N A}$ & NA & 1,560 \\
\hline Underground & $\mathbf{N A}$ & NA & NA & NA & 504 \\
\hline 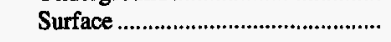 & $\mathbf{N A}$ & NA & NA & NA & 1,056 \\
\hline U.S. Total & 285,619 & 89,521 & 375,140 & 656,274 & $1,032,974$ \\
\hline 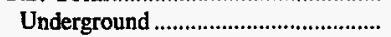 & 181,515 & 2,847 & 184,362 & 211,383 & 396,249 \\
\hline Surface & 104,104 & 86,674 & 190,778 & 444,890 & 636,725 \\
\hline
\end{tabular}

1 For a definition of coal-producing regions, see Appendix $\mathbf{C}$.

2 Includes mines with production of less than 10,000 short tons, which are required to provide only production data.

NA Not available.

Notes: Coal production excludes silt, culm, refuse bank, slurry dam, and dredge operations except for Pennsylvania anthracite. Totals may not equal sum of components due to independent rounding. See Glossary for listing of other unions.

Sources: Energy Information Administration, Form EIA-7A, "Coal Production Report"; State Mining Agency Coal Production Reports; and/or U.S. Department of Labor, Mine Safety and Health Administration, Form 7000-2, "Quarterly Mine Employment and Coal Production Report." 
Table 12. Coal Production by State and Disposition, 1995

(Thousand Short Tons)

\begin{tabular}{|c|c|c|c|}
\hline $\begin{array}{l}\text { Coal-Producing } \\
\text { State and Region }\end{array}$ & Open Market 1 & Captive $^{2}$ & Total \\
\hline Alabama & 23,770 & 810 & 24,579 \\
\hline 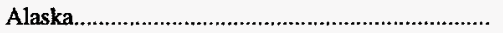 & 1,695 & 3 & 1,698 \\
\hline 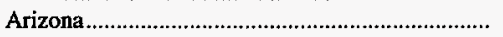 & 11,947 & 0 & 11,947 \\
\hline 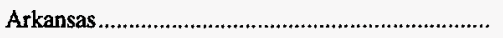 & 0 & 15 & 15 \\
\hline Colorado & 25,710 & 0 & 25,710 \\
\hline Illinois & 47,776 & 403 & 48,180 \\
\hline 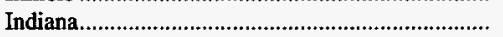 & 25,945 & 38 & 25,983 \\
\hline Kansas & 285 & 0 & 285 \\
\hline 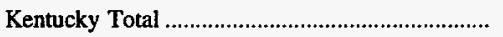 & 151,305 & 2,080 & 153,384 \\
\hline 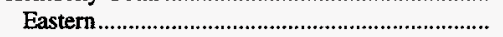 & 116,130 & 2,080 & 118,210 \\
\hline Western & 35,174 & 0 & 35,174 \\
\hline 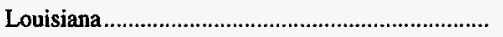 & 3,719 & 0 & 3,719 \\
\hline 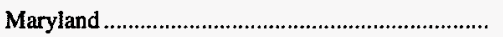 & 3,613 & 30 & 3,643 \\
\hline Missouri & 539 & 0 & 539 \\
\hline 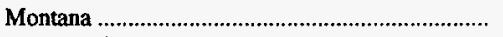 & 35,745 & 3,696 & 39,441 \\
\hline 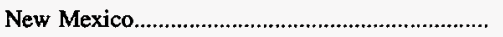 & 26,813 & 0 & 26,813 \\
\hline North Dakota & 29,044 & 1,068 & 30,112 \\
\hline 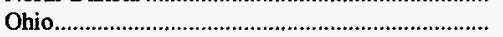 & 19,798 & 6,237 & 26,035 \\
\hline 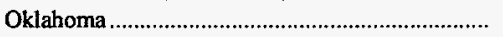 & 1,870 & 0 & 1,870 \\
\hline 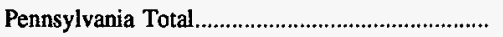 & 59,011 & 1,818 & 60,828 \\
\hline Anthracite & 3,019 & 1,375 & 4,394 \\
\hline 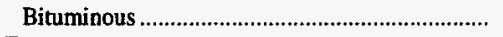 & 55,992 & 442 & 56,434 \\
\hline 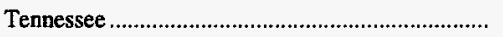 & 3,207 & 0 & 3,207 \\
\hline 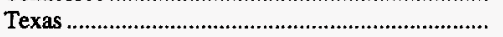 & 14,344 & 38,340 & 52,684 \\
\hline Utah & 17,093 & 8,074 & 25,167 \\
\hline Virginia & 30,986 & 3,043 & 34,029 \\
\hline 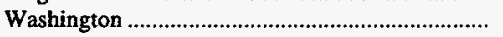 & 241 & 4,627 & 4,868 \\
\hline 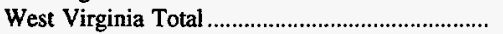 & 159,073 & 3,782 & 162,855 \\
\hline Northern & 44,303 & 1,739 & 46,043 \\
\hline Southern & 114,769 & 2,043 & 116,812 \\
\hline Wyoming & 247,503 & 16,319 & 263,822 \\
\hline 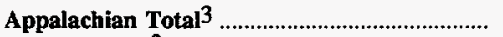 & $\mathbf{4 1 5 , 5 8 8}$ & 17,799 & $\mathbf{4 3 3}, \mathbf{3 8 7}$ \\
\hline Interior Total ${ }^{3}$ & 129,654 & 38,796 & 168,450 \\
\hline Western Total ${ }^{3} \ldots \ldots \ldots \ldots \ldots \ldots$ & 395,790 & 33,786 & 429,577 \\
\hline East of Miss. River & 524,484 & 18,240 & 542,724 \\
\hline West of Miss. River & $\mathbf{4 1 6 , 5 4 8}$ & 72,141 & 488,689 \\
\hline Total 4 & 941,032 & 90,381 & $\mathbf{1 , 0 3 1 , 4 1 3}$ \\
\hline 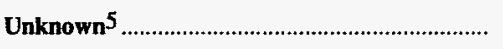 & NA & NA & 1,560 \\
\hline U.S. Total & NA & NA & $1,032,974$ \\
\hline
\end{tabular}

1 Open Market includes all coal sold on the open market to other coal companies or consumers.

2 Captive includes all coal used by the producing company or sold to affiliated or parent companies.

3 For a definition of coal-producing regions, see Appendix C.

4 Excludes mines producing less than 10,000 short tons, which are not required to provide these data.

5 Includes mines producing less than 10,000 short tons, which are required to provide only production data

NA Not available.

Notes: Coal production excludes silt, culm, refuse bank, slurry dam, and dredge operations except for Pennsylvania anthracite.

Sources: Energy Information Administration, Form EIA-7A, "Coal Production Report"; State Mining Agency Coal Production Reports; and/or U.S. Department of Labor, Mine Safety and Health Administration, Form 7000-2, "Quarterly Mine Employment and Coal Production Report." 
Table 13. Coal Mining Acreage, Production and Royalties from Federal and Indian Leases by State, 1995

\begin{tabular}{|c|c|c|c|c|c|c|}
\hline \multirow{2}{*}{$\begin{array}{l}\text { Coal-Producing } \\
\text { State and Region }\end{array}$} & \multicolumn{3}{|c|}{ Federal Leases } & \multicolumn{3}{|c|}{ Indian Leases } \\
\hline & $\begin{array}{l}\text { Acres } \\
\text { Leased }\end{array}$ & $\begin{array}{l}\text { Production } \\
\text { (thousand short tons) }\end{array}$ & $\begin{array}{c}\text { Royalties } \\
\text { (thousand dollars) }\end{array}$ & $\begin{array}{l}\text { Acres } \\
\text { Leased }\end{array}$ & $\begin{array}{l}\text { Production } \\
\text { (thousand short tons) }\end{array}$ & $\begin{array}{c}\text { Royalties } \\
\text { (thousand dollars) }\end{array}$ \\
\hline 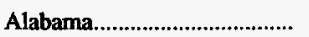 & 3,576 & 38 & 145 & - & - & - \\
\hline Arizona & - & - & - & 64,858 & 12,249 & 32,301 \\
\hline 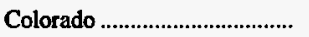 & 44,064 & 18,917 & 24,613 & - & - & - \\
\hline 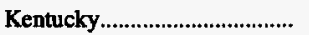 & 820 & 215 & 303 & - & - & - \\
\hline 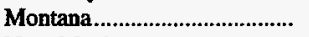 & 36,612 & 28,038 & 38,420 & 14,746 & 4,468 & 2,037 \\
\hline New Mexico................................ & 12,132 & 6,242 & 22,036 & 36,026 & 11,648 & 31,352 \\
\hline 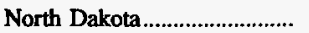 & 6,632 & 2,055 & 891 & - & - & - \\
\hline 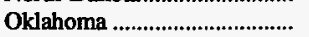 & 15,342 & 521 & 645 & - & - & - \\
\hline Utah & 48,197 & 23,577 & 37,691 & - & - & - \\
\hline 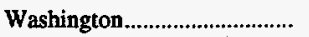 & 241 & 427 & 659 & - & - & - \\
\hline Wyoming & 130,090 & 268,484 & 178,202 & - & - & - \\
\hline Appalachian Total ${ }^{1} \ldots \ldots \ldots \ldots$ & 3,576 & 38 & 145 & - & - & - \\
\hline 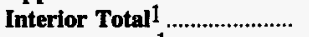 & 16,162 & 736 & 948 & - & - & - \\
\hline Western Total ${ }^{1}$ & 277,968 & 347,740 & 302,512 & 115,630 & 28,365 & 65,690 \\
\hline East of Miss. River............... & 4,396 & 254 & 447 & - & - & - \\
\hline West of Miss. River............... & 293,310 & 348,261 & $\mathbf{3 0 3 , 1 5 8}$ & 115,630 & 28,365 & 65,690 \\
\hline 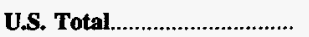 & 297,706 & 348,515 & 303,605 & 115,630 & 28,365 & 65,690 \\
\hline
\end{tabular}

1 For a definition of coal-producing regions, see Appendix $C$.

Notes: U.S. Total for this table represents Federal and Indian Leases only. Output from Federal and Indian Lands is reported as sales volume, the basis for royalties. It is approximately equivalent to production, which includes coai sold and coal added to stockpiles. Totals may not equal sum of components due to independent rounding.

Source: U.S. Department of the Interior, Minerals Management Service (MMS), Mineral Revenues, 1995, Report on Receipts from Federal and Indian Leases. 
Table 14. Major U.S. Coal Mines, 1995

\begin{tabular}{|c|c|c|c|c|}
\hline Rank & Mine Name/Company & Mine Type & State & $\begin{array}{l}\text { Production } \\
\text { (short tons) }\end{array}$ \\
\hline 1 & Black Thunder/Thunder Basin Coal & Surface & Wyoming & $36,180,336$ \\
\hline 2 & Rochelle/Powder River Coal & Surface & Wyoming & $26,089,177$ \\
\hline 3 & Jacobs Ranch/Kerr McGee & Surface & Wyoming & $24,639,356$ \\
\hline 4 & North Antelope/Powder River Coal & Surface & Wyoming & $21,248,991$ \\
\hline 5 & Belle Ayr/Amax Coal West & Surface & Wyoming & $18,807,390$ \\
\hline 6 & Caballo/Caballo Coal & Surface & Wyoming & $18,080,904$ \\
\hline 7 & Eagle Butte/Amax Coal West & Surface & Wyoming & $16,942,228$ \\
\hline 8 & Caballo Rojo/Caballo Rojo & Surface & Wyoming & $16,808,549$ \\
\hline 9 & Rawhide/Caballo Coal & Surface & Wyoming & $15,354,587$ \\
\hline 10 & Freedom-Coteau/Coteau Properties & Surface & North Dakota & $15,112,270$ \\
\hline 11 & Cordero/Cordero Mining & Surface & Wyoming & $14,602,928$ \\
\hline 12 & Buckskin/Triton Coal & Surface & Wyoming & $11,669,531$ \\
\hline 13 & Rosebud \#6/Western Energy & Surface & Montana & $11,255,756$ \\
\hline 14 & Antelope/Antelope Coal & Surface & Wyoming & $10,851,318$ \\
\hline 15 & Spring Creek/Spring Creek Coal & Surface & Montana & $8,514,615$ \\
\hline 16 & West Decker/Decker Coal & Surface & Montana & $8,475,335$ \\
\hline 17 & Navajo/BHP Minerals & Surface & New Mexico & $8,412,000$ \\
\hline 18 & Jewett/Northwestern Resources & Surface & Texas & $8,303,536$ \\
\hline 19 & Enlow Fork/Enlow Fork Mining & Underground & Pennsylvania & $8,035,295$ \\
\hline 20 & Monticello-Winfield/Texas Utilities Mining & Surface & Texas & $7,384,760$ \\
\hline 21 & Bailey No. 1/CONSOL & Underground & Pennsylvania & $7,330,192$ \\
\hline 22 & Keyenta/Peabody Western Coal & Surface & Arizona & $7,119,858$ \\
\hline 23 & Falkirk/Falkirk Mining & Surface & North Dakota & $7,071,425$ \\
\hline 24 & Martin Lake/Texas Utilities Mining & Surface & Texas & $7,003,841$ \\
\hline 25 & Jim Bridger/Bridger Coal & Surface & Wyoming & $6,983,261$ \\
\hline 26 & Mckinley/Pittsburgh \& Midway Coal Mining & Surface & New Mexico & $6,622,935$ \\
\hline 27 & Foidel Creek/Twenty Mile Coal & Underground & Colorado & $5,846,802$ \\
\hline 28 & Sandow-Rockdale/ALCOA & Surface & Texas & $5,827,545$ \\
\hline 29 & Martin Lake-Oak Hill/Texas Utilities Mining & Surface & Texas & $5,536,900$ \\
\hline 30 & Galatia No. 56/Kerr-McGee Coal & Underground & Illinois & $5,510,459$ \\
\hline 31 & Mount Gunnison/Mountain Coal & Underground & Colorado & $5,338,652$ \\
\hline 32 & No. 50 Mine/US Steel Mining & Underground & West Virginia & $5,125,338$ \\
\hline 33 & Mountaineer/Mingo Logan Coal & Underground & West Virginia & $4,996,372$ \\
\hline 34 & Skyline 01/Coastal States Energy & Underground & Utah & $4,883,803$ \\
\hline 35 & Black Mesa/Peabody Western Coal & Surface & Arizona & $4,826,946$ \\
\hline 36 & Big Brown/Texas Utilities Mining & Surface & Texas & $4,759,044$ \\
\hline 37 & Big Sky/Big Sky Coal & Surface & Montana & $4,708,970$ \\
\hline 38 & Cumberland/Cyprus Cumberland Resources & Underground & Pennsylvanja & $4,639,509$ \\
\hline 39 & Peats Branch No. 3/Old Hickory Coal & Surface & West Virginia & $4,401,009$ \\
\hline 40 & Absaloka/Westmoreland Resources & Surface & Montana & $4,386,882$ \\
\hline 41 & Colowyo/Colowyo Coal & Surface & Colorado & $4,363,359$ \\
\hline 42 & No. 13 Baker/Costain Coal & Underground & Kentucky & $4,281,649$ \\
\hline 43 & Federal No. 2/Eastern Associated Coal & Underground & West Virginia & $4,253,239$ \\
\hline 44 & Coal Creek/Thunder Basin Coal & Surface & Wyoming & $4,205,393$ \\
\hline 45 & San Juan/San Juan Coal & Surface & New Mexico & $4,191,335$ \\
\hline 46 & Deer Creek/Pacificorp & Underground & Utah & $4,142,193$ \\
\hline 47 & McElroy/CONSOL & Underground & West Virginia & $4,072,411$ \\
\hline 48 & Center/BNI Coal & Surface & North Dakota & $4,038,689$ \\
\hline 49 & Lee Ranch/Lee Ranch Coal & Surface & New Mexico & $3,973,282$ \\
\hline 50 & Powhatan No. 6/Ohio Valley Coal & Underground & Ohio & $3,946,017$ \\
\hline 51 & No. 37/Arch of Kentucky & Underground & Kentucky & $3,925,000$ \\
\hline 52 & Southern Utah Fuel/Coastal States Energy & Underground & Utah & $3,874,328$ \\
\hline 53 & Shoemaker/CONSOL & Underground & West Virginia & $3,819,586$ \\
\hline 54 & Blacksville No. 2/CONSOL & Underground & West Virginia & $3,811,399$ \\
\hline 55 & Emerald No. 1/Cypruss Emerald Resources & Underground & Pennsylvania & $3,768,145$ \\
\hline 56 & South Hallsville No. 1/Sabine Mining & Surface & Texas & $3,748,956$ \\
\hline 57 & Robinson Run/CONSOL & Underground & West Virginia & $3,666,950$ \\
\hline 58 & Kemmerer/Pittsburg \& Midway Coal & Surface & Wyoming & $3,624,328$ \\
\hline 59 & Samples (Kanawha)/Catenary Coal & Surface & West Virginia & $3,606,926$ \\
\hline 60 & Dry Fork/Dry Fork Coal & Surface & Wyoming & $3,603,601$ \\
\hline 61 & Lynnville/Peabody Coal & Surface & Indiana & $3,495,196$ \\
\hline 62 & Gibbons Creek/Navasota Mining & Surface & Texas & $3,312,452$ \\
\hline 63 & Rend Lake/CONSOL & Underground & Illinois & $3,269,017$ \\
\hline 64 & Dave Johnston/Pacificorp & Surface & Wyoming & $3,268,961$ \\
\hline 65 & Marissa/Peabody Coal & Underground & Illinois & $3,258,994$ \\
\hline 66 & Buchanan No. 1/CONSOL & Underground & Virginia & $3,180,256$ \\
\hline 67 & Shamrock No. 18/Elk River Resources & Underground & Kentucky & $3,157,336$ \\
\hline 68 & Hawthorn/Peabody Coal & Surface & Indiana & $3,151,377$ \\
\hline 69 & Humphrey No. 7/CONSOL & Underground & West Virginia & $3,109,806$ \\
\hline$*$ & Subtotal & & & $527,808,786$ \\
\hline * & All Other Mines & & & $505,164,986$ \\
\hline$*$ & U.S. Total & & & $1,032,973,772$ \\
\hline
\end{tabular}

Notes: Major mines are mines that produced more than 3 million short tons in 1995 . The company is the firm operating the mine.

Sources: Energy Information Administration, Form EIA-7A, "Coal Production Report"; State Mining Agency Coal Production Reports; and/or U.S. Department of Labor, Mine Safety and Health Administration, Form 7000-2, "Quarterly Mine Employment and Production Report." 
Table 15. Major U.S. Coal Producers, 1995

\begin{tabular}{|c|c|c|c|}
\hline Rank & Company Name & $\begin{array}{l}\text { Production } \\
\text { (thousand short tons) }\end{array}$ & Percent of Total Production \\
\hline 1 & Peabody Holding Co., Inc. & 139,048 & 13.5 \\
\hline 2 & Cyprus AMAX Minerals Co. & 76,028 & 7.4 \\
\hline 3 & Consol Energy Inc. & 69,144 & 6.7 \\
\hline 4 & Kennecott Energy Co. & 53,211 & 5.2 \\
\hline 5 & ARCO Coal Co. & 45,724 & 4.4 \\
\hline 6 & Zeigler Coal Holding Co. & 35,507 & 3.4 \\
\hline 7 & Kerr-McGee Coal Corp. & 30,559 & 2.9 \\
\hline 8 & North American Coal Corp. & 26,771 & 2.6 \\
\hline 9 & Texas Utilities Co. & 26,009 & 2.5 \\
\hline 10 & Montana Power Co. & 24,693 & 2.4 \\
\hline 11 & Arch Mineral Corp. & 24,275 & 2.4 \\
\hline 12 & A.T. Massey Coal Co. & 23,927 & 2.3 \\
\hline 13 & Marigold Land Co. & 16,809 & 1.6 \\
\hline 14 & Ashland Coal Inc. & 15,399 & 1.5 \\
\hline 15 & Pittsburg \& Midway Coal Co. & 14,630 & 1.4 \\
\hline 16 & Kiewitt Coal Properties & 14,536 & 1.4 \\
\hline 17 & BHP Utah Minerals & 14,361 & 1.4 \\
\hline 18 & Mapco Coal Inc. & 13,573 & 1.3 \\
\hline 19 & Pittston Coal Group & 13,216 & 1.2 \\
\hline 20 & Costain Coal Inc. & 10,421 & 1.0 \\
\hline 21 & Coastal Corp. & 9,250 & .9 \\
\hline 22 & Jim Walter Resources Inc. & 8,236 & .8 \\
\hline 23 & James River Coal Co. & 8,011 & .8 \\
\hline 24 & U.S. Steel Mining Co. & 7,780 & .8 \\
\hline 25 & PacifiCorp & 7,660 & .7 \\
\hline 26 & Westmoreland Resources Inc. & 7,628 & .7 \\
\hline 27 & Drummond Co. & 7,550 & .7 \\
\hline 28 & Central Ohio Coal Co. & 6,830 & .7 \\
\hline 29 & Black Beauty Coal Co. & 6,682 & .6 \\
\hline 30 & Dal-Tex Coal Corp. & 5,848 & .6 \\
\hline 31 & Rochester \& Pittsburgh Coal & 5,831 & .6 \\
\hline 32 & ALCOA & 5,828 & .6 \\
\hline 33 & Teco Coal Corp. & 5,497 & .5 \\
\hline 34 & Monterey Coal Co. & 5,099 & .5 \\
\hline 35 & Andalex Resources & 4,967 & .5 \\
\hline 36 & General Dynamics Corp. & 4,817 & .5 \\
\hline 37 & Sun Coal Co. & 4,521 & .4 \\
\hline 38 & Golden Oak Mining Co. & 4,346 & .4 \\
\hline 39 & MDU Resources Group Inc. & 4,186 & .4 \\
\hline 40 & Minnesota Power \& Light & 4,039 & .4 \\
\hline 41 & Ohio Valley Resources & 3,946 & .4 \\
\hline 42 & A N R Coal Co. & 3,687 & .4 \\
\hline 43 & Texas Municipal Power & 3,312 & .4 \\
\hline 44 & BethEnergy Mines Inc. & 3,201 & 3 \\
\hline 45 & Black Hills Corp. & 2,934 & 3 \\
\hline 46 & San Miguel Electric CoOp & 2,924 & 3 \\
\hline 47 & Dolet Hills Mining Venture & 2,881 & 3 \\
\hline 48 & Addington Inc. & 2,818 & 3 \\
\hline 49 & Appalachian Mining & 2,775 & .3 \\
\hline 50 & Dorchester Coal Corp. & 2,709 & 3 \\
\hline 51 & Mincorp Inc. & 2,620 & .3 \\
\hline 52 & Anker Group Inc. & 2,422 & .2 \\
\hline 53 & Teco Energy Coal Corp. & 2,215 & .2 \\
\hline 54 & Pen Coal Corp. & 2,122 & .2 \\
\hline 55 & Appolo Fuels Inc. & 2,086 & .2 \\
\hline 56 & Genwal Resources Inc. & 2,081 & .2 \\
\hline 57 & United Coal Co. & 2,042 & .2 \\
\hline 58 & Williams Fork Co. & 2,024 & .2 \\
\hline$*$ & Subtotal & 861,252 & 83.4 \\
\hline * & All other coal producers & 171,722 & 16.6 \\
\hline$*$ & U.S. Total & $1,032,974$ & 100.0 \\
\hline
\end{tabular}

Notes: Major coal producers are companies that produced more than 2 million short tons in 1995 . The company is the firm owning the mineral rights to the mined coal.

Sources: Energy Information Administration, Form EIA-7A "Coal Production Report"; State Mining Agency Coal Production Reports; and/or U.S. Department of Labor, Mine Safety and Health Administration, Form 7000-2, "Quarterly Mine Employment and Production Report." 


\section{Productive Capacity}

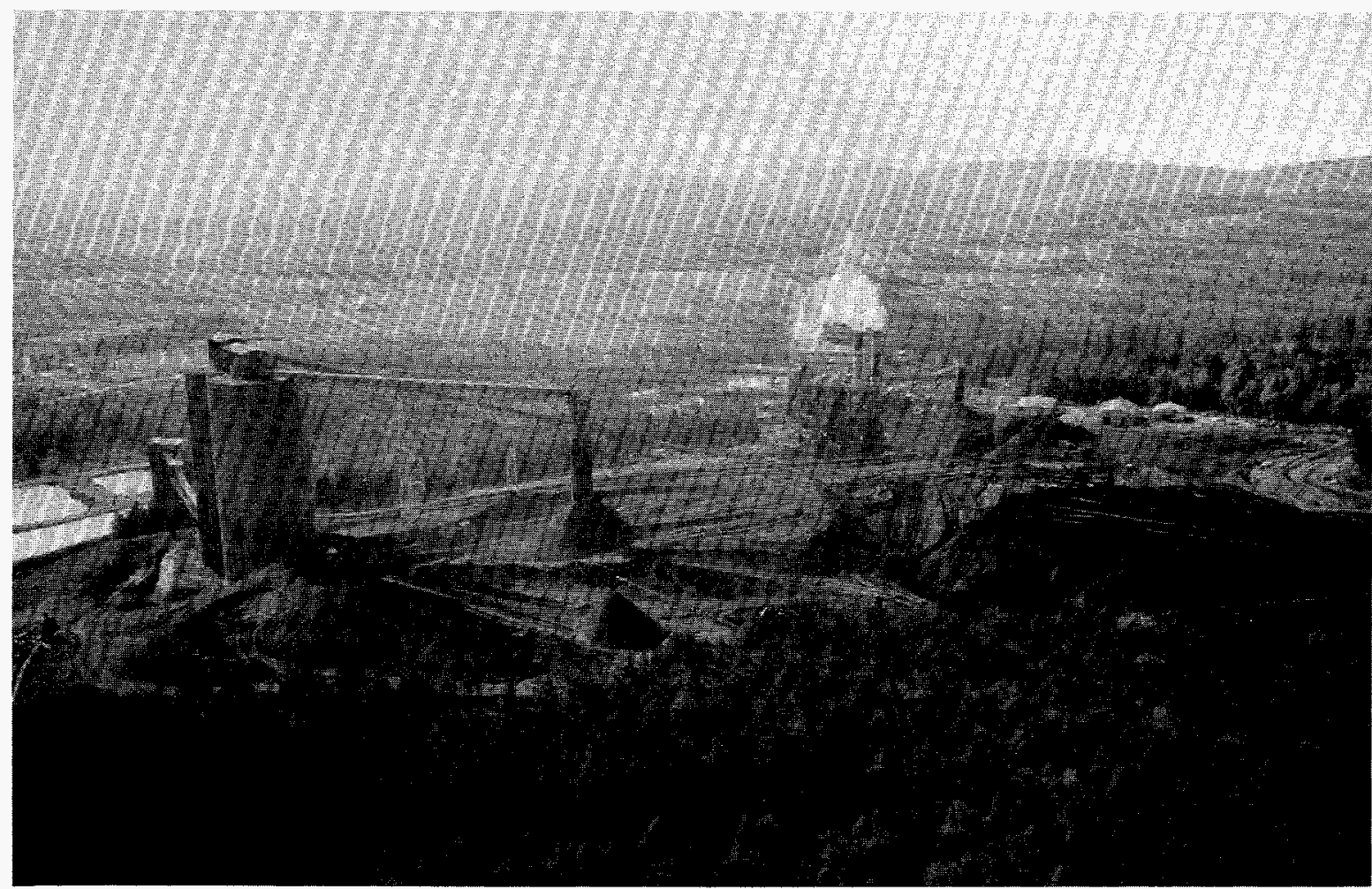

Coal's total productive capacity for 1995 totaled 1,299 million short tons. Surface mines accounted for 62 percent, while underground mines accounted for 38 percent. 
Table 16. Productive Capacity of Coal Mines by State, 1986, 1991-1995

(Thousand Short Tons)

\begin{tabular}{|c|c|c|c|c|c|c|c|c|c|}
\hline \multirow{2}{*}{$\begin{array}{l}\text { Coal-Producing } \\
\text { State and Region }\end{array}$} & \multirow{2}{*}{1995} & \multirow{2}{*}{1994} & \multirow{2}{*}{1993} & \multirow{2}{*}{1992} & \multirow{2}{*}{1991} & \multirow{2}{*}{$1986^{1}$} & \multirow{2}{*}{$\begin{array}{c}\text { Percent } \\
\text { Change } \\
\text { 1994-1995 }\end{array}$} & \multicolumn{2}{|c|}{ Average Annual Percent Change } \\
\hline & & & & & & & & 1991-1995 & 1986-1995 \\
\hline Alabama & 32,546 & 33,049 & 27,916 & 29,815 & 30,195 & 26,818 & -1.5 & 1.9 & 2.2 \\
\hline 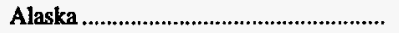 & w & w & w & w & w & 1,570 & w & w & w \\
\hline Arizona & $\mathbf{w}$ & $\mathbf{w}$ & $\mathbf{w}$ & $\mathbf{w}$ & $w$ & 11,556 & $\mathbf{w}$ & $\mathbf{w}$ & $w$ \\
\hline 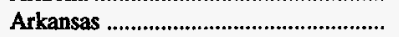 & $\mathbf{w}$ & w & $\mathbf{w}$ & $\mathbf{w}$ & $\mathbf{w}$ & 150 & $\mathbf{w}$ & $\mathbf{w}$ & $w$ \\
\hline California & - & - & - & $\mathbf{w}$ & w & - & w & w & w \\
\hline 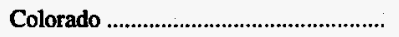 & 32,435 & 31,075 & 30,040 & 25,848 & 23,593 & 16,407 & 4.4 & 8.3 & 7.9 \\
\hline 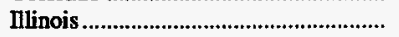 & 56,627 & 69,414 & 69,320 & 75,787 & 75,710 & 66,366 & -18.4 & -7.0 & -1.7 \\
\hline Indiana & 35,256 & 38,931 & 43,955 & 42,990 & 45,155 & 34,880 & -9.4 & -6.0 & .1 \\
\hline Iowa & - & w & $\mathbf{w}$ & $\mathbf{w}$ & $\mathbf{w}$ & 551 & w & $\mathbf{w}$ & w \\
\hline 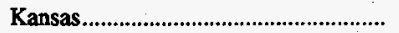 & $\mathbf{w}$ & $\mathbf{w}$ & $\mathbf{w}$ & w & $\mathbf{w}$ & 1,572 & w & $\mathbf{w}$ & $\mathbf{w}$ \\
\hline 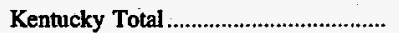 & 203,173 & 213,427 & 204,805 & 195,352 & 199,282 & 161,630 & -4.8 & .5 & 2.6 \\
\hline Eastern & 152,111 & 161,731 & 157,318 & 149,046 & 145,549 & 116,640 & -5.9 & 1.1 & 3.0 \\
\hline Western & 51,062 & 51,696 & 47,486 & 46,306 & 53,734 & 44,991 & -1.2 & -1.3 & 1.4 \\
\hline 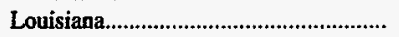 & w & w & $\mathbf{w}$ & w & w & 2,751 & $\mathbf{w}$ & $\mathbf{w}$ & w \\
\hline 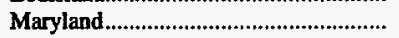 & 4,408 & 4,332 & 3,927 & 3,902 & 4,248 & 3,956 & 1.8 & .9 & 1.2 \\
\hline Missouri & 1,081 & 1,209 & w & w & w & 5,186 & -10.6 & $\mathbf{w}$ & -16.0 \\
\hline Montana & 51,597 & 51,104 & 50,849 & 48,582 & 48,604 & 35,008 & 1.0 & 1.5 & 4.4 \\
\hline 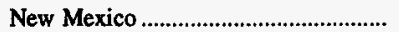 & 32,760 & 32,807 & 33,360 & 29,512 & 28,187 & 23,173 & -.1 & 3.8 & 3.9 \\
\hline North Dakota & 34,464 & 35,920 & 36,371 & 36,986 & 35,976 & 27,480 & -4.0 & -1.1 & 2.5 \\
\hline Ohio & 34,011 & 43,925 & 42,236 & 41,329 & 47,397 & 39,364 & -22.6 & -8.0 & -1.6 \\
\hline 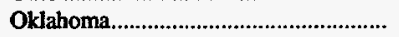 & 2,557 & 2,251 & 2,422 & 2,486 & 2,328 & 3,095 & 13.6 & 2.4 & -2.1 \\
\hline Pennsylvania Total ................................... & 77,187 & 80,975 & 82,148 & 82,968 & 80,418 & 77,674 & -4.7 & -1.0 & -.1 \\
\hline 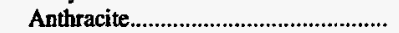 & 6,547 & 5,776 & 5,806 & 4,143 & 4,043 & 4,099 & 13.3 & 12.8 & 5.3 \\
\hline 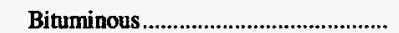 & 70,640 & 75,200 & 76,342 & 78,825 & 76,375 & 73,575 & -6.1 & -1.9 & -.4 \\
\hline 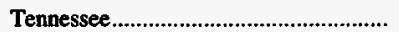 & 3,750 & 3,409 & 3,763 & 3,932 & 5,159 & 7,020 & 10.0 & -7.7 & -6.7 \\
\hline 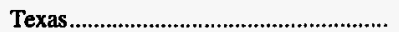 & 54,758 & 55,856 & 57,115 & 58,541 & 59,579 & 51,980 & -2.0 & -2.1 & .6 \\
\hline 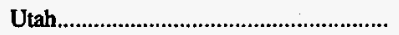 & 30,888 & 27,640 & 25,933 & 25,534 & 25,482 & 18,216 & 11.7 & 4.9 & 6.0 \\
\hline 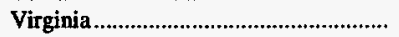 & 43,037 & 46,462 & 50,879 & 54,471 & 55,271 & 41,501 & -7.4 & -6.1 & .4 \\
\hline Washington & $\mathbf{w}$ & $\mathbf{w}$ & w & $\mathbf{w}$ & $\mathbf{w}$ & 4,981 & $\mathbf{w}$ & w & $\mathbf{w}$ \\
\hline West Virginia Total............................... & 204,837 & 201,684 & 191,706 & 198,083 & 200,114 & 138,629 & 1.6 & .6 & 4.4 \\
\hline Northern & 56,355 & 59,295 & 60,015 & 62,811 & 64,717 & 51,192 & -4.9 & -3.4 & 1.1 \\
\hline 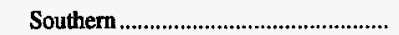 & 148,482 & 142,388 & 131,691 & 135,271 & 135,398 & 87,437 & 4.3 & 2.3 & 6.1 \\
\hline Wyoming & 337,184 & 321,046 & 277,875 & 253,312 & 250,251 & 147,667 & 5.0 & 7.7 & 9.6 \\
\hline 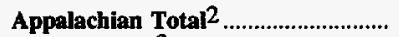 & 551,888 & 575,568 & 559,893 & 563,545 & 568,350 & 451,602 & -4.1 & -.7 & 2.3 \\
\hline Interior Total 2 & 205,393 & 223,897 . & 225,938 & 235,040 & 245,487 & 211,521 & -8.3 & -4.4 & -.3 \\
\hline 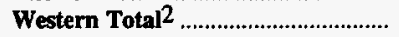 & 541,773 & 521,191 & 476,042 & 442,469 & 434,642 & 286,058 & 3.9 & $\mathbf{5 . 7}$ & 7.3 \\
\hline 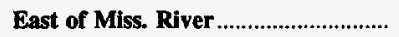 & 694,832 & 735,609 & 720,654 & $\mathbf{7 2 8 , 6 2 8}$ & 742,949 & 597,839 & -5.5 & -1.7 & 1.7 \\
\hline West of Miss. River................................. & 604,222 & 585,047 & 541,219 & 512,426 & 505,529 & $\mathbf{3 5 1 , 3 4 2}$ & 3.3 & 4.5 & 6.2 \\
\hline U.S. Total & $1,299,054$ & $1,320,656$ & $1,261,873$ & $1,241,054$ & $1,248,479$ & 949,182 & -1.6 & 1.0 & 3.5 \\
\hline
\end{tabular}

1 For 1986, the Form EIA-7A solicited data on "Daily Productive Capacity." To obtain annual productive capacity for a mine in 1986, each mine's daily productive capacity was multiplied by the number of days worked during the year.

2 For a definition of coal-producing regions, see Appendix C.

withheld to avoid disclosure of individual company data.

Notes: Productive capacity is the maximum amount of coal that can be produced annually as reported by mining companies on Form EIA-7A. Excludes silt, culm, refuse bank, slurry dam, and dredge operations except for Pennsylvania anthracite. Excludes mines producing less than 10,000 short tons, which are not required to provide these data. Totals may not equal sum of components due to independent rounding. Source: Energy Information Administration, Form EIA-7A, "Coal Production Report." 
Table 17. Capacity Utilization of Coal Mines by State, 1986, 1991-1995 (Percent)

\begin{tabular}{|c|c|c|c|c|c|c|}
\hline $\begin{array}{l}\text { Coal-Producing } \\
\text { State and Region }\end{array}$ & 1995 & 1994 & 1993 & 1992 & 1991 & 19861 \\
\hline Alabama & 75.52 & 70.19 & 88.46 & 86.26 & 89.97 & 95.80 \\
\hline 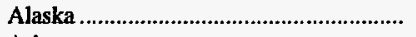 & w & w & $\mathbf{w}$ & $\mathbf{w}$ & $\mathbf{w}$ & 99.97 \\
\hline 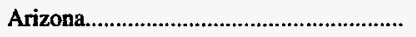 & $\mathbf{w}$ & $\mathbf{w}$ & $\mathbf{w}$ & $\mathbf{w}$ & $\mathbf{w}$ & 100.00 \\
\hline 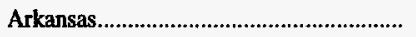 & $\mathbf{w}$ & w & $w$ & $\mathbf{w}$ & w & 98.68 \\
\hline 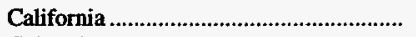 & - & - & - & $\mathbf{w}$ & $\mathbf{w}$ & - \\
\hline 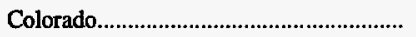 & 79.27 & 81.41 & 72.84 & 74.35 & 75.58 & 92.75 \\
\hline Illinois & 85.08 & 76.06 & 59.28 & 78.98 & 79.58 & 93.21 \\
\hline Indiana & 73.70 & 79.37 & 66.60 & 70.86 & 69.66 & 94.07 \\
\hline Iowa & - & w & $\mathbf{w}$ & $\mathbf{w}$ & $\mathbf{w}$ & 86.57 \\
\hline 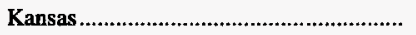 & $\mathbf{w}$ & $\mathbf{w}$ & $\mathbf{w}$ & $\mathbf{w}$ & $\mathbf{w}$ & 94.06 \\
\hline Kentucky Total & 75.49 & 75.54 & 76.11 & 82.15 & 79.45 & 94.22 \\
\hline Eastern. & 77.71 & 76.70 & 76.15 & 79.72 & 80.12 & 95.33 \\
\hline Western & 68.89 & 71.89 & 76.00 & 89.95 & 77.64 & 91.34 \\
\hline Louisiana & $\mathbf{w}$ & $\mathbf{w}$ & $\mathbf{w}$ & $\mathbf{w}$ & $\mathbf{w}$ & 81.93 \\
\hline 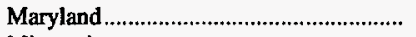 & 82.65 & 83.07 & 85.01 & 84.50 & 88.12 & 97.80 \\
\hline 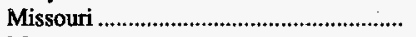 & 49.88 & 69.35 & $\mathbf{w}$ & $\mathbf{w}$ & w & 90.28 \\
\hline 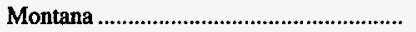 & 76.44 & 81.47 & 70.64 & 80.03 & 78.65 & 97.06 \\
\hline 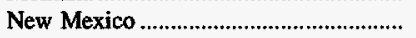 & 81.85 & 85.47 & 84.74 & 83.18 & 76.34 & 92.76 \\
\hline 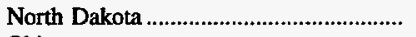 & 87.37 & 89.88 & 87.89 & 85.83 & 82.08 & 93.30 \\
\hline Ohio & 76.55 & 67.87 & 67.94 & 73.19 & 64.27 & 91.98 \\
\hline 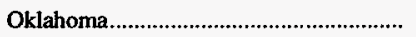 & 73.14 & 83.98 & 71.95 & 69.73 & 79.08 & 98.08 \\
\hline 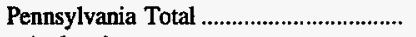 & 78.81 & 75.89 & 71.79 & 82.20 & 80.17 & 90.85 \\
\hline 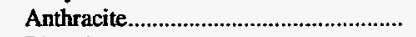 & 67.12 & 74.02 & 68.80 & 75.22 & 74.44 & 93.38 \\
\hline 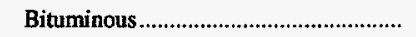 & 79.89 & 76.03 & 72.02 & 82.57 & 80.47 & 90.71 \\
\hline 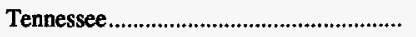 & 85.51 & 87.27 & 79.73 & 86.50 & 81.46 & 96.14 \\
\hline 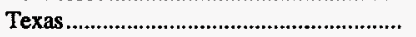 & 96.21 & 93.72 & 95.54 & 94.07 & 90.34 & 93.48 \\
\hline 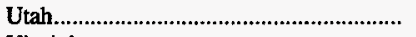 & 81.48 & 88.27 & 84.22 & 83.57 & 86.12 & 78.33 \\
\hline 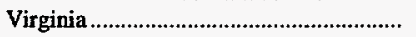 & 79.07 & 79.61 & 77.07 & 78.70 & 75.65 & 98.24 \\
\hline Washington & $\mathbf{w}$ & $w$ & $\mathbf{w}$ & $\mathbf{w}$ & $\mathbf{w}$ & 92.21 \\
\hline 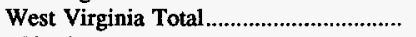 & 79.50 & 80.07 & 67.91 & 81.70 & 83.42 & 93.24 \\
\hline Northern & 81.70 & 83.04 & 56.15 & 79.44 & 80.32 & 93.91 \\
\hline Southern & 78.67 & 78.83 & 73.28 & 82.75 & 84.90 & 92.86 \\
\hline Wyoming & 78.24 & 73.85 & 75.62 & 75.07 & 77.46 & 92.65 \\
\hline Appalachian Total ${ }^{2} \ldots \ldots \ldots \ldots \ldots \ldots \ldots \ldots \ldots$ & $\mathbf{7 8 . 5 3}$ & 77.07 & $\mathbf{7 2 . 8 5}$ & 80.63 & 89.13 & 93.96 \\
\hline Interior Total 2 & 82.01 & 80.29 & 73.95 & 83.22 & 79.57 & 92.87 \\
\hline 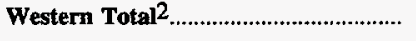 & 79.29 & 78.33 & 77.41 & 78.04 & 78.86 & 92.69 \\
\hline 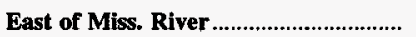 & 78.11 & 76.73 & 71.37 & 80.47 & 79.26 & 93.68 \\
\hline West of Miss. River..................................... & 80.88 & 79.85 & 79.29 & 79.80 & 80.05 & 92.73 \\
\hline 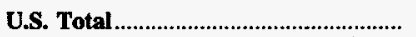 & 79.40 & 78.11 & 74.77 & 80.20 & 79.58 & 93.33 \\
\hline
\end{tabular}

1 For 1986, the Form EIA-7A solicited data on "Daily Productive Capacity." To obtain annual productive capacity for a mine in 1986, each mine's daily productive capacity was multiplied by the number of days worked during the year.

2 For a definition of coal-producing regions, see Appendix C.

w Withbeld to avoid disclosure of individual company data.

Notes: Capacity utilization is the ratio of total production to annual productive capacity as reported by mining companies on Form EIA-7A. Excludes silt, culm, refuse bank, slurry dam, and dredge operations except for Pennsylvania anthracite. Excludes mines producing less than 10,000 short tons, which are not required to provide these data.

Source: Energy Information Administration, Form ELA-7A, "Coal Production Report." 
Table 18. Production, Productive Capacity, and Capacity Utilization of Coal Mines by State and Mine Type, 1995

(Thousand Short Tons)

\begin{tabular}{|c|c|c|c|c|c|c|c|c|c|}
\hline \multirow[b]{2}{*}{$\begin{array}{l}\text { Coal-Producing } \\
\text { State and Region }\end{array}$} & \multicolumn{3}{|c|}{ Underground } & \multicolumn{3}{|c|}{ Surface } & \multicolumn{3}{|c|}{ Total } \\
\hline & Production & $\begin{array}{l}\text { Productive } \\
\text { Capacity }\end{array}$ & $\begin{array}{l}\text { Capacity } \\
\text { Utilization } \\
\text { (percent) }\end{array}$ & Production & $\begin{array}{c}\text { Productive } \\
\text { Capacity }\end{array}$ & $\begin{array}{l}\text { Capacity } \\
\text { Utilization } \\
\text { (percent) }\end{array}$ & Production & $\begin{array}{c}\text { Productive } \\
\text { Capacity }\end{array}$ & $\begin{array}{l}\text { Capacity } \\
\text { Utilization } \\
\text { (percent) }\end{array}$ \\
\hline 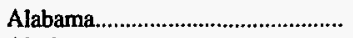 & 17,604 & 22,628 & 77.80 & 6,976 & 9,919 & 70.33 & 24,579 & 32,546 & 75.52 \\
\hline 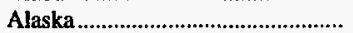 & - & - & - & 1,698 & $\mathbf{w}$ & $w$ & 1,698 & $\mathbf{w}$ & $\mathbf{w}$ \\
\hline Arizona & - & - & - & 11,947 & $\mathbf{w}$ & $\mathbf{w}$ & 11,947 & $\mathbf{w}$ & $w$ \\
\hline 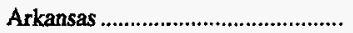 & - & - & - & 15 & $\mathbf{w}$ & $\mathbf{w}$ & 15 & $\mathbf{w}$ & $w$ \\
\hline Colorado & 17,187 & 22,135 & 77.65 & 8,523 & 10,300 & 82.75 & 25,710 & 32,435 & 79.27 \\
\hline 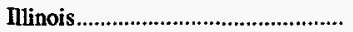 & 41,118 & 48,321 & 85.09 & 7,062 & 8,305 & 85.02 & 48,180 & 56,627 & 85.08 \\
\hline Indiana & 3,540 & w & w & 22,443 & $w$ & w & 25,983 & 35,256 & 73.70 \\
\hline 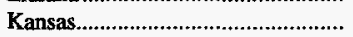 & - & - & - & 285 & $\mathbf{w}$ & w & 285 & $\mathbf{w}$ & w \\
\hline 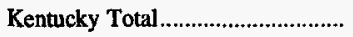 & 94,017 & 125,062 & 75.18 & 59,367 & 78,112 & 76.00 & 153,384 & 203,173 & 75.49 \\
\hline 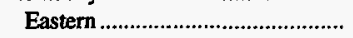 & 69,667 & 89,818 & 77.56 & 48,543 & 62,293 & 77.93 & 118,210 & 152,111 & 77.71 \\
\hline 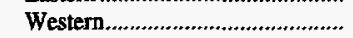 & 24,350 & 35,243 & 69.09 & 10,825 & 15,819 & 68.43 & 35,174 & 51,062 & 68.89 \\
\hline 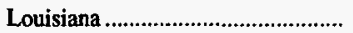 & - & - & - & 3,719 & w & $\mathbf{w}$ & 3,719 & w & w \\
\hline 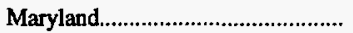 & 2,887 & $w$ & $w$ & 756 & w & w & 3,643 & 4,408 & 82.65 \\
\hline 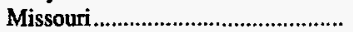 & - & - & - & 539 & 1,081 & 49.88 & 539 & 1,081 & 49.88 \\
\hline Montana & - & - & - & 39,441 & 51,597 & 76.44 & 39,441 & 51,597 & 76.44 \\
\hline 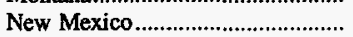 & 640 & $\mathbf{w}$ & $\mathbf{w}$ & 26,173 & $w$ & $\mathbf{w}$ & 26,813 & 32,760 & 81.85 \\
\hline 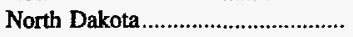 & - & - & - & 30,112 & 34,464 & 87.37 & 30,112 & 34,464 & 87.37 \\
\hline Ohio & 13,077 & 14,823 & 88.23 & 12,958 & 19,188 & 67.53 & 26,035 & 34,011 & 76.55 \\
\hline Oklahoma & 25 & $\mathbf{w}$ & w & 1,845 & $\mathbf{w}$ & w & 1,870 & 2,557 & 73.14 \\
\hline 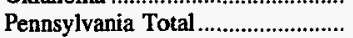 & 41,241 & 50,317 & 81.96 & 19,587 & 26,870 & 72.90 & 60,828 & 77,187 & 78.81 \\
\hline 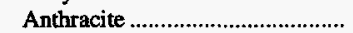 & 270 & 447 & 60.42 & 4,124 & 6,100 & 67.61 & 4,394 & 6,547 & 67.12 \\
\hline 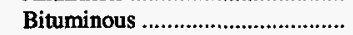 & 40,971 & 49,871 & 82.16 & 15,463 & 20,769 & 74.45 & 56,434 & 70,640 & 79.89 \\
\hline 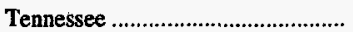 & 1,959 & w & w & 1,248 & w & w & 3,207 & 3,750 & 85.51 \\
\hline 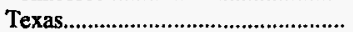 & - & - & - & 52,684 & 54,758 & 96.21 & 52,684 & 54,758 & 96.21 \\
\hline Utah & 25,167 & 30,888 & 81.48 & - & - & - & 25,167 & 30,888 & 81.48 \\
\hline 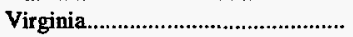 & 25,308 & 30,753 & 82.29 & 8,721 & 12,284 & 71.00 & 34,029 & 43,037 & 79.07 \\
\hline 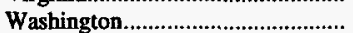 & - & - & - & 4,868 & $\mathbf{w}$ & w & 4,868 & $\mathbf{w}$ & w \\
\hline West Virginia Total ........................ & 109,967 & 132,282 & 83.13 & 52,888 & 72,555 & 72.89 & 162,855 & 204,837 & 79.50 \\
\hline 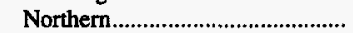 & 40,704 & 48,429 & 84.05 & 5,339 & 7,926 & 67.36 & 46,043 & 56,355 & 81.70 \\
\hline 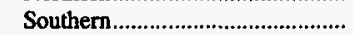 & 69,263 & 83,853 & 82.60 & 47,550 & 64,629 & 73.57 & 116,812 & 148,482 & 78.67 \\
\hline Wyoming & 2,008 & w & w & 261,814 & w & w & 263,822 & 337,184 & 78.24 \\
\hline Appalachian Total 1 .................... & 281,710 & 346,427 & 81.32 & 151,676 & 205,461 & 73.82 & 433,387 & 551,888 & 78.53 \\
\hline Interior Total 1 & 69,033 & 87,940 & $\mathbf{7 8 . 5 0}$ & 99,416 & 117,453 & 84.64 & 168,450 & 205,393 & 82.01 \\
\hline Western Total 1 & 45,001 & 58,023 & $\mathbf{7 7 . 5 6}$ & 384,576 & 483,751 & 79.50 & 429,577 & 541,773 & 79.29 \\
\hline East of Miss. River.......................... & 350,718 & 434,341 & 80.75 & 192,006 & 260,491 & 73.71 & 542,724 & 694,832 & 78.11 \\
\hline West of Miss. River........................... & 45,026 & 58,048 & $\mathbf{7 7 . 5 7}$ & 443,663 & 546,174 & 81.23 & 488,689 & 604,222 & 80.88 \\
\hline 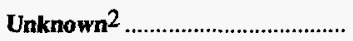 & 504 & NA & NA & 1,056 & NA & NA & 1,560 & NA & NA \\
\hline 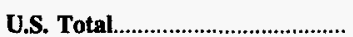 & 396,249 & 492,389 & 80.37 & 636,725 & 806,664 & 78.80 & $1,032,974$ & $1,299,054$ & 79.40 \\
\hline
\end{tabular}

1 For a definition of coal-producing regions, see Appendix $\mathbf{C}$.

2 Includes mines with production of less than 10,000 short tons, which are required to provide only production data.

w Witheld to avoid disclosure of individual company data.

NA Not available.

Notes: Productive capacity is the maximum amount of coal that can be produced annually as reported by mining companies on Form EIA-7A. Capacity utilization is the ratio of total production to annual productive capacity as reported by mining companies on Form EIA-7A. Coal production excludes silt, culm, refuse bank, slurry dam, and dredge operations except for Pennsylvania anthracite. Totals may not equal sum of components due to independent rounding.

Sources: Energy Information Administration, Form EIA-7A, "Coal Production Report"; State Mining Agency Coal Production Reports; and/or U.S. Department of Labor, Mine Safety and Health Administration, Form 7000-2, "Quarterly Mine Employment and Coal Production Report." 
Table 19. Productive Capacity and Capacity Utilization of Underground Coal Mines by State and Mining Method, 1995

(Thousand Short Tons)

\begin{tabular}{|c|c|c|c|c|c|c|c|c|}
\hline \multirow[b]{2}{*}{$\begin{array}{l}\text { Coal-Producing } \\
\text { State and Region }\end{array}$} & \multicolumn{2}{|c|}{ Continuous 1} & \multicolumn{2}{|c|}{ Conventional 1} & \multicolumn{2}{|c|}{ Longwall1 } & \multicolumn{2}{|c|}{ Other 12} \\
\hline & $\begin{array}{l}\text { Productive } \\
\text { Capacity }\end{array}$ & $\begin{array}{c}\text { Capacity } \\
\text { Utilization } \\
\text { (percent) }\end{array}$ & $\begin{array}{c}\text { Productive } \\
\text { Capacity }\end{array}$ & $\begin{array}{c}\text { Capacity } \\
\text { Utilization } \\
\text { (percent) }\end{array}$ & $\begin{array}{l}\text { Productive } \\
\text { Capacity }\end{array}$ & $\begin{array}{c}\text { Capacity } \\
\text { Utilization } \\
\text { (percent) }\end{array}$ & $\begin{array}{c}\text { Productive } \\
\text { Capacity }\end{array}$ & $\begin{array}{c}\text { Capacity } \\
\text { Utilization } \\
\text { (percent) }\end{array}$ \\
\hline 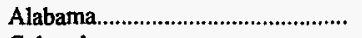 & 7,805 & 70.79 & - & - & 14,823 & 81.49 & - & - \\
\hline Colorado & w & w & $\mathbf{w}$ & $\mathbf{w}$ & 13,697 & 82.60 & $w$ & $\mathbf{w}$ \\
\hline Illinois & 32,374 & 83.05 & - & - & 15,947 & 89.25 & - & - \\
\hline Indiana & w & w & - & - & - & - & - & - \\
\hline 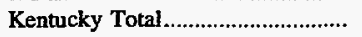 & 87,951 & 78.01 & 19,798 & 67.90 & 16,697 & 68.39 & 615 & 88.33 \\
\hline Eastern & 62,705 & 79.81 & w & $\mathbf{w}$ & $w$ & w & 615 & 88.33 \\
\hline 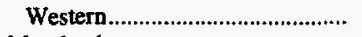 & 25,246 & 73.54 & $\mathbf{w}$ & $\mathbf{w}$ & $\mathbf{w}$ & $\mathbf{w}$ & - & - \\
\hline 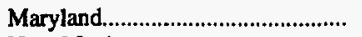 & w & $\mathbf{w}$ & - & - & $w$ & w & - & - \\
\hline 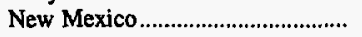 & - & - & - & - & w & w & - & - \\
\hline Ohio & 4,197 & 86.85 & - & - & 10,626 & 88.77 & - & - \\
\hline Oklahoma & $w$ & w & - & - & - & - & - & - \\
\hline Pennsylvania Total ................................ & 22,367 & 73.77 & $\mathbf{w}$ & $\mathbf{w}$ & 26,984 & 89.77 & $\mathbf{w}$ & $\mathbf{w}$ \\
\hline Anthracite & w & w & $\mathbf{w}$ & $\mathbf{w}$ & - & - & $\mathbf{w}$ & $\mathbf{w}$ \\
\hline 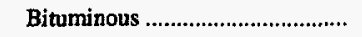 & $\mathbf{w}$ & w & $\mathbf{w}$ & $\mathbf{w}$ & 26,984 & 89.77 & - & - \\
\hline Tennessee & $\mathbf{w}$ & $\mathbf{w}$ & - & - & - & - & - & - \\
\hline Utah & 8,877 & 61.69 & $\mathbf{w}$ & w & 19,878 & 91.24 & $\mathbf{w}$ & $w$ \\
\hline 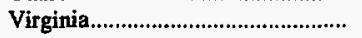 & 16,523 & 84.81 & 6,747 & 78.47 & $\mathbf{w}$ & w & $\mathbf{w}$ & $w$ \\
\hline West Virginia Total ............................ & 69,673 & 80.71 & 17,859 & 77.91 & 44,750 & 88.99 & - & - \\
\hline Northern & 11,928 & 77.26 & 4,042 & 75.75 & 32,459 & 87.58 & - & - \\
\hline 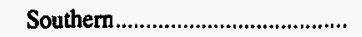 & 57,746 & 81.42 & 13,817 & 78.54 & 12,291 & 92.71 & - & - \\
\hline 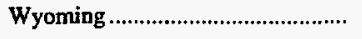 & w & w & $\mathbf{w}$ & $\mathbf{w}$ & $\mathbf{w}$ & $\mathbf{w}$ & - & - \\
\hline Appalachian Total ${ }^{3} \ldots \ldots \ldots \ldots \ldots \ldots$ & 186,527 & 79.67 & $\mathbf{w}$ & $\mathbf{w}$ & $\mathbf{w}$ & $\mathbf{w}$ & $\mathbf{w}$ & $\mathbf{w}$ \\
\hline Interior Total ${ }^{3}$ & 61,996 & 79.06 & $\mathbf{w}$ & $\mathbf{w}$ & $\mathbf{w}$ & $\mathbf{w}$ & - & - \\
\hline 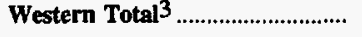 & 15,550 & 68.41 & $\mathbf{w}$ & $\mathbf{w}$ & 37,800 & 84.29 & $\mathbf{w}$ & $\mathbf{w}$ \\
\hline East of Miss. River......................... & 248,498 & 79.52 & $\mathbf{w}$ & $\mathbf{w}$ & 139,766 & 85.40 & $\mathbf{w}$ & $\mathbf{w}$ \\
\hline West of Miss. River......................... & 15,575 & 68.46 & $\mathbf{w}$ & $\mathbf{w}$ & 37,800 & 84.29 & $\mathbf{w}$ & $\mathbf{w}$ \\
\hline 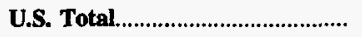 & 264,073 & 78.86 & 48,586 & 71.05 & 177,567 & 85.16 & 2,163 & 81.06 \\
\hline
\end{tabular}

1 Calculated by multiplying reported mining method percentages by the individual mine capacity.

2 Includes shortwall, scoop loading, hand loading and unknown.

3 For a definition of coal-producing regions, see Appendix $\mathbf{C}$.

withheld to avoid disclosure of individual company data.

Notes: Productive capacity is the maximum amount of coal that can be produced annually as reported by mining companies on Form EIA-7A. Capacity utilization is the ratio of total production to annual productive capacity as reported by mining companies on Form EIA-7A. Excludes mines producing less than 10,000 short tons, which are not required to provide these data. Totals may not equal sum of components due to independent rounding.

Source: Energy Information Administration, Form ELA-7A, "Coal Production Report." 
Table 20. Productive Capacity and Capacity Utilization of Coal Mines by State and Coal Rank, 1995

(Thousand Short Tons)

\begin{tabular}{|c|c|c|c|c|c|c|c|c|}
\hline \multirow[b]{2}{*}{$\begin{array}{l}\text { Coal-Producing } \\
\text { State and Region }\end{array}$} & \multicolumn{2}{|c|}{ Bituminous } & \multicolumn{2}{|c|}{ Subbituminous } & \multicolumn{2}{|c|}{ Lignite } & \multicolumn{2}{|c|}{ Anthracite } \\
\hline & $\begin{array}{c}\text { Productive } \\
\text { Capacity }\end{array}$ & $\begin{array}{c}\text { Capacity } \\
\text { Utilization } \\
\text { (percent) }\end{array}$ & $\begin{array}{l}\text { Productive } \\
\text { Capacity }\end{array}$ & $\begin{array}{c}\text { Capacity } \\
\text { Utilization } \\
\text { (percent) }\end{array}$ & $\begin{array}{c}\text { Productive } \\
\text { Capacity }\end{array}$ & $\begin{array}{c}\text { Capacity } \\
\text { Utilization } \\
\text { (percent) }\end{array}$ & $\begin{array}{c}\text { Productive } \\
\text { Capacity }\end{array}$ & $\begin{array}{c}\text { Capacity } \\
\text { Utilization } \\
\text { (percent) }\end{array}$ \\
\hline 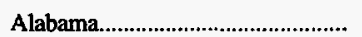 & 32,546 & 75.52 & - & - & - & - & - & - \\
\hline Alaska & - & - & $\mathbf{w}$ & w & - & - & - & - \\
\hline 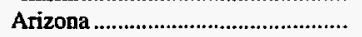 & $\mathbf{w}$ & $\mathbf{w}$ & - & - & - & - & - & - \\
\hline 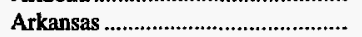 & - & - & - & - & - & - & $\mathbf{w}$ & $\mathbf{w}$ \\
\hline 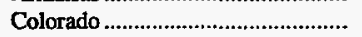 & 21,785 & 78.83 & 10,650 & 80.15 & - & - & - & - \\
\hline 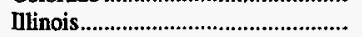 & 56,627 & 85.08 & - & - & - & - & - & - \\
\hline Indiana & 35,256 & 73.70 & - & - & - & - & - & - \\
\hline 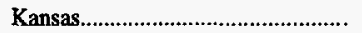 & $\mathbf{w}$ & w & - & - & - & - & - & - \\
\hline 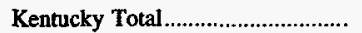 & 203,173 & 75.49 & - & - & - & - & - & - \\
\hline 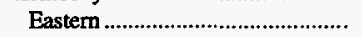 & 152,111 & 77.71 & - & - & - & - & - & - \\
\hline 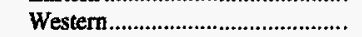 & 51,062 & 68.89 & - & - & - & - & - & - \\
\hline 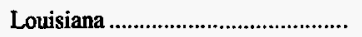 & - & - & - & - & $\mathbf{w}$ & w & - & - \\
\hline 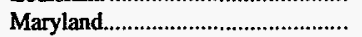 & 4,408 & 82.65 & - & - & - & - & - & - \\
\hline 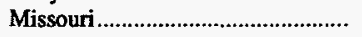 & 1,081 & 49.88 & - & - & - & - & - & - \\
\hline 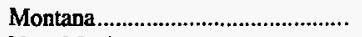 & - & - & $\mathbf{w}$ & $\mathbf{w}$ & $\mathbf{w}$ & $\mathbf{w}$ & - & - \\
\hline 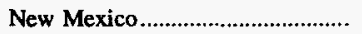 & $w$ & $w$ & $\mathbf{w}$ & $\mathrm{w}$ & - & - & - & - \\
\hline 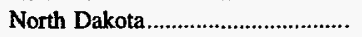 & - & - & - & - & 34,464 & 87.37 & - & - \\
\hline Ohio & 34,011 & 76.55 & - & - & - & - & - & - \\
\hline Oklahoma & 2,557 & 73.14 & - & - & - & - & - & - \\
\hline Pennsylvania Total ............................. & 70,640 & 79.89 & - & - & - & - & 6,547 & 67.12 \\
\hline Anthracite & - & - & - & - & - & - & 6,547 & 67.12 \\
\hline 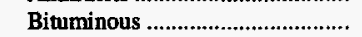 & 70,640 & 79.89 & - & - & - & - & - & - \\
\hline Tennessee & 3,750 & 85.51 & - & - & - & - & - & - \\
\hline 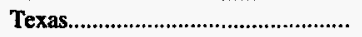 & w & $\mathbf{w}$ & - & - & $\mathbf{w}$ & $\mathbf{w}$ & - & - \\
\hline 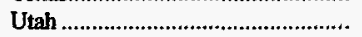 & 30,888 & 81.48 & - & - & - & - & - & - \\
\hline 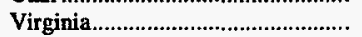 & 43,037 & 79.07 & - & - & - & - & - & - \\
\hline 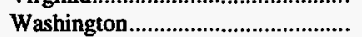 & w & w & $\mathbf{w}$ & $\mathbf{w}$ & - & - & - & - \\
\hline West Virginia Total ......................... & 204,837 & 79.50 & - & - & - & - & - & - \\
\hline 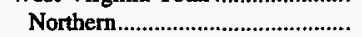 & 56,355 & 81.70 & - & - & - & - & - & - \\
\hline 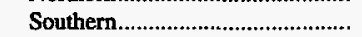 & 148,482 & 78.67 & - & - & - & - & - & - \\
\hline 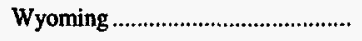 & $w$ & $w$ & $\mathbf{w}$ & $\mathbf{w}$ & - & - & - & - \\
\hline 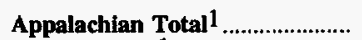 & 545,341 & 78.67 & - & - & - & - & 6,547 & 67.12 \\
\hline 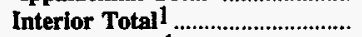 & 147,587 & 76.12 & - & - & $\mathbf{5 7 , 7 8 8}$ & 97.06 & $\mathbf{w}$ & $\mathbf{w}$ \\
\hline 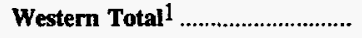 & 91,383 & 77.89 & 415,486 & 78.94 & 34,905 & 87.12 & - & - \\
\hline East of Miss. River........................... & 688,285 & 78.21 & - & - & - & - & 6,547 & 67.12 \\
\hline West of Miss. River......................... & 96,025 & 77.26 & 415,486 & 78.94 & 92,693 & 93.32 & $\mathbf{w}$ & $w$ \\
\hline 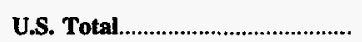 & 784,310 & 78.10 & 415,486 & 78.94 & 92,693 & 93.32 & 26,547 & 267.12 \\
\hline
\end{tabular}

1 For a definition of coal-producing regions, see Appendix $\mathbf{C}$.

2 Does not include Arkansas.

Withheid to avoid disclosure of individual company data.

Notes: Refer to the Classification of Coals by Rank table in Appendix C for coal rank definitions. Productive capacity is the maximum amount of coal that can be produced annually as reported by mining companies on Form EIA-7A. Capacity utilization is the ratio of total production to annual productive capacity as reported by mining companies on Form EIA-7A. Excludes silt, culm, refuse bank, slurry dam, and dredge operations except for Pennsylvania anthracite. Excludes mines producing less than 10,000 short tons, which are not required to provide these data. Totals may not equal sum of components due to independent rounding.

Source: Energy Information Administration, Form EIA-7A, “Coal Production Report." 
Table 21. Productive Capacity and Capacity Utilization of Coal Mines by State and Mine Production Range, 1995

(Thousand Short Tons, Percent)

\begin{tabular}{|c|c|c|c|c|c|c|c|c|c|c|}
\hline \multirow{3}{*}{$\begin{array}{l}\text { Coal-Producing } \\
\text { State and Region }\end{array}$} & \multicolumn{5}{|c|}{ Productive Capacity } & \multicolumn{5}{|c|}{$\begin{array}{c}\text { Capacity } \\
\text { Utilization } \\
\text { (percent) }\end{array}$} \\
\hline & \multicolumn{10}{|c|}{$\begin{array}{l}\text { Mine Production Range } \\
\text { (thousand short tons) }\end{array}$} \\
\hline & $\begin{array}{l}1,000 \\
\text { and } \\
\text { over }\end{array}$ & $\begin{array}{c}500 \\
\text { to } \\
1,000\end{array}$ & $\begin{array}{c}200 \\
\text { to } \\
500\end{array}$ & $\begin{array}{c}100 \\
\text { to } \\
200\end{array}$ & $\begin{array}{c}10 \\
\text { to } \\
109\end{array}$ & $\begin{array}{l}1,000 \\
\text { and } \\
\text { over }\end{array}$ & $\begin{array}{c}500 \\
\text { to } \\
1,000\end{array}$ & $\begin{array}{c}200 \\
\text { to } \\
500\end{array}$ & $\begin{array}{c}100 \\
\text { to } \\
200\end{array}$ & $\begin{array}{c}10 \\
\text { to } \\
100\end{array}$ \\
\hline 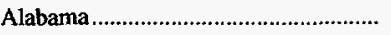 & 21,921 & 5,168 & $\mathbf{w}$ & $\mathbf{w}$ & 2,815 & 79.29 & 71.68 & $w$ & $\mathbf{w}$ & 55.84 \\
\hline Alaska & w & - & - & - & - & w & - & - & - & - \\
\hline Arizona & w & - & - & - & - & w & - & - & - & - \\
\hline 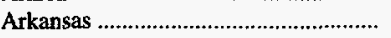 & - & - & - & - & $\mathbf{w}$ & - & - & - & - & $\mathbf{w}$ \\
\hline Colorado & 25,750 & $\mathbf{w}$ & 3,035 & - & $\mathbf{w}$ & 87.68 & $w$ & 58.09 & - & $\mathbf{w}$ \\
\hline Illinois & 51,394 & w & w & w & $\mathbf{w}$ & 87.71 & w & $\mathbf{w}$ & $\mathbf{w}$ & $\mathbf{w}$ \\
\hline Indiana & $\mathbf{w}$ & 6,584 & 7,976 & 2,642 & $\mathbf{w}$ & w & 65.72 & 58.88 & 34.05 & $\mathbf{w}$ \\
\hline Kansas & - & - & $\mathbf{w}$ & - & - & - & - & $\mathbf{w}$ & - & - \\
\hline 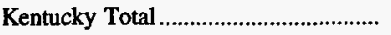 & 57,958 & 61,615 & 43,732 & 19,553 & 20,315 & 82.30 & 78.76 & 75.91 & 70.36 & 50.22 \\
\hline Eastern & 32,100 & 45,230 & 37,609 & 17,559 & 19,614 & 89.29 & 82.57 & 77.76 & 74.76 & 50.11 \\
\hline Western & 25,858 & 16,384 & 6,123 & 1,995 & 702 & 73.62 & 68.24 & 64.54 & 31.61 & 53.34 \\
\hline 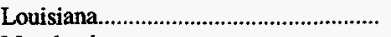 & w & $\mathbf{w}$ & - & - & - & w & $\mathbf{w}$ & - & - & - \\
\hline 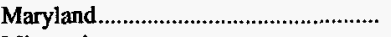 & $\mathbf{w}$ & - & w & $\mathbf{w}$ & 595 & $\mathbf{w}$ & - & w & $\mathbf{w}$ & 68.15 \\
\hline 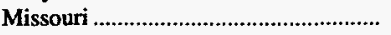 & - & - & - & $\mathbf{w}$ & w & - & - & - & $\mathbf{w}$ & w \\
\hline 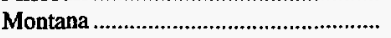 & w & - & $\mathbf{w}$ & - & - & w & - & $\mathbf{w}$ & - & - \\
\hline 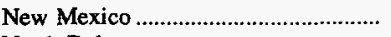 & w & $\mathbf{w}$ & - & - & - & w & $\mathbf{w}$ & - & - & - \\
\hline North Dakota & 34,464 & - & - & - & - & 87.37 & - & - & - & - \\
\hline Ohio & 14,421 & 6,766 & 5,519 & 2,853 & 4,452 & 86.31 & 80.58 & 72.65 & 71.67 & 46.76 \\
\hline 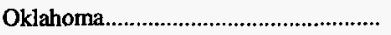 & - & - & 1,770 & w & w & - & - & 82.36 & $\mathbf{w}$ & w \\
\hline 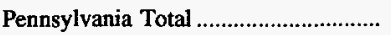 & 38,030 & 7,029 & 14,777 & 6,627 & 10,724 & 84.37 & 90.30 & 71.16 & 75.99 & 63.81 \\
\hline 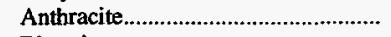 & - & - & 2,648 & 1,177 & 2,722 & - & - & 77.65 & 83.03 & 50.01 \\
\hline 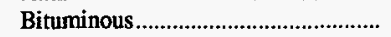 & 38,030 & 7,029 & 12,129 & 5,451 & 8,001 & 84.37 & 90.30 & 69.75 & 74.47 & 68.50 \\
\hline 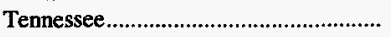 & - & w & 1,693 & 930 & $\mathbf{w}$ & - & w & 89.07 & 80.56 & w \\
\hline Texas & 53,538 & - & $\mathbf{w}$ & - & $\mathbf{w}$ & 97.32 & - & $\mathbf{w}$ & - & w \\
\hline Utah. & 26,810 & w & $w$ & w & - & 88.89 & $\mathbf{w}$ & w & $\mathbf{w}$ & - \\
\hline 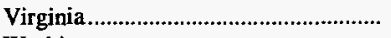 & w & $\mathbf{w}$ & 14,493 & 6,950 & 7,704 & w & $\mathbf{w}$ & 85.23 & 78.19 & 63.43 \\
\hline 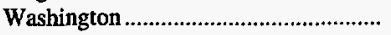 & $\mathbf{w}$ & - & $\mathbf{w}$ & - & - & $\mathbf{w}$ & - & $w$ & - & - \\
\hline West Virginia Total.................................. & 108,796 & 29,759 & 40,542 & 11,290 & 14,451 & 86.93 & 85.09 & 68.61 & 67.58 & 52.00 \\
\hline 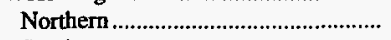 & 39,938 & 6,578 & 4,661 & 2,080 & 3,098 & 86.81 & 73.47 & 76.41 & 67.30 & 50.97 \\
\hline Southern & 68,858 & 23,180 & 35,881 & 9,210 & 11,353 & 87.00 & 88.38 & 67.59 & 67.65 & 52.29 \\
\hline Wyoming & 326,484 & w & $\mathbf{w}$ & - & w & 80.14 & w & w & - & w \\
\hline Appalachian Total ${ }^{1}$ & 227,268 & 99,359 & 117,240 & 47,057 & 60,965 & 85.83 & 83.27 & 74.48 & $\mathbf{7 3 . 7 7}$ & 55.05 \\
\hline 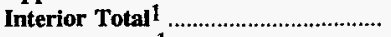 & 151,187 & 27,186 & 18,854 & $\mathbf{w}$ & $\mathbf{w}$ & 89.32 & 66.54 & 63.71 & $\mathbf{w}$ & $\mathbf{w}$ \\
\hline 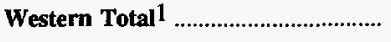 & 517,520 & $\mathbf{9 , 5 3 0}$ & 12,848 & $\mathbf{w}$ & $\mathbf{w}$ & 81.50 & 42.17 & 28.25 & $\boldsymbol{w}$ & $\mathbf{w}$ \\
\hline 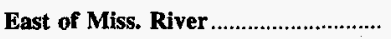 & 322,016 & 125,695 & 132,939 & 51,811 & 62,371 & 85.44 & 79.55 & 73.01 & 70.18 & 54.84 \\
\hline West of Miss. River ............................ & 573,958 & 10,380 & 16,002 & 1,264 & 2,618 & 83.06 & 46.79 & 36.86 & 57.48 & 17.87 \\
\hline 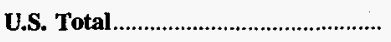 & 895,974 & 136,075 & 148,941 & 53,075 & 64,989 & 83.91 & 77.05 & 69.13 & 69.88 & $\mathbf{5 3 . 3 5}$ \\
\hline
\end{tabular}

1 For a definition of coal-producing regions, see Appendix $C$.

Witheld to avoid disclosure of individual company data.

Notes: Productive capacity is the maximum amount of coal that can be produced annually as reported by mining companies on Form EIA-7A. Capacity utilization is the ratio of total production to annual productive capacity as reported by mining companies on Form EIA-7A. Excludes silt, culm, refuse bank, slurry dam, and dredge operations except for Pennsylvania anthracite. Excludes mines producing less than 10,000 short tons, which are not required to provide these data. Totals may not equal sum of components due to independent rounding.

Source: Energy Information Administration, Form EIA-7A, "Coal Production Report." 
Table 22. Productive Capacity and Productivity of Coal Mines by State and Capacity Utilization Range, 1995

(Thousand Short Tons, Short Tons per Miner per Hour)

\begin{tabular}{|c|c|c|c|c|c|c|c|c|c|c|c|c|}
\hline \multirow{3}{*}{$\begin{array}{l}\text { Coal-Producing } \\
\text { State and Region }\end{array}$} & \multicolumn{6}{|c|}{ Productive Capacity } & \multicolumn{6}{|c|}{ Productivity } \\
\hline & \multicolumn{12}{|c|}{$\begin{array}{l}\text { Capacity Utilization Range } \\
\text { (percent) }\end{array}$} \\
\hline & $\begin{array}{c}90 \\
\text { and } \\
\text { over }\end{array}$ & $\begin{array}{l}80 \\
\text { to } \\
90\end{array}$ & $\begin{array}{l}70 \\
\text { to } \\
80\end{array}$ & $\begin{array}{l}60 \\
\text { to } \\
70\end{array}$ & $\begin{array}{c}\text { Less } \\
\text { than } \\
60\end{array}$ & Total & $\begin{array}{c}90 \\
\text { and } \\
\text { over }\end{array}$ & $\begin{array}{l}80 \\
\text { to } \\
90\end{array}$ & $\begin{array}{l}70 \\
\text { to } \\
80\end{array}$ & $\begin{array}{l}60 \\
\text { to } \\
70\end{array}$ & $\begin{array}{c}\text { Less } \\
\text { than } \\
60\end{array}$ & Total \\
\hline Alabama & 11,127 & 7,213 & 2,414 & 2,691 & 9,102 & 32,546 & 2.25 & 2.98 & 2.26 & 3.16 & 1.48 & 2.24 \\
\hline 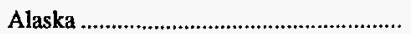 & - & - & - & w & - & $\mathrm{w}$ & - & - & - & 7.46 & - & 7.46 \\
\hline 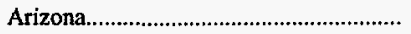 & - & w & - & - & - & w & - & 6.56 & - & - & - & 6.34 \\
\hline 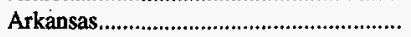 & - & $\mathbf{w}$ & - & - & - & $\mathrm{w}$ & - & 1.47 & - & - & - & 1.47 \\
\hline 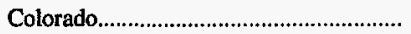 & 13,450 & 8,835 & $\mathbf{w}$ & - & w & 32,435 & 7.66 & 5.89 & 6.38 & - & 3.38 & 6.14 \\
\hline 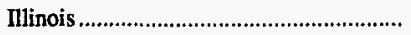 & 22,933 & 16,550 & 13,180 & - & 3,964 & 56,627 & 3.72 & 4.25 & 4.63 & - & 1.83 & 3.87 \\
\hline 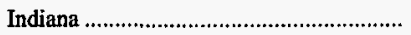 & 18,225 & w & w & $\mathbf{w}$ & 10,993 & 35,256 & 4.72 & 4.68 & 6.22 & 6.15 & 4.06 & 4.68 \\
\hline 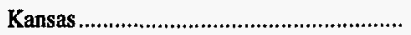 & & - & - & - & - & w & 2.22 & - & - & - & - & 2.22 \\
\hline 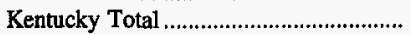 & 82,297 & 30,101 & 25,381 & 12,742 & 52,652 & 203,173 & 4.07 & 4.15 & 4.22 & 4.08 & 1.90 & 3.57 \\
\hline 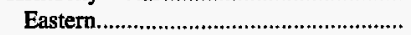 & 69,040 & $\mathrm{w}$ & $\mathbf{w}$ & 6,325 & 35,904 & 152,111 & 3.94 & 4.19 & 4.27 & 4.20 & 1.64 & 3.47 \\
\hline 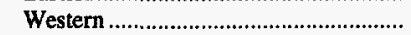 & 13,257 & w & w & 6,417 & 16,748 & 51,062 & 4.92 & 4.04 & 4.12 & 3.97 & 2.79 & 3.97 \\
\hline 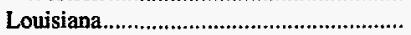 & $\mathrm{w}$ & - & - & - & - & w & 13.25 & - & - & - & - & 13.25 \\
\hline 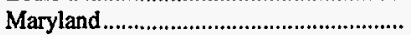 & 254 & w & $w$ & w & 269 & 4,408 & 2.40 & 5.42 & 1.95 & 2.84 & .98 & 3.82 \\
\hline Missouri ........................................................ & w & - & - & w & w & 1,081 & 1.98 & - & - & 2.20 & 3.99 & 2.55 \\
\hline Montana & $\mathrm{w}$ & w & w & w & $\mathrm{w}$ & 51,597 & 16.88 & 26.90 & 27.49 & 18.66 & 9.81 & 21.06 \\
\hline 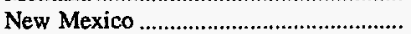 & $\mathrm{w}$ & w & w & - & $\mathrm{w}$ & 32,760 & 8.05 & 3.64 & 7.21 & - & 2.44 & 6.92 \\
\hline 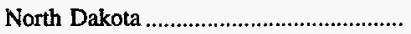 & w & w & - & w & w & 34,464 & 17.89 & 14.26 & - & 13.72 & 16.22 & 16.80 \\
\hline 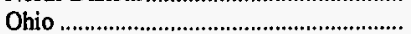 & 11,300 & 6,210 & 5,775 & 3,540 & 7,187 & 34,011 & 3.68 & 4.84 & 3.88 & 2.88 & 2.50 & 3.62 \\
\hline 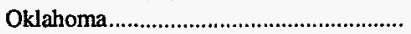 & 1,604 & - & - & - & 953 & 2,557 & 3.23 & - & - & - & 2.15 & 2.97 \\
\hline 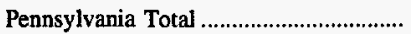 & 37,136 & 16,086 & 3,134 & 4,365 & 16,464 & 77,187 & 4.29 & 3.24 & 2.25 & 4.24 & 1.38 & 3.23 \\
\hline 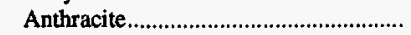 & 2,146 & 1,063 & w & w & 2,539 & 6,547 & 3.07 & 4.07 & 26.05 & 2.81 & .71 & 2.08 \\
\hline 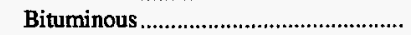 & 34,990 & 15,023 & $\mathrm{w}$ & w & 13,925 & 70,640 & 4.40 & 3.19 & 1.91 & 4.40 & 1.59 & 3.37 \\
\hline 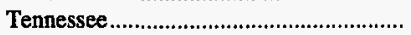 & 1,995 & w & $\mathrm{w}$ & w & w & 3,750 & 3.00 & 4.31 & 2.53 & 1.51 & .56 & 2.36 \\
\hline 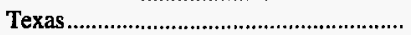 & 47,825 & w & - & - & w & 54,758 & 9.11 & 10.74 & - & - & 3.74 & 9.10 \\
\hline 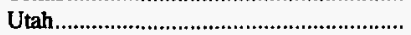 & 12,432 & w & - & w & w & 30,888 & 6.99 & 8.68 & - & 3.98 & 3.58 & 7.02 \\
\hline 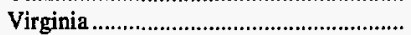 & 20,391 & 6,982 & 1,434 & 5,181 & 9,047 & 43,037 & 2.95 & 3.27 & 2.42 & 2.48 & 1.16 & 2.50 \\
\hline 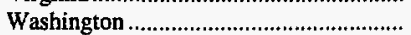 & & - & - & w & - & w & 4.26 & - & - & 3.82 & - & 4.04 \\
\hline West Virginia Total .................................... & 109,050 & 25,188 & 12,167 & 20,032 & 38,400 & 204,837 & 4.31 & 4.30 & 5.07 & 3.91 & 1.54 & 3.74 \\
\hline Northern & 27,439 & 10,671 & 4,120 & 6,445 & 7,680 & 56,355 & 4.17 & 4.11 & 6.61 & 3.36 & 1.57 & 3.72 \\
\hline 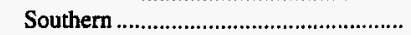 & 81,611 & 14,517 & 8,047 & 13,587 & 30,720 & 148,482 & 4.36 & 4.45 & 4.51 & 4.24 & 1.53 & 3.75 \\
\hline 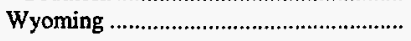 & 134,984 & 56,500 & w & w & 39,400 & 337,184 & 31.85 & 26.79 & 33.02 & 45.01 & 12.21 & 30.06 \\
\hline Appalachian Total $^{1} \ldots$ & 260,293 & 88,501 & 43,740 & 42,605 & 116,748 & 551,888 & 3.87 & $\mathbf{3 . 8 7}$ & 3.79 & 3.52 & 1.52 & $\mathbf{3 . 3 2}$ \\
\hline 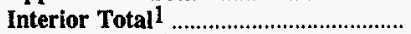 & 108,028 & 33,078 & 22,060 & $\mathbf{7 , 7 1 7}$ & $\mathbf{3 4 , 5 1 0}$ & 205,393 & 5.73 & 4.74 & 4.52 & 4.03 & 2.86 & 4.97 \\
\hline Western Total ${ }^{1} \ldots \ldots \ldots \ldots$ & 205,183 & 117,735 & 74,560 & 84,210 & 60,086 & 541,773 & 17.57 & 13.66 & 16.62 & 21.72 & 6.96 & 15.68 \\
\hline East of Miss. River & 314,708 & 115,849 & 65,800 & 50,022 & 148,453 & 694,832 & 3.93 & 3.95 & 4.01 & 3.60 & 1.69 & 3.45 \\
\hline West of Miss. River ................................. & 258,795 & 123,466 & 74,560 & 84,510 & 62,891 & 604,222 & 14.39 & 13.48 & 16.62 & 21.06 & 6.53 & 14.18 \\
\hline U.S. Total & 573,503 & 239,314 & 140,360 & 134,532 & 211,344 & $1,299,054$ & 5.83 & 6.26 & 6.74 & 7.59 & 2.11 & 5.38 \\
\hline
\end{tabular}

1 For a definition of coal-producing regions, see Appendix C.

w Withheld to avoid disclosure of individual company data.

Notes: Productivity is calculated by dividing total coal production by the total direct labor hours worked by all employees engaged in production, preparation, processing, development, maintenance, repair, and shop or yard work at mining operations. Excludes office workers. Includes mining operations management and all technical and engineering personnel. Productive capacity is the maximum amount of coal that can be produced annually as reported by mining companies on Form EIA-7A. Capacity utilization is the ratio of total production to annual productive capacity as reported by mining companies on Form EIA-7A. Excludes silt, culm, refuse bank, slurry dam, and dredge operations except for Pennsylvania anthracite. Excludes mines producing less than 10,000 short tons and preparation plants with less than 5,000 employee hours, which are not required to provide these data. Totals may not equal sum of components due to independent rounding.

Sources: Energy Information Administration, Form EIA-7A, "Coal Production Report"; State Mining Agency Coal Production Reports; and/or U.S. Department of Labor, Mine Safety and Health Administration, Form 7000-2, "Quarterly Mine Employment and Coal Production Report." 
Table 23. Productive Capacity and Capacity Utilization of Coal Mines by State and Recoverable Reserves Range, 1995 (Thousand Short Tons)

\begin{tabular}{|c|c|c|c|c|c|c|c|c|}
\hline \multirow{3}{*}{$\begin{array}{l}\text { Coal-Producing } \\
\text { State and Region }\end{array}$} & \multicolumn{8}{|c|}{$\begin{array}{l}\text { Recoverable Reserves Range } \\
\text { (million short tons) }\end{array}$} \\
\hline & \multicolumn{2}{|c|}{50 and over } & \multicolumn{2}{|c|}{10 to 50} & \multicolumn{2}{|c|}{0 to 10} & \multicolumn{2}{|c|}{ Total } \\
\hline & $\begin{array}{c}\text { Productive } \\
\text { Capacity }\end{array}$ & $\begin{array}{c}\text { Capacity } \\
\text { Utilization } \\
\text { (percent) }\end{array}$ & $\begin{array}{l}\text { Productive } \\
\text { Capacity }\end{array}$ & $\begin{array}{l}\text { Capacity } \\
\text { Utilization } \\
\text { (percent) }\end{array}$ & $\begin{array}{c}\text { Productive } \\
\text { Capacity }\end{array}$ & $\begin{array}{l}\text { Capacity } \\
\text { Utilization } \\
\text { (percent) }\end{array}$ & $\begin{array}{c}\text { Productive } \\
\text { Capacity }\end{array}$ & $\begin{array}{l}\text { Capacity } \\
\text { Utilization } \\
\text { (percent) }\end{array}$ \\
\hline Alabama. & 11,469 & 77.36 & 11,336 & 74.90 & 9,742 & 74.07 & 32,546 & 75.52 \\
\hline 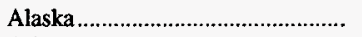 & w & w & - & - & - & - & $w$ & w \\
\hline 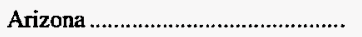 & w & $\mathbf{w}$ & - & - & - & - & $w$ & $w$ \\
\hline 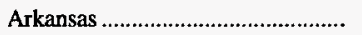 & - & - & - & - & $\mathrm{w}$ & $\mathbf{w}$ & $\mathrm{w}$ & $\mathbf{w}$ \\
\hline Colorado & 18,150 & 92.04 & 10,500 & 68.81 & 3,785 & 47.04 & 32,435 & 79.27 \\
\hline 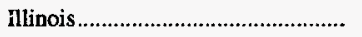 & 16,107 & 92.57 & 26,550 & 81.44 & 13,969 & 83.36 & 56,627 & 85.08 \\
\hline Indiana & w & $\mathbf{w}$ & w & $\mathbf{w}$ & 20,084 & 68.91 & 35,256 & 73.70 \\
\hline 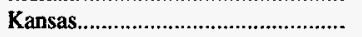 & - & - & - & - & w & w & w & w \\
\hline 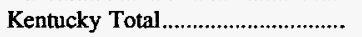 & 18,263 & 65.96 & 33,398 & 71.34 & 151,512 & 77.56 & 203,173 & 75.49 \\
\hline 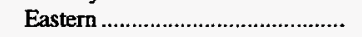 & w & $\mathbf{w}$ & w & $\mathbf{w}$ & 126,767 & 78.76 & 152,111 & 77.71 \\
\hline 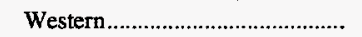 & w & w & $\mathrm{w}$ & $\mathbf{w}$ & 24,745 & 71.39 & 51,062 & 68.89 \\
\hline 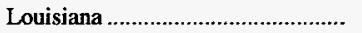 & w & $\mathbf{w}$ & $\mathrm{w}$ & $\mathbf{w}$ & - & - & $w$ & w \\
\hline Maryland & - & - & $\mathrm{w}$ & $\mathbf{w}$ & w & w & 4,408 & 82.65 \\
\hline 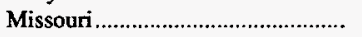 & - & - & - & - & 1,081 & 49.88 & 1,081 & 49.88 \\
\hline 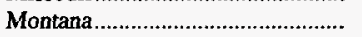 & $w$ & $w$ & $w$ & $w$ & - & - & 51,597 & 76.44 \\
\hline 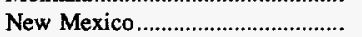 & $\mathbf{w}$ & $\mathbf{w}$ & - & - & $w$ & $\mathbf{w}$ & 32,760 & 81.85 \\
\hline 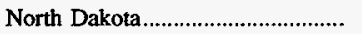 & 34,464 & 87.37 & - & - & - & - & 34,464 & 87.37 \\
\hline Ohio & 10,921 & 89.05 & 6,554 & 71.62 & 16,536 & 70.25 & 34,011 & 76.55 \\
\hline Oklahoma & - & - & w & $\mathbf{w}$ & $\mathbf{w}$ & w & 2,557 & 73.14 \\
\hline 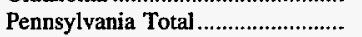 & 24,830 & 85.98 & 16,923 & 74.17 & 35,434 & 75.99 & 77,187 & 78.81 \\
\hline Anthracite & - & - & $\mathrm{w}$ & w & $w$ & w & 6,547 & 67.12 \\
\hline 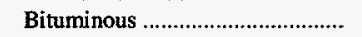 & 24,830 & 85.98 & $w$ & w & $\mathbf{w}$ & w & 70,640 & 79.89 \\
\hline 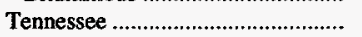 & - & - & $w$ & $\mathbf{w}$ & $w$ & w & 3,750 & 85.51 \\
\hline Texas. & 44,901 & 97.37 & w & w & $\mathbf{w}$ & $w$ & 54,758 & 96.21 \\
\hline 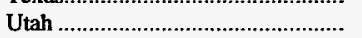 & w & w & 14,956 & 71.11 & $\mathbf{w}$ & w & 30,888 & 81.48 \\
\hline 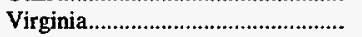 & - & - & 9,412 & 82.05 & 33,624 & 78.24 & 43,037 & 79.07 \\
\hline 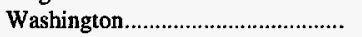 & - & - & $\mathrm{w}$ & $w$ & w & w & w & w \\
\hline West Virginia Total & 34,467 & 83.74 & 58,845 & 83.47 & 111,525 & 76.11 & 204,837 & 79.50 \\
\hline 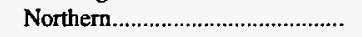 & 22,500 & 87.52 & 18,972 & 79.14 & 14,883 & 76.17 & 56,355 & 81.70 \\
\hline 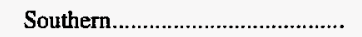 & 11,967 & 76.64 & 39,873 & 85.52 & 96,642 & 76.10 & 148,482 & 78.67 \\
\hline 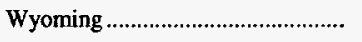 & 326,784 & 79.33 & - & - & 10,400 & 44.22 & 337,184 & 78.24 \\
\hline Appalachian Total 1 .......................... & 82,833 & 84.46 & 130,674 & 78.60 & 338,380 & 77.05 & 551,888 & 78.53 \\
\hline Interior Total 1 & 84,197 & 89.74 & 54,225 & 79.62 & 66,971 & 74.23 & 205,393 & 82.01 \\
\hline 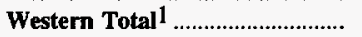 & 487,088 & 80.72 & 36,518 & 75.28 & 18,167 & 48.95 & 541,773 & 79.29 \\
\hline East of Miss. River. & 119,230 & 82.97 & 178,424 & 78.43 & 397,178 & 76.51 & 694,832 & 78.11 \\
\hline West of Miss. River........................ & 534,889 & 82.22 & 42,993 & 77.79 & 26,339 & 58.67 & 604,222 & $\mathbf{8 0 . 8 8}$ \\
\hline 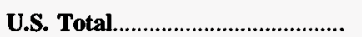 & 654,119 & 82.36 & 221,418 & 78.30 & 423,517 & 75.40 & $1,299,054$ & 79.40 \\
\hline
\end{tabular}

1 For a definition of coal-producing regions, see Appendix C.

w Withheld to avoid disclosure of individual company data.

Notes: Recoverable reserves represent the quantity of coal that can be recovered (i.e., mined) from existing coal reserves at reporting mines. Productive capacity is the maximum amount of coal that can be produced annually as reported by mining companies on Form EIA-7A. Capacity utilization is the ratio of total production to productive capacity as reported by mining companies on Form EIA-7A. Excludes silt, culm, refuse bank, slurry dam, and dredge operations except for Pennsylvania anthracite. Excludes mines producing less than 10,000 short tons, which are not required to provide these data. Totals may not equal sum of components due to independent rounding.

Sources: Energy Information Administration, Form EIA-7A, "Coal Production Report"; State Mining Agency Coal Production Reports; and/or U.S. Department of Labor, Mine Safety and Health Administration, Form 7000-2, "Quarterly Mine Employment and Coal Production Report." 
Table 24. Productive Capacity and Capacity Utilization of Coal Mines by State, Mine Type, and Union Type, 1995

(Thousand Short Tons)

\begin{tabular}{|c|c|c|c|c|c|c|c|c|}
\hline \multirow[b]{2}{*}{$\begin{array}{l}\text { Coal-Producing } \\
\text { State and Region }\end{array}$} & \multicolumn{2}{|c|}{ UMWA } & \multicolumn{2}{|c|}{ Other Unions } & \multicolumn{2}{|c|}{ Nonunion } & \multicolumn{2}{|c|}{ Total } \\
\hline & $\begin{array}{c}\text { Productive } \\
\text { Capacity }\end{array}$ & $\begin{array}{c}\text { Capacity } \\
\text { Utilization } \\
\text { (percent) }\end{array}$ & $\begin{array}{l}\text { Productive } \\
\text { Capacity }\end{array}$ & $\begin{array}{c}\text { Capacity } \\
\text { Utilization } \\
\text { (percent) }\end{array}$ & $\begin{array}{c}\text { Productive } \\
\text { Capacity }\end{array}$ & $\begin{array}{c}\text { Capacity } \\
\text { Utilization } \\
\text { (percent) }\end{array}$ & $\begin{array}{c}\text { Productive } \\
\text { Capacity }\end{array}$ & $\begin{array}{l}\text { Capacity } \\
\text { Utilization } \\
\text { (percent) }\end{array}$ \\
\hline Alabama & 23,512 & 76.14 & - & - & 9,034 & 73.91 & 32,546 & 75.52 \\
\hline 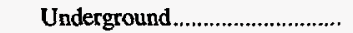 & w & w & - & - & $w$ & w & 22,628 & 77.80 \\
\hline 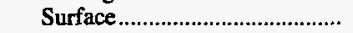 & $\mathbf{w}$ & $\mathbf{w}$ & - & - & $\mathbf{w}$ & w & 9,919 & 70.33 \\
\hline Alaska & - & - & $\mathbf{w}$ & $\mathbf{w}$ & - & - & $\mathbf{w}$ & $\mathbf{w}$ \\
\hline Surface & - & - & w & $\mathbf{w}$ & - & - & $\mathbf{w}$ & $\mathbf{w}$ \\
\hline Arizona & $\mathbf{w}$ & $\mathbf{w}$ & - & - & - & - & $\mathbf{w}$ & $\mathbf{w}$ \\
\hline 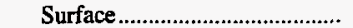 & $\mathbf{w}$ & w & - & - & - & - & $\mathbf{w}$ & $\mathbf{w}$ \\
\hline 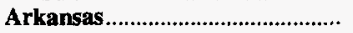 & - & - & - & - & $\mathbf{w}$ & $\mathbf{w}$ & $\mathbf{w}$ & $\mathbf{w}$ \\
\hline Surface & - & - & - & - & $\mathbf{w}$ & w & $\mathbf{w}$ & $\mathbf{w}$ \\
\hline 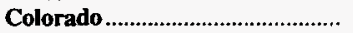 & $\mathbf{w}$ & $\mathbf{w}$ & $\mathbf{w}$ & $\mathbf{w}$ & 21,285 & 85.18 & 32,435 & 79.27 \\
\hline 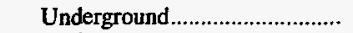 & $\mathbf{w}$ & $\mathrm{w}$ & - & - & $w$ & w & 22,135 & 77.65 \\
\hline 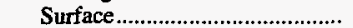 & $\mathbf{w}$ & $\mathbf{w}$ & $\mathbf{w}$ & $\mathbf{w}$ & $\mathbf{w}$ & $\mathbf{w}$ & 10,300 & 82.75 \\
\hline Illinois ................ & 42,494 & 83.67 & $\mathbf{w}$ & $\mathbf{w}$ & $\mathbf{w}$ & $\mathbf{w}$ & 56,627 & 85.08 \\
\hline 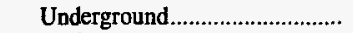 & 35,471 & 83.23 & w & w & $\mathbf{w}$ & w & 48,321 & 85.09 \\
\hline Surface & 7,023 & 85.91 & $\mathbf{w}$ & $\mathbf{w}$ & w & w & 8,305 & 85.02 \\
\hline 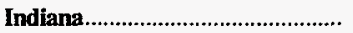 & 17,037 & 74.06 & - & - & 18,218 & 73.36 & 35,256 & 73.70 \\
\hline Underground ................................. & $w$ & w & - & - & $\mathbf{w}$ & w & 4,350 & 81.38 \\
\hline 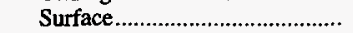 & $w$ & $\mathbf{w}$ & - & - & w & $w$ & 30,906 & 72.62 \\
\hline 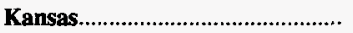 & $\mathbf{w}$ & $\mathbf{w}$ & - & - & - & - & $\mathbf{w}$ & $\mathbf{w}$ \\
\hline Surface & $\mathbf{w}$ & w & - & - & - & - & $\mathbf{w}$ & $\mathbf{w}$ \\
\hline 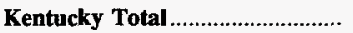 & $\mathbf{w}$ & $\mathbf{w}$ & $\mathbf{w}$ & $\mathbf{w}$ & 172,979 & 74.77 & 203,173 & $\mathbf{7 5 . 4 9}$ \\
\hline 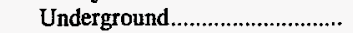 & $w$ & $\mathbf{w}$ & $w$ & $\mathrm{w}$ & 103,928 & 74.35 & 125,062 & 75.18 \\
\hline 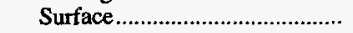 & $\mathbf{w}$ & $\mathbf{w}$ & $\mathbf{w}$ & w & 69,051 & 75.41 & 78,112 & 76.00 \\
\hline Eastern & $\mathbf{w}$ & $\mathbf{w}$ & $\mathbf{w}$ & $\mathbf{w}$ & 134,448 & $\mathbf{7 7 . 2 5}$ & 152,111 & 77.71 \\
\hline 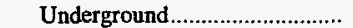 & $\mathbf{w}$ & $\mathbf{w}$ & $\mathbf{w}$ & $\mathbf{w}$ & 79,310 & 76.62 & 89,818 & 77.56 \\
\hline 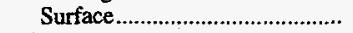 & $\mathbf{w}$ & $\mathbf{w}$ & $\mathbf{w}$ & $\mathbf{w}$ & 55,138 & 78.15 & 62,293 & 77.93 \\
\hline 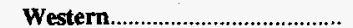 & $\mathbf{w}$ & $\mathbf{w}$ & - & - & $\mathbf{3 8 , 5 3 1}$ & 66.14 & 51,062 & 68.89 \\
\hline 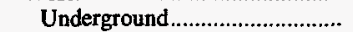 & $\mathbf{w}$ & $\mathbf{w}$ & - & - & w & w & 35,243 & 69.09 \\
\hline 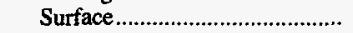 & w & w & - & - & $\mathbf{w}$ & $\mathbf{w}$ & 15,819 & 68.43 \\
\hline Louisiana & - & - & - & - & $\mathbf{w}$ & $\mathbf{w}$ & $\mathbf{w}$ & $\mathbf{w}$ \\
\hline 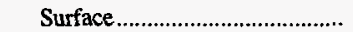 & - & - & - & - & w & w & w & w \\
\hline 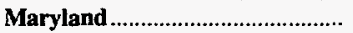 & - & - & - & - & 4,408 & 82.65 & 4,408 & 82.65 \\
\hline 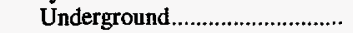 & - & - & - & - & $\mathbf{w}$ & w & w & $w$ \\
\hline 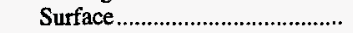 & - & - & - & - & $w$ & w & $\mathbf{w}$ & $\mathbf{w}$ \\
\hline Missouri & - & - & - & - & 1,081 & 49.88 & 1,081 & 49.88 \\
\hline 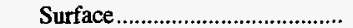 & - & - & - & - & 1,081 & 49.88 & 1,081 & 49.88 \\
\hline Montana & 19,441 & 78.62 & $\mathbf{w}$ & $\mathbf{w}$ & $\mathbf{w}$ & $\mathbf{w}$ & 51,597 & 76.44 \\
\hline Surface & 19,441 & 78.62 & w & $\mathbf{w}$ & $\mathbf{w}$ & $\mathbf{w}$ & 51,597 & 76.44 \\
\hline New Mexico & $\mathbf{w}$ & $\mathbf{w}$ & $\mathbf{w}$ & $\mathbf{w}$ & $\mathbf{w}$ & $\mathbf{w}$ & 32,760 & 81.85 \\
\hline 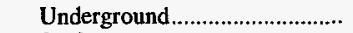 & w & $\mathbf{w}$ & - & - & - & - & w & w \\
\hline Surface & $w$ & $\mathbf{w}$ & w & $\mathbf{w}$ & $\mathbf{w}$ & $w$ & w & $\mathbf{w}$ \\
\hline 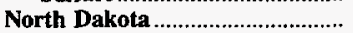 & $\mathbf{w}$ & $\mathbf{w}$ & $\mathbf{w}$ & $\mathbf{w}$ & $\mathbf{w}$ & $\mathbf{w}$ & 34,464 & 87.37 \\
\hline 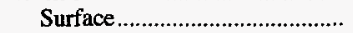 & w & $\mathbf{w}$ & w & $w$ & w & $\mathbf{w}$ & 34,464 & 87.37 \\
\hline 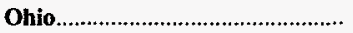 & $\mathbf{w}$ & $\mathbf{w}$ & $\mathbf{w}$ & $\mathbf{w}$ & 18,415 & 69.05 & 34,011 & 76.55 \\
\hline 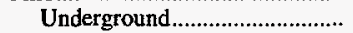 & w & w & - & - & w & w & 14,823 & 88.23 \\
\hline Surface & w & $\mathbf{w}$ & $w$ & $\mathbf{w}$ & w & w & 19,188 & 67.53 \\
\hline 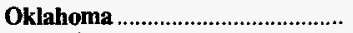 & - & - & - & - & 2,557 & 73.14 & 2,557 & 73.14 \\
\hline 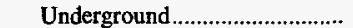 & - & - & - & - & $\mathbf{w}$ & w & $w$ & $\mathbf{w}$ \\
\hline Surface & - & - & - & - & $\mathbf{w}$ & w & $\mathbf{w}$ & w \\
\hline 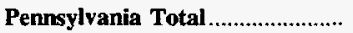 & $\mathbf{w}$ & $\mathbf{w}$ & $\mathbf{w}$ & $\mathbf{w}$ & 49,581 & 78.72 & 77,187 & 78.81 \\
\hline 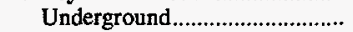 & $w$ & $w$ & - & - & 25,476 & 85.33 & 50,317 & 81.96 \\
\hline Surface & $w$ & $\mathbf{w}$ & w & $\mathbf{w}$ & 24,105 & 71.74 & 26,870 & 72.90 \\
\hline 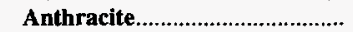 & $\mathbf{w}$ & $\mathbf{w}$ & $\mathbf{w}$ & $\mathbf{w}$ & 4,825 & 64.62 & 6,547 & 67.12 \\
\hline 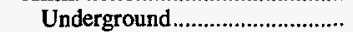 & - & - & - & - & 447 & 60.42 & 447 & 60.42 \\
\hline Surface & w & $\mathbf{w}$ & $w$ & $\mathbf{w}$ & 4,378 & 65.04 & 6,100 & 67.61 \\
\hline 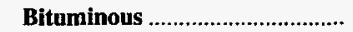 & 25,884 & 79.28 & - & - & 44,756 & 80.24 & 70,640 & 79.89 \\
\hline 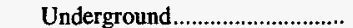 & 24,841 & 78.51 & - & - & 25,029 & 85.78 & 49,871 & 82.16 \\
\hline Surface & 1,042 & 97.60 & - & - & 19,727 & 73.23 & 20,769 & 74.45 \\
\hline 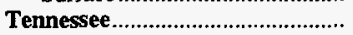 & - & - & - & - & $\mathbf{3 , 7 5 0}$ & $\mathbf{8 5 . 5 1}$ & $\mathbf{3 , 7 5 0}$ & 85.51 \\
\hline 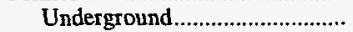 & - & - & - & - & 2,446 & 80.08 & 2,446 & 80.08 \\
\hline Surface & - & - & - & - & 1,304 & 95.69 & 1,304 & 95.69 \\
\hline Texas & - & - & 32,709 & 98.15 & 22,049 & 93.34 & 54,758 & 96.21 \\
\hline Surface & - & - & 32,709 & 98.15 & 22,049 & 93.34 & 54,758 & 96.21 \\
\hline 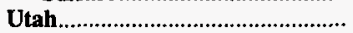 & 9,800 & 89.15 & - & - & 21,088 & 77.91 & 30,888 & 81.48 \\
\hline 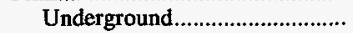 & 9,800 & 89.15 & - & - & 21,088 & 77.91 & 30,888 & 81.48 \\
\hline 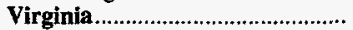 & $\mathbf{w}$ & w & $\mathbf{w}$ & $\mathbf{w}$ & 31,287 & 79.98 & 43,037 & 79.07 \\
\hline Underground ....................................... & $\mathbf{w}$ & w & - & - & w & $\mathbf{w}$ & 30,753 & 82.29 \\
\hline 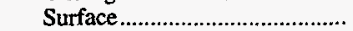 & $\mathbf{w}$ & $\mathbf{w}$ & $w$ & $w$ & w & $\mathbf{w}$ & 12,284 & 71.00 \\
\hline
\end{tabular}

See footnotes at end of table. 
Table 24. Productive Capacity and Capacity Utilization of Coal Mines by State, Mine Type, and Union Type, 1995 (Continued)

(Thousand Short Tons)

\begin{tabular}{|c|c|c|c|c|c|c|c|c|}
\hline \multirow[b]{2}{*}{$\begin{array}{l}\text { Coal-Producing } \\
\text { State and Region }\end{array}$} & \multicolumn{2}{|c|}{ UMWA } & \multicolumn{2}{|c|}{ Other Unions } & \multicolumn{2}{|c|}{ Nonunion } & \multicolumn{2}{|c|}{ Total } \\
\hline & $\begin{array}{c}\text { Productive } \\
\text { Capacity }\end{array}$ & $\begin{array}{l}\text { Capacity } \\
\text { Utilization } \\
\text { (percent) }\end{array}$ & $\begin{array}{c}\text { Productive } \\
\text { Capacity }\end{array}$ & $\begin{array}{c}\text { Capacity } \\
\text { Utilization } \\
\text { (percent) }\end{array}$ & $\begin{array}{c}\text { Productive } \\
\text { Capacity }\end{array}$ & $\begin{array}{c}\text { Capacity } \\
\text { Utilization } \\
\text { (percent) }\end{array}$ & $\begin{array}{l}\text { Productive } \\
\text { Capacity }\end{array}$ & $\begin{array}{l}\text { Capacity } \\
\text { Utilization } \\
\text { (percent) }\end{array}$ \\
\hline 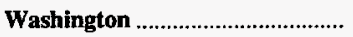 & - & - & $\mathbf{w}$ & $\mathbf{w}$ & $\mathbf{w}$ & $\mathbf{w}$ & $\mathbf{w}$ & $\mathbf{w}$ \\
\hline Surface & - & - & w & $\mathbf{w}$ & w & w & w & $\mathbf{w}$ \\
\hline 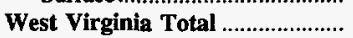 & 115,628 & 81.83 & - & - & 89,209 & 76.49 & 204,837 & 79.50 \\
\hline 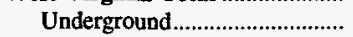 & 79,627 & 84.91 & - & - & 52,655 & 80.44 & 132,282 & 83.13 \\
\hline 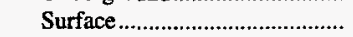 & 36,001 & 75.01 & - & - & 36,554 & 70.81 & 72,555 & 72.89 \\
\hline Northern & 37,744 & 86.55 & - & - & 18,611 & $\mathbf{7 1 . 8 6}$ & 56,355 & 81.70 \\
\hline 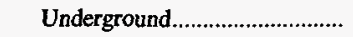 & 37,744 & 86.55 & - & - & 10,685 & 75.20 & 48,429 & 84.05 \\
\hline 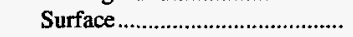 & - & - & - & - & 7,926 & 67.36 & 7,926 & 67.36 \\
\hline Southern & $\mathbf{7 7 , 8 8 4}$ & 79.54 & - & - & 70,598 & $\mathbf{7 7 . 7 1}$ & 148,482 & $\mathbf{7 8 . 6 7}$ \\
\hline 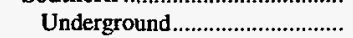 & 41,883 & 83.43 & - & - & 41,970 & 81.77 & 83,853 & 82.60 \\
\hline Surface & 36,001 & 75.01 & - & - & 28,628 & 71.77 & 64,629 & 73.57 \\
\hline Wyoming & $\mathbf{w}$ & $\mathbf{w}$ & $\mathbf{w}$ & $\mathbf{w}$ & 320,800 & 77.65 & 337,184 & 78.24 \\
\hline 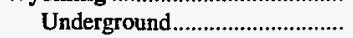 & - & - & - & - & $\mathrm{W}$ & w & w & w \\
\hline Surface $\ldots \ldots \ldots \ldots \ldots \ldots \ldots$ & $\mathbf{w}$ & $\mathbf{w}$ & $\mathbf{w}$ & w & $\mathbf{w}$ & $w$ & w & $\mathbf{w}$ \\
\hline Appalachian Total ${ }^{1} \ldots \ldots \ldots \ldots \ldots \ldots . . . . . . .$. & 209,194 & 80.83 & 2,561 & 74.07 & 340,133 & 77.15 & 551,888 & 78.53 \\
\hline 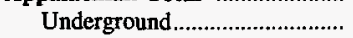 & w & w & $w$ & $\mathbf{w}$ & 189,870 & 79.99 & 346,427 & 81.32 \\
\hline Surface & w & $\mathbf{w}$ & w & $w$ & 150,262 & 73.55 & 205,461 & 73.82 \\
\hline 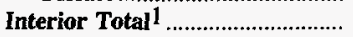 & 72,347 & 80.38 & 36,009 & 97.14 & 97,036 & 77.62 & 205,393 & 82.01 \\
\hline 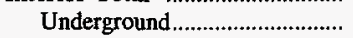 & $\mathbf{w}$ & $\mathbf{w}$ & $\mathbf{w}$ & w & 36,944 & 73.91 & 87,940 & 78.50 \\
\hline Surface & $\mathbf{w}$ & $\mathbf{w}$ & w & w & 60,093 & 79.90 & 117,453 & 84.64 \\
\hline 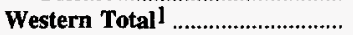 & $\mathbf{7 5 , 4 5 5}$ & 77.36 & 65,096 & 80.87 & 401,223 & 79.40 & 541,773 & 79.29 \\
\hline 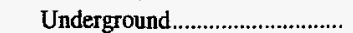 & 17,400 & 73.55 & - & - & 40,623 & 79.28 & 58,023 & 77.56 \\
\hline Surface & 58,055 & 78.51 & 65,096 & 80.87 & 360,600 & 79.41 & 483,751 & 79.50 \\
\hline 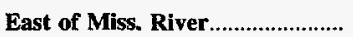 & 281,257 & 80.70 & 5,861 & 81.46 & 407,715 & 76.28 & 694,832 & 78.11 \\
\hline 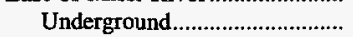 & 204,408 & 82.54 & 3,145 & 90.53 & 226,789 & 79.00 & 434,341 & 80.75 \\
\hline Surface & 76,849 & 75.79 & 2,716 & 70.97 & 180,926 & 72.87 & 260,491 & 73.71 \\
\hline West of Miss. River..................... & $\mathbf{7 5 , 7 4 0}$ & $\mathbf{7 7 . 4 5}$ & 97,805 & 86.65 & 430,677 & 80.17 & 604,222 & 80.88 \\
\hline 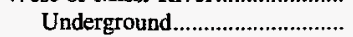 & 17,400 & 73.55 & - & - & 40,648 & 79.29 & 58,048 & 77.57 \\
\hline Surface & 58,340 & 78.61 & 97,805 & 86.65 & 390,029 & 80.26 & 546,174 & 81.23 \\
\hline 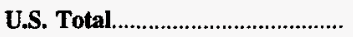 & 356,996 & 80.01 & 103,666 & 86.36 & 838,392 & 78.28 & $1,299,054$ & 79.40 \\
\hline 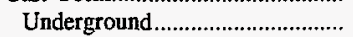 & 221,808 & 81.83 & 3,145 & 90.53 & 267,437 & 79.04 & 492,389 & 80.37 \\
\hline Surface & 135,189 & 77.01 & 100,521 & 86.23 & 570,955 & 77.92 & 806,664 & 78.80 \\
\hline
\end{tabular}

1 For a definition of coal-producing regions, see Appendix C.

w Witheld to avoid disclosure of individual company data.

Notes: Productive capacity is the maximum amount of coal that can be produced annually as reported by mining companies on Form EIA-7A. Capacity utilization is the ratio of total production to annual productive capacity as reported by mining companies on Form EIA-7A. Excludes silt, culm, refuse bank, slurry dam, and dredge operations except for Pennsylvania anthracite. Excludes mines producing less than 10,000 short tons, which are not required to provide these data. Totals may not equal sum of components due to independent rounding. See Glossary for listing of other unions.

Source: Energy Information Administration, Form EIA-7A, "Coal Production Report." 



\section{Recoverable Coal}

Reserves at Mines

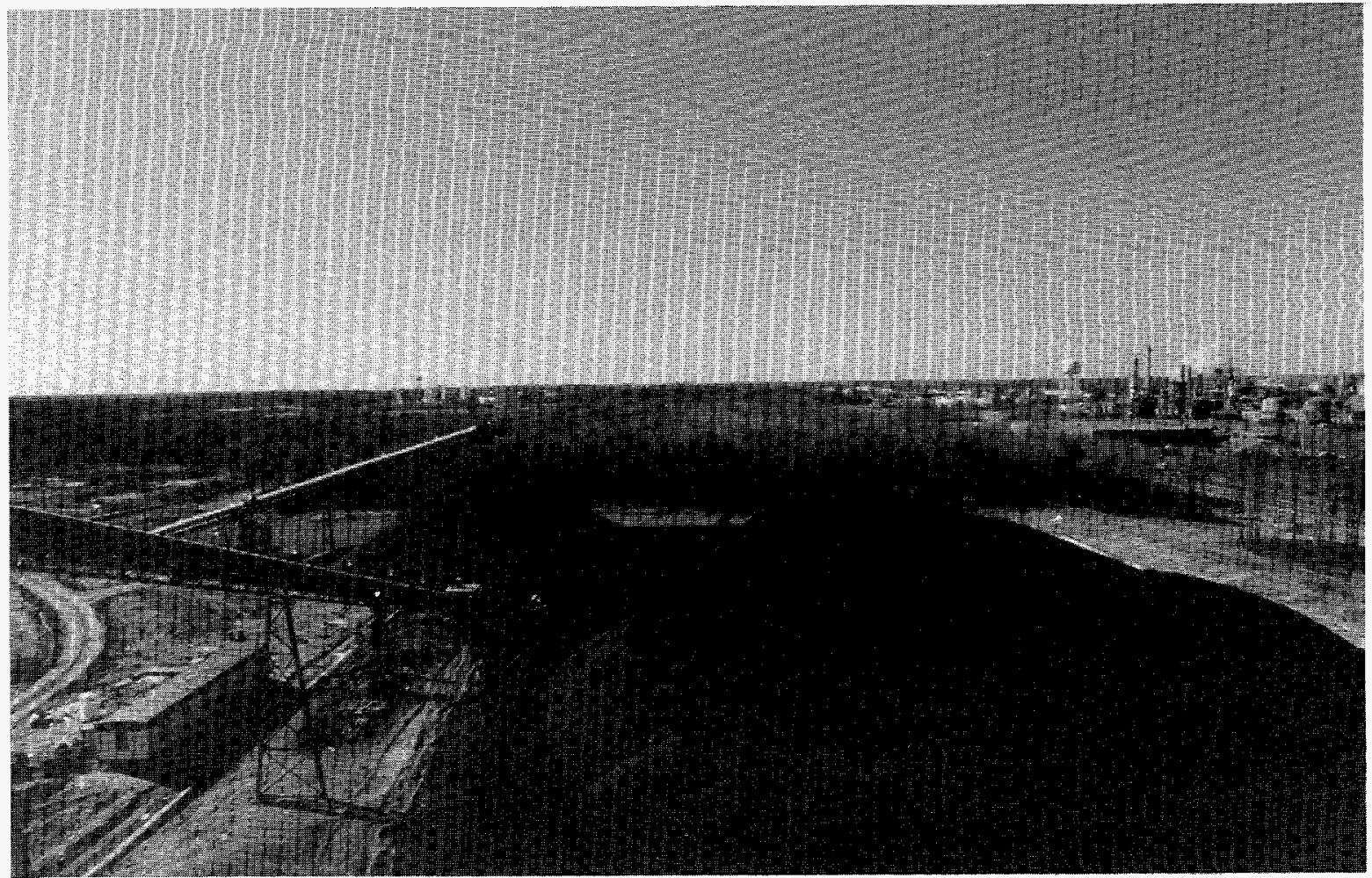

The amount of coal that could be recovered from existing coal mines in 1995 totaled 20.1 billion short tons. 
Figure 2. Recoverable Coal Reserves at U.S. Coal Mines by Mine Type and by Region, 1986-1995
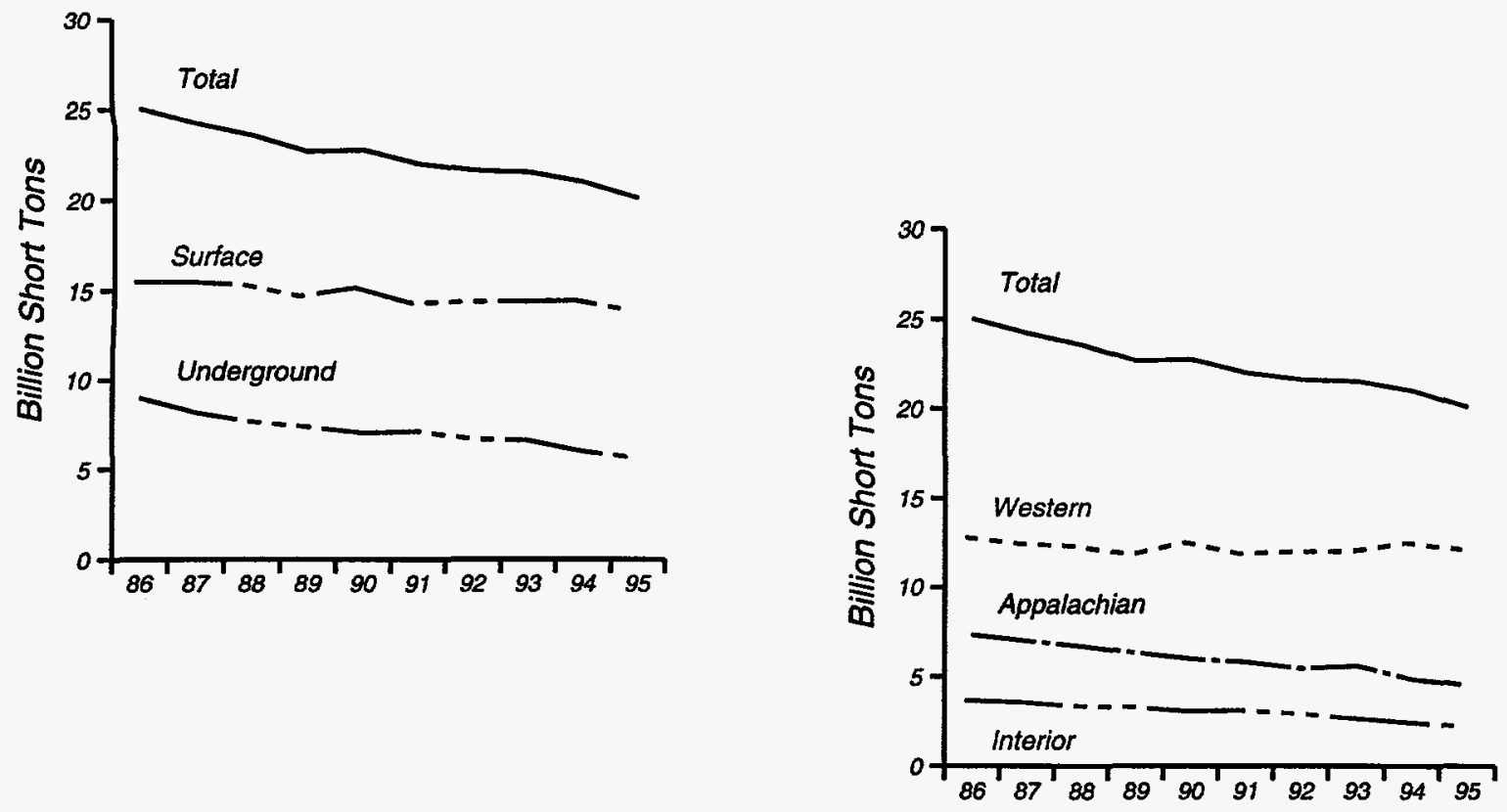

Figure 3. Average Recovery Percentage at U.S. Coal Mines by Mine Type and by Region, 1986-1995
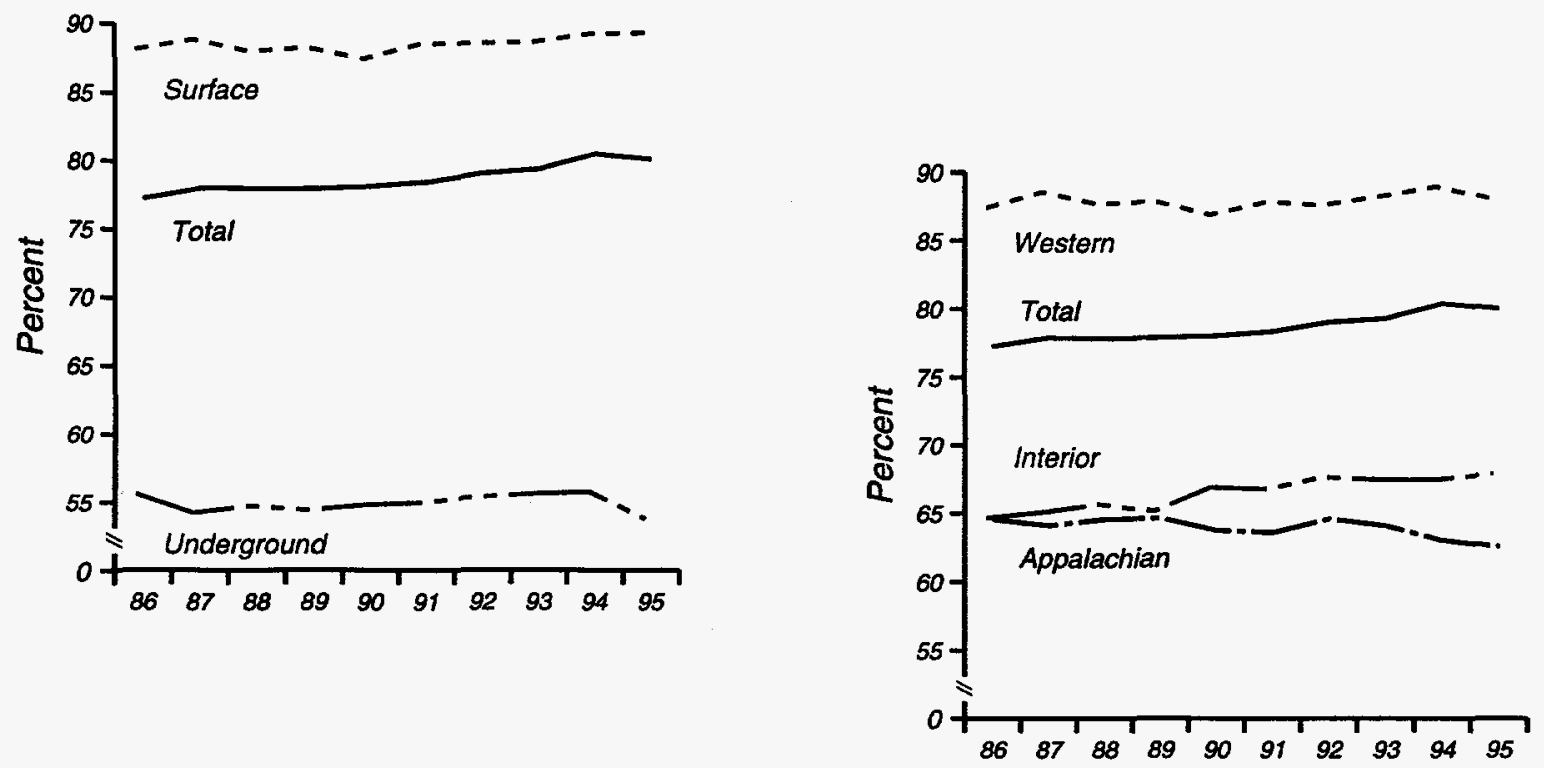

Sources: Energy Information Administration, Form ElA-7A, "Coal Production Report"; State Mining Agency Coal Production Reports; and/or U.S. Department of Labor, Mine Safety and Health Administration, Form 7000-2, "Quarterly Mine Employment and Coal Production Report." 
Table 25. Recoverable Coal Reserves at Producing Mines by State, 1986, 1991-1995 (Million Short Tons)

\begin{tabular}{|c|c|c|c|c|c|c|c|c|c|}
\hline \multirow{2}{*}{$\begin{array}{l}\text { Coal-Producing } \\
\text { State and Region }\end{array}$} & \multirow{2}{*}{1995} & \multirow{2}{*}{1994} & \multirow{2}{*}{1993} & \multirow{2}{*}{1992} & \multirow{2}{*}{1991} & \multirow{2}{*}{1986} & \multirow{2}{*}{$\begin{array}{c}\text { Percent } \\
\text { Change } \\
\text { 1994-1995 }\end{array}$} & \multicolumn{2}{|c|}{ Average Annual Percent Change } \\
\hline & & & & & & & & 1991-1995 & $1986-1995$ \\
\hline Alabama & 510 & 457 & 427 & 468 & 470 & 562 & 11.6 & 2.0 & -1.1 \\
\hline 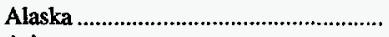 & $\mathbf{w}$ & $\mathbf{w}$ & $w$ & $w$ & w & 92 & $w$ & $w$ & $w$ \\
\hline Arizona & $\mathbf{w}$ & $\mathbf{w}$ & $\mathbf{w}$ & w & $w$ & 285 & $\mathbf{w}$ & $\mathrm{w}$ & $\mathbf{w}$ \\
\hline Arkansas & $\mathbf{w}$ & $\mathbf{w}$ & $\mathbf{w}$ & $\mathrm{w}$ & $\mathbf{w}$ & 1 & $\mathbf{w}$ & $w$ & $w$ \\
\hline 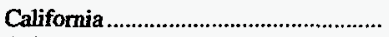 & - & - & - & $w$ & $w$ & - & $w$ & $w$ & $w$ \\
\hline Colorado & 692 & 676 & 609 & 608 & 618 & 611 & 2.3 & 2.9 & 1.4 \\
\hline 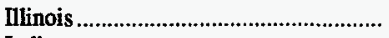 & 882 & 963 & 1,064 & 1,199 & 1,257 & 1,547 & -8.4 & -8.4 & -6.0 \\
\hline Indiana & 324 & 304 & 379 & 405 & 420 & 507 & 6.4 & -6.3 & -4.8 \\
\hline Iowa & - & $\mathbf{w}$ & w & w & $w$ & 9 & $\mathbf{w}$ & $w$ & w \\
\hline 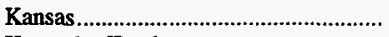 & $\mathbf{w}$ & $\mathbf{w}$ & $\mathbf{w}$ & w & $\mathbf{w}$ & 26 & $\mathbf{w}$ & w & w \\
\hline 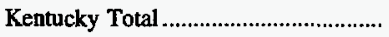 & 1,279 & 1,365 & 1,828 & 1,453 & 1,632 & 1,730 & -6.3 & -5.9 & -3.3 \\
\hline Eastern & 763 & 809 & 1,347 & 955 & 1,084 & 1,129 & -5.7 & -8.4 & -4.3 \\
\hline Western & 516 & 556 & 481 & 498 & 548 & 601 & -7.2 & -1.5 & -1.7 \\
\hline 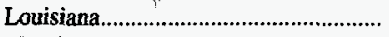 & $w$ & $w$ & $w$ & w & $w$ & 149 & $w$ & w & $w$ \\
\hline 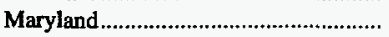 & 58 & 89 & 66 & 59 & 86 & 73 & -35.0 & -9.6 & -2.6 \\
\hline 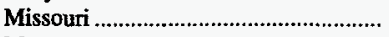 & 2 & 12 & w & w & $\mathbf{w}$ & 161 & -80.9 & w & -37.6 \\
\hline 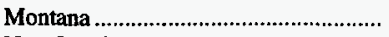 & 1,251 & 1,283 & 1,285 & 1,352 & 1,393 & 1,746 & -2.5 & -2.6 & -3.6 \\
\hline 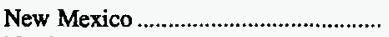 & 1,480 & 1,458 & 1,473 & 1,495 & 1,608 & 1,572 & 1.5 & -2.0 & -.7 \\
\hline North Dakota & 1,668 & 1,695 & 1,411 & 1,335 & 1,386 & 1,533 & -1.6 & 4.7 & .9 \\
\hline Ohio & 468 & 479 & 520 & 576 & 591 & 792 & -2.3 & -5.6 & -5.7 \\
\hline Oklahoma & 19 & 43 & 46 & 48 & 40 & 60 & -56.0 & -17.1 & -12.1 \\
\hline Pennsylvania Total ..................................... & 737 & 913 & 940 & 937 & 986 & 1,530 & -19.3 & -7.0 & -7.8 \\
\hline 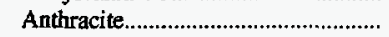 & 49 & 38 & 65 & 70 & 76 & 80 & 30.5 & -10.2 & -5.2 \\
\hline 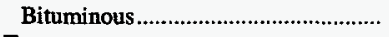 & 687 & 875 & 874 & 867 & 910 & 1,450 & -21.5 & -6.8 & -8.0 \\
\hline Tennessee & 68 & 42 & 29 & 43 & 56 & 84 & 61.5 & 5.0 & -2.4 \\
\hline 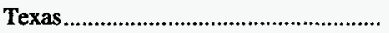 & 940 & 1,026 & 1,105 & 1,188 & 1,225 & 1,261 & -8.4 & -6.4 & -3.2 \\
\hline 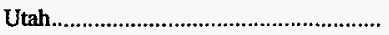 & 375 & 423 & 447 & 488 & 509 & 811 & -11.5 & -7.4 & -8.2 \\
\hline Virginia & 203 & 237 & 336 & 366 & 412 & 499 & -14.2 & -16.2 & -9.5 \\
\hline Washington & w & w & w & w & $\mathbf{w}$ & 112 & w & w & w \\
\hline West Virginia Total............................... & 1,731 & 1,830 & 1,931 & 2,043 & 2,122 & 2,674 & -5.4 & -4.9 & -4.7 \\
\hline Northern & 782 & 861 & 824 & 960 & 1,035 & 1,197 & -9.2 & -6.8 & -4.6 \\
\hline Southern & 949 & 969 & 1,107 & 1,083 & 1,087 & 1,478 & -2.0 & -3.3 & -4.8 \\
\hline 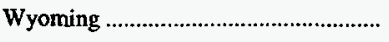 & 6,724 & 6,999 & 6,831 & 6,751 & 6,336 & 6,622 & -3.9 & 1.5 & .2 \\
\hline Appalachian Total 1 .............................. & 4,538 & 4,855 & 5,596 & 5,446 & 5,807 & 7,343 & -6.5 & -6.0 & -5.2 \\
\hline Interior Total 1 & $\mathbf{2 , 8 3 5}$ & 3,069 & $\mathbf{3 , 3 0 0}$ & $\mathbf{3 , 5 5 9}$ & 3,715 & 4,321 & -7.6 & -6.5 & -4.6 \\
\hline Western Total 1 & 12,732 & 13,093 & 12,639 & 12,622 & 12,477 & 13,384 & -2.8 & .5 & -.5 \\
\hline East of Miss. River ........................... & 6,260 & 6,679 & $\mathbf{7 , 5 2 0}$ & 7,549 & $\mathbf{8 , 0 3 1}$ & 9,998 & -6.3 & -6.0 & -5.1 \\
\hline West of Miss. River ................................ & 13,845 & 14,337 & 14,016 & 14,078 & 13,968 & 15,050 & -3.4 & -.2 & -.9 \\
\hline U.S. Total & 20,105 & 21,017 & 21,535 & 21,627 & 21,999 & 25,048 & -4.3 & -2.2 & -2.4 \\
\hline
\end{tabular}

1 For a definition of coal-producing regions, see Appendix $C$.

Withheld to avoid disclosure of individual company data.

Notes: Recoverable reserves represent the quantity of coal that can be recovered (i.e., mined) from existing coal reserves at reporting mines. Excludes silt, culm, refuse bank, slurry dam, and dredge operations except for Pennsylvania anthracite. Excludes mines producing less than 10,000 short tons, which are not required to provide these data. Totals may not equal sum of components due to independent rounding.

Sources: Energy Information Administration, Form EIA-7A, "Coal Production Report"; State Mining Agency Coal Production Reports; and/or U.S. Department of Labor, Mine Safety and Health Administration, Form 7000-2, "Quarterly Mine Employment and Coal Production Report." 
Table 26. Average Recovery Percentage at Producing Coal Mines by State, 1986, 1991-1995

\begin{tabular}{|c|c|c|c|c|c|c|}
\hline $\begin{array}{l}\text { Coal-Producing } \\
\text { State and Region }\end{array}$ & 1995 & 1994 & 1993 & 1992 & 1991 & 1986 \\
\hline 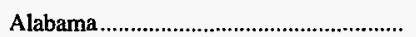 & 58.74 & 60.20 & 63.16 & 61.97 & 60.51 & 64.75 \\
\hline 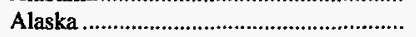 & $\mathbf{w}$ & $\mathbf{w}$ & $w$ & $\mathbf{w}$ & $\mathrm{w}$ & 90.00 \\
\hline 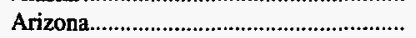 & w & $\mathbf{w}$ & $w$ & $\mathbf{w}$ & $\mathrm{w}$ & 91.00 \\
\hline 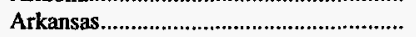 & $w$ & $\mathbf{w}$ & $\mathbf{w}$ & $w$ & $w$ & 71.49 \\
\hline 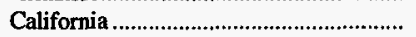 & - & - & - & $\mathbf{w}$ & $\mathbf{w}$ & 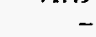 \\
\hline 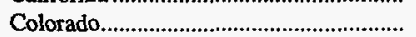 & 66.06 & 77.12 & 71.99 & 71.56 & 69.09 & 64.46 \\
\hline Illinois & 52.05 & 52.92 & 51.19 & 51.53 & 51.57 & 48.17 \\
\hline 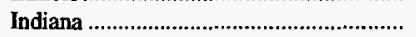 & 76.23 & 72.75 & 73.12 & 74.48 & 72.48 & 72.51 \\
\hline Iowa & - & $w$ & w & w & w & 64.62 \\
\hline Kansas & w & w & w & w & w & 80.15 \\
\hline Kentucky Total & 57.71 & 59.45 & 62.26 & 63.24 & 62.87 & 62.31 \\
\hline 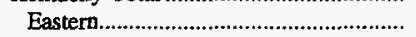 & 60.87 & 62.60 & 64.00 & 66.84 & 65.98 & 66.18 \\
\hline Western & 53.03 & 54.86 & 57.38 & 56.36 & 56.71 & 55.04 \\
\hline 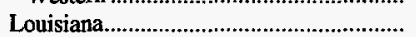 & w & w & $\mathbf{w}$ & w & w & 92.00 \\
\hline 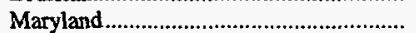 & 58.34 & 55.50 & 67.82 & 66.16 & 72.32 & 73.79 \\
\hline 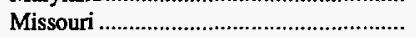 & 61.04 & 81.48 & w & w & w & 89.63 \\
\hline 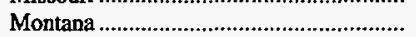 & 90.38 & 90.51 & 90.43 & 89.70 & 89.89 & 90.01 \\
\hline New Mexico & 92.62 & 92.42 & 92.28 & 91.92 & 92.21 & 92.09 \\
\hline 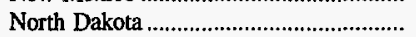 & 89.58 & 89.84 & 90.96 & 90.62 & 90.99 & 89.70 \\
\hline Ohio & 68.73 & 67.81 & 68.86 & 69.15 & 67.58 & 65.86 \\
\hline Oklahoma & 62.39 & 63.89 & 66.11 & 66.73 & 70.48 & 79.93 \\
\hline Pennsylvania Total & 65.26 & 68.23 & 67.95 & 66.12 & 63.77 & 65.89 \\
\hline 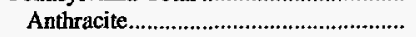 & 64.39 & 65.06 & 61.04 & 62.62 & 62.85 & 49.36 \\
\hline 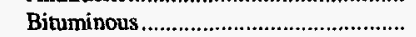 & 65.32 & 68.37 & 68.47 & 66.41 & 63.85 & 66.80 \\
\hline Tennessee & 64.14 & 65.18 & 68.92 & 69.80 & 73.50 & 68.50 \\
\hline 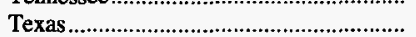 & 87.09 & 86.10 & 84.23 & 85.39 & 84.02 & 81.43 \\
\hline Utah & 46.10 & 46.10 & 47.51 & 49.04 & 52.71 & 57.90 \\
\hline Virginia & 58.14 & 58.37 & 62.72 & 64.78 & 63.61 & 67.26 \\
\hline Washington & w & $\mathbf{w}$ & w & $\mathbf{w}$ & w & 61.00 \\
\hline West Virginia Total. & 62.44 & 61.10 & 61.30 & 62.05 & 61.38 & 61.89 \\
\hline Northern & 55.10 & 53.06 & 52.17 & 54.04 & 51.92 & 53.63 \\
\hline Southern & 68.49 & 68.24 & 68.09 & 69.15 & 70.40 & 68.58 \\
\hline 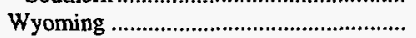 & 92.27 & 92.98 & 91.94 & 91.19 & 91.67 & 92.49 \\
\hline Appalachian Total ${ }^{1} \ldots \ldots \ldots \ldots \ldots \ldots \ldots \ldots \ldots$ & 62.65 & 63.07 & 64.11 & 64.62 & 63.64 & 64.59 \\
\hline Interior Total ${ }^{1}$ & 68.71 & 68.29 & 68.27 & 68.46 & 67.57 & 65.41 \\
\hline Western Total ${ }^{1}$ & $\mathbf{8 8 . 8 1}$ & 89.72 & 88.98 & 88.31 & 88.53 & 88.11 \\
\hline East of Miss. River................................... & 61.06 & 61.36 & 62.31 & 62.52 & 61.74 & 61.88 \\
\hline 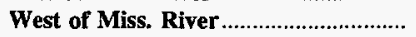 & 88.66 & 89.32 & 88.48 & 87.96 & 88.01 & 87.55 \\
\hline U.S. Total & 80.07 & 80.43 & 79.34 & 79.08 & 78.42 & $\mathbf{7 7 . 3 0}$ \\
\hline
\end{tabular}

1 For a definition of coal-producing regions, see Appendix C.

w Withheld to avoid disclosure of individual company data.

Notes: Average recovery percentage represents the percentage of coal that can be recovered from coal reserves at reporting mines, weighted for all mines in the geographic area. Excludes silt, culm, refuse bank, slurry dam, and dredge operations except for Pennsylvania anthracite. Excludes mines producing less than 10,000 short tons, which are not required to provide these data.

Sources: Energy Information Administration, Form EIA-7A, “Coal Production Report”; State Mining Agency Coal Production Reports; and/or U.S. Department of Labor, Mine Safety and Health Administration, Form 7000-2, "Quarterly Mine Employment and Coal Production Report." 
Table 27. Recoverable Coal Reserves and Average Recovery Percentage at Producing Mines by State and Mine Type, 1995 (Million Short Tons)

\begin{tabular}{|c|c|c|c|c|c|c|}
\hline \multirow[b]{2}{*}{$\begin{array}{l}\text { Coal-Producing } \\
\text { State and Region }\end{array}$} & \multicolumn{2}{|c|}{ Underground } & \multicolumn{2}{|c|}{ Surface } & \multicolumn{2}{|c|}{ Total } \\
\hline & $\begin{array}{c}\text { Recoverable } \\
\text { Coal } \\
\text { Reserves }\end{array}$ & $\begin{array}{c}\text { Average } \\
\text { Recovery } \\
\text { Percentage }\end{array}$ & $\begin{array}{c}\text { Recoverable } \\
\text { Coal } \\
\text { Reserves }\end{array}$ & $\begin{array}{c}\text { Average } \\
\text { Recovery } \\
\text { Percentage }\end{array}$ & $\begin{array}{c}\text { Recoverable } \\
\text { Coal } \\
\text { Reserves }\end{array}$ & $\begin{array}{c}\text { Average } \\
\text { Recovery } \\
\text { Percentage }\end{array}$ \\
\hline 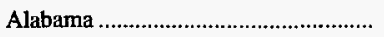 & 457 & 55.30 & 53 & 88.39 & 510 & 58.74 \\
\hline 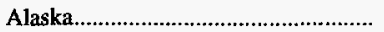 & - & - & $\mathbf{w}$ & $\mathbf{w}$ & $\mathbf{w}$ & w \\
\hline Arizona & - & - & $\mathbf{w}$ & $\mathbf{w}$ & w & w \\
\hline Arkansas & - & - & $w$ & $\mathbf{w}$ & $w$ & $w$ \\
\hline 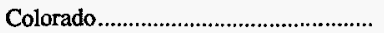 & $w$ & $\mathbf{w}$ & $w$ & w & 692 & 66.06 \\
\hline 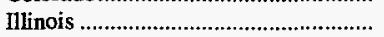 & 834 & 50.45 & 49 & 79.44 & 882 & 52.05 \\
\hline 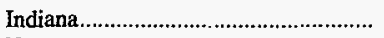 & 60 & 48.18 & 263 & 82.65 & 324 & 76.23 \\
\hline 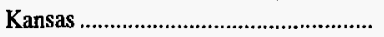 & - & - & $\mathbf{w}$ & w & w & w \\
\hline 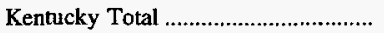 & 1,039 & 52.33 & 240 & 80.95 & 1,279 & 57.71 \\
\hline 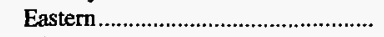 & 598 & 55.11 & 165 & 81.68 & 763 & 60.87 \\
\hline 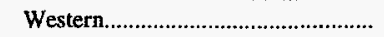 & 441 & 48.56 & 75 & 79.35 & 516 & 53.03 \\
\hline 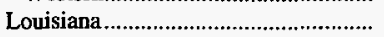 & - & - & $\mathbf{w}$ & w & w & $\mathrm{W}$ \\
\hline Maryland & $w$ & $\mathbf{w}$ & $\mathbf{w}$ & w & 58 & 58.34 \\
\hline 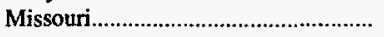 & - & - & 2 & 61.04 & 2 & 61.04 \\
\hline 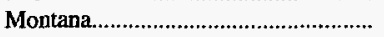 & - & - & 1,251 & 90.38 & 1,251 & 90.38 \\
\hline 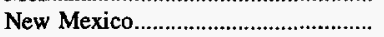 & - & - & 1,480 & 92.62 & 1,480 & 92.62 \\
\hline 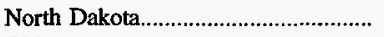 & - & - & 1,668 & 89.58 & 1,668 & 89.58 \\
\hline Ohio & 270 & 57.36 & 198 & 84.24 & 468 & 68.73 \\
\hline 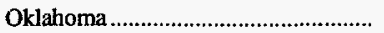 & - & - & 19 & 62.39 & 19 & 62.39 \\
\hline 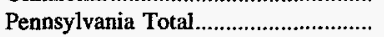 & 594 & 61.78 & 143 & 79.72 & 737 & 65.26 \\
\hline Anthracite & 23 & 73.35 & 26 & 56.58 & 49 & 64.39 \\
\hline 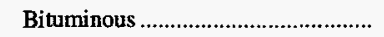 & 571 & 61.32 & 116 & 84.98 & 687 & 65.32 \\
\hline 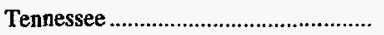 & $w$ & w & $\mathbf{w}$ & w & 68 & 64.14 \\
\hline Texas & $\sim$ & - & 940 & 87.09 & 940 & 87.09 \\
\hline 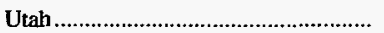 & 375 & 46.10 & - & - & 375 & 46.10 \\
\hline 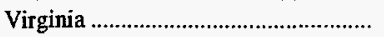 & 192 & 57.18 & 11 & 74.96 & 203 & 58.14 \\
\hline Washington & - & - & $\mathrm{w}$ & w & $w$ & $\mathrm{w}$ \\
\hline 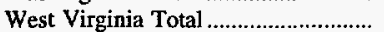 & 1,246 & 56.43 & 486 & 77.86 & 1,731 & 62.44 \\
\hline Northern & 677 & 53.16 & 105 & 67.65 & 782 & 55.10 \\
\hline Southern & 569 & 60.33 & 381 & 80.68 & 949 & 68.49 \\
\hline 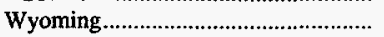 & $\mathbf{w}$ & w & $\mathbf{w}$ & w & 6,724 & 92.27 \\
\hline Appalachian Total 1 .............................. & 3,467 & 57.22 & 1,071 & 80.22 & 4,538 & 62.65 \\
\hline Interior Total 1 & 1,335 & 49.73 & 1,500 & 85.61 & $\mathbf{2 , 8 3 5}$ & 68.71 \\
\hline 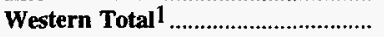 & 832 & 50.73 & 11,900 & 91.47 & 12,732 & 88.81 \\
\hline East of Miss. River & 4,802 & 55.13 & $\mathbf{1 , 4 5 8}$ & 80.59 & 6,260 & 61.06 \\
\hline West of Miss. River ............................. & 832 & 50.73 & 13,014 & 91.09 & 13,845 & 88.66 \\
\hline U.S. Total & 5,633 & 54.48 & 14,472 & 90.03 & 20,105 & 80.07 \\
\hline
\end{tabular}

1 For a definition of coal-producing regions, see Appendix $\mathbf{C}$.

Notes: Recoverable reserves represent the quantity of coal that can be recovered (i.e., mined) from existing coal reserves at reporting mines. Average recovery percentage represents the percentage of coal that can be recovered from coal reserves at reporting mines, weighted for all mines in the reported geographic area. Excludes silt, culm, refuse bank, slurry dam, and dredge operations except for Pennsylvania anthracite. Excludes mines producing less than 10,000 short tons, which are not required to provide these data. Totals may not equal sum of components due to independent rounding.

Sources: Energy Information Administration, Form EIA-7A, "Coal Production Report"; State Mining Agency Coal Production Reports; and/or U.S. Department of Labor, Mine Safety and Health Administration, Form 7000-2, "Quarterly Mine Employment and Coal Production Report." 
Table 28. Recoverable Coal Reserves at Producing Underground Mines by State and Mining Method, 1995

(Million Short Tons)

\begin{tabular}{|c|c|c|c|c|c|}
\hline $\begin{array}{l}\text { Coal-Producing } \\
\text { State and Region }\end{array}$ & Continuous 1 & Conventional 2 & Longwall 3 & Other 4 & Total \\
\hline Alabama & 12 & - & 445 & - & 457 \\
\hline 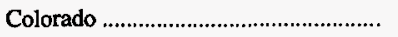 & $w$ & w & 383 & - & w \\
\hline 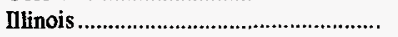 & 434 & - & 400 & - & 834 \\
\hline Indiana & 60 & - & - & - & 60 \\
\hline 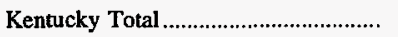 & 755 & w & 240 & $\mathrm{w}$ & 1,039 \\
\hline 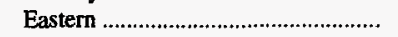 & 527 & w & 27 & w & 598 \\
\hline Western & 228 & - & 213 & - & 441 \\
\hline 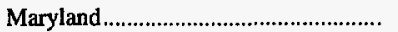 & w & - & $\mathbf{w}$ & - & w \\
\hline Ohio & 27 & - & 243 & - & 270 \\
\hline Pennsylvania Total .................................. & 161 & w & 396 & w & 594 \\
\hline 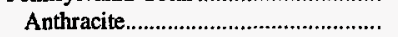 & $\mathbf{w}$ & $w$ & - & $\mathbf{w}$ & 23 \\
\hline 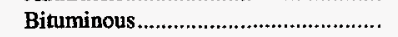 & $\mathbf{w}$ & $\mathbf{w}$ & 396 & - & 571 \\
\hline 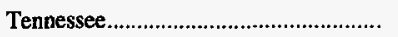 & w & - & - & - & w \\
\hline Utah & $w$ & $w$ & 313 & - & 375 \\
\hline Virginia & 92 & 21 & 79 & * & 192 \\
\hline 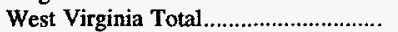 & 452 & 61 & 733 & - & 1,246 \\
\hline 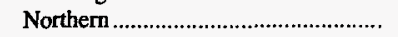 & 49 & 46 & 582 & - & 677 \\
\hline Southern & 403 & 15 & 151 & - & 569 \\
\hline 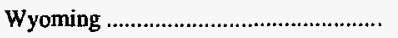 & - & w & w & - & w \\
\hline Appalachian Total ${ }^{5}$................................ & $\mathbf{w}$ & $\mathbf{w}$ & $\mathbf{w}$ & 24 & $\mathbf{3 , 4 6 7}$ \\
\hline Interior Total 5 & $\mathbf{w}$ & - & $\mathbf{w}$ & - & 1,335 \\
\hline 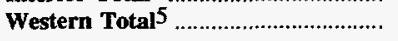 & $\mathbf{w}$ & $\mathbf{w}$ & $\mathbf{w}$ & - & 832 \\
\hline East of Miss. River.................................. & 2,063 & $\mathbf{w}$ & $\mathbf{w}$ & 24 & 4,802 \\
\hline 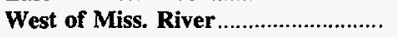 & 102 & $\mathbf{w}$ & $\mathbf{w}$ & - & 832 \\
\hline U.S. Total & 2,165 & 164 & 3,280 & 24 & 5,633 \\
\hline
\end{tabular}

1 Mines that produce greater than 50 percent of coal by continuous mining method.

2 Mines that produce greater than 50 percent of coal by conventional mining method.

3 Mines that have any production from longwall mining method. A typical longwall mining operation uses 80 percent longwall mining and 20 percent continuous mining.

4 Mines that produce coal using shortwall, scoop loading, hand loading, or other mining methods or a $50 / 50$ percent continuous/conventional split in mining method.

5 For a definition of coal-producing regions, see Appendix C.

* Data round to zero.

Notes: Recoverable reserves represent the quantity of coal that can be recovered (i.e., mined) from existing coal reserves at reporting mines. Excludes mines producing less than 10,000 short tons, which are not required to provide these data. Totals may not equal sum of components due to independent rounding.

Sources: Energy Information Administration, Form EIA-7A, "Coal Production Report"; State Mining Agency Coal Production Reports; and/or U.S. Department of Labor, Mine Safety and Health Administration, Form 7000-2, "Quarterly Mine Employment and Coal Production Report." 
Table 29. Average Recovery Percentage at Producing Underground Coal Mines by State and Mining Method, 1995

\begin{tabular}{|c|c|c|c|c|c|}
\hline $\begin{array}{l}\text { Coal-Producing } \\
\text { State and Region }\end{array}$ & Continuous 1 & Conventional 2 & Longwall 3 & Other 4 & Total \\
\hline Alabama & 58.94 & - & 55.20 & - & 55.30 \\
\hline Colorado & $w$ & $\mathbf{w}$ & 56.25 & - & w \\
\hline 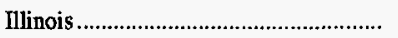 & 44.48 & - & 56.93 & - & 50.45 \\
\hline Indiana & 48.18 & - & - & - & 48.18 \\
\hline 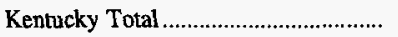 & 52.83 & $w$ & 49.01 & w & 52.33 \\
\hline 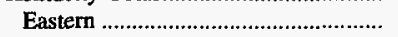 & 54.56 & $\mathbf{w}$ & 54.57 & $\mathbf{w}$ & 55.11 \\
\hline Western & 48.81 & - & 48.30 & - & 48.56 \\
\hline 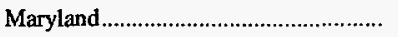 & w & - & $\mathbf{w}$ & - & w \\
\hline Ohio & 50.90 & - & 58.08 & - & 57.36 \\
\hline Pennsylvania Total ...................................... & 67.56 & $\mathbf{w}$ & 58.49 & $w$ & 61.78 \\
\hline Anthracite & $w$ & $\mathbf{w}$ & - & $\mathbf{w}$ & 73.35 \\
\hline 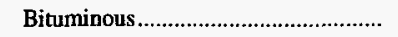 & $\mathbf{w}$ & $\mathbf{w}$ & 58.49 & - & 61.32 \\
\hline 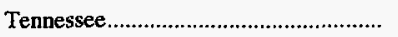 & w & - & - & - & w \\
\hline 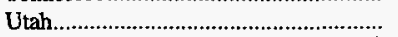 & $\mathbf{w}$ & w & 45.97 & - & 46.10 \\
\hline 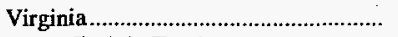 & 59.58 & 58.30 & 54.07 & 65.00 & 57.18 \\
\hline West Virginia Total.................................. & 56.80 & 60.59 & 55.86 & - & 56.43 \\
\hline Northern & 56.79 & 63.26 & 52.05 & - & 53.16 \\
\hline Southern & 56.81 & 52.51 & 70.52 & - & 60.33 \\
\hline Wyoming & - & w & w & - & w \\
\hline Appalachian Total 5 ............................ & $\mathbf{w}$ & $\mathbf{w}$ & $\mathbf{w}$ & 73.65 & 57.22 \\
\hline Interior Totals & $\mathbf{w}$ & - & $\mathbf{w}$ & - & 49.73 \\
\hline Western Total ${ }^{5}$ & $\mathbf{w}$ & $\mathbf{w}$ & $\mathbf{w}$ & - & 50.73 \\
\hline East of Miss. River................................ & 53.58 & $\mathbf{w}$ & $\mathbf{w}$ & 73.65 & 55.13 \\
\hline West of Miss. River............................... & 42.38 & $\mathbf{w}$ & $\mathbf{w}$ & - & 50.73 \\
\hline U.S. Total & 53.05 & 60.80 & 54.97 & 73.65 & 54.48 \\
\hline
\end{tabular}

1 Mines that produce greater than 50 percent of coal by continuous mining method.

2 Mines that produce greater than 50 percent of coal by conventional mining method

3 Mines that have any production from longwall mining method. A typical longwall mining operation uses 80 percent longwall mining and 20 percent continuous mining.

4 Mines that produce coal using shortwall, scoop loading, hand loading, or other mining methods or a $50 / 50$ percent continuous/conventional split in mining method.

5 For a definition of coal-producing regions, see Appendix C.

Notes: Average recovery percentage represents the percentage of coal that can be recovered from coal reserves at reporting mines, weighted for all mines in the reported geographic area. Excludes mines producing less than 10,000 short tons, which are not required to provide these data.

Sources: Energy Information Administration, Form ELA-7A, "Coal Production Report"; State Mining Agency Coal Production Reports; and/or U.S. Department of Labor, Mine Safety and Health Administration, Form 7000-2, "Quarterly Mine Employment and Coal Production Report." 
Table 30. Recoverable Coal Reserves and Average Recovery Percentage at Producing U.S. Mines by Mine Production Range and Mine Type, 1995

(Million Short Tons)

\begin{tabular}{|c|c|c|c|c|c|c|}
\hline \multirow[b]{2}{*}{$\begin{array}{l}\text { Mine Production Range } \\
\text { (thousand short tons) }\end{array}$} & \multicolumn{2}{|c|}{ Underground } & \multicolumn{2}{|c|}{ Surface } & \multicolumn{2}{|c|}{ Total } \\
\hline & $\begin{array}{c}\text { Recoverable } \\
\text { Coal } \\
\text { Reserves }\end{array}$ & $\begin{array}{c}\text { Average } \\
\text { Recovery } \\
\text { Percentage }\end{array}$ & $\begin{array}{c}\text { Recoverable } \\
\text { Coal } \\
\text { Reserves }\end{array}$ & $\begin{array}{c}\text { Average } \\
\text { Recovery } \\
\text { Percentage }\end{array}$ & $\begin{array}{c}\text { Recoverable } \\
\text { Coal } \\
\text { Reserves }\end{array}$ & $\begin{array}{c}\text { Average } \\
\text { Recovery } \\
\text { Percentage }\end{array}$ \\
\hline Over 1,000 & 4,041 & 53.58 & 13,123 & 90.59 & 17,164 & 81.88 \\
\hline 500 to 1,000 & 655 & 54.28 & 486 & 84.99 & 1,142 & 67.36 \\
\hline 200 to 500 & 511 & 56.24 & 657 & 86.16 & 1,168 & 73.07 \\
\hline 100 to 200 & 181 & 60.10 & 74 & 81.52 & 255 & 66.30 \\
\hline 50 to 100 & 152 & 59.76 & 44 & 74.33 & 196 & 63.05 \\
\hline 10 to 50 & 93 & 66.07 & 87 & 78.15 & 180 & 71.94 \\
\hline 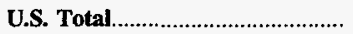 & 5,633 & 54.48 & 14,472 & 90.03 & 20,105 & 80.07 \\
\hline
\end{tabular}

Notes: Recoverable reserves represent the quantity of coal that can be recovered (i.e., mined) from existing coal reserves at reporting mines. Average recovery percentage represents the percentage of coal that can be recovered from coal reserves at reporting mines, weighted for all mines in the reported geographic area. Excludes silt, culm, refuse bank, slurry dam, and dredge operations except for Pennsylvania anthracite. Excludes mines producing less than 10,000 short tons, which are not required to provide these data. Totals may not equal sum of components due to independent rounding.

Sources: Energy Information Administration, Form EIA-7A, "Coal Production Report"; State Mining Agency Coal Production Reports; and/or U.S. Department of Labor, Mine Safety and Health Administration, Form 7000-2, "Quarterly Mine Employment and Coal Production Report."

Table 31. Recoverable Coal Reserves and Average Recovery Percentage at Producing U.S. Mines by Coalbed Thickness and Mine Type, 1995

(Million Short Tons)

\begin{tabular}{|c|c|c|c|c|c|c|}
\hline \multirow[b]{2}{*}{$\begin{array}{c}\text { Coalbed Thickness } \\
\text { (inches) }\end{array}$} & \multicolumn{2}{|c|}{ Underground } & \multicolumn{2}{|c|}{ Surface } & \multicolumn{2}{|c|}{ Total } \\
\hline & $\begin{array}{c}\text { Recoverable } \\
\text { Coal } \\
\text { Reserves }\end{array}$ & $\begin{array}{c}\text { Average } \\
\text { Recovery } \\
\text { Percentage }\end{array}$ & $\begin{array}{c}\text { Recoverable } \\
\text { Coal } \\
\text { Reserves }\end{array}$ & $\begin{array}{c}\text { Average } \\
\text { Recovery } \\
\text { Percentage }\end{array}$ & $\begin{array}{c}\text { Recoverable } \\
\text { Coal } \\
\text { Reserves }\end{array}$ & $\begin{array}{c}\text { Average } \\
\text { Recovery } \\
\text { Percentage }\end{array}$ \\
\hline$<7$ & - & - & $*$ & 57.37 & $*$ & 57.37 \\
\hline $7-12$ & - & - & 12 & 84,54 & 12 & 84.54 \\
\hline 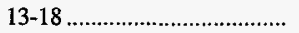 & - & - & 41 & 84.30 & 41 & 84.30 \\
\hline $19-24 \ldots \ldots \ldots \ldots \ldots \ldots \ldots$ & 3 & 59.00 & 146 & 82.73 & 149 & 82.27 \\
\hline 25-30 & 61 & 57.01 & 224 & 82.01 & 285 & 76.64 \\
\hline $31-36$ & 197 & 63.94 & 348 & 82.18 & 545 & 75.58 \\
\hline $37-42 \ldots \ldots \ldots$ & 223 & 58.11 & 196 & 81.15 & 418 & 68.89 \\
\hline $43-48$ & 499 & 61.45 & 384 & 84.66 & 883 & 71.54 \\
\hline 49-54 & 504 & 54.34 & 382 & 91.09 & 886 & 70.18 \\
\hline $55-60 \ldots \ldots \ldots \ldots$ & 530 & 57.25 & 136 & 79.04 & 666 & 61.69 \\
\hline 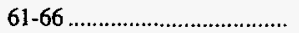 & 707 & 49.13 & 281 & 87.06 & 988 & 59.92 \\
\hline 67-72 & 588 & 51.27 & 256 & 86.49 & 844 & 61.97 \\
\hline 73-78 & 139 & 62.44 & 165 & 87.59 & 304 & 76.08 \\
\hline $79.84 \ldots \ldots \ldots \ldots \ldots$ & 913 & 55.69 & 301 & 82.38 & 1,214 & 62.30 \\
\hline 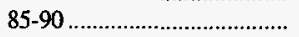 & 153 & 45.87 & 513 & 92.89 & 665 & 82.11 \\
\hline 91-96 & 366 & 52.56 & 114 & 87.13 & 480 & 60.79 \\
\hline $97-102$ & 46 & 49.12 & 68 & 89.17 & 114 & 72.98 \\
\hline 103-108 & 71 & 57.98 & 39 & 89.54 & 110 & 69.19 \\
\hline $109-114$ & 104 & 58.74 & 41 & 78.95 & 144 & 64.43 \\
\hline $115-120$ & 62 & 50.50 & 14 & 81.26 & 76 & 56.07 \\
\hline$>\quad 120$ & 468 & 49.32 & 10,812 & 91.39 & 11,280 & 89.65 \\
\hline U.S. Total & 5,633 & 54.48 & 14,472 & 90.03 & 20,105 & 80.07 \\
\hline
\end{tabular}

* Data round to zero.

Notes: Recoverable reserves represent the quantity of coal that can be recovered (i.e., mined) from existing coal reserves at reporting mines. Average recovery percentage represents thet percentage of coal that can be recovered from coal reserves at reporting mines, weighted for all mines in the reported geographic area. Excludes silt, culm, refuse bank, slurry dam, and dredge operations except for Pennsylvania anthracite. Excludes mines producing less than 10,000 short tons, which are not required to provide these data. Totals may not equal sum of components due to independent rounding.

Sources: Energy Information Administration, Form EIA-7A, "Coal Production Report"; State Mining Agency Coal Production Reports; and/or U.S. Department of Labor, Mine Safety and Health Administration, Form 7000-2, "Quarterly Mine Employment and Coal Production Report." 
Table 32. Recoverable Coal Reserves and Average Recovery Percentage at Producing Mines by State, Mine Type, and Union Type, 1995

(Million Short Tons)

\begin{tabular}{|c|c|c|c|c|c|c|c|c|}
\hline \multirow[b]{2}{*}{$\begin{array}{l}\text { Coal-Producing } \\
\text { State and Region }\end{array}$} & \multicolumn{2}{|c|}{ UMWA } & \multicolumn{2}{|c|}{ Other Unions } & \multicolumn{2}{|c|}{ Nonunion } & \multicolumn{2}{|c|}{ Total } \\
\hline & $\begin{array}{c}\text { Recoverable } \\
\text { Coal } \\
\text { Reserves }\end{array}$ & $\begin{array}{c}\text { Average } \\
\text { Recovery } \\
\text { Percentage }\end{array}$ & $\begin{array}{c}\text { Recoverable } \\
\text { Coal } \\
\text { Reserves }\end{array}$ & $\begin{array}{c}\text { Average } \\
\text { Recovery } \\
\text { Percentage }\end{array}$ & $\begin{array}{c}\text { Recoverable } \\
\text { Coal } \\
\text { Reserves }\end{array}$ & $\begin{array}{c}\text { Average } \\
\text { Recovery } \\
\text { Percentage }\end{array}$ & $\begin{array}{c}\text { Recoverable } \\
\text { Coal } \\
\text { Reserves }\end{array}$ & $\begin{array}{c}\text { Average } \\
\text { Recovery } \\
\text { Percentage }\end{array}$ \\
\hline 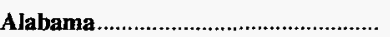 & 448 & $\mathbf{5 7 . 5 8}$ & - & - & 63 & 67.00 & 510 & 58.74 \\
\hline Underground & w & $\mathbf{w}$ & - & - & $\mathbf{w}$ & $w$ & 457 & 55.30 \\
\hline Surface & $\mathrm{w}$ & $\mathbf{w}$ & - & - & w & w & 53 & 88.39 \\
\hline Alaska & - & - & $\mathbf{w}$ & $\mathbf{w}$ & - & - & $\mathbf{w}$ & $\mathbf{w}$ \\
\hline Surface & - & - & $\mathrm{w}$ & w & - & - & w & w \\
\hline 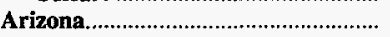 & $\mathbf{w}$ & $\mathbf{w}$ & - & - & - & - & $\mathbf{w}$ & $\mathbf{w}$ \\
\hline 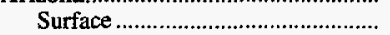 & $\mathrm{w}$ & $w$ & - & - & - & - & $w$ & $\mathbf{w}$ \\
\hline 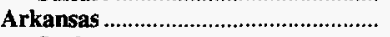 & - & - & - & - & $\mathbf{w}$ & $\mathbf{w}$ & $\mathbf{w}$ & $\mathbf{w}$ \\
\hline Surface & - & - & - & - & $\mathbf{w}$ & w & w & w \\
\hline 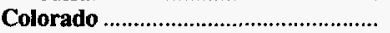 & $w$ & $\mathbf{w}$ & $\mathbf{w}$ & w & 494 & 65.58 & 692 & 66.06 \\
\hline 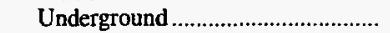 & $\mathrm{w}$ & w & - & - & $\mathbf{w}$ & $\mathbf{w}$ & 442 & 54.25 \\
\hline 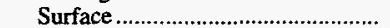 & w & w & $\mathbf{w}$ & w & w & w & 250 & 87.01 \\
\hline 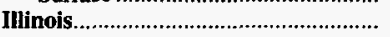 & $\mathbf{5 1 2}$ & 48.24 & $\mathbf{w}$ & $\mathbf{w}$ & $w$ & $\mathbf{w}$ & 882 & 52.05 \\
\hline 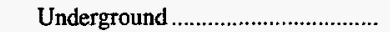 & 475 & 45.86 & $w$ & w & $w$ & $w$ & 834 & 50.45 \\
\hline Surface & 36 & 79.57 & $w$ & $w$ & $w$ & w & 49 & 79.44 \\
\hline Indiana & 207 & 78.78 & - & - & 117 & 71.72 & 324 & 76.23 \\
\hline 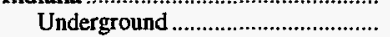 & w & w & - & - & $w$ & $\mathrm{w}$ & 60 & 48.18 \\
\hline Surface & w & $\mathbf{w}$ & - & - & $w$ & $\mathrm{w}$ & 263 & 82.65 \\
\hline 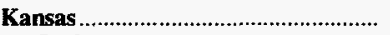 & $\mathbf{w}$ & $\mathbf{w}$ & - & - & - & - & $\mathbf{w}$ & $\mathbf{w}$ \\
\hline Surface & $\mathrm{w}$ & $\mathbf{w}$ & - & - & - & - & w & w \\
\hline Kentucky Total & w & $w$ & $\mathbf{w}$ & $\mathbf{w}$ & 1,108 & 58.84 & 1,279 & $\mathbf{5 7 . 7 1}$ \\
\hline 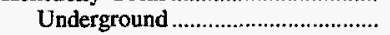 & w & $w$ & $\mathbf{w}$ & $\mathbf{w}$ & 881 & 53.23 & 1,039 & 52.33 \\
\hline Surface & w & $\mathbf{w}$ & $\mathbf{w}$ & $\mathbf{w}$ & 227 & 80.58 & 240 & 80.95 \\
\hline 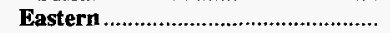 & $\mathrm{w}$ & $\mathbf{w}$ & w & w & 720 & 60.44 & 763 & 60.87 \\
\hline 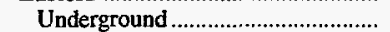 & $\mathrm{w}$ & $\mathbf{w}$ & $w$ & $w$ & 565 & 54.74 & 598 & 55.11 \\
\hline Surface & $\mathrm{w}$ & w & $w$ & w & 154 & 81.31 & 165 & 81.68 \\
\hline Western & w & $w$ & - & - & 388 & $\mathbf{5 5 . 8 7}$ & 516 & 53.03 \\
\hline 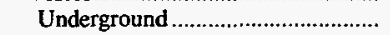 & $w$ & w & - & - & w & w & 441 & 48.56 \\
\hline Surface & $\mathbf{w}$ & w & - & - & $\mathbf{w}$ & w & 75 & 79.35 \\
\hline 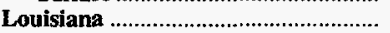 & - & - & - & - & $\mathbf{w}$ & $\mathbf{w}$ & $\mathbf{w}$ & $\mathbf{w}$ \\
\hline 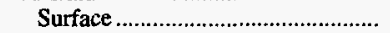 & - & - & - & - & $w$ & w & $\mathbf{w}$ & $\mathbf{w}$ \\
\hline 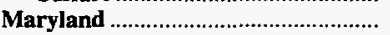 & - & - & - & - & 58 & 58.34 & 58 & 58.34 \\
\hline 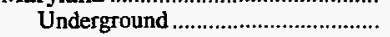 & - & - & - & - & $w$ & w & w & $w$ \\
\hline Surface & - & - & - & - & $\mathbf{w}$ & w & w & w \\
\hline 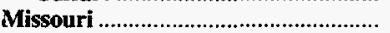 & - & - & - & - & 2 & 61.04 & 2 & 61.04 \\
\hline Surface & - & - & - & - & 2 & 61.04 & 2 & 61.04 \\
\hline Montana & 211 & 90.39 & $\mathbf{w}$ & $\mathbf{w}$ & $\mathbf{w}$ & $\mathbf{w}$ & 1,251 & 90.38 \\
\hline 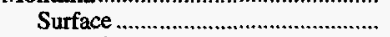 & 211 & 90.39 & w & $\mathrm{w}$ & w & w & 1,251 & 90.38 \\
\hline New Mexico & w & w & $w$ & $\mathbf{w}$ & $w$ & $w$ & 1,480 & 92.62 \\
\hline Surface & $w$ & w & w & $w$ & $w$ & $\mathbf{w}$ & $\mathrm{w}$ & $w$ \\
\hline 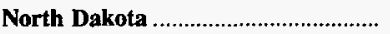 & $w$ & $\mathbf{w}$ & $\mathbf{w}$ & $w$ & $w$ & $\mathbf{w}$ & 1,668 & 89.58 \\
\hline Surface & $\mathrm{w}$ & $\mathbf{w}$ & $\mathbf{w}$ & w & $\mathbf{w}$ & w & 1,668 & 89.58 \\
\hline 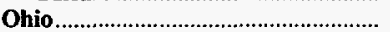 & $w$ & w & - & - & 144 & 77.77 & 468 & 68.73 \\
\hline Underground .......................................... & $\mathbf{w}$ & $\mathbf{w}$ & - & - & $\mathbf{w}$ & w & 270 & 57.36 \\
\hline Surface & $w$ & $w$ & - & - & $\mathbf{w}$ & $w$ & 198 & 84.24 \\
\hline Oklahoma & - & - & - & - & 19 & 62.39 & 19 & 62.39 \\
\hline Surface & - & - & - & - & 19 & 62.39 & 19 & 62.39 \\
\hline Pennsylvania Total & $\mathbf{w}$ & $w$ & $w$ & w & 394 & 67.02 & 737 & 65.26 \\
\hline 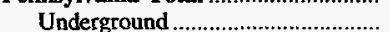 & w & $w$ & - & - & 270 & 60.81 & 594 & 61.78 \\
\hline Surface & $w$ & w & $\mathbf{w}$ & $w$ & 125 & 80.45 & 143 & 79.72 \\
\hline Anthracite & $w$ & w & $w$ & $w$ & 40 & 63.81 & 49 & 64.39 \\
\hline Underground & - & - & - & - & 23 & 73.35 & 23 & 73.35 \\
\hline Surface & w & w & $\mathbf{w}$ & w & 17 & 50.73 & 26 & 56.58 \\
\hline 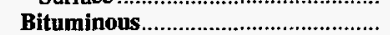 & 333 & 63.12 & - & - & 355 & 67.38 & 687 & 65.32 \\
\hline Underground & 324 & 62.59 & - & - & 247 & 59.64 & 571 & 61.32 \\
\hline Surface & 8 & 83.73 & - & - & 108 & 85.08 & 116 & 84.98 \\
\hline Tennessee & - & - & - & - & 68 & 64.14 & 68 & 64.14 \\
\hline Underground & - & - & - & - & 61 & 63.99 & 61 & 63.99 \\
\hline Surface & - & - & - & - & 7 & 65.35 & 7 & 65.35 \\
\hline Texas & - & - & 507 & 90.15 & 433 & 83.51 & 940 & 87.09 \\
\hline Surface & - & - & 507 & 90.15 & 433 & 83.51 & 940 & 87.09 \\
\hline Utah & 139 & 41.27 & - & - & 236 & 48.93 & 375 & 46.10 \\
\hline Underground & 139 & 41.27 & - & - & 236 & 48.93 & 375 & 46.10 \\
\hline Virginia & w & $\mathrm{w}$ & $w$ & $\mathbf{w}$ & 130 & $\mathbf{5 9 . 3 4}$ & 203 & 58.14 \\
\hline Underground & $\mathbf{w}$ & $\mathrm{w}$ & - & - & w & $\mathbf{w}$ & 192 & 57.18 \\
\hline Surface & $\mathbf{w}$ & $w$ & w & $w$ & $\mathrm{w}$ & $\mathbf{w}$ & 11 & 74.96 \\
\hline
\end{tabular}

See footnotes at end of table. 
Table 32. Recoverable Coal Reserves and Average Recovery Percentage at Producing Mines by State, Mine Type, and Union Type, 1995 (Continued)

(Million Short Tons)

\begin{tabular}{|c|c|c|c|c|c|c|c|c|}
\hline \multirow[b]{2}{*}{$\begin{array}{l}\text { Coal-Producing } \\
\text { State and Region }\end{array}$} & \multicolumn{2}{|c|}{ UMWA } & \multicolumn{2}{|c|}{ Other Inions } & \multicolumn{2}{|c|}{ Nonunion } & \multicolumn{2}{|c|}{ Total } \\
\hline & $\begin{array}{c}\text { Recoverable } \\
\text { Coal } \\
\text { Reserves }\end{array}$ & $\begin{array}{c}\text { Average } \\
\text { Recovery } \\
\text { Percentage }\end{array}$ & $\begin{array}{c}\text { Recoverable } \\
\text { Coal } \\
\text { Reserves }\end{array}$ & $\begin{array}{c}\text { Average } \\
\text { Recovery } \\
\text { Percentage }\end{array}$ & $\begin{array}{c}\text { Recoverable } \\
\text { Coal } \\
\text { Reserves }\end{array}$ & $\begin{array}{c}\text { Average } \\
\text { Recovery } \\
\text { Percentage }\end{array}$ & $\begin{array}{c}\text { Recoverable } \\
\text { Coal } \\
\text { Reserves }\end{array}$ & $\begin{array}{c}\text { Average } \\
\text { Recovery } \\
\text { Percentage }\end{array}$ \\
\hline 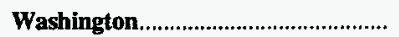 & - & - & $\mathbf{w}$ & $\mathbf{w}$ & $\mathbf{w}$ & $\mathbf{w}$ & $\mathbf{w}$ & $\mathbf{w}$ \\
\hline Surface & - & - & $\mathrm{w}$ & $\mathbf{w}$ & w & w & $\mathbf{w}$ & $\mathbf{w}$ \\
\hline 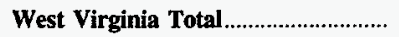 & 1,224 & 61.45 & - & - & 507 & 64.83 & 1,731 & 62.44 \\
\hline 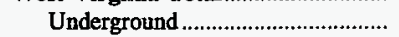 & 939 & 55.84 & - & - & 306 & 58.24 & 1,246 & 56.43 \\
\hline 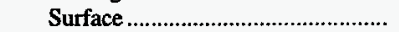 & 285 & 79.94 & - & - & 200 & 74.91 & 486 & 77.86 \\
\hline 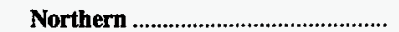 & $\mathbf{5 8 2}$ & 52.05 & - & - & 200 & 63.99 & 782 & 55.10 \\
\hline 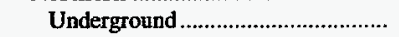 & 582 & 52.05 & - & - & 95 & 59.93 & 677 & 53.16 \\
\hline 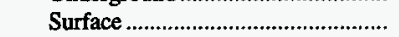 & - & - & - & - & 105 & 67.65 & 105 & 67.65 \\
\hline Southern & 642 & 69.97 & - & - & 307 & 65.38 & 949 & 68.49 \\
\hline 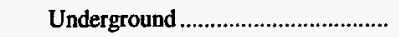 & 357 & 62.02 & - & - & 212 & 57.48 & 569 & 60.33 \\
\hline Surface & 285 & 79.94 & - & - & 95 & 82.89 & 381 & 80.68 \\
\hline Wyoming & $w$ & w & $w$ & $w$ & 6,323 & 92.33 & 6,724 & 92.27 \\
\hline 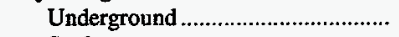 & - & - & - & - & w & $\mathbf{w}$ & w & w \\
\hline Surface & $\mathbf{w}$ & $\mathbf{w}$ & $\mathbf{w}$ & $\mathbf{w}$ & $w$ & w & $\mathbf{w}$ & w \\
\hline 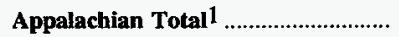 & 2,448 & 61.38 & 7 & 61.15 & 2,083 & 64.15 & 4,538 & 62.65 \\
\hline 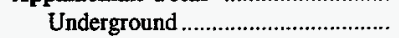 & $w$ & w & $\mathbf{w}$ & w & 1,453 & 57.56 & 3,467 & 57.22 \\
\hline 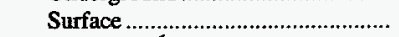 & $\mathbf{w}$ & w & w & $\mathrm{w}$ & 630 & 79.33 & 1,071 & 80.22 \\
\hline Interior Total ${ }^{1}$ & 849 & 55.17 & 536 & 88.97 & 1,450 & 69.15 & 2,835 & 68.71 \\
\hline Underground & w & w & w & w & 691 & 53.22 & 1,335 & 49.73 \\
\hline Surface & $\mathbf{w}$ & $\mathbf{w}$ & w & w & 759 & 83.65 & 1,500 & 85.61 \\
\hline Western Total ${ }^{1}$ & 1,626 & 82.80 & 2,598 & 91.47 & 8,507 & 89.15 & 12,732 & 88.81 \\
\hline 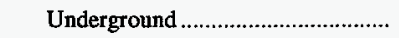 & 273 & 48.69 & - & - & 559 & 51.73 & 832 & 50.73 \\
\hline Surface & 1,354 & 89.66 & 2,598 & 91.47 & 7,948 & 91.78 & 11,900 & 91.47 \\
\hline East of Miss. River .................................. & 3,294 & 59.77 & 36 & 67.09 & 2,930 & 62.44 & 6,260 & 61.06 \\
\hline 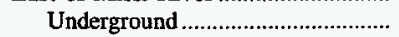 & 2,637 & 54.26 & 20 & 60.10 & 2,144 & 56.16 & 4,802 & 55.13 \\
\hline Surface & 657 & 81.90 & 15 & 76.37 & 786 & 79.58 & 1,458 & 80.59 \\
\hline West of Miss. River................................ & 1,629 & 82.77 & 3,106 & 91.25 & 9,111 & 88.84 & 13,845 & 88.66 \\
\hline Underground & 273 & 48.69 & - & - & 559 & 51.73 & 832 & 50.73 \\
\hline Surface & 1,356 & 89.62 & 3,106 & 91.25 & 8,552 & 91.26 & 13,014 & 91.09 \\
\hline 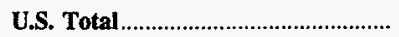 & 4,923 & 67.38 & 3,141 & 90.98 & 12,041 & 82.41 & 20,105 & 80.07 \\
\hline 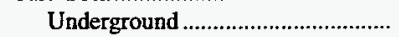 & 2,910 & 53.74 & 20 & 60.10 & 2,703 & 55.25 & 5,633 & 54.48 \\
\hline Surface & 2,013 & 87.10 & 3,121 & 91.18 & 9,338 & 90.28 & 14,472 & 90.03 \\
\hline
\end{tabular}

1 For a definition of coal-producing regions, see Appendix $\mathbf{C}$.

withheld to avoid disclosure of individual company data.

Notes: Recoverable reserves represent the quantity of coal that can be recovered (i.e., mined) from existing coal reserves at reporting mines. Average recovery percentage represents the percentage of coal that can be recovered from coal reserves at reporting mines, weighted for all mines in the reported geographic area. Excludes silt, culm, refuse bank, slurry dam, and dredge operations except for Pennsylvania anthracite. Excludes mines producing less than 10,000 short tons, which are not required to provide these data. Totals may not equal sum of components due to independent rounding. See Glossary for listing of other unions.

Sources: Energy Information Administration, Form EIA-7A, "Coal Production Report"; State Mining Agency Coal Production Reports; and/or U.S. Department of Labor, Mine Safety and Health Administration, Form 7000-2, "Quarterly Mine Employment and Coal Production Report." 
Table 33. Status of Recoverable Coal Reserves and Coal Production from Producing Federal Coal Leases by State, Fiscal Year 1995

(Million Short Tons)

\begin{tabular}{|c|c|c|c|}
\hline Coal-Producing State & Number of Leases & Recoverable Coal Reserves & Production \\
\hline Alabama & 3 & 30 & - \\
\hline 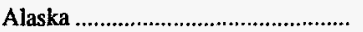 & - & 20 & - \\
\hline 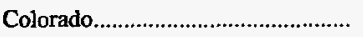 & 33 & 1,525 & 18.9 \\
\hline 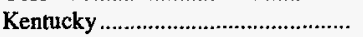 & 2 & 10 & .2 \\
\hline Montana & 13 & 911 & 28.0 \\
\hline New Mexico ........................................ & 6 & 268 & 6.2 \\
\hline North Dakota .................................... & 4 & 146 & 2.1 \\
\hline 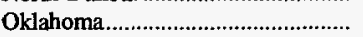 & 6 & 49 & .5 \\
\hline 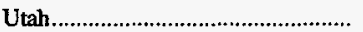 & 34 & 3,014 & 23.6 \\
\hline 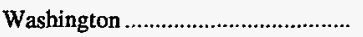 & 1 & - & .4 \\
\hline 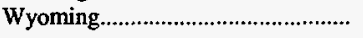 & 44 & 6,397 & 268.5 \\
\hline 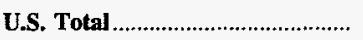 & 146 & 12,370 & 348.5 \\
\hline
\end{tabular}

Notes: Fiscal year 1995 is the period from July 1, 1994, to June 30, 1995. Output from Federal Lands is reported as sales volume, the basis for royalties. It is approximately equivalent to production, which includes coal sold and coal added to stockpiles. Totals may not equal sum of components because of independent rounding.

Source: U.S. Department of the Interior, Minerals Management Service and Bureau of Land Management, Mineral Revenues 1995 

Producer/Distributor Stocks

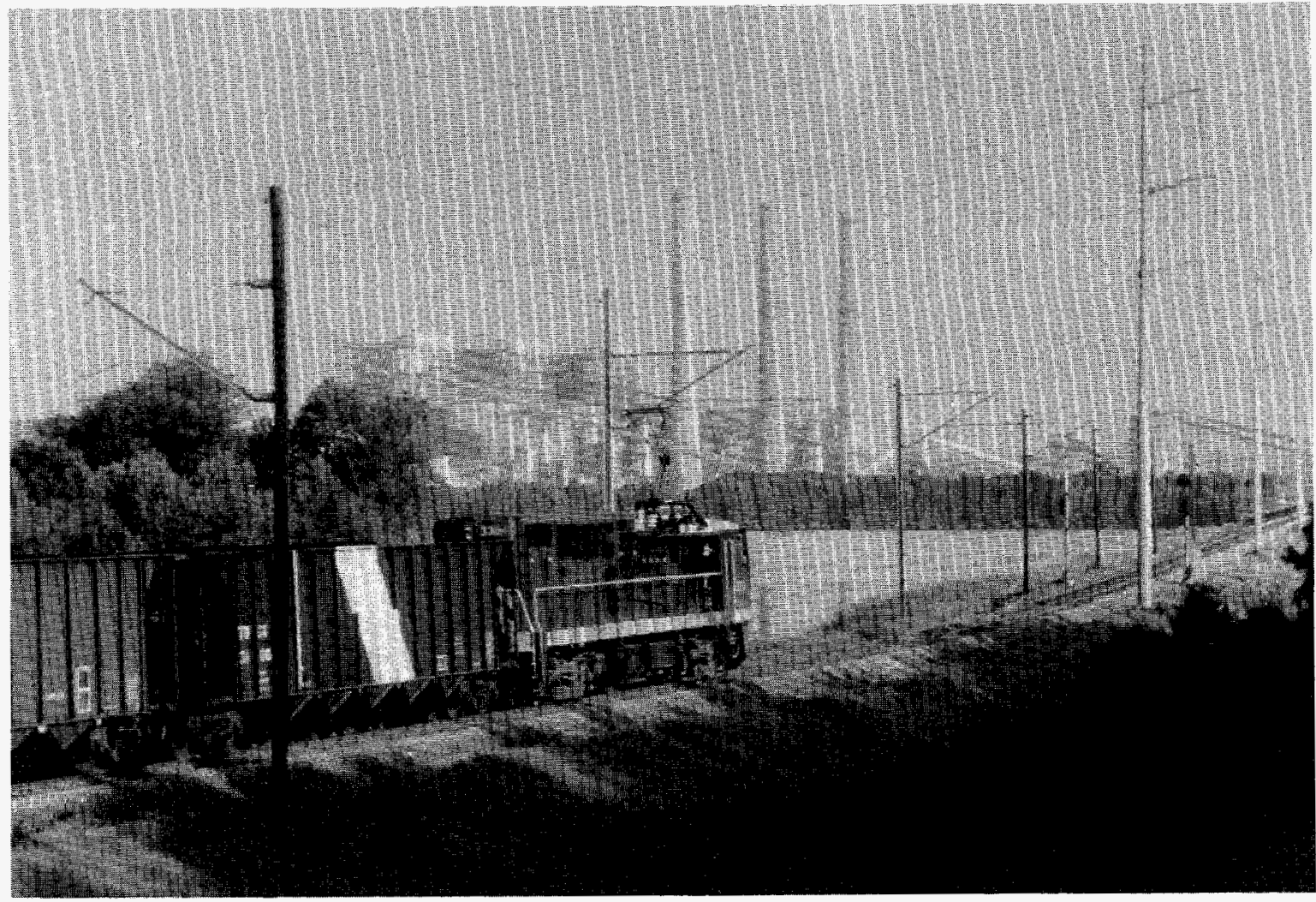

Coal train delivers lignite to a Texas power plant. 
Table 34. Year-End Producer and Distributor Coal Stocks by State, 1991-1995 (Thousand Short Tons)

\begin{tabular}{|c|c|c|c|c|c|c|c|}
\hline \multirow{2}{*}{$\begin{array}{l}\text { Coal-Producing } \\
\text { State and Region }\end{array}$} & \multirow{2}{*}{1995} & \multirow[t]{2}{*}{1994} & \multirow[t]{2}{*}{1993} & \multirow[t]{2}{*}{1992} & \multirow[t]{2}{*}{1991} & \multirow{2}{*}{$\begin{array}{c}\text { Percent } \\
\text { Change } \\
\text { 1994-1995 }\end{array}$} & \multirow{2}{*}{$\begin{array}{c}\begin{array}{c}\text { Average Annual } \\
\text { Percent Change }\end{array} \\
1991-1995\end{array}$} \\
\hline & & & & & & & \\
\hline 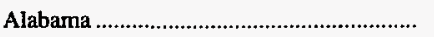 & 1,358 & 1,204 & 1,698 & 2,185 & 2,233 & 12.8 & -11.7 \\
\hline 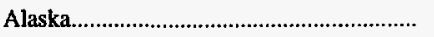 & 26 & 58 & 19 & 71 & 75 & -56.0 & -23.4 \\
\hline Arizona & 2,760 & 2,634 & 1,590 & 1,555 & 1,461 & 4.8 & 17.2 \\
\hline 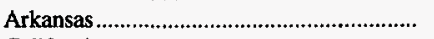 & 4 & 2 & 5 & 6 & - & 111.4 & - \\
\hline 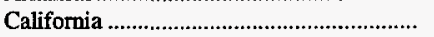 & - & - & - & 36 & 127 & - & - \\
\hline Colorado & 1,063 & 1,575 & 1,155 & 955 & 850 & -32.5 & 5.8 \\
\hline 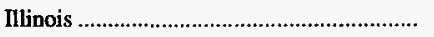 & 2,069 & 1,651 & 713 & 1,969 & 1,418 & 25.3 & 9.9 \\
\hline 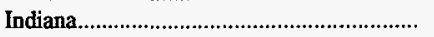 & 611 & 803 & 527 & 1,016 & 525 & -23.9 & 3.9 \\
\hline 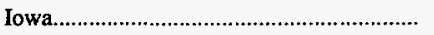 & - & - & - & - & $*$ & - & - \\
\hline 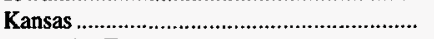 & 27 & 31 & 25 & 28 & 20 & -14.3 & 8.1 \\
\hline 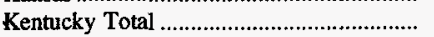 & 4,777 & 5,025 & 3,216 & 3,796 & 4,646 & -4.9 & .7 \\
\hline Eastern & 4,088 & 4,235 & 2,558 & 2,809 & 3,266 & -3.5 & 5.8 \\
\hline Western & 689 & 790 & 658 & 987 & 1,379 & -12.8 & -15.9 \\
\hline 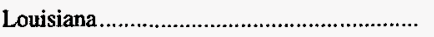 & 309 & 202 & 12 & 2 & 3 & 52.8 & 209.7 \\
\hline Maryland & 269 & 179 & 123 & 59 & 169 & 50.5 & 12.4 \\
\hline 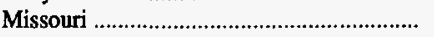 & - & - & 2 & - & 5 & - & - \\
\hline Montana & 718 & 635 & 876 & 694 & 571 & 13.2 & 5.9 \\
\hline 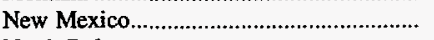 & 2,015 & 1,467 & 2,343 & 1,648 & 1,760 & 37.3 & 3.4 \\
\hline North Dakota & 1,797 & 1,812 & 1,607 & 1,614 & 1,619 & -.8 & 2.6 \\
\hline 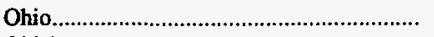 & 1,374 & 833 & 550 & 1,087 & 821 & 64.8 & 13.7 \\
\hline 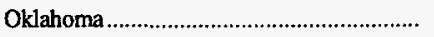 & 2 & 4 & 5 & 19 & 41 & -40.0 & -51.6 \\
\hline Pennsylvania Total & 2,487 & 2,787 & 1,826 & 2,903 & 2,844 & -10.8 & -3.3 \\
\hline Anthracite & 389 & 249 & 234 & 198 & 196 & 56.3 & 18.7 \\
\hline 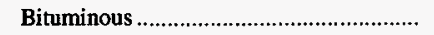 & 2,098 & 2,538 & 1,592 & 2,706 & 2,647 & -17.3 & -5.6 \\
\hline Tennessee & 88 & 57 & 35 & 21 & 94 & 55.7 & -1.4 \\
\hline Texas & 864 & 1,430 & 1,237 & 543 & 400 & -39.6 & 21.3 \\
\hline Utah & 1,946 & 1,301 & 1,203 & 1,827 & 1,676 & 49.6 & 3.8 \\
\hline 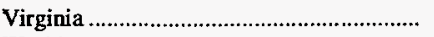 & 1,649 & 1,180 & 1,389 & 1,714 & 2,154 & 39.7 & -6.5 \\
\hline 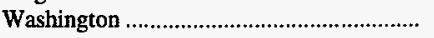 & 59 & 65 & 72 & 47 & 79 & -10.1 & -7.1 \\
\hline 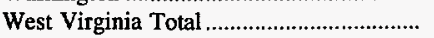 & 6,176 & 6,692 & 4,059 & 7,405 & 6,464 & -7.7 & -1.1 \\
\hline 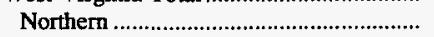 & 1,959 & 1,940 & 685 & 3,222 & 3,083 & .9 & -10.7 \\
\hline Southern & 4,217 & 4,752 & 3,374 & 4,183 & 3,382 & -11.3 & 5.7 \\
\hline Wyoming & 1,997 & 1,592 & 998 & 2,794 & 2,918 & 25.5 & -9.0 \\
\hline Appalachian Total ${ }^{1}$ & 17,489 & 17,166 & 12,239 & 18,183 & 13,891 & 1.9 & -3.1 \\
\hline Interior Total 1 & 4,575 & 4,913 & 3,182 & 4,570 & 3,791 & -6.9 & 4.8 \\
\hline Western Total ${ }^{1}$ & 12,381 & 11,140 & 9,863 & 11,241 & 11,135 & 11.1 & 2.7 \\
\hline 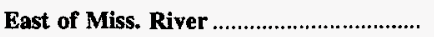 & 20,858 & 20,410 & 14,137 & 22,155 & 17,680 & 2.2 & -5.4 \\
\hline 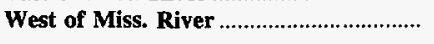 & 13,587 & 12,809 & 11,147 & 11,838 & 11,604 & 6.1 & 4.0 \\
\hline U.S. Total & 34,444 & 33,219 & 25,284 & 33,993 & 32,971 & 3.7 & 1.1 \\
\hline
\end{tabular}

1 For a definition of coal-producing regions, see Appendix $\mathrm{C}$.

* Data round to zero.

Note: Totals may not equal sum of components due to independent rounding

Source: Energy Information Administration, Form EIA-6, "Coal Distribution Report." 


\section{Imports}

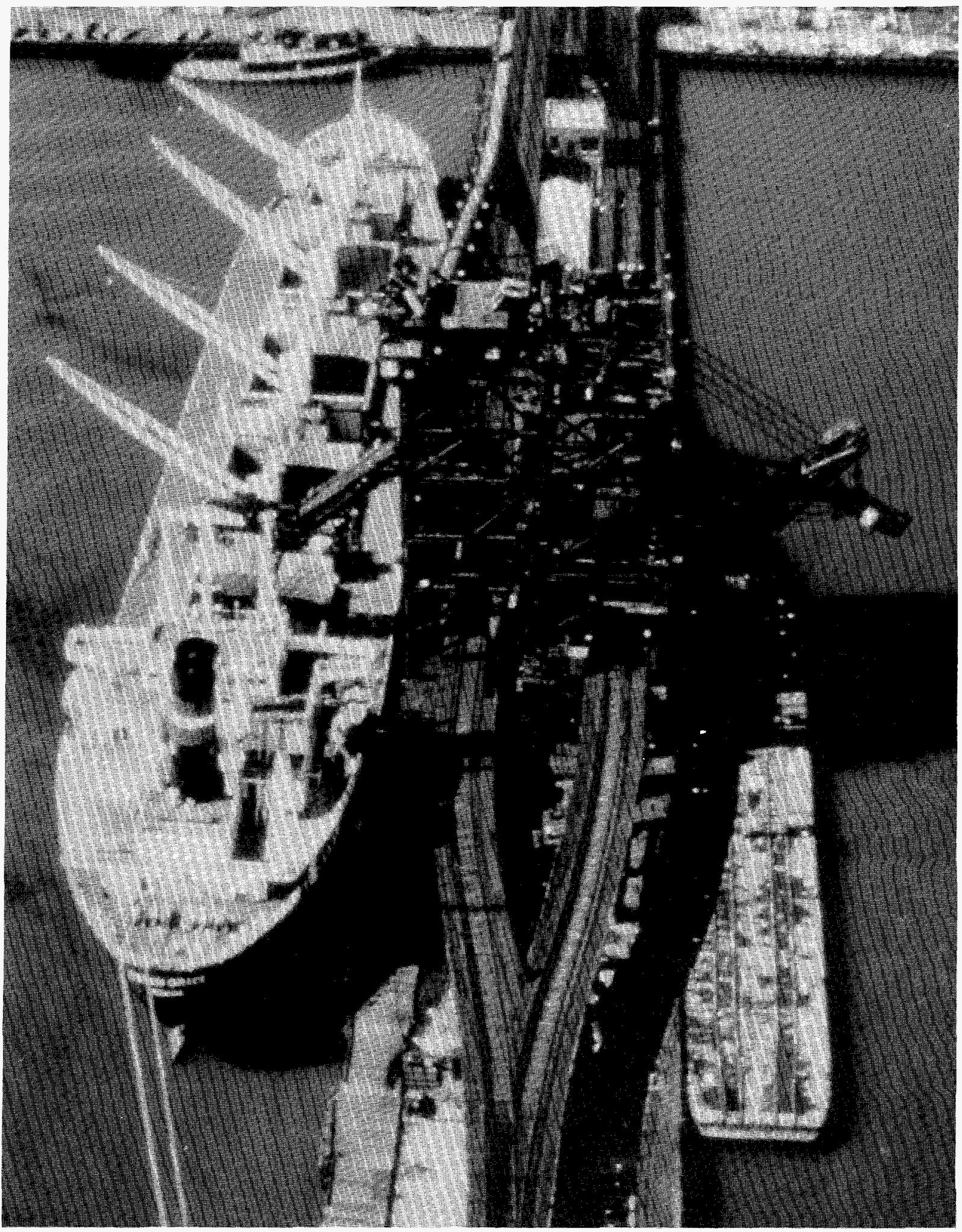

Colombia and Venezuela were the leading sources of imported coal in 1995, which accounted for more than 50 percent of total coal imports. 
Figure 4. U.S. Coal Imports, 1986-1995

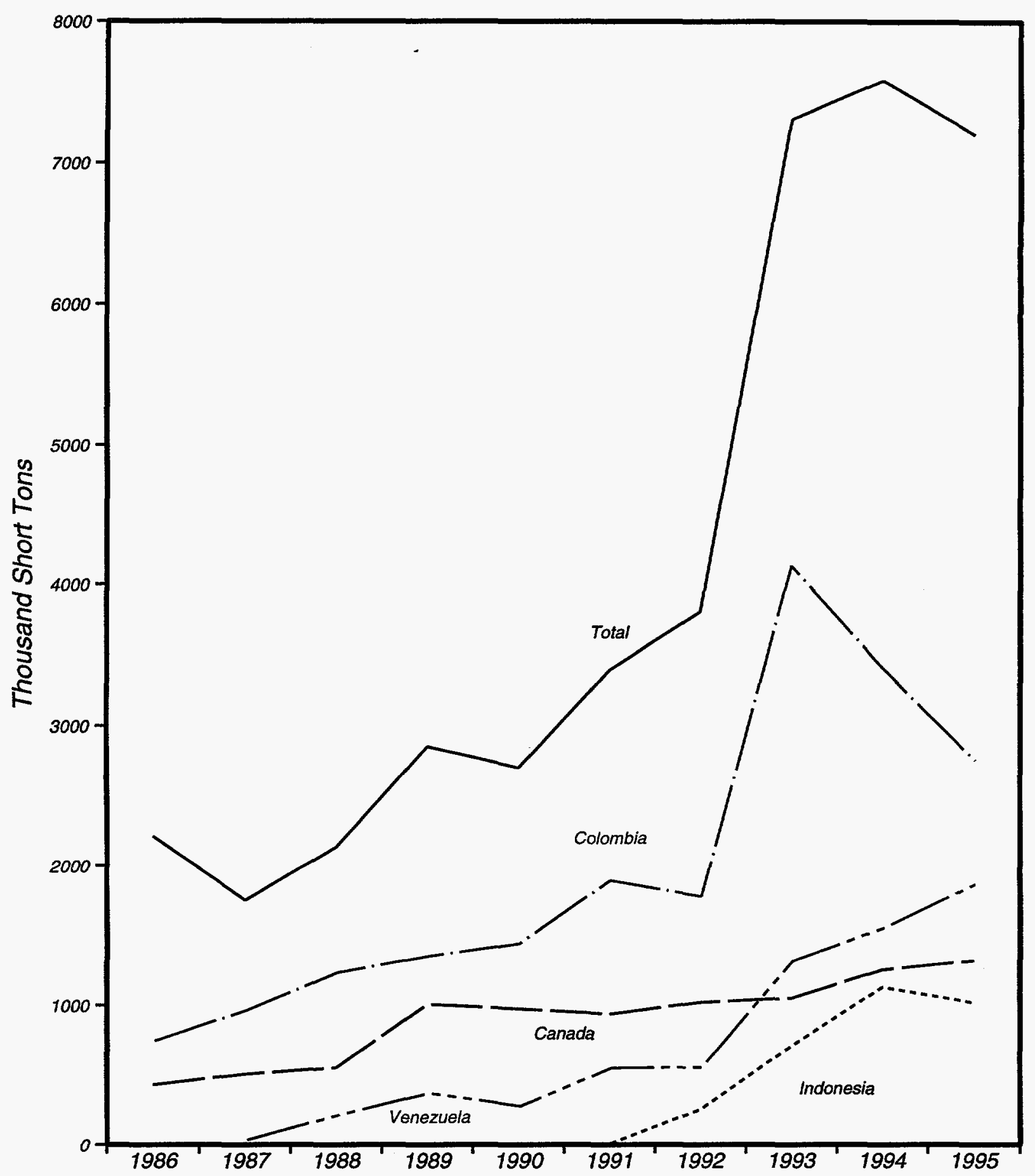

Source: U.S. Department of Commerce, Bureau of the Census, "Monthly Report IM 145." 
Table 35. U.S. Coal Imports by Continent and Country of Origin, 1986, 1991-1995 (Short Tons)

\begin{tabular}{|c|c|c|c|c|c|c|c|c|c|}
\hline \multirow{2}{*}{$\begin{array}{c}\text { Continent and Country } \\
\text { of Origin }\end{array}$} & \multirow{2}{*}{1995} & \multirow{2}{*}{1994} & \multirow{2}{*}{1993} & \multirow{2}{*}{1992} & \multirow{2}{*}{1991} & \multirow{2}{*}{1986} & \multirow{2}{*}{$\begin{array}{c}\text { Percent } \\
\text { Change } \\
\text { 1994-1995 }\end{array}$} & \multicolumn{2}{|c|}{ Average Annual Percent Change } \\
\hline & & & & & & & & 1991-1995 & 1986-1995 \\
\hline North America Total............................. & $1,344,614$ & $1,253,417$ & $1,053,576$ & $1,020,852$ & $\mathbf{9 3 5 , 4 7 7}$ & 433,361 & 7.3 & 9.5 & 13.4 \\
\hline 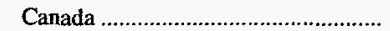 & $1,319,742$ & $1,253,196$ & $1,051,273$ & $1,020,852$ & 935,477 & 432,526 & 5.3 & 9.0 & 13.2 \\
\hline 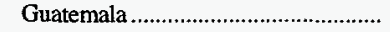 & - & - & 4 & - & - & - & - & - & - \\
\hline Mexico & 216 & 221 & 2,299 & - & - & 835 & -2.3 & - & -13.9 \\
\hline 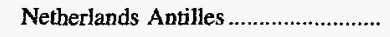 & 24,656 & - & - & - & - & - & - & - & - \\
\hline 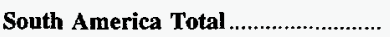 & $4,583,283$ & $4,920,202$ & $5,415,318$ & $2,301,933$ & $2,416,309$ & 742,462 & -6.8 & 17.3 & 22.4 \\
\hline 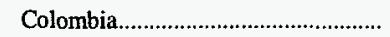 & $2,736,933$ & $3,389,654$ & $4,117,036$ & $1,763,150$ & $1,881,439$ & 742,462 & -19.3 & 9.8 & 15.6 \\
\hline 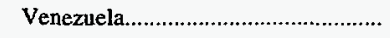 & $1,846,350$ & $1,530,548$ & $1,298,282$ & 538,783 & 534,870 & - & 20.6 & 36.3 & - \\
\hline 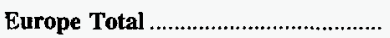 & 522 & 40 & 62 & 89 & 6 & 301 & $\mathbf{N M}$ & 205.4 & 6.3 \\
\hline 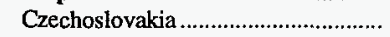 & - & - & - & - & 6 & - & - & -100.0 & - \\
\hline Denmark & 236 & - & 60 & - & - & - & - & - & - \\
\hline Italy & - & - & - & - & - & 241 & - & - & -100.0 \\
\hline 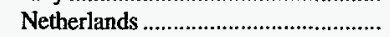 & - & - & - & - & - & 59 & - & - & -100.0 \\
\hline Poland & - & 40 & 2 & - & - & - & -100.0 & - & - \\
\hline 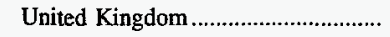 & 286 & - & - & 89 & - & - & - & - & - \\
\hline Asia Total & $1,018,512$ & $1,153,561$ & 708,080 & $\mathbf{3 7 3 , 1 4 5}$ & 6,894 & 139 & -11.7 & 248.6 & 168.8 \\
\hline 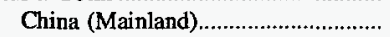 & 53 & 111 & - & 284 & 202 & 99 & -52.3 & -28.4 & -6.7 \\
\hline India & - & - & - & 66,154 & - & - & - & - & - \\
\hline Indonesia & $1,018,433$ & $1,130,468$ & 708,080 & 253,287 & 6,641 & 40 & -9.9 & 251.9 & 208.7 \\
\hline Japan & 26 & 1 & - & - & 1 & - & $\mathrm{NM}$ & 125.8 & - \\
\hline 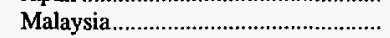 & - & - & - & 53,419 & - & - & - & - & - \\
\hline Thailand & - & - & - & - & 50 & - & - & -100.0 & - \\
\hline 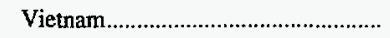 & - & 22,981 & - & - & - & - & -100.0 & - & - \\
\hline Oceania \& Australia Total ................. & 254,141 & 100,313 & 105,452 & 100,986 & 31,106 & 5,857 & 153.3 & 69.1 & $\mathbf{5 2 . 0}$ \\
\hline Australia & 211,702 & 92,204 & 100,076 & 100,986 & 31,106 & 5,857 & 129.6 & 61.5 & 49.0 \\
\hline 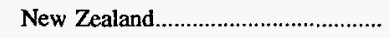 & 42,439 & 8,109 & 5,376 & - & - & - & 423.3 & - & - \\
\hline 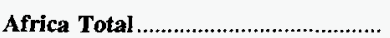 & - & 156,452 & 26,419 & 5,762 & - & $1,029,585$ & -100.0 & - & -100.0 \\
\hline South Africa, Rep of & - & 149,748 & 20,319 & 5,762 & - & $1,029,585$ & -100.0 & - & -100.0 \\
\hline 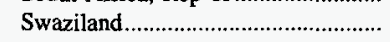 & - & 6,704 & 6,100 & - & - & - & -100.0 & - & - \\
\hline 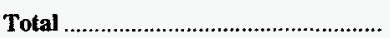 & $7,201,072$ & $7,583,985$ & $7,308,907$ & $3,802,767$ & $3,389,792$ & $2,211,705$ & -5.0 & 20.7 & 14.0 \\
\hline
\end{tabular}

NM Not meaningful as value is greater than 500 percent.

Note: Coal imports include coal to Puerto Rico and the Virgin Islands.

Source: U.S. Department of Commerce, Bureau of the Census, "Monthly Report IM 145." 
Table 36. Coal Imports by Customs District, 1986, 1991-1995

(Short Tons)

\begin{tabular}{|c|c|c|c|c|c|c|c|c|c|}
\hline \multirow{2}{*}{ Customs District } & \multirow{2}{*}{1995} & \multirow{2}{*}{1994} & \multirow{2}{*}{1993} & \multirow{2}{*}{1992} & \multirow{2}{*}{1991} & \multirow{2}{*}{1986} & \multirow{2}{*}{$\begin{array}{c}\text { Percent } \\
\text { Change } \\
\text { 1994-1995 }\end{array}$} & \multicolumn{2}{|c|}{ Average Annual Percent Change } \\
\hline & & & & & & & & 1991-1995 & $1986-1995$ \\
\hline 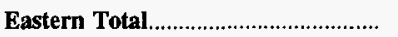 & $1,935,401$ & $1,554,466$ & $1,275,919$ & 340,317 & 361,097 & 656 & 24.5 & 52.1 & 143.0 \\
\hline Boston, MA & $1,484,886$ & 977,473 & 674,086 & 128,667 & 84,310 & 99 & 51.9 & 104.8 & 191.1 \\
\hline Baltimore, MD & 28,328 & 88,668 & 224,579 & - & - & 1 & -68.0 & - & 212.4 \\
\hline 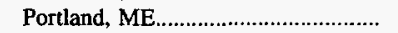 & 364,232 & 385,097 & 236,473 & 108,863 & 207,152 & 150 & -5.4 & 15.1 & 137.8 \\
\hline Buffalo, NY & 2,034 & - & - & - & 134 & 41 & - & 97.4 & 54.3 \\
\hline 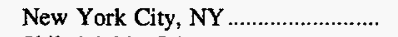 & 522 & - & 2 & - & - & 241 & - & - & 9.0 \\
\hline Philadelphia, PA & 55,399 & 78,387 & 140,779 & 102,787 & 69,501 & 124 & -29.3 & -5.5 & 97.0 \\
\hline 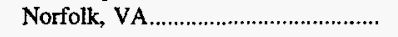 & - & 24,841 & - & - & - & - & -100.0 & - & - \\
\hline 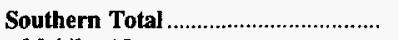 & $3,101,069$ & $4,106,306$ & $4,321,336$ & $2,053,672$ & $2,061,987$ & $1,820,373$ & -24.5 & 10.7 & 6.1 \\
\hline Mobile, AL & $1,108,555$ & $1,033,368$ & 935,232 & - & - & $1,029,585$ & 7.3 & - & .8 \\
\hline Savannah, GA & - & 29,582 & - & - & - & - & -100.0 & - & - \\
\hline Miami, FL & 26,035 & 7,496 & - & - & - & - & 247.3 & - & - \\
\hline Tampa, FL & $1,284,109$ & $2,080,757$ & $2,263,893$ & $1,419,581$ & $1,680,398$ & 45,421 & -38.3 & -6.5 & 45.0 \\
\hline 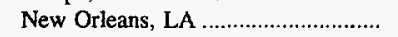 & 387,861 & 524,256 & 675,827 & 154,600 & 23,731 & 488,747 & -26.0 & 101.1 & -2.5 \\
\hline Wilmington, $\mathrm{NC}$ & - & 26,648 & - & - & - & - & -100.0 & - & - \\
\hline 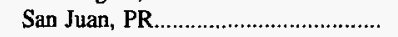 & 272,296 & 80,016 & 107,506 & 169,707 & 211,313 & 255,726 & 240.3 & 6.5 & .7 \\
\hline Houston-Galveston, TX..................... & - & 154,938 & 121,505 & 83,466 & 11,023 & 59 & -100.0 & -100.0 & -100.0 \\
\hline Laredo, TX & 167 & 221 & 2,299 & - & - & 835 & -24.4 & - & -16.4 \\
\hline 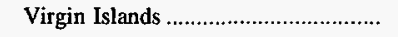 & 22,046 & 169,024 & 215,074 & 226,318 & 135,522 & - & -86.9 & -36.5 & - \\
\hline Western Total & 863,707 & $\mathbf{7 1 0 , 5 7 6}$ & 730,662 & 461,675 & 116,420 & 261,576 & 21.5 & 65.0 & 14.2 \\
\hline Anchorage, AK & - & - & - & - & - & 38 & - & - & -100.0 \\
\hline Los Angeles, CA & - & 12 & - & - & 877 & 40 & -100.0 & -100.0 & -100.0 \\
\hline San Diego, CA & 49 & - & - & - & - & - & - & - & - \\
\hline 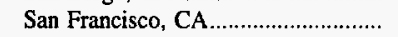 & - & - & - & 284 & - & 48,015 & - & - & -100.0 \\
\hline 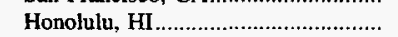 & 844,785 & 670,005 & 660,379 & 407,692 & 31,106 & - & 26.1 & 128.3 & - \\
\hline Great Falls, MT ................................... & 645 & 34,426 & 41,580 & 36,237 & 57,916 & 136,119 & -98.1 & -67.5 & -44.8 \\
\hline Seattle, WA & 18,228 & 6,133 & 28,703 & 17,462 & 26,521 & 77,364 & 197.2 & -8.9 & -14.8 \\
\hline 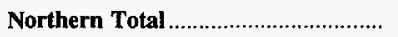 & $1,300,895$ & $1,212,637$ & 980,990 & 947,103 & 850,288 & 129,100 & 7.3 & 11.2 & 29.3 \\
\hline Chicago, IL & 64,394 & 283,106 & 134,485 & 230,677 & 208,021 & 69,149 & -77.3 & -25.4 & -.8 \\
\hline 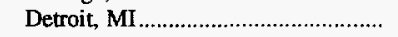 & 421,633 & 312,214 & 203,067 & 58,937 & 275 & - & 35.0 & NM & - \\
\hline 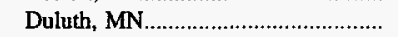 & 244,278 & 77,355 & 12,811 & - & 211 & 1,700 & 215.8 & 483.3 & 73.7 \\
\hline Pembina, ND & 570,590 & 539,962 & 630,627 & 657,400 & 641,781 & 58,251 & 5.7 & -2.9 & 28.8 \\
\hline Cleveland, $\mathrm{OH}$ & - & - & - & 89 & - & - & - & - & - \\
\hline Total & $7, \mathbf{2 0 1 , 0 7 2}$ & $7,583,985$ & $7,308,907$ & $3,802,767$ & $3,389,792$ & $2,211,705$ & -5.0 & 20.7 & 14.0 \\
\hline
\end{tabular}

NM Not meaningful as value is greater than 500 percent.

Source: U.S. Department of Commerce, Bureau of the Census, "Monthly Report IM 145." 
Table 37. U.S. Receipts of Imported Coal by Country of Origin and Destination State, 1986, 1991-1995

(Short Tons)

\begin{tabular}{|c|c|c|c|c|c|c|c|}
\hline $\begin{array}{l}\text { Country of Origin } \\
\text { and Destination State }\end{array}$ & 1995 & 1994 & 1993 & 1992 & 1991 & 1986 & $\begin{array}{c}\text { Percent } \\
\text { Change } \\
\text { 1994-1995 }\end{array}$ \\
\hline Australia Total & $\begin{array}{l}211,099 \\
211,099\end{array}$ & $\begin{array}{l}109,737 \\
109,737\end{array}$ & $\begin{array}{l}98,947 \\
98,947\end{array}$ & $\overline{-}$ & $\overline{-}$ & $\overline{-}$ & $\begin{array}{l}92.4 \\
92.4\end{array}$ \\
\hline $\begin{array}{l}\text { Canada Total } \\
\text { Florida. } \\
\text { Illinois } \\
\text { Indiana .................. } \\
\text { Massachusetts } \\
\text { Michigan } \\
\text { Ohio } \\
\text { Washington }\end{array}$ & $\begin{array}{r}1,401,960 \\
- \\
222,876 \\
760,508 \\
- \\
393,367 \\
1,410 \\
23,799\end{array}$ & $\begin{array}{r}1,317,929 \\
-\overline{3} \\
346,192 \\
592,655 \\
-\overline{371,097} \\
1,635 \\
6,350\end{array}$ & $\begin{array}{r}664,835 \\
5 \overline{-} \\
50,936 \\
582,689 \\
- \\
- \\
2,000 \\
29,210\end{array}$ & $\begin{array}{r}48,090 \\
- \\
- \\
- \\
32,800 \\
- \\
- \\
15,290\end{array}$ & $\begin{array}{r}26,920 \\
- \\
- \\
- \\
- \\
- \\
26,920\end{array}$ & $\begin{array}{r}37,027 \\
37,027 \\
- \\
- \\
- \\
- \\
-\end{array}$ & $\begin{array}{r}6.4 \\
-\overline{-} \\
-35.6 \\
28.3 \\
\overline{-} \\
6.0 \\
-13.8 \\
274.8\end{array}$ \\
\hline 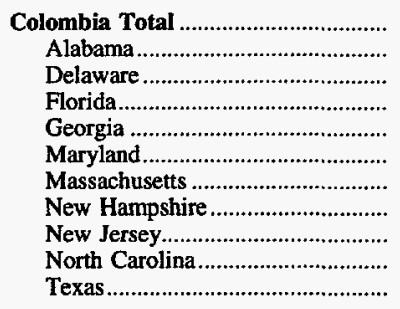 & $\begin{array}{r}2,202,005 \\
161,950 \\
7,143 \\
1,340,640 \\
- \\
- \\
557,900 \\
134,372 \\
- \\
- \\
-\end{array}$ & $\begin{array}{r}3,150,128 \\
178,330 \\
22,031 \\
2,348,550 \\
11,902 \\
88,000 \\
135,500 \\
163,311 \\
22,500 \\
26,600 \\
153,404\end{array}$ & $\begin{array}{r}3,642,728 \\
57,602 \\
- \\
2,999,303 \\
- \\
224,000 \\
187,200 \\
52,143 \\
- \\
- \\
122,480\end{array}$ & $\begin{array}{r}1,504,138 \\
- \\
- \\
1,418,580 \\
- \\
- \\
48,400 \\
- \\
- \\
37,158\end{array}$ & $\begin{array}{r}1,582,600 \\
- \\
1,582,600 \\
- \\
- \\
- \\
- \\
- \\
-\end{array}$ & $\begin{array}{r}418,257 \\
- \\
- \\
418,257 \\
- \\
- \\
- \\
- \\
- \\
-\end{array}$ & $\begin{array}{r}-30.1 \\
-9.2 \\
-67.6 \\
-42.9 \\
-100.0 \\
-100.0 \\
311.7 \\
-17.7 \\
-100.0 \\
-100.0 \\
-100.0\end{array}$ \\
\hline 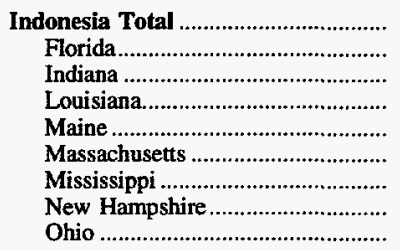 & $\begin{array}{r}428,554 \\
348,854 \\
- \\
- \\
- \\
- \\
- \\
79,700 \\
-\end{array}$ & $\begin{array}{r}437,292 \\
147,215 \\
- \\
169,181 \\
- \\
7,938 \\
- \\
112,958 \\
-\end{array}$ & $\begin{array}{r}118,981 \\
- \\
11,100 \\
- \\
3,135 \\
- \\
67,547 \\
37,199 \\
-\end{array}$ & $\begin{array}{r}13,100 \\
- \\
- \\
- \\
- \\
- \\
- \\
- \\
13,100\end{array}$ & $\begin{array}{r}24,253 \\
24,253 \\
- \\
- \\
- \\
- \\
- \\
-\end{array}$ & $\begin{array}{l}- \\
- \\
- \\
- \\
- \\
- \\
- \\
-\end{array}$ & $\begin{array}{r}-2.0 \\
137.0 \\
-100 . \overline{0} \\
-100 . \overline{0} \\
-\overline{-} \\
-29.4 \\
-\end{array}$ \\
\hline Mexico Total & $\overline{-}$ & $\overline{-}$ & $\begin{array}{l}33,520 \\
33,520\end{array}$ & $\overline{-}$ & $\overline{-}$ & $\overline{-}$ & $\overline{-}$ \\
\hline 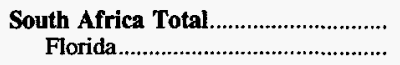 & - & $\begin{array}{l}127,300 \\
127,300\end{array}$ & $\overline{-}$ & - & - & $\begin{array}{l}1,030,500 \\
1,030,500\end{array}$ & $\begin{array}{l}-100.0 \\
-100.0\end{array}$ \\
\hline 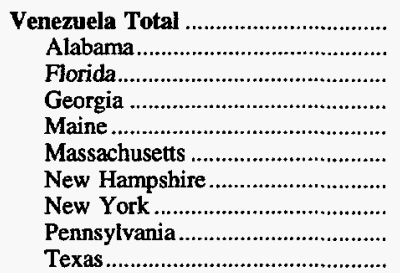 & $\begin{array}{r}2,073,645 \\
- \\
891,400 \\
- \\
81,392 \\
903,700 \\
82,425 \\
28,189 \\
86,539 \\
-\end{array}$ & $\begin{array}{r}1,456,645 \\
- \\
421,674 \\
26,835 \\
91,436 \\
916,700 \\
- \\
- \\
- \\
-\end{array}$ & $\begin{array}{r}936,945 \\
30,278 \\
312,193 \\
- \\
9,123 \\
476,100 \\
109,251 \\
- \\
- \\
-\end{array}$ & $\begin{array}{r}240,584 \\
- \\
- \\
- \\
- \\
163,800 \\
34,300 \\
- \\
- \\
42,484\end{array}$ & $\begin{array}{r}333,027 \\
- \\
42,200 \\
- \\
- \\
83,700 \\
207,127 \\
- \\
- \\
-\end{array}$ & $\begin{array}{l}- \\
- \\
- \\
- \\
- \\
- \\
- \\
- \\
-\end{array}$ & $\begin{array}{r}42.3 \\
- \\
111.4 \\
-100.0 \\
-11.0 \\
-1.4 \\
- \\
- \\
- \\
-\end{array}$ \\
\hline 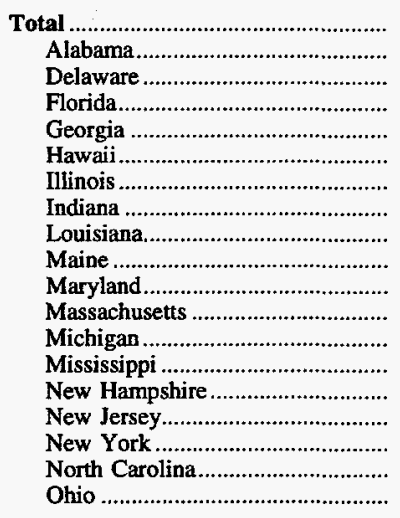 & $\begin{array}{r}6,317,263 \\
161,950 \\
7,143 \\
2,580,894 \\
- \\
211,099 \\
222,876 \\
760,508 \\
- \\
81,39 \overline{-} \\
- \\
1,461,600 \\
393,367 \\
- \\
296,497 \\
- \\
28,189 \\
- \\
1,410\end{array}$ & $\begin{array}{r}6,599,031 \\
178,330 \\
22,031 \\
3,044,739 \\
38,737 \\
109,737 \\
346,192 \\
592,655 \\
169,181 \\
91,436 \\
88,000 \\
1,060,138 \\
371,097 \\
- \\
276,269 \\
22,500 \\
- \\
26,600 \\
1,635\end{array}$ & $\begin{array}{r}5,495,956 \\
87,880 \\
- \\
3,311,496 \\
- \\
98,947 \\
50,936 \\
593,789 \\
- \\
12,258 \\
224,000 \\
663,300 \\
- \\
67,547 \\
198,593 \\
- \\
- \\
- \\
2,000\end{array}$ & $\begin{array}{r}1,805,912 \\
- \\
- \\
1,418,580 \\
- \\
- \\
- \\
- \\
- \\
- \\
- \\
196,600 \\
- \\
- \\
82,700 \\
- \\
- \\
- \\
13,100\end{array}$ & $\begin{array}{r}1,966,800 \\
- \\
- \\
1,649,053 \\
- \\
- \\
- \\
- \\
- \\
- \\
- \\
83,700 \\
- \\
- \\
207,127 \\
- \\
- \\
- \\
-\end{array}$ & $\begin{array}{r}1,485,784 \\
- \\
- \\
1,485,784 \\
- \\
- \\
- \\
- \\
- \\
- \\
- \\
- \\
- \\
- \\
- \\
- \\
- \\
- \\
-\end{array}$ & $\begin{array}{r}-4.3 \\
-9.2 \\
-67.6 \\
-15.2 \\
-100.0 \\
92.4 \\
-35.6 \\
28.3 \\
-100.0 \\
-11.0 \\
-100.0 \\
37.9 \\
6.0 \\
- \\
7.3 \\
-100.0 \\
- \\
-100.0 \\
-13.8\end{array}$ \\
\hline
\end{tabular}

See footnotes at end of table. 
Table 37. U.S. Receipts of Imported Coal by Country of Origin and Destination State, 1986, 1991-1995 (Continued)

(Short Tons)

\begin{tabular}{|c|c|c|c|c|c|c|c|}
\hline $\begin{array}{l}\text { Country of Origin } \\
\text { and Destination State }\end{array}$ & 1995 & 1994 & 1993 & 1992 & 1991 & 1986 & $\begin{array}{c}\text { Percent } \\
\text { Change } \\
\text { 1994-1995 }\end{array}$ \\
\hline \multicolumn{8}{|l|}{ Total (Continued) } \\
\hline Pennsylvania & 86,539 & - & - & - & - & - & - \\
\hline 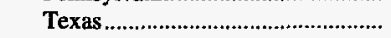 & - & 153,404 & 156,000 & 79,642 & - & - & -100.0 \\
\hline 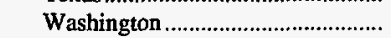 & 23,799 & 6,350 & 29,210 & 15,290 & 26,920 & - & 274.8 \\
\hline
\end{tabular}

Notes: Data for 1986 through 1991 are only for receipts at electric utilities. Data for 1992 through 1995 are for receipts at electric utilities, manufacturing plants and coke plants. See Table 38 and Table 39 for related data. See Technical Note 1 for the difference between receipts of imported coal and U.S. coal imports.

Sources: • 1986-1991: Federal Energy Regulatory Commission (FERC), FERC Form 423, "Monthly Report of Cost and Quality of Fuels for Electric Plants." - 1992-1995: Energy Information Administration, Form EIA-3A, "Annual Coal Quality Report - Manufacturing Plants"; Form EIA-5A, "Annual Coal Quality Report - Coke Plants"; and FERC, FERC Form 423, "Monthly Report of Cost and Quality of Fuels for Electric Plants." 
Table 38. Imported Coal Received at Electric Utilities by Country of Origin and Destination State, 1986, 1991-1995

(Short Tons)

\begin{tabular}{|c|c|c|c|c|c|c|c|c|c|}
\hline \multirow{2}{*}{$\begin{array}{l}\text { Country of Origin } \\
\text { and Destination State }\end{array}$} & \multirow{2}{*}{1995} & \multirow{2}{*}{1994} & \multirow{2}{*}{1993} & \multirow{2}{*}{1992} & \multirow{2}{*}{1991} & \multirow{2}{*}{1986} & \multirow{2}{*}{$\begin{array}{c}\text { Percent } \\
\text { Change } \\
\text { 1994-1995 }\end{array}$} & \multicolumn{2}{|c|}{ Average Annual Percent Change } \\
\hline & & & & & & & & 1991-1995 & 1986-1995 \\
\hline 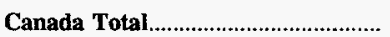 & 23,799 & 63,350 & 29,210 & 48,090 & 26,920 & 37,027 & -62.4 & -3.0 & -4.8 \\
\hline 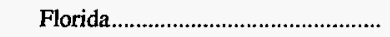 & - & - & - & - & - & 37,027 & - & - & -100.0 \\
\hline 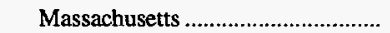 & - & - & - & 32,800 & - & - & - & - & - \\
\hline 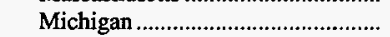 & - & 57,000 & - & - & - & - & -100.0 & - & - \\
\hline 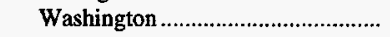 & 23,799 & 6,350 & 29,210 & 15,290 & 26,920 & - & 274.8 & -3.0 & $\rightarrow$ \\
\hline 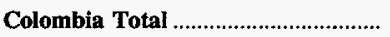 & $2,040,055$ & $2,971,798$ & $3,585,126$ & $1,504,138$ & $1,582,600$ & 418,257 & $-\mathbf{3 1 . 3}$ & 6.5 & 19.3 \\
\hline 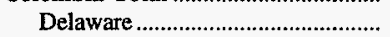 & 7,143 & 22,031 & - & - & - & - & -67.6 & - & - \\
\hline 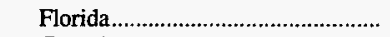 & $1,340,640$ & $2,348,550$ & $2,999,303$ & $1,418,580$ & $1,582,600$ & 418,257 & -42.9 & -4.1 & 13.8 \\
\hline 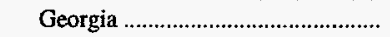 & - & 11,902 & - & - & - & - & -100.0 & - & - \\
\hline 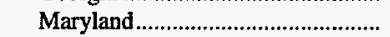 & - & 88,000 & 224,000 & - & - & - & -100.0 & - & - \\
\hline 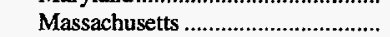 & 557,900 & 135,500 & 187,200 & - & - & - & 311.7 & - & - \\
\hline New Hampshire................................ & 134,372 & 163,311 & 52,143 & 48,400 & - & - & -17.7 & - & - \\
\hline 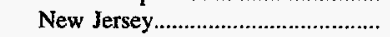 & - & 22,500 & - & - & - & - & -100.0 & - & - \\
\hline 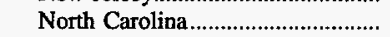 & - & 26,600 & - & - & - & - & -100.0 & - & - \\
\hline Texas & - & 153,404 & 122,480 & 37,158 & - & - & -100.0 & - & - \\
\hline Indonesia Total & 428,554 & 437,292 & 115,846 & 13,100 & 24,253 & - & $-\mathbf{2 . 0}$ & 105.0 & - \\
\hline 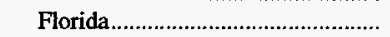 & 348,854 & 147,215 & - & - & 24,253 & - & 137.0 & 94.7 & - \\
\hline 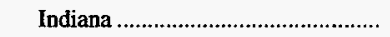 & - & - & 11,100 & - & - & - & - & - & - \\
\hline 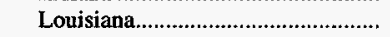 & - & 169,181 & - & - & - & - & -100.0 & - & - \\
\hline 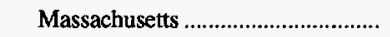 & - & 7,938 & - & - & - & - & -100.0 & - & - \\
\hline 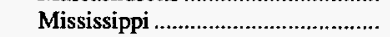 & - & - & 67,547 & - & - & - & - & - & - \\
\hline 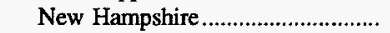 & 79,700 & 112,958 & 37,199 & - & - & - & -29.4 & - & - \\
\hline Ohio & - & - & - & 13,100 & - & - & - & - & - \\
\hline 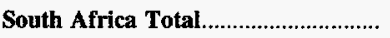 & - & 127,300 & - & - & - & $1, \mathbf{0 3 0}, 50$ & -100.0 & - & -100.0 \\
\hline Florida & - & 127,300 & - & - & - & $1,030,500$ & -100.0 & - & -100.0 \\
\hline 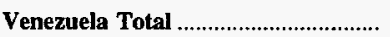 & $1,905,714$ & $1,365,209$ & 897,544 & 240,584 & $\mathbf{3 3 3 , 0 2 7}$ & - & 39.6 & 54.7 & - \\
\hline Florida & 891,400 & 421,674 & 312,193 & - & 42,200 & - & 111.4 & 114.4 & - \\
\hline 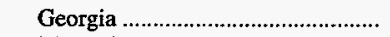 & - & 26,835 & - & - & - & - & -100.0 & - & - \\
\hline 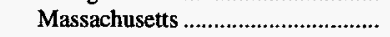 & 903,700 & 916,700 & 476,100 & 163,800 & 83,700 & - & -1.4 & 81.3 & - \\
\hline 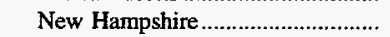 & 82,425 & - & 109,251 & 34,300 & 207,127 & - & - & -20.6 & - \\
\hline 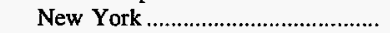 & 28,189 & - & - & - & - & - & - & - & - \\
\hline Texas & - & - & - & 42,484 & - & - & - & - & - \\
\hline Total & $4,398,122$ & $4,964,949$ & $4,627,726$ & $1,805,912$ & $1,966,800$ & $1,485,784$ & -11.4 & 22.3 & 12.8 \\
\hline 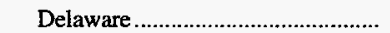 & 7,143 & 22,031 & - & - & - & - & -67.6 & - & - \\
\hline 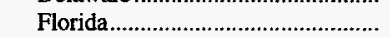 & $2,580,894$ & $3,044,739$ & $3,311,496$ & $1,418,580$ & $1,649,053$ & $1,485,784$ & -15.2 & 11.8 & 6.3 \\
\hline Georgia & - & 38,737 & - & - & - & - & -100.0 & - & - \\
\hline 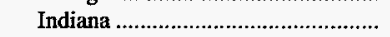 & - & - & 11,100 & - & - & - & - & - & - \\
\hline 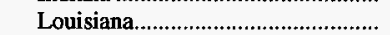 & - & 169,181 & - & - & - & - & -100.0 & - & - \\
\hline 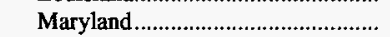 & - & 88,000 & 224,000 & - & - & - & -100.0 & - & - \\
\hline 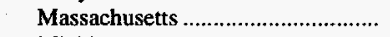 & $1,461,600$ & $1,060,138$ & 663,300 & 196,600 & 83,700 & - & 37.9 & 104.4 & - \\
\hline 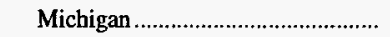 & - & 57,000 & - & - & - & - & -100.0 & - & - \\
\hline Mississippi & - & - & 67,547 & - & - & - & - & - & - \\
\hline 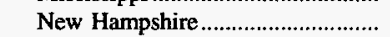 & 296,497 & 276,269 & 198,593 & 82,700 & 207,127 & - & 7.3 & 9.4 & - \\
\hline 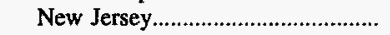 & - & 22,500 & - & - & - & - & -100.0 & - & - \\
\hline 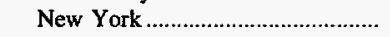 & 28,189 & - & - & - & - & - & - & - & - \\
\hline 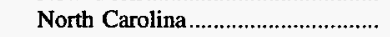 & - & 26,600 & - & - & - & - & -100.0 & - & - \\
\hline Ohio & - & - & - & 13,100 & - & - & - & - & - \\
\hline Texas & - & 153,404 & 122,480 & 79,642 & - & - & -100.0 & - & - \\
\hline Washington & 23,799 & 6,350 & 29,210 & 15,290 & 26,920 & - & 274.8 & -3.0 & - \\
\hline
\end{tabular}

Source: Federal Energy Regulatory Commission, FERC Form 423, "Monthly Report of Cost and Quality of Fuels for Electric Plants." 
Table 39. Imported Coal Received at Manufacturing and Coke Plants by Country of Origin and Destination State, 1993-1995

(Short Tons)

\begin{tabular}{|c|c|c|c|c|c|c|c|c|c|c|}
\hline \multirow{2}{*}{$\begin{array}{l}\text { Country } \\
\text { of Origin } \\
\text { and } \\
\text { Destination } \\
\text { State }\end{array}$} & \multicolumn{3}{|c|}{ Manufacturing } & \multicolumn{3}{|c|}{ Coke Plants } & \multicolumn{4}{|c|}{ Total } \\
\hline & 1995 & 1994 & 1993 & 1995 & 1994 & 1993 & 1995 & 1994 & 1993 & $\begin{array}{c}\text { Percent } \\
\text { Change } \\
\text { 1994-1995 }\end{array}$ \\
\hline Australia Total & 211,099 & 109,737 & 98,947 & - & - & - & 211,099 & 109,737 & $\mathbf{9 8 , 9 4 7}$ & 92.4 \\
\hline 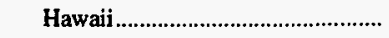 & 211,099 & 109,737 & 98,947 & - & - & - & 211,099 & 109,737 & 98,947 & 92.4 \\
\hline 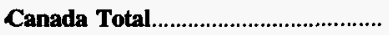 & 1,410 & 1,635 & 2,000 & $1,376,751$ & $1,252,944$ & 633,625 & $1,378,161$ & $1,254,579$ & 635,625 & 9.9 \\
\hline Illinois & - & - & - & 222,876 & 346,192 & 50,936 & 222,876 & 346,192 & 50,936 & -35.6 \\
\hline Indiana & - & - & - & 760,508 & 592,655 & 582,689 & 760,508 & 592,655 & 582,689 & 28.3 \\
\hline 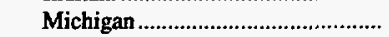 & - & - & - & 393,367 & 314,097 & - & 393,367 & 314,097 & - & 25.2 \\
\hline Ohio & 1,410 & 1,635 & 2,000 & - & - & - & 1,410 & 1,635 & 2,000 & -13.8 \\
\hline 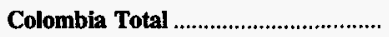 & 161,950 & 178,330 & 57,602 & - & - & - & 161,950 & 178,330 & 57,602 & -9.2 \\
\hline Alabama & 161,950 & 178,330 & 57,602 & - & - & - & 161,950 & 178,330 & 57,602 & -9.2 \\
\hline Indonesia Total & - & - & 3,135 & - & - & - & - & - & 3,135 & - \\
\hline Maine & - & - & 3,135 & - & - & - & - & - & 3,135 & - \\
\hline 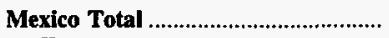 & - & - & 33,520 & - & - & - & - & - & 33,520 & - \\
\hline 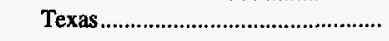 & - & - & 33,520 & - & - & - & - & - & 33,520 & - \\
\hline 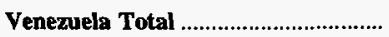 & 167,931 & 91,436 & 39,401 & - & - & - & 167,931 & 91,436 & 39,401 & 83.7 \\
\hline 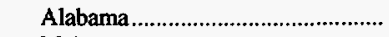 & - & - & 30,278 & - & - & - & - & - & 30,278 & - \\
\hline Maine & 81,392 & 91,436 & 9,123 & - & - & - & 81,392 & 91,436 & 9,123 & -11.0 \\
\hline 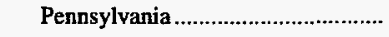 & 86,539 & - & - & - & - & - & 86,539 & - & - & - \\
\hline Total & 542,390 & 381,138 & 234,605 & $1,376,751$ & $1,252,944$ & 633,625 & $1,919,141$ & $1,634,082$ & 868,230 & 17.4 \\
\hline 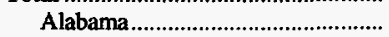 & 161,950 & 178,330 & 87,880 & - & - & - & 161,950 & 178,330 & 87,880 & -9.2 \\
\hline 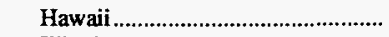 & 211,099 & 109,737 & 98,947 & - & - & - & 211,099 & 109,737 & 98,947 & 92.4 \\
\hline 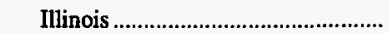 & - & - & - & 222,876 & 346,192 & 50,936 & 222,876 & 346,192 & 50,936 & -35.6 \\
\hline Indiana & - & - & - & 760,508 & 592,655 & 582,689 & 760,508 & 592,655 & 582,689 & 28.3 \\
\hline Maine & 81,392 & 91,436 & 12,258 & - & - & - & 81,392 & 91,436 & 12,258 & -11.0 \\
\hline 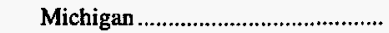 & - & - & - & 393,367 & 314,097 & - & 393,367 & 314,097 & - & 25.2 \\
\hline Ohio & 1,410 & 1,635 & 2,000 & - & - & - & 1,410 & 1,635 & 2,000 & -13.8 \\
\hline Pennsylvania & 86,539 & - & - & - & - & - & 86,539 & - & - & - \\
\hline 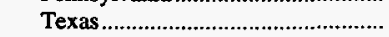 & - & - & 33,520 & - & - & - & - & - & 33,520 & - \\
\hline
\end{tabular}

Sources: Energy Information Administration, Form EIA-3A, “Annual Coal Quality Report - Manufacturing Plants"; and Form EIA-5A, "Annual Coal Quality Report - Coke Plants." 


\section{Employment and Productivity}

\section{Employment}

In 1995, the average number of miners working daily at mines and/or preparation plants producing or processing 10,000 or more short tons of coal dropped 7.4 percent from the 1994 level, to 90,252 miners (Table 40 ). Of the 25 coal-producing States, 18 had a decrease in employment. Although all three regions experienced a decline in employment, the Interior Region accounted for the largest share of the decrease (13.6 percent) in employment. The Appalachian Region had an 7.1 percent decline, while the Western Region showed a slight decline of 0.4 percent.

The average number of miners at underground mines in 1995 totaled 57,879 miners, accounting for 64 percent of all U.S. miners (Table 41). Employment at underground mines decreased 6 percent since 1994, with both Appalachia and Interior Regions showing a decline, while the Western Region showed an increase.

In 1995, the average number of miners at surface mines dropped 9.9 percent from the 1994 level to 32,373 miners (Table 42). All three regions had decreases in surface employment in 1995, with the Interior Region accounting for the largest share (21 percent) of the decline.
Mines that produced 1 million short tons or more of coal in 1995, accounted for 72.8 percent of total production and employed 47 percent of the miners. Of this total, 31 percent worked in underground mines and 16 percent at surface mines. Over 41 thousand miners (45.7 percent) belong to a union.

The United Mine Workers of America (UMWA) represented 41.2 percent of the total coal mining workforce, while 4.4 percent belonged to "other unions" (Table 46).

\section{Productivity}

In 1995 , coal miners working daily averaged 5.38 short tons per miner per hour, an increase of 8 percent from the 1994 level (Table 48). Increases occurred in all regions, with the Interior Region showing the largest increase, 12.1 percent. Over the last decade, productivity has risen at an annual average rate of 6.6 percent, increasing both at underground and surface mines. Underground mines increased 6 percent over the last decade, while surface mines rose 7 percent during the same period. 



\section{Employment}

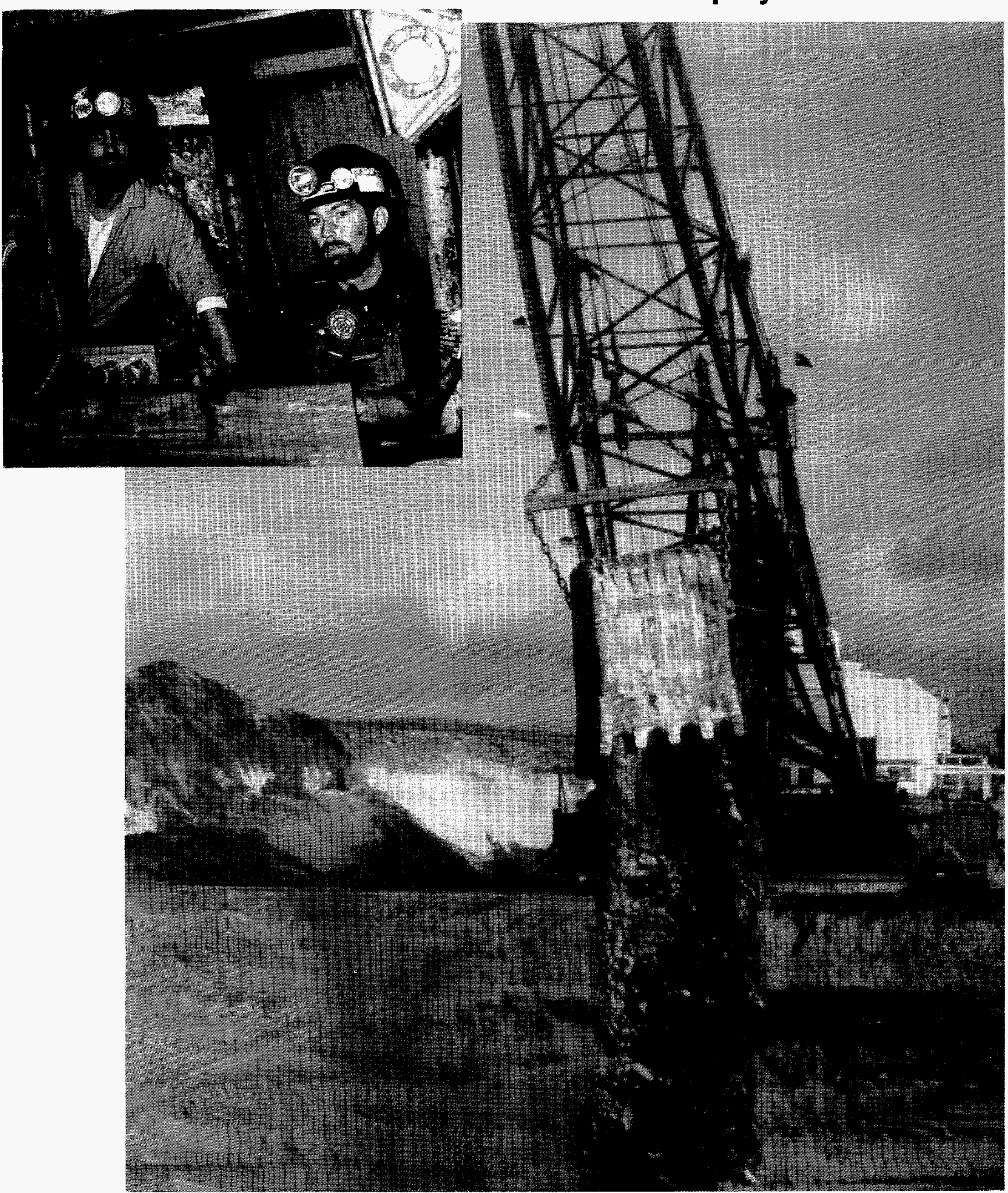

The continuous technological advances in all phases of coal mining in part account for a more productive workforce. Coal miner productivity in 1995 totaled 5.38 short tons per miner per hour, a steady increase since 1991. 

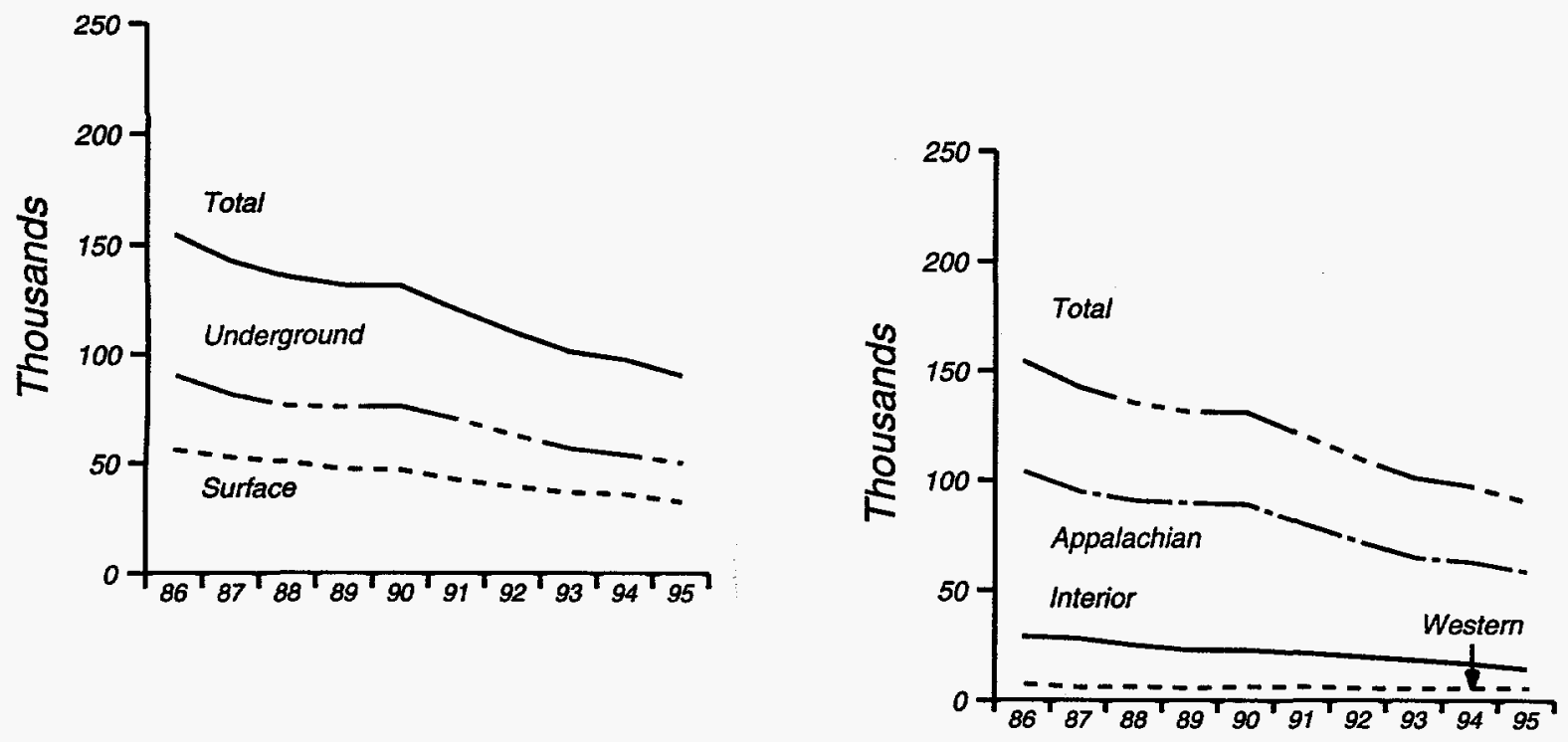

Figure 6. U.S. Coal Mining Productivity by Mine Type and by Region, 1986-1995
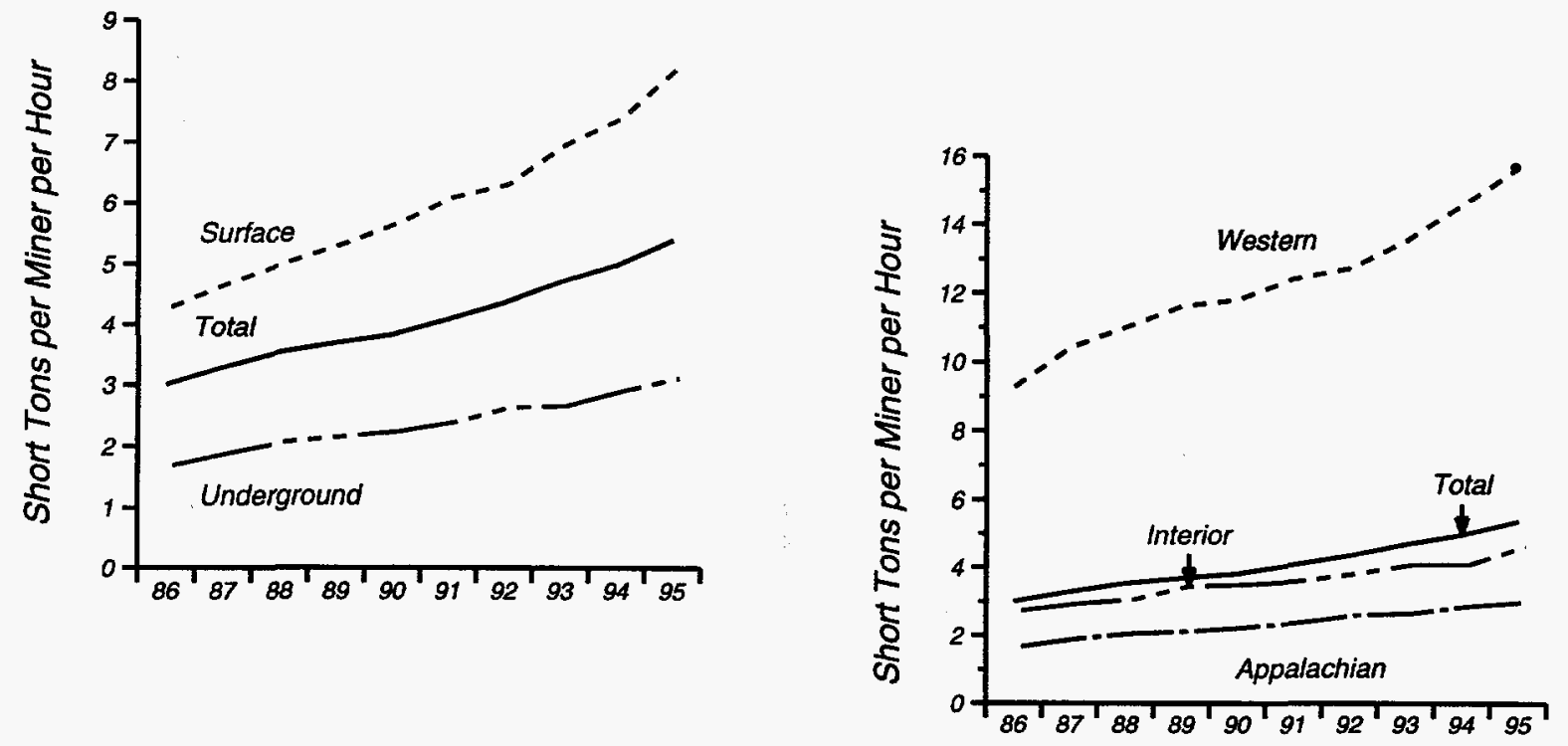

Note: Scale has been enlarged to show detail in the short tons per miner per hour by type of mining plot. Because vertical scales differ, graphs should not be compared. Excludes silt, culm, refuse bank, slurry dam, and dredge operations except for Pennsylvania anthracite. Excludes mines producing less than 10,000 short tons of coal during the year and preparation plants with less than 5,000 employee hours. Includes all employees engaged in production, preparation, processing, development, maintenance, repair, shop or yard work at mining operations. Excludes office workers. Includes mining operations management and all technical and engineering personnel. Short tons produced per miner per hour is calculated by dividing total coal production by the direct labor hours worked by all mine employees.

Sources: Energy Information Administration, Form ElA-7A, "Coal Production Report"; State Mining Agency Coal Production Reports; and/or U.S. Department of Labor, Mine Safety and Health Administration, Form 7000-2, "Quarterly Mine Employment and Coal Production Report." 
Table 40. Average Number of Miners by State, 1986, 1991-1995

\begin{tabular}{|c|c|c|c|c|c|c|c|c|c|}
\hline \multirow{2}{*}{$\begin{array}{l}\text { Coal-Producing } \\
\text { State and Region }\end{array}$} & \multirow{2}{*}{1995} & \multirow{2}{*}{1994} & \multirow{2}{*}{1993} & \multirow{2}{*}{1992} & \multirow{2}{*}{1991} & \multirow{2}{*}{1986} & \multirow{2}{*}{$\begin{array}{c}\text { Percent } \\
\text { Change } \\
\text { 1994-1995 }\end{array}$} & \multicolumn{2}{|c|}{ Average Annual Percent Change } \\
\hline & & & & & & & & 1991-1995 & 1986-1995 \\
\hline Alabama & 5,567 & 5,418 & 5,399 & 5,386 & 6,314 & 7,024 & 2.8 & -3.1 & -2.5 \\
\hline Alaska & 102 & 105 & 96 & 111 & 99 & 96 & -2.8 & .7 & .7 \\
\hline Arizona & 831 & 864 & 876 & 888 & 900 & 915 & -3.8 & -2.0 & -1.1 \\
\hline Arkansas & 4 & 15 & 5 & 14 & 14 & 44 & -73.3 & -26.9 & -23.4 \\
\hline 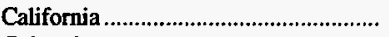 & - & - & - & 8 & 8 & - & - & - & - \\
\hline 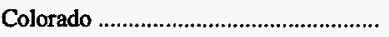 & 1,777 & 1,905 & 1,775 & 1,610 & 2,037 & 2,151 & -6.7 & -3.3 & -2.1 \\
\hline Mlinois & 5,652 & 6,591 & 7,303 & 8,323 & 9,102 & 13,003 & -14.2 & -11.2 & -8.8 \\
\hline Indiana & 2,571 & 3,206 & 3,331 & 3,652 & 3,919 & 4,389 & -19.8 & -10.0 & -5.8 \\
\hline Iowa & - & 20 & 90 & 101 & 97 & 145 & -100.0 & - & - \\
\hline 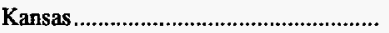 & 54 & 63 & 81 & 96 & 92 & 294 & -14.3 & -12.5 & -17.2 \\
\hline 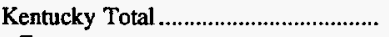 & 21,125 & 23,368 & 24,063 & 24,624 & 26,642 & 32,654 & -9.6 & -5.6 & -4.7 \\
\hline Eastern & 16,840 & 18,577 & 18,711 & 19,419 & 21,129 & 26,030 & -9.3 & -5.5 & -4.7 \\
\hline 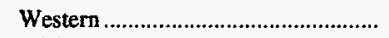 & 4,285 & 4,791 & 5,352 & 5,205 & 5,513 & 6,624 & -10.6 & -6.1 & -4.7 \\
\hline 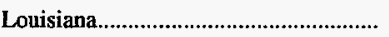 & 114 & 111 & 99 & 77 & 103 & 78 & 2.7 & 2.6 & 4.3 \\
\hline 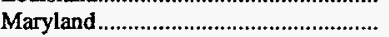 & 458 & 451 & 441 & 438 & 524 & 604 & 1.5 & -3.3 & -3.0 \\
\hline 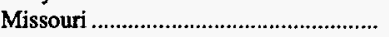 & 92 & 116 & 180 & 323 & 312 & 1,004 & -20.7 & -26.3 & -23.3 \\
\hline 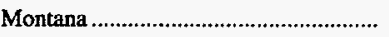 & 722 & 705 & 660 & 715 & 794 & 932 & 2.4 & -2.3 & -2.8 \\
\hline 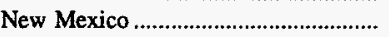 & 1,747 & 1,786 & 1,762 & 1,683 & 1,650 & 1,883 & -2.2 & 1.4 & -.8 \\
\hline 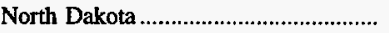 & 716 & 645 & 782 & 744 & 814 & 1,052 & 11.0 & -3.1 & -4.2 \\
\hline Ohio & 3,386 & 3,983 & 3,866 & 4,515 & 5,293 & 8,610 & -15.0 & -10.6 & -9.8 \\
\hline 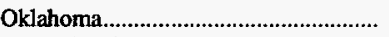 & 241 & 253 & 273 & 334 & 410 & 732 & -4.7 & -12.4 & -11.6 \\
\hline 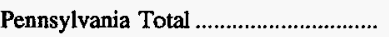 & 8,968 & 9,975 & 10,940 & 12,659 & 13,506 & 19,791 & -10.1 & -9.7 & -8.4 \\
\hline 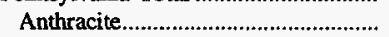 & 1,069 & 1,183 & 1,124 & 1,217 & 1,161 & 1,977 & -9.6 & -2.0 & -6.6 \\
\hline 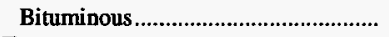 & 7,899 & 8,792 & 9,816 & 11,442 & 12,345 & 17,814 & -10.1 & -10.6 & -8.6 \\
\hline 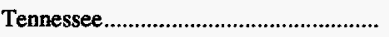 & 681 & 669 & 646 & 804 & 1,242 & 2,240 & 1.8 & -13.9 & -12.4 \\
\hline 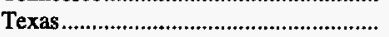 & 1,590 & 1,733 & 1,841 & 2,001 & 2,149 & 3,074 & -8.3 & -7.3 & -7.1 \\
\hline 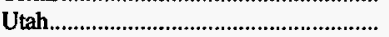 & 1,893 & 1,675 & 1,769 & 1,997 & 2,277 & 2,881 & 13.0 & -4.5 & -4.5 \\
\hline 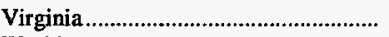 & 6,919 & 8,121 & 8,339 & 9,138 & 10,055 & 13,141 & -14.8 & -8.9 & -6.9 \\
\hline 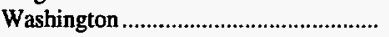 & 566 & 570 & 567 & 612 & 638 & 689 & -.7 & -2.9 & -2.2 \\
\hline West Virginia Total.............................. & 21,334 & 21,861 & 22,979 & 26,017 & 28,310 & 33,540 & -2.4 & -6.8 & -4.9 \\
\hline Northern & 6,114 & 6,659 & 7,274 & 8,481 & 9,468 & 11,030 & -8.2 & -10.3 & -6.3 \\
\hline 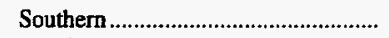 & 15,220 & 15,202 & 15,705 & 17,536 & 18,842 & 22,510 & .1 & -5.2 & -4.3 \\
\hline Wyoming & 3,142 & 3,291 & 3,159 & 3,326 & 3,301 & 3,679 & -4.5 & -1.2 & -1.7 \\
\hline Appalachian Total ${ }^{1} \ldots \ldots \ldots \ldots \ldots \ldots \ldots \ldots$ & 64,153 & 69,055 & $\mathbf{7 1 , 3 2 1}$ & 78,376 & 86,373 & 110,980 & -7.1 & -7.2 & -5.9 \\
\hline Interior Total 1 & 14,603 & 16,899 & 18,555 & 20,126 & 21,711 & 29,387 & -13.6 & -9.4 & -7.5 \\
\hline Western Total ${ }^{1} \ldots \ldots \ldots \ldots \ldots \ldots \ldots \ldots \ldots \ldots$ & 11,496 & 11,546 & 11,446 & 11,694 & 12,518 & 14,278 & -.4 & -2.1 & -2.4 \\
\hline 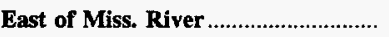 & 76,661 & 83,643 & 87,307 & 95,556 & 104,907 & 134,996 & -8.3 & -7.5 & -6.1 \\
\hline West of Miss. River............................ & 13,591 & 13,857 & 14,015 & 14,640 & 15,695 & 19,649 & -1.9 & -3.5 & -4.0 \\
\hline U.S. Total & 90,252 & 97,500 & 101,322 & 110,196 & 120,602 & 154,645 & -7.4 & -7.0 & -5.8 \\
\hline
\end{tabular}

1 For a definition of coal-producing regions, see Appendix $\mathrm{C}$.

Notes: Includes all employees engaged in production, preparation, processing, development, maintenance, repair, shop or yard work at mining operations. Excludes office workers. Includes mining operations management and all technical and engineering personnel. Excludes silt, culm, refuse bank, slurry dam, and dredge operations except for Pennsylvania anthracite. Excludes mines producing less than 10,000 short tons and preparation plants with less than 5,000 employee hours, which are not required to provide these data.

Sources: Energy Information Administration, Form ElA-7A, "Coal Production Report"; State Mining Agency Coal Production Reports; and/or U.S. Department of Labor, Mine Safety and Health Administration, Form 7000-2, "Quarterly Mine Employment and Coal Production Report." 
Table 41. Average Number of Miners at Underground Mines by State, 1986, 1991-1995

\begin{tabular}{|c|c|c|c|c|c|c|c|c|c|}
\hline \multirow{2}{*}{$\begin{array}{l}\text { Coal-Producing } \\
\text { State and Region }\end{array}$} & \multirow{2}{*}{1995} & \multirow{2}{*}{1994} & \multirow{2}{*}{1993} & \multirow{2}{*}{1992} & \multirow{2}{*}{1991} & \multirow{2}{*}{1986} & \multirow{2}{*}{$\begin{array}{c}\text { Percent } \\
\text { Change } \\
\text { 1994-1995 }\end{array}$} & \multicolumn{2}{|c|}{ Average Annual Percent Change } \\
\hline & & & & & & & & 1991-1995 & 1986-1995 \\
\hline 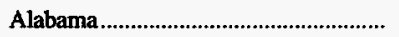 & 4,314 & 3,775 & 3,707 & 3,810 & 4,473 & 4,625 & 14.3 & -0.9 & -0.8 \\
\hline 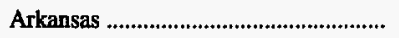 & - & 10 & - & - & - & - & -100.0 & - & - \\
\hline Colorado & 1,301 & 1,248 & 1,119 & 1,085 & 1,530 & 1,218 & 4.2 & -4.0 & .7 \\
\hline 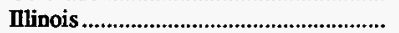 & 4,780 & 5,595 & 6,196 & 6,780 & 7,056 & 9,553 & -14.6 & -9.3 & -7.4 \\
\hline Indiana & 485 & 485 & 545 & 422 & 447 & 460 & - & 2.1 & .6 \\
\hline 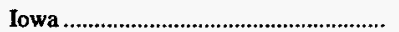 & - & - & - & - & - & 93 & - & - & - \\
\hline 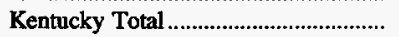 & 14,542 & 15,837 & 16,493 & 16,888 & 18,481 & 21,609 & -8.2 & -5.8 & -4.3 \\
\hline 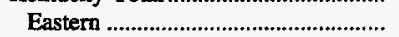 & 11,366 & 12,849 & 13,028 & 13,405 & 14,878 & 17,312 & -11.5 & -6.5 & -4.6 \\
\hline Western & 3,176 & 2,988 & 3,465 & 3,483 & 3,603 & 4,297 & 6.3 & -3.1 & -3.3 \\
\hline 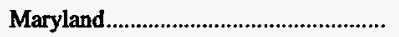 & 293 & 284 & 260 & 228 & 309 & 324 & 3.2 & -1.3 & -1.1 \\
\hline 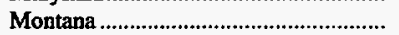 & - & - & 7 & - & - & - & - & - & - \\
\hline 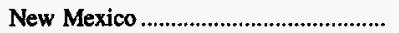 & 132 & 168 & 246 & 141 & 63 & 189 & -21.4 & 20.3 & -3.9 \\
\hline Ohio & 1,670 & 1,694 & 1,601 & 1,926 & 2,359 & 4,110 & -1.4 & -8.3 & -9.5 \\
\hline 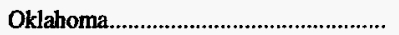 & 12 & 32 & 40 & 24 & 31 & - & -62.5 & -21.1 & - \\
\hline Pennsylvania Total .................................. & 5,659 & 6,192 & 6,853 & 8,113 & 8,751 & 12,415 & -8.6 & -10.3 & -8.3 \\
\hline 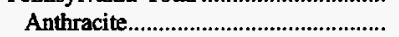 & 152 & 149 & 194 & 147 & 123 & 353 & 2.0 & 5.4 & -8.9 \\
\hline 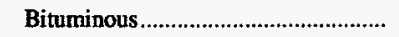 & 5,507 & 6,043 & 6,659 & 7,966 & 8,628 & 12,062 & -8.9 & -10.6 & -8.3 \\
\hline 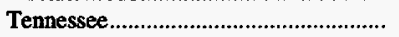 & 473 & 511 & 375 & 599 & 988 & 1,755 & -7.4 & -16.8 & -13.5 \\
\hline 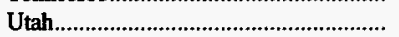 & 1,893 & 1,675 & 1,769 & 1,997 & 2,277 & 2,881 & 13.0 & -4.5 & -4.5 \\
\hline 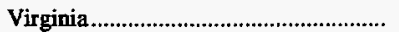 & 5,776 & 6,844 & 7,092 & 7,888 & 8,515 & 11,367 & -15.6 & -9.2 & -7.2 \\
\hline West Virginia Total................................. & 16,347 & 16,956 & 18,040 & 20,738 & 22,512 & 27,839 & -3.6 & -7.7 & -5.7 \\
\hline Northern & 5,561 & 5,997 & 6,414 & 7,513 & 8,509 & 9,224 & -7.3 & -10.1 & -5.5 \\
\hline Southern & 10,786 & 10,959 & 11,626 & 13,225 & 14,003 & 18,615 & -1.6 & -6.3 & -5.9 \\
\hline Wyoming & 202 & 256 & 261 & 268 & 258 & 32 & -21.1 & -5.9 & 22.7 \\
\hline Appalachian Total ${ }^{1}$.............................. & 45,898 & 49,105 & 50,956 & 56,707 & 62,785 & $\mathbf{7 9 , 7 4 7}$ & -6.5 & -7.5 & -5.9 \\
\hline 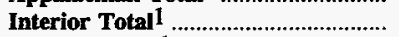 & 8,453 & 9,110 & 10,246 & 10,709 & 11,137 & 14,403 & -7.2 & -6.7 & -5.7 \\
\hline Western Total ${ }^{1}$ & $\mathbf{3 , 5 2 8}$ & 3,347 & 3,402 & 3,491 & 4,128 & 4,320 & 5.4 & -3.8 & -2.2 \\
\hline East of Miss. River................................ & 54,339 & 58,173 & 61,162 & 67,392 & $\mathbf{7 3 , 8 9 1}$ & 94,057 & -6.6 & -7.4 & -5.9 \\
\hline West of Miss. River............................. & 3,540 & 3,389 & 3,442 & 3,515 & 4,159 & 4,413 & 4.4 & -3.9 & -2.4 \\
\hline 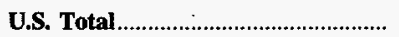 & 57,879 & 61,562 & 64,604 & 70,907 & 78,050 & 98,470 & -6.0 & -7.2 & -5.7 \\
\hline
\end{tabular}

1 For a definition of coal-producing regions, see Appendix $C$.

Notes: Includes all employees engaged in production, preparation, processing, development, maintenance, repair, shop or yard work at mining operations. Excludes office workers. Includes mining operations management and all technical and engineering personnel. Excludes silt, culm, refuse bank, slurry dam, and dredge operations except for Pennsylvania anthracite. Excludes mines producing less than 10,000 short tons and preparation plants with less than 5,000 employee hours, which are not required to provide these data.

Sources: Energy Information Administration, Form EIA-7A, "Coal Production Report"; State Mining Agency Coal Production Reports; and/or U.S. Department of Labor, Mine Safety and Health Administration, Form 7000-2, "Quarterly Mine Employment and Coal Production Report." 
Table 42. Average Number of Miners at Surface Mines by State, 1986, 1991-1995

\begin{tabular}{|c|c|c|c|c|c|c|c|c|c|}
\hline \multirow{2}{*}{$\begin{array}{l}\text { Coal-Producing } \\
\text { State and Region }\end{array}$} & \multirow{2}{*}{1995} & \multirow{2}{*}{1994} & \multirow{2}{*}{1993} & \multirow{2}{*}{1992} & \multirow{2}{*}{1991} & \multirow{2}{*}{1986} & \multirow{2}{*}{$\begin{array}{c}\text { Percent } \\
\text { Change } \\
\text { 1994-1995 }\end{array}$} & \multicolumn{2}{|c|}{ Average Annual Percent Change } \\
\hline & & & & & & & & 1991-1995 & 1986-1995 \\
\hline Alabama & 1,253 & 1,643 & 1,692 & 1,576 & 1,841 & 2,399 & -23.7 & -9.2 & -7.0 \\
\hline 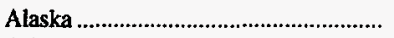 & 102 & 105 & 96 & 111 & 99 & 96 & -2.8 & .7 & .7 \\
\hline Arizona & 831 & 864 & 876 & 888 & 900 & 915 & -3.8 & -2.0 & -1.1 \\
\hline Arkansas & 4 & 5 & 5 & 14 & 14 & 44 & -20.0 & -26.9 & -23.4 \\
\hline California & - & - & - & 8 & 8 & - & - & - & - \\
\hline Colorado & 476 & 657 & 656 & 525 & 507 & 933 & -27.5 & -1.6 & -7.2 \\
\hline 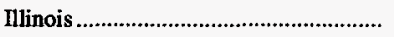 & 872 & 996 & 1,107 & 1,543 & 2,046 & 3,450 & -12.4 & -19.2 & -14.2 \\
\hline Indiana & 2,086 & 2,721 & 2,786 & 3,230 & 3,472 & 3,929 & -23.3 & -11.9 & -6.8 \\
\hline Iowa & - & 20 & 90 & 101 & 97 & 52 & -100.0 & - & - \\
\hline 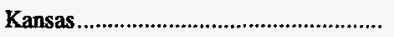 & 54 & 63 & 81 & 96 & 92 & 294 & -14.3 & -12.5 & -17.2 \\
\hline Kentucky Total & 6,583 & 7,531 & 7,570 & 7,736 & 8,161 & 11,045 & -12.6 & -5.2 & -5.6 \\
\hline Eastern & 5,474 & 5,728 & 5,683 & 6,014 & 6,251 & 8,718 & -4.4 & -3.3 & -5.0 \\
\hline Western & 1,109 & 1,803 & 1,887 & 1,722 & 1,910 & 2,327 & -38.5 & -12.7 & -7.9 \\
\hline 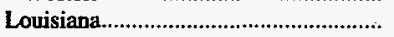 & 114 & 111 & 99 & 77 & 103 & 78 & 2.7 & 2.6 & 4.3 \\
\hline 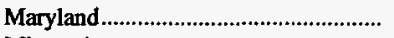 & 165 & 167 & 181 & 210 & 215 & 280 & -1.2 & -6.4 & -5.7 \\
\hline Missouri & 92 & 116 & 180 & 323 & 312 & 1,004 & -20.7 & -26.3 & -23.3 \\
\hline Montana & 722 & 705 & 653 & 715 & 794 & 932 & 2.4 & -2.3 & -2.8 \\
\hline New Mexico & 1,615 & 1,618 & 1,516 & 1,542 & 1,587 & 1,694 & -.2 & .4 & -.5 \\
\hline 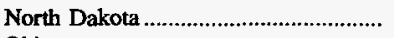 & 716 & 645 & 782 & 744 & 814 & 1,052 & 11.0 & -3.1 & -4.2 \\
\hline Ohio & 1,716 & 2,289 & 2,265 & 2,589 & 2,934 & 4,500 & -25.0 & -12.5 & -10.1 \\
\hline Oklahoma & 229 & 221 & 233 & 310 & 379 & 732 & 3.6 & -11.8 & -12.1 \\
\hline 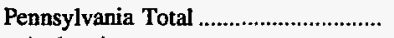 & 3,309 & 3,783 & 4,087 & 4,546 & 4,755 & 7,376 & -12.5 & -8.7 & -8.5 \\
\hline 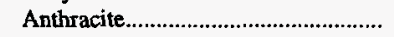 & 917 & 1,034 & 930 & 1,070 & 1,038 & 1,624 & -11.3 & -3.0 & -6.1 \\
\hline 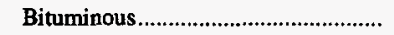 & 2,392 & 2,749 & 3,157 & 3,476 & 3,717 & 5,752 & -13.0 & -10.4 & -9.3 \\
\hline 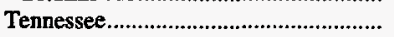 & 208 & 158 & 271 & 205 & 254 & 485 & 31.6 & -4.9 & -9.0 \\
\hline 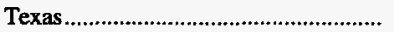 & 1,590 & 1,733 & 1,841 & 2,001 & 2,149 & 3,074 & -8.3 & -7.3 & -7.1 \\
\hline 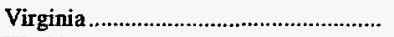 & 1,143 & 1,277 & 1,247 & 1,250 & 1,540 & 1,774 & -10.5 & -7.2 & -4.8 \\
\hline 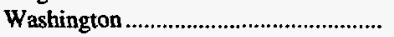 & 566 & 570 & 567 & 612 & 638 & 689 & -.7 & -2.9 & -2.2 \\
\hline West Virginia Total & 4,987 & 4,905 & 4,939 & 5,279 & 5,798 & 5,701 & 1.7 & -3.7 & -1.5 \\
\hline 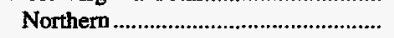 & 553 & 662 & 860 & 968 & 959 & 1,806 & -16.5 & -12.8 & -12.3 \\
\hline 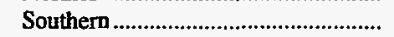 & 4,434 & 4,243 & 4,079 & 4,311 & 4,839 & 3,895 & 4.5 & -2.2 & 1.4 \\
\hline 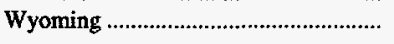 & 2,940 & 3,035 & 2,898 & 3,058 & 3,043 & 3,647 & -3.1 & -.8 & -2.4 \\
\hline Appalachian Total ${ }^{1}$............................... & 18,255 & 19,950 & 20,365 & 21,669 & 23,588 & 31,233 & -8.5 & -6.2 & -5.8 \\
\hline 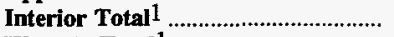 & 6,150 & $\mathbf{7 , 7 8 9}$ & 8,309 & $\mathbf{9 , 4 1 7}$ & 10,574 & 14,984 & -21.0 & -12.7 & -9.4 \\
\hline Western Total ${ }^{1} \ldots \ldots \ldots \ldots \ldots \ldots \ldots \ldots \ldots \ldots \ldots$ & 7,968 & 8,199 & 8,044 & 8,203 & 8,390 & 9,958 & -2.8 & -1.3 & -2.4 \\
\hline 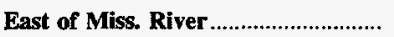 & 22,322 & 25,470 & 26,145 & 28,164 & 31,016 & 40,939 & -12.3 & -7.9 & -6.5 \\
\hline West of Miss. River.............................. & 10,051 & 10,468 & 10,573 & 11,125 & 11,536 & 15,236 & -4.0 & -3.4 & -4.5 \\
\hline 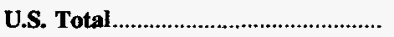 & 32,373 & 35,938 & 36,718 & 39,289 & 42,552 & 56,175 & -9.9 & -6.6 & -5.9 \\
\hline
\end{tabular}

1 For a definition of coal-producing regions, see Appendix C.

Notes: Includes all employees engaged in production, preparation, processing, development, maintenance, repair, shop or yard work at mining operations. Excludes office workers. Includes mining operations management and all technical and engineering personnel. Excludes silt, culm, refuse bank, slurry dam, and dredge operations except for Pennsylvania anthracite. Excludes mines producing less than 10,000 short tons and preparation plants with less than 5,000 employee hours, which are not required to provide these data.

Sources: Energy Infarmation Administration, Form EIA-7A, "Coal Production Report"; State Mining Agency Coal Production Reports; and/or U.S. Department of Labor, Mine Safety and Health Administration, Form 7000-2, "Quarterly Mine Employment and Coal Production Report." 
Table 43. Average Number of Miners by State and Mine Production Range, 1995

\begin{tabular}{|c|c|c|c|c|c|c|c|c|}
\hline \multirow{2}{*}{$\begin{array}{l}\text { Coal-Producing } \\
\text { State and Region }\end{array}$} & \multicolumn{7}{|c|}{$\begin{array}{l}\text { Mine Production Range } \\
\text { (thousand short tons) }\end{array}$} & \multirow{2}{*}{ Total } \\
\hline & $\begin{array}{l}1,000 \\
\text { and over }\end{array}$ & $\begin{array}{c}500 \\
\text { to } 1,000\end{array}$ & $\begin{array}{l}200 \\
\text { to } 500\end{array}$ & $\begin{array}{c}100 \\
\text { to } 200\end{array}$ & $\begin{array}{c}50 \\
\text { to } 100\end{array}$ & $\begin{array}{c}10 \\
\text { to } 50\end{array}$ & Zero 1 & \\
\hline 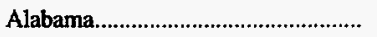 & 4,014 & 582 & 241 & 90 & 167 & 294 & 179 & 5,567 \\
\hline Alaska & 102 & - & - & - & - & - & - & 102 \\
\hline Arizona & 810 & - & - & - & - & - & 21 & 831 \\
\hline 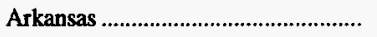 & - & - & - & - & - & 4 & - & 4 \\
\hline 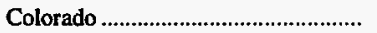 & 1,375 & 126 & 216 & - & - & 9 & 51 & 1,777 \\
\hline 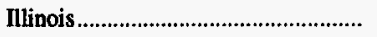 & 4,917 & 256 & 144 & 8 & 93 & 12 & 222 & 5,652 \\
\hline Indiana & 1,450 & 386 & 469 & 171 & 13 & 34 & 48 & 2,571 \\
\hline 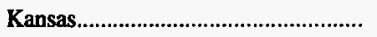 & - & - & 54 & - & - & - & - & 54 \\
\hline 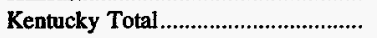 & 4,364 & 4,696 & 3,802 & 2,471 & 2,195 & 1,400 & 2,197 & 21,125 \\
\hline 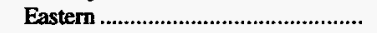 & 2,483 & 3,530 & 3,382 & 2,382 & 1,828 & 1,351 & 1,884 & 16,840 \\
\hline Western & 1,881 & 1,166 & 420 & 89 & 367 & 49 & 313 & 4,285 \\
\hline 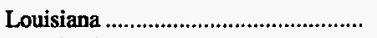 & 87 & 27 & - & - & - & - & - & 114 \\
\hline 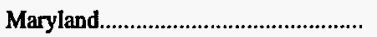 & 234 & - & 83 & 20 & 46 & 42 & 33 & 458 \\
\hline 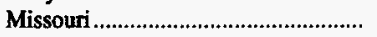 & - & - & - & 69 & 20 & 3 & - & 92 \\
\hline 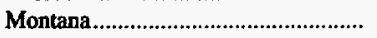 & 709 & - & 13 & - & - & - & - & 722 \\
\hline 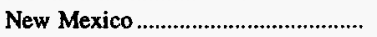 & 1,603 & 123 & - & - & - & - & 21 & 1,747 \\
\hline 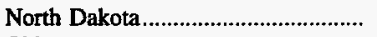 & 711 & - & - & - & - & - & 5 & 716 \\
\hline Ohio & 1,710 & 477 & 451 & 185 & 200 & 204 & 159 & 3,386 \\
\hline 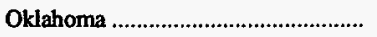 & - & - & 158 & 36 & - & 47 & - & 241 \\
\hline 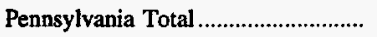 & 3,026 & 1,453 & 1,607 & 696 & 519 & 786 & 881 & 8,968 \\
\hline 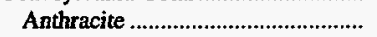 & - & - & 219 & 109 & 84 & 206 & 451 & 1,069 \\
\hline 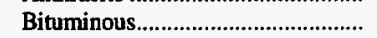 & 3,026 & 1,453 & 1,388 & 587 & 435 & 580 & 430 & 7,899 \\
\hline 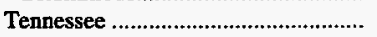 & - & 57 & 216 & 187 & 74 & 38 & 109 & 681 \\
\hline 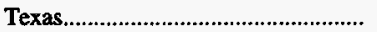 & 1,527 & - & 58 & - & 5 & - & - & 1,590 \\
\hline Utah & 1,605 & 36 & 136 & 86 & - & - & 30 & 1,893 \\
\hline 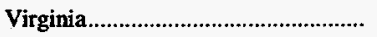 & 1,227 & 389 & 1,640 & 1,060 & 1,157 & 520 & 926 & 6,919 \\
\hline 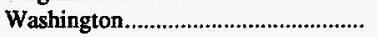 & 522 & - & 44 & - & - & - & - & 566 \\
\hline West Virginia Total ............................ & 9,284 & 2,407 & 3,484 & 1,462 & 1,233 & 1,254 & 2,210 & 21,334 \\
\hline Northern. & 3,996 & 669 & 456 & 189 & 185 & 201 & 418 & 6,114 \\
\hline Southern & 5,288 & 1,738 & 3,028 & 1,273 & 1,048 & 1,053 & 1,792 & 15,220 \\
\hline 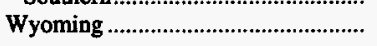 & 2,911 & $\quad 102$ & 40 & - & - & 89 & - & 3,142 \\
\hline Appalachian Total ${ }^{2} \ldots \ldots \ldots \ldots \ldots \ldots \ldots \ldots$ & 21,978 & 8,895 & 11,104 & 6,082 & 5,224 & 4,489 & 6,381 & 64,153 \\
\hline Interior Total 2 & 9,862 & 1,835 & 1,303 & 373 & 498 & 149 & 583 & 14,603 \\
\hline Western Total ${ }^{2} \ldots \ldots \ldots \ldots \ldots \ldots \ldots \ldots \ldots$ & 10,348 & 387 & 449 & 86 & - & 98 & 128 & 11,496 \\
\hline East of Miss. River............................. & 30,226 & 10,703 & 12,137 & 6,350 & 5,697 & 4,584 & 6,964 & 76,661 \\
\hline West of Miss. River............................. & 11,962 & 414 & 719 & 191 & 25 & 152 & 128 & 13,591 \\
\hline 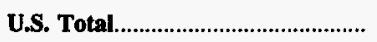 & 42,188 & 11,117 & 12,856 & 6,541 & $\mathbf{5 , 7 2 2}$ & 4,736 & 7,092 & 90,252 \\
\hline
\end{tabular}

1 Includes all employees at preparation plants and tipples not co-located with a mine.

2 For a definition of coal-producing regions, see Appendix C.

Notes: Includes all employees engaged in production, preparation, processing, development, maintenance, repair, shop or yard work at mining operations. Excludes office workers. Includes mining operations management and all technical and engineering personnel. Excludes silt, culm refuse bank, slurry dam, and dredge operations except for Pennsylvania anthracite. Excludes mines producing less than 10,000 short tons and preparation plants with less than 5,000 employee hours, which are not required to provide these data.

Sources: Energy Information Administration, Form EIA-7A, "Coal Production Report"; State Mining Agency Coal Production Reports; and/or U.S. Department of Labor, Mine Safety and Health Administration, Form 7000-2, "Quarterly Mine Employment and Coal Production Report." 
Table 44. Average Number of Miners at Underground Mines by State and Mine Production Range, 1995

\begin{tabular}{|c|c|c|c|c|c|c|c|c|}
\hline \multirow{2}{*}{$\begin{array}{l}\text { Coal-Producing } \\
\text { State and Region }\end{array}$} & \multicolumn{7}{|c|}{$\begin{array}{l}\text { Mine Production Range } \\
\text { (thousand short tons) }\end{array}$} & \multirow{2}{*}{ Total } \\
\hline & $\begin{array}{c}1,000 \\
\text { and over }\end{array}$ & $\begin{array}{c}500 \\
\text { to } 1,000\end{array}$ & $\begin{array}{l}200 \\
\text { to } 500\end{array}$ & $\begin{array}{c}100 \\
\text { to } 200\end{array}$ & $\begin{array}{l}50 \\
\text { to } 100\end{array}$ & $\begin{array}{l}10 \\
\text { to } 50\end{array}$ & Zerol 1 & \\
\hline 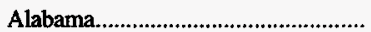 & 3,891 & 321 & - & - & - & - & 102 & 4,314 \\
\hline 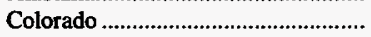 & 930 & 126 & 194 & - & - & - & 51 & 1,301 \\
\hline 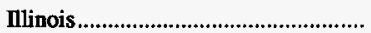 & 4,389 & 216 & - & - & - & - & 175 & 4,780 \\
\hline Indiana & 369 & - & 108 & - & - & - & 8 & 485 \\
\hline 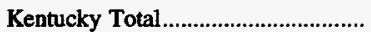 & 3,432 & 2,860 & 2,274 & 1,681 & 1,747 & 925 & 1,623 & 14,542 \\
\hline 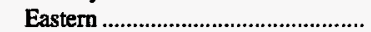 & 1,665 & 2,163 & 2,187 & 1,681 & 1,390 & 925 & 1,355 & 11,366 \\
\hline 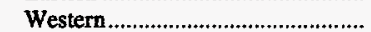 & 1,767 & 697 & 87 & - & 357 & - & 268 & 3,176 \\
\hline 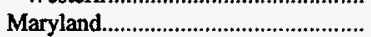 & 234 & - & 26 & - & - & - & 33 & 293 \\
\hline 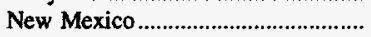 & - & 123 & - & - & - & - & 9 & 132 \\
\hline Ohio & 1,473 & 57 & 76 & - & 40 & - & 24 & 1,670 \\
\hline Oklahoma & - & - & - & - & - & 12 & - & 12 \\
\hline Pennsylvania Total ............................ & 2,879 & 1,156 & 862 & 179 & 98 & 177 & 308 & 5,659 \\
\hline 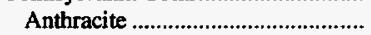 & - & - & - & 36 & - & 44 & 72 & 152 \\
\hline 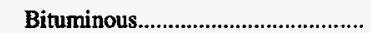 & 2,879 & 1,156 & 862 & 143 & 98 & 133 & 236 & 5,507 \\
\hline Tennessee & - & - & 188 & 160 & 13 & 23 & 89 & 473 \\
\hline Utah & 1,605 & 36 & 136 & 86 & - & - & 30 & 1,893 \\
\hline Virginia & 1,227 & 198 & 1,195 & 876 & 1,074 & 399 & 807 & 5,776 \\
\hline West Virginia Total .......................... & 7,168 & 1,747 & 2,891 & 1,135 & 922 & 871 & 1,613 & 16,347 \\
\hline 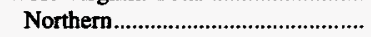 & 3,888 & 639 & 371 & 105 & 168 & 96 & 294 & 5,561 \\
\hline 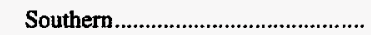 & 3,280 & 1,108 & 2,520 & 1,030 & 754 & 775 & 1,319 & 10,786 \\
\hline Wyoming & 126 & - & - & - & - & 76 & - & 202 \\
\hline Appalachian Total ${ }^{2}$ & 18,537 & 5,642 & $\mathbf{7 , 4 2 5}$ & 4,031 & $\mathbf{3 , 5 3 7}$ & 2,395 & 4,331 & 45,898 \\
\hline Interior Total 2 & 6,525 & 913 & 195 & - & 357 & 12 & 451 & 8,453 \\
\hline Western Total 2 & 2,661 & 285 & 330 & 86 & - & 76 & 90 & $\mathbf{3 , 5 2 8}$ \\
\hline East of Miss. River............................. & 25,062 & 6,555 & 7,620 & 4,031 & 3,894 & 2,395 & 4,782 & 54,339 \\
\hline West of Miss. River............................ & 2,661 & 285 & 330 & 86 & - & 88 & 90 & $\mathbf{3 , 5 4 0}$ \\
\hline 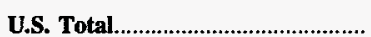 & 27,723 & 6,840 & 7,950 & 4,117 & 3,894 & 2,483 & 4,872 & 57,879 \\
\hline
\end{tabular}

1 Includes all employees at preparation plants and tipples not co-located with a mine.

2 For a definition of coal-producing regions, see Appendix C.

Notes: Includes all employees engaged in production, preparation, processing, development, maintenance, repair, shop or yard work at mining operations. Excludes office workers. Includes mining operations management and all technical and engineering personnel. Excludes silt, culm refuse bank, slurry dam, and dredge operations except for Pennsylvania anthracite. Excludes mines producing less than 10,000 short tons and preparation plants with less than 5,000 employee hours, which are not required to provide these data.

Sources: Energy Information Administration, Form EIA-7A, "Coal Production Report"; State Mining Agency Coal Production Reports; and/or U.S. Department of Labor, Mine Safety and Health Administration, Form 7000-2, "Quarterly Mine Employment and Coal Production Report." 
Table 45. Average Number of Miners at Surface Mines by State and Mine Production Range, 1995

\begin{tabular}{|c|c|c|c|c|c|c|c|c|}
\hline \multirow{2}{*}{$\begin{array}{l}\text { Coal-Producing } \\
\text { State and Region }\end{array}$} & \multicolumn{7}{|c|}{$\begin{array}{l}\text { Mine Production Range } \\
\text { (thousand short tons) }\end{array}$} & \multirow{2}{*}{ Total } \\
\hline & $\begin{array}{c}1,000 \\
\text { and over }\end{array}$ & $\begin{array}{c}500 \\
\text { to } 1,000\end{array}$ & $\begin{array}{c}200 \\
\text { to } 500\end{array}$ & $\begin{array}{c}100 \\
\text { to } 200\end{array}$ & $\begin{array}{c}50 \\
\text { to } 100\end{array}$ & $\begin{array}{c}10 \\
\text { to } 50\end{array}$ & Zero 1 & \\
\hline 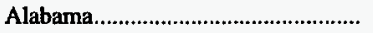 & 123 & 261 & 241 & 90 & 167 & 294 & 77 & 1,253 \\
\hline 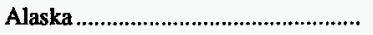 & 102 & - & - & - & - & - & - & 102 \\
\hline Arizona & 810 & - & - & - & - & - & 21 & 831 \\
\hline 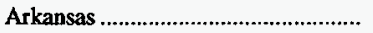 & - & - & - & - & - & 4 & - & 4 \\
\hline 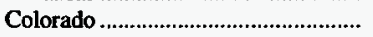 & 445 & - & 22 & - & - & 9 & - & 476 \\
\hline 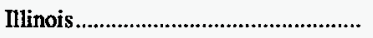 & 528 & 40 & 144 & 8 & 93 & 12 & 47 & 872 \\
\hline Indiana & 1,081 & 386 & 361 & 171 & 13 & 34 & 40 & 2,086 \\
\hline 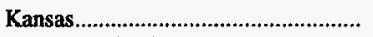 & - & - & 54 & - & - & - & - & 54 \\
\hline 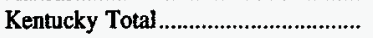 & 932 & 1,836 & 1,528 & 790 & 448 & 475 & 574 & 6,583 \\
\hline 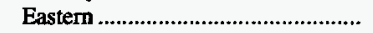 & 818 & 1,367 & 1,195 & 701 & 438 & 426 & 529 & 5,474 \\
\hline Western & 114 & 469 & 333 & 89 & 10 & 49 & 45 & 1,109 \\
\hline 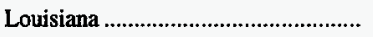 & 87 & 27 & - & - & - & - & - & 114 \\
\hline 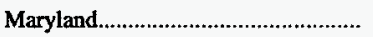 & - & - & 57 & 20 & 46 & 42 & - & 165 \\
\hline 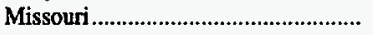 & - & - & - & 69 & 20 & 3 & - & 92 \\
\hline 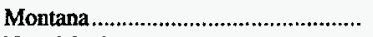 & 709 & - & 13 & - & - & - & - & 722 \\
\hline 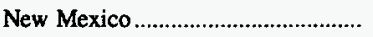 & 1,603 & - & - & - & - & - & 12 & 1,615 \\
\hline North Dakota ........................................ & 711 & - & - & - & - & - & 5 & 716 \\
\hline Ohio & 237 & 420 & 375 & 185 & 160 & 204 & 135 & 1,716 \\
\hline Oklahoma & - & - & 158 & 36 & - & 35 & - & 229 \\
\hline 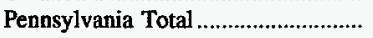 & 147 & 297 & 745 & 517 & 421 & 609 & 573 & 3,309 \\
\hline 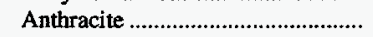 & - & - & 219 & 73 & 84 & 162 & 379 & 917 \\
\hline Bituminous..................................... & 147 & 297 & 526 & 444 & 337 & 447 & 194 & 2,392 \\
\hline 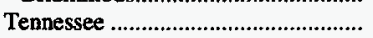 & - & 57 & 28 & 27 & 61 & 15 & 20 & 208 \\
\hline 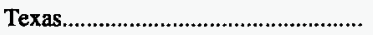 & 1,527 & - & 58 & - & 5 & - & - & 1,590 \\
\hline 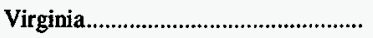 & - & 191 & 445 & 184 & 83 & 121 & 119 & 1,143 \\
\hline 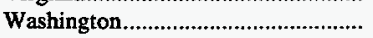 & 522 & - & 44 & - & - & - & - & 566 \\
\hline West Virginia Total ............................. & 2,116 & 660 & 593 & 327 & 311 & 383 & 597 & 4,987 \\
\hline 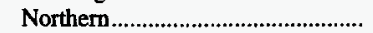 & 108 & 30 & 85 & 84 & 17 & 105 & 124 & 553 \\
\hline 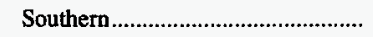 & 2,008 & 630 & 508 & 243 & 294 & 278 & 473 & 4,434 \\
\hline 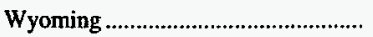 & 2,785 & 102 & 40 & - & - & 13 & - & 2,940 \\
\hline Appalachian Total ${ }^{2} \ldots \ldots \ldots \ldots \ldots \ldots \ldots \ldots$ & 3,441 & 3,253 & 3,679 & 2,051 & 1,687 & 2,094 & 2,050 & 18,255 \\
\hline Interior Total ${ }^{2}$ & $\mathbf{3 , 3 3 7}$ & 922 & 1,108 & 373 & 141 & 137 & 132 & 6,150 \\
\hline 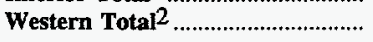 & 7,687 & 102 & 119 & - & - & 22 & 38 & 7,968 \\
\hline East of Miss. River............................. & 5,164 & 4,148 & 4,517 & 2,319 & 1,803 & 2,189 & 2,182 & 22,322 \\
\hline West of Miss. River................................ & 9,301 & 129 & 389 & 105 & 25 & 64 & 38 & 10,051 \\
\hline U.S. Total. & 14,465 & 4,277 & 4,906 & 2,424 & $\mathbf{1 , 8 2 8}$ & 2,253 & 2,220 & 32,373 \\
\hline
\end{tabular}

1 Includes all employees at preparation plants and tipples not co-located with a mine

2 For a definition of coal-producing regions, see Appendix C.

Notes: Includes all employees engaged in production, preparation, processing, development, maintenance, repair, shop or yard work at mining operations. Excludes office workers. Includes mining operations management and all technical and engineering personnel. Excludes silt, culm refuse bank, slurry dam, and dredge operations except for Pennsylvania anthracite. Excludes mines producing less than 10,000 short tons and preparation plants with less than 5,000 employee hours, which are not required to provide these data.

Sources: Energy Information Administration, Form ELA-7A, "Coal Production Report"; State Mining Agency Coal Production Reports; and/or U.S. Department of Labor, Mine Safety and Health Administration, Form 7000-2, "Quarterly Mine Employment and Coal Production Report." 
Table 46. Average Number of Miners by State, Mine Type, and Union Type, 1995

\begin{tabular}{|c|c|c|c|c|c|}
\hline $\begin{array}{l}\text { Coal-Producing } \\
\text { State and Region }\end{array}$ & UMWA & Other Unions & Union Total & Nonunion & Total \\
\hline 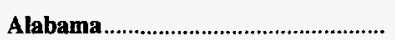 & 4,255 & _ & 4,255 & 1,312 & 5,567 \\
\hline 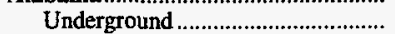 & 3,646 & - & 3,646 & 668 & 4,314 \\
\hline Surface & 609 & - & 609 & 644 & 1,253 \\
\hline 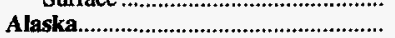 & - & 102 & 102 & - & 102 \\
\hline Surface & - & 102 & 102 & - & 102 \\
\hline 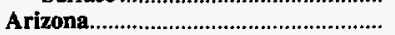 & 831 & - & 831 & - & 831 \\
\hline Surface & 831 & - & 831 & - & 831 \\
\hline 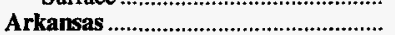 & - & - & - & 4 & 4 \\
\hline 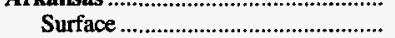 & - & - & - & 4 & 4 \\
\hline Colorado & 693 & 147 & 840 & 937 & 1,777 \\
\hline Underground & 560 & - & 560 & 741 & 1,301 \\
\hline 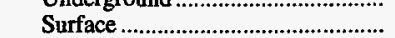 & 133 & 147 & 280 & 196 & 476 \\
\hline Illinois. & $\mathbf{4 , 5 6 6}$ & 287 & $\mathbf{4 , 8 5 3}$ & 799 & 5,652 \\
\hline Underground & 3,784 & 260 & 4,044 & 736 & 4,780 \\
\hline Surface & 782 & 27 & 809 & 63 & 872 \\
\hline 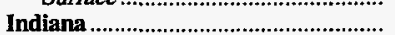 & 1,325 & - & 1,325 & 1,246 & 2,571 \\
\hline Underground & 279 & - & 279 & 206 & 485 \\
\hline Surface & 1,046 & - & 1,046 & 1,040 & 2,086 \\
\hline 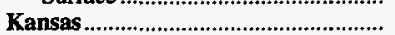 & 54 & - & 54 & - & 54 \\
\hline Surface & 54 & - & 54 & - & 54 \\
\hline Kentucky Total & $\mathbf{2 , 5 7 0}$ & 131 & 2,701 & 18,424 & 21,125 \\
\hline 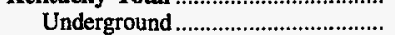 & 2,089 & 75 & 2,164 & 12,378 & 14,542 \\
\hline 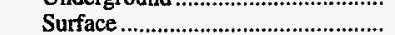 & 481 & 56 & 537 & 6,046 & 6,583 \\
\hline Eastern & 1,428 & 131 & 1,559 & 15,281 & 16,840 \\
\hline 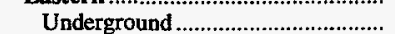 & 1,118 & 75 & 1,193 & 10,173 & 11,366 \\
\hline 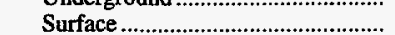 & 310 & 56 & 366 & 5,108 & 5,474 \\
\hline Western & 1,142 & - & $\mathbf{1 , 1 4 2}$ & $\mathbf{3 , 1 4 3}$ & $\mathbf{4 , 2 8 5}$ \\
\hline Underground & 971 & - & 971 & 2,205 & 3,176 \\
\hline 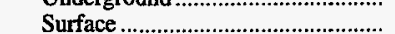 & 171 & - & 171 & 938 & 1,109 \\
\hline 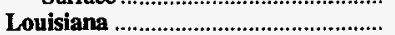 & - & - & - & 114 & 114 \\
\hline Surface & - & - & - & 114 & 114 \\
\hline Maryland & _ & - & - & 458 & 458 \\
\hline Underground & - & - & - & 293 & 293 \\
\hline Surface & _- & _- & - & 165 & 165 \\
\hline Missouri & - & - & - & 92 & 92 \\
\hline Surface & - & & - & 92 & 92 \\
\hline 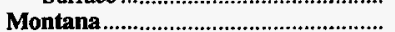 & 367 & 291 & 658 & 64 & 722 \\
\hline Surface .............. & 367 & 291 & 658 & 64 & 722 \\
\hline New Mexico & 705 & 841 & $\begin{array}{r}038 \\
1,546\end{array}$ & 201 & 1,747 \\
\hline Underground & 132 & - & 132 & - & 132 \\
\hline 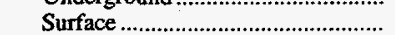 & 573 & 841 & 1,414 & 201 & 1,615 \\
\hline & 155 & 102 & 257 & 459 & 716 \\
\hline 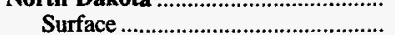 & 155 & 102 & 257 & 459 & 716 \\
\hline Ohio & 1,823 & 14 & 1,837 & 1,549 & 3,386 \\
\hline Underground & 1,473 & - & 1,473 & 197 & 1,670 \\
\hline 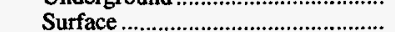 & 350 & 14 & 364 & 1,352 & 1,716 \\
\hline Oklahoma & - & - & - & 241 & 241 \\
\hline Underground & - & - & - & 12 & 12 \\
\hline Surface & - & - & - & 229 & 229 \\
\hline Pennsylvania Total ................................ & $\mathbf{4 , 0 4 2}$ & 16 & 4,058 & 4,910 & 8,968 \\
\hline 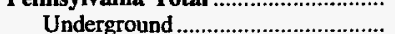 & 3,507 & 6 & 3,513 & 2,146 & 5,659 \\
\hline Surface & 535 & 10 & 545 & 2,764 & 3,309 \\
\hline Anthracite & 399 & 10 & 409 & 660 & 1,069 \\
\hline 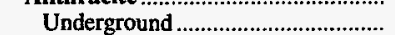 & - & 3 & 3 & 149 & 152 \\
\hline 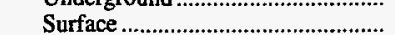 & 399 & 7 & 406 & 511 & 917 \\
\hline 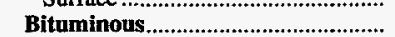 & 3,643 & 6 & 3,649 & 4,250 & $\mathbf{7 , 8 9 9}$ \\
\hline 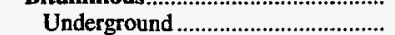 & 3,507 & 3 & 3,510 & 1,997 & 5,507 \\
\hline Surface & 136 & 3 & 139 & 2,253 & 2,392 \\
\hline Tennessee .............. & - & - & - & 681 & 681 \\
\hline 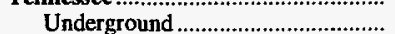 & _- & - & - & 473 & 473 \\
\hline Surface & - & - & - & 208 & 208 \\
\hline Texas & - & 934 & 934 & 656 & 1,590 \\
\hline Surface ...... & - & 934 & 934 & 656 & 1.590 \\
\hline Utah & 637 & - & 637 & 1,256 & 1,893 \\
\hline Underground & 637 & - & 637 & 1,256 & 1,893 \\
\hline Virginia & 1,994 & 44 & $\mathbf{2 , 0 3 8}$ & $\mathbf{4 , 8 8 1}$ & 6,919 \\
\hline Underground & 1,922 & 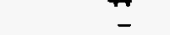 & 1,922 & 3,854 & 5,776 \\
\hline 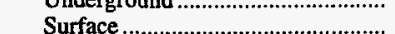 & 72 & 44 & 116 & 1,027 & 1,143 \\
\hline Washington. & 12 & 522 & 522 & 44 & 566 \\
\hline 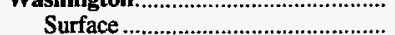 & - & 522 & 522 & 44 & 566 \\
\hline West Virginia Total & $12,84 \overline{2}$ & 26 & 12,868 & 8,466 & 21,334 \\
\hline 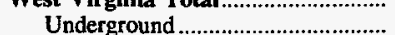 & 10,468 & 7 & 10,475 & 5,872 & 16,347 \\
\hline 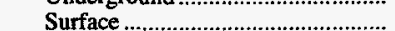 & 2,374 & 19 & 2,393 & 2,594 & 4,987 \\
\hline
\end{tabular}

See footnotes at end of table. 
Table 46. Average Number of Miners by State, Mine Type, and Union Type, 1995 (Continued)

\begin{tabular}{|c|c|c|c|c|c|}
\hline $\begin{array}{l}\text { Coal-Producing } \\
\text { State and Region }\end{array}$ & UMWA & Other Unions & Union Total & Nonunion & Total \\
\hline Northern & 4,359 & - & 4,359 & 1,755 & 6,114 \\
\hline 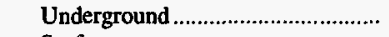 & 4,343 & - & 4,343 & 1,218 & 5,561 \\
\hline Surface & 16 & - & 16 & 537 & 553 \\
\hline 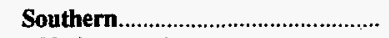 & 8,483 & 26 & 8,509 & 6,711 & 15,220 \\
\hline 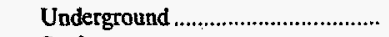 & 6,125 & 7 & 6,132 & 4,654 & 10,786 \\
\hline Surface & 2,358 & 19 & 2,377 & 2,057 & 4,434 \\
\hline Wyoming & 367 & 534 & 901 & 2,241 & 3,142 \\
\hline 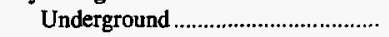 & - & - & - & 202 & 202 \\
\hline Surface & 367 & 534 & 901 & 2,039 & 2,940 \\
\hline Appalachian Total ${ }^{1}$ & 26,384 & 231 & 26,615 & $\mathbf{3 7 , 5 3 8}$ & 64,153 \\
\hline 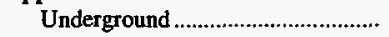 & 22,134 & 88 & 22,222 & 23,676 & 45,898 \\
\hline 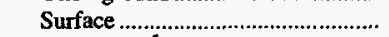 & 4,250 & 143 & 4,393 & 13,862 & 18,255 \\
\hline 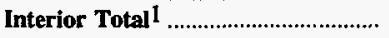 & 7,087 & 1,221 & 8,308 & 6,295 & 14,603 \\
\hline 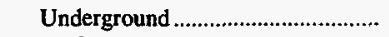 & 5,034 & 260 & 5,294 & 3,159 & 8,453 \\
\hline 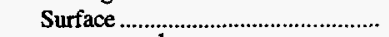 & 2,053 & 961 & 3,014 & 3,136 & 6,150 \\
\hline Western Total ${ }^{1}$ & 3,755 & 2,539 & 6,294 & 5,202 & 11,496 \\
\hline Underground & 1,329 & - & 1,329 & 2,199 & 3,528 \\
\hline Surface & 2,426 & 2,539 & 4,965 & 3,003 & 7,968 \\
\hline 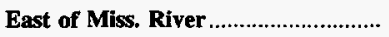 & 33,417 & 518 & 33,935 & 42,726 & 76,661 \\
\hline Underground & 27,168 & 348 & 27,516 & 26,823 & 54,339 \\
\hline Surface & 6,249 & 170 & 6,419 & 15,903 & 22,322 \\
\hline West of Miss. River................................ & 3,809 & 3,473 & $\mathbf{7 , 2 8 2}$ & 6,309 & 13,591 \\
\hline Underground & 1,329 & - & 1,329 & 2,211 & 3,540 \\
\hline Surface & 2,480 & 3,473 & 5,953 & 4,098 & 10,051 \\
\hline 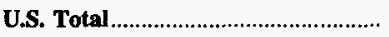 & 37,226 & 3,991 & 41,217 & 49,035 & 90,252 \\
\hline 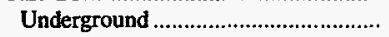 & 28,497 & 348 & 28,845 & 29,034 & 57,879 \\
\hline Surface & 8,729 & 3,643 & 12,372 & 20,001 & 32,373 \\
\hline
\end{tabular}

1 For a definition of coal-producing regions, see Appendix $\mathbf{C}$.

Notes: Includes all employees engaged in production, preparation, processing, development, maintenance, repair, shop or yard work at mining operations. Excludes office workers. Includes mining operations management and all technical and engineering personnel. Excludes silt, culm, refuse bank, slurry dam, and dredge operations except for Pennsylvania anthracite. Excludes mines producing less than 10,000 short tons and preparation plants with less than 5,000 employee hours, which are not required to provide these data. See Glossary for listing of other unions.

Sources: Energy Information Administration, Form EIA-7A, "Coal Production Report"; State Mining Agency Coal Production Reports; and/or U.S. Department of Labor, Mine Safety and Health Administration, Form 7000-2, "Quarterly Mine Employment and Coal Production Report."

Table 47. U.S. Coal Mine Injuries, 1986, 1991-1995

\begin{tabular}{|c|c|c|c|c|c|c|c|c|c|}
\hline \multirow{2}{*}{ Injury Type } & \multirow{2}{*}{1995} & \multirow{2}{*}{1994} & \multirow{2}{*}{1993} & \multirow{2}{*}{1992} & \multirow{2}{*}{1991} & \multirow{2}{*}{1986} & \multirow{2}{*}{$\begin{array}{c}\text { Percent } \\
\text { Change } \\
\text { 1994-1995 }\end{array}$} & \multicolumn{2}{|c|}{ Average Annual Percent Change } \\
\hline & & & & & & & & 1991-1995 & 1986-1995 \\
\hline 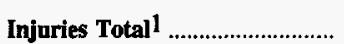 & 7,097 & R 8,764 & ${ }^{R} 8,446$ & 10,109 & 11,386 & 9,506 & -19.0 & -11.1 & -3.2 \\
\hline Fatal & 47 & 44 & 47 & 54 & 61 & 87 & 6.8 & 6.3 & -6.6 \\
\hline Nonfatal ${ }^{2} \ldots \ldots \ldots \ldots \ldots \ldots \ldots \ldots \ldots$ & 7,050 & R 8,720 & ${ }^{R} 8,399$ & 10,055 & 11,325 & 9,419 & -19.2 & -11.2 & -3.2 \\
\hline Injuries per $\mathbf{2 0 0 , 0 0 0}$ & & & & & & & & & \\
\hline Employee-Hours Total............ & 6.03 & ${ }^{R} 6.89$ & ${ }^{R} 6.86$ & 7.33 & 7.90 & 5.65 & -12.5 & -6.5 & .7 \\
\hline Nonfatal 2 & 6.00 & $\mathrm{R}_{6.86}$ & $R_{6.82}$ & 7.29 & 7.86 & 5.60 & -12.5 & -6.5 & .8 \\
\hline
\end{tabular}

1 Includes contractors and office workers.

2 Includes only non-fatal injuries that resulted in absence from work.

Revised.

Note: Calculations of growth rate are based using unrounded values.

Source: U.S. Department of Labor, Mine Safety and Health Administration, Mine Injuries and Worktime, Quarterly, various issues. 
Figure 7. U.S. Coal Mine Injuries, 1986-1995

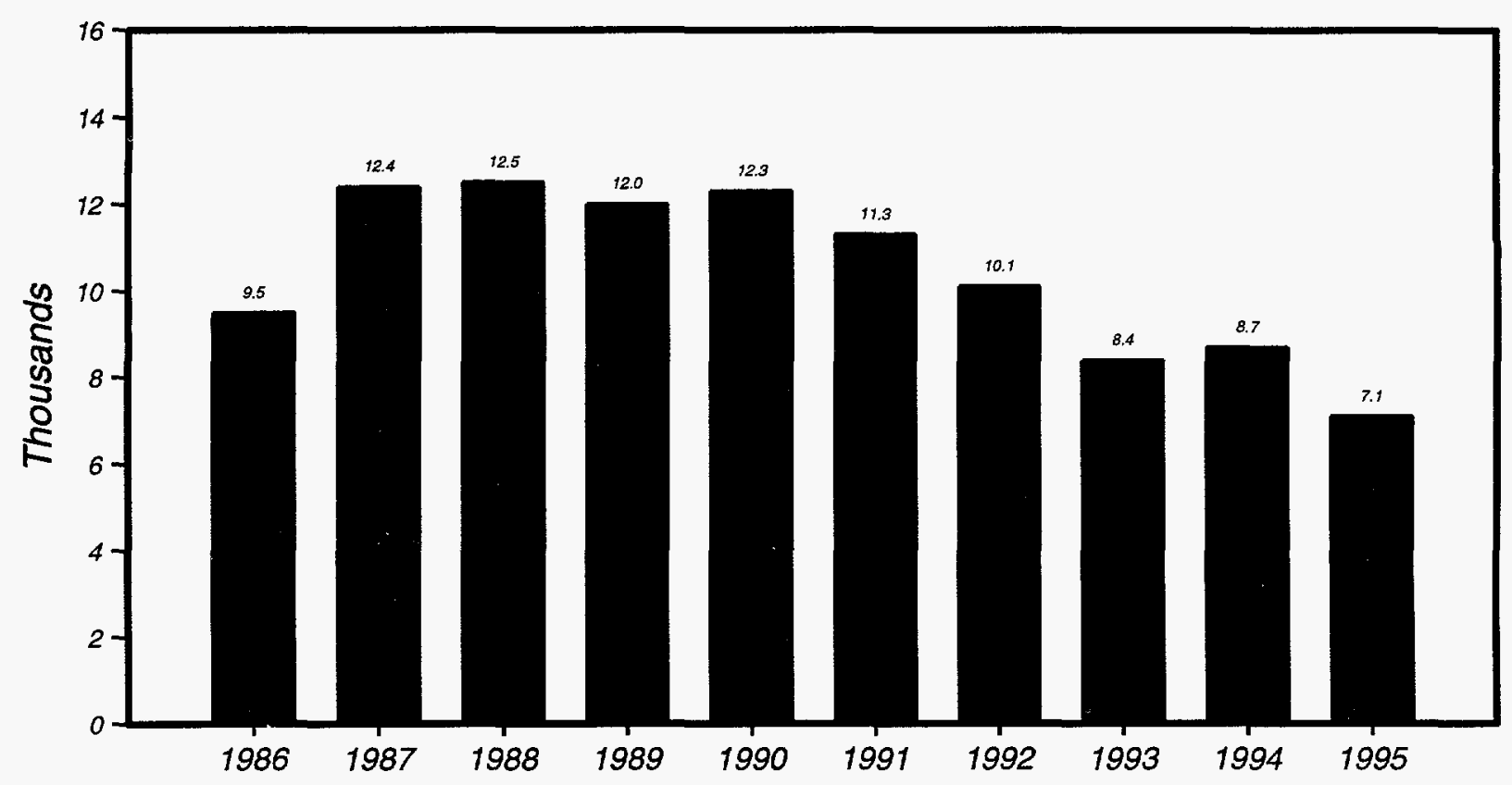

Figure 8. U.S. Coal Mine Fatalities, 1986-1995

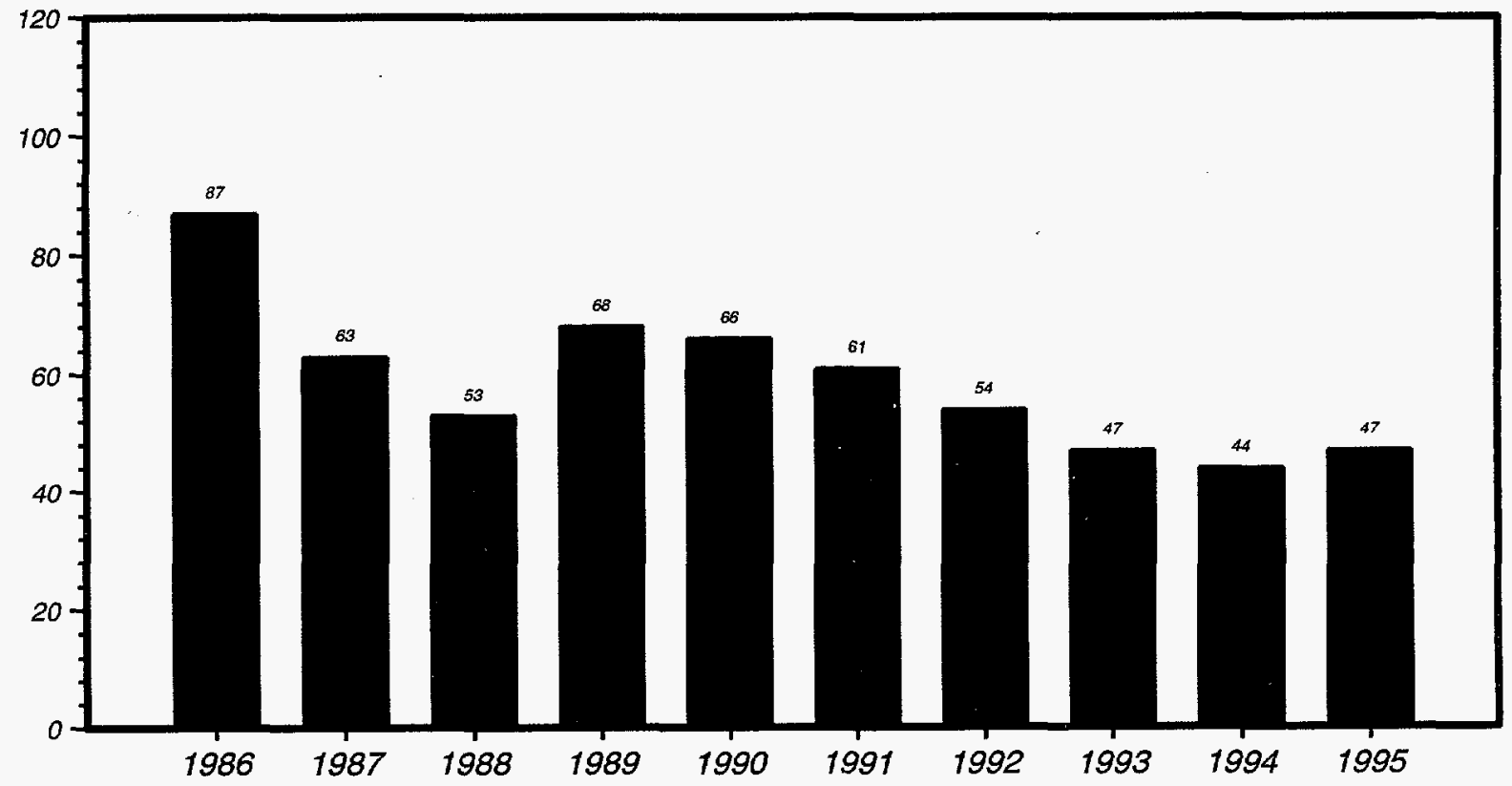

Sources: U.S. Department of Labor, Mine Safety and Health Administration, Mine Injuries and Worktime, Quarterly, various issues. 


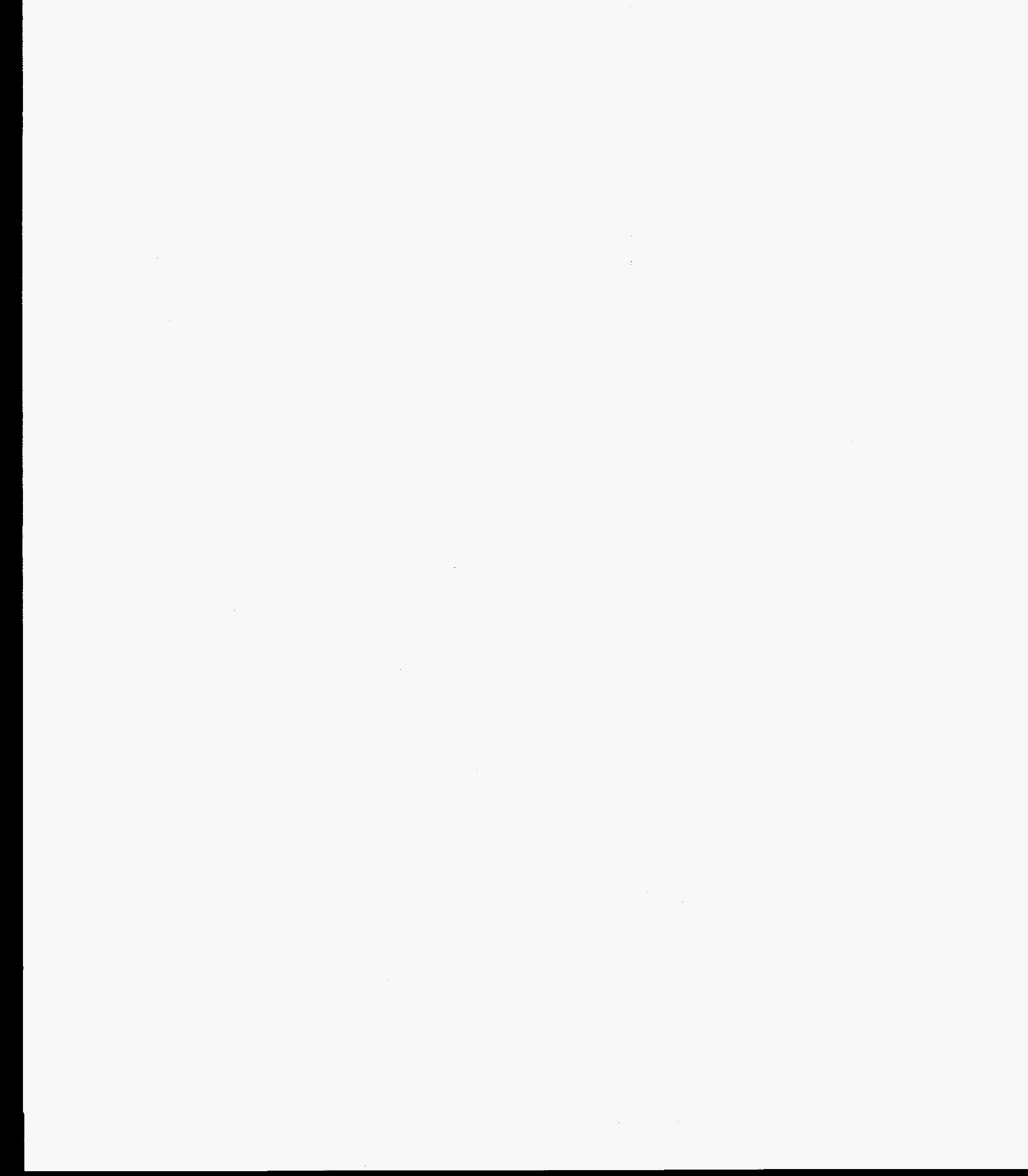




\section{Productivity}



Coal is being taken from a storage facility by conveyor. 
Table 48. Coal Mining Productivity by State, 1986, 1991-1995

(Short Tons of Coal Produced per Miner per Hour)

\begin{tabular}{|c|c|c|c|c|c|c|c|c|c|}
\hline \multirow{2}{*}{$\begin{array}{l}\text { Coal-Producing } \\
\text { State and Region }\end{array}$} & \multirow{2}{*}{1995} & \multirow{2}{*}{1994} & \multirow{2}{*}{1993} & \multirow{2}{*}{1992} & \multirow{2}{*}{1991} & \multirow{2}{*}{1986} & \multirow{2}{*}{$\begin{array}{c}\text { Percent } \\
\text { Change } \\
\text { 1994-1995 }\end{array}$} & \multicolumn{2}{|c|}{ Average Annual Percent Change } \\
\hline & & & & & & & & 1991-1995 & 1986-1995 \\
\hline 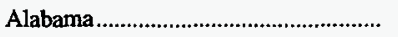 & 2.24 & 2.25 & 2.35 & 2.49 & 2.17 & 1.88 & -0.6 & 0.8 & 1.9 \\
\hline 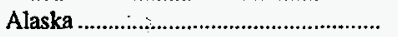 & 7.46 & 6.94 & 7.40 & 6.93 & 6.82 & 6.77 & 7.5 & 2.3 & 1.1 \\
\hline Arizona & 6.34 & 6.71 & 6.21 & 6.29 & 6.64 & 6.68 & -5.4 & -1.1 & -.6 \\
\hline Arkansas & 1.47 & 1.52 & 1.39 & 1.68 & 2.27 & 1.75 & -3.7 & -10.3 & -1.9 \\
\hline 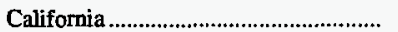 & - & - & - & 11.12 & 11.31 & - & - & - & - \\
\hline Colorado & 6.14 & 6.20 & 5.85 & 5.27 & 5.05 & 3.84 & -.9 & 5.0 & 5.3 \\
\hline 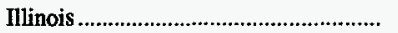 & 3.87 & 3.59 & 3.23 & 3.42 & 3.18 & 2.37 & 7.6 & 5.0 & 5.6 \\
\hline Indiana & 4.68 & 4.28 & 4.46 & 4.09 & 4.02 & 3.36 & 9.4 & 3.8 & 3.7 \\
\hline Iowa & - & 1.52 & .72 & 1.14 & 1.29 & 1.56 & -100.0 & - & - \\
\hline 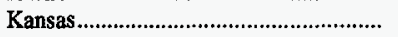 & 2.22 & 1.93 & 2.30 & 2.21 & 2.15 & 2.83 & 14.9 & .8 & -2.7 \\
\hline 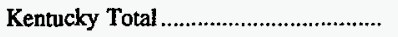 & 3.57 & 3.25 & 3.25 & 3.20 & 3.01 & 2,45 & 9.8 & 4.3 & 4.3 \\
\hline 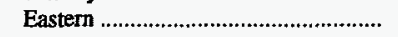 & 3.47 & 3.24 & 3.18 & 3.10 & 2.90 & 2.31 & 6.9 & 4.5 & 4.6 \\
\hline 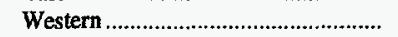 & 3.97 & 3.28 & 3.49 & 3.49 & 3.37 & 2.94 & 21.2 & 4.2 & 3.4 \\
\hline 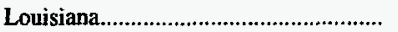 & 13.25 & 13.00 & 12.14 & 12.43 & 12.56 & 12.45 & 1.9 & 1.3 & .7 \\
\hline 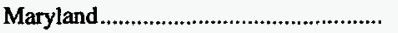 & 3.82 & 3.68 & 3.41 & 2.91 & 3.11 & 3.50 & 3.6 & 5.2 & 1.0 \\
\hline 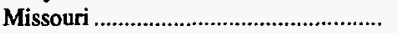 & 2.55 & 3.59 & 1.84 & 3.10 & 2.69 & 2.20 & -29.0 & -1.4 & 1.6 \\
\hline 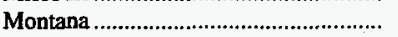 & 21.06 & 21.92 & 19.49 & 20.16 & 18.99 & 17.63 & -3.9 & 2.6 & 2.0 \\
\hline 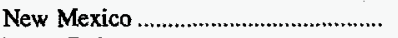 & 6.92 & 6.77 & 6.68 & 6.68 & 6.25 & 5.81 & 2.1 & 2.6 & 2.0 \\
\hline 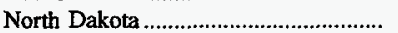 & 16.80 & 18.84 & 17.66 & 18.12 & 17.64 & 12.27 & -10.8 & -1.2 & 3.5 \\
\hline Ohio & 3.62 & 3.42 & 3.46 & 3.04 & 2.67 & 2.13 & 6.1 & 7.9 & 6.1 \\
\hline Oklahoma & 2.97 & 2.68 & 2.80 & 2.17 & 2.30 & 1.74 & 10.8 & 6.6 & 6.1 \\
\hline 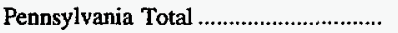 & 3.23 & 2.98 & 2.80 & 2.67 & 2.43 & 1.83 & 8.3 & 7.3 & 6.5 \\
\hline 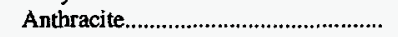 & 2.08 & 1.93 & 1.85 & 1.33 & 1.39 & 1.03 & 8.0 & 10.6 & 8.1 \\
\hline 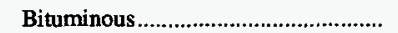 & 3.37 & 3.11 & 2.91 & 2.81 & 2.52 & 1.92 & 8.5 & 7.5 & 6.5 \\
\hline 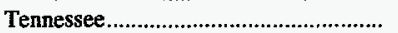 & 2.36 & 2.23 & 2.47 & 2.19 & 1.88 & 1.57 & 5.6 & 5.8 & 4.6 \\
\hline Texas & 9.10 & 8.82 & 8.42 & 7.34 & 7.17 & 6.33 & 3.2 & 6.1 & 4.1 \\
\hline Utah & 7.02 & 6.59 & 5.96 & 5.46 & 4.80 & 3.08 & 6.5 & 10.0 & 9.6 \\
\hline 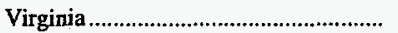 & 2.50 & 2.51 & 2.41 & 2.37 & 2.23 & 1.90 & -.1 & 2.9 & 3.1 \\
\hline 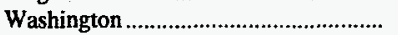 & 4.04 & 4.11 & 4.00 & 4.51 & 3.97 & 2.83 & -1.6 & .5 & 4.0 \\
\hline West Virginia Total ................................. & 3.74 & 3.69 & 3.27 & 3.27 & 3.11 & 2.19 & 1.5 & 4.7 & 6.1 \\
\hline 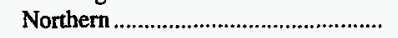 & 3.72 & 3.63 & 2.98 & 3.17 & 2.84 & 2.35 & 2.7 & 7.0 & 5.3 \\
\hline Southern & 3.75 & 3.72 & 3.39 & 3.32 & 3.26 & 2.11 & .9 & 3.6 & 6.6 \\
\hline Wyoming & 30.06 & 26.05 & 24.46 & 21.50 & 21.87 & 15.73 & 15.4 & 8.3 & 7.5 \\
\hline 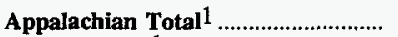 & 3.32 & 3.20 & 3.00 & 2.95 & 2.74 & 2.09 & 3.9 & 4.9 & 5.3 \\
\hline 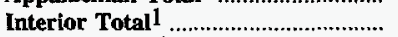 & 4.97 & 4.43 & 4.43 & 4.18 & 3.98 & 3.14 & 12.1 & 5.7 & 5.2 \\
\hline Western Total $^{1}$ & 15.68 & 14.58 & 13.53 & 12.73 & 12.42 & 9.27 & 7.5 & 6.0 & 6.0 \\
\hline 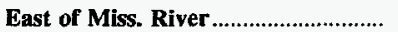 & 3.45 & 3.28 & 3.11 & 3.07 & 2.86 & 2.21 & 5.1 & 4.7 & 5.0 \\
\hline West of Miss. River............................... & 14.18 & 13.22 & 12.14 & 11.03 & 10.79 & 7.90 & 7.3 & 7.1 & 6.7 \\
\hline U.S. Total & 5.38 & 4.98 & 4.70 & 4.36 & 4.09 & 3.01 & 8.0 & 7.1 & 6.6 \\
\hline
\end{tabular}

1 For a definition of coal-producing regions, see Appendix $\mathbf{C}$.

Notes: Productivity is calculated by dividing total coal production by the total direct labor hours worked by all employees engaged in production, preparation, processing, development, maintenance, repair, shop or yard work at mining operations, but excludes office workers. Excludes silt, culm, refuse bank, slurry dam, and dredge operations except for Pennsylvania anthracite. Excludes mines producing less than 10,000 short tons, which are not required to provide these data.

Sources: Energy Information Administration, Form EIA-7A “Coal Production Report"; State Mining Agency Coal Production Reports; and/or U.S. Department of Labor, Mine Safety and Health Administration, Form 7000-2, "Quarterly Mine Employment and Coal Production Report." 
Table 49. Underground Coal Mining Productivity by State, 1986, 1991-1995

(Short Tons of Coal Produced per Miner per Hour)

\begin{tabular}{|c|c|c|c|c|c|c|c|c|c|}
\hline \multirow{2}{*}{$\begin{array}{l}\text { Coal-Producing } \\
\text { State and Region }\end{array}$} & \multirow{2}{*}{1995} & \multirow{2}{*}{1994} & \multirow{2}{*}{1993} & \multirow{2}{*}{1992} & \multirow{2}{*}{1991} & \multirow{2}{*}{1986} & \multirow{2}{*}{$\begin{array}{c}\text { Percent } \\
\text { Change } \\
\text { 1994-1995 }\end{array}$} & \multicolumn{2}{|c|}{ Average Annual Percent Change } \\
\hline & & & & & & & & 1991-1995 & 1986-1995 \\
\hline 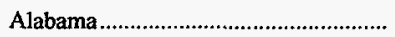 & 2.02 & 1.94 & 2.09 & 2.17 & 1.90 & 1.54 & 4.1 & 1.5 & 3.1 \\
\hline Arkansas & - & 1.62 & - & - & - & - & -100.0 & - & - \\
\hline Colorado & 5.86 & 5.81 & 5.21 & 4.52 & 4.23 & 2.38 & 1.0 & 8.5 & 10.5 \\
\hline Illinois & 3.86 & 3.49 & 3.11 & 3.21 & 2.88 & 2.06 & 10.5 & 7.6 & 7.2 \\
\hline Indiana & 3.22 & 2.82 & 2.49 & 2.80 & 2.97 & 2.11 & 14.2 & 2.1 & 4.8 \\
\hline 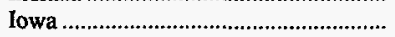 & - & - & - & - & - & 1.15 & - & - & - \\
\hline Kentucky Total & 3.25 & 2.89 & 2.93 & 2.91 & 2.71 & 2.23 & 12.4 & 4.7 & 4.3 \\
\hline 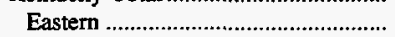 & 3.12 & 2.87 & 2.89 & 2.86 & 2.66 & 2.09 & 8.6 & 4.0 & 4.5 \\
\hline 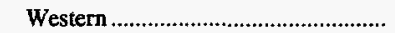 & 3.70 & 2.96 & 3.10 & 3.05 & 2.86 & 2.78 & 25.0 & 6.6 & 3.2 \\
\hline 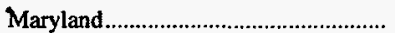 & 4.77 & 4.52 & 4.34 & 3.50 & 3.73 & 4.09 & 5.6 & 6.3 & 1.7 \\
\hline 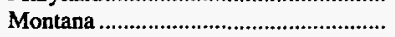 & - & - & 1.06 & - & - & - & - & - & - \\
\hline 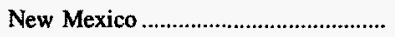 & 2.68 & 2.57 & 1.63 & .40 & .71 & 2.29 & 4.2 & 39.2 & 1.8 \\
\hline 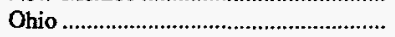 & 3.81 & 3.51 & 3.27 & 3.01 & 2.55 & 1.78 & 8.4 & 10.5 & 8.8 \\
\hline 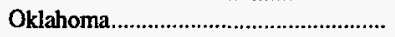 & .74 & 1.70 & 1.03 & 1.04 & .52 & - & -56.6 & 9.0 & - \\
\hline 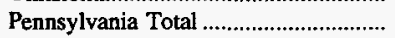 & 3.49 & 3.18 & 2.91 & 2.81 & 2.38 & 1.57 & 9.6 & 10.1 & 9.3 \\
\hline Anthracite. & .86 & .64 & .74 & .84 & .51 & .56 & 34.6 & 13.8 & 4.8 \\
\hline 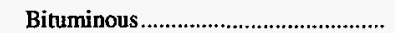 & 3.56 & 3.25 & 2.98 & 2.84 & 2.40 & 1.60 & 9.8 & 10.3 & 9.3 \\
\hline 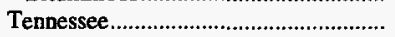 & 2.02 & 1.90 & 2.34 & 1.81 & 1.72 & 1.53 & 6.2 & 4.1 & 3.1 \\
\hline 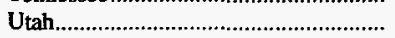 & 7.02 & 6.59 & 5.96 & 5.46 & 4.80 & 3.08 & 6.5 & 10.0 & 9.6 \\
\hline Virginia & 2.25 & 2.27 & 2.19 & 2.20 & 2.12 & 1.81 & -.7 & 1.6 & 2.4 \\
\hline 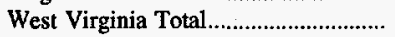 & 3.40 & 3.38 & 2.92 & 2.99 & 2.83 & 2.07 & .5 & 4.7 & 5.7 \\
\hline Northern & 3.66 & 3.61 & 2.84 & 3.12 & 2.80 & 2.30 & 1.4 & 6.9 & 5.3 \\
\hline Southern & 3.27 & 3.25 & 2.96 & 2.92 & 2.84 & 1.94 & .4 & 3.5 & 6.0 \\
\hline 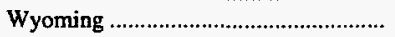 & 5.97 & 5.07 & 3.56 & 4.19 & 4.17 & 2.98 & 17.7 & 9.4 & 8.0 \\
\hline Appalachian Total ${ }^{1}$............................... & 3.08 & 2.96 & 2.75 & 2.76 & 2.54 & 1.90 & 3.9 & 5.0 & 5.5 \\
\hline Interior Total 1 . & 3.76 & 3.26 & 3.06 & 3.14 & 2.87 & 2.26 & 15.4 & 6.9 & 5.8 \\
\hline 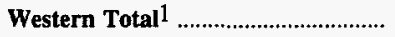 & 6.35 & 5.98 & 5.23 & 4.88 & 4.56 & 2.82 & 6.1 & 8.6 & 9.4 \\
\hline East of Miss. River ................................ & 3.19 & 3.02 & 2.81 & 2.82 & 2.59 & 1.96 & 5.8 & 5.3 & 5.6 \\
\hline 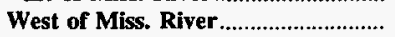 & 6.32 & 5.93 & 5.18 & 4.85 & 4.53 & 2.80 & 6.5 & 8.7 & 9.5 \\
\hline 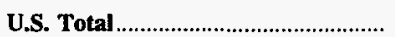 & 3.39 & 3.19 & 2.95 & 2.93 & 2.69 & 2.00 & 6.0 & 5.9 & 6.0 \\
\hline
\end{tabular}

1 For a definition of coal-producing regions, see Appendix $\mathbf{C}$.

Notes: Productivity is calculated by dividing total coal production by the total direct labor hours worked by all mine employees engaged in production, preparation, processing, development, maintenance, repair, shop or yard work at mining operations, but excludes office workers. Excludes silt, culm, refuse bank, slurry dam, and dredge operations except for Pennsylvania anthracite. Excludes mines producing less than 10,000 short tons, which are not required to provide these data.

Sources: Energy Information Administration, Form EIA-7A, “Coal Production Report"; State Mining Agency Coal Production Reports; and/or U.S. Department of Labor, Mine Safety and Health Administration, Form 7000-2, “Quarterly Mine Employment and Coal Production Report." 
Table 50. Surface Coal Mining Productivity by State, 1986, 1991-1995 (Short Tons of Coal Produced per Miner per Hour)

\begin{tabular}{|c|c|c|c|c|c|c|c|c|c|}
\hline \multirow{2}{*}{$\begin{array}{l}\text { Coal-Producing } \\
\text { State and Region }\end{array}$} & \multirow{2}{*}{1995} & \multirow{2}{*}{1994} & \multirow{2}{*}{1993} & \multirow{2}{*}{1992} & \multirow{2}{*}{1991} & \multirow{2}{*}{1986} & \multirow{2}{*}{$\begin{array}{c}\text { Percent } \\
\text { Change } \\
\text { 1994-1995 }\end{array}$} & \multicolumn{2}{|c|}{ Average Annual Percent Change } \\
\hline & & & & & & & & 1991-1995 & 1986-1995 \\
\hline 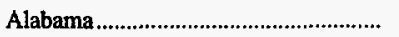 & 3.07 & 3.07 & 3.01 & 3.28 & 2.84 & 2.47 & $*$ & 1.9 & 2.4 \\
\hline Alaska & 7.46 & 6.94 & 7.40 & 6.93 & 6.82 & 6.77 & 7.5 & 2.3 & 1.1 \\
\hline Arizona & 6.34 & 6.71 & 6.21 & 6.29 & 6.64 & 6.68 & -5.4 & -1.1 & -.6 \\
\hline 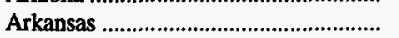 & 1.47 & 1.46 & 1.39 & 1.68 & 2.27 & 1.75 & .2 & -10.3 & -1.9 \\
\hline 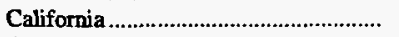 & - & - & - & 11.12 & 11.31 & - & - & - & - \\
\hline Colorado & 6.79 & 7.06 & 7.07 & 6.52 & 6.51 & 5.85 & -3.9 & 1.0 & 1.6 \\
\hline 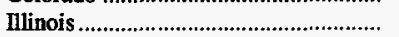 & 3.89 & 4.12 & 3.86 & 4.47 & 4.30 & 3.22 & -5.5 & -2.4 & 2.1 \\
\hline Indiana & 5.04 & 4.56 & 4.82 & 4.28 & 4.17 & 3.49 & 10.5 & 4.8 & 4.2 \\
\hline 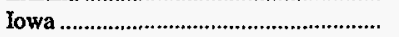 & - & 1.52 & .72 & 1.14 & 1.29 & 1.78 & -100.0 & - & - \\
\hline 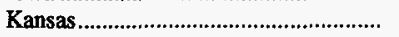 & 2.22 & 1.93 & 2.30 & 2.21 & 2.15 & 2.83 & 14.9 & .8 & -2.7 \\
\hline 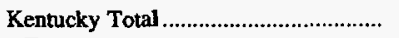 & 4.23 & 3.96 & 3.84 & 3.75 & 3.66 & 2.84 & 6.8 & 3.7 & 4.5 \\
\hline Eastern & 4.13 & 3.97 & 3.74 & 3.57 & 3.43 & 2.72 & 3.9 & 4.8 & 4.7 \\
\hline Western & 4.77 & 3.93 & 4.14 & 4.31 & 4.42 & 3.16 & 21.5 & 1.9 & 4.7 \\
\hline 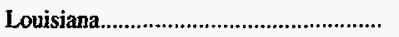 & 13.25 & 13.00 & 12.14 & 12.43 & 12.56 & 12.45 & 1.9 & 1.3 & .7 \\
\hline 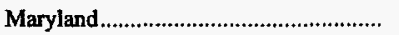 & 2.16 & 2.18 & 2.07 & 2.13 & 2.23 & 2.81 & -.8 & -.8 & -2.8 \\
\hline 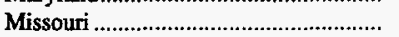 & 2.55 & 3.59 & 1.84 & 3.10 & 2.69 & 2.20 & -29.0 & -1.4 & 1.6 \\
\hline 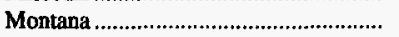 & 21.06 & 21.92 & 19.59 & 20.16 & 18.99 & 17.63 & -3.9 & 2.6 & 2.0 \\
\hline 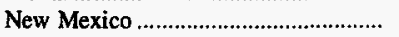 & 7.19 & 7.18 & 7.26 & 7.11 & 6.31 & 6.15 & .1 & 3.3 & 1.8 \\
\hline 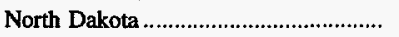 & 16.80 & 18.84 & 17.66 & 18.12 & 17.64 & 12.27 & -10.8 & -1.2 & 3.5 \\
\hline Ohio & 3.46 & 3.34 & 3.58 & 3.06 & 2.76 & 2.44 & 3.5 & 5.8 & 3.9 \\
\hline 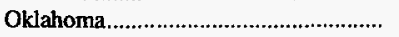 & 3.10 & 2.80 & 3.12 & 2.26 & 2.42 & 1.74 & 10.5 & 6.4 & 6.6 \\
\hline Pennsylvania Total ................................. & 2.79 & 2.67 & 2.63 & 2.45 & 2.53 & 2.26 & 4.3 & 2.4 & 2.3 \\
\hline 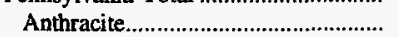 & 2.30 & 2.13 & 2.09 & 1.39 & 1.51 & 1.15 & 7.9 & 11.1 & 8.0 \\
\hline 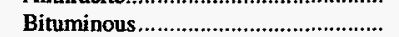 & 2.95 & 2.84 & 2.78 & 2.74 & 2.80 & 2.54 & 4.0 & 1.4 & 1.7 \\
\hline 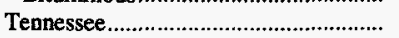 & 3.20 & 3.19 & 2.71 & 3.06 & 2.45 & 1.73 & .3 & 6.9 & 7.0 \\
\hline Texas & 9.10 & 8.82 & 8.42 & 7.34 & 7.17 & 6.33 & 3.2 & 6.1 & 4.1 \\
\hline Virginia & 3.73 & 3.73 & 3.55 & 3.50 & 2.95 & 2.48 & -1 & 6.0 & 4.6 \\
\hline Washington & 4.04 & 4.11 & 4.00 & 4.51 & 3.97 & 2.83 & -1.6 & .5 & 4.0 \\
\hline 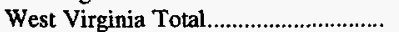 & 4.74 & 4.62 & 4.35 & 4.27 & 4.18 & 2.88 & 2.6 & 3.1 & 5.7 \\
\hline Northern & 4.31 & 3.78 & 3.70 & 3.52 & 3.16 & 2.64 & 14.0 & 8.0 & 5.6 \\
\hline 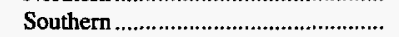 & 4.79 & 4.75 & 4.49 & 4.43 & 4.38 & 3.00 & .8 & 2.3 & 5.3 \\
\hline Wyoming & 31.02 & 27.37 & 26.03 & 22.76 & 23.11 & 15.81 & 13.3 & 7.6 & 7.8 \\
\hline Appalachian Total ${ }^{1}$ & 3.88 & 3.72 & 3.55 & 3.40 & 3.24 & 2.54 & 4.3 & 4.6 & 4.8 \\
\hline Interior Total 1 & 6.39 & 5.71 & $\mathbf{5 . 7 1}$ & 5.21 & 5.08 & 3.87 & 11.9 & 5.9 & 5.7 \\
\hline 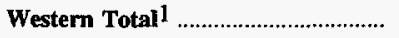 & 18.93 & 17.68 & 16.49 & 15.46 & 15.33 & 11.49 & 7.1 & 5.4 & 5.7 \\
\hline 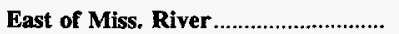 & 4.03 & $\mathbf{3 . 8 5}$ & 3.74 & 3.61 & 3.49 & 2.75 & 4.9 & 3.7 & 4.3 \\
\hline West of Miss. River................................. & 16.23 & 15.19 & 13.94 & 12.49 & 12.36 & 9.02 & 6.9 & 7.0 & 6.8 \\
\hline 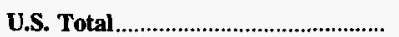 & 8.48 & 7.67 & 7.23 & 6.59 & 6.38 & 4.60 & 10.7 & 7.4 & 7.0 \\
\hline
\end{tabular}

1 For a definition of coal-producing regions, see Appendix $C$.

* Data round to zero.

Notes: Productivity is calculated by dividing total coal production by the total direct labor hours worked by all mine employees engaged in production, preparation, processing, development, maintenance, repair, shop or yard work at mining operations, but excludes office workers. Excludes silt, culm, refuse bank, slurry dam, and dredge operations except for Pennsylvania anthracite. Excludes mines producing less than 10,000 short tons, which are not required to provide these data.

Sources: Energy Information Administration, Form EIA-7A, "Coal Production Report'; State Mining Agency Coal Production Reports; and/or U.S. Department of Labor, Mine Safety and Health Administration, Form 7000-2, "Quarterly Mine Employment and Coal Production Report." 
Table 51. Coal Mining Productivity by State and Mine Type, 1995

\begin{tabular}{|c|c|c|c|c|}
\hline $\begin{array}{l}\text { Coal-Producing } \\
\text { State and Region }\end{array}$ & $\begin{array}{l}\text { Number of } \\
\text { Mining } \\
\text { Operations } 1\end{array}$ & $\begin{array}{c}\text { Average Number } \\
\text { of Miners } \\
\text { Working Daily } 2\end{array}$ & $\begin{array}{l}\text { Average Production } \\
\text { per Miner per Hour } \\
\text { (short tons) }\end{array}$ & $\begin{array}{l}\text { Average Production } \\
\text { per Miner per Shift } \\
\text { (short tons) }\end{array}$ \\
\hline Alabama & 67 & 5,567 & 2.24 & 18.86 \\
\hline 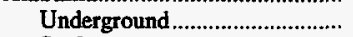 & 14 & 4,314 & 2.02 & 16.53 \\
\hline 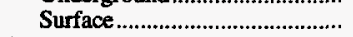 & 53 & 1,253 & 3.07 & 26.05 \\
\hline 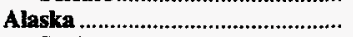 & 1 & 102 & 7.46 & 67.17 \\
\hline 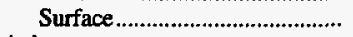 & 1 & 102 & 7.46 & 67.17 \\
\hline Arizona & 3 & 831 & 6.34 & 62.46 \\
\hline Surface & 3 & 831 & 6.34 & 62.46 \\
\hline 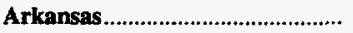 & 1 & 4 & 1.47 & 14.67 \\
\hline Surface & 1 & 4 & 1.47 & 14.67 \\
\hline 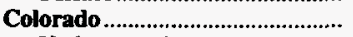 & 19 & 1,777 & 6.14 & $\mathbf{5 2 . 8 4}$ \\
\hline 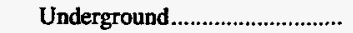 & 14 & 1,301 & 5.86 & 50.28 \\
\hline 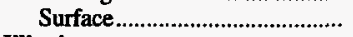 & 5 & 476 & 6.79 & 58.97 \\
\hline 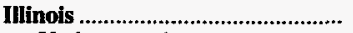 & 40 & 5,652 & 3.87 & 32.86 \\
\hline 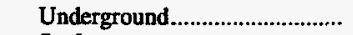 & 25 & 4,780 & 3.86 & 32.15 \\
\hline 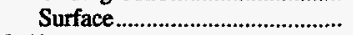 & 15 & 872 & 3.89 & 34.22 \\
\hline Indiana & 44 & 2,571 & 4.68 & 43.51 \\
\hline 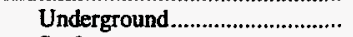 & 6 & 485 & 3.22 & 28.12 \\
\hline 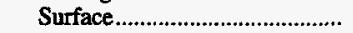 & 38 & 2,086 & 5.04 & 47.31 \\
\hline 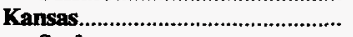 & 1 & 54 & 2.22 & 17.76 \\
\hline Surface & 1 & 54 & 2.22 & 17.76 \\
\hline 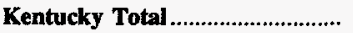 & 690 & 21,125 & 3.57 & 32.36 \\
\hline 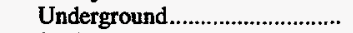 & 410 & 14,542 & 3.25 & 28.49 \\
\hline Surface & 280 & 6,583 & 4.23 & 40.18 \\
\hline Eastern & 626 & 16,840 & $\mathbf{3 . 4 7}$ & 31.60 \\
\hline 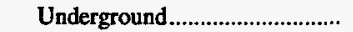 & 380 & 11,366 & 3.12 & 27.35 \\
\hline 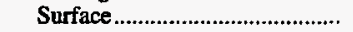 & 246 & 5,474 & 4.13 & 39.83 \\
\hline Western & 64 & 4,285 & 3.97 & 33.89 \\
\hline 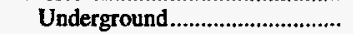 & 30 & 3,176 & 3.70 & 32.20 \\
\hline 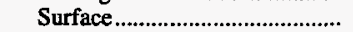 & 34 & 1,109 & 4.77 & 39.96 \\
\hline Louisiana & 2 & 114 & 13.25 & 125.86 \\
\hline Surface & 2 & 114 & 13.25 & 125.86 \\
\hline 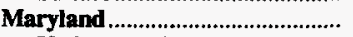 & 16 & 458 & 3.82 & 32.39 \\
\hline 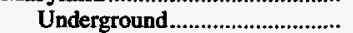 & 3 & 293 & 4.77 & 38.97 \\
\hline 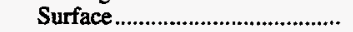 & 13 & 165 & 2.16 & 18.51 \\
\hline 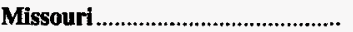 & $\mathbf{5}$ & 92 & 2.55 & 21.98 \\
\hline 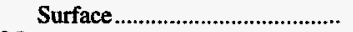 & 5 & 92 & 2.55 & 21.98 \\
\hline 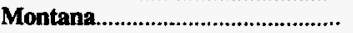 & 7 & 722 & 21.06 & 179.64 \\
\hline Surface & 7 & 722 & 21.06 & 179.64 \\
\hline 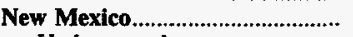 & 9 & 1,747 & 6.92 & 54.01 \\
\hline 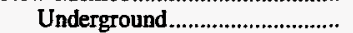 & 2 & 132 & 2.68 & 20.07 \\
\hline 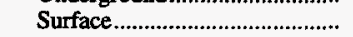 & 7 & 1,615 & 7.19 & 56.82 \\
\hline 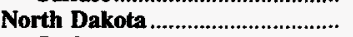 & 7 & 716 & 16.80 & 136.69 \\
\hline Surface. & 7 & 716 & 16.80 & 136.69 \\
\hline 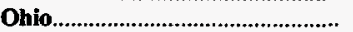 & 109 & 3,386 & 3.62 & 34.06 \\
\hline 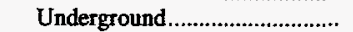 & 14 & 1,670 & 3.81 & 34.87 \\
\hline 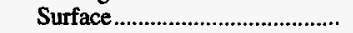 & 95 & 1,716 & 3.46 & 32.60 \\
\hline Oklahoma & 12 & 241 & 2.97 & 26.79 \\
\hline 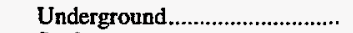 & 1 & 12 & .74 & 6.62 \\
\hline 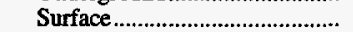 & 11 & 229 & 3.10 & 27.96 \\
\hline Pennsylvania Total ........................ & 378 & 8,968 & 3.23 & 27.75 \\
\hline 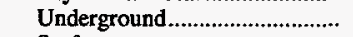 & 98 & 5,659 & 3.49 & 29.26 \\
\hline 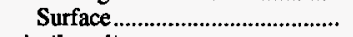 & 280 & 3,309 & 2.79 & 24.15 \\
\hline 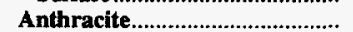 & 102 & 1,069 & 2.08 & 16.92 \\
\hline 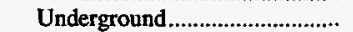 & 22 & 152 & .86 & 7.01 \\
\hline Surface & 80 & 917 & 2.30 & 18.63 \\
\hline 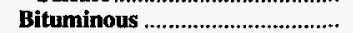 & 276 & 7,899 & $\mathbf{3 . 3 7}$ & 29.58 \\
\hline 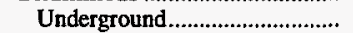 & 76 & 5,507 & 3.56 & 30.11 \\
\hline Surface & 200 & 2,392 & 2.95 & 26.27 \\
\hline 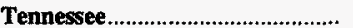 & 33 & 681 & 2.36 & 20.75 \\
\hline 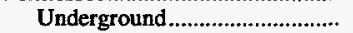 & 20 & 473 & 2.02 & 17.20 \\
\hline Surface & 13 & 208 & 3.20 & 29.54 \\
\hline Texas & 14 & 1,590 & 9.10 & 90.97 \\
\hline Surface & 14 & 1,590 & 9.10 & 90.97 \\
\hline 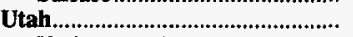 & 16 & 1,893 & 7.02 & 60.50 \\
\hline Underground & 16 & 1,893 & 7.02 & 60.50 \\
\hline 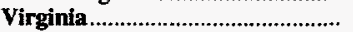 & 232 & 6,919 & 2.50 & 22.04 \\
\hline 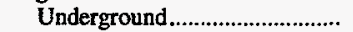 & 170 & 5,776 & 2.25 & 19.36 \\
\hline Surface ...................................... & 62 & 1,143 & 3.73 & 34.77 \\
\hline Washington & 3 & 566 & 4.04 & 33.71 \\
\hline Surface & 3 & 566 & 4.04 & 33.71 \\
\hline West Virginia Total ................ & 542 & 21,334 & 3.74 & 32.80 \\
\hline 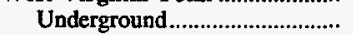 & 351 & 16,347 & 3.40 & 29.01 \\
\hline Surface & 191 & 4,987 & 4.74 & 43.51 \\
\hline
\end{tabular}

See footnotes at end of table. 
Table 51. Coal Mining Productivity by State and Mine Type, 1995 (Continued)

\begin{tabular}{|c|c|c|c|c|}
\hline $\begin{array}{l}\text { Coal-Producing } \\
\text { State and Region }\end{array}$ & $\begin{array}{l}\text { Number of } \\
\text { Mining } \\
\text { Operations } 1\end{array}$ & $\begin{array}{c}\text { Average Number } \\
\text { of Miners } \\
\text { Working Daily } 2\end{array}$ & $\begin{array}{c}\text { Average Production } \\
\text { per Miner per Hour } \\
\text { (short tons) }^{3}\end{array}$ & $\begin{array}{l}\text { Average Production } \\
\text { per Miner per Shift } \\
\text { (short tons) } 4\end{array}$ \\
\hline 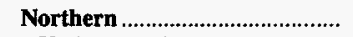 & 116 & 6,114 & 3.72 & 31.63 \\
\hline 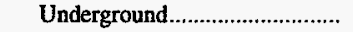 & 68 & 5,561 & 3.66 & 29.96 \\
\hline 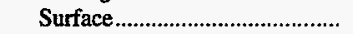 & 48 & 553 & 4.31 & 38.43 \\
\hline 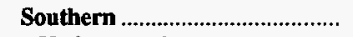 & 426 & 15,220 & 3.75 & 33.15 \\
\hline Underground & 283 & 10,786 & 3.27 & 28.13 \\
\hline 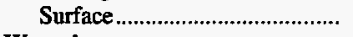 & 143 & 4,434 & 4.79 & 44.42 \\
\hline Wyoming & 27 & 3,142 & 30.06 & 294.01 \\
\hline Underground & 2 & 202 & 5.97 & 59.75 \\
\hline Surface & 25 & 2,940 & 31.02 & 302.85 \\
\hline Appalachian Total 5 ...................... & 2,003 & 64,153 & 3.32 & 29.44 \\
\hline 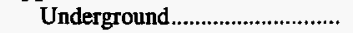 & 1,050 & 45,898 & 3.08 & 26.55 \\
\hline Surface & 953 & 18,255 & 3.88 & 35.49 \\
\hline Interior Total $5 \ldots \ldots \ldots \ldots \ldots \ldots \ldots$ & 183 & 14,603 & 4.97 & 44.06 \\
\hline 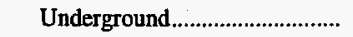 & 62 & 8,453 & 3.76 & 32.18 \\
\hline 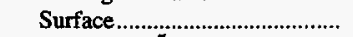 & 121 & 6,150 & 6.39 & 57.72 \\
\hline 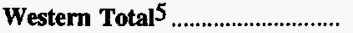 & 92 & 11,496 & 15.68 & 139.02 \\
\hline 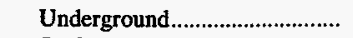 & 34 & 3,528 & 6.35 & 54.68 \\
\hline 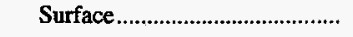 & 58 & 7,968 & 18.93 & 170.72 \\
\hline 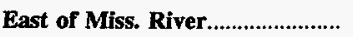 & 2,151 & 76,661 & 3.45 & 30.55 \\
\hline Underground & 1,111 & 54,339 & 3.19 & 27.52 \\
\hline 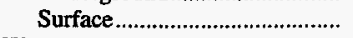 & 1,040 & 22,322 & 4.03 & 36.79 \\
\hline West of Miss. River. & 127 & 13,591 & 14.18 & 127.79 \\
\hline 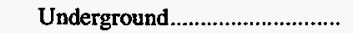 & 35 & 3,540 & 6.32 & 54.51 \\
\hline Surface & 92 & 10,051 & 16.23 & 148.63 \\
\hline 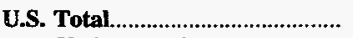 & 2,278 & 90,252 & 5.38 & 47.67 \\
\hline 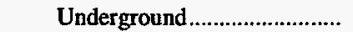 & 1,146 & 57,879 & 3.39 & 29.16 \\
\hline 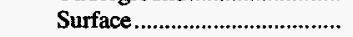 & 1,132 & 32,373 & 8.48 & 77.40 \\
\hline
\end{tabular}

1 Mining operations that consist of a mine and preparation plant, or a preparation plant only processing both underground and surface coal will be counted as two operations.

2 Includes all employees engaged in production, preparation, processing, development, maintenance, repair, shop or yard work at mining operations. Excludes office workers. Includes mining operations management and all technical and engineering employees.

3 Calculated by dividing total coal production by the total direct labor hours worked by all employees engaged in production, preparation, processing, development, maintenance, repair, shop or yard work at mining operations, but excludes office workers.

4 Calculated by multiplying average production per miner per hour by the average length of a miner shift

5 For a definition of coal-producing regions, see Appendix $C$.

Notes: Excludes silt, culm, refuse bank, slurry dam, and dredge operations except for Pennsylvania anthracite. Excludes mines producing less than 10,000 short tons of coal during the year, and preparation plants with less than 5,000 employee hours, which are not required to provide these data.

Sources: Energy Information Administration, Form EIA-7A, "Coal Production Report"; State Mining Agency Coal Production Reports; and/or U.S. Department of Labor, Mine Safety and Health Administration, Form 7000-2, "Quarterly Mine Employment and Coal Production Report." 
Table 52. Weighted Average Number of Days Worked by State and Mine Type, 1986, 1991-1995

\begin{tabular}{|c|c|c|c|c|c|c|c|c|c|}
\hline \multirow{2}{*}{$\begin{array}{l}\text { Coal-Producing } \\
\text { State and Region }\end{array}$} & \multirow{2}{*}{1995} & \multirow{2}{*}{1994} & \multirow{2}{*}{1993} & \multirow{2}{*}{1992} & \multirow{2}{*}{1991} & \multirow{2}{*}{1986} & \multirow{2}{*}{$\begin{array}{c}\text { Percent } \\
\text { Change } \\
\text { 1994-1995 }\end{array}$} & \multicolumn{2}{|c|}{ Average Annual Percent Change } \\
\hline & & & & & & & & 1991-1995 & 1986-1995 \\
\hline 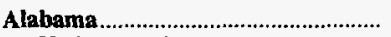 & 260 & 240 & 250 & 245 & 239 & 252 & 8.2 & 2.1 & 0.3 \\
\hline Underground & 259 & 246 & 249 & 236 & 231 & 232 & 5.4 & 2.9 & 1.3 \\
\hline Surface & 262 & 231 & 253 & 258 & 254 & 274 & 13.4 & .8 & -.5 \\
\hline 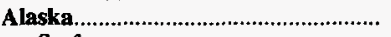 & 251 & 246 & 250 & 224 & 265 & 260 & 2.0 & -1.3 & -.4 \\
\hline 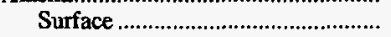 & 251 & 246 & 250 & 224 & 265 & 260 & 2.0 & -1.3 & -.4 \\
\hline 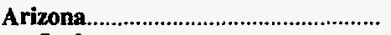 & 211 & 217 & 211 & 225 & 230 & 221 & -2.6 & -2.1 & -.5 \\
\hline 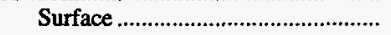 & 211 & 217 & 211 & 225 & 230 & 221 & -2.6 & -2.1 & -.5 \\
\hline 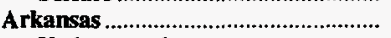 & 240 & 176 & 240 & 228 & 161 & 285 & 36.4 & 10.5 & -1.9 \\
\hline Underground & - & 85 & - & - & - & - & -100.0 & - & - \\
\hline 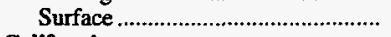 & 240 & 240 & 240 & 228 & 161 & 285 & - & 10.5 & -1.9 \\
\hline 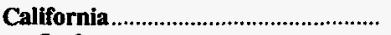 & - & - & - & 116 & 63 & - & - & - & - \\
\hline Surface & - & - & - & 116 & 63 & - & - & - & - \\
\hline 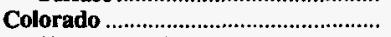 & 287 & 258 & 243 & 278 & 275 & 259 & 11.0 & 1.1 & 1.1 \\
\hline 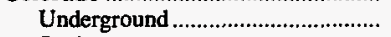 & 287 & 288 & 282 & 268 & 260 & 232 & -.3 & 2.5 & 2.4 \\
\hline 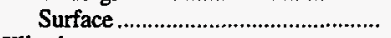 & 286 & 204 & 188 & 290 & 292 & 275 & 40.1 & -.5 & .5 \\
\hline 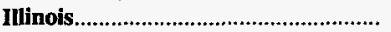 & 260 & 253 & 220 & 253 & 252 & 246 & 2.8 & .7 & .6 \\
\hline Underground & 264 & 257 & 222 & 258 & 259 & 240 & 2.7 & .5 & 1.1 \\
\hline Surface & 233 & 231 & 212 & 233 & 235 & 256 & .8 & -.2 & -1.0 \\
\hline 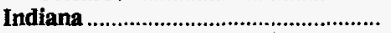 & 266 & 274 & 254 & 250 & 245 & 268 & -2.9 & 2.0 & -.1 \\
\hline 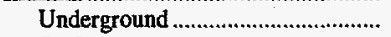 & 277 & 285 & 246 & 263 & 284 & 309 & -2.8 & -.6 & -1.2 \\
\hline Surface & 264 & 272 & 255 & 249 & 241 & 266 & -3.1 & 2.2 & -1 \\
\hline Iowa . & - & 150 & 260 & 259 & 250 & 254 & -100.0 & - & - \\
\hline Underground & - & - & - & - & - & 280 & - & - & - \\
\hline Surface & - & 150 & 260 & 259 & 250 & 245 & -100.0 & - & - \\
\hline 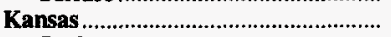 & 265 & 269 & 259 & 242 & 277 & 241 & -1.5 & -1.1 & 1.0 \\
\hline 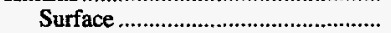 & 265 & 269 & 259 & 242 & 277 & 241 & -1.5 & -1.1 & 1.0 \\
\hline 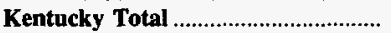 & 254 & 261 & 255 & 259 & 248 & 234 & -2.8 & .6 & .9 \\
\hline 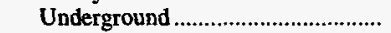 & 252 & 257 & 247 & 254 & 249 & 231 & -2.1 & .3 & 1.0 \\
\hline Surface & 258 & 268 & 267 & 267 & 247 & 239 & -3.6 & 1.1 & .8 \\
\hline 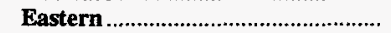 & 250 & 257 & 253 & 250 & 241 & 225 & -2.9 & .8 & 1.2 \\
\hline Underground & 246 & 249 & 244 & 246 & 241 & 220 & -1.1 & .6 & 1.3 \\
\hline Surface & 254 & 268 & 266 & 255 & 242 & 231 & -5.3 & 1.3 & 1.0 \\
\hline Western & 269 & 276 & 263 & 286 & 268 & 260 & $\mathbf{- 2 . 5}$ & .1 & .4 \\
\hline 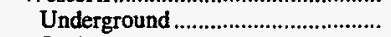 & 267 & 283 & 256 & 278 & 273 & 262 & -5.9 & -.6 & .2 \\
\hline 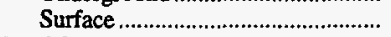 & 276 & 266 & 270 & 296 & 261 & 258 & 3.8 & 1.4 & .7 \\
\hline 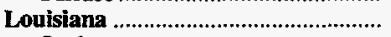 & 299 & 290 & 293 & 335 & 268 & 324 & 3.1 & 2.7 & -.9 \\
\hline Surface & 299 & 290 & 293 & 335 & 268 & 324 & 3.1 & 2.7 & -.9 \\
\hline 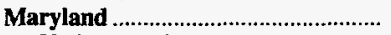 & 237 & 237 & 249 & 311 & 282 & 240 & $*$ & -4.2 & -.1 \\
\hline Underground & 241 & 236 & 249 & 339 & 291 & 241 & 2.3 & -4.6 & * \\
\hline Surface & 223 & 243 & 250 & 251 & 260 & 238 & -8.6 & -3.8 & -.8 \\
\hline 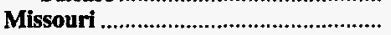 & 278 & 263 & 181 & 345 & 344 & 234 & 5.8 & $-\mathbf{5 . 2}$ & 1.9 \\
\hline Surface & 278 & 263 & 181 & 345 & 344 & 234 & 5.8 & -5.2 & 1.9 \\
\hline Montana & 341 & 341 & 341 & 344 & 319 & 300 & -.2 & 1.7 & 1.4 \\
\hline 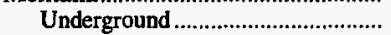 & - & - & 158 & - & - & - & - & - & - \\
\hline 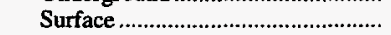 & 341 & 341 & 341 & 344 & 319 & 300 & -.2 & 1.7 & 1.4 \\
\hline New Mexico & 282 & 283 & 297 & 284 & 279 & 283 & -.1 & .3 & $*$ \\
\hline 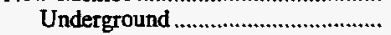 & 241 & 241 & 241 & 241 & 71 & 205 & - & 35.7 & 1.8 \\
\hline Surface & 283 & 284 & 299 & 284 & 279 & 285 & -.2 & .4 & -.1 \\
\hline North Dakota & 289 & 303 & 307 & 287 & 265 & 246 & -4.5 & 2.3 & 1.8 \\
\hline 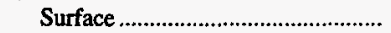 & 289 & 303 & 307 & 287 & 265 & 246 & -4.5 & 2.3 & 1.8 \\
\hline Ohio & 242 & 257 & 266 & 268 & 258 & 235 & -5.8 & -1.6 & 3 \\
\hline Underground & 239 & 265 & 266 & 268 & 260 & 236 & -9.7 & -2.1 & 1 \\
\hline Surface & 245 & 251 & 266 & 267 & 257 & 234 & -2.2 & -1.2 & .5 \\
\hline Oklahoma & 296 & 305 & 294 & 282 & 289 & 252 & -2.8 & .6 & 1.8 \\
\hline 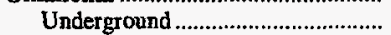 & 309 & 310 & 300 & 260 & 162 & - & -.3 & 17.5 & - \\
\hline 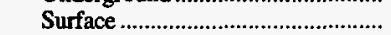 & 296 & 305 & 293 & 283 & 291 & 252 & -2.8 & .5 & 1.8 \\
\hline Pennsylvania Total & 262 & 257 & 246 & 249 & 243 & 236 & 2.0 & 1.9 & 1.2 \\
\hline Underground & 266 & 256 & 238 & 245 & 240 & 226 & 4.0 & 2.7 & 1.9 \\
\hline 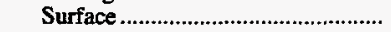 & 253 & 259 & 258 & 257 & 250 & 247 & -2.2 & .4 & .3 \\
\hline Anthracite & 253 & 264 & 247 & 246 & 256 & 241 & -4.3 & -.3 & .5 \\
\hline 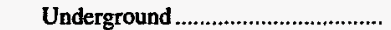 & 256 & 264 & 263 & 227 & 256 & 240 & -3.1 & -.1 & .7 \\
\hline Surface & 252 & 264 & 246 & 247 & 256 & 241 & -4.3 & -.3 & .5 \\
\hline 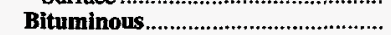 & 263 & 256 & 246 & 249 & 243 & 235 & 2.5 & 2.0 & 1.2 \\
\hline 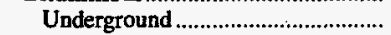 & 266 & 256 & 238 & 245 & 240 & 225 & 4.1 & 2.7 & 1.9 \\
\hline Surface & 253 & 258 & 261 & 258 & 249 & 248 & -1.6 & .5 & .2 \\
\hline 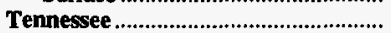 & 236 & 230 & 223 & 243 & 236 & 236 & 2.7 & * & * \\
\hline 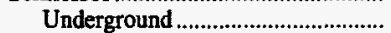 & 245 & 233 & 254 & 241 & 239 & 242 & 5.0 & .7 & .2 \\
\hline 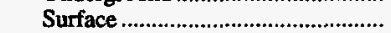 & 221 & 223 & 173 & 246 & 228 & 218 & -.9 & -.8 & .2 \\
\hline
\end{tabular}

See footnotes at end of table. 
Table 52. Weighted Average Number of Days Worked by State and Mine Type, 1986, 1991-1995 (Continued)

\begin{tabular}{|c|c|c|c|c|c|c|c|c|c|}
\hline \multirow{2}{*}{$\begin{array}{l}\text { Coal-Producing } \\
\text { State and Region }\end{array}$} & \multirow{2}{*}{1995} & \multirow{2}{*}{1994} & \multirow{2}{*}{1993} & \multirow{2}{*}{1992} & \multirow{2}{*}{1991} & \multirow{2}{*}{1986} & \multirow{2}{*}{$\begin{array}{l}\text { Percent } \\
\text { Change } \\
\text { 1994-1995 }\end{array}$} & \multicolumn{2}{|c|}{ Average Annual Percent Change } \\
\hline & & & & & & & & 1991-1995 & 1986-1995 \\
\hline 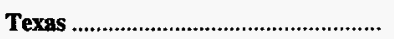 & 345 & 346 & 350 & 348 & 341 & 320 & -0.3 & 0.2 & 0.8 \\
\hline Surface & 345 & 346 & 350 & 348 & 341 & 320 & -.3 & .2 & .8 \\
\hline 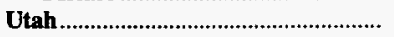 & 241 & 257 & 249 & 246 & 252 & 217 & -6.4 & -1.1 & 1.2 \\
\hline Underground & 241 & 257 & 249 & 246 & 252 & 217 & -6.4 & -1.1 & 1.2 \\
\hline Virginia & 254 & 245 & 246 & 253 & 241 & 220 & 3.8 & 1.4 & 1.6 \\
\hline Underground & 254 & 243 & 245 & 256 & 243 & 219 & 4.5 & 1.1 & 1.6 \\
\hline Surface & 255 & 251 & 247 & 241 & 231 & 224 & 1.5 & 2.5 & 1.4 \\
\hline 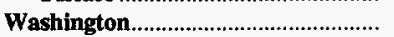 & 262 & 263 & 252 & 231 & 230 & 355 & -.3 & 3.3 & -3.3 \\
\hline Surface & 262 & 263 & 252 & 231 & 230 & 355 & -.3 & 3.3 & -3.3 \\
\hline West Virginia Total.................................. & 255 & 252 & 227 & 246 & 241 & 223 & 1.1 & 1.3 & 1.5 \\
\hline 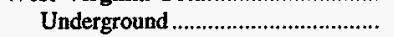 & 244 & 244 & 217 & 243 & 240 & 222 & -.2 & .4 & 1.1 \\
\hline Surface & 278 & 270 & 248 & 255 & 244 & 228 & 3.1 & 3.2 & 2.2 \\
\hline 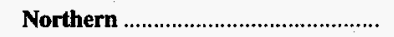 & 242 & 246 & 194 & 235 & 240 & 222 & -1.6 & .2 & 1.0 \\
\hline 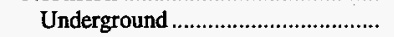 & 237 & 242 & 175 & 235 & 241 & 223 & -2.1 & -.4 & .7 \\
\hline 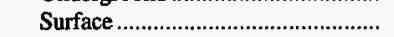 & 283 & 278 & 271 & 236 & 238 & 217 & 1.6 & 4.4 & 3.0 \\
\hline 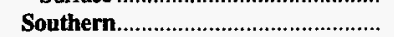 & 260 & 255 & 238 & 251 & 242 & 223 & 2.0 & 1.8 & 1.7 \\
\hline 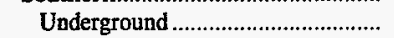 & 248 & 246 & 235 & 248 & 240 & 221 & .8 & .8 & 1.3 \\
\hline 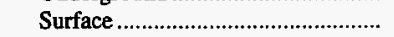 & 277 & 268 & 243 & 258 & 245 & 232 & 3.3 & 3.1 & 2.0 \\
\hline Wyoming & 352 & 341 & 345 & 339 & 339 & 313 & 3.2 & .9 & 1.3 \\
\hline 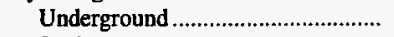 & 245 & 243 & 258 & 238 & 232 & 175 & .7 & 1.4 & 3.8 \\
\hline Surface & 353 & 342 & 346 & 341 & 341 & 313 & 3.1 & .9 & 1.3 \\
\hline 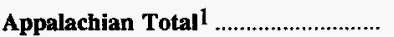 & 254 & 253 & 243 & 250 & 243 & 228 & .2 & 1.1 & 1.2 \\
\hline 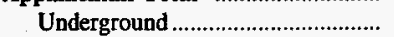 & 249 & 248 & 235 & 247 & 241 & 223 & .6 & .8 & 1.2 \\
\hline Surface & 261 & 262 & 257 & 256 & 246 & 238 & -.2 & 1.5 & 1.0 \\
\hline Interior Total 1 & 291 & 290 & 280 & 289 & 281 & 272 & 3 & .8 & .7 \\
\hline Underground & 266 & 267 & 236 & 265 & 265 & 250 & -.5 & .1 & .7 \\
\hline Surface & 308 & 304 & 302 & 304 & 290 & 282 & 1.4 & 1.5 & 1.0 \\
\hline Western Total ${ }^{1} \ldots \ldots \ldots \ldots \ldots$ & 326 & 319 & 320 & 316 & 312 & 291 & 2.4 & 1.2 & 1.3 \\
\hline Underground & 259 & 267 & 261 & 252 & 253 & 220 & -3.3 & .6 & 1.8 \\
\hline 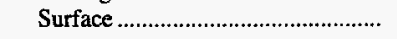 & 334 & 325 & 327 & 323 & 318 & 297 & 2.9 & 1.2 & 1.3 \\
\hline 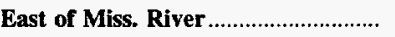 & 256 & 256 & 243 & 253 & 246 & 235 & $*$ & 1.0 & 9 \\
\hline 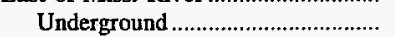 & 253 & 252 & 235 & 250 & 246 & 228 & .4 & .7 & 1.1 \\
\hline Surface & 261 & 262 & 256 & 257 & 246 & 245 & -.3 & 1.6 & .7 \\
\hline West of Miss. River & 328 & 321 & 323 & 320 & 315 & 294 & 2.1 & 1.0 & 1.2 \\
\hline 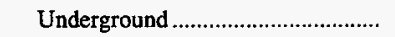 & 259 & 267 & 261 & 252 & 253 & 221 & -3.3 & 6 & 1.8 \\
\hline Surface & 335 & 327 & 329 & 326 & 321 & 299 & 2.5 & 1.1 & 1.3 \\
\hline U.S. Total & 290 & 285 & 280 & 280 & 274 & 257 & 1.6 & 1.4 & 1.4 \\
\hline 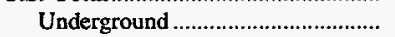 & 253 & 253 & 238 & 251 & 246 & 228 & -.1 & .7 & 1.2 \\
\hline Surface & 313 & 305 & 304 & 301 & 293 & 276 & 2.4 & 1.6 & 1.4 \\
\hline
\end{tabular}

1 For a definition of coal-producing regions, see Appendix C.

* Data round to zero.

Notes: Weighted average number of days worked is calculated by multiplying average days worked for each mine times its production and then summing these products over all mines in the region/State and then dividing the sum by the total production for the region/State. Excludes silt, culm, refuse bank, slurry dam, and dredge operations except for Pennsylvania anthracite. Excludes mines producing less than 10,000 short tons, which are not required to provide these data.

Sources: Energy Information Administration, Form EIA-7A "Coal Production Report"; State Mining Agency Coal Production Reports; and/or U.S. Department of Labor, Mine Safety and Health Administration, Form 7000-2, "Quarterly Mine Employment and Coal Production Report." 
Table 53. Weighted Average Number of Days Worked by Mine Production Range, 1995

\begin{tabular}{|c|c|c|c|c|c|c|c|}
\hline \multirow{2}{*}{$\begin{array}{l}\text { Coal-Producing } \\
\text { State and Region }\end{array}$} & \multicolumn{6}{|c|}{$\begin{array}{l}\text { Mine Production Range } \\
\text { (thousand short tons) }\end{array}$} & \multirow{2}{*}{ Total ${ }^{1}$} \\
\hline & $\begin{array}{l}1,000 \\
\text { and over }\end{array}$ & $\begin{array}{l}500 \\
\text { to } 1,000\end{array}$ & $\begin{array}{l}200 \\
\text { to } 500\end{array}$ & $\begin{array}{c}100 \\
\text { to } 200\end{array}$ & $\begin{array}{c}50 \\
\text { to } 100\end{array}$ & $\begin{array}{l}10 \\
\text { to } 50\end{array}$ & \\
\hline Alabama. & 261 & 297 & 214 & 278 & 215 & 178 & 260 \\
\hline Alaska & 251 & - & - & - & - & - & 251 \\
\hline Arizona & 211 & - & - & - & - & - & 211 \\
\hline Arkansas & - & - & - & - & - & 240 & 240 \\
\hline 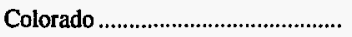 & 291 & 290 & 233 & - & - & 241 & 287 \\
\hline 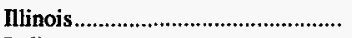 & 261 & 220 & 303 & 81 & 22 & 186 & 260 \\
\hline Indiana & 291 & 241 & 230 & 139 & 90 & 97 & 266 \\
\hline 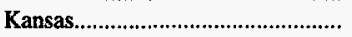 & - & - & 265 & - & - & - & 265 \\
\hline Kentucky Total & 282 & 256 & 245 & 220 & 198 & 175 & 254 \\
\hline 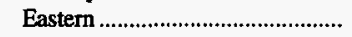 & 285 & 253 & 242 & 222 & 202 & 175 & 250 \\
\hline 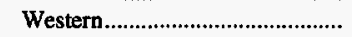 & 278 & 266 & 266 & 178 & 87 & 185 & 269 \\
\hline 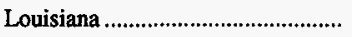 & 313 & 250 & - & - & - & - & 299 \\
\hline 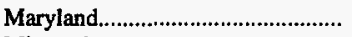 & 240 & - & 232 & 235 & 240 & 215 & 237 \\
\hline 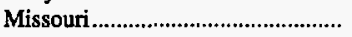 & - & - & - & 293 & 249 & 264 & 278 \\
\hline 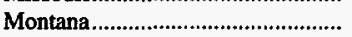 & 341 & - & 245 & - & - & - & 341 \\
\hline New Mexico & 283 & 241 & - & - & - & - & 282 \\
\hline 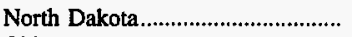 & 289 & - & - & - & - & - & 289 \\
\hline 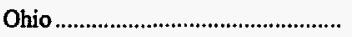 & 229 & 263 & 265 & 264 & 215 & 180 & 242 \\
\hline Oklahoma & - & - & 308 & 260 & - & 245 & 296 \\
\hline Pennsylvania Total ............................. & 274 & 261 & 250 & 247 & 248 & 225 & 262 \\
\hline Anthracite & - & - & 280 & 208 & 243 & 243 & 253 \\
\hline 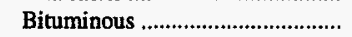 & 274 & 261 & 243 & 256 & 249 & 221 & 263 \\
\hline 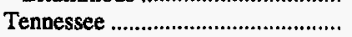 & - & 244 & 241 & 250 & 179 & 194 & 236 \\
\hline 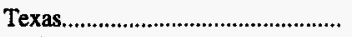 & 345 & - & 284 & - & 145 & - & 345 \\
\hline 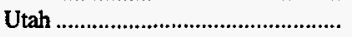 & 242 & 237 & 190 & 234 & - & - & 241 \\
\hline 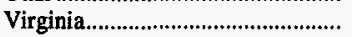 & 254 & 299 & 266 & 239 & 215 & 177 & 254 \\
\hline 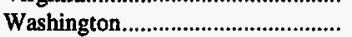 & 260 & - & 305 & - & - & - & 262 \\
\hline West Virginia Total ......................... & 272 & 256 & 231 & 193 & 196 & 178 & 255 \\
\hline 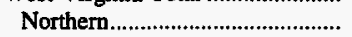 & 247 & 227 & 241 & 232 & 217 & 192 & 242 \\
\hline 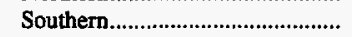 & 286 & 263 & 230 & 184 & 192 & 173 & 260 \\
\hline 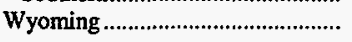 & 353 & 248 & 166 & - & - & 172 & 352 \\
\hline 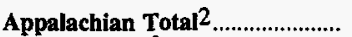 & 269 & 259 & 243 & 226 & 211 & 191 & 254 \\
\hline Interior Total 2 & 301 & 255 & 261 & 185 & 128 & 198 & 291 \\
\hline Western Total ${ }^{2}$ & 328 & 260 & 219 & 234 & - & 188 & 326 \\
\hline East of Miss. River......................... & 270 & 258 & 244 & 222 & 209 & 191 & 256 \\
\hline West of Miss. River........................ & 330 & 258 & 249 & 272 & 222 & 233 & 328 \\
\hline 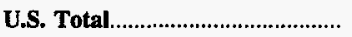 & 308 & 258 & 245 & 223 & 209 & 192 & 290 \\
\hline
\end{tabular}

1 Includes stand alone preparation plants.

2 For a definition of coal-producing regions, see Appendix C.

Notes: Weighted average number of days worked is calculated by multiplying average days worked for each mine times its production and then summing these products over all mines in the region/State and then dividing the sum by the total production for the region/State. Excludes silt, culm refuse bank, slurry dam, and dredge operations except for Pennsylvania anthracite. Excludes mines producing less than 10,000 short tons and preparation plants with less than 5,000 employee hours, which are not required to provide these data.

Sources: Energy Information Administration, Form EIA-7A, "Coal Production Report"; State Mining Agency Coal Production Reports; and/or U.S. Department of Labor, Mine Safety and Health Administration, Form 7000-2, "Quarterly Mine Employment and Coal Production Report." 
Table 54. Underground Coal Mining Productivity by State and Mining Method, 1995 (Short Tons of Coal Produced per Miner per Hour)

\begin{tabular}{|c|c|c|c|c|c|}
\hline $\begin{array}{l}\text { Coal-Producing } \\
\text { State and Region }\end{array}$ & Continuous 1 & Conventional 2 & Longwall ${ }^{3}$ & Other ${ }^{4}$ & Total \\
\hline Alabama & 2.03 & - & 2.02 & - & 2.02 \\
\hline 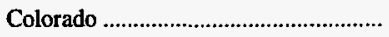 & 4.24 & 3.66 & 6.44 & - & 5.86 \\
\hline 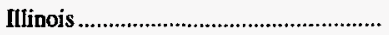 & 3.75 & - & 4.03 & - & 3.86 \\
\hline Indiana & 3.22 & - & - & - & 3.22 \\
\hline 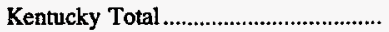 & 3.36 & 2.60 & 3.63 & 1.86 & 3.25 \\
\hline Eastern & 3.19 & 2.56 & 4.28 & 1.86 & 3.12 \\
\hline Western & 3.95 & 3.67 & 3.28 & - & 3.70 \\
\hline 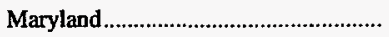 & 5.14 & - & 4.73 & - & 4.77 \\
\hline 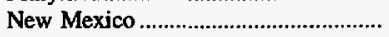 & - & - & 2.68 & - & 2.68 \\
\hline Ohio & 3.96 & - & 3.79 & - & 3.81 \\
\hline Oklahoma & .74 & - & - & - & .74 \\
\hline 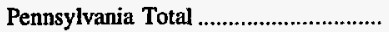 & 2.29 & 2.09 & 4.51 & .63 & 3.49 \\
\hline Anthracite & .94 & .81 & - & .63 & .86 \\
\hline 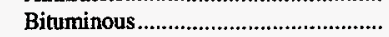 & 2.32 & 2.39 & 4.51 & - & 3.56 \\
\hline 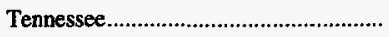 & 2.02 & - & - & - & 2.02 \\
\hline Utah & 3.87 & 2.08 & 7.69 & - & 7.02 \\
\hline 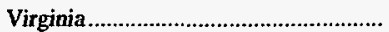 & 2.17 & 2.11 & 2.51 & 1.74 & 2.25 \\
\hline 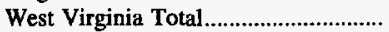 & 3.25 & 3.07 & 3.68 & - & 3.40 \\
\hline Northern & 3.34 & 2.99 & 3.80 & - & 3.66 \\
\hline Southern & 3.25 & 3.09 & 3.45 & - & 3.27 \\
\hline Wyoming & - & .81 & 6.34 & - & 5.97 \\
\hline 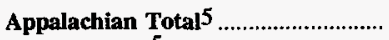 & 2.94 & 2.67 & 3.39 & 1.80 & 3.08 \\
\hline 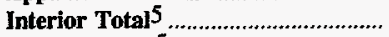 & 3.76 & 3.67 & 3.75 & - & 3.76 \\
\hline Western Total 5 & 4.12 & 2.60 & 6.92 & - & 6.35 \\
\hline East of Miss. River................................ & $\mathbf{3 . 1 2}$ & 2.69 & 3.45 & 1.80 & 3.19 \\
\hline West of Miss. River................................ & 4.01 & 2.60 & 6.92 & - & 6.32 \\
\hline 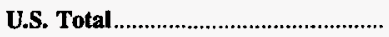 & 3.14 & 2.69 & 3.85 & 1.80 & 3.39 \\
\hline
\end{tabular}

1 Mines that produce greater than 50 percent of coal by continuous mining method.

2 Mines that produce greater than 50 percent of coal by conventional mining method.

3 Mines that have any production from longwall mining method. A typical longwall mining operation uses 80 percent longwall mining and 20 percent continuous mining.

4 Mines that produce coal using shortwall, scoop loading, hand loading, or other mining methods or a $50 / 50$ percent continuous/conventional split in mining method.

5 For a definition of coal-producing regions, see Appendix C.

Notes: For each State, stand alone preparation plant hours are distributed across the mining methods by the proportion of production for all stand alone mines. Productivity is calculated by dividing total coal production by the total direct labor hours worked by all ernployees engaged in production, preparation, processing, development, maintenance, repair, shop or yard work at mining operations, but excludes office workers. Excludes mines producing less than 10,000 short tons of coal during the year, and preparation plants with less than 5,000 employee hours, which are not required to provide these data.

Sources: Energy Information Administration, Form ElA-7A, “Coal Production Report”; State Mining Agency Coal Production Reports; and/or U.S. Department of Labor, Mine Safety and Health Administration, Form 7000-2, "Quarterly Mine Employment and Coal Production Report." 
Table 55. U.S. Coal Mining Productivity by Coalbed Thickness and Mining Method, 1995

(Short Tons of Coal Produced per Miner per Hour)

\begin{tabular}{|c|c|c|c|c|c|c|}
\hline \multirow{2}{*}{$\begin{array}{c}\text { Coalbed Thickness } \\
\text { (inches) }\end{array}$} & \multicolumn{4}{|c|}{ Underground } & \multirow{2}{*}{ Surface } & \multirow{2}{*}{ Total } \\
\hline & Continuous 1 & Conventional 2 & Longwall 3 & Other ${ }^{4}$ & & \\
\hline$<7$ & - & - & - & - & 3.00 & 3.00 \\
\hline 7-12 & - & - & - & - & 3.89 & 3.89 \\
\hline 13-18 & - & - & - & - & 4.66 & 4.66 \\
\hline $19-24$ & 3.02 & 1.88 & - & 1.71 & 4.31 & 4.25 \\
\hline $25-30$ & 2.39 & 2.68 & - & 2.94 & 4.19 & 3.94 \\
\hline 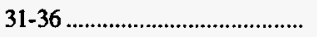 & 2.70 & 3.12 & - & 1.89 & 4.93 & 4.09 \\
\hline $37-42 \ldots \ldots \ldots \ldots \ldots \ldots \ldots$ & 3.09 & 2.23 & - & 1.47 & 4.96 & 4.05 \\
\hline 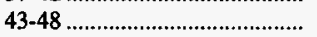 & 3.73 & 2.94 & 3.12 & - & 5.04 & 4.17 \\
\hline 49-54 & 3.31 & 2.97 & 2.39 & - & 5.86 & 4.26 \\
\hline $55-60 \ldots \ldots \ldots \ldots \ldots$ & 4.00 & 3.76 & 3.14 & 2.50 & 5.70 & 4.49 \\
\hline $61-66$ & 3.31 & 3.37 & 4.48 & - & 6.15 & 4.65 \\
\hline 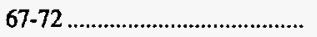 & 4.20 & 3.14 & 4.00 & - & 5.64 & 4.75 \\
\hline 73-78 & 4.53 & - & 3.45 & - & 6.77 & 4.88 \\
\hline 79-84 & 4.73 & 3.42 & 3.92 & - & 6.83 & 4.90 \\
\hline $85-90$ & 6.83 & - & 3.64 & - & 6.70 & 6.07 \\
\hline 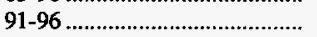 & 4.08 & - & 4.06 & .95 & 5.33 & 4.43 \\
\hline 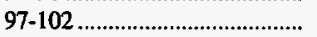 & 5.02 & - & 4.92 & - & 5.57 & 5.39 \\
\hline 103-108 & 4.02 & 2.94 & 7.36 & - & 5.62 & 5.58 \\
\hline $109-114$ & 3.98 & - & 9.43 & - & 4.38 & 5.63 \\
\hline $115-120 \ldots \ldots \ldots \ldots$ & 5.94 & 2.01 & 6.55 & - & 5.70 & 6.11 \\
\hline$>\quad 120$ & 4.64 & 4.61 & 8.25 & - & 17.78 & 16.49 \\
\hline U.S. Total 5 & $\mathbf{3 . 1 4}$ & 2.69 & $\mathbf{3 . 8 5}$ & 1.80 & 8.48 & $\mathbf{5 . 3 8}$ \\
\hline
\end{tabular}

1 Mines that produce greater than 50 percent of coal by continuous mining method.

2 Mines that produce greater than 50 percent of coal by conventional mining method.

3 Mines that have any production from longwall mining method. A typical longwall mining operation uses 80 percent longwall mining and 20 percent continuous mining.

4 Mines that produce coal using shortwall, scoop loading, hand loading, or other mining methods or a $50 / 50$ percent continuous/conventional split in mining method.

5 Includes stand alone preparation plants.

Notes: Productivity is calculated by dividing total coal production by the total direct labor hours worked by all employees engaged in production, preparation, processing, development, maintenance, repair, shop or yard work at mining operations, but excludes office workers. Excludes silt, culm, refuse bank, slurry dam, and dredge operations except for Pennsylvania anthracite. Excludes mines producing less than 10,000 short tons and preparation plants with less than 5,000 employee hours, which are not required to provide these data.

Sources: Energy Information Administration, Form EIA-7A, "Coal Production Report"; State Mining Agency Coal Production Reports; and/or U.S. Department of Labor, Mine Safety and Health Administration, Form 7000-2, "Quarterly Mine Employment and Coal Production Report." 
Table 56. Coal Mining Productivity by State, Mine Type, and Mine Production Range, 1995

(Short Tons of Coal Produced per Miner per Hour)

\begin{tabular}{|c|c|c|c|c|c|c|c|}
\hline \multirow{2}{*}{$\begin{array}{l}\text { Coal-Producing } \\
\text { State and Region }\end{array}$} & \multicolumn{6}{|c|}{$\begin{array}{l}\text { Mine Production Range } \\
\text { (thousand short tons) }\end{array}$} & \multirow{2}{*}{ Total 1} \\
\hline & $\begin{array}{c}1,000 \\
\text { and over }\end{array}$ & $\begin{array}{l}500 \\
\text { to } 1,000\end{array}$ & $\begin{array}{c}200 \\
\text { to } 500\end{array}$ & $\begin{array}{l}100 \\
\text { to } 200\end{array}$ & $\begin{array}{c}50 \\
\text { to } 100\end{array}$ & $\begin{array}{c}10 \\
\text { to } 50\end{array}$ & \\
\hline 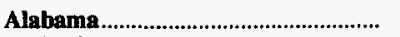 & 2.16 & 2.73 & 3.46 & 2.67 & 3.03 & 2.65 & 2.24 \\
\hline 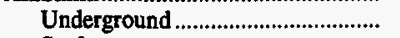 & 2.07 & 2.13 & - & - & - & - & 2.02 \\
\hline 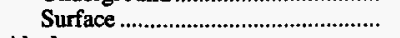 & 4.43 & 3.37 & 3.46 & 2.67 & 3.03 & 2.65 & 3.07 \\
\hline 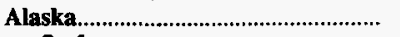 & 7.46 & - & - & - & - & - & 7.46 \\
\hline Surface & 7.46 & - & - & - & - & - & 7.46 \\
\hline 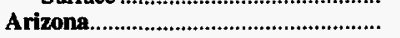 & 6.56 & - & - & - & - & - & 6.34 \\
\hline 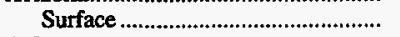 & 6.56 & - & - & - & - & - & 6.34 \\
\hline 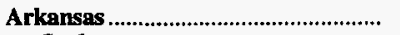 & - & - & - & - & - & 1.47 & 1.47 \\
\hline Surface & - & - & - & - & - & 1.47 & 1.47 \\
\hline 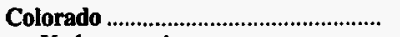 & 6.81 & 4.06 & 4.17 & - & - & 1.05 & 6.14 \\
\hline 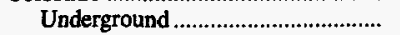 & 6.82 & 4.06 & 3.67 & - & - & - & 5.86 \\
\hline Surface & 6.81 & - & 8.42 & - & - & 1.05 & 6.79 \\
\hline 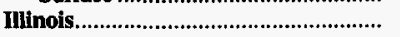 & 4.06 & 3.71 & 3.35 & 18.16 & .90 & 6.53 & 3.87 \\
\hline 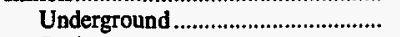 & 4.02 & 3.28 & - & - & - & - & 3.86 \\
\hline 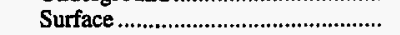 & 4.37 & 5.47 & 3.35 & 18.16 & .90 & 6.53 & 3.89 \\
\hline Indiana & 4.76 & 4.71 & 4.81 & 4.52 & $\mathbf{4 . 5 7}$ & 4.89 & 4.68 \\
\hline 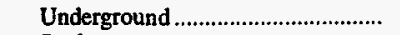 & 3.42 & - & 2.56 & - & - & - & 3.22 \\
\hline Surface & 5.25 & 4.71 & 5.36 & 4.52 & 4.57 & 4.89 & 5.04 \\
\hline 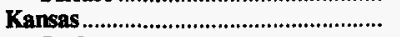 & - & - & 2.22 & - & - & - & 2.22 \\
\hline 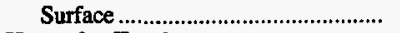 & - & - & 2.22 & - & - & - & 2.22 \\
\hline 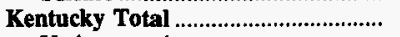 & 4.52 & 4.50 & 3.93 & 3.07 & 2.67 & 2.40 & 3.57 \\
\hline 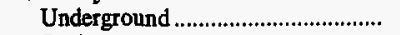 & 4.21 & 4.10 & 3.60 & 2.86 & 2.54 & 2.11 & 3.25 \\
\hline 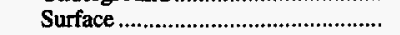 & 5.55 & 5.07 & 4.39 & 3.53 & 3.14 & 2.93 & 4.23 \\
\hline 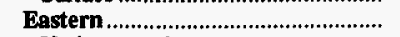 & 4.84 & 4.50 & 3.86 & 3.02 & 2.65 & 2.34 & 3.47 \\
\hline 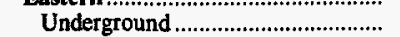 & 4.44 & 4.14 & 3.56 & 2.86 & 2.52 & 2.11 & 3.12 \\
\hline Surface & 5.52 & 5.06 & 4.38 & 3.38 & 3.12 & 2.80 & 4.13 \\
\hline Western & 4.11 & 4,48 & 4.49 & 5.09 & 3.99 & 4.39 & 3.97 \\
\hline 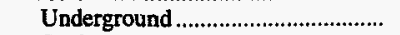 & 4.02 & 3.98 & 4.59 & - & 4.18 & - & 3.70 \\
\hline 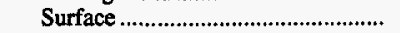 & 5.79 & 5.13 & 4.46 & 5.09 & 3.65 & 4.39 & 4.77 \\
\hline 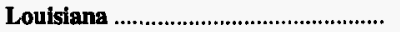 & 13.38 & 12.81 & - & - & - & - & 13.25 \\
\hline 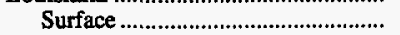 & 13.38 & 12.81 & - & - & - & - & 13.25 \\
\hline 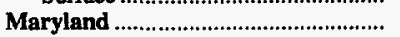 & 5.37 & - & 3.27 & 2.19 & 2.05 & 2.28 & 3.82 \\
\hline 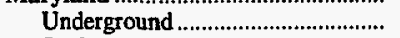 & 5.37 & - & 5.90 & - & - & - & 4.77 \\
\hline 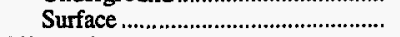 & - & - & 2.15 & 2.19 & 2.05 & 2.28 & 2.16 \\
\hline 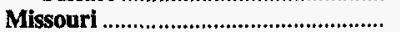 & - & - & - & 2.10 & 3.59 & 6.14 & 2.55 \\
\hline 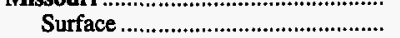 & - & - & - & 2.10 & 3.59 & 6.14 & 2.55 \\
\hline Montana & 21.12 & - & 14.99 & - & - & - & 21.06 \\
\hline 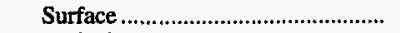 & 21.12 & - & 14.99 & - & - & - & 21.06 \\
\hline 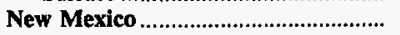 & 7.24 & 2.86 & - & - & - & - & 6.92 \\
\hline 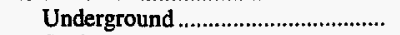 & - & 2.86 & - & - & - & - & 2.68 \\
\hline Surface & 7.24 & - & - & - & - & - & 7.19 \\
\hline 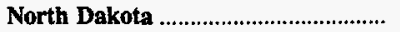 & 16.86 & - & - & - & - & - & 16.80 \\
\hline 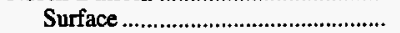 & 16.86 & - & - & - & - & - & 16.80 \\
\hline Ohio & 3.71 & 4.50 & 3.61 & 4.30 & 3.26 & 2.87 & 3.62 \\
\hline Underground & 3.83 & 4.80 & 3.88 & - & 2.22 & - & 3.81 \\
\hline 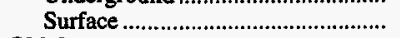 & 2.74 & 4.44 & 3.56 & 4.30 & 3.36 & 2.87 & 3.46 \\
\hline 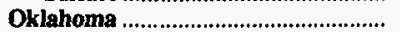 & - & - & 3.21 & 3.92 & - & 1.24 & 2.97 \\
\hline 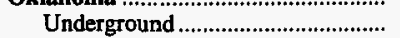 & - & - & - & - & - & .74 & .74 \\
\hline 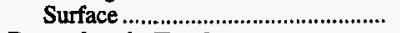 & - & - & 3.21 & 3.92 & - & 1.50 & 3.10 \\
\hline Pennsylvania Total ................................... & 4.76 & 2.15 & 3.13 & 3.44 & 2.92 & 2.60 & 3.23 \\
\hline 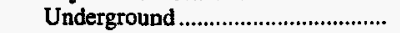 & 4.74 & 1.97 & 2.72 & 2.54 & 2.11 & 1.64 & 3.49 \\
\hline 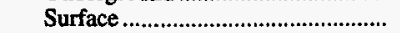 & 5.24 & 2.74 & 3.54 & 3.73 & 3.09 & 2.78 & 2.79 \\
\hline Anthracite & - & - & 4.47 & 4.49 & 3.97 & 1.74 & 2.08 \\
\hline Underground & - & - & - & 1.92 & - & 1.31 & .86 \\
\hline Surface & - & - & 4.47 & 6.06 & 3.97 & 1.86 & 2.30 \\
\hline 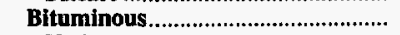 & 4.76 & 2.15 & 2.92 & 3.25 & 2.74 & 2.95 & 3.37 \\
\hline 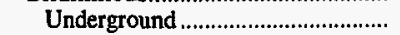 & 4.74 & 1.97 & 2.72 & 2.72 & 2.11 & 1.85 & 3.56 \\
\hline Surface & 5.24 & 2.74 & 3.19 & 3.40 & 2.90 & 3.13 & 2.95 \\
\hline 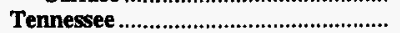 & - & 3.99 & 3.46 & 1.86 & 3.24 & 1.74 & 2.36 \\
\hline 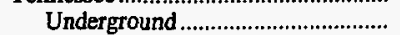 & - & - & 3.27 & 1.75 & 2.75 & 1.78 & 2.02 \\
\hline 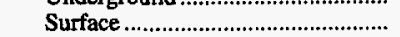 & - & 3.99 & 4.51 & 2.48 & 3.48 & 1.68 & 3.20 \\
\hline 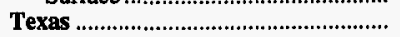 & 9.25 & - & 3.54 & - & 9.01 & - & 9.10 \\
\hline 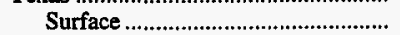 & 9.25 & - & 3.54 & - & 9.01 & - & 9.10 \\
\hline Utah & $\mathbf{7 . 5 3}$ & 6.68 & 3.12 & 2.01 & - & - & 7.02 \\
\hline 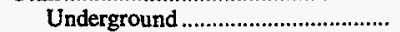 & 7.53 & 6.68 & 3.12 & 2.01 & - & - & 7.02 \\
\hline Virginia & 2.85 & 3.88 & $\mathbf{3 . 3 3}$ & 2.54 & 2.03 & 2.07 & 2.50 \\
\hline Underground & 2.85 & 3.00 & 2.91 & 2.41 & 1.98 & 1.92 & 2.25 \\
\hline Surface & - & 4.97 & 4.32 & 3.24 & 2.84 & 2.74 & 3.73 \\
\hline
\end{tabular}

See footnotes at end of table. 
Table 56. Coal Mining Productivity by State, Mine Type, and Mine Production Range, 1995 (Continued)

(Short Tons of Coal Produced per Miner per Hour)

\begin{tabular}{|c|c|c|c|c|c|c|c|}
\hline \multirow{2}{*}{$\begin{array}{l}\text { Coal-Producing } \\
\text { State and Region }\end{array}$} & \multicolumn{6}{|c|}{$\begin{array}{l}\text { Mine Production Range } \\
\text { (thousand short tons) }\end{array}$} & \multirow{2}{*}{ Total 1} \\
\hline & $\begin{array}{c}1,000 \\
\text { and over }\end{array}$ & $\begin{array}{c}500 \\
\text { to } 1,000\end{array}$ & $\begin{array}{c}200 \\
\text { to } 500\end{array}$ & $\begin{array}{l}100 \\
\text { to } 200\end{array}$ & $\begin{array}{c}50 \\
\text { to } 100\end{array}$ & $\begin{array}{c}10 \\
\text { to } 50\end{array}$ & \\
\hline 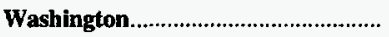 & 4.20 & - & 2.36 & - & - & - & 4.04 \\
\hline 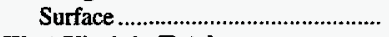 & 4.20 & - & 2.36 & - & - & - & 4.04 \\
\hline West Virginia Total & 4.52 & 4.68 & 3.97 & $\mathbf{3 . 2 5}$ & 2.73 & 2.46 & 3.74 \\
\hline 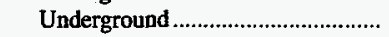 & 4.01 & 4.29 & 3.77 & 3.03 & 2.58 & 2.21 & 3.40 \\
\hline Surface & 5.88 & 5.59 & 4.93 & 3.96 & 3.39 & 2.94 & 4.74 \\
\hline Northern & 4.19 & 3.81 & 3.85 & 3.37 & 2.45 & 2.57 & 3.72 \\
\hline 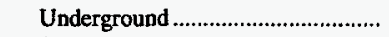 & 4.05 & 3.38 & 3.86 & 3.16 & 2.30 & 1.90 & 3.66 \\
\hline 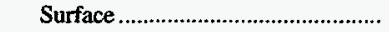 & 7.19 & 10.30 & 3.78 & 3.68 & 3.81 & 3.06 & 4.31 \\
\hline Southern & 4.74 & 4.95 & 3.99 & 3.22 & 2.80 & 2.43 & 3.75 \\
\hline Underground & 3.98 & 4.71 & 3.75 & 3.01 & 2.67 & 2.27 & 3.27 \\
\hline Surface & 5.79 & 5.35 & 5.12 & 4.09 & 3.33 & 2.85 & 4.79 \\
\hline Wyoming & 31.07 & 6.53 & 8.43 & - & - & 1.10 & 30.06 \\
\hline Underground & 6.34 & - & - & - & - & .81 & 5.97 \\
\hline 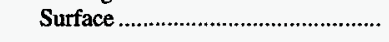 & 32.03 & 6.53 & 8.43 & - & - & 1.32 & 31.02 \\
\hline Appalachian Total ${ }^{2} \ldots \ldots \ldots \ldots \ldots \ldots$ & 4.06 & 4.06 & 3.68 & 3.03 & 2.60 & 2.45 & 3.32 \\
\hline Underground & 3.72 & 3.63 & 3.44 & 2.72 & 2.35 & 2.06 & 3.08 \\
\hline Surface & 5.59 & 4.72 & 4.12 & 3.58 & 3.12 & 2.77 & 3.88 \\
\hline Interior Total $2 \ldots \ldots \ldots \ldots \ldots \ldots \ldots \ldots \ldots \ldots \ldots \ldots \ldots \ldots \ldots$ & 5.42 & 4.58 & 4.12 & 4.03 & 2.64 & 2.68 & 4.97 \\
\hline 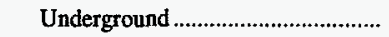 & 3.99 & 3.83 & 3.62 & - & 4.18 & .74 & 3.76 \\
\hline Surface & 7.65 & 5.20 & 4.20 & 4.03 & 2.36 & 3.13 & 6.39 \\
\hline Western Total ${ }^{2}$ & 16.67 & 4.64 & 4.30 & 2.01 & - & 1.09 & 15.68 \\
\hline 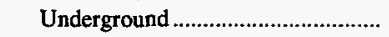 & 7.20 & 3.96 & 3.46 & 2.01 & - & .81 & 6.35 \\
\hline Surface & 19.36 & 6.53 & 6.39 & - & - & 1.22 & 18.93 \\
\hline East of Miss. River & 4.10 & 4.12 & 3.74 & 3.09 & 2.60 & 2.48 & $\mathbf{3 . 4 5}$ \\
\hline Underground & 3.79 & 3.66 & 3.44 & 2.72 & 2.36 & 2.06 & 3.19 \\
\hline 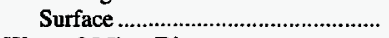 & 5.41 & 4.77 & 4.21 & 3.69 & 3.06 & 2.82 & 4.03 \\
\hline West of Miss. River............................... & 15.30 & 5.22 & 3.74 & 2.55 & 4.23 & 1.40 & $\mathbf{1 4 . 1 8}$ \\
\hline Underground & 7.20 & 3.96 & 3.46 & 2.01 & - & .76 & 6.32 \\
\hline Surface & 17.08 & 7.92 & 3.92 & 2.66 & 4.23 & 1.68 & 16.23 \\
\hline 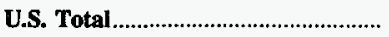 & 7.65 & 4.16 & 3.74 & 3.07 & 2.61 & 2.44 & $\mathbf{5 . 3 8}$ \\
\hline 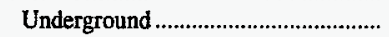 & 4.11 & 3.67 & 3.45 & 2.72 & 2.36 & 2.03 & 3.39 \\
\hline Surface & 13.21 & 4.86 & 4.18 & 3.64 & 3.08 & 2.77 & 8.48 \\
\hline
\end{tabular}

1 Includes stand alone preparation plants.

2 For a definition of coal-producing regions, see Appendix C.

Notes: Productivity is calculated by dividing total coal production by the total direct labor hours worked by all employees engaged in production, preparation, processing, development, maintenance, repair, shop or yard work at mining operations, but excludes office workers. Excludes silt, culm, refuse bank, slurry dam, and dredge operations except for Pennsylvania anthracite. Excludes mines producing less than 10,000 short tons and preparation plants with less than 5,000 employee hours, which are not required to provide these data.

Sources: Energy Information Administration, Form ELA-7A, "Coal Production Report"; State Mining Agency Coal Production Reports; and/or U.S. Department of Labor, Mine Safety and Health Administration, Form 7000-2, "Quarterly Mine Employment and Coal Production Report." 
Table 57. Coal Mining Productivity by State, Mine Type, and Union Type,1995 (Short Tons of Coal Produced per Miner per Hour)

\begin{tabular}{|c|c|c|c|c|c|}
\hline $\begin{array}{l}\text { Coal-Producing } \\
\text { State and Region }\end{array}$ & UMWA & Other Unions & Union Total & Nonunion & Total \\
\hline 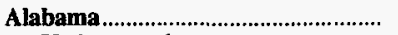 & 2.14 & - & 2.14 & 2.54 & 2.24 \\
\hline 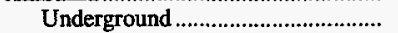 & 2.01 & - & 2.01 & 2.09 & 2.02 \\
\hline Surface & 2.92 & - & 2.92 & 3.24 & 3.07 \\
\hline 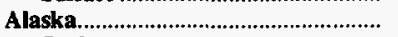 & - & 7.46 & 7.46 & - & 7.46 \\
\hline 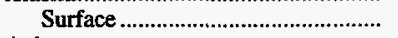 & - & 7.46 & 7.46 & - & 7.46 \\
\hline 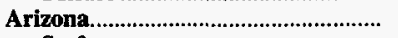 & 6.34 & - & 6.34 & - & 6.34 \\
\hline Surface & 6.34 & - & 6.34 & - & 6.34 \\
\hline 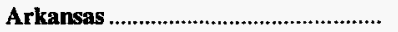 & - & - & - & 1.47 & 1.47 \\
\hline Surface & - & - & - & 1.47 & 1.47 \\
\hline Colorado & 3.84 & 7.11 & 4.38 & 7.38 & 6.14 \\
\hline 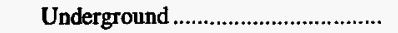 & 2.91 & - & 2.91 & 7.83 & 5.86 \\
\hline 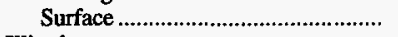 & 7.83 & 7.11 & 7.46 & 6.25 & 6.79 \\
\hline 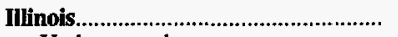 & 3.73 & 4.20 & 3.76 & 4.34 & 3.87 \\
\hline 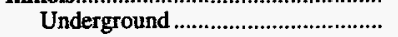 & 3.73 & 4.21 & 3.76 & 4.25 & 3.86 \\
\hline 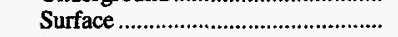 & 3.74 & 4.16 & 3.76 & 5.88 & 3.89 \\
\hline Indiana & 4.62 & - & 4.62 & 4.74 & 4.68 \\
\hline 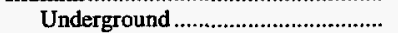 & 3.13 & - & 3.13 & 3.33 & 3.22 \\
\hline 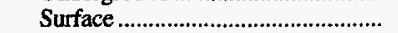 & 5.02 & - & 5.02 & 5.05 & 5.04 \\
\hline 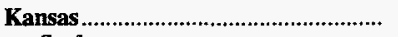 & 2.22 & - & 2.22 & - & 2.22 \\
\hline Surface & 2.22 & - & 2.22 & - & 2.22 \\
\hline Kentucky Total & 3.94 & 4.89 & 3.98 & 3.50 & 3.57 \\
\hline 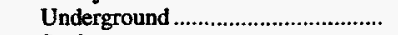 & 3.58 & 2.55 & 3.56 & 3.19 & 3.25 \\
\hline Surface & 5.29 & 6.98 & 5.47 & 4.10 & 4.23 \\
\hline Eastern & 4.27 & 4.89 & 4.32 & $\mathbf{3 . 3 7}$ & 3.47 \\
\hline 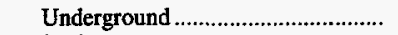 & 3.84 & 2.55 & 3.77 & 3.04 & 3.12 \\
\hline 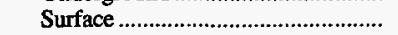 & 5.43 & 6.98 & 5.66 & 3.99 & 4.13 \\
\hline Western & 3.56 & - & 3.56 & 4.16 & 3.97 \\
\hline 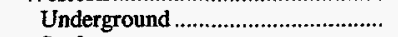 & 3.34 & - & 3.34 & 3.90 & 3.70 \\
\hline 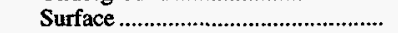 & 4.98 & - & 4.98 & 4.73 & 4.77 \\
\hline 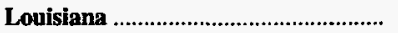 & - & - & - & 13.25 & 13.25 \\
\hline 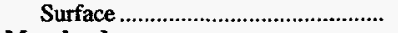 & - & - & - & 13.25 & 13.25 \\
\hline 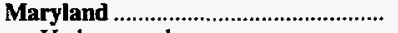 & - & - & - & 3.82 & 3.82 \\
\hline 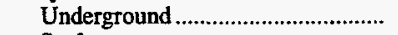 & - & - & - & 4.77 & 4.77 \\
\hline Surface & - & - & - & 2.16 & 2.16 \\
\hline 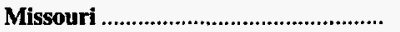 & - & - & - & 2.55 & 2.55 \\
\hline 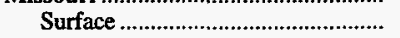 & - & - & - & 2.55 & 2.55 \\
\hline Montana & 17.28 & 20.61 & 18.82 & 37.05 & 21.06 \\
\hline 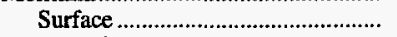 & 17.28 & 20.61 & 18.82 & 37.05 & 21.06 \\
\hline 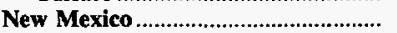 & 6.06 & 7.14 & 6.70 & 8.50 & 6.92 \\
\hline Underground & 2.68 & - & 2.68 & - & 2.68 \\
\hline Surface & 6.76 & 7.14 & 7.00 & 8.50 & 7.19 \\
\hline 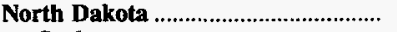 & 14.80 & 14.26 & 14.52 & 17.80 & 16.80 \\
\hline 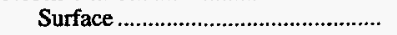 & 14.80 & 14.26 & 14.52 & 17.80 & 16.80 \\
\hline Ohio & 3.67 & 2.51 & 3.66 & 3.59 & 3.62 \\
\hline 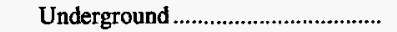 & 3.83 & - & 3.83 & 3.66 & 3.81 \\
\hline 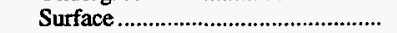 & 2.92 & 2.51 & 2.91 & 3.57 & 3.46 \\
\hline Oklahoma & - & - & - & 2.97 & 2.97 \\
\hline 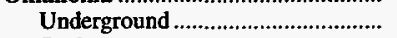 & - & - & - & .74 & .74 \\
\hline Surface & - & - & - & 3.10 & 3.10 \\
\hline 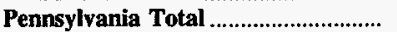 & 2.57 & 1.73 & 2.56 & 3.78 & 3.23 \\
\hline Underground & 2.63 & - & 2.63 & 4.95 & 3.49 \\
\hline Surface & 2.11 & 2.80 & 2.12 & 2.91 & 2.79 \\
\hline 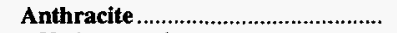 & 1.60 & 3.08 & 1.64 & 2.35 & 2.08 \\
\hline 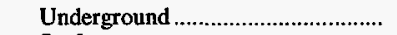 & - & - & - & .88 & .86 \\
\hline 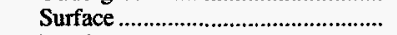 & 1.60 & 4.33 & 1.65 & 2.79 & 2.30 \\
\hline 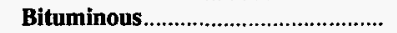 & 2.66 & - & 2.66 & 3.99 & 3.37 \\
\hline 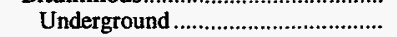 & 2.63 & - & 2.63 & 5.26 & 3.56 \\
\hline Surface & 3.39 & - & 3.30 & 2.93 & 2.95 \\
\hline 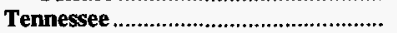 & - & - & - & 2.36 & 2.36 \\
\hline 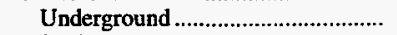 & - & - & - & 2.02 & 2.02 \\
\hline 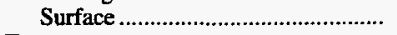 & - & - & - & 3.20 & 3.20 \\
\hline Texas & - & 8.48 & 8.48 & 10.26 & 9.10 \\
\hline Surface & - & 8.48 & 8.48 & 10.26 & 9.10 \\
\hline 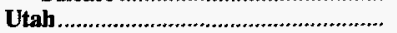 & 6.65 & - & 6.65 & 7.23 & 7.02 \\
\hline 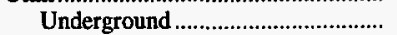 & 6.65 & - & 6.65 & 7.23 & 7.02 \\
\hline 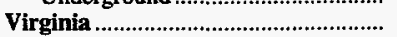 & 2.40 & 3.55 & 2.44 & 2.53 & 2.50 \\
\hline Underground & 2.39 & - & 2.39 & 2.19 & 2.25 \\
\hline 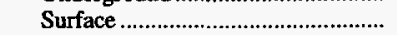 & 2.58 & 3.55 & 3.08 & 3.81 & 3.73 \\
\hline 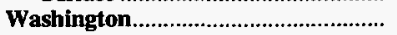 & - & 4.20 & 4.20 & 2.36 & 4.04 \\
\hline 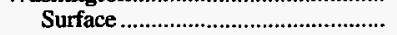 & - & 4.20 & 4.20 & 2.36 & 4.04 \\
\hline West Virginia Total ............................ & 3.53 & - & 3.52 & 4.10 & 3.74 \\
\hline Underground ..................................... & 3.19 & - & 3.19 & 3.80 & 3.40 \\
\hline Surface & 4.80 & - & 4.76 & 4.71 & 4.74 \\
\hline
\end{tabular}

See footnotes at end of table. 
Table 57. Coal Mining Productivity by State, Mine Type, and Union Type,1995 (Continued) (Short Tons of Coal Produced per Miner per Hour)

\begin{tabular}{|c|c|c|c|c|c|}
\hline $\begin{array}{l}\text { Coal-Producing } \\
\text { State and Region }\end{array}$ & UMWA & Other Unions & Union Total & Nonunion & Total \\
\hline Northern & 3.76 & - & 3.76 & 3.63 & 3.72 \\
\hline 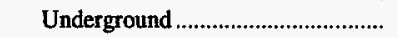 & 3.78 & - & 3.78 & 3.23 & 3.66 \\
\hline Surface & - & - & - & 4.45 & 4.31 \\
\hline Southern & 3.42 & - & 3.41 & 4.24 & 3.75 \\
\hline 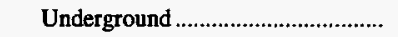 & 2.79 & - & 2.79 & 3.96 & 3.27 \\
\hline Surface & 4.83 & - & 4.79 & 4.79 & 4.79 \\
\hline Wyoming & 5.66 & 9.84 & 8.04 & 35.88 & 30.06 \\
\hline 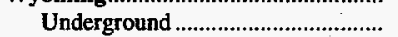 & - & - & - & 5.97 & 5.97 \\
\hline Surface & 5.66 & 9.84 & 8.04 & 37.40 & 31.02 \\
\hline 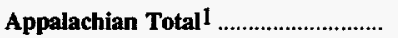 & 3.14 & 3.60 & 3.14 & 3.45 & 3.32 \\
\hline Underground & 2.92 & 2.05 & 2.92 & 3.23 & 3.08 \\
\hline Surface & 4.15 & 4.24 & 4.16 & 3.79 & 3.88 \\
\hline Interior Total 1 & 3.85 & $\mathbf{7 . 8 3}$ & 4.76 & 5.25 & 4.97 \\
\hline Underground & 3.61 & 4.21 & 3.64 & 3.95 & 3.76 \\
\hline 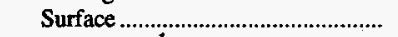 & 4.45 & 8.39 & 6.32 & 6.47 & 6.39 \\
\hline Western Total ${ }^{1}$ & $\mathbf{7 . 3 2}$ & 9.22 & 8.11 & 23.23 & 15.68 \\
\hline 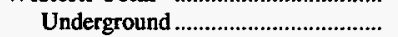 & 4.69 & - & 4.69 & 7.38 & 6.35 \\
\hline Surface & 8.67 & 9.22 & 8.96 & 30.63 & 18.93 \\
\hline 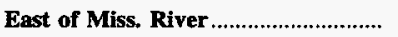 & $\mathbf{3 . 3 0}$ & 3.94 & 3.31 & 3.56 & 3.45 \\
\hline 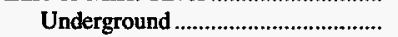 & 3.06 & 3.77 & 3.07 & 3.33 & 3.19 \\
\hline Surface & 4.26 & 4.23 & 4.26 & 3.94 & 4.03 \\
\hline West of Miss. River............................. & 7.23 & 8.93 & 8.15 & 20.49 & 14.18 \\
\hline Underground & 4.69 & - & 4.69 & 7.32 & 6.32 \\
\hline Surface & 8.52 & 8.93 & 8.78 & 25.14 & 16.23 \\
\hline U.S. Total & 3.71 & 8.36 & 4.28 & 6.30 & 5.38 \\
\hline 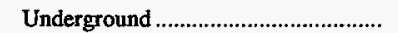 & 3.14 & 3.77 & 3.14 & 3.63 & 3.39 \\
\hline 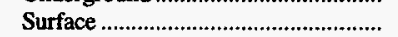 & 5.47 & 8.71 & 6.58 & 9.69 & 8.48 \\
\hline
\end{tabular}

1 For a definition of coal-producing regions, see Appendix C.

Notes: Productivity is calculated by dividing total coal production by the total direct labor hours worked by all employees engaged in production, preparation, processing, development, maintenance, repair, shop or yard work at mining operations, but excludes office workers. Excludes silt, culm, refuse bank, slurry dam, and dredge operations except for Pennsylvania anthracite. Excludes mines producing less than 10,000 short tons and preparation plants with less than 5,000 employee hours, which are not required to provide these data. See Glossary for listing of other unions.

Sources: Energy Information Administration, Form EIA-7A, "Coal Production Report"; State Mining Agency Coal Production Reports; and/or U.S. Department of Labor, Mine Safety and Health Administration, Form 7000-2, "Quarterly Mine Employment and Coal Production Report." 



\section{Distribution}

The amount of U.S. coal distributed during 1995 reached a record 1,030 million short tons, surpassing by 1 percent the previous record of 1,023 million short tons distributed during 1994 (Table 58). Compared with 1994, distribution of U.S. coal to domestic consumers declined 1 percent to 940 million short tons, while foreign distribution increased 24 percent to 90 million short tons (Table 59).

Texas was the leading destination for coal distributed domestically during 1995, with shipments totaling 94 million short tons, representing 9 percent of total distribution (Table 61). Of the coal distributed in Texas during 1995, 56 percent was indigenous, with virtually all of the balance arriving from Wyoming (38 million short tons) and Colorado ( 2 million short tons) (Table 61).

Other major destination States included Indiana, Pennsylvania, and Ohio, which collectively received 170 million short tons, representing 16.5 percent of total distribution. As with distributions of coal in Texas, a substantial portion of the coal distributed in each of these States was indigenous (Table 61).
West Virginia was the leading source of U.S. coal distributed abroad during 1995, with foreign shipments totaling 44 million short tons, representing 49 percent of total foreign distributions. Other leading sources of U.S. coal distributed abroad during 1995 were Virginia and Kentucky, each with foreign distributions totaling 10 million short tons, and Pennsylvania, with foreign distributions totaling 8 million short tons. Collectively, foreign distributions of coal mined in these four States totaled 72 million short tons, accounting for 80 percent of the U.S. coal shipped abroad during 1995 .

Rail continued to be the primary method of transporting coal during 1995, accounting for 591 million short tons, or 57 percent, of coal shipments (Table 65). Coal transported by water (including shipments by river, shipments on the Great Lakes, and shipments through tidewater ports) accounted for 233 million short tons or 23 percent of total coal distributed. Distributions of coal by truck totaled 104 million short tons (10 percent of the total), while distributions by tramway and conveyor totaled 100 million short tons, or 10 percent of the total. 
Figure 9. Coal Distribution, 1986-1995

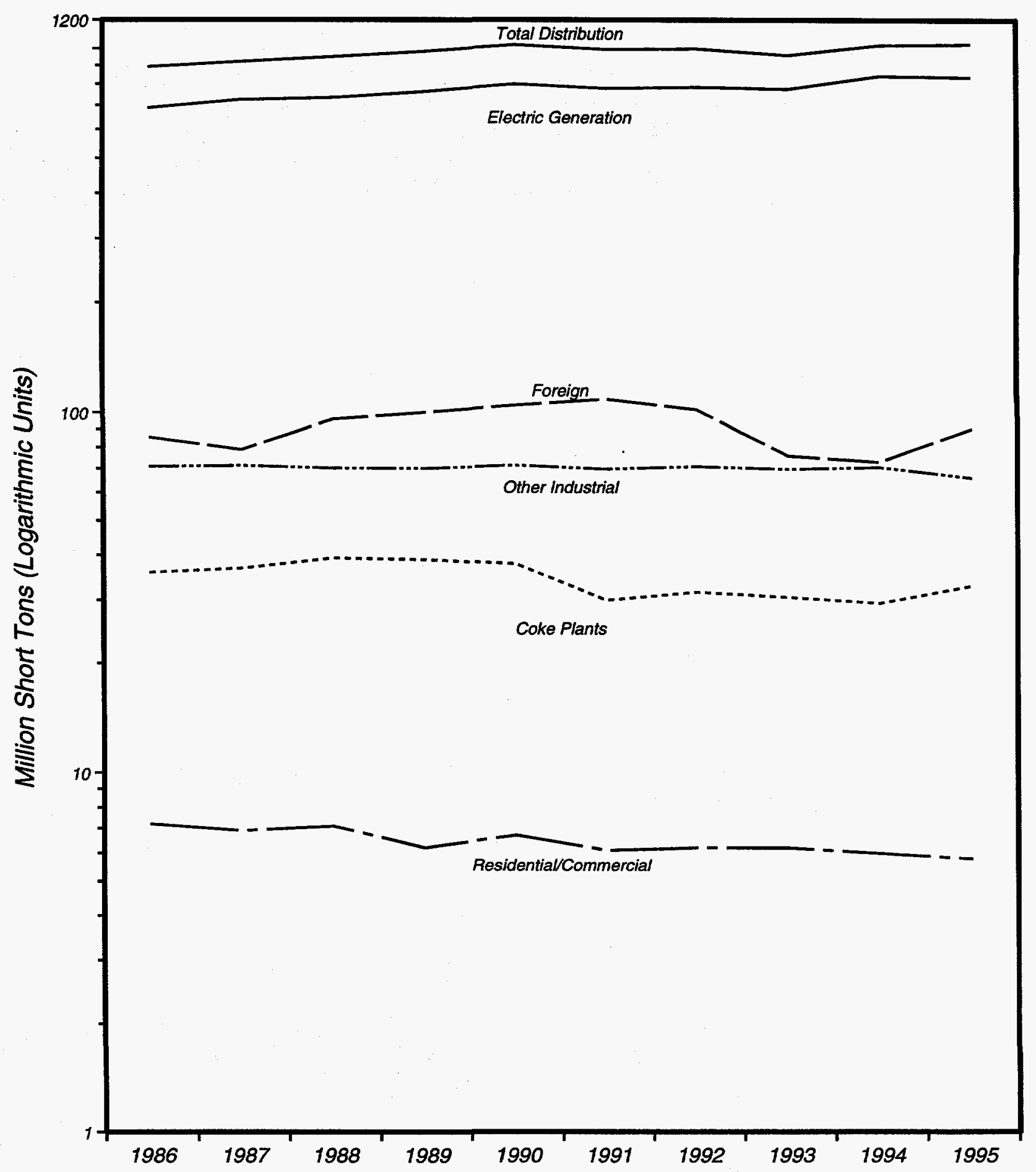

Source: Energy Information Administration, Form EIA-6, "Coal Distribution Report." 
Table 58. Distribution of U.S. Coal by State of Origin, 1991-1995 (Thousand Short Tons)

\begin{tabular}{|c|c|c|c|c|c|c|c|}
\hline \multirow{2}{*}{$\begin{array}{l}\text { Coal-Producing } \\
\text { State and Region }\end{array}$} & \multirow{2}{*}{1995} & \multirow{2}{*}{1994} & \multirow{2}{*}{1993} & \multirow{2}{*}{1992} & \multirow{2}{*}{1991} & \multirow{2}{*}{$\begin{array}{c}\text { Percent } \\
\text { Change } \\
1994-1995\end{array}$} & \multirow{2}{*}{$\begin{array}{c}\text { Average Annual } \\
\text { Percent Change } \\
1991-1995\end{array}$} \\
\hline & & & & & & & \\
\hline Alabama & 25,159 & 23,750 & 25,556 & 25,491 & 27,679 & 5.9 & -2.4 \\
\hline 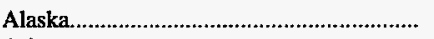 & 1,670 & 1,505 & 1,598 & 1,531 & 1,483 & 10.9 & 3.0 \\
\hline 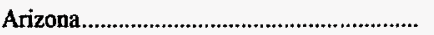 & 11,783 & 12,011 & 12,138 & 12,418 & 12,933 & -1.9 & -2.3 \\
\hline 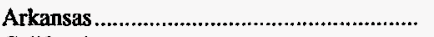 & 11 & 28 & 25 & 32 & 1 & -59.2 & 112.9 \\
\hline 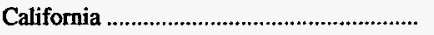 & - & - & - & 142 & 23 & - & - \\
\hline Colorado & 25,635 & 24,810 & 21,465 & 18,864 & 17,757 & 3.3 & 9.6 \\
\hline 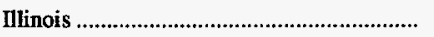 & 47,869 & 51,973 & 42,000 & 58,913 & 58,553 & -7.9 & -4.9 \\
\hline 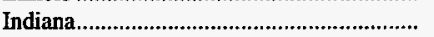 & 25,695 & 30,684 & 29,664 & 31,393 & 31,412 & -16.3 & -4.9 \\
\hline Iowa. & - & 46 & 175 & 287 & 350 & -100.0 & - \\
\hline 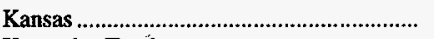 & 291 & 282 & 345 & 354 & 424 & 3.3 & -9.0 \\
\hline 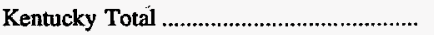 & 151,466 & 159,130 & 160,395 & 161,860 & 158,290 & -4.8 & -1.1 \\
\hline Eastern & 117,831 & 124,257 & 125,041 & 120,186 & 117,462 & -5.2 & .1 \\
\hline Western & 33,635 & 34,873 & 35,354 & 41,674 & 40,828 & -3.5 & -4.7 \\
\hline 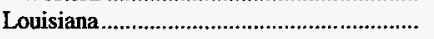 & 3,426 & 3,463 & 3,103 & 3,208 & 3,151 & -1.1 & 2.1 \\
\hline 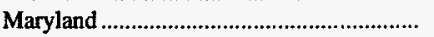 & 3,570 & 3,460 & 3,572 & 3,480 & 3,773 & 3.2 & -1.4 \\
\hline 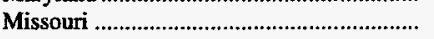 & 464 & 679 & 638 & 2,795 & 2,203 & -31.7 & -32.3 \\
\hline Montana & 39,620 & 41,916 & 35,916 & 38,866 & 38,119 & -5.5 & 1.0 \\
\hline 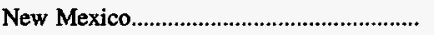 & 26,154 & 28,570 & 27,942 & 24,827 & 22,378 & -8.5 & 4.0 \\
\hline 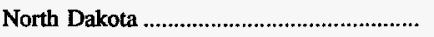 & 30,118 & 32,056 & 32,372 & 31,702 & 29,741 & -6.0 & .3 \\
\hline 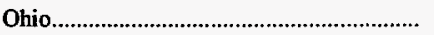 & 24,345 & 28,749 & 28,315 & 29,550 & 30,023 & -15.3 & -5.1 \\
\hline Oklahoma & 2,158 & 1,925 & 2,309 & 1,954 & 1,867 & 12.1 & 3.7 \\
\hline Pennsylvania Total........................................ & 62,240 & 61,508 & 58,990 & 67,649 & 65,454 & 1.2 & -1.3 \\
\hline 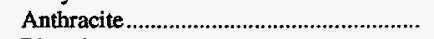 & 3,994 & 4,700 & 3,331 & 3,554 & 3,182 & -15.0 & 5.8 \\
\hline 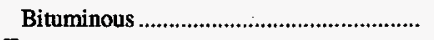 & 58,246 & 56,808 & 55,659 & 64,095 & 62,272 & 2.5 & -1.7 \\
\hline Tennessee & 2,627 & 2,547 & 2,577 & 2,837 & 4,274 & 3.1 & -11.4 \\
\hline 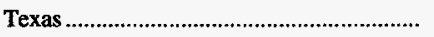 & 52,832 & 52,256 & 54,224 & 54,447 & 54,243 & 1.1 & -.7 \\
\hline Utah & 25,521 & 23,225 & 22,243 & 21,052 & 21,444 & 9.9 & 4.4 \\
\hline Virginia & 34,024 & 38,548 & 41,639 & 45,728 & 43,495 & -11.7 & -5.9 \\
\hline Washington & 4,863 & 4,877 & 4,714 & 5,283 & 5,096 & -.3 & -1.2 \\
\hline 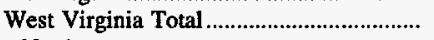 & 165,187 & 158,985 & 135,818 & 163,723 & 165,576 & 3.9 & -.1 \\
\hline Northern & 42,615 & 45,535 & 37,100 & 50,646 & 52,633 & -6.4 & -5.1 \\
\hline Southern & 122,572 & 113,449 & 98,718 & 113,077 & 112,943 & 8.0 & 2.1 \\
\hline 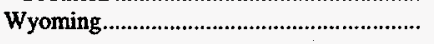 & 263,601 & 235,540 & 211,713 & 190,260 & 194,407 & 11.9 & 7.9 \\
\hline 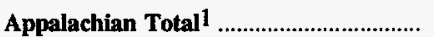 & 434,984 & 441,805 & 421,508 & 458,645 & 457,735 & -1.5 & -1.3 \\
\hline Interior Total 1 & 166,380 & 176,208 & 167,836 & 195,057 & 193,030 & -5.6 & -3.6 \\
\hline 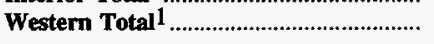 & 428,966 & 404,510 & 370,102 & 344,945 & 343,381 & 6.0 & $\mathbf{5 . 7}$ \\
\hline East of Miss. River & 542,182 & 559,334 & 528,525 & 590,624 & $\mathbf{5 8 8 , 5 2 7}$ & -3.1 & $-\mathbf{2 . 0}$ \\
\hline West of Miss. River ......................................... & 488,148 & 463,190 & 430,920 & 408,023 & 405,619 & 5.4 & 4.7 \\
\hline U.S. Total & $\mathbf{1 , 0 3 0 , 3 3 0}$ & $1,022,523$ & 959,445 & 998,647 & 994,146 & .8 & .9 \\
\hline
\end{tabular}

1 For a definition of coal-producing regions, see Appendix $C$. rounding.

Notes: See Technical Note 1 for the difference between production and distribution. Totals may not equal sum of components due to independent Source: Energy Information Administration, Form EIA-6, “Coal Distribution Report.” 
Table 59. Domestic and Foreign Distribution of U.S. Coal by State of Origin, 1991-1995 (Thousand Short Tons)

\begin{tabular}{|c|c|c|c|c|c|c|c|}
\hline \multirow{2}{*}{$\begin{array}{l}\text { Coal-Producing } \\
\text { State and Region }\end{array}$} & \multirow[t]{2}{*}{1995} & \multirow[t]{2}{*}{1994} & \multirow[t]{2}{*}{1993} & \multirow[t]{2}{*}{1992} & \multirow[t]{2}{*}{1991} & \multirow{2}{*}{$\begin{array}{l}\text { Percent } \\
\text { Change } \\
\text { 1994-1995 }\end{array}$} & \multirow{2}{*}{$\begin{array}{c}\text { Average Annual } \\
\text { Percent Change } \\
1991-1995\end{array}$} \\
\hline & & & & & & & \\
\hline & \multicolumn{7}{|c|}{ Domestic } \\
\hline Alabama & 19,127 & 19,220 & 19,668 & 19,560 & 20,667 & -0.5 & -1.9 \\
\hline 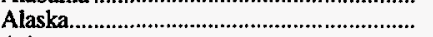 & 815 & 789 & 855 & 797 & 811 & 3.3 & .1 \\
\hline 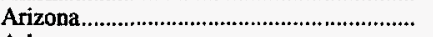 & 11,783 & 12,011 & 12,138 & 12,418 & 12,933 & -1.9 & -2.3 \\
\hline 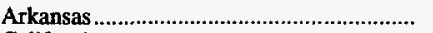 & 11 & 28 & 25 & 29 & 1 & -59.2 & 112.9 \\
\hline 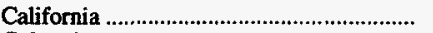 & - & - & - & 142 & 23 & - & - \\
\hline Colorado & 24,734 & 24,059 & 20,338 & 18,195 & 17,132 & 2.8 & 9.6 \\
\hline 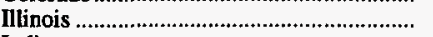 & 45,170 & 51,737 & 41,330 & 57,670 & 57,290 & -12.7 & -5.8 \\
\hline 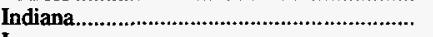 & 25,625 & 30,477 & 29,475 & 31,216 & 31,204 & -15.9 & -4.8 \\
\hline Iowa & - & 46 & 175 & 287 & 350 & -100.0 & - \\
\hline 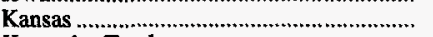 & 291 & 282 & 345 & 354 & 424 & 3.3 & -9.0 \\
\hline 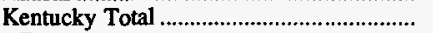 & 141,771 & 151,963 & 150,874 & 147,825 & 142,811 & -6.7 & -.2 \\
\hline Eastern & 108,781 & 117,234 & 115,723 & 106,372 & 102,416 & -7.2 & 1.5 \\
\hline 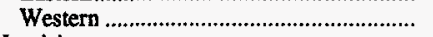 & 32,990 & 34,729 & 35,151 & 41,453 & 40,395 & -5.0 & -4.9 \\
\hline 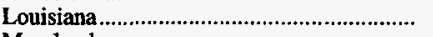 & 3,426 & 3,463 & 3,103 & 3,208 & 3,151 & -1.1 & 2.1 \\
\hline Maryland & 3,382 & 3,277 & 3,278 & 3,246 & 3,594 & 3.2 & -1.5 \\
\hline 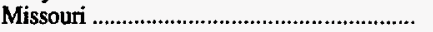 & 464 & 679 & 638 & 2,795 & 2,203 & -31.7 & -32.3 \\
\hline Montana & 39,362 & 41,672 & 35,795 & 38,804 & 37,812 & -5.5 & 1.0 \\
\hline 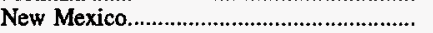 & 25,640 & 28,540 & 27,942 & 24,823 & 22,378 & -10.2 & 3.5 \\
\hline 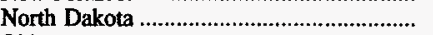 & 30,118 & 32,056 & 32,372 & 31,702 & 29,741 & -6.0 & .3 \\
\hline Ohio & 24,318 & 28,688 & 28,315 & 29,549 & 30,001 & -15.2 & -5.1 \\
\hline 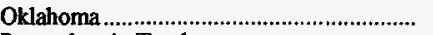 & 2,158 & 1,925 & 2,297 & 1,940 & 1,867 & 12.1 & 3.7 \\
\hline 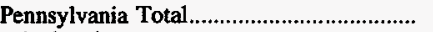 & 53,961 & 55,207 & 53,482 & 61,208 & 58,151 & $-2,3$ & -1.8 \\
\hline Anthracite & 3,497 & 4,346 & 3,015 & 3,230 & 2,821 & -19.5 & 5.5 \\
\hline 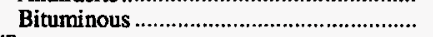 & 50,464 & 50,861 & 50,467 & 57,979 & 55,330 & -.8 & -2.3 \\
\hline 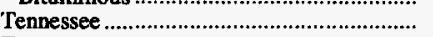 & 2,627 & 2,547 & 2,577 & 2,835 & 4,243 & 3.1 & -11.3 \\
\hline 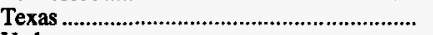 & 52,812 & 52,256 & 54,224 & 54,447 & 54,243 & 1.1 & -.7 \\
\hline 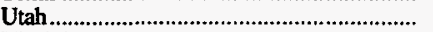 & 21,591 & 20,527 & 19,283 & 18,792 & 19,358 & 5.2 & 2.8 \\
\hline Virginia & 24,283 & 26,866 & 27,388 & 28,504 & 25,390 & -9.6 & -1.1 \\
\hline Washington & 4,756 & 4,731 & 4,621 & 5,020 & 4,894 & .5 & -.7 \\
\hline 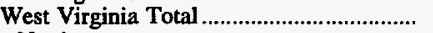 & 120,866 & 122,779 & 102,659 & 112,917 & 111,785 & -1.6 & 2.0 \\
\hline 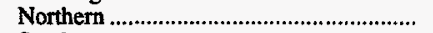 & 36,073 & 39,985 & 34,573 & 44,093 & 45,586 & -9.8 & -5.7 \\
\hline 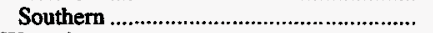 & 84,793 & 82,794 & 68,086 & 68,824 & 66,199 & 2.4 & 6.4 \\
\hline Wyoming & 261,333 & 234,016 & 210,739 & 188,983 & 193,425 & 11.7 & 7.8 \\
\hline Appalachian Total ${ }^{1} \ldots \ldots \ldots \ldots \ldots \ldots \ldots$ & 357,344 & 375,819 & 353,089 & 364,191 & 356,247 & -4.9 & .1 \\
\hline Interior Total $1 . \ldots \ldots$ & 162,947 & 175,622 & 166,763 & 193,400 & 191,126 & -7.2 & -3.9 \\
\hline 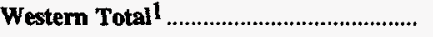 & 420,132 & 398,402 & 364,083 & 339,676 & 338,508 & 5.4 & 5.5 \\
\hline East of Miss. River ..................................... & 461,128 & 492,762 & 459,045 & 494,530 & 485,136 & -6.4 & -1.3 \\
\hline 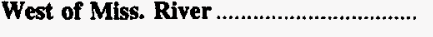 & 479,294 & 457,081 & 424,890 & 402,737 & 400,746 & 4.9 & 4.6 \\
\hline \multirow[t]{2}{*}{ U.S. Total } & 940,423 & 949,843 & 883,934 & 897,267 & 885,882 & -1.0 & 1.5 \\
\hline & \multicolumn{7}{|c|}{ Foreign } \\
\hline Alabama & 6,032 & 4,529 & 5,888 & 5,931 & 7,012 & 33.2 & -3.7 \\
\hline 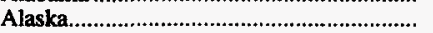 & 855 & 716 & 743 & 734 & 672 & 19.3 & 6.2 \\
\hline 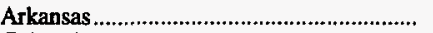 & - & - & - & 3 & - & - & - \\
\hline Colorado & 900 & 752 & 1,128 & 669 & 625 & 19.8 & 9.6 \\
\hline Illinois & 2,699 & 236 & 670 & 1,242 & 1,263 & $\mathbf{N M}$ & 20.9 \\
\hline 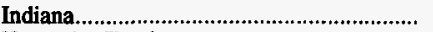 & 70 & 206 & 188 & 177 & 208 & -66.2 & -23.9 \\
\hline 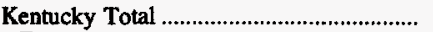 & 9,695 & 7,167 & 9,521 & 14,036 & 15,478 & 35.3 & -11.0 \\
\hline Eastern & 9,051 & 7,023 & 9,318 & 13,815 & 15,046 & 28.9 & -11.9 \\
\hline Western & 645 & 144 & 204 & 221 & 433 & 348.8 & 10.5 \\
\hline 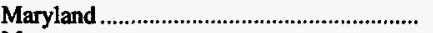 & 188 & 184 & 295 & 234 & 178 & 2.5 & 1.4 \\
\hline 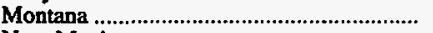 & 259 & 243 & 121 & 62 & 306 & 6.4 & -4.1 \\
\hline 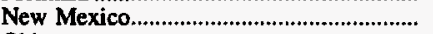 & 514 & 30 & - & 5 & - & $\mathrm{NM}$ & - \\
\hline Ohio & 28 & 61 & - & 2 & 22 & -54.7 & 5.9 \\
\hline 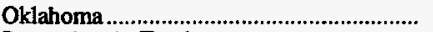 & - & - & 11 & 14 & - & - & - \\
\hline Pennsylvania Total & 8,279 & 6,301 & 5,508 & 6,440 & 7,303 & 31.4 & 3.2 \\
\hline 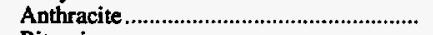 & 497 & 354 & 316 & 324 & 361 & 40.4 & 8.3 \\
\hline 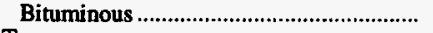 & 7,782 & 5,947 & 5,192 & 6,116 & 6,942 & 30.9 & 2.9 \\
\hline 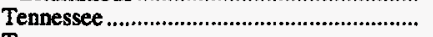 & - & - & - & 2 & 31 & - & - \\
\hline 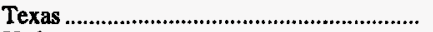 & 20 & - & - & - & - & - & - \\
\hline 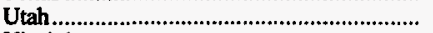 & 3,930 & 2,698 & 2,959 & 2,260 & 2,086 & 45.7 & 17.2 \\
\hline Virginia & 9,742 & 11,683 & 14,251 & 17,224 & 18,105 & -16.6 & -14.3 \\
\hline 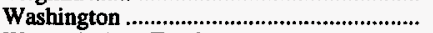 & 107 & 146 & 94 & 264 & 202 & -26.5 & -14.7 \\
\hline 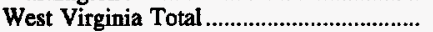 & 44,321 & 36,205 & 33,159 & 50,806 & 53,791 & 22.4 & -4.7 \\
\hline Northern & 6,542 & 5,550 & 2,527 & 6,553 & 7,047 & 17.9 & -1.8 \\
\hline Southern & 37,779 & 30,655 & 30,632 & 44,254 & 46,744 & 23.2 & -5.2 \\
\hline Wyoming & 2,269 & 1,524 & 974 & 1,277 & 982 & 48.9 & 23.3 \\
\hline
\end{tabular}

See footnotes at end of table. 
Table 59. Domestic and Foreign Distribution of U.S. Coal by State of Origin, 1991-1995 (Continued) (Thousand Short Tons)

\begin{tabular}{|c|c|c|c|c|c|c|c|}
\hline \multirow{2}{*}{$\begin{array}{l}\text { Coal-Producing } \\
\text { State and Region }\end{array}$} & \multirow{2}{*}{1995} & \multirow{2}{*}{1994} & \multirow{2}{*}{1993} & \multirow{2}{*}{1992} & \multirow{2}{*}{1991} & \multirow{2}{*}{$\begin{array}{c}\text { Percent } \\
\text { Change } \\
\text { 1994-1995 }\end{array}$} & \multirow{2}{*}{$\begin{array}{c}\begin{array}{c}\text { Average Annual } \\
\text { Percent Change }\end{array} \\
1991-1995\end{array}$} \\
\hline & & & & & & & \\
\hline & \multicolumn{7}{|c|}{ Foreign } \\
\hline 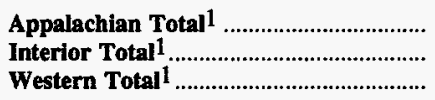 & $\begin{array}{r}77,640 \\
3,433 \\
\mathbf{8 , 8 3 4}\end{array}$ & $\begin{array}{r}65,986 \\
586 \\
6,108\end{array}$ & $\begin{array}{r}68,419 \\
1,073 \\
6,018\end{array}$ & $\begin{array}{r}94,454 \\
1,657 \\
5,269\end{array}$ & $\begin{array}{r}101,488 \\
1,904 \\
4,873\end{array}$ & $\begin{array}{r}17.7 \\
485.9 \\
44.6\end{array}$ & $\begin{array}{r}-6.5 \\
15.9 \\
16.0\end{array}$ \\
\hline 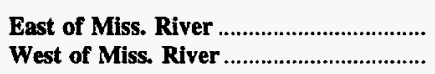 & $\begin{array}{r}81,054 \\
8,854\end{array}$ & $\begin{array}{r}66,572 \\
6,108\end{array}$ & $\begin{array}{r}69,481 \\
6,030\end{array}$ & $\begin{array}{r}96,094 \\
5,286\end{array}$ & $\begin{array}{r}103,392 \\
4,873\end{array}$ & $\begin{array}{l}21.8 \\
44.9\end{array}$ & $\begin{array}{r}-5.9 \\
16.1\end{array}$ \\
\hline 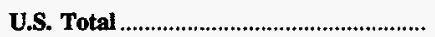 & 89,907 & 72,680 & $\mathbf{7 5 , 5 1 0}$ & 101,380 & 108,264 & 23.7 & -4.5 \\
\hline
\end{tabular}

1 For a definition of coal-producing regions, see Appendix C.

NM Not meaningful as value is greater than 500 percent.

Note: Totals may not equal sum of components due to independent rounding.

Source: Energy Information Administration, Form EIA-6, "Coal Distribution Report."

Table 60. Major U.S. Coal Distributors, 1995

\begin{tabular}{|c|c|}
\hline \multicolumn{2}{|c|}{ Company Name } \\
\hline \multicolumn{2}{|c|}{ Top Ten Distributors } \\
\hline $\begin{array}{l}\text { Atlantic Richfield Co. } \\
\text { A.T. Massey Coal Co., Inc. } \\
\text { Consol Energy Inc. } \\
\text { Cyprus AMAX Minerals Co. } \\
\text { Kennecott Energy Co. }\end{array}$ & $\begin{array}{l}\text { Kerr-McGee Coal Corp. } \\
\text { North American Coal Corp. } \\
\text { Peabody Holding Co. } \\
\text { Texas Utilities Mining Co. } \\
\text { Zeigler Coal Holding Co. }\end{array}$ \\
\hline \multicolumn{2}{|c|}{ Other Major Distributors } \\
\hline $\begin{array}{l}\text { AEP Service Corp. } \\
\text { Aluminum Co. of America } \\
\text { American Coal Sales, Inc. } \\
\text { American Eagle Coal Co. } \\
\text { American Metals \& Coal }\end{array}$ & $\begin{array}{l}\text { Knife River Coal Mining Co. } \\
\text { Mapco Coal Inc. } \\
\text { Marigold Land Corp. } \\
\text { Montana Power Co. } \\
\text { Monterey Coal Co. }\end{array}$ \\
\hline $\begin{array}{l}\text { Andalex Resources Inc. } \\
\text { Anker Energy Corp. } \\
\text { Arch Mineral Corp. } \\
\text { Ashland Coal Inc. } \\
\text { Bethlehem Steel Corp. }\end{array}$ & $\begin{array}{l}\text { Mincorp, Inc. } \\
\text { Minnesota Power \& Light } \\
\text { Pardee Coal Co., Inc. } \\
\text { Pen Holdings } \\
\text { Phibro Energy Inc. }\end{array}$ \\
\hline $\begin{array}{l}\text { BHP Minerals Int 1 } \\
\text { Black Beauty Coal Co. } \\
\text { Blue Diamond Coal Co. } \\
\text { Central Coal Co. } \\
\text { Chevron Corp. }\end{array}$ & $\begin{array}{l}\text { Quaker Coal Co. } \\
\text { Rheinbraun Thyssen Energy } \\
\text { Rochester \& Pittsburgh Co. } \\
\text { San Miguel Electric CoOp. } \\
\text { Sun Coal Co. }\end{array}$ \\
\hline $\begin{array}{l}\text { Coal Arbed Int '1 Trading Co. } \\
\text { Costain America Inc. } \\
\text { Dolet Hills Mining Venture } \\
\text { Drummond Co. } \\
\text { Electric Fuels Corp. }\end{array}$ & $\begin{array}{l}\text { Teco Coal Corp. } \\
\text { The Coastal Corp. } \\
\text { The Pittston Co. } \\
\text { TMPA } \\
\text { Transco Energy Inc. }\end{array}$ \\
\hline $\begin{array}{l}\text { General Dynamics Corp. } \\
\text { Golden Oak Mining Co. } \\
\text { James River Coal Co. } \\
\text { Jim Walter Resources, Inc. } \\
\text { Kiewit Coal Properties }\end{array}$ & $\begin{array}{l}\text { United Coal Co. } \\
\text { Usibelli Coal Mine, Inc. } \\
\text { USX Corp. } \\
\text { Westmoreland Coal Co. } \\
\text { Wyodak Resources Dev 't. }\end{array}$ \\
\hline
\end{tabular}

Notes: The top 10 distribution companies accounted for 51 percent of the total distribution. Companies are listed in alphabetical order to ensure nondisclosure of company data.

Source: Energy Information Administration, Form EIA-6, "Coal Distribution Report." 
Table 61. Domestic Distribution of U.S. Coal by Coal-Producing Region and State, and Destination Census Division and State, 1991-1995 (Thousand Short Tons)

\begin{tabular}{|c|c|c|c|c|c|c|c|}
\hline \multirow{2}{*}{$\begin{array}{c}\text { Coal-Producing Region and State, } \\
\text { and Destination Census Division } \\
\text { and State }\end{array}$} & \multirow[t]{2}{*}{1995} & \multirow[t]{2}{*}{1994} & \multirow[t]{2}{*}{1993} & \multirow[t]{2}{*}{1992} & \multirow[t]{2}{*}{1991} & \multirow{2}{*}{$\begin{array}{c}\text { Percent } \\
\text { Change } \\
\text { 1994-1995 }\end{array}$} & \multirow{2}{*}{$\begin{array}{c}\text { Average Annual } \\
\text { Percent Change } \\
1991-1995\end{array}$} \\
\hline & & & & & & & \\
\hline Appalachian Total & 357,344 & 375,819 & 353,089 & 364,191 & 356,247 & -4.9 & 0.1 \\
\hline Alabama & 19,127 & 19,220 & 19,668 & 19,560 & 20,667 & -.5 & -1.9 \\
\hline 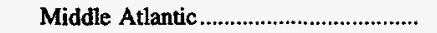 & 616 & 496 & 492 & 228 & * & 24.3 & $\mathbf{N M}$ \\
\hline 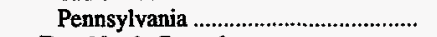 & 616 & 496 & 492 & 228 & * & 24.3 & NM \\
\hline 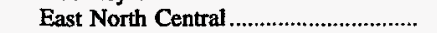 & - & - & - & - & 6 & - & - \\
\hline 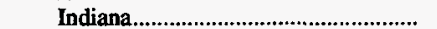 & - & - & - & - & 6 & - & - \\
\hline West North Central.................................. & - & - & - & 1 & - & - & - \\
\hline 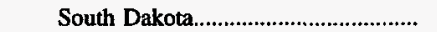 & - & - & - & 1 & - & - & - \\
\hline 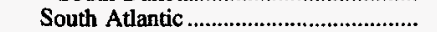 & 253 & 89 & 207 & 287 & 112 & 184.9 & 22.6 \\
\hline 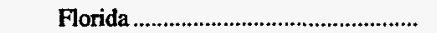 & 115 & 85 & 202 & 185 & 69 & 35.2 & 13.7 \\
\hline 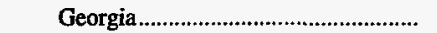 & 134 & * & 1 & 102 & 43 & NM & 32.9 \\
\hline 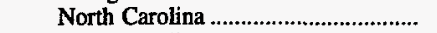 & - & - & - & - & $*$ & - & - \\
\hline 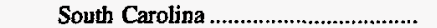 & 4 & 3 & 4 & - & - & 16.6 & - \\
\hline 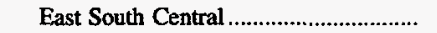 & 18,182 & 18,484 & 18,851 & 18,918 & 20,181 & -1.6 & -2.6 \\
\hline Alabama & 18,024 & 18,351 & 18,716 & 18,849 & 20,150 & -1.8 & -2.8 \\
\hline Mississippi & 156 & 129 & 132 & 68 & 28 & 21.2 & 53.6 \\
\hline 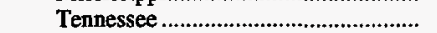 & 2 & 4 & 3 & * & 3 & -49.3 & -11.8 \\
\hline 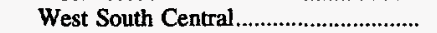 & 24 & 63 & 18 & 33 & 5 & -61.0 & 47.7 \\
\hline 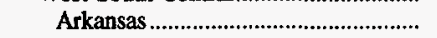 & 24 & 60 & 9 & 12 & 5 & -59.1 & 47.7 \\
\hline Texas . & - & 3 & 8 & 21 & * & -100.0 & - \\
\hline 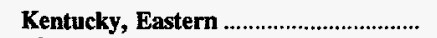 & 108,781 & 117,234 & 115,723 & 106,372 & 102,416 & -7.2 & 1.5 \\
\hline New England & 1,764 & 1,447 & 1,106 & 2,337 & 1,572 & 21.9 & 2.9 \\
\hline 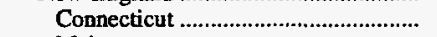 & 811 & 787 & 576 & 837 & 912 & 3.0 & -2.9 \\
\hline 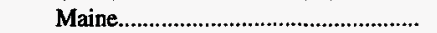 & 258 & 433 & 380 & 771 & 187 & -40.5 & 8.4 \\
\hline 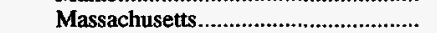 & 695 & 227 & 71 & 729 & 465 & 206.2 & 10.6 \\
\hline New Hampshire ...................................... & - & - & 79 & - & 8 & - & - \\
\hline 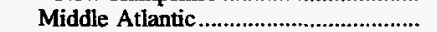 & 4,145 & 4,522 & 3,679 & 3,901 & 4,238 & -8.3 & -.6 \\
\hline 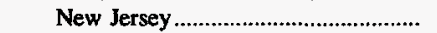 & 381 & 63 & 61 & 204 & 36 & $\mathbf{N M}$ & 80.0 \\
\hline New York & 996 & 1,288 & 1,188 & 1,288 & 2,040 & -22.6 & -16.4 \\
\hline 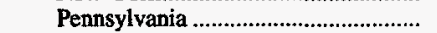 & 2,767 & 3,171 & 2,429 & 2,409 & 2,162 & -12.7 & 6.4 \\
\hline 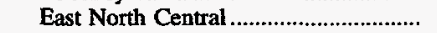 & 22,832 & 24,669 & 25,309 & 21,902 & 23,622 & -7.4 & -.8 \\
\hline 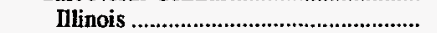 & 1,442 & 1,555 & 2,333 & 1,575 & 1,766 & -7.2 & -4.9 \\
\hline 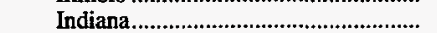 & 2,397 & 2,109 & 2,074 & 2,645 & 2,299 & 13.7 & 1.0 \\
\hline 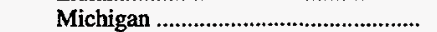 & 6,977 & 9,524 & 8,857 & 8,083 & 8,648 & -26.8 & -5.2 \\
\hline Ohio & 11,200 & 10,532 & 11,294 & 8,648 & 9,915 & 6.3 & 3.1 \\
\hline 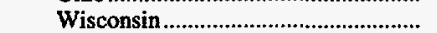 & 816 & 949 & 751 & 951 & 995 & -14.0 & -4.8 \\
\hline 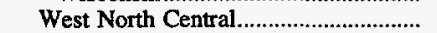 & 611 & 564 & 482 & 444 & 641 & 8.4 & -1.2 \\
\hline 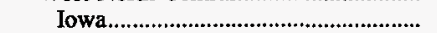 & 160 & 40 & 25 & 65 & 232 & 298.0 & -8.8 \\
\hline 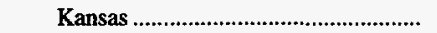 & - & - & - & 5 & 15 & - & - \\
\hline 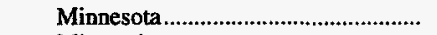 & 211 & 172 & 124 & 125 & 184 & 22.7 & 3.5 \\
\hline 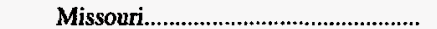 & 238 & 351 & 334 & 249 & 211 & -32.3 & 3.0 \\
\hline 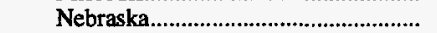 & 3 & - & - & - & - & - & - \\
\hline 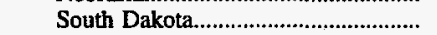 & - & 1 & - & - & - & -100.0 & - \\
\hline 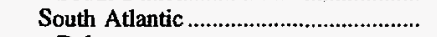 & 57,820 & 60,914 & 57,532 & 52,997 & 50,747 & -5.1 & 3.3 \\
\hline Delaware & - & 37 & 27 & - & 52 & -100.0 & - \\
\hline 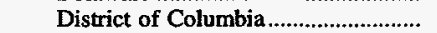 & - & 13 & 16 & 18 & 47 & -100.0 & - \\
\hline Florida & 12,121 & 12,069 & 11,311 & 12,584 & 11,302 & .4 & 1.8 \\
\hline 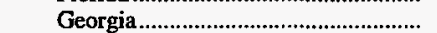 & 15,803 & 15,649 & 14,163 & 11,770 & 12,277 & 1.0 & 6.5 \\
\hline 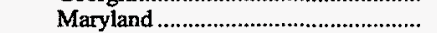 & 29 & 423 & 958 & 296 & 449 & -93.2 & -49.7 \\
\hline 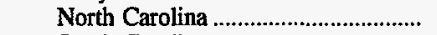 & 12,902 & 13,590 & 13,491 & 12,517 & 11,334 & -5.1 & 3.3 \\
\hline 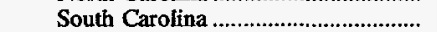 & 10,007 & 11,443 & 9,962 & 9,847 & 9,587 & -12.5 & 1.1 \\
\hline 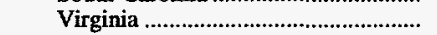 & 6,129 & 6,539 & 6,672 & 5,246 & 4,872 & -6.3 & 5.9 \\
\hline 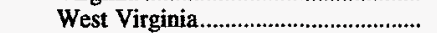 & 829 & 1,151 & 932 & 721 & 826 & -28.0 & .1 \\
\hline 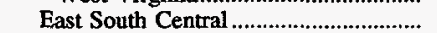 & 20,332 & 22,813 & 26,008 & 22,917 & 19,666 & -10.9 & .8 \\
\hline Alabama & 1,434 & 2,402 & 2,320 & 2,703 & 2,164 & -40.3 & -9.8 \\
\hline 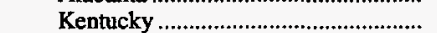 & 9,653 & 10,317 & 10,145 & 10,486 & 8,563 & -6.4 & 3.0 \\
\hline 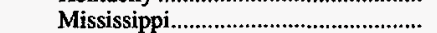 & 815 & 1,006 & 1,731 & 1,616 & 2,105 & -18.9 & -21.1 \\
\hline 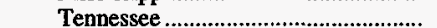 & 8,430 & 9,088 & 11,811 & 8,112 & 6,835 & -7.3 & 5.4 \\
\hline 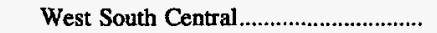 & 513 & 802 & 104 & 95 & 237 & -36.0 & 21.3 \\
\hline Arkansas ................................................ & - & * & - & - & - & -100.0 & - \\
\hline 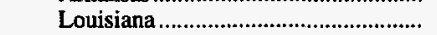 & 500 & 791 & 104 & 95 & 235 & -36.8 & 20.7 \\
\hline Oklahoma & 4 & - & - & - & - & - & - \\
\hline Texas & 8 & 10 & - & - & 1 & -15.5 & 62.1 \\
\hline Mountain & 2 & 10 & - & 2 & - & -80.3 & - \\
\hline 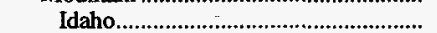 & 2 & - & - & 2 & - & - & - \\
\hline 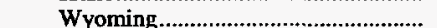 & - & 10 & - & - & - & -100.0 & _- \\
\hline 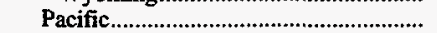 & 15 & 15 & 4 & - & - & 2.0 & - \\
\hline 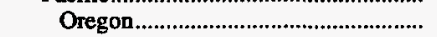 & 15 & 15 & 4 & - & - & 2.0 & - \\
\hline
\end{tabular}

See footnotes at end of table. 
Table 61. Domestic Distribution of U.S. Coal by Coal-Producing Region and State, and Destination Census Division and State, 1991-1995 (Continued) (Thousand Short Tons)

\begin{tabular}{|c|c|c|c|c|c|c|c|}
\hline \multirow{2}{*}{$\begin{array}{l}\text { Coal-Producing Region and State, } \\
\text { and Destination Census Division } \\
\text { and State }\end{array}$} & \multirow{2}{*}{1995} & \multirow{2}{*}{1994} & \multirow{2}{*}{1993} & \multirow{2}{*}{1992} & \multirow{2}{*}{1991} & \multirow{2}{*}{$\begin{array}{l}\text { Percent } \\
\text { Change } \\
\text { 1994-1995 }\end{array}$} & \multirow{2}{*}{$\begin{array}{c}\text { Average Annual } \\
\text { Percent Change } \\
1991-1995\end{array}$} \\
\hline & & & & & & & \\
\hline 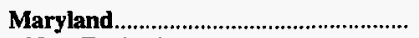 & 3,382 & 3,277 & 3,278 & 3,246 & 3,594 & 3.2 & -1.5 \\
\hline New England & 32 & - & 11 & - & - & - & - \\
\hline 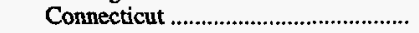 & 32 & - & - & - & - & - & - \\
\hline 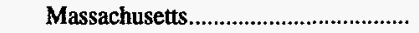 & - & - & 11 & - & - & - & - \\
\hline 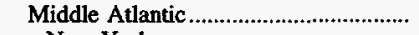 & 45 & - & 19 & 6 & 22 & - & 19.3 \\
\hline 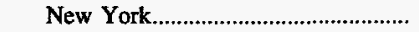 & - & - & - & 4 & 8 & - & - \\
\hline Pennsylvania & 45 & - & 19 & 2 & 14 & - & 34.6 \\
\hline South Atlantic & 3,297 & 3,277 & 3,230 & 3,166 & 3,553 & .6 & -1.8 \\
\hline 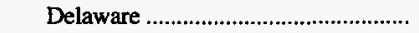 & 97 & 125 & 37 & - & 15 & -22.7 & 59.2 \\
\hline 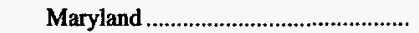 & 1,216 & 1,034 & 820 & 1,159 & 1,363 & 17.7 & -2.8 \\
\hline 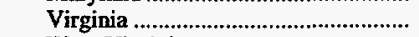 & 12 & 1 & - & 1 & 37 & NM & -23.6 \\
\hline 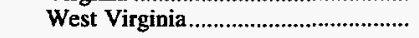 & 1,971 & 2,116 & 2,373 & 2,006 & 2,139 & -6.8 & -2.0 \\
\hline Ohio & 24,318 & 28,688 & 28,315 & 29,549 & 30,001 & -15.2 & -5.1 \\
\hline New England & - & * & 17 & 4 & * & -100.0 & - \\
\hline 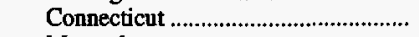 & - & $*$ & $*$ & 1 & - & -100.0 & - \\
\hline 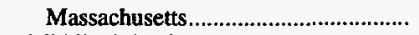 & - & - & 17 & 4 & * & - & - \\
\hline 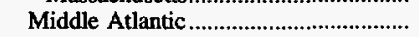 & 1,568 & 2,443 & 1,388 & 556 & 934 & -35.8 & 13.8 \\
\hline New York & 25 & 124 & 52 & 47 & 45 & -79.9 & -13.9 \\
\hline 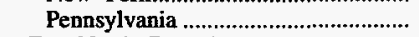 & 1,543 & 2,318 & 1,336 & 508 & 889 & -33.4 & 14.8 \\
\hline 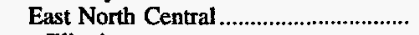 & 20,912 & 24,810 & 25,119 & 27,497 & 27,875 & -15.7 & -6.9 \\
\hline 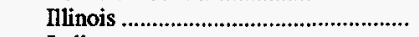 & - & 17 & $*$ & 2 & - & -100.0 & - \\
\hline 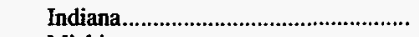 & 243 & 345 & 274 & 134 & 181 & -29.7 & 7.5 \\
\hline 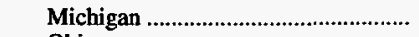 & 431 & 522 & 474 & 419 & 627 & -17.4 & -8.9 \\
\hline 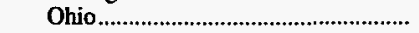 & 20,228 & 23,907 & 24,370 & 26,941 & 27,061 & -15.4 & -7.0 \\
\hline Wisconsin & 10 & 18 & - & - & 6 & -45.4 & 12.0 \\
\hline West North Central.................................. & 10 & 33 & 83 & - & $*$ & -70.2 & 330.1 \\
\hline Iowa & - & 15 & 67 & - & - & -100.0 & - \\
\hline 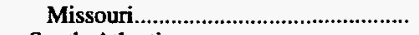 & 10 & 18 & 16 & - & $*$ & -44.5 & 330.1 \\
\hline 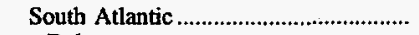 & 1,620 & 971 & 1,041 & 1,385 & 995 & 66.8 & 13.0 \\
\hline 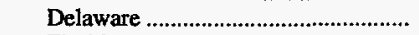 & - & 29 & 48 & - & - & -100.0 & - \\
\hline 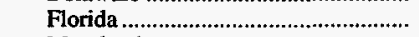 & - & - & - & - & 172 & - & - \\
\hline 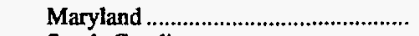 & - & - & - & - & 7 & - & - \\
\hline South Carolina & - & - & - & - & 5 & - & - \\
\hline 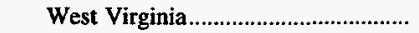 & 1,620 & 942 & 993 & 1,385 & 811 & 72.0 & 18.9 \\
\hline 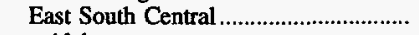 & 53 & 130 & 261 & - & 49 & -59.5 & 2.0 \\
\hline 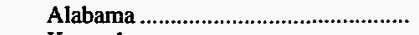 & 18 & 37 & 151 & - & 48 & -50.5 & -21.8 \\
\hline 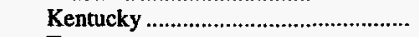 & 14 & 93 & 29 & - & - & -85.1 & - \\
\hline 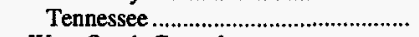 & 21 & - & 81 & - & * & - & 354.8 \\
\hline 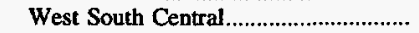 & - & - & - & $*$ & 1 & - & - \\
\hline 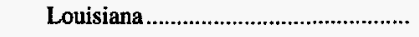 & - & - & - & * & 1 & - & - \\
\hline \multicolumn{8}{|l|}{ Pennsylvania, } \\
\hline Anthracite & 3,497 & 4,346 & 3,015 & $\mathbf{3 , 2 3 0}$ & $\mathbf{2 , 8 2 1}$ & -19.5 & 5.5 \\
\hline 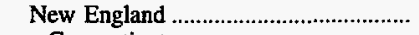 & 37 & 54 & 64 & 73 & 74 & -30.6 & -15.8 \\
\hline 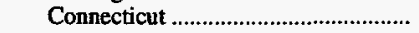 & 8 & 11 & 13 & 14 & 13 & -28.2 & -10.3 \\
\hline 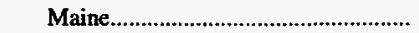 & 3 & 6 & 8 & 12 & 11 & -50.9 & -27.0 \\
\hline 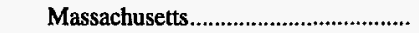 & 16 & 20 & 24 & 22 & 20 & -22.2 & -5.7 \\
\hline 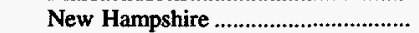 & 5 & 8 & 10 & 14 & 22 & -37.8 & -31.1 \\
\hline 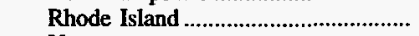 & 3 & 3 & 3 & 5 & 4 & -18.4 & -10.3 \\
\hline 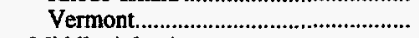 & 3 & 5 & 6 & 7 & 5 & -42.4 & -14.3 \\
\hline 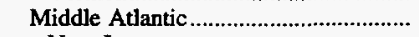 & 2,922 & 3,227 & 2,618 & 2,916 & 2,554 & -9.4 & 3.4 \\
\hline 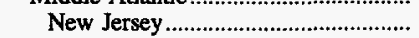 & 15 & 17 & 20 & 17 & 18 & -13.1 & -5.4 \\
\hline 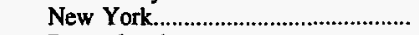 & 140 & 121 & 179 & 215 & 164 & 15.9 & -4.0 \\
\hline 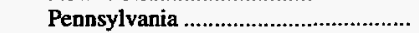 & 2,768 & 3,089 & 2,420 & 2,685 & 2,371 & -10.4 & 3.9 \\
\hline 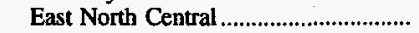 & 37 & 36 & 39 & 40 & 31 & 1.7 & 4,5 \\
\hline 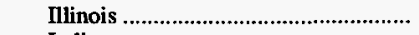 & 9 & 8 & 14 & 8 & 11 & 17.8 & -4.7 \\
\hline 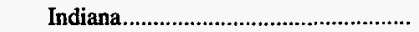 & 6 & 4 & 5 & 5 & 5 & 51.8 & 1.5 \\
\hline 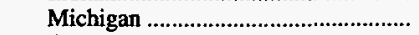 & 9 & 2 & $*$ & 1 & 2 & 269.6 & 54.4 \\
\hline Ohio ... & 8 & 21 & 19 & 26 & 13 & -60.2 & -10.9 \\
\hline 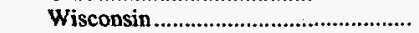 & 6 & 2 & 1 & * & * & 159.4 & 134.8 \\
\hline West North Central .................................. & 46 & 31 & 34 & 35 & 33 & 46.4 & 8.4 \\
\hline 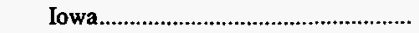 & 39 & 26 & 28 & 27 & 29 & 50.2 & 7.5 \\
\hline 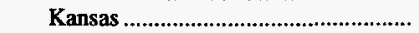 & - & - & * & 6 & $*$ & - & - \\
\hline 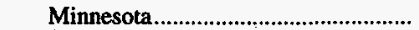 & 7 & 3 & 3 & * & * & 105.2 & 188.2 \\
\hline 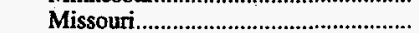 & * & 2 & 2 & * & * & -90.6 & 47.6 \\
\hline 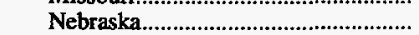 & * & * & 2 & 2 & 4 & - & -87.2 \\
\hline 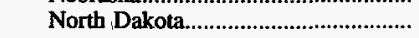 & * & $*$ & * & $*$ & * & 68.4 & 7.5 \\
\hline
\end{tabular}

See footnotes at end of table. 
Table 61. Domestic Distribution of U.S. Coal by Coal-Producing Region and State, and Destination Census Division and State, 1991-1995 (Continued) (Thousand Short Tons)

\begin{tabular}{|c|c|c|c|c|c|c|c|}
\hline \multirow{2}{*}{$\begin{array}{l}\text { Coal-Producing Region and State, } \\
\text { and Destination Census Division } \\
\text { and State }\end{array}$} & \multirow[t]{2}{*}{1995} & \multirow[t]{2}{*}{1994} & \multirow[t]{2}{*}{1993} & \multirow[t]{2}{*}{1992} & \multirow[t]{2}{*}{1991} & \multirow{2}{*}{$\begin{array}{l}\text { Percent } \\
\text { Change } \\
1994-1995\end{array}$} & \multirow{2}{*}{$\begin{array}{c}\begin{array}{c}\text { Average Annual } \\
\text { Percent Change }\end{array} \\
1991-1995\end{array}$} \\
\hline & & & & & & & \\
\hline \multicolumn{8}{|l|}{ Pennsylvania, } \\
\hline 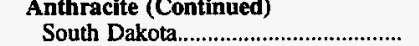 & - & * & - & * & * & -100.0 & - \\
\hline South Atlantic & 91 & 79 & 64 & 51 & 39 & 14.9 & 23.8 \\
\hline 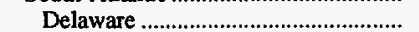 & 11 & 11 & 11 & 7 & $*$ & 4.0 & 152.3 \\
\hline 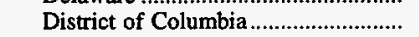 & $*$ & $*$ & * & * & * & 215.1 & 32.3 \\
\hline 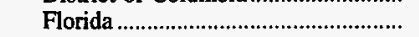 & 9 & 8 & 6 & 7 & 4 & 13.5 & 25.2 \\
\hline 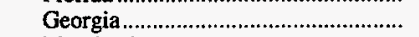 & $*$ & $*$ & * & 1 & 1 & 53.3 & -13.3 \\
\hline 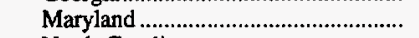 & 27 & 6 & 4 & 3 & 9 & 323.3 & 30.7 \\
\hline 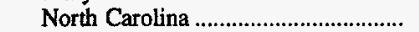 & * & * & * & * & * & -83.3 & 24.7 \\
\hline 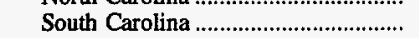 & 3 & 8 & 11 & * & 3 & -66.4 & .3 \\
\hline 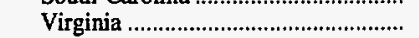 & 9 & 11 & 14 & 13 & 16 & -13.6 & -12.7 \\
\hline 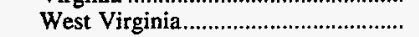 & 31 & 35 & 18 & 20 & 6 & -10.7 & 49.7 \\
\hline 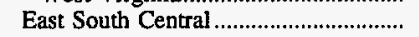 & 44 & 28 & 34 & 42 & 44 & 59.8 & .1 \\
\hline 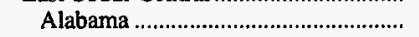 & 1 & 1 & 3 & 6 & 1 & 22.4 & 17.4 \\
\hline Kentucky & 21 & 12 & 18 & 24 & 27 & 75.3 & -6.0 \\
\hline 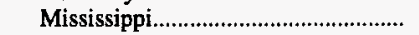 & * & $*$ & $*$ & * & $*$ & -30.0 & -36.8 \\
\hline 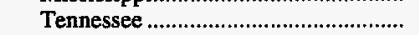 & 22 & 15 & 14 & 12 & 16 & 50.4 & 7.6 \\
\hline 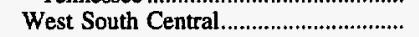 & 12 & 8 & 11 & 10 & 5 & 65.6 & 28.0 \\
\hline 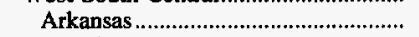 & $*$ & 1 & $*$ & 4 & 3 & -81.7 & -53.3 \\
\hline 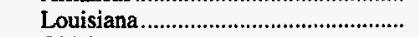 & 10 & 3 & 8 & $*$ & $*$ & 201.4 & 264.4 \\
\hline 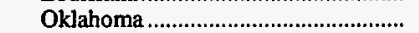 & $*$ & * & $*$ & 1 & $*$ & -5.9 & 10.3 \\
\hline 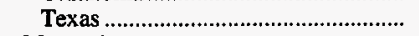 & 2 & 3 & 3 & 5 & 1 & -44.5 & 8.4 \\
\hline 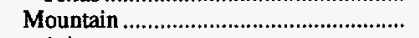 & 13 & 18 & 3 & * & 1 & -28.4 & 116.1 \\
\hline 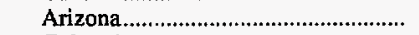 & * & $*$ & $*$ & $*$ & $*$ & -44.4 & -23.4 \\
\hline 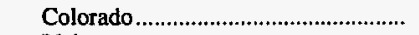 & 12 & 15 & 3 & $*$ & * & -18.1 & 137.2 \\
\hline 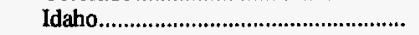 & $*$ & 3 & $*$ & - & - & -94.9 & - \\
\hline 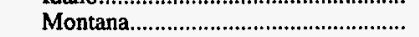 & - & $*$ & $*$ & - & - & -100.0 & - \\
\hline 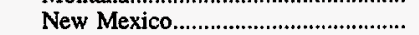 & $*$ & * & $*$ & $*$ & - & 172.7 & - \\
\hline Utah & $*$ & * & $*$ & - & $*$ & NM & 149.6 \\
\hline 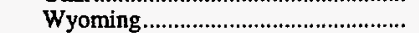 & - & $*$ & $*$ & $*$ & $*$ & -100.0 & - \\
\hline 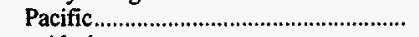 & 12 & 7 & 10 & $*$ & $*$ & 69.9 & 173.9 \\
\hline 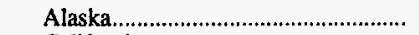 & - & * & - & - & - & -100.0 & - \\
\hline 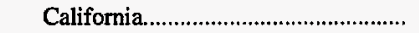 & * & * & $*$ & $*$ & $*$ & -94.8 & -35.4 \\
\hline 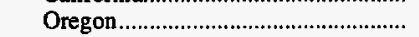 & 12 & 7 & 9 & - & $*$ & 81.6 & 313.9 \\
\hline 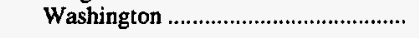 & $*$ & - & - & $*$ & $*$ & - & -49.7 \\
\hline \multicolumn{8}{|l|}{ Pennsylvania, } \\
\hline 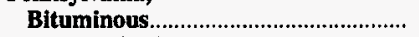 & 50,464 & 50,861 & 50,467 & 57,979 & $\mathbf{5 5 , 3 3 0}$ & -.8 & -2.3 \\
\hline 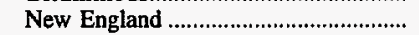 & 1,009 & 1,025 & 989 & 1,124 & 1,113 & -1.5 & -2.4 \\
\hline 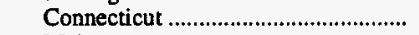 & 516 & 12 & $*$ & 131 & 87 & $\mathrm{NM}$ & 56.1 \\
\hline 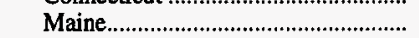 & 32 & 24 & 2 & 19 & 1 & 32.8 & 119.2 \\
\hline 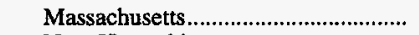 & 4 & 292 & 330 & 294 & 290 & -98.7 & -66.3 \\
\hline 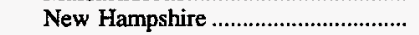 & 458 & 698 & 656 & 681 & 734 & -34.4 & -11.1 \\
\hline 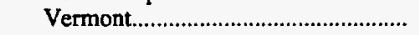 & - & - & * & - & * & - & - \\
\hline Middle Atlantic .......................................... & 37,612 & 38,188 & 37,417 & 43,559 & 40,806 & -1.5 & -2.0 \\
\hline 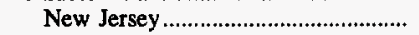 & 558 & 537 & 153 & 15 & 23 & 3.8 & 121.5 \\
\hline New York & 3,675 & 5,551 & 6,228 & 8,532 & 6,893 & -33.8 & -14.5 \\
\hline 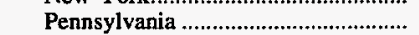 & 33,379 & 32,100 & 31,036 & 35,012 & 33,889 & 4.0 & -.4 \\
\hline 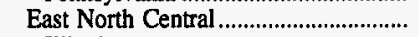 & 6,682 & 6,288 & 6,502 & 7,466 & 7,138 & 6.3 & -1.6 \\
\hline 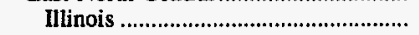 & - & 206 & 43 & 69 & 154 & -100.0 & - \\
\hline 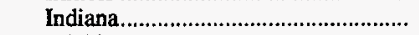 & 222 & 629 & 505 & 380 & 277 & -64.8 & -5.4 \\
\hline Michigan & 2,650 & 1,756 & 1,607 & 1,862 & 1,682 & 50.9 & 12.0 \\
\hline Ohio & 2,707 & 2,769 & 3,848 & 3,567 & 3,411 & -2.2 & -5.6 \\
\hline 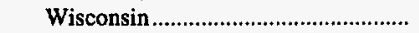 & 1,103 & 928 & 500 & 1,589 & 1,615 & 18.8 & -9.1 \\
\hline 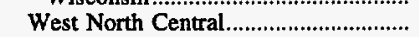 & 228 & 46 & 205 & 191 & 558 & 397.2 & -20.1 \\
\hline 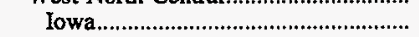 & 227 & 46 & 103 & 162 & 67 & 394.8 & 35.8 \\
\hline 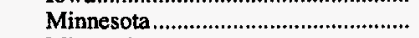 & - & - & - & 28 & 492 & - & - \\
\hline 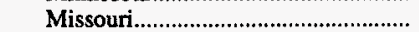 & 1 & - & 102 & 1 & - & - & - \\
\hline 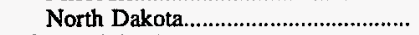 & - & - & $*$ & - & - & - & - \\
\hline 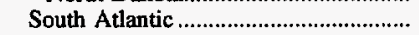 & 3,377 & 3,783 & 3,955 & 4,090 & 4,957 & -10.8 & -9.1 \\
\hline 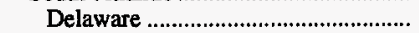 & 452 & 314 & 252 & 226 & 576 & 44.1 & -5.9 \\
\hline 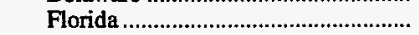 & - & 135 & - & - & 66 & -100.0 & - \\
\hline Maryland & 1,741 & 2,278 & 2,372 & 2,437 & 2,624 & -23.6 & -9.7 \\
\hline South Carolina & 6 & - & - & 1 & 2 & - & 30.2 \\
\hline 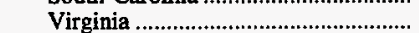 & 20 & 55 & 29 & 5 & $7 \overline{1}$ & -63.4 & -27.2 \\
\hline 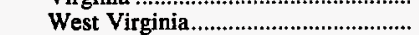 & 1,157 & 1,002 & 1,302 & 1,421 & 1,616 & 15.5 & -8.0 \\
\hline 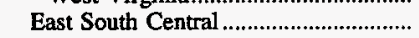 & 1,078 & 993 & 772 & 83 & 6 & 8.5 & 263.2 \\
\hline
\end{tabular}

See footnotes at end of table. 
Table 61. Domestic Distribution of U.S. Coal by Coal-Producing Region and State, and Destination Census Division and State, 1991-1995 (Continued) (Thousand Short Tons)

\begin{tabular}{|c|c|c|c|c|c|c|c|}
\hline \multirow{2}{*}{$\begin{array}{c}\text { Coal-Producing Region and State, } \\
\text { and Destination Census Division } \\
\text { and State }\end{array}$} & \multirow{2}{*}{1995} & \multirow{2}{*}{1994} & \multirow{2}{*}{1993} & \multirow{2}{*}{1992} & \multirow{2}{*}{1991} & \multirow{2}{*}{$\begin{array}{l}\text { Percent } \\
\text { Change } \\
\text { 1994-1995 }\end{array}$} & \multirow{2}{*}{$\begin{array}{c}\begin{array}{c}\text { Average Annual } \\
\text { Percent Change }\end{array} \\
1991-1995\end{array}$} \\
\hline & & & & & & & \\
\hline \multicolumn{8}{|l|}{$\begin{array}{l}\text { Pennsylvania, } \\
\text { Bituminous (Continued) }\end{array}$} \\
\hline 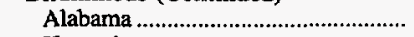 & 39 & 34 & 46 & 1 & $*$ & 15.7 & 261.4 \\
\hline 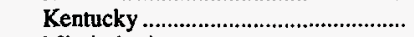 & 363 & 460 & 225 & 31 & - & -21.0 & - \\
\hline 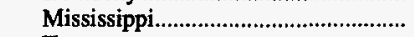 & - & - & 3 & 6 & - & - & - \\
\hline 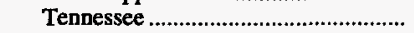 & 675 & 500 & 498 & 46 & 6 & 35.2 & 226.2 \\
\hline 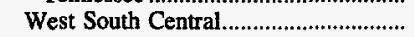 & 6 & - & * & * & 2 & - & 36.2 \\
\hline 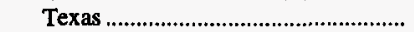 & 6 & - & * & * & $\overline{2}$ & - & 36.2 \\
\hline 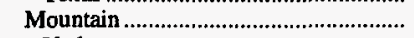 & 215 & 183 & 240 & 290 & 265 & 16.9 & -5.1 \\
\hline Utah & 215 & 183 & 240 & 290 & 265 & 16.9 & -5.1 \\
\hline Pacific & - & - & - & - & $*$ & - & - \\
\hline 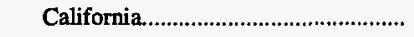 & - & - & - & - & * & - & - \\
\hline 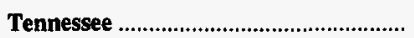 & 2,627 & 2,547 & 2,577 & 2,835 & 4,243 & 3.1 & -11.3 \\
\hline 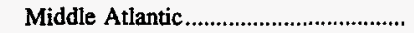 & - & - & - & - & 5 & - & - \\
\hline New York & - & - & - & - & 5 & - & - \\
\hline 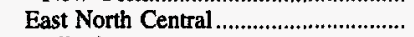 & * & 1 & $*$ & 24 & 63 & -82.8 & -76.0 \\
\hline 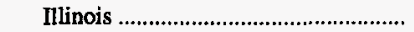 & - & 1 & - & - & 29 & -100.0 & - \\
\hline 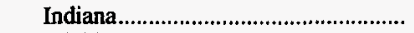 & - & - & $*$ & 3 & 2 & - & - \\
\hline 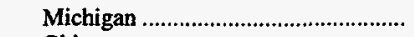 & * & - & - & 21 & 31 & - & -78.8 \\
\hline 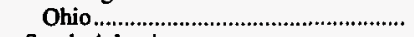 & $*$ & - & - & - & 1 & - & -41.1 \\
\hline South Atlantic & 251 & 301 & 303 & 387 & 820 & -16.7 & -25.6 \\
\hline Florida & 39 & 40 & - & - & 242 & -2.7 & -36.5 \\
\hline 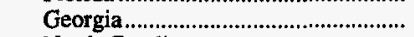 & 189 & 202 & 204 & 259 & 384 & -6.6 & -16.3 \\
\hline North Carolina ..................................... & 23 & 58 & 96 & 114 & 150 & -61.4 & -37.8 \\
\hline 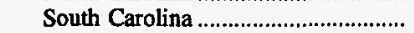 & - & - & 3 & 13 & 34 & - & - \\
\hline Virginia & - & - & * & 1 & 10 & - & - \\
\hline 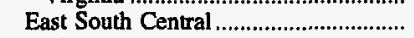 & 2,363 & 2,223 & 2,215 & 2,376 & 3,292 & 6.3 & -7.9 \\
\hline 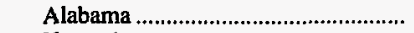 & 936 & 710 & 592 & 734 & 934 & 31.8 & * \\
\hline 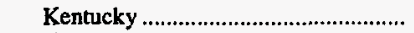 & 5 & 135 & 122 & 39 & 436 & -96.4 & -67.6 \\
\hline 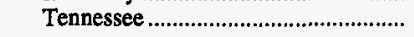 & 1,422 & 1,378 & 1,501 & 1,603 & 1,922 & 3.2 & -7.3 \\
\hline Virginia & 24,283 & 26,866 & 27,388 & 28,504 & 25,390 & -9.6 & -1.1 \\
\hline 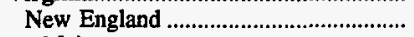 & 19 & - & - & 281 & 886 & - & -61.7 \\
\hline 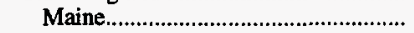 & - & - & - & 44 & - & - & - \\
\hline 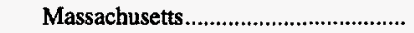 & - & - & - & 237 & 693 & - & - \\
\hline 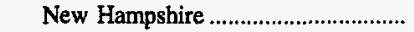 & 19 & - & - & - & 186 & - & -43.4 \\
\hline 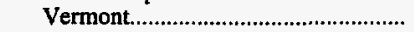 & - & - & - & - & 7 & - & - \\
\hline 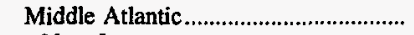 & 2,311 & 1,490 & 2,073 & 2,957 & 1,812 & 55.1 & 6.3 \\
\hline 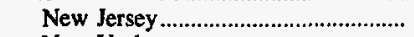 & 635 & 190 & 359 & 734 & 551 & 234.0 & 3.6 \\
\hline New York & 362 & 156 & 108 & 96 & 128 & 131.5 & 29.8 \\
\hline 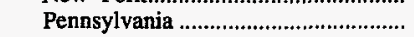 & 1,314 & 1,143 & 1,607 & 2,127 & 1,134 & 15.0 & 3.8 \\
\hline 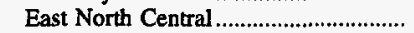 & 3,557 & 2,455 & 3,475 & 3,794 & 2,574 & 44.9 & 8.4 \\
\hline 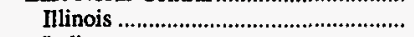 & 578 & 302 & 260 & 478 & 380 & 91.2 & 11.1 \\
\hline 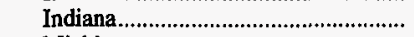 & 2,395 & 1,202 & 2,045 & 2,240 & 1,339 & 99.2 & 15.6 \\
\hline 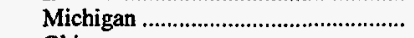 & 83 & 376 & 188 & 68 & 34 & -78.0 & 24.6 \\
\hline 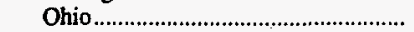 & 493 & 488 & 875 & 940 & 772 & .9 & -10.6 \\
\hline 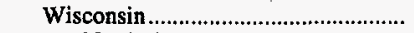 & 9 & 86 & 108 & 68 & 50 & -89.5 & -34.7 \\
\hline 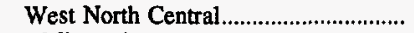 & 8 & - & - & * & - & - & - \\
\hline 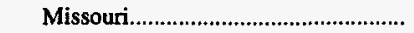 & 8 & - & - & - & - & - & - \\
\hline 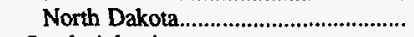 & - & - & - & * & - & - & - \\
\hline 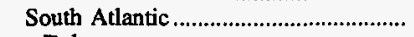 & 14,600 & 18,898 & 18,553 & 16,839 & 16,290 & -22.7 & -2.7 \\
\hline 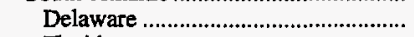 & 152 & 203 & 155 & 208 & 137 & -25.2 & 2.5 \\
\hline Florida & 377 & 531 & 457 & 451 & 455 & -28.9 & -4.6 \\
\hline Georgia & 2,064 & 3,038 & 3,496 & 2,779 & 3,219 & -32.1 & -10.5 \\
\hline 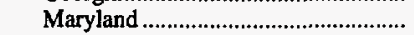 & 392 & 90 & 8 & 79 & 115 & 337.2 & 36.0 \\
\hline 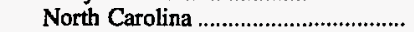 & 4,056 & 5,634 & 5,867 & 5,207 & 4,520 & -28.0 & -2.7 \\
\hline 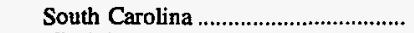 & 1,468 & 1,604 & 1,663 & 1,356 & 1,523 & -8.5 & -.9 \\
\hline 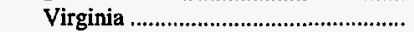 & 5,657 & 6,867 & 6,076 & 6,082 & 5,457 & -17.6 & .9 \\
\hline 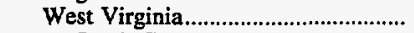 & 433 & 930 & 832 & 677 & 864 & -53.4 & -15.8 \\
\hline East South Central .................................... & 3,413 & 3,658 & 3,026 & 3,432 & 3,312 & -6.7 & .8 \\
\hline Alabama & 1,083 & 1,156 & 887 & 1,017 & 763 & -6.3 & 9.1 \\
\hline 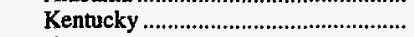 & 142 & 41 & 1 & $*$ & - & 249.8 & - \\
\hline 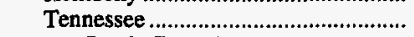 & 2,187 & 2,462 & 2,138 & 2,415 & 2,549 & -11.1 & -3.8 \\
\hline 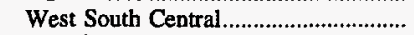 & 21 & - & 2 & 2 & 10 & - & 20.5 \\
\hline 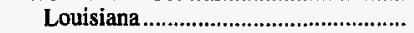 & 21 & - & - & - & - & - & - \\
\hline 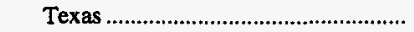 & - & - & 2 & 2 & 10 & - & - \\
\hline 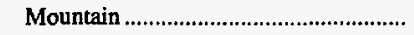 & 313 & 320 & 103 & 192 & 198 & -2.3 & 12.1 \\
\hline
\end{tabular}

See footnotes at end of table. 
Table 61. Domestic Distribution of U.S. Coal by Coal-Producing Region and State, and Destination Census Division and State, 1991-1995 (Continued) (Thousand Short Tons)

\begin{tabular}{|c|c|c|c|c|c|c|c|}
\hline \multirow{2}{*}{$\begin{array}{c}\text { Coal-Producing Region and State, } \\
\text { and Destination Census Division } \\
\text { and State }\end{array}$} & \multirow{2}{*}{1995} & \multirow{2}{*}{1994} & \multirow{2}{*}{1993} & \multirow{2}{*}{1992} & \multirow{2}{*}{1991} & \multirow{2}{*}{$\begin{array}{l}\text { Percent } \\
\text { Change } \\
\text { 1994-1995 }\end{array}$} & \multirow{2}{*}{$\begin{array}{c}\text { Average Annual } \\
\text { Percent Change } \\
1991-1995\end{array}$} \\
\hline & & & & & & & \\
\hline \multicolumn{8}{|l|}{ Virginia (Continued) } \\
\hline 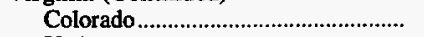 & - & - & $*$ & - & $*$ & - & - \\
\hline Utah & 313 & 320 & 103 & 192 & 198 & -2.3 & 12.1 \\
\hline West Virginia, Northern & 36,073 & 39,985 & $\mathbf{3 4 , 5 7 3}$ & 44,093 & 45,586 & -9.8 & -5.7 \\
\hline 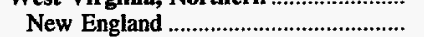 & 918 & 1,086 & 1,002 & 2,776 & 2,216 & -15.4 & -19.8 \\
\hline 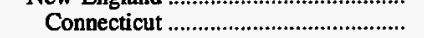 & 572 & 166 & - & $*$ & - & 245.5 & - \\
\hline 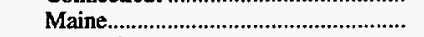 & 9 & - & - & - & - & - & - \\
\hline 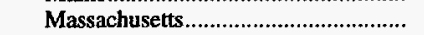 & 113 & 648 & 613 & 2,379 & 1,965 & -82.6 & -51.1 \\
\hline New Hampshire .................................. & 225 & 272 & 389 & 397 & 251 & -17.5 & -2.8 \\
\hline 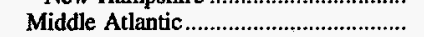 & 13,740 & 12,566 & 9,769 & 13,804 & 15,039 & 9.3 & -2.2 \\
\hline 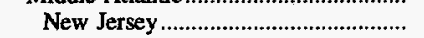 & 1,182 & 1,096 & 1,234 & 1,213 & 1,094 & 7.9 & 2.0 \\
\hline 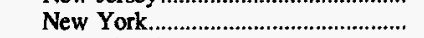 & 4,040 & 3,079 & 1,336 & 2,530 & 3,393 & 31.2 & 4.5 \\
\hline 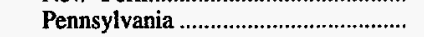 & 8,518 & 8,391 & 7,200 & 10,061 & 10,553 & 1.5 & -5.2 \\
\hline 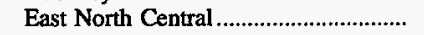 & 2,887 & 5,924 & 4,220 & 5,803 & 6,435 & -51.3 & -18.2 \\
\hline 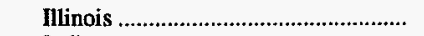 & 51 & 23 & 44 & - & - & 116.9 & - \\
\hline Indiana & 38 & 1,060 & 216 & 70 & 313 & -96.4 & -40.8 \\
\hline 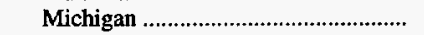 & 437 & 392 & 113 & 114 & 81 & 11.4 & 52.5 \\
\hline Ohio & 1,977 & 4,098 & 3,659 & 5,365 & 5,513 & -51.8 & -22.6 \\
\hline 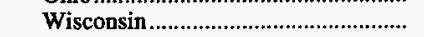 & 384 & 350 & 188 & 254 & 528 & 9.7 & -7.6 \\
\hline West North Central................................. & 3 & 12 & 54 & 9 & 20 & -72.6 & -37.1 \\
\hline 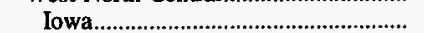 & - & 2 & - & - & 1 & -100.0 & - \\
\hline 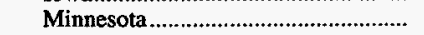 & 3 & 10 & - & 9 & 19 & -67.9 & -36.4 \\
\hline 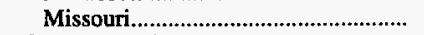 & - & - & 54 & - & - & - & - \\
\hline 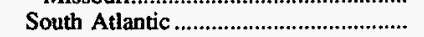 & 17,213 & 19,270 & 17,772 & 20,988 & 21,434 & -10.7 & -5.3 \\
\hline 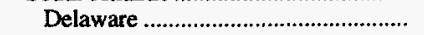 & 737 & 969 & 1,096 & 1,111 & 714 & -24.0 & .8 \\
\hline District of Columbia & 5 & 10 & 12 & 6 & 9 & -43.2 & -11.9 \\
\hline 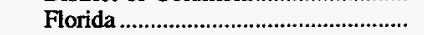 & 259 & 449 & 580 & 452 & 454 & -42.3 & -13.1 \\
\hline 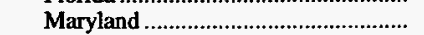 & 3,341 & 3,443 & 4,314 & 3,466 & 4,425 & -3.0 & -6.8 \\
\hline 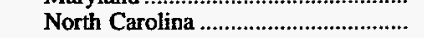 & - & 7 & - & $*$ & 2 & -100.0 & - \\
\hline South Carolina ...................................... & - & 1 & - & - & - & -100.0 & - \\
\hline Virginia & 30 & 75 & 306 & 243 & 276 & -59.9 & -42.7 \\
\hline 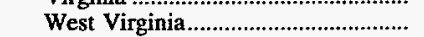 & 12,841 & 14,316 & 11,464 & 15,710 & 15,553 & -10.3 & -4.7 \\
\hline 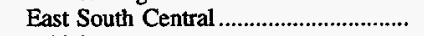 & 1,178 & 492 & 749 & 167 & 53 & 139.4 & 117.3 \\
\hline Alabama & 604 & 34 & 27 & - & * & NM & NM \\
\hline 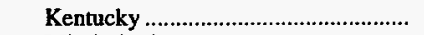 & 527 & 160 & 130 & 4 & 9 & 229.1 & 176.4 \\
\hline 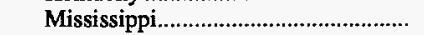 & - & 5 & 5 & 4 & 13 & -100.0 & - \\
\hline 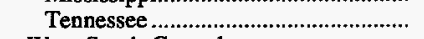 & 46 & 293 & 589 & 159 & 31 & -84.1 & 10.6 \\
\hline 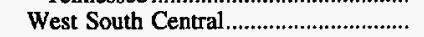 & - & 368 & 154 & - & - & -100.0 & - \\
\hline 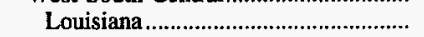 & - & 368 & 154 & - & - & -100.0 & - \\
\hline Mountain & $*$ & - & - & - & - & - & - \\
\hline 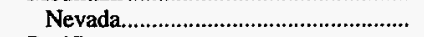 & $*$ & - & - & - & - & - & - \\
\hline 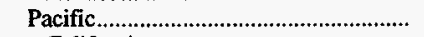 & - & - & * & * & $*$ & - & - \\
\hline 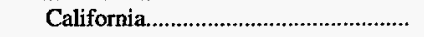 & - & - & $*$ & $*$ & $*$ & - & - \\
\hline \multicolumn{8}{|l|}{ West Virginia, } \\
\hline Southern & 84,793 & 82,794 & 68,086 & 68,824 & 66,199 & 2.4 & 6.4 \\
\hline 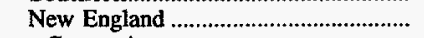 & 1,404 & 1,351 & 917 & 1,109 & 416 & 3.9 & 35.5 \\
\hline 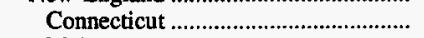 & - & - & 106 & 21 & * & - & - \\
\hline 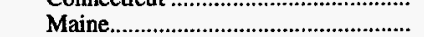 & - & * & 14 & 20 & 11 & -100.0 & - \\
\hline 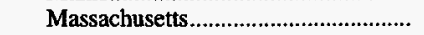 & 1,330 & 1,351 & 739 & 971 & 304 & -1.6 & 44.6 \\
\hline 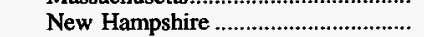 & 74 & - & 58 & 97 & 101 & - & -7.5 \\
\hline 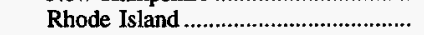 & - & - & $*$ & - & - & - & - \\
\hline 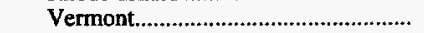 & - & * & - & - & - & -100.0 & - \\
\hline 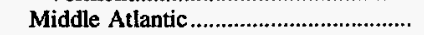 & 7,170 & 6,738 & 6,965 & 5,160 & 4,570 & 6.4 & 11.9 \\
\hline 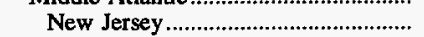 & 165 & 260 & 343 & 250 & 155 & -36.4 & 1.6 \\
\hline New York & 1,466 & 1,345 & 1,860 & 1,265 & 980 & 9.0 & 10.6 \\
\hline 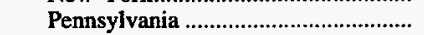 & 5,538 & 5,133 & 4,763 & 3,644 & 3,434 & 7.9 & 12.7 \\
\hline East North Central & 28,905 & 27,503 & 22,906 & 25,560 & 24,205 & 5.1 & 4.5 \\
\hline 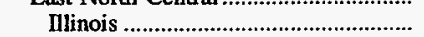 & 1,400 & 1,403 & 1,971 & 1,472 & 1,668 & -.2 & -4.3 \\
\hline Indiana & 5,142 & 4,918 & 3,401 & 4,920 & 4,131 & 4.5 & 5.6 \\
\hline 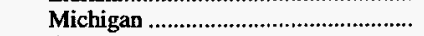 & 4,416 & 5,903 & 4,048 & 6,120 & 6,951 & -25.2 & -10.7 \\
\hline Ohio & 17,566 & 14,802 & 12,485 & 12,457 & 11,163 & 18.7 & 12.0 \\
\hline Wisconsin & 381 & 478 & 1,002 & 592 & 293 & -20.4 & 6.8 \\
\hline 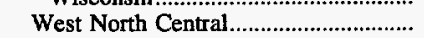 & 313 & 237 & 223 & 364 & 253 & 32.2 & 5.5 \\
\hline 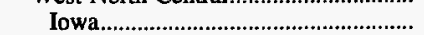 & 119 & 70 & 69 & 77 & 39 & 71.3 & 32.1 \\
\hline 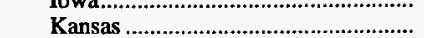 & - & - & - & 34 & 60 & - & - \\
\hline 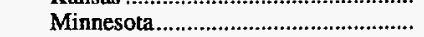 & 107 & 92 & 11 & 24 & 22 & 17.2 & 49.2 \\
\hline
\end{tabular}

See footnotes at end of table. 
Table 61. Domestic Distribution of U.S. Coal by Coal-Producing Region and

State, and Destination Census Division and State, 1991-1995 (Continued)

(Thousand Short Tons)

\begin{tabular}{|c|c|c|c|c|c|c|c|}
\hline \multirow{2}{*}{$\begin{array}{l}\text { Coal-Producing Region and State, } \\
\text { and Destination Census Division } \\
\text { and State }\end{array}$} & \multirow[t]{2}{*}{1995} & \multirow{2}{*}{1994} & \multirow[t]{2}{*}{1993} & \multirow[t]{2}{*}{1992} & \multirow{2}{*}{1991} & \multirow{2}{*}{$\begin{array}{c}\text { Percent } \\
\text { Change } \\
\text { 1994-1995 }\end{array}$} & \multirow{2}{*}{$\begin{array}{c}\begin{array}{c}\text { Average Annual } \\
\text { Percent Change }\end{array} \\
1991-1995\end{array}$} \\
\hline & & & & & & & \\
\hline \multicolumn{8}{|l|}{$\begin{array}{l}\text { West Virginia, } \\
\text { Southern (Continued) }\end{array}$} \\
\hline 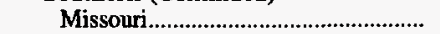 & 85 & 70 & 142 & 228 & 126 & 21.6 & -9.3 \\
\hline 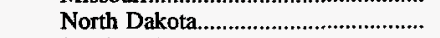 & $*$ & * & - & - & 1 & 92.6 & -52.9 \\
\hline 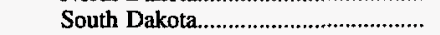 & 2 & 6 & - & - & 5 & -73.8 & -25.6 \\
\hline 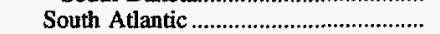 & 36,164 & 36,045 & 26,459 & 28,512 & 28,761 & .3 & 5.9 \\
\hline 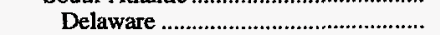 & 485 & 621 & 617 & 361 & 723 & -21.9 & -9.5 \\
\hline District of Columbia ............................... & - & 24 & 23 & 27 & 10 & -100.0 & - \\
\hline Florida …….................................. & 1,341 & 1,372 & 817 & 1,010 & 1,507 & -2.3 & -2.9 \\
\hline Georgia & 4,159 & 4,106 & 2,958 & 2,477 & 2,517 & 1.3 & 13.4 \\
\hline 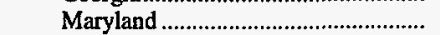 & 3,209 & 2,855 & 1,376 & 2,296 & 1,721 & 12.4 & 16.9 \\
\hline 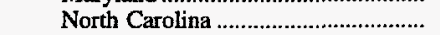 & 7,169 & 6,910 & 6,167 & 7,963 & 6,047 & 3.8 & 4.3 \\
\hline South Carolina ....................................... & 257 & 394 & 107 & 121 & 178 & -34.7 & 9.6 \\
\hline 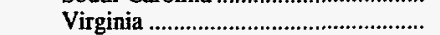 & 3,367 & 3,188 & 2,971 & 3,323 & 4,420 & 5.6 & -6.6 \\
\hline 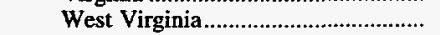 & 16,177 & 16,575 & 11,423 & 10,934 & 11,638 & -2.4 & 8.6 \\
\hline East South Central ..................................... & 10,433 & 10,551 & 10,095 & 7,054 & 7,469 & -1.1 & 8.7 \\
\hline Alabama & 3,487 & 4,392 & 3,736 & 2,362 & 2,335 & -20.6 & 10.5 \\
\hline 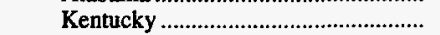 & 5,330 & 4,744 & 4,704 & 3,755 & 4,062 & 12.3 & 7.0 \\
\hline Mississippi....1. & 44 & 93 & 87 & 34 & 4 & -52.4 & 86.8 \\
\hline 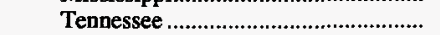 & 1,571 & 1,322 & 1,569 & 904 & 1,067 & 18.8 & 10.1 \\
\hline West South Central.................................. & 48 & 60 & 49 & 22 & 31 & -19.8 & 11.5 \\
\hline 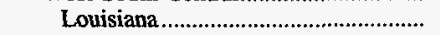 & - & 4 & 2 & - & 14 & -100.0 & - \\
\hline Oklahoma .............................................. & 48 & 56 & 48 & 22 & 18 & -13.3 & 28.9 \\
\hline 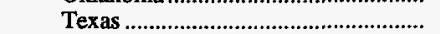 & - & 1 & - & - & - & -100.0 & - \\
\hline 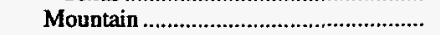 & 206 & 212 & 135 & - & 35 & -3.0 & 55.5 \\
\hline Idaho & - & $*$ & - & - & - & -100.0 & - \\
\hline 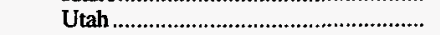 & 206 & 211 & 135 & - & 35 & -2.8 & 55.5 \\
\hline 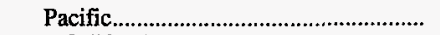 & 2 & 1 & * & - & * & 77.3 & 112.4 \\
\hline 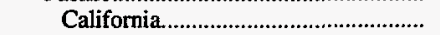 & - & - & * & - & * & - & - \\
\hline 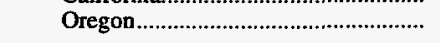 & 2 & 1 & - & - & - & 77.3 & - \\
\hline Interior Total & 162,947 & 175,622 & 166,763 & 193,400 & 191,126 & -7.2 & -3.9 \\
\hline 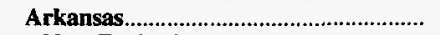 & 11 & 28 & 25 & 29 & 1 & -59.2 & 112.9 \\
\hline 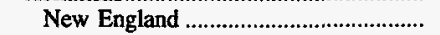 & - & - & - & 13 & - & - & - \\
\hline 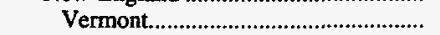 & - & - & - & 13 & - & - & - \\
\hline 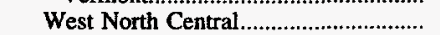 & - & 4 & 8 & 11 & - & -100.0 & - \\
\hline Missouri................................................... & - & 4 & 8 & 11 & - & -100.0 & - \\
\hline West South Central...................................... & 11 & 24 & 17 & 5 & 1 & -53.1 & 112.9 \\
\hline Arkansas & 11 & 13 & 12 & - & $\mathrm{i}$ & -10.0 & 112.9 \\
\hline 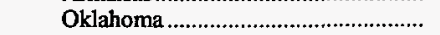 & - & * & 5 & 5 & - & -100.0 & - \\
\hline 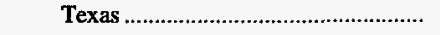 & - & 12 & - & - & - & -100.0 & - \\
\hline Hlinois ... & 45,170 & 51,737 & 41,330 & 57,670 & 57,290 & -12.7 & -5.8 \\
\hline 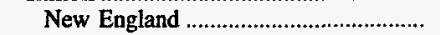 & $*$ & - & - & - & * & - & .6 \\
\hline 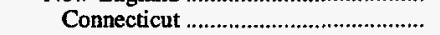 & * & - & - & - & * & - & .6 \\
\hline 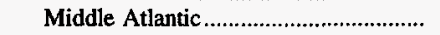 & * & $*$ & * & * & 1 & 44.5 & -10.5 \\
\hline 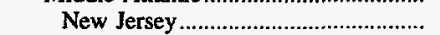 & * & - & - & * & - & - & - \\
\hline 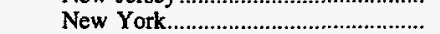 & * & * & * & * & * & 25.8 & 23.7 \\
\hline Pennsylvania …………………………....... & * & * & - & - & * & 108.2 & -32.7 \\
\hline 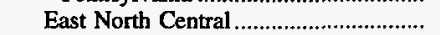 & 25,629 & 28,299 & 20,483 & 28,821 & 29,022 & -9.4 & -3.1 \\
\hline 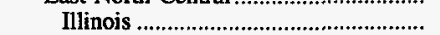 & 15,587 & 17,517 & 15,206 & 18,167 & 18,787 & -11.0 & -4.6 \\
\hline 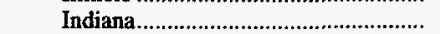 & 8,559 & 9,574 & 4,541 & 9,595 & 9,185 & -10.6 & -1.8 \\
\hline 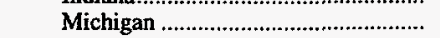 & 70 & 51 & - & 6 & 5 & 37.5 & 93.1 \\
\hline Ohio & 1 & 18 & - & - & 73 & -95.8 & -68.0 \\
\hline Wisconsin & 1,412 & 1,139 & 736 & 1,053 & 971 & 24.0 & 9.8 \\
\hline West North Central...................................... & 6,270 & $\mathbf{9 , 4 4 8}$ & 7,783 & 13,499 & 15,470 & -33.6 & -20.2 \\
\hline Iowa. & 1,216 & 1,535 & 1,534 & 1,175 & 1,473 & -20.8 & -4.7 \\
\hline 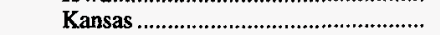 & 128 & 193 & 179 & 640 & 1,320 & -33.7 & -44.2 \\
\hline 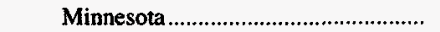 & 111 & 179 & 43 & 58 & 40 & -38.1 & 29.0 \\
\hline 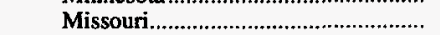 & 4,815 & 7,541 & 6,027 & 11,625 & 12,637 & -36.2 & -21.4 \\
\hline 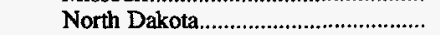 & - & - & * & - & $*$ & & - \\
\hline South Atlantic .................................. & 6,651 & 8,403 & 8,137 & 10,485 & 8,811 & -20.8 & -6.8 \\
\hline Florida & 6,056 & 5,846 & 4,782 & 5,529 & 4,464 & 3.6 & 7.9 \\
\hline 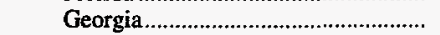 & 584 & 2,557 & 3,355 & 4,955 & 4,347 & -77.1 & -39.4 \\
\hline 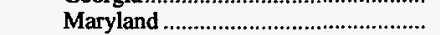 & 5 & - & - & - & - & - & - \\
\hline North Carolina ......................................... & * & - & - & - & - & - & - \\
\hline South Carolina & - & - & - & 1 & - & - & - \\
\hline 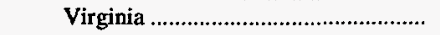 & * & - & * & - & * & - & 21.8 \\
\hline
\end{tabular}

See footnotes at end of table. 
Table 61. Domestic Distribution of U.S. Coal by Coal-Producing Region and State, and Destination Census Division and State, 1991-1995 (Continued) (Thousand Short Tons)

\begin{tabular}{|c|c|c|c|c|c|c|c|}
\hline \multirow{2}{*}{$\begin{array}{c}\text { Coal-Producing Region and State, } \\
\text { and Destination Census Division } \\
\text { and State }\end{array}$} & \multirow{2}{*}{1995} & \multirow{2}{*}{1994} & \multirow{2}{*}{1993} & \multirow{2}{*}{1992} & \multirow{2}{*}{1991} & \multirow{2}{*}{$\begin{array}{c}\text { Percent } \\
\text { Change } \\
\text { 1994-1995 }\end{array}$} & \multirow{2}{*}{$\begin{array}{c}\text { Average Annua } \\
\text { Percent Change } \\
1991-1995\end{array}$} \\
\hline & & & & & & & \\
\hline \multicolumn{8}{|l|}{ Illinois (Continued) } \\
\hline 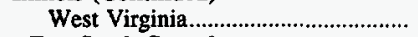 & 6 & * & - & - & - & $\mathbf{N M}$ & - \\
\hline 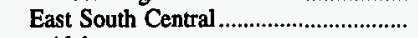 & 6,510 & 5,453 & 4,823 & 4,780 & 3,681 & 19.4 & 15.3 \\
\hline 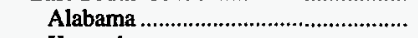 & 1,146 & 750 & 401 & 632 & 474 & 52.8 & 24.7 \\
\hline 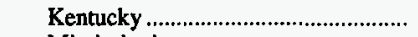 & 274 & 343 & 535 & 7 & 15 & -20.3 & 106.6 \\
\hline Mississippi................................................. & 1,304 & 1,164 & 1,106 & 1,879 & 1,518 & 12.0 & -3.7 \\
\hline 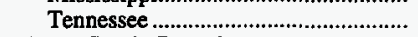 & 3,787 & 3,195 & 2,780 & 2,261 & 1,673 & 18.5 & 22.6 \\
\hline West South Central .................................. & 86 & 46 & 58 & 81 & 99 & 87.8 & -3.5 \\
\hline 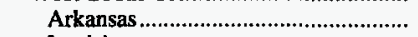 & 76 & 30 & 37 & 81 & 99 & 153.0 & -6.3 \\
\hline 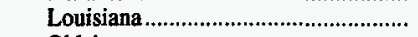 & - & - & 21 & - & - & - & - \\
\hline Oklahoma & 10 & 16 & - & - & - & -37.4 & - \\
\hline Texas & - & - & - & - & * & - & - \\
\hline Mountain & - & - & - & - & 201 & - & - \\
\hline 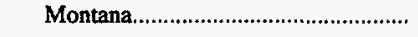 & - & - & - & - & 201 & - & - \\
\hline Indiana & 25,625 & 30,477 & 29,475 & 31,216 & 31,204 & -15.9 & -4.8 \\
\hline 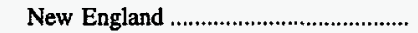 & - & * & 21 & - & - & -100.0 & - \\
\hline 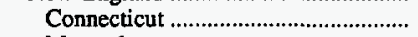 & - & $*$ & - & - & - & -100.0 & - \\
\hline Massachusetts........................................ & - & - & 21 & - & - & - & - \\
\hline East North Central .................................... & 22,461 & 27,088 & 26,347 & 27,677 & 27,023 & -17.1 & -4.5 \\
\hline 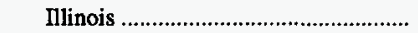 & 963 & 1,597 & 1,562 & 1,021 & 1,698 & -39.7 & -13.2 \\
\hline 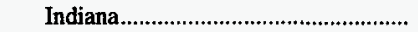 & 21,185 & 24,733 & 23,913 & 24,655 & 22,619 & -14.3 & -1.6 \\
\hline 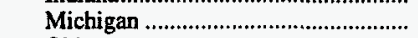 & 180 & 135 & 184 & 338 & 311 & 33.5 & -12.8 \\
\hline 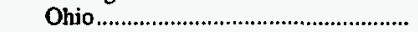 & 26 & 36 & 60 & 135 & * & -26.9 & 340.5 \\
\hline 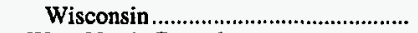 & 106 & 587 & 628 & 1,528 & 2,394 & -81.9 & -54.1 \\
\hline 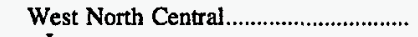 & 454 & 973 & 1,249 & 756 & 1,289 & -53.3 & -23.0 \\
\hline 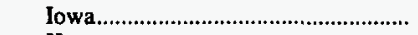 & 435 & 426 & 646 & 746 & 1,027 & 2.0 & -19.3 \\
\hline 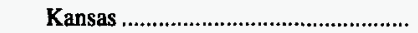 & - & - & 18 & - & 6 & - & - \\
\hline 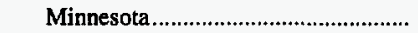 & - & 43 & - & - & 91 & -100.0 & - \\
\hline 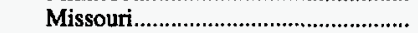 & 19 & 504 & 585 & 11 & 165 & -96.1 & -41.4 \\
\hline 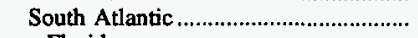 & - & 19 & 264 & 548 & 316 & -100.0 & - \\
\hline Florida & - & - & 96 & - & 171 & - & - \\
\hline Georgia & - & 19 & 153 & 548 & 144 & -100.0 & - \\
\hline Virginia & - & - & 15 & - & - & - & - \\
\hline 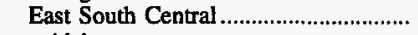 & 2,586 & 2,313 & 1,506 & 2,071 & 2,401 & 11.8 & 1.9 \\
\hline 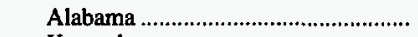 & 1 & 56 & 60 & 127 & 127 & -98.2 & -70.3 \\
\hline 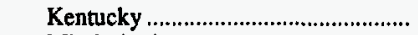 & 2,466 & 2,219 & 1,313 & 1,694 & 2,039 & 11.1 & 4.9 \\
\hline 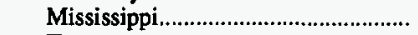 & - & - & - & 3 & 24 & - & - \\
\hline 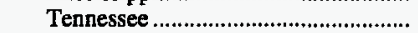 & 119 & 38 & 132 & 247 & 210 & 211.0 & -13.3 \\
\hline 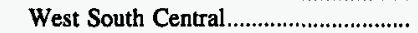 & 1 & 4 & 1 & - & 2 & -75.2 & -14.4 \\
\hline 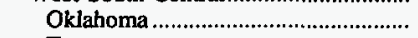 & - & 3 & $i$ & - & 2 & -100.0 & - \\
\hline 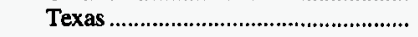 & 1 & $*$ & - & - & * & 84.3 & 148.0 \\
\hline Iowa & - & 46 & 175 & 287 & 350 & -100.0 & - \\
\hline 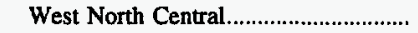 & - & 46 & 175 & 287 & 350 & -100.0 & - \\
\hline Iowa $\ldots \ldots \ldots$ & - & 46 & 175 & 287 & 347 & -100.0 & - \\
\hline 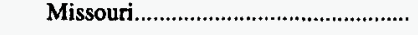 & - & - & - & - & 2 & - & - \\
\hline 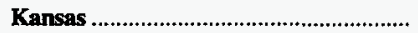 & 291 & 282 & 345 & 354 & 424 & 3.3 & -9.0 \\
\hline West North Central..................................... & 291 & 282 & 345 & 345 & 424 & 3.3 & -9.0 \\
\hline 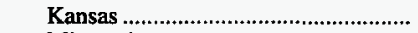 & 160 & 165 & 167 & 165 & 136 & -2.9 & 4.3 \\
\hline 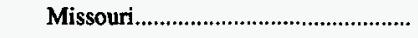 & 131 & 117 & 178 & 181 & 288 & 12.1 & -17.9 \\
\hline Kentucky, Western & 32,990 & 34,729 & 35,151 & 41,453 & 40,395 & $-\mathbf{5 . 0}$ & -4.9 \\
\hline 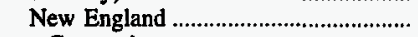 & - & - & - & - & * & - & - \\
\hline 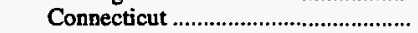 & - & - & - & - & * & - & - \\
\hline Middle Atlantic & - & * & $*$ & * & - & -100.0 & - \\
\hline Pennsylvania & - & * & * & * & - & -100.0 & - \\
\hline East North Central.................................... & 542 & 2,762 & 4,169 & 4,346 & 5,204 & -80.4 & -43.2 \\
\hline 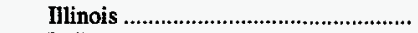 & - & 192 & 659 & 214 & 470 & -100.0 & - \\
\hline 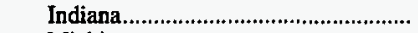 & 243 & 2,214 & 3,229 & 3,275 & 3,482 & -89.0 & -48.6 \\
\hline 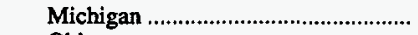 & - & 12 & 28 & 8 & 74 & -100.0 & - \\
\hline 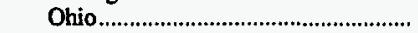 & 103 & 180 & 146 & 514 & 786 & -42.6 & -39.8 \\
\hline 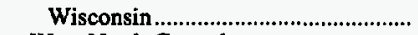 & 196 & 165 & 108 & 336 & 391 & 18.9 & -15.9 \\
\hline West North Central.................................. & 101 & 1,170 & 352 & 896 & 1,027 & -91.4 & -44.1 \\
\hline 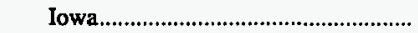 & 75 & 377 & 217 & 271 & 383 & -80.1 & -33.4 \\
\hline Kansas & - & - & - & - & 3 & - & - \\
\hline Minnesota & 19 & 26 & 32 & 27 & 2 & -27.1 & 84.3 \\
\hline
\end{tabular}

See footnotes at end of table. 
Table 61. Domestic Distribution of U.S. Coal by Coal-Producing Region and State, and Destination Census Division and State, 1991-1995 (Continued) (Thousand Short Tons)

\begin{tabular}{|c|c|c|c|c|c|c|c|}
\hline \multirow{2}{*}{$\begin{array}{c}\text { Coal-Producing Region and State, } \\
\text { and Destination Census Division } \\
\text { and State }\end{array}$} & \multirow{2}{*}{1995} & \multirow{2}{*}{1994} & \multirow{2}{*}{1993} & \multirow{2}{*}{1992} & \multirow{2}{*}{1991} & \multirow{2}{*}{$\begin{array}{l}\text { Percent } \\
\text { Change } \\
\text { 1994-1995 }\end{array}$} & \multirow{2}{*}{$\begin{array}{c}\begin{array}{c}\text { Average Annual } \\
\text { Percent Change }\end{array} \\
1991-1995\end{array}$} \\
\hline & & & & & & & \\
\hline \multicolumn{8}{|l|}{ Kentucky, Western (Continued) } \\
\hline 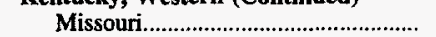 & 6 & 766 & 102 & 598 & 640 & -99.2 & -68.6 \\
\hline South Atlantic & 2,375 & 2,709 & 3,040 & 5,260 & 5,849 & -12.3 & -20.2 \\
\hline Florida & 2,375 & 2,706 & 3,039 & 3,910 & 4,472 & -12.2 & -14.6 \\
\hline Georgia & - & - & - & 1,350 & 1,358 & - & - \\
\hline 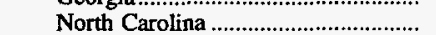 & - & * & - & - & - & -100.0 & - \\
\hline 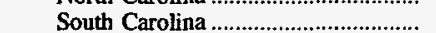 & - & * & - & - & - & -100.0 & - \\
\hline 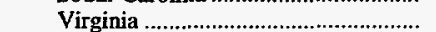 & - & - & - & * & - & - & - \\
\hline 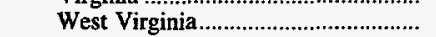 & - & 3 & 1 & * & 19 & -100.0 & - \\
\hline 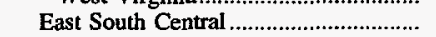 & 29,927 & 28,048 & 27,512 & 30,682 & 27,911 & 6.7 & 1.8 \\
\hline Alabama & 1,717 & 2,038 & 1,761 & 2,310 & 1,627 & -15.8 & 1.3 \\
\hline 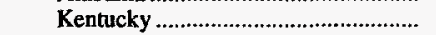 & 17,488 & 16,401 & 17,990 & 17,455 & 16,268 & 6.6 & 1.8 \\
\hline 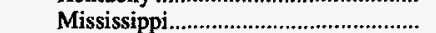 & - & - & 10 & 190 & 169 & - & - \\
\hline 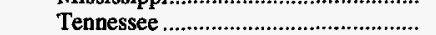 & 10,723 & 9,609 & 7,751 & 10,727 & 9,847 & 11.6 & 2.1 \\
\hline 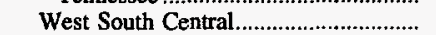 & 13 & 8 & 12 & 204 & 311 & 58.8 & -54.7 \\
\hline 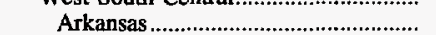 & 13 & 8 & 12 & 8 & 11 & 58.8 & 4.6 \\
\hline 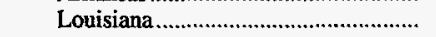 & - & - & - & 196 & 300 & - & - \\
\hline Louisiana & $\mathbf{3 , 4 2 6}$ & 3,463 & 3,103 & 3,208 & 3,151 & -1.1 & 2.1 \\
\hline 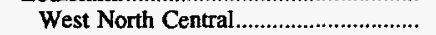 & - & - & * & - & - & - & - \\
\hline 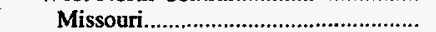 & - & - & * & - & - & - & - \\
\hline 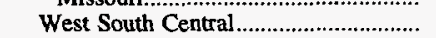 & 3,426 & 3,463 & 3,103 & 3,208 & 3,151 & -1.1 & 2.1 \\
\hline 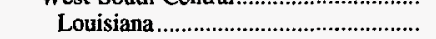 & 3,426 & 3,463 & 3,103 & 3,208 & 3,151 & -1.1 & 2.1 \\
\hline 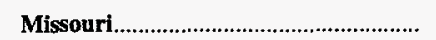 & 464 & 679 & 638 & 2,795 & 2,203 & -31.7 & -32.3 \\
\hline East North Central & - & - & - & - & * & - & - \\
\hline 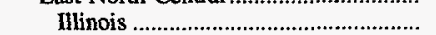 & - & - & - & - & * & - & - \\
\hline 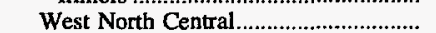 & 464 & 679 & 614 & 2,783 & 2,183 & -31.7 & -32.1 \\
\hline 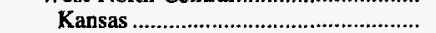 & 91 & 77 & 23 & 54 & 19 & 18.6 & 48.3 \\
\hline 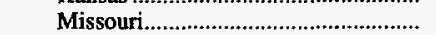 & 373 & 602 & 592 & 2,729 & 2,164 & -38.1 & -35.6 \\
\hline 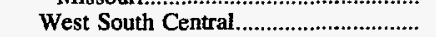 & - & - & 2 & - & - & - & - \\
\hline 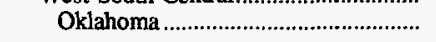 & - & - & 2 & - & - & - & - \\
\hline 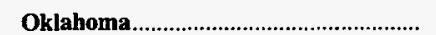 & 2,158 & 1,925 & 2,297 & 1,940 & 1,867 & 12.1 & $\mathbf{3 . 7}$ \\
\hline West North Central........................... & 31 & 27 & 57 & 83 & 23 & 18.3 & 8.4 \\
\hline Kansas & 31 & 22 & 41 & 73 & 23 & 43.2 & 8.4 \\
\hline 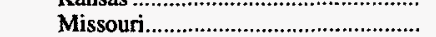 & $*$ & 5 & 16 & 10 & $*$ & -95.3 & 19.4 \\
\hline 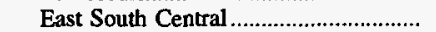 & 2 & - & - & - & - & - & - \\
\hline 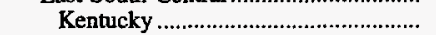 & 2 & - & - & - & - & - & - \\
\hline 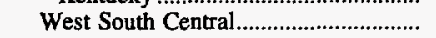 & 2,121 & 1,896 & 2,240 & 1,857 & 1,841 & 11.8 & 3.6 \\
\hline Arkansas & 159 & 205 & 196 & 130 & 94 & -22.4 & 13.9 \\
\hline 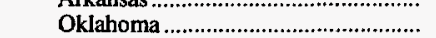 & 1,790 & 1,532 & 1,869 & 1,601 & 1,569 & 16.9 & 3.3 \\
\hline Texas & 171 & 159 & 175 & 126 & 178 & 7.4 & -.9 \\
\hline 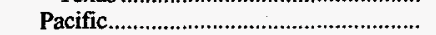 & - & * & * & - & - & -100.0 & - \\
\hline 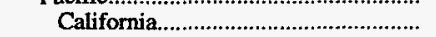 & - & $*$ & $*$ & - & - & -100.0 & - \\
\hline 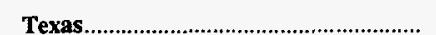 & 52,812 & 52,256 & $\mathbf{5 4 , 2 2 4}$ & $\mathbf{5 4 , 4 4 7}$ & 54,243 & 1.1 & -.7 \\
\hline 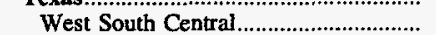 & 52,812 & 52,256 & 54,224 & 54,447 & 54,243 & 1.1 & -.7 \\
\hline 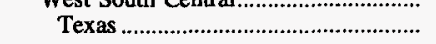 & 52,812 & 52,256 & 54,224 & 54,447 & 54,243 & 1.1 & -.7 \\
\hline Western Total & 420,132 & 398,402 & 364,083 & 339,676 & $\mathbf{3 3 8 , 5 0 8}$ & 5.4 & 5.5 \\
\hline Alaska & 815 & 789 & 855 & 797 & 811 & 3.3 & .1 \\
\hline 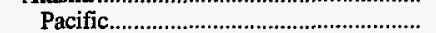 & 815 & 789 & 855 & 797 & 811 & 3.3 & .1 \\
\hline 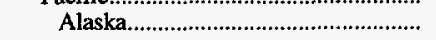 & 815 & 789 & 855 & 797 & 811 & 3.3 & .1 \\
\hline Arizona & 11,783 & 12,011 & 12,138 & 12,418 & 12,933 & -1.9 & -2.3 \\
\hline 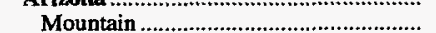 & 11,783 & 12,011 & 12,138 & 12,418 & 12,933 & -1.9 & -2.3 \\
\hline Arizona & 6,956 & 7,580 & 7,566 & 7,441 & 7,869 & -8.2 & -3.0 \\
\hline 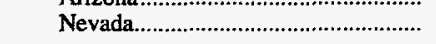 & 4,827 & 4,431 & 4,572 & 4,977 & 5,064 & 8.9 & -1.2 \\
\hline 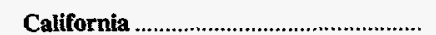 & - & - & - & 142 & 23 & - & - \\
\hline 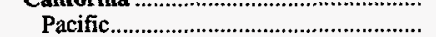 & - & - & - & 142 & 23 & - & - \\
\hline 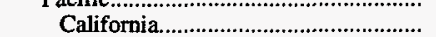 & - & - & - & 142 & 23 & - & - \\
\hline Colorado & 24,734 & 24,059 & 20,338 & 18,195 & $\mathbf{1 7 , 1 3 2}$ & 2.8 & 9.6 \\
\hline East North Central & 2,333 & 2,357 & 2,471 & 1,322 & 1,033 & -1.0 & 22.6 \\
\hline 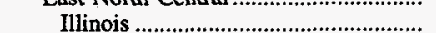 & 1,628 & 1,439 & 1,246 & 518 & 460 & 13.1 & 37.1 \\
\hline 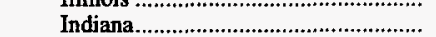 & 20 & 457 & 978 & 794 & 573 & -95.6 & -56.7 \\
\hline
\end{tabular}

See footnotes at end of table. 
Table 61. Domestic Distribution of U.S. Coal by Coal-Producing Region and State, and Destination Census Division and State, 1991-1995 (Continued) (Thousand Short Tons)

\begin{tabular}{|c|c|c|c|c|c|c|c|}
\hline \multirow{2}{*}{$\begin{array}{l}\text { Coal-Producing Region and State, } \\
\text { and Destination Census Division } \\
\text { and State }\end{array}$} & \multirow{2}{*}{1995} & \multirow{2}{*}{1994} & \multirow{2}{*}{1993} & \multirow{2}{*}{1992} & \multirow{2}{*}{1991} & \multirow{2}{*}{$\begin{array}{c}\text { Percent } \\
\text { Change } \\
\text { 1994-1995 }\end{array}$} & \multirow{2}{*}{$\begin{array}{c}\begin{array}{c}\text { Average Annual } \\
\text { Percent Change }\end{array} \\
1991-1995\end{array}$} \\
\hline & & & & & & & \\
\hline \multicolumn{8}{|l|}{ Colorado (Continued) } \\
\hline 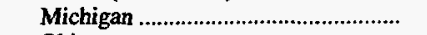 & 44 & - & - & - & - & - & - \\
\hline 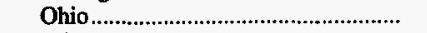 & - & - & - & - & $*$ & - & - \\
\hline Wisconsin & 641 & 462 & 246 & 10 & - & 38.9 & - \\
\hline West North Central.................................... & 3,109 & 2,194 & 871 & 1,013 & 445 & 41.7 & 62.6 \\
\hline 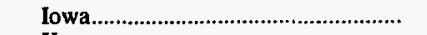 & 550 & 171 & 135 & 122 & - & 221.5 & - \\
\hline 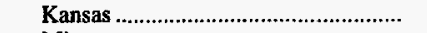 & 1,436 & 1,148 & 90 & 298 & - & 25.2 & - \\
\hline Minnesota & 13 & 23 & 8 & - & - & -44.5 & - \\
\hline 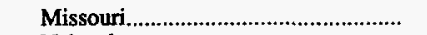 & 1,005 & 775 & 566 & 526 & 386 & 29.6 & 27.0 \\
\hline 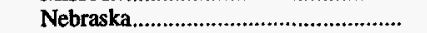 & 104 & 77 & 72 & 67 & 59 & 36.0 & 15.1 \\
\hline 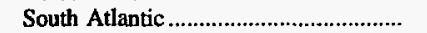 & 811 & 435 & 44 & 181 & - & 86.3 & - \\
\hline Florida & 811 & 423 & - & 181 & - & 91.8 & - \\
\hline 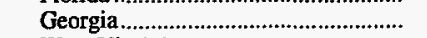 & - & 11 & 44 & - & - & -100.0 & - \\
\hline 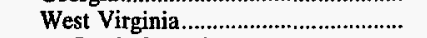 & - & 2 & - & - & - & -100.0 & - \\
\hline East South Central .................................... & 2,797 & 2,038 & 711 & - & - & 37.2 & - \\
\hline 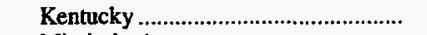 & 1,098 & 710 & - & - & - & 54.7 & - \\
\hline 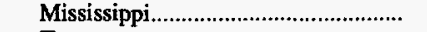 & 963 & 735 & 170 & - & - & 30.9 & - \\
\hline 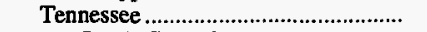 & 736 & 593 & 541 & - & - & 24.2 & - \\
\hline West South Central.................................... & 2,258 & 2,563 & 2,628 & 2,250 & 2,267 & -11.9 & -.1 \\
\hline 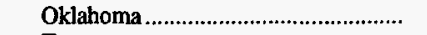 & 31 & 26 & 121 & - & 58 & 17.5 & -14.8 \\
\hline Texas & 2,228 & 2,537 & 2,507 & 2,250 & 2,208 & -12.2 & .2 \\
\hline Mountain & 13,353 & 14,362 & 13,497 & 13,369 & 13,229 & -7.0 & .2 \\
\hline 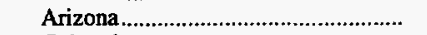 & 105 & 219 & 117 & 226 & 868 & -51.8 & -41.0 \\
\hline 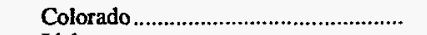 & 11,820 & 12,035 & 11,181 & 11,241 & 10,911 & -1.8 & 2.0 \\
\hline Idaho & 3 & - & - & - & 12 & - & -28.6 \\
\hline 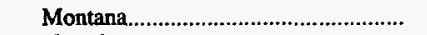 & - & - & - & - & 6 & - & - \\
\hline Nevada & 161 & 306 & 514 & 169 & - & -47.4 & - \\
\hline 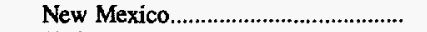 & 97 & 84 & 84 & 69 & 64 & 15.3 & 11.1 \\
\hline Utah & 1,113 & 1,714 & 1,598 & 1,604 & 1,300 & -35.0 & -3.8 \\
\hline 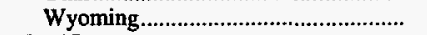 & 53 & 4 & 3 & 60 & 67 & NM & -5.6 \\
\hline Pacific & 37 & 73 & 80 & 60 & 158 & -49.0 & -30.2 \\
\hline 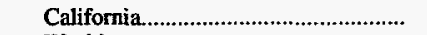 & 1 & 22 & 33 & 24 & 107 & -95.8 & -69.7 \\
\hline 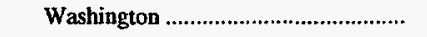 & 36 & 52 & 47 & 37 & 50 & -29.5 & -7.8 \\
\hline 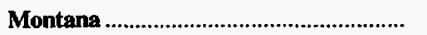 & 39,362 & 41,672 & 35,795 & 38,804 & 37,812 & -5.5 & 1.0 \\
\hline 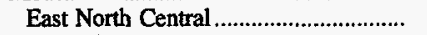 & 16,582 & 17,875 & 15,841 & 15,717 & 16,771 & -7.2 & -.3 \\
\hline 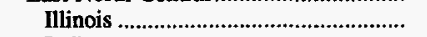 & 2,713 & 4,338 & 3,295 & 3,013 & 3,203 & -37.5 & -4.1 \\
\hline 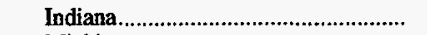 & 720 & 749 & 433 & 451 & 725 & -3.8 & -.2 \\
\hline 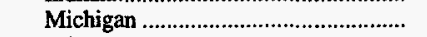 & 11,014 & 10,481 & 10,055 & 10,376 & 10,838 & 5.1 & .4 \\
\hline Wisconsin & 2,135 & 2,307 & 2,057 & 1,878 & 2,005 & -7.4 & 1.6 \\
\hline 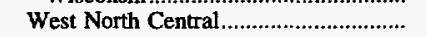 & 11,338 & 10,668 & 9,411 & 9,152 & 10,243 & 6.3 & 2.6 \\
\hline 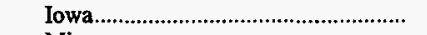 & 2 & $*$ & 1 & - & - & NM & - \\
\hline 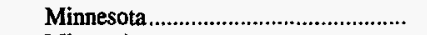 & 10,199 & 10,038 & 8,852 & 8,566 & 9,668 & 1.6 & 1.3 \\
\hline 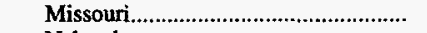 & 6 & - & - & - & - & - & - \\
\hline 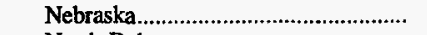 & 205 & 71 & 136 & 142 & 150 & 190.5 & 8.1 \\
\hline 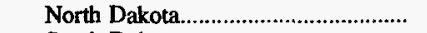 & 469 & 559 & 422 & 444 & 425 & -16.2 & 2.5 \\
\hline 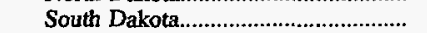 & 457 & - & - & - & - & - & - \\
\hline East South Central .............................. & 1,234 & 1,314 & 178 & 84 & 105 & -6.1 & 84.9 \\
\hline 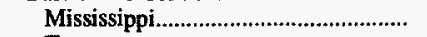 & 1,234 & 1,314 & 178 & 82 & 105 & -6.1 & 84.9 \\
\hline 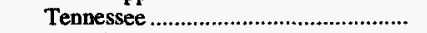 & - & - & - & 2 & - & - & - \\
\hline 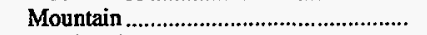 & 9,611 & 10,718 & 9,233 & 11,276 & 10,687 & -10.3 & -2.6 \\
\hline Colorado & 63 & 89 & 86 & 106 & 101 & -29.1 & -11.1 \\
\hline 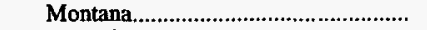 & 9,477 & 10,581 & 9,115 & 11,159 & 10,578 & -10.4 & -2.7 \\
\hline 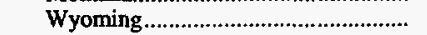 & 71 & 49 & 31 & 11 & 8 & 45.3 & 71.4 \\
\hline 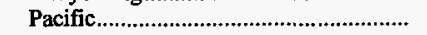 & 583 & 1,097 & 1,108 & 2,549 & - & -46.8 & - \\
\hline 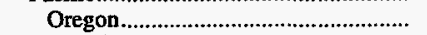 & - & - & 355 & 1,835 & - & - & - \\
\hline 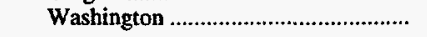 & 583 & 1,097 & 753 & 715 & - & -46.8 & - \\
\hline New Mexico & 25,640 & 28,540 & 27,942 & 24,823 & 22,378 & -10.2 & 3.5 \\
\hline 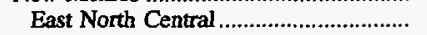 & 1,591 & 1,495 & 1,392 & 590 & 129 & 6.4 & 87.4 \\
\hline Wisconsin & 1,591 & 1,495 & 1,392 & 590 & 129 & 6.4 & 87.4 \\
\hline 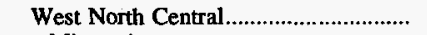 & - & - & - & - & 8 & - & - \\
\hline 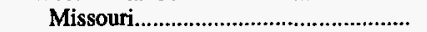 & - & - & - & - & 8 & - & - \\
\hline 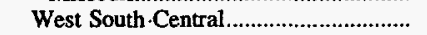 & 160 & 296 & 350 & 216 & 182 & -46.1 & -3.3 \\
\hline 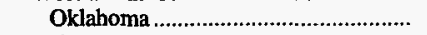 & - & - & 5 & - & - & - & - \\
\hline 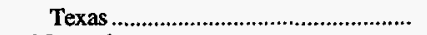 & 160 & 296 & 345 & 216 & 182 & -46.1 & -3.3 \\
\hline 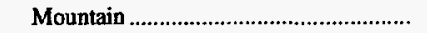 & 23,889 & 26,749 & 26,201 & 24,005 & 21,973 & -10.7 & 2.1 \\
\hline 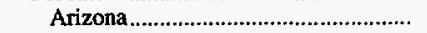 & 9,259 & 11,284 & 11,263 & 9,175 & 8,843 & -17.9 & 1.2 \\
\hline
\end{tabular}

See footnotes at end of table. 
Table 61. Domestic Distribution of U.S. Coal by Coal-Producing Region and State, and Destination Census Division and State, 1991-1995 (Continued) (Thousand Short Tons)

\begin{tabular}{|c|c|c|c|c|c|c|c|}
\hline \multirow{2}{*}{$\begin{array}{c}\text { Coal-Producing Region and State, } \\
\text { and Destination Census Division } \\
\text { and State }\end{array}$} & \multirow[t]{2}{*}{1995} & \multirow[t]{2}{*}{1994} & \multirow[t]{2}{*}{1993} & \multirow[t]{2}{*}{1992} & \multirow[t]{2}{*}{1991} & \multirow{2}{*}{$\begin{array}{l}\text { Percent } \\
\text { Change } \\
\text { 1994-1995 }\end{array}$} & \multirow{2}{*}{$\begin{array}{c}\begin{array}{c}\text { Average Annua } \\
\text { Percent Change }\end{array} \\
1991-1995\end{array}$} \\
\hline & & & & & & & \\
\hline \multicolumn{8}{|l|}{ New Mexico (Continued) } \\
\hline Colorado & - & - & - & - & * & - & - \\
\hline 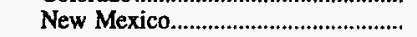 & 14,630 & 15,464 & 14,938 & 14,829 & 13,130 & -5.4 & 2.7 \\
\hline Pacific & - & - & - & 12 & 87 & - & - \\
\hline 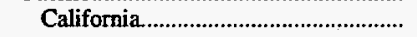 & - & - & - & 12 & 87 & - & - \\
\hline 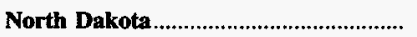 & 30,118 & 32,056 & 32,372 & 31,702 & 29,741 & -6.0 & $\mathbf{3}$ \\
\hline 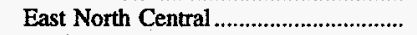 & - & * & * & - & - & -100.0 & - \\
\hline Wisconsin & - & $*$ & $*$ & - & - & -100.0 & - \\
\hline 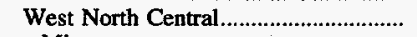 & 30,113 & 32,055 & 32,367 & 31,702 & 29,741 & -6.1 & .3 \\
\hline 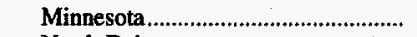 & - & - & - & - & 1 & - & - \\
\hline 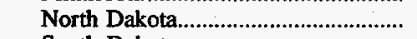 & 28,838 & 29,731 & 30,215 & 29,573 & 27,405 & -3.0 & 1.3 \\
\hline 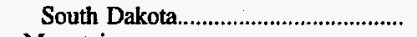 & 1,276 & 2,325 & 2,153 & 2,129 & 2,335 & -45.1 & -14.0 \\
\hline 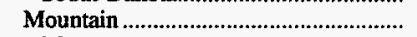 & - & - & $*$ & - & - & - & - \\
\hline 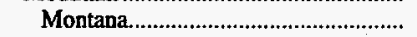 & - & - & * & - & - & - & - \\
\hline Utah & 21,591 & 20,527 & 19,283 & 18,792 & 19,358 & 5.2 & 2.8 \\
\hline 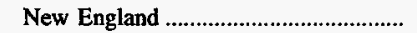 & 17 & - & - & - & - & - & - \\
\hline Connecticut & 17 & - & - & - & - & - & - \\
\hline 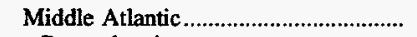 & 20 & 68 & - & - & - & -71.0 & - \\
\hline 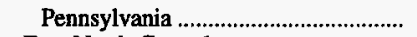 & 20 & 68 & - & - & - & -71.0 & - \\
\hline 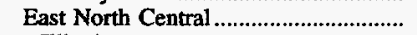 & 1,932 & 656 & 421 & 233 & 10 & 194.7 & 269.3 \\
\hline 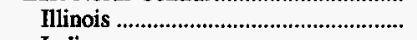 & 1,776 & 369 & 207 & 233 & 10 & 381.5 & 261.6 \\
\hline Indiana & - & 178 & 204 & - & - & -100.0 & - \\
\hline 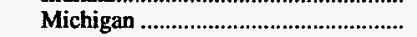 & 76 & 66 & - & - & - & 15.6 & - \\
\hline Ohio & - & - & * & - & - & - & - \\
\hline 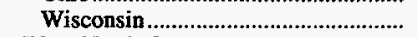 & 81 & 43 & 10 & - & - & 87.0 & - \\
\hline 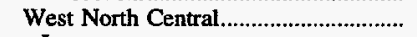 & 395 & 414 & 382 & 80 & $*$ & -4.8 & NM \\
\hline 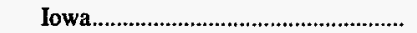 & - & - & - & - & $*$ & - & - \\
\hline 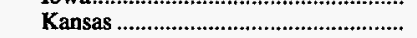 & * & 2 & - & - & - & -96.5 & - \\
\hline Minnesota & 1 & - & - & - & - & - & - \\
\hline 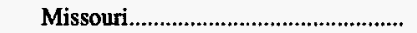 & 393 & 412 & 382 & 79 & - & -4.5 & - \\
\hline 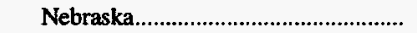 & - & - & * & $*$ & - & - & - \\
\hline 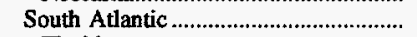 & - & - & 159 & 32 & - & - & - \\
\hline Florida & - & - & 155 & 32 & - & - & - \\
\hline 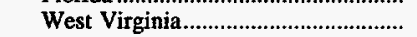 & - & - & 4 & - & - & - & - \\
\hline 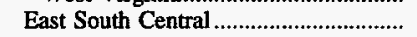 & 1,095 & 218 & - & - & - & 402.3 & - \\
\hline 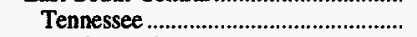 & 1,095 & 218 & - & - & - & 402.3 & - \\
\hline 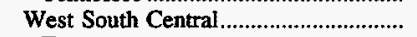 & 4 & 33 & - & - & - & -87.9 & - \\
\hline 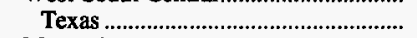 & 4 & 33 & - & - & - & -87.9 & - \\
\hline 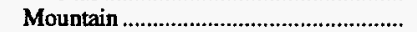 & 15,163 & 15,793 & 15,456 & 15,289 & 16,632 & -4.0 & -2.3 \\
\hline 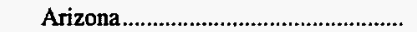 & 80 & 86 & 89 & 101 & 111 & -6.9 & -7.9 \\
\hline Colorado & 6 & 4 & 14 & 34 & 19 & 40.9 & -26.4 \\
\hline 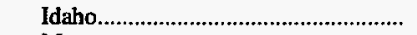 & 141 & 59 & 95 & 61 & 89 & 137.9 & 12.2 \\
\hline 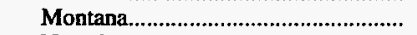 & 9 & 29 & 42 & 42 & 34 & -69.9 & -28.6 \\
\hline 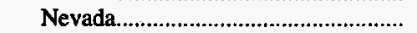 & 2,150 & 2,027 & 1,781 & 1,979 & 2,711 & 6.1 & -5.6 \\
\hline Utah & 12,755 & 13,586 & 13,418 & 13,035 & 13,605 & -6.1 & -1.6 \\
\hline 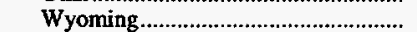 & 22 & 2 & 18 & 37 & 62 & $\mathbf{N M}$ & -22.5 \\
\hline 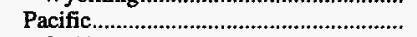 & 2,965 & 3,317 & 2,843 & 3,143 & 2,708 & -10.6 & 2.3 \\
\hline 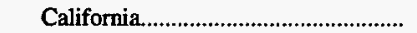 & 2,838 & 3,074 & 2,575 & 2,777 & 2,556 & -7.7 & 2.6 \\
\hline Oregon & 2 & 127 & 123 & 113 & 2 & -98.8 & -.2 \\
\hline 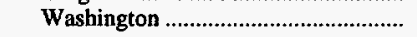 & 126 & 115 & 145 & 253 & 150 & 9.0 & -4.4 \\
\hline Washington & 4,756 & 4,731 & 4,621 & 5,020 & 4,894 & 5 & -.7 \\
\hline 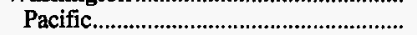 & 4,756 & 4,731 & 4,621 & 5,020 & 4,894 & .5 & -.7 \\
\hline 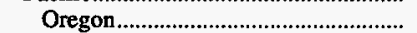 & 2 & - & - & - & - & - & - \\
\hline 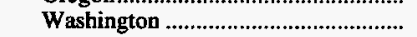 & 4,754 & 4,731 & 4,621 & 5,020 & 4,894 & .5 & -.7 \\
\hline 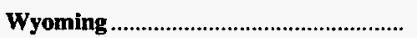 & 261,333 & 234,016 & 210,739 & 188,983 & 193,425 & $\mathbf{1 1 . 7}$ & 7.8 \\
\hline 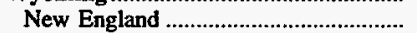 & - & $*$ & 14 & - & $*$ & -100.0 & - \\
\hline 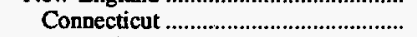 & - & * & - & - & * & -100.0 & - \\
\hline 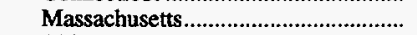 & - & - & 14 & - & - & - & - \\
\hline 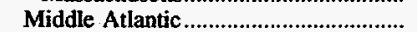 & - & - & - & * & 9 & - & - \\
\hline 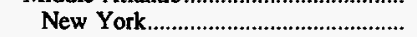 & - & - & - & - & 9 & - & - \\
\hline 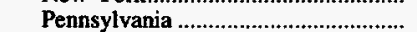 & - & - & - & * & - & - & - \\
\hline 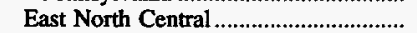 & 55,223 & 42,685 & 37,648 & 30,920 & 31,402 & 29.4 & 15.2 \\
\hline 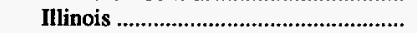 & 15,480 & 9,779 & 7,593 & 4,417 & 4,394 & 58.3 & 37.0 \\
\hline Indiana & 18,306 & 15,417 & 12,635 & 11,804 & 11,967 & 18.7 & 11.2 \\
\hline
\end{tabular}

See footnotes at end of table. 
Table 61. Domestic Distribution of U.S. Coal by Coal-Producing Region and State, and Destination Census Division and State, 1991-1995 (Continued) (Thousand Short Tons)

\begin{tabular}{|c|c|c|c|c|c|c|c|}
\hline \multirow{2}{*}{$\begin{array}{l}\text { Coal-Producing Region and State, } \\
\text { and Destination Census Division } \\
\text { and State }\end{array}$} & \multirow[t]{2}{*}{1995} & \multirow[t]{2}{*}{1994} & \multirow[t]{2}{*}{1993} & \multirow[t]{2}{*}{1992} & \multirow[t]{2}{*}{1991} & \multirow{2}{*}{$\begin{array}{c}\text { Percent } \\
\text { Change } \\
\text { 1994-1995 }\end{array}$} & \multirow{2}{*}{$\begin{array}{c}\begin{array}{c}\text { Average Annual } \\
\text { Percent Change }\end{array} \\
1991-1995\end{array}$} \\
\hline & & & & & & & \\
\hline \multicolumn{8}{|l|}{ Wyoming (Continued) } \\
\hline 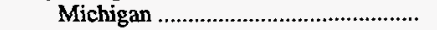 & 7,543 & 4,267 & 4,487 & 2,948 & 2,762 & 76.8 & 28.5 \\
\hline Ohio & - & - & - & 124 & 45 & - & - \\
\hline 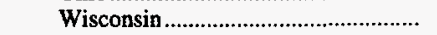 & 13,895 & 13,221 & 12,934 & 11,627 & 12,234 & 5.1 & 3.2 \\
\hline 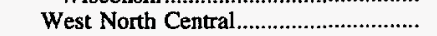 & 77,051 & 67,523 & 61,641 & 52,269 & 53,239 & 14.1 & 9.7 \\
\hline 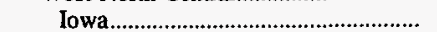 & 16,955 & 15,505 & 15,950 & 14,056 & 14,614 & 9.3 & 3.8 \\
\hline 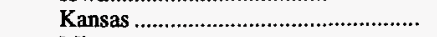 & 14,243 & 16,490 & 16,484 & 13,526 & 14,016 & -13.6 & .4 \\
\hline 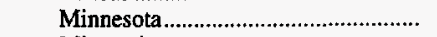 & 8,816 & 9,911 & 9,093 & 8,385 & 7,531 & -11.0 & 4.0 \\
\hline 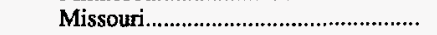 & 25,731 & 16,112 & 10,815 & 7,958 & 7,976 & 59.7 & 34.0 \\
\hline 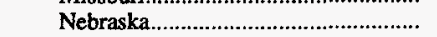 & 10,065 & 8,908 & 8,801 & 7,882 & 8,583 & 13.0 & 4.1 \\
\hline 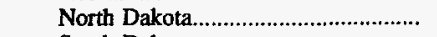 & * & - & 5 & 5 & * & - & 182.8 \\
\hline 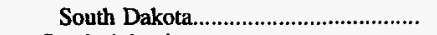 & 1,241 & 597 & 493 & 456 & 519 & 107.8 & 24.4 \\
\hline 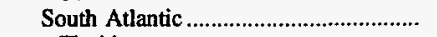 & 7,432 & 5,836 & 938 & 12 & 1,128 & 27.3 & 60.2 \\
\hline 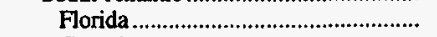 & - & 93 & - & 12 & - & -100.0 & - \\
\hline 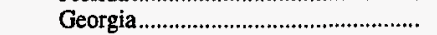 & 6,796 & 4,914 & 726 & - & 1,128 & 38.3 & 56.7 \\
\hline 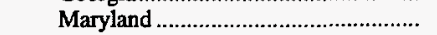 & 636 & 829 & 213 & - & - & -23.3 & - \\
\hline 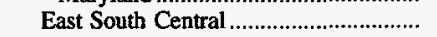 & 2,970 & 594 & 317 & 156 & 991 & 400.3 & 31.6 \\
\hline 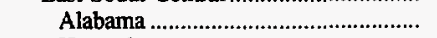 & 2,950 & 251 & - & - & 115 & NM & 125.3 \\
\hline 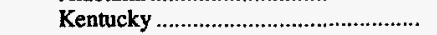 & - & - & 248 & 1 & 238 & - & - \\
\hline 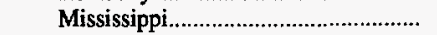 & - & - & - & 83 & - & - & - \\
\hline 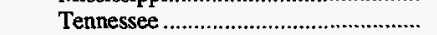 & 20 & 342 & 68 & 72 & 639 & -94.2 & -58.0 \\
\hline West South Central..................................... & 82,918 & 80,246 & 76,692 & 72,339 & 72,484 & 3.3 & 3.4 \\
\hline 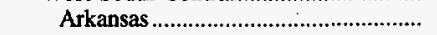 & 14,033 & 12,184 & 10,826 & 11,571 & 12,268 & 15.2 & 3.4 \\
\hline 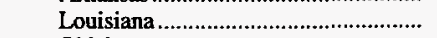 & 10,309 & 11,215 & 11,133 & 10,039 & 9,690 & -8.1 & 1.6 \\
\hline 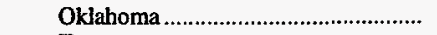 & 20,326 & 17,577 & 16,726 & 16,733 & 15,489 & 15.6 & 7.0 \\
\hline Texas & 38,250 & 39,270 & 38,007 & 33,995 & 35,036 & -2.6 & 2.2 \\
\hline 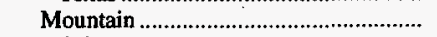 & 32,950 & 34,935 & 32,195 & 33,165 & 32,177 & -5.7 & .6 \\
\hline 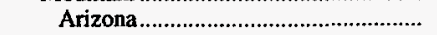 & - & - & 5 & - & - & - & - \\
\hline Colorado & 5,602 & 5,132 & 5,538 & 4,952 & 5,321 & 9.2 & 1.3 \\
\hline 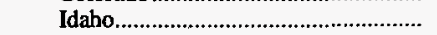 & 293 & 337 & 343 & 393 & 455 & -13.1 & -10.4 \\
\hline 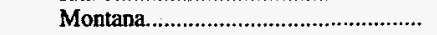 & 193 & 119 & 37 & 44 & 104 & 62.7 & 16.6 \\
\hline 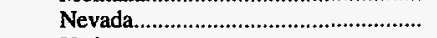 & 342 & 1,014 & 753 & 802 & 797 & -66.3 & -19.1 \\
\hline 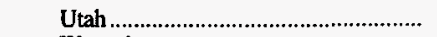 & $*$ & - & - & 586 & 216 & - & -88.8 \\
\hline Wyoming & 26,521 & 28,334 & 25,519 & 26,388 & 25,284 & -6.4 & 1.2 \\
\hline 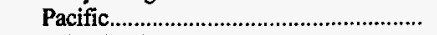 & 2,775 & 2,198 & 1,271 & 120 & 1,982 & 26.3 & 8.8 \\
\hline 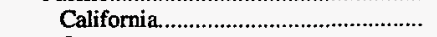 & - & - & - & - & 58 & - & - \\
\hline 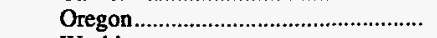 & 1,485 & 2,197 & 1,270 & 114 & 1,892 & -32.4 & -5.9 \\
\hline 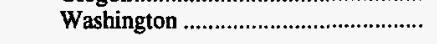 & 1,290 & 1 & 1 & 6 & 33 & NM & 151.0 \\
\hline U.S. Total & 940,423 & 949,843 & 883,934 & 897,267 & $\mathbf{8 8 5 , 8 8 2}$ & -1.0 & 1.5 \\
\hline 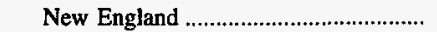 & 5,199 & 4,963 & 4,141 & 7,717 & 6,276 & 4.8 & -4.6 \\
\hline 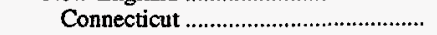 & 1,955 & 976 & 695 & 1,003 & 1,012 & 100.3 & 17.9 \\
\hline 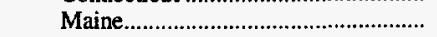 & 302 & 463 & 405 & 866 & 209 & -34.8 & 9.6 \\
\hline 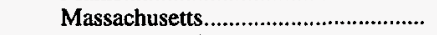 & 2,157 & 2,538 & 1,840 & 4,634 & 3,737 & -15.0 & -12.8 \\
\hline 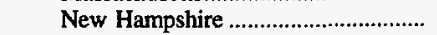 & 780 & 978 & 1,192 & 1,189 & 1,302 & -20.2 & -12.0 \\
\hline 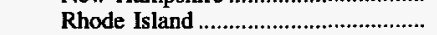 & 3 & 3 & 3 & 5 & 4 & -18.4 & -10.3 \\
\hline 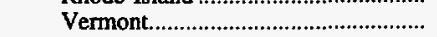 & 3 & 5 & 6 & 20 & 12 & -43.0 & -30.9 \\
\hline 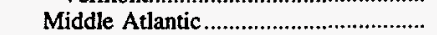 & 70,149 & 69,737 & 64,421 & 73,087 & 69,990 & .6 & .1 \\
\hline 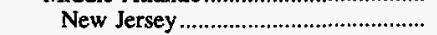 & 2,936 & 2,163 & 2,169 & 2,433 & 1,878 & 35.7 & 11.8 \\
\hline 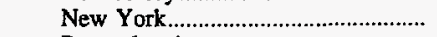 & 10,705 & 11,664 & 10,950 & 13,977 & 13,666 & -8.2 & -5.9 \\
\hline 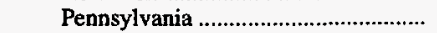 & 56,509 & 55,910 & 51,302 & 56,677 & 54,446 & 1.1 & .9 \\
\hline 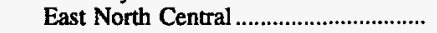 & 212,105 & 214,903 & 196,343 & 201,713 & 202,545 & -1.3 & 1.2 \\
\hline 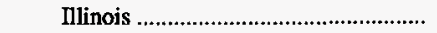 & 41,626 & 38,745 & 34,433 & 31,186 & 33,031 & 7.4 & 5.9 \\
\hline 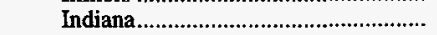 & 59,476 & 63,589 & 54,452 & 60,970 & 57,105 & -6.5 & 1.0 \\
\hline 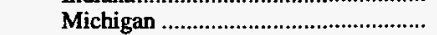 & 33,928 & 33,487 & 30,041 & 30,363 & 32,046 & 1.3 & 1.4 \\
\hline Ohio & 54,310 & 56,850 & 56,755 & 58,716 & 58,752 & -4.5 & -1.9 \\
\hline Wisconsin & 22,766 & 22,231 & 20,662 & 20,477 & 21,611 & 2.4 & 1.3 \\
\hline 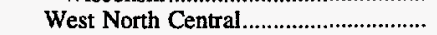 & 130,836 & 126,407 & 116,337 & 113,921 & 115,949 & 3.5 & 3.1 \\
\hline Iowa & 19,777 & 18,259 & 18,950 & 16,988 & 18,212 & 8.3 & 2.1 \\
\hline 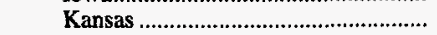 & 16,091 & 18,097 & 17,002 & 14,801 & 15,599 & -11.1 & .8 \\
\hline 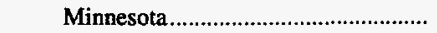 & 19,488 & 20,498 & 18,166 & 17,224 & 18,051 & -4.9 & 1.9 \\
\hline 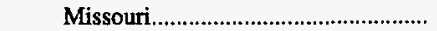 & 32,821 & 27,278 & 19,921 & 24,207 & 24,602 & 20.3 & 7.5 \\
\hline 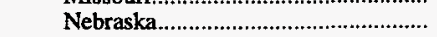 & 10,377 & 9,055 & 9,011 & 8,093 & 8,796 & 14.6 & 4.2 \\
\hline 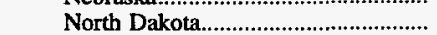 & 29,307 & 30,290 & 30,642 & 30,022 & 27,831 & -3.3 & 1.3 \\
\hline 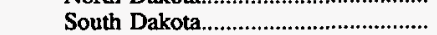 & 2,976 & 2,929 & 2,646 & 2,586 & 2,859 & 1.6 & 1.0 \\
\hline 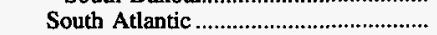 & 151,954 & 161,029 & 141,701 & 145,219 & 143,809 & -5.6 & 1.4 \\
\hline Delaware & 1,933 & 2,309 & 2,242 & 1,913 & 2,219 & -16.3 & -3.4 \\
\hline District of Columbia & 6 & 47 & 51 & 50 & 66 & -87.9 & -45.8 \\
\hline
\end{tabular}

See footnotes at end of table. 
Table 61. Domestic Distribution of U.S. Coal by Coal-Producing Region and State, and Destination Census Division and State, 1991-1995 (Continued) (Thousand Short Tons)

\begin{tabular}{|c|c|c|c|c|c|c|c|}
\hline \multirow{2}{*}{$\begin{array}{c}\text { Coal-Producing Region and State, } \\
\text { and Destination Census Division } \\
\text { and State }\end{array}$} & \multirow{2}{*}{1995} & \multirow{2}{*}{1994} & \multirow{2}{*}{1993} & \multirow{2}{*}{1992} & \multirow{2}{*}{1991} & \multirow{2}{*}{$\begin{array}{c}\text { Percent } \\
\text { Change } \\
\text { 1994-1995 }\end{array}$} & \multirow{2}{*}{$\begin{array}{c}\begin{array}{c}\text { Average Annual } \\
\text { Percent Change }\end{array} \\
1991-1995\end{array}$} \\
\hline & & & & & & & \\
\hline \multicolumn{8}{|l|}{ U.S. Total (Continued) } \\
\hline Florida & 23,505 & 23,757 & 21,446 & 24,351 & 23,378 & -1.1 & 0.1 \\
\hline Georgia & 29,730 & 30,497 & 25,101 & 24,242 & 25,418 & -2.5 & 4.0 \\
\hline 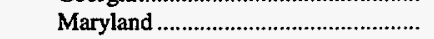 & 10,596 & 10,959 & 10,063 & 9,735 & 10,712 & -3.3 & -.3 \\
\hline North Carolina & 24,149 & 26,199 & 25,621 & 25,801 & 22,054 & -7.8 & 2.3 \\
\hline 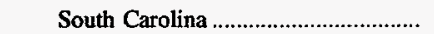 & 11,745 & 13,454 & 11,749 & 11,338 & 11,332 & -12.7 & .9 \\
\hline 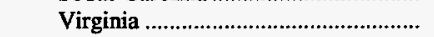 & 15,225 & 16,735 & 16,084 & 14,915 & 15,159 & -9.0 & .1 \\
\hline 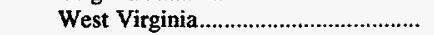 & 35,065 & 37,071 & 29,343 & 32,874 & 33,473 & -5.4 & 1.2 \\
\hline 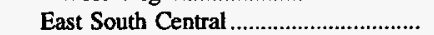 & 104,194 & 99,350 & 97,057 & 92,761 & 89,160 & 4.9 & 4.0 \\
\hline Alabama & 31,440 & 30,210 & 28,698 & 28,741 & 28,739 & 4.1 & 2.3 \\
\hline Kentucky & 37,382 & 35,636 & 35,461 & 33,497 & 31,656 & 4.9 & 4.2 \\
\hline 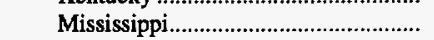 & 4,516 & 4,446 & 3,421 & 3,965 & 3,966 & 1.6 & 3.3 \\
\hline Tennessee & 30,856 & 29,057 & 29,477 & 26,559 & 24,799 & 6.2 & 5.6 \\
\hline 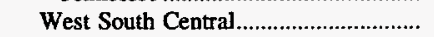 & 144,435 & 142,136 & 139,664 & 134,770 & 134,870 & 1.6 & 1.7 \\
\hline 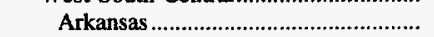 & 14,317 & 12,501 & 11,094 & 11,807 & 12,481 & 14.5 & 3.5 \\
\hline Louisiana & 14,267 & 15,844 & 14,524 & 13,539 & 13,392 & -10.0 & 1.6 \\
\hline Oklahoma & 22,210 & 19,210 & 18,777 & 18,362 & 17,136 & 15.6 & 6.7 \\
\hline 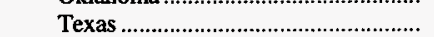 & 93,642 & 94,581 & 95,269 & 91,062 & 91,862 & -1.0 & .5 \\
\hline 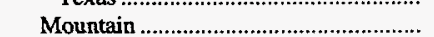 & 107,497 & 115,311 & 109,200 & 110,007 & 108,329 & -6.8 & -.2 \\
\hline Arizona & 16,401 & 19,169 & 19,039 & 16,944 & 17,691 & -14.4 & -1.9 \\
\hline Colorado & 17,502 & 17,274 & 16,821 & 16,333 & 16,352 & 1.3 & 1.7 \\
\hline 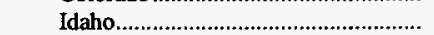 & 440 & 400 & 438 & 456 & 556 & 9.9 & -5.7 \\
\hline 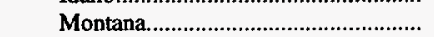 & 9,678 & 10,729 & 9,194 & 11,245 & 10,924 & -9.8 & -3.0 \\
\hline 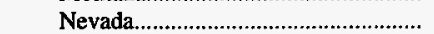 & 7,479 & 7,777 & 7,621 & 7,926 & 8,572 & -3.8 & -3.3 \\
\hline New Mexico & 14,727 & 15,549 & 15,022 & 14,899 & 13,194 & -5.3 & 2.8 \\
\hline Utah & 14,602 & 16,015 & 15,493 & 15,707 & 15,619 & -8.8 & -1.7 \\
\hline 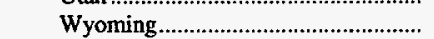 & 26,668 & 28,399 & 25,571 & 26,497 & 25,421 & -6.1 & 1.2 \\
\hline 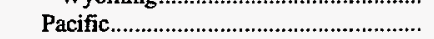 & 11,962 & 12,228 & 10,791 & 11,844 & 10,664 & -2.2 & 2.9 \\
\hline 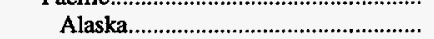 & 815 & 789 & 855 & 797 & 811 & 3.3 & .1 \\
\hline California & 2,839 & 3,096 & 2,608 & 2,955 & 2,833 & -8.3 & .1 \\
\hline Oregon & 1,518 & 2,347 & 1,761 & 2,062 & 1,893 & -35.3 & -5.4 \\
\hline Washington & 6,790 & 5,996 & 5,567 & 6,030 & 5,127 & 13.2 & 7.3 \\
\hline
\end{tabular}

* Data round to zero.

NM Not meaningful as value is greater than 500 percent.

Note: Totals may not equal sum of components due to independent rounding.

Source: Energy Information Administration, Form EIA-6, "Coal Distribution Report." 
Table 62. Foreign Distribution of U.S. Coal by Major Coal-Exporting States and Destination, 1991-1995

(Thousand Short Tons)

\begin{tabular}{|c|c|c|c|c|c|c|c|}
\hline \multirow{2}{*}{$\begin{array}{l}\text { Coal-Exporting State } \\
\text { and Destination }\end{array}$} & \multirow[t]{2}{*}{1995} & \multirow[t]{2}{*}{1994} & \multirow[t]{2}{*}{1993} & \multirow[t]{2}{*}{1992} & \multirow[t]{2}{*}{1991} & \multirow{2}{*}{$\begin{array}{c}\text { Percent } \\
\text { Change } \\
\text { 1994-1995 }\end{array}$} & \multirow{2}{*}{$\begin{array}{c}\begin{array}{c}\text { Average Annual } \\
\text { Percent Change }\end{array} \\
1991-1995\end{array}$} \\
\hline & & & & & & & \\
\hline 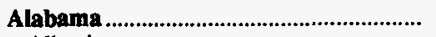 & 6,032 & 4,529 & 5,888 & 5,931 & 7,012 & 33.2 & -3.7 \\
\hline 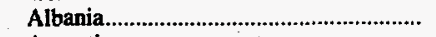 & - & - & - & 22 & 63 & - & - \\
\hline 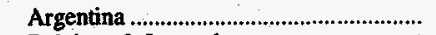 & 306 & 268 & 351 & 170 & 223 & 14.4 & 8.3 \\
\hline Belgium \& Luxembourg ............................... & 574 & 627 & 952 & 856 & 1,074 & -8.3 & -14.5 \\
\hline 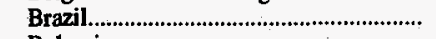 & 564 & 42 & 212 & - & 112 & NM & 49.7 \\
\hline 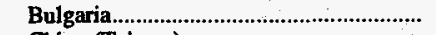 & 128 & 35 & 120 & - & $\overline{-}$ & 260.0 & - \\
\hline China (Taiwan) & - & - & 46 & 49 & 87 & - & - \\
\hline 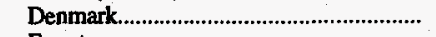 & 26 & - & - & - & - & - & - \\
\hline 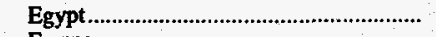 & 111 & - & - & - & - & - & - \\
\hline France & - & $*$ & - & - & - & -100.0 & - \\
\hline Germany, FR & 59 & - & - & - & - & - & - \\
\hline 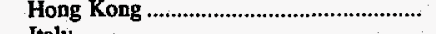 & - & $\overline{s e}$ & - & - & 89 & - & - \\
\hline Italy & $\begin{array}{r}930 \\
1358\end{array}$ & $\begin{array}{r}565 \\
266\end{array}$ & $2^{*}$ & 138 & 443 & 64.7 & 20.3 \\
\hline & 1,358 & 1,266 & 2,422 & 2,536 & 2,920 & 7.2 & -17.4 \\
\hline Morocco & $4 \overline{18}$ & $\overline{88}$ & 43 & $\overline{77}$ & - & - & - \\
\hline & $\begin{array}{l}418 \\
492\end{array}$ & 602 & 188 & $\begin{array}{r}7 \\
199\end{array}$ & $3 \overline{3}$ & 374.4 & $\overline{1}$ \\
\hline South Africa, Rep of & - & - & - & 229 & $\begin{array}{r}373 \\
-\end{array}$ & -18.3 & 7.1 \\
\hline Spain & 48 & 27 & - & - & - & 80.7 & - \\
\hline 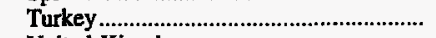 & 302 & 137 & 278 & 459 & 386 & 119.6 & -6.0 \\
\hline United Kingdom & 717 & 872 & 1,277 & 1,197 & 1,213 & -17.8 & -12.3 \\
\hline 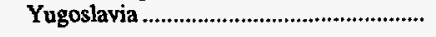 & - & - & - & - & 29 & - & - \\
\hline 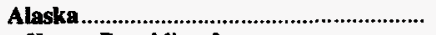 & 855 & 716 & 743 & 734 & 672 & 19.3 & 6.2 \\
\hline 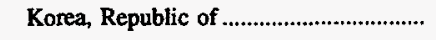 & 855 & 716 & 743 & 734 & 672 & 19.3 & 6.2 \\
\hline 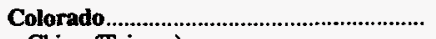 & 900 & 752 & 1,128 & 669 & 625 & 19.8 & 9.6 \\
\hline China (Taiwan) & 235 & 134 & - & - & - & 75.0 & - \\
\hline 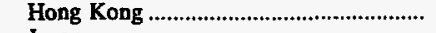 & - & 46 & - & - & - & -100.0 & - \\
\hline Japan & 651 & 395 & 918 & 669 & 625 & 65.0 & 1.0 \\
\hline Korea, Republic of ..................................... & - & 177 & 209 & - & - & -100.0 & - \\
\hline 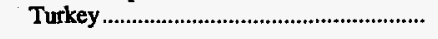 & 14 & - & - & - & - & - & - \\
\hline 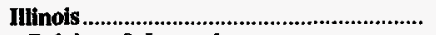 & 2,699 & 236 & 670 & 1,242 & 1,263 & NM & 20.9 \\
\hline Belgium \& Luxembourg .............................. & - & - & - & 58 & 79 & - & - \\
\hline 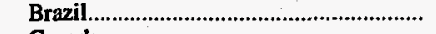 & - & - & - & 298 & 249 & - & - \\
\hline 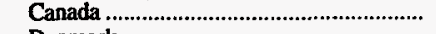 & - & - & - & $\tilde{-}$ & 4 & - & - \\
\hline Denmark & 516 & - & - & 45 & - & - & - \\
\hline 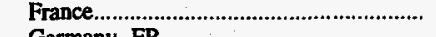 & 57 & - & - & - & - & - & - \\
\hline Germany, FR & 722 & - & - & - & - & - & - \\
\hline Ireland & - & - & 108 & 213 & 225 & - & - \\
\hline Italy & 42 & - & - & - & - & - & - \\
\hline Japan & 49 & 236 & 109 & 53 & 59 & -79.2 & -4.7 \\
\hline 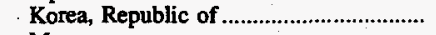 & $\overline{-}$ & - & - & 50 & 34 & - & - \\
\hline 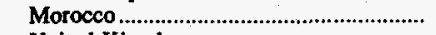 & 775 & - & 452 & 350 & 464 & - & 13.7 \\
\hline United Kingdom & 538 & - & - & 175 & 148 & - & 38.0 \\
\hline 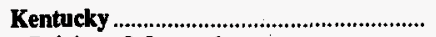 & 9,695 & 7,167 & $\mathbf{9 , 5 2 1}$ & 14,036 & 15,478 & 35.3 & -11.0 \\
\hline 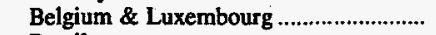 & 363 & 583 & 601 & 651 & 973 & -37.7 & -21.9 \\
\hline 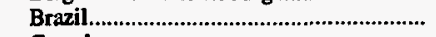 & 52 & 29 & 478 & 110 & 77 & 82.0 & -9.3 \\
\hline Canada & 777 & 1,099 & 1,416 & 2,078 & 1,560 & -29.3 & -16.0 \\
\hline China (Taiwan) & 2,376 & 3,260 & 4,419 & 3,758 & 4,329 & -27.1 & -13.9 \\
\hline Denmark & - & - & 40 & 193 & 779 & - & - \\
\hline Finland & - & - & 2 & - & - & - & - \\
\hline 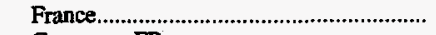 & 260 & 196 & 446 & 1,764 & 2,304 & 32.7 & -42.0 \\
\hline 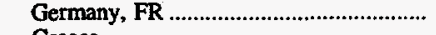 & 186 & - & - & - & - & - & - \\
\hline Greece & - & - & - & - & 91 & - & - \\
\hline 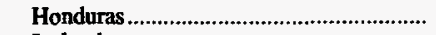 & - & - & - & - & * & - & - \\
\hline 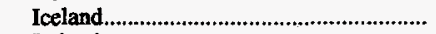 & 76 & 9 & - & 35 & - & $\mathbf{N M}$ & - \\
\hline 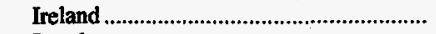 & 57 & - & 19 & 379 & 13 & - & 44.0 \\
\hline 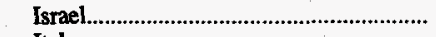 & 215 & - & - & - & - & - & - \\
\hline Italy & 1,698 & 993 & 998 & 930 & 1,013 & 71.0 & 13.8 \\
\hline 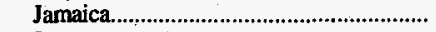 & 62 & 32 & 47 & 39 & 59 & 94.7 & 1.3 \\
\hline 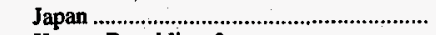 & 133 & 124 & 328 & 342 & 539 & 7.3 & -29.5 \\
\hline Korea, Republic of & 1,509 & - & - & - & - & - & - \\
\hline 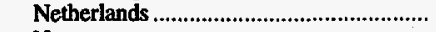 & 616 & 331 & 294 & 2,229 & 1,649 & 86.1 & -21.8 \\
\hline 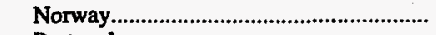 & 140 & 91 & 110 & 110 & 158 & 54.6 & -2.9 \\
\hline 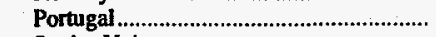 & - & 30 & - & - & 183 & -100.0 & - \\
\hline 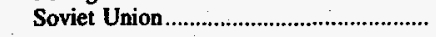 & - & - & - & - & 64 & - & - \\
\hline
\end{tabular}

See footnotes at end of table. 
Table 62. Foreign Distribution of U.S. Coal by Major Coal-Exporting States and Destination, 1991-1995 (Continued)

(Thousand Short Tons)

\begin{tabular}{|c|c|c|c|c|c|c|c|}
\hline \multirow{2}{*}{$\begin{array}{l}\text { Coal-Exporting State } \\
\text { and Destination }\end{array}$} & \multirow{2}{*}{1995} & \multirow{2}{*}{1994} & \multirow{2}{*}{1993} & \multirow{2}{*}{1992} & \multirow{2}{*}{1991} & \multirow{2}{*}{$\begin{array}{c}\text { Percent } \\
\text { Change } \\
\text { 1994-1995 }\end{array}$} & \multirow{2}{*}{$\begin{array}{c}\begin{array}{c}\text { Average Annua } \\
\text { Percent Change }\end{array} \\
1991-1995\end{array}$} \\
\hline & & & & & & & \\
\hline \multicolumn{8}{|l|}{ Kentucky (Continued) } \\
\hline Spain & 229 & - & - & 111 & 170 & - & 3.8 \\
\hline Sweden & - & 19 & 238 & 514 & 343 & -100.0 & - \\
\hline 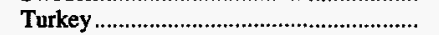 & 195 & - & - & 170 & 260 & - & -6.9 \\
\hline 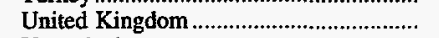 & 752 & 371 & 86 & 581 & 835 & 102.7 & -2.6 \\
\hline 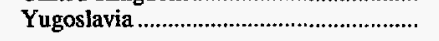 & - & - & - & 42 & 79 & - & - \\
\hline 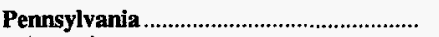 & 8,279 & 6,301 & 5,508 & 6,440 & 7,303 & 31.4 & 3.2 \\
\hline 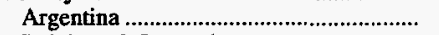 & - & - & 46 & - & - & & - \\
\hline Belgium \& Luxembourg ……………….......... & - & 29 & - & 91 & 305 & -100.0 & - \\
\hline 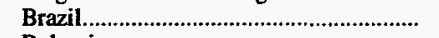 & 380 & 338 & 156 & 101 & 23 & 12.5 & 102.3 \\
\hline Bulgaria & - & - & 71 & 73 & 106 & - & - \\
\hline 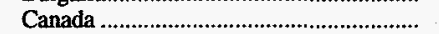 & 713 & 844 & 597 & 1,599 & 2,338 & -15.5 & -25.7 \\
\hline Chile & - & - & - & 38 & 206 & - & - \\
\hline 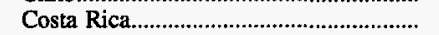 & - & - & - & * & $*$ & - & - \\
\hline 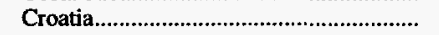 & - & - & 45 & - & - & - & - \\
\hline 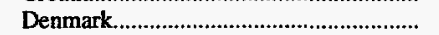 & 1,589 & 508 & 187 & 1,456 & 795 & 212.5 & 18.9 \\
\hline Dominican Republic..................................... & 18 & 65 & 59 & 158 & 201 & -71.5 & -45.0 \\
\hline 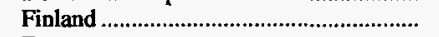 & 544 & 71 & 56 & - & - & NM & - \\
\hline 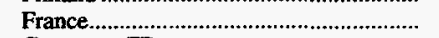 & - & - & 188 & 186 & - & - & - \\
\hline Germany, FR & 202 & 197 & 102 & 153 & 29 & 2.6 & 62.3 \\
\hline Indonesia & - & & & * & - & - & - \\
\hline 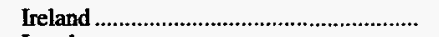 & 1,161 & 1,015 & 911 & - & - & 14.4 & - \\
\hline 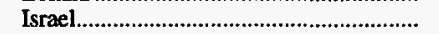 & 995 & 922 & 940 & 1,101 & 987 & 7.9 & .2 \\
\hline Italy …………… & - & 601 & 164 & 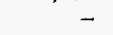 & - & -100.0 & - \\
\hline 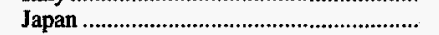 & 916 & 834 & 1,384 & 931 & 1,389 & 9.9 & -9.9 \\
\hline 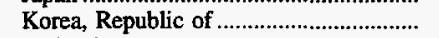 & 109 & 214 & 91 & - & - & -49.1 & - \\
\hline Malaysia & - & - & - & * & * & - & - \\
\hline 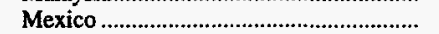 & - & - & - & 18 & - & - & - \\
\hline Morocco & - & - & - & $\overline{-}$ & 185 & - & - \\
\hline 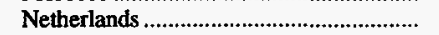 & 783 & 261 & - & 2 & - & 199.8 & - \\
\hline 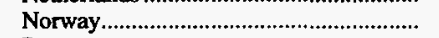 & 28 & 14 & 30 & 49 & 66 & 98.9 & -19.1 \\
\hline 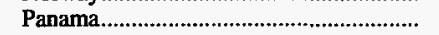 & - & - & - & * & $*$ & - & - \\
\hline 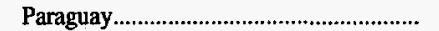 & - & - & - & 3 & - & - & - \\
\hline 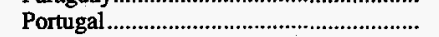 & 472 & 378 & 467 & 305 & 492 & 24.9 & -1.0 \\
\hline 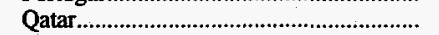 & - & - & - & * & - & - & - \\
\hline Saudi Arabia & - & - & * & - & - & - & - \\
\hline 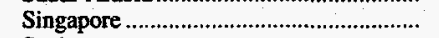 & - & - & - & - & * & - & - \\
\hline 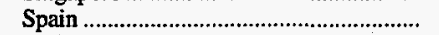 & 18 & - & - & - & 130 & - & -38.8 \\
\hline 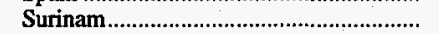 & - & - & $*$ & * & - & - & - \\
\hline Sweden & - & - & - & 1 & - & - & - \\
\hline 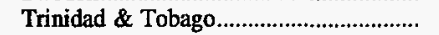 & - & - & 1 & 2 & 2 & - & - \\
\hline 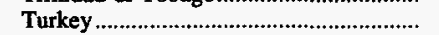 & 43 & - & - & - & 11 & - & 39.6 \\
\hline United Arab Emirates...................................... & - & - & * & - & - & - & \\
\hline 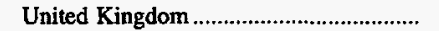 & 299 & - & $*$ & - & * & - & NM \\
\hline 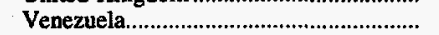 & 9 & 11 & 15 & 39 & 39 & -18.5 & -30.1 \\
\hline 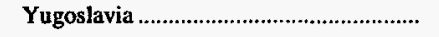 & - & - & - & 134 & - & - & - \\
\hline 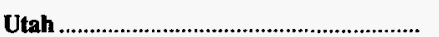 & $\mathbf{3 , 9 3 0}$ & 2,698 & 2,959 & 2,260 & 2,086 & 45.7 & 17.2 \\
\hline 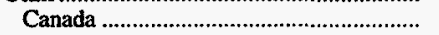 & - & - & 346 & - & - & - & - \\
\hline Chile & 117 & - & - & - & $-\bar{s}$ & - & $\bar{\varepsilon}$ \\
\hline 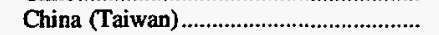 & 355 & 321 & 849 & 721 & 506 & 10.7 & -8.5 \\
\hline 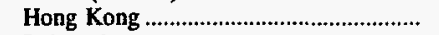 & - & - & - & 359 & 433 & - & - \\
\hline Indonesia & - & - & - & - & & - & - \\
\hline 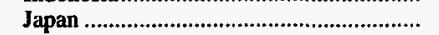 & 2,977 & 2,377 & 1,764 & 1,180 & 1,147 & 25.3 & 26.9 \\
\hline Korea, Republic of $\ldots$ & 481 & - & - & - & - & - & - \\
\hline Mexico & - & - & * & - & - & - & - \\
\hline Virginia & $\mathbf{9 , 7 4 2}$ & 11,683 & 14,251 & 17,224 & 18,105 & -16.6 & -14.3 \\
\hline Albania & & & - & - & 20 & - & \\
\hline Algeria & 167 & 250 & 466 & 670 & 427 & -33.3 & -20.9 \\
\hline 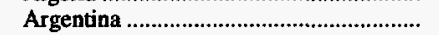 & - & 49 & 70 & - & 59 & -100.0 & - \\
\hline Austria & - & - & - & - & 104 & - & - \\
\hline 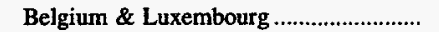 & 748 & 822 & 1,447 & 2,650 & 2,473 & -8.9 & -25.8 \\
\hline 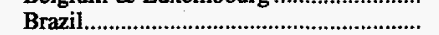 & 1,096 & 1,131 & 2,469 & 2,826 & 2,660 & -3.1 & -19.9 \\
\hline 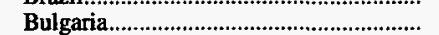 & - & & 328 & 216 & 349 & - & - \\
\hline 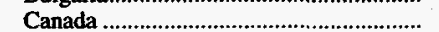 & 445 & 786 & 1,229 & 1,331 & 1,404 & -43.4 & -25.0 \\
\hline China (Taiwan) ................................. & - & - & - & - & 13 & - & - \\
\hline
\end{tabular}

See footnotes at end of table. 
Table 62. Foreign Distribution of U.S. Coal by Major Coal-Exporting States and Destination, 1991-1995 (Continued)

(Thousand Short Tons)

\begin{tabular}{|c|c|c|c|c|c|c|c|}
\hline \multirow{2}{*}{$\begin{array}{l}\text { Coal-Exporting State } \\
\text { and Destination }\end{array}$} & \multirow{2}{*}{1995} & \multirow{2}{*}{1994} & \multirow{2}{*}{1993} & \multirow{2}{*}{1992} & \multirow{2}{*}{1991} & \multirow{2}{*}{$\begin{array}{c}\text { Percent } \\
\text { Change } \\
1994-1995\end{array}$} & \multirow{2}{*}{$\begin{array}{c}\begin{array}{c}\text { Average Annual } \\
\text { Percent Change }\end{array} \\
1991-1995\end{array}$} \\
\hline & & & & & & & \\
\hline \multicolumn{8}{|l|}{ Virginia (Continued) } \\
\hline Croatia & - & - & 339 & - & - & - & - \\
\hline 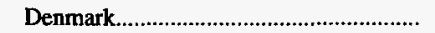 & - & - & - & 75 & - & - & - \\
\hline Egypt & 335 & 405 & 316 & 300 & 182 & -17.3 & 16.4 \\
\hline 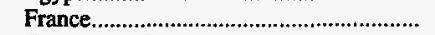 & 628 & 523 & 822 & 877 & 1,027 & 19.9 & -11.6 \\
\hline Germany, FR & 68 & 9 & 68 & 47 & 22 & $\mathrm{NM}$ & 32.0 \\
\hline 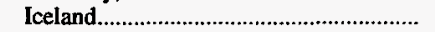 & - & - & - & - & 34 & - & - \\
\hline Ireland & - & - & - & - & 12 & - & - \\
\hline Italy & 1,480 & 1,676 & 1,903 & 1,688 & 2,073 & -11.7 & -8.1 \\
\hline Japan & 1,804 & 1,964 & 403 & 632 & 474 & -8.2 & 39.7 \\
\hline 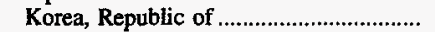 & 569 & 1,287 & 1,675 & 1,863 & 1,974 & -55.8 & -26.7 \\
\hline 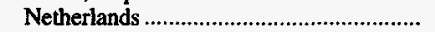 & 797 & 712 & 546 & 1,185 & 1,641 & 12.0 & -16.5 \\
\hline 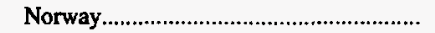 & - & - & - & - & 31 & - & - \\
\hline 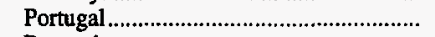 & 105 & 85 & - & 356 & 298 & 24.2 & -22.9 \\
\hline 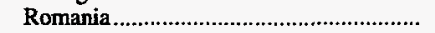 & - & 207 & - & 182 & 61 & -100.0 & - \\
\hline Spain & 851 & 1,205 & 1,782 & 1,363 & 1,344 & -29.4 & -10.8 \\
\hline Sweden & 115 & 34 & - & - & 96 & 237.0 & 4.7 \\
\hline 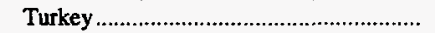 & - & - & - & - & 470 & - & - \\
\hline United Kingdom & 534 & 538 & 389 & 910 & 746 & -.7 & -8.0 \\
\hline 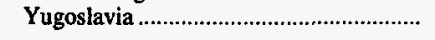 & - & - & - & 55 & 108 & - & - \\
\hline 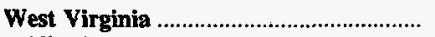 & 44,321 & 36,205 & 33,159 & 50,806 & 53,792 & 22.4 & -4.7 \\
\hline 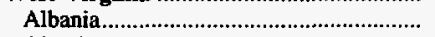 & - & - & - & - & 20 & - & - \\
\hline Algeria & - & $*$ & - & - & * & -100.0 & - \\
\hline 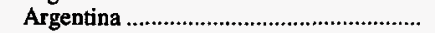 & - & 35 & 110 & 149 & 113 & -100.0 & - \\
\hline 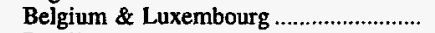 & 1,923 & 2,307 & 1,911 & 1,382 & 1,625 & -16.7 & 4.3 \\
\hline 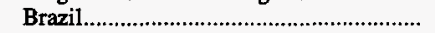 & 4,328 & 4,101 & 2,101 & 2,599 & 3,395 & 5.5 & 6.3 \\
\hline 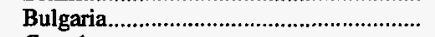 & 1,360 & 1,568 & 535 & 547 & 440 & -13.3 & 32.6 \\
\hline 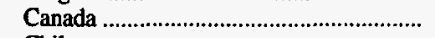 & 5,784 & 5,644 & 4,108 & 8,911 & 7,464 & 2.5 & -6.2 \\
\hline Chile & 118 & - & - & - & 27 & - & 44.4 \\
\hline 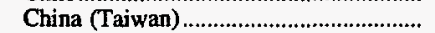 & 355 & 308 & 117 & 250 & 229 & 15.3 & 11.5 \\
\hline 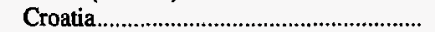 & 72 & - & 52 & 151 & - & - & - \\
\hline Denmark & 189 & - & 140 & 2,118 & 3,640 & - & -52.3 \\
\hline 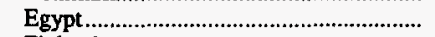 & 714 & 592 & 499 & 492 & 510 & 20.6 & 8.8 \\
\hline 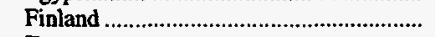 & 792 & 375 & 176 & 183 & 517 & 111.4 & 11.3 \\
\hline France & 4,408 & 3,492 & 3,140 & 5,991 & 5,602 & 26.3 & -5.8 \\
\hline 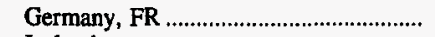 & 997 & 381 & 527 & 847 & 1,354 & 161.7 & -7.3 \\
\hline 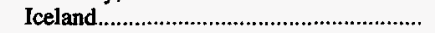 & - & - & - & 7 & 5 & - & - \\
\hline Ireland & - & - & 97 & 726 & 764 & - & - \\
\hline 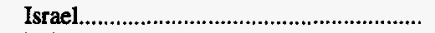 & - & - & - & 185 & 150 & - & - \\
\hline Italy & 5,138 & 3,626 & 3,476 & 5,980 & 7,144 & 41.7 & -7.9 \\
\hline Japan & 3,373 & 2,590 & 4,437 & 5,348 & 5,052 & 30.2 & -9.6 \\
\hline 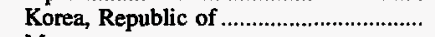 & 1,171 & 575 & 660 & 514 & 558 & 103.6 & 20.3 \\
\hline 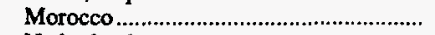 & 275 & 101 & 66 & 150 & 182 & 173.1 & 10.9 \\
\hline 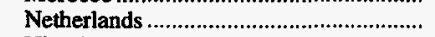 & 3,737 & 3,333 & 3,269 & 4,027 & 4,175 & 12.1 & -2.7 \\
\hline 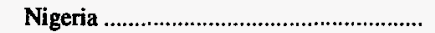 & - & - & 36 & - & - & - & - \\
\hline 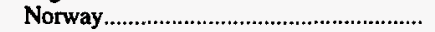 & - & - & - & 13 & - & - & - \\
\hline 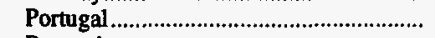 & 1,390 & 673 & 950 & 850 & 800 & 106.6 & 14.8 \\
\hline 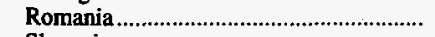 & 1,623 & 923 & 598 & 332 & 622 & 75.8 & 27.1 \\
\hline 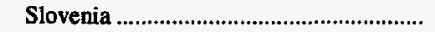 & - & - & - & 61 & - & - & - \\
\hline 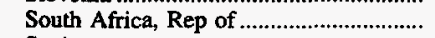 & 946 & 769 & 479 & 361 & 218 & 23.0 & 44.3 \\
\hline Spain & 1,083 & 1,253 & 1,371 & 1,611 & 1,841 & -13.5 & -12.4 \\
\hline 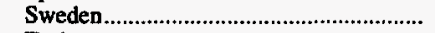 & 1,351 & 885 & 501 & 724 & 758 & 52.8 & 15.5 \\
\hline 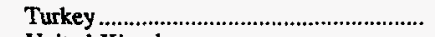 & 1,560 & 1,465 & 1,137 & 1,262 & 707 & 6.5 & 21.9 \\
\hline United Kingdom & 1,633 & 1,210 & 2,668 & 4,649 & 5,148 & 34.9 & -25.0 \\
\hline 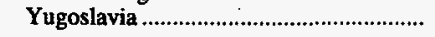 & - & - & - & 385 & 730 & - & - \\
\hline Wyoming & 2,269 & 1,524 & 974 & 1,277 & 982 & 48.9 & 23.3 \\
\hline Canada & 32 & - & - & - & 20 & - & 11.7 \\
\hline Japan & - & - & * & - & * & - & - \\
\hline Spain & 2,237 & 1,524 & 974 & 1,277 & 791 & 46.8 & 29.7 \\
\hline 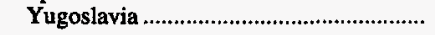 & - & - & - & - & 170 & - & - \\
\hline 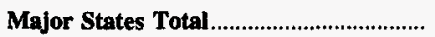 & $\mathbf{8 8 , 7 2 2}$ & 71,811 & 74,801 & 100,619 & 107,318 & 23.5 & -4.6 \\
\hline 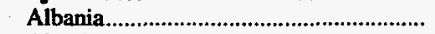 & - & - & - & 22 & 103 & - & - \\
\hline Algeria & 167 & 250 & 466 & 670 & 427 & -33.3 & -20.9 \\
\hline 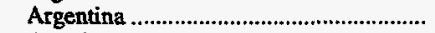 & 306 & 352 & 576 & 319 & 395 & -13.1 & -6.1 \\
\hline 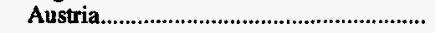 & - & - & - & - & 104 & - & - \\
\hline
\end{tabular}

See footnotes at end of table. 
Table 62. Foreign Distribution of U.S. Coal by Major Coal-Exporting States and Destination, 1991-1995 (Continued)

(Thousand Short Tons)

\begin{tabular}{|c|c|c|c|c|c|c|c|}
\hline \multirow{2}{*}{$\begin{array}{l}\text { Coal-Exporting State } \\
\text { and Destination }\end{array}$} & \multirow[t]{2}{*}{1995} & \multirow[t]{2}{*}{1994} & \multirow[t]{2}{*}{1993} & \multirow[t]{2}{*}{1992} & \multirow[t]{2}{*}{1991} & \multirow{2}{*}{$\begin{array}{c}\text { Percent } \\
\text { Change } \\
\text { 1994-1995 }\end{array}$} & \multirow{2}{*}{$\begin{array}{c}\begin{array}{c}\text { Average Annual } \\
\text { Percent Change }\end{array} \\
1991-1995\end{array}$} \\
\hline & & & & & & & \\
\hline \multicolumn{8}{|l|}{ Major States Total (Continued) } \\
\hline Belgium \& Luxembourg ........................... & 3,608 & 4,367 & 4,911 & 5,688 & 6,529 & -17.4 & -13.8 \\
\hline 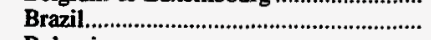 & 6,420 & 5,641 & 5,416 & 5,935 & 6,517 & 13.8 & -.4 \\
\hline 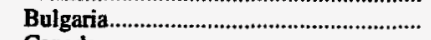 & 1,488 & 1,604 & 1,052 & 836 & 896 & -7.2 & 13.5 \\
\hline Canada & 7,750 & 8,373 & 7,696 & 13,919 & 12,790 & -7.4 & -11.8 \\
\hline 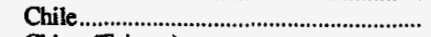 & 234 & - & - & 38 & 233 & - & .2 \\
\hline China (Taiwan) & 3,321 & 4,023 & 5,430 & 4,778 & 5,165 & -17.5 & -10.4 \\
\hline 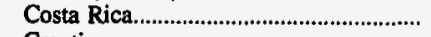 & - & - & - & $*$ & $*$ & - & - \\
\hline Croatia & 72 & - & 437 & 151 & - & - & - \\
\hline Denmark & 2,320 & 508 & 366 & 3,888 & 5,213 & 356.2 & -18.3 \\
\hline Dominican Republic ..................................... & 18 & 65 & 59 & 158 & 201 & -71.5 & -45.0 \\
\hline 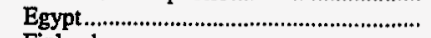 & 1,159 & 997 & 814 & 792 & 693 & 16.3 & 13.7 \\
\hline Finland & 1,337 & 445 & 234 & 183 & 517 & 200.2 & 26.8 \\
\hline 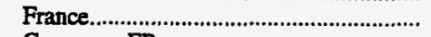 & 5,353 & 4,211 & 4,595 & 8,817 & 8,933 & 27.1 & -12.0 \\
\hline 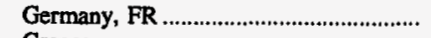 & 2,234 & 586 & 697 & 1,047 & 1,405 & 280.9 & 12.3 \\
\hline 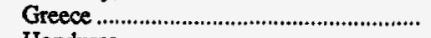 & - & - & - & - & 91 & - & - \\
\hline 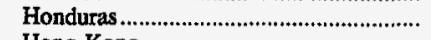 & - & - & - & - & $*$ & - & - \\
\hline 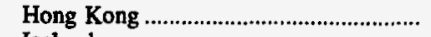 & $\overline{-}$ & 46 & - & 359 & 522 & -100.0 & - \\
\hline Iceland & 76 & 9 & - & 42 & 39 & NM & 17.8 \\
\hline Indonesia & - & - & - & $*$ & $*$ & - & - \\
\hline Ireland & 1,218 & 1,015 & 1,135 & 1,318 & 1,015 & 20.0 & 4.7 \\
\hline 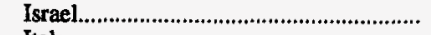 & 1,210 & 922 & 940 & 1,287 & 1,137 & 31.3 & 1.6 \\
\hline Italy & 9,289 & 7,462 & 6,540 & 8,735 & 10,674 & 24.5 & -3.4 \\
\hline 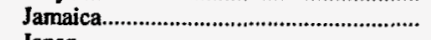 & 62 & 32 & 47 & 39 & 59 & 94.7 & 1.3 \\
\hline Japan & 11,262 & 9,786 & 11,765 & 11,690 & 12,206 & 15.1 & -2.0 \\
\hline Korea, Republic of & 4,694 & 2,970 & 3,378 & 3,160 & 3,238 & 58.1 & 9.7 \\
\hline 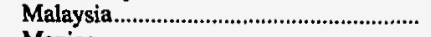 & - & - & - & * & $*$ & - & - \\
\hline 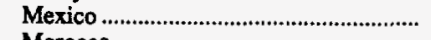 & - & - & $*$ & 18 & - & - & - \\
\hline Morocco & 1,050 & 101 & 561 & 500 & 831 & NM & 6.0 \\
\hline 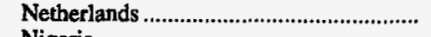 & 6,350 & 4,725 & 4,109 & 7,520 & 7,466 & 34.4 & -4.0 \\
\hline Nigeria & - & $\overline{-}$ & 36 & $\overline{-}$ & - & - & - \\
\hline 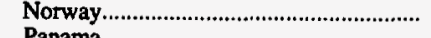 & 168 & 105 & 141 & 172 & 254 & 60.6 & -9.8 \\
\hline 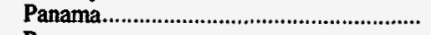 & - & - & - & * & $*$ & - & - \\
\hline 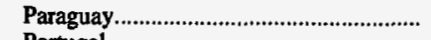 & - & - & - & 3 & - & - & - \\
\hline 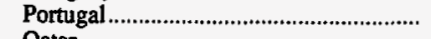 & 1,967 & 1,165 & 1,417 & 1,511 & 1,773 & 68.8 & 2.6 \\
\hline 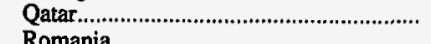 & $\bar{\pi}$ & - & - & $\stackrel{*}{*}$ & - & - & - \\
\hline & 2,115 & 1,732 & 786 & 713 & 1,056 & 22.1 & 19.0 \\
\hline 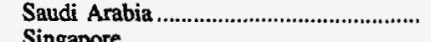 & - & - & $*$ & - & - & - & - \\
\hline & - & - & - & - & * & - & - \\
\hline & $0 \overline{6}$ & 760 & $\bar{a}$ & 61 & $\overline{8}$ & $\bar{a}$ & $\bar{n}$ \\
\hline $\begin{array}{l}\text { South Africa, Rep of } \\
\text { Soviet Union }\end{array}$ & $\begin{array}{r}946 \\
-\end{array}$ & $\begin{array}{r}769 \\
-\end{array}$ & $\begin{array}{r}479 \\
-\end{array}$ & $\begin{aligned} 590 \\
-\end{aligned}$ & $\begin{array}{r}218 \\
64\end{array}$ & 23.0 & 44.3 \\
\hline & $4,46 \overline{6}$ & $4,00 \overline{8}$ & $4,12 \overline{7}$ & $4,36 \overline{2}$ & $\begin{array}{r}64 \\
4,275\end{array}$ & $11 \overline{4}$ & $\overline{1}$ \\
\hline & $\begin{array}{r}4,400 \\
-\end{array}$ & $\begin{array}{r}4,008 \\
-\end{array}$ & $\begin{array}{r}4,127 \\
*\end{array}$ & $\begin{array}{r}4,362 \\
*\end{array}$ & 4,275 & 11.4 & 1.1 \\
\hline $\begin{array}{l}\text { Surinam } \\
\text { Sweden }\end{array}$ & 1,467 & 938 & 738 & 1,239 & 1,197 & 56.3 & $5 . \overline{2}$ \\
\hline 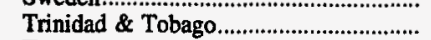 & - & - & 1 & 2 & 2 & - & - \\
\hline 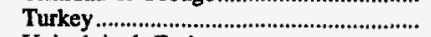 & 2,114 & 1,603 & 1,416 & $1,89 \overline{1}$ & 1,834 & 31.9 & 3.6 \\
\hline United Arab Emirates................................. & - & - & $*$ & - & - & - & - \\
\hline United Kingdom ...................................... & 4,471 & 2,990 & 4,420 & 7,512 & 8,090 & 49.5 & -13.8 \\
\hline 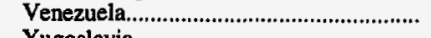 & 9 & 11 & 15 & 39 & 39 & -18.5 & -30.1 \\
\hline 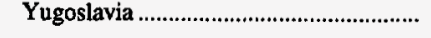 & - & - & - & 616 & 1,116 & - & - \\
\hline Other States Total & 1,185 & 870 & 709 & 761 & 947 & 36.3 & 5.8 \\
\hline 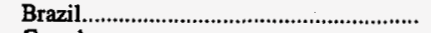 & 188 & 184 & 295 & - & - & 2.5 & - \\
\hline 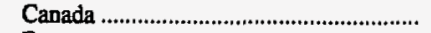 & 273 & 93 & 55 & $*$ & 38 & 192.3 & 63.4 \\
\hline France, & - & - & - & 10 & 39 & - & - \\
\hline Ireland & - & - & - & 10 & - & - & - \\
\hline Japan & - & - & - & - & 202 & - & - \\
\hline 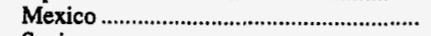 & 498 & - & 11 & - & - & - & - \\
\hline Spain & - & 153 & - & 52 & 258 & -100.0 & - \\
\hline 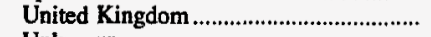 & $\overline{-}$ & - & - & 4 & - & - & - \\
\hline 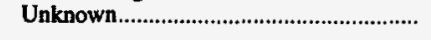 & 226 & 439 & 348 & 685 & 410 & -48.4 & -13.8 \\
\hline U.S. Total & 89,907 & 72,680 & $\mathbf{7 5 , 5 1 0}$ & 101,380 & 108,264 & 23.7 & -4.5 \\
\hline 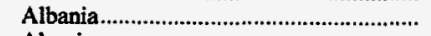 & - & - & - & 22 & 103 & - & - \\
\hline Algeria & 167 & 250 & 466 & 670 & 427 & -33.3 & -20.9 \\
\hline Argentina & 306 & 352 & 576 & 319 & 395 & -13.1 & -6.1 \\
\hline 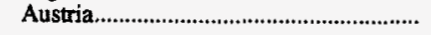 & - & - & - & - & 104 & - & - \\
\hline
\end{tabular}

See footnotes at end of table. 
Table 62. Foreign Distribution of U.S. Coal by Major Coal-Exporting States and Destination, 1991-1995 (Continued)

(Thousand Short Tons)

\begin{tabular}{|c|c|c|c|c|c|c|c|}
\hline \multirow{2}{*}{$\begin{array}{l}\text { Coal-Exporting State } \\
\text { and Destination }\end{array}$} & \multirow{2}{*}{1995} & \multirow{2}{*}{1994} & \multirow{2}{*}{1993} & \multirow{2}{*}{1992} & \multirow{2}{*}{1991} & \multirow{2}{*}{$\begin{array}{l}\text { Percent } \\
\text { Change } \\
\text { 1994-1995 }\end{array}$} & \multirow{2}{*}{$\begin{array}{c}\begin{array}{c}\text { Average Annual } \\
\text { Percent Change }\end{array} \\
1991-1995\end{array}$} \\
\hline & & & & & & & \\
\hline \multicolumn{8}{|l|}{ U.S. Total (Continued) } \\
\hline Belgium \& Luxembourg .............................. & 3,608 & 4,367 & 4,911 & 5,688 & 6,529 & -17.4 & -13.8 \\
\hline 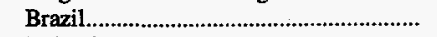 & 6,609 & 5,824 & 5,711 & 5,935 & 6,517 & 13.5 & .3 \\
\hline 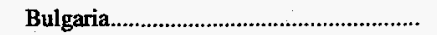 & 1,488 & 1,604 & 1,052 & 836 & 896 & -7.2 & 13.5 \\
\hline 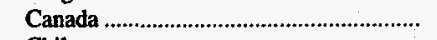 & 8,023 & 8,467 & 7,751 & 13,919 & 12,828 & -5.2 & -11.1 \\
\hline Chile & 234 & - & - & 38 & 233 & - & .2 \\
\hline 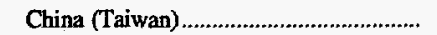 & 3,321 & 4,023 & 5,430 & 4,778 & 5,165 & -17.5 & -10.4 \\
\hline Costa Rica................. & - & - & - & $*$ & * & - & - \\
\hline 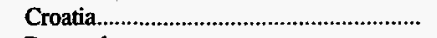 & 72 & - & 437 & 151 & - & - & - \\
\hline 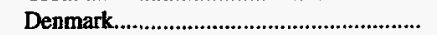 & 2,320 & 508 & 366 & 3,888 & 5,213 & 356.2 & -18.3 \\
\hline 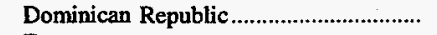 & 18 & 65 & 59 & 158 & 201 & -71.5 & -45.0 \\
\hline Egypt & 1,159 & 997 & 814 & 792 & 693 & 16.3 & 13.7 \\
\hline Finland & 1,337 & 445 & 234 & 183 & 517 & 200.2 & 26.8 \\
\hline 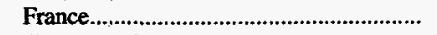 & 5,353 & 4,211 & 4,595 & 8,827 & 8,972 & 27.1 & -12.1 \\
\hline Germany, FR & 2,234 & 586 & 697 & 1,047 & 1,405 & 280.9 & 12.3 \\
\hline 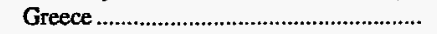 & - & - & - & - & 91 & - & - \\
\hline 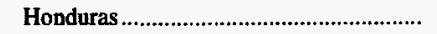 & - & - & - & - & * & - & - \\
\hline 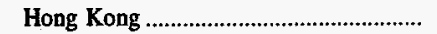 & - & 46 & - & 359 & 522 & -100.0 & - \\
\hline Iceland & 76 & 9 & - & 42 & 39 & NM & 17.8 \\
\hline Indonesia & - & - & - & * & * & - & - \\
\hline 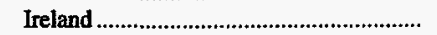 & 1,218 & 1,015 & 1,135 & 1,328 & 1,015 & 20.0 & 4.7 \\
\hline 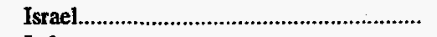 & 1,210 & 922 & 940 & 1,287 & 1,137 & 31.3 & 1.6 \\
\hline 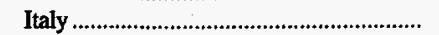 & 9,289 & 7,462 & 6,540 & 8,735 & 10,674 & 24.5 & -3.4 \\
\hline 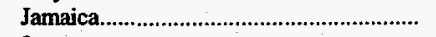 & 62 & 32 & 47 & 39 & 59 & 94.7 & 1.3 \\
\hline 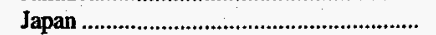 & 11,262 & 9,786 & 11,765 & 11,690 & 12,408 & 15.1 & -2.4 \\
\hline 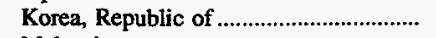 & 4,694 & 2,970 & 3,378 & 3,160 & 3,238 & 58.1 & 9.7 \\
\hline 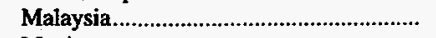 & - & - & - & * & * & - & - \\
\hline Mexico & 498 & - & 11 & 18 & - & - & - \\
\hline Morocco & 1,050 & 101 & 561 & 500 & 831 & NM & 6.0 \\
\hline 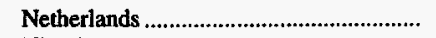 & 6,350 & 4,725 & 4,109 & 7,520 & 7,466 & 34.4 & -4.0 \\
\hline 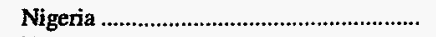 & - & - & 36 & - & - & - & - \\
\hline Norway & 168 & 105 & 141 & 172 & 254 & 60.6 & -9.8 \\
\hline 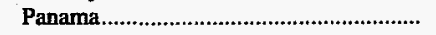 & - & - & - & * & * & - & - \\
\hline Paraguay & - & - & - & 3 & - & - & - \\
\hline Portugal & 1,967 & 1,165 & 1,417 & 1,511 & 1,773 & 68.8 & 2.6 \\
\hline 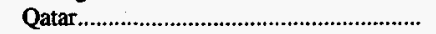 & - & - & - & * & - & - & - \\
\hline 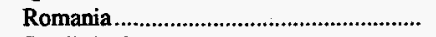 & 2,115 & 1,732 & 786 & 713 & 1,056 & 22.1 & 19.0 \\
\hline 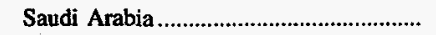 & - & - & * & - & - & - & - \\
\hline 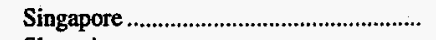 & - & - & - & - & * & - & - \\
\hline 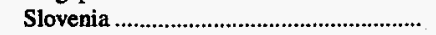 & - & - & - & 61 & - & - & - \\
\hline South Africa, Rep of & 946 & 769 & 479 & 590 & 218 & 23.0 & 44.3 \\
\hline 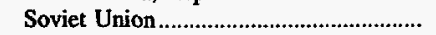 & - & - & - & - & 64 & - & - \\
\hline Spain & 4,466 & 4,162 & 4,127 & 4,414 & 4,533 & 7.3 & -.4 \\
\hline 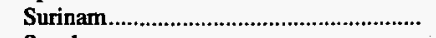 & - & - & * & * & - & - & - \\
\hline Sweden & 1,467 & 938 & 738 & 1,239 & 1,197 & 56.3 & 5.2 \\
\hline 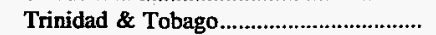 & - & - & 1 & 2 & 2 & - & - \\
\hline Turkey & 2,114 & 1,603 & 1,416 & 1,891 & 1,834 & 31.9 & 3.6 \\
\hline 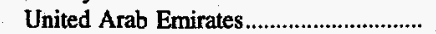 & - & - & * & - & - & - & - \\
\hline 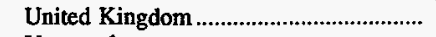 & 4,471 & 2,990 & 4,420 & 7,516 & 8,090 & 49.5 & -13.8 \\
\hline Venezuela & 9 & 11 & 15 & 39 & 39 & -18.5 & -30.1 \\
\hline 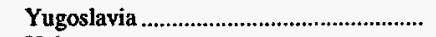 & - & - & - & 616 & 1,116 & - & - \\
\hline 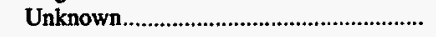 & 226 & 439 & 348 & 685 & 410 & -48.4 & -13.8 \\
\hline
\end{tabular}

* Data round to zero.

Not meaningful as value is greater than 500 percent.

Notes: Major coal-exporting States are those with total coal exports of over 600,000 short tons in 1994. Totals may not equal sum of components due to independent rounding.

Sources: Values shown for destinations other than Canada and Mexico are estimates based upon information reported on Energy Information Administration Form EIA-6, "Coal Distribution Report," and coal export data presented in King's COALBASE (King Publishing Corporation, Knoxville, Tennessee). See the Explanatory Notes for a complete description of the methodology used to develop these estimates. Values shown for Canada and Mexico are based upon Form EIA-6. 
Table 63. Foreign Distribution of U.S. Metallurgical Coal by Major Coal-Exporting States and Destination, 1991-1995

(Thousand Short Tons)

\begin{tabular}{|c|c|c|c|c|c|c|c|}
\hline \multirow{2}{*}{$\begin{array}{l}\text { Coal-Exporting State } \\
\text { and Destination }\end{array}$} & \multirow[t]{2}{*}{1995} & \multirow[t]{2}{*}{1994} & \multirow[t]{2}{*}{1993} & \multirow[t]{2}{*}{1992} & \multirow{2}{*}{1991} & \multirow{2}{*}{$\begin{array}{c}\text { Percent } \\
\text { Change } \\
\text { 1994-1995 }\end{array}$} & \multirow{2}{*}{$\begin{array}{c}\begin{array}{c}\text { Average Annual } \\
\text { Percent Change }\end{array} \\
1991-1995\end{array}$} \\
\hline & & & & & & & \\
\hline Alabama & 5,330 & 4,359 & 5,841 & 5,789 & 6,476 & 22.3 & -4.8 \\
\hline 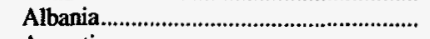 & - & - & - & 22 & 63 & - & - \\
\hline 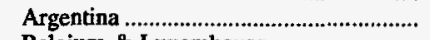 & 306 & 268 & 351 & 170 & 223 & 14.4 & 8.3 \\
\hline 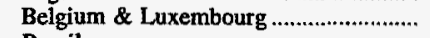 & 574 & 627 & 952 & 856 & 1,074 & -8.3 & -14.5 \\
\hline 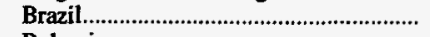 & 564 & 42 & 212 & - & 112 & NM & 49.7 \\
\hline 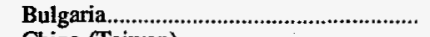 & 128 & 35 & 120 & - & - & 260.0 & - \\
\hline China (Taiwan) & - & - & 46 & 49 & 87 & - & - \\
\hline 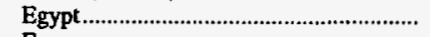 & 111 & - & - & - & - & - & - \\
\hline 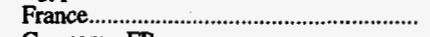 & - & $*$ & - & - & - & -100.0 & - \\
\hline Germany, FR & 59 & - & - & - & - & - & - \\
\hline Italy & 314 & 421 & $*$ & $*$ & $\overline{-}$ & -25.6 & - \\
\hline 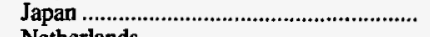 & 1,358 & 1,266 & 2,418 & 2,532 & 2,917 & 7.2 & -17.4 \\
\hline 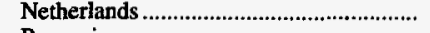 & 358 & 88 & - & 77 & - & 307.0 & - \\
\hline 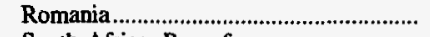 & 492 & 602 & 188 & 199 & 373 & -18.3 & 7.1 \\
\hline 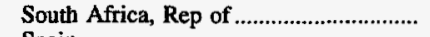 & - & - & - & 229 & - & - & - \\
\hline 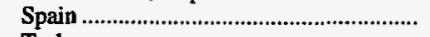 & 48 & - & - & - & - & - & - \\
\hline 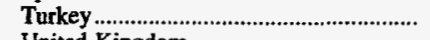 & 302 & 137 & 278 & 459 & 386 & 119.6 & -6.0 \\
\hline 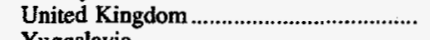 & 717 & 872 & 1,277 & 1,197 & 1,213 & -17.8 & -12.3 \\
\hline 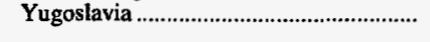 & - & - & - & - & 29 & - & - \\
\hline 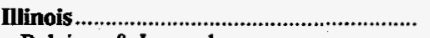 & 49 & 236 & 109 & 614 & 546 & -79.2 & -45.3 \\
\hline Belgium \& Luxembourg .......................... & - & - & - & 58 & 79 & - & - \\
\hline 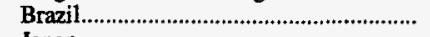 & - & - & - & 298 & 249 & - & - \\
\hline Japan & 49 & 236 & 109 & 32 & 36 & -79.2 & 8.3 \\
\hline 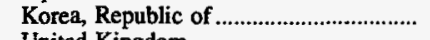 & - & - & - & 50 & 34 & - & - \\
\hline 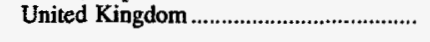 & - & - & - & 175 & 148 & - & - \\
\hline Kentucky & 3,695 & 2,180 & $\mathbf{3 , 3 1 0}$ & 4,971 & 4,354 & 69.5 & -4.0 \\
\hline 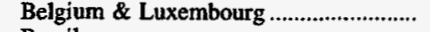 & - & 81 & 68 & - & 546 & -100.0 & - \\
\hline 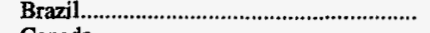 & 52 & 29 & 478 & 110 & 77 & 82.0 & -9.3 \\
\hline 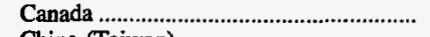 & 777 & 1,073 & 1,356 & 1,373 & 1,125 & -27.6 & -8.8 \\
\hline & 76 & 120 & 171 & 139 & 198 & -37.3 & -21.4 \\
\hline France & 260 & 196 & 122 & 202 & 23 & 32.7 & 83.9 \\
\hline 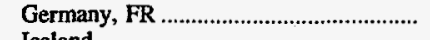 & 92 & $\overline{0}$ & - & - & - & - & - \\
\hline 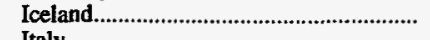 & 76 & 9 & - & 35 & - & NM & - \\
\hline & - & 52 & 121 & 22 & 54 & -100.0 & - \\
\hline & 133 & 83 & 266 & 342 & 506 & 60.1 & -28.4 \\
\hline $\begin{array}{l}\text { Korea, Republic of } \\
\text { Netherlands }\end{array}$ & 1,509 & - & - & - & - & - & - \\
\hline & 101 & 55 & 294 & 1,613 & 866 & 83.8 & -41.5 \\
\hline & 140 & 91 & 110 & 110 & 83 & 54.6 & 14.0 \\
\hline 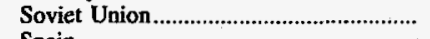 & - & - & - & - & 64 & - & - \\
\hline Spain & - & - & - & - & 29 & - & - \\
\hline Sweden & - & 19 & 238 & 514 & 287 & -100.0 & - \\
\hline 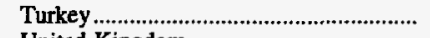 & - & $\overline{1}$ & - & 170 & 260 & - & $\overline{-}$ \\
\hline 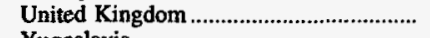 & 479 & 371 & 86 & 299 & 156 & 29.1 & 32.3 \\
\hline 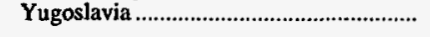 & - & - & - & 42 & 79 & - & - \\
\hline 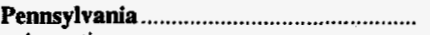 & 1,467 & 1,624 & 1,919 & 1,266 & 1,332 & -9.7 & 2.4 \\
\hline 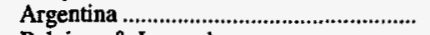 & - & - & 46 & - & - & - & - \\
\hline 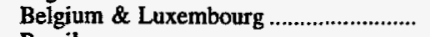 & - & 29 & - & - & - & -100.0 & - \\
\hline 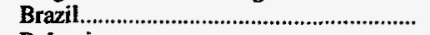 & 371 & 333 & 148 & 90 & - & 11.5 & - \\
\hline 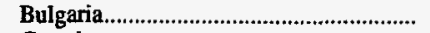 & - & - & 71 & 73 & 106 & - & - \\
\hline Canada & 4 & - & 10 & - & 8 & - & -14.2 \\
\hline Chile & - & - & - & 38 & - & - & - \\
\hline Dominican Republic & - & 23 & - & - & - & -100.0 & - \\
\hline Finland & - & - & 56 & - & - & - & - \\
\hline Germany, FR & 66 & 174 & - & - & - & -61.7 & - \\
\hline Italy & - & - & 114 & - & - & - & - \\
\hline Japan & 916 & 834 & 1,384 & 931 & 1,218 & 9.9 & -6.9 \\
\hline 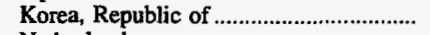 & 109 & 214 & 91 & - & - & -49.1 & - \\
\hline 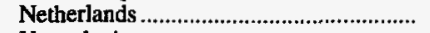 & - & 17 & - & - & - & -100.0 & - \\
\hline Yugoslavia & - & - & - & 134 & - & - & - \\
\hline Virginia & 8,917 & 11,193 & 13,512 & 14,005 & 14,403 & -20.3 & -11.3 \\
\hline 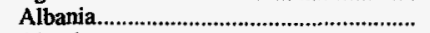 & 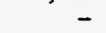 & - & - & - & 20 & - & - \\
\hline Algeria & 167 & 250 & 466 & 670 & 427 & -33.3 & -20.9 \\
\hline Argentina & - & 49 & 70 & - & 59 & -100.0 & - \\
\hline 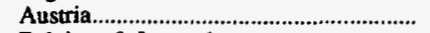 & - & - & - & - & 104 & - & - \\
\hline Belgium \& Luxembourg ............................ & 748 & 822 & 1,447 & 2,259 & 2,112 & -8.9 & -22.8 \\
\hline
\end{tabular}

See footnotes at end of table. 
Table 63. Foreign Distribution of U.S. Metallurgical Coal by Major Coal-Exporting States and Destination, 1991-1995 (Continued)

(Thousand Short Tons)

\begin{tabular}{|c|c|c|c|c|c|c|c|}
\hline \multirow{2}{*}{$\begin{array}{l}\text { Coal-Exporting State } \\
\text { and Destination }\end{array}$} & \multirow[t]{2}{*}{1995} & \multirow[t]{2}{*}{1994} & \multirow[t]{2}{*}{1993} & \multirow[t]{2}{*}{1992} & \multirow[t]{2}{*}{1991} & \multirow{2}{*}{$\begin{array}{c}\text { Percent } \\
\text { Change } \\
\text { 1994-1995 }\end{array}$} & \multirow{2}{*}{$\begin{array}{c}\begin{array}{c}\text { Average Annual } \\
\text { Percent Change }\end{array} \\
1991-1995\end{array}$} \\
\hline & & & & & & & \\
\hline \multicolumn{8}{|l|}{ Virginia (Continued) } \\
\hline 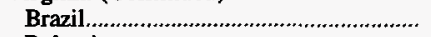 & 1,096 & 1,131 & 2,469 & 2,826 & 2,660 & -3.1 & -19.9 \\
\hline 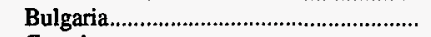 & - & - & 328 & 216 & 349 & - & - \\
\hline 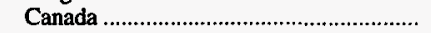 & 445 & 786 & 1,229 & - & - & -43.4 & - \\
\hline China (Taiwan) & - & - & - & - & 13 & - & - \\
\hline 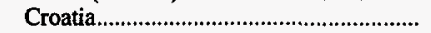 & - & - & 339 & - & - & - & - \\
\hline 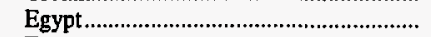 & 335 & 405 & 316 & 300 & 182 & -17.3 & 16.4 \\
\hline 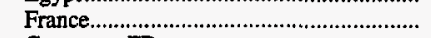 & 628 & 523 & 822 & 791 & 871 & 19.9 & -7.9 \\
\hline 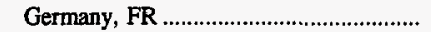 & 68 & 9 & 68 & 47 & 22 & NM & 32.0 \\
\hline 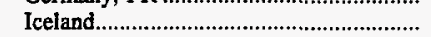 & - & - & - & - & 34 & - & - \\
\hline Italy & 761 & 1,343 & 1,286 & 780 & 545 & -43.3 & 8.7 \\
\hline 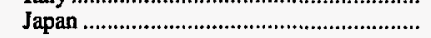 & 1,804 & 1,893 & 329 & 550 & 474 & -4.7 & 39.7 \\
\hline 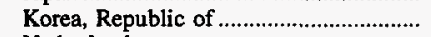 & 569 & 1,287 & 1,675 & 1,863 & 1,974 & -55.8 & -26.7 \\
\hline 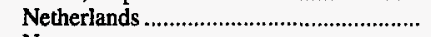 & 797 & 712 & 498 & 1,185 & 1,641 & 12.0 & -16.5 \\
\hline 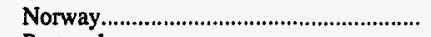 & - & - & - & - & 31 & - & - \\
\hline 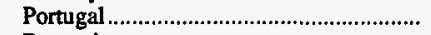 & - & - & - & 92 & 65 & - & - \\
\hline 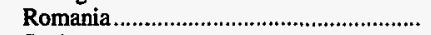 & - & 207 & - & 182 & 61 & -100.0 & - \\
\hline Spain & 851 & 1,205 & 1,782 & 1,281 & 1,337 & -29.4 & -10.7 \\
\hline 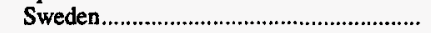 & 115 & 34 & - & - & 96 & 237.0 & 4.7 \\
\hline 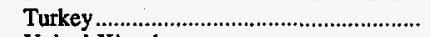 & - & - & - & - & 470 & - & - \\
\hline United Kingdom & 534 & 538 & 389 & 910 & 746 & -.7 & -8.0 \\
\hline 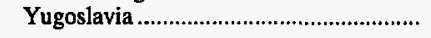 & - & - & - & 55 & 108 & - & - \\
\hline 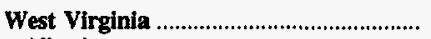 & 34,633 & 31,612 & 27,627 & 35,337 & 35,883 & 9.6 & -.9 \\
\hline 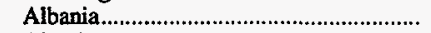 & - & - & - & - & 20 & - & - \\
\hline Algeria & - & $*$ & - & - & $*$ & -100.0 & - \\
\hline Argentina & - & 35 & 110 & 149 & 113 & -100.0 & - \\
\hline Belgium \& Luxembourg ......................... & 1,078 & 1,299 & 1,236 & 281 & 786 & -17.1 & 8.2 \\
\hline 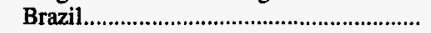 & 4,328 & 4,101 & 2,101 & 2,599 & 3,395 & 5.5 & 6.3 \\
\hline 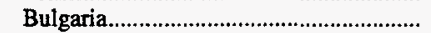 & 1,360 & 1,568 & 535 & 547 & 440 & -13.3 & 32.6 \\
\hline Canada & 5,759 & 5,605 & 4,071 & 8,021 & 7,351 & 2.8 & -5.9 \\
\hline 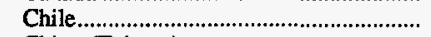 & - & - & - & - & 27 & - & - \\
\hline 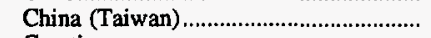 & 355 & 308 & 117 & 250 & 229 & 15.3 & 11.5 \\
\hline 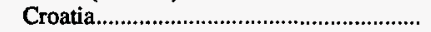 & - & - & 52 & 151 & - & - & - \\
\hline Egypt & 714 & 592 & 499 & 492 & 510 & 20.6 & 8.8 \\
\hline Finland & 683 & 375 & 176 & 183 & 388 & 82.3 & 15.2 \\
\hline 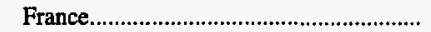 & 3,593 & 3,492 & 2,695 & 3,257 & 3,196 & 2.9 & 3.0 \\
\hline Germany, FR & 254 & 381 & 237 & 278 & 404 & -33.5 & -11.0 \\
\hline Iceland & - & - & - & 7 & 5 & - & - \\
\hline 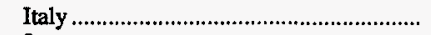 & 2,872 & 2,921 & 2,891 & 4,743 & 5,165 & -1.7 & -13.6 \\
\hline Japan & 3,164 & 2,143 & 4,079 & 4,979 & 4,562 & 47.6 & -8.7 \\
\hline 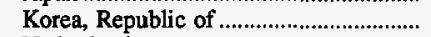 & 1,171 & 575 & 660 & 514 & 558 & 103.6 & 20.3 \\
\hline 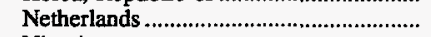 & 1,523 & 1,713 & 2,280 & 2,863 & 2,252 & -11.1 & -9.3 \\
\hline 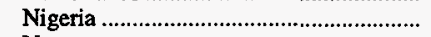 & - & - & 36 & - & - & - & - \\
\hline 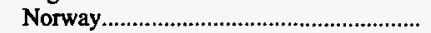 & - & - & - & 13 & - & - & - \\
\hline Portugal & 33 & - & 126 & 96 & - & - & - \\
\hline Romania & 1,623 & 923 & 598 & 332 & 490 & 75.8 & 34.9 \\
\hline 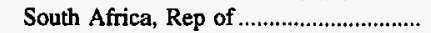 & 946 & 769 & 479 & 361 & 218 & 23.0 & 44.3 \\
\hline Spain & 1,083 & 1,253 & 1,255 & 1,374 & 1,569 & -13.5 & -8.8 \\
\hline Sweden & 1,351 & 885 & 501 & 724 & 751 & 52.8 & 15.8 \\
\hline Turkey & 1,560 & 1,465 & 1,137 & 1,262 & 707 & 6.5 & 21.9 \\
\hline 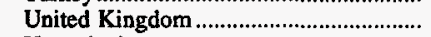 & 1,182 & 1,210 & 1,757 & 1,477 & 2,016 & -2.3 & -12.5 \\
\hline Yugoslavia & - & - & - & 385 & 730 & - & - \\
\hline Major States Total & 54,091 & 51,205 & 52,318 & 61,982 & 62,995 & 5.6 & -3.7 \\
\hline 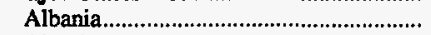 & - & - & - & 22 & 103 & - & - \\
\hline Algeria & 167 & 250 & 466 & 670 & 427 & -33.3 & -20.9 \\
\hline Argentina & 306 & 352 & 576 & 319 & 395 & -13.1 & -6.1 \\
\hline 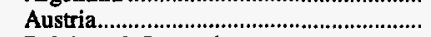 & - & - & - & - & 104 & - & - \\
\hline Belgium \& Luxembourg ............................ & 2,400 & 2,858 & 3,703 & 3,453 & 4,597 & -16.0 & -15.0 \\
\hline 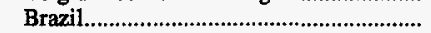 & 6,411 & 5,636 & 5,409 & 5,923 & 6,494 & 13.8 & -.3 \\
\hline 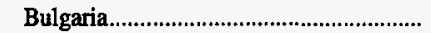 & 1,488 & 1,604 & 1,052 & 836 & 896 & -7.2 & 13.5 \\
\hline 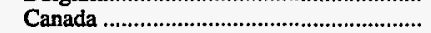 & 6,985 & 7,464 & 6,666 & 9,394 & 8,483 & -6.4 & -4.7 \\
\hline 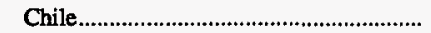 & - & - & - & 38 & 27 & - & - \\
\hline 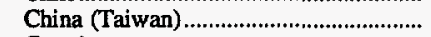 & 430 & 428 & 334 & 437 & 528 & .5 & -5.0 \\
\hline 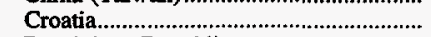 & - & - & 392 & 151 & - & - & - \\
\hline 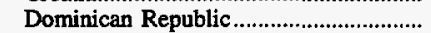 & - & 23 & - & - & - & -100.0 & - \\
\hline Egypt. & 1,159 & 997 & 814 & 792 & 693 & 16.3 & 13.7 \\
\hline
\end{tabular}

See footnotes at end of table. 
Table 63. Foreign Distribution of U.S. Metallurgical Coal by Major Coal-Exporting States and Destination, 1991-1995 (Continued)

(Thousand Short Tons)

\begin{tabular}{|c|c|c|c|c|c|c|c|}
\hline \multirow{2}{*}{$\begin{array}{l}\text { Coal-Exporting State } \\
\text { and Destination }\end{array}$} & \multirow{2}{*}{1995} & \multirow{2}{*}{1994} & \multirow{2}{*}{1993} & \multirow[t]{2}{*}{1992} & \multirow{2}{*}{1991} & \multirow{2}{*}{$\begin{array}{c}\text { Percent } \\
\text { Change } \\
\text { 1994-1995 }\end{array}$} & \multirow{2}{*}{$\begin{array}{c}\begin{array}{c}\text { Average Annual } \\
\text { Percent Change }\end{array} \\
1991-1995\end{array}$} \\
\hline & & & & & & & \\
\hline \multicolumn{8}{|l|}{ Major States Total (Continued) } \\
\hline Finland & 683 & 375 & 232 & 183 & 388 & 82.3 & 15.2 \\
\hline France. & 4,481 & 4,211 & 3,639 & 4,250 & 4,090 & 6.4 & 2.3 \\
\hline Germany, FR & 539 & 563 & 305 & 325 & 426 & -4.4 & 6.0 \\
\hline 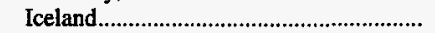 & 76 & 9 & - & 42 & 39 & NM & 17.8 \\
\hline 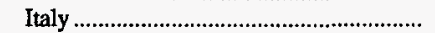 & 3,947 & 4,737 & 4,412 & 5,544 & 5,764 & -16.7 & -9.0 \\
\hline Japan & 7,424 & 6,455 & 8,586 & 9,366 & 9,713 & 15.0 & -6.5 \\
\hline Korea, Republic of & 3,358 & 2,076 & 2,425 & 2,427 & 2,566 & 61.8 & 6.9 \\
\hline 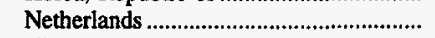 & 2,779 & 2,586 & 3,071 & 5,738 & 4,760 & 7.5 & -12.6 \\
\hline 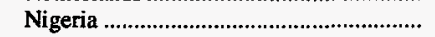 & - & - & 36 & - & - & - & - \\
\hline 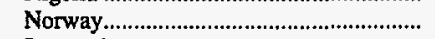 & 140 & 91 & 110 & 124 & 114 & 54.6 & 5.4 \\
\hline 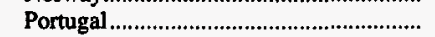 & 33 & - & 126 & 188 & 65 & - & -15.2 \\
\hline Romania & 2,115 & 1,732 & 786 & 713 & 924 & 22.1 & 23.0 \\
\hline South Africa, Rep of & 946 & 769 & 479 & 590 & 218 & 23.0 & 44.3 \\
\hline Soviet Union & - & - & - & - & 64 & - & - \\
\hline Spain & 1,982 & 2,458 & 3,037 & 2,654 & 2,935 & -19.3 & -9.3 \\
\hline 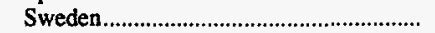 & 1,467 & 938 & 738 & 1,238 & 1,133 & 56.3 & 6.6 \\
\hline 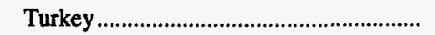 & 1,862 & 1,603 & 1,416 & 1,891 & 1,823 & 16.2 & .5 \\
\hline 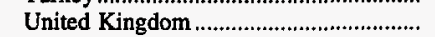 & 2,911 & 2,990 & 3,509 & 4,059 & 4,279 & -2.6 & -9.2 \\
\hline 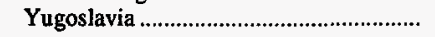 & - & - & - & 616 & 946 & - & - \\
\hline Other States Total & 188 & 184 & 295 & - & - & 2.5 & - \\
\hline 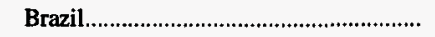 & 188 & 184 & 295 & - & - & 2.5 & - \\
\hline U.S. Total & 54,279 & 51,388 & 52,613 & 61,982 & 62,995 & 5.6 & -3.6 \\
\hline 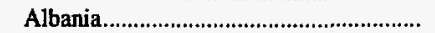 & - & - & - & 22 & 103 & - & - \\
\hline 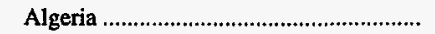 & 167 & 250 & 466 & 670 & 427 & -33.3 & -20.9 \\
\hline 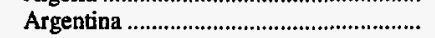 & 306 & 352 & 576 & 319 & 395 & -13.1 & -6.1 \\
\hline 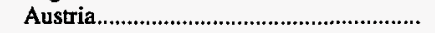 & - & - & - & - & 104 & - & - \\
\hline 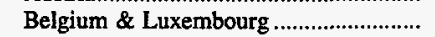 & 2,400 & 2,858 & 3,703 & 3,453 & 4,597 & -16.0 & -15.0 \\
\hline 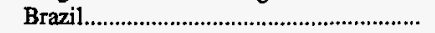 & 6,600 & 5,819 & 5,703 & 5,923 & 6,494 & 13.4 & .4 \\
\hline 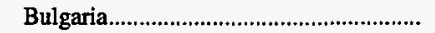 & 1,488 & 1,604 & 1,052 & 836 & 896 & -7.2 & 13.5 \\
\hline Canada & 6,985 & 7,464 & 6,666 & 9,394 & 8,483 & -6.4 & -4.7 \\
\hline 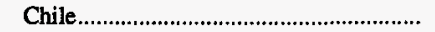 & - & - & - & 38 & 27 & - & - \\
\hline 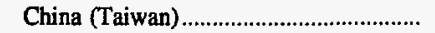 & 430 & 428 & 334 & 437 & 528 & .5 & -5.0 \\
\hline 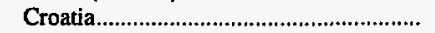 & - & - & 392 & 151 . & - & - & - \\
\hline Dominican Republic................................... & - & 23 & - & - & - & -100.0 & - \\
\hline 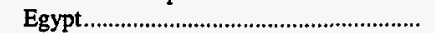 & 1,159 & 997 & 814 & 792 & 693 & 16.3 & 13.7 \\
\hline 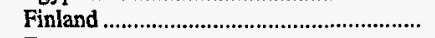 & 683 & 375 & 232 & 183 & 388 & 82.3 & 15.2 \\
\hline 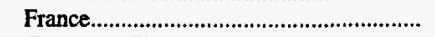 & 4,481 & 4,211 & 3,639 & 4,250 & 4,090 & 6.4 & 2.3 \\
\hline Germany, FR & 539 & 563 & 305 & 325 & 426 & -4.4 & 6.0 \\
\hline 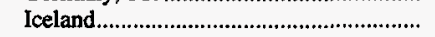 & 76 & 9 & - & 42 & 39 & NM & 17.8 \\
\hline Italy & 3,947 & 4,737 & 4,412 & 5,544 & 5,764 & -16.7 & -9.0 \\
\hline 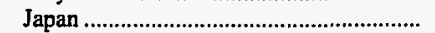 & 7,424 & 6,455 & 8,586 & 9,366 & 9,713 & 15.0 & -6.5 \\
\hline 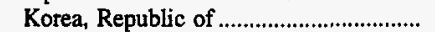 & 3,358 & 2,076 & 2,425 & 2,427 & 2,566 & 61.8 & 6.9 \\
\hline 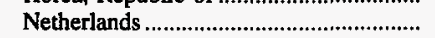 & 2,779 & 2,586 & 3,071 & 5,738 & 4,760 & 7.5 & -12.6 \\
\hline 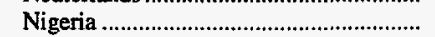 & - & - & 36 & - & - & - & - \\
\hline 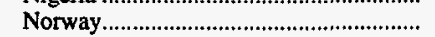 & 140 & 91 & 110 & 124 & 114 & 54.6 & 5.4 \\
\hline Portugal & 33 & - & 126 & 188 & 65 & - & -15.2 \\
\hline 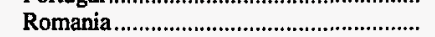 & 2,115 & 1,732 & 786 & 713 & 924 & 22.1 & 23.0 \\
\hline 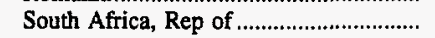 & 946 & 769 & 479 & 590 & 218 & 23.0 & 44.3 \\
\hline Soviet Union & - & - & - & - & 64 & - & - \\
\hline Spain & 1,982 & 2,458 & 3,037 & 2,654 & 2,935 & -19.3 & -9.3 \\
\hline Sweden & 1,467 & 938 & 738 & 1,238 & 1,133 & 56.3 & 6.6 \\
\hline Turkey & 1,862 & 1,603 & 1,416 & 1,891 & 1,823 & 16.2 & .5 \\
\hline United Kingdom & 2,911 & 2,990 & 3,509 & 4,059 & 4,279 & -2.6 & -9.2 \\
\hline Yugoslavia & - & - & - & 616 & 946 & - & - \\
\hline
\end{tabular}

* Data round to zero.

Not meaningful as value is greater than 500 percent.

Notes: Major coal-exporting States are those with total coal exports of over 600,000 short tons in 1994. Totals may not equal sum of components due to independent rounding.

Sources: Values shown for destinations other than Canada and Mexico are estimates based upon information reported on Energy Information Administration Form EIA-6, "Coal Distribution Report," and coal export data presented in King's COALBASE (King Publishing Corporation, Knoxville, Tennessee). See the Explanatory Notes for a complete description of the methodology used to develop these estimates. Values shown for Canada and Mexico are based upon Form EIA-6. 
Table 64. Foreign Distribution of U.S. Steam Coal by Major Coal-Exporting States and Destination, 1991-1995

(Thousand Short Tons)

\begin{tabular}{|c|c|c|c|c|c|c|c|}
\hline \multirow{2}{*}{$\begin{array}{l}\text { Coal-Exporting State } \\
\text { and Destination }\end{array}$} & \multirow[t]{2}{*}{1995} & \multirow[t]{2}{*}{1994} & \multirow[t]{2}{*}{1993} & \multirow[t]{2}{*}{1992} & \multirow[t]{2}{*}{1991} & \multirow{2}{*}{$\begin{array}{l}\text { Percent } \\
\text { Change } \\
\text { 1994-1995 }\end{array}$} & \multirow{2}{*}{$\begin{array}{c}\begin{array}{c}\text { Average Annual } \\
\text { Percent Change }\end{array} \\
1991-1995\end{array}$} \\
\hline & & & & & & & \\
\hline 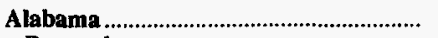 & 702 & 170 & 47 & 142 & 536 & $\mathbf{3 1 3 . 0}$ & 7.0 \\
\hline Denmark & 26 & - & - & - & - & - & - \\
\hline 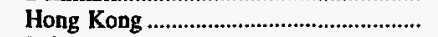 & - & - & - & - & 89 & - & - \\
\hline Italy & 617 & 144 & - & 138 & 443 & 329.6 & 8.6 \\
\hline 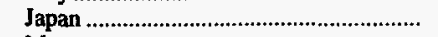 & - & - & 4 & 4 & 3 & - & - \\
\hline 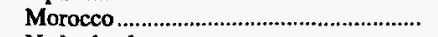 & - & - & 43 & - & - & $=$ & - \\
\hline 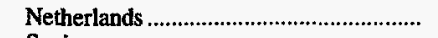 & 59 & $\overline{7}$ & - & - & - & - & - \\
\hline Spain & - & 27 & - & - & - & -100.0 & - \\
\hline Alaska & 855 & 716 & 743 & 734 & 672 & 19.3 & 6.2 \\
\hline Korea, Republic of .................................... & 855 & 716 & 743 & 734 & 672 & 19.3 & 6.2 \\
\hline Colorado & 900 & 752 & 1,128 & 669 & 625 & 19.8 & 9.6 \\
\hline China (Taiwan) & 235 & 134 & - & - & - & 75.0 & - \\
\hline 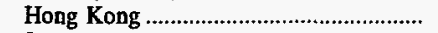 & - & 46 & - & - & - & -100.0 & - \\
\hline Japan & 651 & 395 & 918 & 669 & 625 & 65.0 & 1.0 \\
\hline 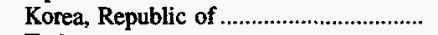 & - & 177 & 209 & - & - & -100.0 & - \\
\hline 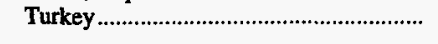 & 14 & - & - & - & - & - & - \\
\hline 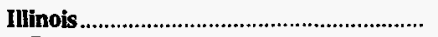 & 2,650 & - & 561 & 629 & 717 & - & 38.7 \\
\hline 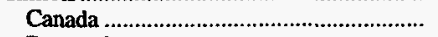 & - & - & - & - & 4 & - & - \\
\hline Denmark & 516 & - & - & 45 & - & - & - \\
\hline 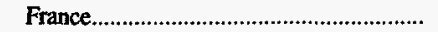 & 57 & - & - & - & - & - & - \\
\hline 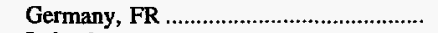 & 722 & - & - & - & - & - & $=$ \\
\hline Ireland & - & - & 108 & 213 & 225 & - & - \\
\hline Italy & 42 & - & - & - & - & - & $=$ \\
\hline Japan & - & - & - & 21 & 24 & - & $\div$ \\
\hline Morocco & 775 & - & 452 & 350 & 464 & $=-$ & $13: 7$ \\
\hline United Kingdom & 538 & - & - & - & - & $=$ & $=$ \\
\hline Kentucky & 6,001 & 4,987 & 6,212 & 9,065 & 11,124 & 20.3 & -14.3 \\
\hline 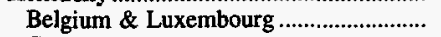 & 363 & 501 & 533 & 651 & 427 & -27.6 & -4.0 \\
\hline 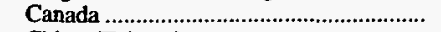 & - & 26 & 60 & 705 & 435 & -100.0 & - \\
\hline China (Taiwan) & 2,300 & 3,140 & 4,248 & 3,620 & 4,131 & -26.7 & -13.6 \\
\hline 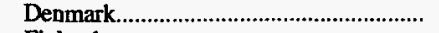 & - & - & 40 & 193 & 779 & - & - \\
\hline 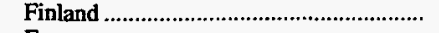 & - & - & 2 & - & - & - & - \\
\hline 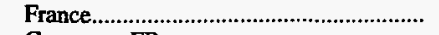 & - & - & 324 & 1,561 & 2,281 & - & - \\
\hline 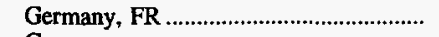 & 94 & - & - & - & - & - & - \\
\hline 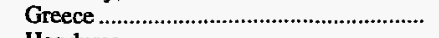 & - & - & - & - & 91 & - & - \\
\hline 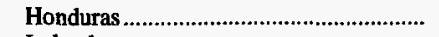 & - & - & - & - & $*$ & - & $\ddot{-}$ \\
\hline Ireland & 57 & - & 19 & 379 & 13 & - & 44.0 \\
\hline 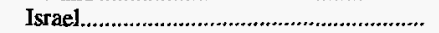 & 215 & - & - & - & - & - & - \\
\hline Italy & 1,698 & 941 & 877 & 908 & 959 & 80.5 & 15.4 \\
\hline 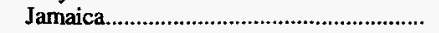 & 62 & 32 & 47 & 39 & 59 & 94.7 & 1.3 \\
\hline Japan & - & 41 & 61 & - & 33 & -100.0 & $=$ \\
\hline 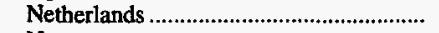 & 514 & 276 & - & 616 & 783 & 86.6 & $=10.0$ \\
\hline 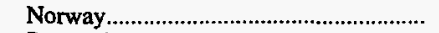 & - & - & - & - & 75 & $=$ & - \\
\hline 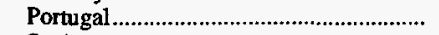 & - & 30 & - & - & 183 & -100.0 & - \\
\hline Spain & 229 & - & - & 111 & 140 & - & 12.9 \\
\hline Sweden & - & - & - & - & 56 & - & - \\
\hline Turkey & 195 & - & - & - & - & - & - \\
\hline 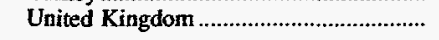 & 273 & - & - & 282 & 679 & - & -20.4 \\
\hline Pennsylvania & 6,812 & 4,677 & 3,589 & 5,175 & 5,971 & 45.6 & $\mathbf{3 . 3}$ \\
\hline 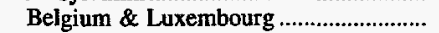 & - & - & - & 91 & 305 & - & $=$ \\
\hline 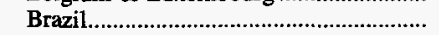 & 9 & 5 & 7 & 12 & 23 & 80.1 & -20.8 \\
\hline 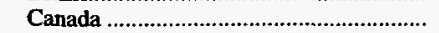 & 708 & 844 & 587 & 1,599 & 2,330 & -16.0 & -25.7 \\
\hline 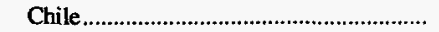 & - & - & - & - & 206 & - & $=$ \\
\hline 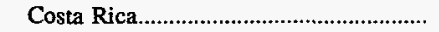 & - & - & - & $*$ & * & - & $\ddot{-}$ \\
\hline Croatia & - & - & 45 & - & - & - & - \\
\hline Denmark & 1,589 & 508 & 187 & 1,456 & 795 & 212.5 & 18.9 \\
\hline 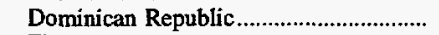 & 18 & 42 & 59 & 158 & 201 & -55.6 & -45.0 \\
\hline Finland & 544 & 71 & - & - & - & NM & - \\
\hline 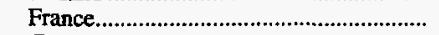 & - & - & 188 & 186 & - & - & - \\
\hline 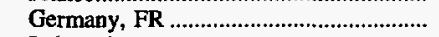 & 135 & 23 & 102 & 153 & 29 & 483.7 & 46.9 \\
\hline 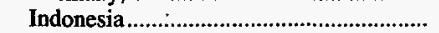 & - & - & - & * & - & - & - \\
\hline Ireland & 1,161 & 1,015 & 911 & - & - & 14.4 & - \\
\hline 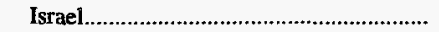 & 995 & 922 & 940 & 1,101 & 987 & 7.9 & .2 \\
\hline Italy & - & 601 & 50 & - & - & -100.0 & - \\
\hline
\end{tabular}

See footnotes at end of table. 
Table 64. Foreign Distribution of U.S. Steam Coal by Major Coal-Exporting States and Destination, 1991-1995 (Continued)

(Thousand Short Tons)

\begin{tabular}{|c|c|c|c|c|c|c|c|}
\hline \multirow{2}{*}{$\begin{array}{l}\text { Coal-Exporting State } \\
\text { and Destination }\end{array}$} & \multirow{2}{*}{1995} & \multirow{2}{*}{1994} & \multirow{2}{*}{1993} & \multirow{2}{*}{1992} & \multirow{2}{*}{1991} & \multirow{2}{*}{$\begin{array}{c}\text { Percent } \\
\text { Change } \\
\text { 1994-1995 }\end{array}$} & $\begin{array}{l}\text { Average Annual } \\
\text { Percent Change }\end{array}$ \\
\hline & & & & & & & 1991-1995 \\
\hline
\end{tabular}

Pennsylvania (Continued)

Japan .

Malaysia

Mexico .

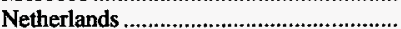

.

Paraguay.

Portugal

Qatar.........

Saudi Arabia

Singapore

Spain ......

Surinam

Trinidad

Turkey.

United Arab Emirates............................

United King

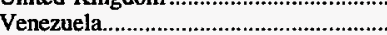

Utah

.

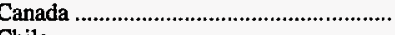

Chile.

China (Taiwan).

Hong Kong

Indonesia.

(1)

Japan ............................................................

Korea, Republic of ................................

Mexico

Virginia

Belgium \& Luxembourg ..........................

Canada

Denmark.

France.

Irelan

Italy

Japan .......

Netherlands .

Portugal...

Spain

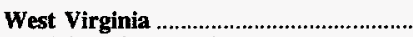

Belgium \& Luxembourg

Brazil.

Canada

Chile.

Croatia...

Denmark.

Finland

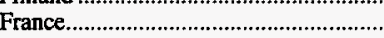

Germany, FR

Ireland

Israel

Italy.

Japan...

Morocco.

Netherlands

Portugal

Romani

Slovenia

Spain .

Sweden

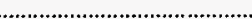

United Kingdom.

See footnotes at end of table.

\begin{tabular}{|c|c|c|c|c|c|c|}
\hline & & & & & & \\
\hline - & - & - & - & 171 & - & - \\
\hline$\overline{-}$ & - & - & $\stackrel{*}{*}$ & ${ }^{*}$ & - & - \\
\hline$\overline{-}$ & $\overline{-}$ & $\overline{-}$ & 18 & $18 \overline{5}$ & $\overline{-}$ & $\overline{-}$ \\
\hline 783 & 244 & - & 2 & - & $221 . \overline{2}$ & - \\
\hline 28 & 14 & 30 & 49 & 66 & 98.9 & -19.1 \\
\hline- & - & - & * & * & - & - \\
\hline- & - & - & 3 & - & - & - \\
\hline 472 & 378 & 467 & 305 & 492 & 24.9 & -1.0 \\
\hline - & - & - & * & - & - & - \\
\hline$\overline{-}$ & - & * & - & $\bar{x}$ & - & - \\
\hline$\overline{18}$ & - & - & - & ${ }^{*}$ & - & - \\
\hline 18 & - & $\bar{*}$ & $\bar{*}$ & 130 & - & -38.8 \\
\hline - & _- & - & 1 & - & - & - \\
\hline - & - & 1 & 2 & 2 & _- & - \\
\hline 43 & - & - & - & 11 & - & 39.6 \\
\hline- & - & * & - & - & - & - \\
\hline $\begin{array}{r}299 \\
9\end{array}$ & - & * & - & * & - & NM \\
\hline 9 & 11 & 15 & 39 & 39 & -18.5 & -30.1 \\
\hline 3,930 & 2,698 & 2,959 & 2,260 & 2,086 & 45.7 & 17.2 \\
\hline $11 \overline{7}$ & - & 346 & - & - & - & - \\
\hline 355 & 321 & 849 & 721 & 506 & 107 & -8.5 \\
\hline - & - & - & 359 & 433 & - & - \\
\hline - & - & - & - & * & - & - \\
\hline 2,977 & 2,377 & 1,764 & 1,180 & 1,147 & 25.3 & 26.9 \\
\hline 481 & - & - & - & - & - & - \\
\hline- & - & & - & - & - & - \\
\hline 825 & 490 & 739 & 3,219 & 3,701 & 68.3 & -31.3 \\
\hline- & - & - & 391 & 361 & - & - \\
\hline - & - & - & 1,331 & 1,404 & - & - \\
\hline - & - & - & 75 & - & - & - \\
\hline - & - & - & 85 & 156 & - & - \\
\hline 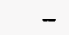 & - & - & & 12 & - & - \\
\hline 719 & 333 & 616 & 908 & 1,528 & 115.8 & -17.2 \\
\hline- & 72 & 74 & 82 & - & -100.0 & - \\
\hline- & - & 49 & - & - & - & - \\
\hline 105 & 85 & - & 264 & 233 & 24.2 & -18.0 \\
\hline- & - & - & 83 & 7 & - & - \\
\hline 9,688 & 4,593 & $\mathbf{5 , 5 3 2}$ & 15,469 & 17,908 & 110.9 & -14.2 \\
\hline 845 & 1,008 & 675 & 1,101 & 839 & -16.1 & .2 \\
\hline & - & - & - & - & - & - \\
\hline 25 & 40 & 37 & 890 & 113 & -37.6 & -31.5 \\
\hline 118 & - & - & - & - & - & - \\
\hline 72 & - & - & - & - & - & - \\
\hline 189 & - & 140 & 2,118 & 3,640 & - & -52.3 \\
\hline 109 & - & - & - & 129 & - & -4.1 \\
\hline 815 & - & 445 & 2,734 & 2,406 & - & -23.7 \\
\hline 744 & - & 290 & 569 & 950 & - & -5.9 \\
\hline$\ldots$ & - & 97 & 726 & 764 & - & - \\
\hline - & - & - & 185 & 150 & - & - \\
\hline 2,266 & 705 & 585 & 1,237 & 1,980 & 221.2 & 3.4 \\
\hline 209 & 447 & 358 & 370 & 490 & -53.3 & -19.2 \\
\hline 275 & 101 & 66 & 150 & 182 & 173.1 & 10.9 \\
\hline 2,214 & 1,619 & 989 & 1,164 & 1,923 & 36.7 & 3.6 \\
\hline 1,357 & 673 & 824 & 754 & 800 & 101.7 & 14.1 \\
\hline 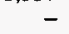 & . & - & - & 131 & - & - \\
\hline - & - & - & 61 & - & - & - \\
\hline- & - & 116 & 238 & 272 & - & - \\
\hline - & - & - & - & 7 & - & - \\
\hline 451 & - & 911 & 3,172 & 3,132 & - & -38.4 \\
\hline
\end{tabular}


Table 64. Foreign Distribution of U.S. Steam Coal by Major Coal-Exporting States and Destination, 1991-1995 (Continued)

(Thousand Short Tons)

\begin{tabular}{|c|c|c|c|c|c|c|c|}
\hline \multirow{2}{*}{$\begin{array}{l}\text { Coal-Exporting State } \\
\text { and Destination }\end{array}$} & \multirow{2}{*}{1995} & \multirow{2}{*}{1994} & \multirow{2}{*}{1993} & \multirow{2}{*}{1992} & \multirow{2}{*}{1991} & \multirow{2}{*}{$\begin{array}{c}\text { Percent } \\
\text { Change } \\
\text { 1994-1995 }\end{array}$} & \multirow{2}{*}{$\begin{array}{c}\begin{array}{c}\text { Average Annual } \\
\text { Percent Change }\end{array} \\
1991-1995\end{array}$} \\
\hline & & & & & & & \\
\hline 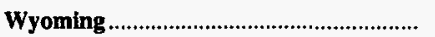 & 2,269 & 1,524 & 974 & 1,277 & 982 & 48.9 & $\mathbf{2 3 . 3}$ \\
\hline 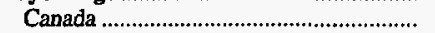 & 32 & - & - & - & 20 & - & 11.7 \\
\hline Japan & - & - & * & - & $*$ & - & - \\
\hline Spain & 2,237 & 1,524 & 974 & 1,277 & 791 & 46.8 & 29.7 \\
\hline 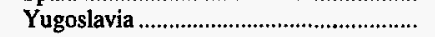 & - & - & - & - & 170 & - & - \\
\hline 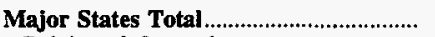 & 34,632 & 20,606 & 22,483 & 38,637 & 44,323 & 68.1 & -6.0 \\
\hline Belgium \& Luxembourg ................................ & 1,208 & 1,509 & 1,208 & 2,235 & 1,932 & -20.0 & -11.1 \\
\hline 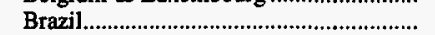 & 9 & 5 & 7 & 12 & 23 & 80.2 & -20.8 \\
\hline Canada & 765 & 910 & 1,030 & 4,525 & 4,306 & -15.9 & -35.1 \\
\hline Chile & 234 & - & - & - & 206 & - & 3.3 \\
\hline China (Taiwan) & 2,890 & 3,595 & 5,097 & 4,341 & 4,637 & -19.6 & -11.1 \\
\hline 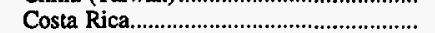 & - & - & - & $*$ & * & - & - \\
\hline 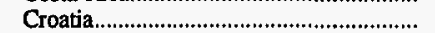 & 72 & - & 45 & - & - & - & - \\
\hline 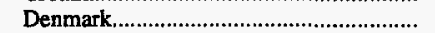 & 2,320 & 508 & 366 & 3,888 & 5,213 & 356.2 & -18.3 \\
\hline Dominican Republic.................................... & 18 & 42 & 59 & 158 & 201 & -55.6 & -45.0 \\
\hline Finland & 654 & 71 & 2 & - & 129 & NM & 50.0 \\
\hline 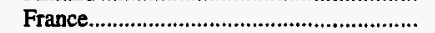 & 872 & - & 957 & 4,567 & 4,843 & - & -34.9 \\
\hline 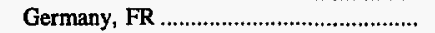 & 1,695 & 23 & 392 & 722 & 979 & NM & 14.7 \\
\hline Greece & - & - & - & - & 91 & - & - \\
\hline 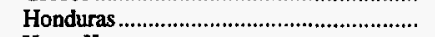 & - & - & - & - & * & - & - \\
\hline Hong Kong & - & 46 & - & 359 & 522 & -100.0 & - \\
\hline Indonesia & - & - & - & * & * & - & - \\
\hline Ireland & 1,218 & 1,015 & 1,135 & 1,318 & 1,015 & 20.0 & 4.7 \\
\hline 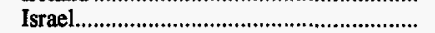 & 1,210 & 922 & 940 & 1,287 & 1,137 & 31.3 & 1.6 \\
\hline Italy & 5,342 & 2,725 & 2,128 & 3,191 & 4,910 & 96.1 & 2.1 \\
\hline 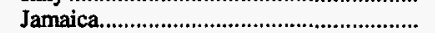 & 62 & 32 & 47 & 39 & 59 & 94.7 & 1.3 \\
\hline 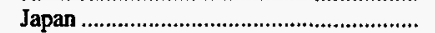 & 3,837 & 3,331 & 3,179 & 2,324 & 2,494 & 15.2 & 11.4 \\
\hline 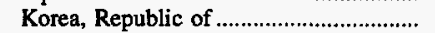 & 1,336 & 894 & 952 & 734 & 672 & 49.5 & 18.7 \\
\hline 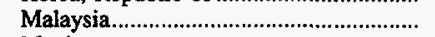 & - & - & - & $*$ & $*$ & - & - \\
\hline Mexico & - & - & * & 18 & - & - & - \\
\hline Morocco & 1,050 & 101 & 561 & 500 & 831 & NM & 6.0 \\
\hline 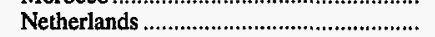 & 3,571 & 2,139 & 1,038 & 1,782 & 2,706 & 67.0 & 7.2 \\
\hline Norway & 28 & 14 & 30 & 49 & 140 & 98.9 & -33.1 \\
\hline 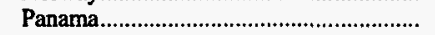 & - & - & - & $*$ & $*$ & - & - \\
\hline 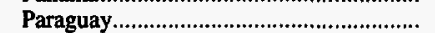 & - & - & - & 3 & - & - & - \\
\hline 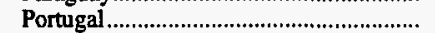 & 1,934 & 1,165 & 1,292 & 1,323 & 1,708 & 65.9 & 3.1 \\
\hline 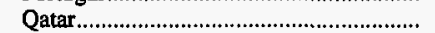 & - & - & - & * & - & - & - \\
\hline 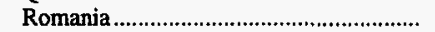 & - & - & - & - & 131 & - & - \\
\hline 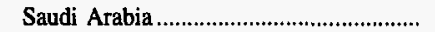 & - & - & $*$ & - & - & - & - \\
\hline Singapore & - & - & - & - & * & - & - \\
\hline 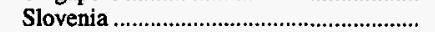 & - & - & - & 61 & - & - & - \\
\hline Spain & 2,484 & 1,550 & 1,090 & 1,708 & 1,340 & 60.2 & 16.7 \\
\hline 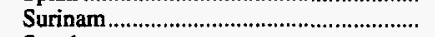 & - & - & $*$ & $*$ & - & - & - \\
\hline 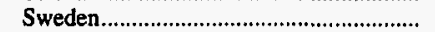 & - & - & - & 1 & 64 & - & - \\
\hline 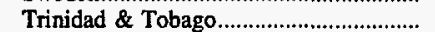 & - & - & 1 & 2 & 2 & - & - \\
\hline 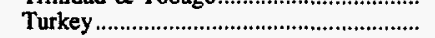 & 252 & - & - & - & 11 & - & 117.6 \\
\hline United Arab Emirates............................... & - & - & * & - & - & - & - \\
\hline United Kingdom & 1,560 & - & 911 & 3,454 & 3,811 & - & -20.0 \\
\hline 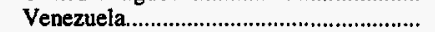 & 9 & 11 & 15 & 39 & 39 & -18.5 & -30.1 \\
\hline 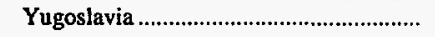 & - & - & - & - & 170 & - & - \\
\hline 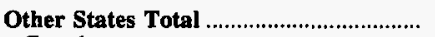 & 997 & 686 & 414 & 761 & 947 & 45.3 & 1.3 \\
\hline Canada & 273 & 93 & 55 & $*$ & 38 & 192.3 & 63.4 \\
\hline 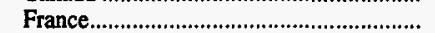 & - & - & - & 10 & 39 & - & - \\
\hline Ireland & - & - & - & 10 & - & - & - \\
\hline 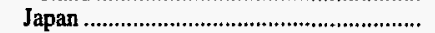 & - & - & - & - & 202 & - & - \\
\hline 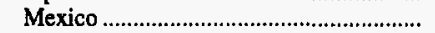 & 498 & - & 11 & - & - & - & - \\
\hline 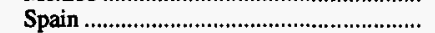 & - & 153 & - & 52 & 258 & -100.0 & - \\
\hline 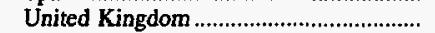 & - & - & - & 4 & - & - & - \\
\hline Unknown & 226 & 439 & 348 & 685 & 410 & -48.4 & -13.8 \\
\hline 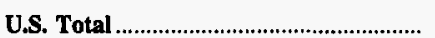 & 35,628 & 21,292 & 22,897 & 39,398 & 45,269 & 67.3 & $-\mathbf{5 . 8}$ \\
\hline 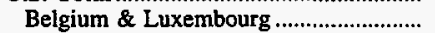 & 1,208 & 1,509 & 1,208 & 2,235 & 1,932 & -20.0 & -11.1 \\
\hline 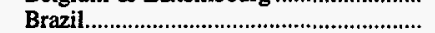 & 9 & 5 & 7 & 12 & 23 & 80.2 & -20.8 \\
\hline 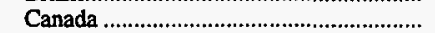 & 1,037 & 1,003 & 1,085 & 4,525 & 4,345 & 3.4 & -30.1 \\
\hline 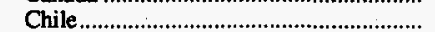 & 234 & - & - & - & 206 & - & 3.3 \\
\hline China (Taiwan) & 2,890 & 3,595 & 5,097 & 4,341 & 4,637 & -19.6 & -11.1 \\
\hline
\end{tabular}

See footnotes at end of table. 
Table 64. Foreign Distribution of U.S. Steam Coal by Major Coal-Exporting States and Destination, 1991-1995 (Continued)

(Thousand Short Tons)

\begin{tabular}{|c|c|c|c|c|c|c|c|}
\hline \multirow{2}{*}{$\begin{array}{l}\text { Coal-Exporting State } \\
\text { and Destination }\end{array}$} & \multirow{2}{*}{1995} & \multirow{2}{*}{1994} & \multirow{2}{*}{1993} & \multirow{2}{*}{1992} & \multirow{2}{*}{1991} & \multirow{2}{*}{$\begin{array}{c}\text { Percent } \\
\text { Change } \\
\text { 1994-1995 }\end{array}$} & \multirow{2}{*}{$\begin{array}{c}\begin{array}{c}\text { Average Annual } \\
\text { Percent Change }\end{array} \\
1991-1995\end{array}$} \\
\hline & & & & & & & \\
\hline \multicolumn{8}{|l|}{ U.S. Total (Continued) } \\
\hline 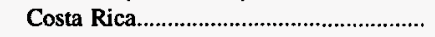 & - & - & - & $*$ & * & - & - \\
\hline 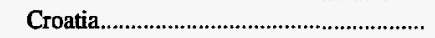 & 72 & - & 45 & - & - & - & - \\
\hline 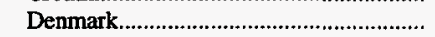 & 2,320 & 508 & 366 & 3,888 & 5,213 & 356.2 & -18.3 \\
\hline 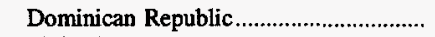 & 18 & 42 & 59 & 158 & 201 & -55.6 & -45.0 \\
\hline Finland & 654 & 71 & 2 & - & 129 & NM & 50.0 \\
\hline 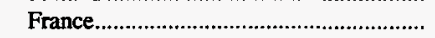 & 872 & - & 957 & 4,577 & 4,883 & - & -35.0 \\
\hline Germany, FR & 1,695 & 23 & 392 & 722 & 979 & NM & 14.7 \\
\hline Greece & - & - & - & - & 91 & - & - \\
\hline 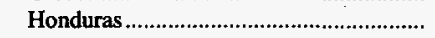 & - & - & - & - & $*$ & - & - \\
\hline 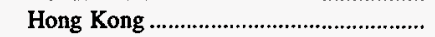 & - & 46 & - & 359 & 522 & -100.0 & - \\
\hline 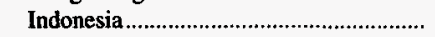 & - & - & - & * & * & - & - \\
\hline Ireland & 1,218 & 1,015 & 1,135 & 1,328 & 1,015 & 20.0 & 4.7 \\
\hline 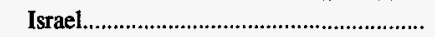 & 1,210 & 922 & 940 & 1,287 & 1,137 & 31.3 & 1.6 \\
\hline Italy & 5,342 & 2,725 & 2,128 & 3,191 & 4,910 & 96.1 & 2.1 \\
\hline 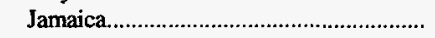 & 62 & 32 & 47 & 39 & 59 & 94.7 & 1.3 \\
\hline Japan & 3,837 & 3,331 & 3,179 & 2,324 & 2,696 & 15.2 & 9.2 \\
\hline 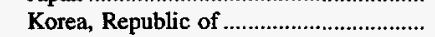 & 1,336 & 894 & 952 & 734 & 672 & 49.5 & 18.7 \\
\hline 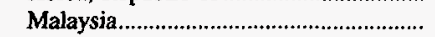 & - & - & - & $*$ & * & - & - \\
\hline Mexico & 498 & - & 11 & 18 & - & - & - \\
\hline 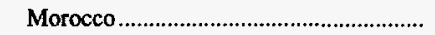 & 1,050 & 101 & 561 & 500 & 831 & NM & 6.0 \\
\hline 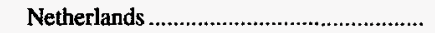 & 3,571 & 2,139 & 1,038 & 1,782 & 2,706 & 67.0 & 7.2 \\
\hline 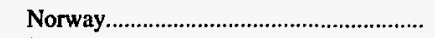 & 28 & 14 & 30 & 49 & 140 & 98.9 & -33.1 \\
\hline 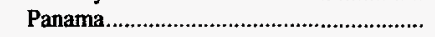 & - & - & - & * & $*$ & - & - \\
\hline 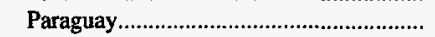 & - & - & - & 3 & - & - & - \\
\hline 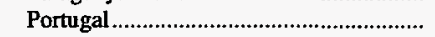 & 1,934 & 1,165 & 1,292 & 1,323 & 1,708 & 65.9 & 3.1 \\
\hline 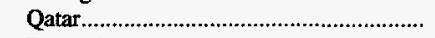 & - & - & - & * & - & - & - \\
\hline Romania & - & - & - & - & 131 & - & - \\
\hline 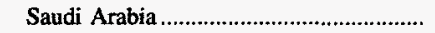 & - & - & * & - & - & - & - \\
\hline Singapore & - & - & - & - & * & - & - \\
\hline 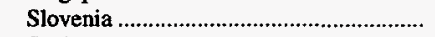 & - & - & - & 61 & - & - & - \\
\hline Spain & 2,484 & 1,704 & 1,090 & 1,760 & 1,598 & 45.8 & 11.7 \\
\hline Surinam & - & - & $*$ & * & - & - & - \\
\hline 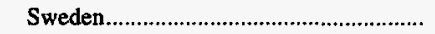 & - & - & - & 1 & 64 & - & - \\
\hline 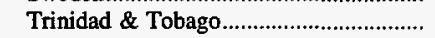 & - & - & 1 & 2 & 2 & - & - \\
\hline 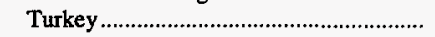 & 252 & - & - & - & 11 & - & 117.6 \\
\hline United Arab Emirates................................ & - & - & * & - & - & - & - \\
\hline United Kingdom & 1,560 & - & 911 & 3,458 & 3,811 & - & -20.0 \\
\hline Venezuela. & 9 & 11 & 15 & 39 & 39 & -18.5 & -30.1 \\
\hline 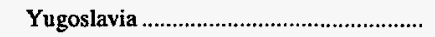 & - & - & - & - & 170 & - & - \\
\hline Unknown & 226 & 439 & 348 & 685 & 410 & -48.4 & -13.8 \\
\hline
\end{tabular}

* Data round to zero.

NM Not meaningful as value is greater than 500 percent.

Notes: Major coal-exporting States are those with total coal exports of over 600,000 short tons in 1994. Totals may not equal sum of components due to independent rounding.

Sources: Values shown for destinations other than Canada and Mexico are estimates based upon information reported on Energy Information Administration Form EIA-6, "Coal Distribution Report," and coal export data presented in King's COALBASE (King Publishing Corporation, Knoxville, Tennessee). See the Explanatory Notes for a complete description of the methodology used to develop these estimates. Values shown for Canada and Mexico are based upon Form EIA-6. 
Table 65. Distribution of U.S. Coal by Origin, Destination, and Method of Transportation, 1995

(Thousand Short Tons)

\begin{tabular}{|c|c|c|c|c|c|c|c|c|c|}
\hline \multirow{2}{*}{$\begin{array}{l}\text { Origin State and } \\
\text { Destination State }\end{array}$} & \multirow[b]{2}{*}{ Railroad } & \multicolumn{4}{|c|}{ Water } & \multirow[b]{2}{*}{ Truck } & \multirow{2}{*}{$\begin{array}{l}\text { Tramway, } \\
\text { Conveyor, } \\
\text { and Slurry } \\
\text { Pipeline }\end{array}$} & \multirow[b]{2}{*}{ Unknown } & \multirow[b]{2}{*}{ Total } \\
\hline & & River & $\begin{array}{l}\text { Great } \\
\text { Lakes }\end{array}$ & Tidewater & Total & & & & \\
\hline Alabama & 7,086 & 4,122 & - & 6,032 & 10,154 & 6,573 & 1,295 & 50 & 25,159 \\
\hline Alabama & 6,764 & 3,506 & - & - & 3,506 & 6,458 & 1,295 & - & 18,024 \\
\hline 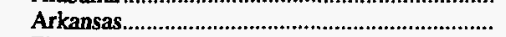 & 13 & - & - & - & - & 11 & 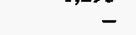 & - & 24 \\
\hline Florida & 87 & - & - & - & - & 28 & - & - & 115 \\
\hline 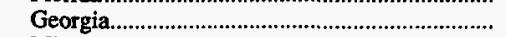 & 133 & - & - & - & - & 1 & - & - & 134 \\
\hline 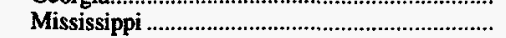 & 89 & - & - & - & - & 68 & - & - & 156 \\
\hline Pennsylvania & - & 616 & - & - & 616 & - & - & - & 616 \\
\hline South Carolina & - & - & - & - & - & 4 & - & - & 4 \\
\hline 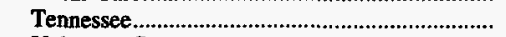 & - & - & - & - & - & 2 & - & - & 2 \\
\hline 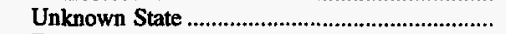 & - & - & - & - & - & - & - & 50 & 50 \\
\hline 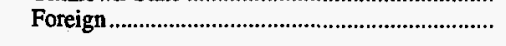 & - & - & - & 6,032 & 6,032 & - & - & - & 6,032 \\
\hline Alaska & 583 & - & - & 855 & 855 & 232 & - & - & 1,670 \\
\hline Alaska & 583 & - & - & - & - & 232 & _- & - & 815 \\
\hline 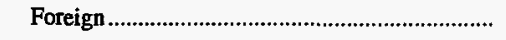 & - & - & - & 855 & 855 & - & - & - & 855 \\
\hline 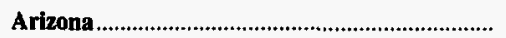 & 6,956 & - & - & - & - & - & 4,827 & - & 11,783 \\
\hline Arizona..... & 6,956 & - & - & - & - & - & & - & 6,956 \\
\hline 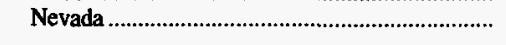 & - & - & - & - & - & - & 4,827 & - & 4,827 \\
\hline Arkansas & - & - & - & - & - & 11 & - & - & 11 \\
\hline Arkansas & - & - & - & - & - & 11 & - & - & 11 \\
\hline Colorado & 19,153 & $\mathbf{7 1 7}$ & - & 900 & 1,617 & 4,828 & - & 36 & 25,635 \\
\hline Arizona & 69 & - & - & - & - & 37 & - & - & 105 \\
\hline 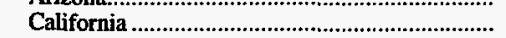 & 1 & - & - & - & - & - & - & - & 1 \\
\hline Colorado & 7,182 & - & - & - & - & 4,638 & - & - & 11,820 \\
\hline Florida & 811 & - & - & - & - & - & - & - & 811 \\
\hline 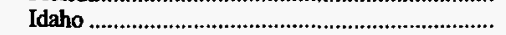 & 3 & - & - & - & - & - & - & - & 3 \\
\hline 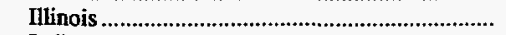 & 1,255 & 372 & - & - & 372 & - & - & - & 1,628 \\
\hline Indiana & 20 & - & - & - & - & - & - & - & 20 \\
\hline IOWa & 550 & - & - & - & - & - & - & - & 550 \\
\hline Kansas & 1,436 & - & - & - & - & - & - & - & 1,436 \\
\hline Kentucky & 1,098 & - & - & - & - & - & - & - & 1,098 \\
\hline 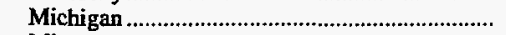 & 44 & - & - & - & - & - & - & - & 44 \\
\hline 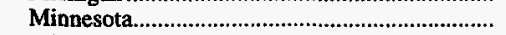 & 13 & - & - & - & - & - & - & - & 13 \\
\hline 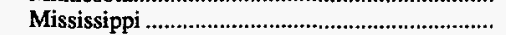 & 963 & - & - & - & - & - & - & - & 963 \\
\hline 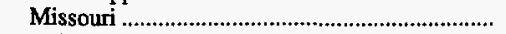 & 1,001 & - & - & - & - & 4 & - & - & 1,005 \\
\hline 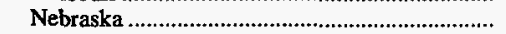 & 104 & - & - & - & - & - & - & - & 104 \\
\hline 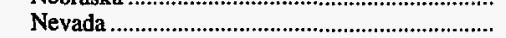 & 161 & - & - & - & - & - & - & - & 161 \\
\hline New Mexico & - & - & - & - & - & 97 & - & - & 97 \\
\hline Oklahoma & 31 & - & - & - & - & - & - & - & 31 \\
\hline 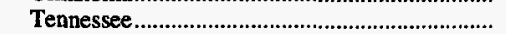 & 736 & - & - & - & - & - & - & - & 736 \\
\hline 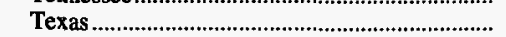 & 2,225 & - & - & - & - & 3 & - & - & 2,228 \\
\hline Utah.....1. & 1,113 & - & - & - & - & $*$ & - & - & 1,113 \\
\hline Washington & 36 & - & - & - & - & - & - & - & 36 \\
\hline Wisconsin & 296 & 345 & - & - & 345 & - & - & - & 641 \\
\hline 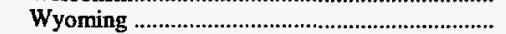 & 4 & - & - & - & - & 49 & - & - & 53 \\
\hline Unknown State ........................................................ & - & - & - & - & - & - & - & 36 & 36 \\
\hline Foreign & - & - & - & 900 & 900 & - & - & - & 900 \\
\hline 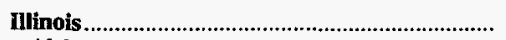 & 25,242 & 12,728 & 93 & $\mathbf{3 , 9 7 2}$ & 16,794 & $\mathbf{5 , 7 9 8}$ & 11 & 23 & 47,869 \\
\hline 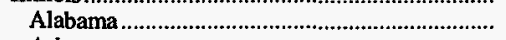 & & 1,146 & - & - & 1,146 & - & - & - & 1,146 \\
\hline 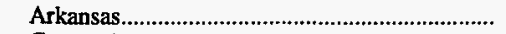 & 76 & - & - & - & - & - & - & - & 76 \\
\hline 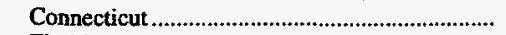 & - & - & - & - & - & * & - & - & * \\
\hline Florida & 156 & 4,500 & - & 1,231 & 5,732 & 168 & - & - & 6,056 \\
\hline 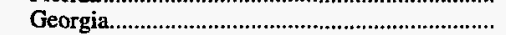 & 584 & - & - & - & - & - & - & - & 584 \\
\hline 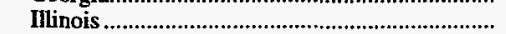 & 10,607 & 676 & - & - & 676 & 4,293 & 11 & - & 15,587 \\
\hline Indiana ………………………………………... & 8,311 & 186 & - & - & 186 & 62 & - & - & 8,559 \\
\hline 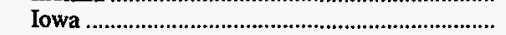 & 87 & 948 & - & - & 948 & 180 & - & - & 1,216 \\
\hline 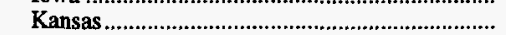 & 128 & - & - & - & - & - & - & - & 128 \\
\hline 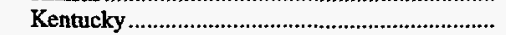 & 274 & - & - & - & - & - & - & - & 274 \\
\hline 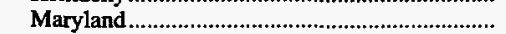 & - & - & - & - & - & 5 & - & - & 5 \\
\hline Michigan & 10 & - & 60 & - & 60 & - & - & - & 70 \\
\hline 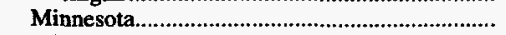 & 102 & - & - & - & - & 9 & - & - & 111 \\
\hline 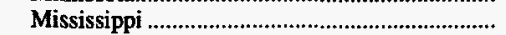 & - & 1,024 & - & 42 & 1,066 & 238 & - & - & 1,304 \\
\hline 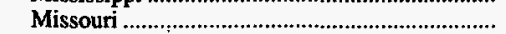 & 3,801 & 479 & - & - & 479 & 534 & - & - & 4,815 \\
\hline 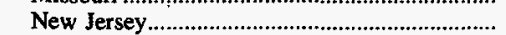 & - & - & - & - & - & * & - & - & * \\
\hline 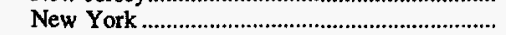 & - & - & - & - & - & * & - & - & * \\
\hline
\end{tabular}

See footnotes at end of table. 
Table 65. Distribution of U.S. Coal by Origin, Destination, and Method of Transportation, 1995

(Continued)

(Thousand Short Tons)

\begin{tabular}{|c|c|c|c|c|c|c|c|c|c|}
\hline \multirow{2}{*}{$\begin{array}{l}\text { Origin State and } \\
\text { Destination State }\end{array}$} & \multirow[b]{2}{*}{ Railroad } & \multicolumn{4}{|c|}{ Water } & \multirow[b]{2}{*}{ Truck } & \multirow{2}{*}{$\begin{array}{l}\text { Tramway, } \\
\text { Conveyor, } \\
\text { and Slurry } \\
\text { Pipeline }\end{array}$} & \multirow[b]{2}{*}{ Unknown } & \multirow[b]{2}{*}{ Total } \\
\hline & & River & $\begin{array}{l}\text { Great } \\
\text { Lakes }\end{array}$ & Tidewater & Total & & & & \\
\hline
\end{tabular}

Illinois (Continued)

North Carolina

Ohio ..

Oklahoma

Pennsylvania

Tennessee

Virginia

West Virginia

Wisconsin.......

Unknown State

Foreign

Indiana .

Alabama

Illinois

Indiana .

Iowa ...

Kentucky

Michigan

Missouri

Ohio .

Tennessee

Texas...

Wisconsin.

Unknown State

Foreign..

Kansas .

Kansas

Missouri.

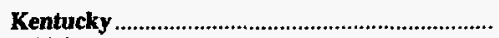

Alabama

Arkansas..

Connecticut

Florida..

Georgia.

Idaho.

Illinois

Indiana

lowa ...

Kentucky

Louisiana.

Maine ...

Maryland..

Michigan

Minnesota

Mississippi

Missouri .

Nebraska .

New Jersey

New York ...

North Carolina........

Ohio

Oklahoma

Oregon .

Pennsylvania

South Carolina

Tennessee

Texas...

Virginia...

West Virginia

Wisconsin...

Unknown State

Foreign

$\begin{array}{rrrr}- & - & - & - \\ - & \overline{-} & - & - \\ - & 10 & - & - \\ - & - & - & - \\ 438 & 3,046 & - & - \\ - & - & - & - \\ - & - & - & - \\ 667 & 712 & 33 & - \\ - & - & - & - \\ - & - & - & 2,699\end{array}$

-
$\overline{10}$
$\overline{-}$
$3,04 \overline{6}$
$\overline{-}$
746
$\overline{-}$
2,699

$*$
1
-
303
$*$
6
-
-
-

13,804

471
2,910

12,910

18

94

29

29

94,191

2,234

27

11,255

15,601

879

2,127

8
10,318
-

29

352
5511

5,511
79

79
387

24
3

973

12,668

4,714

4,714
15

1,088
9,911

9,494
2

6,065

62
286

286

76
2,786

2,339

-

16

127

2,050

19

26

100

377

201

$20 \overline{1}$

176

8,229

491

7,306

414

2,050

176

19
26

100

-

$\overline{69}$

- 262

160
102

$43,796 \quad 12,698$

30,207

1,925

2,121

561

389

227

6,861
500

-

539

539
120

427

204

-14
14

5,744

1,602

$0, \overline{3}$

9,043

2
740

225

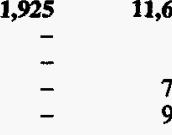

11,664
-
784
974

868
784
3,095

48

13

146

19

561

389

227

6,861
500

258

343

1,224

151
427

204

381

5,778

$1,60 \overline{2}$

$9,04 \overline{3}$

9,043
3

13
740

701

$9,61 \overline{7}$

961

-
-
-
-
$=$
$=$
-
-
7
753
$=$
753
-
-
-
-
-
-
-
-
-

$\begin{array}{rr}- & * \\ - & 1 \\ - & 10 \\ - & * \\ - & 3,787 \\ - & * \\ - & 6 \\ - & 1,412 \\ 23 & 23 \\ - & 2,699\end{array}$

$\begin{array}{rr}123 & 25,695 \\ - & 1 \\ - & 963 \\ - & 21,185 \\ - & 435 \\ - & 2,466 \\ - & 180 \\ - & 19 \\ - & 26 \\ - & 119 \\ - & 1 \\ - & 106 \\ 123 & 123 \\ - & 70\end{array}$

291

160

151,466

3,150

13
811

14,497

15,803

2
1,442

2,640

$\begin{array}{rrrr}- & - & - & 235 \\ 9,961 & - & 1 & 27,140\end{array}$

500
258

29

695

6,977

230

815

3

381
996

12,902

11,304

See footnotes at end of table. 
Table 65. Distribution of U.S. Coal by Origin, Destination, and Method of Transportation, 1995 (Continued)

(Thousand Short Tons)

\begin{tabular}{|c|c|c|c|c|c|c|c|c|c|}
\hline \multirow{2}{*}{$\begin{array}{l}\text { Origin State and } \\
\text { Destination State }\end{array}$} & \multirow{2}{*}{ Railroad } & \multicolumn{4}{|c|}{ Water } & \multirow{2}{*}{ Truck } & \multirow{2}{*}{$\begin{array}{l}\text { Tramway, } \\
\text { Conveyor, } \\
\text { and Slurry } \\
\text { Pipeline }\end{array}$} & \multirow{2}{*}{ Unknown } & \multirow[b]{2}{*}{ Total } \\
\hline & & River & $\begin{array}{l}\text { Great } \\
\text { Lakes }\end{array}$ & Tidewater & Total & & & & \\
\hline Kentucky, Eastern & 84,140 & 13,786 & 1,925 & 10,795 & 26,506 & 6,438 & - & 748 & $\mathbf{1 1 7 , 8 3 1}$ \\
\hline 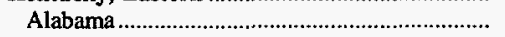 & 1,261 & 124 & - & - & 124 & 48 & - & - & 1,434 \\
\hline 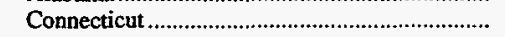 & 27 & - & - & 784 & 784 & - & - & - & 811 \\
\hline 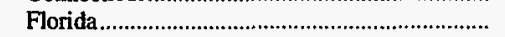 & 11,255 & 116 & - & 750 & 866 & - & - & - & 12,121 \\
\hline 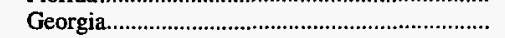 & 15,601 & 3 & - & - & 3 & 199 & - & - & 15,803 \\
\hline 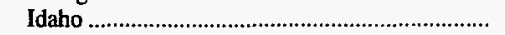 & - & - & - & - & - & 2 & - & - & 2 \\
\hline Illinois & 879 & 561 & - & - & 561 & 2 & - & - & $1,44 \overline{2}$ \\
\hline Indiana & 2,127 & 147 & - & - & 147 & 123 & - & - & 2,397 \\
\hline Iowa & 8 & 152 & - & - & 152 & - & - & - & 160 \\
\hline Kentucky & 3,867 & 1,733 & - & - & 1,733 & 4,052 & - & 1 & 9,653 \\
\hline 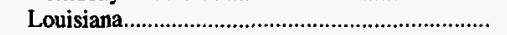 & - & 500 & - & - & 500 & - & - & - & 500 \\
\hline Maine & - & - & - & 258 & 258 & - & - & - & 258 \\
\hline 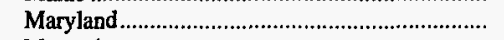 & 29 & - & - & - & - & - & - & - & 29 \\
\hline 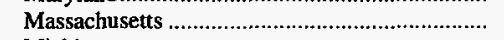 & 352 & - & - & 343 & 343 & * & - & - & 695 \\
\hline 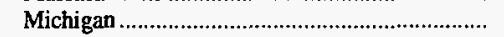 & 5,511 & 539 & 685 & - & 1,224 & 242 & - & - & 6,977 \\
\hline 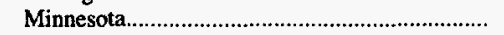 & 79 & 101 & 31 & - & 131 & 1 & - & - & 211 \\
\hline 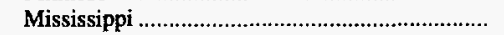 & 387 & 427 & - & - & 427 & 2 & - & - & 815 \\
\hline 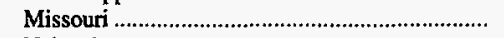 & 24 & 198 & - & - & 198 & $1 \overline{6}$ & - & - & 238 \\
\hline 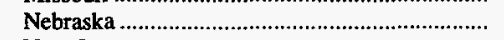 & 3 & - & - & - & - & - & - & - & 3 \\
\hline 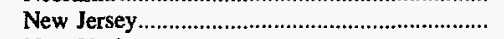 & - & 14 & - & 367 & 381 & - & - & - & 381 \\
\hline New York & 973 & 14 & - & 9 & 23 & - & - & - & 996 \\
\hline North Carolina & 12,668 & - & - & - & - & 234 & - & - & 12,902 \\
\hline Ohio & 4,678 & 5,677 & 34 & - & 5,711 & 812 & - & - & 11,200 \\
\hline 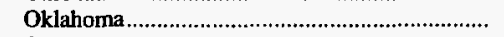 & 4 & - & - & - & - & - & - & - & 4 \\
\hline 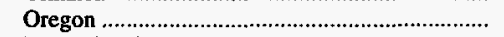 & 15 & - & - & - & - & - & - & - & 15 \\
\hline 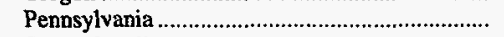 & 1,088 & 1,602 & - & - & 1,602 & 78 & - & - & 2,767 \\
\hline South Carolina & 9,911 & - & - & - & - & 96 & - & - & 10,007 \\
\hline 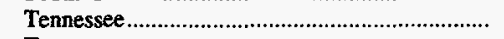 & 7,085 & 921 & - & - & 921 & 424 & - & - & 8,430 \\
\hline 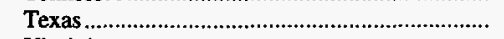 & 2 & 3 & - & - & 3 & 3 & - & - & 8 \\
\hline Virginia & 6,065 & 2 & - & 11 & 13 & 51 & - & - & 6,129 \\
\hline 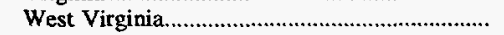 & 62 & 740 & - & - & 740 & 28 & - & - & 829 \\
\hline Wisconsin & 104 & 211 & 476 & - & 688 & 24 & - & - & 816 \\
\hline Unknown State & - & - & - & - & - & - & - & 747 & 747 \\
\hline Foreign & 76 & - & 699 & 8,273 & 8,972 & 3 & - & - & 9,051 \\
\hline Kentucky, Western & 10,051 & 16,422 & - & 869 & 17,291 & 6,260 & - & 33 & 33,635 \\
\hline 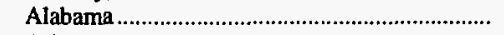 & 972 & 744 & - & - & 744 & - & - & - & 1,717 \\
\hline 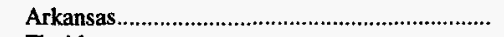 & - & - & - & - & - & 13 & - & - & 13 \\
\hline Florida & - & 2,005 & - & 224 & 2,229 & 146 & - & - & 2,375 \\
\hline Indiana & - & 242 & - & - & 242 & 1 & - & - & 243 \\
\hline Iowa & - & 75 & - & - & 75 & - & - & - & 75 \\
\hline Kentucky & 6,450 & 5,128 & - & - & 5,128 & 5,909 & - & - & 17,488 \\
\hline 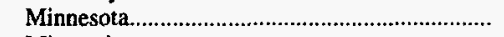 & - & 19 & - & - & 19 & - & - & - & 19 \\
\hline 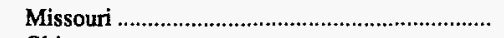 & - & 6 & - & - & 6 & - & - & - & 6 \\
\hline Ohio & 36 & 67 & - & - & 67 & - & - & - & 103 \\
\hline 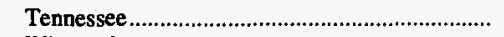 & 2,410 & 8,122 & - & - & 8,122 & 191 & - & - & 10,723 \\
\hline Wisconsin & 182 & 14 & - & - & 14 & - & - & - & 196 \\
\hline 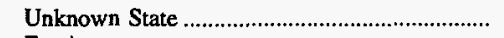 & - & - & - & - & - & - & - & 33 & 33 \\
\hline Foreign & - & - & - & 645 & 645 & - & - & - & 645 \\
\hline Louisiana & - & - & - & - & - & 838 & 2,588 & - & 3,426 \\
\hline 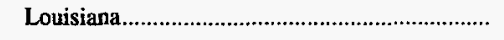 & - & - & - & - & - & 838 & 2,588 & - & 3,426 \\
\hline Maryland & 2,045 & 286 & - & 188 & 474 & 1,042 & - & 9 & $\mathbf{3 , 5 7 0}$ \\
\hline 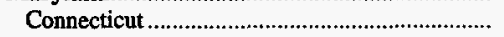 & - & - & - & - & - & 32 & - & - & 32 \\
\hline Delaware & 97 & - & - & - & - & - & - & - & 97 \\
\hline 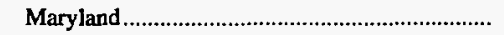 & 827 & - & - & - & - & 390 & - & - & 1,216 \\
\hline Pennsylvania & - & - & - & - & - & 45 & - & - & 45 \\
\hline Virginia & - & - & - & - & - & 12 & - & - & 12 \\
\hline 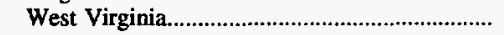 & 1,122 & 286 & - & - & 286 & 564 & - & - & 1,971 \\
\hline Unknown State & - & - & - & - & - & - & - & 9 & 9 \\
\hline Foreign & - & - & - & 188 & 188 & - & - & - & 188 \\
\hline Missouri & 85 & - & - & - & - & 379 & - & - & 464 \\
\hline
\end{tabular}

See footnotes at end of table. 
Table 65. Distribution of U.S. Coal by Origin, Destination, and Method of Transportation, 1995 (Continued)

(Thousand Short Tons)

\begin{tabular}{|c|c|c|c|c|c|c|c|c|c|}
\hline \multirow{2}{*}{$\begin{array}{l}\text { Origin State and } \\
\text { Destination State }\end{array}$} & \multirow{2}{*}{ Railroad } & \multicolumn{4}{|c|}{ Water } & \multirow{2}{*}{ Truck } & \multirow{2}{*}{$\begin{array}{c}\text { Tramway, } \\
\text { Conveyor, } \\
\text { and Slurry } \\
\text { Pipeline }\end{array}$} & \multirow[b]{2}{*}{ Unknown } & \multirow[b]{2}{*}{ Total } \\
\hline & & River & $\begin{array}{l}\text { Great } \\
\text { Lakes }\end{array}$ & Tidewater & Total & & & & \\
\hline \multicolumn{10}{|l|}{ Missouri (Continued) } \\
\hline 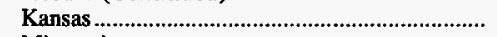 & - & - & - & - & - & 91 & - & - & 91 \\
\hline 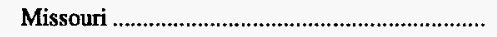 & 85 & - & - & - & - & 288 & - & - & 373 \\
\hline Montana & 22,961 & 4 & 7,464 & - & 7,468 & 413 & 8,764 & 14 & 39,620 \\
\hline 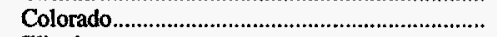 & 63 & - & - & - & - & - & - & - & 63 \\
\hline 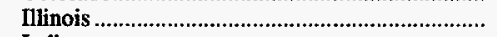 & 2,713 & - & - & - & - & - & - & - & 2,713 \\
\hline Indiana & 720 & - & - & - & - & - & - & - & 720 \\
\hline Iowa & 2 & - & - & - & - & - & - & - & 2 \\
\hline Michigan & 3,784 & - & 7,230 & - & 7,230 & - & - & - & 11,014 \\
\hline Minnesota. & 10,096 & * & 84 & - & 84 & 19 & - & - & 10,199 \\
\hline 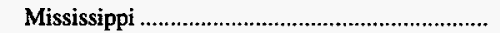 & 1,234 & - & - & - & - & - & - & - & 1,234 \\
\hline 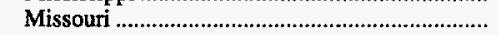 & - & - & - & - & - & 6 & - & - & 6 \\
\hline Montana & 391 & - & - & - & - & 321 & 8,764 & - & 9,477 \\
\hline Nebraska & 199 & - & - & - & - & 6 & - & - & 205 \\
\hline North Dakota & 463 & - & - & - & - & 6 & - & - & 469 \\
\hline South Dakota & 451 & - & - & - & - & 6 & - & - & 457 \\
\hline Washington & 583 & - & - & - & - & - & - & - & 583 \\
\hline Wisconsin & 2,131 & 4 & - & - & 4 & - & - & - & 2,135 \\
\hline Wyoming & 22 & - & - & - & - & 49 & - & - & 71 \\
\hline Unknown State & - & - & - & - & - & - & - & 14 & 14 \\
\hline Foreign & 108 & - & 151 & - & 151 & - & - & - & 259 \\
\hline New Mexico & 20,491 & - & - & 16 & 16 & 2,730 & 2,917 & - & 26,154 \\
\hline 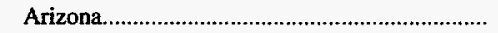 & 9,259 & - & - & - & - & $*$ & - & - & 9,259 \\
\hline New Mexico & 8,983 & - & - & - & - & 2,730 & 2,917 & - & 14,630 \\
\hline 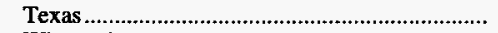 & 160 & - & - & - & - & - & - & - & 160 \\
\hline Wisconsin & 1,591 & - & - & - & - & - & - & - & 1,591 \\
\hline Foreign & 498 & - & - & 16 & 16 & - & - & - & 514 \\
\hline North Dakota. & $\mathbf{1 , 5 1 0}$ & - & - & - & - & 4,043 & 24,561 & 5 & 30,118 \\
\hline 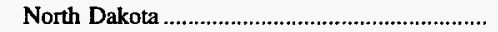 & 235 & - & - & - & - & 4,043 & 24,561 & - & 28,838 \\
\hline South Dakota & 1,276 & - & - & - & - & - & - & - & 1,276 \\
\hline Unknown State & - & - & - & - & - & - & - & 5 & 5 \\
\hline Ohio & 1,827 & 8,326 & 44 & 15 & 8,385 & $\mathbf{8 , 5 4 1}$ & 5,436 & 156 & 24,345 \\
\hline 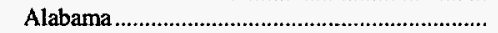 & - & 18 & - & - & 18 & - & - & - & 18 \\
\hline Indiana & 77 & 68 & - & - & 68 & 98 & - & - & 243 \\
\hline Kentucky & - & 12 & - & - & 12 & 2 & - & - & 14 \\
\hline 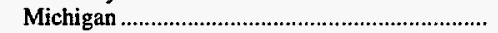 & 20 & - & 21 & - & 21 & 390 & - & - & 431 \\
\hline Missouri & - & - & - & - & - & 10 & - & - & 10 \\
\hline New York & 12 & - & - & - & - & 13 & - & - & 25 \\
\hline Ohio & 1,719 & 5,747 & - & - & 5,747 & 7,326 & 5,436 & 1 & 20,228 \\
\hline Pennsylvania & - & 867 & - & - & 867 & 676 & - & - & 1,543 \\
\hline Tennessee & - & 21 & - & - & 21 & - & - & - & 21 \\
\hline 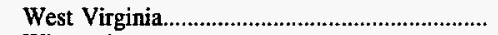 & - & 1,594 & - & - & 1,594 & 26 & - & - & 1,620 \\
\hline Wisconsin & - & - & 10 & - & 10 & - & - & - & 10 \\
\hline Unknown State & - & - & - & - & - & - & - & 156 & 156 \\
\hline Foreign & - & - & 13 & 15 & 28 & - & - & - & 28 \\
\hline 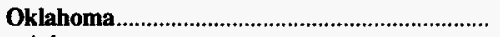 & - & 2 & - & - & 2 & 2,152 & - & 4 & 2,158 \\
\hline 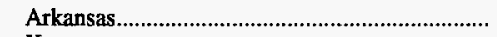 & - & - & - & - & - & 159 & - & - & 159 \\
\hline 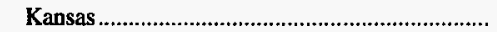 & - & - & - & - & - & 31 & - & - & 31 \\
\hline 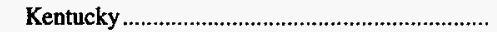 & - & 2 & - & - & 2 & - & - & - & 2 \\
\hline Missouri & - & - & - & - & - & * & - & - & * \\
\hline 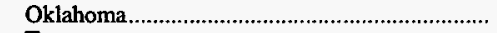 & - & - & - & - & - & 1,790 & - & - & 1,790 \\
\hline Texas & - & - & - & - & - & 171 & - & - & 171 \\
\hline 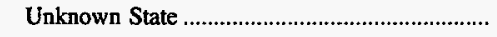 & - & - & - & - & - & - & - & 4 & 4 \\
\hline Pennsylvania & 22,370 & 9,027 & 1,700 & 7,688 & 18,415 & 15,197 & 5,701 & 557 & 62,240 \\
\hline 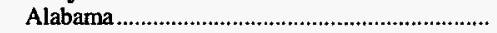 & 2 & 38 & - & - & 38 & 1 & - & - & 41 \\
\hline 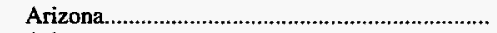 & * & - & - & - & - & * & - & - & * \\
\hline 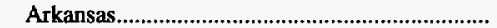 & * & - & - & - & - & * & - & - & * \\
\hline California & $*$ & - & - & - & - & - & - & - & * \\
\hline Colorado. & 12 & - & - & - & - & * & - & - & 12 \\
\hline 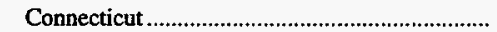 & 515 & - & - & - & - & 8 & - & - & 524 \\
\hline 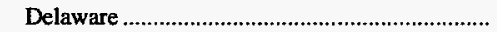 & 433 & 13 & - & - & 13 & 17 & - & - & 463 \\
\hline 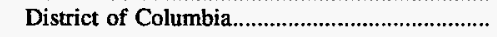 & - & - & - & - & - & $*$ & - & - & * \\
\hline
\end{tabular}

See footnotes at end of table. 
Table 65. Distribution of U.S. Coal by Origin, Destination, and Method of Transportation, 1995 (Continued)

(Thousand Short Tons)

\begin{tabular}{|c|c|c|c|c|c|c|c|c|c|}
\hline \multirow{2}{*}{$\begin{array}{l}\text { Origin State and } \\
\text { Destination State }\end{array}$} & \multirow[b]{2}{*}{ Railroad } & \multicolumn{4}{|c|}{ Water } & \multirow[b]{2}{*}{ Truck } & \multirow{2}{*}{$\begin{array}{c}\text { Tramway, } \\
\text { Conveyor, } \\
\text { and Slurry } \\
\text { Pipeline }\end{array}$} & \multirow[b]{2}{*}{ Unknown } & \multirow[b]{2}{*}{ Total } \\
\hline & & River & $\begin{array}{l}\text { Great } \\
\text { Lakes }\end{array}$ & Tidewater & Total & & & & \\
\hline
\end{tabular}

Pennsylvania (Continued)

Florida.

Georgia

Idaho.

Illinois

Indiana

Iowa .........

Kentucky

Louisiana

Maine

Maryland......

Massachusetts

Michigan

Minnesota.

Mississippi

Missouri .

New Hampshir.

New Jersey.

New Mexico

North Carolin

North Dakota

Ohio

Oklahoma.

Oregon ..

Pennsylvania

Rhode Island

South Carolina

Tennessee

Texas.

Utah

Vermont

Virginia....

Washington ..

West Virginia

Wisconsin.........

Unknown State

Foreign...

Pennsylvania Anthracite Alabama

Arizona.

Arkansas.

California .

Colorado...

Connecticut

Delaware

District of Columbia

Florida

Georgia.

Idaho.

nlinois

Indiana

Iowa ...

Kentucky

Louisiana.

Maine.

Maryland.

Massachusett

Michigan ...

Minnesota.

Mississippi

Missouri .

Nebraska

New Jersey...

New Mexico

See footnotes at end of table.

\begin{tabular}{|c|c|c|c|c|c|c|c|c|}
\hline 8 & - & - & - & - & 1 & - & - & 9 \\
\hline * & - & - & - & - & $*$ & - & - & $*$ \\
\hline- & - & - & - & - & * & - & - & * \\
\hline 8 & $\overline{7}$ & - & - & $\overline{7}$ & 1 & - & - & 9 \\
\hline $\begin{array}{r}* \\
38\end{array}$ & $\begin{array}{l}222 \\
227\end{array}$ & $\overline{-}$ & - & 222 & 6 & - & - & 227 \\
\hline $\begin{array}{l}38 \\
17\end{array}$ & 363 & $=$ & $\overline{-}$ & $\begin{array}{l}227 \\
363\end{array}$ & $\begin{array}{l}1 \\
4\end{array}$ & $=$ & $\overline{-}$ & $\begin{array}{l}266 \\
384\end{array}$ \\
\hline 7 & 3 & - & - & 3 & $i$ & - & - & $\begin{array}{r}0.4 \\
10\end{array}$ \\
\hline 21 & - & - & - & - & 14 & - & - & 35 \\
\hline 1,582 & - & - & - & - & 187 & - & - & 1,768 \\
\hline 1 & - & - & - & - & 18 & - & - & 19 \\
\hline 2,478 & - & 180 & - & 180 & 1 & - & - & 2,659 \\
\hline$*$ & 3 & - & - & 3 & 4 & - & - & 7 \\
\hline * & - & - & - & - & * & - & - & * \\
\hline * & - & - & - & - & 1 & - & - & 1 \\
\hline - & - & - & - & - & * & - & - & * \\
\hline 457 & - & - & - & - & 5 & - & - & 463 \\
\hline 556 & 2 & - & - & 2 & 15 & - & - & 573 \\
\hline${ }^{*}$ & $\overline{0}$ & - & - & - & ${ }^{*}$ & - & - & * \\
\hline 2,906 & 210 & - & - & 210 & 699 & - & - & 3,815 \\
\hline * & - & - & - & - & * & - & & * \\
\hline * & - & - & - & - & * & - & - & * \\
\hline 1,683 & 742 & 230 & - & 972 & 60 & - & - & 2,715 \\
\hline & - & - & - & - & * & - & - & * \\
\hline 12 & - & - & - & - & $*$ & - & - & 12 \\
\hline 10,990 & 5,439 & - & 23 & 5,462 & 13,976 & 5,701 & 17 & 36,147 \\
\hline- & - & - & - & - & 3 & - & - & 3 \\
\hline 6 & - & - & - & - & 3 & - & - & 9 \\
\hline 9 & 675 & - & - & 675 & 13 & - & - & 697 \\
\hline 2 & 6 & - & - & 6 & $*$ & - & - & 7 \\
\hline 215 & - & - & - & - & * & - & - & 215 \\
\hline * & - & - & - & - & 3 & - & - & 3 \\
\hline * & - & - & - & - & 29 & - & - & 29 \\
\hline$*$ & - & - & - & - & - & - & - & * \\
\hline 19 & 1,064 & - & - & 1,064 & 105 & - & - & 1,188 \\
\hline 220 & 19 & 853 & - & 872 & 16 & - & - & 1,108 \\
\hline- & - & - & - & - & - & - & 540 & 540 \\
\hline 172 & - & 437 & 7,665 & 8,102 & 6 & - & - & 8,279 \\
\hline 397 & 6 & 71 & 251 & 328 & 2,395 & 592 & 283 & 3,994 \\
\hline 1 & - & - & - & - & 1 & - & - & 1 \\
\hline * & - & - & - & - & * & - & - & * \\
\hline * & - & - & - & - & * & - & - & * \\
\hline${ }^{*}$ & - & - & - & - & - & - & - & $*$ \\
\hline 12 & - & - & - & - & * & - & - & 12 \\
\hline * & - & - & - & - & 8 & - & - & 8 \\
\hline * & - & - & - & - & 11 & - & - & 11 \\
\hline- & - & - & - & - & $*$ & - & - & \\
\hline 8 & - & - & - & - & 1 & - & - & 9 \\
\hline$*$ & - & - & - & - & * & - & - & * \\
\hline- & - & - & - & - & * & - & - & * \\
\hline 8 & - & - & - & - & 1 & - & - & 9 \\
\hline$*$ & - & - & - & - & 6 & - & - & 6 \\
\hline 38 & - & - & - & - & 1 & - & - & 39 \\
\hline 17 & - & - & - & - & 4 & - & - & 21 \\
\hline 7 & 3 & - & - & 3 & 1 & - & - & 10 \\
\hline$*$ & - & - & - & - & 3 & - & - & 3 \\
\hline 24 & - & - & - & - & 3 & - & - & 27 \\
\hline 1 & - & - & - & - & 15 & - & - & 16 \\
\hline 8 & - & - & - & - & 1 & - & - & 9 \\
\hline$*$ & 3 & - & - & 3 & 4 & - & - & 7 \\
\hline * & - & - & - & - & * & - & - & * \\
\hline * & - & - & - & - & $*$ & - & - & * \\
\hline - & - & - & - & - & * & - & - & * \\
\hline 1 & - & - & - & - & 4 & - & - & 5 \\
\hline * & - & - & - & - & 15 & - & - & 15 \\
\hline * & - & - & - & - & $*$ & - & - & * \\
\hline
\end{tabular}


Table 65. Distribution of U.S. Coal by Origin, Destination, and Method of Transportation, 1995

(Continued)

(Thousand Short Tons)

\begin{tabular}{|c|c|c|c|c|c|c|c|c|c|}
\hline \multirow{2}{*}{$\begin{array}{l}\text { Origin State and } \\
\text { Destination State }\end{array}$} & \multirow[b]{2}{*}{ Railroad } & \multicolumn{4}{|c|}{ Water } & \multirow[b]{2}{*}{ Truck } & \multirow{2}{*}{$\begin{array}{c}\text { Tramway, } \\
\text { Conveyor, } \\
\text { and Slurry } \\
\text { Pipeline }\end{array}$} & \multirow[b]{2}{*}{ Unknown } & \multirow[b]{2}{*}{ Total } \\
\hline & & River & $\begin{array}{l}\text { Great } \\
\text { Lakes }\end{array}$ & Tidewater & Total & & & & \\
\hline
\end{tabular}

Pennsylvania Anthracite (Continued)

New York

North Carolina

North Dakot

Ohio

Oklahoma

Oregon ....

Pennsylvania

Rhode Island.

South Carolina

Tennessee.

Texas.

Utah.

Vermont

Virginia....

Washington

West Virginia.

Wisconsin.

Unknown State

Foreign.

Pennsylvania Bituminous

Alabama ....

Connecticut

Delaware.

Indiana

Iowa

Kentucky

Maine ..

Maryland

Massachusett

Michigan .

Missouri

New Hampshire

New Jersey

New York

Ohio .

Pennsylvania...

South Carolina

Tennessee.

Texas

Utah.

Virginia

West Virgini

Wisconsin.

Unknown State

Foreign

Tennessee ..
Alabama..

Florida...

Georgia.

Kentucky

Michigan

North Carolin

Ohio

Tennessee.

Unknown State

Texas

Texas.

Foreign.

Utah

See footnotes at end of table.

\begin{tabular}{|c|c|c|c|c|c|c|c|c|}
\hline 22 & - & - & - & - & 118 & - & - & 140 \\
\hline * & - & - & - & - & * & - & - & * \\
\hline * & - & - & - & - & * & - & - & * \\
\hline$*$ & - & - & - & - & 8 & - & - & 8 \\
\hline$*$ & - & - & - & - & * & - & - & * \\
\hline 12 & - & - & - & - & * & - & - & 12 \\
\hline 57 & - & - & - & - & 2,119 & 592 & - & 2,768 \\
\hline- & - & - & - & - & 3 & - & - & 3 \\
\hline * & - & - & - & - & 3 & - & - & 3 \\
\hline 9 & - & - & - & - & 13 & - & - & 22 \\
\hline 2 & - & - & - & - & * & - & - & 2 \\
\hline * & - & - & - & - & * & - & - & * \\
\hline * & - & - & - & - & 3 & - & - & 3 \\
\hline * & - & - & - & - & 9 & - & - & 9 \\
\hline * & - & - & - & - & - & - & - & * \\
\hline * & - & - & - & - & 31 & - & - & 31 \\
\hline$*$ & - & - & - & - & 6 & - & - & 6 \\
\hline - & - & - & - & - & - & - & 283 & 283 \\
\hline 170 & - & 71 & 251 & 322 & 6 & - & - & 497 \\
\hline 21,973 & 9,020 & 1,629 & 7,437 & 18,087 & 12,802 & 5,109 & 275 & 58,246 \\
\hline 2 & 38 & - & - & 38 & 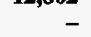 & - & - & 39 \\
\hline 515 & - & - & - & - & * & - & - & 516 \\
\hline 433 & 13 & - & - & 13 & 6 & - & - & 452 \\
\hline- & 222 & - & - & 222 & - & - & - & 222 \\
\hline- & 227 & - & - & 227 & - & - & - & 227 \\
\hline - & 363 & - & - & 363 & - & - & - & 363 \\
\hline 21 & - & - & - & - & 11 & - & - & 32 \\
\hline 1,558 & - & - & - & - & 184 & - & - & 1,741 \\
\hline - & - & - & - & - & 4 & - & - & 4 \\
\hline $2,47 \overline{0}$ & - & 180 & - & 180 & $*$ & - & - & 2,650 \\
\hline- & - & - & - & - & 1 & - & - & 1 \\
\hline 456 & - & - & - & - & 2 & - & - & 458 \\
\hline 556 & 2 & - & - & 2 & - & - & - & 558 \\
\hline 2,884 & 210 & - & - & 210 & 581 & - & - & 3,675 \\
\hline 1,683 & 742 & 230 & - & 972 & $\quad 53$ & - & - & 2,707 \\
\hline $\begin{array}{r}1,003 \\
10,934\end{array}$ & 5,439 & - & 23 & 5,462 & 11,857 & 5,109 & 17 & 33,379 \\
\hline 6 & - & - & - & - & - & - & - & 6 \\
\hline- & $67 \overline{5}$ & - & - & 675 & - & - & - & 675 \\
\hline - & 6 & - & - & 6 & - & - & - & 6 \\
\hline $21 \overline{4}$ & - & - & - & - & - & - & - & 214 \\
\hline $\begin{array}{r}214 \\
-\end{array}$ & - & - & - & - & 20 & - & - & 20 \\
\hline 19 & 1,064 & - & - & 1,064 & 74 & - & - & 1,157 \\
\hline 220 & $\begin{array}{r}1,004 \\
19\end{array}$ & 853 & - & 872 & 10 & - & - & 1,103 \\
\hline $\begin{array}{r}220 \\
-\end{array}$ & 19 & - & - & - & - & - & 257 & 257 \\
\hline 2 & - & 366 & 7,414 & 7,780 & * & - & - & 7,782 \\
\hline 1,376 & 749 & - & - & 749 & 489 & - & 13 & 2,627 \\
\hline 143 & 749 & - & - & 749 & 45 & - & - & 936 \\
\hline 39 & - & - & - & - & - & - & - & 39 \\
\hline 99 & - & - & - & - & 90 & - & - & 189 \\
\hline 5 & - & - & - & - & - & - & - & 5 \\
\hline- & - & - & - & - & * & - & - & * \\
\hline 23 & - & - & - & - & - & - & - & 23 \\
\hline 2 & - & - & - & - & * & - & - & * \\
\hline 1,068 & - & - & - & - & 354 & - & * & 1,422 \\
\hline - & - & - & - & - & - & - & 13 & 13 \\
\hline 21,250 & - & - & 20 & 20 & 13,801 & 17,761 & - & 52,832 \\
\hline 21,250 & - & - & - & - & 13,801 & 17,761 & - & 52,812 \\
\hline- & - & - & 20 & 20 & - & - & - & 20 \\
\hline 12,917 & 9 & 59 & 3,930 & 3,998 & 6,064 & 2,542 & * & 25,521 \\
\hline
\end{tabular}


Table 65. Distribution of U.S. Coal by Origin, Destination, and Method of Transportation, 1995 (Continued)

(Thousand Short Tons)

\begin{tabular}{|c|c|c|c|c|c|c|c|c|c|}
\hline \multirow{2}{*}{$\begin{array}{l}\text { Origin State and } \\
\text { Destination State }\end{array}$} & \multirow[b]{2}{*}{ Railroad } & \multicolumn{4}{|c|}{ Water } & \multirow{2}{*}{ Truck } & \multirow{2}{*}{$\begin{array}{c}\text { Tramway, } \\
\text { Conveyor, } \\
\text { and Slurry } \\
\text { Pipeline }\end{array}$} & \multirow[b]{2}{*}{ Unknown } & \multirow[b]{2}{*}{ Total } \\
\hline & & River & $\begin{array}{l}\text { Great } \\
\text { Lakes }\end{array}$ & Tidewater & Total & & & & \\
\hline
\end{tabular}

Utah (Continued)

Arizona........

California.

Colorado....

Idaho.

Illinois

Kansas

Michigan .

Minnesota.

Missouri

Montana

Nevada.

Pennsylvania

Tennessee.

Texas.

Utah.....

Washington

Wisconsin

Wyoming

Unknown State

Foreign.

Virginia .

Alabama.

Delaware

Florida.

Georgia

Illinois

Indiana ...

Kentucky

Louisiana.

Maryland..

Michigan

Missouri

New Hampshire

New Jersey

New York

North Carolina

Ohio

Pennsylvania

South Carolina

Tennessee..

Utah...

Virginia.

West Virginia.

Wisconsin...........

Unknown State

Foreign..

Washington

Oregon ..........

Washington

Foreign...

West Virginia

Alabama........

Connecticut

Delaware .............

District of Columbia

Florida..

Georgia.

Illinois

Indiana

lowa

Kentucky

Maine

80

2,837

101

1,776

17

393

6
2,047

10

1,095

4,332

125

T

19,684

19,684

1,08

152
377

2,055

27
1,511
46

21

392

52

19
228

362

3,996

351
526

1,444

2,167

313

4,118

428

(

* $\quad-$

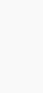

65,208

2,025

107

1,168

1,033

4,141

4,126

4,126
48

1,866

529

883

-
-
-

-
142

$$
\text { I }
$$$$
\overline{6}
$$

40,621

2,057

245
19
807
1,055
71
3,736

$\begin{array}{ll}- & - \\ - & - \\ - & - \\ - & - \\ - & - \\ - & - \\ - & - \\ - & 59 \\ - & - \\ - & - \\ - & - \\ - & - \\ - & - \\ - & - \\ - & - \\ - & - \\ - & - \\ - & - \\ 9 & - \\ - & - \\ - & - \\ - & -\end{array}$

$-$

-
$\overline{-}$
$\overline{-}$
$\overline{-}$
59
-
-
$\overline{-}$
$\overline{-}$
$\overline{-}$
$\overline{-}$
$\overline{9}$
$\overline{-}$
3,930

-
1
-
41
-
$*$
-
-
-
3
103
$*$
9
-
-
5,882
1
-
22
-
-

-
-
-
-
-
-
-
-
-
-
-
-
-
-
-
2,542
-
-
-
-
-

$-\quad 80$
$-\quad 2838$

- $\quad 2,838$

199

$\mathbf{9 , 7 0 3}$

12,251

1,050

999

$$
\overline{-}
$$

$$
2
$$

2

$\overline{9}$

21

-

$\overline{-}$

$\begin{aligned} 142 & - \\ - & - \\ - & -\end{aligned}$$$
\text { - } \quad-\quad 106
$$$$
\begin{array}{r}
106 \\
- \\
-
\end{array}
$$$$
106
$$$$
\text { - }
$$

106

106

88,894

465

$\mathbf{8 8 , 8 9 4} \quad 5,316$

88,057
465

54

323

See footnotes at end of table. 
Table 65. Distribution of U.S. Coal by Origin, Destination, and Method of Transportation, 1995 (Continued)

(Thousand Short Tons)

\begin{tabular}{|c|c|c|c|c|c|c|c|c|c|}
\hline \multirow{2}{*}{$\begin{array}{l}\text { Origin State and } \\
\text { Destination State }\end{array}$} & \multirow{2}{*}{ Railroad } & \multicolumn{4}{|c|}{ Water } & \multirow{2}{*}{ Truck } & \multirow{2}{*}{$\begin{array}{c}\text { Tramway, } \\
\text { Conveyor, } \\
\text { and Slurry } \\
\text { Pipeline }\end{array}$} & \multirow{2}{*}{ Unknown } & \multirow[b]{2}{*}{ Total } \\
\hline & & River & $\begin{array}{l}\text { Great } \\
\text { Lakes }\end{array}$ & Tidewater & Total & & & & \\
\hline \multicolumn{10}{|l|}{ West Virginia (Continued) } \\
\hline 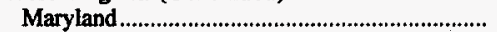 & 5,548 & 2 & - & 892 & 894 & 109 & - & - & 6,550 \\
\hline 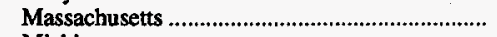 & 148 & 30 & - & 1,264 & 1,294 & * & - & - & 1,442 \\
\hline 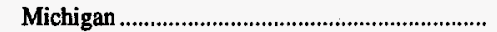 & 4,783 & 24 & 41 & - & 65 & 4 & - & - & 4,853 \\
\hline 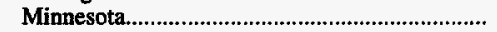 & 40 & 66 & - & - & 66 & 4 & - & - & 111 \\
\hline 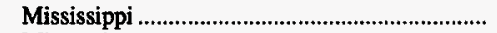 & 21 & 17 & - & - & 17 & 6 & - & - & 44 \\
\hline 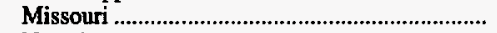 & - & 85 & - & - & 85 & - & - & - & 85 \\
\hline 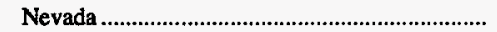 & $*$ & - & - & - & - & - & - & - & * \\
\hline New Hampshire & 296 & - & - & - & - & 2 & - & - & 299 \\
\hline 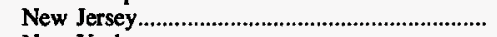 & 723 & 333 & - & 292 & 625 & * & - & - & 1,348 \\
\hline 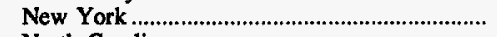 & 5,348 & 154 & - & - & 154 & 4 & - & - & 5,506 \\
\hline 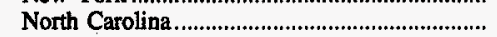 & 7,132 & 36 & - & - & 36 & 1 & - & - & 7,169 \\
\hline 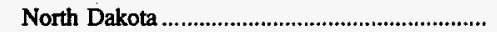 & - & * & - & - & * & - & - & - & * \\
\hline Ohio & 6,698 & 11,594 & 736 & - & 12,330 & 516 & - & - & 19,543 \\
\hline 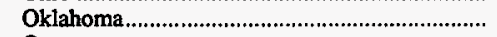 & 3 & 21 & - & - & 21 & 24 & - & - & 48 \\
\hline Oregon & 2 & - & - & - & - & - & - & - & 2 \\
\hline 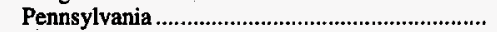 & 3,757 & 9,804 & - & - & 9,804 & 495 & - & - & 14,056 \\
\hline 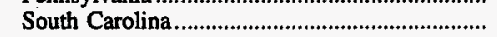 & 254 & - & - & - & - & 3 & - & - & 257 \\
\hline 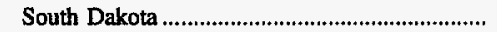 & - & 1 & - & - & 1 & * & - & - & 1 \\
\hline Tennessee. & 99 & 1,518 & - & - & 1,518 & - & - & - & 1,618 \\
\hline 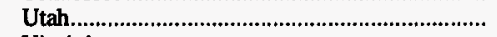 & 206 & - & - & - & - & - & - & - & 206 \\
\hline 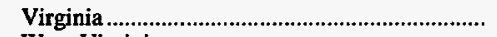 & 3,130 & 48 & - & * & 48 & 218 & - & - & 3,397 \\
\hline 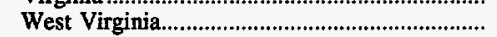 & 11,052 & 8,830 & - & - & 8,830 & 3,649 & 5,487 & - & 29,018 \\
\hline 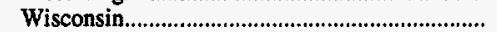 & 371 & 15 & 378 & - & 394 & $*$ & - & - & 765 \\
\hline Unknown State & - & - & - & - & - & - & - & 282 & 282 \\
\hline Foreign & 459 & - & 5,250 & 38,612 & 43,862 & - & - & - & 44,321 \\
\hline West Virginia, Northern & 12,998 & 14,653 & 1,991 & 6,010 & 22,655 & 2,983 & $\mathbf{3 , 8 4 6}$ & 134 & 42,615 \\
\hline Alabama & - & 604 & - & - & 604 & - & - & - & 604 \\
\hline 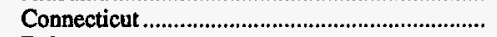 & 107 & - & - & 465 & 465 & - & - & - & 572 \\
\hline Delaware & 683 & 54 & - & - & 54 & $*$ & - & - & 737 \\
\hline District of Columbia & - & - & - & - & - & 5 & - & - & 5 \\
\hline Florida & 259 & - & - & - & - & - & - & - & 259 \\
\hline Illinois & 51 & - & - & - & - & - & - & - & 51 \\
\hline Indiana & 13 & 26 & - & - & 26 & - & - & - & 38 \\
\hline 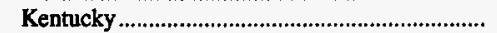 & - & 527 & - & - & 527 & - & - & - & 527 \\
\hline 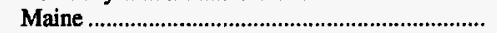 & - & - & - & - & - & 9 & - & - & 9 \\
\hline 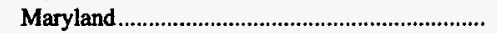 & 2,870 & - & - & 362 & 362 & 109 & - & - & 3,341 \\
\hline 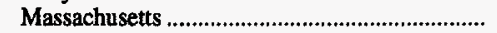 & 113 & - & - & - & - & - & - & - & 113 \\
\hline 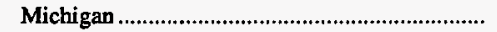 & 407 & - & 30 & - & 30 & - & - & - & 437 \\
\hline 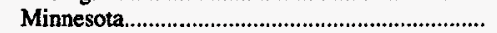 & 3 & - & - & - & - & - & - & - & 3 \\
\hline 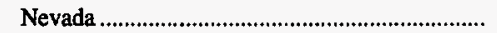 & * & - & - & - & - & - & - & - & * \\
\hline New Hampshire & 222 & - & - & - & - & 2 & - & - & 225 \\
\hline 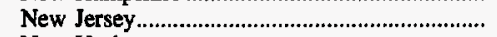 & 558 & 333 & - & 292 & 625 & $*$ & - & - & 1,182 \\
\hline New York & 4,039 & 1 & - & - & 1 & - & - & - & 4,040 \\
\hline Ohio & 587 & 1,386 & - & - & 1,386 & 4 & - & - & 1,977 \\
\hline 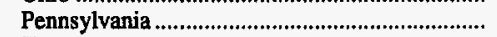 & 798 & 7,248 & - & - & 7,248 & 471 & - & - & 8,518 \\
\hline 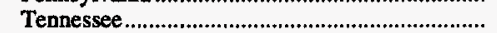 & - & 46 & - & - & 46 & - & - & - & 46 \\
\hline 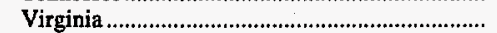 & 17 & - & - & - & - & 13 & - & - & 30 \\
\hline 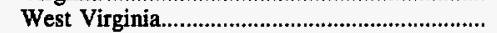 & 2,204 & 4,422 & - & - & 4,422 & 2,369 & 3,846 & - & 12,841 \\
\hline 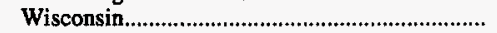 & $*$ & 6 & 378 & - & 384 & - & - & - & 384 \\
\hline 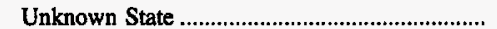 & - & - & - & - & - & - & - & 134 & 134 \\
\hline Foreign & 68 & - & 1,583 & 4,891 & 6,474 & - & - & - & 6,542 \\
\hline 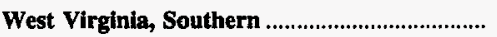 & 52,210 & 25,968 & 4,433 & 35,838 & 66,239 & 2,333 & 1,642 & 149 & 122,572 \\
\hline 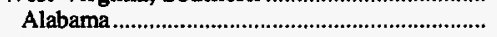 & 2,025 & 1,453 & - & - & 1,453 & 10 & - & - & 3,487 \\
\hline Delaware & 485 & - & - & - & - & - & - & - & 485 \\
\hline 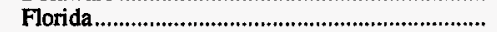 & 774 & 245 & - & 323 & 568 & - & - & - & 1,341 \\
\hline 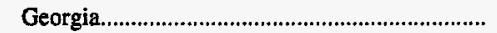 & 4,141 & 19 & - & - & 19 & - & - & - & 4,159 \\
\hline 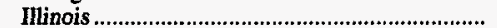 & 574 & 807 & 19 & - & 826 & - & - & - & 1,400 \\
\hline Indiana & 4,113 & 1,029 & - & - & 1,029 & - & - & - & 5,142 \\
\hline Iowa & 48 & 71 & - & - & 71 & - & - & - & 119 \\
\hline 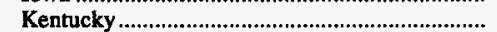 & 1,866 & 3,208 & - & - & 3,208 & 255 & - & - & 5,330 \\
\hline Maryland & 2,678 & 2 & - & 530 & 531 & - & - & - & 3,209 \\
\hline 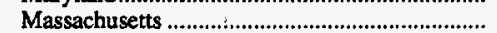 & 36 & 30 & - & 1,264 & 1,294 & * & - & - & 1,330 \\
\hline Michigan & 4,376 & 24 & 11 & - & 35 & 4 & - & - & 4,416 \\
\hline 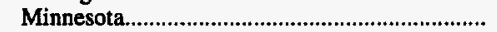 & 37 & 66 & - & - & 66 & 4 & - & - & 107 \\
\hline Mississippi & 21 & 17 & - & - & 17 & 6 & - & - & 44 \\
\hline
\end{tabular}

See footnotes at end of table. 
Table 65. Distribution of U.S. Coal by Origin, Destination, and Method of Transportation, 1995 (Continued)

(Thousand Short Tons)

\begin{tabular}{|c|c|c|c|c|c|c|c|c|c|}
\hline \multirow{2}{*}{$\begin{array}{l}\text { Origin State and } \\
\text { Destination State }\end{array}$} & \multirow[b]{2}{*}{ Railroad } & \multicolumn{4}{|c|}{ Water } & \multirow{2}{*}{ Truck } & \multirow{2}{*}{$\begin{array}{c}\text { Tramway, } \\
\text { Conveyor, } \\
\text { and Slurry } \\
\text { Pipeline }\end{array}$} & \multirow{2}{*}{ Unknown } & \multirow[b]{2}{*}{ Total } \\
\hline & & River & $\begin{array}{l}\text { Great } \\
\text { Lakes }\end{array}$ & Tidewater & Total & & & & \\
\hline
\end{tabular}

West Virginia, Southern (Continued)

Missouri

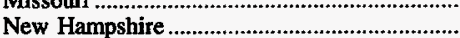
New Jersey..

New York

North Carolina

North Dakota

Ohio

Oklahoma

Oregon

Pennsylvania

South Carolina.

South Dakota

Tennessee

Utah.

Virginia .

.

West Virginia.

Wisconsin.

Unknown State

Foreign.

Wyoming

Alabama

Arkansas

Colorado

Georgia

Idaho

Illinois .

Indiana

lowa

Kansas

Louisian

Maryland

Michigan

Missouri .

Montana .

Nebraska

Nevada

North Dakota

Oklahoma

Oregon .

South Dakota.

Tennessee

Texas

Utah.

Washington

Wisconsin.

Wyoming

Unknown s

Foreign.

U.S. Total

Alabama.

Alaska

Arizona.

Arkansas.

California

Colorado..........

Connecticut .

Delaware.

District of

.

Georgia .

Idaho ....

Illinois ...

Indiana

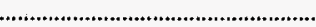

See footnotes at end of table.

\begin{tabular}{|c|c|c|c|c|c|c|c|c|}
\hline - & 85 & - & - & 85 & - & - & - & 85 \\
\hline 74 & - & - & - & - & - & - & - & 74 \\
\hline 165 & - & - & - & - & - & - & - & 165 \\
\hline 1,309 & 153 & - & - & 153 & 4 & - & - & 1,466 \\
\hline 7,132 & 36 & - & - & 36 & 1 & - & - & 7,169 \\
\hline- & * & - & - & * & - & - & - & * \\
\hline 6,111 & 10,208 & 736 & - & 10,943 & 512 & - & - & 17,566 \\
\hline 3 & 21 & - & - & 21 & 24 & - & - & 48 \\
\hline 2 & - & - & - & - & - & - & - & 2 \\
\hline 2,959 & 2,556 & - & - & 2,556 & 23 & - & - & 5,538 \\
\hline 254 & - & - & - & - & 3 & - & - & 257 \\
\hline- & 1 & - & - & 1 & * & - & - & 1 \\
\hline 99 & 1,472 & - & - & 1,472 & - & - & - & 1,571 \\
\hline 206 & - & - & - & - & - & - & - & 206 \\
\hline 3,114 & 48 & - & * & 48 & 205 & - & - & 3,367 \\
\hline 8,848 & 4,408 & - & - & 4,408 & 1,279 & 1,642 & - & 16,177 \\
\hline 371 & 10 & - & - & 10 & * & - & - & 381 \\
\hline - & - & - & - & - & - & - & 149 & 149 \\
\hline 391 & - & 3,667 & 33,721 & 37,388 & - & - & - & 37,779 \\
\hline 231,897 & 13,617 & 538 & 2,237 & 16,392 & 3,565 & 11,734 & 13 & 263,601 \\
\hline 2,950 & - & - & - & - & - & - & - & 2,950 \\
\hline 14,033 & - & - & - & - & - & - & - & 14,033 \\
\hline 5,602 & - & - & - & - & - & - & - & 5,602 \\
\hline 6,796 & - & - & - & - & - & - & - & 6,796 \\
\hline 266 & - & - & - & - & 27 & - & - & 293 \\
\hline 15,480 & - & - & - & - & - & - & - & 15,480 \\
\hline 18,306 & - & - & - & - & - & - & - & 18,306 \\
\hline 16,448 & 507 & - & - & 507 & - & - & - & 16,955 \\
\hline 14,243 & - & - & - & - & - & - & - & 14,243 \\
\hline 6,185 & 4,123 & - & - & 4,123 & - & - & - & 10,309 \\
\hline 636 & - & - & - & - & - & - & - & 636 \\
\hline 7,543 & - & - & - & - & - & - & - & 7,543 \\
\hline 8,353 & - & 463 & - & 463 & - & - & - & 8,816 \\
\hline 16,774 & 8,948 & - & - & 8,948 & 9 & - & - & 25,731 \\
\hline 193 & - & - & - & - & $*$ & - & - & 193 \\
\hline 10,065 & - & - & - & - & $*$ & - & - & 10,065 \\
\hline 342 & - & - & - & - & - & - & - & 342 \\
\hline- & - & - & - & - & * & - & - & * \\
\hline 20,326 & - & - & - & - & - & - & - & 20,326 \\
\hline 1,485 & - & - & - & - & - & - & - & 1,485 \\
\hline 770 & - & - & - & - & 471 & - & - & 1,241 \\
\hline 4 & 16 & - & - & 16 & - & - & - & 20 \\
\hline 38,227 & 23 & - & - & 23 & - & - & - & 38,250 \\
\hline- & - & - & - & - & $*$ & - & - & * \\
\hline 1,290 & - & - & - & - & - & - & - & 1,290 \\
\hline 13,852 & - & 43 & - & 43 & - & - & - & 13,895 \\
\hline 11,728 & - & - & - & - & 3,058 & 11,734 & - & 26,521 \\
\hline- & - & - & - & - & - & - & 13 & 13 \\
\hline- & - & 32 & 2,237 & 2,269 & - & - & - & 2,269 \\
\hline 590,665 & 125,101 & 18,824 & 89,245 & 233,170 & 104,384 & 100,002 & 2,110 & $1,030,330$ \\
\hline 15,199 & 8,382 & - & - & 8,382 & 6,564 & 1,295 & - & 31,440 \\
\hline 583 & - & - & - & - & 232 & - & - & 815 \\
\hline 16,364 & - & - & - & - & 37 & - & - & 16,401 \\
\hline 14,122 & - & - & - & - & 195 & - & - & 14,317 \\
\hline 2,838 & - & - & - & - & 1 & - & - & 2,839 \\
\hline 12,862 & - & - & - & - & 4,640 & - & - & 17,502 \\
\hline 667 & - & - & 1,248 & 1,248 & 40 & - & - & 1,955 \\
\hline 1,850 & 67 & - & - & 67 & 17 & - & - & 1,933 \\
\hline & - & - & - & - & 6 & - & - & 6 \\
\hline 13,766 & 6,866 & - & 2,529 & 9,395 & 344 & - & - & 23,505 \\
\hline 29,409 & 21 & - & - & 21 & 300 & - & - & 29,730 \\
\hline 370 & - & - & - & - & 70 & - & - & 440 \\
\hline 33,841 & 2,946 & 40 & - & 2,987 & 4,788 & 11 & - & 41,626 \\
\hline 48,109 & 2,819 & 201 & - & 3,019 & 7,595 & 753 & - & 59,476 \\
\hline 17,489 & 2,108 & - & - & 2,108 & 181 & - & - & 19,777 \\
\hline
\end{tabular}


Table 65. Distribution of U.S. Coal by Origin, Destination, and Method of Transportation, 1995 (Continued)

(Thousand Short Tons)

\begin{tabular}{|c|c|c|c|c|c|c|c|c|c|}
\hline \multirow{2}{*}{$\begin{array}{l}\text { Origin State and } \\
\text { Destination State }\end{array}$} & \multirow{2}{*}{ Reiltroad } & \multicolumn{4}{|c|}{ Water } & \multirow{2}{*}{ Truck } & \multirow{2}{*}{$\begin{array}{c}\text { Tramway, } \\
\text { Conveyor, } \\
\text { and Slurry } \\
\text { Pipeline }\end{array}$} & \multirow{2}{*}{ Unknown } & \multirow{2}{*}{ Total } \\
\hline & & River & $\begin{array}{l}\text { Great } \\
\text { Lakes }\end{array}$ & Tidewater & Total & & & & \\
\hline
\end{tabular}

U.S. Total (Continued)

Kansas.

Kentucky.

Louisiana.

Maine.

Maryland

Massachusetts

Michigan

Minnesota.

Mississippi

Missouri .

Montana

Nebraska .

Nevada

New Hampshire

New Jersey

New Mexico

New York

North Carolin

North Dakota

Ohio

Oklahoma

Oregon ...

Pennsylvania

Rhode Island

South Carolina

South Dakota

Tennessee.

Texas

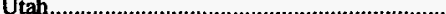

Vermont

Virginia.

Washington

West Virginia

Wisconsin

Wyoming .

Unknown State

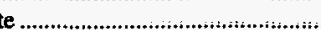

Foreign ....................................:
15,807

13,625
6,213

6,213
21

9,012

502

24,241

18,684

2,694

22,116

590

10,371

2,550

773

1,507

8,983

9,600

23,818

697

15,164

20,364

1,515

16,371

$11, \hat{61 \hat{5}}$

2,497

15,129

61,869

6,178

13,314

2,035

12,682

19,590

11,755

$1,31 \overline{2}$
13,024

4,627

$-$

2
30
563

563

189
1,468

9,736

-

349

379

36
$*$

23,994

31

19,117

-

14,419

32

-

50

12,519

1,329

1,329

-
-

- $\quad$ - 13,024

- 258

$\begin{array}{rr}- & 892 \\ - & 1,607\end{array}$

8,483

578

- 42

- $\quad-$

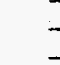

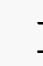

-

-

1,000

-

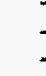

$-$

$-$

-
-

$-$

-

1,793

-

6,728

81,560
4,627

258

894

1,637

9,046

767
1,510

9,736

-

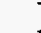

1,415

388

38
$*$

24,995

31

19,140

-

14,419

32

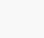

61

3,123

88,287
283
10733

10,733
839

23

690
18

641

37

312

969

324

6
103

8

15
2,827

717

295
4,049

1,815

1,815
3

15,279

3

129
477

1,308
13,979

5,882

851
129

4,377

3,179

308
2,588

$-$

-

-

-

8,764

4,827

2,917

24,561

5,436

5,701

$-$

-

17,761

2,542

999

4,627

5,487

$11,73 \overline{4}$

-
16,091

37,382

14,267

302

10,596

2,157

33,928

19,488

4,516

32,821

9.678

10,377

7,479

780

2,936

14,727

10,705

24,149

29,307

54,310

22,210

1,518

56,509

11,745

2,976

30,856

93,642

14,602

15,225

15,225

6,790

35,065

22,766

26,668

2,091

* Data round to zero.

Note: Totals may not equal sum of components due to independent rounding.

Source: Energy Information Administration, Form EIA-6, "Codal Distribution Report," 



\section{Demand}

\section{Domestic Markets}

U.S. coal consumption during 1995 reached a record 941 million short tons, surpassing by 1.1 percent the previous record of 930 million short tons consumed during 1994. Consumption of coal by electric utilities rose 1.4 percent to a record 829 million short tons, accounting for 88 percent of total domestic coal consumption (Table 69). Compared with 1994, coal consumption at U.S. coke plants during 1995 rose 4 percent to 33 million short tons, while consumption at other industrial plants declined 3 percent to 73 million short tons (Table 71 and Table 73).

\section{Electric Utility Use}

Leading the 1995 increase in electric utility coal consumption was the additional 16 million short tons of coal consumed by coal-fired electricity generators in the East South Central, West North Central, and East North Central Divisions. Collectively, electric utilities in these three Census divisions accounted for 48 percent of total electric utility coal consumption in 1995. Electric utility coal consumption in the East South Central Division rose 8 percent to 92 million short tons, reflecting the increased use of coal-fired generators to meet a 5-percent increase in electricity demand. In the West North Central Division, electric utility coal consumption rose 5 percent to 117 million short tons as coal-fired electricity generation in Missouri and Nebraska increased 10.3 percent and 14.8 percent, respectively. Similarly, in the East North Central Division, rising electricity demand, notably in Michigan and Ohio, boosted electric utility coal consumption 2 percent to 187 million short tons. The increased 1995 electric utility coal consumption in these three Census Divisions was offset by declines totaling 11 million short tons of coal consumed by electric utilities in the Mountain and Pacific Divisions. Electric utility coal consumption in the Mountain Division declined 7 percent to 101 million short tons as reduced electricity demand, coupled with increased hydroelectric generation, led to a decline of 8 percent in coal-fired electricity generation. In the Pacific Division, electric utility coal consumption declined 41 percent to 5 million short tons as increased hydroelectric generation in Washington and Oregon replaced coal-fired generation.

\section{Coke Plant Use}

The increase in the total amount of coal consumed at U.S. coke plants during 1995 was attributable primarily to plants in Indiana, where consumption of coking coals rose by more than 1 million short tons (22 percent) to 6 million short tons. This increase, coupled with increased coal consumption at coke plants in Illinois and Kentucky, more than offset a 10-percent decline in coal consumption at coke plants in Ohio.

\section{Other Uses}

Compared with 1994, coal consumption in the other industrial sector declined 3.2 percent to 72.8 million short tons. Coal consumption in the residential and commercial sectors during 1995 totaled 6 million short tons, 3 percent lower than in 1994.

\section{Coal Stocks}

Stocks of coal held by consumers at the close of 1995 totaled 134.6 million short tons, a decline of 1 percent compared with stocks at the close of 1994. Compared with year-end 1994, coal stocks at electric utility power plants and coke plants at the end of 1995 remained relatively constant at approximately 127 million short tons and 3 million short tons, respectively, while coal stocks held by other industrial consumers declined 13 percent to 5.7 million short tons.

\section{Foreign Markets}

U.S. coal exports during 1995 totaled 88.5 million short tons, 17.2 million short tons ( 24.1 percent) more than in 1994. The primary reason for the increase was the substantial growth in export demand for U.S. steam coal. Compared with 1994, exports of steam coal to Europe more than doubled to 21 million short tons, reflecting sharp increases in steam coal shipments to most European destinations except Romania and Belgium and Luxembourg. Steam coal exports to Denmark, Germany, and the United Kingdom, which totaled less than one million short tons in 1994, increased to 5.6 million short tons, accounting for 46 percent of the total increase in steam coal shipments to Europe. Similarly, compared with 1994, steam coal shipments to Italy increased 82.5 percent to 4.6 million short tons while shipments to the Netherlands more than doubled to 3.3 million short tons. In the Far East, increases in steam coal shipments to Japan and the Republic of Korea offset a decline in shipments to Taiwan. U.S. metallurgical coal exports during 1995 
rose 10 percent to 52 million short tons, accounting for 59 percent of total coal exports. Shipments of metallurgical coal to Europe, which accounted for over half of total metallurgical coal exports, increased 8 percent to 27 million short tons as increased shipments to Romania, Turkey, and the Netherlands offset declines in shipments to Spain, Italy, and the United
Kingdom. Metallurgical coal shipments to Japan increased 10 percent to 8 million short tons, while shipments to Brazil increased 16 percent to 6 million short tons. 


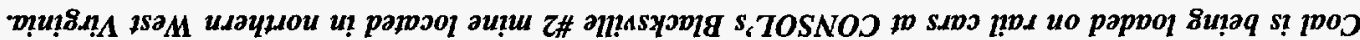

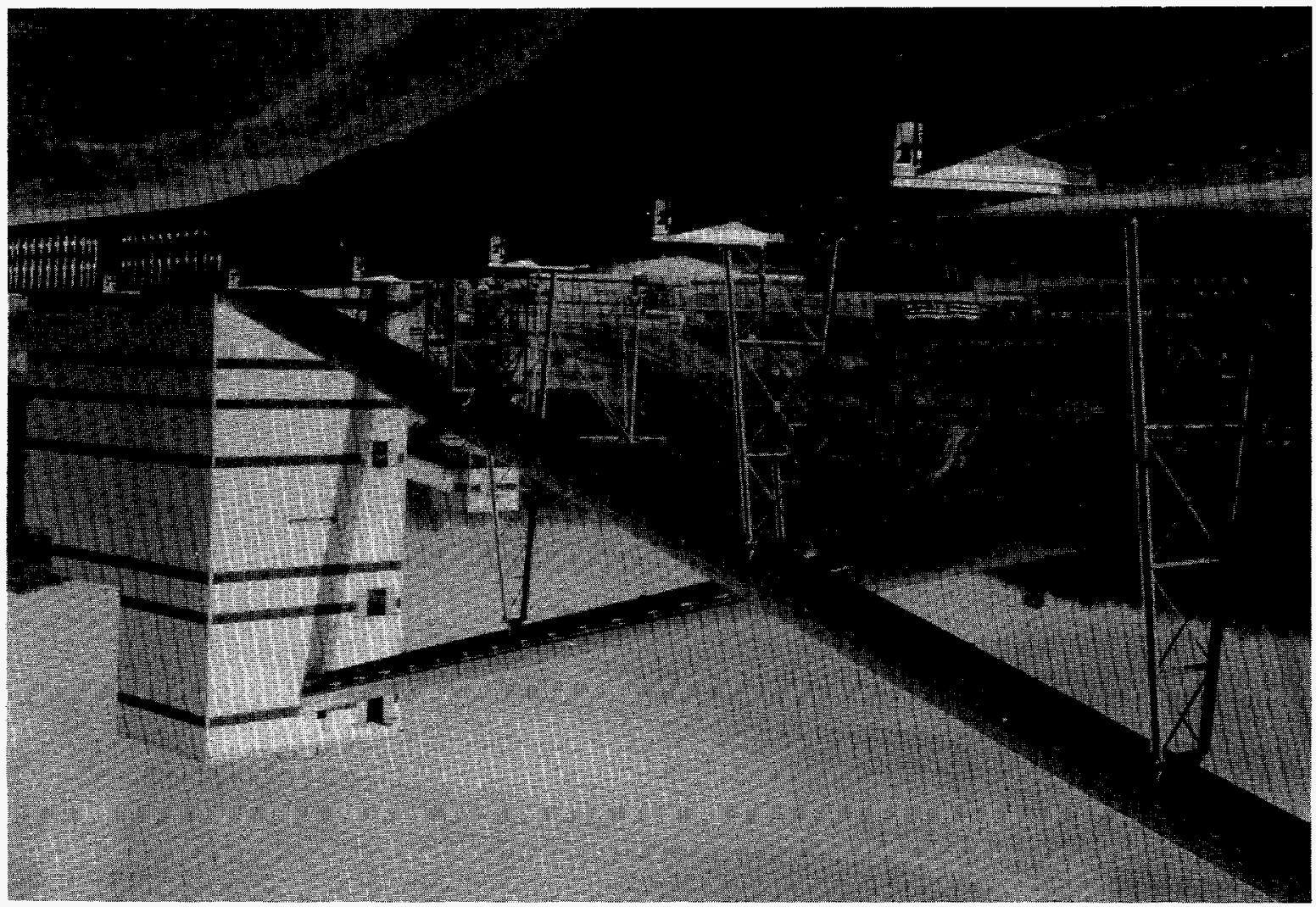

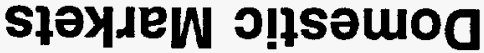


Figure 10. U.S. Coal Consumption, 1986-1995

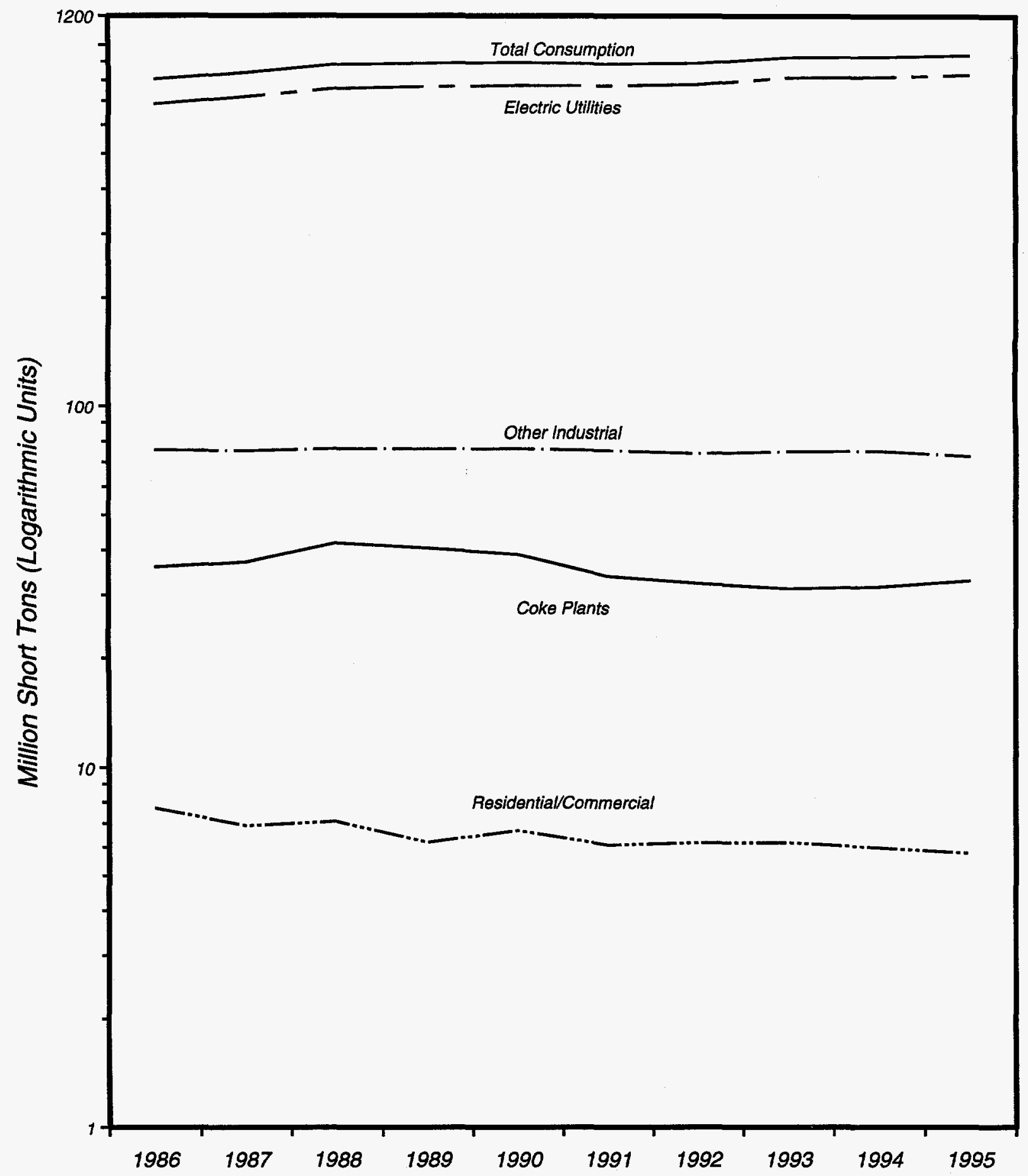

Note: Total coal consumption does not include coal consumed by independent power producers.

Sources: Energy Information Administration, •Electric Utilities: Form ElA-759, "Monthly Power Plant Report." •Coke Plants: Form EIA-5, "Coke Plant Report - Quarterly." -Other Industrial: Form EIA-3, "Quarterly Coal Consumption Report - Manufacturing Plants" and Form EIA-6, "Coal Distribution Report." •Residential and Commercial: Form EIA-6, "Coal Distribution Report." 
Table 66. Major U.S. Coal Consumers, 1995

\begin{tabular}{|c|c|c|}
\hline Rank & Company Name & Plant Locations \\
\hline & \multicolumn{2}{|c|}{ Electric Utilities } \\
\hline 1 & Tennessee Valley Auth & (AL) (KY) (TN) \\
\hline 2 & Texas Utilities Elec Co & (TX) \\
\hline 3 & Georgia Power Co & (GA) \\
\hline 4 & Pacificorp & (UT) (WA) (WY) \\
\hline 5 & Detroit Edison Co (The) & (MI) \\
\hline 6 & Alabama Power Co & (AL) \\
\hline 7 & Houston Lighting \& Pwr Co & (TX) \\
\hline 8 & GPU Service Corporation & $(\mathbf{P A})$ \\
\hline 9 & Basin Elec Power Coop & (ND) (WY) \\
\hline 10 & PSI Energy, Inc & (IN) \\
\hline 11 & Ohio Power Co & $(\mathrm{OH})(\mathrm{WV})$ \\
\hline 12 & Commonwealth Edison Co & (IL) \\
\hline 13 & Northern States Power Co & $(\mathrm{MN})(\mathrm{WI})$ \\
\hline 14 & Union Electric Co & (MO) \\
\hline 15 & Indiana Michigan Power Co & (IN) \\
\hline 16 & Duke Power Co & (NC) $(\mathrm{SC})$ \\
\hline 17 & Virginia Elec \& Power Co & (VA) (WV) \\
\hline 18 & Arkansas Power \& Light Co & (AR) \\
\hline 19 & Arizona Public Service Co & (AZ) (NM) \\
\hline 20 & Monongahela Power Co & (WV) \\
\hline 21 & MidAmerican Energy & (IA) \\
\hline 22 & Southwestern Elec Pwr Co & (AR) (TX) \\
\hline 23 & Cincinnati Gas Elec Co & (KY) (OH) \\
\hline 24 & Kansas City Pwr \& Lgt Co & (KS) (MO) \\
\hline 25 & Carolina Power \& Light Co & (NC) (SC) \\
\hline 26 & Wisconsin Electric Pwr Co & (MI) (WI) \\
\hline 27 & Oklahoma Gas \& Elec Co & $(\mathrm{OK})$ \\
\hline 28 & Public Service Co of Colo & (CO) \\
\hline 29 & Salt River Project & (AZ) \\
\hline 30 & Montana Power Co (The) & (MT) \\
\hline 31 & Appalachian Power Co & (VA) (WV) \\
\hline 32 & KPL - Western Resources & (KS) \\
\hline 33 & Associated Elec Coop & (MO) \\
\hline 34 & Southwestern Pub Serv Co & (TX) \\
\hline 35 & Pennsylvania Pwr \& Lgt Co & (PA) \\
\hline 36 & Northern Ind Pub Serv Co & (IN) \\
\hline 37 & Wisconsin Pwr \& Lgt Co & (WI) \\
\hline 38 & Dayton Pwr \& Lgt Co (The) & $(\mathrm{OH})$ \\
\hline 39 & Tampa Electric Co & (FL) \\
\hline 40 & Cooperative Power Asso & (ND) \\
\hline 41 & Ohio Edison Co & $(\mathrm{OH})$ \\
\hline 42 & Consumers Power Co & (MI) \\
\hline 43 & Illinois Power Co & (IL) \\
\hline 44 & Indianapolis Pwr \& Lgt Co & (IN) \\
\hline 45 & Kentucky Utilities Co & $(\mathrm{KY})$ \\
\hline 46 & Lower Colorado River Auth & (TX) \\
\hline 47 & Louisville Gas \& Elec Co & $(\mathrm{KY})$ \\
\hline 48 & Potomac Electric Pwr Co & (MD) (VA) \\
\hline 49 & Pub Serv Co of New Mexico & (NM) \\
\hline 50 & Pennsylvania Power Co & (PA) \\
\hline 51 & Cajun Elec Power Coop Ine & (LA) \\
\hline 52 & Nebraska Pub Power Dist & (NE) \\
\hline 53 & Florida Power Corporation & $(\mathrm{FL})$ \\
\hline 54 & Central Ill Public Ser Co & (IL) \\
\hline 55 & Baltimore Gas \& Elec Co & (MD) \\
\hline 56 & Central Louisiana Elec Co & (LA) \\
\hline 57 & So Carolina Pub Serv Auth & (SC) \\
\hline 58 & Big Rivers Electric Corp & $(\mathrm{KY})$ \\
\hline 59 & Southern Calif Edison Co & (NV) \\
\hline 60 & Electric Energy Inc & (IL) \\
\hline 61 & Cleveland Elec Illum Co & (OH) \\
\hline 62 & Indiana-Kentucky El Corp & (IN) \\
\hline 63 & Scana Corporation & (SC) \\
\hline 64 & West Penn Power Co & (PA) \\
\hline 65 & I E S Utilities Co & (IA) \\
\hline 66 & San Antonio Pub Serv Brd & (TX) \\
\hline 67 & Tri-state G \& T Assn Inc & (CO) \\
\hline 68 & Cardinal Operating Co & (OH) \\
\hline 69 & Los Angeles (City of) & (UT) \\
\hline 70 & Minnesota Power \& Lgt Co & (MN) \\
\hline 71 & Minnkota Power Coop Inc & (ND) \\
\hline 72 & Grand River Dam Authority & (OK) \\
\hline 73 & Omaha Public Power Dist & (NE) \\
\hline
\end{tabular}

See footnotes at end of table. 
Table 66. Major U.S. Coal Consumers, 1995 (Continued)

\begin{tabular}{|c|c|}
\hline Company Name & Plant Location \\
\hline \multicolumn{2}{|c|}{ Top Ten Manufacturers } \\
\hline $\begin{array}{l}\text { Aluminum Company of America } \\
\text { Archer Daniels Midland Co } \\
\text { Basin Electric Power Coop } \\
\text { Champion International Corp } \\
\text { E I Du Pont De Nemours \& Co } \\
\text { Eastman Kodak Company } \\
\text { General Motors Corporation } \\
\text { Hoechst AG } \\
\text { Holnam Inc } \\
\text { Westvaco Corporation }\end{array}$ & $\begin{array}{l}\text { (IN) (OH) (TN) (TX) } \\
\text { (IA) (IL) (MN) (NE) } \\
\text { (ND) } \\
\text { (AL) (FL) (ME) (MI) (MN) (NC) (NY) (OH) } \\
\text { (DE) (KY) (MS) (NC) (SC) (TN) (VA) (WV) } \\
\text { (AR) (NY) (SC) (TN) (TX) } \\
\text { (AL) (IL) (IN) (MI) (MO) (NY) (OH) (WI) } \\
\text { (SC) (TX) (VA) } \\
\text { (AL) (CO) (IA) (MI) (MO) (MS) (MT) (SC) (UT) (WA) } \\
\text { (MD) (PA) (SD) (VA) }\end{array}$ \\
\hline \multicolumn{2}{|c|}{ Other Major Manufacturers } \\
\hline $\begin{array}{l}\text { A E Staley Manufacturing Co } \\
\text { American Crystal Sugar Co } \\
\text { Applied Energy Services Inc } \\
\text { Ash Grove Cement Company } \\
\text { Blue Circle Inc } \\
\text { Cargill Incorporated } \\
\text { Dravo Corporation } \\
\text { Elkem A/S } \\
\text { Florida Crushed Stone } \\
\text { Fort Howard Corporation } \\
\text { FMC Corporation } \\
\text { G E Company } \\
\text { General Chemical Corporation } \\
\text { Heidelberger Zement Ag-Heidelb } \\
\text { Inland Steel Industries Inc } \\
\text { International Paper Company } \\
\text { Jefferson Smurfit Corporation } \\
\text { Kerr-McGee Corporation } \\
\text { Lafarge Corporation } \\
\text { Lone Star Industries Inc. } \\
\text { Marblehead Lime Co } \\
\text { Monsanto Company } \\
\text { New King Fuel Co Inc } \\
\text { P H Glatfelter Co } \\
\text { PPG Industries Inc } \\
\text { Southdown Inc } \\
\text { Stone Container Corporation } \\
\text { Tenneco Inc } \\
\text { Union Camp Corporation } \\
\text { Willamette Industries Inc }\end{array}$ & $\begin{array}{l}\text { (IL) (IN) (TN) } \\
\text { (MN) (ND) } \\
\text { (PA) } \\
\text { (AR) (KS) (MT) (NE) (OR) (TX) (UT) } \\
\text { (AL) (GA) (NY) (OK) (SC) } \\
\text { (GA) (IA) (MN) (NC) (OH) (TN) (VA) } \\
\text { (AL) (KY) } \\
\text { (OH) (WV) } \\
\text { (FL) } \\
\text { (GA) (OK) (WI) } \\
\text { (NC) (WV) (WY) } \\
\text { (IN) (KY) (PA) } \\
\text { (WY) } \\
\text { (AL) (IA) (IN) (MD) (NY) } \\
\text { (IN) } \\
\text { (AL) (LA) (PA) (SC) (WI) } \\
\text { (AL) (FL) (IL) (IN) (OH) (PA) } \\
\text { (CA) } \\
\text { (IA) (IL) (KS) (MI) (MO) (OH) (PA) } \\
\text { (FL) (IL) (IN) (MO) (OK) (TX) } \\
\text { (IL) (IN) (MI) } \\
\text { (AL) (IA) (ID) (IL) (MA) (WV) } \\
\text { (IA) (NC) (OH) (PA) (UT) (VA) } \\
\text { (NC) (PA) } \\
\text { (WV) } \\
\text { (CA) (CO) (FL) (OH) (TN) } \\
\text { (AZ) (FL) (MI) (SC) (VA) } \\
\text { (OH) (TN) (WY) } \\
\text { (AL) (GA) (OH) (SC) (VA) } \\
\text { (OH) (TN) }\end{array}$ \\
\hline
\end{tabular}

Top Ten Coke Producers

\begin{tabular}{ll}
\hline AK Steel Corp & (KY) (OH) \\
Bethlehem Steel Corp & (IN) (MD) (NY) (PA) \\
Citizens Gas \& Coke Utility & (IN) \\
Drummond Company Inc & (AL) \\
Geneva Steel Company & (UT) \\
LTV Steel Company Inc & (IL) (IN) (OH) (PA) \\
National Steel Corp & (IL) (MI) \\
Sun Co Inc & (VA) \\
USX Corporation & (IN) (PA) \\
Wheeling Pittsburgh Steel Corp & (WV)
\end{tabular}

Note: Major electric utility coal consumers are companies that consumed more than 3.6 million short tons of coal in 1995 . Major manufacturers are the top 40 coal consumers in the manufacturing sector. Major coke producers are the top 10 coal consumers in the coke plant sector. Electric utilities are ranked by consumption and manufacturers and coke producers are listed in alphabetical order.

Sources: Energy Information Administration, - Electric Utilities: Form EIA-759, "Monthly Power Plant Report." - Manufacturers: Form EIA-3, "Quarterly Coal Consumption Report - Manufacturing Plants." - Coke Plants: Form EIA-5, "Coke Plant Report - Quarterly," 
Table 67. Coal Consumption by Census Division and State, 1986, 1991-1995 (Thousand Short Tons)

\begin{tabular}{|c|c|c|c|c|c|c|c|c|c|}
\hline \multirow{2}{*}{ Census Division and State } & \multirow{2}{*}{1995} & \multirow{2}{*}{1994} & \multirow{2}{*}{1993} & \multirow{2}{*}{1992} & \multirow{2}{*}{1991} & \multirow{2}{*}{1986} & \multirow{2}{*}{$\begin{array}{c}\text { Percent } \\
\text { Change } \\
\text { 1994-1995 }\end{array}$} & \multicolumn{2}{|c|}{ Average Annual Percent Change } \\
\hline & & & & & & & & 1991-1995 & $1986-1995$ \\
\hline New England Total.............................. & 6,662 & 6,553 & 6,485 & 7,298 & 7,012 & 5,956 & 1.6 & -1.3 & 1.3 \\
\hline Connecticut & 906 & 862 & 788 & 849 & 856 & 809 & 5.1 & 1.4 & 1.3 \\
\hline 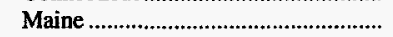 & 282 & 464 & 449 & 856 & 374 & 375 & -39.3 & -6.8 & -3.1 \\
\hline 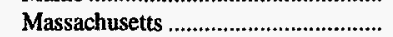 & 4,113 & 3,932 & 3,811 & - 4,257 & 4,451 & 3,785 & 4.6 & -1.9 & .9 \\
\hline 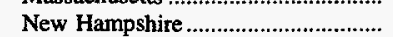 & 1,355 & 1,287 & 1,428 & 1,311 & 1,315 & 933 & 5.3 & .8 & 4.2 \\
\hline 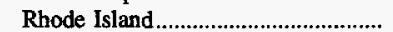 & 3 & 3 & 3 & 5 & 4 & 28 & -18.4 & -10.3 & -23.0 \\
\hline 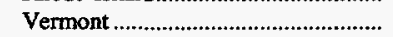 & 3 & 5 & 6 & 20 & 12 & 26 & -43.0 & -30.9 & -22.2 \\
\hline Middle Atlantic Total............................ & 68,387 & 67,536 & 70,389 & 71,418 & 70,594 & 65,864 & 1.3 & -.8 & .4 \\
\hline 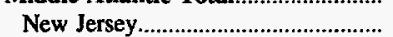 & 2,074 & 1,969 & 2,353 & 2,348 & 2,326 & 2,961 & 5.3 & -2.8 & -3.9 \\
\hline New York & 11,025 & 11,474 & 11,878 & 12,996 & 13,338 & 9,931 & -3.9 & -4.6 & 1.2 \\
\hline 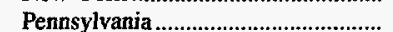 & 55,289 & 54,094 & 56,158 & 56,074 & 54,931 & 52,972 & 2.2 & .2 & .5 \\
\hline East North Central Total ....................... & 217,486 & 213,188 & 210,632 & 200,660 & 208,583 & 199,793 & 2.0 & 1.0 & .9 \\
\hline 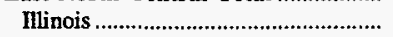 & 39,623 & 39,077 & 38,135 & 31,599 & 34,677 & 37,082 & 1.4 & 3.4 & .7 \\
\hline 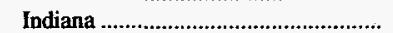 & 62,631 & 59,996 & 60,353 & 58,765 & 60,790 & 50,643 & 4.4 & .7 & 2.4 \\
\hline Michigan & 35,586 & 35,674 & 32,217 & 31,554 & 33,879 & 33,999 & -.2 & 1.2 & .5 \\
\hline Ohio & 56,580 & 56,711 & 59,031 & 58,671 & 58,578 & 59,324 & -.2 & -.9 & -.5 \\
\hline Wisconsin & 23,066 & 21,731 & 20,897 & 20,071 & 20,659 & 18,743 & 6.1 & 2.8 & 2.3 \\
\hline West North Central Total ................. & 131,082 & 125,591 & 120,940 & 115,505 & 116,707 & 95,526 & 4.4 & 2.9 & 3.6 \\
\hline lowa & 20,636 & 19,341 & 19,188 & 17,992 & 18,741 & 13,862 & 6.7 & 2.4 & 4.5 \\
\hline 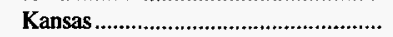 & 16,575 & 17,158 & 17,386 & 14,227 & 14,881 & 14,359 & -3.4 & 2.7 & 1.6 \\
\hline 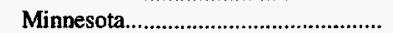 & 18,947 & 18,729 & 18,321 & 16,924 & 16,993 & 11,327 & 1.2 & 2.8 & 5.9 \\
\hline 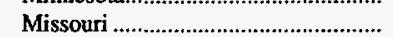 & 31,753 & 27,663 & 23,381 & 25,180 & 25,773 & 23,821 & 14.8 & 5.3 & 3.2 \\
\hline 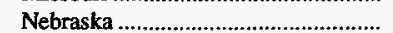 & 10,396 & 9,300 & 9,666 & 8,212 & 8,859 & 6,288 & 11.8 & 4.1 & 5.7 \\
\hline 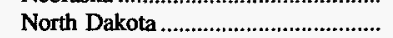 & 30,237 & 30,363 & 30,302 & 30,301 & 28,597 & 23,587 & -.4 & 1.4 & 2.8 \\
\hline South Dakota & 2,537 & 3,036 & 2,696 & 2,670 & 2,863 & 2,281 & -16.4 & -3.0 & 1.2 \\
\hline 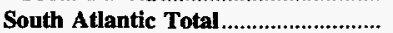 & 155,306 & 151,935 & 150,580 & 144,178 & 144,073 & 141,098 & 2.2 & 1.9 & 1.1 \\
\hline 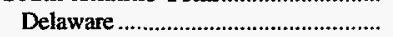 & 2,011 & 2,226 & 2,446 & 1,770 & 2,186 & 2,565 & -9.6 & -2.1 & -2.7 \\
\hline District of Columbia........................... & 6 & 47 & 51 & 50 & 66 & 54 & -87.9 & -45.8 & -22.2 \\
\hline Florida & 26,526 & 26,082 & 26,430 & 26,368 & 26,004 & 18,699 & 1.7 & .5 & 4.0 \\
\hline 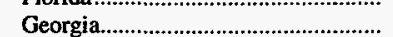 & 31,288 & 29,254 & 27,081 & 25,481 & 26,957 & 28,460 & 6.9 & 3.8 & 1.0 \\
\hline 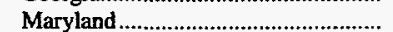 & 11,198 & 10,491 & 10,268 & 9,713 & 10,709 & 10,750 & 6.7 & 1.1 & .4 \\
\hline North Carolina & 24,084 & 23,282 & 25,760 & 24,075 & 20,877 & 23,242 & 3.4 & 3.6 & .4 \\
\hline South Carolina & 12,279 & 12,993 & 12,914 & 11,285 & 11,451 & 10,461 & -5.5 & 1.8 & 1.8 \\
\hline 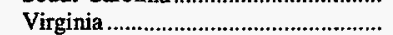 & 13,425 & 12,792 & 13,584 & 13,418 & 13,980 & 11,857 & 4.9 & -1.0 & 1.4 \\
\hline 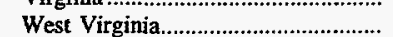 & 34,489 & 34,767 & 32,046 & 32,019 & 31,843 & 35,009 & -.8 & 2.0 & -.2 \\
\hline East South Central Total................... & 105,849 & 99,289 & 104,027 & 93,804 & 90,785 & 88,532 & 6.6 & 3.9 & 2.0 \\
\hline 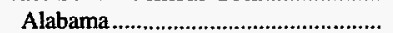 & 34,327 & 31,473 & 33,047 & 31,510 & 29,349 & 26,759 & 9.1 & 4.0 & 2.8 \\
\hline Kentucky & 39,516 & 38,090 & 39,095 & 34,704 & 34,517 & 32,111 & 3.7 & 3.4 & 2.3 \\
\hline Mississippi & 4,606 & 4,285 & 4,030 & 3,485 & 3,812 & 4,454 & 7.5 & 4.8 & .4 \\
\hline 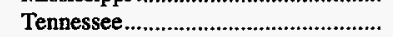 & 27,399 & 25,440 & 27,854 & 24,106 & 23,107 & 25,208 & 7.7 & 4.3 & .9 \\
\hline West South Central Total ................ & 139,106 & 138,251 & 140,797 & 135,210 & 133,635 & 114,962 & .6 & 1.0 & 2.1 \\
\hline 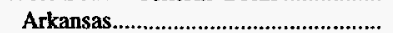 & 13,540 & 12,596 & 11,447 & 12,538 & 12,261 & 12,849 & 7.5 & 2.5 & 6 \\
\hline 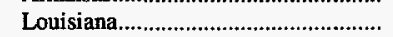 & 13,357 & 14,100 & 13,676 & 13,674 & 12,965 & 10,459 & -5.3 & .7 & 2.8 \\
\hline 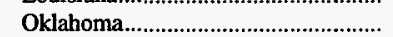 & 19,596 & 17,726 & 18,866 & 17,430 & 16,345 & 12,395 & 10.5 & 4.6 & 5.2 \\
\hline Texas & 92,612 & 93,829 & 96,809 & 91,568 & 92,064 & 79,259 & -1.3 & .1 & 1.7 \\
\hline Mountain Total & 107,923 & 115,695 & 110,673 & 112,163 & 105,177 & 85,314 & -6.7 & .6 & 2.6 \\
\hline 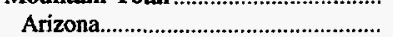 & 16,682 & 19,580 & 18,991 & 17,915 & 16,805 & 14,150 & -14.8 & -.2 & 1.8 \\
\hline 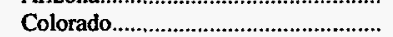 & 16,971 & 17,475 & 17,070 & 16,696 & 16,218 & 15,029 & -2.9 & 1.1 & 1.3 \\
\hline Idaho & 465 & 534 & 528 & 535 & 673 & 466 & -13.0 & -8.8 & $*$ \\
\hline 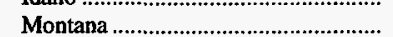 & 10,005 & 11,089 & 9,247 & 11,040 & 10,549 & 7,780 & -9.8 & -1.3 & 2.8 \\
\hline Nevada & 7,340 & 7,968 & 7,806 & 8,088 & 8,091 & 7,195 & -7.9 & -2.4 & .2 \\
\hline New Mexico & 15,221 & 15,374 & 15,012 & 14,832 & 12,858 & 13,245 & -1.0 & 4.3 & 1.5 \\
\hline 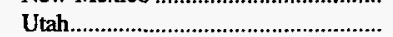 & 15,307 & 16,216 & 15,848 & 15,719 & 14,834 & 8,112 & -5.6 & .8 & 7.3 \\
\hline Wyoming & 25,933 & 27,459 & 26,171 & 27,339 & 25,150 & 19,337 & -5.5 & .8 & 3.3 \\
\hline Pacific Total & 8,837 & 12,162 & 11,422 & 12,186 & 11,055 & 6,603 & -27.3 & -5.4 & 3.3 \\
\hline Alaska & 815 & 796 & 863 & 792 & 802 & 769 & 2.4 & .4 & .6 \\
\hline California & 2,618 & 2,498 & 2,453 & 2,821 & 2,816 & 1,865 & 4.8 & -1.8 & 3.8 \\
\hline Hawaii & 121 & 86 & 73 & 47 & 37 & 16 & 41.0 & 34.5 & 24.9 \\
\hline Oregon & 1,125 & 2,479 & 2,099 & 2,124 & 1,940 & 163 & -54.6 & -12.7 & 23.9 \\
\hline Washington & 4,158 & 6,303 & 5,934 & 6,402 & 5,461 & 3,790 & -34.0 & -6.6 & 1.0 \\
\hline 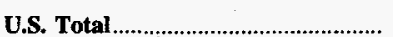 & 940,638 & 930,201 & 925,944 & 892,421 & 887,621 & 804,169 & 1.1 & 1.5 & 1.8 \\
\hline
\end{tabular}

\footnotetext{
* Data sound to zero.
}

Notes: U.S. Total does not include coal consumed by independent power producers. Totals may not equal sum of components due to independent rounding.

Sources: Energy Information Administration, Form EIA-759, "Monthly Power Plant Report"; Form EIA-3, "Quarterly Coal Consumption Report - Manufacturing Plants"; Form EIA-5, "Coke Plant Report - Quarterly"; and Form EIA-6, "Coal Distribution Report." 
Figure 11. U.S. Consumer Coal Stocks, 1986-1995

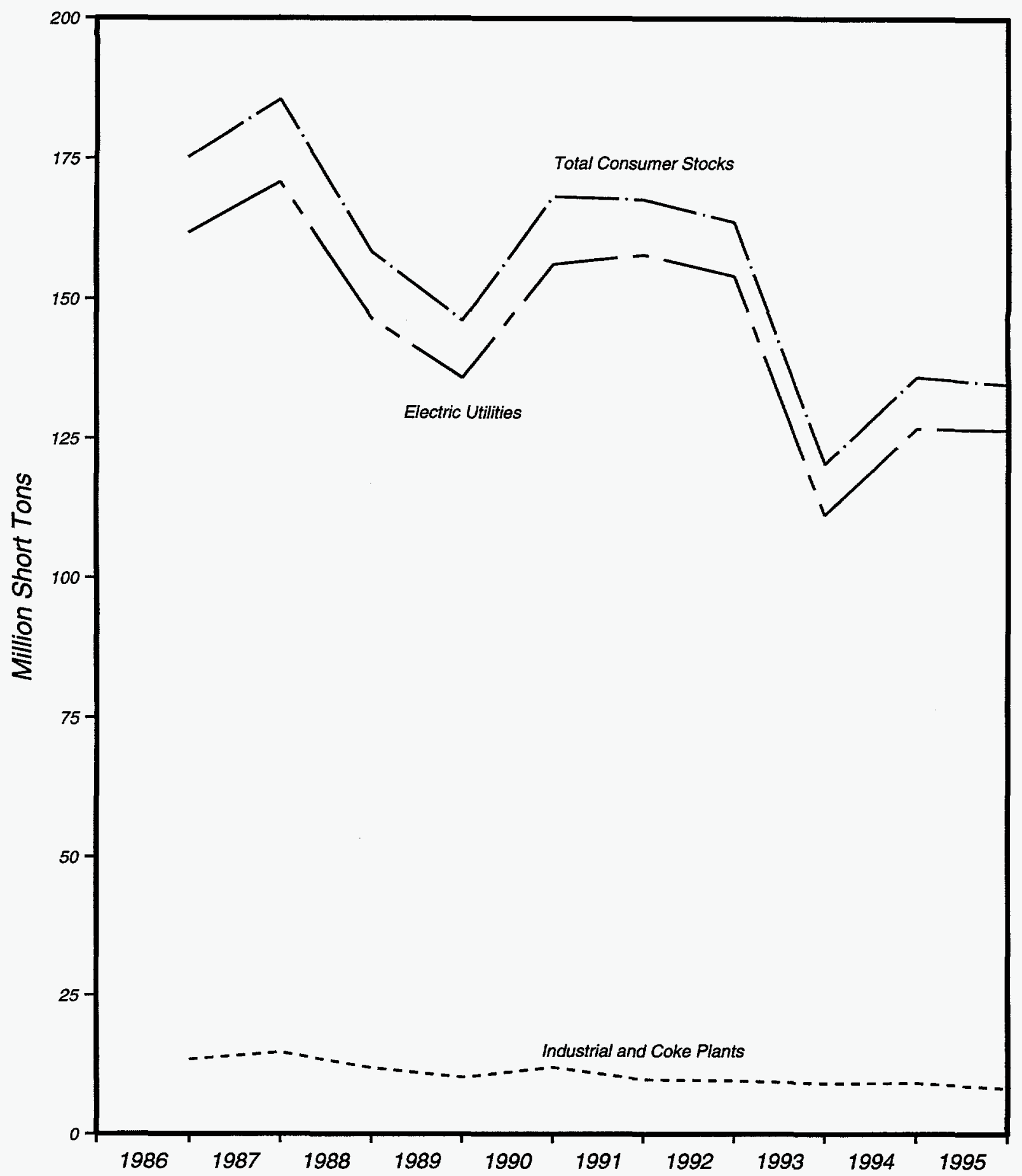

Note: Each increment represents end-of-year data. Industrial stocks reflect manufacturing plants and coke plants.

Sources: Energy Information Administration, •Electric Utilities: Form EIA-759, "Monthly Power Plant Report." •Industrial: Form EIA-5, "Coke Plant Report - Quarterly" and Form EIA-3, "Quarterly Coal Consumption Report - Manufacturing Plants." 
Table 68. Year-End Consumer Coal Stocks by Census Division and State, 1986, 1991-1995

(Thousand Short Tons)

\begin{tabular}{|c|c|c|c|c|c|c|c|c|c|}
\hline \multirow{2}{*}{ Census Division and State } & \multirow{2}{*}{1995} & \multirow{2}{*}{1994} & \multirow{2}{*}{1993} & \multirow{2}{*}{1992} & \multirow{2}{*}{1991} & \multirow{2}{*}{1986} & \multirow{2}{*}{$\begin{array}{c}\text { Percent } \\
\text { Change } \\
\text { 1994-1995 }\end{array}$} & \multicolumn{2}{|c|}{ Average Annual Percent Change } \\
\hline & & & & & & & & 1991-1995 & 1986-1995 \\
\hline New England Total................................. & 969 & 1,117 & 989 & 1,253 & 1,134 & 1,377 & -13.3 & -3.9 & -3.8 \\
\hline Connecticut & $\mathbf{w}$ & $w$ & w & w & 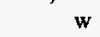 & w & w & w & w \\
\hline Maine & $w$ & w & $w$ & $\mathbf{w}$ & w & w & $w$ & $w$ & $\mathbf{w}$ \\
\hline 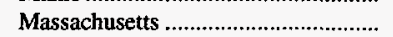 & $w$ & $\mathbf{w}$ & w & $w$ & $\mathbf{w}$ & $\mathrm{w}$ & $w$ & $w$ & $\mathbf{w}$ \\
\hline 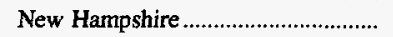 & $w$ & $w$ & $w$ & $\mathbf{w}$ & $w$ & $w$ & $w$ & $w$ & $w$ \\
\hline 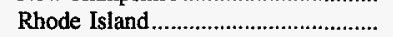 & - & - & - & - & - & $\mathrm{w}$ & $\mathrm{w}$ & $w$ & $w$ \\
\hline 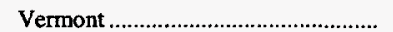 & - & - & - & - & - & $\mathrm{w}$ & w & w & w \\
\hline Middle Atlantic Total........................... & 12,404 & 14,068 & 14,060 & 19,486 & 18,246 & 18,059 & -11.8 & -9.2 & -4.1 \\
\hline 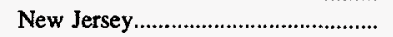 & w & w & w & $w$ & w & $w$ & w & w & w \\
\hline 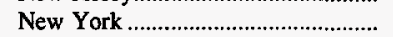 & w & w & w & $\mathbf{w}$ & w & w & w & w & w \\
\hline Pennsylvania & 10,303 & 12,060 & 12,265 & 15,976 & 15,546 & 14,691 & -14.6 & -9.8 & -3.9 \\
\hline East North Central Total .................... & 33,818 & $\mathbf{3 5 , 8 3 3}$ & 30,162 & 43,661 & 43,179 & 48,873 & $-\mathbf{5 . 6}$ & -5.9 & -4.0 \\
\hline 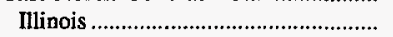 & w & $w$ & $w$ & $\mathbf{w}$ & w & 9,670 & w & w & w \\
\hline Indiana & 9,298 & 11,707 & 7,798 & 12,507 & 11,387 & 12,587 & -20.6 & -4.9 & -3.3 \\
\hline 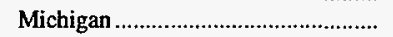 & $\mathrm{w}$ & w & $\mathbf{w}$ & $\mathbf{w}$ & w & $w$ & w & w & $w$ \\
\hline Ohio & 5,936 & 7,815 & 7,630 & 10,804 & 10,618 & 9,508 & -24.0 & -13.5 & -5.1 \\
\hline 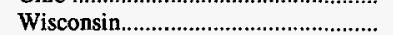 & 3,656 & 3,436 & w & w & $\mathrm{W}$ & $w$ & 6.4 & w & $\mathbf{w}$ \\
\hline West North Central Total .................... & 18,713 & 17,717 & $\mathbf{w}$ & $\mathbf{w}$ & $\mathbf{w}$ & $\mathbf{w}$ & 5.6 & $\mathbf{w}$ & $\mathbf{w}$ \\
\hline Iowa & 4,447 & 4,178 & 3,819 & 4,857 & 5,133 & 4,479 & 6.4 & -3.5 & -.1 \\
\hline 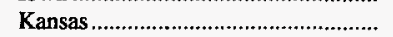 & 3,860 & 2,623 & 2,024 & 2,759 & 3,321 & 3,369 & 47.1 & 3.8 & 1.5 \\
\hline 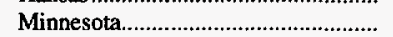 & 1,985 & 2,234 & 1,250 & 2,252 & 2,693 & 3,649 & -11.1 & -7.3 & -6.5 \\
\hline 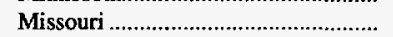 & 4,779 & 4,570 & $w$ & $w$ & $w$ & w & 4.6 & w & w \\
\hline 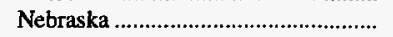 & w & $w$ & w & $\mathbf{w}$ & w & 2,007 & w & $\mathbf{w}$ & $w$ \\
\hline 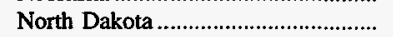 & w & w & w & $\mathbf{w}$ & w & $\mathrm{w}$ & w & $w$ & $w$ \\
\hline 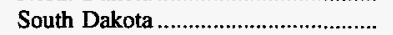 & w & $\mathbf{w}$ & w & w & w & w & $\mathbf{w}$ & w & w \\
\hline 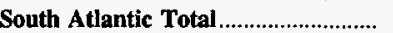 & 19,707 & 24,427 & 19,092 & 29,282 & 29,952 & 25,384 & -19.3 & -9.9 & -2.8 \\
\hline 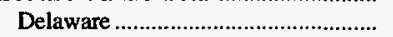 & $w$ & $w$ & w & $w$ & w & w & $\mathbf{w}$ & $w$ & w \\
\hline 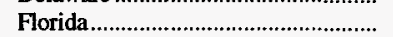 & 3,268 & 3,914 & 3,541 & 4,121 & 4,880 & 3,289 & -16.5 & -9.5 & -.1 \\
\hline Georgia & 3,786 & 4,843 & 2,926 & 4,836 & 5,419 & 5,966 & -21.8 & -8.6 & -4.9 \\
\hline 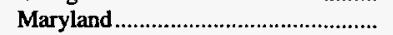 & w & $w$ & $w$ & $\mathbf{w}$ & w & w & w & $\mathbf{w}$ & w \\
\hline North Carolina & 2,855 & 4,318 & 3,059 & 4,715 & 4,866 & 4,009 & -33.9 & -12.5 & -3.7 \\
\hline 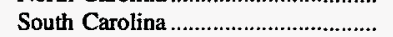 & 2,194 & 2,533 & 1,893 & 2,451 & 2,241 & 2,127 & -13.4 & -.5 & .3 \\
\hline Virginia & $w$ & w & W & W & $\mathrm{w}$ & $w$ & w & $w$ & $w$ \\
\hline 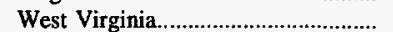 & w & w & $w$ & $\mathbf{w}$ & $\mathrm{w}$ & $w$ & w & w & $w$ \\
\hline 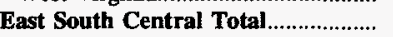 & 10,940 & 11,267 & 9,277 & 14,400 & 15,026 & 17,527 & -2.9 & -7.6 & -5.1 \\
\hline 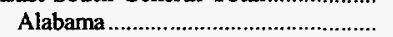 & 3,648 & 4,132 & 2,797 & 4,529 & 4,671 & 5,208 & -11.7 & -6.0 & -3.9 \\
\hline Kentucky & w & $w$ & $\mathrm{w}$ & $\mathrm{W}$ & w & w & w & $\mathrm{w}$ & $\mathrm{w}$ \\
\hline 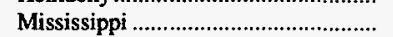 & w & w & w & $\mathbf{w}$ & $w$ & w & w & $w$ & $w$ \\
\hline 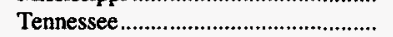 & 1,884 & 1,764 & w & 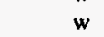 & $w$ & w & 6.8 & w & $\mathrm{w}$ \\
\hline 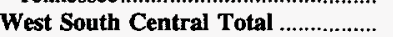 & 20,564 & 15,959 & 15,105 & 16,898 & $\mathbf{w}$ & 18,956 & 28.8 & $\mathbf{w}$ & .9 \\
\hline 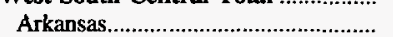 & 2,820 & 1,777 & 1,881 & 1,591 & 2,165 & 1,954 & 58.7 & 6.8 & 4.1 \\
\hline 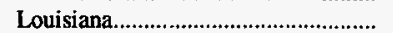 & 2,669 & 1,922 & 2,000 & 1,755 & 2,301 & $w$ & 38.8 & 3.8 & w \\
\hline 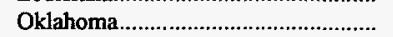 & 4,246 & 2,467 & 2,052 & 3,161 & 2,919 & 4,415 & 72.1 & 9.8 & -.4 \\
\hline Texas & 10,829 & 9,793 & 9,172 & 10,392 & $w$ & w & 10.6 & $w$ & w \\
\hline Mountain Total & w & $\mathbf{w}$ & $w$ & w & $\mathbf{w}$ & $\mathbf{w}$ & $w$ & $\mathbf{w}$ & $w$ \\
\hline 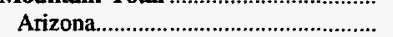 & 3,032 & 3,242 & 3,717 & 3,596 & 4,229 & w & -6.5 & -8.0 & $w$ \\
\hline Colorado & 3,682 & 3,145 & 3,454 & 3,439 & 3,487 & w & 17.1 & 1.4 & $\mathrm{w}$ \\
\hline Idaho & 118 & 78 & 86 & 101 & 79 & 109 & 51.1 & 10.6 & .8 \\
\hline 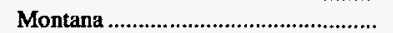 & $w$ & $\mathbf{w}$ & $\mathbf{w}$ & w & $\mathbf{w}$ & w & w & w & $\mathbf{w}$ \\
\hline 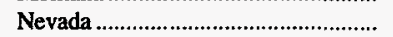 & $\mathbf{w}$ & $\mathbf{w}$ & $w$ & $w$ & w & $w$ & $\mathbf{w}$ & $\mathbf{w}$ & $w$ \\
\hline 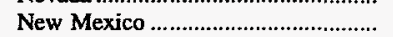 & $w$ & $\mathbf{w}$ & $\mathbf{w}$ & w & $w$ & w & $w$ & $w$ & $w$ \\
\hline Utah & $\mathbf{w}$ & $\mathbf{w}$ & $w$ & w & w & w & $w$ & 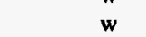 & $w$ \\
\hline Wyoming & 2,936 & 2,553 & 1,841 & 2,242 & 2,835 & w & 15.0 & .9 & $w$ \\
\hline 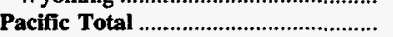 & $w$ & $\mathbf{w}$ & $\mathbf{w}$ & $\mathbf{w}$ & $\mathbf{w}$ & $\mathbf{w}$ & $\mathbf{w}$ & $\mathbf{w}$ & $\mathbf{w}$ \\
\hline Alaska & 1 & 2 & 5 & 6 & w & w & -67.4 & $w$ & $w$ \\
\hline California & 133 & 126 & 93 & 109 & 139 & $w$ & 6.2 & -.9 & $w$ \\
\hline 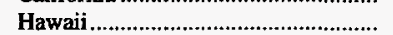 & w & $\mathbf{w}$ & $w$ & w & w & 25 & $w$ & w & $\mathrm{w}$ \\
\hline Oregon & w & $\mathbf{w}$ & $\mathbf{w}$ & $w$ & w & $\mathbf{w}$ & w & w & w \\
\hline 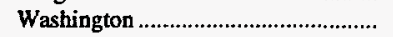 & 1,969 & 569 & 459 & 736 & 1,194 & 1,755 & 246.1 & 13.3 & 1.3 \\
\hline 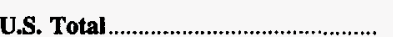 & 134,639 & 136,139 & 120,458 & 163,692 & 167,711 & 175,226 & -1.1 & -5.3 & -2.9 \\
\hline
\end{tabular}

Withheld to avoid disclosure of individual company data.

Notes: Stocks for the residential and commercial sector are not included. Totals may not equal sum of components due to independent rounding.

Sources: Energy Information Administration, Form EIA-759, "Monthly Power Plant Report"; Form EIA-5, "Coke Plant Report - Quarterly"; and Form

EIA-3, "Quarterly Coal Consumption Report - Manufacturing Plants." 
Table 69. Coal Consumption at Electric Utility Plants by Census Division and State, 1986, 1991-1995

(Thousand Short Tons)

\begin{tabular}{|c|c|c|c|c|c|c|c|c|c|}
\hline \multirow{2}{*}{ Census Division and State } & \multirow{2}{*}{1995} & \multirow{2}{*}{1994} & \multirow{2}{*}{1993} & \multirow{2}{*}{1992} & \multirow{2}{*}{1991} & \multirow{2}{*}{1986} & \multirow{2}{*}{$\begin{array}{c}\text { Percent } \\
\text { Change } \\
\text { 1994-1995 }\end{array}$} & \multicolumn{2}{|c|}{ Average Annual Percent Change } \\
\hline & & & & & & & & 1991-1995 & 1986-1995 \\
\hline 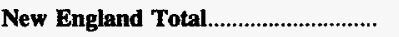 & 6,272 & 5,945 & 5,736 & 6,112 & 6,421 & 5,288 & 5.5 & -0.6 & 1.9 \\
\hline 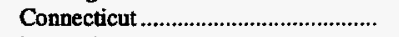 & 881 & 821 & 745 & 817 & 840 & 763 & 7.3 & 1.2 & 1.6 \\
\hline 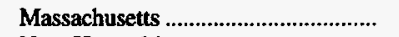 & 4,044 & 3,845 & 3,652 & 4,044 & 4,339 & 3,596 & 5.2 & -1.7 & 1.3 \\
\hline New Hampshire ................................... & 1,346 & 1,279 & 1,339 & 1,251 & 1,242 & 917 & 5.3 & 2.0 & 4.3 \\
\hline 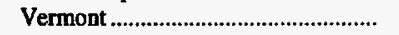 & - & - & - & - & - & 12 & - & - & - \\
\hline Middle Atlantic Total. & 49,357 & 48,326 & 51,079 & 52,488 & 52,617 & 49,523 & 2.1 & -1.6 & * \\
\hline New Jersey............................................ & 2,054 & 1,887 & 2,123 & 2,118 & 2,081 & 2,637 & 8.8 & -.3 & -2.7 \\
\hline New York & 8,051 & 8,395 & 8,699 & 9,963 & 9,874 & 6,298 & -4.1 & -5.0 & 2.8 \\
\hline Pennsylvania & 39,252 & 38,044 & 40,257 & 40,407 & 40,662 & 40,589 & 3.2 & -.9 & -.4 \\
\hline East North Central Total .................. & 187,490 & 183,282 & 179,833 & 169,029 & 173,718 & 159,601 & 2.3 & 1.9 & 1.8 \\
\hline 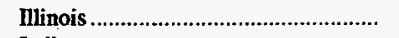 & 33,463 & 32,599 & 31,744 & 25,264 & 27,754 & 30,844 & 2.6 & 4.8 & .9 \\
\hline 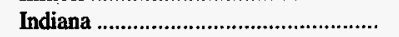 & 52,089 & 50,554 & 48,836 & 46,937 & 47,720 & 37,309 & 3.0 & 2.2 & 3.8 \\
\hline Michigan & 31,165 & 31,106 & 28,749 & 28,238 & 29,896 & 27,061 & .2 & 1.0 & 1.6 \\
\hline 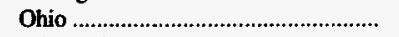 & 49,785 & 49,326 & 51,456 & 50,358 & 49,577 & 47,785 & .9 & .1 & .4 \\
\hline 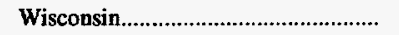 & 20,987 & 19,696 & 19,049 & 18,231 & 18,771 & 16,601 & 6.5 & 2.8 & 2.6 \\
\hline West North Central Total .................. & 116,720 & 111,672 & 107,584 & 102,557 & 104,246 & 83,595 & 4.5 & 2.9 & 3.8 \\
\hline Iowa & 17,785 & 16,565 & 16,623 & 15,357 & 15,846 & 12,044 & 7.4 & 2.9 & 4.4 \\
\hline 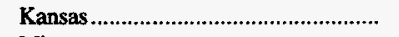 & 16,345 & 16,989 & 17,226 & 14,068 & 14,732 & 14,097 & -3.8 & 2.6 & 1.6 \\
\hline 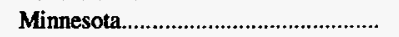 & 17,282 & 17,046 & 16,844 & 15,841 & 16,114 & 10,170 & 1.4 & 1.8 & 6.1 \\
\hline 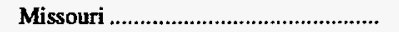 & 30,440 & 26,375 & 21,945 & 23,815 & 24,286 & 22,029 & 15.4 & 5.8 & 3.6 \\
\hline 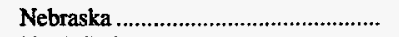 & 10,048 & 8,879 & 9,297 & 7,881 & 8,524 & 5,945 & 13.2 & 4.2 & 6.0 \\
\hline 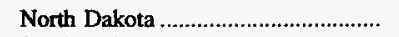 & 22,680 & 23,248 & 23,290 & 23,192 & 22,174 & 17,291 & -2.4 & .6 & 3.1 \\
\hline South Dakota & 2,137 & 2,570 & 2,360 & 2,402 & 2,570 & 2,018 & -16.8 & -4.5 & .6 \\
\hline South Atlantic Total............................. & 138,134 & 133,984 & 132,885 & 126,093 & 123,729 & 121,239 & 3.1 & 2.8 & 1.5 \\
\hline 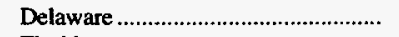 & 1,816 & 2,007 & 2,223 & 1,628 & 1,958 & 2,350 & -9.5 & -1.9 & -2.8 \\
\hline Florida & 25,200 & 24,758 & 25,108 & 25,016 & 24,870 & 17,822 & 1.8 & .3 & 3.9 \\
\hline 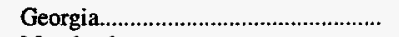 & 29,280 & 27,293 & 25,339 & 23,656 & 24,848 & 26,652 & 7.3 & 4.2 & 1.0 \\
\hline 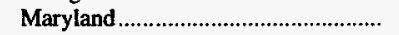 & 10,141 & 9,717 & 9,521 & 8,993 & 8,632 & 7,961 & 4.4 & 4.1 & 2.7 \\
\hline 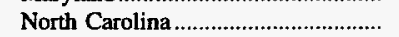 & 21,424 & 20,624 & 23,055 & 21,011 & 18,078 & 20,542 & 3.9 & 4.3 & .5 \\
\hline South Carolina & 10,074 & 10,597 & 10,410 & 9,078 & 9,218 & 7,777 & -4.9 & 2.2 & 2.9 \\
\hline 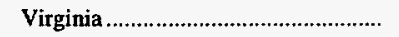 & 9,543 & 8,670 & 9,447 & 8,661 & 8,568 & 7,345 & 10.1 & 2.7 & 2.9 \\
\hline 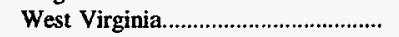 & 30,657 & 30,318 & 27,782 & 28,050 & 27,557 & 30,790 & 1.1 & 2.7 & $*$ \\
\hline East South Central Total................... & 92,262 & 85,622 & 90,365 & $\mathbf{8 0 , 2 0 3}$ & 77,917 & 75,231 & 7.8 & 4.3 & $\mathbf{2 . 3}$ \\
\hline Alabama & 28,759 & 25,817 & 27,533 & 24,988 & 23,700 & 21,436 & 11.4 & 4.9 & 3.3 \\
\hline 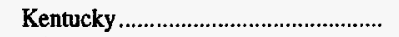 & 35,707 & 34,564 & 35,264 & 31,715 & 31,432 & 28,535 & 3.3 & 3.2 & 2.5 \\
\hline 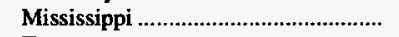 & 4,319 & 3,989 & 3,767 & 3,237 & 3,570 & 4,208 & 8.3 & 4.9 & .3 \\
\hline 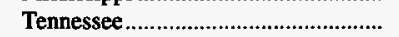 & 23,477 & 21,253 & 23,801 & 20,263 & 19,216 & 21,051 & 10.5 & 5.1 & 1.2 \\
\hline West South Central Total ..................... & 132,633 & 131,168 & 134,009 & 129,351 & 127,908 & 109,086 & 1.1 & .9 & 2.2 \\
\hline 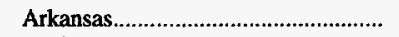 & 13,216 & 12,250 & 11,116 & 12,241 & 11,978 & 12,505 & 7.9 & 2.5 & .6 \\
\hline 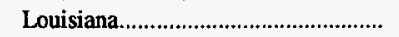 & 12,930 & 13,479 & 13,089 & 13,077 & 12,406 & 10,196 & -4.1 & 1.0 & 2.7 \\
\hline 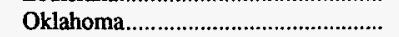 & 18,130 & 16,961 & 17,668 & 16,699 & 15,668 & 11,628 & 6.9 & 3.7 & 5.0 \\
\hline 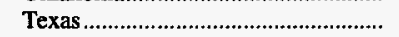 & 88,358 & 88,479 & 92,135 & 87,333 & 87,856 & 74,757 & -.1 & .1 & 1.9 \\
\hline 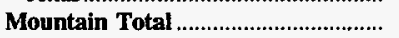 & 101,013 & 108,651 & 104,093 & 105,609 & 98,400 & 77,891 & $-\mathbf{7 . 0}$ & .6 & 2.9 \\
\hline 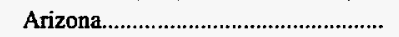 & 16,021 & 18,853 & 18,316 & 17,280 & 16,116 & 11,861 & -15.0 & -.1 & 3.4 \\
\hline 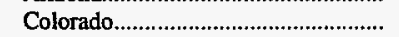 & 16,222 & 16,596 & 16,252 & 15,902 & 15,416 & 14,150 & -2.2 & 1.3 & 1.5 \\
\hline 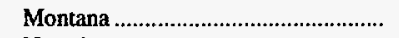 & 9,373 & 10,513 & 8,869 & 10,768 & 10,223 & 7,438 & -10.8 & -2.1 & 2.6 \\
\hline Nevada & 7,084 & 7,772 & 7,608 & 7,914 & 7,892 & 7,086 & -8.8 & -2.7 & $*$ \\
\hline New Mexico & 15,137 & 15,297 & 14,942 & 14,775 & 12,809 & 13,147 & -1.0 & 4.3 & 1.6 \\
\hline 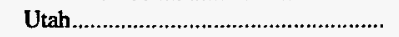 & 13,325 & 14,269 & 13,995 & 13,857 & 12,829 & 6,756 & -6.6 & .9 & 7.8 \\
\hline 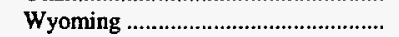 & 23,850 & 25,350 & 24,111 & 25,114 & 23,115 & 17,452 & -5.9 & .8 & 3.5 \\
\hline Pacific Total & $\mathbf{5 , 1 2 7}$ & 8,621 & 7,924 & 8,419 & 7,313 & 3,602 & -40.5 & -8.5 & 4.0 \\
\hline 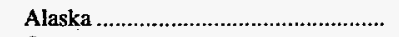 & 293 & 271 & 298 & 277 & 298 & 272 & 7.8 & -.5 & .8 \\
\hline Oregon & 977 & 2,333 & 1,981 & 1,994 & 1,831 & - & -58.1 & -14.5 & - \\
\hline 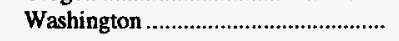 & 3,857 & 6,016 & 5,646 & 6,148 & 5,184 & 3,329 & -35.9 & -7.1 & 1.6 \\
\hline U.S. Total & 829,007 & 817,270 & 813,508 & 779,860 & 772,268 & 685,056 & 1.4 & 1.8 & 2.1 \\
\hline
\end{tabular}

* Data round to zero.

Note: Totals may not equal sum of components due to independent rounding.

Source: Energy Information Administration, Form EIA-759, "Monthly Power Plant Report." 
Table 70. Year-End Coal Stocks at Electric Utility Plants by Census Division and State, 1986, 1991-1995

(Thousand Short Tons)

\begin{tabular}{|c|c|c|c|c|c|c|c|c|c|}
\hline \multirow{2}{*}{ Census Division and State } & \multirow{2}{*}{1995} & \multirow{2}{*}{1994} & \multirow{2}{*}{1993} & \multirow{2}{*}{1992} & \multirow{2}{*}{1991} & \multirow{2}{*}{1986} & \multirow{2}{*}{$\begin{array}{c}\text { Percent } \\
\text { Change } \\
\text { 1994-1995 }\end{array}$} & \multicolumn{2}{|c|}{ Average Annual Percent Change } \\
\hline & & & & & & & & 1991-1995 & 1986-1995 \\
\hline New England Total............................... & 908 & 1,079 & 967 & 1,237 & 1,128 & 1,354 & -15.8 & -5.3 & -4.3 \\
\hline 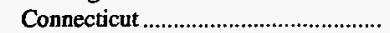 & 164 & 202 & 160 & 150 & 173 & 5 & -18.7 & -1.3 & 47.7 \\
\hline 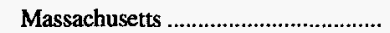 & 425 & 629 & 449 & 727 & 583 & 964 & -32.4 & -7.6 & -8.7 \\
\hline New Hampshire ..................................... & 319 & 248 & 358 & 361 & 373 & 357 & 28.8 & -3.8 & -1.2 \\
\hline 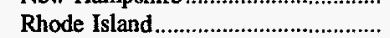 & - & - & - & - & - & 28 & -20.0 & - & -1.2 \\
\hline Middle Atlantic Total.......................... & 11,064 & 12,687 & 12,564 & $\mathbf{1 7 , 7 4 3}$ & 16,638 & 16,381 & -12.8 & -9.7 & -4.3 \\
\hline 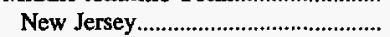 & 804 & 688 & 501 & 771 & 681 & 995 & 16.9 & 4.3 & -2.3 \\
\hline New York & 1,015 & 999 & 953 & 2,106 & 1,546 & 1,768 & 1.6 & -10.0 & -6.0 \\
\hline 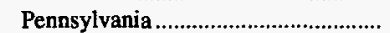 & 9,244 & 11,000 & 11,110 & 14,866 & 14,412 & 13,619 & -16.0 & -10.5 & -4.2 \\
\hline East North Central Total .................. & 30,505 & 32,088 & 27,296 & 40,250 & 39,394 & $\mathbf{4 3 , 8 5 8}$ & -4.9 & -6.2 & -3.9 \\
\hline 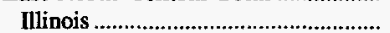 & 5,331 & 4,526 & 4,019 & 7,399 & 6,977 & 8,960 & 17.8 & -6.5 & -5.6 \\
\hline Indiana & 8,435 & 10,449 & 6,935 & 11,294 & 9,953 & 11,248 & -19.3 & -4.0 & -3.1 \\
\hline 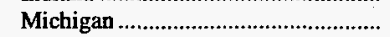 & 7,708 & 6,505 & 6,206 & 7,402 & 8,099 & 9,824 & 18.5 & -1.2 & -2.7 \\
\hline Ohio & 5,661 & 7,499 & 7,249 & 10,395 & 10,213 & 8,785 & -24.5 & -13.7 & -4.8 \\
\hline 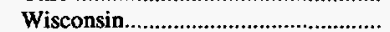 & 3,371 & 3,109 & 2,887 & 3,760 & 4,151 & 5,041 & 8.4 & -5.1 & -4.4 \\
\hline West North Central Total .................. & $\mathbf{1 7 , 7 3 2}$ & 16,739 & 14,123 & 19,712 & 20,169 & 21,231 & 5.9 & -3.2 & -2.0 \\
\hline Iowa & 3,923 & 3,642 & 3,401 & 4,301 & 4,499 & 3,832 & 7.7 & -3.4 & .3 \\
\hline 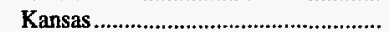 & 3,850 & 2,610 & 2,008 & 2,747 & 3,310 & 3,319 & 47.5 & 3.8 & 1.7 \\
\hline Minnesota. & 1,898 & 2,134 & 1,182 & 2,175 & 2,616 & 3,560 & -11.1 & -7.7 & -6.8 \\
\hline 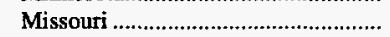 & 4,641 & 4,410 & 3,555 & 6,211 & 5,458 & 5,374 & 5.2 & -4.0 & -1.6 \\
\hline 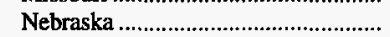 & 1,409 & 1,276 & 1,272 & 1,798 & 1,976 & 1,962 & 10.4 & -8.1 & -3.6 \\
\hline North Dakota & 1,858 & 2,406 & 2,417 & 2,194 & 1,999 & 2,879 & -22.8 & -1.8 & -4.7 \\
\hline South Dakota & 153 & 259 & 287 & 285 & 312 & 306 & -40.8 & -16.3 & -7.4 \\
\hline 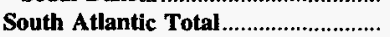 & 18,851 & 23,226 & 17,877 & 27,977 & 28,746 & $\mathbf{2 3 , 7 1 2}$ & -18.8 & -10.0 & -2.5 \\
\hline Delaware & 363 & 470 & 192 & 361 & 458 & 452 & -22.8 & -5.6 & -2.4 \\
\hline 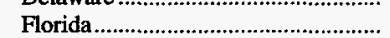 & 3,204 & 3,813 & 3,451 & 4,021 & 4,781 & 3,201 & -16.0 & -9.5 & * \\
\hline 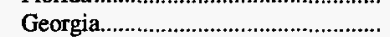 & 3,657 & 4,699 & 2,825 & 4,692 & 5,251 & 5,773 & -22.2 & -8.6 & -4.9 \\
\hline Maryland & 1,038 & 1,306 & 1,455 & 2,400 & 2,220 & 1,507 & -20.5 & -17.3 & -4.0 \\
\hline North Carolina & 2,715 & 4,139 & 2,887 & 4,512 & 4,657 & 3,744 & -34.4 & -12.6 & -3.5 \\
\hline South Carolina & 2,033 & 2,255 & 1,648 & 2,206 & 1,988 & 1,767 & -9.8 & .6 & 1.6 \\
\hline 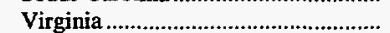 & 1,098 & 2,064 & 1,418 & 1,922 & 1,685 & 1,602 & -46.8 & -10.2 & -4.1 \\
\hline 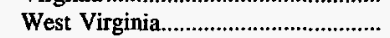 & 4,744 & 4,479 & 4,001 & 7,863 & 7,707 & 5,667 & 5.9 & -11.4 & -1.9 \\
\hline East South Central Total.................... & 10,148 & 10,317 & 8,370 & 13,401 & 14,210 & 16,470 & -1.6 & -8.1 & -5.2 \\
\hline Alabama & 3,282 & 3,652 & 2,331 & 4,071 & 4,247 & 4,738 & -10.1 & -6.2 & -4.0 \\
\hline 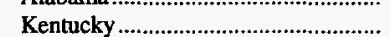 & 4,472 & 4,466 & 3,990 & 5,415 & 5,881 & 5,604 & .1 & -6.6 & -2.5 \\
\hline Mississippi & 724 & 690 & 417 & 899 & 933 & 978 & 4.9 & -6.1 & -3.3 \\
\hline 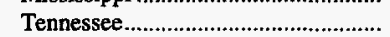 & 1,670 & 1,509 & 1,632 & 3,016 & 3,148 & 5,149 & 10.7 & -14.7 & -11.8 \\
\hline West South Central Total & 20,195 & 15,520 & 13,867 & 16,483 & 17,694 & 17,025 & 30.1 & 3.4 & 1.9 \\
\hline 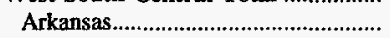 & 2,790 & 1,751 & 1,866 & 1,572 & 2,150 & 1,906 & 59.4 & 6.7 & 4.3 \\
\hline 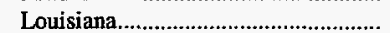 & 2,659 & 1,872 & 1,932 & 1,701 & 2,235 & 1,886 & 42.1 & 4.4 & 3.9 \\
\hline Oklahoma & 4,118 & 2,319 & 1,944 & 3,066 & 2,835 & 4,219 & 77.5 & 9.8 & -.3 \\
\hline 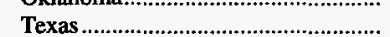 & 10,628 & 9,578 & 8,125 & 10,143 & 10,474 & 9,014 & 10.9 & .4 & 1.8 \\
\hline Mountain Total & 14,562 & 14,559 & 15,529 & 16,009 & 18,086 & 19,929 & * & -5.3 & -3.4 \\
\hline 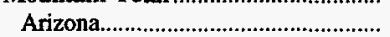 & 2,998 & 3,197 & 3,687 & 3,543 & 4,177 & 3,718 & -6.2 & -8.0 & -2.4 \\
\hline Colorado & 3,622 & 3,118 & 3,428 & 3,410 & 3,466 & 4,520 & 16.2 & 1.1 & -2.4 \\
\hline 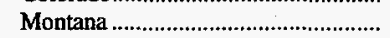 & 511 & 517 & 721 & 735 & 741 & 788 & -1.2 & -8.8 & -4.7 \\
\hline Nevada & 1,356 & 1,034 & 1,195 & 1,447 & 1,412 & 1,832 & 31.2 & -1.0 & -3.3 \\
\hline New Mexico & 967 & 1,462 & 1,506 & 1,570 & 1,399 & 1,442 & -33.9 & -8.8 & -4.3 \\
\hline Utah & 2,250 & 2,753 & 3,264 & 3,153 & 4,123 & 2,344 & -18.3 & -14.0 & -.4 \\
\hline Wyoming & 2,857 & 2,476 & 1,728 & 2,153 & 2,767 & 5,284 & 15.4 & .8 & -6.6 \\
\hline Pacific Total & 2,341 & 683 & 748 & 1,317 & 1,812 & 1,846 & 242.6 & 6.6 & 2.7 \\
\hline Alaska & 1 & 2 & 5 & 6 & 8 & 8 & -67.4 & -42.9 & -22.3 \\
\hline Oregon & 399 & 150 & 312 & 615 & 660 & 184 & 166.7 & -11.8 & 9.0 \\
\hline Washington & 1,941 & 531 & 431 & 697 & 1,145 & 1,654 & 265.4 & 14.1 & 1.8 \\
\hline U.S. Total & 126,304 & 126,897 & 111,341 & 154,130 & 157,876 & 161,806 & -.5 & -5.4 & -2.7 \\
\hline
\end{tabular}

* Data round to zero.

Note: Totals may not equal sum of components due to independent rounding.

Source: Energy Information Administration, Form EIA-759, "Monthly Power Plant Report." 
Table 71. Coal Consumption at Other Industrial Plants by Census Division and State, 1986, 1991-1995

(Thousand Short Tons)

\begin{tabular}{|c|c|c|c|c|c|c|c|c|c|}
\hline \multirow{2}{*}{ Census Division and State } & \multirow{2}{*}{1995.} & \multirow{2}{*}{1994} & \multirow{2}{*}{1993} & \multirow{2}{*}{1992} & \multirow{2}{*}{1991} & \multirow{2}{*}{1986} & \multirow{2}{*}{$\begin{array}{c}\text { Percent } \\
\text { Change } \\
\text { 1994-1995 }\end{array}$} & \multicolumn{2}{|c|}{ Average Annual Percent Change } \\
\hline & & & & & & & & 1991-1995 & 1986-1995 \\
\hline 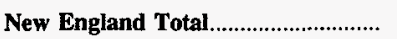 & 321 & 553 & 647 & 1,045 & 507 & 463 & -42.0 & -10.8 & -4.0 \\
\hline Connecticut & w & w & w & $\mathrm{w}$ & $w$ & $\mathbf{w}$ & $w$ & $w$ & w \\
\hline 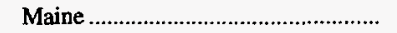 & $\mathbf{w}$ & $\mathbf{w}$ & w & w & w & $w$ & w & w & $w$ \\
\hline 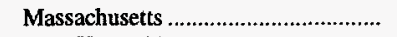 & $\mathbf{w}$ & $\mathbf{w}$ & $\mathbf{w}$ & $\mathbf{w}$ & $\mathbf{w}$ & $\mathbf{w}$ & $\mathbf{w}$ & $\mathbf{w}$ & $\mathbf{w}$ \\
\hline 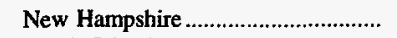 & $\mathbf{w}$ & - & $\mathbf{w}$ & $w$ & $\mathbf{w}$ & $w$ & w & $\mathbf{w}$ & $\mathbf{w}$ \\
\hline 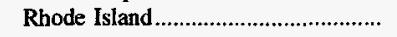 & - & - & $\mathbf{w}$ & - & - & $w$ & $\mathbf{w}$ & $\mathbf{w}$ & $\mathbf{w}$ \\
\hline 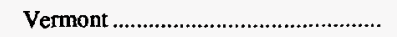 & - & - & w & $\mathrm{w}$ & $\mathbf{w}$ & $\mathbf{w}$ & $w$ & $\mathbf{w}$ & $\mathbf{w}$ \\
\hline Middle Atlantic Total............................. & $\mathbf{w}$ & $\mathbf{w}$ & $\mathbf{w}$ & $\mathbf{w}$ & $\mathbf{w}$ & $\mathbf{w}$ & $\mathbf{w}$ & $\mathbf{w}$ & $\mathbf{w}$ \\
\hline 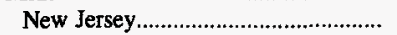 & w & $\mathbf{w}$ & w & $w$ & w & $w$ & $\mathbf{w}$ & w & $\mathbf{w}$ \\
\hline 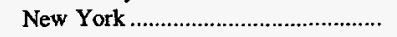 & 1,370 & 1,559 & 1,704 & 1,610 & 1,959 & 1,940 & -12.1 & -8.5 & -3.8 \\
\hline 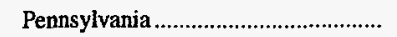 & 4,027 & 4,044 & 4,311 & 4,173 & 4,049 & 3,975 & -.4 & -.1 & .1 \\
\hline East North Central Total .................. & 16,351 & 17,098 & 17,699 & 16,931 & 18,670 & 20,145 & -4.4 & -3.3 & -2.3 \\
\hline Illinois & 3,653 & 4,187 & 3,970 & 3,736 & 4,426 & 3,759 & -12.8 & -4.7 & -.3 \\
\hline Indiana & 4,373 & 4,244 & 4,587 & 4,263 & 4,404 & 4,980 & 3.0 & -.2 & -1.4 \\
\hline 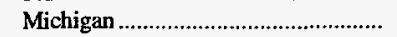 & 2,767 & 2,890 & 3,230 & 3,127 & 3,149 & 3,994 & -4.2 & -3.2 & -4.0 \\
\hline Ohio & 3,609 & 3,794 & 4,100 & 3,970 & 4,813 & 5,303 & -4.9 & -6.9 & -4.2 \\
\hline 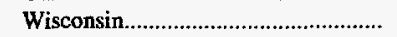 & 1,949 & 1,984 & 1,811 & 1,835 & 1,878 & 2,109 & -1.7 & .9 & -.9 \\
\hline West North Central Total ..................... & 13,581 & 13,238 & 12,753 & 12,505 & 11,741 & 10,944 & 2.6 & 3.7 & 2.4 \\
\hline lowa & 2,761 & 2,735 & 2,494 & 2,571 & 2,672 & 1,563 & .9 & .8 & 6.5 \\
\hline 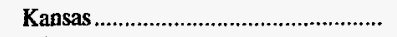 & 138 & 137 & 137 & 158 & 148 & 261 & .3 & -1.9 & -6.9 \\
\hline 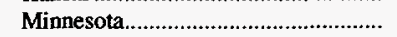 & 1,401 & 1,455 & 1,370 & 1,059 & 785 & 964 & -3.7 & 15.6 & 4.2 \\
\hline 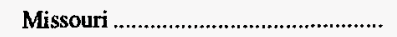 & 1,102 & 1,070 & 1,177 & 1,137 & 1,235 & 1,457 & 3.1 & -2.8 & -3.0 \\
\hline 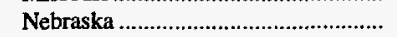 & $w$ & $\mathbf{w}$ & $w$ & $w$ & $\mathbf{w}$ & 339 & w & w & w \\
\hline 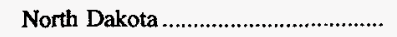 & $\mathbf{w}$ & $w$ & $\mathbf{w}$ & $\mathbf{w}$ & w & $\mathbf{w}$ & $\mathbf{w}$ & w & $\mathbf{w}$ \\
\hline 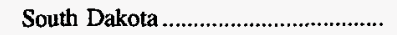 & $w$ & $\mathbf{w}$ & w & $w$ & $w$ & $w$ & w & w & w \\
\hline 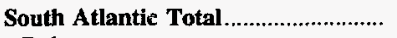 & $\mathbf{w}$ & $\mathbf{w}$ & $\mathbf{w}$ & $\mathbf{w}$ & $\mathbf{w}$ & $\mathbf{w}$ & $\mathbf{w}$ & $\mathbf{w}$ & $\mathbf{w}$ \\
\hline Delaware & w & w & w & w & w & w & $\mathbf{w}$ & w & $w$ \\
\hline Florida & 1,325 & 1,303 & 1,307 & 1,335 & 1,133 & 782 & 1.6 & 4.0 & 6.0 \\
\hline 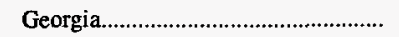 & 1,949 & 1,933 & 1,720 & 1,787 & 2,101 & 1,801 & .8 & -1.9 & .9 \\
\hline 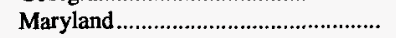 & 760 & 738 & 731 & 706 & 1,040 & 726 & 3.0 & -7.5 & .5 \\
\hline 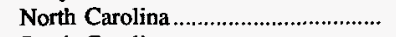 & 2,437 & 2,396 & 2,476 & 2,860 & 2,702 & 2,545 & 1.7 & -2.5 & -.5 \\
\hline 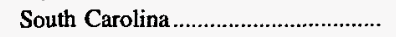 & 2,188 & 2,334 & 2,395 & 2,177 & 2,212 & 2,465 & -6.3 & -.3 & -1.3 \\
\hline 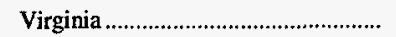 & 2,631 & 2,838 & 2,863 & 3,592 & 4,301 & 3,373 & -7.3 & -11.5 & -2.7 \\
\hline West Virginia...................................... & 1,984 & 2,637 & 2,406 & 2,266 & 2,310 & 2,234 & -24.8 & -3.7 & -1.3 \\
\hline East South Central Total..................... & $\mathbf{w}$ & $\mathbf{w}$ & $w$ & $\mathbf{w}$ & $\mathbf{w}$ & $\mathbf{w}$ & $\mathbf{w}$ & $\mathbf{w}$ & $\mathbf{w}$ \\
\hline 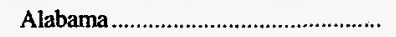 & 2,304 & 2,394 & 2,268 & 3,136 & 2,468 & 2,536 & -3.7 & -1.7 & -1.1 \\
\hline 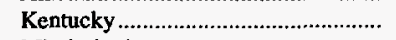 & 2,250 & 1,994 & 2,392 & 1,648 & 2,044 & 2,192 & 12.8 & 2.4 & .3 \\
\hline 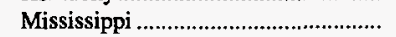 & $\mathrm{w}$ & w & w & $\mathbf{w}$ & w & $\mathbf{w}$ & w & w & $\mathbf{w}$ \\
\hline 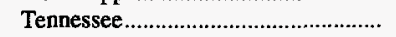 & 3,777 & 4,097 & 3,942 & 3,686 & 3,702 & 3,984 & -7.8 & .5 & -.6 \\
\hline West South Central Total .................. & 6,456 & $\mathbf{7 , 0 8 2}$ & 6,780 & 5,846 & 5,716 & $\mathbf{w}$ & -8.8 & 3.1 & $\mathbf{w}$ \\
\hline 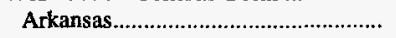 & 325 & 346 & 330 & 295 & 283 & 344 & -6.2 & 3.5 & -.6 \\
\hline 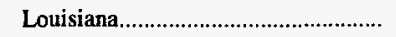 & $\mathbf{w}$ & w & w & $\mathbf{w}$ & w & $\mathbf{w}$ & w & w & $\mathrm{w}$ \\
\hline 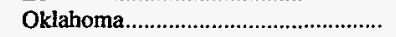 & w & w & w & w & w & 763 & w & $\mathbf{w}$ & w \\
\hline 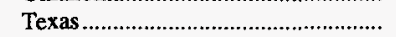 & 4,255 & 5,350 & 4,667 & 4,225 & 4,198 & 4,411 & -20.5 & 3 & -.4 \\
\hline 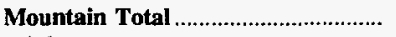 & 5,615 & 5,614 & 5,163 & 4,973 & 4,954 & 6,176 & $*$ & 3.2 & -1.0 \\
\hline 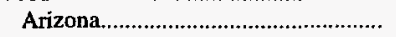 & 657 & 727 & 674 & 632 & 689 & $w$ & -9.7 & -1.2 & w \\
\hline 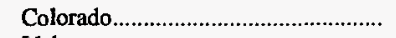 & 729 & 857 & 780 & 735 & 738 & 773 & -14.9 & -.3 & -.6 \\
\hline 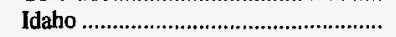 & 426 & 494 & 486 & 484 & 604 & 429 & -13.8 & -8.4 & -.1 \\
\hline Montana & $\mathbf{w}$ & w & $\mathbf{w}$ & w & w & w & w & w & w \\
\hline Nevada & w & $\mathbf{w}$ & $\mathbf{w}$ & $\mathbf{w}$ & w & $\mathbf{w}$ & w & w & $\mathbf{w}$ \\
\hline 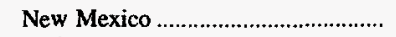 & $\mathbf{w}$ & w & $w$ & w & w & $\mathbf{w}$ & $w$ & w & $\mathbf{w}$ \\
\hline 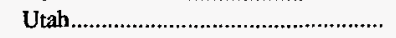 & 915 & 835 & 727 & 525 & 508 & 380 & 9.5 & 15.8 & 10.3 \\
\hline 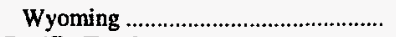 & 1,937 & 1,867 & 1,873 & 2,126 & 1,896 & w & 3.8 & .5 & w \\
\hline 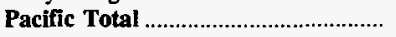 & 2,976 & 2,769 & 2,677 & 3,161 & 3,113 & 2,415 & 7.5 & -1.1 & 2.3 \\
\hline 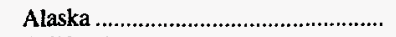 & - & 5 & 2 & - & - & - & $w$ & w & w \\
\hline 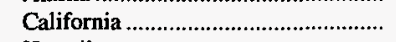 & 2,485 & 2,332 & 2,311 & 2,821 & 2,771 & 1,865 & 6.6 & -2.7 & 3.2 \\
\hline 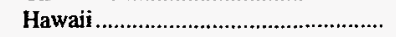 & $w$ & $w$ & $w$ & $w$ & w & 16 & $w$ & $w$ & w \\
\hline Oregon & $w$ & $w$ & w & w & $w$ & w & $\mathbf{w}$ & $\mathbf{w}$ & $\mathbf{w}$ \\
\hline 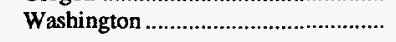 & 223 & 201 & 174 & 163 & 197 & 372 & 10.7 & 3.1 & -5.5 \\
\hline 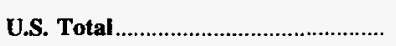 & $\mathbf{7 2 , 7 9 6}$ & 75,179 & 74,892 & 74,042 & 75,405 & $\mathbf{7 5 , 5 8 3}$ & -3.2 & -.9 & -.4 \\
\hline
\end{tabular}

* Data round to zero.

W Withheld to avoid disclosure of individual company data

Note: Totals may not equal sum of components due to independent rounding.

Sources: Energy Information Administration, Form ElA-3, "Quarterly Coal Consumption Report - Manufacturing Plants"; and Form EIA-6, "Coal Distribution Report." 
Table 72. Year-End Coal Stocks at Other Industrial Plants by Census Division and State, 1986, 1991-1995

(Thousand Short Tons)

\begin{tabular}{|c|c|c|c|c|c|c|c|c|c|}
\hline \multirow{2}{*}{ Census Division and State } & \multirow{2}{*}{1995} & \multirow{2}{*}{1994} & \multirow{2}{*}{1993} & \multirow{2}{*}{1992} & \multirow{2}{*}{1991} & \multirow{2}{*}{1986} & \multirow{2}{*}{$\begin{array}{c}\text { Percent } \\
\text { Change } \\
\text { 1994-1995 }\end{array}$} & \multicolumn{2}{|c|}{ Average Annual Percent Change } \\
\hline & & & & & & & & 1991-1995 & 1986-1995 \\
\hline New England Total................................ & 60 & 38 & 21 & 16 & 6 & 23 & 57.1 & 80.4 & 11.3 \\
\hline 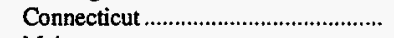 & - & - & - & - & - & w & w & $\mathbf{w}$ & w \\
\hline Maine & $\mathbf{w}$ & $w$ & $w$ & $\mathbf{w}$ & $w$ & $\mathbf{w}$ & $w$ & w & $w$ \\
\hline 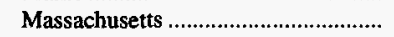 & $\mathbf{w}$ & $w$ & $\mathbf{w}$ & $\mathbf{w}$ & w & $\mathrm{w}$ & w & w & w \\
\hline 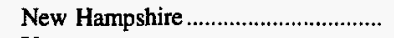 & - & - & - & - & - & w & $w$ & $\mathbf{w}$ & $w$ \\
\hline 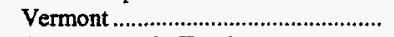 & - & - & - & - & - & $w$ & $w$ & $\mathbf{w}$ & $\mathbf{w}$ \\
\hline Middle Atlantic Total .............................. & $\mathbf{w}$ & $\mathbf{w}$ & $\mathbf{w}$ & $\mathbf{w}$ & $\mathbf{w}$ & $\mathbf{w}$ & $\mathbf{w}$ & $\mathbf{w}$ & $\mathbf{w}$ \\
\hline New Jersey............................................. & w & w & w & $\mathbf{w}$ & $\mathbf{w}$ & w & $w$ & w & w \\
\hline 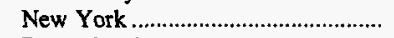 & 203 & 250 & 321 & 402 & 296 & 334 & -18.8 & -8.9 & -5.4 \\
\hline 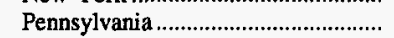 & 218 & 298 & 287 & 306 & 282 & 506 & -26.8 & -6.3 & -8.9 \\
\hline East North Central Total .................... & 2,031 & 2,462 & 2,044 & 2,505 & $\mathbf{2 , 5 8 3}$ & 3,345 & $-\mathbf{1 7 . 5}$ & -5.8 & -5.4 \\
\hline 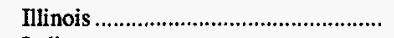 & 333 & 426 & 368 & 389 & 422 & 498 & -21.8 & -5.7 & -4.4 \\
\hline Indiana & 451 & 690 & 470 & 732 & 764 & 692 & -34.6 & -12.3 & -4.6 \\
\hline 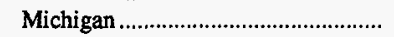 & 822 & 865 & 702 & 882 & 891 & 1,070 & -4.9 & -2.0 & -2.9 \\
\hline Ohio & 138 & 153 & 198 & 228 & 190 & 419 & -9.4 & -7.6 & -11.6 \\
\hline 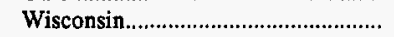 & 286 & 328 & 306 & 274 & 315 & 666 & -12.8 & -2.4 & -9.0 \\
\hline West North Central Total .................. & 981 & 978 & 775 & 1,020 & 1,163 & 1,235 & .3 & -4.1 & -2.5 \\
\hline Iowa & 524 & 535 & 418 & 556 & 635 & 648 & -2.1 & -4.7 & -2.3 \\
\hline 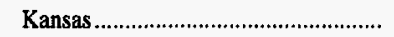 & 10 & 13 & 16 & 11 & 10 & 50 & -27.7 & -1.2 & -16.6 \\
\hline 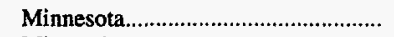 & 87 & 99 & 68 & 77 & 78 & 89 & -12.3 & 2.9 & -.2 \\
\hline 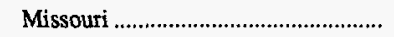 & 138 & 159 & 148 & 227 & 210 & 237 & -13.3 & -9.9 & -5.8 \\
\hline 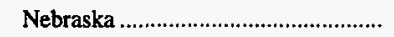 & $w$ & $w$ & $\mathbf{w}$ & $\mathbf{w}$ & $w$ & 45 & $\mathbf{w}$ & w & w \\
\hline 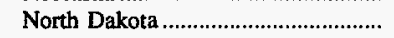 & $w$ & w & $\mathbf{w}$ & $\mathbf{w}$ & w & w & $\mathrm{w}$ & $\mathrm{w}$ & $\mathrm{w}$ \\
\hline 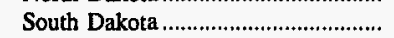 & $\mathbf{w}$ & w & $\mathbf{w}$ & $w$ & w & $\mathrm{w}$ & w & $w$ & $\mathrm{w}$ \\
\hline South Atlantic Total ............................... & $\mathbf{w}$ & $\mathbf{w}$ & $\mathbf{w}$ & $\mathbf{w}$ & $\mathbf{w}$ & $\mathbf{w}$ & $\mathbf{w}$ & $\mathbf{w}$ & $\mathbf{w}$ \\
\hline 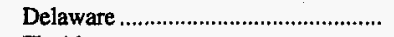 & $\mathbf{w}$ & $\mathbf{w}$ & $\mathbf{w}$ & $w$ & $\mathbf{w}$ & w & w & w & w \\
\hline Florida & 64 & 101 & 90 & 100 & 99 & 88 & -36.7 & -10.4 & -3.5 \\
\hline 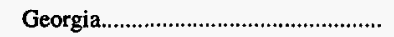 & 129 & 144 & 101 & 144 & 167 & 193 & -10.1 & -6.3 & -4.3 \\
\hline 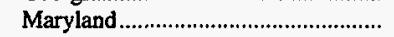 & 24 & 36 & 41 & 35 & 37 & 33 & -33.8 & -10.1 & -3.6 \\
\hline 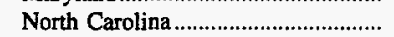 & 140 & 179 & 172 & 203 & 209 & 265 & -21.8 & -9.5 & -6.8 \\
\hline 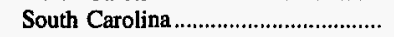 & 160 & 278 & 245 & 245 & 253 & 360 & -42.4 & -10.8 & -8.6 \\
\hline 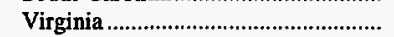 & 177 & 217 & 216 & 275 & 206 & 378 & -18.1 & -3.7 & -8.1 \\
\hline 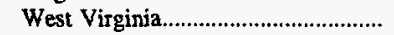 & 105 & 130 & 167 & 156 & 131 & 156 & -19.4 & -5.4 & -4.3 \\
\hline East South Central Total ........................ & $\mathbf{w}$ & $\mathbf{w}$ & $\mathbf{w}$ & $\mathbf{w}$ & $\mathbf{w}$ & $\mathbf{w}$ & $\mathbf{w}$ & w & $\mathbf{w}$ \\
\hline Alabama & 133 & 183 & 132 & 127 & 131 & 223 & -27.0 & .5 & -5.5 \\
\hline 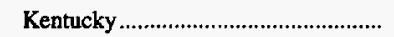 & 120 & 112 & 73 & 127 & 123 & 170 & 7.0 & -.7 & -3.8 \\
\hline 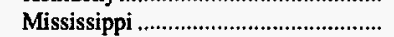 & w & w & w & $\mathbf{w}$ & w & $w$ & w & $w$ & w \\
\hline 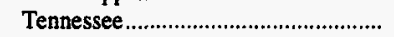 & 215 & 256 & 246 & 270 & 219 & 315 & -16.0 & -.5 & -4.2 \\
\hline West South Central Total .................. & 370 & 439 & 1,218 & 395 & 603 & $\mathbf{w}$ & -15.9 & -11.5 & $\mathbf{w}$ \\
\hline 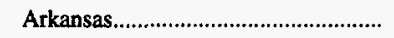 & 29 & 26 & 15 & 19 & 15 & 48 & 14.3 & 17.9 & -5.4 \\
\hline 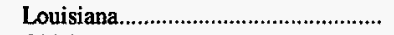 & w & $w$ & w & $\mathbf{w}$ & w & $w$ & w & w & w \\
\hline 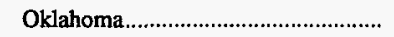 & w & $\mathbf{w}$ & w & $\mathbf{w}$ & w & 196 & w & $\mathbf{w}$ & w \\
\hline 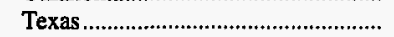 & 201 & 215 & 1,026 & 228 & 437 & 1,652 & -6.4 & -17.6 & -20.8 \\
\hline 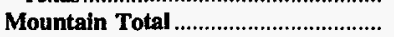 & 313 & 267 & 332 & 360 & 307 & 503 & 17.5 & .5 & -5.1 \\
\hline Arizona. & 34 & 45 & 30 & 53 & 52 & $w$ & -23.0 & -9.8 & $w$ \\
\hline 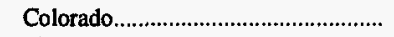 & 59 & 26 & 25 & 29 & 21 & 53 & 127.2 & 30.4 & 1.2 \\
\hline Idaho & 118 & 78 & 86 & 101 & 79 & 109 & 51.1 & 10.6 & .8 \\
\hline Montana & $\mathbf{w}$ & w & $\mathbf{w}$ & $\mathbf{w}$ & w & w & w & w & w \\
\hline 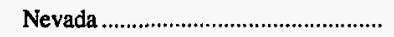 & $\mathbf{w}$ & $w$ & $\mathbf{w}$ & $w$ & w & $\mathbf{w}$ & $\mathbf{w}$ & w & $\mathbf{w}$ \\
\hline 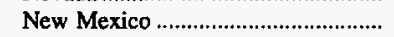 & $\mathbf{w}$ & $\mathbf{w}$ & $\mathbf{w}$ & $\mathbf{w}$ & w & $\mathbf{w}$ & $\mathbf{w}$ & w & w \\
\hline 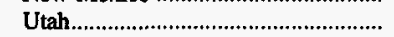 & 7 & 13 & 20 & 26 & 39 & 147 & -49.4 & -35.5 & -29.0 \\
\hline 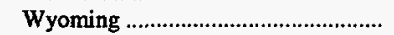 & 79 & 77 & 113 & 89 & 68 & $\mathbf{w}$ & 3.3 & 4.0 & w \\
\hline 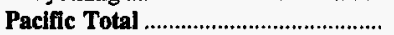 & 245 & 194 & 170 & 211 & 222 & 287 & 26.3 & 2.4 & -1.7 \\
\hline 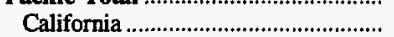 & 133 & 126 & 93 & 109 & 139 & 130 & 6.2 & -.9 & .3 \\
\hline 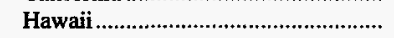 & w & w & $\mathbf{w}$ & w & w & 25 & w & w & $w$ \\
\hline Oregon & $\mathbf{w}$ & w & $\mathbf{w}$ & $\mathbf{w}$ & $\mathbf{w}$ & w & w & w & w \\
\hline 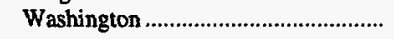 & 28 & 38 & 27 & 39 & 49 & 101 & -26.7 & -13.4 & -13.4 \\
\hline 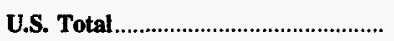 & 5,702 & 6,585 & 6,716 & 6,965 & 7,061 & 10,429 & -13.4 & -5.2 & -6.5 \\
\hline
\end{tabular}

Withheld to avoid disclosure of individual company data.

Notes: Other industrial plants include manufacturing plants only. Totals may not equal sum of components due to independent rounding. Source: Energy Information Administration, Form EIA-3, "Quarterly Coal Consumption Report - Manufacturing Plants." 
Table 73. Coal Carbonized at Coke Plants by Census Division and State, 1986, 1991-1995

(Thousand Short Tons)

\begin{tabular}{|c|c|c|c|c|c|c|c|c|c|}
\hline \multirow{2}{*}{ Census Division and State } & \multirow{2}{*}{1995} & \multirow{2}{*}{1994} & \multirow{2}{*}{1993} & \multirow{2}{*}{1992} & \multirow{2}{*}{1991} & \multirow{2}{*}{1986} & \multirow{2}{*}{$\begin{array}{c}\text { Percent } \\
\text { Change } \\
\text { 1994-1995 }\end{array}$} & \multicolumn{2}{|c|}{ Average Annual Percent Change } \\
\hline & & & & & & & & $1991-1995$ & 1986-1995 \\
\hline Middle Atlantic Total ............................ & $\mathbf{w}$ & $\mathbf{w}$ & $\mathbf{w}$ & $\mathbf{w}$ & $\mathbf{w}$ & $\mathbf{w}$ & $\mathbf{w}$ & $w$ & $\mathbf{w}$ \\
\hline 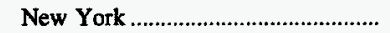 & w & $\mathbf{w}$ & w & w & $\mathbf{w}$ & w & w & $\mathbf{w}$ & $\mathbf{w}$ \\
\hline 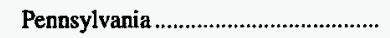 & 10,858 & 10,849 & 10,333 & 9,868 & 8,812 & 7,007 & 0.1 & 5.4 & 5.0 \\
\hline East North Central Total ...................... & 12,345 & 11,356 & 11,643 & 13,224 & 14,742 & 17,913 & 8.7 & -4.3 & -4.0 \\
\hline 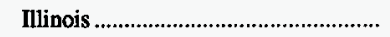 & $\mathrm{w}$ & $\mathbf{w}$ & $\mathrm{w}$ & $\mathrm{w}$ & $\mathbf{w}$ & 2,234 & $\mathbf{w}$ & $\mathbf{w}$ & w \\
\hline Indiana & 5,883 & 4,841 & 6,591 & 7,153 & 8,234 & 7,808 & 21.5 & -8.1 & -3.1 \\
\hline 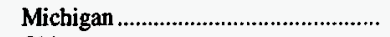 & w & $\mathbf{w}$ & - & - & $\mathbf{w}$ & w & $\mathbf{w}$ & $\mathbf{w}$ & $\mathbf{w}$ \\
\hline Ohio & 2,777 & 3,092 & 2,892 & 3,755 & 3,698 & 5,184 & -10.2 & -6.9 & -6.7 \\
\hline West North Central Total ................ & - & - & - & - & - & $\mathbf{w}$ & - & $\mathbf{w}$ & $\mathbf{w}$ \\
\hline 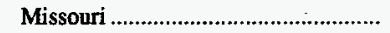 & - & - & - & - & - & $\mathbf{w}$ & - & $\mathbf{w}$ & $\mathbf{w}$ \\
\hline South Atlantic Total ............................... & $w$ & $\mathbf{w}$ & $\mathbf{w}$ & $\mathbf{w}$ & $\mathbf{w}$ & $\mathbf{w}$ & $\mathbf{w}$ & $\mathbf{w}$ & $\mathbf{w}$ \\
\hline 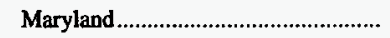 & - & - & - & - & $\mathbf{w}$ & w & $\mathbf{w}$ & w & $w$ \\
\hline 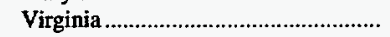 & $\mathbf{w}$ & $\mathbf{w}$ & w & $\mathbf{w}$ & $w$ & w & $\mathbf{w}$ & w & $\mathbf{w}$ \\
\hline 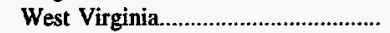 & $\mathbf{w}$ & $\mathbf{w}$ & $w$ & $w$ & $\mathbf{w}$ & $\mathbf{w}$ & $\mathbf{w}$ & w & $\mathbf{w}$ \\
\hline East South Central Total.................... & $\mathbf{w}$ & $\mathbf{w}$ & $\mathbf{w}$ & $\mathbf{w}$ & $\mathbf{w}$ & $\mathbf{w}$ & $\mathbf{w}$ & $\mathbf{w}$ & $\mathbf{w}$ \\
\hline 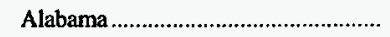 & 3,257 & 3,253 & 3,206 & 3,297 & 3,166 & 2,674 & .1 & .7 & 2.2 \\
\hline Kentucky & w & w & $\mathbf{w}$ & $\mathbf{w}$ & $\mathbf{w}$ & w & $\mathbf{w}$ & $\mathbf{w}$ & w \\
\hline 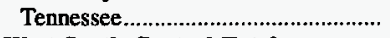 & - & - & - & - & $\mathbf{w}$ & $\mathbf{w}$ & - & w & w \\
\hline West South Central Total ..................... & - & - & - & - & - & $\mathbf{w}$ & - & $\mathbf{w}$ & $\mathbf{w}$ \\
\hline Texas & - & - & - & - & - & $\mathbf{w}$ & - & $\mathbf{w}$ & $\mathbf{w}$ \\
\hline 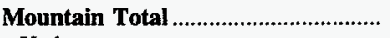 & $\mathbf{w}$ & $\mathbf{w}$ & $\mathbf{w}$ & $\mathbf{w}$ & $\mathbf{w}$ & $\mathbf{w}$ & $\mathbf{w}$ & $\mathbf{w}$ & $\mathbf{w}$ \\
\hline Utah & $\mathbf{w}$ & $\mathbf{w}$ & $\mathbf{w}$ & $\mathbf{w}$ & $\mathbf{w}$ & $\mathbf{w}$ & $\mathbf{w}$ & $\mathbf{w}$ & $\mathbf{w}$ \\
\hline U.S. Total & 33,011 & 31,740 & 31,323 & 32,366 & $\mathbf{3 3 , 8 5 4}$ & 35,862 & 4.0 & -.6 & -.9 \\
\hline
\end{tabular}

W Withheld to avoid disclosure of individual company data.

Note: Totals may not equal sum of components due to independent rounding

Source: Energy Information Administration, Form EIA-5, "Coke Plant Report - Quarterly."

Table 74. Year-End Coal Stocks at Coke Plants by Census Division and State, 1986, 1991-1995

(Thousand Short Tons)

\begin{tabular}{|c|c|c|c|c|c|c|c|c|c|}
\hline \multirow{2}{*}{ Census Division and State } & \multirow{2}{*}{1995} & \multirow{2}{*}{1994} & \multirow{2}{*}{1993} & \multirow{2}{*}{1992} & \multirow{2}{*}{1991} & \multirow{2}{*}{1986} & \multirow{2}{*}{$\begin{array}{c}\text { Percent } \\
\text { Change } \\
\text { 1994-1995 }\end{array}$} & \multicolumn{2}{|c|}{ Average Annual Percent Change } \\
\hline & & & & & & & & $1991-1995$ & $1986-1995$ \\
\hline Middle Atlantic Total & $\mathbf{w}$ & $\mathbf{w}$ & $\mathbf{w}$ & $\mathbf{w}$ & $\mathbf{w}$ & $\mathbf{w}$ & $\mathbf{w}$ & $\mathbf{w}$ & $\mathbf{w}$ \\
\hline 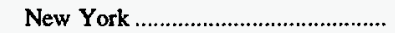 & $\mathbf{w}$ & w & $\mathbf{w}$ & $\mathbf{w}$ & w & $\mathbf{w}$ & $w$ & w & $w$ \\
\hline 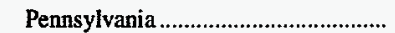 & 841 & 762 & 868 & 804 & 852 & 567 & 10.3 & -0.3 & 4.5 \\
\hline East North Central Total .................... & 1,282 & 1,282 & 822 & 906 & 1,203 & 1,670 & * & 1.6 & -2.9 \\
\hline 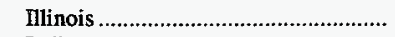 & $\mathbf{w}$ & $\mathrm{w}$ & w & w & $\mathrm{w}$ & 212 & w & w & $\mathbf{w}$ \\
\hline Indiana & 412 & 567 & 394 & 481 & 669 & 648 & -27.4 & -11.4 & -4.9 \\
\hline 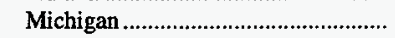 & w & w & - & - & w & $w$ & w & w & w \\
\hline Ohio & 136 & 163 & 183 & 180 & 215 & 304 & -16.4 & -10.7 & -8.5 \\
\hline West North Central Total ................. & - & - & - & - & - & $\mathbf{w}$ & - & $\mathbf{w}$ & $\mathbf{w}$ \\
\hline 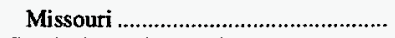 & - & - & - & - & - & $\mathbf{w}$ & - & $\mathbf{w}$ & w \\
\hline 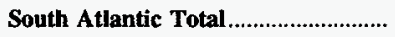 & $\mathbf{w}$ & $\mathbf{w}$ & $\mathbf{w}$ & $\mathbf{w}$ & $\mathbf{w}$ & $\mathbf{w}$ & $\mathbf{w}$ & $\mathbf{w}$ & $\mathbf{w}$ \\
\hline 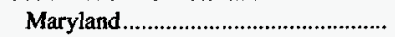 & - & - & - & - & $\mathbf{w}$ & $\mathbf{w}$ & $w$ & $w$ & $\mathbf{w}$ \\
\hline 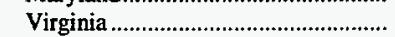 & - & - & - & w & - & - & $\mathbf{w}$ & $\mathbf{w}$ & $\mathrm{w}$ \\
\hline West Virginia & $\mathbf{w}$ & $\mathbf{w}$ & $\mathbf{w}$ & $\mathrm{w}$ & $\mathbf{w}$ & w & $\mathbf{w}$ & $w$ & w \\
\hline East South Central Total................... & $\mathbf{w}$ & $\mathbf{w}$ & $\mathbf{w}$ & $\mathbf{w}$ & $\mathbf{w}$ & $\mathbf{w}$ & $\mathbf{w}$ & $\mathbf{w}$ & $\mathbf{w}$ \\
\hline 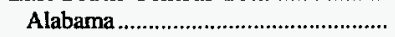 & 233 & 297 & 333 & 332 & 293 & 246 & -21.6 & -5.6 & -.6 \\
\hline Kentucky & w & w & w & w & w & $\mathbf{w}$ & w & w & $\mathbf{w}$ \\
\hline 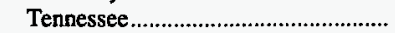 & - & - & - & - & - & $\mathbf{w}$ & $\mathbf{w}$ & $\mathbf{w}$ & w \\
\hline West South Central Total .................... & - & - & 21 & 21 & $\mathbf{w}$ & $\mathbf{w}$ & - & $\mathbf{w}$ & $\mathbf{w}$ \\
\hline 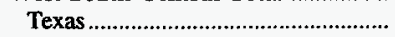 & - & - & 21 & 21 & $\mathbf{w}$ & $\mathbf{w}$ & - & $\mathbf{w}$ & $\mathbf{w}$ \\
\hline Mountain Total & $\mathbf{w}$ & $\mathbf{w}$ & w & w & $\mathbf{w}$ & $\mathbf{w}$ & $\mathbf{w}$ & $\mathbf{w}$ & $\mathbf{w}$ \\
\hline 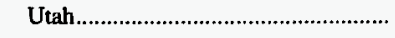 & $\mathbf{w}$ & $\mathbf{w}$ & $\mathrm{w}$ & $\mathbf{w}$ & $\mathbf{w}$ & $\mathrm{w}$ & w & $\mathbf{w}$ & $\mathbf{w}$ \\
\hline U.S. Total & 2,632 & 2,657 & 2,401 & 2,597 & 2,773 & 2,992 & -.9 & -1.3 & -1.4 \\
\hline
\end{tabular}

* Data round to zero.

Withbeld to avoid disclosure of individual company data

Note: Totals may not equal sum of components due to independent rounding.

Source: Energy Information Administration, Form EIA-5, "Coke Plant Report - Quarterly." 
Table 75. Coal Consumption by Residential and Commercial Sector, by Census Division and State, 1986, 1991-1995

(Thousand Short Tons)

\begin{tabular}{|c|c|c|c|c|c|c|c|c|c|}
\hline \multirow{2}{*}{ Census Division and State } & \multirow{2}{*}{1995} & \multirow{2}{*}{1994} & \multirow{2}{*}{1993} & \multirow{2}{*}{1992} & \multirow{2}{*}{1991} & \multirow{2}{*}{1986} & \multirow{2}{*}{$\begin{array}{c}\text { Percent } \\
\text { Change } \\
\text { 1994-1995 }\end{array}$} & \multicolumn{2}{|c|}{ Average Annual Percent Change } \\
\hline & & & & & & & & 1991-1995 & 1986-1995 \\
\hline 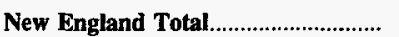 & 69 & 56 & 102 & 141 & 84 & 204 & 24.4 & -4.6 & -11.3 \\
\hline Connecticut & w & $w$ & w & w & w & w & w & $\mathbf{w}$ & $\mathbf{w}$ \\
\hline 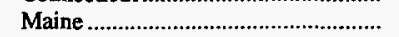 & $w$ & w & $\mathbf{w}$ & $\mathbf{w}$ & w & $\mathbf{w}$ & w & $\mathbf{w}$ & $\mathbf{w}$ \\
\hline Massachusetts & $\mathrm{w}$ & $\mathrm{w}$ & $\mathbf{w}$ & $\hat{w}$ & $w$ & $\mathbf{w}$ & w & $\mathbf{w}$ & $\mathbf{w}$ \\
\hline 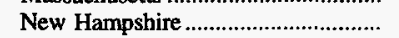 & w & $w$ & $\mathbf{w}$ & $\mathbf{w}$ & $\mathbf{w}$ & w & $\mathbf{w}$ & $\mathbf{w}$ & $\mathbf{w}$ \\
\hline Rhode Island & w & $\mathbf{w}$ & $w$ & $w$ & w & $\mathbf{w}$ & $\mathbf{w}$ & $w$ & $\mathbf{w}$ \\
\hline 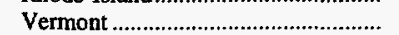 & $w$ & w & $\mathbf{w}$ & w & w & $\mathbf{w}$ & $\mathbf{w}$ & $\mathbf{w}$ & w \\
\hline Middle Atlantic Total. & 1,379 & 1,351 & 1,498 & 1,916 & 1,698 & 1,928 & 2.0 & -5.1 & -3.6 \\
\hline 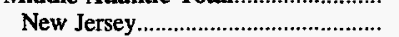 & $w$ & w & $w$ & $w$ & $w$ & $\mathbf{w}$ & w & $w$ & w \\
\hline 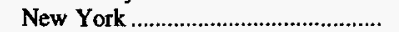 & w & $\mathbf{w}$ & $\mathbf{w}$ & W & $w$ & $\mathbf{w}$ & $w$ & $\mathbf{w}$ & w \\
\hline Pennsylvania & 1,151 & 1,156 & 1,257 & 1,626 & 1,408 & 1,402 & -.4 & -4.9 & -2.2 \\
\hline 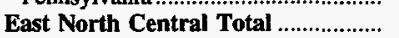 & 1,301 & 1,452 & 1,458 & 1,476 & 1,453 & 2,134 & -10.3 & -2.7 & -5.3 \\
\hline Illinois & $w$ & $\mathrm{w}$ & $\mathrm{w}$ & $w$ & w & 245 & w & w & w \\
\hline Indiana & 287 & 356 & 339 & 411 & 433 & 546 & -19.5 & -9.8 & -6.9 \\
\hline 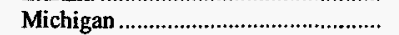 & w & w & $\mathbf{w}$ & $\mathbf{w}$ & $\mathbf{w}$ & $\mathbf{w}$ & w & $w$ & w \\
\hline Ohio & 409 & 498 & 584 & 588 & 489 & 1,052 & -18.0 & -4.4 & -10.0 \\
\hline 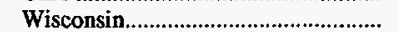 & w & w & $w$ & w & w & w & w & w & w \\
\hline West North Central Total .................. & $\mathbf{w}$ & $\mathbf{w}$ & $\mathbf{w}$ & $\mathbf{w}$ & $\mathbf{w}$ & $\mathbf{w}$ & $\mathbf{w}$ & $\mathbf{w}$ & $\mathbf{w}$ \\
\hline Iowa & 90 & 40 & 70 & 64 & 223 & 255 & 121.9 & -20.3 & -10.9 \\
\hline 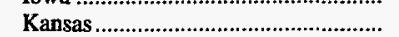 & 92 & 32 & 23 & $*$ & $*$ & 1 & 183.5 & 303.6 & 65.6 \\
\hline 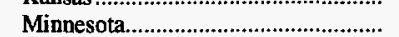 & 264 & 229 & 107 & 25 & 94 & 193 & 15.2 & 29.3 & 3.5 \\
\hline 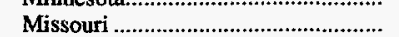 & $w$ & $w$ & w & $\mathbf{w}$ & $w$ & w & w & w & $\mathbf{w}$ \\
\hline 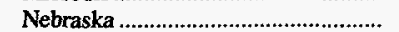 & $\mathbf{w}$ & $\mathbf{w}$ & $w$ & $w$ & $w$ & 4 & $w$ & $\mathbf{w}$ & $\mathbf{w}$ \\
\hline 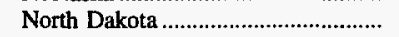 & w & $\mathbf{w}$ & w & $\mathbf{w}$ & $\mathbf{w}$ & w & w & $\mathbf{w}$ & w \\
\hline 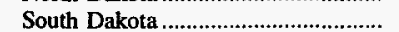 & $\mathbf{w}$ & $\mathbf{w}$ & w & w & $\mathbf{w}$ & w & $w$ & $w$ & $\mathbf{w}$ \\
\hline South Atlantic Total ............................. & 954 & 887 & 904 & 632 & 490 & 1,002 & 7.6 & 18.1 & -.5 \\
\hline Delaware & $\mathbf{w}$ & $\mathbf{w}$ & w & $\mathbf{w}$ & $\mathbf{w}$ & w & w & $\mathbf{w}$ & w \\
\hline 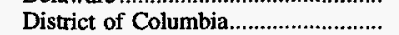 & 6 & 47 & 51 & 50 & 66 & 54 & -87.9 & -45.8 & -22.2 \\
\hline Florida & 1 & 20 & 16 & 16 & $*$ & 95 & -93.5 & 66.6 & -37.9 \\
\hline 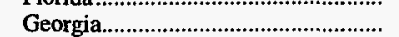 & 59 & 28 & 22 & 38 & 8 & 8 & 108.8 & 65.5 & 25.8 \\
\hline 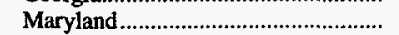 & $\mathbf{w}$ & $\mathbf{w}$ & w & $\mathbf{w}$ & $\mathbf{w}$ & $\mathbf{w}$ & NM & w & w \\
\hline 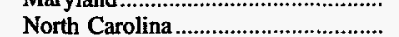 & 224 & 263 & 229 & 204 & 97 & 155 & -14.8 & 23.1 & 4.2 \\
\hline 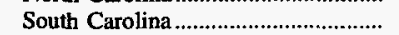 & 17 & 61 & 109 & 31 & 22 & 219 & -72.5 & -6.6 & -24.8 \\
\hline Virginia & w & $\mathbf{w}$ & w & $\mathbf{w}$ & $w$ & $\mathbf{w}$ & w & $w$ & w \\
\hline 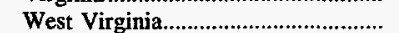 & w & $\mathbf{w}$ & w & $\mathbf{w}$ & $\mathbf{w}$ & $w$ & w & $w$ & w \\
\hline East South Central Total................... & 283 & 386 & 417 & 458 & 376 & 452 & -26.8 & -6.8 & -5.1 \\
\hline Alabama & 7 & 11 & 40 & 89 & 17 & 112 & -32.5 & -18.9 & -26.3 \\
\hline 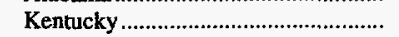 & $\mathbf{w}$ & $\mathbf{w}$ & $\mathbf{w}$ & w & $\mathbf{w}$ & $w$ & w & $\mathbf{w}$ & w \\
\hline 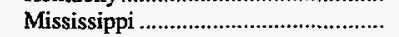 & - & - & $\mathbf{w}$ & $w$ & $w$ & $w$ & $\mathrm{w}$ & $w$ & w \\
\hline 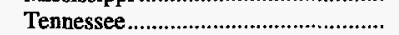 & $\mathbf{w}$ & $\mathbf{w}$ & $\mathbf{w}$ & w & $w$ & $\mathrm{w}$ & w & $w$ & $\mathbf{w}$ \\
\hline West South Central Total .................. & 17 & 1 & 8 & 13 & 12 & 18 & $\mathbf{N M}$ & 8.9 & -1.2 \\
\hline 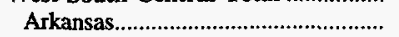 & - & * & $\mathbf{1}$ & 2 & * & $*$ & - & - & - \\
\hline 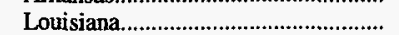 & w & - & $w$ & - & $w$ & $w$ & w & NM & $w$ \\
\hline 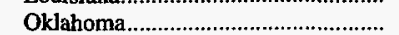 & $\mathbf{w}$ & $w$ & $\mathbf{w}$ & $\mathbf{w}$ & 1 & 4 & $\mathrm{NM}$ & w & $w$ \\
\hline 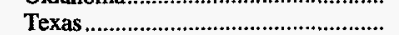 & - & $*$ & 6 & 10 & $\mathbf{w}$ & $\mathbf{w}$ & - & $\mathbf{w}$ & $\mathbf{w}$ \\
\hline 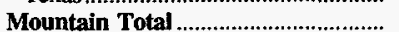 & $\mathbf{w}$ & $\mathbf{w}$ & $\mathbf{w}$ & $\mathbf{w}$ & $\mathbf{w}$ & $\mathbf{w}$ & $\mathbf{w}$ & $\mathbf{w}$ & $\mathbf{w}$ \\
\hline 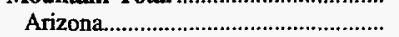 & 5 & * & 1 & 4 & $\mathbf{w}$ & $\mathbf{w}$ & NM & w & $w$ \\
\hline Colorado & 20 & 23 & 38 & 58 & w & $\mathbf{w}$ & -14.0 & $w$ & $\mathbf{w}$ \\
\hline Idaho & 39 & 40 & 43 & 51 & 68 & 37 & -3.3 & -13.1 & .5 \\
\hline Montana & w & $w$ & $w$ & w & w & $\mathbf{w}$ & w & w & $\mathbf{w}$ \\
\hline Nevada & $w$ & $w$ & $w$ & $w$ & $w$ & $w$ & $w$ & $\mathbf{w}$ & $\mathbf{w}$ \\
\hline New Mexico & $\mathbf{w}$ & $w$ & $w$ & $\mathbf{w}$ & $w$ & $w$ & $w$ & w & $\mathbf{w}$ \\
\hline Utah & $\tilde{w}$ & $w$ & $w$ & $\mathbf{w}$ & w & $w$ & $\mathbf{w}$ & $w$ & $\mathbf{w}$ \\
\hline Wyoming & 146 & 242 & 187 & 99 & $\mathrm{w}$ & w & -39.8 & $w$ & $\mathbf{w}$ \\
\hline Pacific Total & 734 & 773 & 821 & 607 & $\mathbf{w}$ & $\mathbf{w}$ & -5.0 & $\mathbf{w}$ & $\mathbf{w}$ \\
\hline Alaska & 523 & 520 & 563 & 514 & w & $\mathbf{w}$ & .6 & w & $\mathbf{w}$ \\
\hline California & 133 & 166 & 142 & * & $\mathrm{w}$ & $w$ & -20.0 & $\mathbf{w}$ & $\mathbf{w}$ \\
\hline 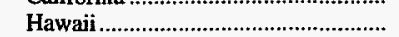 & w & w & w & $\mathbf{w}$ & $w$ & $\mathbf{w}$ & w & $w$ & $\mathbf{w}$ \\
\hline Oregon & w & $\mathbf{w}$ & $\mathbf{w}$ & $w$ & $w$ & $\mathbf{w}$ & w & w & w \\
\hline Washington & 78 & 86 & 114 & 91 & 80 & 88 & -9.4 & -.7 & -1.4 \\
\hline U.S. Total & 5,824 & 6,013 & 6,221 & 6,153 & 6,094 & 7,667 & -3.1 & -1.1 & $-\mathbf{3 . 0}$ \\
\hline
\end{tabular}

* Data round to zero.

W Witheld to avoid disclosure of individual company data.

NM Not meaningful as value is greater than 500 percent

Note: Totals may not equal sum of components due to independent rounding.

Source: Energy Information Administration, Form ELA-6, "Coal Distribution Report." 



\section{Foreign Markets}

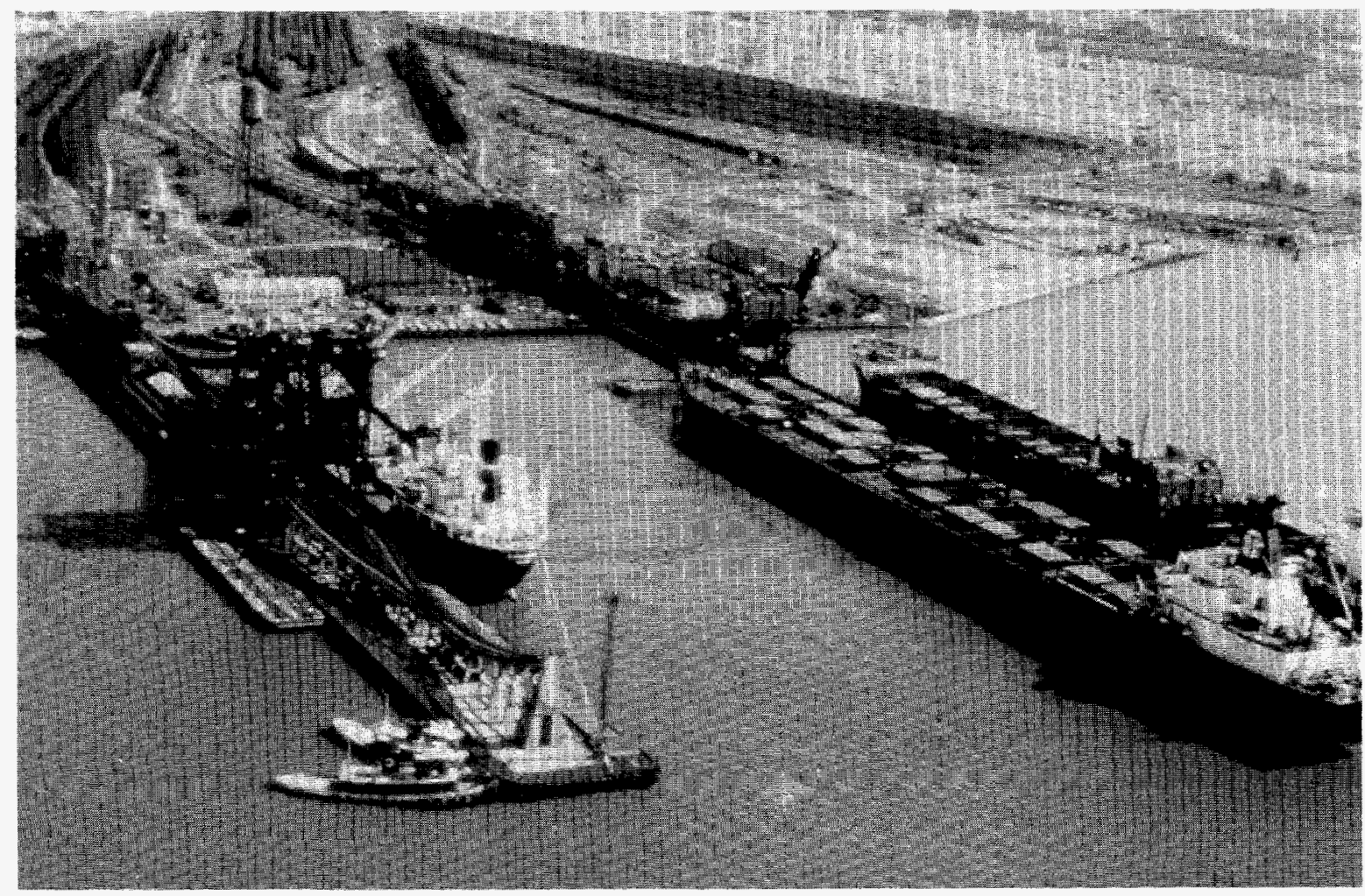

The demand for U.S. coal has increased (88,547 thousand short tons) since last year (71,359 thousand short tons). Europe accounted for the largest share of exported coal. 
Figure 12. U.S. Coal Exports, 1986-1995

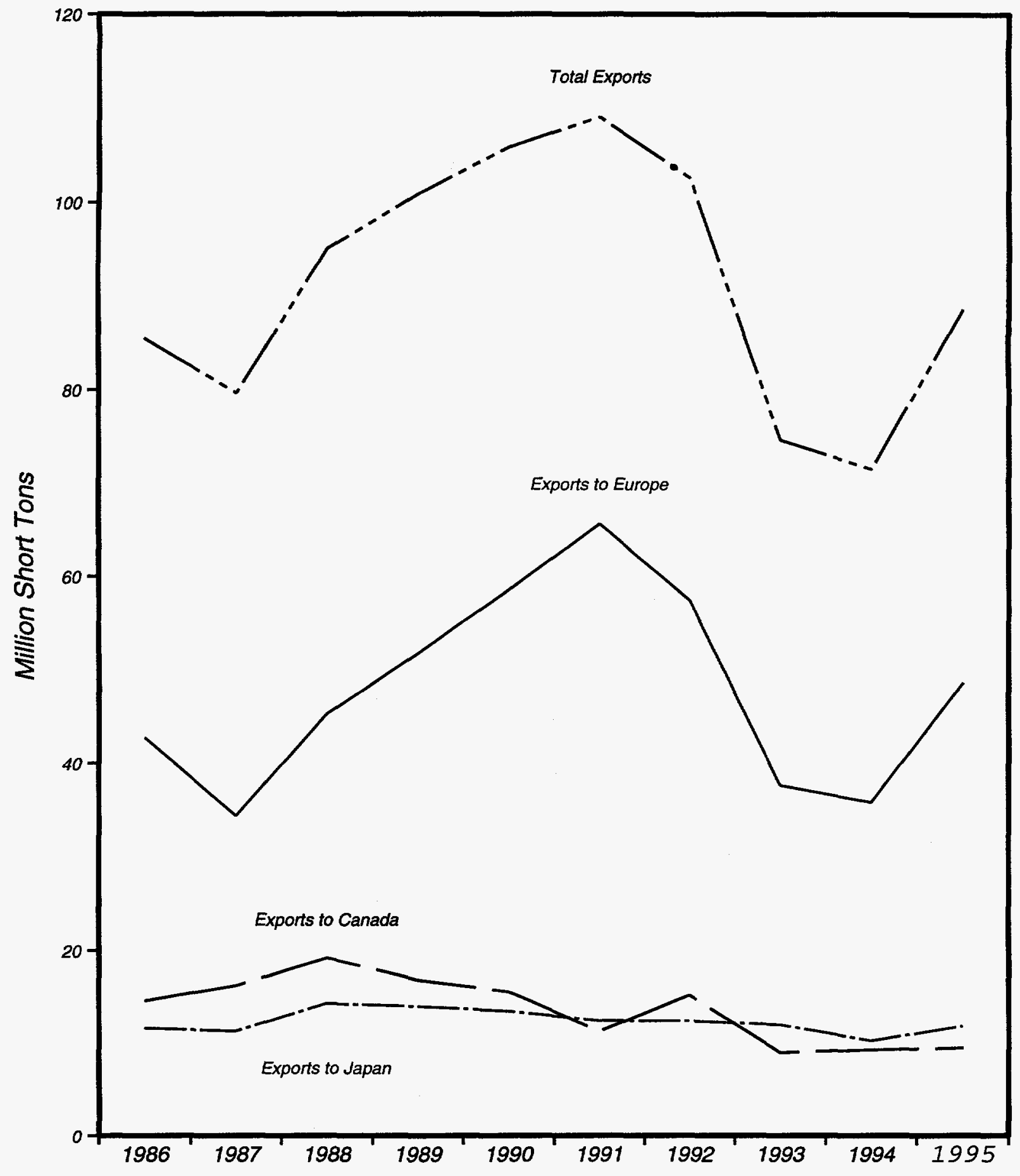

Source: U.S. Department of Commerce; Bureau of the Census, "Monthly Report EM 545." 
Table 76. U.S. Coal Exports by Destination, 1986, 1991-1995

(Thousand Short Tons)

\begin{tabular}{|c|c|c|c|c|c|c|c|c|c|}
\hline \multirow{2}{*}{$\begin{array}{l}\text { Continent and Country } \\
\text { of Destination }\end{array}$} & \multirow{2}{*}{1995} & \multirow{2}{*}{1994} & \multirow{2}{*}{1993} & \multirow{2}{*}{1992} & \multirow{2}{*}{1991} & \multirow{2}{*}{1986} & \multirow{2}{*}{$\begin{array}{c}\text { Percent } \\
\text { Change } \\
\text { 1994-1995 }\end{array}$} & \multicolumn{2}{|c|}{ Average Annual Percent Change } \\
\hline & & & & & & & & 1991-1995 & 1986-1995 \\
\hline North America Total & 10,411 & 9,505 & 9,219 & 15,331 & 11,454 & 14,774 & 9.5 & -2.4 & -3.8 \\
\hline Canada 1 & 9,427 & 9,193 & 8,889 & 15,140 & 11,178 & 14,478 & 2.5 & -4.2 & -4.6 \\
\hline Mexico & 871 & 241 & 250 & 77 & 92 & 225 & 261.7 & 75.3 & 16.2 \\
\hline Other $^{2}$ & 113 & 71 & 81 & 115 & 184 & 72 & 58.1 & -11.6 & 5.2 \\
\hline 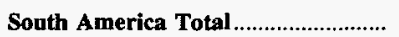 & 6,968 & 5,946 & 5,750 & 6,769 & 7,661 & 6,636 & 17.2 & -2.3 & .5 \\
\hline 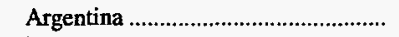 & 342 & 453 & 524 & 335 & 429 & 752 & -24.4 & -5.5 & -8.4 \\
\hline 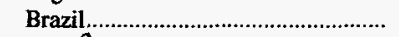 & 6,351 & 5,482 & 5,197 & 6,370 & 7,052 & 5,720 & 15.8 & -2.6 & 1.2 \\
\hline Other ${ }^{2}$ & 276 & 12 & 28 & 64 & 180 & 164 & NM & 11.3 & 5.9 \\
\hline 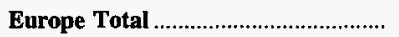 & 48,620 & 35,825 & 37,575 & 57,255 & 65,520 & 42,552 & 35.7 & -7.2 & 1.5 \\
\hline 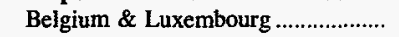 & 4,501 & 4,911 & 5,229 & 7,196 & 7,464 & 4,362 & -8.3 & -11.9 & 3 \\
\hline 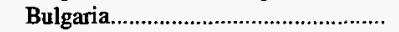 & 1,339 & 1,238 & 906 & 602 & 946 & 32 & 8.1 & 9.1 & 51.5 \\
\hline 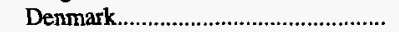 & 2,100 & 477 & 336 & 3,832 & 4,658 & 2,123 & 340.6 & -18.0 & -.1 \\
\hline Finland & 1,308 & 377 & 252 & 205 & 530 & 49 & 247.2 & 25.3 & 43.9 \\
\hline 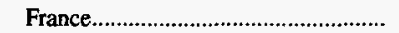 & 3,659 & 2,875 & 3,972 & 8,060 & 9,509 & 5,389 & 27.3 & -21.2 & -4.2 \\
\hline Germany, FR & 1,953 & 323 & 508 & 1,003 & 1,742 & 810 & NM & 2.9 & 10.3 \\
\hline Ireland & 914 & 974 & 985 & 1,449 & 1,313 & 770 & -6.1 & -8.6 & 1.9 \\
\hline 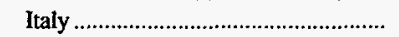 & 9,063 & 7,543 & 6,918 & 9,344 & 11,274 & 10,359 & 20.1 & -5.3 & -1.5 \\
\hline 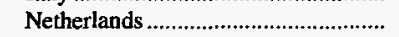 & 7,301 & 4,874 & 5,562 & 9,148 & 9,625 & 5,631 & 49.8 & -6.7 & 2.9 \\
\hline 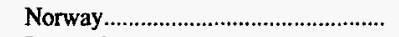 & 120 & 87 & 101 & 118 & 200 & 267 & 38.5 & -12.0 & -8.5 \\
\hline 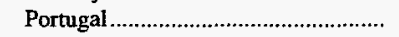 & 1,752 & 1,057 & 1,491 & 1,479 & 1,629 & 1,103 & 65.9 & 1.8 & 5.3 \\
\hline 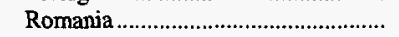 & 1,984 & 1,553 & 720 & 753 & 1,147 & 1,111 & 27.7 & 14.7 & 6.6 \\
\hline Spain & 4,653 & 4,132 & 4,064 & 4,535 & 4,694 & 2,620 & 12.6 & -.2 & 6.6 \\
\hline 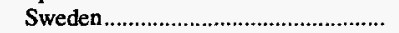 & 1,117 & 702 & 736 & 1,165 & 1,239 & 1,097 & 59.3 & -2.5 & .2 \\
\hline 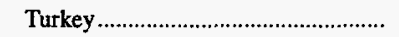 & 2,011 & 1,335 & 1,605 & 1,990 & 2,186 & 2,418 & 50.7 & -2.1 & -2.0 \\
\hline 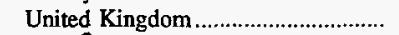 & 4,726 & 3,363 & 4,111 & 5,595 & 6,171 & 2,907 & 40.5 & -6.4 & 5.5 \\
\hline Other $^{2}$ & 117 & 8 & 78 & 781 & 1,192 & 1,505 & NM & -44.0 & -24.7 \\
\hline Asia Total & 19,095 & 17,957 & 19,500 & 20,540 & 21,788 & 19,639 & 6.3 & -3.2 & -.3 \\
\hline China (Taiwan) & 2,533 & 3,374 & 3,435 & 3,560 & 4,547 & 3,690 & -24.9 & -13.6 & -4.1 \\
\hline 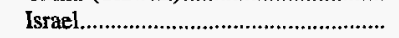 & 760 & 864 & 849 & 824 & 651 & 381 & -12.0 & 3.9 & 8.0 \\
\hline Japan & 11,787 & 10,158 & 11,878 & 12,304 & 12,269 & 11,370 & 16.0 & -1.0 & .4 \\
\hline 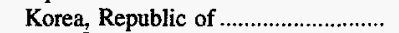 & 4,012 & 3,558 & 3,316 & 3,352 & 3,711 & 3,555 & 12.8 & 2.0 & 1.3 \\
\hline Other ${ }^{2}$ & 2 & 3 & 22 & 500 & 611 & 643 & -30.3 & -75.2 & -46.5 \\
\hline Oceania \& Australia Total ................ & * & $\mathbf{1}$ & 1 & * & * & $*$ & -60.1 & 12.9 & -2.9 \\
\hline Other 2 & $*$ & 1 & 1 & * & * & $*$ & -60.1 & 12.9 & -2.9 \\
\hline 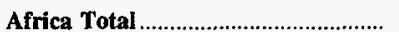 & 3,453 & 2,124 & 2,474 & 2,621 & 2,545 & 1,916 & 62.5 & 7.9 & 6.8 \\
\hline Algeria & 220 & 355 & 409 & 611 & 522 & 685 & -38.0 & -19.4 & -11.8 \\
\hline 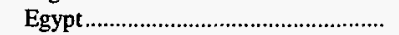 & 1,235 & 1,048 & 868 & 848 & 769 & 408 & 17.8 & 12.6 & 13.1 \\
\hline Morocco & 1,212 & 83 & 587 & 737 & 1,013 & 737 & NM & 4.6 & 5.7 \\
\hline 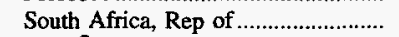 & 786 & 638 & 567 & 425 & 239 & - & 23.3 & 34.7 & - \\
\hline Other ${ }^{2}$ & - & - & 42 & - & 2 & 86 & - & -100.0 & -100.0 \\
\hline Total & 88,547 & 71,359 & 74,519 & 102,516 & 108,969 & 85,518 & 24.1 & -5.0 & .4 \\
\hline
\end{tabular}

1 Based on the U.S. - Canada Free Trade Agreement, as of January 1990, the U.S. Department of Commerce began reporting statistics on U.S. exports to Canada based on information on imports provided monthly by the Canadian government.

2 Includes countries with exports less than or equal to 50,000 short tons in 1994.

* Data round to zero.

NM Not meaningful as value is greater than 500 percent.

Note: Totals may not equal sum of components due to independent rounding.

Source: U.S. Department of Commerce, Bureau of the Census, "Monthly Report EM 545." 
Table 77. U.S. Metallurgical Coal Exports by Destination, 1986, 1991-1995

(Thousand Short Tons)

\begin{tabular}{|c|c|c|c|c|c|c|c|c|c|}
\hline \multirow{2}{*}{$\begin{array}{l}\text { Continent and Country } \\
\text { of Destination }\end{array}$} & \multirow{2}{*}{1995} & \multirow{2}{*}{1994} & \multirow{2}{*}{1993} & \multirow{2}{*}{1992} & \multirow{2}{*}{1991} & \multirow{2}{*}{1986} & \multirow{2}{*}{$\begin{array}{c}\text { Percent } \\
\text { Change } \\
\text { 1994-1995 }\end{array}$} & \multicolumn{2}{|c|}{ Average Annual Percent Change } \\
\hline & & & & & & & & 1991-1995 & 1986-1995 \\
\hline North America Total ............................. & 4,776 & 4,246 & 4,853 & 4,957 & 4,060 & 6,624 & 12.5 & 4.1 & -3.6 \\
\hline 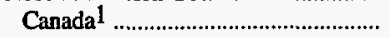 & 4,452 & 4,032 & 4,663 & 4,920 & 4,019 & 6,440 & 10.4 & 2.6 & -4.0 \\
\hline Mexico & 324 & 214 & 190 & 36 & 41 & 135 & 51.2 & 68.0 & 10.2 \\
\hline Other ${ }^{2}$ & - & - & $*$ & * & * & 48 & - & -100.0 & -100.0 \\
\hline 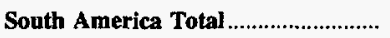 & 6,778 & 5,926 & 5,514 & 6,524 & 7,416 & 6,414 & 14.4 & -2.2 & .6 \\
\hline 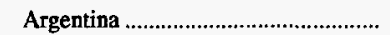 & 336 & 449 & 487 & 332 & 428 & 751 & -25.2 & -5.9 & -8.6 \\
\hline 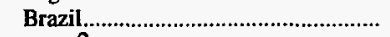 & 6,336 & 5,477 & 5,027 & 6,165 & 6,958 & 5,524 & 15.7 & -2.3 & 1.5 \\
\hline Other ${ }^{2}$ & 106 & - & - & 27 & 30 & 139 & - & 37.6 & -2.9 \\
\hline Europe Total & 27,282 & 25,245 & 26,791 & 33,365 & 38,544 & 27,824 & 8.1 & -8.3 & -2 \\
\hline 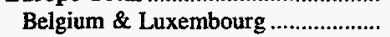 & 3,468 & 3,706 & 4,030 & 5,165 & 5,531 & 3,577 & -6.4 & -11.0 & -3 \\
\hline 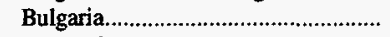 & 1,339 & 1,184 & 849 & 602 & 946 & - & 13.1 & 9.1 & - \\
\hline Denmark & - & - & 106 & - & 568 & 405 & - & -100.0 & -100.0 \\
\hline Finland & 724 & 311 & 252 & 205 & 389 & - & 133.1 & 16.8 & - \\
\hline 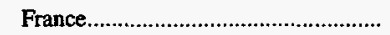 & 3,155 & 2,816 & 3,101 & 4,314 & 5,851 & 3,864 & 12.0 & -14.3 & -2.2 \\
\hline 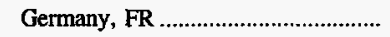 & 231 & 288 & 203 & 247 & 561 & 597 & -19.9 & -19.9 & -10.0 \\
\hline Italy & 4,504 & 5,045 & 4,965 & 6,180 & 6,489 & 5,491 & -10.7 & -8.7 & -2.2 \\
\hline Netherlands & 3,978 & 3,231 & 3,743 & 4,836 & 4,598 & 3,139 & 23.1 & -3.5 & 2.7 \\
\hline 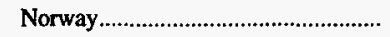 & 92 & 73 & 70 & 91 & 101 & 176 & 25.4 & -2.3 & -6.9 \\
\hline 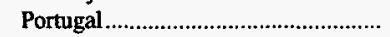 & 30 & - & 149 & 135 & 74 & 172 & - & -20.1 & -17.6 \\
\hline Romania & 1,685 & 663 & 373 & 753 & 1,003 & 1,103 & 153.9 & 13.8 & 4.8 \\
\hline 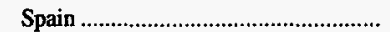 & 2,178 & 2,656 & 2,994 & 2,795 & 3,402 & 2,232 & -18.0 & -10.5 & -.3 \\
\hline 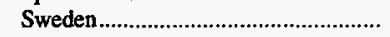 & 1,109 & 702 & 736 & 1,165 & 1,231 & 1,053 & 58.0 & -2.6 & .6 \\
\hline 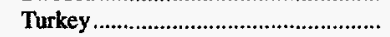 & 1,806 & 1,335 & 1,604 & 1,989 & 2,066 & 2,283 & 35.3 & -3.3 & -2.6 \\
\hline United Kingdom & 2,932 & 3,228 & 3,573 & 4,175 & 4,657 & 2,788 & -9.2 & -10.9 & .5 \\
\hline Other $^{2}$ & 52 & 7 & 43 & 712 & 1,076 & 944 & NM & -53.1 & -27.6 \\
\hline Asia Total & 11,014 & 9,877 & 10,608 & 12,655 & 12,894 & 12,936 & 11.5 & -3.9 & -1.8 \\
\hline China (Taiwan) & 370 & 296 & 285 & 424 & 459 & 248 & 25.3 & -5.2 & 4.5 \\
\hline Israel. & 141 & - & - & 143 & 109 & - & - & 6.5 & - \\
\hline Japan & 7,929 & 7,195 & 8,028 & 9,480 & 9,395 & 10,479 & 10.2 & -4.1 & -3.0 \\
\hline 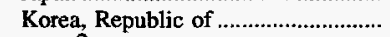 & 2,574 & 2,386 & 2,276 & 2,608 & 2,931 & 1,962 & 7.8 & -3.2 & 3.1 \\
\hline Other 2 & - & - & 19 & - & - & 248 & - & - & -100.0 \\
\hline 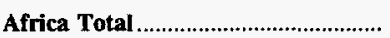 & 2,239 & 2,040 & 1,886 & 1,925 & 1,731 & 1,178 & 9.8 & 6.6 & 7.4 \\
\hline Algeria & 220 & 355 & 409 & 611 & 522 & 684 & -38.0 & -19.4 & -11.8 \\
\hline Egypt & 1,233 & 1,047 & 868 & 848 & 768 & 408 & 17.8 & 12.6 & 13.1 \\
\hline Morocco & - & - & - & 41 & 202 & - & - & -100.0 & - \\
\hline South Africa, Rep of .......................... & 786 & 638 & 567 & 425 & 239 & - & 23.2 & 34.7 & - \\
\hline Other ${ }^{2}$ & - & - & 42 & - & - & 86 & - & - & -100.0 \\
\hline Total & 52,089 & 47,334 & 49,652 & 59,426 & 64,645 & 54,977 & 10.0 & -5.3 & -.6 \\
\hline
\end{tabular}

1 Based on the U.S. - Canada Free Trade Agreement, as of January 1990, the U.S. Department of Commerce began reporting statistics on U.S. exports to Canada based an information on imports provided monthly by the Canadian government.

2 Includes countries with exports less than or equal to 50,000 short tons in 1994.

* Data round to zero.

NM Not meaningful as value is greater than 500 percent.

Note: Totals may not equal sum of components due to independent rounding

Source: U.S. Department of Commerce, Bureau of the Census, "Monthly Report EM 545." 
Table 78. U.S. Steam Coal Exports by Destination, 1986, 1991-1995 (Thousand Short Tons)

\begin{tabular}{|c|c|c|c|c|c|c|c|c|c|}
\hline \multirow{2}{*}{$\begin{array}{c}\text { Continent and Country } \\
\text { of Destination }\end{array}$} & \multirow{2}{*}{1995} & \multirow{2}{*}{1994} & \multirow{2}{*}{1993} & \multirow{2}{*}{1992} & \multirow{2}{*}{1991} & \multirow{2}{*}{1986} & \multirow{2}{*}{$\begin{array}{c}\text { Percent } \\
\text { Change } \\
\text { 1994-1995 }\end{array}$} & \multicolumn{2}{|c|}{ Average Annual Percent Change } \\
\hline & & & & & & & & 1991-1995 & 1986-1995 \\
\hline North America Total ........................... & 5,635 & 5,259 & 4,366 & 10,374 & 7,394 & 8,150 & 7.1 & -6.6 & -4.0 \\
\hline Canada 1 & 4,975 & 5,161 & 4,225 & 10,219 & 7,159 & 8,037 & -3.6 & -8.7 & -5.2 \\
\hline 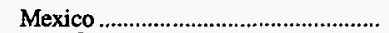 & 547 & 26 & 60 & 40 & 52 & 90 & NM & 80.5 & 22.2 \\
\hline Other ${ }^{2}$ & 113 & 71 & 81 & 115 & 184 & 23 & 58.1 & -11.6 & 19.2 \\
\hline South America Total ........................... & 190 & 20 & 236 & 245 & 245 & 222 & $\mathbf{N M}$ & -6.1 & -1.7 \\
\hline Argentina & 6 & 4 & 38 & 3 & 1 & 1 & 70.7 & 57.3 & 30.3 \\
\hline 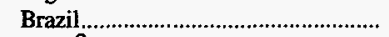 & 15 & 5 & 170 & 205 & 94 & 196 & 216.7 & -37.1 & -25.0 \\
\hline Other ${ }^{2}$ & 169 & 12 & 28 & 37 & 150 & 26 & NM & 3.1 & 23.3 \\
\hline Europe Total & 21,338 & $\mathbf{1 0 , 5 8 0}$ & 10,784 & 23,891 & 26,975 & 14,728 & 101.7 & -5.7 & 4.2 \\
\hline Belgium \& Luxembourg ....................... & 1,033 & 1,205 & 1,199 & 2,031 & 1,933 & 786 & -14.2 & -14.5 & 3.1 \\
\hline 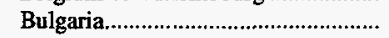 & - & 54 & 57 & - & - & 32 & -100.0 & - & -100.0 \\
\hline 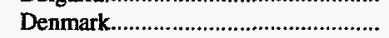 & 2,100 & 477 & 230 & 3,832 & 4,090 & 1,717 & 340.6 & -15.3 & 2.3 \\
\hline 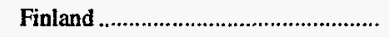 & 584 & 66 & 1 & - & 142 & 49 & NM & 42.5 & 31.6 \\
\hline 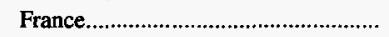 & 503 & 58 & 870 & 3,745 & 3,658 & 1,525 & $\mathbf{N M}$ & -39.1 & -11.6 \\
\hline Germany, FR & 1,722 & 35 & 305 & 756 & 1,181 & 214 & NM & 9.9 & 26.1 \\
\hline Ireland & 914 & 974 & 985 & 1,449 & 1,313 & 770 & -6.1 & -8.6 & 1.9 \\
\hline 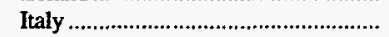 & 4,559 & 2,498 & 1,954 & 3,164 & 4,785 & 4,867 & 82.5 & -1.2 & -.7 \\
\hline 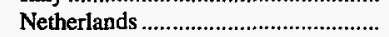 & 3,323 & 1,643 & 1,819 & 4,312 & 5,027 & 2,492 & 102.3 & -9.8 & 3.2 \\
\hline 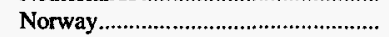 & 28 & 13 & 31 & 27 & 99 & 92 & 111.4 & -27.0 & -12.3 \\
\hline 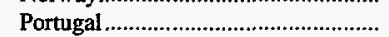 & 1,722 & 1,057 & 1,342 & 1,344 & 1,555 & 931 & 63.0 & 2.6 & 7.1 \\
\hline 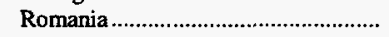 & 299 & 890 & 347 & - & 144 & 8 & -66.4 & 20.1 & 50.1 \\
\hline Spain & 2,475 & 1,476 & 1,070 & 1,740 & 1,292 & 387 & 67.7 & 17.6 & 22.9 \\
\hline Sweden & 9 & - & - & * & 8 & 43 & - & 1.7 & -16.4 \\
\hline Turkey & 206 & - & $*$ & * & 120 & 135 & - & 14.4 & 4.8 \\
\hline 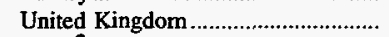 & 1,795 & 135 & 538 & 1,421 & 1,514 & 119 & NM & 4.3 & 35.2 \\
\hline Other ${ }^{2}$ & 66 & * & 35 & 68 & 116 & 562 & NM & -13.3 & -21.2 \\
\hline Asia Total & 8,081 & 8,080 & 8,892 & 7,885 & 8,894 & 6,702 & $*$ & -2.4 & 2.1 \\
\hline China (Taiwan) & 2,163 & 3,078 & 3,150 & 3,136 & 4,088 & 3,442 & -29.7 & -14.7 & -5.0 \\
\hline 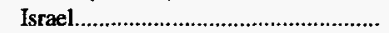 & 620 & 864 & 849 & 681 & 542 & 381 & -28.2 & 3.4 & 5.5 \\
\hline 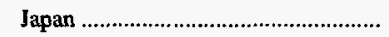 & 3,858 & 2,963 & 3,850 & 2,823 & 2,874 & 891 & 30.2 & 7.6 & 17.7 \\
\hline 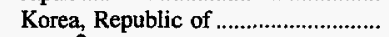 & 1,438 & 1,172 & 1,040 & 744 & 779 & 1,593 & 22.8 & 16.5 & -1.1 \\
\hline Other 2 & 2 & 3 & 3 & 500 & 611 & 394 & -30.3 & -75.2 & -43.5 \\
\hline Oceania \& Australia Total ................ & * & 1 & 1 & * & * & * & -60.1 & 12.9 & -2.9 \\
\hline Other ${ }^{2}$ & $*$ & 1 & 1 & * & * & * & -60.1 & 12.9 & -2.9 \\
\hline Africa Total & 1,214 & 85 & 588 & 696 & 814 & 738 & $\mathbf{N M}$ & 10.5 & 5.7 \\
\hline Algeria & - & - & - & - & - & 1 & - & - & -100.0 \\
\hline Egypt & 2 & 1 & 1 & 1 & 1 & * & 28.3 & 9.6 & 42.9 \\
\hline Morocco & 1,212 & 83 & 587 & 695 & 811 & 737 & NM & 10.6 & 5.7 \\
\hline South Africa, Rep of & $*$ & - & - & - & - & - & - & - & - \\
\hline Other ${ }^{2}$ & - & - & - & - & 2 & - & - & -100.0 & - \\
\hline Total & 36,458 & 24,025 & 24,867 & 43,090 & 44,323 & 30,541 & 51.8 & -4.8 & 2.0 \\
\hline
\end{tabular}

1 Based on the U.S. - Canada Free Trade Agreement, as of January 1990, the U.S. Department of Commerce began reporting statistics on U.S. exports to Canada based on information on imports provided monthly by the Canadian government.

2 Includes countries with exports less than or equal to 50,000 short tons in 1994.

* Data round to zero.

NM Not meaningful as value is greater than 500 percent.

Note: Totals may not equal sum of components due to independent rounding.

Source: U.S. Department of Commerce, Bureau of the Census, "Monthly Report EM 545." 
Table 79. Coal Exports by Customs District, 1986, 1991-1995

(Thousand Short Tons)

\begin{tabular}{|c|c|c|c|c|c|c|c|c|c|}
\hline \multirow{2}{*}{ Customs District } & \multirow{2}{*}{1995} & \multirow{2}{*}{1994} & \multirow{2}{*}{1993} & \multirow{2}{*}{1992} & \multirow{2}{*}{1991} & \multirow{2}{*}{1986} & \multirow{2}{*}{$\begin{array}{c}\text { Percent } \\
\text { Change } \\
\text { 1994-1995 }\end{array}$} & \multicolumn{2}{|c|}{ Average Annual Percent Change } \\
\hline & & & & & & & & 1991-1995 & 1986-1995 \\
\hline 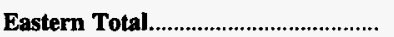 & 55,374 & 43,474 & 45,138 & 64,826 & 69,185 & 50,859 & 27,4 & -5.4 & 0.9 \\
\hline Boston, MA & 33 & - & 33 & - & - & - & - & - & - \\
\hline 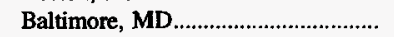 & 11,313 & 7,912 & 7,354 & 9,450 & 9,790 & 6,807 & 43.0 & 3.7 & 5.8 \\
\hline 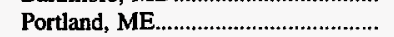 & 57 & 1 & 1 & * & - & $*$ & NM & - & 86.8 \\
\hline Buffalo, NY & 1,574 & 166 & 67 & 1,971 & 773 & 30 & NM & 19.5 & 55.5 \\
\hline 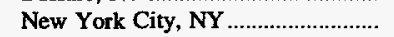 & 87 & 1 & 1 & 4 & 5 & 380 & NM & 104.3 & -15.1 \\
\hline Ogdensburg, NY & 163 & 337 & 44 & 66 & 110 & 17 & -51.6 & 10.3 & 28.4 \\
\hline Philadelphia, PA & 339 & 213 & 190 & 252 & 639 & 4,018 & 59.3 & -14.7 & -24.0 \\
\hline 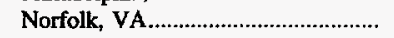 & 41,808 & 34,845 & 37,448 & 53,083 & 57,868 & 39,608 & 20.0 & -7.8 & .6 \\
\hline 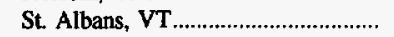 & $*$ & $*$ & * & * & * & $*$ & -62.2 & -40.0 & -4.2 \\
\hline 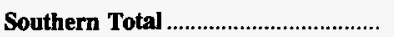 & 19,936 & 15,607 & 16,658 & 21,343 & 25,667 & 18,582 & 27.7 & -6.1 & .8 \\
\hline Mobile, AL & 8,283 & 4,997 & 6,262 & 6,853 & 8,743 & 7,615 & 65.7 & -1.3 & .9 \\
\hline 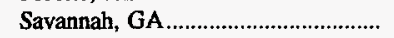 & 4 & 1 & - & 37 & * & * & 406.6 & 125.8 & 77.4 \\
\hline 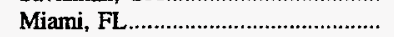 & 2 & 3 & 2 & 2 & 2 & 1 & -37.7 & -5.3 & 3.6 \\
\hline Tampa, FL & 1 & * & $*$ & - & 11 & * & $\mathrm{NM}$ & -42.1 & 22.8 \\
\hline 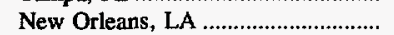 & 10,522 & 9,475 & 9,705 & 13,480 & 15,494 & 9,504 & 11.0 & -9.2 & 1.1 \\
\hline Wilmington, NC & - & - & - & - & $\quad-$ & 266 & - & - & -100.0 \\
\hline San Juan, PR & * & 26 & * & - & * & 1 & -99.7 & -15.7 & -22.4 \\
\hline Charleston, SC & 401 & 957 & 475 & 791 & 1,161 & 1,038 & -58.1 & -23.3 & -10.0 \\
\hline El Paso, TX & $*$ & - & $*$ & 1 & $*$ & 2 & - & 57.7 & -15.7 \\
\hline Houston-Galveston, TX & 179 & 121 & 155 & 145 & 206 & 94 & 47.8 & -3.4 & 7.4 \\
\hline Laredo, TX & 542 & 26 & 59 & 36 & 49 & 62 & NM & 82.3 & 27.3 \\
\hline 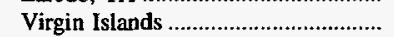 & - & - & - & $*$ & - & * & - & - & -100.0 \\
\hline 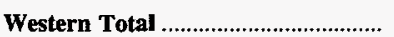 & 5,527 & 3,813 & 4,201 & 3,651 & 4,430 & 1,732 & 44.9 & 5.7 & 13.8 \\
\hline 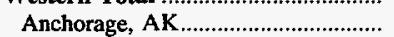 & 919 & 719 & 733 & 728 & 777 & 691 & 27.8 & 4.3 & 3.2 \\
\hline Nogales, $\mathrm{AZ}$ & - & $*$ & $*$ & $*$ & - & - & -100.0 & - & - \\
\hline Las Angeles, CA & 4,475 & 2,963 & 3,358 & 2,721 & 2,840 & 1,029 & 51.0 & 12.0 & 17.7 \\
\hline San Diego, CA & * & 1 & 1 & 4 & 2 & 4 & -90.4 & -57.3 & -36.4 \\
\hline San Francisco, CA & * & 1 & - & 60 & 33 & 2 & -61.1 & -67.4 & -14.4 \\
\hline Great Falls, MT & * & 1 & * & - & - & 1 & -56.4 & - & -15.0 \\
\hline Portland, OR & - & - & 2 & - & 576 & - & - & -100.0 & - \\
\hline Seattle, WA & 132 & 128 & 106 & 137 & 202 & 6 & 3.3 & -10.0 & 41.1 \\
\hline 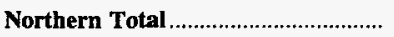 & 7,688 & 8,437 & 8,495 & $\mathbf{1 2 , 5 5 7}$ & 9,635 & 14,344 & -8.9 & -5.5 & -6.7 \\
\hline Chicago, IL & - & 24 & - & * & * & 15 & -100.0 & -100.0 & -100.0 \\
\hline Detroit, MI & 1,845 & 2,600 & 609 & 2,181 & 520 & 48 & -29.0 & 37.2 & 49.9 \\
\hline Duluth, MN & 210 & 161 & 134 & 119 & 130 & $*$ & 30.5 & 12.7 & 109.6 \\
\hline Pembina, ND & 19 & 10 & 1 & * & $*$ & * & 79.7 & 287.6 & 61.5 \\
\hline Cleveland, $\mathrm{OH}$ & 5,614 & 5,642 & 7,751 & 10,258 & 8,985 & 14,280 & -.5 & -11.1 & -9.8 \\
\hline 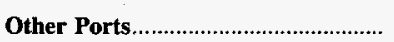 & 22 & 28 & 26 & 138 & $\mathbf{5 1}$ & - & -19.3 & -18.7 & - \\
\hline Total & 88,547 & 71,359 & 74,519 & 102,516 & 108,969 & 85,518 & 24.1 & -5.0 & .4 \\
\hline
\end{tabular}

* Data round to zero.
NM Not meaningful as value is greater than 500 percent.

Note: Totals may not equal sum of components due to independent rounding

Source: U.S. Department of Commerce, Bureau of the Census, "Monthly Report EM 545." 


\section{Coal Prices}

\section{Mine Prices}

The average mine price of U.S. coal in 1995 was $\$ 18.83$ per short ton, a drop of 3 percent from 1994 and the 13th straight year of decline (Table 80). Since 1986 the average mine price of U.S. coal has declined at an average annual rate of 2.6 percent. During this period, the average mine price of coal east of the Mississippi River decreased at an annual average rate of 1.2 percent, whereas west of the Mississippi River the decline was 3.3 percent. In real dollars, the decline over this period for the United States was 5.6 percent (Table 81).

Compared with 1994 , the average mine price of coal in both the Interior and Western Regions declined 5.3 percent and 4.0 percent, respectively, while the average price in Appalachia increased 0.3 percent. In the three largest coal-producing States, prices fell slightly in West Virginia and Kentucky, while declining in Wyoming by almost 4 percent.

\section{Consumer Prices}

The average price of coal delivered to electric utilities during 1995 was $\$ 27.01$ per short ton, 3.6 percent less than the average price of $\$ 28.03$ per short ton reported for 1994 (Table 92). Similarly, compared with 1994, the average price of coal delivered to industrial consumers other than coke plants declined 0.4 percent to $\$ 32.42$ per short ton, while the average price of coal delivered to coke plants increased 1.7 percent to $\$ 47.34$ per short ton (Tables 94 and 96 ).

The average price of U.S. coal imports in 1995 was $\$ 34.13$ per short ton, a 13 -percent increase compared with the 1994 average of $\$ 30.21$ per short ton.

The average price of U.S. coal exported during 1995 was $\$ 40.27$ per short ton, 0.8 percent above the average of $\$ 39.93$ per short ton reported for 1994 (Table 99). Compared with 1994, the average price of metallurgical coal exported during 1995 increased 3.6 percent to $\$ 44.30$ per short ton, while the average price of steam coal rose 0.5 percent to $\$ 34.51$ per short ton (Tables 101 and 103). 
Figure 13. Coal Prices, 1986-1995

(Nominal Dollars per Short Ton)

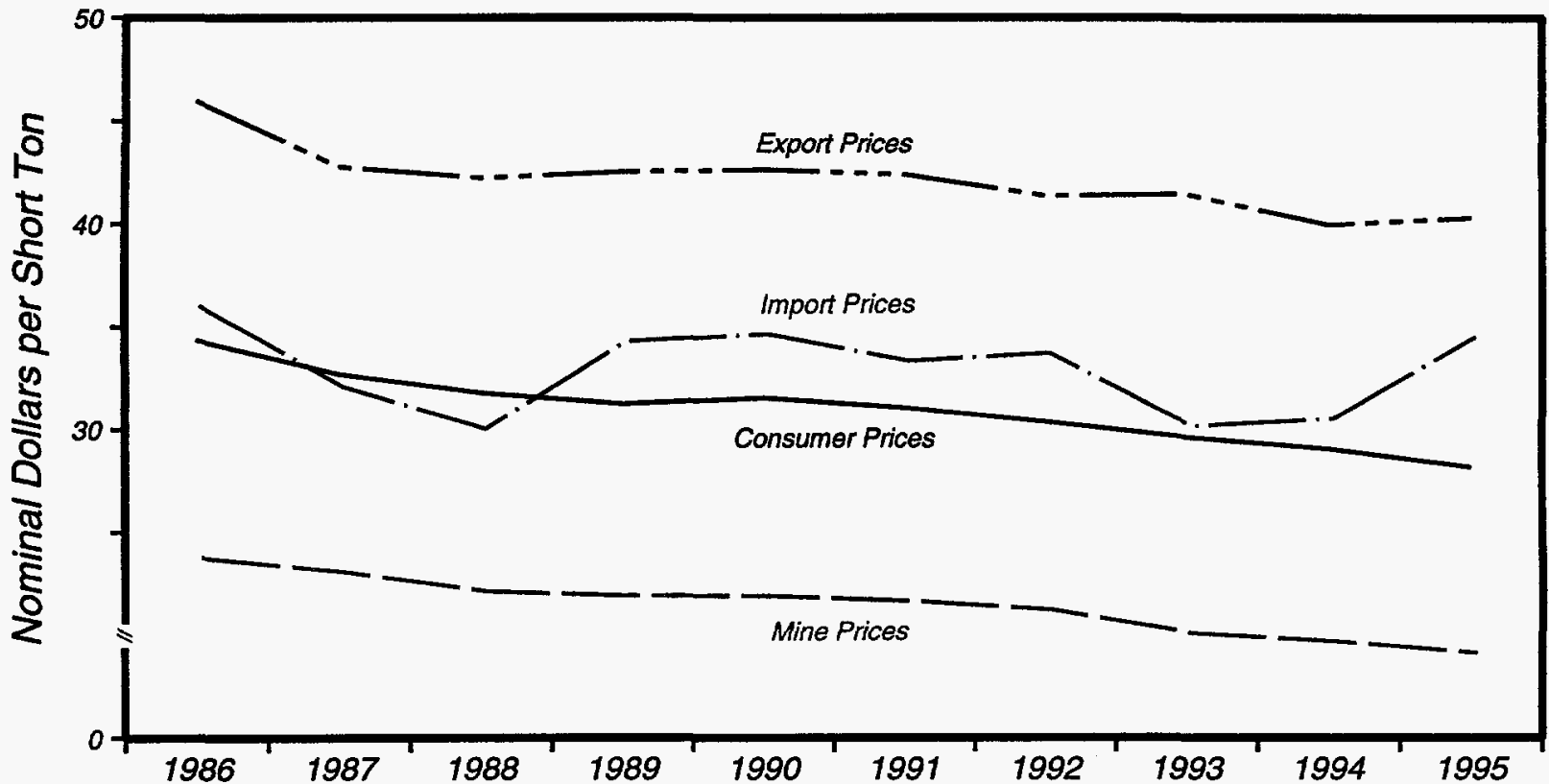

Note: Average mine prices exclude mines producing less than 10,000 short tons of coal during the year. Mine Price is calculated by dividing the total free on board (f.o.b.) mine value of the coal produced by the total production. Consumer Price is based on the cost including insurance and freight (c.i.f.) for electric utilities, and insurance, freight and taxes for manufacturing and coke plants, and does not include the residential and commercial sector. Export Price is based on the free alongside ship (f.a.s.) value. Import Price is based on the customs import value

Sources: -Mine Price: Energy Information Administration (EIA), Form ElA-7A, "Coal Production Report"; State Mining Agency Coal Production Reports; and/or U.S. Department of Labor, Mine Safety and Health Administration, Form 7000-2, "Quarterly Mine Employment and Coal Production Report." •Consumer Prices: Federal Regulatory Commission (FERC), FERC Form 423, "Monthly Report of Cost and Quality of Fuels for Electric Plants"; EIA, Form EIA-5, "Coke Plant Report - Quarterly" and Form ElA-3, "Quarterly Coal Consumption Report - Manufacturing Plants." •Export Prices: U.S. Department of Commerce, Bureau of the Census, "Monthly Report EM545." -Import Prices: U.S. Department of Commerce, Bureau of the Census, "Monthly Report IM 145." 


\section{Mine Prices}

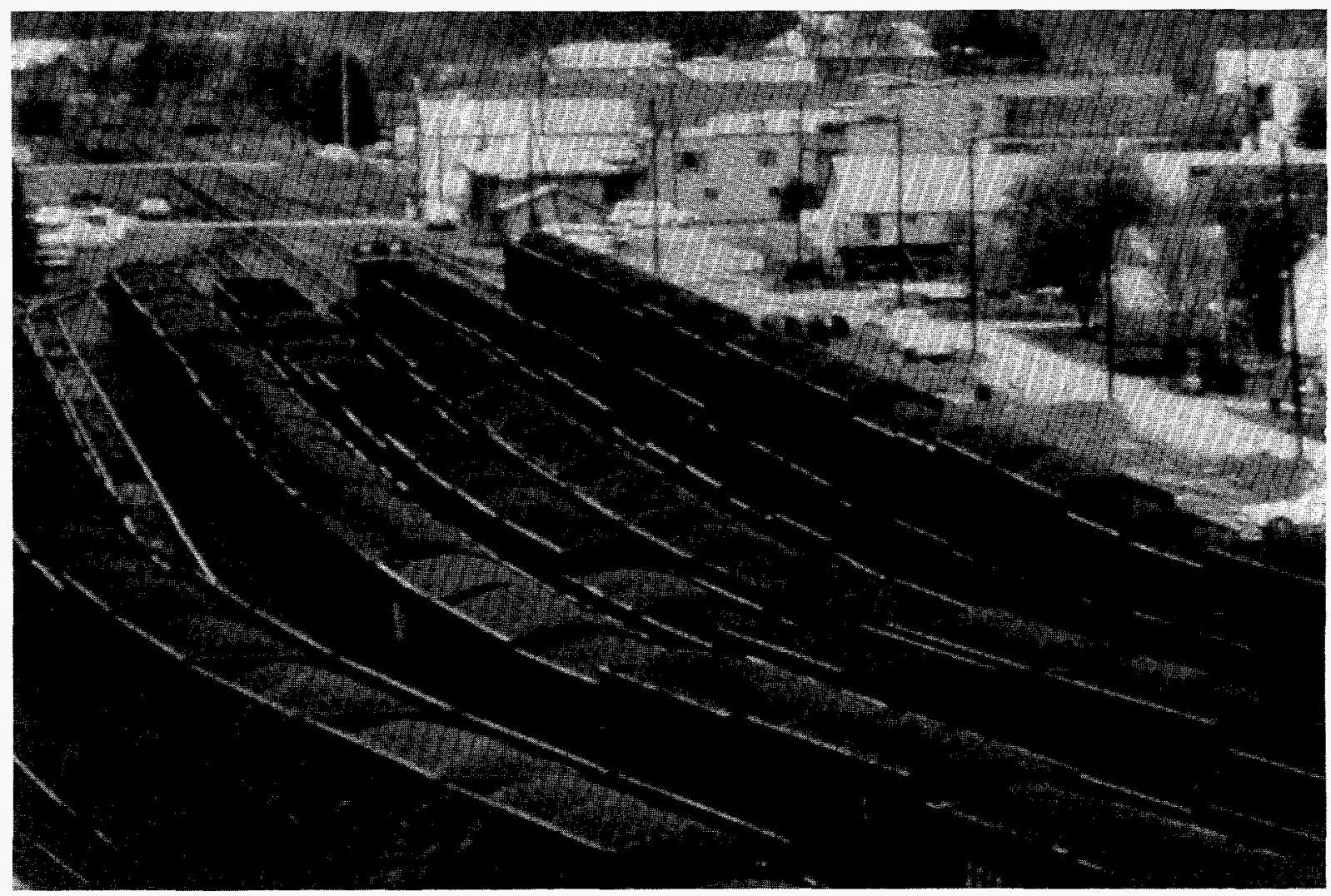

Since 1991, the average mine price of U.S. coal has declined. In 1995, the average U.S. mine price for underground and surface methods totaled $\$ 26.18$ and $\$ 14.25$, respectively. 
Table 80. Average Mine Price of Coal by State, 1986, 1991-1995

(Nominal Dollars per Short Ton)

\begin{tabular}{|c|c|c|c|c|c|c|c|c|c|}
\hline \multirow{2}{*}{$\begin{array}{l}\text { Coal-Producing } \\
\text { State and Region }\end{array}$} & \multirow{2}{*}{1995} & \multirow{2}{*}{1994} & \multirow{2}{*}{1993} & \multirow{2}{*}{1992} & \multirow{2}{*}{1991} & \multirow{2}{*}{1986} & \multirow{2}{*}{$\begin{array}{c}\text { Percent } \\
\text { Change } \\
\text { 1994-1995 }\end{array}$} & \multicolumn{2}{|c|}{ Average Annual Percent Change } \\
\hline & & & & & & & & 1991-1995 & 1986-1995 \\
\hline 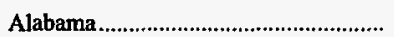 & $\$ 38.44$ & $\$ 40.12$ & $\$ 42.34$ & $\$ 40.82$ & $\$ 41.14$ & $\$ 41.27$ & -4.2 & -1.7 & -0.8 \\
\hline 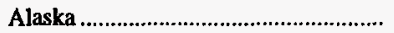 & w & w & w & w & w & 25.70 & w & $\mathbf{w}$ & w \\
\hline Arizona & $\mathbf{w}$ & $\mathbf{w}$ & $\mathbf{w}$ & $\mathbf{w}$ & $\mathbf{w}$ & 16.50 & w & $\mathbf{w}$ & $\mathrm{w}$ \\
\hline 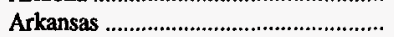 & w & $w$ & $\mathbf{w}$ & $w$ & $\mathbf{w}$ & 15.03 & $w$ & $\mathrm{w}$ & w \\
\hline 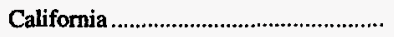 & - & - & - & $\mathbf{w}$ & $\mathbf{w}$ & - & w & w & w \\
\hline Colorado & 19.26 & 19.76 & 20.35 & 21.33 & 22.18 & 23.44 & -2.5 & -3.5 & -2.2 \\
\hline 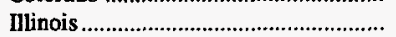 & 23.05 & 23.14 & 25.27 & 27.66 & 28.35 & 29.99 & -.3 & -5.0 & -2.9 \\
\hline Indiana & 21.71 & 22.28 & 22.89 & 23.41 & 23.58 & 25.37 & -2.5 & -2.0 & -1.7 \\
\hline 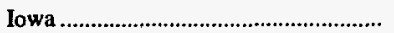 & - & $\mathbf{w}$ & $\mathbf{w}$ & $w$ & $\mathbf{w}$ & 27.26 & w & w & w \\
\hline 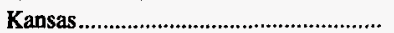 & w & w & $\mathbf{w}$ & $\mathbf{w}$ & w & 25.65 & $\mathbf{w}$ & w & w \\
\hline 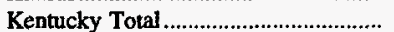 & 24.79 & 24.88 & 24.77 & 24.50 & 25.45 & 26.09 & -.3 & -.6 & -.6 \\
\hline Eastern & 26.00 & 25.25 & 25.50 & 25.00 & 26.37 & 26.38 & 2.9 & -.3 & -.2 \\
\hline Western & 20.75 & 23.63 & 22.36 & 23.10 & 22.88 & 25.31 & -12.2 & -2.4 & -2.2 \\
\hline 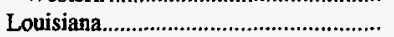 & w & $w$ & w & $\mathbf{w}$ & w & 16.70 & $w$ & w & $\mathbf{w}$ \\
\hline Maryland & 24.69 & 26.34 & 25.21 & 25.39 & 25.73 & 25.96 & -6.3 & -1.0 & -5 \\
\hline 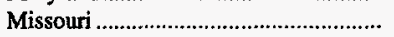 & 18.91 & 21.78 & w & w & $w$ & 29.67 & -13.2 & $\begin{array}{r}-1.0 \\
w\end{array}$ & -4.9 \\
\hline 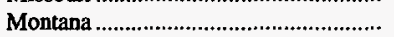 & 9.62 & 10.39 & 11.05 & 10.20 & 10.76 & 12.93 & -7.4 & -2.7 & -3.2 \\
\hline 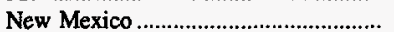 & 23.80 & 23.29 & 22.96 & 23.14 & 23.25 & 22.26 & 2.2 & .6 & .7 \\
\hline North Dakota & 7.99 & 7.62 & 7.63 & 7.48 & 7.84 & 8.49 & 4.9 & .5 & -.7 \\
\hline Ohio & 25.97 & 29.13 & 28.04 & 26.93 & 27.75 & 32.80 & -10.8 & -1.6 & -2.6 \\
\hline Oklahoma & 24.13 & 25.57 & 24.91 & 25.76 & 28.52 & 28.23 & -5.6 & -4.1 & -1.7 \\
\hline Pennsylvania Total & 26.78 & 26.18 & 26.50 & 28.61 & 29.40 & 30.30 & 2.3 & -2.3 & -1.4 \\
\hline 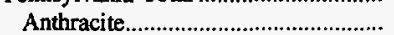 & 39.78 & 36.07 & 32.94 & 34.24 & 36.34 & 44.12 & 10.3 & 2.3 & -1.1 \\
\hline Bituminous & 25.77 & 25.45 & 26.03 & 28.34 & 29.06 & 29.50 & 1.3 & -2.9 & -1.5 \\
\hline Tennessee & 26.94 & 27.17 & 27.23 & 27.11 & 26.74 & 28.00 & -.8 & .2 & -.4 \\
\hline Texas & 12.16 & 12.38 & 12.87 & 12.42 & 12.21 & 11.60 & -1.8 & -.1 & .5 \\
\hline 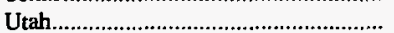 & 19.10 & 19.27 & 20.81 & 21.11 & 22.59 & 27.64 & -.9 & -4.1 & -4.0 \\
\hline Virginia & 28.47 & 26.84 & 26.80 & 27.55 & 27.45 & 28.13 & 6.1 & $\begin{array}{r}7 \\
.9\end{array}$ & .1 \\
\hline 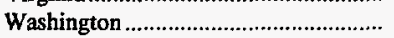 & $\mathbf{w}$ & w & $w$ & $\mathbf{w}$ & w & 25.73 & w & w & w \\
\hline West Virginia Total & 27.18 & 27.42 & 27.58 & 28.15 & 28.62 & 30.90 & -.9 & -1.3 & -1.4 \\
\hline Northern & 24.91 & 26.77 & 28.09 & 29.03 & 30.16 & 29.95 & -6.9 & -4.7 & -2.0 \\
\hline Southern & 28.07 & 27.71 & 27.40 & 27.76 & 27.93 & 31.47 & 1.3 & .1 & -1.3 \\
\hline Wyoming & 6.58 & 6.83 & 7.32 & 8.14 & 8.09 & 10.85 & -3.7 & -5.0 & -5.4 \\
\hline 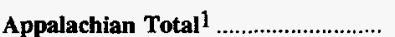 & 27.45 & 27.36 & 27.64 & 27.95 & 28.69 & 30.05 & .3 & -1.1 & -1.0 \\
\hline Interior Total 1 & 18.81 & 19.87 & 20.03 & 21.61 & 21.86 & 23.45 & -5.3 & -3.7 & -2.4 \\
\hline 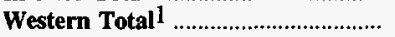 & 10.15 & 10.57 & 11.14 & 11.60 & 11.71 & 14.03 & -4.0 & $-\mathbf{3 . 5}$ & -3.5 \\
\hline East of Miss. River .............................. & 26.35 & 26.44 & 26.81 & 27.34 & 27.97 & 29.42 & -.4 & -1.5 & -1.2 \\
\hline West of Miss. River & 10.48 & 10.91 & 11.50 & 11.98 & 12.06 & 14.12 & -3.9 & -3.4 & $-\mathbf{3 . 3}$ \\
\hline 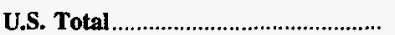 & 18.83 & 19.41 & 19.85 & 21.03 & 21.49 & 23.79 & -3.0 & -3.3 & -2.6 \\
\hline
\end{tabular}

1 For a definition of coal-producing regions, see Appendix C.

Withheld to avoid disclosure of individual company data.

Notes: Average mine price is calculated by dividing the total free on board (f.o.b.) mine value of the coal produced by the total production. A measure of dispersion of these average prices at the State level (interquartile range) is given in Appendix D, Table D2. Excludes silt, culm, refuse bank, slurry dam, and dredge operations except for Pennsylvania anthracite. Excludes mines producing less than 10,000 short tons, which are not required to provide these data.

Sources: Energy Information Administration, Form EIA-7A, “Coal Production Report”; State Mining Agency Coal Production Reports; and/or U.S. Department of Labor, Mine Safety and Health Administration, Form 7000-2, "Quarterly Mine Employment and Coal Production Report." 
Table 81. Average Real Mine Price of Coal by State, 1986, 1991-1995 (Real Dollars per Short Ton)

\begin{tabular}{|c|c|c|c|c|c|c|c|c|c|}
\hline \multirow{2}{*}{$\begin{array}{l}\text { Coal-Producing } \\
\text { State and Region }\end{array}$} & \multirow{2}{*}{1995} & \multirow{2}{*}{1994} & \multirow{2}{*}{1993} & \multirow{2}{*}{1992} & \multirow{2}{*}{1991} & \multirow{2}{*}{1986} & \multirow{2}{*}{$\begin{array}{c}\text { Percent } \\
\text { Change } \\
\text { 1994-1995 }\end{array}$} & \multicolumn{2}{|c|}{ Average Annual Percent Change } \\
\hline & & & & & & & & 1991-1995 & 1986-1995 \\
\hline 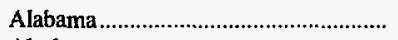 & $\$ 35.76$ & $\$ 38.21$ & $\$ 41.27$ & $\$ 40.82$ & $\$ 42.28$ & $\$ 51.20$ & -6.4 & -4.1 & -3.9 \\
\hline 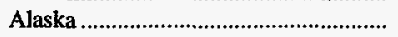 & w & w & w & w & $\mathbf{w}$ & 31.89 & w & w & $w$ \\
\hline 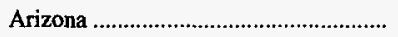 & w & w & w & w & $w$ & 20.48 & $\mathbf{w}$ & $\mathbf{w}$ & $\mathbf{w}$ \\
\hline 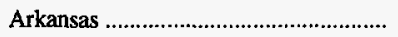 & w & w & w & w & $\mathbf{w}$ & 18.65 & $\mathbf{w}$ & $w$ & w \\
\hline California & - & - & - & $\mathbf{w}$ & $w$ & - & $w$ & $\mathbf{w}$ & $w$ \\
\hline Colorado & 17.91 & 18.82 & 19.84 & 21.33 & 22.80 & 29.08 & -4.8 & -5.8 & -5.2 \\
\hline Illinois & 21.45 & 22.03 & 24.63 & 27.66 & 29.14 & 37.21 & -2.7 & -7.4 & -5.9 \\
\hline Indiana & 20.20 & 21.22 & 22.31 & 23.41 & 24.24 & 31.47 & -4.8 & -4.4 & -4.8 \\
\hline Iowa & - & w & w & $\mathbf{w}$ & w & 33.82 & w & $w$ & w \\
\hline 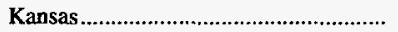 & w & w & w & $\mathbf{w}$ & $\mathbf{w}$ & 31.82 & w & $w$ & $\mathbf{w}$ \\
\hline 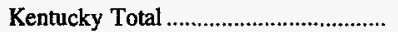 & 23.06 & 23.69 & 24.15 & 24.50 & 26.16 & 32.38 & -2.6 & -3.1 & -3.7 \\
\hline Eastern & 24.18 & 24.05 & 24.86 & 25.00 & 27.10 & 32.74 & .5 & -2.8 & -3.3 \\
\hline Western & 19.31 & 22.51 & 21.79 & 23.10 & 23.52 & 31.40 & -14.2 & -4.8 & -5.3 \\
\hline 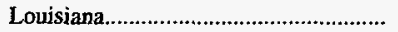 & w & $w$ & $\mathbf{w}$ & $\mathbf{w}$ & $\mathbf{w}$ & 20.72 & w & w & $w$ \\
\hline 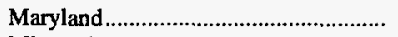 & 22.97 & 25.09 & 24.57 & 25.39 & 26.44 & 32.21 & -8.4 & -3.4 & -3.7 \\
\hline 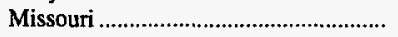 & 17.59 & 20.74 & w & $w$ & w & 36.81 & -15.2 & w & -7.9 \\
\hline Montana & 8.95 & 9.90 & 10.77 & 10.20 & 11.05 & 16.04 & -9.5 & -5.1 & -6.3 \\
\hline New Mexico & 22.14 & 22.18 & 22.38 & 23.14 & 23.90 & 27.61 & -.2 & -1.9 & -2.4 \\
\hline 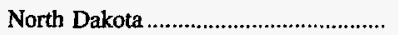 & 7.44 & 7.26 & 7.44 & 7.48 & 8.05 & 10.53 & 2.4 & -2.0 & -3.8 \\
\hline Ohio & 24.15 & 27.74 & 27.33 & 26.93 & 28.52 & 40.69 & -12.9 & -4.1 & -5.6 \\
\hline Oklahoma & 22.45 & 24.35 & 24.28 & 25.76 & 29.31 & 35.03 & -7.8 & -6.4 & -4.8 \\
\hline Pennsylvania Total ....................................... & 24.91 & 24.94 & 25.83 & 28.61 & 30.21 & 37.59 & -.1 & -4.7 & -4.5 \\
\hline Anthracite. & 37.01 & 34.35 & 32.11 & 34.24 & 37.35 & 54.74 & 7.7 & -.2 & -4.3 \\
\hline 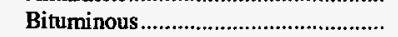 & 23.97 & 24.23 & 25.37 & 28.34 & 29.86 & 36.61 & -1.1 & -5.3 & -4.6 \\
\hline 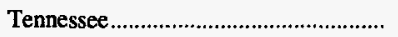 & 25.06 & 25.87 & 26.54 & 27.11 & 27.48 & 34.74 & -3.1 & -2.3 & -3.6 \\
\hline 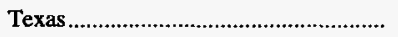 & 11.31 & 11.79 & 12.54 & 12.42 & 12.55 & 14.40 & -4.1 & -2.5 & -2.6 \\
\hline 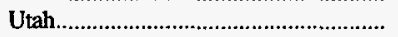 & 17.77 & 18.35 & 20.28 & 21.11 & 23.22 & 34.29 & -3.2 & -6.5 & -7.0 \\
\hline 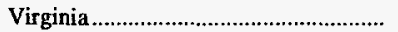 & 26.48 & 25.56 & 26.13 & 27.55 & 28.21 & 34.90 & 3.6 & -1.6 & -3.0 \\
\hline 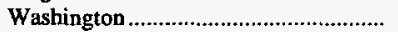 & $\mathbf{w}$ & $\mathbf{w}$ & $\mathbf{w}$ & $\mathbf{w}$ & w & 31.92 & w & w & w \\
\hline West Virginia Total................................. & 25.28 & 26.12 & 26.88 & 28.15 & 29.42 & 38.34 & -3.2 & -3.7 & -4.5 \\
\hline Northern & 23.18 & 25.50 & 27.38 & 29.03 & 31.00 & 37.15 & -9.1 & -7.0 & -5.1 \\
\hline 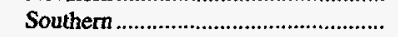 & 26.11 & 26.39 & 26.70 & 27.76 & 28.70 & 39.04 & -1.0 & -2.3 & -4.4 \\
\hline Wyoming & 6.12 & 6.50 & 7.13 & 8.14 & 8.32 & 13.47 & -5.9 & -7.4 & -8.4 \\
\hline Appalachian Total ${ }^{1}$ & 25.53 & 26.06 & 26.94 & 27.95 & 29.49 & 37.28 & -2.0 & -3.5 & -4.1 \\
\hline Interior Total 1 & 17.50 & 18.92 & 19.52 & 21.61 & 22.47 & 29.10 & -7.5 & -6.1 & -5.5 \\
\hline 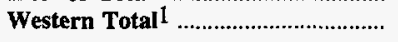 & 9.44 & 10.07 & 10.86 & 11.60 & 12.04 & 17.41 & -6.2 & -5.9 & -6.6 \\
\hline East of Miss. River. & 24.51 & 25.18 & 26.13 & 27.34 & 28.75 & 36.50 & -2.7 & -3.9 & -4.3 \\
\hline West of Miss. River............................... & 9.75 & 10.39 & 11.21 & 11.98 & 12.39 & 17.52 & -6.2 & -5.8 & -6.3 \\
\hline U.S. Total & 17.52 & 18.49 & 19.35 & 21.03 & 22.09 & 29.52 & -5.2 & -5.6 & -5.6 \\
\hline
\end{tabular}

1 For a definition of coal-producing regions, see Appendix $\mathrm{C}$.

withheld to avoid disclosure of individual company data.

Notes: Real prices are in 1992 dollars, calculated using implicit Gross Domestic Product price deflators. See Appendix D, Table D3. Average mine price is calculated by dividing the total free on board (f.o.b.) mine value of the coal produced by the total production. Excludes silt, culm, refuse bank, slurry dam, and dredge operations except for Pennsylvania anthracite. Excludes mines producing less than 10,000 short tons, which are not required to provide these data.

Sources: Energy Information Administration, Form EIA-7A, "Coal Production Report"; State Mining Agency Coal Production Reports; and/or U.S. Department of Labor, Mine Safety and Health Administration, Form 7000-2, "Quarterly Mine Employment and Coal Production Report." 
Table 82. Average Mine Price of Coal by State and Mine Type, 1995 (Dollars per Short Ton)

\begin{tabular}{|c|c|c|c|c|c|c|}
\hline \multirow{2}{*}{$\begin{array}{l}\text { Coal-Producing } \\
\text { State and Region }\end{array}$} & \multicolumn{2}{|c|}{ Underground } & \multicolumn{2}{|c|}{ Surface } & \multicolumn{2}{|c|}{ Total } \\
\hline & Nominal & Real ${ }^{1}$ & Nominal & Real 1 & Nominal & Real ${ }^{1}$ \\
\hline Alabama & $\$ 39.26$ & $\$ 36.52$ & $\$ 36.38$ & $\$ 33.84$ & $\$ 38.44$ & $\$ 35.76$ \\
\hline Alaska & - & - & $\mathbf{w}$ & w & $w$ & w \\
\hline Arizona & - & - & $\mathbf{w}$ & $w$ & $\mathbf{w}$ & w \\
\hline 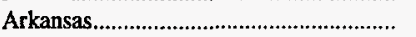 & - & - & $\mathbf{w}$ & $\mathbf{w}$ & $\mathbf{w}$ & w \\
\hline 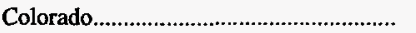 & 18.58 & 17.28 & 20.63 & 19.19 & 19.26 & 17.91 \\
\hline Illinois & 22.88 & 21.29 & 24.04 & 22.36 & 23.05 & 21.45 \\
\hline Indiana & w & w & $\mathbf{w}$ & $\mathbf{w}$ & 21.71 & 20.20 \\
\hline 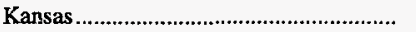 & - & - & $\mathbf{w}$ & $\mathbf{w}$ & $\mathbf{w}$ & w \\
\hline 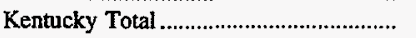 & 25.18 & 23.42 & 24.19 & 22.50 & 24.79 & 23.06 \\
\hline 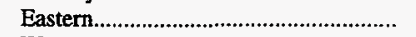 & 26.52 & 24.67 & 25.24 & 23.48 & 26.00 & 24.18 \\
\hline 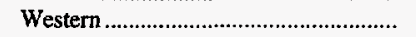 & 21.33 & 19.84 & 19.46 & 18.10 & 20.75 & 19.31 \\
\hline 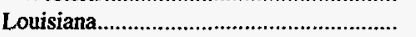 & - & - & w & $w$ & w & w \\
\hline Maryland & w & $w$ & $\mathbf{w}$ & $\mathbf{w}$ & 24.69 & 22.97 \\
\hline 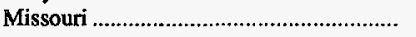 & - & - & 18.91 & 17.59 & 18.91 & 17.59 \\
\hline 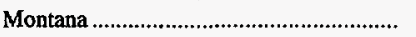 & - & - & 9.62 & 8.95 & 9.62 & 8.95 \\
\hline 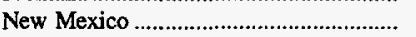 & w & $\mathbf{w}$ & $\mathbf{w}$ & $\mathbf{w}$ & 23.80 & 22.14 \\
\hline 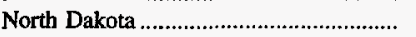 & - & - & 7.99 & 7.44 & 7.99 & 7.44 \\
\hline Ohio & 28.98 & 26.96 & 22.92 & 21.32 & 25.97 & 24.15 \\
\hline 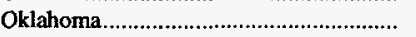 & w & $w$ & $w$ & $w$ & 24.13 & 22.45 \\
\hline 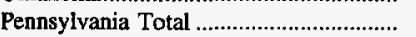 & 27.09 & 25.20 & 26.14 & 24.31 & 26.78 & 24.91 \\
\hline Anthracite & 36.91 & 34.33 & 39.97 & 37.18 & 39.78 & 37.01 \\
\hline 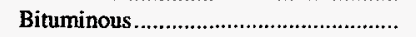 & 27.02 & 25.14 & 22.45 & 20.88 & 25.77 & 23.97 \\
\hline 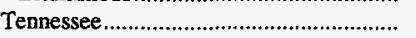 & w & $w$ & $w$ & $w$ & 26.94 & 25.06 \\
\hline Texas & - & - & 12.16 & 11.31 & 12.16 & 11.31 \\
\hline 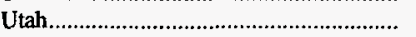 & 19.10 & 17.77 & - & - & 19.10 & 17.77 \\
\hline 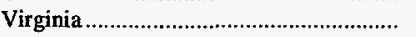 & 29.20 & 27.16 & 26.34 & 24.51 & 28.47 & 26.48 \\
\hline Washington & - & - & w & w & $w$ & $\mathbf{w}$ \\
\hline 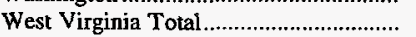 & 27.77 & 25.83 & 25.95 & 24.14 & 27.18 & 25.28 \\
\hline Northern & 25.17 & 23.41 & 22.97 & 21.37 & 24.91 & 23.18 \\
\hline Southern & 29.30 & 27.25 & 26.29 & 24.45 & 28.07 & 26.11 \\
\hline Wyoming & w & $w$ & $\mathbf{w}$ & $w$ & 6.58 & 6.12 \\
\hline Appalachian Total 2 ............................ & 28.24 & 26.27 & 25.97 & 24.16 & 27.45 & 25.53 \\
\hline Interior Total ${ }^{2}$ & 22.57 & 21.00 & 16.19 & 15.06 & 18.81 & 17.50 \\
\hline Western Total 2 & 18.85 & $\mathbf{1 7 . 5 3}$ & 9.13 & 8.49 & 10.15 & 9.44 \\
\hline East of Miss. River ................................. & 27.12 & 25.23 & 24.93 & 23.19 & 26.35 & 24.51 \\
\hline West of Miss. River .................................... & 18.85 & 17.53 & 9.63 & 8.96 & 10.48 & 9.75 \\
\hline U.S. Total & 26.18 & 24.36 & 14.25 & 13.26 & 18.83 & 17.52 \\
\hline
\end{tabular}

1 Real prices are in 1992 dollars, calculated using implicit Gross Domestic Product price deflators. See Appendix D, Table D3.

2 For a definition of coal-producing regions, see Appendix C.

withheld to avoid disclosure of individual company data.

Notes: Average mine price is calculated by dividing the total free on board (f.o.b.) mine value of the coal produced by the total production. A measure of dispersion of these average nominal prices at the State level (interquartile range) is given in Appendix D, Table D2. Excludes silt, culm, refuse bank, slurry dam, and dredge operations except for Pennsylvania anthracite. Excludes mines producing less than 10,000 short tons, which are not required to provide these data.

Sources: Energy Information Administration, Form ElA-7A, "Coal Production Report"; State Mining Agency Coal Production Reports; and/or U.S. Department of Labor, Mine Safety and Health Administration, Form 7000-2, "Quarterly Mine Employment and Coal Production Report." 
Table 83. Average Mine Price of Coal by State and Underground Mining Method, 1995 (Nominal Dollars per Short Ton)

\begin{tabular}{|c|c|c|c|c|c|}
\hline $\begin{array}{l}\text { Coal-Producing } \\
\text { State and Region }\end{array}$ & Continuous 1 & Conventional 2 & Longwall 3 & Other 4 & Total \\
\hline Alabama & $\$ 37.44$ & - & $\$ 39.43$ & - & $\$ 39.26$ \\
\hline Colorado & $\mathrm{w}$ & $\mathbf{w}$ & 18.91 & - & 18.58 \\
\hline 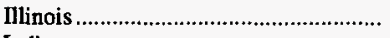 & $\$ 23.13$ & - & 22.56 & - & 22.88 \\
\hline Indiana & w & - & - & - & $w$ \\
\hline 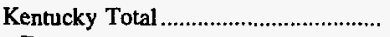 & 25.37 & $\$ 25.61$ & 24.21 & $\$ 20.19$ & 25.18 \\
\hline Eastern & 26.93 & $\mathbf{w}$ & w & 20.19 & 26.52 \\
\hline 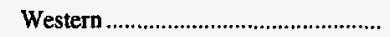 & 20.83 & w & $\mathrm{w}$ & - & 21.33 \\
\hline Maryland & w & - & $w$ & - & w \\
\hline 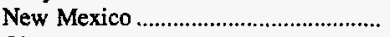 & - & - & $\mathrm{w}$ & - & w \\
\hline Ohio & 21.34 & - & $\$ 30.12$ & - & 28.98 \\
\hline Oklahoma & w & - & - & - & w \\
\hline 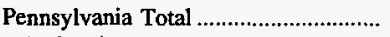 & $\mathbf{w}$ & $\$ 29.25$ & $w$ & $w$ & 27.09 \\
\hline 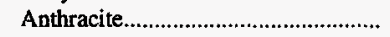 & w & 35.25 & - & $w$ & 36.91 \\
\hline 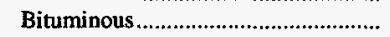 & $\mathbf{w}$ & 27.93 & $w$ & - & 27.02 \\
\hline Tennessee. & $\mathbf{w}$ & - & - & - & w \\
\hline 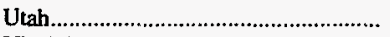 & w & $\mathbf{w}$ & $\$ 19.35$ & - & 19.10 \\
\hline 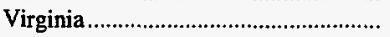 & $\$ 28.06$ & $\mathbf{w}$ & 31.41 & $\mathbf{w}$ & 29.20 \\
\hline 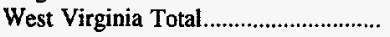 & 28.24 & $\$ 27.31$ & 27.43 & - & 27.77 \\
\hline 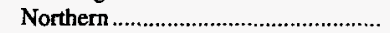 & 21.11 & 20.20 & 26.28 & - & 25.17 \\
\hline 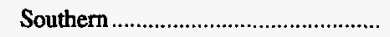 & 29.10 & 29.33 & 29.81 & - & 29.30 \\
\hline Wyoming & - & w & w & - & w \\
\hline Appalachian Total ${ }^{5}$ & 27.98 & $\mathbf{w}$ & $\mathbf{w}$ & 21.35 & 28.24 \\
\hline Interior Total 5 & 22.62 & $\mathbf{w}$ & $\mathbf{w}$ & - & $\mathbf{2 2 . 5 7}$ \\
\hline 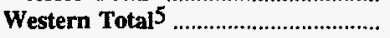 & 16.42 & 17.98 & 19.12 & - & 18.85 \\
\hline 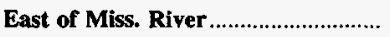 & 26.60 & 26.86 & 27.80 & 21.35 & 27.12 \\
\hline 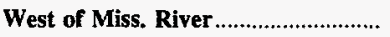 & 16.45 & 17.98 & 19.12 & - & 18.85 \\
\hline U.S. Total & 26.35 & 26.66 & 25.96 & 21.35 & 26.18 \\
\hline
\end{tabular}

1 Mines that produce greater than 50 percent of coal by continuous mining method.

2 Mines that produce greater than 50 percent of coal by conventional mining method.

3 Mines that have any production from longwall mining method. A typical longwall mining operation uses 80 percent longwall mining and 20 percent continuous mining.

4 Mines that produce coal using shortwall, scoop loading, hand loading, or other mining methods or a $50 / 50$ percent continuous/conventional split in mining method.

5 For a definition of coal-producing regions, see Appendix C.

w Witheld to avoid disclosure of individual company data.

Notes: Average mine price is calculated by dividing the total free on board (f.o.b.) mine value of the coal produced by the total production. Excludes mines producing less than 10,000 short tons, which are not required to provide these data.

Sources: Energy Information Administration, Form EIA-7A, "Coal Production Report"; State Mining Agency Coal Production Reports; and/or U.S. Department of Labor, Mine Safety and Health Administration, Form 7000-2, "Quarterly Mine Employment and Coal Production Report." 
Table 84. Coal Production, Number of Mines, and Average Mine Price, by State and County, 1995

(Thousand Short Tons, Nominal Dollars per Short Ton)

\begin{tabular}{|c|c|c|c|}
\hline $\begin{array}{l}\text { Coal-Producing } \\
\text { State and County }\end{array}$ & Number of Mines & Production & Average Mine Price \\
\hline Alabama & 73 & 24,640 & 38.44 \\
\hline 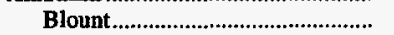 & 2 & 20 & w \\
\hline 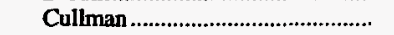 & 3 & 90 & $\mathrm{w}$ \\
\hline Fayette & 1 & 1,984 & $\mathrm{w}$ \\
\hline 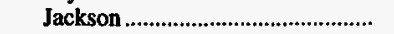 & 1 & 46 & w \\
\hline 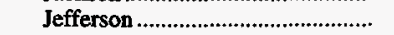 & 10 & 8,225 & $\$ 41.77$ \\
\hline 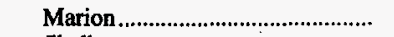 & 6 & 214 & 30.13 \\
\hline 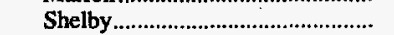 & 1 & 857 & w \\
\hline Tuscaloosa & 10 & 8,265 & w \\
\hline 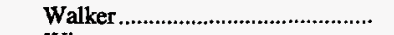 & 35 & 4,631 & 36.76 \\
\hline 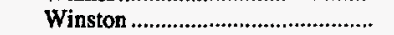 & 4 & 310 & w \\
\hline 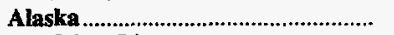 & 1 & 1,698 & $\mathbf{w}$ \\
\hline 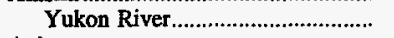 & 1 & 1,698 & w \\
\hline Arizona & 2 & 11,947 & $\mathbf{w}$ \\
\hline Navajo & 2 & 11,947 & $\mathrm{w}$ \\
\hline 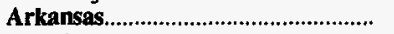 & 3 & 29 & $\mathbf{w}$ \\
\hline Johnson ................................................ & 2 & 24 & w \\
\hline Sebastian ………………………........ & 1 & 5 & - \\
\hline Colorado & 17 & 25,710 & 19.26 \\
\hline Delta & 1 & 405 & w \\
\hline 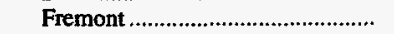 & 1 & 302 & w \\
\hline 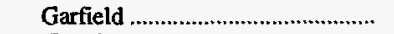 & 1 & * & - \\
\hline 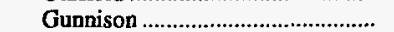 & 3 & 6,870 & w \\
\hline La Plata & 1 & 209 & w \\
\hline 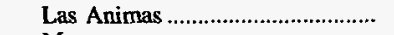 & 1 & 1,156 & w \\
\hline 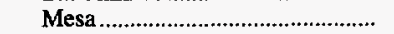 & 1 & 607 & $\mathrm{w}$ \\
\hline 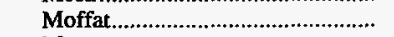 & 3 & 7,133 & w \\
\hline 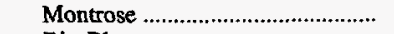 & 1 & 374 & w \\
\hline 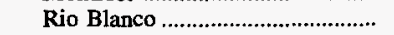 & 1 & 1,047 & w \\
\hline Routt & 3 & 7,608 & w \\
\hline 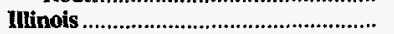 & 31 & 48,180 & 23.05 \\
\hline 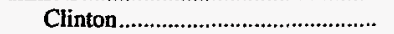 & 1 & 2,998 & w \\
\hline 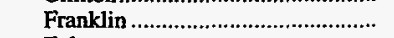 & 2 & 5,298 & $w$ \\
\hline Fulton & 1 & 469 & w \\
\hline 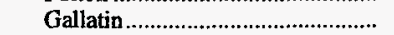 & 1 & 1,086 & w \\
\hline 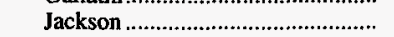 & 1 & 19 & $\mathrm{w}$ \\
\hline Jefferson ....................................... & 2 & 4,705 & $\mathbf{w}$ \\
\hline 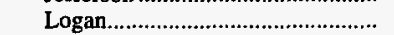 & 1 & 1,745 & w \\
\hline Macoupin & 3 & 4,815 & $\mathbf{w}$ \\
\hline McDonough & 1 & 278 & w \\
\hline Perry & 6 & 6,647 & w \\
\hline 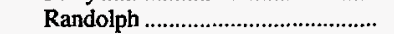 & 2 & 2,891 & $\mathbf{w}$ \\
\hline 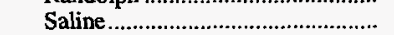 & 4 & 7,809 & $\$ 23.49$ \\
\hline 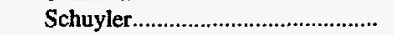 & 1 & 504 & $\mathbf{w}$ \\
\hline 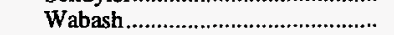 & 1 & 2,557 & w \\
\hline 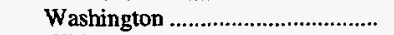 & 1 & 3,259 & $\mathbf{w}$ \\
\hline White & 1 & 1,808 & $\mathbf{w}$ \\
\hline 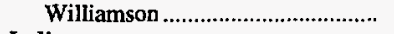 & 2 & 1,293 & w \\
\hline Indiana & 42 & 26,007 & 21.71 \\
\hline 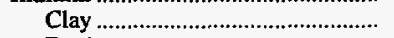 & 5 & 1,178 & $\$ 22.47$ \\
\hline 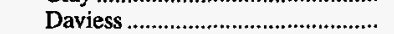 & 6 & 3,341 & 19.02 \\
\hline 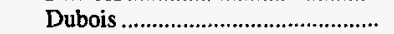 & 1 & 458 & w \\
\hline 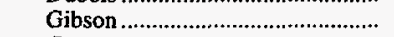 & 2 & 1,550 & w \\
\hline 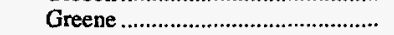 & 5 & 2,741 & 20.68 \\
\hline 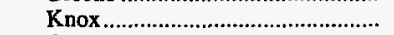 & 4 & 2,828 & w \\
\hline Owen & 1 & 354 & w \\
\hline Pike & 5 & 2,492 & 17.76 \\
\hline 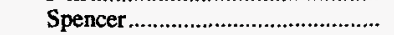 & 2 & 196 & w \\
\hline Sullivan & 3 & 3,823 & $\mathrm{w}$ \\
\hline Vigo & 1 & 1,204 & $\mathrm{w}$ \\
\hline 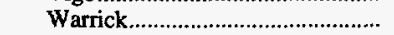 & 7 & 5,841 & $w$ \\
\hline 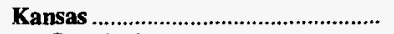 & $\mathbf{1}$ & 285 & w \\
\hline 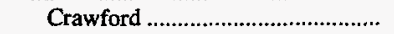 & 1 & 285 & $\mathbf{w}$ \\
\hline Kentucky & 598 & 153,739 & 24.79 \\
\hline 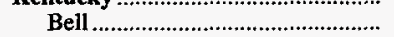 & 24 & 4,977 & $\$ 26.50$ \\
\hline 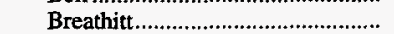 & 8 & 2,505 & 22.16 \\
\hline 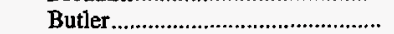 & 1 & 170 & w \\
\hline 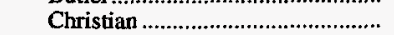 & 1 & 3 & - \\
\hline 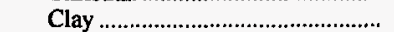 & 8 & 257 & w \\
\hline 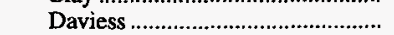 & 6 & 742 & w \\
\hline Floyd & 52 & 6,451 & 23.09 \\
\hline
\end{tabular}

See footnotes at end of table. 
Table 84. Coal Production, Number of Mines, and Average Mine Price, by State and County, 1995 (Continued)

(Thousand Short Tons, Nominal Dollars per Short Ton)

\begin{tabular}{|c|c|c|c|}
\hline $\begin{array}{l}\text { Coal-Producing } \\
\text { State and County }\end{array}$ & Number of Mines & Production & Average Mine Price \\
\hline \multicolumn{4}{|l|}{ Kentucky (Continued) } \\
\hline 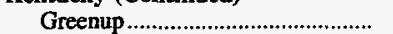 & 1 & 1 & - \\
\hline 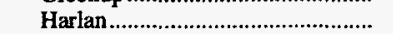 & 56 & 10,926 & $\$ 29.89$ \\
\hline Henderson & 6 & 2,702 & w \\
\hline 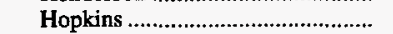 & 14 & 8,466 & 21.13 \\
\hline 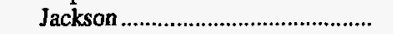 & 1 & 31 & $w$ \\
\hline 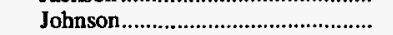 & 12 & 1,258 & 23.42 \\
\hline 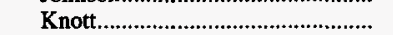 & 48 & 11,994 & 25.92 \\
\hline 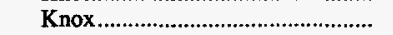 & 22 & 725 & 27.85 \\
\hline 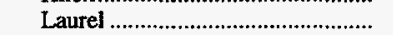 & 1 & 11 & w \\
\hline 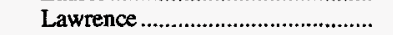 & 3 & 242 & $\mathrm{w}$ \\
\hline 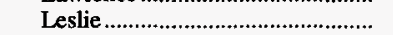 & 12 & 8,752 & 24.33 \\
\hline 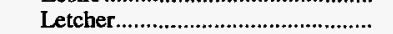 & 42 & 8,159 & 25.56 \\
\hline 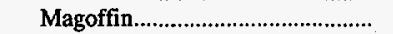 & 5 & 1,005 & $\mathrm{w}$ \\
\hline 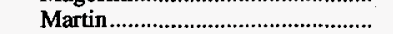 & 34 & 12,108 & 29.72 \\
\hline McLean ……………………............. & 2 & 448 & w \\
\hline 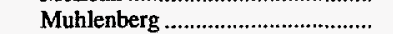 & 5 & 2,983 & 15.98 \\
\hline 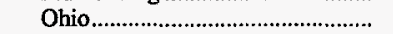 & 12 & 2,763 & 17.62 \\
\hline 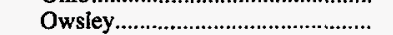 & 2 & 161 & $\mathbf{w}$ \\
\hline 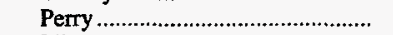 & 29 & 12,105 & 25.44 \\
\hline 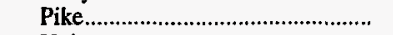 & 161 & 35,525 & 25.00 \\
\hline 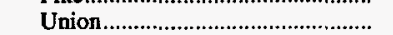 & 4 & 6,398 & $\mathbf{w}$ \\
\hline 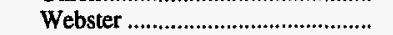 & 7 & 10,524 & 22.55 \\
\hline 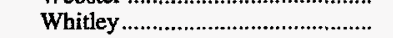 & 18 & 1,337 & 33.04 \\
\hline 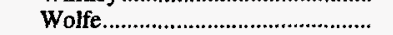 & 1 & 12 & w \\
\hline 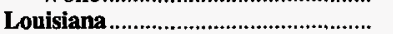 & 2 & 3,719 & $\mathbf{w}$ \\
\hline De Soto & 1 & 2,881 & w \\
\hline 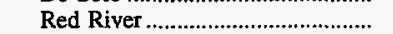 & 1 & 838 & w \\
\hline 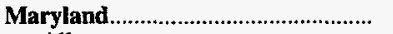 & 20 & 3,667 & 24.69 \\
\hline 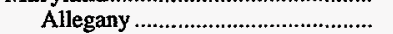 & 12 & 623 & $\mathrm{w}$ \\
\hline 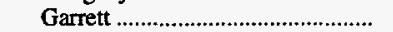 & 8 & 3,045 & $\mathbf{w}$ \\
\hline 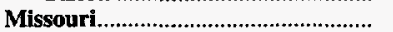 & 6 & 548 & 18.91 \\
\hline 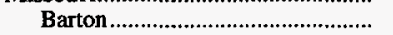 & 1 & 198 & w \\
\hline 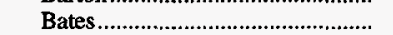 & 2 & 102 & w \\
\hline 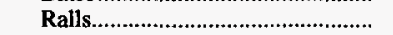 & 1 & 57 & w \\
\hline 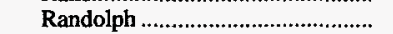 & 1 & 141 & w \\
\hline Vernon & 1 & 49 & w \\
\hline 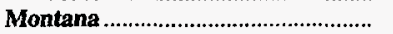 & 8 & 39,451 & 9.62 \\
\hline 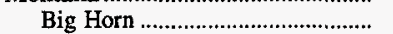 & 4 & 23,179 & $\$ 11.17$ \\
\hline 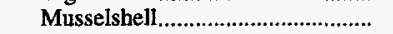 & 1 & 10 & - \\
\hline 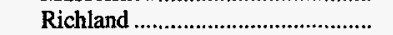 & 1 & 297 & $\mathbf{w}$ \\
\hline 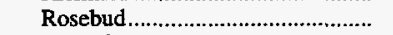 & 2 & 15,965 & $w$ \\
\hline New Mexico …………………… & 7 & 26,813 & 23.80 \\
\hline 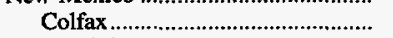 & 2 & 1,855 & w \\
\hline 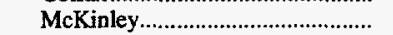 & 2 & 10,596 & w \\
\hline 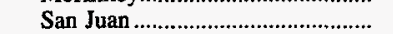 & 3 & 14,361 & w \\
\hline North Dakota................................. & 6 & 30,112 & 7.99 \\
\hline 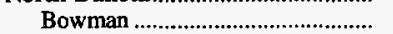 & 1 & 1,267 & w \\
\hline 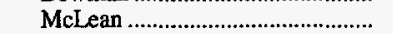 & 1 & 7,071 & w \\
\hline 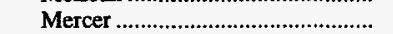 & 2 & 16,502 & w \\
\hline Oliver................................................... & 2 & 5,271 & $w$ \\
\hline Ohio & 113 & 26,118 & 25.97 \\
\hline 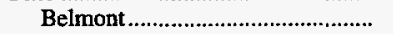 & 10 & 5,164 & $\mathbf{w}$ \\
\hline 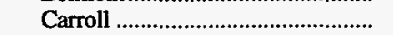 & 6 & 203 & $\$ 18.28$ \\
\hline 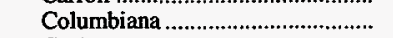 & 10 & 956 & 17.45 \\
\hline 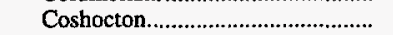 & 6 & 884 & $\mathbf{w}$ \\
\hline 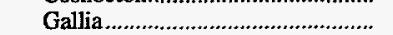 & 1 & 189 & $w$ \\
\hline 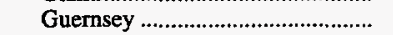 & 6 & 272 & 20.18 \\
\hline 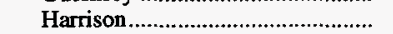 & 12 & 2,172 & 20.90 \\
\hline 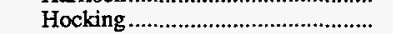 & 1 & 1 & - \\
\hline Holmes & 3 & 206 & w \\
\hline Jackson & 4 & 1,084 & w \\
\hline Jefferson & 12 & 766 & 23.58 \\
\hline 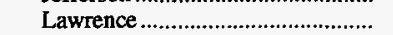 & 1 & 3 & - \\
\hline 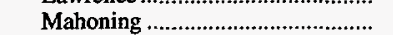 & 2 & 12 & - \\
\hline 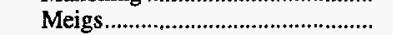 & 2 & 4,721 & $\mathbf{w}$ \\
\hline Monroe ............... & 1 & 2,722 & $w$ \\
\hline Morgan & 1 & 1,058 & $w$ \\
\hline 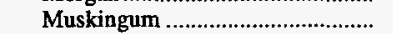 & 2 & 180 & w \\
\hline Noble , & 1 & 823 & w \\
\hline 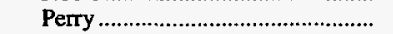 & 6 & 763 & 19.11 \\
\hline
\end{tabular}

See footnotes at end of table. 
Table 84. Coal Production, Number of Mines, and Average Mine Price, by State and County, 1995 (Continued)

(Thousand Short Tons, Nominal Dollars per Short Ton)

\begin{tabular}{|c|c|c|c|}
\hline $\begin{array}{l}\text { Coal-Producing } \\
\text { State and County }\end{array}$ & Number of Mines & Production & Average Mine Price \\
\hline \multicolumn{4}{|l|}{ Ohio (Continued) } \\
\hline Stark & 6 & 312 & $\$ 15.60$ \\
\hline 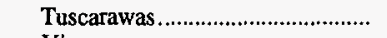 & 15 & 1,518 & 17.82 \\
\hline Vinton & 5 & 2,110 & 22.28 \\
\hline 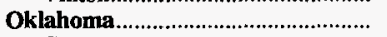 & 13 & 1,876 & 24.13 \\
\hline 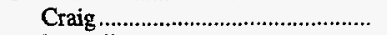 & 1 & 139 & w \\
\hline 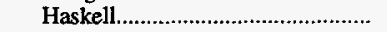 & 1 & 16 & $\mathbf{w}$ \\
\hline 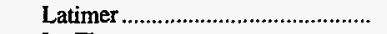 & 1 & 222 & w \\
\hline 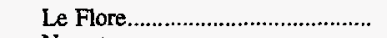 & 6 & 1,309 & $\$ 23.31$ \\
\hline Nowata & 2 & 159 & $w$ \\
\hline Okmulgee & 2 & 31 & w \\
\hline 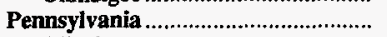 & 459 & 61,576 & 26.78 \\
\hline 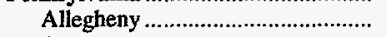 & 5 & 27 & w \\
\hline 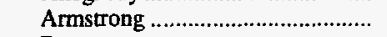 & 41 & 5,374 & $\$ 32.31$ \\
\hline 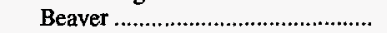 & 2 & 125 & w \\
\hline 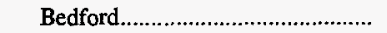 & 1 & 2 & - \\
\hline 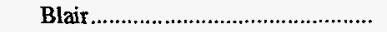 & 1 & 69 & w \\
\hline 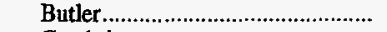 & 7 & 135 & 21.90 \\
\hline 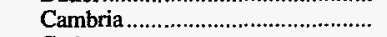 & 14 & 1,484 & 24.80 \\
\hline 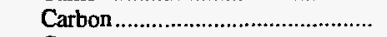 & 1 & 432 & w \\
\hline Centre & 3 & 142 & $\mathbf{w}$ \\
\hline 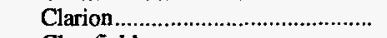 & 6 & 624 & w \\
\hline 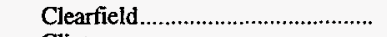 & 56 & 4,499 & 24.73 \\
\hline 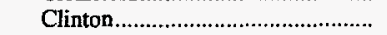 & 1 & 1 & - \\
\hline Columbia & 4 & 203 & $\mathbf{w}$ \\
\hline 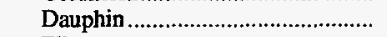 & 1 & 2 & - \\
\hline 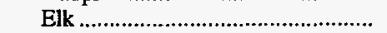 & 5 & 369 & 25.04 \\
\hline Fayette & 27 & 375 & 21.88 \\
\hline Greene & 14 & 28,390 & 25.38 \\
\hline 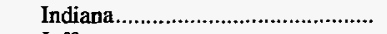 & 32 & 4,243 & 32.74 \\
\hline Jefferson & 33 & 1,461 & 25.61 \\
\hline 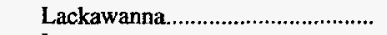 & 7 & 414 & w \\
\hline 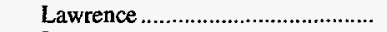 & 5 & 165 & $\mathbf{w}$ \\
\hline 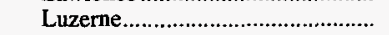 & 16 & 879 & 45.82 \\
\hline 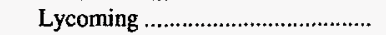 & 1 & 290 & $w$ \\
\hline Mercer & 2 & 8 & - \\
\hline 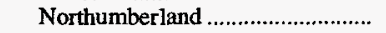 & $2 \overline{1}$ & 334 & $\mathbf{w}$ \\
\hline 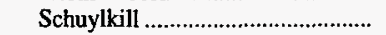 & 82 & 2,392 & 48.23 \\
\hline 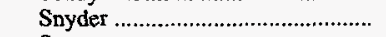 & 1 & 1 & - \\
\hline 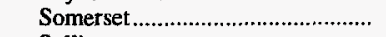 & 42 & 5,611 & 18.81 \\
\hline 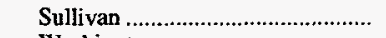 & 1 & 27 & $\mathbf{w}$ \\
\hline 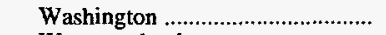 & 10 & 2,749 & 24.93 \\
\hline 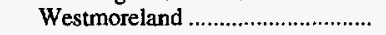 & 17 & 751 & 20.05 \\
\hline 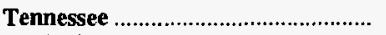 & 25 & 3,221 & 26.94 \\
\hline 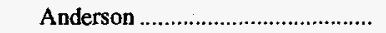 & 3 & 216 & $\mathbf{w}$ \\
\hline 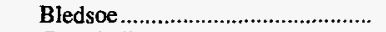 & 1 & 8 & - \\
\hline Campbell & 10 & 1,252 & $\$ 27.79$ \\
\hline 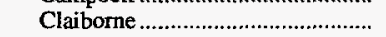 & 2 & 364 & w \\
\hline 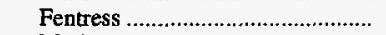 & 1 & 95 & $\mathbf{w}$ \\
\hline 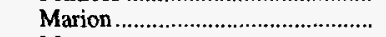 & 2 & 111 & $\mathbf{w}$ \\
\hline 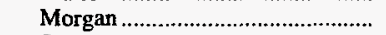 & $\overline{1}$ & 25 & $w$ \\
\hline 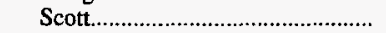 & 1 & 215 & w \\
\hline 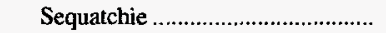 & 4 & 936 & w \\
\hline 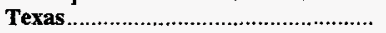 & 14 & 52,684 & 12.16 \\
\hline 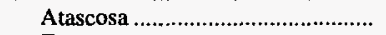 & 1 & 2,924 & w \\
\hline Freestone & 1 & 4,759 & $w$ \\
\hline Grimes & 1 & 3,312 & $w$ \\
\hline 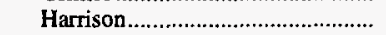 & 2 & 4,015 & $\mathbf{w}$ \\
\hline 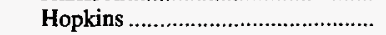 & 1 & 1,325 & w \\
\hline 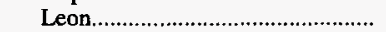 & 1 & 8,304 & $\mathbf{w}$ \\
\hline 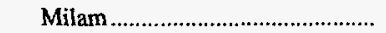 & 1 & 5,828 & $\mathbf{w}$ \\
\hline 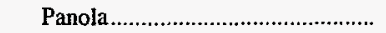 & 1 & 7,004 & $\mathbf{w}$ \\
\hline Robertson & 1 & 1,980 & $\mathbf{w}$ \\
\hline Rusk & 1 & 5,537 & w \\
\hline Titus & 1 & 7,385 & $\mathbf{w}$ \\
\hline 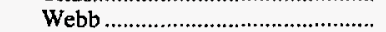 & 2 & 312 & $\mathbf{w}$ \\
\hline Utah & 13 & 25,167 & 19.10 \\
\hline 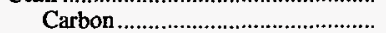 & 6 & 11,227 & $\$ 21.12$ \\
\hline Emery & 6 & 10,066 & w \\
\hline 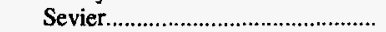 & 1 & 3,874 & $w$ \\
\hline
\end{tabular}

See footnotes at end of table. 
Table 84. Coal Production, Number of Mines, and Average Mine Price, by State and County, 1995 (Continued)

(Thousand Short Tons, Nominal Dollars per Short Ton)

\begin{tabular}{|c|c|c|c|}
\hline $\begin{array}{l}\text { Coal-Producing } \\
\text { State and County }\end{array}$ & Number of Mines & Production & Average Mine Price \\
\hline 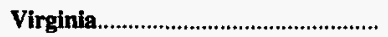 & 194 & 34,099 & 28.47 \\
\hline 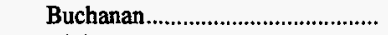 & 73 & 13,792 & $\$ 28.47$ \\
\hline 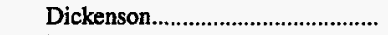 & 24 & 2,704 & 30.00 \\
\hline 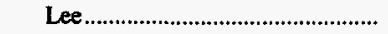 & 13 & 1,984 & 27.70 \\
\hline 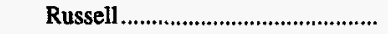 & 12 & 1,729 & w \\
\hline 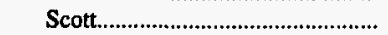 & 1 & 21 & $\mathrm{w}$ \\
\hline 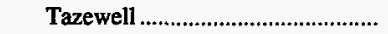 & 16 & 2,156 & 32.64 \\
\hline Wise & 55 & 11,713 & 27.39 \\
\hline Washington & $\mathbf{3}$ & $\mathbf{4 , 8 6 8}$ & $\mathbf{w}$ \\
\hline King & 1 & 241 & $\mathrm{w}$ \\
\hline Lewis ............................................. & 1 & 2,617 & $\mathrm{w}$ \\
\hline 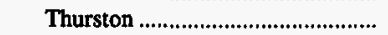 & 1 & 2,009 & $\mathrm{w}$ \\
\hline West Virginia & 424 & 162,997 & 27.18 \\
\hline Barbour............................................. & 8 & 1,570 & w \\
\hline Boone …………………………....... & 43 & 26,801 & $\$ 29.24$ \\
\hline 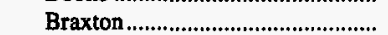 & 1 & 201 & w \\
\hline Brooke ................................................. & 1 & 1,051 & $\mathrm{w}$ \\
\hline Clay & 8 & 4,392 & w \\
\hline 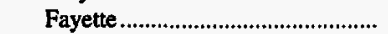 & 12 & 4,265 & 28.69 \\
\hline Gilmer & 1 & 32 & $\mathbf{w}$ \\
\hline 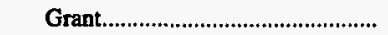 & 5 & 3,510 & w \\
\hline 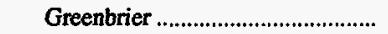 & 8 & 338 & 22.51 \\
\hline Harrison.............................................. & 14 & 4,078 & w \\
\hline Kanawha............................................... & 15 & 10,609 & 23.93 \\
\hline 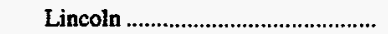 & 1 & 3 & - \\
\hline Logan.................................................. & 35 & 18,644 & 26.19 \\
\hline Marion .................................. & 7 & 3,623 & w \\
\hline Marshall............................................. & 2 & 7,892 & w \\
\hline 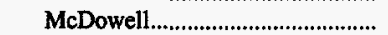 & 79 & 5,489 & 26.57 \\
\hline Mineral ............................................... & 2 & 121 & w \\
\hline 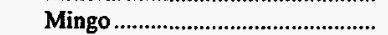 & 54 & 23,888 & 29.17 \\
\hline Monongalia ....................................... & 14 & 14,880 & w \\
\hline Nicholas............................... & 20 & 3,930 & 29.40 \\
\hline 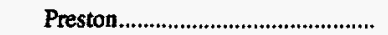 & 16 & 1,906 & 24.27 \\
\hline 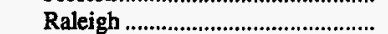 & 22 & 7,693 & 29.56 \\
\hline 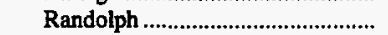 & 4 & 676 & 25.93 \\
\hline 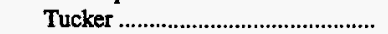 & 1 & 128 & w \\
\hline 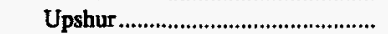 & 12 & 1,607 & 18.78 \\
\hline 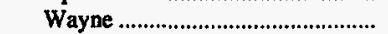 & 7 & 2,717 & w \\
\hline 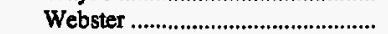 & 10 & 4,840 & 21.72 \\
\hline Wyoming ................................................. & 22 & 8,114 & 29.80 \\
\hline Wyoming & 29 & 263,822 & 6.58 \\
\hline 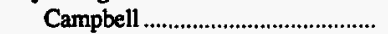 & 17 & 232,255 & $\$ 5.68$ \\
\hline 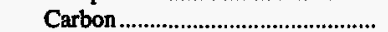 & 4 & 3,723 & w \\
\hline 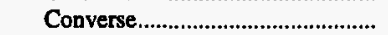 & 2 & 14,120 & w \\
\hline 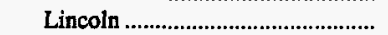 & 2 & 4,444 & w \\
\hline 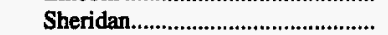 & 1 & 38 & w \\
\hline 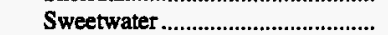 & 3 & 9,242 & $w$ \\
\hline 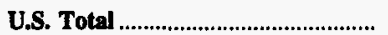 & 2,104 & $1,032,974$ & 18.83 \\
\hline
\end{tabular}

* Data round to zero.

Withheld to avoid disclosure of individual company data.

Notes: Average mine price is calculated by dividing the total free on board (f.o.b.) mine value of the coal produced by the total production and excludes mines producing less than 10,000 short tons, which are not required to provide these data. Coal production excludes silt, culm, refuse bank, slurry dam, and dredge operations except for Pennsylvania anthracite. Totals may not equal sum of components due to independent rounding.

Sources: Energy Information Administration, Form EIA-7A, "Coal Production Report"; State Mining Agency Coal Production Reports; and/or U.S. Department of Labor, Mine Safety and Health Administration, Form 7000-2, "Quarterly Mine Employment and Coal Production Report." 
Table 85. Average Mine Price by State and Coal Rank, 1995

(Nominal Dollars per Short Ton)

\begin{tabular}{|c|c|c|c|c|c|}
\hline $\begin{array}{l}\text { Coal-Producing } \\
\text { State and Region }\end{array}$ & Bituminous & Subbituminous & Lignite & Anthracite & Total \\
\hline 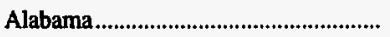 & $\$ 38.44$ & - & - & - & $\$ 38.44$ \\
\hline Alaska & - & $\mathbf{w}$ & - & - & w \\
\hline Arizona & $\mathbf{w}$ & - & - & - & $\mathbf{w}$ \\
\hline 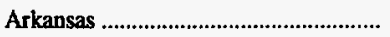 & - & - & - & $\mathbf{w}$ & $\mathbf{w}$ \\
\hline Colorado & 18.48 & $\$ 20.82$ & - & - & 19.26 \\
\hline Illinois & 23.05 & - & - & - & 23.05 \\
\hline Indiana & 21.71 & - & - & - & 21.71 \\
\hline 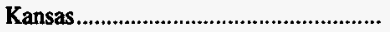 & $\mathbf{w}$ & - & - & - & w \\
\hline Kentucky Total & 24.79 & - & - & - & 24.79 \\
\hline 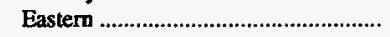 & 26.00 & - & - & - & 26.00 \\
\hline 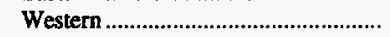 & 20.75 & - & - & - & 20.75 \\
\hline 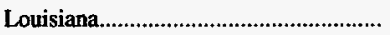 & - & - & w & - & $\mathbf{w}$ \\
\hline 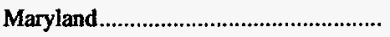 & 24.69 & - & - & - & 24.69 \\
\hline 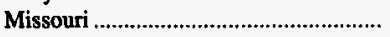 & 18.91 & - & - & - & 18.91 \\
\hline Montana & - & $\mathbf{w}$ & w & - & 9.62 \\
\hline New Mexico & $\mathrm{w}$ & $w$ & - & - & 23.80 \\
\hline 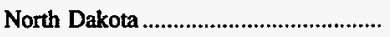 & - & - & $\$ 7.99$ & - & 7.99 \\
\hline 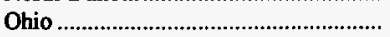 & $\$ 25.97$ & - & - & - & 25.97 \\
\hline Oklahoma & 24.13 & - & - & - & 24.13 \\
\hline Pennsylvania Total .................................. & 25.77 & - & - & $\$ 39.78$ & 26.78 \\
\hline 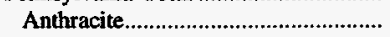 & - & - & - & 39.78 & 39.78 \\
\hline 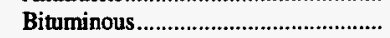 & 25.77 & - & - & - & 25.77 \\
\hline 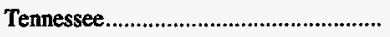 & 26.94 & - & - & - & 26.94 \\
\hline 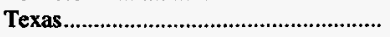 & w & - & $\mathbf{w}$ & - & 12.16 \\
\hline 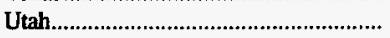 & $\$ 19.10$ & - & - & - & 19.10 \\
\hline Virginia & 28.47 & - & - & - & 28.47 \\
\hline 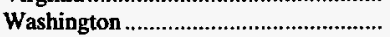 & w & $w$ & - & - & w \\
\hline West Virginia Total ............................. & 27.18 & - & - & - & 27.18 \\
\hline 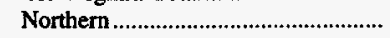 & 24.91 & - & - & - & 24.91 \\
\hline Southern & 28.07 & - & - & - & 28.07 \\
\hline Wyoming & w & $\mathbf{w}$ & - & - & 6.58 \\
\hline Appalachian Total ${ }^{1}$ & $\mathbf{2 7 . 3 2}$ & - & - & 39.78 & 27.45 \\
\hline Interior Total 1 . & 22.03 & $\mathbf{w}$ & 12.35 & $\mathbf{w}$ & 18.81 \\
\hline Western Total ${ }^{1}$ & 20.51 & $\mathbf{w}$ & 8.02 & - & 10.15 \\
\hline 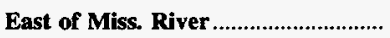 & 26.24 & - & - & 39.78 & 26.35 \\
\hline West of Miss. River............................ & 20.62 & 8.10 & 10.83 & $\mathbf{w}$ & 10.48 \\
\hline U.S. Total & 25.56 & 8.10 & 10.83 & 239.78 & 18.83 \\
\hline
\end{tabular}

I For a definition of coal-producing regions, see Appendix C.

2 Does not include Arkansas.

withheld to avoid disclosure of individual company data.

Notes: Average mine price is calculated by dividing the total free on board (f.o.b.) mine value of the coal produced by the total production. Excludes silt, culm, refuse bank, slurry dam, and dredge operations except for Pennsylvania anthracite. Excludes mines producing less than 10,000 short tons, which are not required to provide these data.

Sources: Energy Information Administration, Form ElA-7A, "Coal Production Report"; State Mining Agency Coal Production Reports; and/or U.S. Department of Labor, Mine Safety and Health Administration, Form 7000-2, "Quarterly Mine Employment and Coal Production Report." 
Table 86. Average Mine Price of U.S. Coal by Mine Production Range and Mine Type, 1995 (Nominal Dollars per Short Ton)

\begin{tabular}{|c|c|c|c|}
\hline $\begin{array}{l}\text { Mine Production Range } \\
\text { (thousand short tons) }\end{array}$ & Underground & Surface & Total \\
\hline Over 1,000 & $\$ 25.82$ & $\$ 11.75$ & $\$ 16.37$ \\
\hline 500 to 1,000 & 27.09 & 23.59 & 25.41 \\
\hline 200 to $500 \ldots \ldots$ & 27.22 & 23.96 & 25.74 \\
\hline 100 to 200 & 25.42 & 24.06 & 24.80 \\
\hline 50 to 100 & 25.95 & 25.61 & 25.81 \\
\hline 10 to 50 & 26.18 & 23.87 & 24.73 \\
\hline U.S. Total & 26.18 & 14.25 & 18.83 \\
\hline
\end{tabular}

Notes: Average mine price is calculated by dividing the total free on board (f.o.b.) mine value of the coal produced by the total production. Excludes silt, culm, refuse bank, slurry dam, and dredge operations except for Pennsylvania anthracite. Excludes mines producing less than 10,000 short tons, which are not required to provide these data.

Sources: Energy Information Administration, Form EIA-7A, “Coal Production Report"; State Mining Agency Coal Production Reports; and/or U.S. Department of Labor, Mine Safety and Health Administration, Form 7000-2, "Quarterly Mine Employment and Coal Production Report."

Table 87. Average Mine Price of U.S. Coal by Coalbed Thickness and Mine Type, 1995 (Nominal Dollars per Short Ton)

\begin{tabular}{|c|c|c|c|}
\hline $\begin{array}{c}\text { Coalbed Thickness } \\
\text { (inches) }\end{array}$ & Underground & Surface & Total \\
\hline$<7$ & - & $\$ 21.97$ & $\$ 21.97$ \\
\hline $7-12$ & - & 29.30 & 29.30 \\
\hline $13-18$ & - & 25.85 & 25.85 \\
\hline $19-24$ & $\$ 24.71$ & 23.80 & 23.83 \\
\hline $25-30$ & 28.01 & 23.08 & 24.05 \\
\hline 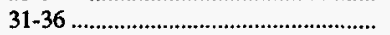 & 27.81 & 23.68 & 25.59 \\
\hline $37-42$ & 27.31 & 24.13 & 25.95 \\
\hline 43-48 & 28.08 & 23.88 & 26.66 \\
\hline $49-54$ & 28.56 & 24.27 & 27.03 \\
\hline $55-60$ & 29.02 & 22.18 & 27.24 \\
\hline $61-66$ & 26.29 & 25.16 & 26.00 \\
\hline $67-72$ & 26.23 & 19.40 & 24.11 \\
\hline $73-78$ & 24.84 & 20.30 & 23.13 \\
\hline 79-84 & 24.10 & 19.20 & 22.54 \\
\hline $85-90$ & 24.08 & 16.39 & 18.95 \\
\hline $91-96$ & 27.96 & 24.22 & 27.23 \\
\hline $97-102$ & 20.62 & 24.66 & 22.91 \\
\hline 103-108 & 26.84 & 21.55 & 25.43 \\
\hline $109-114$ & 14.85 & 28.28 & 18.76 \\
\hline $115-120$ & 27.15 & 23.98 & 26.47 \\
\hline 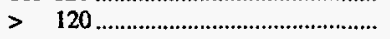 & 16.78 & 8.59 & 9.05 \\
\hline U.S. Total & 26.18 & 14.25 & 18.83 \\
\hline
\end{tabular}

Notes: Average mine price is calculated by dividing the total free on board (f.o.b.) mine value of the coal produced by the total production. Excludes silt, culm, refuse bank, slurry dam, and dredge operations except for Pennsylvania anthracite. Excludes mines producing less than 10,000 short tons, which are not required to provide these data.

Sources: Energy Information Administration, Form EIA-7A, "Coal Production Report"; State Mining Agency Coal Production Reports; and/or U.S. Department of Labor, Mine Safety and Health Administration, Form 7000-2, "Quarterly Mine Employment and Coal Production Report." 
Table 88. Average Mine Price of Coal by State and Productivity Range, 1995

(Nominal Dollars per Short Ton)

\begin{tabular}{|c|c|c|c|c|c|c|}
\hline \multirow{2}{*}{$\begin{array}{l}\text { Coal-Producing } \\
\text { State and Region }\end{array}$} & \multicolumn{6}{|c|}{$\begin{array}{l}\text { Productivity Range } \\
\text { (short tons per miner per hour) }\end{array}$} \\
\hline & $>=16$ & 8 to 16 & 4 to 8 & 2 to 4 & 0 to 2 & Total \\
\hline Alabama & - & $\mathbf{w}$ & $\$ 33.07$ & $\$ 37.58$ & w & $\$ 38.44$ \\
\hline Alaska & - & - & w & - & - & $\mathbf{w}$ \\
\hline 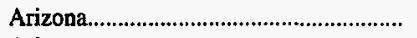 & - & - & w & - & - & w \\
\hline 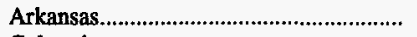 & - & - & - & - & $\mathbf{w}$ & w \\
\hline 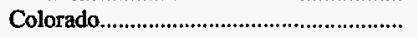 & - & $\$ 15.59$ & 19.82 & w & $\mathbf{w}$ & 19.26 \\
\hline 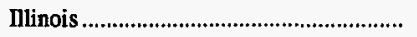 & $\mathrm{w}$ & $\mathbf{w}$ & 21.46 & $\$ 25.05$ & $w$ & 23.05 \\
\hline Indiana & - & $\mathbf{w}$ & 20.63 & w & - & 21.71 \\
\hline 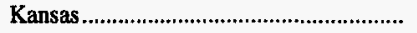 & - & - & - & w & - & w \\
\hline 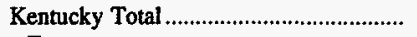 & $\$ 23.67$ & $\$ 25.26$ & 24.57 & $\$ 25.01$ & $\$ 25.18$ & 24.79 \\
\hline 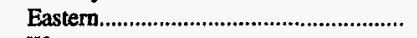 & $\mathrm{w}$ & $\mathbf{w}$ & 25.88 & 26.11 & w & 26.00 \\
\hline 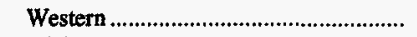 & $\mathbf{w}$ & $\mathbf{w}$ & 19.94 & 21.68 & $\mathbf{w}$ & 20.75 \\
\hline 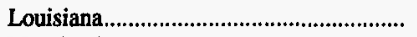 & - & $\mathbf{w}$ & - & - & - & $\mathbf{w}$ \\
\hline Maryland & - & - & $\mathbf{w}$ & 20.77 & $w$ & 24.69 \\
\hline 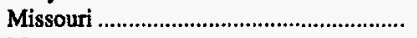 & - & $\mathbf{w}$ & $w$ & w & $\mathbf{w}$ & 18.91 \\
\hline Montana & $\mathbf{w}$ & $\mathbf{w}$ & - & - & - & 9.62 \\
\hline 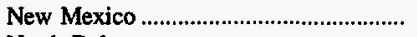 & - & $\mathbf{w}$ & $\$ 24.95$ & $\mathbf{w}$ & - & 23.80 \\
\hline 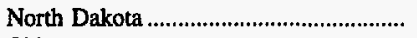 & $\mathbf{w}$ & $w$ & - & - & - & 7.99 \\
\hline Ohio & - & $\$ 18.63$ & 24.68 & $\$ 27.89$ & $\$ 18.90$ & 25.97 \\
\hline 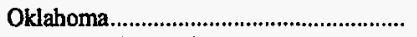 & - & - & w & w & w & 24.13 \\
\hline Pennsylvania Total ........................................ & $\$ 28.89$ & 24.68 & $\$ 23.65$ & $\$ 28.62$ & $\$ 35.04$ & 26.78 \\
\hline 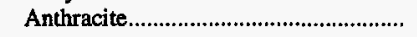 & 28.89 & 34.96 & 21.42 & 60.43 & 47.07 & 39.78 \\
\hline 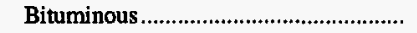 & - & 24.32 & 23.76 & 26.10 & 33.95 & 25.77 \\
\hline 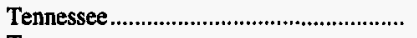 & w & - & 25.95 & 27.40 & w & 26.94 \\
\hline 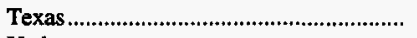 & - & 11.73 & $\mathbf{w}$ & w & - & 12.16 \\
\hline 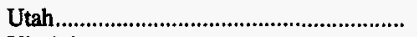 & - & 17.76 & $\$ 21.62$ & $\$ 15.99$ & - & 19.10 \\
\hline 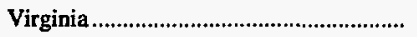 & - & $\mathbf{w}$ & w & 28.53 & $\$ 28.64$ & 28.47 \\
\hline 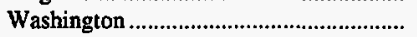 & - & - & w & w & - & w \\
\hline West Virginia Total...................................... & $\mathbf{w}$ & $\mathbf{w}$ & $\$ 25.75$ & 29.38 & 28.40 & 27.18 \\
\hline Northern & - & $\mathbf{w}$ & 24.06 & 26.91 & 26.98 & 24.91 \\
\hline 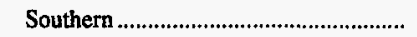 & $\mathbf{w}$ & $\$ 27.30$ & 26.58 & 30.32 & 29.13 & 28.07 \\
\hline Wyoming & $w$ & $\mathbf{w}$ & 17.70 & - & w & 6.58 \\
\hline Appalachian Total ${ }^{1}$ & 28.42 & 25.93 & 25.72 & 28.72 & 33.74 & 27.45 \\
\hline 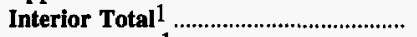 & 18.95 & 12.34 & 18.90 & 23.87 & 17.49 & 18.81 \\
\hline 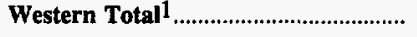 & 6.21 & 15.89 & 21.35 & 24.81 & 10.36 & 10.15 \\
\hline East of Miss. River .................................. & 27.28 & 25.54 & 24.53 & 27.73 & 33.68 & 26.35 \\
\hline West of Miss. River.................................. & 6.21 & 14.55 & 19.27 & 24.71 & 16.18 & 10.48 \\
\hline U.S. Total & 6.30 & 17.37 & 23.29 & 27.60 & 33.40 & 18.83 \\
\hline
\end{tabular}

1 For a definition of coal-producing regions, see Appendix C.

w Witheld to avoid disclosure of individual company data.

Notes: Average mine price is calculated by dividing the total free on board (f.o.b.) mine value of the coal produced by the total production. Excludes silt, culm, refuse bank, slurry dam, and dredge operations except for Pennsylvania anthracite. Excludes mines producing less than 10,000 short tons, which are not required to provide these data.

Sources: Energy Information Administration, Form EIA-7A, “Coal Production Report”; State Mining Agency Coal Production Reports; and/or U.S. Department of Labor, Mine Safety and Health Administration, Form 7000-2, "Quarterly Mine Employment and Coal Production Report." 
Table 89. Average Mine Price of Underground Coal by State and Productivity Range, 1995 (Nominal Dollars per Short Ton)

\begin{tabular}{|c|c|c|c|c|c|c|}
\hline \multirow{2}{*}{$\begin{array}{l}\text { Coal-Producing } \\
\text { State and Region }\end{array}$} & \multicolumn{6}{|c|}{$\begin{array}{l}\text { Productivity Range } \\
\text { (short tons per miner per hour) }\end{array}$} \\
\hline & $>=16$ & 8 to 16 & 4 to 8 & 2 to 4 & 0 to 2 & Total \\
\hline Alabama & - & - & $w$ & $\$ 37.88$ & $\mathbf{w}$ & $\$ 39.26$ \\
\hline 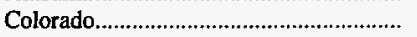 & - & w & w & w & - & 18.58 \\
\hline 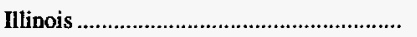 & - & - & $\$ 21.08$ & $w$ & - & 22.88 \\
\hline Indiana & - & - & - & $\mathbf{w}$ & - & w \\
\hline 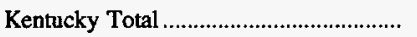 & - & w & 24.80 & $\$ 25.33$ & $\$ 25.28$ & 25.18 \\
\hline 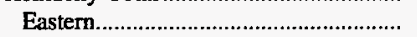 & - & $\mathbf{w}$ & 26.26 & 26.69 & 25.28 & 26.52 \\
\hline Western & - & - & 20.39 & 21.97 & - & 21.33 \\
\hline 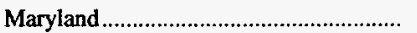 & - & - & w & - & - & W \\
\hline 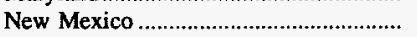 & - & - & - & w & - & w \\
\hline Ohio & - & - & w & w & - & 28.98 \\
\hline 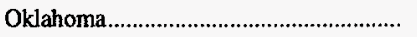 & - & - & - & - & w & w \\
\hline 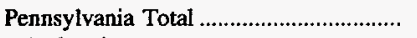 & - & $\mathbf{w}$ & $\$ 24.88$ & $\mathbf{w}$ & 37.29 & 27.09 \\
\hline 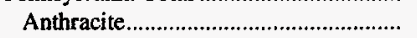 & - & - & - & $w$ & 37.84 & 36.91 \\
\hline 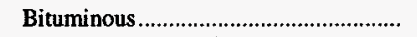 & - & w & 24.88 & $\mathbf{w}$ & 37.26 & 27.02 \\
\hline 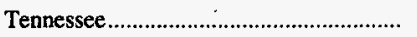 & - & - & w & w & w & $\mathbf{w}$ \\
\hline 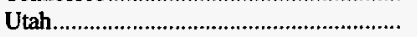 & - & $\$ 17.76$ & 21.62 & $\$ 15.99$ & - & 19.10 \\
\hline Virginia & - & - & 32.13 & 28.96 & 28.53 & 29.20 \\
\hline 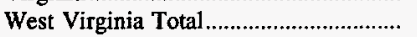 & - & 27.38 & 25.87 & 29.52 & 28.39 & 27.77 \\
\hline 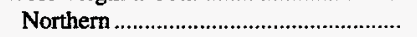 & - & w & 24.12 & 27.11 & w & 25.17 \\
\hline 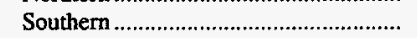 & - & $\mathbf{w}$ & 27.75 & 30.49 & $\mathbf{w}$ & 29.30 \\
\hline 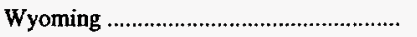 & - & - & $\mathbf{w}$ & - & $\mathbf{w}$ & w \\
\hline Appalachian Total ${ }^{1}$.............................. & - & $\mathbf{w}$ & 26.19 & 29.13 & 34.64 & 28.24 \\
\hline Interior Total ${ }^{1}$ & - & - & 20.87 & 24.09 & $\mathbf{w}$ & 22.57 \\
\hline 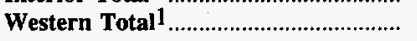 & - & $\mathbf{w}$ & 19.83 & 26.00 & $\mathbf{w}$ & 18.85 \\
\hline East of Miss. River .............................. & - & $\mathbf{w}$ & 24.95 & 28.05 & 34.64 & 27.12 \\
\hline 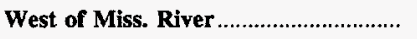 & - & $\mathbf{w}$ & 19.83 & 26.00 & $\mathbf{w}$ & 18.85 \\
\hline U.S. Total & - & 21.02 & 24.49 & 27.98 & 34.61 & 26.18 \\
\hline
\end{tabular}

1 For a definition of coal-producing regions, see Appendix $\mathrm{C}$.

Withheld to avoid disclosure of individual company data.

Notes: Average mine price is calculated by dividing the total free on board (f.o.b.) mine value of the coal produced by the total production. Excludes silt, culm, refuse bank, slurry dam, and dredge operations except for Pennsylvania anthracite. Excludes mines producing less than 10,000 short tons, which are not required to provide these data.

Sources: Energy Information Administration, Form EIA-7A, "Coal Production Report"; State Mining Agency Coal Production Reports; and/or U.S. Department of Labor, Mine Safety and Health Administration, Form 7000-2, "Quarterly Mine Employment and Coal Production Report." 
Table 90. Average Mine Price of Surface Coal by State and Productivity Range, 1995 (Nominal Dollars per Short Ton)

\begin{tabular}{|c|c|c|c|c|c|c|}
\hline \multirow{2}{*}{ Coal-Producing State and Region } & \multicolumn{6}{|c|}{$\begin{array}{l}\text { Productivity Range } \\
\text { (short tons per miner per hour) }\end{array}$} \\
\hline & $>=16$ & 8 to 16 & 4 to 8 & 2 to 4 & 0 to 2 & Total \\
\hline Alabama & - & $\mathbf{w}$ & $\$ 36.18$ & $\$ 36.81$ & $\mathbf{w}$ & $\$ 36.38$ \\
\hline Alaska & - & - & $\mathbf{w}$ & - & - & w \\
\hline 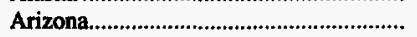 & - & - & $\mathbf{w}$ & - & - & $w$ \\
\hline 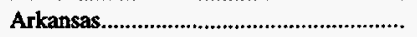 & - & - & - & - & $\mathbf{w}$ & $\mathbf{w}$ \\
\hline 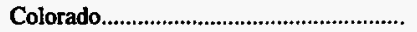 & - & $\mathbf{w}$ & $\mathbf{w}$ & - & $w$ & 20.63 \\
\hline Itlinois & $\mathbf{w}$ & $\mathbf{w}$ & $\mathbf{w}$ & 24.68 & $\mathbf{w}$ & 24.04 \\
\hline Indiana & - & $\mathbf{w}$ & $\mathbf{w}$ & $\mathbf{w}$ & - & $\mathbf{w}$ \\
\hline 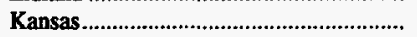 & - & - & - & $\mathbf{w}$ & - & $\mathbf{w}$ \\
\hline 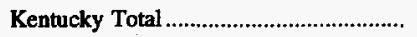 & $\$ 23.67$ & $\$ 22.97$ & $\$ 24,32$ & 24.09 & $\$ 24.68$ & 24.19 \\
\hline 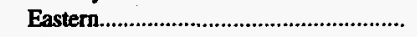 & w & $\mathbf{w}$ & 25.52 & 24.75 & w & 25.24 \\
\hline 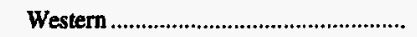 & $\mathbf{w}$ & $\mathbf{w}$ & 19.34 & 20.05 & $\mathbf{w}$ & 19.46 \\
\hline 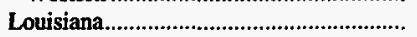 & - & $\mathbf{w}$ & - & - & - & $\mathbf{w}$ \\
\hline 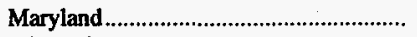 & - & - & - & $w$ & $\mathbf{w}$ & $\mathbf{w}$ \\
\hline 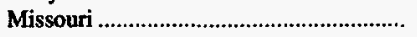 & - & $\mathbf{w}$ & $\mathbf{w}$ & w & $\mathbf{w}$ & 18.91 \\
\hline 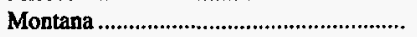 & $\mathbf{w}$ & $\$ 16.74$ & - & - & - & 9.62 \\
\hline 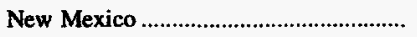 & - & $\mathbf{w}$ & $\mathbf{w}$ & $\mathbf{w}$ & - & w \\
\hline 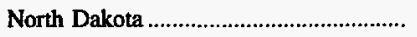 & w & $w$ & - & - & - & 7.99 \\
\hline Ohio & - & $\$ 18.63$ & 20.25 & $\$ 26.38$ & $\$ 18.90$ & 22.92 \\
\hline Oklahoma & - & - & $\mathbf{w}$ & $\mathbf{w}$ & $\mathbf{w}$ & $\mathbf{w}$ \\
\hline Pennsylvania Total & $\$ 28.89$ & 22.17 & 20.46 & 29.76 & 31.12 & 26.14 \\
\hline 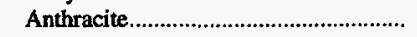 & 28.89 & 34.96 & 21.42 & 61.35 & 52.60 & 39.97 \\
\hline 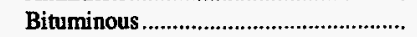 & - & 18.35 & 20.26 & 23.25 & 27.52 & 22.45 \\
\hline Tennessee & $\mathbf{w}$ & - & w & w & w & w \\
\hline 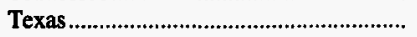 & - & 11.73 & $\mathbf{w}$ & w & - & 12.16 \\
\hline 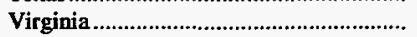 & - & $\mathbf{w}$ & $\mathbf{w}$ & $\$ 26.29$ & 32.39 & 26.34 \\
\hline 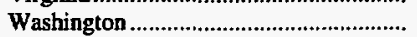 & - & - & $\mathbf{w}$ & w & - & $w$ \\
\hline West Virginia Total..................................... & $\mathbf{w}$ & $\mathbf{w}$ & $\$ 25.61$ & 28.30 & 28.44 & 25.95 \\
\hline 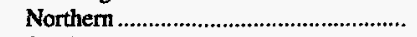 & - & $\mathbf{w}$ & 23.63 & 24.22 & w & 22.97 \\
\hline Southern & $\mathbf{w}$ & $\$ 26.24$ & 25.81 & 29.14 & $\mathbf{w}$ & 26.29 \\
\hline Wyoming & $w$ & $\mathbf{w}$ & $\mathbf{w}$ & - & $\mathbf{w}$ & $\mathbf{w}$ \\
\hline 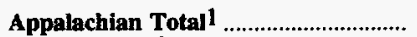 & 28.42 & 24.93 & 25.16 & 27.49 & 29.57 & 25.97 \\
\hline Interior Total 1 & 18.95 & 12.34 & 17.60 & 23.14 & 17.27 & 16.19 \\
\hline 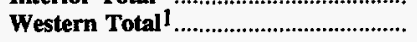 & 6.21 & 15.47 & $\mathbf{2 1 . 8 1}$ & $\mathbf{w}$ & 8.39 & 9.13 \\
\hline East of Miss. River ................................ & 27.28 & 24.15 & 24.03 & 26.72 & 29.31 & 24.93 \\
\hline West of Miss. River ................................... & 6.21 & 13.91 & 19.15 & 22.69 & 15.84 & 9.63 \\
\hline U.S. Total & 6.30 & 15.63 & 22.27 & 26.45 & 28.31 & 14.25 \\
\hline
\end{tabular}

1 For a definition of coal-producing regions, see Appendix $C$.

w Withheld to avoid disclosure of individual company data.

Notes: Average mine price is calculated by dividing the total free on board (f.o.b.) mine value of the coal produced by the total production. Excludes silt, culm, refuse bank, slurry dam, and dredge operations except for Pennsylvania anthracite. Excludes mines producing less than 10,000 short tons, which are not required to provide these data.

Sources: Energy Information Administration, Form EIA-7A, "Coal Production Report"; State Mining Agency Coal Production Reports; and/or U.S. Department of Labor, Mine Safety and Health Administration, Form 7000-2, "Quarterly Mine Employment and Coal Production Report." 
Table 91. Average Mine Price by State and Disposition, 1995 (Nominal Dollars per Short Ton)

\begin{tabular}{|c|c|c|c|}
\hline $\begin{array}{l}\text { Coal-Producing } \\
\text { State and Region }\end{array}$ & Open Market 1 & Captive 2 & Total \\
\hline Alabama & $w$ & $\mathbf{w}$ & 38.44 \\
\hline 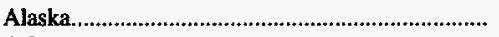 & w & $\mathbf{w}$ & $\mathbf{w}$ \\
\hline 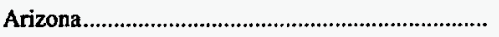 & $w$ & - & $\mathbf{w}$ \\
\hline 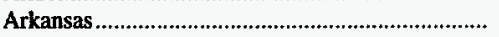 & - & $\mathbf{w}$ & $\mathbf{w}$ \\
\hline 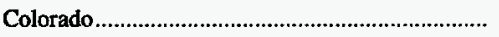 & 19.26 & - & 19.26 \\
\hline 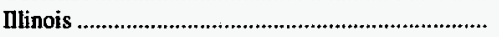 & w & $w$ & 23.05 \\
\hline 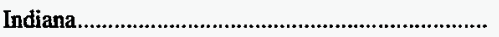 & 21.72 & 15.76 & 21.71 \\
\hline 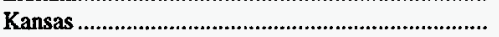 & $w$ & - & $w$ \\
\hline Kentucky Total & 24.74 & 28.41 & 24.79 \\
\hline Eastern & 25.95 & 28.41 & 26.00 \\
\hline 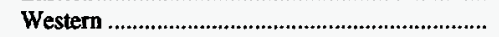 & 20.75 & - & 20.75 \\
\hline 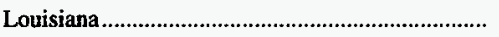 & w & - & w \\
\hline 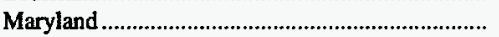 & w & $\mathbf{w}$ & 24.69 \\
\hline 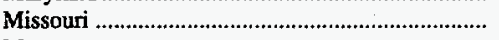 & 18.91 & - & 18.91 \\
\hline Montana & $w$ & $\mathbf{w}$ & 9.62 \\
\hline New Mexico & 23.80 & - & 23.80 \\
\hline 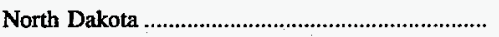 & w & $\mathbf{w}$ & 7.99 \\
\hline 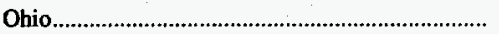 & 21.53 & 40.04 & 25.97 \\
\hline Oklahoma & 24.13 & - & 24.13 \\
\hline 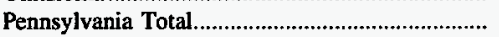 & 27.00 & 19.78 & 26.78 \\
\hline Anthracite & 48.90 & 19.78 & 39.78 \\
\hline 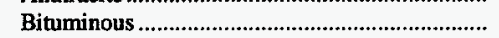 & 25.82 & 19.77 & 25.77 \\
\hline 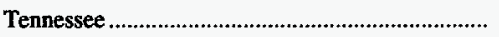 & 26.94 & - & 26.94 \\
\hline 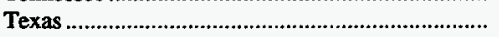 & 13.47 & 11.67 & 12.16 \\
\hline Utah & 20.41 & 16.33 & 19.10 \\
\hline Virginia & 28.30 & 30.24 & 28.47 \\
\hline Washington & w & $\mathbf{w}$ & $\mathbf{w}$ \\
\hline West Virginia Total & 27.01 & 34.37 & 27.18 \\
\hline 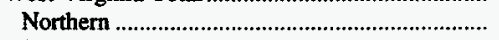 & 24.47 & 36.28 & 24.91 \\
\hline Southern & 27.99 & 32.74 & 28.07 \\
\hline Wyoming & 6.14 & 13.13 & 6.58 \\
\hline Appalachian Total ${ }^{3}$ & 27.19 & 33.52 & 27.45 \\
\hline Interior Total ${ }^{3}$ & 20.88 & 11.88 & 18.81 \\
\hline Western Total ${ }^{3}$ & 9.77 & 14.54 & 10.15 \\
\hline East of Miss. River & 26.10 & 33.43 & 26.35 \\
\hline West of Miss. River & 10.04 & 13.02 & 10.48 \\
\hline U.S. Total & 18.99 & 17.14 & 18.83 \\
\hline
\end{tabular}

1 Open Market includes all coal sold on the open market to other coal companies or consumers.

2 Captive includes all coal used by the producing company or sold to affiliated or parent companies.

3 For a definition of coal-producing regions, see Appendix C.

Witheld to avoid disclosure of individual company data.

Notes: Average mine price is calculated by dividing the total free on board (f.o.b.) mine value of the coal produced by the total production. Excludes silt, culm, refuse bank, slurry dam, and dredge operations except for Pennsylvania anthracite. Excludes mines producing less than 10,000 short tons, which are not required to provide these data.

Sources: Energy Information Administration, Form EIA-7A, “Coal Production Report”; State Mining Agency Coal Production Reports; and/or U.S. Department of Labor, Mine Safety and Health Administration, Form 7000-2, "Quarterly Mine Employment and Coal Production Report." 



\section{Consumer Prices}

Figure 14. U.S. Coal Prices by Sector, 1986-1995

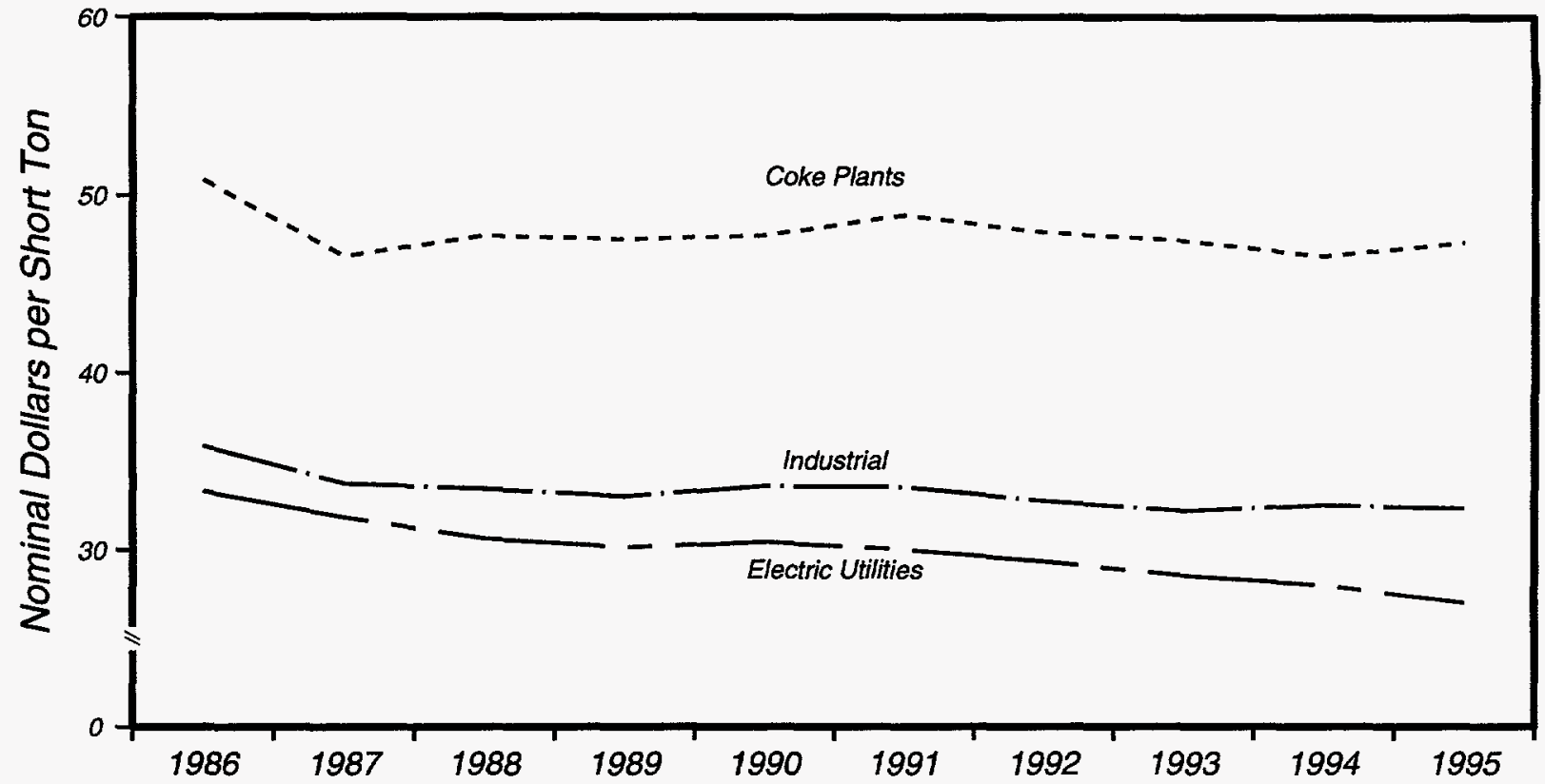

Sources: •Electric Utilities: Federal Energy Regulatory Commission (FERC), FERC Form 423, "Monthly Report of Cost and Quality of Fuels for Electric Plants." -Coke Plants: Energy Information Administration (EIA), Form EIA-5, "Coke Plant Report - Quarterly." -Industrial: EIA, Form EIA-3, "Quarterly Coal Consumption Report - Manufacturing Plants." 
Table 92. Average Price of Coal Delivered to Electric Utilities by Census Division and State, 1986, 1991-1995

(Nominal Dollars per Short Ton)

\begin{tabular}{|c|c|c|c|c|c|c|c|c|c|}
\hline \multirow{2}{*}{ Census Division and State } & \multirow{2}{*}{1995} & \multirow{2}{*}{1994} & \multirow{2}{*}{1993} & \multirow{2}{*}{1992} & \multirow{2}{*}{1991} & \multirow{2}{*}{1986} & \multirow{2}{*}{$\begin{array}{c}\text { Percent } \\
\text { Change } \\
\text { 1994-1995 }\end{array}$} & \multicolumn{2}{|c|}{ Average Annual Percent Change } \\
\hline & & & & & & & & 1991-1995 & 1986-1995 \\
\hline 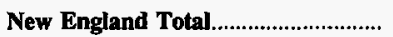 & $\$ 43.34$ & $\$ 42.81$ & $\$ 43.34$ & $\$ 45.14$ & $\$ 47.13$ & $\$ 49.92$ & 1.2 & -2.1 & -1.5 \\
\hline 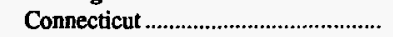 & 49.33 & 46.45 & 44.80 & 51.30 & 57.35 & 59.83 & 6.2 & -3.7 & -2.1 \\
\hline 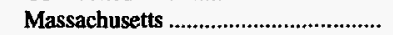 & 42.63 & 43.00 & 43.39 & 44.11 & 45.33 & 47.00 & -.8 & -1.5 & -1.1 \\
\hline 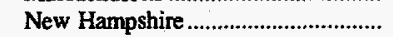 & 41.67 & 39.66 & 42.39 & 44.69 & 46.20 & 53.51 & 5.0 & -2.5 & -2.7 \\
\hline Middle Atlantic Total......................... & 34.63 & 36.33 & 36.66 & $\mathbf{3 7 . 5 6}$ & 38.99 & 37.90 & -4.7 & -2.9 & -1.0 \\
\hline 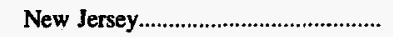 & 47.17 & 48.49 & 47.50 & 46.62 & 47.76 & 47.84 & -2.7 & -.3 & -1 \\
\hline New York & 36.86 & 37.63 & 38.63 & 38.62 & 41.19 & 41.96 & -2.1 & -2.7 & -1.4 \\
\hline 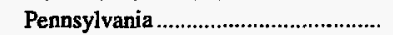 & 33.48 & 35.39 & 35.73 & 36.81 & 38.05 & 36.65 & -5.4 & -3.1 & -1.0 \\
\hline East North Central Total ................... & 29.67 & 30.56 & 30.98 & 32.05 & 32.63 & 38.69 & -2.9 & -2.3 & -2.9 \\
\hline 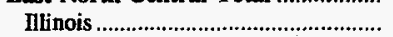 & 32.58 & 32.69 & 35.30 & 37.06 & 36.76 & 44.71 & -.3 & -3.0 & -3.4 \\
\hline Indiana & 25.94 & 26.79 & 26.73 & 27.89 & 28.41 & 33.70 & -3.2 & -2.2 & -2.9 \\
\hline 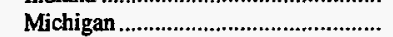 & 30.95 & 32.90 & 33.17 & 34.23 & 35.20 & 42.48 & -5.9 & -3.2 & -3.4 \\
\hline Ohio & 34.44 & 34.70 & 34.05 & 34.40 & 35.33 & 38.80 & -.8 & -.6 & -1.3 \\
\hline Wisconsin & 21.23 & 23.13 & 22.96 & 25.92 & 26.19 & 31.37 & -8.2 & -5.1 & -4.2 \\
\hline West North Central Total .................... & 16.10 & 16.76 & 16.88 & 18.92 & 19.44 & 22.41 & -3.9 & -4.6 & -3.6 \\
\hline Iowa & 17.13 & 17.39 & 17.53 & 19.58 & 19.62 & 25.20 & -1.5 & -3.3 & -4.2 \\
\hline 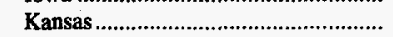 & 17.83 & 17.85 & 17.69 & 20.99 & 22.06 & 23.02 & -.1 & -5.2 & -2.8 \\
\hline 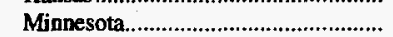 & 20.12 & 20.09 & 20.07 & 20.96 & 22.18 & 23.74 & .2 & -2.4 & -1.8 \\
\hline 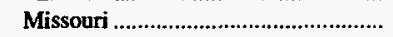 & 18.14 & 21.39 & 24.40 & 27.57 & 27.65 & 31.35 & -15.2 & -10.0 & -5.9 \\
\hline Nebraska & 12.86 & 13.11 & 12.92 & 12.77 & 12.73 & 18.12 & -1.9 & .3 & -3.7 \\
\hline 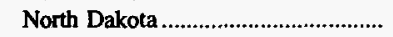 & 9.65 & 9.28 & 9.38 & 9.45 & 9.37 & 10.81 & 4.0 & .7 & -1.3 \\
\hline 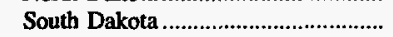 & 14.35 & 13.10 & 13.30 & 13.68 & 13.65 & 15.10 & 9.6 & 1.3 & -.6 \\
\hline 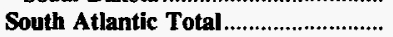 & 38.25 & 39.53 & 40.80 & 41.28 & 42.18 & 43.28 & $-\mathbf{3 . 2}$ & -2.4 & -1.4 \\
\hline 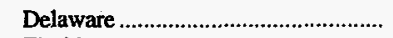 & 42.27 & 41.98 & 44.02 & 45.31 & 46.51 & 49.75 & .7 & -2.3 & -1.8 \\
\hline 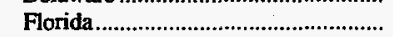 & 43.93 & 43.71 & 43.58 & 45.03 & 45.87 & 47.36 & .5 & -1.1 & -8 \\
\hline Georgia & 38.62 & 39.82 & 43.29 & 43.36 & 42.95 & 43.95 & -3.0 & -2.6 & -1.4 \\
\hline 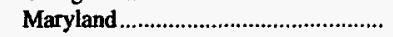 & 39.00 & 39.84 & 40.78 & 40.68 & 41.83 & 42.28 & -2.1 & -1.7 & -.9 \\
\hline 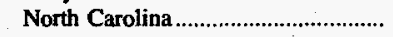 & 40.57 & 41.77 & 42.36 & 43.00 & 44.49 & 46.40 & -2.9 & -2.3 & -1.5 \\
\hline 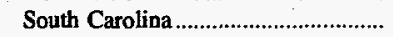 & 38.86 & 39.84 & 40.17 & 39.13 & 41.37 & 46.23 & -2.5 & -1.5 & -1.9 \\
\hline 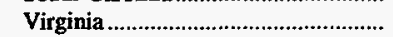 & 36.90 & 37.05 & 37.57 & 37.81 & 38.87 & 43.86 & -.4 & -1.3 & -1.9 \\
\hline 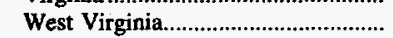 & 31.61 & 34.70 & 35.42 & 36.88 & 37.93 & 37.25 & -8.9 & -4.4 & -1.8 \\
\hline East South Central Total.................... & 30.08 & 32.43 & 33.30 & 33.05 & 33.93 & 37.59 & -7.2 & -3.0 & -2.4 \\
\hline 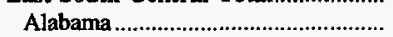 & 37.00 & 40.42 & 42.56 & 41.67 & 43.82 & 46.51 & -8.4 & -4.1 & -2.5 \\
\hline Kentucky & 25.71 & 27.16 & 27.29 & 27.01 & 27.19 & 30.84 & -5.4 & -1.4 & -2.0 \\
\hline Mississippi & 34.40 & 35.54 & 40.51 & 39.94 & 41.92 & 56.11 & -3.2 & -4.8 & -5.3 \\
\hline 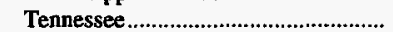 & 27.94 & 30.61 & 30.94 & 31.01 & 30.48 & 33.79 & -8.7 & -2.1 & -2.1 \\
\hline West South Central Total .................. & 20.66 & 20.79 & 22.14 & 22.55 & 22.98 & 24.38 & -.6 & -2.6 & -1.8 \\
\hline 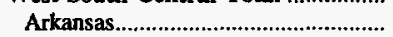 & 27.99 & 27.91 & 29.50 & 28.84 & 27.90 & 27.10 & .3 & .1 & .3 \\
\hline 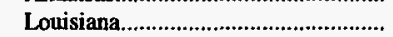 & 25.13 & 25.04 & 25.65 & 24.93 & 27.09 & 29.14 & .3 & -1.9 & -1.6 \\
\hline Oklahoma & 17.00 & 17.50 & 21.32 & 21.47 & 23.17 & 28.36 & -2.8 & -7.4 & -5.5 \\
\hline 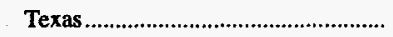 & 19.65 & 19.84 & 20.91 & 21.58 & 21.66 & 22.59 & -.9 & -2.4 & -1.5 \\
\hline Mountain Total & 21.51 & 21.83 & 22.11 & 21.64 & 22.22 & 22.47 & -1.5 & -.8 & -.5 \\
\hline 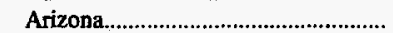 & 28.65 & 28.26 & 27.78 & 28.31 & 29.16 & 28.70 & 1.4 & -.4 & * \\
\hline 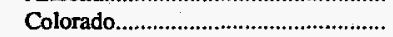 & 20.73 & 21.01 & 21.59 & 21.67 & 21.49 & 22.96 & -1.3 & -.9 & -1.1 \\
\hline Montana & 11.47 & 11.79 & 11.78 & 12.14 & .11 .44 & 11.53 & -2.7 & * & -.1 \\
\hline Nevada & 29.02 & 32.37 & 32.34 & 32.32 & 31.28 & 31.23 & -10.3 & -1.8 & -.8 \\
\hline New Mexico & 25.59 & 25.48 & 24.61 & 23.83 & 25.02 & 20.64 & .4 & .6 & 2.4 \\
\hline Utah & 25.27 & 26.10 & 27.34 & 27.54 & 27.40 & 32.32 & -3.2 & -2.0 & -2.7 \\
\hline 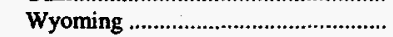 & 14.29 & 14.09 & 14.03 & 13.42 & 14.55 & 16.06 & 1.4 & -.4 & -1.3 \\
\hline 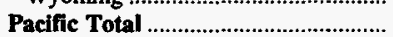 & 22.83 & 21.93 & $\mathbf{2 1 . 5 5}$ & 22.17 & 23.16 & 27.31 & 4.1 & -3 & -2.0 \\
\hline 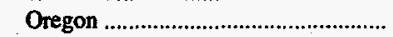 & 18.79 & 19.18 & 19.75 & 21.23 & 18.28 & - & -2.0 & .7 & - \\
\hline Washington & 23.74 & 22.93 & 22.09 & 22.48 & 24.86 & 27.31 & 3.5 & -1.1 & -1.5 \\
\hline 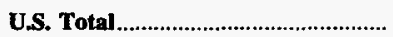 & 27.01 & 28.03 & 28.58 & 29.36 & 30.02 & $\mathbf{3 3 . 3 0}$ & -3.6 & -2.6 & -2.3 \\
\hline
\end{tabular}

* Data round to zero.

Note: Average prices are based on the cost including insurance and freight.

Source: Federal Energy Regulatory Commission, FERC Form 423, "Monthly Report of Cost and Quality of Fuels for Electric Plants." 
Table 93. Average Real Price of Coal Delivered to Electric Utilities by Census Division and State, 1986, 1991-1995

(Real Dollars per Short Ton)

\begin{tabular}{|c|c|c|c|c|c|c|c|c|c|}
\hline \multirow{2}{*}{ Census Division and State } & \multirow{2}{*}{1995} & \multirow{2}{*}{1994} & \multirow{2}{*}{1993} & \multirow{2}{*}{1992} & \multirow{2}{*}{1991} & \multirow{2}{*}{1986} & \multirow{2}{*}{$\begin{array}{c}\text { Percent } \\
\text { Change } \\
\text { 1994-1995 }\end{array}$} & \multicolumn{2}{|c|}{ Average Annual Percent Change } \\
\hline & & & & & & & & 1991-1995 & 1986-1995 \\
\hline New England Total................................ & $\$ 40.32$ & $\$ 40.77$ & $\$ 42.24$ & $\$ 45.14$ & $\$ 48.44$ & $\$ 61.93$ & -1.1 & -4.5 & -4.6 \\
\hline Connecticut & 45.89 & 44.24 & 43.66 & 51.30 & 58.94 & 74.23 & 3.7 & -6.1 & -5.2 \\
\hline 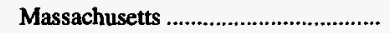 & 39.66 & 40.96 & 42.29 & 44.11 & 46.59 & 58.31 & -3.2 & -3.9 & -4.2 \\
\hline 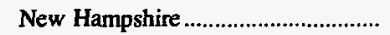 & 38.76 & 37.77 & 41.32 & 44.69 & 47.49 & 66.39 & 2.6 & -4.9 & -5.8 \\
\hline 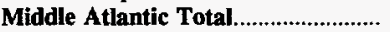 & 32.21 & 34.60 & 35.73 & $\mathbf{3 7 . 5 6}$ & 40.07 & 47.02 & -6.9 & -5.3 & -4.1 \\
\hline 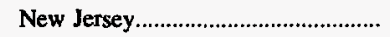 & 43.88 & 46.18 & 46.30 & 46.62 & 49.08 & 59.35 & -5.0 & -2.8 & -3.3 \\
\hline 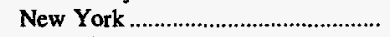 & 34.29 & 35.84 & 37.65 & 38.62 & 42.34 & 52.06 & -4.3 & -5.1 & -4.5 \\
\hline Pennsylvania & 31.15 & 33.70 & 34.82 & 36.81 & 39.11 & 45.47 & -7.6 & -5.5 & -4.1 \\
\hline East North Central Total ................... & 27.60 & 29.11 & 30.20 & 32.05 & 33.54 & 48.00 & -5.2 & -4.8 & -6.0 \\
\hline 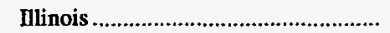 & 30.30 & 31.14 & 34.41 & 37.06 & 37.78 & 55.47 & -2.7 & -5.4 & -6.5 \\
\hline 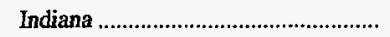 & 24.13 & 25.52 & 26.06 & 27.89 & 29.19 & 41.81 & -5.4 & -4.6 & -5.9 \\
\hline 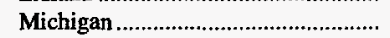 & 28.79 & 31.33 & 32.33 & 34.23 & 36.17 & 52.71 & -8.1 & -5.5 & -6.5 \\
\hline Ohio & 32.03 & 33.05 & 33.19 & 34.40 & 36.31 & 48.14 & -3.0 & -3.1 & -4.4 \\
\hline 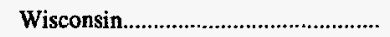 & 19.75 & 22.03 & 22.38 & 25.92 & 26.92 & 38.92 & -10.3 & -7.4 & -7.3 \\
\hline West North Central Total .................. & 14.98 & 15.96 & 16.45 & 18.92 & 19.98 & 27.81 & -6.1 & -6.9 & -6.6 \\
\hline lowa & 15.93 & 16.57 & 17.08 & 19.58 & 20.17 & 31.27 & -3.8 & -5.7 & -7.2 \\
\hline 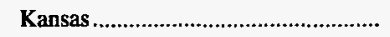 & 16.59 & 17.00 & 17.24 & 20.99 & 22.67 & 28.56 & -2.4 & -7.5 & -5.9 \\
\hline 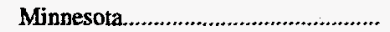 & 18.72 & 19.13 & 19.56 & 20.96 & 22,80 & 29.45 & -2.1 & -4.8 & -4.9 \\
\hline 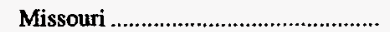 & 16.88 & 20.37 & 23.78 & 27.57 & 28.41 & 38.89 & -17.2 & -12.2 & -8.8 \\
\hline Nebraska & 11.97 & 12.49 & 12.59 & 12.77 & 13.08 & 22.49 & -4.2 & -2.2 & -6.8 \\
\hline 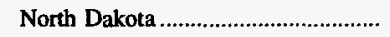 & 8.98 & 8.84 & 9.14 & 9.45 & 9.63 & 13.41 & 1.6 & -1.7 & -4.4 \\
\hline 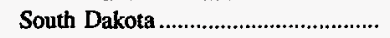 & 13.35 & 12.47 & 12.96 & 13.68 & 14.03 & 18.73 & 7.0 & -1.2 & -3.7 \\
\hline 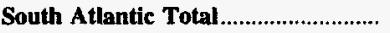 & $\mathbf{3 5 . 5 8}$ & 37.65 & 39.77 & 41.28 & 43.35 & 53.69 & -5.5 & -4.8 & -4.5 \\
\hline Delaware & 39.32 & 39.98 & 42.91 & 45.31 & 47.80 & 61.73 & -1.6 & -4.8 & -4.9 \\
\hline Florida & 40.86 & 41.63 & 42.48 & 45.03 & 47.14 & 58.76 & -1.8 & -3.5 & -3.9 \\
\hline 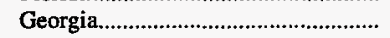 & 35.92 & 37.92 & 42.19 & 43.36 & 44.14 & 54.52 & -5.3 & -5.0 & -4.5 \\
\hline 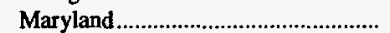 & 36.28 & 37.95 & 39.74 & 40.68 & 42.99 & 52.46 & -4.4 & -4.1 & -4.0 \\
\hline North Carolina & 37.74 & 39.78 & 41.28 & 43.00 & 45.73 & 57.56 & -5.1 & -4.7 & -4.6 \\
\hline 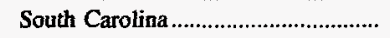 & 36.14 & 37.94 & 39.15 & 39.13 & 42.52 & 57.35 & -4.7 & -4.0 & -5.0 \\
\hline 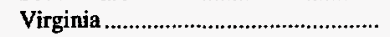 & 34.33 & 35.29 & 36.62 & 37.81 & 39.95 & 54.42 & -2.7 & -3.7 & -5.0 \\
\hline 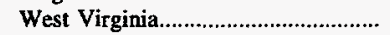 & 29.40 & 33.05 & 34.53 & 36.88 & 38.98 & 46.22 & -11.0 & -6.8 & -4.9 \\
\hline 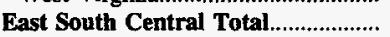 & 27.98 & 30.89 & 32.46 & 33.05 & 34.87 & 46.64 & -9.4 & -5.3 & -5.5 \\
\hline 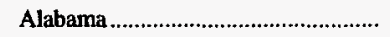 & 34.42 & 38.49 & 41.48 & 41.67 & 45.04 & 57.70 & -10.6 & -6.5 & -5.6 \\
\hline 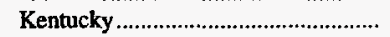 & 23.91 & 25.87 & 26.60 & 27.01 & 27.95 & 38.26 & -7.6 & -3.8 & -5.1 \\
\hline 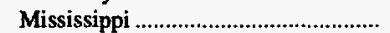 & 32.00 & 33.85 & 39.48 & 39.94 & 43.08 & 69.62 & -5.4 & -7.2 & -8.3 \\
\hline Tennessee & 26.00 & 29.16 & 30.16 & 31.01 & 31.32 & 41.92 & -10.8 & -4.5 & -5.2 \\
\hline West South Central Total .................. & 19.22 & 19.80 & 21.58 & 22.55 & 23.62 & 30.25 & -2.9 & $-\mathbf{5 . 0}$ & -4.9 \\
\hline 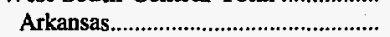 & 26.03 & 26.58 & 28.75 & 28.84 & 28.67 & 33.62 & -2.0 & -2.4 & -2.8 \\
\hline 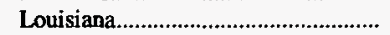 & 23.37 & 23.85 & 25.00 & 24.93 & 27.84 & 36.15 & -2.0 & -4.3 & -4.7 \\
\hline 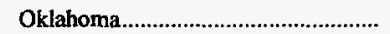 & 15.82 & 16.66 & 20.78 & 21.47 & 23.82 & 35.19 & -5.1 & -9.7 & -8.5 \\
\hline 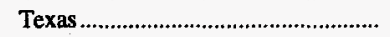 & 18.28 & 18.89 & 20.38 & 21.58 & 22.26 & 28.02 & -3.3 & -4.8 & -4.6 \\
\hline Mountain Total & 20.01 & 20.79 & 21.55 & 21.64 & 22.83 & 27.87 & -3.8 & -3.2 & -3.6 \\
\hline 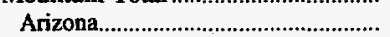 & 26.65 & 26.91 & 27.08 & 28.31 & 29.97 & 35.61 & -.9 & -2.9 & -3.2 \\
\hline 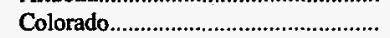 & 19.28 & 20.01 & 21.04 & 21.67 & 22.09 & 28.49 & -3.6 & -3.3 & -4.2 \\
\hline Montana & 10.67 & 11.23 & 11.48 & 12.14 & 11.76 & 14.31 & -5.0 & -2.4 & -3.2 \\
\hline Nevada & 26.99 & 30.83 & 31.52 & 32.32 & 32.15 & 38.75 & -12.4 & -4.3 & -3.9 \\
\hline 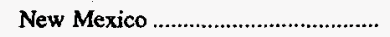 & 23.81 & 24.26 & 23.98 & 23.83 & 25.72 & 25.61 & -1.9 & -1.9 & -.8 \\
\hline 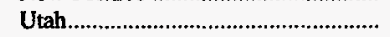 & 23.51 & 24.86 & 26.64 & 27.54 & 28.16 & 40.10 & -5.4 & -4.4 & -5.8 \\
\hline Wyoming & 13.29 & 13.42 & 13.68 & 13.42 & 14.95 & 19.92 & -.9 & -2.9 & -4.4 \\
\hline 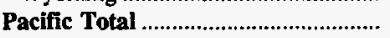 & 21.24 & 20.89 & 21.00 & 22.17 & 23.80 & 33.88 & 1.6 & -2.8 & $-\mathbf{5 . 0}$ \\
\hline Oregon & 17.48 & 18.26 & 19.25 & 21.23 & 18.79 & - & -4.3 & -1.8 & - \\
\hline 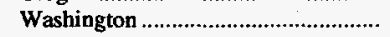 & 22.08 & 21.83 & 21.53 & 22.48 & 25.55 & 33.88 & 1.1 & -3.6 & -4.6 \\
\hline 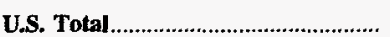 & 25.12 & 26.69 & 27.86 & 29.36 & 30.86 & 41.31 & -5.9 & $-\mathbf{5 . 0}$ & -5.4 \\
\hline
\end{tabular}

Notes: Real prices are in 1992 dollars, calculated using implicit Gross Domestic Product price deflators. See Appendix D, Table D3. Average prices are based on the cost including insurance and freight.

Source: Federal Energy Regulatory Commission, FERC Form 423, "Monthly Report of Cost and Quality of Fuels for Electric Plants." 
Table 94. Average Price of Coal Delivered to Other Industrial Plants By Census Division and State, 1986, 1991-1995

(Nominal Dollars per Short Ton)

\begin{tabular}{|c|c|c|c|c|c|c|c|c|c|}
\hline \multirow{2}{*}{ Census Division and State } & \multirow{2}{*}{1995} & \multirow{2}{*}{1994} & \multirow{2}{*}{1993} & \multirow{2}{*}{1992} & \multirow{2}{*}{1991} & \multirow{2}{*}{1986} & \multirow{2}{*}{$\begin{array}{c}\text { Percent } \\
\text { Change } \\
\text { 1994-1995 }\end{array}$} & \multicolumn{2}{|c|}{ Average Annual Percent Change } \\
\hline & & & & & & & & 1991-1995 & 1986-1995 \\
\hline New England Total.................................. & $\$ 56.90$ & $\$ 55.73$ & $\$ 57.83$ & $\$ 65.71$ & $\$ 66.74$ & $\$ 63.97$ & 2.1 & -3.9 & -1.3 \\
\hline 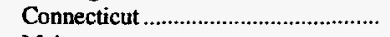 & - & - & - & - & - & w & w & $\mathbf{w}$ & w \\
\hline Maine & w & $w$ & $\mathbf{w}$ & $\mathbf{w}$ & $\mathbf{w}$ & $\mathbf{w}$ & $\mathbf{w}$ & $\mathbf{w}$ & $\mathbf{w}$ \\
\hline 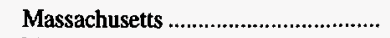 & $w$ & $w$ & w & $\mathbf{w}$ & $\mathbf{w}$ & w & $\mathbf{w}$ & $\mathbf{w}$ & $\mathbf{w}$ \\
\hline 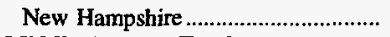 & - & - & - & - & - & w & $\mathbf{w}$ & $\mathbf{w}$ & $\mathbf{w}$ \\
\hline Middle Atlantic Total............................ & $\mathbf{w}$ & $\mathbf{w}$ & $\mathbf{w}$ & $\mathbf{w}$ & $\mathbf{w}$ & $\mathbf{w}$ & $w$ & $\mathbf{w}$ & $\mathbf{w}$ \\
\hline 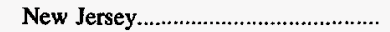 & w & $\mathbf{w}$ & w & $\mathbf{w}$ & $\mathbf{w}$ & $\mathbf{w}$ & w & w & $\mathbf{w}$ \\
\hline 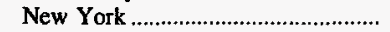 & 41.91 & 42.20 & 42.15 & 43.95 & 43.79 & 43.75 & -.7 & -1.1 & -.5 \\
\hline Pennsylvania & 34.07 & 33.66 & 34.04 & 35.70 & 35.89 & 38.17 & 1.2 & -1.3 & -1.3 \\
\hline East North Central Total .................... & 34.89 & 34.72 & 34.54 & 35.05 & 35.98 & 39.09 & .5 & -.8 & -1.3 \\
\hline 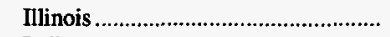 & 29.03 & 29.13 & 29.42 & 29.24 & 30.81 & 36.91 & -.3 & -1.5 & -2.6 \\
\hline Indiana & 33.14 & 31.35 & 30.91 & 31.58 & 33.01 & 34.30 & 5.7 & .1 & -4 \\
\hline 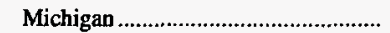 & 41.18 & 41.20 & 41.46 & 42.17 & 43.69 & 47.24 & -.1 & -1.5 & -1.5 \\
\hline 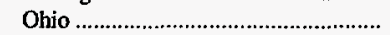 & 35.18 & 35.75 & 34.82 & 35.24 & 34.85 & 35.49 & -1.6 & .2 & -.1 \\
\hline 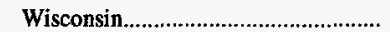 & 40.21 & 41.23 & 40.85 & 42.27 & 43.31 & 47.72 & -2.5 & -1.8 & -1.9 \\
\hline West North Central Total ................. & 18.92 & 18.61 & 18.00 & 17.76 & 18.34 & 19.32 & 1.7 & .8 & -.2 \\
\hline Iowa & 29.24 & 28.52 & 28.01 & 27.17 & 29.15 & 35.55 & 2.5 & .1 & -2.1 \\
\hline 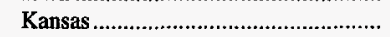 & 32.42 & 32.25 & 33.06 & 31.96 & 30.81 & 37.27 & .5 & 1.3 & -1.5 \\
\hline 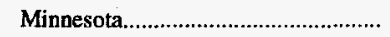 & 34.40 & 35.66 & 35.81 & 35.63 & 36.26 & 40.60 & -3.5 & -1.3 & -1.8 \\
\hline 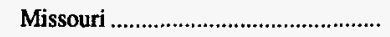 & 32.81 & 32.87 & 32.12 & 31.48 & 31.28 & 31.26 & -.2 & 1.2 & .5 \\
\hline 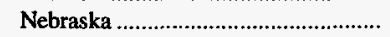 & $w$ & $\mathbf{w}$ & w & w & $w$ & 30.77 & $\mathbf{w}$ & w & $w$ \\
\hline North Dakota & $\mathbf{w}$ & $\mathbf{w}$ & w & $\mathbf{w}$ & $\mathbf{w}$ & w & $\mathbf{w}$ & $\mathbf{w}$ & $w$ \\
\hline 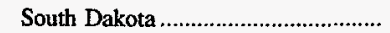 & w & $\mathbf{w}$ & $\mathbf{w}$ & $\mathbf{w}$ & $\mathbf{w}$ & $\mathbf{w}$ & $\mathbf{w}$ & $\mathbf{w}$ & $w$ \\
\hline South Atlantic Total ............................... & $\mathbf{w}$ & $\mathbf{w}$ & $\mathbf{w}$ & $\mathbf{w}$ & $\mathbf{w}$ & $\mathbf{w}$ & $\mathbf{w}$ & $\mathbf{w}$ & $\mathbf{w}$ \\
\hline 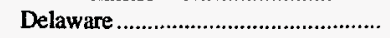 & w & w & w & w & w & w & $\mathbf{w}$ & $\mathbf{w}$ & $\mathbf{w}$ \\
\hline Florida & 46.63 & 46.60 & 48.28 & 48.27 & 47.42 & 49.31 & .1 & -.4 & -.6 \\
\hline 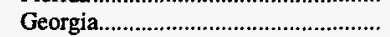 & 44.64 & 45.71 & 45.20 & 45.18 & 44.95 & 43.81 & -2.3 & -.2 & .2 \\
\hline 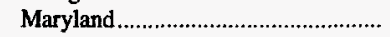 & 31.66 & 32.18 & 32.18 & 32.83 & 33.71 & 32.92 & -1.6 & -1.5 & -.4 \\
\hline 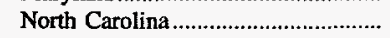 & 43.29 & 43.62 & 43.44 & 43.46 & 43.05 & 45.72 & -.7 & .1 & -.6 \\
\hline 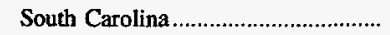 & 43.16 & 43.84 & 43.35 & 43.31 & 43.19 & 44.09 & -1.5 & * & -.2 \\
\hline 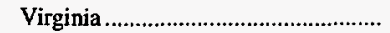 & 42.50 & 41.56 & 41.27 & 40.97 & 40.53 & 40.29 & 2.2 & 1.2 & .6 \\
\hline 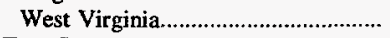 & 33.61 & 32.73 & 32.91 & 31.93 & 32.08 & 33.37 & 2.7 & 1.2 & .1 \\
\hline East South Central Total ...................... & $\mathbf{w}$ & $\mathbf{w}$ & $\mathbf{w}$ & $\mathbf{w}$ & $\mathbf{w}$ & $\mathbf{w}$ & $\mathbf{w}$ & $\mathbf{w}$ & $\mathbf{w}$ \\
\hline 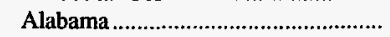 & 39.53 & 38.74 & 39.01 & 39.76 & 40.43 & 43.30 & 2.0 & -.6 & -1.0 \\
\hline 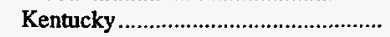 & 44.09 & 43.22 & 42.30 & 43.78 & 46.54 & 43.98 & 2.0 & -1.3 & * \\
\hline 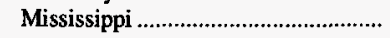 & w & w & w & w & w & w & w & w & $\mathbf{w}$ \\
\hline 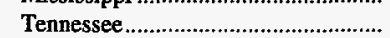 & 35.68 & 35.34 & 35.41 & 35.52 & 35.66 & 38.12 & 1.0 & * & -.7 \\
\hline West South Central Total ..................... & 22.04 & 22.95 & 21.38 & 22.80 & 23.31 & $\mathbf{w}$ & -4.0 & -1.4 & $\vec{w}$ \\
\hline 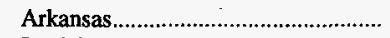 & 43.52 & 44.28 & 44.06 & 44.61 & 45.09 & 45.82 & -1.7 & -.9 & -.6 \\
\hline 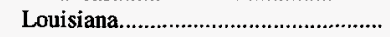 & $\mathbf{w}$ & w & $\mathbf{w}$ & $\mathbf{w}$ & $w$ & w & w & w & w \\
\hline 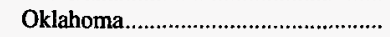 & $\mathbf{w}$ & w & w & w & w & 39.76 & w & $\mathbf{w}$ & w \\
\hline Texas & 18.76 & 19.54 & 17.58 & 18.14 & 18.61 & 23.95 & -4.0 & .2 & -2.7 \\
\hline 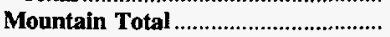 & 27.06 & 28.78 & 28.51 & 28.66 & 29.60 & 30.94 & -6.0 & -2.2 & -1.5 \\
\hline 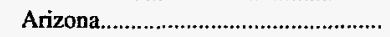 & 40.46 & 41.35 & 40.51 & 40.95 & 40.09 & w & -2.1 & .2 & w \\
\hline 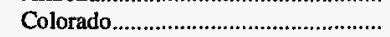 & 26.11 & 28.96 & 28.63 & 30.34 & 29.27 & 26.58 & -9.8 & -2.8 & -.2 \\
\hline Idaho & 34.11 & 33.35 & 32.78 & 33.29 & 33.91 & 36.53 & 2.3 & .1 & -.8 \\
\hline 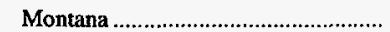 & w & w & w & $\mathbf{w}$ & w & $w$ & w & w & w \\
\hline 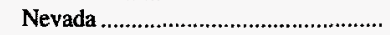 & $\mathbf{w}$ & w & $\mathbf{w}$ & $\mathbf{w}$ & w & $\mathbf{w}$ & w & $\mathbf{w}$ & $w$ \\
\hline 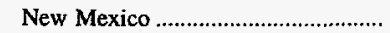 & $\mathbf{w}$ & w & $w$ & $\mathbf{w}$ & w & w & $\mathbf{w}$ & w & w \\
\hline Utah & 19.74 & 26.57 & 26.51 & 25.35 & 26.43 & 27.59 & -25.7 & -7.0 & -3.6 \\
\hline 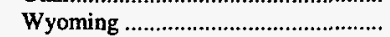 & 22.72 & 22.87 & 23.43 & 23.67 & 25.19 & w & -.6 & -2.5 & $w$ \\
\hline Pacific Total & 43.68 & 44.92 & $\mathbf{4 3 . 8 3}$ & $\mathbf{4 3 . 3 7}$ & 45.75 & 49.02 & -2.8 & -1.1 & -1.3 \\
\hline 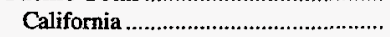 & 41.11 & 43.39 & 42.86 & 42.05 & 44.79 & 51.52 & -5.3 & -2.1 & -2.5 \\
\hline 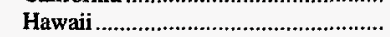 & $w$ & w & $w$ & $\mathbf{w}$ & $\mathbf{w}$ & 58.35 & w & w & w \\
\hline Oregon & $\mathbf{w}$ & $\mathbf{w}$ & w & w & w & w & $w$ & $w$ & w \\
\hline Washington & 59.15 & 58.86 & 53.11 & 56.25 & 59.16 & 41.48 & .5 & * & 4.0 \\
\hline 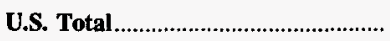 & 32.42 & 32.55 & 32.23 & 32.78 & 33.54 & 35.84 & -.4 & -.8 & -1.1 \\
\hline
\end{tabular}

* Data round to zero.

witheld to avoid disclosure of individual company data.

Notes: Price data are for manufacturing plants only. Average prices are based on the cost including insurance, freight, and taxes.

Source: Energy Information Administration, Form EIA-3, "Quarterly Coal Consumption - Manufacturing Plants." 
Table 95. Average Real Price of Coal Delivered to Other Industrial Plants by Census Division and State, 1986, 1991-1995

(Real Dollars per Short Ton)

\begin{tabular}{|c|c|c|c|c|c|c|c|c|c|}
\hline \multirow{2}{*}{ Census Division and State } & \multirow{2}{*}{1995} & \multirow{2}{*}{1994} & \multirow{2}{*}{1993} & \multirow{2}{*}{1992} & \multirow{2}{*}{1991} & \multirow{2}{*}{1986} & \multirow{2}{*}{$\begin{array}{c}\text { Percent } \\
\text { Change } \\
\text { 1994-1995 }\end{array}$} & \multicolumn{2}{|c|}{ Average Annual Percent Change } \\
\hline & & & & & & & & 1991-1995 & $1986-1995$ \\
\hline New England Total................................... & $\$ 52.93$ & $\$ 53.08$ & $\$ 56.37$ & $\$ 65.71$ & $\$ 68.59$ & $\$ 79.37$ & -0.3 & -6.3 & -4.4 \\
\hline 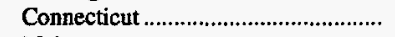 & - & - & - & - & - & w & $\mathbf{w}$ & $\mathbf{w}$ & w \\
\hline Maine & $w$ & $\mathbf{w}$ & w & $\mathbf{w}$ & $\mathbf{w}$ & w & $\mathbf{w}$ & $\mathbf{w}$ & $\mathbf{w}$ \\
\hline Massachusetts ....................................... & w & $w$ & $\mathbf{w}$ & $\mathbf{w}$ & $\mathbf{w}$ & $\mathbf{w}$ & $\mathbf{w}$ & $\mathbf{w}$ & $\mathbf{w}$ \\
\hline New Hampshire & - & - & - & - & - & $w$ & $\mathbf{w}$ & $w$ & w \\
\hline 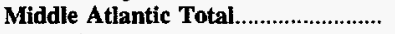 & $\mathbf{w}$ & $\mathbf{w}$ & $\mathbf{w}$ & $\mathbf{w}$ & $\mathbf{w}$ & $\mathbf{w}$ & $w$ & $\mathbf{w}$ & $\mathbf{w}$ \\
\hline 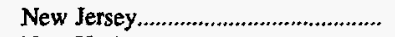 & $w$ & w & w & w & $\mathbf{w}$ & $\mathbf{w}$ & $\mathbf{w}$ & $w$ & $\mathbf{w}$ \\
\hline 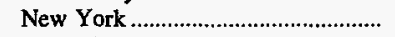 & 38.98 & 40.19 & 41.09 & 43.95 & 45.01 & 54.28 & -3.0 & -3.5 & -3.6 \\
\hline Pennsylvania & 31.69 & 32.06 & 33.18 & 35.70 & 36.89 & 47.36 & -1.1 & -3.7 & -4.4 \\
\hline East North Central Total ...................... & 32.45 & 33.07 & 33.66 & 35.05 & 36.98 & 48.50 & -1.9 & -3.2 & -4.4 \\
\hline 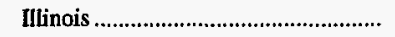 & 27.01 & 27.74 & 28.67 & 29.24 & 31.66 & 45.80 & -2.6 & -3.9 & -5.7 \\
\hline Indiana & 30.83 & 29.86 & 30.13 & 31.58 & 33.92 & 42.55 & 3.2 & -2.4 & -3.5 \\
\hline 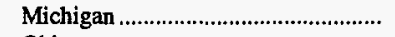 & 38.30 & 39.24 & 40.41 & 42.17 & 44.91 & 58.61 & -2.4 & -3.9 & -4.6 \\
\hline Ohio & 32.73 & 34.04 & 33.94 & 35.24 & 35.81 & 44.03 & -3.9 & -2.2 & -3.2 \\
\hline 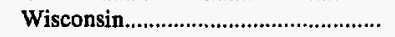 & 37.40 & 39.27 & 39.81 & 42.27 & 44.51 & 59.21 & -4.8 & -4.3 & -5.0 \\
\hline West North Central Total .................... & 17.60 & 17.73 & 17.54 & 17.76 & 18.84 & 23.97 & -.7 & -1.7 & -3.4 \\
\hline Iowa & 27.20 & 27.16 & 27.30 & 27.17 & 29.96 & 44.10 & .1 & -2.4 & -5.2 \\
\hline 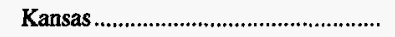 & 30.16 & 30.71 & 32.22 & 31.96 & 31.67 & 46.24 & -1.8 & -1.2 & -4.6 \\
\hline 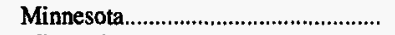 & 32.00 & 33.96 & 34.90 & 35.63 & 37.26 & 50.37 & -5.8 & -3.7 & -4.9 \\
\hline 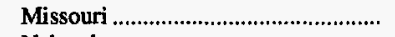 & 30.52 & 31.31 & 31.30 & 31.48 & 32.15 & 38.79 & -2.5 & -1.3 & -2.6 \\
\hline 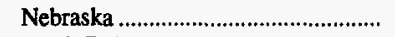 & w & w & w & w & w & 38.18 & w & w & w \\
\hline 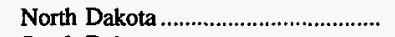 & w & $\mathbf{w}$ & w & w & w & $\mathbf{w}$ & w & w & w \\
\hline 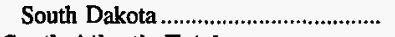 & w & $w$ & $\mathbf{w}$ & w & $\mathbf{w}$ & $\mathbf{w}$ & $w$ & $\mathbf{w}$ & w \\
\hline South Atlantic Total & $\mathbf{w}$ & $\mathbf{w}$ & $\mathbf{w}$ & $\mathbf{w}$ & $\mathbf{w}$ & $\mathbf{w}$ & $\mathbf{w}$ & $\mathbf{w}$ & $\mathbf{w}$ \\
\hline Delaware & $w$ & $\mathbf{w}$ & $w$ & w & $w$ & w & $\mathbf{w}$ & $w$ & w \\
\hline Florida & 43.38 & 44.39 & 47.06 & 48.27 & 48.74 & 61.18 & -2.3 & -2.9 & -3.7 \\
\hline 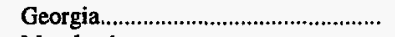 & 41.52 & 43.53 & 44.05 & 45.18 & 46.20 & 54.36 & -4.6 & -2.6 & -2.9 \\
\hline 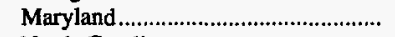 & 29.45 & 30.64 & 31.36 & 32.83 & 34.65 & 40.85 & -3.9 & -4.0 & -3.6 \\
\hline North Carolina & 40.27 & 41.54 & 42.34 & 43.46 & 44.24 & 56.73 & -3.0 & -2.3 & -3.7 \\
\hline 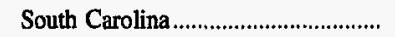 & 40.15 & 41.75 & 42.25 & 43.31 & 44.39 & 54.70 & -3.8 & -2.5 & -3.4 \\
\hline 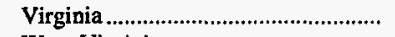 & 39.53 & 39.58 & 40.23 & 40.97 & 41.65 & 49.98 & -.1 & -1.3 & -2.6 \\
\hline 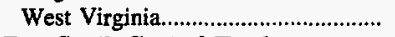 & 31.27 & 31.17 & 32.08 & 31.93 & 32.97 & 41.40 & .3 & -1.3 & -3.1 \\
\hline East South Central Total...................... & $\mathbf{w}$ & $\mathbf{w}$ & $\mathbf{w}$ & $\mathbf{w}$ & $\mathbf{w}$ & $\mathbf{w}$ & $\mathbf{w}$ & $\mathbf{w}$ & $\mathbf{w}$ \\
\hline 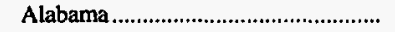 & 36.77 & 36.90 & 38.02 & 39.76 & 41.56 & 53.72 & -.3 & -3.0 & -4.1 \\
\hline 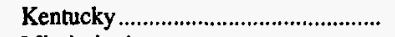 & 41.01 & 41.16 & 41.23 & 43.78 & 47.83 & 54.57 & -.4 & -3.8 & -3.1 \\
\hline 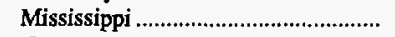 & w & w & w & $w$ & w & $w$ & $w$ & w & $w$ \\
\hline 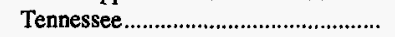 & 33.19 & 33.66 & 34.52 & 35.52 & 36.65 & 47.30 & -1.4 & $-2,4$ & -3.8 \\
\hline West South Central Total ................ & 20.50 & 21.85 & 20.84 & 22.80 & 23.96 & $\mathbf{w}$ & -6.2 & -3.8 & $\mathbf{w}$ \\
\hline 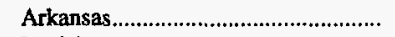 & 40.48 & 42.17 & 42.95 & 44.61 & 46.35 & 56.85 & -4.0 & -3.3 & -3.7 \\
\hline 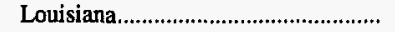 & $\mathbf{w}$ & $\mathbf{w}$ & $\mathbf{w}$ & w & w & w & $\mathbf{w}$ & $\mathbf{w}$ & w \\
\hline 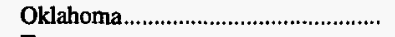 & w & w & w & w & w & 49.33 & w & $\mathbf{w}$ & w \\
\hline 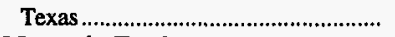 & 17.45 & 18.61 & 17.13 & 18.14 & 19.12 & 29.72 & -6.2 & -2.3 & -5.7 \\
\hline Mountain Total & 25.17 & 27.41 & 27.79 & 28.66 & 30.42 & 38.38 & -8.2 & -4.6 & -4.6 \\
\hline 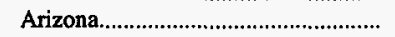 & 37.64 & 39.38 & 39.48 & 40.95 & 41.21 & w & -4.4 & -2.2 & w \\
\hline 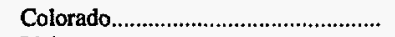 & 24.29 & 27.58 & 27.90 & 30.34 & 30.08 & 32.98 & -11.9 & -5.2 & -3.3 \\
\hline Idaho & 31.73 & 31.76 & 31.95 & 33.29 & 34.85 & 45.32 & -.1 & -2.3 & -3.9 \\
\hline Montana & w & $\mathbf{w}$ & $\mathbf{w}$ & w & w & $\mathbf{w}$ & w & w & $\mathbf{w}$ \\
\hline Nevada & $\mathbf{w}$ & w & $w$ & $\mathbf{w}$ & $\mathbf{w}$ & $w$ & w & $\mathbf{w}$ & $\mathbf{w}$ \\
\hline 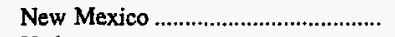 & w & $\mathbf{w}$ & $\mathbf{w}$ & w & $w$ & w & $\mathbf{w}$ & $\mathbf{w}$ & $w$ \\
\hline Utah & 18.36 & 25.31 & 25.84 & 25.35 & 27.16 & 34.23 & -27.4 & -9.3 & -6.7 \\
\hline Wyoming & 21.14 & 21.79 & 22.84 & 23.67 & 25.89 & $w$ & -3.0 & -4.9 & $\mathbf{w}$ \\
\hline Pacific Total & 40.64 & 42.78 & 42.72 & 43.37 & 47.02 & 60.81 & -5.0 & -3.6 & -4.4 \\
\hline 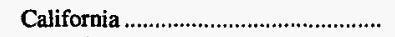 & 38.24 & 41.33 & 41.78 & 42.05 & 46.03 & 63.92 & -7.4 & -4.5 & -5.5 \\
\hline 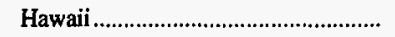 & w & w & w & w & w & 72.39 & $\mathbf{w}$ & $\mathbf{w}$ & w \\
\hline Oregon & w & $\mathbf{w}$ & $\mathbf{w}$ & w & w & $\mathbf{w}$ & $w$ & w & w \\
\hline Washington & 55.02 & 56.06 & 51.76 & 56.25 & 60.81 & 51.47 & -1.8 & -2.5 & .7 \\
\hline U.S. Total & 30.16 & 31.00 & 31.41 & 32.78 & 34.47 & $\mathbf{4 4 . 4 7}$ & -2.7 & -3.3 & -4.2 \\
\hline
\end{tabular}

W Withheld to avoid disclosure of individual company data.

Notes: Price data are for manufacturing plants only. Real prices are in 1992 dollars, calculated using implicit Gross Domestic Product price deflators.

See Appendix D, Table D3. Average prices are based on the cost including insurance, freight, and taxes.

Source: Energy Information Administration, Form ElA-3, "Quarterly Coal Consumption - Manufacturing Plants." 
Table 96. Average Price of Coal Delivered to Coke Plants by Census Division and State, 1986, 1991-1995

(Nominal Dollars per Short Ton)

\begin{tabular}{|c|c|c|c|c|c|c|c|c|c|}
\hline \multirow{2}{*}{ Census Division and State } & \multirow{2}{*}{1995} & \multirow{2}{*}{1994} & \multirow{2}{*}{1993} & \multirow{2}{*}{1992} & \multirow{2}{*}{1991} & \multirow{2}{*}{1986} & \multirow{2}{*}{$\begin{array}{c}\text { Percent } \\
\text { Change } \\
\text { 1994-1995 }\end{array}$} & \multicolumn{2}{|c|}{ Average Annual Percent Change } \\
\hline & & & & & & & & 1991-1995 & 1986-1995 \\
\hline Middle Atlantic Total......................... & $w$ & $\mathbf{w}$ & $\mathbf{w}$ & $\mathbf{w}$ & $\mathbf{w}$ & $\mathbf{w}$ & $\mathbf{w}$ & $\mathbf{w}$ & $\mathbf{w}$ \\
\hline 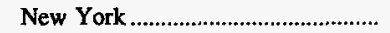 & $\mathbf{w}$ & $\mathbf{w}$ & $\mathbf{w}$ & $\mathrm{w}$ & $\mathbf{w}$ & w & $\mathbf{w}$ & w & $\mathbf{w}$ \\
\hline 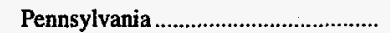 & $\$ 46.11$ & $\$ 46.25$ & $\$ 46.41$ & $\$ 46.49$ & $\$ 46.86$ & $\$ 47.23$ & -0.3 & -0.4 & -0.3 \\
\hline East North Central Total ................... & 49.09 & 47.23 & 49.52 & $\mathbf{5 0 . 3 7}$ & 50.70 & 53.94 & 3.9 & -.8 & -1.0 \\
\hline 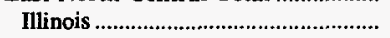 & w & $w$ & w & w & w & 49.11 & $\mathbf{w}$ & $\mathbf{w}$ & w \\
\hline 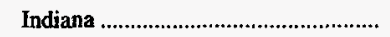 & 52.74 & 50.90 & 52.29 & 53.72 & 53.31 & 55.00 & 3.6 & -.3 & -.5 \\
\hline 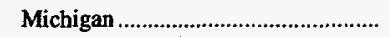 & w & w & - & - & $\mathbf{w}$ & $\mathbf{w}$ & $\mathbf{w}$ & $\mathbf{w}$ & w \\
\hline Ohio & 42.18 & 42.02 & 45.07 & 46.68 & 46.15 & 54.59 & .4 & -2.2 & -2.8 \\
\hline West North Central Total ..................... & - & - & - & - & - & $\mathbf{w}$ & - & $\mathbf{w}$ & $\mathbf{w}$ \\
\hline 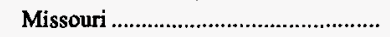 & - & - & - & - & - & w & - & $\mathbf{w}$ & $\mathbf{w}$ \\
\hline 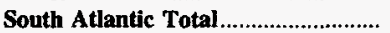 & $\mathbf{w}$ & $\mathbf{w}$ & $w$ & $\mathbf{w}$ & $\mathbf{w}$ & $\mathbf{w}$ & $\mathbf{w}$ & $\mathbf{w}$ & $\mathbf{w}$ \\
\hline 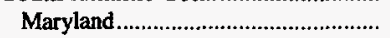 & - & - & - & - & $w$ & $w$ & w & $\mathbf{w}$ & $w$ \\
\hline Virginia & $w$ & w & $\mathbf{w}$ & $w$ & $w$ & $\mathbf{w}$ & $w$ & $w$ & $w$ \\
\hline 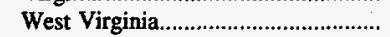 & w & $\mathbf{w}$ & w & $w$ & $\mathbf{w}$ & $w$ & $w$ & $\mathbf{w}$ & $w$ \\
\hline 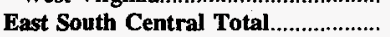 & $\mathbf{w}$ & $\mathbf{w}$ & $\mathbf{w}$ & $\mathbf{w}$ & $\mathbf{w}$ & $\mathbf{w}$ & $\mathbf{w}$ & $\mathbf{w}$ & $\mathbf{w}$ \\
\hline 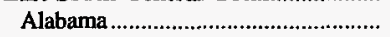 & 48.42 & 47.45 & 47.50 & 47.80 & 48.35 & 48.42 & 2.0 & $*$ & $*$ \\
\hline 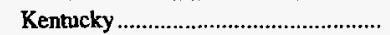 & $w$ & w & $w$ & w & $\mathbf{w}$ & w & $\mathbf{w}$ & $\mathbf{w}$ & $\mathbf{w}$ \\
\hline 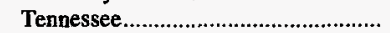 & - & - & - & - & $\mathbf{w}$ & w & - & $\mathbf{w}$ & $w$ \\
\hline West South Central Total ................... & - & - & - & - & - & $\mathbf{w}$ & - & $\mathbf{w}$ & $\mathbf{w}$ \\
\hline 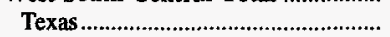 & - & - & - & - & + & w & - & $w$ & $\mathbf{w}$ \\
\hline 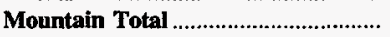 & $\mathbf{w}$ & $\mathbf{w}$ & $\mathbf{w}$ & $\mathbf{w}$ & $\mathbf{w}$ & $\mathbf{w}$ & $\mathbf{w}$ & $\mathbf{w}$ & $\mathbf{w}$ \\
\hline Utah & $\mathbf{w}$ & $\mathbf{w}$ & w & $\mathbf{w}$ & $w$ & $\mathbf{w}$ & $\mathbf{w}$ & w & w \\
\hline 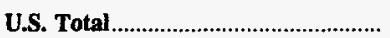 & $\mathbf{4 7 . 3 4}$ & $\mathbf{4 6 . 5 6}$ & 47.44 & 47.92 & 48.88 & $\mathbf{5 0 . 8 5}$ & 1.7 & -.8 & -.8 \\
\hline
\end{tabular}

* Data round to zero.

Withheld to avoid disclosure of individual company data.

Note: Average prices are based on the cost including insurance, freight, and taxes.

Source: Energy Information Administration, Form ELA-5, "Coke Plant Report - Quarterly." 
Table 97. Average Real Price of Coal Delivered to Coke Plants by Census

Division and State, 1986, 1991-1995

(Real Dollars per Short Ton)

\begin{tabular}{|c|c|c|c|c|c|c|c|c|c|}
\hline \multirow{2}{*}{ Census Division and State } & \multirow{2}{*}{1995} & \multirow{2}{*}{1994} & \multirow{2}{*}{1993} & \multirow{2}{*}{1992} & \multirow{2}{*}{1991} & \multirow{2}{*}{1986} & \multirow{2}{*}{$\begin{array}{c}\text { Percent } \\
\text { Change } \\
\text { 1994-1995 }\end{array}$} & \multicolumn{2}{|c|}{ Average Annual Percent Change } \\
\hline & & & & & & & & 1991-1995 & 1986-1995 \\
\hline Middle Atlantic Total........................... & $\mathbf{w}$ & $\mathbf{w}$ & $\mathbf{w}$ & $\mathbf{w}$ & $\mathbf{w}$ & $\mathbf{w}$ & $\mathbf{w}$ & $\mathbf{w}$ & $\mathbf{w}$ \\
\hline 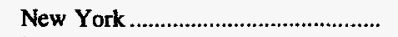 & $\mathbf{w}$ & w & w & $\mathbf{w}$ & $\mathbf{w}$ & $\mathbf{w}$ & $\mathbf{w}$ & $w$ & $\mathbf{w}$ \\
\hline 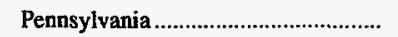 & $\$ 42.89$ & $\$ 44.05$ & $\$ 45.24$ & $\$ 46.49$ & $\$ 48.16$ & $\$ 58.60$ & -2.6 & -2.8 & -3.4 \\
\hline East North Central Total ..................... & 45.67 & 44.98 & 48.26 & 50.37 & 52.11 & 66.92 & 1.5 & -3.2 & -4.1 \\
\hline 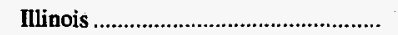 & w & $w$ & $w$ & $w$ & $\mathbf{w}$ & 60.93 & $\mathbf{w}$ & $\mathbf{w}$ & w \\
\hline 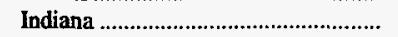 & 49.06 & 48.48 & 50.97 & 53.72 & 54.79 & 68.24 & 1.2 & -2.7 & -3.6 \\
\hline 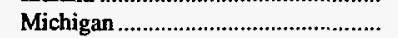 & $w$ & $w$ & - & - & $w$ & $w$ & $w$ & w & w \\
\hline Ohio & 39.24 & 40.02 & 43.92 & 46.68 & 47.43 & 67.73 & -1.9 & -4.6 & -5.9 \\
\hline West North Central Total .................. & - & - & - & - & - & $\mathbf{w}$ & - & $\mathbf{w}$ & $\mathbf{w}$ \\
\hline 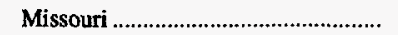 & - & - & - & - & - & $\mathbf{w}$ & - & $\mathbf{w}$ & $\mathbf{w}$ \\
\hline South Atlantic Total ............................... & $\mathbf{w}$ & $\mathbf{w}$ & $\mathbf{w}$ & $\mathbf{w}$ & $\mathbf{w}$ & $\mathbf{w}$ & $\mathbf{w}$ & $\mathbf{w}$ & $\mathbf{w}$ \\
\hline Maryland & - & - & - & - & $\mathbf{w}$ & $\mathbf{w}$ & $\mathbf{w}$ & $\mathbf{w}$ & $w$ \\
\hline 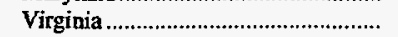 & $\mathbf{w}$ & $\mathbf{w}$ & $\mathbf{w}$ & $\mathbf{w}$ & $w$ & $w$ & $w$ & $\mathbf{w}$ & w \\
\hline 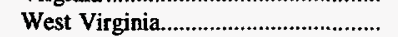 & $\mathbf{w}$ & $\mathbf{w}$ & $\mathbf{w}$ & $\mathbf{w}$ & $\mathbf{w}$ & $\mathbf{w}$ & $w$ & $\mathbf{w}$ & w \\
\hline East South Central Total..................... & $w$ & $\mathbf{w}$ & $\mathbf{w}$ & $\mathbf{w}$ & w & $\mathbf{w}$ & $\mathbf{w}$ & $\mathbf{w}$ & $\mathbf{w}$ \\
\hline Alabama & 45.04 & 45.19 & 46.30 & 47.80 & 49.69 & 60.07 & -.3 & -2.4 & -3.1 \\
\hline 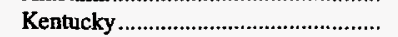 & $w$ & $\mathbf{w}$ & $w$ & w & $\mathbf{w}$ & $\mathbf{w}$ & $w$ & w & w \\
\hline 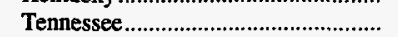 & - & - & - & - & $w$ & $\mathbf{w}$ & - & $w$ & $\mathbf{w}$ \\
\hline West South Central Total ................. & - & - & - & - & - & $\mathbf{w}$ & - & $\mathbf{w}$ & $\mathbf{w}$ \\
\hline 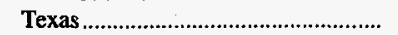 & - & - & - & - & - & $\mathbf{w}$ & - & $\mathbf{w}$ & w \\
\hline Mountain Total & $\mathbf{w}$ & $\mathbf{w}$ & $\mathbf{w}$ & $\mathbf{w}$ & $\mathbf{w}$ & $\mathbf{w}$ & $\mathbf{w}$ & $\mathbf{w}$ & $\mathbf{w}$ \\
\hline Utah & $\mathbf{w}$ & $\mathbf{w}$ & $\mathbf{w}$ & $w$ & $w$ & $\mathbf{w}$ & w & $w$ & $\mathbf{w}$ \\
\hline 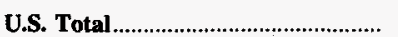 & 44.04 & 44.34 & 46.24 & 47.92 & 50.24 & 63.09 & -.7 & -3.2 & -3.9 \\
\hline
\end{tabular}

w Withheld to avoid disclosure of individual company data.

Notes: Real prices are in 1992 dollars, calculated using implicit Gross Domestic Product price deflators. See Appendix D, Table D3. Average prices are based on the cost including insurance, freight, and taxes.

Source: Energy Information Administration, Form EIA-5, "Coke Plant Report - Quarterly." 



\section{Import/Export Prices}

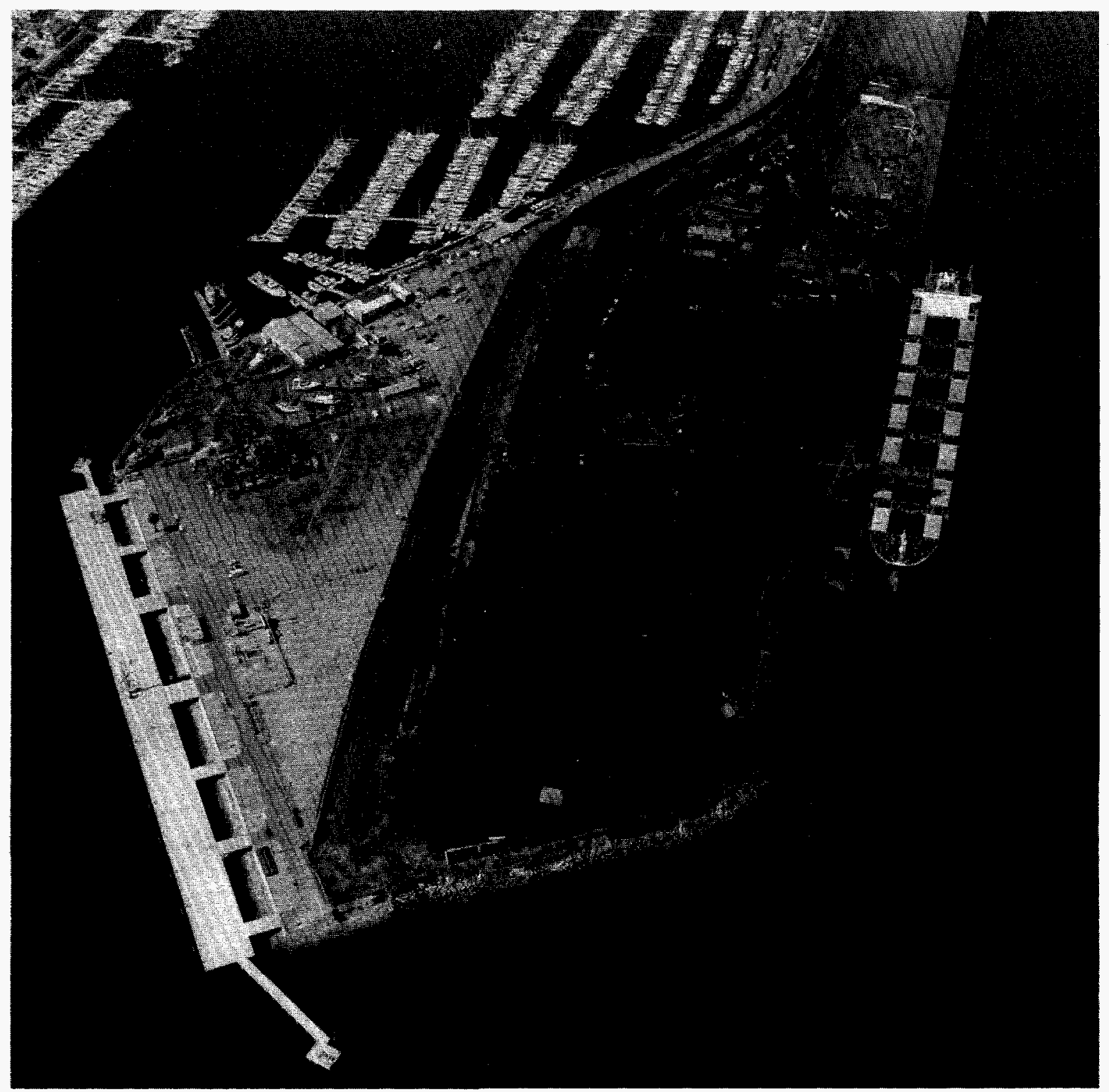

Three transcontinental railroads link the Port of Los Angeles (Southern Pacific, Union Pacific and Atchinson, Topeka and Sante Fe) to destinations across America. The railroad interchange with other U.S., Mexican, and Canadian railroads. Los Angeles' dry bulk facility operated by Kaiser International Corporation handles coal and Petroleum coke. 
Table 98. Average Price of U.S. Coal Imports by Continent and Country of Origin, 1986, 1991-1995

(Nominal Dollars per Short Ton)

\begin{tabular}{|c|c|c|c|c|c|c|c|c|c|}
\hline \multirow{2}{*}{$\begin{array}{c}\text { Continent and Country } \\
\text { of Origin }\end{array}$} & \multirow{2}{*}{1995} & \multirow{2}{*}{1994} & \multirow{2}{*}{1993} & \multirow{2}{*}{1992} & \multirow{2}{*}{1991} & \multirow{2}{*}{1986} & \multirow{2}{*}{$\begin{array}{c}\text { Percent } \\
\text { Change } \\
\text { 1994-1995 }\end{array}$} & \multicolumn{2}{|c|}{ Average Annual Percent Change } \\
\hline & & & & & & & & 1991-1995 & 1986-1995 \\
\hline 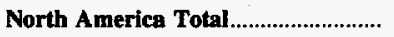 & $\$ 32.59$ & $\$ 30.61$ & $\$ 29.00$ & $\$ 27.88$ & $\$ 25.10$ & $\$ 29.84$ & 6.5 & 6.7 & 1.0 \\
\hline 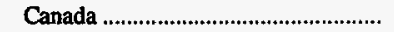 & 32.59 & 30.61 & 29.02 & 27.88 & 25.10 & 29.84 & 6.5 & 6.7 & 1.0 \\
\hline 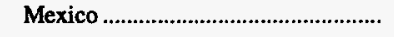 & - & - & 21.19 & - & - & - & - & - & - \\
\hline 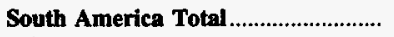 & 32.76 & 29.00 & 27.64 & 33.04 & 34.64 & $\mathbf{3 5 . 2 0}$ & 13.0 & -1.4 & -.8 \\
\hline 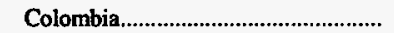 & 31.15 & 27.46 & 27.26 & 32.25 & 32.87 & 35.20 & 13.4 & -1.3 & -1.3 \\
\hline 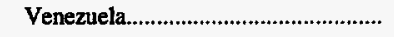 & 35.14 & 32.41 & 28.87 & 35.61 & 40.87 & - & 8.4 & -3.7 & - \\
\hline Europe Total & 25.70 & - & 32.08 & 34.12 & - & - & - & - & - \\
\hline 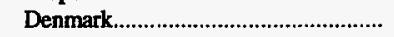 & - & - & 32.08 & - & - & - & - & - & - \\
\hline United Kingdom ............................... & 25.70 & - & - & 34.12 & - & - & - & - & - \\
\hline Asia Total & 35.13 & 34.09 & 42.70 & 38.75 & - & - & 3.1 & - & - \\
\hline India & - & - & - & 23.65 & - & - & - & - & - \\
\hline 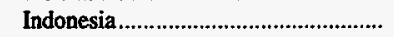 & 35.13 & 33.80 & 42.70 & 40.94 & - & - & 3.9 & - & - \\
\hline 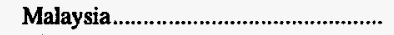 & - & - & - & 47.06 & - & - & - & - & - \\
\hline Vietnam & - & 48.08 & - & - & - & - & -100.0 & - & - \\
\hline Oceania \& Australia Total ................ & 33.57 & 31.16 & 31.56 & 36.07 & 37.97 & 46.64 & 7.7 & $-\mathbf{3 . 0}$ & -3.6 \\
\hline Australia & 30.99 & 30.02 & 31.56 & 36.07 & 37.97 & 46.64 & 3.2 & -4.9 & -4.4 \\
\hline 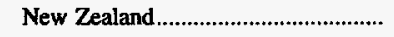 & 46.42 & 44.15 & - & - & - & - & 5.1 & - & - \\
\hline Africa Total & - & 25.33 & 27.81 & 49.29 & - & 37.41 & -100.0 & - & -100.0 \\
\hline 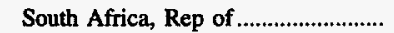 & - & 25.33 & - & 49.29 & - & 37.41 & -100.0 & - & -100.0 \\
\hline 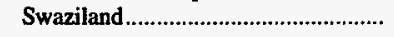 & - & - & 27.81 & - & - & - & - & - & - \\
\hline Total ${ }^{1}$ & 33.11 & 29.98 & 29.36 & 32.48 & 32.34 & 35.61 & 10.4 & .6 & -.8 \\
\hline U.S. Total ${ }^{2}$ & 34.13 & 30.21 & 29.89 & 33.46 & 33.12 & 36.02 & 13.0 & .7 & -.6 \\
\hline
\end{tabular}

1 The average prices presented in this table, with the exception of U.S. Total, are considered to be representative prices for coal imports and fall within the range of $\$ 20$ to $\$ 50$ per short ton, inclusively.

2 U.S. Total is the average price of all coal imports.

Notes: Average price is based on the customs import value. Coal imports include coal to Puerto Rico and the Virgin Islands.

Source: U.S. Department of Commerce, Bureau of the Census, "Monthly Report IM 145." 
Table 99. Average Price of U.S. Coal Exports by Destination, 1986, 1991-1995 (Nominal Dollars per Short Ton)

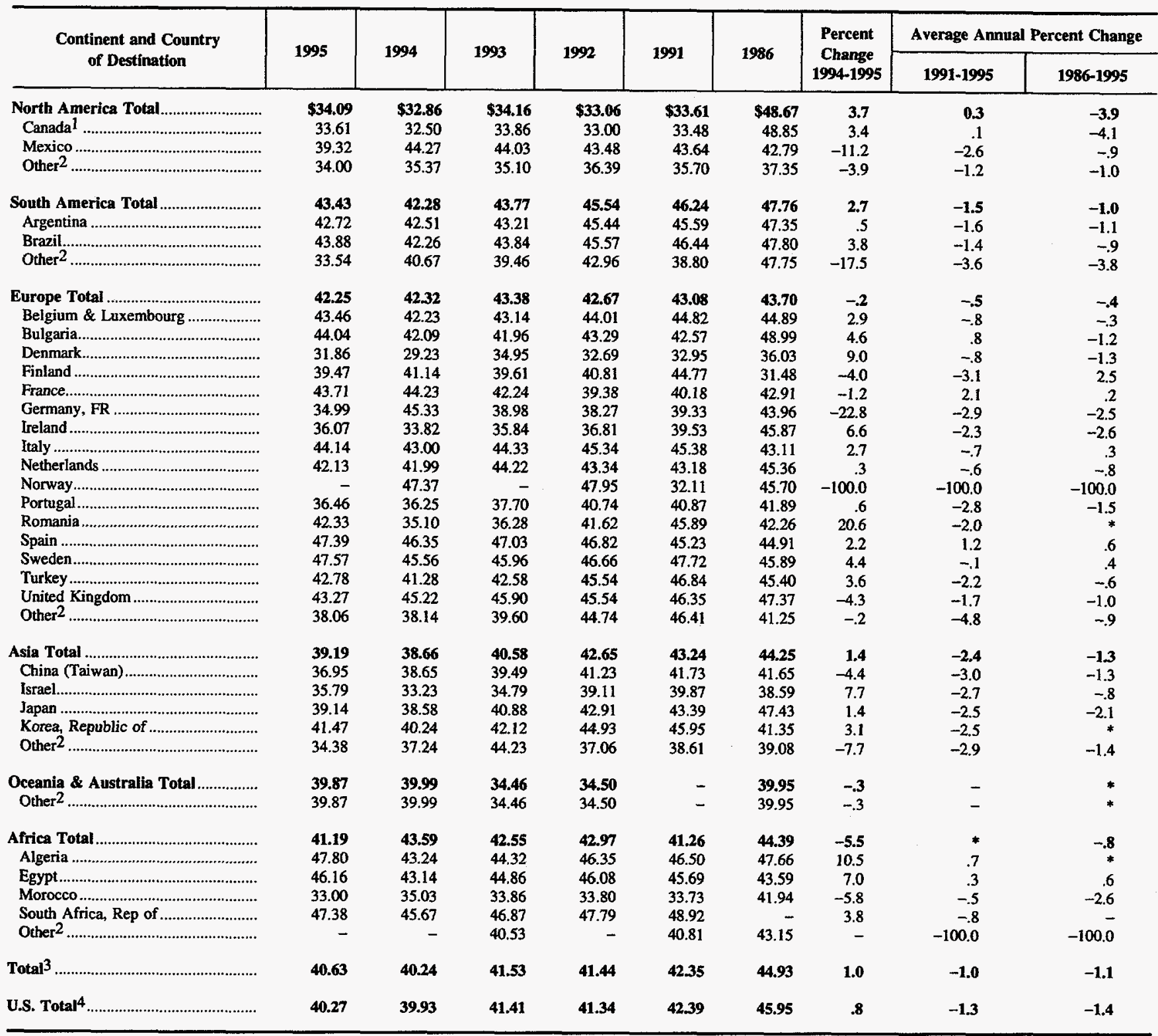

1 Based on the U.S. - Canada Free Trade Agreement, as of January 1990, the U.S. Department of Commerce began reporting statistics on U.S. exports to Canada based on information on imports provided monthly by the Canadian government.

2 Includes countries with exports less than or equal to 50,000 short tons in 1994.

3 The average prices presented in this table, with the exception of U.S. Total, are considered to be representative prices for coal exports and fall within the range of $\$ 28$ to $\$ 50$ per short ton, inclusively.

4 U.S. Total is the average price of all coal exports

* Data round to zero.

Note: Average price is based on the free alongside ship (f.a.s.) value.

Source: U.S. Department of Commerce, Bureau of the Census, "Monthly Report EM 545." 
Table 100. Average Real Price of U.S. Coal Exports by Destination, 1986, 1991-1995

(Real Dollars per Short Ton)

\begin{tabular}{|c|c|c|c|c|c|c|c|c|c|}
\hline \multirow{2}{*}{$\begin{array}{l}\text { Continent and Country } \\
\text { of Destination }\end{array}$} & \multirow{2}{*}{1995} & \multirow{2}{*}{1994} & \multirow{2}{*}{1993} & \multirow{2}{*}{1992} & \multirow{2}{*}{1991} & \multirow{2}{*}{1986} & \multirow{2}{*}{$\begin{array}{c}\text { Percent } \\
\text { Change } \\
\text { 1994-1995 }\end{array}$} & \multicolumn{2}{|c|}{ Average Annual Percent Change } \\
\hline & & & & & & & & 1991-1995 & $1986-1995$ \\
\hline North America Total.............................. & $\$ 31.71$ & $\$ 31.29$ & $\$ 33.29$ & $\$ 33.06$ & $\$ 34.54$ & $\$ 60.39$ & 1.3 & -2.1 & -6.9 \\
\hline 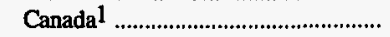 & 31.26 & 30.96 & 33.00 & 33.00 & 34.41 & 60.60 & 1.0 & -2.4 & -7.1 \\
\hline 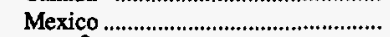 & 36.58 & 42.17 & 42.91 & 43.48 & 44.85 & 53.09 & -13.3 & -5.0 & -4.0 \\
\hline Other ${ }^{2}$ & 31.62 & 33.68 & 34.21 & 36.39 & 36.69 & 46.34 & -6.1 & -3.6 & -4.1 \\
\hline 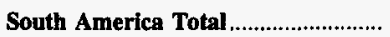 & 40.40 & 40.27 & 42.66 & 45.54 & 47.52 & 59.25 & .3 & -4.0 & -4.2 \\
\hline 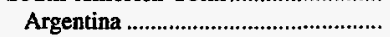 & 39.74 & 40.48 & 42.12 & 45.44 & 46.86 & 58.75 & -1.8 & -4.0 & -4.3 \\
\hline 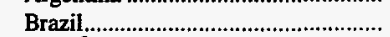 & 40.82 & 40.25 & 42.73 & 45.57 & 47.73 & 59.31 & 1.4 & -3.8 & -4.1 \\
\hline Other $^{2}$ & 31.20 & 38.73 & 38.46 & 42.96 & 39.88 & 59.24 & -19.4 & -5.9 & -6.9 \\
\hline Europe Total ......................................... & 39.30 & 40.31 & 42.28 & 42.67 & 44.28 & 54.22 & -2.5 & -2.9 & -3.5 \\
\hline Belgium \& Luxembourg ....................... & 40.43 & 40.22 & 42.05 & 44.01 & 46.06 & 55.69 & .5 & -3.2 & -3.5 \\
\hline 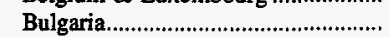 & 40.96 & 40.09 & 40.90 & 43.29 & 43.75 & 60.78 & 2.2 & -1.6 & -4.3 \\
\hline 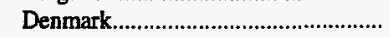 & 29.63 & 27.84 & 34.07 & 32.69 & 33.87 & 44.70 & 6.4 & -3.3 & -4.5 \\
\hline Finland & 36.72 & 39.18 & 38.61 & 40.81 & 46.02 & 39.06 & -6.3 & -5.5 & -.7 \\
\hline France & 40.66 & 42.13 & 41.17 & 39.38 & 41.29 & 53.24 & -3.5 & -.4 & -2.9 \\
\hline Germany, FR & 32.55 & 43.17 & 37.99 & 38.27 & 40.42 & 54.54 & -24.6 & -5.3 & -5.6 \\
\hline Ireland & 33.55 & 32.21 & 34.93 & 36.81 & 40.63 & 56.91 & $\begin{array}{r}-24.0 \\
4.1\end{array}$ & -4.7 & $\begin{array}{l}-3.0 \\
-5.7\end{array}$ \\
\hline Italy & 41.06 & 40.95 & 43.20 & 45.34 & 46.64 & 53.48 & .3 & -3.1 & -2.9 \\
\hline Netherlands & 39.19 & 39.99 & 43.10 & 43.34 & 44.38 & 56.28 & -2.0 & -3.1 & -3.9 \\
\hline Norway & - & 45.12 & - & 47.95 & 33.01 & 56.71 & -100.0 & -100.0 & -100.0 \\
\hline 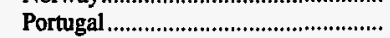 & 33.91 & 34.53 & 36.74 & 40.74 & 42.00 & 51.98 & -1.8 & -5.2 & -4.6 \\
\hline 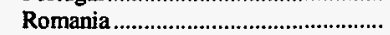 & 39.37 & 33.42 & 35.36 & 41.62 & 47.16 & 52.43 & 17.8 & -4.4 & -3.1 \\
\hline Spain & 44.08 & 44.14 & 45.84 & 46.82 & 46.48 & 55.73 & -.1 & -1.3 & -2.6 \\
\hline Sweden & 44.25 & 43.39 & 44.80 & 46.66 & 49.04 & 56.93 & 2.0 & -2.5 & -2.8 \\
\hline 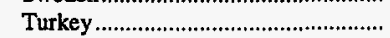 & 39.80 & 39.32 & 41.50 & 45.54 & 48.14 & 56.33 & 1.2 & -4.6 & -3.8 \\
\hline United Kingdom & 40.25 & 43.06 & 44.74 & 45.54 & 47.64 & 58.77 & -6.5 & -4.1 & -4.1 \\
\hline Other ${ }^{2} \ldots \ldots \ldots$ & 35.41 & 36.33 & 38.59 & 44.74 & 47.69 & 51.18 & -2.5 & -7.2 & -4.0 \\
\hline Asia Total & 36.46 & 36.82 & 39.55 & 42.65 & 44.44 & 54.90 & -1.0 & -4.8 & -4.4 \\
\hline China (Taiwan) & 34.37 & 36.81 & 38.49 & 41.23 & 42.88 & 51.68 & -6.6 & -5.4 & -4.4 \\
\hline 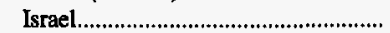 & 33.29 & 31.65 & 33.91 & 39.11 & 40.98 & 47.87 & 5.2 & -5.0 & -3.9 \\
\hline Japan & 36.41 & 36.74 & 39.84 & 42.91 & 44.60 & 58.85 & -.9 & -4.9 & -5.2 \\
\hline 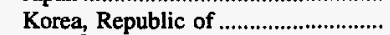 & 38.58 & 38.32 & 41.05 & 44.93 & 47.23 & 51.30 & .7 & -4.9 & -3.1 \\
\hline Other $^{2}$ & 31.98 & 35.46 & 43.11 & 37.06 & 39.69 & 48.48 & -9.8 & -5.3 & -4.5 \\
\hline Oceania \& Australia Total ................. & 37.09 & 38.08 & 33.59 & 34.50 & - & 49.57 & -2.6 & - & -3.2 \\
\hline Other $^{2}$ & 37.09 & 38.08 & 33.59 & 34.50 & - & 49.57 & -2.6 & - & -3.2 \\
\hline 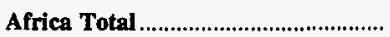 & 38.31 & 41.52 & 41.47 & 42.97 & 42.41 & 55.87 & -7.7 & -2.5 & -3.9 \\
\hline Algeria & 44.46 & 41.18 & 43.20 & 46.35 & 47.80 & 59.13 & 8.0 & -1.8 & -3.1 \\
\hline Egypt & 42.94 & 41.08 & 43.72 & 46.08 & 46.96 & 54.09 & 4.5 & -2.2 & -2.5 \\
\hline Morocco & 30.69 & 33.36 & 33.00 & 33.80 & 34.67 & 52.03 & -8.0 & -3.0 & -5.7 \\
\hline 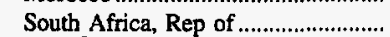 & 44.08 & 43.49 & 45.69 & 47.79 & 50.28 & - & 1.3 & -3.2 & - \\
\hline Other $^{2}$ & - & - & 39.51 & - & 41.94 & 53.54 & - & -100.0 & -100.0 \\
\hline Total ${ }^{3}$ & 37.79 & 38.33 & 40.47 & 41.44 & 43.53 & 55.74 & -1.4 & -3.5 & -4.2 \\
\hline U.S. Total 4 & 37.46 & 38.03 & 40.36 & 41.34 & 43.57 & 57.01 & -1.5 & -3.7 & -4.5 \\
\hline
\end{tabular}

1 Based on the U.S. - Canada Free Trade Agreement, as of January 1990, the U.S. Department of Commerce began reporting statistics on U.S. exports to Canada based on information on imports provided monthly by the Canadian government.

2 Includes countries with exports less than or equal to 50,000 short tons in 1994 .

3 The average prices presented in this table, with the exception of U.S. Total, are considered to be representative prices for coal exports and fall within the range of $\$ 28$ to $\$ 50$ (nominal) per short ton, inclusively.

4 U.S. Total is the average price of all coal exports.

Notes: Real prices are in 1992 dollars, calculated using implicit Gross Domestic Product price deflators. See Appendix D, Table D3. Average prices are based on the free alongside ship (f.a.s.) value.

Source: U.S. Department of Commerce, Bureau of the Census, "Monthly Report EM 545." 
Table 101. Average Price of U.S. Metallurgical Coal Exports by Destination, 1986, 1991-1995

(Nominal Dollars per Short Ton)

\begin{tabular}{|c|c|c|c|c|c|c|c|c|c|}
\hline \multirow{2}{*}{$\begin{array}{l}\text { Continent and Country } \\
\text { of Destination }\end{array}$} & \multirow{2}{*}{1995} & \multirow{2}{*}{1994} & \multirow{2}{*}{1993} & \multirow{2}{*}{1992} & \multirow{2}{*}{1991} & \multirow{2}{*}{1986} & \multirow{2}{*}{$\begin{array}{c}\text { Percent } \\
\text { Change } \\
\text { 1994-1995 }\end{array}$} & \multicolumn{2}{|c|}{ Average Annual Percent Change } \\
\hline & & & & & & & & 1991-1995 & 1986-1995 \\
\hline North America Total............................. & $\$ 37.19$ & $\$ 35.08$ & $\$ 36.03$ & $\$ 36.43$ & $\$ 38.54$ & $\$ 49.10$ & 6.0 & -0.9 & $-\mathbf{3 . 0}$ \\
\hline 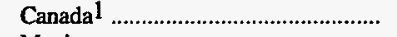 & 36.57 & 34.58 & 35.66 & 36.34 & 38.43 & 49.36 & 5.8 & -1.2 & -3.3 \\
\hline Mexico & 46.45 & 44.53 & 45.16 & 47.92 & 49.20 & 44.71 & 4.3 & -1.4 & .4 \\
\hline Other $^{2}$ & - & - & - & - & - & 41.10 & - & - & -100.0 \\
\hline South America Total ........................... & 43.63 & 42.29 & 43.91 & 45.65 & 46.44 & 47.75 & 3.2 & -1.5 & -1.0 \\
\hline Argentina & 42.85 & 42.56 & 43.63 & 45.45 & 45.62 & 47.35 & .7 & -1.5 & -1.1 \\
\hline 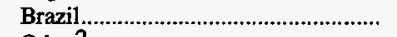 & 43.89 & 42.26 & 43.94 & 45.65 & 46.48 & 47.80 & 3.8 & -1.4 & -.9 \\
\hline Other $^{2}$ & 30.26 & - & - & 47.40 & 49.78 & 47.85 & - & -11.7 & -5.0 \\
\hline Europe Total & 45.75 & 44.54 & 45.57 & 46.57 & 46.91 & 46.33 & 2.7 & -.6 & -.1 \\
\hline Belgium \& Luxembourg ....................... & 45.97 & 45.07 & 45.88 & 47.02 & 47.52 & 46.06 & 2.0 & -.8 & * \\
\hline 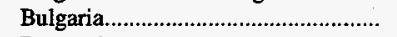 & 44.04 & 42.13 & 41.99 & 43.29 & 42.57 & - & 4.5 & .8 & - \\
\hline 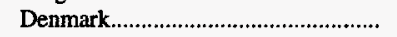 & - & - & 36.29 & - & 29.37 & 39.43 & - & -100.0 & -100.0 \\
\hline Finland & 42.65 & 42.34 & 39.61 & 40.81 & 46.21 & - & .7 & -2.0 & - \\
\hline 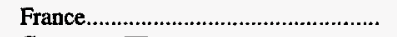 & 45.08 & 44.36 & 45.17 & 44.39 & 43.96 & 45.69 & 1.6 & .6 & -.1 \\
\hline Germany, FR & 47.52 & 45.88 & 46.17 & 45.79 & 48.55 & 46.14 & 3.5 & -.5 & .3 \\
\hline Italy & 46.61 & 45.32 & 45.93 & 47.44 & 48.28 & 47.38 & 2.8 & -.9 & -.2 \\
\hline 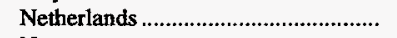 & 46.56 & 45.07 & 46.17 & 47.00 & 47.11 & 47.10 & 3.3 & -.3 & -.1 \\
\hline 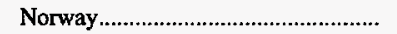 & - & 47.37 & - & 47.95 & - & 45.29 & -100.0 & - & -100.0 \\
\hline 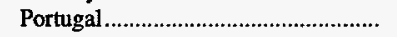 & 46.45 & - & 38.43 & 44.33 & 48.11 & 46.99 & - & -.9 & -.1 \\
\hline 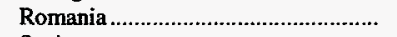 & 42.91 & 32.65 & 36.26 & 41.62 & 46.47 & 42.26 & 31.4 & -2.0 & .2 \\
\hline Spain & 47.76 & 46.36 & 47.47 & 48.48 & 48.45 & 46.70 & 3.0 & -.3 & .2 \\
\hline 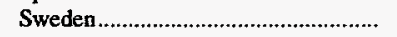 & 47.57 & 45.56 & 45.96 & 46.67 & 47.76 & 45.84 & 4.4 & -.1 & .4 \\
\hline 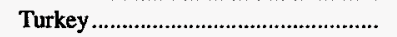 & 43.47 & 41.28 & 42.58 & 45.54 & 46.87 & 45.81 & 5.3 & -1.9 & -.6 \\
\hline 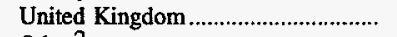 & 46.81 & 45.15 & 46.64 & 47.72 & 48.46 & 47.78 & 3.7 & -.9 & -.2 \\
\hline Other 2 & - & 38.00 & 48.31 & 44.75 & 46.41 & 45.84 & -100.0 & -100.0 & -100.0 \\
\hline Asia Total & 42.38 & $\mathbf{4 1 . 4 5}$ & 44.04 & 45.39 & 46.00 & 47.95 & 2.2 & -2.0 & -1.4 \\
\hline 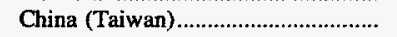 & 44.48 & 42.48 & 44.86 & 45.63 & 46.80 & 46.62 & 4.7 & -1.3 & -.5 \\
\hline 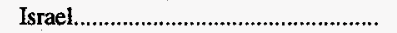 & 40.91 & - & - & 40.13 & 42.18 & - & - & -.8 & - \\
\hline Japan & 41.14 & 40.57 & 43.53 & 44.98 & 45.52 & 48.40 & 1.4 & -2.5 & -1.8 \\
\hline 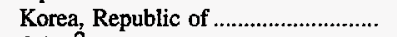 & 45.98 & 44.00 & 45.67 & 47.13 & 47.54 & 46.48 & 4.5 & -.8 & -.1 \\
\hline Other ${ }^{2}$ & - & - & 45.13 & - & - & 44.57 & - & - & -100.0 \\
\hline Africa Total & 46.93 & 43.95 & 45.25 & 46.29 & 45.03 & 45.93 & 6.8 & 1.0 & .2 \\
\hline Algeria & 47.80 & 43.24 & 44.32 & 46.35 & 46.50 & 47.67 & 10.5 & .7 & $*$ \\
\hline 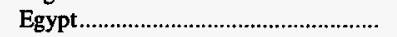 & 46.17 & 43.14 & 44.86 & 46.09 & 45.70 & 43.59 & 7.0 & .3 & .6 \\
\hline Morocco & - & - & - & 34.22 & 34.54 & - & - & -100.0 & - \\
\hline South Africa, Rep of & 47.38 & 45.67 & 46.87 & 47.79 & 48.92 & - & 3.8 & -.8 & - \\
\hline Other ${ }^{2}$ & - & - & 40.53 & - & - & 43.15 & - & - & -100.0 \\
\hline Total $^{3}$ & 43.96 & $\mathbf{4 2 . 7 4}$ & 44.11 & 45.36 & 46.07 & 47.02 & 2.9 & -1.2 & -.7 \\
\hline U.S. Total ${ }^{4}$ & 44.30 & $\mathbf{4 2 . 7 7}$ & 44.11 & 45.41 & 46.15 & 47.81 & 3.6 & -1.0 & -.8 \\
\hline
\end{tabular}

1 Based on the U.S. - Canada Free Trade Agreement, as of January 1990, the U.S. Department of Commerce began reporting statistics on U.S. exports to Canada based on information on imports provided monthly by the Canadian government.

2 Includes countries with exports less than or equal to 50,000 short tons in 1994.

3 The average prices presented in this table, with the exception of U.S. Total, are considered to be representative prices for coal exports and fall within the range of $\$ 28$ to $\$ 50$ per short ton, inclusively.

4 U.S. Total is the average price of all coal exports.

* Data round to zero.

Note: Average price is based on the free alongside ship (f.a.s.) value.

Source: U.S. Department of Commerce, Bureau of the Census, "Monthly Report EM 545." 
Table 102. Average Real Price of U.S. Metallurgical Coal Exports by Destination, 1986, 1991-1995

(Real Dollars per Short Ton)

\begin{tabular}{|c|c|c|c|c|c|c|c|c|c|}
\hline \multirow{2}{*}{$\begin{array}{l}\text { Continent and Country } \\
\text { of Destination }\end{array}$} & \multirow{2}{*}{1995} & \multirow{2}{*}{1994} & \multirow{2}{*}{1993} & \multirow{2}{*}{1992} & \multirow{2}{*}{1991} & \multirow{2}{*}{1986} & \multirow{2}{*}{$\begin{array}{c}\text { Percent } \\
\text { Change } \\
\text { 1994-1995 }\end{array}$} & \multicolumn{2}{|c|}{ Average Annual Percent Change } \\
\hline & & & & & & & & 1991-1995 & 1986-1995 \\
\hline 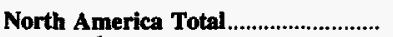 & $\$ 34.60$ & $\$ 33.41$ & $\mathbf{\$ 3 5 . 1 2}$ & $\$ 36.43$ & $\$ 39.61$ & $\$ 60.91$ & 3.6 & -3.3 & -6.1 \\
\hline 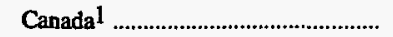 & 34.02 & 32.93 & 34.76 & 36.34 & 39.50 & 61.24 & 3.3 & -3.7 & -6.3 \\
\hline Mexico & 43.21 & 42.41 & 44.02 & 47.92 & 50.56 & 55.48 & 1.9 & -3.8 & -2.7 \\
\hline 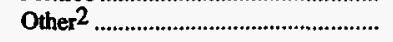 & - & - & - & - & - & 51.00 & - & - & -100.0 \\
\hline South America Total ........................... & 40.59 & 40.27 & 42.80 & 45.65 & 47.73 & 59.25 & .8 & -4.0 & -4.1 \\
\hline Argentina & 39.86 & 40.53 & 42.52 & 45.45 & 46.88 & 58.75 & -1.6 & -4.0 & -4.2 \\
\hline 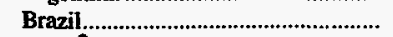 & 40.83 & 40.25 & 42.83 & 45.65 & 47.77 & 59.31 & 1.4 & -3.8 & -4.1 \\
\hline Other 2 & 28.15 & - & - & 47.40 & 51.16 & 59.36 & - & -13.9 & -7.9 \\
\hline 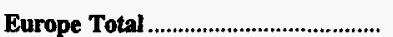 & 42.56 & 42.42 & 44.42 & $\mathbf{4 6 . 5 7}$ & 48.21 & $\mathbf{5 7 . 4 8}$ & .3 & -3.1 & -3.3 \\
\hline 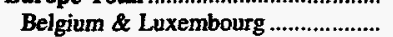 & 42.76 & 42.93 & 44.71 & 47.02 & 48.84 & 57.14 & -.4 & -3.3 & -3.2 \\
\hline 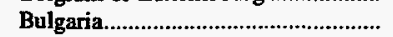 & 40.96 & 40.12 & 40.93 & 43.29 & 43.75 & - & 2.1 & -1.6 & - \\
\hline 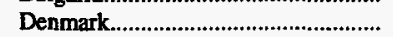 & - & - & 35.37 & - & 30.18 & 48.92 & - & -100.0 & -100.0 \\
\hline Finland & 39.67 & 40.33 & 38.60 & 40.81 & 47.49 & - & -1.6 & -4.4 & - \\
\hline 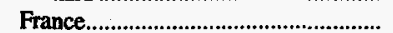 & 41.94 & 42.25 & 44.02 & 44.39 & 45.18 & 56.69 & -.7 & -1.8 & -3.3 \\
\hline 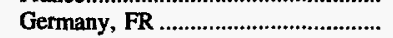 & 44.20 & 43.70 & 45.00 & 45.79 & 49.89 & 57.24 & 1.1 & -3.0 & -2.8 \\
\hline Italy & 43.36 & 43.17 & 44.76 & 47.44 & 49.62 & 58.79 & .4 & -3.3 & -3.3 \\
\hline Netherlands & 43.31 & 42.92 & 45.00 & 47.00 & 48.41 & 58.44 & .9 & -2.7 & -3.3 \\
\hline Norway & - & 45.12 & - & 47.95 & - & 56.19 & -100.0 & - & -100.0 \\
\hline 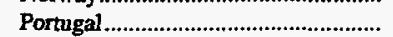 & 43.21 & - & 37.46 & 44.33 & 49.44 & 58.30 & - & -3.3 & -3.3 \\
\hline Romania & 39.92 & 31.09 & 35.34 & 41.62 & 47.76 & 52.43 & 28.4 & -4.4 & -3.0 \\
\hline Spain & 44.43 & 44.15 & 46.27 & 48.48 & 49.79 & 57.94 & .6 & -2.8 & -2.9 \\
\hline 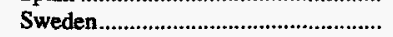 & 44.25 & 43.39 & 44.80 & 46.67 & 49.09 & 56.87 & 2.0 & -2.6 & -2.8 \\
\hline Turkey & 40.43 & 39.32 & 41.51 & 45.54 & 48.17 & 56.83 & 2.8 & -4.3 & -3.7 \\
\hline United Kingdom & 43.55 & 43.00 & 45.46 & 47.72 & 49.80 & 59.27 & 1.3 & -3.3 & -3.4 \\
\hline Other ${ }^{2}$ & - & 36.20 & 47.08 & 44.75 & 47.70 & 56.87 & -100.0 & -100.0 & -100.0 \\
\hline 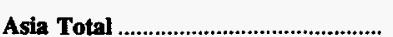 & 39.42 & 39.48 & 42.92 & 45.39 & 47.27 & 59.49 & -1 & -4.4 & -4.5 \\
\hline 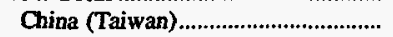 & 41.37 & 40.46 & 43.73 & 45.63 & 48.09 & 57.85 & 2.3 & -3.7 & -3.6 \\
\hline Israel & 38.06 & - & - & 40.13 & 43.35 & - & - & -3.2 & - \\
\hline 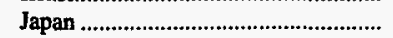 & 38.27 & 38.63 & 42.43 & 44.98 & 46.78 & 60.05 & -.9 & -4.9 & -4.9 \\
\hline 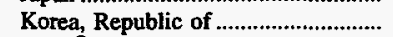 & 42.77 & 41.90 & 44.52 & 47.13 & 48.86 & 57.66 & 2.1 & -3.3 & -3.3 \\
\hline Other 2 & - & - & 43.99 & - & - & 55.30 & - & - & -100.0 \\
\hline 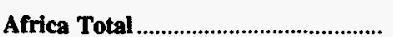 & 43.65 & 41.85 & 44.11 & 46.29 & 46.28 & 56.98 & 4.3 & -1.4 & -2.9 \\
\hline Algeria & 44.46 & 41.18 & 43.20 & 46.35 & 47.80 & 59.15 & 8.0 & -1.8 & -3.1 \\
\hline 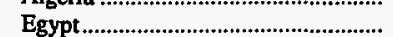 & 42.95 & 41.08 & 43.73 & 46.09 & 46.96 & 54.09 & 4.5 & -2.2 & -2.5 \\
\hline Morocco & - & - & - & 34.22 & 35.49 & - & - & -100.0 & - \\
\hline 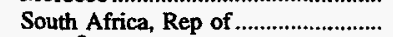 & 44.08 & 43.49 & 45.69 & 47.79 & 50.28 & - & 1.3 & -3.2 & - \\
\hline Other ${ }^{2}$ & - & - & 39.51 & - & - & 53.54 & - & - & -100.0 \\
\hline Tota| 3 & 40.89 & 40.70 & 42.99 & 45.36 & 47.35 & $\mathbf{5 8 . 3 4}$ & .5 & -3.6 & -3.9 \\
\hline U.S. Total ${ }^{4}$ & 41.21 & $\mathbf{4 0 . 7 3}$ & 42.99 & 45.41 & $\mathbf{4 7 . 4 4}$ & 59.32 & 1.2 & -3.4 & -4.0 \\
\hline
\end{tabular}

1 Based on the U.S. - Canada Free Trade Agreement, as of January 1990, the U.S. Department of Commerce began reporting statistics on U.S. exports to Canada based on information on imports provided monthly by the Canadian government.

2 Includes countries with exports less than or equal to 50,000 short tons in 1994.

3 The average prices presented in this table, with the exception of U.S. Total, are considered to be representative prices for coal exports and fall within the range of $\$ 28$ to $\$ 50$ (nominal) per short ton, inclusively.

4 U.S. Total is the average price of all coal exports.

Notes: Real prices are in 1992 dollars, calculated using implicit Gross Domestic Product price deflators. See Appendix D, Table D3. Average prices are based on the free alongside ship (f.a.s.) value.

Source: U.S. Department of Commerce, Bureau of the Census, "Monthly Report EM 545." 
Table 103. Average Price of U.S. Steam Coal Exports by Destination, 1986, 1991-1995

(Nominal Dollars per Short Ton)

\begin{tabular}{|c|c|c|c|c|c|c|c|c|c|}
\hline \multirow{2}{*}{$\begin{array}{c}\text { Continent and Country } \\
\text { of Destination }\end{array}$} & \multirow{2}{*}{1995} & \multirow{2}{*}{1994} & \multirow{2}{*}{1993} & \multirow{2}{*}{1992} & \multirow{2}{*}{1991} & \multirow{2}{*}{1986} & \multirow{2}{*}{$\begin{array}{l}\text { Percent } \\
\text { Change } \\
\text { 1994-1995 }\end{array}$} & \multicolumn{2}{|c|}{ Average Annual Percent Change } \\
\hline & & & & & & & & 1991-1995 & 1986-1995 \\
\hline North America Total .............................. & $\$ 31.13$ & $\$ 30.54$ & $\$ 31.85$ & $\mathbf{\$ 3 1 . 4 2}$ & $\mathbf{\$ 3 0 . 4 9}$ & $\$ 48.46$ & 1.9 & 0.5 & -4.8 \\
\hline 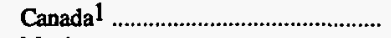 & 30.59 & 30.41 & 31.66 & 31.35 & 30.28 & 48.60 & .6 & .3 & -5.0 \\
\hline 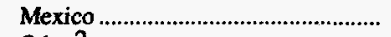 & 35.11 & 41.42 & 40.40 & 38.19 & 39.16 & 38.96 & -15.2 & -2.7 & -1.1 \\
\hline Other ${ }^{2}$ & 34.00 & 35.37 & 35.10 & 36.39 & 35.70 & 28.49 & -3.9 & -1.2 & 2.0 \\
\hline South America Total & 35.66 & 37.59 & 40.05 & 42.38 & 39.13 & 47.80 & -5.1 & -2.3 & -3.2 \\
\hline Argentina & 36.09 & 34.46 & 37.81 & 38.57 & 35.95 & - & 4.7 & .1 & - \\
\hline 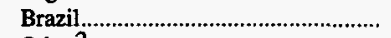 & 34.81 & - & 40.61 & 42.95 & 43.64 & 47.86 & - & -5.5 & -3.5 \\
\hline Other $^{2}$ & 35.68 & 40.67 & 39.46 & 36.96 & 36.32 & 39.95 & -12.3 & -.4 & -1.2 \\
\hline 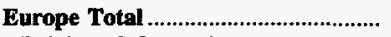 & 36.93 & 36.01 & 37.47 & 36.99 & 37.74 & 38.42 & 2.6 & -.5 & -.4 \\
\hline Belgium \& Luxembourg ..................... & 35.07 & 33.49 & 34.09 & 36.35 & 37.08 & 39.50 & 4.7 & -1.4 & -1.3 \\
\hline Bulgaria & - & 41.40 & 41.51 & - & - & 48.99 & -100.0 & - & -100.0 \\
\hline Denmark & 31.86 & 29.23 & 34.34 & 32.69 & 33.14 & 35.16 & 9.0 & -1.0 & -1.1 \\
\hline Finland & 35.53 & 35.47 & 40.85 & - & 40.82 & 31.48 & .2 & -3.4 & 1.3 \\
\hline 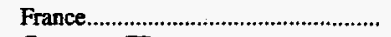 & 35.13 & 38.11 & 31.83 & 33.64 & 33.93 & 35.87 & -7.8 & .9 & -.2 \\
\hline Germany, FR & 33.31 & 40.67 & 34.19 & 35.82 & 34.95 & 42.91 & -18.1 & -1.2 & -2.8 \\
\hline Ireland & 36.07 & 33.82 & 35.84 & 36.81 & 39.53 & 45.87 & 6.6 & -2.3 & -2.6 \\
\hline Italy & 41.70 & 38.30 & 40.26 & 41.32 & 41.71 & 38.28 & 8.9 & * & .9 \\
\hline 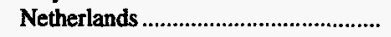 & 36.68 & 35.95 & 40.21 & 39.24 & 39.59 & 41.50 & 2.0 & -1.9 & -1.4 \\
\hline 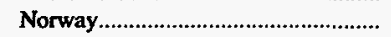 & - & - & - & - & 32.11 & 48.76 & - & -100.0 & -100.0 \\
\hline Portugal & 36.28 & 36.25 & 37.62 & 40.38 & 40.52 & 41.51 & .1 & -2.7 & -1.5 \\
\hline 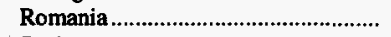 & 39.08 & 37.26 & 36.29 & - & 42.64 & - & 4.9 & -2.1 & - \\
\hline Spain & 41.25 & 40.84 & 39.22 & 38.47 & 34.32 & 35.26 & 1.0 & 4.7 & 1.8 \\
\hline 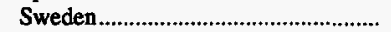 & 48.54 & - & - & 34.53 & 41.64 & 47.92 & - & 3.9 & .1 \\
\hline Turkey & 30.98 & - & 40.84 & 40.72 & 46.38 & 38.85 & - & -9.6 & -2.5 \\
\hline United Kingdom & 33.39 & 47.21 & 40.87 & 38.96 & 39.69 & 38.02 & -29.3 & -4.2 & -1.4 \\
\hline Other ${ }^{2}$ & 38.06 & 40.92 & 29.07 & 42.07 & 40.81 & 32.79 & -7.0 & -1.7 & 1.7 \\
\hline Asia Total & 34.79 & 35.24 & 36.52 & $\mathbf{3 8 . 2 7}$ & 39.18 & 39.59 & -1.3 & -2.9 & -1.4 \\
\hline 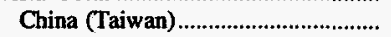 & 35.66 & 38.28 & 39.01 & 40.63 & 41.16 & 41.36 & -6.8 & -3.5 & -1.6 \\
\hline 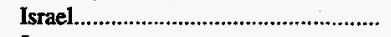 & 34.63 & 33.23 & 34.79 & 38.89 & 39.41 & 38.59 & 4.2 & -3.2 & -1.2 \\
\hline Japan & 35.03 & 33.69 & 35.45 & 35.98 & 36.05 & 39.80 & 4.0 & -.7 & -1.4 \\
\hline 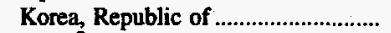 & 32.78 & 32.56 & 34.34 & 37.24 & 39.97 & 36.53 & .7 & -4.8 & -1.2 \\
\hline Other ${ }^{2}$ & 34.38 & 37.24 & 37.31 & 37.06 & 38.61 & 37.14 & -7.7 & -2.9 & -.8 \\
\hline Oceania \& Australia Total ................ & 39.87 & 39.99 & 34.46 & $\mathbf{3 4 . 5 0}$ & - & 39.95 & -.3 & - & $*$ \\
\hline Other ${ }^{2}$ & 39.87 & 39.99 & 34.46 & 34.50 & - & 39.95 & -.3 & - & $*$ \\
\hline Africa Total & 33.01 & 35.12 & 33.86 & 33.78 & 33.56 & 41.94 & -6.0 & -.4 & -2.6 \\
\hline 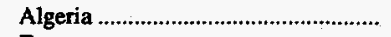 & - & - & - & - & - & 40.01 & - & - & -100.0 \\
\hline Egypt & 40.81 & 40.89 & 40.88 & 39.92 & 40.81 & - & -.2 & $*$ & - \\
\hline Morocco & 33.00 & 35.03 & 33.86 & 33.77 & 33.53 & 41.94 & -5.8 & -.4 & -2.6 \\
\hline 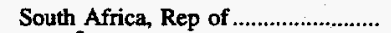 & 39.80 & - & - & - & - & - & - & - & - \\
\hline Other $^{2}$ & - & - & - & - & 40.81 & - & - & -100.0 & - \\
\hline Total $^{3}$ & 35.31 & 34.65 & 36.10 & 35.86 & 36.86 & 41.48 & 1.9 & -1.1 & -1.8 \\
\hline U.S. Total 4 & 34.51 & 34.34 & 36.03 & 35.73 & 36.91 & 42.60 & .5 & -1.7 & $-\mathbf{2 . 3}$ \\
\hline
\end{tabular}

1 Based on the U.S. - Canada Free Trade Agreement, as of January 1990, the U.S. Department of Commerce began reporting statistics on U.S. exports to Canada based on information on imports provided monthly by the Canadian government.

2 Includes countries with exports less than or equal to 50,000 short tons in 1994.

3 The average prices presented in this table, with the exception of U.S. Total, are considered to be representative prices for coal exports and fall within the range of $\$ 28$ to $\$ 50$ per short ton, inclusively.

4 U.S. Total is the average price of all coal exports.

* Data round to zero.

Notes: Average price is based on the free alongside ship (f.a.s.) value. Steam coal includes bituminous, subbituminous, lignite, and anthracite Source: U.S. Department of Commerce, Bureau of the Census, "Monthly Report EM 545." 
Table 104. Average Real Price of U.S. Steam Coal Exports by Destination, 1986, 1991-1995

(Real Dollars per Short Ton)

\begin{tabular}{|c|c|c|c|c|c|c|c|c|c|}
\hline \multirow{2}{*}{$\begin{array}{l}\text { Continent and Country } \\
\text { of Destination }\end{array}$} & \multirow{2}{*}{1995} & \multirow{2}{*}{1994} & \multirow{2}{*}{1993} & \multirow{2}{*}{1992} & \multirow{2}{*}{1991} & \multirow{2}{*}{1986} & \multirow{2}{*}{$\begin{array}{c}\text { Percent } \\
\text { Change } \\
\text { 1994-1995 }\end{array}$} & \multicolumn{2}{|c|}{ Average Annual Percent Change } \\
\hline & & & & & & & & 1991-1995 & 1986-1995 \\
\hline North America Total.............................. & $\$ 28.96$ & $\$ 29.09$ & $\$ 31.04$ & $\$ 31.42$ & $\$ 31.34$ & $\$ 60.13$ & -0.4 & -1.9 & -7.8 \\
\hline 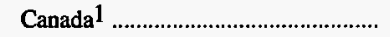 & 28.46 & 28.96 & 30.86 & 31.35 & 31.13 & 60.30 & -1.7 & -2.2 & -8.0 \\
\hline 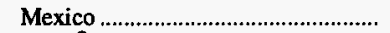 & 32.66 & 39.44 & 39.37 & 38.19 & 40.24 & 48.34 & -17.2 & -5.1 & -4.3 \\
\hline Other $^{2}$ & 31.62 & 33.68 & 34.21 & 36.39 & 36.69 & 35.34 & -6.1 & -3.6 & -1.2 \\
\hline South America Total .......................... & 33.17 & 35.80 & 39.04 & 42.38 & 40.22 & $\mathbf{5 9 . 3 0}$ & -7.3 & -4.7 & -6.3 \\
\hline 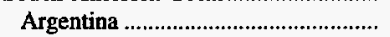 & 33.57 & 32.82 & 36.85 & 38.57 & 36.94 & - & 2.3 & -2.4 & - \\
\hline 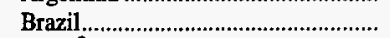 & 32.39 & - & 39.58 & 42.95 & 44.85 & 59.38 & - & -7.8 & -6.5 \\
\hline Other $^{2}$ & 33.19 & 38.73 & 38.46 & 36.96 & 37.33 & 49.57 & -14.3 & -2.9 & -4.3 \\
\hline 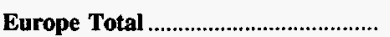 & $\mathbf{3 4 . 3 6}$ & $\mathbf{3 4 . 3 0}$ & 36.52 & 36.99 & 38.79 & 47.66 & .2 & $-\mathbf{3 . 0}$ & -3.6 \\
\hline 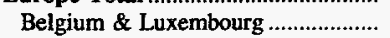 & 32.62 & 31.89 & 33.23 & 36.35 & 38.11 & 49.01 & 2.3 & -3.8 & -4.4 \\
\hline 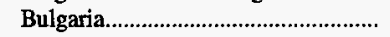 & - & 39.42 & 40.46 & - & - & 60.78 & -100.0 & - & -100.0 \\
\hline Denmark & 29.63 & 27.84 & 33.47 & 32.69 & 34.06 & 43.62 & 6.4 & -3.4 & -4.2 \\
\hline 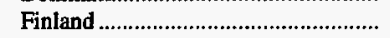 & 33.05 & 33.78 & 39.81 & - & 41.96 & 39.06 & -2.1 & -5.8 & -1.8 \\
\hline 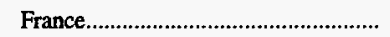 & 32.68 & 36.29 & 31.02 & 33.64 & 34.87 & 44.50 & -10.0 & -1.6 & -3.4 \\
\hline Germany, FR & 30.99 & 38.74 & 33.33 & 35.82 & 35.92 & 53.24 & -20.0 & -3.6 & -5.8 \\
\hline Ireland & 33.55 & 32.21 & 34.93 & 36.81 & 40.63 & 56.91 & 4.1 & -4.7 & -5.7 \\
\hline 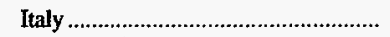 & 38.79 & 36.47 & 39.24 & 41.32 & 42.87 & 47.49 & 6.4 & -2.5 & -2.2 \\
\hline Netherlands & 34.13 & 34.24 & 39.19 & 39.24 & 40.69 & 51.49 & -3 & -4.3 & -4.5 \\
\hline 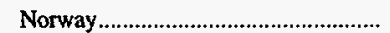 & - & - & - & - & 33.01 & 60.50 & - & -100.0 & -100.0 \\
\hline 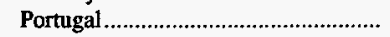 & 33.75 & 34.53 & 36.67 & 40.38 & 41.64 & 51.51 & -2.3 & -5.1 & -4.6 \\
\hline Romania & 36.35 & 35.48 & 35.37 & - & 43.82 & - & 2.4 & -4.6 & - \\
\hline 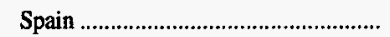 & 38.37 & 38.90 & 38.22 & 38.47 & 35.27 & 43.75 & -1.3 & 2.1 & -1.4 \\
\hline Sweden & 45.15 & - & - & 34.53 & 42.80 & 59.45 & - & 1.3 & -3.0 \\
\hline Turkey & 28.82 & - & 39.80 & 40.72 & 47.67 & 48.20 & - & -11.8 & -5.5 \\
\hline United Kingdom & 31.06 & 44.96 & 39.83 & 38.96 & 40.79 & 47.17 & -30.9 & -6.6 & -4.5 \\
\hline Other ${ }^{2}$ & 35.41 & 38.98 & 28.34 & 42.07 & 41.95 & 40.68 & -9.1 & -4.1 & -1.5 \\
\hline 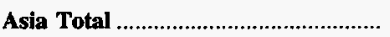 & 32.36 & 33.56 & 35.59 & 38.27 & 40.26 & 49.12 & -3.5 & -5.3 & -4.5 \\
\hline 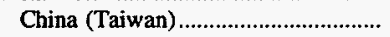 & 33.17 & 36.46 & 38.02 & 40.63 & 42.30 & 51.31 & -9.0 & -5.9 & -4.7 \\
\hline 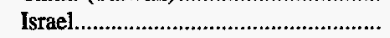 & 32.21 & 31.65 & 33.91 & 38.89 & 40.50 & 47.87 & 1.8 & -5.6 & -4.3 \\
\hline Japan & 32.58 & 32.09 & 34.55 & 35.98 & 37.05 & 49.38 & 1.5 & -3.1 & -4.5 \\
\hline 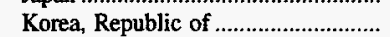 & 30.49 & 31.01 & 33.47 & 37.24 & 41.08 & 45.32 & -1.7 & -7.2 & -4.3 \\
\hline Other $^{2}$ & 31.98 & 35.46 & 36.37 & 37.06 & 39.69 & 46.08 & -9.8 & -5.3 & -4.0 \\
\hline Oceania \& Australia Total ................ & 37.09 & 38.08 & 33.59 & $\mathbf{3 4 . 5 0}$ & - & 49.57 & -2.6 & - & -3.2 \\
\hline Other ${ }^{2}$ & 37.09 & 38.08 & 33.59 & 34.50 & - & 49.57 & -2.6 & - & -3.2 \\
\hline Africa Total & 30.70 & 33.44 & 33.01 & 33.78 & 34.49 & $\mathbf{5 2 . 0 3}$ & -8.2 & -2.9 & -5.7 \\
\hline Algeria & - & - & - & - & - & 49.64 & - & - & -100.0 \\
\hline 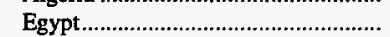 & 37.96 & 38.94 & 39.85 & 39.92 & 41.95 & - & -2.5 & -2.5 & - \\
\hline Morocco & 30.69 & 33,36 & 33.00 & 33.77 & 34.46 & 52.03 & -8.0 & -2.8 & -5.7 \\
\hline 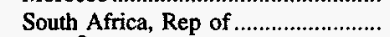 & 37.03 & - & - & - & - & - & - & - & - \\
\hline Other ${ }^{2}$ & - & - & - & - & 41.94 & - & - & -100.0 & - \\
\hline Total $^{3}$ & 32.85 & 33.00 & 35.18 & 35.86 & 37.89 & 51.46 & -.5 & -3.5 & -4.9 \\
\hline U.S. Total ${ }^{4} \ldots \ldots \ldots \ldots \ldots \ldots \ldots \ldots \ldots \ldots \ldots \ldots \ldots \ldots$ & 32.11 & 32.71 & 35.12 & 35.73 & 37.93 & 52.86 & -1.8 & -4.1 & -5.4 \\
\hline
\end{tabular}

1 Based on the U.S. - Canada Free Trade Agreement, as of January 1990, the U.S. Department of Commerce began reporting statistics on U.S. exports to Canada based on information on imports provided monthly by the Canadian government.

2 Includes countries with exports less than or equal to 50,000 short tons in 1994.

3 The average prices presented in this table, with the exception of U.S. Total, are considered to be representative prices for coal exports and fall within the range of $\$ 28$ to $\$ 50$ (nominal) per short ton, inclusively.

4 U.S. Total is the average price of all coal exports.

Notes: Real prices are in 1992 dollars, calculated using implicit Gross Domestic Product price deflators. See Appendix D, Table D3. Average prices are based on the free alongside ship (f.a.s.) value. Steam coal includes bituminous, subbituminous, lignite, and anthracite.

Source: U.S. Department of Commerce, Bureau of the Census, "Monthly Report EM 545." 


\section{Coal Quality and Emissions}

As of January 1, 1995, recoverable U.S. coal reserves were estimated to total 274 billion short tons (Table 105). Reserves of low sulfur coal (defined as coal containing from 0 to 0.60 pounds of sulfur per million Btu) are estimated to represent 37 percent of all reserves, with medium sulfur coal (containing 0.61 to 1.67 pounds sulfur per million $B t u$ ) and high sulfur coal (containing more than 1.67 pounds sulfur per million Btu) each accounting for 32 percent of total reserves.

Compared with 1994, the average sulfur content of coal delivered to the Nation's electric utilities during
1995 declined 7.3 percent to 1.08 percent sulfur by weight, while the average ash content declined 1.4 percent to 9.23 percent by weight (Table 106). The average heat content of electric utility coal declined 1 percent to 10,248 Btu per pound.

By comparison, the average heat content of coal delivered to manufacturing and coke plants during 1995 increased 0.4 percent to $11,367 \mathrm{Btu}$ per pound, while the average ash content and average sulfur content of industrial coal remained relatively constant at 7.61 percent and 1.15 percent, respectively. 
Table 105. Estimate of Recoverable Reserves of Coal by Sulfur Range, State, and Mine Type

(Million Short Tons Remaining as of January 1, 1995)

\begin{tabular}{|c|c|c|c|c|c|c|c|}
\hline \multirow{2}{*}{ State and Type of Mining } & \multicolumn{6}{|c|}{$\begin{array}{l}\text { Sulfur Content } \\
\text { (pounds of sulfur per million Btu) }\end{array}$} & \multirow{2}{*}{ Total } \\
\hline & $<=0.40$ & $0.41-0.60$ & $0.61-0.83$ & $0.84-1.67$ & $1.68-2.50$ & $>2.50$ & \\
\hline Alabama & - & 413 & 374 & 2,036 & 194 & - & 3,018 \\
\hline 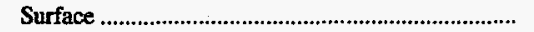 & - & 255 & 269 & 1,670 & 137 & - & 2,332 \\
\hline 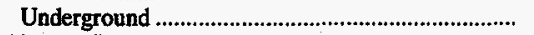 & - & 158 & 105 & 366 & 57 & - & 686 \\
\hline 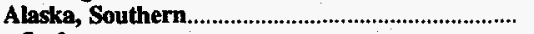 & 2,453 & 94 & - & - & - & - & 2,548 \\
\hline 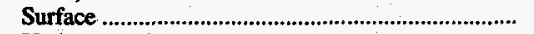 & 386 & 41 & - & - & - & - & 427 \\
\hline 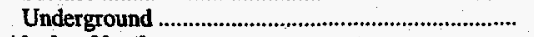 & 2,067 & 53 & - & - & - & - & 2,120 \\
\hline Alaska, Northern & - & - & - & - & - & - & - \\
\hline Surface & - & - & - & - & - & - & - \\
\hline 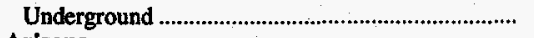 & - & - & - & - & - & - & - \\
\hline Arizona & - & 121 & - & - & - & - & 121 \\
\hline Surface & - & 69 & - & - & - & - & 69 \\
\hline 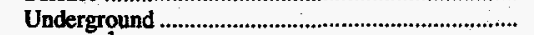 & - & 51 & - & $\sim$ & - & - & 51 \\
\hline 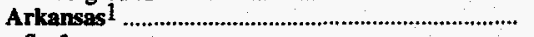 & - & 8 & 163 & 44 & 6 & 7 & 228 \\
\hline Surface & - & 2 & 73 & 24 & 1 & 1 & 101 \\
\hline 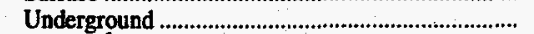 & - & 7 & 90 & 20 & 5 & 6 & 127 \\
\hline 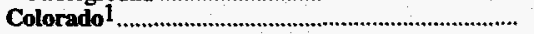 & 3,674 & 2,316 & 3,684 & 326 & 96 & - & 10,096 \\
\hline Surface & 273 & 120 & 3,325 & 41 & 14 & - & 3,772 \\
\hline 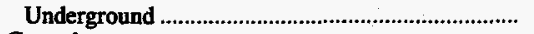 & 3,400 & 2,196 & 359 & 285 & 82 & $=$ & 6,323 \\
\hline Georgia & 1 & 1 & * & $\bullet$ & - & * & 2 \\
\hline 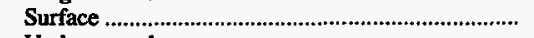 & * & * & * & * & * & * & 1 \\
\hline 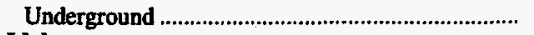 & * & * & * & * & * & * & 1 \\
\hline Idaho & * & 1 & 1 & 1 & - & - & 2 \\
\hline 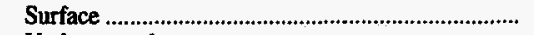 & - & - & - & - & - & - & - \\
\hline 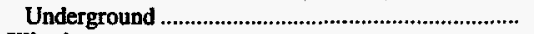 & * & 1 & 1 & 1 & - & - & 2 \\
\hline Hlinois & 5 & 113 & 350 & 1,456 & 1,567 & $\mathbf{3 0 , 4 7 7}$ & 33,969 \\
\hline 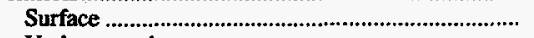 & $=$ & - & 1 & 43 & 291 & 9,031 & 9,366 \\
\hline 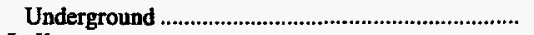 & 5 & 113 & 349 & 1,413 & 1,276 & 21,445 & 24,603 \\
\hline Indiana & - & 312 & 185 & 674 & 1,125 & $\mathbf{2 , 0 3 3}$ & 4,329 \\
\hline 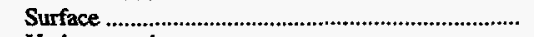 & - & 63 & 27 & 107 & 149 & 307 & 654 \\
\hline 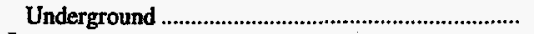 & - & 248 & 158 & 567 & 976 & 1,727 & 3,675 \\
\hline lowa & - & - & - & - & 407 & 720 & $\mathbf{1 , 1 2 7}$ \\
\hline 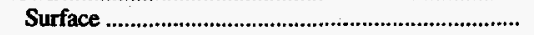 & - & - & - & - & 320 & - & 320 \\
\hline 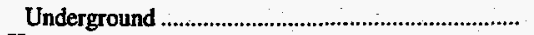 & - & - & - & - & 87 & 720 & 807 \\
\hline 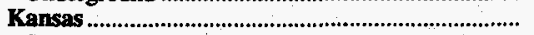 & - & - & - & - & 226 & 457 & 683 \\
\hline 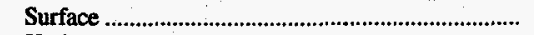 & - & - & - & - & 226 & 457 & 683 \\
\hline 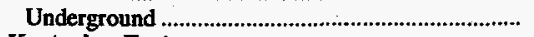 & - & - & - & & & & \\
\hline Kentucky, Eastern & 175 & 2,077 & 1,434 & 1,769 & 860 & 657 & 6,973 \\
\hline Surface & 140 & 1,657 & 1,144 & 1,412 & 686 & 525 & 5,563 \\
\hline & 35 & 420 & 290 & 358 & 174 & 133 & 1,410 \\
\hline Kentucky, Western & - & - & - & 156 & $\mathbf{2 , 7 2 3}$ & 6,406 & 9,285 \\
\hline 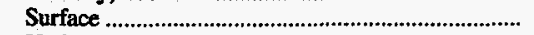 & - & - & - & 125 & 926 & 1,276 & 2,327 \\
\hline 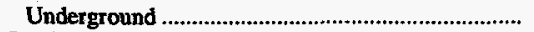 & - & - & - & 32 & 1,798 & 5,130 & 6,959 \\
\hline Loulsiana & - & - & - & 349 & - & - & 349 \\
\hline 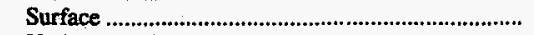 & - & - & - & 349 & - & - & 349 \\
\hline 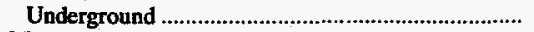 & - & - & - & - & - & - & - \\
\hline Maryland & - & 31 & 58 & 118 & 205 & - & 412 \\
\hline 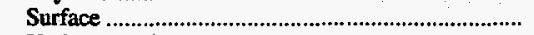 & - & 3 & 8 & 13 & 32 & - & 56 \\
\hline 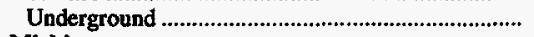 & - & 28 & 50 & 105 & 173 & - & 356 \\
\hline Michigan & - & - & 8 & 23 & 16 & 11 & 59 \\
\hline 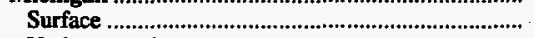 & - & - & 1 & 2 & 1 & * & 3 \\
\hline 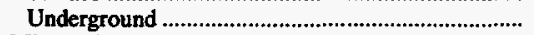 & - & - & 8 & 21 & 16 & 11 & 55 \\
\hline Missouri ……………………………………………... & - & - & - & - & 170 & 3,681 & $\mathbf{3 , 8 5 1}$ \\
\hline Surface & - & - & - & - & 150 & 3,012 & 3,162 \\
\hline 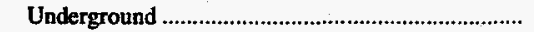 & - & - & - & - & 20 & 670 & 689 \\
\hline Montana & 33,577 & 16,828 & 16,776 & 4,815 & 2,022 & 1,371 & 75,389 \\
\hline Surface & 18,014 & 6,995 & 9,499 & 2,444 & 1,610 & 906 & 39,466 \\
\hline 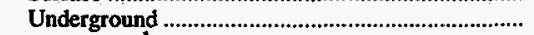 & 15,563 & 9,833 & 7,277 & 2,371 & 413 & 466 & 35,923 \\
\hline New Mexicol ${ }^{1} . . . .1$. & 62 & $\mathbf{2 , 6 3 8}$ & 1,890 & 3,625 & - & - & 8,215 \\
\hline Surface & 37 & 1,229 & 1,348 & 2,587 & - & - & 5,202 \\
\hline 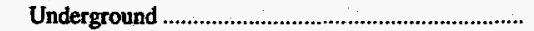 & 25 & 1,409 & 542 & 1,038 & - & - & 3,013 \\
\hline North Carolina & - & - & * & 2 & 2 & 1 & 5 \\
\hline Surface & - & - & - & - & - & - & - \\
\hline 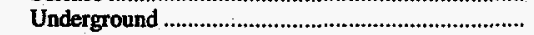 & - & - & * & 2 & 2 & 1 & 5 \\
\hline 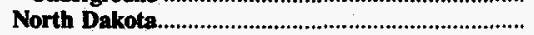 & 436 & 750 & 1,375 & $\mathbf{3 , 4 3 8}$ & 857 & 369 & 7,224 \\
\hline 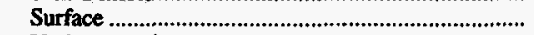 & 436 & 750 & 1,375 & 3,438 & 857 & 369 & 7,224 \\
\hline 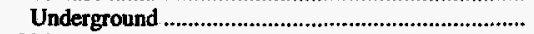 & - & - & & & & & \\
\hline Ohio & 82 & 169 & 335 & 1,049 & 2,636 & $\mathbf{7 , 4 4 7}$ & 11,718 \\
\hline Surface & 20 & 96 & 168 & 416 & 863 & 2,304 & 3,867 \\
\hline 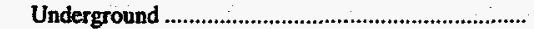 & 62 & 73 & 168 & 634 & 1,773 & 5,143 & 7,852 \\
\hline
\end{tabular}

See footnotes at end of table. 
Table 105. Estimate of Recoverable Reserves of Coal by Sulfur Range, State, and Mine Type (Continued)

(Million Short Tons Remaining as of January 1, 1995)

\begin{tabular}{|c|c|c|c|c|c|c|c|}
\hline \multirow{2}{*}{ State and Type of Mining } & \multicolumn{6}{|c|}{$\begin{array}{l}\text { Sulfur Content } \\
\text { (pounds of sulfur per million Btu) }\end{array}$} & \multirow{2}{*}{ Total } \\
\hline & $<=0.40$ & $0.41-0.60$ & $0.61-0.83$ & $0.84-1.67$ & $1.68 \cdot 2.50$ & $>2.50$ & \\
\hline Oklahoma. & - & 220 & 123 & 178 & 114 & 181 & 816 \\
\hline 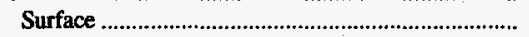 & - & 66 & 25 & 34 & 33 & 82 & 240 \\
\hline Underground & - & 154 & 98 & 145 & 80 & 100 & 577 \\
\hline Oregon & 4 & 1 & 3 & - & 1 & 1 & 9 \\
\hline Surface & 1 & * & 1 & - & * & $*$ & 2 \\
\hline 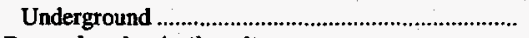 & 3 & 1 & 2 & - & 1 & 1 & 7 \\
\hline Pennsylvania, Anthracite & 180 & 468 & 96 & 17 & 2 & * & 763 \\
\hline 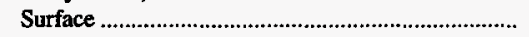 & 85 & 263 & 62 & 11 & 1 & * & 422 \\
\hline Underground & 95 & 205 & 35 & 6 & 1 & - & 341 \\
\hline 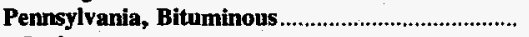 & - & 283 & 807 & 5,001 & 4,151 & $\mathbf{1 , 5 1 7}$ & 11,757 \\
\hline 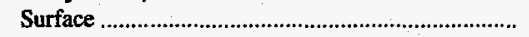 & - & 22 & 64 & 307 & 227 & 135 & 756 \\
\hline 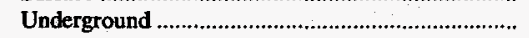 & - & 260 & 742 & 4,694 & 3,923 & 1,381 & 11,001 \\
\hline 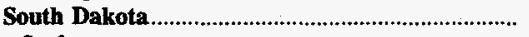 & - & - & 104 & 1 & 172 & - & $\mathbf{2 7 7}$ \\
\hline Surface & - & - & 104 & 1 & 172 & - & 277 \\
\hline 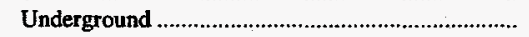 & - & - & - & - & - & - & - \\
\hline Tennessee & - & 106 & 62 & 224 & 99 & - & 491 \\
\hline Surface & - & 39 & 23 & 93 & 40 & - & 195 \\
\hline Underground & - & 67 & 38 & 131 & 59 & - & 295 \\
\hline 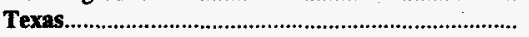 & - & - & 590 & 5,681 & 3,409 & 376 & 10,057 \\
\hline Surface & - & - & 590 & 5,681 & 3,409 & 376 & 10,057 \\
\hline 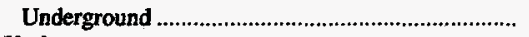 & - & - & - & - & - & - & - \\
\hline Utah & 378 & 678 & 554 & 864 & 225 & 301 & 3,001 \\
\hline Surface & 8 & 32 & 19 & 85 & 35 & 34 & 212 \\
\hline Underground & 370 & 646 & 535 & 780 & 190 & 267 & 2,788 \\
\hline Virginia1 & 195 & 632 & 403 & 133 & - & - & 1,362 \\
\hline Surface & 43 & 197. & 161 & 46 & - & - & 447 \\
\hline Underground & 152 & 435 & 241 & 87 & - & - & 915 \\
\hline Washington & 63 & 92 & 86 & 489 & - & - & 729 \\
\hline Surface & - & - & - & 54 & - & - & 54 \\
\hline 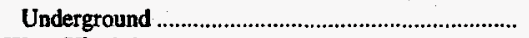 & 63 & 92 & 86 & 434 & - & - & 675 \\
\hline West Virginia & 653 & 6,497 & 2,736 & 4,036 & 2,516 & 3,211 & 19,649 \\
\hline 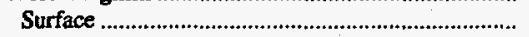 & 116 & 1,464 & 568 & 447 & 180 & 107 & 2,881 \\
\hline Underground & 537 & 5,034 & 2,168 & 3,589 & 2,337 & 3,104 & 16,769 \\
\hline 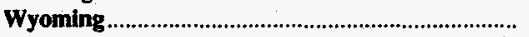 & 6,786 & 17,586 & 10,186 & $\mathbf{7 , 4 3 8}$ & 1,168 & 2,241 & 45,403 \\
\hline Surface & 4,434 & 8,822 & 4,134 & 2,314 & 527 & 2,208 & 22,439 \\
\hline Underground & 2,352 & 8,764 & 6,052 & 5,123 & 641 & 33 & 22,964 \\
\hline U.S. Total & 48,723 & 52,434 & 42,380 & 43,943 & 24,968 & 61,466 & 273,913 \\
\hline 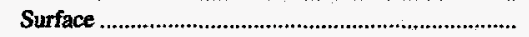 & 23,993 & 22,185 & 22,987 & 21,742 & 10,888 & 21,130 & 122,925 \\
\hline 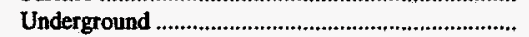 & 24,730 & 30,249 & 19,393 & 22,201 & 14,080 & 40,336 & 150,988 \\
\hline
\end{tabular}

1 Data include minor amounts of anthracite (all occurring in heat content categories greater than 23.00 million short tons) as follows: Arkansas 52.2 , Colorado 13.4, New Mexico 1.2, and Virginia 70.5, expressed in million short tons.

* Data round to zero.

Note: Totals may not equal sum of components due to independent rounding.

Source: Energy Information Administration, U.S. Coal Reserves: A Review and Update (DOE/EIA-0529(95)), August, 1996. 
Table 106. Average Quality of Coal Received at Electric Utilities by Census Division and State, 1986, 1991-1995

\begin{tabular}{|c|c|c|c|c|c|c|c|c|c|}
\hline \multirow{2}{*}{$\begin{array}{c}\text { Census Division and State } \\
\text { and Quality } 1\end{array}$} & \multirow{2}{*}{1995} & \multirow{2}{*}{1994} & \multirow{2}{*}{1993} & \multirow{2}{*}{1992} & \multirow{2}{*}{1991} & \multirow{2}{*}{1986} & \multirow{2}{*}{$\begin{array}{c}\text { Percent } \\
\text { Change } \\
\text { 1994-1995 }\end{array}$} & \multicolumn{2}{|c|}{ Average Annual Percent Change } \\
\hline & & & & & & & & 1991-1995 & 1986-1995 \\
\hline \multicolumn{10}{|l|}{ New England } \\
\hline 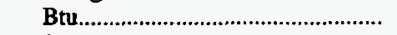 & 12,848 & 12,897 & 13,033 & 13,120 & 13,176 & 13,249 & * & -1 & * \\
\hline 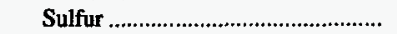 & .84 & .98 & 1.11 & 1.18 & 1.17 & 1.23 & -14.3 & -7.8 & -4.1 \\
\hline 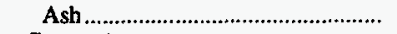 & 7.48 & 7.49 & 7.62 & 7.66 & 7.52 & 7.49 & -.2 & -.1 & * \\
\hline \multicolumn{10}{|l|}{ Connecticut } \\
\hline 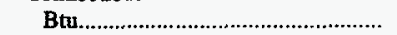 & 13,110 & 13,094 & 13,144 & 13,167 & 13,238 & 13,172 & * & * & * \\
\hline Sulfur & .56 & .54 & .55 & .55 & .55 & .51 & 4.7 & .7 & 1.1 \\
\hline Ash & 7.05 & 7.38 & 6.95 & 6.25 & 6.07 & 6.76 & -4.5 & 3.8 & .5 \\
\hline Massachusetts & & & & & & & & & \\
\hline 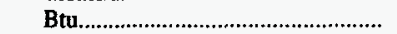 & 12,698 & 12,814 & 12,951 & 13,070 & 13,141 & 13,219 & -1 & -1 & * \\
\hline Sulfur & .71 & .91 & 1.03 & 1.17 & 1.22 & 1.19 & -22.0 & -12.5 & -5.5 \\
\hline Ash & 7.83 & 7.85 & 8.10 & 8.26 & 8.14 & 7.65 & -.2 & -1.0 & .3 \\
\hline New Hampshire & & & & & & & & & \\
\hline 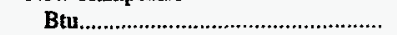 & 13,111 & 13,032 & 13,179 & 13,260 & 13,247 & 13,443 & 1 & * & * \\
\hline Sulfur & 1.38 & 1.52 & 1.62 & 1.61 & 1.43 & 2.03 & -9.1 & -.8 & -4.2 \\
\hline Ash & 6.74 & 6.40 & 6.75 & 6.52 & 6.43 & 7.44 & 5.4 & 1.2 & -1.1 \\
\hline Middle Atlantic & & & & & & & & & \\
\hline 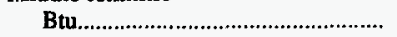 & 12,474 & 12,509 & 12,556 & 12,555 & 12,455 & 12,381 & * & * & * \\
\hline Sulfur & 2.03 & 2.01 & 1.96 & 1.99 & 2.04 & 2.07 & 1.0 & -.1 & -.2 \\
\hline Ash & 11.93 & 11.52 & 11.29 & 11.45 & 12.28 & 12.71 & 3.6 & -.7 & -.7 \\
\hline New Jersey & & & & & & & & & \\
\hline 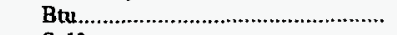 & 13,282 & 13,341 & 13,397 & 13,465 & 13,402 & 13,229 & * & * & * \\
\hline Sulfur & 1.21 & 1.29 & 1.29 & 1.29 & 1.27 & 1.35 & -6.2 & -1.2 & -1.2 \\
\hline Ash & 7.51 & 7.44 & 7.21 & 6.84 & 7.31 & 7.99 & .9 & .7 & -.7 \\
\hline New York & & & & & & & & & \\
\hline 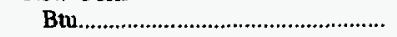 & 13,051 & 12,959 & 12,914 & 12,978 & 12,923 & 12,722 & 1 & * & * \\
\hline 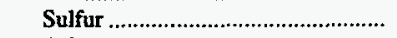 & 1.79 & 1.71 & 1.55 & 1.65 & 1.77 & 1.92 & 5.2 & .3 & -.7 \\
\hline Ash & 7.90 & 7.98 & 8.15 & 8.02 & 8.88 & 10.13 & -1.1 & -2.9 & -2.7 \\
\hline Pennsylvania & & & & & & & & & \\
\hline Btu. & 12,315 & 12,368 & 12,443 & 12,399 & 12,302 & 12,275 & * & * & * \\
\hline 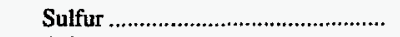 & 2.12 & 2.11 & 2.07 & 2.12 & 2.14 & 2.13 & .4 & -.2 & * \\
\hline Ash & 12.97 & 12.49 & 12.11 & 12.56 & 13.30 & 13.40 & 3.9 & -.6 & -.4 \\
\hline East North Central & & & & & & & & & \\
\hline 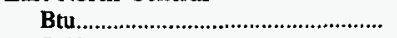 & 10,676 & 10,837 & 10,885 & 11,005 & 10,971 & 11,082 & -1 & -1 & * \\
\hline 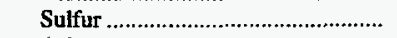 & 1.28 & 1.55 & 1.61 & 1.77 & 1.82 & 1.87 & -17.0 & -8.4 & -4.1 \\
\hline Ash $\ldots \ldots \ldots \ldots \ldots \ldots$ & 8.00 & 8.34 & 8.41 & 8.72 & 8.75 & 9.00 & -4.0 & -2.2 & -1.3 \\
\hline Illinois & & & & & & & & & \\
\hline 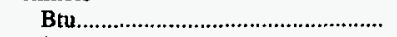 & 9,970 & 10,181 & 10,362 & 10,666 & 10,721 & 10,537 & -2 & -2 & -1 \\
\hline Sulfur & 1.14 & 1.46 & 1.63 & 1.91 & 2.00 & 1.82 & -22.0 & -13.1 & -5.1 \\
\hline Ash & 7.01 & 7.44 & 7.51 & 8.19 & 8.54 & 8.34 & -5.8 & -4.8 & -1.9 \\
\hline Indiana & & & & & & & & & \\
\hline Btu. & 10,338 & 10,535 & 10,539 & 10,628 & 10,570 & 10,679 & -2 & -1 & * \\
\hline 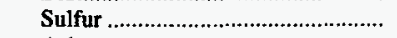 & 1.57 & 1.76 & 1.78 & 1.88 & 1.98 & 2.39 & -11.1 & -5.7 & -4.6 \\
\hline Ash & 7.65 & 8.09 & 8.23 & 8.43 & 8.45 & 9.11 & -5.5 & -2.5 & -1.9 \\
\hline Michigan & & & & & & & & & \\
\hline Btu. & 10,677 & 10,925 & 10,853 & 10,995 & 11,052 & 11,721 & -2 & -1 & -1 \\
\hline Sulfur & .63 & .68 & .68 & .69 & .70 & .82 & -7.4 & -2.5 & -2.9 \\
\hline Ash & 6.66 & 6.97 & 6.61 & 6.76 & 6.58 & 7.20 & -4.4 & .3 & -.9 \\
\hline Ohio & & & & & & & & & \\
\hline Btu & 12,122 & 12,052 & 12,049 & 11,983 & 11,945 & 11,910 & 1 & * & * \\
\hline Sulfur & 1.89 & 2.34 & 2.39 & 2.57 & 2.63 & 2.45 & -19.2 & -7.9 & -2.8 \\
\hline Ash & 10.84 & 10.91 & 11.01 & 11.24 & 11.39 & 11.30 & -.6 & -1.2 & -.4 \\
\hline Wisconsin & & & & & & & & & \\
\hline Btu. & 9,351 & 9,565 & 9,490 & 9,725 & 9,643 & 9,662 & -2 & -1 & ${ }^{*}$ \\
\hline Sulfur & .46 & .51 & .49 & .71 & .81 & .90 & -9.7 & -13.1 & -7.1 \\
\hline Ash & 6.03 & 6.27 & 6.11 & 6.18 & 6.20 & 6.48 & -3.8 & -.7 & -.8 \\
\hline West North Central & & & & & & & & & \\
\hline 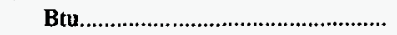 & 8,418 & 8,480 & 8,366 & 8,602 & 8,665 & 8,778 & -1 & -1 & * \\
\hline 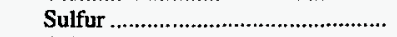 & .54 & .68 & .63 & .91 & .92 & 1.22 & -20.2 & -12.5 & -8.6 \\
\hline 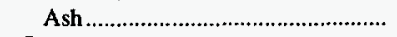 & 6.41 & 6.82 & 6.74 & 7.18 & 7.23 & 8.08 & -6.0 & -2.9 & -2.5 \\
\hline Lowa & & & & & & & & & \\
\hline 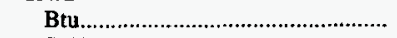 & 8,678 & 8,783 & 8,660 & 8,867 & 8,890 & 9,186 & -1 & -1 & -1 \\
\hline Sulfur & .49 & .57 & .52 & .67 & .67 & .92 & -13.5 & -7.5 & -6.8 \\
\hline Ash & 5.60 & 5.59 & 5.47 & 5.78 & 5.99 & 6.44 & .2 & -1.7 & -1.5 \\
\hline
\end{tabular}

See footnotes at end of table. 
Table 106. Average Quality of Coal Received at Electric Utilities by Census Division and State, 1986, 1991-1995 (Continued)

\begin{tabular}{|c|c|c|c|c|c|c|c|c|c|}
\hline \multirow{2}{*}{$\begin{array}{c}\text { Census Division and State } \\
\text { and Quality } 1\end{array}$} & \multirow{2}{*}{1995} & \multirow{2}{*}{1994} & \multirow{2}{*}{1993} & \multirow{2}{*}{1992} & \multirow{2}{*}{1991} & \multirow{2}{*}{1986} & \multirow{2}{*}{$\begin{array}{c}\text { Percent } \\
\text { Change } \\
\text { 1994-1995 }\end{array}$} & \multicolumn{2}{|c|}{ Average Annual Percent Change } \\
\hline & & & & & & & & 1991-1995 & 1986-1995 \\
\hline Kansas & & & & & & & & & \\
\hline 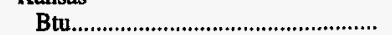 & 8,730 & 8,708 & 8,654 & 8,900 & 8,998 & 8,728 & * & -1 & * \\
\hline 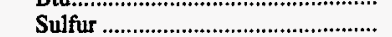 & .43 & .49 & .43 & .49 & .59 & .93 & -11.3 & -7.6 & -8.2 \\
\hline Ash & 5.46 & 5.63 & 5.19 & 5.74 & 5.63 & 7.44 & -3.1 & -.8 & -3.4 \\
\hline Minnesota & & & & & & & & & \\
\hline 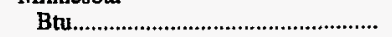 & 8,828 & 8,821 & 8,844 & 8,838 & 8,802 & 8,725 & $*$ & * & $*$ \\
\hline 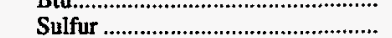 & $\begin{array}{r}0,020 \\
.47\end{array}$ & $\begin{array}{r}0,0<1 \\
.46\end{array}$ & .44 & $\begin{array}{r}0,0.30 \\
.45\end{array}$ & $\begin{array}{r}0, \text { ove } \\
.48\end{array}$ & .63 & .8 & -.4 & -3.3 \\
\hline Ash & 6.71 & 6.64 & 6.40 & 6.57 & 7.08 & 7.65 & 1.0 & -1.3 & -1.4 \\
\hline Missouri & & & & & & & & & \\
\hline 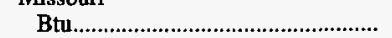 & 9,216 & 9,718 & 9,860 & 10,321 & 10,298 & 10,689 & -5 & -3 & -2 \\
\hline Sulfur & .57 & 1.03 & 1.02 & 1.80 & 1.84 & 2.50 & -44.3 & -25.3 & -15.1 \\
\hline 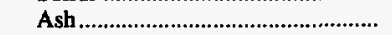 & 5.69 & 6.65 & 6.54 & 7.71 & 8.00 & 9.82 & -14.4 & -8.2 & -5.9 \\
\hline Nebraska & & & & & & & & & \\
\hline 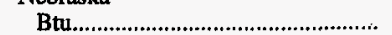 & 8,594 & 8,571 & 8,561 & 8,553 & 8,542 & 8,713 & $*$ & * & $*$ \\
\hline Sulfur & .33 & .35 & .35 & .37 & .35 & .34 & -3.5 & -1.4 & -.2 \\
\hline Ash & 5.16 & 5.17 & 5.11 & 5.00 & 4.97 & 4.95 & -.1 & 9 & .5 \\
\hline North Dakota & & & & & & & & & \\
\hline 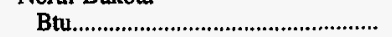 & 6,585 & 6,593 & 6,570 & 6,558 & 6,606 & 6,579 & * & * & $*$ \\
\hline Sulfur & .74 & .75 & .74 & .87 & .83 & .71 & -1.7 & -2.9 & .5 \\
\hline Ash & 9.29 & 9.39 & 9.47 & 9.29 & 9.17 & 8.82 & -1.0 & .3 & .6 \\
\hline South Dakota & & & & & & & & & \\
\hline 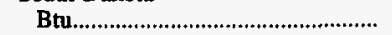 & 6,972 & 6,049 & 6,057 & 6,034 & 6,025 & 6,085 & 15 & 4 & 2 \\
\hline Sulfur & .87 & .91 & .90 & .92 & .87 & .93 & -5.0 & -.1 & -.7 \\
\hline Ash & 4.96 & 8.81 & 8.82 & 9.34 & 9.04 & 8.09 & -43.7 & -13.9 & -5.3 \\
\hline South Atlantic & & & & & & & & & \\
\hline 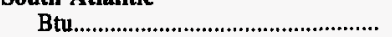 & 12,324 & 12,362 & 12,465 & 12,461 & 12,425 & 12,432 & $*$ & * & $*$ \\
\hline Sulfur & 1.27 & 1.33 & 1.39 & 1.52 & 1.51 & 1.50 & -4.8 & -4.2 & -1.9 \\
\hline Ash & 9.71 & 9.72 & 9.81 & 9.95 & 9.94 & 10.10 & -.2 & -.6 & -.4 \\
\hline Delaware & & & & & & & & & \\
\hline 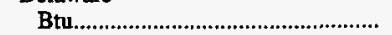 & 13,085 & 12,954 & 13,027 & 13,064 & 13,053 & 13,000 & 1 & $*$ & $*$ \\
\hline 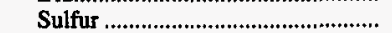 & 1.00 & .92 & .94 & 1.03 & .96 & .96 & 8.4 & .8 & .4 \\
\hline Ash & 8.56 & 9.09 & 9.08 & 8.83 & 8.66 & 8.80 & -5.8 & -.3 & -.3 \\
\hline Florida & & & & & & & & & \\
\hline 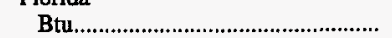 & 12,296 & 12,293 & 12,332 & 12,370 & 12,351 & 12,276 & * & * & * \\
\hline Sulfur & 1.47 & 1.60 & 1.57 & 1.68 & 1.73 & 1.80 & -8.2 & -3.9 & -2.2 \\
\hline Ash & 8.09 & 8.19 & 8.04 & 8.33 & 8.42 & 8.84 & -1.1 & -1.0 & -1.0 \\
\hline Georgia & & & & & & & & & \\
\hline 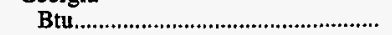 & 11,576 & 11,774 & 12,148 & 12,039 & 11,936 & 12,146 & -2 & -1 & -1 \\
\hline Sulfur & .81 & 1.05 & 1.37 & 1.68 & 1.63 & 1.78 & -22.9 & -15.9 & -8.3 \\
\hline Ash & 8.87 & 8.99 & 9.94 & 10.29 & 10.00 & 9.80 & -1.4 & -2.9 & -1.1 \\
\hline Maryland & & & & & & & & & \\
\hline 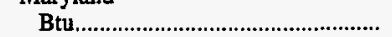 & 12,965 & 12,824 & 12,752 & 12,753 & 12,796 & 12,689 & 1 & * & * \\
\hline 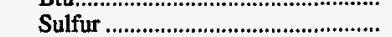 & 1.06 & $\begin{array}{r}12,024 \\
1.16\end{array}$ & 1.31 & 1.36 & 1.34 & 1.39 & -8.6 & -5.7 & -3.0 \\
\hline Ash & 9.32 & 9.91 & 10.02 & 10.47 & 10.46 & 10.63 & -6.0 & -2.8 & -1.4 \\
\hline North Carolina & & & & & & & & & \\
\hline Btu. & 12,461 & 12,416 & 12,465 & 12,456 & 12,506 & 12,554 & * & * & * \\
\hline 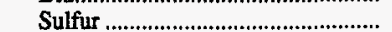 & .86 & .95 & .96 & .92 & .94 & .90 & -9.5 & -2.3 & -.5 \\
\hline Ash & 10.20 & 10.27 & 10.12 & $10.1 \overline{7}$ & 9.96 & 9.82 & -.7 & .6 & .4 \\
\hline South Carolina & & & & & & & & & \\
\hline 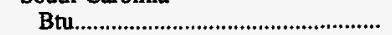 & 12,852 & 12,771 & 12,802 & 12,817 & 12,724 & 12,663 & 1 & * & * \\
\hline Sulfur & 1.19 & 1.21 & 1.17 & 1.14 & 1.19 & 1.22 & -2.0 & -.1 & -.3 \\
\hline Ash & 8.53 & 8.87 & 8.92 & 8.77 & 9.09 & 9.48 & -3.9 & -1.6 & -1.2 \\
\hline Virginia & & & & & & & & & \\
\hline 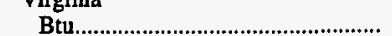 & 12,743 & 12,778 & 12,817 & 12,830 & 12,768 & 12,854 & * & * & * \\
\hline Sulfur & 1.03 & .99 & 1.00 & 1.03 & 1.00 & .94 & 3.5 & .5 & 1.0 \\
\hline Ash & 10.21 & 9.91 & 9.60 & 9.48 & 9.79 & 9.19 & 3.0 & 1.0 & 1.2 \\
\hline West Virginia & & & & & & & & & \\
\hline 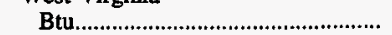 & 12,418 & 12,468 & 12,489 & 12,524 & 12,505 & 12,439 & * & $*$ & * \\
\hline 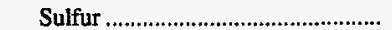 & 1.98 & 1.87 & 1.94 & 2.05 & 1.92 & 1.73 & 6.0 & .8 & 1.5 \\
\hline Ash & 11.88 & 11.50 & 11.61 & 11.32 & 11.41 & 11.59 & 3.3 & 1.0 & .3 \\
\hline East South Central & & & & & & & & & \\
\hline 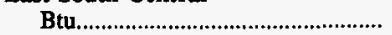 & 11,808 & 11,909 & 11,988 & 11,933 & 11,924 & 11,862 & -1 & $*$ & * \\
\hline Sulfur & 1.87 & 1.88 & 1.91 & 1.99 & 2.01 & 2.02 & -.3 & -1.7 & -.8 \\
\hline Ash & 10.58 & 10.66 & 10.92 & 10.70 & 10.83 & 10.98 & -.8 & -.6 & -.4 \\
\hline Alabama & & & & & & & & & \\
\hline 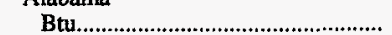 & 11,861 & 12,088 & 12,092 & 12,061 & 12,107 & 12,175 & -2 & -1 & * \\
\hline 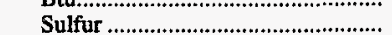 & 1.20 & 1.30 & 1.33 & 1.43 & 1.40 & 1.54 & -8.2 & -3.8 & -2.7 \\
\hline Ash & 10.74 & 11.54 & 11.79 & 11.80 & 11.77 & 11.59 & -6.9 & -2.3 & -.8 \\
\hline
\end{tabular}

See footnotes at end of table. 
Table 106. Average Quality of Coal Received at Electric Utilities by Census Division and State, 1986, 1991-1995 (Continued)

\begin{tabular}{|c|c|c|c|c|c|c|c|c|c|}
\hline \multirow{2}{*}{$\begin{array}{l}\text { Census Division and State } \\
\text { and Quality } 1\end{array}$} & \multirow{2}{*}{1995} & \multirow{2}{*}{1994} & \multirow{2}{*}{1993} & \multirow{2}{*}{1992} & \multirow{2}{*}{1991} & \multirow{2}{*}{1986} & \multirow{2}{*}{$\begin{array}{c}\text { Percent } \\
\text { Change } \\
\text { 1994-1995 }\end{array}$} & \multicolumn{2}{|c|}{ Average Annual Percent Change } \\
\hline & & & & & & & & 1991-1995 & 1986-1995 \\
\hline \multicolumn{10}{|l|}{ Kentucky } \\
\hline 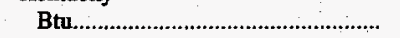 & 11,625 & 11,683 & 11,697 & 11,620 & 11,552 & $11 ; 523$ & * & * & * \\
\hline Sulfur & 2.42 & 2.34 & 2.39 & 2.44 & 2.53 & 2.49 & 3.5 & -1.1 & -0.3 \\
\hline Ash & 11.91 & 11.35 & 11.64 & 11.60 & 11.80 & 11.79 & 4.9 & .2 & .1 \\
\hline \multicolumn{10}{|l|}{ Mississippi } \\
\hline 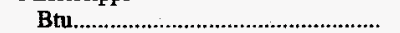 & 11,221 & 11,312 & 12,338 & 12,507 & 12,555 & 12,228 & -1 & -3 & -1 \\
\hline Sulfur & 1.04 & 1.02 & 1.41 & 1.69 & 1.56 & 1.51 & 1.2 & -9.8 & -4.1 \\
\hline Ash & 7.81 & 7.88 & 8.52 & 8.31 & 8.09 & 8.08 & -1.0 & -.9 & -.4 \\
\hline Tennessee & & & & & & & & & \\
\hline 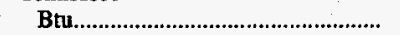 & 12,130 & 12,186 & 12,268 & 12,182 & 12,169 & 11,908 & * & * & * \\
\hline 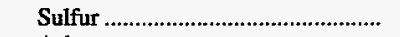 & 1.97 & 2.00 & 1.92 & 2.02 & 2.04 & 2.01 & -1.3 & -.8 & -.2 \\
\hline Ash & 8.83 & 8.94 & 9.14 & 8.31 & 8.59 & 9.92 & -1.2 & .7 & -1.3 \\
\hline West South Central & & & & & & & & & \\
\hline 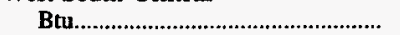 & 7,733 & 7,709 & 7,646 & 7,648 & 7,662 & 7,682 & * & * & * \\
\hline Sulfur & .64 & .62 & .64 & .65 & .65 & .58 & 3.4 & * & 1.2 \\
\hline Ash & 9.53 & 9.50 & 10.06 & 10.25 & 10.42 & 9.79 & .3 & -2.2 & -3 \\
\hline Arkansas & & & & & & & & & \\
\hline 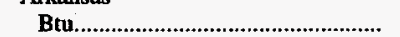 & 8,687 & 8,707 & 8,665 & 8,724 & 8,734 & 8,670 & * & * & * \\
\hline 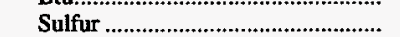 & .33 & .32 & .32 & .32 & .32 & .32 & 2.4 & .3 & .1 \\
\hline Ash & 5.10 & 4.92 & 5.06 & 5.12 & 5.14 & 5.38 & 3.6 & -.2 & -.6 \\
\hline Louisiana & & & & & & & & & \\
\hline 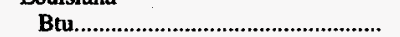 & 8,110 & 8,136 & 8,092 & 8,122 & 8,223 & 8,121 & * & * & * \\
\hline Sulfur & .58 & 51 & .52 & .50 & .49 & .50 & 14.9 & 4.7 & 1.8 \\
\hline Ash & 7.42 & 7.16 & 7.13 & 7.20 & 7.24 & 7.63 & 3.6 & .6 & -.3 \\
\hline Oklahoma & & & & & & & & & \\
\hline 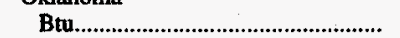 & 8,557 & 8,573 & 8,621 & 8,700 & 8,792 & 8,663 & * & -1 & * \\
\hline Sulfur & .36 & .35 & .37 & .42 & .44 & .40 & 3.3 & -4.5 & -1.1 \\
\hline Ash & 5.20 & 5.07 & 5.21 & 5.24 & 5.27 & 5.26 & 2.6 & -.3 & -1 \\
\hline Texas & & & & & & & & & \\
\hline Btu. & 7,346 & 7,346 & 7,284 & 7,234 & 7,225 & 7,291 & * & * & * \\
\hline Sulfur & .77 & .73 & .75 & .76 & .75 & .66 & 4.4 & .5 & 1.6 \\
\hline 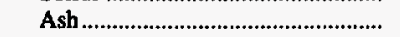 & 11.50 & 11.31 & 11.95 & 12.33 & 12.56 & 11.59 & 1.6 & -2.2 & -.1 \\
\hline Mountain & & & & & & & & & \\
\hline 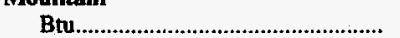 & 9,736 & 9,755 & 9,751 & 9,722 & 9,777 & 9,713 & * & * & * \\
\hline Sulfur & .54 & .55 & .54 & .55 & .53 & .55 & -1.1 & 3 & -.1 \\
\hline Ash & 11.16 & 11.11 & 11.19 & 11.15 & 10.90 & 11.20 & $\begin{array}{r}-1.1 \\
.5\end{array}$ & .0 & $*$ \\
\hline Arizona & & & & & & & & & \\
\hline 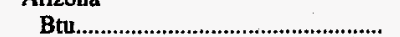 & 10,274 & 10,281 & 10,271 & 10,303 & 10,356 & 10,522 & * & * & * \\
\hline Sulfur & .53 & .51 & .49 & .51 & .51 & .53 & 2.5 & .6 & $*$ \\
\hline Ash & 12.13 & 11.97 & 12.08 & 12.19 & 12.49 & 11.48 & 1.4 & -.7 & .6 \\
\hline Colorado & & 1.67 & 12.00 & & & & & & \\
\hline 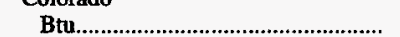 & 9,895 & 9,946 & 9,888 & 9,920 & 9,888 & 9,770 & -1 & * & * \\
\hline 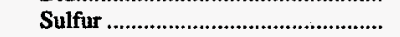 & .39 & .40 & .38 & .38 & .37 & .38 & -2.5 & 1.4 & .4 \\
\hline Ash & 7.16 & 7.12 & 6.97 & 7.01 & 6.76 & 6.79 & .5 & 1.4 & .6 \\
\hline Montana & & & & & & & & & \\
\hline 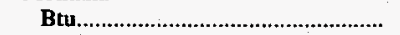 & 8,520 & 8,500 & 8,496 & 8,576 & 8,522 & 8,550 & * & * & $*$ \\
\hline Sulfur & .68 & 66 & .65 & .66 & .65 & .65 & 3.4 & 1.1 & .6 \\
\hline Ash & 9.15 & 9.05 & 8.99 & 8.92 & 8.81 & 9.72 & 1.1 & 1.0 & -.7 \\
\hline Nevada & & & & & & & & & \\
\hline 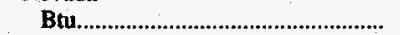 & 11,075 & 11,291 & 11,012 & 11,051 & 11,121 & 11,222 & -2 & * & * \\
\hline Sulfur & .48 & .49 & .49 & .49 & .50 & .52 & -2.5 & -.7 & -.8 \\
\hline Ash & 9.70 & 9.57 & 9.73 & 9.67 & 9.80 & 9.44 & 1.3 & -.3 & .3 \\
\hline New Mexico & & & & & & & & & \\
\hline 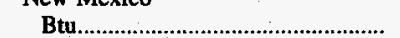 & 9,033 & 9,043 & 8,992 & 9,013 & 9,092 & 9,107 & * & * & * \\
\hline Sulfur & .80 & .82 & .81 & .81 & 80 & .78 & -2.1 & -.2 & 3 \\
\hline Ash : & 22.51 & 22.44 & 22.77 & 22.49 & 22.26 & 21.16 & .3 & .3 & .7 \\
\hline Utah & & & & & & & & & \\
\hline 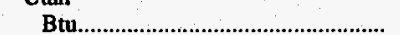 & 11,550 & 11,491 & 11,489 & 11,384 & 11,469 & 11,488 & 1 & * & * \\
\hline Sulfur & .47 & .47 & $\begin{array}{r}11,707 \\
.48\end{array}$ & .47 & .46 & .49 & .8 & 1.0 & -.3 \\
\hline Ash & 10.27 & 10.25 & 10.47 & 11.24 & 10.52 & 10.87 & .2 & -.6 & -.6 \\
\hline Wyoming & & & & & & & & & \\
\hline 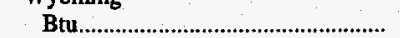 & 8,738 & 8,766 & 8,779 & 8,840 & 8,756 & 8,706 & * & * & * \\
\hline Sulfur & .50 & .52 & .51 & .52 & .51 & .52 & -3.9 & -.6 & -.4 \\
\hline Ash & 8.06 & 8.00 & 7.78 & 7.54 & 7.64 & 8.26 & .7 & 1.3 & -.3 \\
\hline
\end{tabular}

See footnotes at end of table. 
Table 106. Average Quality of Coal Received at Electric Utilities by Census Division and State, 1986, 1991-1995 (Continued)

\begin{tabular}{|c|c|c|c|c|c|c|c|c|c|}
\hline \multirow{2}{*}{$\begin{array}{c}\text { Census Division and State } \\
\text { and Quality } 1\end{array}$} & \multirow{2}{*}{1995} & \multirow{2}{*}{1994} & \multirow{2}{*}{1993} & \multirow{2}{*}{1992} & \multirow{2}{*}{1991} & \multirow{2}{*}{1986} & \multirow{2}{*}{$\begin{array}{c}\text { Percent } \\
\text { Change } \\
\text { 1994-1995 }\end{array}$} & \multicolumn{2}{|c|}{ Average Annual Percent Change } \\
\hline & & & & & & & & 1991-1995 & 1986-1995 \\
\hline \multicolumn{10}{|l|}{ Pacific } \\
\hline 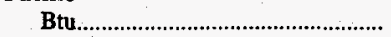 & 8,380 & 8,543 & 8,283 & 8,550 & 8,122 & 8,100 & -2 & 1 & * \\
\hline Sulfur & .62 & .57 & .63 & .59 & .57 & .69 & 7.3 & 1.8 & -1.3 \\
\hline Ash & 11.79 & 11.14 & 12.58 & 11.39 & 12.22 & 14.64 & 5.8 & -9 & -2.4 \\
\hline \multicolumn{10}{|l|}{ Oregon } \\
\hline 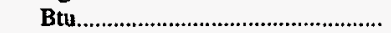 & 8,882 & 8,937 & 8,801 & 9,642 & 8,429 & - & -1 & 1 & - \\
\hline Sulfur & .30 & .37 & .38 & .40 & .32 & - & -19.1 & -1.0 & - \\
\hline Ash & 5.52 & 5.89 & 4.98 & 4.42 & 4.60 & - & -6.3 & 4.7 & - \\
\hline \multicolumn{10}{|l|}{ Washington } \\
\hline 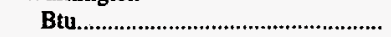 & 8,267 & 8,400 & 8,125 & 8,189 & 8,014 & 8,100 & -2 & 1 & * \\
\hline 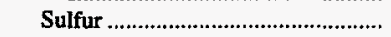 & .69 & .65 & .71 & .66 & .66 & .69 & $6 . \overline{3}$ & .9 & -.1 \\
\hline Ash & 13.20 & 13.04 & 14.90 & 13.69 & 14.88 & 14.64 & 1.3 & -2.9 & -1.1 \\
\hline \multicolumn{10}{|l|}{ U.S. Total } \\
\hline 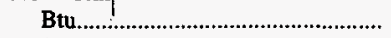 & 10,248 & 10,338 & 10,315 & 10,395 & 10,378 & 10,543 & -1 & * & $*$ \\
\hline Sulfur & 1.08 & 1.17 & 1.18 & 1.29 & 1.30 & 1.39 & -7.3 & -4.6 & -2.8 \\
\hline Ash & 9.23 & 9.36 & 9.55 & 9.71 & 9.76 & 9.98 & -1.4 & -1.4 & -.9 \\
\hline
\end{tabular}

1 Quality units are: Btu (per pound); sulfur (percent by weight); and ash (percent by weight).

* Data round to zero.

Source: Federal Energy Regulatory Commission, FERC Form 423, "Monthly Report of Cost and Quality of Fuels for Electric Plants." 
Table 107. Average Quality of Coal Received at Manufacturing and Coke Plants by Census Division and State, 1993-1995

\begin{tabular}{|c|c|c|c|c|}
\hline $\begin{array}{l}\text { Census Division and State } \\
\text { and Quality } 1\end{array}$ & 1995 & 1994 & 1993 & $\begin{array}{c}\text { Percent } \\
\text { Change } \\
\text { 1994-1995 }\end{array}$ \\
\hline \multicolumn{5}{|l|}{ New England } \\
\hline Bto & 13,410 & 13,383 & 13,232 & 0.2 \\
\hline 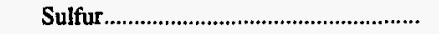 & 1.32 & .72 & 1.20 & 83.3 \\
\hline 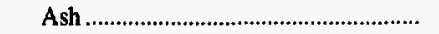 & 6.99 & 5.86 & 6.87 & 19.3 \\
\hline \multicolumn{5}{|l|}{ Maine } \\
\hline Btu & 13,392 & 13,162 & 13,062 & 1.8 \\
\hline 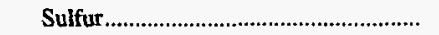 & 1.39 & .71 & 1.29 & 95.8 \\
\hline Ash & 6.93 & 5.30 & 7.18 & 30.8 \\
\hline \multicolumn{5}{|l|}{ Massachusetts } \\
\hline Btu & 13,556 & 13,939 & 13,992 & -2.8 \\
\hline 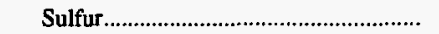 & .75 & .73 & .78 & 2.7 \\
\hline Ash & 7.55 & 7.26 & 5.48 & 4.0 \\
\hline \multicolumn{5}{|l|}{ Middle Atlantic } \\
\hline Btu & 12,559 & 12,549 & 12,367 & .1 \\
\hline 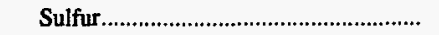 & 1.15 & 1.14 & 1.06 & .9 \\
\hline Ash & 7.11 & 6.95 & 7.37 & 2.3 \\
\hline \multicolumn{5}{|l|}{ New Jersey } \\
\hline Btu & 12,575 & 12,515 & 11,685 & .5 \\
\hline 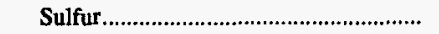 & .96 & .93 & .83 & 3.2 \\
\hline Ash & 11.34 & 12.40 & 10.71 & -8.5 \\
\hline \multicolumn{5}{|l|}{ New York ${ }^{2}$} \\
\hline 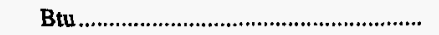 & 13,122 & 13,203 & 13,044 & -.6 \\
\hline 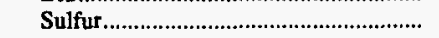 & 1.29 & 1.34 & 1.69 & -3.7 \\
\hline Ash & 7.20 & 6.69 & 7.73 & 7.6 \\
\hline \multicolumn{5}{|l|}{ Pennsylvania 2} \\
\hline Btu & 12,366 & 12,326 & 12,117 & .3 \\
\hline 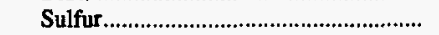 & 1.12 & 1.10 & .99 & 1.8 \\
\hline Ash & 7.09 & 7.00 & 7.30 & 1.3 \\
\hline \multicolumn{5}{|l|}{ East North Central } \\
\hline Btu & 12,022 & 11,947 & $\mathrm{R}_{11,956}$ & .6 \\
\hline 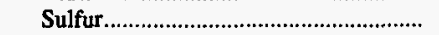 & 1.46 & 1.52 & 2.40 & -3.9 \\
\hline Ash & 7.59 & 7.61 & 6.98 & -.3 \\
\hline \multicolumn{5}{|l|}{ Illinois 2} \\
\hline Btu. & 11,290 & 11,387 & R 11,372 & -.8 \\
\hline 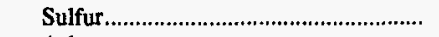 & 1.82 & 1.94 & 4.83 & -6.2 \\
\hline \multirow{2}{*}{\multicolumn{5}{|c|}{ Indiana ${ }^{2}$}} \\
\hline & & & & \\
\hline Btu & 11,894 & 11,641 & R 11,591 & 2.2 \\
\hline 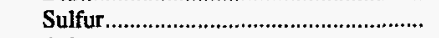 & 1.20 & 1.45 & 1.28 & -17.2 \\
\hline 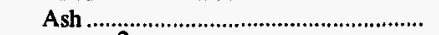 & 7.38 & 7.50 & 6.77 & -1.6 \\
\hline \multicolumn{5}{|l|}{ Michigan $^{2}$} \\
\hline Btu & 12,386 & 12,470 & 12,547 & -.7 \\
\hline 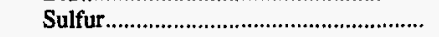 & .96 & 1.02 & 1.05 & -5.9 \\
\hline 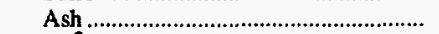 & 7.60 & 7.82 & 6.98 & -2.8 \\
\hline \multicolumn{5}{|l|}{ Ohio $^{2}$} \\
\hline Btu, & 12,424 & 12,429 & R 12,476 & * \\
\hline 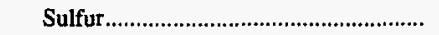 & 1.69 & 1.60 & 1.40 & 5.6 \\
\hline 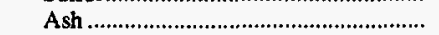 & 7.79 & 7.66 & 7.10 & 1.7 \\
\hline \multicolumn{5}{|l|}{ Wisconsin } \\
\hline Btu & 12,450 & 11,873 & 11,745 & 4.9 \\
\hline 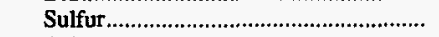 & 2.14 & 1.52 & 1.83 & 40.8 \\
\hline Ash & 8.40 & 7.30 & 7.25 & 15.1 \\
\hline \multicolumn{5}{|l|}{ West North Central } \\
\hline Btu & 8,669 & 8,710 & 8,589 & -.5 \\
\hline 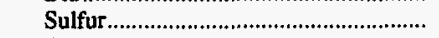 & .89 & .88 & .90 & 1.1 \\
\hline Ash & 6.54 & 6.56 & 6.46 & -.3 \\
\hline lowa & & & & \\
\hline Btu & 10,332 & 10,480 & 10,344 & $-1,4$ \\
\hline Sulfur & .96 & .98 & 1.13 & -2.0 \\
\hline Ash & 6.44 & 6.38 & 6.41 & .9 \\
\hline Kansas & & & & \\
\hline Btu & 12,197 & 12,249 & 12,479 & -.4 \\
\hline 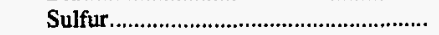 & 3.41 & 3.11 & 3.14 & 9.6 \\
\hline 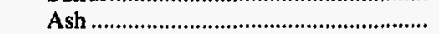 & 11.48 & 11.04 & 11.78 & 4.0 \\
\hline
\end{tabular}

See footnotes at end of table. 
Table 107. Average Quality of Coal Received at Manufacturing and Coke

Plants by Census Division and State, 1993-1995 (Continued)

\begin{tabular}{|c|c|c|c|c|}
\hline $\begin{array}{l}\text { Census Division and State } \\
\text { and Quality }{ }^{1}\end{array}$ & 1995 & 1994 & 1993 & $\begin{array}{c}\text { Percent } \\
\text { Change } \\
\text { 1994-1995 }\end{array}$ \\
\hline \multicolumn{5}{|l|}{ Minnesota } \\
\hline Btu & 10,280 & 10,112 & 10,003 & 1.7 \\
\hline 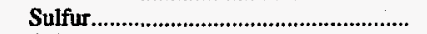 & .56 & .54 & .51 & 3.7 \\
\hline \multirow{2}{*}{\multicolumn{5}{|c|}{ Missouri 2}} \\
\hline & & & & \\
\hline Btu & 11,644 & 11,510 & 11,527 & 1.2 \\
\hline 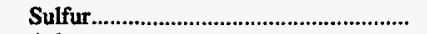 & 1.91 & 1.92 & 2.01 & -.5 \\
\hline \multirow{2}{*}{\multicolumn{5}{|c|}{ Nebraska }} \\
\hline & & & & \\
\hline Вtu & 10,096 & 9,931 & 9,979 & 1.7 \\
\hline 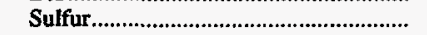 & .42 & .40 & .37 & 5.0 \\
\hline Ash & 5.73 & 5.95 & 5.20 & -3.7 \\
\hline \multicolumn{5}{|l|}{ North Dakota } \\
\hline 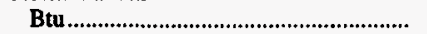 & 7,171 & 7,142 & 7,172 & .4 \\
\hline 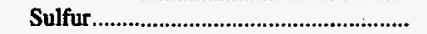 & .71 & .71 & .70 & - \\
\hline \multirow{2}{*}{\multicolumn{5}{|c|}{ South Dakota }} \\
\hline & & & & \\
\hline Btu & 9,504 & 9,418 & 8,159 & .9 \\
\hline Sulfur & .86 & .77 & .39 & 11.7 \\
\hline Ash & 7.72 & 7.15 & 5.42 & 8.0 \\
\hline \multicolumn{5}{|l|}{ South Atlantic } \\
\hline 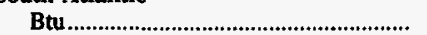 & 12,992 & R 13,043 & ${ }^{R} 12,973$ & -.4 \\
\hline 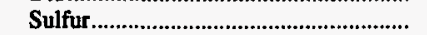 & 1.10 & $\mathrm{R}_{1.15}$ & $\hat{\mathrm{R}}_{1.12}$ & -4.3 \\
\hline 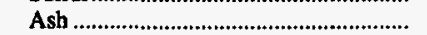 & 8.07 & R 7.96 & $\mathrm{R} 7.62$ & 1.4 \\
\hline \multicolumn{5}{|l|}{ Delaware } \\
\hline Btu & 13,483 & 13,300 & 13,087 & 1.4 \\
\hline 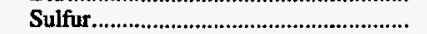 & 1.87 & 1.89 & 2.00 & -1.1 \\
\hline \multirow{2}{*}{\multicolumn{5}{|c|}{ Florida }} \\
\hline & & & & \\
\hline Btu & 12,865 & 12,933 & 12,742 & -.5 \\
\hline 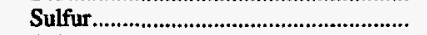 & .91 & .93 & .97 & -2.1 \\
\hline 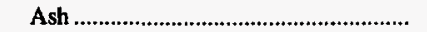 & 8.14 & 8.93 & 9.46 & -8.8 \\
\hline \multicolumn{5}{|l|}{ Georgia } \\
\hline Btu & 12,895 & 13,267 & 12,870 & -2.8 \\
\hline 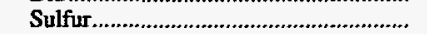 & 1.23 & 1.29 & 1.30 & -4.6 \\
\hline 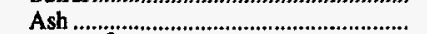 & 8.78 & 8.90 & 7.73 & -1.3 \\
\hline \multicolumn{5}{|l|}{ Maryland 2} \\
\hline 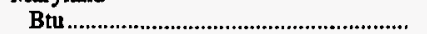 & 12,598 & 12,330 & 12,422 & 2.2 \\
\hline 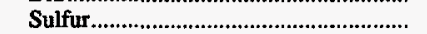 & 1.92 & 1.89 & 1.93 & 1.6 \\
\hline Ash & 14.52 & 10.90 & 13.32 & 33.2 \\
\hline \multicolumn{5}{|l|}{ North Carolina } \\
\hline 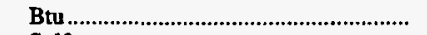 & 13,250 & 13,188 & 13,321 & .5 \\
\hline Sulfur.......... & .97 & .91 & .92 & 6.6 \\
\hline Ash ........... & 6.99 & 7.20 & 6.89 & -2.9 \\
\hline \multicolumn{5}{|l|}{ South Carolina } \\
\hline Btu & 13,051 & 12,994 & 12,955 & .4 \\
\hline Sulfur ......... & 1.08 & 1.12 & 1.22 & -3.6 \\
\hline 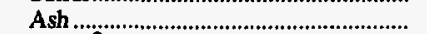 & 8.24 & 7.95 & 8.67 & 3.6 \\
\hline Virginia $^{2}$ & & & & \\
\hline Btu & 13,067 & R 13,215 & $\mathrm{R}_{13,193}$ & -1.1 \\
\hline 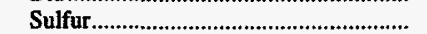 & 1.05 & $\mathrm{R}_{1.05}$ & $\widetilde{R} .99$ & - \\
\hline 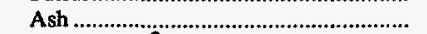 & 7.75 & R 7.44 & $R_{7.20}$ & 4.2 \\
\hline West Virginia 2 & & & & \\
\hline Btu & 12,765 & 12,780 & R 12,721 & -.1 \\
\hline 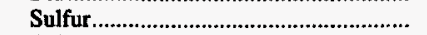 & 1.06 & 1.24 & 1.06 & -14.5 \\
\hline Ash & 7.28 & 7.54 & 6.32 & -3.4 \\
\hline East South Central & & & & \\
\hline 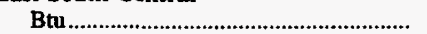 & 12,941 & 12,869 & R 12,838 & .6 \\
\hline Sulfur & 1.09 & 1.09 & .74 & - \\
\hline 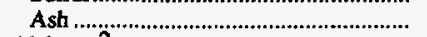 & 7.32 & 7.51 & 4.54 & -2.5 \\
\hline Alabama $^{2}$ & & & & $-2 . J$ \\
\hline 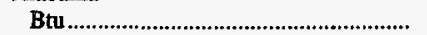 & 12,612 & 12,694 & R 12,628 & -.6 \\
\hline 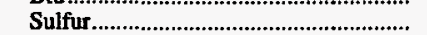 & .94 & .98 & .71 & -4.1 \\
\hline 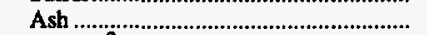 & 7.07 & 7.27 & 4.25 & -2.8 \\
\hline Kentucky 2 & & & & \\
\hline Btu & 13,086 & 13,150 & R 12,686 & -.5 \\
\hline 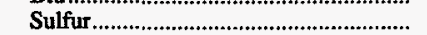 & 1.03 & .99 & .90 & 4.0 \\
\hline 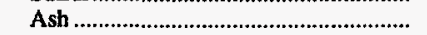 & 6.61 & 6.59 & 6.04 & .3 \\
\hline
\end{tabular}

See footnotes at end of table. 
Table 107. Average Quality of Coal Received at Manufacturing and Coke Plants by Census Division and State, 1993-1995 (Continued)

\begin{tabular}{|c|c|c|c|c|}
\hline $\begin{array}{l}\text { Census Division and State } \\
\text { and Quality } 1\end{array}$ & 1995 & 1994 & 1993 & $\begin{array}{c}\text { Percent } \\
\text { Change } \\
\text { 1994-1995 }\end{array}$ \\
\hline \multicolumn{5}{|l|}{ Mississippi } \\
\hline Btu & 11,897 & 11,786 & 12,013 & 0.9 \\
\hline 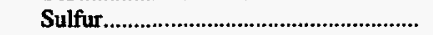 & 1.41 & 1.44 & 2.52 & -2.1 \\
\hline Ash & 10.66 & 9.98 & 10.33 & 6.8 \\
\hline \multicolumn{5}{|l|}{ Tennessee 2} \\
\hline Btu & 13,160 & 12,958 & 13,059 & 1.6 \\
\hline 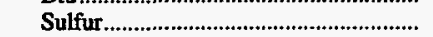 & 1.35 & 1.32 & 1.08 & 2.3 \\
\hline 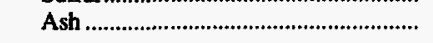 & 8.04 & 8.47 & 8.38 & -5.1 \\
\hline \multicolumn{5}{|l|}{ West South Central } \\
\hline Btu & 9,116 & 8,925 & R 9,143 & 2.1 \\
\hline 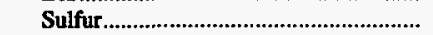 & 1.00 & 1.00 & 1.05 & - \\
\hline Ash & 10.78 & 11.18 & 11.55 & -3.6 \\
\hline \multicolumn{5}{|l|}{ Arkansas } \\
\hline Btu & 12,573 & 12,646 & 12,850 & -.6 \\
\hline 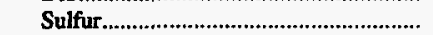 & 2.02 & 2.10 & 1.97 & -3.8 \\
\hline Ash & 9.96 & 10.11 & 9.78 & -1.5 \\
\hline \multicolumn{5}{|l|}{ Louisiana } \\
\hline Btu & 9,292 & 9,051 & 9,255 & 2.7 \\
\hline 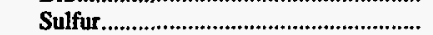 & .39 & .35 & .39 & 11.4 \\
\hline \multirow{2}{*}{\multicolumn{5}{|c|}{ Oklahoma }} \\
\hline & & & & \\
\hline Btu & 9,995 & 10,118 & 11,263 & -1.2 \\
\hline 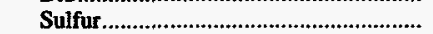 & .72 & .73 & .89 & -1.4 \\
\hline Ash & 5.60 & 6.18 & 6.86 & -9.4 \\
\hline \multicolumn{5}{|l|}{ Texas $^{2}$} \\
\hline Btu & 8,690 & 8,447 & ${ }^{R} 8,585$ & 2.9 \\
\hline 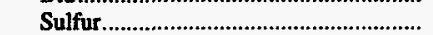 & 1.02 & 1.04 & 1.09 & -1.9 \\
\hline Ash & 12.20 & 12.72 & 12.98 & -4.1 \\
\hline \multicolumn{5}{|l|}{ Mountain } \\
\hline Btu & 10,698 & 10,601 & $R_{10,443}$ & .9 \\
\hline 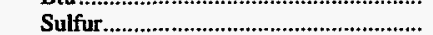 & .71 & .68 & .56 & 4.4 \\
\hline Ash & 7.28 & 7.05 & 6.75 & 3.3 \\
\hline \multicolumn{5}{|l|}{ Arizona } \\
\hline Btu & 10,969 & 11,072 & R 10,690 & -.9 \\
\hline Sulfur...1. & & .48 & .46 & 12.5 \\
\hline Ash & 12.23 & 11.09 & 11.17 & 10.3 \\
\hline \multicolumn{5}{|l|}{ Colorado } \\
\hline Btu & 11,262 & 10,785 & R 10,564 & 4.4 \\
\hline Sulfur & .61 & .58 & .54 & 5.2 \\
\hline 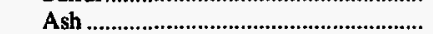 & 7.24 & 7.00 & 6.52 & 3.4 \\
\hline \multicolumn{5}{|l|}{ Idaho } \\
\hline Btu & 10,232 & 9,988 & R 10,089 & 2.4 \\
\hline 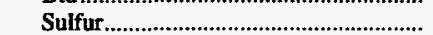 & .78 & .79 & .71 & -1.3 \\
\hline Ash & 6.22 & 5.72 & 6.03 & 8.7 \\
\hline \multicolumn{5}{|l|}{ Montana } \\
\hline Btu & 8,368 & 8,496 & 8,065 & -1.5 \\
\hline Sulfur & .59 & .57 & .46 & 3.5 \\
\hline Ash & 7.93 & 7.65 & 9.31 & 3.7 \\
\hline \multicolumn{5}{|l|}{ Nevada } \\
\hline Btu & 11,698 & 11,907 & R 12,042 & -1.8 \\
\hline Sulfur & .48 & .26 & .28 & 84.6 \\
\hline Ash & 7.13 & 4.01 & 4.63 & 77.8 \\
\hline \multicolumn{5}{|l|}{ New Mexico } \\
\hline 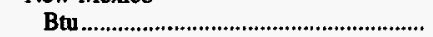 & 12,518 & 12,688 & 12,776 & -1.3 \\
\hline Sulfur & .79 & .94 & .74 & -16.0 \\
\hline 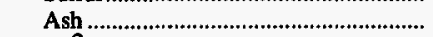 & 10.26 & 9.87 & 9.95 & 3.9 \\
\hline $\mathbf{U t a h}^{2}$ & & & & \\
\hline 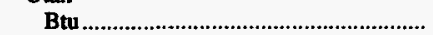 & 11,671 & 11,679 & 11,530 & -.1 \\
\hline Sulfur..... & .84 & .82 & .44 & 2.4 \\
\hline Ash & 8.08 & 7.68 & 7.12 & 5.2 \\
\hline Wyoming & & & & \\
\hline Btu & 10,170 & 10,098 & R 10,074 & .7 \\
\hline 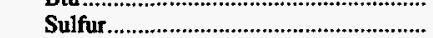 & .71 & .70 & .72 & 1.4 \\
\hline Ash & 4.84 & 5.27 & 5.12 & -8.2 \\
\hline
\end{tabular}

See footnotes at end of table. 
Table 107. Average Quality of Coal Received at Manufacturing and Coke

Plants by Census Division and State, 1993-1995 (Continued)

\begin{tabular}{|c|c|c|c|c|}
\hline $\begin{array}{l}\text { Census Division and State } \\
\text { and Quality } 1\end{array}$ & 1995 & 1994 & 1993 & $\begin{array}{c}\text { Percent } \\
\text { Change } \\
\text { 1994-1995 }\end{array}$ \\
\hline \multicolumn{5}{|l|}{ Pacific } \\
\hline Btu & 11,551 & R 11,749 & ${ }^{R} 12,218$ & -1.7 \\
\hline 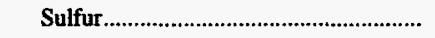 & .53 & .52 & .49 & 1.9 \\
\hline Ash & 9.17 & ${ }^{R} 8.98$ & ${ }^{R} 8.65$ & 2.1 \\
\hline \multicolumn{5}{|l|}{ California } \\
\hline Btu & 11,912 & 11,950 & 12,441 & -.3 \\
\hline Sulfur & .52 & .52 & .49 & - \\
\hline Ash & 8.62 & 8.93 & 8.35 & -3.5 \\
\hline \multicolumn{5}{|l|}{ Hawaii } \\
\hline Btu & 9,275 & R 9,576 & R 9,776 & -3.1 \\
\hline Sulfur & .53 & ${ }^{R} .51$ & ${ }^{R} .52$ & 3.9 \\
\hline Ash & 15.37 & R 16.72 & ${ }_{15.96}$ & -8.1 \\
\hline \multicolumn{5}{|l|}{ Oregon } \\
\hline Btu & 10,188 & 10,704 & 10,216 & -4.8 \\
\hline 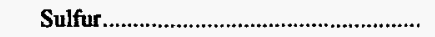 & .54 & .53 & .51 & 1.9 \\
\hline 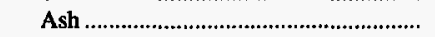 & 5.98 & 6.48 & 5.94 & -7.7 \\
\hline \multicolumn{5}{|l|}{ Washington } \\
\hline Btu & 11,846 & 11,818 & 12,151 & .2 \\
\hline Sulfur & .57 & .58 & .56 & -1.7 \\
\hline Ash & 9.62 & 5.63 & 9.47 & 70.9 \\
\hline \multicolumn{5}{|l|}{ U.S. Total } \\
\hline Btu & 11,367 & 11,316 & R 11,303 & .4 \\
\hline Sulfur & 1.15 & 1.16 & 1.23 & -.9 \\
\hline Ash & 7.61 & 7.63 & 6.34 & -.3 \\
\hline
\end{tabular}

1 Quality units are: Btu (per pound); sulfur (percent by weight); and ash (percent by weight).

2 Includes sulfur and ash data for coke plants.

* Data round to zero.

R Revised data.

Notes: Btu data are for manufacturing plants only. The national average of coke plant data ranges from .51 to 1.70 for sulfur and 2.6 to 10.1 for ash. The magnitude of changes due to revisions in this table ranges between 0.03 and 72.2 percent.

Sources: Energy Information Administration, Form ElA-3A, "Annual Coal Quality Report - Manufacturing Plants"; and Form EIA-5A, "Annual Coal Quality Report - Coke Plants." 

Major Coal Producing States

Table A1. Alabama Coal Statistics, 1986, 1991-1995

\begin{tabular}{|c|c|c|c|c|c|c|c|c|c|}
\hline \multirow{2}{*}{ Category } & \multirow{2}{*}{1995} & \multirow{2}{*}{1994} & \multirow{2}{*}{1993} & \multirow{2}{*}{1992} & \multirow{2}{*}{1991} & \multirow{2}{*}{1986} & \multirow{2}{*}{$\begin{array}{c}\text { Percent } \\
\text { Change } \\
\text { 1994-1995 }\end{array}$} & \multicolumn{2}{|c|}{$\begin{array}{l}\text { Average Annual } \\
\text { Percent Change }\end{array}$} \\
\hline & & & & & & & & 1991-1995 & 1986-1995 \\
\hline \multicolumn{10}{|l|}{ Supply (thousand short tons) } \\
\hline 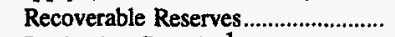 & 510,406 & 457,144 & 426,635 & 467,741 & 470,434 & 562,467 & 11.6 & 2.0 & -1.1 \\
\hline Productive Capacity 1 ........................... & 32,546 & 33,049 & 27,916 & 29,815 & NA & 26,818 & -1.5 & NA & 2.2 \\
\hline 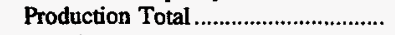 & 24,640 & 23,266 & 24,768 & 25,796 & 27,269 & 25,826 & 5.9 & -2.5 & -.5 \\
\hline 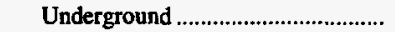 & 17,605 & 14,471 & 15,557 & 15,944 & 17,070 & 13,284 & 21.6 & .8 & 3.2 \\
\hline Surface & 7,036 & 8,795 & 9,211 & 9,852 & 10,199 & 12,542 & -20.0 & -8.9 & -6.2 \\
\hline $\begin{array}{l}\text { Capacity Utilization }{ }^{2} \\
\text { Ratio of Recoverable................... }\end{array}$ & 75.52 & 70.19 & 88.46 & 86.26 & NA & 95.80 & 7.6 & $\mathrm{NA}$ & -2.6 \\
\hline Reserves to Production......................... & 20.7 & 19.6 & 17.2 & 18.1 & 17.3 & 21.8 & 5.4 & 4.7 & -.5 \\
\hline Number of Miners & 5,567 & 5,418 & 5,399 & 5,386 & 6,314 & 7,024 & 2.8 & -3.1 & -2.5 \\
\hline Productivity Total ${ }^{2} \ldots \ldots \ldots \ldots \ldots \ldots \ldots \ldots \ldots$ & 2.24 & 2.25 & 2.35 & 2.49 & 2.17 & 1.88 & -.6 & .8 & 1.9 \\
\hline 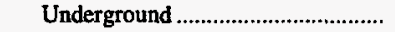 & 2.02 & 1.94 & 2.09 & 2.17 & 1.90 & 1.54 & 4.1 & 1.5 & 3.1 \\
\hline Surface & 3.07 & 3.07 & 3.01 & 3.28 & 2.84 & 2.47 & * & 1.9 & 2.4 \\
\hline Producer/Distributor Stocks ............... & 1,358 & 1,204 & 1,698 & 2,185 & 2,233 & - & 12.8 & -11.7 & - \\
\hline Imports $^{3}$ & 162 & 178 & 88 & - & - & - & -9.2 & - & - \\
\hline \multicolumn{10}{|l|}{ Distribution (thousand short tons) } \\
\hline 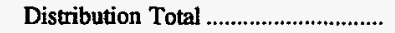 & 25,159 & 23,750 & 25,556 & 25,491 & 27,679 & NA & 5.9 & -2.4 & NA \\
\hline Domestic Distribution Total.......... & 19,127 & 19,220 & 19,668 & 19,560 & 20,667 & $\mathbf{N A}$ & -.5 & -1.9 & NA \\
\hline Within State & 18,024 & 18,351 & 18,716 & 18,849 & 20,150 & NA & -1.8 & -2.8 & NA \\
\hline 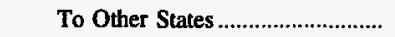 & 1,103 & 870 & 952 & 711 & 517 & NA & 26.8 & 20.8 & NA \\
\hline Foreign Distribution Total............... & 6,032 & 4,529 & 5,888 & 5,931 & 7,012 & NA & 33.2 & -3.7 & $N A$ \\
\hline Metallurgical ............................. & 5,330 & 4,359 & 5,841 & 5,789 & 6,476 & NA & 22.3 & -4.8 & NA \\
\hline Steam & 702 & 170 & 47 & 142 & 536 & NA & 313.0 & 7.0 & NA \\
\hline 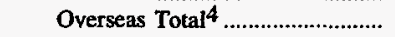 & 6,032 & 4,529 & 5,888 & 5,931 & 7,012 & NA & 33.2 & -3.7 & $\mathbf{N A}$ \\
\hline 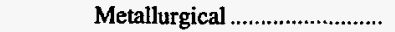 & 5,330 & 4,359 & 5,841 & 5,789 & 6,476 & NA & 22.3 & -4.8 & NA \\
\hline Steam & 702 & 170 & 47 & 142 & 536 & NA & 313.0 & 7.0 & NA \\
\hline \multicolumn{10}{|l|}{ Demand (thousand short tons) } \\
\hline 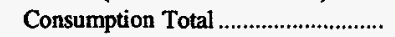 & 34,327 & 31,473 & 33,047 & 31,510 & 29,349 & 26,759 & 9.1 & 4.0 & 2.8 \\
\hline 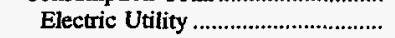 & 28,759 & 25,817 & 27,533 & 24,988 & 23,700 & 21,436 & 11.4 & 4.9 & 3.3 \\
\hline 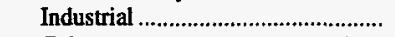 & 2,304 & 2,394 & 2,268 & 3,136 & 2,468 & 2,536 & -3.7 & -1.7 & -1.1 \\
\hline Coke & 3,257 & 3,253 & 3,206 & 3,297 & 3,166 & 2,674 & .1 & .7 & 2.2 \\
\hline Residential/Commercial................... & 7 & 11 & 40 & 89 & 17 & 112 & -32.5 & -18.9 & -26.3 \\
\hline Consumer Stocks Total ....................... & 3,648 & 4,132 & 2,797 & 4,529 & 4,671 & 5,208 & -11.7 & -6.0 & -3.9 \\
\hline 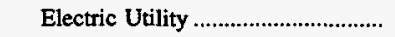 & 3,282 & 3,652 & 2,331 & 4,071 & 4,247 & 4,738 & -10.1 & -6.2 & -4.0 \\
\hline 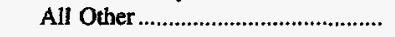 & 366 & 480 & 466 & 458 & 424 & 469 & -23.6 & -3.6 & -2.7 \\
\hline \multicolumn{10}{|c|}{ Coal Prices (nominal dollars per short ton) } \\
\hline 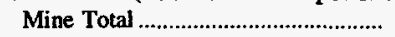 & $\$ 38.44$ & $\$ 40.12$ & $\$ 42.34$ & $\$ 40.82$ & $\$ 41.14$ & $\$ 41.27$ & -4.2 & -1.7 & -.8 \\
\hline 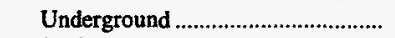 & 39.26 & 39.92 & 42.00 & 40.70 & 40.19 & 41.36 & -1.6 & -.6 & -.6 \\
\hline 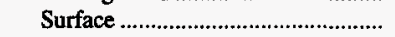 & 36.38 & 40.45 & 42.91 & 41.02 & 42.72 & 41.17 & -10.1 & -3.9 & -1.4 \\
\hline \multicolumn{10}{|l|}{ Consumer } \\
\hline 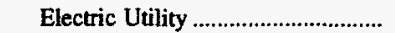 & 37.00 & 40.42 & 42.56 & 41.67 & 43.82 & 46.51 & -8.4 & -4.1 & -2.5 \\
\hline Industrial & 39.53 & 38.74 & 39.01 & 39.76 & 40.43 & 43.30 & 2.0 & -.6 & -1.0 \\
\hline Coke & 48.42 & 47.45 & 47.50 & 47.80 & 48.35 & 48.42 & 2.0 & * & * \\
\hline
\end{tabular}

1 For 1986, the Form EIA-7A solicited data on "Daily Productive Capacity." To obtain annual productive capacity for a mine in 1986, each mine's daily productive capacity was multiplied by the number of days worked during the year.

2 Capacity utilization (percent) is the ratio of total production to annual productive capacity as reported by mining companies on Form EIA-7A. Productivity (short tons per miner per hour) is calculated by dividing total coal production by the total direct labor hours worked by all employees engaged in production, preparation, processing, development, maintenance, repair, and shop or yard work at mining operations.

3 Imports for 1992 through 1995 include imports to electric utilities, manufacturing plants and coke plants. Imports for 1986 through 1991 include only imports to electric utilities.

4 Includes Mexico.

* Data round to zero.

NA Not available.

Notes: Excludes silt, culm, refuse bank, slurry dam, and dredge operations except for Pennsylvania anthracite. Consumption Total does not include coal consumed by independent power producers. Totals may not equal sum of components due to independent rounding.

Sources: Energy Information Administration, Form EIA-3, "Quarterly Coal Consumption Report - Manufacturing Plants"; Form EIA-5, "Coke Plant Report - Quarterly"; Form EIA-6, "Coal Distribution Report"; Form EIA-7A, "Coal Production Report"; Form EIA-759, "Monthly Power Plant Report"; and U.S. Department of Commerce, Bureau of the Census, "Monthly Report EM 545" and "Monthly Report IM 145." 


\begin{tabular}{|c|c|c|c|c|c|c|c|c|c|}
\hline \multirow{2}{*}{ Category } & \multirow{2}{*}{1995} & \multirow{2}{*}{1994} & \multirow{2}{*}{1993} & \multirow{2}{*}{1992} & \multirow{2}{*}{1991} & \multirow{2}{*}{1986} & \multirow{2}{*}{$\begin{array}{c}\text { Percent } \\
\text { Change } \\
1994-1995\end{array}$} & \multicolumn{2}{|c|}{$\begin{array}{l}\text { Average Annual } \\
\text { Percent Change }\end{array}$} \\
\hline & & & & & & & & 1991-1995 & $1986-1995$ \\
\hline \multicolumn{10}{|l|}{ Supply (thousand short tons) } \\
\hline 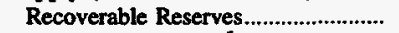 & $\mathbf{w}$ & $\mathbf{w}$ & $\mathbf{w}$ & w & $\mathbf{w}$ & $\mathbf{w}$ & w & $\mathbf{w}$ & $\mathbf{w}$ \\
\hline Productive Capacity 1 ............................. & w & $w$ & w & 13,800 & NA & $\mathbf{w}$ & -0.6 & NA & w \\
\hline Production Total & 11,947 & 13,056 & 12,173 & 12,512 & 13,203 & 11,556 & -8.5 & -2.5 & 0.4 \\
\hline 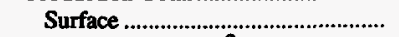 & 11,947 & 13,056 & 12,173 & 12,512 & 13,203 & 11,556 & -8.5 & -2.5 & .4 \\
\hline Capacity Utilization ${ }^{2}$ & w & $w$ & w & 90.67 & NA & w & -7.9 & NA & $\mathbf{w}$ \\
\hline \multicolumn{10}{|l|}{ Ratio of Recoverable } \\
\hline Reserves to Production............................ & w & $\mathbf{w}$ & w & w & $\mathbf{w}$ & $\mathbf{w}$ & w & $\mathbf{w}$ & $\mathbf{w}$ \\
\hline Number of Miners & 831 & 864 & 876 & 888 & 900 & 915 & -3.8 & -2.0 & -1.1 \\
\hline 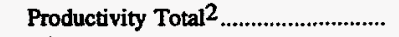 & 6.34 & 6.71 & 6.21 & 6.29 & 6.64 & 6.68 & -5.4 & -1.1 & -.6 \\
\hline 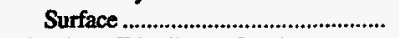 & 6.34 & 6.71 & 6.21 & 6.29 & 6.64 & 6.68 & -5.4 & -1.1 & -.6 \\
\hline Producer/Distributor Stocks ................ & 2,760 & 2,634 & 1,590 & 1,555 & 1,461 & - & 4.8 & 17.2 & - \\
\hline \multicolumn{10}{|l|}{ Distribution (thousand short tons) } \\
\hline 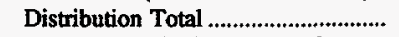 & 11,783 & 12,011 & 12,138 & 12,418 & 12,933 & NA & -1.9 & -2.3 & NA \\
\hline Domestic Distribution Total........... & 11,783 & 12,011 & 12,138 & 12,418 & 12,933 & NA & -1.9 & -2.3 & NA \\
\hline Within State ........................................ & 6,956 & 7,580 & 7,566 & 7,441 & 7,869 & NA & -8.2 & -3.0 & NA \\
\hline To Other States & 4,827 & 4,431 & 4,572 & 4,977 & 5,064 & NA & 8.9 & -1.2 & NA \\
\hline \multicolumn{10}{|l|}{ Demand (thousand short tons) } \\
\hline 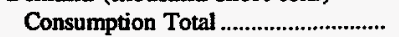 & 16,682 & 19,580 & 18,991 & 17,915 & 16,805 & 14,150 & -14.8 & -.2 & 1.8 \\
\hline 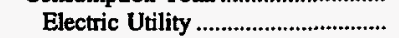 & 16,021 & 18,853 & 18,316 & 17,280 & 16,116 & 11,861 & -15.0 & -.1 & 3.4 \\
\hline 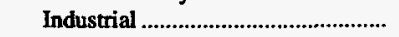 & 657 & 727 & 674 & 632 & 689 & w & -9.7 & -1.2 & $\mathbf{w}$ \\
\hline Residential/Commercial................... & 5 & * & 1 & 4 & $\mathbf{w}$ & $\mathbf{w}$ & NM & w & $\mathbf{w}$ \\
\hline Consumer Stocks Total ........................ & 3,032 & 3,242 & 3,717 & 3,596 & 4,229 & $\mathbf{w}$ & -6.5 & -8.0 & $\mathbf{w}$ \\
\hline 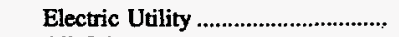 & 2,998 & 3,197 & 3,687 & 3,543 & 4,177 & 3,718 & -6.2 & -8.0 & -2.4 \\
\hline 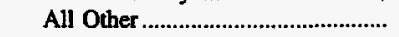 & 34 & 45 & 30 & 53 & 52 & w & -23.0 & -9.8 & w \\
\hline \multicolumn{10}{|c|}{ Coal Prices (nominal dollars per short ton) } \\
\hline 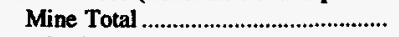 & $\mathbf{w}$ & $\mathbf{w}$ & $\mathbf{w}$ & $\mathbf{w}$ & $\mathbf{w}$ & $\mathbf{w}$ & $\mathbf{w}$ & $\mathbf{w}$ & $\mathbf{w}$ \\
\hline 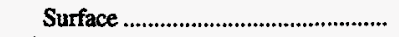 & $\mathbf{w}$ & $\mathbf{w}$ & $\mathbf{w}$ & $\mathbf{w}$ & $w$ & $\mathbf{w}$ & $\mathbf{w}$ & $\mathbf{w}$ & $\mathbf{w}$ \\
\hline \multicolumn{10}{|l|}{ Consumer } \\
\hline 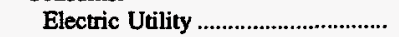 & $\$ 28.65$ & $\$ 28.26$ & $\$ 27.78$ & $\$ 28.31$ & $\$ 29.16$ & $\$ 28.70$ & 1.4 & -.4 & * \\
\hline 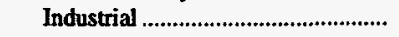 & 40.46 & 41.35 & 40.51 & 40.95 & 40.09 & w & -2.1 & .2 & $\mathbf{w}$ \\
\hline
\end{tabular}

1 For 1986, the Form EIA-7A solicited data on "Daily Productive Capacity." To obtain annual productive capacity for a mine in 1986, each mine's daily productive capacity was multiplied by the number of days worked during the year.

2 Capacity utilization (percent) is the ratio of total production to annual productive capacity as reported by mining companies on Form EIA-7A. Productivity (short tons per miner per hour) is calculated by dividing total coal production by the total direct labor hours worked by all employees engaged in production, preparation, processing, development, maintenance, repair, and shop or yard work at mining operations.

* Data round to zero.

witheld to avoid disclosure of individual company data.

NM Not meaningful as value is greater than 500 percent.

NA Not available.

Notes: Excludes silt, culm, refuse bank, slurry dam, and dredge operations except for Pennsylvania anthracite. Consumption Total does not include coal consumed by independent power producers. Totals may not equal sum of components due to independent rounding.

Sources: Energy Information Administration, Form EIA-3, "Quarterly Coal Consumption Report - Manufacturing Plants"; Form EIA-5, "Coke Plant Report - Quarterly"; Form EIA-6, "Coal Distribution Report"; Form EIA-7A, "Coal Production Report"; Form EIA-759, "Monthly Power Plant Report"; and U.S. Department of Commerce, Bureau of the Census, "Monthly Report EM 545" and "Monthly Report IM 145." 
Table A3. Colorado Coal Statistics, 1986, 1991-1995

\begin{tabular}{|c|c|c|c|c|c|c|c|c|c|}
\hline \multirow{2}{*}{ Category } & \multirow{2}{*}{1995} & \multirow{2}{*}{1994} & \multirow{2}{*}{1993} & \multirow{2}{*}{1992} & \multirow{2}{*}{1991} & \multirow{2}{*}{1986} & \multirow{2}{*}{$\begin{array}{c}\text { Percent } \\
\text { Change } \\
\text { 1994-1995 }\end{array}$} & \multicolumn{2}{|c|}{$\begin{array}{l}\text { Average Annual } \\
\text { Percent Change }\end{array}$} \\
\hline & & & & & & & & 1991-1995 & $1986-1995$ \\
\hline \multicolumn{10}{|l|}{ Supply (thousand short tons) } \\
\hline Recoverable Reserves........................... & 692,030 & 676,272 & 608,815 & 608,067 & 617,752 & 611,003 & 2.3 & 2.9 & 1.4 \\
\hline Productive Capacity 1 ............................. & 32,435 & 31,075 & 30,040 & 25,848 & NA & 16,407 & 4.4 & NA & 7.9 \\
\hline 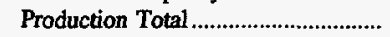 & 25,710 & 25,304 & 21,886 & 19,226 & 17,834 & 15,237 & 1.6 & 9.6 & 6.0 \\
\hline 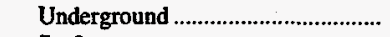 & 17,187 & 16,332 & 12,843 & 10,246 & 9,601 & 5,472 & 5.2 & 15.7 & 13.6 \\
\hline Surface & 8,523 & 8,972 & 9,043 & 8,981 & 8,233 & 9,766 & -5.0 & .9 & -1.5 \\
\hline 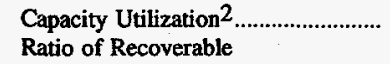 & 79.27 & 81.41 & 72.84 & 74.35 & NA & 92.75 & -2.6 & NA & -1.7 \\
\hline Reserves to Production........................ & 26.9 & 26.7 & 27.8 & 31.6 & 34.6 & 40.1 & .7 & -6.1 & -4.3 \\
\hline 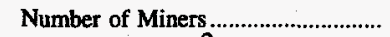 & 1,777 & 1,905 & 1,775 & 1,610 & 2,037 & 2,151 & -6.7 & -3.3 & -2.1 \\
\hline 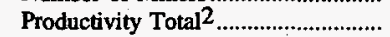 & 6.14 & 6.20 & 5.85 & 5.27 & 5.05 & 3.84 & -.9 & 5.0 & 5.3 \\
\hline 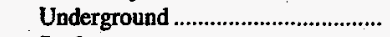 & 5.86 & 5.81 & 5.21 & 4.52 & 4.23 & 2.38 & 1.0 & 8.5 & 10.5 \\
\hline 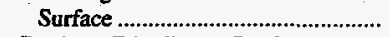 & 6.79 & 7.06 & 7.07 & 6.52 & 6.51 & 5.85 & -3.9 & 1.0 & 1.6 \\
\hline Producer/Distributor Stocks ............... & 1,063 & 1,575 & 1,155 & 955 & 850 & - & -32.5 & 5.8 & - \\
\hline \multicolumn{10}{|l|}{ Distribution (thousand short tons) } \\
\hline 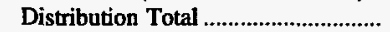 & 25,635 & 24,810 & 21,465 & 18,864 & 17.757 & NA & 3.3 & 9.6 & NA \\
\hline Domestic Distribution Total........... & 24,734 & 24,059 & 20,338 & 18,195 & 17,132 & NA & 2.8 & 9.6 & NA \\
\hline 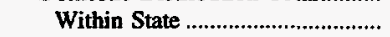 & 11,820 & 12,035 & 11,181 & 11,241 & 10,911 & NA & -1.8 & 2.0 & NA \\
\hline 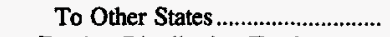 & 12,915 & 12,024 & 9,157 & 6,954 & 6,222 & NA & 7.4 & 20.0 & NA \\
\hline Foreign Distribution Total.............. & 900 & 752 & 1,128 & 669 & 625 & NA & 19.8 & 9.6 & NA \\
\hline 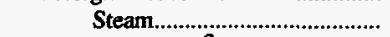 & 900 & 752 & 1,128 & 669 & 625 & $N A$ & 19.8 & 9.6 & $N A$ \\
\hline Overseas Total ${ }^{3} \ldots \ldots \ldots \ldots \ldots \ldots \ldots \ldots$ & 900 & 752 & 1,128 & 669 & 625 & NA & 19.8 & 9.6 & NA \\
\hline Steam & 900 & 752 & 1,128 & 669 & 625 & NA & 19.8 & 9.6 & NA \\
\hline \multicolumn{10}{|l|}{ Demand (thousand short tons) } \\
\hline 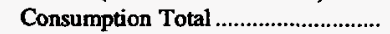 & 16,971 & 17,475 & 17,070 & 16,696 & 16,218 & 15,029 & -2.9 & 1.1 & 1.3 \\
\hline Electric Utility & 16,222 & 16,596 & 16,252 & 15,902 & 15,416 & 14,150 & -2.2 & 1.3 & 1.5 \\
\hline 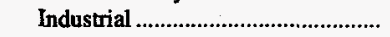 & 729 & 857 & 780 & 735 & 738 & 773 & -14.9 & -3 & -6 \\
\hline Residential/Commercial.................... & 20 & 23 & 38 & 58 & w & $\mathbf{w}$ & $-14: 0$ & $\mathbf{w}$ & $\mathbf{w}$ \\
\hline Consumer Stocks Total ......................... & 3,682 & 3,145 & 3,454 & 3,439 & 3,487 & w & 17.1 & 1.4 & $w$ \\
\hline 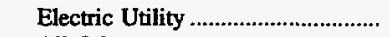 & 3,622 & 3,118 & 3,428 & 3,410 & 3,466 & 4,520 & 16.2 & 1.1 & -2.4 \\
\hline 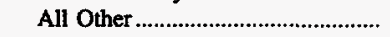 & 59 & 26 & 25 & 29 & 21 & w & 127.2 & 30.4 & w \\
\hline \multicolumn{10}{|c|}{ Coal Prices (nominal dollars per short ton) } \\
\hline Mine Total & $\$ 19.26$ & $\$ 19.76$ & $\$ 20.35$ & $\$ 21.33$ & $\$ 22.18$ & $\$ 23.44$ & -2.5 & -3.5 & -2.2 \\
\hline 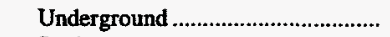 & 18.58 & 19.05 & 20.53 & 21.80 & 24.09 & 26.92 & -2.5 & -6.3 & -4.0 \\
\hline 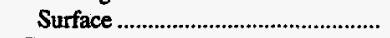 & 20.63 & 21.05 & 20.10 & 20.80 & 19.96 & 21.49 & -2.0 & .8 & -.4 \\
\hline \multicolumn{10}{|l|}{ Consumer } \\
\hline 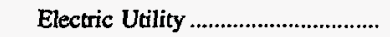 & 20.73 & 21.01 & 21.59 & 21.67 & 21.49 & 22.96 & -1.3 & -.9 & -1.1 \\
\hline 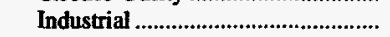 & 26.11 & 28.96 & 28.63 & 30.34 & 29.27 & 26.58 & -9.8 & -2.8 & -.2 \\
\hline
\end{tabular}

1 For 1986, the Form EIA-7A solicited data on "Daily Productive Capacity." To obtain annual productive capacity for a mine in 1986, each mine's daily productive capacity was multiplied by the number of days worked during the year.

2 Capacity utilization (percent) is the ratio of total production to annual productive capacity as reported by mining companies on Form EIA-7A. Productivity (short tons per miner per hour) is calculated by dividing total coal production by the total direct labor hours worked by all employees engaged in production, preparation, processing, development, maintenance, repair, and shop or yard work at mining operations.

3 Includes Mexico.

Withheld to avoid disclosure of individual company data

NA Not available.

Notes: Excludes silt, culm, refuse bank, slurry dam, and dredge operations except for Pennsylvania anthracite. Consumption Total does not include coal consumed by independent power producers. Totals may not equal sum of components due to independent rounding.

Sources: Energy Information Administration, Form EIA-3, "Quarterly Coal Consumption Report - Manufacturing Plants"; Form ELA-5, "Coke Plant Report - Quarterly"; Form EIA-6, "Coal Distribution Report"; Form EIA-7A, "Coal Production Report"; Form EIA-759, "Monthly Power Plant Report"; and U.S. Department of Commerce, Bureau of the Census, "Monthly Report EM 545" and "Monthly Report IM 145." 
Table A4. Illinois Coal Statistics, 1986, 1991-1995

\begin{tabular}{|c|c|c|c|c|c|c|c|c|c|}
\hline \multirow{2}{*}{ Category } & \multirow{2}{*}{1995} & \multirow{2}{*}{1994} & \multirow{2}{*}{1993} & \multirow{2}{*}{1992} & \multirow{2}{*}{1991} & \multirow{2}{*}{1986} & \multirow{2}{*}{$\begin{array}{c}\text { Percent } \\
\text { Change } \\
\text { 1994-1995 }\end{array}$} & \multicolumn{2}{|c|}{$\begin{array}{l}\text { Average Annual } \\
\text { Percent Change }\end{array}$} \\
\hline & & & & & & & & 1991-1995 & 1986-1995 \\
\hline \multicolumn{10}{|l|}{ Supply (thousand short tons) } \\
\hline Recoverable Reserves............................. & 882,323 & 963,470 & $1,063,832$ & $1,199,313$ & $1,256,524$ & $1,547,385$ & -8.4 & -8.4 & -6.0 \\
\hline Productive Capacityl ........................... & 56,627 & 69,414 & 69,320 & 75,787 & NA & 66,366 & -18.4 & NA & -1.7 \\
\hline Production Total ..................................... & 48,180 & 52,797 & 41,098 & 59,857 & 60,258 & 61,866 & -8.7 & -5.4 & -2.7 \\
\hline 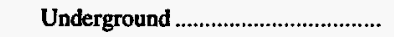 & 41,118 & 43,281 & 33,096 & 46,965 & 43,134 & 39,719 & -5.0 & -1.2 & .4 \\
\hline Surface & 7,062 & 9,516 & 8,002 & 12,892 & 17,124 & 22,147 & -25.8 & -19.9 & -11.9 \\
\hline Capacity Utilization ${ }^{2}$ & 85.08 & 76.06 & 59.28 & 78.98 & NA & 93.21 & 11.9 & NA & -1.0 \\
\hline \multicolumn{10}{|l|}{ Ratio of Recoverable } \\
\hline Reserves to Production.......................... & 18.3 & 18.3 & 25.9 & 20.0 & 20.8 & 25.0 & .3 & -3.2 & -3.4 \\
\hline 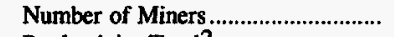 & 5,652 & 6,591 & 7,303 & 8,323 & 9,102 & 13,003 & -14.2 & -11.2 & -8.8 \\
\hline 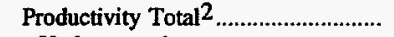 & 3.87 & 3.59 & 3.23 & 3.42 & 3.18 & 2.37 & 7.6 & 5.0 & 5.6 \\
\hline 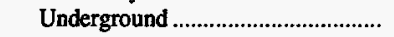 & 3.86 & 3.49 & 3.11 & 3.21 & 2.88 & 2.06 & 10.5 & 7.6 & 7.2 \\
\hline 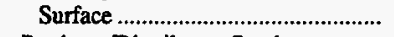 & 3.89 & 4.12 & 3.86 & 4.47 & 4.30 & 3.22 & -5.5 & -2.4 & 2.1 \\
\hline Producer/Distributor Stocks .............. & 2,069 & 1,651 & 713 & 1,969 & 1,418 & - & 25.3 & 9.9 & - \\
\hline 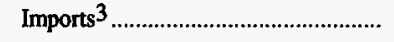 & 223 & 346 & 51 & - & - & - & -35.6 & - & - \\
\hline \multicolumn{10}{|l|}{ Distribution (thousand short tons) } \\
\hline 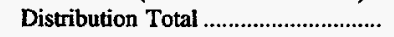 & 47,869 & 51,973 & 42,000 & 58,913 & 58,553 & NA & -7.9 & -4.9 & NA \\
\hline Domestic Distribution Total ........... & 45,170 & 51,737 & 41,330 & 57,670 & 57,290 & NA & -12.7 & -5.8 & NA \\
\hline Within State ....................................... & 15,587 & 17,517 & 15,206 & 18,167 & 18,787 & NA & -11.0 & -4.6 & NA \\
\hline 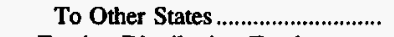 & 29,582 & 34,220 & 26,124 & 39,503 & 38,503 & NA & -13.5 & -6.4 & NA \\
\hline Foreign Distribution Total.............. & 2,699 & 236 & 670 & 1,242 & 1,263 & NA & $\mathbf{N M}$ & 20.9 & NA \\
\hline 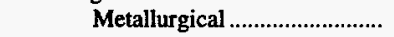 & 49 & 236 & 109 & 614 & 546 & NA & -79.2 & -45.3 & NA \\
\hline Steam & 2,650 & - & 561 & 629 & 717 & NA & - & 38.7 & NA \\
\hline 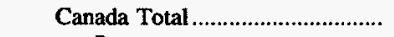 & - & - & - & - & 4 & NA & - & - & NA \\
\hline Steam & - & - & - & - & 4 & NA & - & - & NA \\
\hline 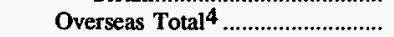 & 2,699 & 236 & 670 & 1,242 & 1,259 & NA & $\mathbf{N M}$ & 21.0 & NA \\
\hline 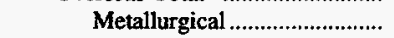 & 49 & 236 & 109 & 614 & 546 & NA & -79.2 & -45.3 & NA \\
\hline Steam & 2,650 & - & 561 & 629 & 713 & NA & - & 38.9 & NA \\
\hline \multicolumn{10}{|l|}{ Demand (thousand short tons) } \\
\hline 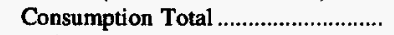 & 39,623 & 39,077 & 38,135 & 31,599 & 34,677 & 37,082 & 1.4 & 3.4 & .7 \\
\hline 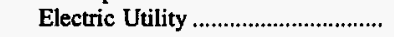 & 33,463 & 32,599 & 31,744 & 25,264 & 27,754 & 30,844 & 2.6 & 4.8 & .9 \\
\hline 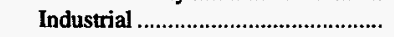 & 3,653 & 4,187 & 3,970 & 3,736 & 4,426 & 3,759 & -12.8 & -4.7 & -.3 \\
\hline Coke & $\mathbf{w}$ & $\mathbf{w}$ & $\mathbf{w}$ & $\mathbf{w}$ & $\mathbf{w}$ & 2,234 & $\mathbf{w}$ & w & w \\
\hline Residential/Commercial................... & $\mathbf{w}$ & $\mathbf{w}$ & $\mathbf{w}$ & $\mathbf{w}$ & $\mathbf{w}$ & 245 & w & $\mathbf{w}$ & $w$ \\
\hline Consumer Stocks Total ........................ & $\mathbf{w}$ & $\mathbf{w}$ & $\mathbf{w}$ & $\mathbf{w}$ & $\mathbf{w}$ & 9,670 & $\mathbf{w}$ & $\mathbf{w}$ & w \\
\hline Electric Utility ................................... & 5,331 & 4,526 & 4,019 & 7,399 & 6,977 & 8,960 & 17.8 & -6.5 & -5.6 \\
\hline 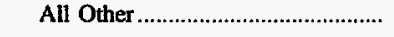 & w & w & w & w & $\mathbf{w}$ & 710 & $w$ & $\mathbf{w}$ & $\mathbf{w}$ \\
\hline \multicolumn{10}{|c|}{ Coal Prices (nominal dollars per short ton) } \\
\hline 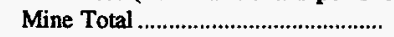 & $\$ 23.05$ & $\$ 23.14$ & $\$ 25.27$ & $\$ 27.66$ & $\$ 28.35$ & $\$ 29.99$ & -.3 & -5.0 & -2.9 \\
\hline 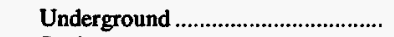 & 22.88 & 23.18 & 25.54 & 27.93 & 29.05 & 30.66 & -1.3 & -5.8 & -3.2 \\
\hline 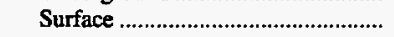 & 24.04 & 22.92 & 24.18 & 26.69 & 26.59 & 28.79 & 4.9 & -2.5 & -2.0 \\
\hline \multicolumn{10}{|l|}{ Consumer } \\
\hline Electric Utility ..................................... & 32.58 & 32.69 & 35.30 & 37.06 & 36.76 & 44.71 & -.3 & -3.0 & -3.4 \\
\hline 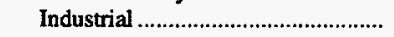 & 29.03 & 29.13 & 29.42 & 29.24 & 30.81 & 36.91 & -.3 & -1.5 & -2.6 \\
\hline 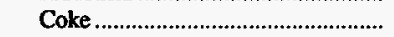 & $\mathbf{w}$ & w & $\mathbf{w}$ & $\mathbf{w}$ & w & 49.11 & $\mathbf{w}$ & $\mathbf{w}$ & w \\
\hline
\end{tabular}

1 For 1986, the Form EIA-7A solicited data on "Daily Productive Capacity." To obtain annual productive capacity for a mine in 1986, each mine's daily productive capacity was multiplied by the number of days worked during the year.

2 Capacity utilization (percent) is the ratio of total production to annual productive capacity as reported by mining companies on Form EIA-7A. Productivity (short tons per miner per hour) is calculated by dividing total coal production by the total direct labor hours worked by all employees engaged in production, preparation, processing, development, maintenance, repair, and shop or yard work at mining operations.

3 Imports for 1992 through 1995 include imports to electric utilities, manufacturing plants and coke plants. Imports for 1986 through 1991 include only imports to electric utilities.

4 Includes Mexico.

Withheld to avoid disclosure of individual company data

NM Not meaningful as value is greater than 500 percent.

NA Not available.

Notes: Excludes silt, culm, refuse bank, slurry dam, and dredge operations except for Pennsylvania anthracite. Consumption Total does not include coal consumed by independent power producers. Totals may not equal sum of components due to independent rounding.

Sources: Energy Information Administration, Form EIA-3, "Quarterly Coal Consumption Report - Manufacturing Plants"; Form EIA-5, "Coke Plant Re-

port - Quarterly"; Form EIA-6, "Coal Distribution Report"; Form EIA-7A, "Coal Production Report"; Form EIA-759, "Monthly Power Plant Report"; and U.S.

Department of Commerce, Bureau of the Census, "Monthly Report EM 545" and "Monthly Report IM 145." 


\begin{tabular}{|c|c|c|c|c|c|c|c|c|c|}
\hline \multirow{2}{*}{ Category } & \multirow{2}{*}{1995} & \multirow{2}{*}{1994} & \multirow{2}{*}{1993} & \multirow{2}{*}{1992} & \multirow{2}{*}{1991} & \multirow{2}{*}{1986} & \multirow{2}{*}{$\begin{array}{c}\text { Percent } \\
\text { Change } \\
\text { 1994-1995 }\end{array}$} & \multicolumn{2}{|c|}{$\begin{array}{l}\text { Average Annual } \\
\text { Percent Change }\end{array}$} \\
\hline & & & & & & & & $1991-1995$ & $1986-1995$ \\
\hline \multicolumn{10}{|l|}{ Supply (thousand short tons) } \\
\hline Recoverable Reserves........................... & 323,667 & 304,225 & 379,294 & 404,549 & 419,790 & 506,785 & 6.4 & -6.3 & -4.8 \\
\hline 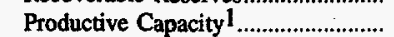 & 35,256 & 38,931 & 43,955 & 42,990 & NA & 34,880 & -9.4 & NA & .1 \\
\hline 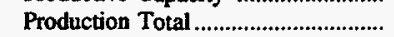 & 26,007 & 30,927 & 29,295 & 30,466 & 31,468 & 32,852 & -15.9 & -4.6 & -2.6 \\
\hline 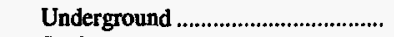 & 3,540 & 3,324 & 2,583 & 2,641 & 2,832 & 1,860 & 6.5 & 5.7 & 7.4 \\
\hline 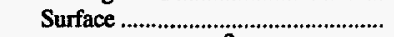 & 22,467 & 27,603 & 26,713 & 27,825 & 28,636 & 30,991 & -18.6 & -5.9 & -3.5 \\
\hline Capacity Utilization 2 & 73.70 & 79.37 & 66.60 & 70.86 & NA & 94.07 & -7.1 & NA & -2.7 \\
\hline Reserves to Production......................... & 12.4 & 9.8 & 12.9 & 13.3 & 13.3 & 15.4 & 26.5 & -1.7 & -2.3 \\
\hline Number of Miners & 2,571 & 3,206 & 3,331 & 3,652 & 3,919 & 4,389 & -19.8 & -10.0 & -5.8 \\
\hline 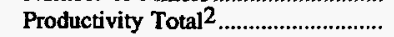 & 4.68 & 4.28 & 4.46 & 4.09 & 4.02 & 3.36 & 9.4 & 3.8 & 3.7 \\
\hline 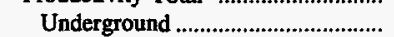 & 3.22 & 2.82 & 2.49 & 2.80 & 2.97 & 2.11 & 14.2 & 2.1 & 4.8 \\
\hline 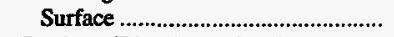 & 5.04 & 4.56 & 4.82 & 4.28 & 4.17 & 3.49 & 10.5 & 4.8 & 4.2 \\
\hline Producer/Distributor Stocks ............... & 611 & 803 & 527 & 1,016 & 525 & - & -23.9 & 3.9 & - \\
\hline Imports 3 & 761 & 593 & 594 & - & - & - & 28.3 & - & - \\
\hline \multicolumn{10}{|l|}{ Distribution (thousand short tons) } \\
\hline 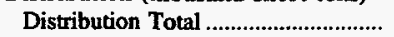 & 25,695 & 30,684 & 29,664 & 31,393 & 31,412 & NA & -16.3 & -4.9 & NA \\
\hline Domestic Distribution Total........... & 25,625 & 30,477 & 29,475 & 31,216 & 31,204 & NA & -15.9 & -4.8 & NA \\
\hline 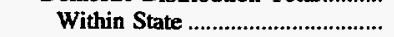 & 21,185 & 24,733 & 23,913 & 24,655 & 22,619 & NA & -14.3 & -1.6 & NA \\
\hline To Other States & 4,439 & 5,744 & 5,562 & 6,561 & 8,585 & NA & -22.7 & -15.2 & $N A$ \\
\hline Foreign Distribution Total............. & 70 & 206 & 188 & 177 & 208 & NA & -66.2 & -23.9 & $\mathrm{NA}$ \\
\hline Steam & 70 & 206 & 188 & 177 & 208 & NA & -66.2 & -23.9 & NA \\
\hline 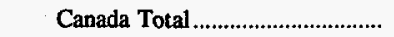 & $*$ & - & - & - & 3 & NA & - & -42.8 & NA \\
\hline Steam & * & - & - & - & 3 & NA & - & -42.8 & NA \\
\hline 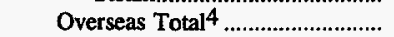 & 69 & 206 & 188 & 177 & 204 & NA & -66.3 & -23.7 & NA \\
\hline Steam & 69 & 206 & 188 & 177 & 204 & NA & -66.3 & -23.7 & NA \\
\hline \multicolumn{10}{|l|}{ Demand (thousand short tons) } \\
\hline 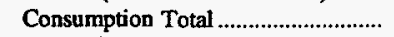 & 62,631 & 59,996 & 60,353 & 58,765 & 60,790 & 50,643 & 4.4 & .7 & 2.4 \\
\hline 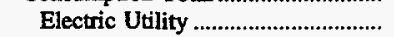 & 52,089 & 50,554 & 48,836 & 46,937 & 47,720 & 37,309 & 3.0 & 2.2 & 3.8 \\
\hline Industrial & 4,373 & 4,244 & 4,587 & 4,263 & 4,404 & 4,980 & 3.0 & -.2 & -1.4 \\
\hline Coke & 5,883 & 4,841 & 6,591 & 7,153 & 8,234 & 7,808 & 21.5 & -8.1 & -3.1 \\
\hline Residential/Commercial................... & 287 & 356 & 339 & 411 & 433 & 546 & -19.5 & -9.8 & -6.9 \\
\hline Consumer Stocks Total ...................... & 9,298 & 11,707 & 7,798 & 12,507 & 11,387 & 12,587 & -20.6 & -4.9 & -3.3 \\
\hline 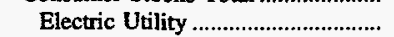 & 8,435 & 10,449 & 6,935 & 11,294 & 9,953 & 11,248 & -19.3 & -4.0 & -3.1 \\
\hline 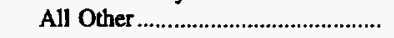 & 863 & 1,258 & 863 & 1,214 & 1,434 & 1,340 & -31.4 & -11.9 & -4.8 \\
\hline \multicolumn{10}{|c|}{ Coal Prices (nominal dollars per short ton) } \\
\hline 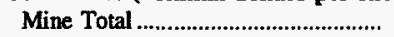 & $\$ 21.71$ & $\$ 22.28$ & $\$ 22.89$ & $\$ 23.41$ & $\$ 23.58$ & $\$ 25.37$ & -2.5 & -2.0 & -1.7 \\
\hline 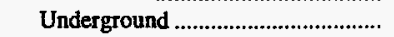 & w & $\mathbf{w}$ & w & w & $\mathbf{w}$ & w & $w$ & $\mathbf{w}$ & w \\
\hline 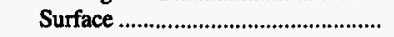 & $\mathbf{w}$ & $\mathbf{w}$ & $\mathbf{w}$ & $w$ & $\mathbf{w}$ & $\mathbf{w}$ & $\mathbf{w}$ & $w$ & $\mathbf{w}$ \\
\hline \multicolumn{10}{|l|}{ Consumer } \\
\hline 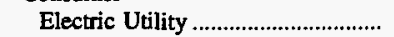 & $\$ 25.94$ & $\$ 26.79$ & $\$ 26.73$ & $\$ 27.89$ & $\$ 28.41$ & $\$ 33.70$ & -3.2 & -2.2 & -2.9 \\
\hline 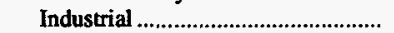 & 33.14 & 31.35 & 30.91 & 31.58 & 33.01 & 34.30 & 5.7 & .1 & -.4 \\
\hline Coke & 52.74 & 50.90 & 52.29 & 53.72 & 53.31 & 55.00 & 3.6 & -.3 & -.5 \\
\hline
\end{tabular}

1 For 1986, the Form EIA-7A solicited data on "Daily Productive Capacity." To obtain annual productive capacity for a mine in 1986, each mine's daily productive capacity was multiplied by the number of days worked during the year.

2 Capacity utilization (percent) is the ratio of total production to annual productive capacity as reported by mining companies on Form ElA-7A. Productivity (short tons per miner per hour) is calculated by dividing total coal production by the total direct labor hours worked by all employees engaged in production, preparation, processing, development, maintenance, repair, and shop or yard work at mining operations.

3 Imports for 1992 through 1995 include imports to electric utilities, manufacturing plants and coke plants. Imports for 1986 through 1991 include only imports to electric utilities.

4 Includes Mexico.

* Data round to zero.

Withheld to avoid disclosure of individual company data.

NA Not available.

Notes: Excludes silt, culm, refuse bank, slurry dam, and dredge operations except for Pennsylvania anthracite. Consumption Total does not include coal consumed by independent power producers. Totals may not equal sum of components due to independent rounding.

Sources: Energy Information Administration, Form EIA-3, "Quarterly Coal Consumption Report - Manufacturing Plants"; Form EIA-5, "Coke Plant Report - Quarterly"; Form EIA-6, "Coal Distribution Report"; Form EIA-7A, "Coal Production Report"; Form EIA-759, "Monthly Power Plant Report"; and U.S. Department of Commerce, Bureau of the Census, "Monthly Report EM 545" and "Monthly Report IM 145." 


\begin{tabular}{|c|c|c|c|c|c|c|c|c|c|}
\hline \multirow{2}{*}{ Category } & \multirow{2}{*}{1995} & \multirow{2}{*}{1994} & \multirow{2}{*}{1993} & \multirow{2}{*}{1992} & \multirow{2}{*}{1991} & \multirow{2}{*}{1986} & \multirow{2}{*}{$\begin{array}{c}\text { Percent } \\
\text { Change } \\
\text { 1994-1995 }\end{array}$} & \multicolumn{2}{|c|}{$\begin{array}{l}\text { Average Annual } \\
\text { Percent Change }\end{array}$} \\
\hline & & & & & & & & 1991-1995 & 1986-1995 \\
\hline \multicolumn{10}{|l|}{ Supply (thousand short tons) } \\
\hline 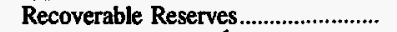 & $1,279,011$ & $1,365,188$ & $1,828,072$ & $1,452,789$ & $1,632,076$ & $1,729,815$ & -6.3 & -5.9 & -3.3 \\
\hline Productive Capacity 1 ............................... & 203,173 & 213,427 & 204,805 & 195,352 & NA & 161,630 & -4.8 & NA & 2.6 \\
\hline 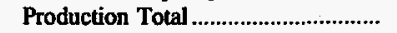 & 153,739 & 161,642 & 156,299 & 161,068 & 158,980 & 153,933 & -4.9 & -.8 & * \\
\hline 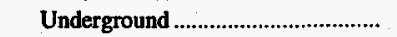 & 94,207 & 95,414 & 92,207 & 96,053 & 97,332 & 87,852 & -1.3 & -.8 & .8 \\
\hline 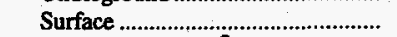 & 59,532 & 66,227 & 64,092 & 65,016 & 61,647 & 66,081 & -10.1 & -.9 & -1.1 \\
\hline 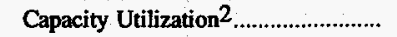 & 75.49 & 75.54 & 76.11 & 82.15 & NA & 94.22 & $*$ & NA & -2.4 \\
\hline Ratio of Recoverable & & & & & & & & & \\
\hline Reserves to Production........................... & 8.3 & 8.4 & 11.7 & 9.0 & 10.3 & 11.2 & -1.5 & -5.1 & -3.3 \\
\hline 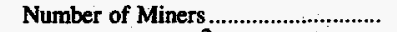 & 21,125 & 23,368 & 24,063 & 24,624 & 26,642 & 32,654 & -9.6 & -5.6 & -4.7 \\
\hline Productivity Total ${ }^{2}$ & 3.57 & 3.25 & 3.25 & 3.20 & 3.01 & 2.45 & 9.8 & 4.3 & 4.3 \\
\hline 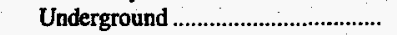 & 3.25 & 2.89 & 2.93 & 2.91 & 2.71 & 2.23 & 12.4 & 4.7 & 4.3 \\
\hline Surface & 4.23 & 3.96 & 3.84 & 3.75 & 3.66 & 2.84 & 6.8 & 3.7 & 4.5 \\
\hline Producer/Distributor Stocks ............... & 4,777 & 5,025 & 3,216 & 3,796 & 4,646 & - & -4.9 & .7 & - \\
\hline \multicolumn{10}{|l|}{ Distribution (thousand short tons) } \\
\hline 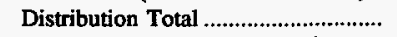 & 151,466 & 159,130 & 160,395 & 161,860 & 158,290 & NA & -4.8 & -1.1 & NA \\
\hline Domestic Distribution Total........... & 141,771 & 151,963 & 150,874 & 147,825 & 142,811 & NA & -6.7 & -.2 & NA \\
\hline Within State ....................................... & 27,140 & 26,719 & 28,136 & 27,941 & 24,831 & NA & 1.6 & 2.3 & NA \\
\hline 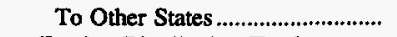 & 114,631 & 125,244 & 122,738 & 119,883 & 117,980 & NA & -8.5 & -.7 & NA \\
\hline Foreign Distribution Total............. & 9,695 & 7,167 & 9,521 & 14,036 & 15,478 & NA & 35.3 & -11.0 & NA \\
\hline 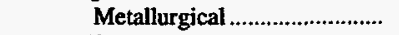 & 3,695 & 2,180 & 3,310 & 4,971 & 4,354 & NA & 69.5 & -4.0 & NA \\
\hline Steam & 6,001 & 4,987 & 6,212 & 9,065 & 11,124 & NA & 20.3 & -14.3 & NA \\
\hline 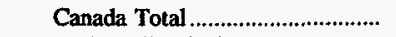 & 777 & 1,099 & 1,416 & 2,078 & 1,560 & NA & -29.3 & -16.0 & NA \\
\hline 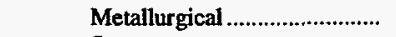 & 777 & 1,073 & 1,356 & 1,373 & 1,125 & NA & -27.6 & -8.8 & NA \\
\hline 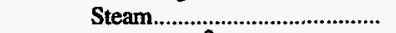 & - & 26 & 60 & 705 & 435 & NA & -100.0 & - & NA \\
\hline Overseas Total ${ }^{3}$ & 8,918 & 6,067 & 8,106 & 11,958 & 13,918 & NA & 47.0 & -10.5 & NA \\
\hline 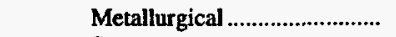 & 2,917 & 1,107 & 1,954 & 3,599 & 3,229 & NA & 163.6 & -2.5 & NA \\
\hline 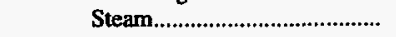 & 6,001 & 4,961 & 6,152 & 8,359 & 10,689 & NA & 21.0 & -13.4 & NA \\
\hline \multicolumn{10}{|l|}{ Demand (thousand short tons) } \\
\hline 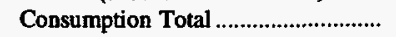 & 39,516 & 38,090 & 39,095 & 34,704 & 34,517 & 32,111 & 3.7 & 3.4 & 2.3 \\
\hline Electric Utility & 35,707 & 34,564 & 35,264 & 31,715 & 31,432 & 28,535 & 3.3 & 3.2 & 2.5 \\
\hline 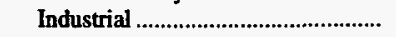 & 2,250 & 1,994 & 2,392 & 1,648 & 2,044 & 2,192 & 12.8 & 2.4 & .3 \\
\hline 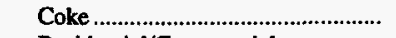 & w & $\mathbf{w}$ & w & $\mathbf{w}$ & $\mathbf{w}$ & w & $\mathbf{w}$ & $\mathbf{w}$ & $\mathbf{w}$ \\
\hline Residential/Commercial................... & w & $\mathbf{w}$ & w & $\mathbf{w}$ & $\mathbf{w}$ & $\mathbf{w}$ & $\mathbf{w}$ & $\mathbf{w}$ & $\mathbf{w}$ \\
\hline Consumer Stocks Total ......................... & w & $\mathbf{w}$ & $\mathbf{w}$ & $\mathbf{w}$ & $\mathbf{w}$ & $\mathbf{w}$ & $\mathbf{w}$ & w & w \\
\hline 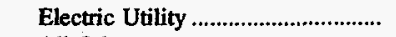 & 4,472 & 4,466 & 3,990 & 5,415 & 5,881 & 5,604 & .1 & -6.6 & -2.5 \\
\hline All Other & w & $\mathbf{w}$ & w & $\mathbf{w}$ & $\mathbf{w}$ & $\mathbf{w}$ & $\mathbf{w}$ & $\mathbf{w}$ & $\mathbf{w}$ \\
\hline \multicolumn{10}{|c|}{ Coal Prices (nominal dollars per short ton) } \\
\hline 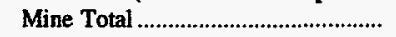 & $\$ 24.79$ & $\$ 24.88$ & $\$ 24.77$ & $\$ 24.50$ & $\$ 25.45$ & $\$ 26.09$ & -.3 & -.6 & -.6 \\
\hline 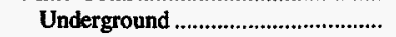 & 25.18 & 26.14 & 25.07 & 25.18 & 25.92 & 26.22 & -3.7 & -.7 & -.4 \\
\hline Surface & 24.19 & 23.07 & 24.35 & 23.50 & 24.70 & 25.93 & 4.8 & -.5 & -.8 \\
\hline \multicolumn{10}{|l|}{ Consumer } \\
\hline 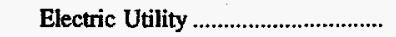 & 25.71 & 27.16 & 27.29 & 27.01 & 27.19 & 30.84 & -5.4 & -1.4 & -2.0 \\
\hline 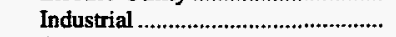 & 44.09 & 43.22 & 42.30 & 43.78 & 46.54 & 43.98 & 2.0 & -1.3 & $*$ \\
\hline Coke & w & w & $\mathbf{w}$ & $\mathbf{w}$ & w & w & w & $\mathbf{w}$ & $w$ \\
\hline
\end{tabular}

1 For 1986, the Form EIA-7A solicited data on "Daily Productive Capacity." To obtain annual productive capacity for a mine in 1986, each mine's daily productive capacity was multiplied by the number of days worked during the year.

2 Capacity utilization (percent) is the ratio of total production to annual productive capacity as reported by mining companies on Form EIA-7A. Productivity (short tons per miner per hour) is calculated by dividing total coal production by the total direct labor hours worked by all employees engaged in production, preparation, processing, development, maintenance, repair, and shop or yard work at mining operations.

3 Includes Mexico.

* Data round to zero.

Withheld to avoid disclosure of individual company data

NA Not available.

Notes: Excludes silt, culm, refuse bank, slurry dam, and dredge operations except for Pennsylvania anthracite. Consumption Total does not include coal consumed by independent power producers. Totals may not equal sum of components due to independent rounding.

Sources: Energy Information Administration, Form EIA-3, "Quarterly Coal Consumption Report - Manufacturing Plants"; Form EIA-5, "Coke Plant Report - Quarterly"; Form EIA-6, "Coal Distribution Report"; Form EIA-7A, "Coal Production Report"; Form EIA-759, "Monthly Power Plant Report"; and U.S. Department of Commerce, Bureau of the Census, "Monthly Report EM 545" and "Monthly Report IM 145." 
Table A7. Montana Coal Statistics, 1986, 1991-1995

\begin{tabular}{|c|c|c|c|c|c|c|c|c|c|}
\hline \multirow{2}{*}{ Category } & \multirow{2}{*}{1995} & \multirow{2}{*}{1994} & \multirow{2}{*}{1993} & \multirow{2}{*}{1992} & \multirow{2}{*}{1991} & \multirow{2}{*}{1986} & \multirow{2}{*}{$\begin{array}{c}\text { Percent } \\
\text { Change } \\
\text { 1994-1995 }\end{array}$} & \multicolumn{2}{|c|}{$\begin{array}{l}\text { Average Annual } \\
\text { Percent Change }\end{array}$} \\
\hline & & & & & & & & 1991-1995 & 1986-1995 \\
\hline \multicolumn{10}{|l|}{ Supply (thousand short tons) } \\
\hline Recoverable Reserves.......................... & $1,250,866$ & $1,282,525$ & $1,284,806$ & $1,351,609$ & $1,392,795$ & $1,746,243$ & -2.5 & -2.6 & -3.6 \\
\hline Productive Capacity ${ }^{1}$.............................. & 51,597 & 51,104 & 50,849 & 48,582 & NA & 35,008 & 1.0 & NA & 4.4 \\
\hline 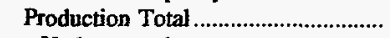 & 39,451 & 41,640 & 35,917 & 38,889 & 38,237 & 33,978 & -5.3 & .8 & 1.7 \\
\hline 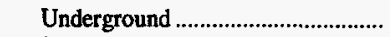 & 10 & 3 & 10 & 10 & 3 & - & 206.8 & 38.8 & - \\
\hline Surface & 39,441 & 41,636 & 35,907 & 38,879 & 38,235 & 33,978 & -5.3 & 8 & 1.7 \\
\hline $\begin{array}{l}\text { Capacity Utilization } 2 \\
\text { Ratio of Recoverable }\end{array}$ & 76.44 & 81.47 & 70.64 & 80.03 & NA & 97.06 & -6.2 & NA & -2.6 \\
\hline Reserves to Production....................... & 31.7 & 30.8 & 35.8 & 34.8 & 36.4 & 51.4 & 2.9 & -3.4 & -5.2 \\
\hline 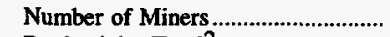 & 722 & 705 & 660 & 715 & 794 & 932 & 2.4 & -2.3 & -2.8 \\
\hline Productivity Total $2 \ldots \ldots \ldots \ldots \ldots \ldots \ldots \ldots$ & 21.06 & 21.92 & 19.49 & 20.16 & 18.99 & 17.63 & -3.9 & 2.6 & 2.0 \\
\hline 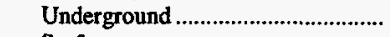 & - & - & 1.06 & - & - & - & - & - & - \\
\hline Surface & 21.06 & 21.92 & 19.59 & 20.16 & 18.99 & 17.63 & -3.9 & 2.6 & 2.0 \\
\hline Producer/Distributor Stocks .............. & 718 & 635 & 876 & 694 & 571 & - & 13.2 & 5.9 & - \\
\hline \multicolumn{10}{|l|}{ Distribution (thousand short tons) } \\
\hline 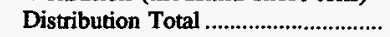 & 39,620 & 41,916 & 35,916 & 38,866 & 38,119 & NA & -5.5 & 1.0 & NA \\
\hline Domestic Distribution Total........... & 39,362 & 41,672 & 35,795 & 38,804 & 37,812 & NA & -5.5 & 1.0 & NA \\
\hline 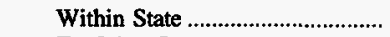 & 9,477 & 10,581 & 9,115 & 11,159 & 10,578 & NA & -10.4 & -2.7 & NA \\
\hline To Other States & 29,885 & 31,092 & 26,680 & 27,645 & 27,234 & NA & -3.9 & 2.3 & NA \\
\hline Foreign Distribution Total.............. & 259 & 243 & 121 & 62 & 306 & NA & 6.4 & -4.1 & NA \\
\hline Steam. & 259 & 243 & 121 & 62 & 306 & NA & 6.4 & -4.1 & NA \\
\hline 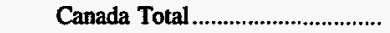 & 259 & 90 & 54 & - & 10 & NA & 187.8 & 127.8 & NA \\
\hline Steam & 259 & 90 & 54 & - & 10 & NA & 187.8 & 127.8 & NA \\
\hline Overseas Total 3 & - & 153 & 67 & 62 & 297 & NA & -100.0 & - & NA \\
\hline 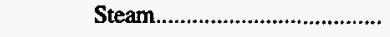 & - & 153 & 67 & 62 & 297 & NA & -100.0 & - & NA \\
\hline \multicolumn{10}{|l|}{ Demand (thousand short tons) } \\
\hline 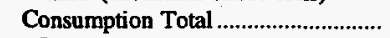 & 10,005 & 11,089 & 9,247 & 11,040 & 10,549 & 7,780 & -9.8 & -1.3 & 2.8 \\
\hline 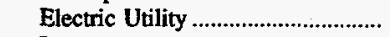 & 9,373 & 10,513 & 8,869 & 10,768 & 10,223 & 7,438 & -10.8 & -2.1 & 2.6 \\
\hline 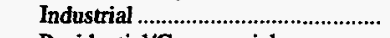 & $w$ & $w$ & $w$ & $w$ & $w$ & $w$ & $\mathbf{w}$ & $\mathbf{w}$ & $w$ \\
\hline Residential/Commercial.................... & $\mathbf{w}$ & $\mathbf{w}$ & $\mathbf{w}$ & w & $\mathbf{w}$ & $\mathbf{w}$ & $\mathbf{w}$ & $\mathbf{w}$ & $\mathbf{w}$ \\
\hline Consumer Stocks Total ....................... & $\mathbf{w}$ & $\mathbf{w}$ & $w$ & $\mathbf{w}$ & $\mathbf{w}$ & $\mathbf{w}$ & $\mathbf{w}$ & w & w \\
\hline Electric Utility ................................... & 511 & 517 & 721 & 735 & 741 & 788 & -1.2 & -8.8 & -4.7 \\
\hline All Other & $\mathbf{w}$ & $\mathbf{w}$ & w & w & w & $\mathbf{w}$ & $\mathbf{w}$ & w & $\mathbf{w}$ \\
\hline \multicolumn{10}{|c|}{ Coal Prices (nominal dollars per short ton) } \\
\hline 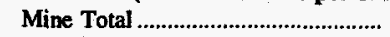 & $\$ 9.62$ & $\$ 10.39$ & $\$ 11.05$ & $\$ 10.20$ & $\$ 10.76$ & $\$ 12.93$ & -7.4 & -2.7 & -3.2 \\
\hline Underground ..................................... & - & $\mathbf{w}$ & 11.22 & - & - & - & - & - & - \\
\hline 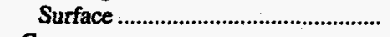 & 9.62 & $\mathbf{w}$ & 11.05 & 10.20 & 10.76 & 12.93 & $\mathbf{w}$ & $w$ & $\mathbf{w}$ \\
\hline \multicolumn{10}{|l|}{ Consumer } \\
\hline 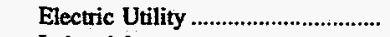 & 11.47 & $\$ 11.79$ & 11.78 & 12.14 & 11.44 & 11.53 & -2.7 & * & -.1 \\
\hline 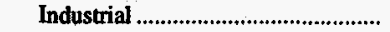 & $\mathbf{w}$ & $\mathbf{w}$ & $\mathbf{w}$ & $\mathbf{w}$ & $\mathbf{w}$ & $\mathbf{w}$ & $\mathbf{w}$ & $\mathbf{w}$ & w \\
\hline
\end{tabular}

1 For 1986, the Form EIA-7A solicited data on "Daily Productive Capacity." To obtain annual productive capacity for a mine in 1986, each mine's daily productive capacity was multiplied by the number of days worked during the year.

2 Capacity utilization (percent) is the ratio of total production to annual productive capacity as reported by mining companies on Form ElA-7A. Productivity (short tons per miner per hour) is calculated by dividing total coal production by the total direct labor hours worked by all employees engaged in production, preparation, processing, development, maintenance, repair, and shop or yard work at mining operations.

3 Includes Mexico.

* Data round to zero.

Witheld to avoid disclosure of individual company data.

NA Not available.

Notes: Excludes silt, culm; refuse bank, slurry dam, and dredge operations except for Pennsylvania anthracite. Consumption Total does not include coal consumed by independent power producers. Totals may not equal sum of components due to independent rounding.

Sources: Energy Information Administration, Form EIA-3, "Quarterly Coal Consumption Report - Manufacturing Plants"; Form EIA-5, "Coke Plant Re-

port - Quarterly"; Form EIA-6, "Coal Distribution Report"; Form ElA-7A, "Coal Production Report"; Form ElA-759, "Monthly Power Plant Report"; and U.S.

Department of Commerce, Bureau of the Census, "Monthly Report EM 545" and "Monthly Report IM 145." 


\begin{tabular}{|c|c|c|c|c|c|c|c|c|c|}
\hline \multirow{2}{*}{ Category } & \multirow{2}{*}{1995} & \multirow{2}{*}{1994} & \multirow{2}{*}{1993} & \multirow{2}{*}{1992} & \multirow{2}{*}{1991} & \multirow{2}{*}{1986} & \multirow{2}{*}{$\begin{array}{c}\text { Percent } \\
\text { Change } \\
\text { 1994-1995 }\end{array}$} & \multicolumn{2}{|c|}{$\begin{array}{l}\text { Average Annual } \\
\text { Percent Change }\end{array}$} \\
\hline & & & & & & & & 1991-1995 & 1986-1995 \\
\hline \multicolumn{10}{|l|}{ Supply (thousand short tons) } \\
\hline Recoverable Reserves........................... & $1,479,956$ & $1,457,523$ & $1,472,927$ & $1,494,541$ & $1,608,287$ & $1,571,755$ & 1.5 & -2.0 & -0.7 \\
\hline 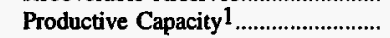 & 32,760 & 32,807 & 33,360 & 29,512 & NA & 23,173 & -.1 & NA & 3.9 \\
\hline 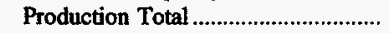 & 26,813 & 28,041 & 28,268 & 24,549 & 21,518 & 21,496 & -4.4 & 5.6 & 2.5 \\
\hline 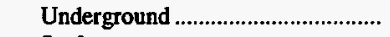 & 640 & 950 & 719 & 93 & 25 & 753 & -32.6 & 124.2 & -1.8 \\
\hline Surface & 26,173 & 27,091 & 27,549 & 24,456 & 21,492 & 20,743 & -3.4 & 5.0 & 2.6 \\
\hline $\begin{array}{l}\text { Capacity Utilization }{ }^{2} . \ldots \ldots \ldots . . . . . . . . . . . . . . . . \\
\text { Ratio of Recoverable }\end{array}$ & 81.85 & 85.47 & 84.74 & 83.18 & NA & 92.76 & -4.2 & NA & -1.4 \\
\hline Reserves to Production....................... & 55.2 & 52.0 & 52.1 & 60.9 & 74.7 & 73.1 & 6.2 & -7.3 & -3.1 \\
\hline Number of Miners .................................. & 1,747 & 1,786 & 1,762 & 1,683 & 1,650 & 1,883 & -2.2 & 1.4 & -.8 \\
\hline Productivity Total 2 ............................ & 6.92 & 6.77 & 6.68 & 6.68 & 6.25 & 5.81 & 2.1 & 2.6 & 2.0 \\
\hline 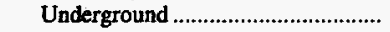 & 2.68 & 2.57 & 1.63 & .40 & .71 & 2.29 & 4.2 & 39.2 & 1.8 \\
\hline Surface & 7.19 & 7.18 & 7.26 & 7.11 & 6.31 & 6.15 & .1 & 3.3 & 1.8 \\
\hline Producer/Distributor Stocks ............... & 2,015 & 1,467 & 2,343 & 1,648 & 1,760 & - & 37.3 & 3.4 & - \\
\hline \multicolumn{10}{|l|}{ Distribution (thousand short tons) } \\
\hline Distribution Total & 26,154 & 28,570 & 27,942 & 24,827 & 22,378 & NA & -8.5 & 4.0 & NA \\
\hline Domestic Distribution Total ............ & 25,640 & 28,540 & 27,942 & 24,823 & 22,378 & NA & -10.2 & 3.5 & NA \\
\hline 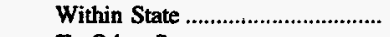 & 14,630 & 15,464 & 14,938 & 14,829 & 13,130 & NA & -5.4 & 2.7 & NA \\
\hline To Other States & 11,010 & 13,076 & 13,004 & 9,993 & 9,248 & NA & -15.8 & 4.5 & NA \\
\hline Foreign Distribution Total ............... & 514 & 30 & - & 5 & - & NA & NM & - & NA \\
\hline Steam & 514 & 30 & - & 5 & - & NA & NM & - & NA \\
\hline 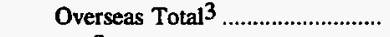 & 514 & 30 & - & 5 & - & NA & NM & - & NA \\
\hline Steam & 514 & 30 & - & 5 & - & NA & NM & - & NA \\
\hline \multicolumn{10}{|l|}{ Demand (thousand short tons) } \\
\hline 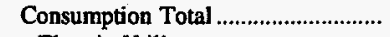 & 15,221 & 15,374 & 15,012 & 14,832 & 12,858 & 13,245 & -1.0 & 4.3 & 1.5 \\
\hline Electric Utility & 15,137 & 15,297 & 14,942 & 14,775 & 12,809 & 13,147 & -1.0 & 4.3 & 1.6 \\
\hline 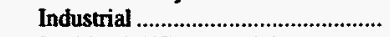 & $\mathbf{w}$ & $\mathbf{w}$ & w & w & w & $w$ & $\mathbf{w}$ & $\mathbf{w}$ & $\mathbf{w}$ \\
\hline Residential/Commercial................. & $\mathbf{w}$ & w & $\mathbf{w}$ & $\mathbf{w}$ & $w$ & $\mathbf{w}$ & $\mathbf{w}$ & $w$ & $\mathbf{w}$ \\
\hline Consumer Stocks Total ...................... & $w$ & w & w & w & w & $\mathbf{w}$ & $\mathbf{w}$ & $\mathbf{w}$ & w \\
\hline 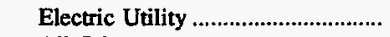 & 967 & 1,462 & 1,506 & 1,570 & 1,399 & 1,442 & -33.9 & -8.8 & -4.3 \\
\hline All Other & $\mathbf{w}$ & $\mathbf{w}$ & w & w & $w$ & w & w & w & $w$ \\
\hline \multicolumn{10}{|c|}{ Coal Prices (nominal dollars per short ton) } \\
\hline Mine Total & $\$ 23.80$ & $\$ 23.29$ & $\$ 22.96$ & $\$ 23.14$ & $\$ 23.25$ & $\$ 22.26$ & 2.2 & .6 & .7 \\
\hline 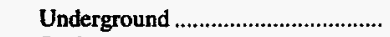 & $\mathbf{w}$ & w & w & w & w & w & $\mathbf{w}$ & w & $\mathbf{w}$ \\
\hline 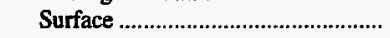 & $\mathbf{w}$ & $\mathbf{w}$ & $\mathbf{w}$ & $\mathbf{w}$ & $\mathbf{w}$ & $\mathbf{w}$ & w & w & $w$ \\
\hline \multicolumn{10}{|l|}{ Consumer } \\
\hline 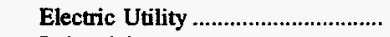 & $\$ 25.59$ & $\$ 25.48$ & $\$ 24.61$ & $\$ 23.83$ & $\$ 25.02$ & $\$ 20.64$ & .4 & .6 & 2.4 \\
\hline 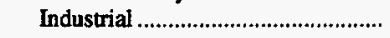 & $\mathbf{w}$ & w & w & $\mathbf{w}$ & $\mathbf{w}$ & $w$ & $\mathbf{w}$ & $\mathbf{w}$ & $\mathbf{w}$ \\
\hline
\end{tabular}

1 For 1986, the Form EIA-7A solicited data on "Daily Productive Capacity." To obtain annual productive capacity for a mine in 1986, each mine's daily productive capacity was multiplied by the number of days worked during the year.

2 Capacity utilization (percent) is the ratio of total production to annual productive capacity as reported by mining companies on Form EIA-7A. Productivity (short tons per miner per hour) is calculated by dividing total coal production by the total direct labor hours worked by all employees engaged in production, preparation, processing, development, maintenance, repair, and shop or yard work at mining operations.

3 Includes Mexico.

Withheld to avoid disclosure of individual company data

NM Not meaningful as value is greater than 500 percent.

NA Not available.

Notes: Excludes silt, culm, refuse bank, slurry dam, and dredge operations except for Pennsylvania anthracite. Consumption Total does not include coal consumed by independent power producers. Totals may not equal sum of components due to independent rounding.

Sources: Energy Information Administration, Form EIA-3, "Quarterly Coal Consumption Report - Manufacturing Plants"; Form EIA-5, "Coke Plant Report - Quarterly"; Form EIA-6, "Coal Distribution Report"; Form EIA-7A, "Coal Production Report"; Form ELA-759, "Monthly Power Plant Report"; and U.S Department of Commerce, Bureau of the Census, "Monthly Report EM 545" and "Monthly Report IM 145." 
Table A9. North Dakota Coal Statistics, 1986, 1991-1995

\begin{tabular}{|c|c|c|c|c|c|c|c|c|c|}
\hline \multirow{2}{*}{ Category } & \multirow{2}{*}{1995} & \multirow{2}{*}{1994} & \multirow{2}{*}{1993} & \multirow{2}{*}{1992} & \multirow{2}{*}{1991} & \multirow{2}{*}{1986} & \multirow{2}{*}{$\begin{array}{c}\text { Percent } \\
\text { Change } \\
\text { 1994-1995 }\end{array}$} & \multicolumn{2}{|c|}{$\begin{array}{l}\text { Average Annual } \\
\text { Percent Change }\end{array}$} \\
\hline & & & & & & & & 1991-1995 & 1986-1995 \\
\hline \multicolumn{10}{|l|}{ Supply (thousand short tons) } \\
\hline Recoverable Reserves........................... & $1,667,596$ & $1,694,548$ & $1,411,026$ & $1,335,164$ & $1,385,624$ & $1,532,651$ & -1.6 & 4.7 & 0.9 \\
\hline Productive Capacity 1 .......................... & 34,464 & 35,920 & 36,371 & 36,986 & NA & 27,480 & -4.0 & NA & 2.5 \\
\hline 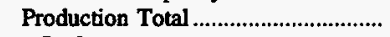 & 30,112 & 32,286 & 31,973 & 31,744 & 29,530 & 25,640 & -6.7 & .5 & 1.8 \\
\hline Surface & 30,112 & 32,286 & 31,973 & 31,744 & 29,530 & 25,640 & -6.7 & .5 & 1.8 \\
\hline & 87.37 & 89.88 & 87.89 & 85.83 & NA & 93.30 & -2.8 & NA & -.7 \\
\hline $\begin{array}{l}\text { Ratio of Recoverable } \\
\text { Reserves to Production.................... }\end{array}$ & 55.4 & 525 & 44.1 & 42.1 & 46.9 & 59.8 & 5.5 & 42 & -8 \\
\hline Number of Miners ............................ & 716 & 645 & 782 & 744 & 814 & 1,052 & 11.0 & $\begin{array}{r}4.2 \\
-3.1\end{array}$ & -4.2 \\
\hline Productivity Total 2 & 16.80 & 18.84 & 17.66 & 18.12 & 17.64 & 12.27 & -10.8 & -1.2 & 3.5 \\
\hline 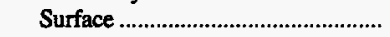 & 16.80 & 18.84 & 17.66 & 18.12 & 17.64 & 12.27 & -10.8 & -1.2 & 3.5 \\
\hline Producer/Distributor Stocks ............... & 1,797 & 1,812 & 1,607 & 1,614 & 1,619 & - & -.8 & 2.6 & - \\
\hline \multicolumn{10}{|l|}{ Distribution (thousand short tons) } \\
\hline 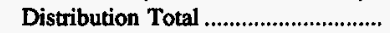 & 30,118 & 32,056 & 32,372 & 31,702 & 29,741 & NA & -6.0 & .3 & NA \\
\hline Domestic Distribution Total ............ & 30,118 & 32,056 & 32,372 & 31,702 & 29,741 & NA & -6.0 & .3 & NA \\
\hline 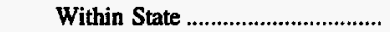 & 28,838 & 29,731 & 30,215 & 29,573 & 27,405 & NA & -3.0 & 1.3 & NA \\
\hline To Other States ................................. & 1,281 & 2,325 & 2,157 & 2,129 & 2,337 & NA & -44.9 & -14.0 & $\mathbf{N A}$ \\
\hline \multicolumn{10}{|l|}{ Demand (thousand short tons) } \\
\hline 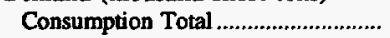 & 30,237 & 30,363 & 30,302 & 30,301 & 28,597 & 23,587 & -.4 & 1.4 & 2.8 \\
\hline 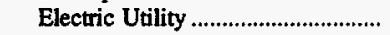 & 22,680 & 23,248 & 23,290 & 23,192 & 22,174 & 17,291 & -2.4 & .6 & 3.1 \\
\hline Industrial & $\mathbf{w}$ & $\mathbf{w}$ & $\mathbf{w}$ & $w$ & w & $\mathbf{w}$ & $\mathbf{w}$ & $\mathbf{w}$ & w \\
\hline Residential/Commercial................... & $\mathbf{w}$ & $w$ & $\mathbf{w}$ & $w$ & $\mathbf{w}$ & $w$ & w & $\mathbf{w}$ & $\mathbf{w}$ \\
\hline 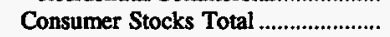 & $\mathbf{w}$ & $w$ & $w$ & w & $\mathbf{w}$ & $w$ & $\mathbf{w}$ & w & w \\
\hline 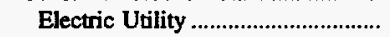 & 1,858 & 2,406 & 2,417 & 2,194 & 1,999 & 2,879 & -22.8 & -1.8 & -4.7 \\
\hline 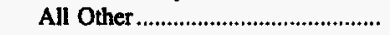 & $\mathbf{w}$ & $\mathbf{w}$ & w & $w$ & w & $w$ & w & w & w \\
\hline \multicolumn{10}{|c|}{ Coal Prices (nominal dollars per short ton) } \\
\hline 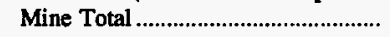 & $\$ 7.99$ & $\$ 7.62$ & $\$ 7.63$ & $\$ 7.48$ & $\$ 7.84$ & $\$ 8.49$ & 4.9 & .5 & -.7 \\
\hline Surface & 7.99 & 7.62 & 7.63 & 7.48 & 7.84 & 8.49 & 4.9 & .5 & -.7 \\
\hline \multicolumn{10}{|l|}{ Consumer } \\
\hline 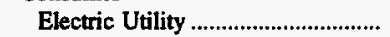 & 9.65 & 9.28 & 9.38 & 9.45 & 9.37 & 10.81 & 4.0 & .7 & -1.3 \\
\hline 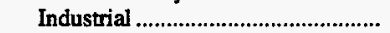 & w & w & $w$ & $\mathbf{w}$ & w & w & $\mathbf{w}$ & $\mathbf{w}$ & $w$ \\
\hline
\end{tabular}

1 For 1986, the Form EIA-7A solicited data on "Daily Productive Capacity." To obtain annual productive capacity for a mine in 1986, each mine's daily productive capacity was multiplied by the number of days worked during the year.

2 Capacity utilization (percent) is the ratio of total production to annual productive capacity as reported by mining companies on Form EIA-7A. Productivity (short tons per miner per hour) is calculated by dividing total coal production by the total direct labor hours worked by all employees engaged in production, preparation, processing, development, maintenance, repair, and shop or yard work at mining operations.

Withheld to avoid disclosure of individual company data.

NA Not available.

Notes: Excludes silt, culm, refuse bank, slurry dam, and dredge operations except for Pennsylvania anthracite. Consumption Total does not include coal consumed by independent power producers. Totals may not equal sum of components due to independent rounding.

Sources: Energy Information Administration, Form EIA-3, "Quarterly Coal Consumption Report - Manufacturing Plants"; Form ElA-5, "Coke Plant Report - Quarterly"; Form EIA-6, "Coal Distribution Report"; Form EIA-7A, "Coal Production Report"; Form EIA-759, "Monthly Power Plant Report"; and U.S. Department of Commerce, Bureau of the Census, "Monthly Report EM 545" and "Monthly Report IM 145." 
Table A10. Ohio Coal Statistics, 1986, 1991-1995

\begin{tabular}{|c|c|c|c|c|c|c|c|c|c|}
\hline \multirow{2}{*}{ Category } & \multirow{2}{*}{1995} & \multirow{2}{*}{1994} & \multirow{2}{*}{1993} & \multirow{2}{*}{1992} & \multirow{2}{*}{1991} & \multirow{2}{*}{1986} & \multirow{2}{*}{$\begin{array}{c}\text { Percent } \\
\text { Change } \\
\text { 1994-1995 }\end{array}$} & \multicolumn{2}{|c|}{$\begin{array}{l}\text { Average Annual } \\
\text { Percent Change }\end{array}$} \\
\hline & & & & & & & & $1991-1995$ & 1986-1995 \\
\hline \multicolumn{10}{|l|}{ Supply (thousand short tons) } \\
\hline Recoverable Reserves............................ & 467,984 & 479,235 & $519 ; 768$ & 575,973 & 590,604 & 791,792 & -2.3 & -5.6 & -5.7 \\
\hline 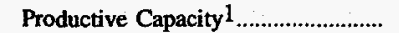 & 34,011 & 43,925 & 42,236 & 41,329 & NA & 39,364 & -22.6 & NA & -1.6 \\
\hline 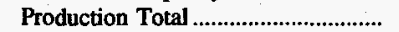 & 26,118 & 29,897 & 28,816 & 30,403 & 30,569 & 36,441 & -12.6 & -3.8 & -3.6 \\
\hline 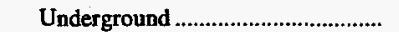 & 13,077 & 13,607 & 10,437 & 12,031 & 12,237 & 14,359 & -3.9 & 1.7 & -1.0 \\
\hline 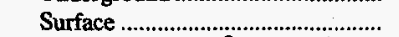 & 13,041 & 16,290 & 18,379 & 18,371 & 18,333 & 22,082 & -19.9 & -8.2 & -5.7 \\
\hline Capacity Utilization 2 & 76.55 & 67.87 & 67.94 & 73.19 & NA & 91.98 & 12.8 & NA & -2.0 \\
\hline Ratio of Recoverable & & & & & & & & & \\
\hline Reserves to Production....................... & 17.9 & 16.0 & 18.0 & 18.9 & 19.3 & 21.7 & 11.8 & -1.9 & -2.1 \\
\hline 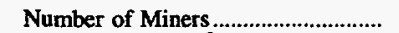 & 3,386 & 3,983 & 3,866 & 4,515 & 5,293 & 8,610 & -15.0 & -10.6 & -9.8 \\
\hline 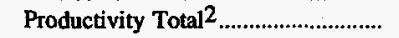 & 3.62 & 3.42 & 3.46 & 3.04 & 2.67 & 2.13 & 6.1 & 7.9 & 6.1 \\
\hline 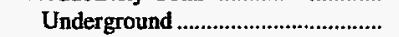 & 3.81 & 3.51 & 3.27 & 3.01 & 2.55 & 1.78 & 8.4 & 10.5 & 8.8 \\
\hline 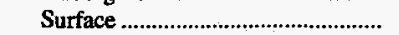 & 3.46 & 3.34 & 3.58 & 3.06 & 2.76 & 2.44 & 3.5 & 5.8 & 3.9 \\
\hline Producer/Distributor Stocks .............. & 1,374 & 833 & 550 & 1,087 & 821 & - & 64.8 & 13.7 & - \\
\hline Imports ${ }^{3}$ & 1 & 2 & 2 & 13 & - & - & -13.8 & - & - \\
\hline \multicolumn{10}{|l|}{ Distribution (thousand short tons) } \\
\hline 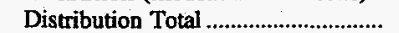 & 24,345 & 28,749 & 28,315 & 29,550 & 30,023 & NA & -15.3 & -5.1 & NA \\
\hline Domestic Distribution Total........... & 24,318 & 28,688 & 28,315 & 29,549 & 30,001 & NA & -15.2 & -5.1 & NA \\
\hline 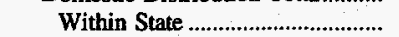 & 20,228 & 23,907 & 24,370 & 26,941 & 27,061 & NA & -15.4 & -7.0 & NA \\
\hline To Other States & 4,090 & 4,782 & 3,944 & 2,608 & 2,940 & NA & -14.5 & 8.6 & NA \\
\hline Foreign Distribution Total.............. & 28 & 61 & - & 2 & 22 & NA & -54.7 & 5.9 & NA \\
\hline Steam & 28 & 61 & - & 2 & 22 & NA & -54.7 & 5.9 & NA \\
\hline 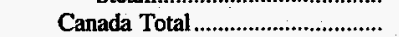 & 13 & - & - & * & 3 & NA & - & 49.1 & NA \\
\hline 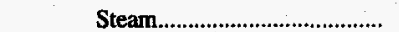 & 13 & - & - & $*$ & 3 & NA & - & 49.1 & NA \\
\hline 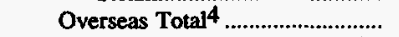 & 15 & 61 & - & 1 & 19 & NA & -75.7 & -6.5 & NA \\
\hline 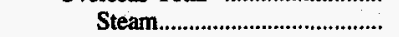 & 15 & 61 & - & 1 & 19 & NA & -75.7 & -6.5 & NA \\
\hline \multicolumn{10}{|l|}{ Demand (thousand short tons) } \\
\hline 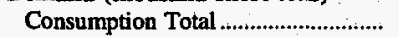 & 56,580 & 56,711 & 59,031 & 58,671 & 58,578 & 59,324 & -.2 & -.9 & -.5 \\
\hline 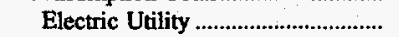 & 49,785 & 49,326 & 51,456 & 50,358 & 49,577 & 47,785 & .9 & .1 & .4 \\
\hline 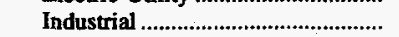 & 3,609 & 3,794 & 4,100 & 3,970 & 4,813 & 5,303 & -4.9 & -6.9 & -4.2 \\
\hline Coke & 2,777 & 3,092 & 2,892 & 3,755 & 3,698 & 5,184 & -10.2 & -6.9 & -6.7 \\
\hline Residential/Commercial................... & 409 & 498 & 584 & 588 & 489 & 1,052 & -18.0 & -4.4 & -10.0 \\
\hline Consumer Stocks Total ......................... & 5,936 & 7,815 & 7,630 & 10,804 & 10,618 & 9,508 & -24.0 & -13.5 & -5.1 \\
\hline 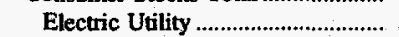 & 5,661 & 7,499 & 7,249 & 10,395 & 10,213 & 8,785 & -24.5 & -13.7 & -4.8 \\
\hline 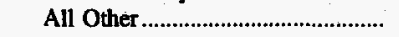 & 275 & 316 & 381 & 408 & 405 & 723 & -13.0 & -9.2 & -10.2 \\
\hline \multicolumn{10}{|c|}{ Coal Prices (nominal dollars per short ton) } \\
\hline 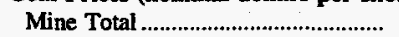 & $\$ 25.97$ & $\$ 29.13$ & $\$ 28.04$ & $\$ 26.93$ & $\$ 27.75$ & $\$ 32.80$ & -10.8 & -1.6 & -2.6 \\
\hline 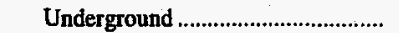 & 28.98 & 31.61 & 30.73 & 30.11 & 31.52 & 39.17 & -8.3 & -2.1 & -3.3 \\
\hline 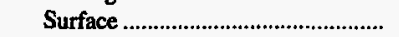 & 22.92 & 27.04 & 26.51 & 24.84 & 25.22 & 28.61 & -15.2 & -2.4 & -2.4 \\
\hline \multicolumn{10}{|l|}{ Consumer } \\
\hline 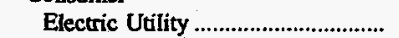 & 34.44 & 34.70 & 34.05 & 34.40 & 35.33 & 38.80 & -.8 & -.6 & -1.3 \\
\hline 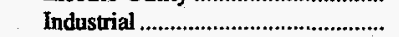 & 35.18 & 35.75 & 34.82 & 35.24 & 34.85 & 35.49 & -1.6 & .2 & -.1 \\
\hline 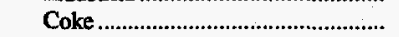 & 42.18 & 42.02 & 45.07 & 46.68 & 46.15 & 54.59 & .4 & -2.2 & -2.8 \\
\hline
\end{tabular}

1 For 1986, the Form EIA-7A solicited data on "Daily Productive Capacity." To obtain annual productive capacity for a mine in 1986, each mine's daily productive capacity was multiplied by the number of days worked during the year.

2 Capacity utilization (percent) is the ratio of total production to annual productive capacity as reported by mining companies on Form EIA-7A. Productivity (short tons per miner per hour) is calculated by dividing total coal production by the total direct labor hours worked by all employees engaged in production, preparation, processing, development, maintenance, repair, and shop or yard work at mining operations.

3 Imports for 1992 through 1995 include imports to electric utilities, manufacturing plants and coke plants. Imports for 1986 through 1991 include only imports to electric utilities.

4 Includes Mexico.

* Data round to zero.

NA Not available.

Notes: Excludes silt, culm, refuse bank, slurry dam, and dredge operations except for Pennsylvania anthracite. Totals may not equal sum of components due to independent rounding.

Sources: Energy Information Administration, Form EIA-3, "Quarterly Coal Consumption Report - Manufacturing Plants"; Form ELA-5, "Coke Plant Report - Quarterly"; Form EIA-6, "Coal Distribution Report"; Form EIA-7A, "Coal Production Report"; Form EIA-759, "Monthly Power Plant Report"; and U.S. Department of Commerce, Bureau of the Census, "Monthly Report EM 545" and "Monthly Report IM 145." 
Table A11. Pennsylvania Coal Statistics, 1986, 1991-1995

\begin{tabular}{|c|c|c|c|c|c|c|c|c|c|}
\hline \multirow{2}{*}{ Category } & \multirow{2}{*}{1995} & \multirow{2}{*}{1994} & \multirow{2}{*}{1993} & \multirow{2}{*}{1992} & \multirow{2}{*}{1991} & \multirow{2}{*}{1986} & \multirow{2}{*}{$\begin{array}{c}\text { Percent } \\
\text { Change } \\
\text { 1994-1995 }\end{array}$} & \multicolumn{2}{|c|}{$\begin{array}{l}\text { Average Annual } \\
\text { Percent Change }\end{array}$} \\
\hline & & & & & & & & 1991-1995 & $1986-1995$ \\
\hline \multicolumn{10}{|l|}{ Supply (thousand short tons) } \\
\hline 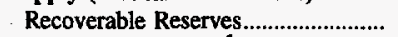 & 736,601 & 912,870 & 939,706 & 937,427 & 985,784 & $1,529,533$ & -19.3 & -7.0 & -7.8 \\
\hline Productive Capacityl............................. & 77,187 & 80,975 & 82,148 & 82,968 & NA & 77,674 & -4.7 & NA & -.1 \\
\hline 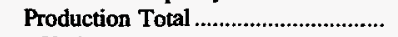 & 61,576 & 62,237 & 59,700 & 68,981 & 65,381 & 71,648 & -1.1 & -1.5 & -1.7 \\
\hline 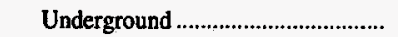 & 41,409 & 39,974 & 36,934 & 45,112 & 40,953 & 37,418 & 3.6 & .3 & 1.1 \\
\hline Surface & 20,167 & 22,263 & 22,766 & 23,868 & 24,429 & 34,230 & -9.4 & -4.7 & -5.7 \\
\hline 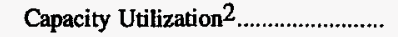 & 78.81 & 75.89 & 71.79 & 82.20 & NA & 90.85 & 3.8 & NA & -1.6 \\
\hline \multicolumn{10}{|l|}{ Ratio of Recoverable } \\
\hline Reserves to Production........................... & 12.0 & 14.7 & 15.7 & 13.6 & 15.1 & 21.3 & -18.4 & -5.6 & -6.2 \\
\hline 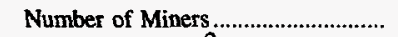 & 8,968 & 9,975 & 10,940 & 12,659 & 13,506 & 19,791 & -10.1 & -9.7 & -8.4 \\
\hline 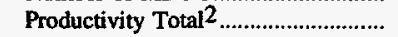 & 3.23 & 2.98 & 2.80 & 2.67 & 2.43 & 1.83 & 8.3 & 7.3 & 6.5 \\
\hline 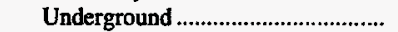 & 3.49 & 3.18 & 2.91 & 2.81 & 2.38 & 1.57 & 9.6 & 10.1 & 9.3 \\
\hline Surface & 2.79 & 2.67 & 2.63 & 2.45 & 2.53 & 2.26 & 4.3 & 2.4 & 2.3 \\
\hline Producer/Distributor Stocks ............... & 2,487 & 2,787 & 1,826 & 2,903 & 2,844 & - & -10.8 & -3.3 & - \\
\hline Imports ${ }^{3}$ & 87 & - & - & - & - & - & - & - & - \\
\hline \multicolumn{10}{|l|}{ Distribution (thousand short tons) } \\
\hline 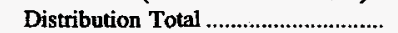 & 62,240 & 61,508 & 58,990 & 67,649 & 65,454 & NA & 1.2 & -1.3 & NA \\
\hline Domestic Distribution Total............ & 53,961 & 55,207 & 53,482 & 61,208 & 58,151 & NA & -2.3 & -1.8 & NA \\
\hline 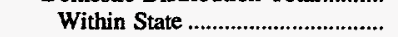 & 36,147 & 35,189 & 33,456 & 37,696 & 36,260 & NA & 2.7 & -.1 & NA \\
\hline 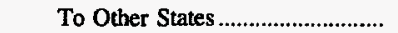 & 17,814 & 20,018 & 20,026 & 23,512 & 21,891 & NA & -11.0 & -5.0 & NA \\
\hline Foreign Distribution Total.............. & 8,279 & 6,301 & 5,508 & 6,440 & 7,303 & NA & 31.4 & 3.2 & NA \\
\hline 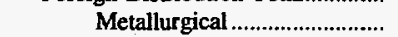 & 1,467 & 1,624 & 1,919 & 1,266 & 1,332 & NA & -9.7 & 2.4 & NA \\
\hline 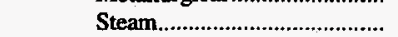 & 6,812 & 4,677 & 3,589 & 5,175 & 5,971 & NA & 45.6 & 3.3 & NA \\
\hline 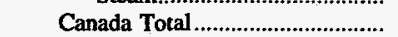 & 713 & 844 & 597 & 1,599 & 2,338 & NA & -15.5 & -25.7 & NA \\
\hline 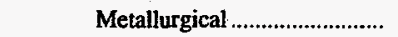 & 4 & - & 10 & - & 8 & NA & - & -14.2 & NA \\
\hline 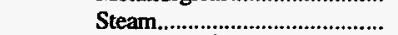 & 708 & 844 & 587 & 1,599 & 2,330 & NA & -16.0 & -25.7 & NA \\
\hline 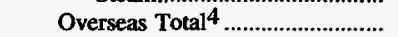 & 7,566 & 5,457 & 4,911 & 4,841 & 4,966 & NA & 38.6 & 11.1 & NA \\
\hline 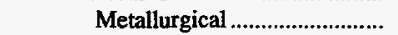 & 1,463 & 1,624 & 1,909 & 1,266 & 1,324 & NA & -9.9 & 2.5 & NA \\
\hline 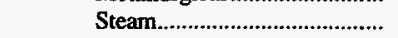 & 6,103 & 3,833 & 3,002 & 3,575 & 3,641 & NA & 59.2 & 13.8 & NA \\
\hline \multicolumn{10}{|l|}{ Demand (thousand short tons) } \\
\hline 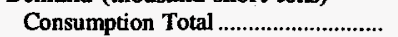 & 55,289 & 54,094 & 56,158 & 56,074 & 54,931 & 52,972 & 2.2 & .2 & .5 \\
\hline 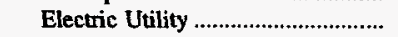 & 39,252 & 38,044 & 40,257 & 40,407 & 40,662 & 40,589 & 3.2 & -.9 & -.4 \\
\hline 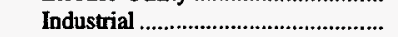 & 4,027 & 4,044 & 4,311 & 4,173 & 4,049 & 3,975 & -.4 & -1 & .1 \\
\hline 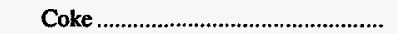 & 10,858 & 10,849 & 10,333 & 9,868 & 8,812 & 7,007 & .1 & 5.4 & 5.0 \\
\hline Residential/Commercial................... & 1,151 & 1,156 & 1,257 & 1,626 & 1,408 & 1,402 & -.4 & -4.9 & -2.2 \\
\hline Consumer Stocks Total ......................... & 10,303 & 12,060 & 12,265 & 15,976 & 15,546 & 14,691 & -14.6 & -9.8 & -3.9 \\
\hline 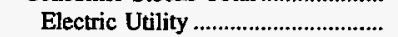 & 9,244 & 11,000 & 11,110 & 14,866 & 14,412 & 13,619 & -16.0 & -10.5 & -4.2 \\
\hline All Other & 1,059 & 1,060 & 1,155 & 1,110 & 1,135 & 1,072 & -.1 & -1.7 & -.1 \\
\hline \multicolumn{10}{|c|}{ Coal Prices (nominal dollars per short ton) } \\
\hline 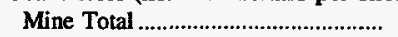 & $\$ 26.78$ & $\$ 26.18$ & $\$ 26.50$ & $\$ 28.61$ & $\$ 29.40$ & $\$ 30.30$ & 2.3 & -2.3 & -1.4 \\
\hline 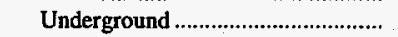 & 27.09 & 26.59 & 27.35 & 30.23 & 31.72 & 33.07 & 1.9 & -3.9 & -2.2 \\
\hline 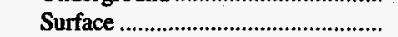 & 26.14 & 25.43 & 25.09 & 25.48 & 25.42 & 27.22 & 2.8 & .7 & -.4 \\
\hline \multicolumn{10}{|l|}{ Consumer } \\
\hline 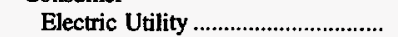 & 33.48 & 35.39 & 35.73 & 36.81 & 38.05 & 36.65 & -5.4 & -3.1 & -1.0 \\
\hline 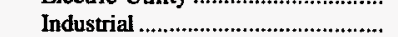 & 34.07 & 33.66 & 34.04 & 35.70 & 35.89 & 38.17 & 1.2 & -1.3 & -1.3 \\
\hline 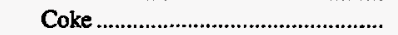 & 46.11 & 46.25 & 46.41 & 46.49 & 46.86 & 47.23 & -.3 & -.4 & -.3 \\
\hline
\end{tabular}

1 For 1986, the Form EIA-7A solicited data on "Daily Productive Capacity." To obtain annual productive capacity for a mine in 1986, each mine's daily productive capacity was multiplied by the number of days worked during the year.

2 Capacity utilization (percent) is the ratio of total production to annual productive capacity as reported by mining companies on Form ElA-7A. Productivity (short tons per miner per hour) is calculated by dividing total coal production by the total direct labor hours worked by all employees engaged in production, preparation, processing, development, maintenance, repair, and shop or yard work at mining operations.

3 Imports for 1992 through 1995 include imports to electric utilities, manufacturing plants and coke plants. Imports for 1986 through 1991 include only imports to electric utilities.

4 Includes Mexico.

NA Not available.

Notes: Excludes silt, culm, refuse bank, slurry dam, and dredge operations except for Pennsylvania anthracite. Totals may not equal sum of components due to independent rounding.

Sources: Energy Information Administration, Form EIA-3, “Quarterly Coal Consumption Report - Manufacturing Plants"; Form EIA-5, “Coke Plant Report - Quarterly"; Form EIA-6, “Coal Distribution Report"; Form EIA-7A, "Coal Production Report"; Form EIA-759, "Monthly Power Plant Report"; and U.S. Department of Commerce, Bureau of the Census, "Monthly Report EM 545" and "Monthly Report IM 145." 
Table A12. Texas Coal Statistics, 1986, 1991-1995

\begin{tabular}{|c|c|c|c|c|c|c|c|c|c|}
\hline \multirow{2}{*}{ Category } & \multirow{2}{*}{1995} & \multirow{2}{*}{1994} & \multirow{2}{*}{1993} & \multirow{2}{*}{1992} & \multirow{2}{*}{1991} & \multirow{2}{*}{1986} & \multirow{2}{*}{$\begin{array}{c}\text { Percent } \\
\text { Change } \\
\text { 1994-1995 }\end{array}$} & \multicolumn{2}{|c|}{$\begin{array}{l}\text { Average Annual } \\
\text { Percent Change }\end{array}$} \\
\hline & & & & & & & & 1991-1995 & $1986-1995$ \\
\hline \multicolumn{10}{|l|}{ Supply (thousand short tons) } \\
\hline Recoverable Reserves........................... & 939,901 & $1,026,309$ & $1,104,864$ & $1,188,240$ & $1,225,124$ & $1,260,715$ & -8.4 & -6.4 & -3.2 \\
\hline Productive Capacity 1 .......................... & 54,758 & 55,856 & 57,115 & 58,541 & NA & 51,980 & -2.0 & NA & .6 \\
\hline 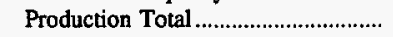 & 52,684 & 52,346 & 54,567 & 55,071 & 53,825 & 48,590 & .6 & -.5 & .9 \\
\hline Surface & 52,684 & 52,346 & 54,567 & 55,071 & 53,825 & 48,590 & .6 & -.5 & .9 \\
\hline Capacity Utilization 2 ........................... & 96.21 & 93.72 & 95.54 & 94.07 & NA & 93.48 & 2.7 & NA & 3 \\
\hline \multicolumn{10}{|l|}{ Ratio of Recoverable } \\
\hline Reserves to Production......................... & 17.8 & 19.6 & 20.3 & 21.6 & 22.8 & 25.9 & -9.0 & -5.9 & -4.1 \\
\hline 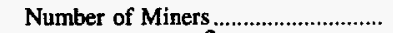 & 1,590 & 1,733 & 1,841 & 2,001 & 2,149 & 3,074 & -8.3 & -7.3 & -7.1 \\
\hline Productivity Total 2 .............................. & 9.10 & 8.82 & 8.42 & 7.34 & 7.17 & 6.33 & 3.2 & 6.1 & 4.1 \\
\hline 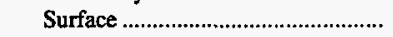 & 9.10 & 8.82 & 8.42 & 7.34 & 7.17 & 6.33 & 3.2 & 6.1 & 4.1 \\
\hline Producer/Distributor Stocks .............. & 864 & 1,430 & 1,237 & 543 & 400 & - & -39.6 & 21.3 & - \\
\hline Imports $^{3} \ldots \ldots \ldots \ldots \ldots \ldots$ & - & 153 & 156 & 80 & - & - & -100.0 & - & - \\
\hline \multicolumn{10}{|l|}{ Distribution (thousand short tons) } \\
\hline 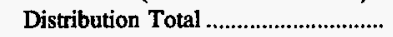 & 52,832 & 52,256 & 54,224 & 54,447 & 54,243 & NA & 1.1 & -.7 & NA \\
\hline Domestic Distribution Total........... & 52,812 & 52,256 & 54,224 & 54,447 & 54,243 & NA & 1.1 & -.7 & NA \\
\hline 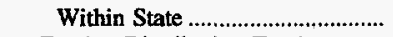 & 52,812 & 52,256 & 54,224 & 54,447 & 54,243 & NA & 1.1 & -.7 & NA \\
\hline Foreign Distribution Total.............. & 20 & - & - & - & - & NA & - & - & NA \\
\hline Steam & 20 & - & - & - & - & NA & - & - & $\mathrm{NA}$ \\
\hline 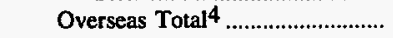 & 20 & - & - & - & - & NA & - & - & $\mathrm{NA}$ \\
\hline 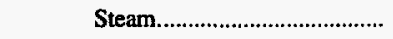 & 20 & - & - & - & - & NA & - & - & NA \\
\hline \multicolumn{10}{|l|}{ Demand (thousand short tons) } \\
\hline Consumption Total .................................. & 92,612 & 93,829 & 96,809 & 91,568 & 92,064 & 79,259 & -1.3 & .1 & 1.7 \\
\hline 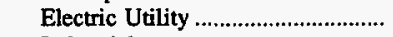 & 88,358 & 88,479 & 92,135 & 87,333 & 87,856 & 74,757 & -.1 & .1 & 1.9 \\
\hline 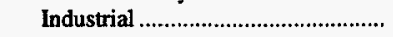 & 4,255 & 5,350 & 4,667 & 4,225 & 4,198 & 4,411 & -20.5 & .3 & -.4 \\
\hline Coke & - & - & - & - & - & $w$ & - & - & - \\
\hline Residential/Commercial...................... & - & * & 6 & 10 & $\mathbf{w}$ & $\mathbf{w}$ & - & $\mathbf{w}$ & $\mathbf{w}$ \\
\hline 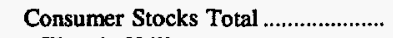 & 10,829 & 9,793 & 9,172 & 10,392 & w & $\mathbf{w}$ & 10.6 & $w$ & $\mathbf{w}$ \\
\hline 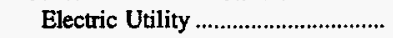 & 10,628 & 9,578 & 8,125 & 10,143 & 10,474 & 9,014 & 10.9 & .4 & 1.8 \\
\hline 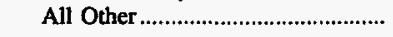 & 201 & 215 & 1,047 & 248 & $\mathbf{w}$ & $\mathbf{w}$ & -6.4 & $\mathbf{w}$ & $\mathbf{w}$ \\
\hline \multicolumn{10}{|c|}{ Coal Prices (nominal dollars per short ton) } \\
\hline 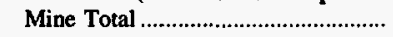 & $\$ 12.16$ & $\$ 12.38$ & $\$ 12.87$ & $\$ 12.42$ & $\$ 12.21$ & $\$ 11.60$ & -1.8 & -.1 & .5 \\
\hline 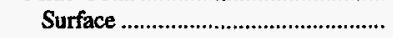 & 12.16 & 12.38 & 12.87 & 12.42 & 12.21 & 11.60 & -1.8 & -.1 & .5 \\
\hline \multicolumn{10}{|l|}{ Consumer } \\
\hline 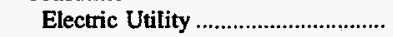 & 19.65 & 19.84 & 20.91 & 21.58 & 21.66 & 22.59 & -.9 & -2.4 & -1.5 \\
\hline 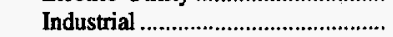 & 18.76 & 19.54 & 17.58 & 18.14 & 18.61 & 23.95 & -4.0 & .2 & -2.7 \\
\hline Coke & - & - & - & - & - & $\mathbf{w}$ & - & - & - \\
\hline
\end{tabular}

1 For 1986, the Form EIA-7A solicited data on "Daily Productive Capacity." To obtain annual productive capacity for a mine in 1986, each mine's daily productive capacity was multiplied by the number of days worked during the year.

2 Capacity utilization (percent) is the ratio of total production to annual productive capacity as reported by mining companies on Form EIA-7A. Productivity (short tons per miner per hour) is calculated by dividing total coal production by the total direct labor hours worked by all employees engaged in production, preparation, processing, development, maintenance, repair, and shop or yard work at mining operations.

3 Imports for 1992 through 1995 include imports to electric utilities, manufacturing plants and coke plants. Imports for 1986 through 1991 include only imports to electric utilities.

4 Includes Mexico.

* Data round to zero.

Withheld to avoid disclosure of individual company data.

NA Not available.

Notes: Excludes silt, culm, refuse bank, slurry dam, and dredge operations except for Pennsylvania anthracite. Totals may not equal sum of components due to independent rounding.

Sources: Energy Information Administration, Form ELA-3, "Quarterly Coal Consumption Report - Manufacturing Plants"; Form EIA-5, "Coke Plant Report - Quarterly"; Form EIA-6, "Coal Distribution Report"; Form EIA-7A, "Coal Production Report"; Form EIA-759. "Monthly Power Plant Report"; and U.S. Department of Commerce, Bureau of the Census, "Monthly Report EM 545" and "Monthly Report IM 145." 
Table A13. Utah Coal Statistics, 1986, 1991-1995

\begin{tabular}{|c|c|c|c|c|c|c|c|c|c|}
\hline \multirow{2}{*}{ Category } & \multirow{2}{*}{1995} & \multirow{2}{*}{1994} & \multirow{2}{*}{1993} & \multirow{2}{*}{1992} & \multirow{2}{*}{1991} & \multirow{2}{*}{1986} & \multirow{2}{*}{$\begin{array}{c}\text { Percent } \\
\text { Change } \\
\text { 1994-1995 }\end{array}$} & \multicolumn{2}{|c|}{$\begin{array}{l}\text { Average Annual } \\
\text { Percent Change }\end{array}$} \\
\hline & & & & & & & & 1991-1995 & 1986-1995 \\
\hline \multicolumn{10}{|l|}{ Supply (thousand short tons) } \\
\hline Recoverable Reserves.......................... & 374,750 & 423,349 & 446,729 & 488,109 & 508,872 & 810,968 & -11.5 & -7.4 & -8.2 \\
\hline 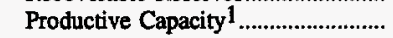 & 30,888 & 27,640 & 25,933 & 25,534 & NA & 18,216 & 11.7 & $\mathrm{NA}$ & 6.0 \\
\hline 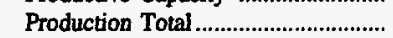 & 25,167 & 24,399 & 21,847 & 21,339 & 21,945 & 14,269 & 3.1 & 3.5 & 6.5 \\
\hline 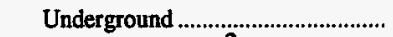 & 25,167 & 24,399 & 21,847 & 21,339 & 21,945 & 14,269 & 3.1 & 3.5 & 6.5 \\
\hline Capacity Utilization $2 \ldots \ldots \ldots \ldots \ldots \ldots \ldots$ & 81.48 & 88.27 & 84.22 & 83.57 & NA & 78.33 & -7.7 & NA & .4 \\
\hline \multicolumn{10}{|l|}{ Ratio of Recoverable } \\
\hline Reserves to Production.......................... & 14.9 & 17.3 & 20.4 & 22.9 & 23.2 & 56.8 & -14.2 & -10.5 & -13.8 \\
\hline Number of Miners ................................ & 1,893 & 1,675 & 1,769 & 1,997 & 2,277 & 2,881 & 13.0 & -4.5 & -4.5 \\
\hline 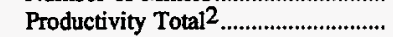 & 7.02 & 6.59 & 5.96 & 5.46 & 4.80 & 3.08 & 6.5 & 10.0 & 9.6 \\
\hline 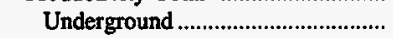 & 7.02 & 6.59 & 5.96 & 5.46 & 4.80 & 3.08 & 6.5 & 10.0 & 9.6 \\
\hline Producer/Distributor Stocks .............. & 1,946 & 1,301 & 1,203 & 1,827 & 1,676 & - & 49.6 & 3.8 & - \\
\hline \multicolumn{10}{|l|}{ Distribution (thousand short tons) } \\
\hline 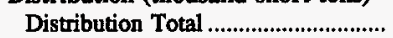 & 25,521 & 23,225 & 22,243 & 21,052 & 21,444 & NA & 9.9 & 4.4 & NA \\
\hline Domestic Distribution Total ............ & 21,591 & 20,527 & 19,283 & 18,792 & 19,358 & NA & 5.2 & 2.8 & NA \\
\hline 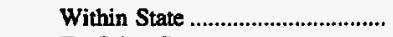 & 12,755 & 13,586 & 13,418 & 13,035 & 13,605 & NA & -6.1 & -1.6 & NA \\
\hline 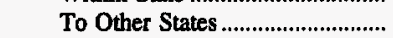 & 8,836 & 6,941 & 5,866 & 5,758 & 5,753 & NA & 27.3 & 11.3 & NA \\
\hline Foreign Distribution Total.............. & 3,930 & 2,698 & 2,959 & 2,260 & 2,086 & NA & 45.7 & 17.2 & NA \\
\hline 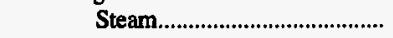 & 3,930 & 2,698 & 2,959 & 2,260 & 2,086 & NA & 45.7 & 17.2 & $\mathrm{NA}$ \\
\hline 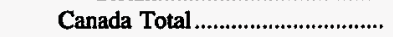 & - & - & 346 & - & - & NA & - & - & NA \\
\hline 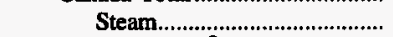 & - & - & 346 & - & - & NA & - & - & NA \\
\hline Overseas Total $3 \ldots \ldots \ldots \ldots \ldots \ldots \ldots \ldots$ & 3,930 & 2,698 & 2,613 & 2,260 & 2,086 & NA & 45.7 & 17.2 & $\mathrm{NA}$ \\
\hline 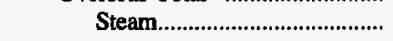 & 3,930 & 2,698 & 2,613 & 2,260 & 2,086 & NA & 45.7 & 17.2 & NA \\
\hline \multicolumn{10}{|l|}{ Demand (thousand short tons) } \\
\hline 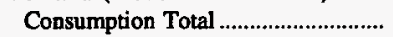 & 15,307 & 16,216 & 15,848 & 15,719 & 14,834 & 8,112 & -5.6 & .8 & 7.3 \\
\hline 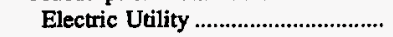 & 13,325 & 14,269 & 13,995 & 13,857 & 12,829 & 6,756 & -6.6 & .9 & 7.8 \\
\hline 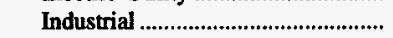 & 915 & 835 & 727 & 525 & 508 & 380 & 9.5 & 15.8 & 10.3 \\
\hline 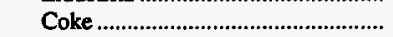 & w & w & w & $\mathbf{w}$ & $\mathbf{w}$ & $w$ & w & w & w \\
\hline 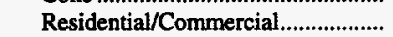 & $\mathbf{w}$ & $\mathbf{w}$ & $\mathbf{w}$ & $\mathbf{w}$ & $\mathbf{w}$ & w & $\mathbf{w}$ & w & $\mathbf{w}$ \\
\hline Consumer Stocks Total ......................... & w & w & $\mathbf{w}$ & $\mathbf{w}$ & $\mathbf{w}$ & $\mathbf{w}$ & w & w & w \\
\hline 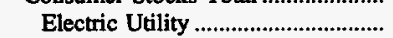 & 2,250 & 2,753 & 3,264 & 3,153 & 4,123 & 2,344 & -18.3 & -14.0 & -.4 \\
\hline 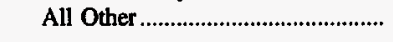 & w & $\mathbf{w}$ & w & w & $\mathbf{w}$ & $\mathbf{w}$ & $w$ & $w$ & w \\
\hline \multicolumn{10}{|c|}{ Coal Prices (nominal dollars per short ton) } \\
\hline 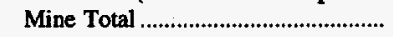 & $\$ 19.10$ & $\$ 19.27$ & $\$ 20.81$ & $\$ 21.11$ & $\$ 22.59$ & $\$ 27.64$ & -.9 & -4.1 & -4.0 \\
\hline 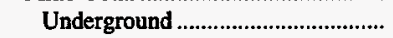 & 19.10 & 19.27 & 20.81 & 21.11 & 22.59 & 27.64 & -.9 & -4.1 & -4.0 \\
\hline \multicolumn{10}{|l|}{ Consumer } \\
\hline 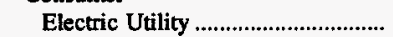 & 25.27 & 26.10 & 27.34 & 27.54 & 27.40 & 32.32 & -3.2 & -2.0 & -2.7 \\
\hline 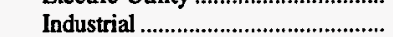 & 19.74 & 26.57 & 26.51 & 25.35 & 26.43 & 27.59 & -25.7 & -7.0 & -3.6 \\
\hline Coke & $\mathbf{w}$ & $\mathbf{w}$ & $\mathbf{w}$ & $\mathbf{w}$ & $\mathbf{w}$ & $\mathbf{w}$ & $\mathbf{w}$ & w & $w$ \\
\hline
\end{tabular}

1 For 1986, the Form EIA-7A solicited data on "Daily Productive Capacity." To obtain annual productive capacity for a mine in 1986, each mine's daily productive capacity was multiplied by the number of days worked during the year.

2 Capacity utilization (percent) is the ratio of total production to annual productive capacity as reported by mining companies on Form EIA-7A. Productivity (short tons per miner per hour) is calculated by dividing total coal production by the total direct labor hours worked by all employees engaged in production, preparation, processing, development, maintenance, repair, and shop or yard work at mining operations.

3 Includes Mexico.

Withheld to avoid disclosure of individual company data.

NA Not available.

Notes: Excludes silt, culm, refuse bank, slurry dam, and dredge operations except for Pennsylvania anthracite. Totals may not equal sum of components due to independent rounding.

Sources: Energy Information Administration, Form EIA-3, "Quarterly Coal Consumption Report - Manufacturing Plants"; Form EIA-5, "Coke Plant Report - Quarterly"; Form EIA-6, "Coal Distribution Report"; Form EIA-7A, "Coal Production Report"; Form EIA-759, "Monthly Power Plant Report"; and U.S. Department of Commerce, Bureau of the Census, "Monthly Report EM 545" and "Monthly Report IM 145." 
Table A14. Virginia Coal Statistics, 1986, 1991-1995

\begin{tabular}{|c|c|c|c|c|c|c|c|c|c|}
\hline \multirow{2}{*}{ Category } & \multirow{2}{*}{1995} & \multirow{2}{*}{1994} & \multirow{2}{*}{1993} & \multirow{2}{*}{1992} & \multirow{2}{*}{1991} & \multirow{2}{*}{1986} & \multirow{2}{*}{$\begin{array}{c}\text { Percent } \\
\text { Change } \\
\text { 1994-1995 }\end{array}$} & \multicolumn{2}{|c|}{$\begin{array}{l}\text { Average Annual } \\
\text { Percent Change }\end{array}$} \\
\hline & & & & & & & & 1991-1995 & $1986-1995$ \\
\hline \multicolumn{10}{|l|}{ Supply (thousand short tons) } \\
\hline 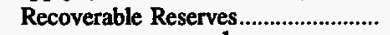 & 203,159 & 236,773 & 335,883 & 365,823 & 411,849 & 499,068 & -14.2 & -16.2 & -9.5 \\
\hline Productive Capacity 1 .......................... & 43,037 & 46,462 & 50,879 & 54,471 & NA & 41,501 & -7.4 & NA & .4 \\
\hline 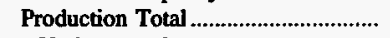 & 34,099 & 37,129 & 39,317 & 43,024 & 41,954 & 41,178 & -8.2 & -5.0 & -2.1 \\
\hline 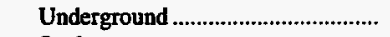 & 25,372 & 28,054 & 30,166 & 34,715 & 34,138 & 34,054 & -9.6 & -7.1 & -3.2 \\
\hline 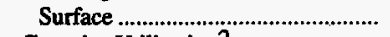 & 8,727 & 9,075 & 9,151 & 8,308 & 7,816 & 7,124 & -3.8 & 2.8 & 2.3 \\
\hline $\begin{array}{l}\text { Capacity Utilization }{ }^{2} \\
\text { Ratio of Recoverable.................... }\end{array}$ & 79.07 & 79.61 & 77.07 & 78.70 & NA & 98.24 & -.7 & NA & -2.4 \\
\hline Reserves to Production.......................... & 6.0 & 6.4 & 8.5 & 8.5 & 9.8 & 12.1 & -6.6 & -11.7 & -7.6 \\
\hline 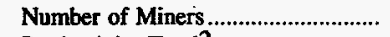 & 6,919 & 8,121 & 8,339 & 9,138 & 10,055 & 13,141 & -14.8 & -8.9 & -6.9 \\
\hline Productivity Total 2 & 2.50 & 2.51 & 2.41 & 2.37 & 2.23 & 1.90 & -.1 & 2.9 & 3.1 \\
\hline 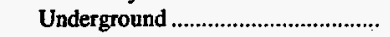 & 2.25 & 2.27 & 2.19 & 2.20 & 2.12 & 1.81 & -.7 & 1.6 & 2.4 \\
\hline Surface & 3.73 & 3.73 & 3.55 & 3.50 & 2.95 & 2.48 & -.1 & 6.0 & 4.6 \\
\hline Producer/Distributor Stocks ............... & 1,649 & 1,180 & 1,389 & 1,714 & 2,154 & - & 39.7 & -6.5 & - \\
\hline \multicolumn{10}{|l|}{ Distribution (thousand short tons) } \\
\hline 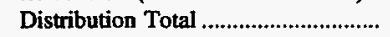 & 34,024 & 38,548 & 41,639 & 45,728 & 43,495 & NA & -11.7 & -5.9 & NA \\
\hline Domestic Distribution Total........... & 24,283 & 26,866 & 27,388 & 28,504 & 25,390 & NA & -9.6 & -1.1 & NA \\
\hline 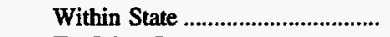 & 5,657 & 6,867 & 6,076 & 6,082 & 5,457 & NA & -17.6 & .9 & NA \\
\hline 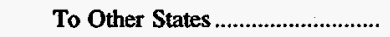 & 18,625 & 19,999 & 21,312 & 22,422 & 19,933 & NA & -6.9 & -1.7 & NA \\
\hline Foreign Distribution Total.............. & 9,742 & 11,683 & 14,251 & 17,224 & 18,105 & NA & -16.6 & -14.3 & NA \\
\hline 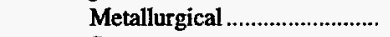 & 8,917 & 11,193 & 13,512 & 14,005 & 14,403 & NA & -20.3 & -11.3 & NA \\
\hline Steam & 825 & 490 & 739 & 3,219 & 3,701 & NA & 68.3 & -31.3 & NA \\
\hline 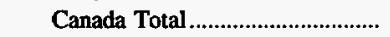 & 445 & 786 & 1,229 & 1,331 & 1,404 & NA & -43.4 & -25.0 & NA \\
\hline Metallurgical ............................. & 445 & 786 & 1,229 & - & - & NA & -43.4 & - & NA \\
\hline Steam & - & - & - & 1,331 & 1,404 & NA & - & - & NA \\
\hline Overseas Total $^{3} \ldots \ldots \ldots \ldots \ldots \ldots$ & 9,297 & $10 ; 897$ & 13,021 & 15,893 & 16,701 & NA & -14.7 & -13.6 & NA \\
\hline 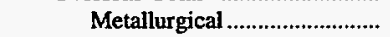 & 8,472 & 10,407 & 12,283 & 14,005 & 14,403 & NA & -18.6 & -12.4 & NA \\
\hline Steam & 825 & 490 & 739 & 1,888 & 2,298 & NA & 68.3 & -22.6 & NA \\
\hline \multicolumn{10}{|l|}{ Demand (thousand short tons) } \\
\hline 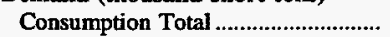 & 13,425 & 12,792 & 13,584 & 13,418 & 13,980 & 11,857 & 4.9 & -1.0 & 1.4 \\
\hline 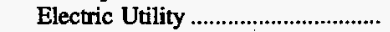 & 9,543 & 8,670 & 9,447 & 8,661 & 8,568 & 7,345 & 10.1 & 2.7 & 2.9 \\
\hline Industrial & 2,631 & 2,838 & 2,863 & 3,592 & 4,301 & 3,373 & -7.3 & -11.5 & -2.7 \\
\hline Coke & $\mathbf{w}$ & $\mathbf{w}$ & $\mathbf{w}$ & $\mathbf{w}$ & w & $\mathbf{w}$ & $\mathbf{w}$ & $\mathbf{w}$ & $\mathbf{w}$ \\
\hline Residential/Commercial.................... & $\mathbf{w}$ & $\mathbf{w}$ & $w$ & $\mathbf{w}$ & $\mathbf{w}$ & $\mathbf{w}$ & $w$ & $w$ & $\mathbf{w}$ \\
\hline Consumer Stocks Total ........................ & $\mathbf{w}$ & $\mathbf{w}$ & $\mathbf{w}$ & $\mathbf{w}$ & $\mathbf{w}$ & $\mathbf{w}$ & w & $\mathbf{w}$ & w \\
\hline 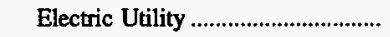 & 1,098 & 2,064 & 1,418 & 1,922 & 1,685 & 1,602 & -46.8 & -10.2 & -4.1 \\
\hline All Other & w & w & w & w & w & w & w & $\mathbf{w}$ & $\mathbf{w}$ \\
\hline \multicolumn{10}{|c|}{ Coal Prices (nominal dollars per short ton) } \\
\hline 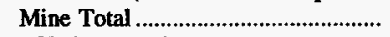 & $\$ 28.47$ & $\$ 26.84$ & $\$ 26.80$ & $\$ 27.55$ & $\$ 27.45$ & $\$ 28.13$ & 6.1 & .9 & .1 \\
\hline 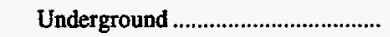 & 29.20 & 27.33 & 27.26 & 27.88 & 27.76 & 28.31 & 6.8 & 1.3 & .3 \\
\hline 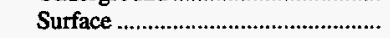 & 26.34 & 25.33 & 25.29 & 26.17 & 26.12 & 27.27 & 4.0 & .2 & -.4 \\
\hline \multicolumn{10}{|l|}{ Consumer } \\
\hline 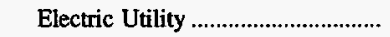 & 36.90 & 37.05 & 37.57 & 37.81 & 38.87 & 43.86 & -.4 & -1.3 & -1.9 \\
\hline 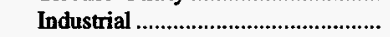 & 42.50 & 41.56 & 41.27 & 40.97 & 40.53 & 40.29 & 2.2 & 1.2 & .6 \\
\hline Coke & $\mathbf{w}$ & $\mathbf{w}$ & $\mathbf{w}$ & w & w & $\mathbf{w}$ & w & $\mathbf{w}$ & $\mathbf{w}$ \\
\hline
\end{tabular}

1 For 1986, the Form EIA-7A solicited data on "Daily Productive Capacity." To obtain annual productive capacity for a mine in 1986, each mine's daily productive capacity was multiplied by the number of days worked during the year.

2 Capacity utilization (percent) is the ratio of total production to annual productive capacity as reported by mining companies on Form EIA-7A. Productivity (short tons per miner per hour) is calculated by dividing total coal production by the total direct labor hours worked by all employees engaged in production, preparation, processing, development, maintenance, repair, and shop or yard work at mining operations.

3 Includes Mexico.

Witheld to avoid disclosure of individual company data.

NA Not available.

Notes: Excludes silt, culm, refuse bank, slurry dam, and dredge operations except for Pennsylvania anthracite. Totals may not equal sum of components due to independent rounding.

Sources: Energy Information Administration, Form EIA-3, "Quarterly Coal Consumption Report - Manufacturing Plants"; Form EIA-5, "Coke Plant Report - Quarterly"; Form EIA-6, “Coal Distribution Report"; Form EIA-7A, "Coal Production Report"; Form EIA-759, "Monthly Power Plant Report"; and U.S. Department of Commerce, Bureau of the Census, "Monthly Report EM 545" and "Monthly Report IM 145." 


\begin{tabular}{|c|c|c|c|c|c|c|c|c|c|}
\hline \multirow{2}{*}{ Category } & \multirow{2}{*}{1995} & \multirow{2}{*}{1994} & \multirow{2}{*}{1993} & \multirow{2}{*}{1992} & \multirow{2}{*}{1991} & \multirow{2}{*}{1986} & \multirow{2}{*}{$\begin{array}{c}\text { Percent } \\
\text { Change } \\
\text { 1994-1995 }\end{array}$} & \multicolumn{2}{|c|}{$\begin{array}{l}\text { Average Annual } \\
\text { Percent Change }\end{array}$} \\
\hline & & & & & & & & 1991-1995 & 1986-1995 \\
\hline \multicolumn{10}{|l|}{ Supply (thousand short tons) } \\
\hline 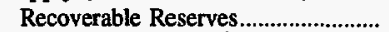 & $1,731,400$ & $1,829,630$ & $1,930,903$ & $2,043,062$ & $2,122,087$ & $2,674,207$ & -5.4 & -4.9 & -4.7 \\
\hline Productive Capacity 1 .......................... & 204,837 & 201,684 & 191,706 & 198,083 & NA & 138,629 & 1.6 & NA & 4.4 \\
\hline 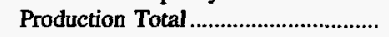 & 162,997 & 161,776 & 130,525 & 162,164 & 167,352 & 129,907 & .8 & -.6 & 2.5 \\
\hline 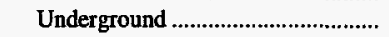 & 110,029 & 111,679 & 87,997 & 115,212 & 119,821 & 103,369 & -1.5 & -2.1 & .7 \\
\hline 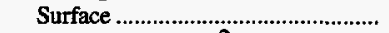 & 52,968 & 50,097 & 42,528 & 46,952 & 47,530 & 26,539 & 5.7 & 2.7 & 8.0 \\
\hline 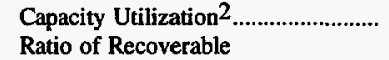 & 79.50 & 80.07 & 67.91 & 81.70 & NA & 93.24 & -.7 & NA & -1.8 \\
\hline Reserves to Production........................... & 10.6 & 11.3 & 14.8 & 12.6 & 12.7 & 20.6 & -6.1 & -4.3 & -7.1 \\
\hline 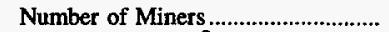 & 21,334 & 21,861 & 22,979 & 26,017 & 28,310 & 33,540 & -2.4 & -6.8 & -4.9 \\
\hline Productivity Total $2 \ldots \ldots \ldots \ldots \ldots \ldots \ldots \ldots$ & 3.74 & 3.69 & 3.27 & 3.27 & 3.11 & 2.19 & 1.5 & 4.7 & 6.1 \\
\hline 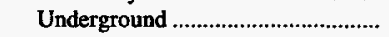 & 3.40 & 3.38 & 2.92 & 2.99 & 2.83 & 2.07 & .5 & 4.7 & 5.7 \\
\hline Surface & 4.74 & 4.62 & 4.35 & 4.27 & 4.18 & 2.88 & 2.6 & 3.1 & 5.7 \\
\hline Producer/Distributor Stocks ............... & 6,176 & 6,692 & 4,059 & 7,405 & 6,464 & - & -7.7 & -1.1 & - \\
\hline \multicolumn{10}{|l|}{ Distribution (thousand short tons) } \\
\hline 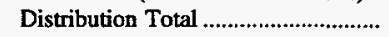 & 165,187 & 158,985 & 135,818 & 163,723 & 165,576 & NA & 3.9 & -.1 & NA \\
\hline Domestic Distribution Total........... & 120,866 & 122,779 & 102,659 & 112,917 & 111,785 & NA & -1.6 & 2.0 & NA \\
\hline 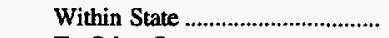 & 29,018 & 30,891 & 22,887 & 26,644 & 27,191 & NA & -6.1 & 1.6 & NA \\
\hline 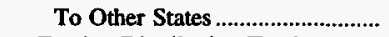 & 91,848 & 91,888 & 79,772 & 86,273 & 84,593 & NA & $*$ & 2.1 & NA \\
\hline Foreign Distribution Total.............. & 44,321 & 36,205 & 33,159 & 50,806 & 53,792 & NA & 22.4 & -4.7 & NA \\
\hline 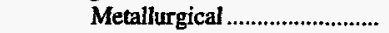 & 34,633 & 31,612 & 27,627 & 35,337 & 35,883 & NA & 9.6 & -.9 & NA \\
\hline 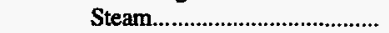 & 9,688 & 4,593 & 5,532 & 15,469 & 17,908 & NA & 110.9 & -14.2 & NA \\
\hline 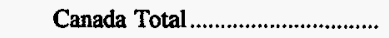 & 5,784 & 5,644 & 4,108 & 8,911 & 7,464 & NA & 2.5 & -6.2 & NA \\
\hline 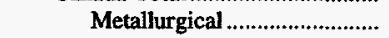 & 5,759 & 5,605 & 4,071 & 8,021 & 7,351 & NA & 2.8 & -5.9 & NA \\
\hline 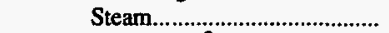 & 25 & 40 & 37 & 890 & 113 & NA & -37.6 & -31.5 & NA \\
\hline 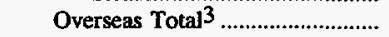 & 38,537 & 30,561 & 29,052 & 41,895 & 46,328 & NA & 26.1 & -4.5 & NA \\
\hline 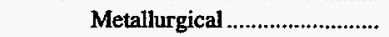 & 28,874 & 26,008 & 23,556 & 27,316 & 28,532 & NA & 11.0 & 3 & NA \\
\hline Steam & 9,663 & 4,553 & 5,495 & 14,580 & 17,796 & NA & 112.2 & -14.2 & NA \\
\hline \multicolumn{10}{|l|}{ Demand (thousand short tons) } \\
\hline 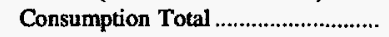 & 34,489 & 34,767 & 32,046 & 32,019 & 31,843 & 35,009 & -.8 & 2.0 & -.2 \\
\hline 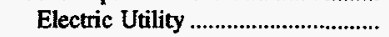 & 30,657 & 30,318 & 27,782 & 28,050 & 27,557 & 30,790 & 1.1 & 2.7 & * \\
\hline 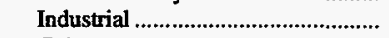 & 1,984 & 2,637 & 2,406 & 2,266 & 2,310 & 2,234 & -24.8 & -3.7 & -1.3 \\
\hline Coke & $\mathbf{w}$ & w & w & $\mathbf{w}$ & $w$ & w & w & $\mathbf{w}$ & $w$ \\
\hline Residential/Commercial................... & $\mathbf{w}$ & $w$ & $w$ & $\mathbf{w}$ & $\mathbf{w}$ & $w$ & w & $w$ & $\mathbf{w}$ \\
\hline 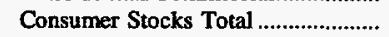 & $\mathbf{w}$ & $\mathbf{w}$ & w & $\mathbf{w}$ & $\mathbf{w}$ & w & w & w & $\mathbf{w}$ \\
\hline 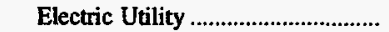 & 4,744 & 4,479 & 4,001 & 7,863 & 7,707 & 5,667 & 5.9 & -11.4 & -1.9 \\
\hline All Other & w & $w$ & w & $\mathbf{w}$ & w & w & w & w & $\mathbf{w}$ \\
\hline \multicolumn{10}{|c|}{ Coal Prices (nominal dollars per short ton) } \\
\hline 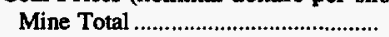 & $\$ 27.18$ & $\$ 27.42$ & $\$ 27.58$ & $\$ 28.15$ & $\$ 28.62$ & $\$ 30.90$ & -.9 & -1.3 & -1.4 \\
\hline 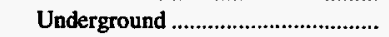 & 27.77 & 27.93 & 28.54 & 29.14 & 29.63 & 31.46 & -.6 & -1.6 & -1.4 \\
\hline Surface & 25.95 & 26.29 & 25.57 & 25.72 & 26.08 & 28.70 & -1.3 & -.1 & -1.1 \\
\hline \multicolumn{10}{|l|}{ Consumer } \\
\hline 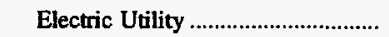 & 31.61 & 34.70 & 35.42 & 36.88 & 37.93 & 37.25 & -8.9 & -4.4 & -1.8 \\
\hline Industrial & 33.61 & 32.73 & 32.91 & 31.93 & 32.08 & 33.37 & 2.7 & 1.2 & .1 \\
\hline Coke & $\mathbf{w}$ & w & $\mathbf{w}$ & $\mathbf{w}$ & w & $\mathbf{w}$ & w & $\mathbf{w}$ & $\mathbf{w}$ \\
\hline
\end{tabular}

1 For 1986, the Form EIA-7A solicited data on "Daily Productive Capacity." To obtain annual productive capacity for a mine in 1986, each mine's daily productive capacity was multiplied by the number of days worked during the year.

2 Capacity utilization (percent) is the ratio of total production to annual productive capacity as reported by mining companies on Form EIA-7A. Productivity (short tons per miner per hour) is calculated by dividing total coal production by the total direct labor hours worked by all employees engaged in production, preparation, processing, development, maintenance, repair, and shop or yard work at mining operations.

3 Includes Mexico.

* Data round to zero.

Witheld to avoid disclosure of individual company data

NA Not available.

Notes: Excludes silt, culm, refuse bank, slurry dam, and dredge operations except for Pennsylvania anthracite. Totals may not equal sum of components due to independent rounding.

Sources: Energy Information Administration, Form EIA-3, "Quarterly Coal Consumption Report - Manufacturing Plants"; Form EIA-5, "Coke Plant Report - Quarterly"; Form EIA-6, "Coal Distribution Report"; Form EIA-7A, "Coal Production Report"; Form EIA-759, "Monthly Power Plant Report"; and U.S. Department of Commerce, Bureau of the Census, "Monthly Report EM 545" and "Monthly Report IM 145." 
Table A16. Wyoming Coal Statistics, 1986, 1991-1995

\begin{tabular}{|c|c|c|c|c|c|c|c|c|c|}
\hline \multirow{2}{*}{ Category } & \multirow{2}{*}{1995} & \multirow{2}{*}{1994} & \multirow{2}{*}{1993} & \multirow{2}{*}{1992} & \multirow{2}{*}{1991} & \multirow{2}{*}{1986} & \multirow{2}{*}{$\begin{array}{c}\text { Percent } \\
\text { Change } \\
\text { 1994-1995 }\end{array}$} & \multicolumn{2}{|c|}{$\begin{array}{l}\text { Average Annual } \\
\text { Percent Change }\end{array}$} \\
\hline & & & & & & & & 1991-1995 & 1986-1995 \\
\hline \multicolumn{10}{|l|}{ Supply (thousand short tons) } \\
\hline Recoverable Reserves............................ & $6,723,963$ & $6,999,460$ & $6,830,720$ & $6,750,718$ & $6,336,075$ & $6,622,345$ & -3.9 & 1.5 & 0.2 \\
\hline Productive Capacity 1 ........................... & 337,184 & 321,046 & 277,875 & 253,312 & NA & 147,667 & 5.0 & NA & 9.6 \\
\hline 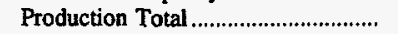 & 263,822 & 237,092 & 210,129 & 190,172 & 193,854 & 136,826 & 11.3 & 8.0 & 7.6 \\
\hline Underground & 2,008 & 2,735 & 2,136 & 2,511 & 2,418 & 164 & -26.6 & -4.5 & 32.0 \\
\hline 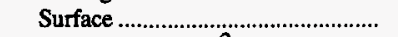 & 261,814 & 234,357 & 207,993 & 187,661 & 191,437 & 136,661 & 11.7 & 8.1 & 7.5 \\
\hline $\begin{array}{l}\text { Capacity Utilization } 2 \\
\text { Ratio of Recoverable }\end{array}$ & 78.24 & 73.85 & 75.62 & 75.07 & NA & 92.65 & 5.9 & NA & -1.9 \\
\hline Reserves to Production........................... & 25.5 & 29.5 & 32.5 & 35.5 & 32.7 & 48.4 & -13.7 & -6.0 & -6.9 \\
\hline 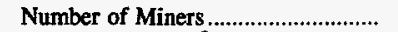 & 3,142 & 3,291 & 3,159 & 3,326 & 3,301 & 3,679 & -4.5 & -1.2 & -1.7 \\
\hline Productivity Total ${ }^{2}$ & 30.06 & 26.05 & 24.46 & 21.50 & 21.87 & 15.73 & 15.4 & 8.3 & 7.5 \\
\hline 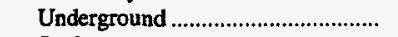 & 5.97 & 5.07 & 3.56 & 4.19 & 4.17 & 2.98 & 17.7 & 9.4 & 8.0 \\
\hline 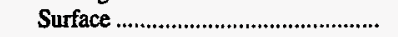 & 31.02 & 27.37 & 26.03 & 22.76 & 23.11 & 15.81 & 13.3 & 7.6 & 7.8 \\
\hline Producer/Distributor Stocks .............. & 1,997 & 1,592 & 998 & 2,794 & 2,918 & - & 25.5 & -9.0 & - \\
\hline \multicolumn{10}{|l|}{ Distribution (thousand short tons) } \\
\hline 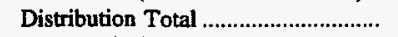 & 263,601 & 235,540 & 211,713 & 190,260 & 194,407 & NA & 11.9 & 7.9 & NA \\
\hline Domestic Distribution Total ........... & 261,333 & 234,016 & 210,739 & 188,983 & 193,425 & NA & 11.7 & 7.8 & NA \\
\hline Within State & 26,521 & 28,334 & 25,519 & 26,388 & 25,284 & NA & -6.4 & 1.2 & NA \\
\hline 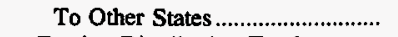 & 234,812 & 205,682 & 185,220 & 162,595 & 168,141 & NA & 14.2 & 8.7 & NA \\
\hline Foreign Distribution Total............... & 2,269 & 1,524 & 974 & 1,277 & 982 & NA & 48.9 & 23.3 & NA \\
\hline Steam & 2,269 & 1,524 & 974 & 1,277 & 982 & NA & 48.9 & 23.3 & NA \\
\hline 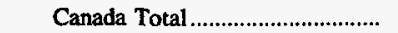 & 32 & - & - & - & 20 & NA & - & 11.7 & NA \\
\hline Steam & 32 & - & - & - & 20 & NA & - & 11.7 & NA \\
\hline Overseas Total ${ }^{3} \ldots \ldots \ldots \ldots \ldots \ldots \ldots \ldots \ldots$ & 2,237 & 1,524 & 974 & 1,277 & 962 & NA & 46.8 & 23.5 & NA \\
\hline Steam & 2,237 & 1,524 & 974 & 1,277 & 962 & NA & 46.8 & 23.5 & NA \\
\hline \multicolumn{10}{|l|}{ Demand (thousand short tons) } \\
\hline 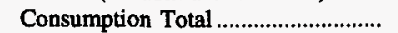 & 25,933 & 27,459 & 26,171 & 27,339 & 25,150 & 19,337 & -5.5 & .8 & 3.3 \\
\hline 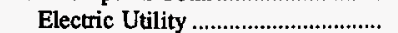 & 23,850 & 25,350 & 24,111 & 25,114 & 23,115 & 17,452 & -5.9 & .8 & 3.5 \\
\hline Industrial & 1,937 & 1,867 & 1,873 & 2,126 & 1,896 & w & 3.8 & .5 & w \\
\hline Residential/Commercial................... & 146 & 242 & 187 & 99 & $\mathbf{w}$ & $\mathbf{w}$ & -39.8 & $\mathbf{w}$ & $\mathbf{w}$ \\
\hline Consumer Stocks Total ....................... & 2,936 & 2,553 & 1,841 & 2,242 & 2,835 & w & 15.0 & .9 & w \\
\hline 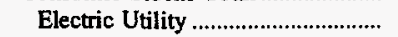 & 2,857 & 2,476 & 1,728 & 2,153 & 2,767 & 5,284 & 15.4 & .8 & -6.6 \\
\hline All Other & 79 & 77 & 113 & 89 & 68 & w & 3.3 & 4.0 & $\mathbf{w}$ \\
\hline \multicolumn{10}{|c|}{ Coal Prices (nominal dollars per short ton) } \\
\hline Mine Total & $\$ 6.58$ & $\$ 6.83$ & $\$ 7.32$ & $\$ 8.14$ & $\$ 8.09$ & $\$ 10.85$ & -3.7 & -5.0 & -5.4 \\
\hline 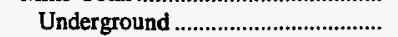 & w & $w$ & w & w & w & w & w & w & w \\
\hline Surface & $w$ & $\mathbf{w}$ & $w$ & $\mathbf{w}$ & w & $\mathrm{w}$ & $w$ & $w$ & $w$ \\
\hline \multicolumn{10}{|l|}{ Consumer } \\
\hline 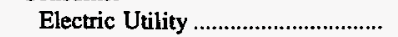 & $\$ 14.29$ & $\$ 14.09$ & $\$ 14.03$ & $\$ 13.42$ & $\$ 14.55$ & $\$ 16.06$ & 1.4 & -.4 & -1.3 \\
\hline Industrial & 22.72 & 22.87 & 23.43 & 23.67 & 25.19 & w & -.6 & -2.5 & $w$ \\
\hline
\end{tabular}

1 For 1986, the Form EIA-7A solicited data on "Daily Productive Capacity." To obtain annual productive capacity for a mine in 1986, each mine's daily productive capacity was multiplied by the number of days worked during the year.

2 Capacity utilization (percent) is the ratio of total production to annual productive capacity as reported by mining companies on Form EIA-7A. Productivity (short tons per miner per hour) is calculated by dividing total coal production by the total direct labor hours worked by all employees engaged in production, preparation, processing, development, maintenance, repair, and shop or yard work at mining operations.

3 Includes Mexico.

W Witheld to avoid disclosure of individual company data.

NA Not available.

Notes: Excludes silt, culm, refuse bank, slurry dam, and dredge operations except for Pennsylvania anthracite. Totals may not equal sum of components due to independent rounding.

Sources: Energy Information Administration, Form EIA-3, "Quarterly Coal Consumption Report - Manufacturing Plants"; Form EIA-5, "Coke Plant Report - Quarterly"; Form EIA-6, "Coal Distribution Report"; Form EIA-7A, "Coal Production Report"; Form EIA-759, "Monthly Power Plant Report"; and U.S. Department of Commerce, Bureau of the Census, "Monthly Report EM 545" and "Monthly Report IM 145." 


\begin{tabular}{|c|c|c|c|c|c|c|c|c|c|}
\hline \multirow{2}{*}{ Category } & \multirow{2}{*}{1995} & \multirow{2}{*}{1994} & \multirow{2}{*}{1993} & \multirow{2}{*}{1992} & \multirow{2}{*}{1991} & \multirow{2}{*}{1986} & \multirow{2}{*}{$\begin{array}{c}\text { Percent } \\
\text { Change } \\
\text { 1994-1995 }\end{array}$} & \multicolumn{2}{|c|}{$\begin{array}{l}\text { Average Annual } \\
\text { Percent Change }\end{array}$} \\
\hline & & & & & & & & 1991-1995 & 1986-1995 \\
\hline \multicolumn{10}{|l|}{ Supply (thousand short tons) } \\
\hline 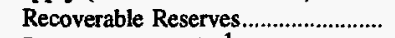 & 420,998 & 476,538 & 506,419 & 514,739 & 577,615 & 766,018 & -11.6 & -7.6 & -6.4 \\
\hline Productive Capacity 1 .............................. & 24,495 & 23,451 & 23,567 & 28,144 & NA & 30,832 & 4.4 & NA & -2.5 \\
\hline 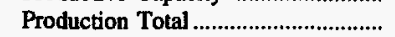 & 19,911 & 19,672 & 18,848 & 22,284 & 22,807 & 29,072 & 1.2 & -3.3 & -4.1 \\
\hline 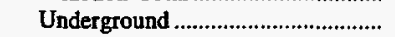 & 4,880 & 4,880 & 4,523 & 4,367 & 5,717 & 7,863 & $*$ & -3.9 & -5.2 \\
\hline Surface & 15,031 & 14,792 & 14,325 & 17,917 & 17,089 & 21,209 & 1.6 & -3.1 & -3.8 \\
\hline 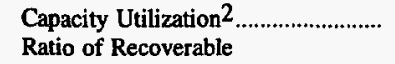 & 81.02 & 83.52 & 79.48 & 78.65 & NA & 93.59 & -3.0 & NA & -1.6 \\
\hline Reserves to Production......................... & 21.1 & 24.2 & 26.9 & 23.1 & 25.3 & 26.3 & -12.7 & -4.4 & -2.4 \\
\hline 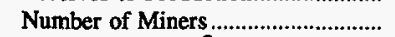 & 2,312 & 2,373 & 2,478 & 2,918 & 3,539 & 5,926 & -2.6 & -10.1 & -9.9 \\
\hline Productivity Total $2 \ldots \ldots \ldots \ldots \ldots \ldots \ldots \ldots$ & 3.96 & 3.82 & 3.59 & 3.40 & 3.09 & 2.36 & 3.8 & 6.4 & 5.9 \\
\hline 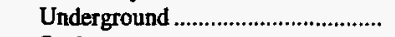 & 3.03 & 2.86 & 3.05 & 2.40 & 2.27 & 1.89 & 6.0 & 7.5 & 5.3 \\
\hline 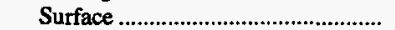 & 4.41 & 4.30 & 3.80 & 3.78 & 3.52 & 2.60 & 2.5 & 5.8 & 6.0 \\
\hline Producer/Distributor Stocks ............... & 784 & 598 & 297 & 289 & 612 & - & 31.0 & 6.4 & - \\
\hline Imports $^{3} \ldots \ldots \ldots \ldots$ & 5,084 & 5,327 & 4,605 & 1,713 & 1,967 & 1,486 & -4.5 & 26.8 & 14.6 \\
\hline \multicolumn{10}{|l|}{ Distribution (thousand short tons) } \\
\hline 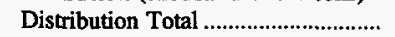 & 397,974 & 398,435 & 374,259 & 415,136 & 411,964 & NA & -.1 & -.9 & NA \\
\hline Domestic Distribution Total ........... & 17,930 & 17,767 & 17,914 & 20,654 & 21,561 & NA & .9 & -4.5 & NA \\
\hline 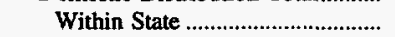 & 26 & 25 & 100 & 143 & 106 & NA & 6.9 & -29.4 & NA \\
\hline 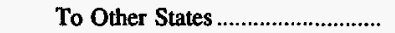 & 17,904 & 17,743 & 17,814 & 20,511 & 21,454 & NA & .9 & -4.4 & NA \\
\hline Foreign Distribution Total............... & 1,150 & 1,046 & 1,142 & 1,250 & 1,083 & NA & 10.0 & 1.5 & NA \\
\hline 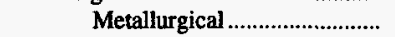 & 38 & 109 & 51 & 25 & $*$ & NA & -65.3 & $\mathrm{NM}$ & NA \\
\hline 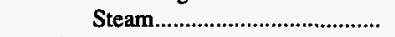 & 1,112 & 937 & 1,092 & 1,225 & 1,083 & NA & 18.7 & .7 & NA \\
\hline 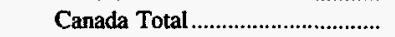 & 1 & 3 & 1 & - & 23 & NA & -79.8 & -58.5 & NA \\
\hline 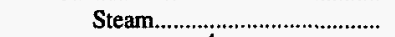 & 1 & 3 & 1 & - & 23 & NA & -79.8 & -58.5 & NA \\
\hline Overseas Total ${ }^{4} \ldots \ldots \ldots \ldots \ldots$ & 1,149 & 1,042 & 1,142 & 1,250 & 1,060 & NA & 10.3 & 2.0 & NA \\
\hline 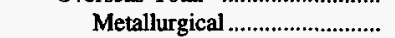 & 38 & 109 & 51 & 25 & $*$ & NA & -65.3 & $\mathrm{NM}$ & NA \\
\hline 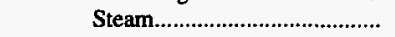 & 1,112 & 934 & 1,091 & 1,225 & 1,060 & NA & 19.0 & 1.2 & NA \\
\hline \multicolumn{10}{|l|}{ Demand (thousand short tons) } \\
\hline 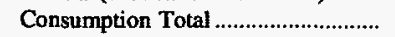 & 381,790 & 371,817 & 365,045 & 350,253 & 351,883 & 317,390 & 2.7 & 2.1 & 2.1 \\
\hline 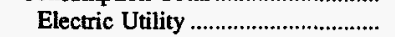 & 344,785 & 334,773 & 329,279 & 315,259 & 314,762 & 277,569 & 3.0 & 2.3 & 2.4 \\
\hline 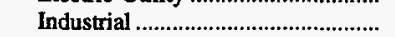 & 31,328 & 31,795 & 31,970 & 31,728 & 31,954 & 30,664 & -1.5 & -.5 & .2 \\
\hline Coke & 2,784 & 2,723 & 1,243 & 1,148 & 2,806 & 6,195 & 2.2 & -.2 & -8.5 \\
\hline 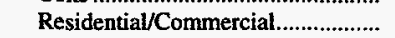 & 2,892 & 2,526 & 2,552 & 2,119 & 2,360 & 2,962 & 14.5 & 5.2 & -.3 \\
\hline Consumer Stocks Total ....................... & 62,368 & 57,506 & 48,850 & 68,257 & 71,836 & 77,604 & 8.4 & -3.5 & -2.4 \\
\hline 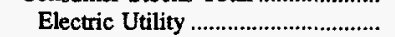 & 58,348 & 53,253 & 45,411 & 64,006 & 67,654 & 71,594 & 9.6 & -3.6 & -2.2 \\
\hline 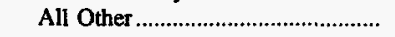 & 4,021 & 4,252 & 3,439 & 4,251 & 4,182 & 6,011 & -5.4 & -1.0 & -4.4 \\
\hline \multicolumn{10}{|c|}{ Coal Prices (nominal dollars per short ton) } \\
\hline 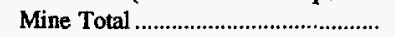 & $\$ 22.80$ & $\$ 23.19$ & $\$ 23.14$ & $\$ 24.39$ & $\$ 25.57$ & $\$ 26.45$ & -1.7 & -2.8 & -1.6 \\
\hline 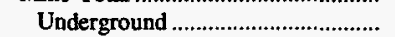 & 26.25 & 26.97 & 26.72 & 27.26 & 26.50 & 28.42 & -2.7 & -.2 & -.9 \\
\hline 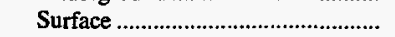 & 21.67 & 21.94 & 22.02 & 23.69 & 25.26 & 25.72 & -1.2 & -3.8 & -1.9 \\
\hline \multicolumn{10}{|l|}{ Consumer } \\
\hline 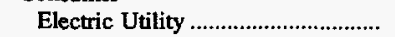 & 28.22 & 29.70 & 30.68 & 31.79 & 32.31 & 36.22 & -5.0 & -3.3 & -2.7 \\
\hline Industrial & 38.51 & 38.72 & 38.95 & 39.35 & 39.70 & 42.67 & -.5 & -8 & -1.1 \\
\hline Coke & 51.67 & 50.89 & 58.19 & 53.10 & 56.62 & 52.25 & 1.5 & -2.3 & -.1 \\
\hline
\end{tabular}

1 For 1986, the Form EIA-7A solicited data on "Daily Productive Capacity." To obtain annual productive capacity for a mine in 1986, each mine's daily productive capacity was multiplied by the number of days worked during the year.

2 Capacity utilization (percent) is the ratio of total production to annual productive capacity as reported by mining companies on Form EIA-7A. Productivity (short tons per miner per hour) is calculated by dividing total coal production by the total direct labor hours worked by all employees engaged in production, preparation, processing, development, maintenance, repair, and shop or yard work at mining operations.

3 Imports for 1992 through 1995 include imports to electric utilities, manufacturing plants and coke plants. Imports for 1986 through 1991 include only imports to electric utilities.

4 Includes Mexico.

* Data round to zero.

NM Not meaningful as value is greater than 500 percent.
Not available.

Notes: Other States include Alaska, Arkansas, California, Iowa, Kansas, Louisiana, Maryland, Missouri, Oklahoma, Tennessee, and Washington. Excludes silt, culm, refuse bank, slurry dam, and dredge operations except for Pennsylvania anthracite. Totals may not equal sum of components due to independent rounding.

Sources: Energy Information Administration, Form EIA-3 "Quarterly Coal Consumption Report - Manufacturing Plants"; Form EIA-5, "Coke Plant Report - Quarterly"; Form EIA-6, "Coal Distribution Report"; Form EIA-7A, "Coal Production Report"; Form EIA-759, "Monthly Power Plant Report"; and U.S. Department of Commerce, Bureau of the Census, "Monthly Report EM 545" and "Monthly Report IM 145." 


\begin{tabular}{|c|c|c|c|c|c|c|c|c|c|}
\hline \multirow{2}{*}{ Category } & \multirow{2}{*}{1995} & \multirow{2}{*}{1994} & \multirow{2}{*}{1993} & \multirow{2}{*}{1992} & \multirow{2}{*}{1991} & \multirow{2}{*}{1986} & \multirow{2}{*}{$\begin{array}{l}\text { Percent } \\
\text { Change } \\
\text { 1994-1995 }\end{array}$} & \multicolumn{2}{|c|}{$\begin{array}{l}\text { Average Annual } \\
\text { Percent Change }\end{array}$} \\
\hline & & & & & & & & 1991-1995 & 1986-1995 \\
\hline \multicolumn{10}{|l|}{ Supply (thousand short tons) } \\
\hline Recoverable Reserves............................ & $20,105,197$ & $21,016,526$ & $21,535,283$ & $21,626,971$ & $21,998,540$ & $25,047,698$ & -4.3 & -2.2 & -2.4 \\
\hline Productive Capacity 1 ........................... & $1,299,054$ & $1,320,656$ & $1,261,873$ & $1,241,054$ & NA & 949,182 & -1.6 & NA & 3.5 \\
\hline 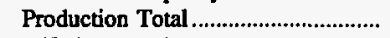 & $1,032,974$ & $1,033,504$ & 945,424 & 997,545 & 995,984 & 890,315 & $*$ & .9 & 1.7 \\
\hline 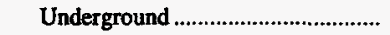 & 396,249 & 399,103 & 351,053 & 407,239 & 407,225 & 360,438 & -.7 & -.7 & 1.0 \\
\hline Surface & 636,725 & 634,401 & 594,371 & 590,306 & 588,759 & 529,877 & .4 & 2.0 & 2.1 \\
\hline 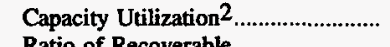 & 79.40 & 78.11 & 74.77 & 80.20 & NA & 93.33 & 1.6 & NA & -1.8 \\
\hline Ratio of Recoverable & & & & & & & & & \\
\hline Reserves to Production........................... & 19.5 & 20.3 & 22.8 & 21.7 & 22.1 & 28.1 & -4.3 & -3.1 & -4.0 \\
\hline 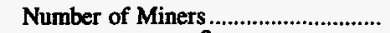 & 90,252 & 97,500 & 101,322 & 110,196 & 120,602 & 154,645 & -7.4 & -7.0 & -5.8 \\
\hline Productivity Total 2 & 5.38 & 4.98 & 4.70 & 4.36 & 4.09 & 3.01 & 8.0 & 7.1 & 6.6 \\
\hline Underground & 3.39 & 3.19 & 2.95 & 2.93 & 2.69 & 2.00 & 6.0 & 5.9 & 6.0 \\
\hline Surface & 8.48 & 7.67 & 7.23 & 6.59 & 6.38 & 4.60 & 10.7 & 7.4 & 7.0 \\
\hline Producer/Distributor Stocks .............. & 34,444 & 33,219 & 25,284 & 33,993 & 32,971 & 34,090 & 3.7 & 1.1 & .1 \\
\hline Imports $^{3}$ & 6,317 & 6,599 & 5,496 & 1,806 & 1,967 & 1,486 & -4.3 & 33.9 & 17.4 \\
\hline \multicolumn{10}{|l|}{ Distribution (thousand short tons) } \\
\hline 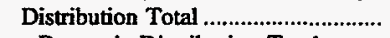 & $1,030,330$ & $1,022,523$ & 959,445 & 998,647 & 994,146 & NA & .8 & .9 & NA \\
\hline Domestic Distribution Total........... & 940,423 & 949,843 & 883,934 & 897,267 & 885,882 & NA & -1.0 & 1.5 & NA \\
\hline 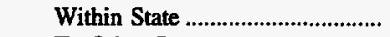 & 336,821 & 353,765 & 339,034 & 355,232 & 345,486 & NA & -4.8 & -.6 & NA \\
\hline To Other States & 603,602 & 596,078 & 544,900 & 542,035 & 540,395 & NA & 1.3 & 2.8 & NA \\
\hline Foreign Distribution Total............... & 89,907 & 72,680 & 75,510 & 101,380 & 108,264 & NA & 23.7 & -4.5 & NA \\
\hline 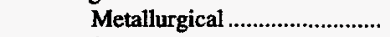 & 54,128 & 51,313 & 52,369 & 62,007 & 62,995 & NA & 5.5 & -3.7 & NA \\
\hline 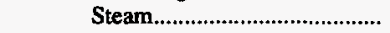 & 35,779 & 21,367 & 23,141 & 39,373 & 45,269 & NA & 67.4 & -5.7 & NA \\
\hline 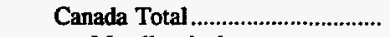 & 8,023 & 8,467 & 7,751 & 13,919 & 12,828 & NA & -5.2 & -11.1 & NA \\
\hline 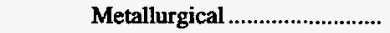 & 6,985 & 7,464 & 6,666 & 9,394 & 8,483 & NA & -6.4 & -4.7 & NA \\
\hline 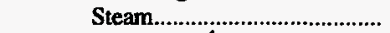 & 1,037 & 1,003 & 1,085 & 4,525 & 4,345 & NA & 3.4 & -30.1 & NA \\
\hline 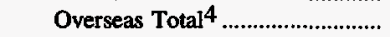 & 81,884 & 64,214 & 67,759 & 87,461 & 95,436 & NA & 27.5 & -3.8 & NA \\
\hline Metallurgical ........................... & 47,143 & 43,849 & 45,703 & 52,614 & 54,511 & NA & 7.5 & -3.6 & NA \\
\hline Steam & 34,742 & 20,364 & 22,057 & 34,848 & 40,925 & NA & 70.6 & -4.0 & NA \\
\hline \multicolumn{10}{|l|}{ Demand (thousand short tons) } \\
\hline 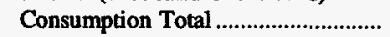 & 940,638 & 930,201 & 925,944 & 892,421 & 887,621 & 804,169 & 1.1 & 1.5 & 1.8 \\
\hline 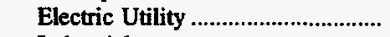 & 829,007 & 817,270 & 813,508 & 779,860 & 772,268 & 685,056 & 1.4 & 1.8 & 2.1 \\
\hline 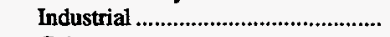 & 72,796 & 75,179 & 74,892 & 74,042 & 75,405 & 75,583 & -3.2 & -.9 & -.4 \\
\hline 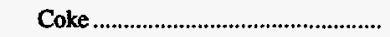 & 33,011 & 31,740 & 31,323 & 32,366 & 33,854 & 35,862 & 4.0 & -.6 & -.9 \\
\hline Residential/Commercial ................... & 5,824 & 6,013 & 6,221 & 6,153 & 6,094 & 7,667 & -3.1 & -1.1 & -3.0 \\
\hline Consumer Stocks Total ...................... & 134,639 & 136,139 & 120,458 & 163,692 & 167,711 & 175,226 & -1.1 & -5.3 & -2.9 \\
\hline 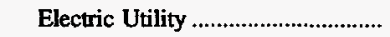 & 126,304 & 126,897 & 111,341 & 154,130 & 157,876 & 161,806 & -.5 & -5.4 & -2.7 \\
\hline 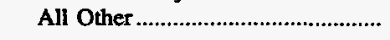 & 8,334 & 9,243 & 9,117 & 9,562 & 9,835 & 13,420 & -9.8 & -4.0 & -5.1 \\
\hline \multicolumn{10}{|c|}{ Coal Prices (nominal dollars per short ton) } \\
\hline 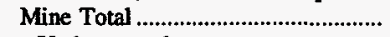 & $\$ 18.83$ & $\$ 19.41$ & $\$ 19.85$ & $\$ 21.03$ & $\$ 21.49$ & $\$ 23.79$ & -3.0 & -3.3 & -2.6 \\
\hline 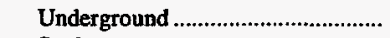 & 26.18 & 26.39 & 26.92 & 27.83 & 28.56 & 30.33 & -.8 & -2.1 & -1.6 \\
\hline 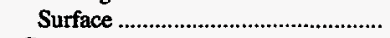 & 14.25 & 15.02 & 15.67 & 16.34 & 16.60 & 19.34 & -5.1 & -3.7 & -3.3 \\
\hline \multicolumn{10}{|l|}{ Consumer } \\
\hline 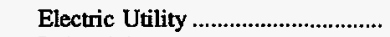 & 27.01 & 28.03 & 28.58 & 29.36 & 30.02 & 33.30 & -3.6 & -2.6 & -2.3 \\
\hline 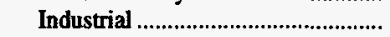 & 32,42 & 32.55 & 32.23 & 32.78 & 33.54 & 35.84 & -.4 & -.8 & -1.1 \\
\hline Coke & 47.34 & 46.56 & 47.44 & 47.92 & 48.88 & 50.85 & 1.7 & -.8 & -.8 \\
\hline
\end{tabular}

1 For 1986, the Form EIA-7A solicited data on "Daily Productive Capacity." To obtain annual productive capacity for a mine in 1986, each mine's daily productive capacity was multiplied by the number of days worked during the year.

2 Capacity utilization (percent) is the ratio of total production to annual productive capacity as reported by mining companies on Form EIA-7A. Productivity (short tons per miner per hour) is calculated by dividing total coal production by the total direct labor hours worked by all employees engaged in production, preparation, processing, development, maintenance, repair, and shop or yard work at mining operations.

3 Imports for 1992 through 1995 include imports to electric utilities, manufacturing plants and coke plants. Imports for 1986 through 1991 include only imports to electric utilities.

4 Includes Mexico.

* Data round to zero.

NA Not available.

Notes: Excludes silt, culm, refuse bank, slurry dam, and dredge operations except for Pennsylvania anthracite. Consumption Total does not include coal consumed by independent power producers. Totals may not equal sum of components due to independent rounding.

Sources: Energy Information Administration, Form EIA-3, "Ouarterly Coal Consumption Report - Manufacturing Plants"; Form EIA-5, "Coke Plant Report - Quarterly"; Form EIA-6, "Coal Distribution Report"; Form EIA-7A, "Coal Production Report"; Form EIA-759, "Monthly Power Plant Report"; and U.S. Department of Commerce, Bureau of the Census, "Monthly Report EM 545" and "Monthly Report IM 145." 


\section{Metric Tables}

In response to requests from international users of U.S. coal statistics, certain summary data have been converted from the customary short tons to metric. This enables U.S. statistics to be compared with data published by countries using the metric system. The conversion to metric tons is made by multiplying short tons by 0.907185 .

The data converted to metric tons are from Tables ES3, $1,16,25,35,48,67,68,76,80,92,94,96,98$, and 99.

Table B1. Trends in U.S. Coal Production, Imports, Consumption, Exports, and

Stocks, 1986, 1991-1995

(Million Metric Tons)

\begin{tabular}{|c|c|c|c|c|c|c|c|c|c|}
\hline \multirow{2}{*}{ Activity } & \multirow{2}{*}{1995} & \multirow{2}{*}{1994} & \multirow{2}{*}{1993} & \multirow{2}{*}{1992} & \multirow{2}{*}{1991} & \multirow{2}{*}{1986} & \multirow{2}{*}{$\begin{array}{c}\text { Percent } \\
\text { Change } \\
\text { 1994-1995 }\end{array}$} & \multicolumn{2}{|c|}{ Average Annual Percent Change } \\
\hline & & & & & & & & 1991-1995 & 1986-1995 \\
\hline 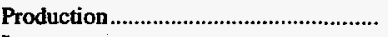 & 937 & 938 & 858 & 905 & 904 & 808 & $*$ & 0.9 & 1.7 \\
\hline 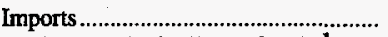 & 7 & 7 & 7 & 3 & 3 & 2 & -5.0 & 20.7 & 14.0 \\
\hline Producer and Distributor Stocks 1 . & 31 & 30 & 23 & 31 & 30 & 31 & 3.7 & 1.1 & .1 \\
\hline Consumption & 853 & 844 & 840 & 810 & 805 & 730 & 1.1 & 1.5 & 1.8 \\
\hline 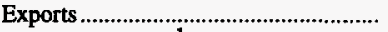 & 80 & 65 & 68 & 93 & 99 & 78 & 24.1 & -5.0 & .4 \\
\hline Consumer Stocks 1 . & 122 & 124 & 109 & 148 & 152 & 159 & -1.1 & -5.3 & -2.9 \\
\hline
\end{tabular}

1 Reported as of the last day of the quarter.

* Data round to zero.

Note: Consumption does not include coal consumed by independent power producers.

Sources: • Production: Energy Information Administration (EIA), Form EIA-7A, "Coal Production Report"; U.S. Department of Labor, Mine Safety and Health Administration, Form 7000-2, "Quarterly Mine Employment and Coal Production Report"; and State Mining Agency Coal Production Reports. • Imports: U.S. Department of Commerce, Bureau of the Census, "Monthly Report IM 145." - Producer and Distributor Stocks: EIA, Form EIA-6, "Coal Distribution Report." - Exports: U.S. Department of Commerce, Bureau of the Census, "Monthly Report EM 545."

- Consumption and Consumer Stocks: EIA, Form ElA-759, "Monthly Power Plant Report"; Form EIA-3. Quarterly Coal Consumption Report - Manufacturing Plants"; Form EIA-5, "Coke Plant Report - Quarterly"; and Form EIA-6, "Coal Distribution Report." 
Table B2. Coal Production by State, 1986, 1991-1995

(Thousand Metric Tons)

\begin{tabular}{|c|c|c|c|c|c|c|c|c|c|}
\hline \multirow{2}{*}{$\begin{array}{l}\text { Coal-Producing } \\
\text { State and Region }\end{array}$} & \multirow{2}{*}{1995} & \multirow{2}{*}{1994} & \multirow{2}{*}{1993} & \multirow{2}{*}{1992} & \multirow{2}{*}{1991} & \multirow{2}{*}{1986} & \multirow{2}{*}{$\begin{array}{c}\text { Percent } \\
\text { Change } \\
\text { 1994-1995 }\end{array}$} & \multicolumn{2}{|c|}{ Average Annual Percent Change } \\
\hline & & & & & & & & 1991.1995 & 1986-1995 \\
\hline Alabama & 22,353 & 21,106 & 22,469 & 23,401 & 24,738 & 23,429 & 5.9 & -2.5 & -0.5 \\
\hline Alaska & 1,540 & 1,422 & 1,452 & 1,392 & 1,303 & 1,424 & 8.4 & 4.3 & .9 \\
\hline Arizona & 10,838 & 11,844 & 11,043 & 11,351 & 11,977 & 10,483 & -8.5 & -2.5 & .4 \\
\hline 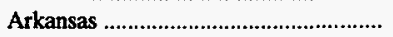 & 26 & 46 & 40 & 53 & 47 & 152 & -42.8 & -13.5 & -17.6 \\
\hline 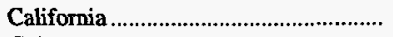 & - & - & - & 94 & 51 & - & - & - & - \\
\hline Colorado & 23,324 & 22,955 & 19,854 & 17,442 & 16,179 & 13,823 & 1.6 & 9.6 & 6.0 \\
\hline 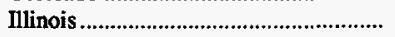 & 43,708 & 47,896 & 37,283 & 54,301 & 54,665 & 56,124 & -8.7 & -5.4 & -2.7 \\
\hline Indiana & 23,593 & 28,057 & 26,576 & 27,639 & 28,547 & 29,803 & -15.9 & -4.6 & -2.6 \\
\hline Iowa & - & 42 & 159 & 262 & 312 & 439 & -100.0 & - & - \\
\hline 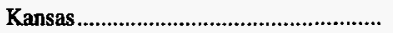 & 258 & 258 & 310 & 329 & 377 & 1,348 & .1 & -9.0 & -16.8 \\
\hline 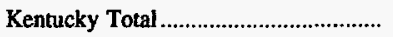 & 139,470 & 146,639 & 141,792 & 146,119 & 144,224 & 139,646 & -4.9 & -.8 & * \\
\hline Eastern & 107,539 & 112,897 & 109,036 & 108,302 & 106,340 & 102,269 & -4.7 & .3 & .5 \\
\hline 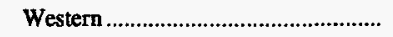 & 31,931 & 33,742 & 32,756 & 37,817 & 37,884 & 37,377 & -5.4 & -4.2 & -1.7 \\
\hline 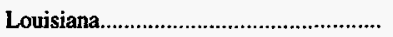 & 3,374 & 3,141 & 2,843 & 2,939 & 2,858 & 2,044 & 7.4 & 4.2 & 5.7 \\
\hline Maryland & 3,327 & 3,295 & 3,044 & 3,031 & 3,423 & 3,543 & 1.0 & -.7 & -.7 \\
\hline 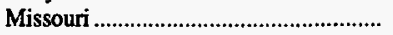 & 497 & 761 & 592 & 2,618 & 2,090 & 4,252 & -34.7 & -30.2 & -21.2 \\
\hline Montana & 35,789 & 37,775 & 32,583 & 35,280 & 34,688 & 30,824 & -5.3 & .8 & 1.7 \\
\hline New Mexico & 24,324 & 25,438 & 25,644 & 22,270 & 19,520 & 19,501 & -4.4 & 5.6 & 2.5 \\
\hline 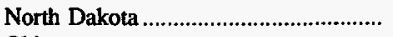 & 27,317 & 29,289 & 29,005 & 28,797 & 26,790 & 23,260 & -6.7 & .5 & 1.8 \\
\hline Ohio & 23,694 & 27,122 & 26,141 & 27,581 & 27,732 & 33,059 & -12.6 & -3.8 & -3.6 \\
\hline 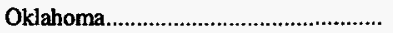 & 1,702 & 1,733 & 1,595 & 1,580 & 1,670 & 2,765 & -1.8 & .5 & -5.2 \\
\hline 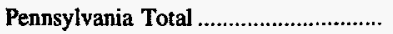 & 55,860 & 56,460 & 54,159 & 62,578 & 59,313 & 64,998 & -1.1 & -1.5 & -1.7 \\
\hline 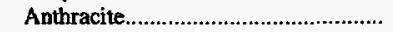 & 4,248 & 4,192 & 3,906 & 3,160 & 3,125 & 3,894 & 1.3 & 8.0 & 1.0 \\
\hline 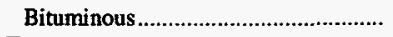 & 51,613 & 52,268 & 50,253 & 59,419 & 56,188 & 61,104 & -1.3 & -2.1 & -1.8 \\
\hline 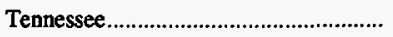 & 2,922 & 2,709 & 2,764 & 3,154 & 3,892 & 6,232 & 7.8 & -6.9 & -8.1 \\
\hline 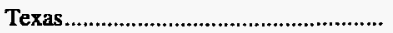 & 47,794 & 47,488 & 49,502 & 49,960 & 48,829 & 44,080 & .6 & -.5 & .9 \\
\hline 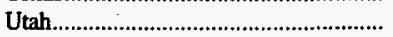 & 22,831 & 22,135 & 19,819 & 19,359 & 19,908 & 12,945 & 3.1 & 3.5 & 6.5 \\
\hline Virginia & 30,934 & 33,683 & 35,668 & 39,030 & 38,060 & 37,356 & -8.2 & -5.0 & -2.1 \\
\hline 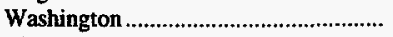 & 4,416 & 4,439 & 4,300 & 4,764 & 4,666 & 4,174 & -.5 & -1.4 & .6 \\
\hline 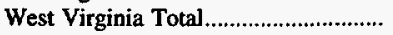 & 147,869 & 146,760 & 118,411 & 147,112 & 151,819 & 117,850 & .8 & -.6 & 2.5 \\
\hline 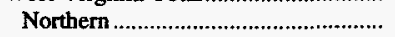 & 41,834 & 44,739 & 30,665 & 45,379 & 47,314 & 43,867 & -6.5 & -3.0 & -.5 \\
\hline Southern & 106,034 & 102,022 & 87,746 & 101,734 & 104,504 & 73,983 & 3.9 & .4 & 4.1 \\
\hline 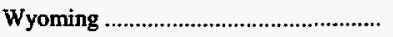 & 239,336 & 215,086 & 190,626 & 172,522 & 175,862 & 124,126 & 11.3 & 8.0 & 7.6 \\
\hline 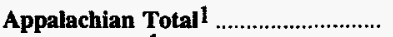 & 394,499 & 404,033 & 371,690 & 414,189 & 415,317 & 388,736 & -2.3 & -1.3 & 2 \\
\hline 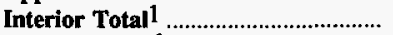 & 152,884 & 163,164 & 151,658 & 177,499 & 177,280 & 178,384 & -6.3 & -3.6 & -1.7 \\
\hline Western Total ${ }^{1}$ & 389,715 & 370,382 & 334,327 & 313,270 & 310,945 & 240,560 & 5.2 & 5.8 & 5.5 \\
\hline East of Miss. River............................... & 493,732 & $\mathbf{5 1 3 , 7 2 9}$ & 468,306 & 533,946 & 536,413 & 512,040 & -3.9 & -2.0 & -.4 \\
\hline 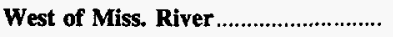 & 443,367 & 423,851 & 389,369 & 371,012 & 367,129 & 295,640 & 4.6 & 4.8 & 4.6 \\
\hline U.S. Total & 937,098 & 937,580 & 857,675 & 904,958 & 903,542 & 807,680 & * & .9 & 1.7 \\
\hline
\end{tabular}

1 For a definition of coal-producing regions, see Appendix C.

* Data round to zero.

Notes: Coal production excludes silt, culm, refuse bank, slurry dam, and dredge operations except for Pennsylvania anthracite. Totals may not equal sum of components due to independent rounding.

Sources: Energy Information Administration, Form EIA-7A, "Coal Production Report'; State Mining Agency Coal Production Reports; and/or U.S. Department of Labor, Mine Safety and Health Administration, Form 7000-2, "Quarterly Mine Employment and Coal Production Report." 
Table B3. Productive Capacity of Coal Mines by State, 1986, 1991-1995

(Thousand Metric Tons)

\begin{tabular}{|c|c|c|c|c|c|c|c|c|c|}
\hline \multirow{2}{*}{$\begin{array}{l}\text { Coal-Producing } \\
\text { State and Region }\end{array}$} & \multirow{2}{*}{1995} & \multirow{2}{*}{1994} & \multirow{2}{*}{1993} & \multirow{2}{*}{1992} & \multirow{2}{*}{1991} & \multirow{2}{*}{$1986^{1}$} & \multirow{2}{*}{$\begin{array}{c}\text { Percent } \\
\text { Change } \\
\text { 1994-1995 }\end{array}$} & \multicolumn{2}{|c|}{ Average Annual Percent Change } \\
\hline & & & & & & & & 1991-1995 & 1986-1995 \\
\hline 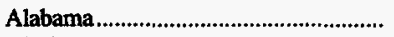 & 29,526 & 29,982 & 25,325 & 27,048 & 27,392 & 24,329 & -1.5 & 1.9 & 2.2 \\
\hline 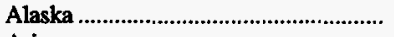 & $\mathbf{w}$ & w & $\mathbf{w}$ & w & $\mathbf{w}$ & 1,424 & w & $\mathbf{w}$ & $\mathbf{w}$ \\
\hline Arizona & $\mathbf{w}$ & $\mathbf{w}$ & $\mathbf{w}$ & $\mathbf{w}$ & $\mathbf{w}$ & 10,483 & $\mathbf{w}$ & $\mathbf{w}$ & $\mathbf{w}$ \\
\hline 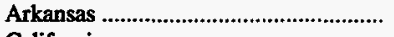 & $\mathbf{w}$ & $\mathbf{w}$ & $\mathbf{w}$ & $\mathbf{w}$ & $\mathbf{w}$ & 136 & $\mathbf{w}$ & $\mathbf{w}$ & $\mathbf{w}$ \\
\hline 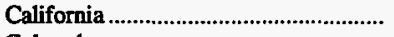 & - & - & - & $\mathbf{w}$ & $\mathbf{w}$ & - & w & $\mathbf{w}$ & w \\
\hline Colorado & 29,425 & 28,191 & 27,252 & 23,449 & 21,403 & 14,884 & 4.4 & 8.3 & 7.9 \\
\hline Illinois & 51,371 & 62,971 & 62,886 & 68,753 & 68,683 & 60,206 & -18.4 & -7.0 & -1.7 \\
\hline Indiana & 31,984 & 35,318 & 39,875 & 39,000 & 40,964 & 31,643 & -9.4 & -6.0 & .1 \\
\hline Iowa & - & $\mathbf{w}$ & w & w & $w$ & 500 & w & w & $\mathbf{w}$ \\
\hline 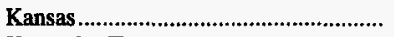 & $\mathbf{w}$ & $\mathbf{w}$ & $\mathbf{w}$ & $\mathbf{w}$ & $\mathbf{w}$ & 1,426 & w & $\mathbf{w}$ & $\mathbf{w}$ \\
\hline 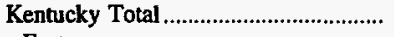 & 184,316 & 193,618 & 185,796 & 177,220 & 180,786 & 146,629 & -4.8 & .5 & 2.6 \\
\hline 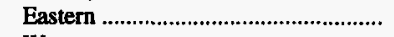 & 137,993 & 146,720 & 142,717 & 135,212 & 132,040 & 105,814 & -5.9 & 1.1 & 3.0 \\
\hline Western & 46,323 & 46,897 & 43,079 & 42,008 & 48,747 & 40,815 & -1.2 & -1.3 & 1.4 \\
\hline 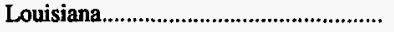 & w & w & w & w & $\mathbf{w}$ & 2,495 & w & w & w \\
\hline Maryland & 3,999 & 3,930 & 3,563 & 3,539 & 3,854 & 3,589 & 1.8 & .9 & 1.2 \\
\hline Missouri & 980 & 1,097 & w & w & $w$ & 4,705 & -10.6 & $\mathbf{w}$ & -16.0 \\
\hline 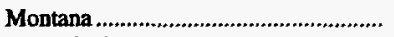 & 46,808 & 46,361 & 46,129 & 44,073 & 44,093 & 31,759 & 1.0 & 1.5 & 4.4 \\
\hline 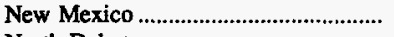 & 29,719 & 29,762 & 30,264 & 26,773 & 25,571 & 21,022 & -.1 & 3.8 & 3.9 \\
\hline North Dakota & 31,265 & 32,586 & 32,995 & 33,553 & 32,637 & 24,929 & -4.0 & -1.1 & 2.5 \\
\hline Ohio & 30,854 & 39,848 & 38,315 & 37,493 & 42,997 & 35,711 & -22.6 & -8.0 & -1.6 \\
\hline Oklahoma & 2,320 & 2,042 & 2,197 & 2,256 & 2,112 & 2,808 & 13.6 & 2.4 & -2.1 \\
\hline Pennsylvania Total ................................... & 70,023 & 73,460 & 74,524 & 75,267 & 72,954 & 70,465 & -4.7 & -1.0 & -.1 \\
\hline Anthracite & 5,939 & 5,240 & 5,267 & 3,758 & 3,668 & 3,719 & 13.3 & 12.8 & 5.3 \\
\hline 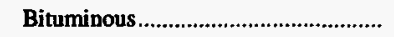 & 64,083 & 68,220 & 69,256 & 71,509 & 69,286 & 66,746 & -6.1 & -1.9 & -.4 \\
\hline 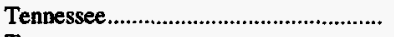 & 3,402 & 3,092 & 3,414 & 3,567 & 4,680 & 6,368 & 10.0 & -7.7 & -6.7 \\
\hline 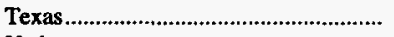 & 49,676 & 50,672 & 51,814 & 53,108 & 54,049 & 47,155 & -2.0 & -2.1 & .6 \\
\hline Utah & 28,021 & 25,075 & 23,526 & 23,164 & 23,117 & 16,525 & 11.7 & 4.9 & 6.0 \\
\hline 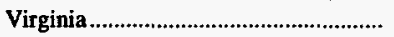 & 39,042 & 42,150 & 46,156 & 49,415 & 50,141 & 37,649 & -7.4 & -6.1 & .4 \\
\hline Washington & $\mathbf{w}$ & $\mathbf{w}$ & $w$ & $w$ & $\mathbf{w}$ & 4,519 & $w$ & $w$ & $w$ \\
\hline 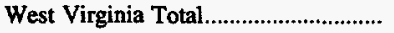 & 185,825 & 182,964 & 173,913 & 179,697 & 181,541 & 125,762 & 1.6 & .6 & 4.4 \\
\hline Northern & 51,124 & 53,792 & 54,445 & 56,981 & 58,710 & 46,441 & -4.9 & -3.4 & 1.1 \\
\hline 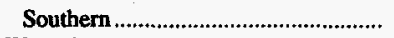 & 134,701 & 129,172 & 119,468 & 122,716 & 122,831 & 79,321 & 4.3 & 2.3 & 6.1 \\
\hline 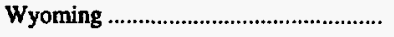 & 305,888 & 291,248 & 252,084 & 229,801 & 227,024 & 133,962 & 5.0 & 7.7 & 9.6 \\
\hline Appalachian Total2 & 500,664 & 522,147 & 507,927 & $\mathbf{5 1 1 , 2 3 9}$ & 515,599 & 409,687 & -4.1 & -7 & 2.3 \\
\hline Interior Total2 & 186,329 & 203,116 & 204,968 & 213,225 & 222,702 & 191,889 & -8.3 & -4.4 & -3 \\
\hline Western Total ${ }^{2}$ & 491,489 & 472,817 & 431,858 & 401,401 & 394,300 & 259,507 & 3.9 & 5.7 & 7.3 \\
\hline East of Miss. River................................ & 630,341 & 667,333 & 653,767 & 661,000 & 673,992 & 542,351 & -5.5 & -1.7 & 1.7 \\
\hline West of Miss. River............................... & 548,141 & 530,746 & 490,986 & 464,865 & 458,609 & 318,732 & $\mathbf{3 . 3}$ & 4.5 & 6.2 \\
\hline U.S. Total & $1,178,482$ & $1,198,079$ & $1,144,753$ & $1,125,866$ & $1,132,601$ & 861,083 & -1.6 & 1.0 & 3.5 \\
\hline
\end{tabular}

1 For 1986, Form EIA-7A solicited data on "Daily Productive Capacity." To obtain annual productive capacity for a mine in 1986, each mine's daily productive capacity was multiplied by the number of days worked during the year.

2 For a definition of coal-producing regions, see Appendix $\mathbf{C}$

withheld to avoid disclosure of individual company data.

Notes: Productive capacity is the maximum amount of coal that can be produced as reported by mining companies on Form EIA-7A. Excludes silt, culm, refuse bank, slurry dam, and dredge operations except for Pennsylvania anthracite. Excludes mines producing less than 10,000 short tons, which are not required to provide these data.

Source: Energy Information Administration, Form EIA-7A, "Coal Production Report." 
Table B4. Recoverable Coal Reserves at Producing Mines by State, 1986, 1991-1995 (Million Metric Tons)

\begin{tabular}{|c|c|c|c|c|c|c|c|c|c|}
\hline \multirow{2}{*}{$\begin{array}{l}\text { Coal-Producing } \\
\text { State and Region }\end{array}$} & \multirow{2}{*}{1995} & \multirow{2}{*}{1994} & \multirow{2}{*}{1993} & \multirow{2}{*}{1992} & \multirow{2}{*}{1991} & \multirow{2}{*}{1986} & \multirow{2}{*}{$\begin{array}{c}\text { Percent } \\
\text { Change } \\
\text { 1994-1995 }\end{array}$} & \multicolumn{2}{|c|}{ Average Annual Percent Change } \\
\hline & & & & & & & & 1991-1995 & 1986-1995 \\
\hline Alabama & 463 & 415 & 387 & 424 & 427 & 510 & 11.6 & 2.0 & -1.1 \\
\hline 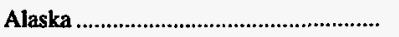 & $\mathbf{w}$ & $\mathbf{w}$ & $\mathbf{w}$ & $\mathbf{w}$ & w & 83 & w & $\mathbf{w}$ & $\mathbf{w}$ \\
\hline Arizona & $\mathbf{w}$ & $\mathbf{w}$ & $\mathbf{w}$ & $\mathbf{w}$ & $\mathbf{w}$ & 259 & $w$ & $w$ & $\mathbf{w}$ \\
\hline Arkansas & $\mathbf{w}$ & w & $\mathbf{w}$ & $\mathbf{w}$ & $w$ & 1 & $\mathbf{w}$ & $\mathbf{w}$ & $\mathbf{w}$ \\
\hline 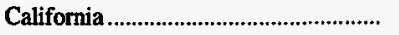 & - & - & - & $\mathbf{w}$ & $\mathbf{w}$ & - & $\mathbf{w}$ & $\mathbf{w}$ & $\mathbf{w}$ \\
\hline Colorado & 628 & 614 & 552 & 552 & 560 & 554 & 2.3 & 2.9 & 1.4 \\
\hline 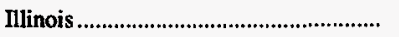 & 800 & 874 & 965 & 1,088 & 1,140 & 1,404 & -8.4 & -8.4 & -6.0 \\
\hline Indiana & 294 & 276 & 344 & 367 & 381 & 460 & 6.4 & -6.3 & -4.8 \\
\hline Iowa & - & $\mathbf{w}$ & $\mathbf{w}$ & w & w & 8 & $\mathbf{w}$ & $\mathbf{w}$ & $\mathbf{w}$ \\
\hline Kansas & w & w & $\mathbf{w}$ & $\mathbf{w}$ & w & 23 & w & $\mathbf{w}$ & w \\
\hline 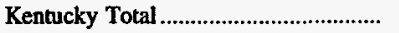 & 1,160 & 1,238 & 1,658 & 1,318 & 1,481 & 1,569 & -6.3 & -5.9 & -3.3 \\
\hline Eastern & 692 & 734 & 1,222 & 866 & 983 & 1,024 & -5.7 & -8.4 & -4.3 \\
\hline Western & 468 & 505 & 436 & 452 & 497 & 545 & -7.2 & -1.5 & -1.7 \\
\hline 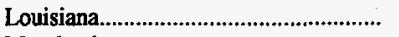 & w & $\mathbf{w}$ & $\mathbf{w}$ & $\mathbf{w}$ & $\mathbf{w}$ & 135 & $\mathbf{w}$ & $\mathbf{w}$ & $w$ \\
\hline 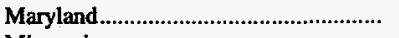 & 52 & 80 & 60 & 53 & 78 & 66 & -35.0 & -9.6 & -2.6 \\
\hline 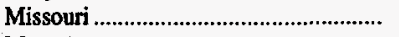 & 2 & 11 & w & $\mathbf{w}$ & $w$ & 146 & -80.9 & $\mathbf{w}$ & -37.6 \\
\hline Montana & 1,135 & 1,163 & 1,166 & 1,226 & 1,264 & 1,584 & -2.5 & -2.6 & -3.6 \\
\hline New Mexico & 1,343 & 1,322 & 1,336 & 1,356 & 1,459 & 1,426 & 1.5 & -2.0 & -.7 \\
\hline 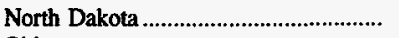 & 1,513 & 1,537 & 1,280 & 1,211 & 1,257 & 1,390 & -1.6 & 4.7 & .9 \\
\hline Ohio & 425 & 435 & 472 & 523 & 536 & 718 & -2.3 & -5.6 & -5.7 \\
\hline 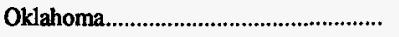 & 17 & 39 & 42 & 44 & 36 & 55 & -56.0 & -17.1 & -12.1 \\
\hline 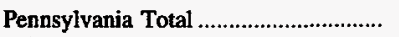 & 668 & 828 & 852 & 850 & 894 & 1,388 & -19.3 & -7.0 & -7.8 \\
\hline 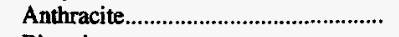 & 45 & 34 & 59 & 64 & 69 & 72 & 30.5 & -10.2 & -5.2 \\
\hline 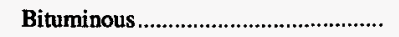 & 623 & 794 & 793 & 787 & 825 & 1,315 & -21.5 & -6.8 & -8.0 \\
\hline 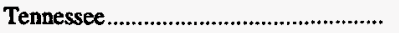 & 62 & 38 & 27 & 39 & 51 & 76 & 61.5 & 5.0 & -2.4 \\
\hline Texas & 853 & 931 & 1,002 & 1,078 & 1,111 & 1,144 & -8.4 & -6.4 & -3.2 \\
\hline Utah & 340 & 384 & 405 & 443 & 462 & 736 & -11.5 & -7.4 & -8.2 \\
\hline Virginia & 184 & 215 & 305 & 332 & 374 & 453 & -14.2 & -16.2 & -9.5 \\
\hline Washington & $\mathbf{w}$ & $\mathbf{w}$ & $\mathbf{w}$ & w & $\mathbf{w}$ & 102 & $\mathbf{w}$ & $\mathbf{w}$ & $\mathbf{w}$ \\
\hline 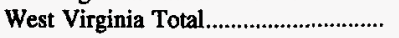 & 1,571 & 1,660 & 1,752 & 1,853 & 1,925 & 2,426 & -5.4 & -4.9 & -4.7 \\
\hline 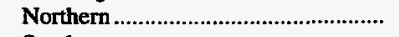 & 709 & 781 & 747 & 871 & 939 & 1,086 & -9.2 & -6.8 & -4.6 \\
\hline Southern & 861 & 879 & 1,005 & 982 & 986 & 1,340 & -2.0 & -3.3 & -4.8 \\
\hline 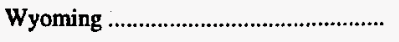 & 6,100 & 6,350 & 6,197 & 6,124 & 5,748 & 6,008 & -3.9 & 1.5 & .2 \\
\hline Appalachian Total ${ }^{1}$ & 4,117 & 4,404 & 5,076 & 4,941 & 5,268 & 6,661 & -6.5 & -6.0 & -5.2 \\
\hline 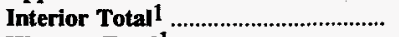 & 2,572 & $\mathbf{2 , 7 8 4}$ & 2,994 & $\mathbf{3 , 2 2 8}$ & $\mathbf{3 , 3 7 0}$ & 3,920 & -7.6 & -6.5 & -4.6 \\
\hline Western Total ${ }^{1}$ & 11,550 & 11,878 & 11,466 & 11,450 & 11,319 & 12,142 & -2.8 & .5 & -.5 \\
\hline East of Miss. River ................................. & 5,679 & 6,059 & 6,822 & 6,848 & 7,286 & 9,070 & -6.3 & -6.0 & -5.1 \\
\hline West of Miss. River .................................. & 12,560 & 13,007 & 12,715 & 12,772 & 12,671 & 13,653 & -3.4 & -.2 & -.9 \\
\hline U.S. Total & 18,239 & 19,066 & 19,536 & 19,620 & 19,957 & 22,723 & -4.3 & -2.2 & $-2,4$ \\
\hline
\end{tabular}

1 For a definition of coal-producing regions, see Appendix C

Withheld to avoid disclosure of individual company data.

Notes: Recoverable reserves represent the quantity of coal that can be recovered (i.e., mined) from existing coal reserves at reporting mines. Ex cludes silt, culm, refuse bank, slurry dam, and dredge operations except for Pennsylvania anthracite. Excludes mines producing less than 10,000 short tons, which are not required to provide these data. Totals may not equal sum of components due to independent rounding.

Sources: Energy Information Administration, Form EIA-7A, "Coal Production Report"; State Mining Agency Coal Production Reports; and/or U.S. Department of Labor, Mine Safety and Health Administration, Form 7000-2, "Quarterly Mine Employment and Coal Production Report." 
Table B5. U.S. Coal Imports by Continent and Country of Origin, 1986, 1991-1995 (Metric Tons)

\begin{tabular}{|c|c|c|c|c|c|c|c|c|c|}
\hline \multirow{2}{*}{$\begin{array}{c}\text { Continent and Country } \\
\text { of Origin }\end{array}$} & \multirow{2}{*}{1995} & \multirow{2}{*}{1994} & \multirow{2}{*}{1993} & \multirow{2}{*}{1992} & \multirow{2}{*}{1991} & \multirow{2}{*}{1986} & \multirow{2}{*}{$\begin{array}{c}\text { Percent } \\
\text { Change } \\
\text { 1994-1995 }\end{array}$} & \multicolumn{2}{|c|}{ Average Annual Percent Change } \\
\hline & & & & & & & & 1991-1995 & 1986-1995 \\
\hline North America Total ............................. & $1,219,813$ & $1,137,082$ & 955,786 & 926,102 & 848,646 & 393,136 & 7.3 & 9.5 & 13.4 \\
\hline 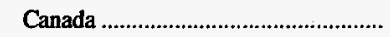 & $1,197,249$ & $1,136,881$ & 953,696 & 926,102 & 848,646 & 392,379 & 5.3 & 9.0 & 13.2 \\
\hline 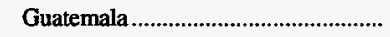 & - & - & 4 & - & - & - & - & - & - \\
\hline Mexico & 196 & 201 & 2,086 & - & - & 757 & -2.5 & - & -13.9 \\
\hline Netherlands Antilles ................................ & 22,368 & - & - & - & - & - & - & - & - \\
\hline South America Total ............................. & $4,157,892$ & $4,463,532$ & $4,912,696$ & $2,088,277$ & $2,192,038$ & 673,548 & -6.8 & 17.3 & 22.4 \\
\hline 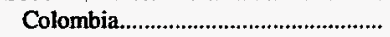 & $2,482,908$ & $3,075,043$ & $3,734,915$ & $1,599,502$ & $1,706,814$ & 673,548 & -19.3 & 9.8 & 15.6 \\
\hline 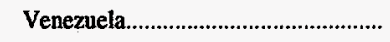 & $1,674,984$ & $1,388,489$ & $1,177,781$ & 488,775 & 485,224 & - & 20.6 & 36.3 & - \\
\hline Europe Total & 474 & 36 & 56 & 81 & 5 & 274 & NM & 212.0 & 6.3 \\
\hline 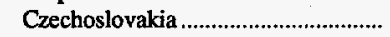 & - & - & - & - & 5 & - & - & -100.0 & - \\
\hline 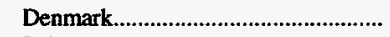 & 214 & - & 54 & - & - & - & - & - & - \\
\hline 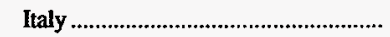 & - & - & - & - & - & 219 & - & - & -100.0 \\
\hline Netherlands & - & - & - & - & - & 54 & - & - & -100.0 \\
\hline 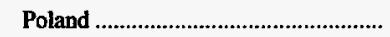 & - & 36 & 2 & - & - & - & -100.0 & - & - \\
\hline United Kingdom & 260 & - & - & 81 & - & - & - & - & - \\
\hline Asia Total & 923,980 & $1,046,493$ & 642,359 & 338,513 & 6,254 & 126 & -11.7 & 248.6 & 168.8 \\
\hline China (Mainland) & 48 & 101 & - & 258 & 183 & 90 & -52.5 & -28.4 & -6.7 \\
\hline India & - & - & - & 60,014 & - & - & - & - & - \\
\hline 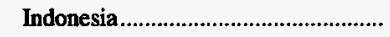 & 923,908 & $1,025,543$ & 642,359 & 229,779 & 6,025 & 36 & -9.9 & 251.9 & 209.0 \\
\hline 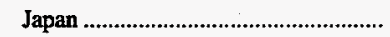 & 24 & 1 & - & - & 1 & - & NM & 121.3 & - \\
\hline Malaysia.............................................. & - & - & - & 48,461 & - & - & - & - & - \\
\hline 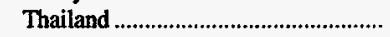 & - & - & - & - & 45 & - & - & -100.0 & - \\
\hline Vietnam & - & 20,848 & - & - & - & - & -100.0 & - & - \\
\hline Oceania \& Australia Total ................. & 230,554 & 91,002 & 95,664 & 91,613 & 28,219 & 5,313 & 153.3 & 69.1 & 52.0 \\
\hline 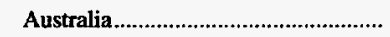 & 192,054 & 83,646 & 90,787 & 91,613 & 28,219 & 5,313 & 129.6 & 61.5 & 49.0 \\
\hline 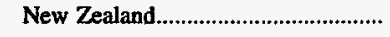 & 38,500 & 7,356 & 4,877 & - & - & - & 423.4 & - & - \\
\hline Africa Total & - & 141,931 & 23,967 & 5,227 & - & 934,025 & -100.0 & - & -100.0 \\
\hline South Africa, Rep of & - & 135,849 & 18,433 & 5,227 & - & 934,025 & -100.0 & - & -100.0 \\
\hline 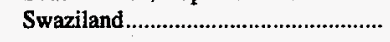 & - & 6,082 & 5,534 & - & - & - & -100.0 & - & - \\
\hline Total & $6,532,713$ & $6,880,076$ & $6,630,528$ & $3,449,813$ & $3,075,162$ & $2,006,422$ & -5.0 & 20.7 & 14.0 \\
\hline
\end{tabular}

NM Not meaningful as value is greater than 500 percent.

Note: Coal imports include coal to Puerto Rico and the Virgin Islands.

Source: U.S. Department of Commerce, Bureau of the Census, "Monthly Report IM 145." 
Table B6. Coal Mining Productivity by State, 1986, 1991-1995

(Metric Tons of Coal Produced per Miner per Hour)

\begin{tabular}{|c|c|c|c|c|c|c|c|c|c|}
\hline \multirow{2}{*}{$\begin{array}{l}\text { Coal-Producing } \\
\text { State and Region }\end{array}$} & \multirow{2}{*}{1995} & \multirow{2}{*}{1994} & \multirow{2}{*}{1993} & \multirow{2}{*}{1992} & \multirow{2}{*}{1991} & \multirow{2}{*}{1986} & \multirow{2}{*}{$\begin{array}{c}\text { Percent } \\
\text { Change } \\
\text { 1994-1995 }\end{array}$} & \multicolumn{2}{|c|}{ Average Annual Percent Chang } \\
\hline & & & & & & & & 1991-1995 & 1986-1995 \\
\hline Alabama & 2.03 & 2.04 & 2.14 & 2.26 & 1.97 & 1.71 & -0.6 & 0.8 & 1.9 \\
\hline 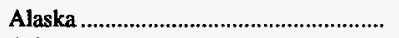 & 6.77 & 6.30 & 6.71 & 6.29 & 6.19 & 6.14 & 7.5 & 2.3 & 1.1 \\
\hline Arizona & 5.75 & 6.08 & 5.63 & 5.71 & 6.03 & 6.06 & -5.4 & -1.1 & -.6 \\
\hline 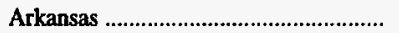 & 1.33 & 1.38 & 1.26 & 1.52 & 2.06 & 1.58 & -3.7 & -10.3 & -1.9 \\
\hline 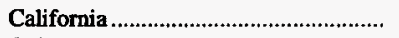 & - & - & - & 10.09 & 10.26 & - & - & - & - \\
\hline 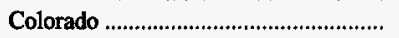 & 5.57 & 5.62 & 5.30 & 4.78 & 4.58 & 3.48 & -.9 & 5.0 & 5.3 \\
\hline 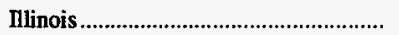 & 3.51 & 3.26 & 2.93 & 3.10 & 2.89 & 2.15 & 7.6 & 5.0 & 5.6 \\
\hline Indiana & 4.24 & 3.88 & 4.04 & 3.71 & 3.65 & 3.05 & 9.4 & 3.8 & 3.7 \\
\hline lowa & - & 1.38 & .65 & 1.03 & 1.17 & 1.42 & -100.0 & - & - \\
\hline 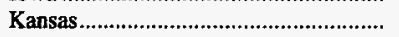 & 2.01 & 1.75 & 2.09 & 2.00 & 1.95 & 2.57 & 14.9 & .8 & -2.7 \\
\hline 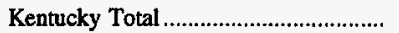 & 3.24 & 2.95 & 2.94 & 2.90 & 2.73 & 2.23 & 9.8 & 4.3 & 4.3 \\
\hline Eastern & 3.14 & 2.94 & 2.88 & 2.82 & 2.63 & 2.10 & 6.9 & 4.5 & 4.6 \\
\hline 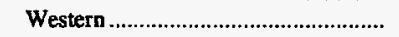 & 3.60 & 2.97 & 3.16 & 3.17 & 3.05 & 2.67 & 21.2 & 4.2 & 3.4 \\
\hline 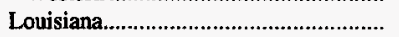 & 12.02 & 11.79 & 11.02 & 11.27 & 11.40 & 11.30 & 1.9 & 1.3 & .7 \\
\hline 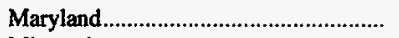 & 3.46 & 3.34 & 3.10 & 2.64 & 2.82 & 3.18 & 3.6 & 5.2 & 1.0 \\
\hline 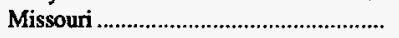 & 2.31 & 3.26 & 1.67 & 2.81 & 2.44 & 2.00 & -29.0 & -1.4 & 1.6 \\
\hline Montana & 19.10 & 19.89 & 17.68 & 18.29 & 17.23 & 16.00 & -3.9 & 2.6 & 2.0 \\
\hline New Mexico & 6.27 & 6.14 & 6.06 & 6.06 & 5.67 & 5.27 & 2.1 & 2.6 & 2.0 \\
\hline 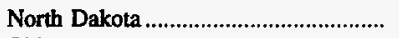 & 15.24 & 17.09 & 16.02 & 16.43 & 16.00 & 11.13 & -10.8 & -1.2 & 3.5 \\
\hline 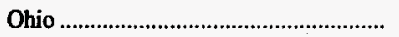 & 3.29 & 3.10 & 3.14 & 2.76 & 2.43 & 1.93 & 6.1 & 7.9 & 6.1 \\
\hline Oklahoma & 2.69 & 2.43 & 2.54 & 1.97 & 2.08 & 1.58 & 10.8 & 6.6 & 6.1 \\
\hline Pennsylvania Total ................................ & 2.93 & 2.71 & 2.54 & 2.43 & 2.21 & 1.66 & 8.3 & 7.3 & 6.5 \\
\hline Anthracite. & 1.89 & 1.75 & 1.68 & 1.21 & 1.26 & .94 & 8.0 & 10.6 & 8.1 \\
\hline 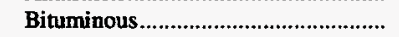 & 3.06 & 2.82 & 2.64 & 2.55 & 2.29 & 1.74 & 8.5 & 7.5 & 6.5 \\
\hline 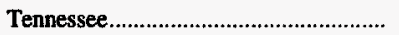 & 2.14 & 2.03 & 2.24 & 1.98 & 1.71 & 1.43 & 5.6 & 5.8 & 4.6 \\
\hline Texas & 8.25 & 8.00 & 7.63 & 6.66 & 6.50 & 5.74 & 3.2 & 6.1 & 4.1 \\
\hline 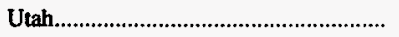 & 6.37 & 5.98 & 5.41 & 4.96 & 4.35 & 2.79 & 6.5 & 10.0 & 9.6 \\
\hline 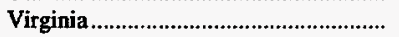 & 2.27 & 2.28 & 2.18 & 2.15 & 2.02 & 1.72 & -1 & 2.9 & 3.1 \\
\hline Washington & 3.67 & 3.73 & 3.62 & 4.09 & 3.60 & 2.57 & -1.6 & .5 & 4.0 \\
\hline West Virginia Total. & 3.40 & 3.35 & 2.97 & 2.97 & 2.82 & 1.99 & 1.5 & 4.7 & 6.1 \\
\hline Northern & 3.38 & 3.29 & 2.70 & 2.88 & 2.57 & 2.13 & 2.7 & 7.0 & 5.3 \\
\hline Southern & 3.40 & 3.37 & 3.07 & 3.01 & 2.95 & 1.91 & .9 & 3.6 & 6.6 \\
\hline 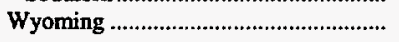 & 27.27 & 23.63 & 22.19 & 19.50 & 19.84 & 14.27 & 15.4 & 8.3 & 7.5 \\
\hline Appalachian Total 1 .............................. & 3.01 & 2.90 & 2.73 & 2.67 & 2.48 & 1.89 & 3.9 & 4.9 & 5.3 \\
\hline 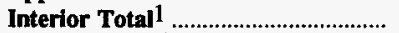 & 4.51 & 4.02 & 4.01 & 3.79 & 3.61 & 2.85 & 12.1 & 5.7 & 5.2 \\
\hline 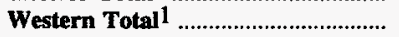 & 14.22 & 13.23 & 12.27 & 11.55 & 11.27 & 8.41 & 7.5 & 6.0 & 6.0 \\
\hline East of Miss. River............................... & $\mathbf{3 . 1 3}$ & 2.98 & 2.82 & 2.78 & 2.60 & 2.01 & 5.1 & 4.7 & $\mathbf{5 . 0}$ \\
\hline West of Miss. River ............................... & 12.87 & 11.99 & 11.01 & 10.01 & 9.79 & 7.16 & 7.3 & 7.1 & 6.7 \\
\hline U.S. Total & 4.88 & 4.51 & 4.26 & 3.96 & 3.71 & 2.73 & 8.0 & 7.1 & 6.6 \\
\hline
\end{tabular}

1 For a definition of coal-producing regions, see Appendix C.

Notes: Productivity is calculated by dividing total coal production by the total direct labor hours worked by all mine employees. Excludes silt, culm, refuse bank, slurry dam, and dredge operations except for Pennsylvania anthracite. Excludes mines producing less than 10,000 short tons, which are not required to provide these data.

Sources: Energy Information Administration, Form EIA-7A, "Coal Production Report"; State Mining Agency Coal Production Reports; and/or U.S. Department of Labor, Mine Safety and Health Administration, Form 7000-2, "Quarterly Mine Employment and Coal Production Report." 
Table B7. Coal Consumption by Census Division and State, 1986, 1991-1995 (Thousand Metric Tons)

\begin{tabular}{|c|c|c|c|c|c|c|c|c|c|}
\hline \multirow{2}{*}{ Census Division and State } & \multirow{2}{*}{1995} & \multirow{2}{*}{1994} & \multirow{2}{*}{1993} & \multirow{2}{*}{1992} & \multirow{2}{*}{1991} & \multirow{2}{*}{1986} & \multirow{2}{*}{$\begin{array}{c}\text { Percent } \\
\text { Change } \\
\text { 1994-1995 }\end{array}$} & \multicolumn{2}{|c|}{ Average Annual Percent Change } \\
\hline & & & & & & & & 1991-1995 & 1986-1995 \\
\hline 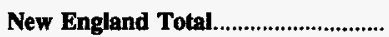 & 6,043 & $\mathbf{5 , 9 4 5}$ & 5,883 & 6,620 & 6,361 & 5,403 & 1.6 & -1.3 & 1.3 \\
\hline 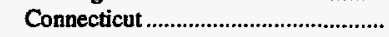 & 822 & 782 & 715 & 770 & 777 & 734 & 5.1 & 1.4 & 1.3 \\
\hline 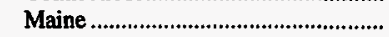 & 256 & 421 & 407 & 777 & 339 & 340 & -39.3 & -6.8 & -3.1 \\
\hline 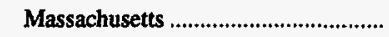 & 3,732 & 3,567 & 3,457 & 3,862 & 4,038 & 3,434 & 4.6 & -1.9 & .9 \\
\hline 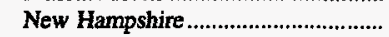 & 1,229 & 1,167 & 1,295 & 1,189 & 1,193 & 846 & 5.3 & .8 & 4.2 \\
\hline 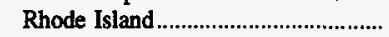 & 2 & 3 & 3 & 4 & 4 & 26 & -18.4 & -10.3 & -23.0 \\
\hline 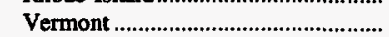 & 3 & 4 & 5 & 18 & 11 & 24 & -43.0 & -30.9 & -22.2 \\
\hline Middle Atlantic Total.......................... & 62,040 & 61,268 & 63,856 & 64,790 & 64,042 & 59,751 & 1.3 & -.8 & .4 \\
\hline 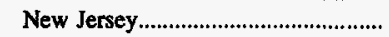 & 1,881 & 1,786 & 2,135 & 2,130 & 2,110 & 2,686 & 5.3 & -2.8 & -3.9 \\
\hline New York & 10,002 & 10,409 & 10,776 & 11,790 & 12,100 & 9,009 & -3.9 & -4.6 & 1.2 \\
\hline 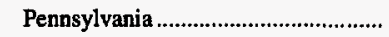 & 50,157 & 49,073 & 50,945 & 50,870 & 49,832 & 48,056 & 2.2 & .2 & .5 \\
\hline East North Central Total ................... & 197,300 & 193,401 & 191,082 & 182,035 & 189,223 & 181,249 & 2.0 & 1.0 & .9 \\
\hline 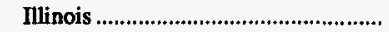 & 35,946 & 35,450 & 34,595 & 28,667 & 31,459 & 33,641 & 1.4 & 3.4 & .7 \\
\hline Indiana & 56,818 & 54,427 & 54,751 & 53,310 & 55,148 & 45,943 & 4.4 & .7 & 2.4 \\
\hline 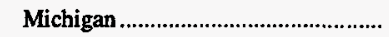 & 32,283 & 32,363 & 29,226 & 28,626 & 30,734 & 30,844 & -.2 & 1.2 & .5 \\
\hline Ohio & 51,328 & 51,447 & 53,552 & 53,225 & 53,141 & 53,818 & -.2 & -.9 & -.5 \\
\hline 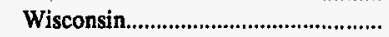 & 20,925 & 19,714 & 18,957 & 18,208 & 18,741 & 17,004 & 6.1 & 2.8 & 2.3 \\
\hline West North Central Total ................. & 118,916 & 113,935 & 109,715 & 104,785 & 105,875 & 86,659 & 4.4 & 2.9 & 3.6 \\
\hline Iowa & 18,721 & 17,546 & 17,407 & 16,322 & 17,001 & 12,576 & 6.7 & 2.4 & 4.5 \\
\hline 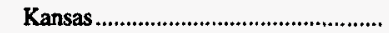 & 15,037 & 15,566 & 15,772 & 12,906 & 13,500 & 13,026 & -3.4 & 2.7 & 1.6 \\
\hline 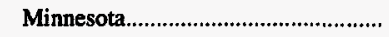 & 17,189 & 16,991 & 16,620 & 15,354 & 15,416 & 10,276 & 1.2 & 2.8 & 5.9 \\
\hline 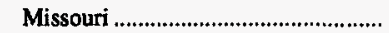 & 28,806 & 25,096 & 21,211 & 22,843 & 23,381 & 21,610 & 14.8 & 5.3 & 3.2 \\
\hline 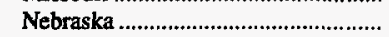 & 9,431 & 8,437 & 8,769 & 7,450 & 8,037 & 5,705 & 11.8 & 4.1 & 5.7 \\
\hline North Dakota & 27,431 & 27,545 & 27,490 & 27,488 & 25,943 & 21,397 & -.4 & 1.4 & 2.8 \\
\hline 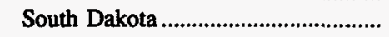 & 2,302 & 2,754 & 2,445 & 2,422 & 2,598 & 2,069 & -16.4 & -3.0 & 1.2 \\
\hline 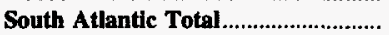 & 140,891 & 137,833 & 136,604 & 130,796 & 130,701 & 128,002 & 2.2 & 1.9 & 1.1 \\
\hline 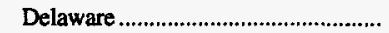 & 1,825 & 2,020 & 2,219 & 1,605 & 1,983 & 2,327 & -9.6 & -2.1 & -2.7 \\
\hline District of Columbia.............................. & 5 & 43 & 46 & 46 & 60 & 49 & -87.9 & -45.8 & -22.2 \\
\hline 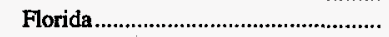 & 24,064 & 23,661 & 23,977 & 23,921 & 23,590 & 16,964 & $1: 7$ & .5 & 4.0 \\
\hline 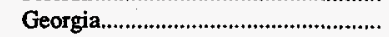 & 28,384 & 26,539 & 24,567 & 23,116 & 24,455 & 25,819 & 6.9 & 3.8 & 1.0 \\
\hline 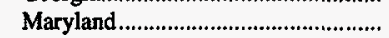 & 10,159 & 9,518 & 9,315 & 8,811 & 9,715 & 9,753 & 6.7 & 1.1 & .4 \\
\hline 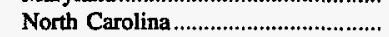 & 21,849 & 21,121 & 23,369 & 21,840 & 18,940 & 21,084 & 3.4 & 3.6 & .4 \\
\hline 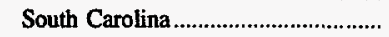 & 11,139 & 11,787 & 11,715 & 10,238 & 10,388 & 9,490 & -5.5 & 1.8 & 1.8 \\
\hline 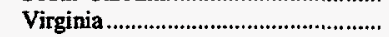 & 12,179 & 11,605 & 12,323 & 12,173 & 12,682 & 10,756 & 4.9 & -1.0 & 1.4 \\
\hline 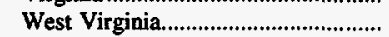 & 31,288 & 31,540 & 29,072 & 29,047 & 28,887 & 31,760 & -.8 & 2.0 & -.2 \\
\hline East South Central Total.................... & 96,024 & 90,074 & 94,371 & 85,097 & 82,359 & 80,315 & 6.6 & 3.9 & 2.0 \\
\hline 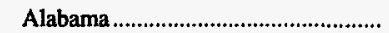 & 31,141 & 28,552 & 29,980 & 28,585 & 26,625 & 24,275 & 9.1 & 4.0 & 2.8 \\
\hline 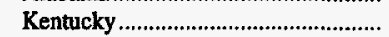 & 35,849 & 34,555 & 35,467 & 31,483 & 31,313 & 29,130 & 3.7 & 3.4 & 2.3 \\
\hline 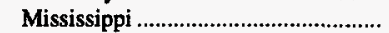 & 4,178 & 3,887 & 3,656 & 3,161 & 3,458 & 4,040 & 7.5 & 4.8 & .4 \\
\hline 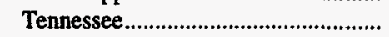 & 24,856 & 23,079 & 25,268 & 21,868 & 20,962 & 22,869 & 7.7 & 4.3 & .9 \\
\hline West South Central Total .................. & 126,195 & 125,419 & 127,729 & 122,660 & 121,232 & 104,291 & .6 & 1.0 & 2.1 \\
\hline 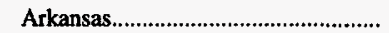 & 12,284 & 11,427 & 10,384 & 11,374 & 11,123 & 11,656 & 7.5 & 2.5 & .6 \\
\hline 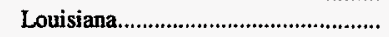 & 12,118 & 12,791 & 12,407 & 12,405 & 11,762 & 9,488 & -5.3 & .7 & 2.8 \\
\hline 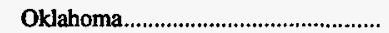 & 17,777 & 16,080 & 17,115 & 15,812 & 14,828 & 11,244 & 10.5 & 4.6 & 5.2 \\
\hline Texas & 84,017 & 85,121 & 87,823 & 83,069 & 83,519 & 71,903 & -1.3 & .1 & 1.7 \\
\hline 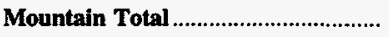 & 97,906 & 104,957 & 100,401 & 101,752 & 95,415 & 77,396 & -6.7 & .6 & 2.6 \\
\hline 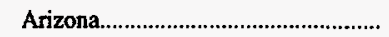 & 15,134 & 17,763 & 17,228 & 16,252 & 15,245 & 12,837 & -14.8 & -.2 & 1.8 \\
\hline 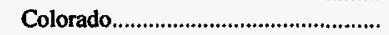 & 15,395 & 15,853 & 15,485 & 15,146 & 14,713 & 13,634 & -2.9 & 1.1 & 1.3 \\
\hline Idaho & 421 & 484 & 479 & 485 & 610 & 422 & -13.0 & -8.8 & * \\
\hline 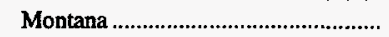 & 9,076 & 10,060 & 8,389 & 10,015 & 9,570 & 7,058 & -9.8 & -1.3 & 2.8 \\
\hline 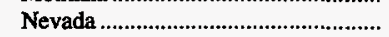 & 6,659 & 7,229 & 7,081 & 7,338 & 7,340 & 6,527 & -7.9 & -2.4 & .2 \\
\hline 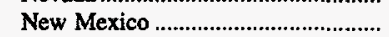 & 13,809 & 13,947 & 13,619 & 13,455 & 11,664 & 12,015 & -1.0 & 4.3 & 1.5 \\
\hline Utah & 13,886 & 14,711 & 14,377 & 14,260 & 13,457 & 7,359 & -5.6 & .8 & 7.3 \\
\hline Wyoming & 23,526 & 24,910 & 23,742 & 24,801 & 22,815 & 17,542 & -5.5 & .8 & 3.3 \\
\hline 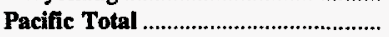 & 8,017 & 11,034 & 10,362 & 11,055 & 10,029 & 5,990 & -27.3 & -5.4 & 3.3 \\
\hline Alaska & 740 & 722 & 783 & 718 & 728 & 697 & 2.4 & .4 & 6 \\
\hline 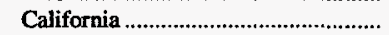 & 2,375 & 2,266 & 2,226 & 2,559 & 2,554 & 1,692 & 4.8 & -1.8 & 3.8 \\
\hline 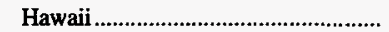 & 110 & 78 & 66 & 42 & 33 & 15 & 41.0 & 34.5 & 24.9 \\
\hline 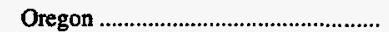 & 1,020 & 2,249 & 1,904 & 1,927 & 1,760 & 148 & -54.6 & -12.7 & 23.9 \\
\hline 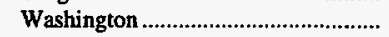 & 3,772 & 5,718 & 5,383 & 5,808 & 4,954 & 3,438 & -34.0 & -6.6 & 1.0 \\
\hline U.S. Total & 853,333 & 843,865 & 840,003 & 809,591 & 805,236 & $\mathbf{7 2 9 , 5 3 0}$ & 1.1 & 1.5 & 1.8 \\
\hline
\end{tabular}

\footnotetext{
* Data round to zero.

Note: U.S. Total does not include coal consumed by independent power producers. Totals may not equal sum of components due to independent rounding.

Sources: Energy Information Administration, Form EIA-759, "Monthly Power Plant Report”; Form EIA-3, "Quarterly Coal Consumption Report - Manufacturing Plants"; Form EIA-5, "Coke Plant Report - Quarterly"; and Form EIA-6, "Coal Distribution Report."
} 
Table B8. Year-End Consumer Coal Stocks by Census Division and State, 1986, 1991-1995 (Thousand Metric Tons)

\begin{tabular}{|c|c|c|c|c|c|c|c|c|c|}
\hline \multirow{2}{*}{ Census Division and State } & \multirow{2}{*}{1995} & \multirow{2}{*}{1994} & \multirow{2}{*}{1993} & \multirow{2}{*}{1992} & \multirow{2}{*}{1991} & \multirow{2}{*}{1986} & \multirow{2}{*}{$\begin{array}{c}\text { Percent } \\
\text { Change } \\
\text { 1994-1995 }\end{array}$} & \multicolumn{2}{|c|}{ Average Annual Percent Change } \\
\hline & & & & & & & & 1991-1995 & 1986-1995 \\
\hline New England Total............................. & 879 & 1,013 & 897 & $\mathbf{1 , 1 3 7}$ & 1,029 & 1,249 & -13.3 & -3.9 & $-\mathbf{3 . 8}$ \\
\hline 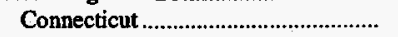 & $w$ & w & $\mathbf{w}$ & $w$ & $w$ & $\mathbf{w}$ & $\mathbf{w}$ & $\mathbf{w}$ & $\mathbf{w}$ \\
\hline Maine & $\mathbf{w}$ & $\mathbf{w}$ & $\mathbf{w}$ & $\mathbf{w}$ & $\mathbf{w}$ & $\mathbf{w}$ & $\mathbf{w}$ & $\mathbf{w}$ & $\mathbf{w}$ \\
\hline 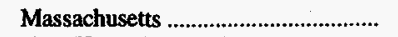 & $\mathbf{w}$ & $\mathbf{w}$ & $\mathbf{w}$ & $\mathbf{w}$ & $\mathbf{w}$ & $\mathbf{w}$ & $\mathbf{w}$ & $\mathbf{w}$ & $\mathbf{w}$ \\
\hline 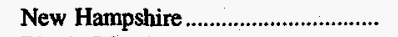 & $\mathbf{w}$ & $\mathbf{w}$ & $\mathbf{w}$ & $\mathbf{w}$ & $\mathbf{w}$ & $\mathbf{w}$ & w & $w$ & $w$ \\
\hline 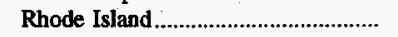 & - & - & - & - & - & $\mathbf{w}$ & $\mathbf{w}$ & $\mathbf{w}$ & $\mathbf{w}$ \\
\hline 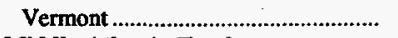 & - & - & - & - & - & $\mathbf{w}$ & $\mathbf{w}$ & $\mathbf{w}$ & w \\
\hline Middle Atlantic Total.......................... & 11,253 & 12,762 & 12,755 & 17,678 & 16,553 & 16,383 & -11.8 & -9.2 & -4.1 \\
\hline 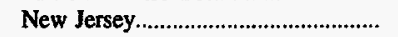 & w & $w$ & $w$ & w & w & $\mathrm{w}$ & $\mathbf{w}$ & $\mathbf{w}$ & $\mathbf{w}$ \\
\hline 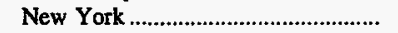 & $\mathbf{w}$ & $\mathbf{w}$ & $\mathbf{w}$ & $\mathbf{w}$ & $\mathbf{w}$ & $\mathbf{w}$ & $\mathbf{w}$ & $\mathbf{w}$ & $\mathbf{w}$ \\
\hline Pennsylvania & 9,346 & 10,940 & 11,127 & 14,493 & 14,104 & 13,328 & -14.6 & -9.8 & -3.9 \\
\hline East North Central Total .................... & 30,679 & 32,507 & 27,363 & 39,609 & 39,172 & 44,337 & -5.6 & -5.9 & -4.0 \\
\hline 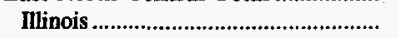 & w & $w$ & $w$ & w & w & 8,773 & $\mathbf{w}$ & $w$ & $\mathbf{w}$ \\
\hline 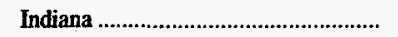 & 8,435 & 10,620 & 7,074 & 11,347 & 10,330 & 11,419 & -20.6 & -4.9 & -3.3 \\
\hline 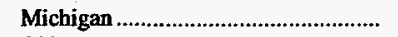 & $w$ & $\mathbf{w}$ & $w$ & w & $\mathbf{w}$ & w & w & $\mathbf{w}$ & $\mathbf{w}$ \\
\hline Ohio & 5,385 & 7,090 & 6,922 & 9,801 & 9,632 & 8,626 & -24.0 & -13.5 & -5.1 \\
\hline 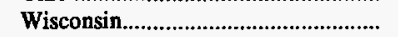 & 3,317 & 3,118 & w & $w$ & $\mathbf{w}$ & $w$ & 6.4 & $\mathbf{w}$ & w \\
\hline West North Central Total .................... & 16,976 & 16,072 & $\mathbf{w}$ & $\mathbf{w}$ & $\mathbf{w}$ & $\mathbf{w}$ & 5.6 & $\mathbf{w}$ & $\mathbf{w}$ \\
\hline Iowa & 4,034 & 3,790 & 3,465 & 4,406 & 4,657 & 4,064 & 6.4 & -3.5 & -.1 \\
\hline 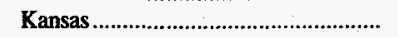 & 3,501 & 2,380 & 1,836 & 2,503 & 3,012 & 3,056 & 47.1 & 3.8 & 1.5 \\
\hline 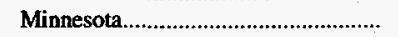 & 1,800 & 2,026 & 1,134 & 2,043 & 2,443 & 3,310 & -11.1 & -7.3 & -6.5 \\
\hline 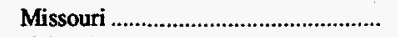 & 4,335 & 4,145 & w & $\mathbf{w}$ & $\mathbf{w}$ & $\mathbf{w}$ & 4.6 & $\mathbf{w}$ & $\mathbf{w}$ \\
\hline 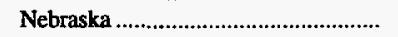 & w & w & $\mathbf{w}$ & $\mathbf{w}$ & $w$ & 1,821 & w & $\mathbf{w}$ & $w$ \\
\hline 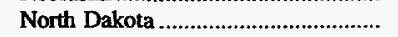 & w & $\mathbf{w}$ & $\mathbf{w}$ & $\mathbf{w}$ & $\mathbf{w}$ & w & $\mathbf{w}$ & $\mathbf{w}$ & $\mathbf{w}$ \\
\hline 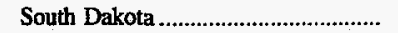 & w & $\mathbf{w}$ & $\mathbf{w}$ & $\mathbf{w}$ & w & $\mathrm{w}$ & $\mathbf{w}$ & $\mathbf{w}$ & $\mathbf{w}$ \\
\hline South Atlantic Total .............................. & 17,878 & 22,160 & 17,320 & 26,565 & 27,172 & 23,028 & -19.3 & -9.9 & -2.8 \\
\hline Delaware & w & w & w & $\mathbf{w}$ & $\mathbf{w}$ & $\mathbf{w}$ & $\mathbf{w}$ & w & $\mathbf{w}$ \\
\hline Florida & 2,965 & 3,550 & 3,212 & 3,739 & 4,427 & 2,983 & -16.5 & -9.5 & -.1 \\
\hline 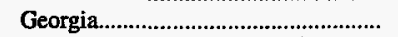 & 3,435 & 4,394 & 2,655 & 4,387 & 4,916 & 5,413 & -21.8 & -8.6 & -4.9 \\
\hline Maryland & w & $w$ & w & $\mathbf{w}$ & $\mathbf{w}$ & w & $\mathbf{w}$ & $\mathbf{w}$ & w \\
\hline 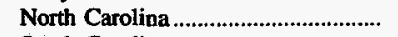 & 2,590 & 3,917 & 2,776 & 4,277 & 4,415 & 3,637 & -33.9 & -12.5 & -3.7 \\
\hline 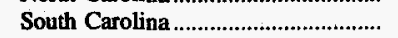 & 1,990 & 2,298 & 1,717 & 2,223 & 2,033 & 1,930 & -13.4 & -.5 & .3 \\
\hline Virginia & $\mathbf{w}$ & $\mathbf{w}$ & $w$ & $\mathbf{w}$ & $\mathbf{w}$ & w & $\mathbf{w}$ & $\mathbf{w}$ & $\mathbf{w}$ \\
\hline West Virginia......................................... & $\mathbf{w}$ & w & $w$ & $\mathbf{w}$ & $\mathbf{w}$ & $\mathbf{w}$ & $\mathbf{w}$ & $w$ & $\mathbf{w}$ \\
\hline East South Central Total.................... & 9,925 & 10,221 & 8,416 & 13,063 & 13,631 & 15,900 & -2.9 & -7.6 & -5.1 \\
\hline 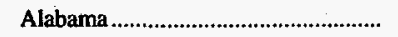 & 3,310 & 3,748 & 2,538 & 4,109 & 4,237 & 4,725 & -11.7 & -6.0 & -3.9 \\
\hline Kentucky & w & w & $\mathbf{w}$ & $\mathbf{w}$ & w & $\mathbf{w}$ & $\mathbf{w}$ & $\mathbf{w}$ & $\mathbf{w}$ \\
\hline 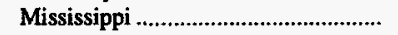 & w & $\mathbf{w}$ & $\mathbf{w}$ & $\mathbf{w}$ & $\mathbf{w}$ & $w$ & $w$ & $\mathbf{w}$ & $w$ \\
\hline 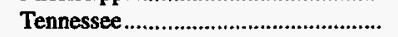 & 1,709 & 1,601 & $w$ & $\mathbf{w}$ & $\mathbf{w}$ & $\mathbf{w}$ & 6.8 & $w$ & $\mathbf{w}$ \\
\hline West South Central Total ................. & 18,656 & 14,478 & 13,703 & 15,330 & $w$ & 17,196 & 28.8 & $\mathbf{w}$ & 9 \\
\hline 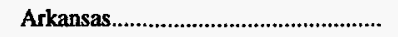 & 2,558 & 1,612 & 1,706 & 1,444 & 1,964 & 1,773 & 58.7 & 6.8 & 4.1 \\
\hline 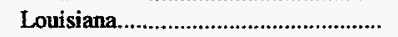 & 2,422 & 1,744 & 1,814 & 1,592 & 2,087 & $w$ & 38.8 & 3.8 & $\mathbf{w}$ \\
\hline 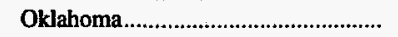 & 3,852 & 2,238 & 1,862 & 2,867 & 2,648 & 4,005 & 72.1 & 9.8 & -.4 \\
\hline 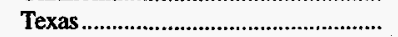 & 9,824 & 8,884 & 8,320 & 9,427 & $\mathbf{w}$ & $\mathbf{w}$ & 10.6 & $w$ & $\mathbf{w}$ \\
\hline 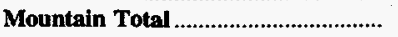 & $\mathbf{w}$ & $w$ & $w$ & $w$ & $\mathbf{w}$ & $\mathbf{w}$ & $\mathbf{w}$ & $\mathbf{w}$ & $\mathbf{w}$ \\
\hline 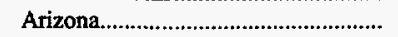 & 2,751 & 2,941 & 3,372 & 3,263 & 3,837 & $\mathbf{w}$ & -6.5 & -8.0 & $w$ \\
\hline 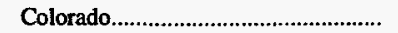 & 3,340 & 2,853 & 3,133 & 3,120 & 3,163 & $\mathbf{w}$ & 17.1 & 1.4 & $\mathbf{w}$ \\
\hline Idaho & 107 & 71 & 78 & 92 & 71 & 99 & 51.1 & 10.6 & .8 \\
\hline 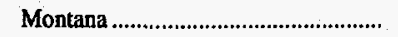 & $\mathbf{w}$ & w & $w$ & $\mathbf{w}$ & $w$ & $w$ & $w$ & $w$ & $w$ \\
\hline Nevada & $w$ & $\mathbf{w}$ & w & $\mathbf{w}$ & $\mathbf{w}$ & $\mathbf{w}$ & $\mathbf{w}$ & w & w \\
\hline 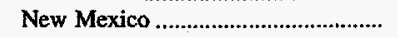 & $\mathbf{w}$ & $\mathbf{w}$ & $\mathbf{w}$ & w & $\mathbf{w}$ & $w$ & $\mathbf{w}$ & $w$ & $\mathbf{w}$ \\
\hline 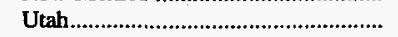 & $\mathbf{w}$ & $\mathbf{w}$ & w & $\mathbf{w}$ & $\mathbf{w}$ & $\mathbf{w}$ & $\mathbf{w}$ & $\mathbf{w}$ & $\mathbf{w}$ \\
\hline 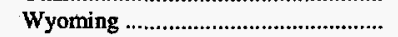 & 2,664 & 2,316 & 1,670 & 2,034 & 2,571 & $\mathbf{w}$ & 15.0 & .9 & $\mathbf{w}$ \\
\hline 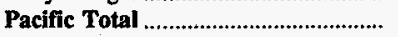 & 2,346 & 796 & 833 & 1,387 & 1,846 & $\mathbf{w}$ & 194.8 & 6.2 & $\mathbf{w}$ \\
\hline Alaska & 1 & 2 & 4 & 5 & $w$ & $\mathbf{w}$ & -67.4 & $\mathbf{w}$ & $w$ \\
\hline 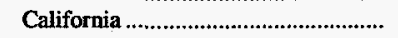 & 121 & 114 & 84 & 99 & 126 & $\mathbf{w}$ & 6.2 & -.9 & $\mathbf{w}$ \\
\hline Hawaii & $\mathbf{w}$ & $\mathbf{w}$ & $\mathbf{w}$ & w & $w$ & 22 & $\mathbf{w}$ & $\mathbf{w}$ & $\mathbf{w}$ \\
\hline Oregon & w & $\mathbf{w}$ & $\mathbf{w}$ & $\mathbf{w}$ & $\mathbf{w}$ & $\mathbf{w}$ & $\mathbf{w}$ & $\mathbf{w}$ & $\mathbf{w}$ \\
\hline 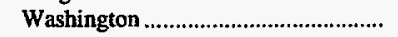 & 1,786 & 516 & 416 & 667 & 1,083 & 1,592 & 246.1 & 13.3 & 1.3 \\
\hline 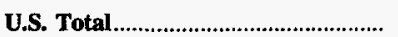 & 122,142 & 123,504 & 109,278 & 148,499 & 152,145 & 158,963 & -1.1 & -5.3 & -2.9 \\
\hline
\end{tabular}

W Witheld to avoid disclosure of individual company data.

Notes: Totals may not equal sum of components due to independent rounding. Stocks for Residential and Commercial Sector are not included.

Sources: Energy Information Administration, Form EIA-759, "Monthly Power Plant Report"; Form EIA-5, "Coke Plant Report - Quarterly"; and Form EIA-3, "Quarterly Coal Consumption Report - Manufacturing Plants." 
Table B9. U.S. Coal Exports by Destination, 1986, 1991-1995

(Thousand Metric Tons)

\begin{tabular}{|c|c|c|c|c|c|c|c|c|c|}
\hline \multirow{2}{*}{$\begin{array}{l}\text { Continent and Country } \\
\text { of Destination }\end{array}$} & \multirow{2}{*}{1995} & \multirow{2}{*}{1994} & \multirow{2}{*}{1993} & \multirow{2}{*}{1992} & \multirow{2}{*}{1991} & \multirow{2}{*}{1986} & \multirow{2}{*}{$\begin{array}{c}\text { Percent } \\
\text { Change } \\
\text { 1994-1995 }\end{array}$} & \multicolumn{2}{|c|}{ Average Annual Percent Change } \\
\hline & & & & & & & & 1991-1995 & 1986-1995 \\
\hline North America Total .......................... & 9,444 & 8,623 & 8,363 & 13,908 & 10,391 & 13,403 & 9.5 & -2.4 & -3.8 \\
\hline 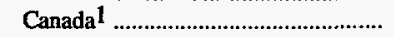 & 8,552 & 8,340 & 8,064 & 13,734 & 10,140 & 13,134 & 2.5 & -4.2 & -4.6 \\
\hline Mexico & 790 & 218 & 226 & 70 & 84 & 204 & 261.7 & 75.3 & 16.2 \\
\hline Other $^{2}$ & 102 & 65 & 73 & 104 & 167 & 65 & 58.1 & -11.6 & 5.2 \\
\hline South America Total ......................... & 6,321 & 5,394 & 5,216 & 6,141 & 6,950 & 6,020 & 17.2 & -2.3 & $\mathbf{s}$ \\
\hline Argentina & 310 & 411 & 476 & 304 & 390 & 682 & -24.4 & -5.5 & -8.4 \\
\hline 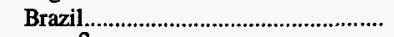 & 5,761 & 4,973 & 4,715 & 5,778 & 6,397 & 5,189 & 15.8 & -2.6 & 1.2 \\
\hline Other ${ }^{2}$ & 250 & 11 & 26 & 58 & 163 & 149 & NM & 11.3 & 5.9 \\
\hline 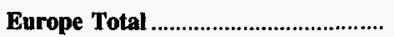 & 44,107 & 32,500 & 34,088 & 51,941 & $\mathbf{5 9 , 4 3 9}$ & 38,603 & 35.7 & -7.2 & 1.5 \\
\hline Belgium \& Luxembourg ................... & 4,084 & 4,455 & 4,744 & 6,528 & 6,771 & 3,958 & -8.3 & -11.9 & 3 \\
\hline 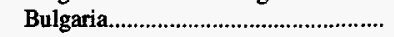 & 1,214 & 1,123 & 822 & 546 & 858 & 29 & 8.1 & 9.1 & 51.5 \\
\hline Denmark & 1,905 & 432 & 305 & 3,477 & 4,225 & 1,926 & 340.6 & -18.0 & -.1 \\
\hline Finland & 1,187 & 342 & 229 & 186 & 481 & 45 & 247.2 & 25.3 & 43.9 \\
\hline 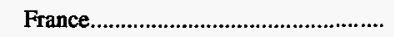 & 3,319 & 2,608 & 3,603 & 7,312 & 8,626 & 4,889 & 27.3 & -21.2 & -4.2 \\
\hline 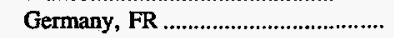 & 1,772 & 293 & 461 & 910 & 1,581 & 735 & NM & 2.9 & 10.3 \\
\hline Ireland & 829 & 883 & 894 & 1,314 & 1,191 & 698 & -6.1 & -8.6 & 1.9 \\
\hline 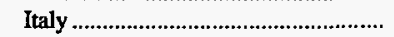 & 8,222 & 6,843 & 6,276 & 8,476 & 10,227 & 9,397 & 20.1 & -5.3 & -1.5 \\
\hline 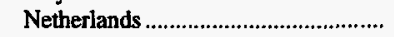 & 6,624 & 4,421 & 5,046 & 8,299 & 8,732 & 5,108 & 49.8 & -6.7 & 2.9 \\
\hline Norway & 109 & 79 & 92 & 107 & 181 & 242 & 38.5 & -12.0 & -8.5 \\
\hline 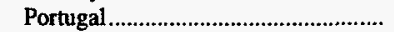 & 1,590 & 958 & 1,353 & 1,342 & 1,478 & 1,001 & 65.9 & 1.8 & 5.3 \\
\hline 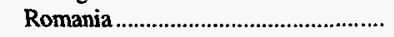 & 1,800 & 1,409 & 653 & 683 & 1,040 & 1,008 & 27.7 & 14.7 & 6.6 \\
\hline Spain & 4,221 & 3,748 & 3,687 & 4,114 & 4,258 & 2,377 & 12.6 & -.2 & 6.6 \\
\hline 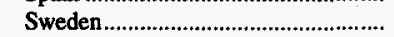 & 1,014 & 636 & 668 & 1,057 & 1,124 & 995 & 59.3 & -2.5 & .2 \\
\hline 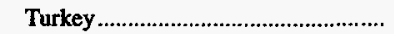 & 1,825 & 1,211 & 1,456 & 1,805 & 1,983 & 2,193 & 50.7 & -2.1 & -2.0 \\
\hline 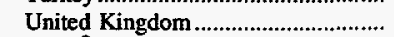 & 4,288 & 3,051 & 3,730 & 5,076 & 5,598 & 2,637 & 40.5 & -6.4 & 5.5 \\
\hline Other ${ }^{2}$ & 107 & 7 & 71 & 708 & 1,082 & 1,366 & NM & -44.0 & -24.7 \\
\hline Asia Total & 17,323 & 16,290 & 17,690 & 18,633 & 19,766 & 17,816 & 6.3 & -3.2 & -.3 \\
\hline China (Taiwan) & 2,298 & 3,061 & 3,117 & 3,230 & 4,125 & 3,348 & -24.9 & -13.6 & -4.1 \\
\hline Israel. & 690 & 784 & 770 & 748 & 591 & 346 & -12.0 & 3.9 & 8.0 \\
\hline 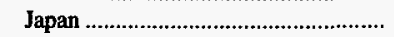 & 10,693 & 9,215 & 10,776 & 11,162 & 11,130 & 10,315 & 16.0 & -1.0 & .4 \\
\hline Korea, Republic of & 3,640 & 3,228 & 3,008 & 3,041 & 3,366 & 3,225 & 12.8 & 2.0 & 1.3 \\
\hline Other ${ }^{2}$ & 2 & 3 & 20 & 454 & 554 & 583 & -30.4 & -75.2 & -46.5 \\
\hline Oceania \& Australia Total ................ & * & - & $\mathbf{1}$ & * & * & * & -60.1 & 13.0 & -3.0 \\
\hline Other ${ }^{2}$ & $*$ & $*$ & 1 & $*$ & $*$ & * & -60.1 & 13.0 & -3.0 \\
\hline Africa Total & $\mathbf{3 , 1 3 3}$ & 1,927 & 2,245 & 2,378 & 2,309 & 1,738 & 62.5 & 7.9 & 6.8 \\
\hline 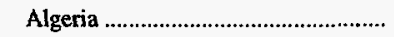 & 200 & 322 & 371 & 555 & 474 & 621 & -38.0 & -19.4 & -11.8 \\
\hline 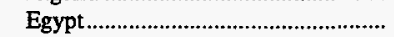 & 1,120 & 951 & 788 & 770 & 698 & 370 & 17.8 & 12.6 & 13.1 \\
\hline Morocco & 1,099 & 76 & 533 & 668 & 919 & 668 & NM & 4.6 & 5.7 \\
\hline 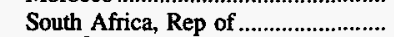 & 713 & 578 & 515 & 385 & 217 & - & 23.3 & 34.7 & - \\
\hline Other ${ }^{2}$ & - & - & 38 & - & 2 & 78 & - & -100.0 & -100.0 \\
\hline Total & 80,329 & 64,735 & 67,603 & 93,001 & 98,855 & 77,581 & 24.1 & -5.0 & .4 \\
\hline
\end{tabular}

1 Based on the U.S. - Canada Free Trade Agreement, as of January 1990, the U.S. Department of Commerce began reporting statistics on U.S. exports to Canada based on information on imports provided monthly by the Canadian government.

2 Includes countries with exports less than or equal to 50,000 short tons (45,359 metric tons) in 1994.

* Data round to zero.

NM Not meaningful as value is greater than 500 percent

Note: Total may not equal sum of components due to independent rounding.

Source: U.S. Department of Commerce, Bureau of the Census, "Monthly Report EM 545.' 
Table B10. Average Mine Price of Coal by State, 1986, 1991-1995 (Nominal Dollars per Metric Ton)

\begin{tabular}{|c|c|c|c|c|c|c|c|c|c|}
\hline \multirow{2}{*}{$\begin{array}{l}\text { Coal-Producing } \\
\text { State and Region }\end{array}$} & \multirow{2}{*}{1995} & \multirow{2}{*}{1994} & \multirow{2}{*}{1993} & \multirow{2}{*}{1992} & \multirow{2}{*}{1991} & \multirow{2}{*}{1986} & \multirow{2}{*}{$\begin{array}{c}\text { Percent } \\
\text { Change } \\
\text { 1994-1995 }\end{array}$} & \multicolumn{2}{|c|}{ Average Annual Percent Change } \\
\hline & & & & & & & & 1991-1995 & 1986-1995 \\
\hline 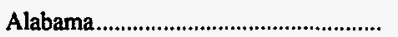 & $\$ 42.38$ & $\$ 44.22$ & $\$ 46.67$ & $\$ 45.00$ & $\$ 45.34$ & $\$ 45.49$ & -4.2 & -1.7 & -0.8 \\
\hline Alaska & $\mathbf{w}$ & w & w & $\mathbf{w}$ & $\mathbf{w}$ & 28.33 & $\mathbf{w}$ & $\mathbf{w}$ & $\mathbf{w}$ \\
\hline Arizona & $\mathbf{w}$ & $\mathbf{w}$ & $\mathbf{w}$ & $w$ & w & 18.19 & $\mathbf{w}$ & $\mathbf{w}$ & $w$ \\
\hline Arkansas & $\mathbf{w}$ & $\mathbf{w}$ & $w$ & $\mathbf{w}$ & $\mathbf{w}$ & 16.57 & $\mathbf{w}$ & $\mathbf{w}$ & $w$ \\
\hline California & - & - & - & w & $\mathbf{w}$ & - & w & w & w \\
\hline 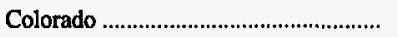 & 21.23 & 21.78 & 22.44 & 23.51 & 24.45 & 25.84 & -2.5 & -3.5 & -2.2 \\
\hline 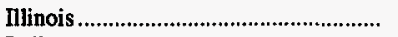 & 25.41 & 25.50 & 27.86 & 30.49 & 31.25 & 33.06 & -.3 & -5.0 & -2.9 \\
\hline Indiana & 23.93 & 24.56 & 25.23 & 25.81 & 26.00 & 27.96 & -2.5 & -2.0 & -1.7 \\
\hline lowa & - & w & w & $\mathbf{w}$ & $\mathbf{w}$ & 30.05 & $\mathbf{w}$ & w & $\mathbf{w}$ \\
\hline 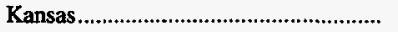 & $\mathbf{w}$ & w & w & w & $\mathbf{w}$ & 28.27 & $\mathbf{w}$ & $w$ & w \\
\hline 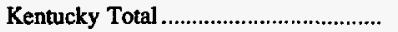 & 27.33 & 27.42 & 27.31 & 27.01 & 28.05 & 28.76 & -.3 & -.6 & -.6 \\
\hline Eastern & 28.66 & 27.83 & 28.11 & 27.55 & 29.07 & 29.08 & 2.9 & -.3 & -.2 \\
\hline 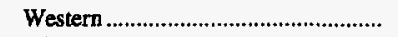 & 22.88 & 26.05 & 24.64 & 25.46 & 25.22 & 27.90 & -12.2 & -2.4 & -2.2 \\
\hline 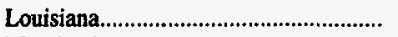 & w & w & w & $\mathbf{w}$ & $\mathbf{w}$ & 18.41 & $\mathbf{w}$ & $\mathbf{w}$ & $\mathbf{w}$ \\
\hline 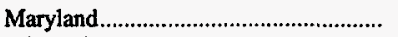 & 27.22 & 29.04 & 27.79 & 27.99 & 28.36 & 28.62 & -6.3 & -1.0 & -.5 \\
\hline 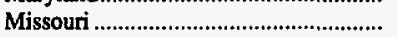 & 20.84 & 24.01 & w & w & w & 32.70 & -13.2 & $\mathbf{w}$ & -4.9 \\
\hline Montana & 10.61 & 11.46 & 12.18 & 11.24 & 11.86 & 14.25 & -7.4 & -2.7 & -3.2 \\
\hline 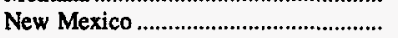 & 26.24 & 25.68 & 25.31 & 25.51 & 25.63 & 24.53 & 2.2 & .6 & .7 \\
\hline North Dakota & 8.81 & 8.40 & 8.41 & 8.25 & 8.64 & 9.36 & 4.9 & .5 & -.7 \\
\hline Ohio & 28.62 & 32.11 & 30.91 & 29.69 & 30.59 & 36.15 & -10.8 & -1.6 & -2.6 \\
\hline Otlahoma & 26.60 & 28.18 & 27.46 & 28.40 & 31.44 & 31.12 & -5.6 & -4.1 & -1.7 \\
\hline 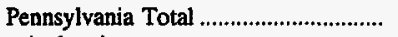 & 29.52 & 28.86 & 29.21 & 31.53 & 32.40 & 33.40 & 2.3 & -2.3 & -1.4 \\
\hline Anthracite. & 43.85 & 39.76 & 36.31 & 37.74 & 40.05 & 48.64 & 10.3 & 2.3 & -1.1 \\
\hline 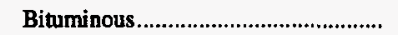 & 28.41 & 28.05 & 28.70 & 31.24 & 32.03 & 32.52 & 1.3 & -2.9 & -1.5 \\
\hline 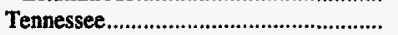 & 29.70 & 29.95 & 30.01 & 29.88 & 29.48 & 30.86 & -.8 & .2 & -.4 \\
\hline 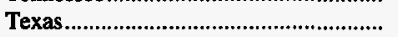 & 13.41 & 13.65 & 14.19 & 13.69 & 13.46 & 12.79 & -1.8 & -.1 & .5 \\
\hline 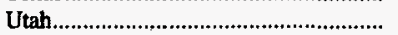 & 21.06 & 21.24 & 22.94 & 23.27 & 24.91 & 30.46 & -.9 & -4.1 & -4.0 \\
\hline 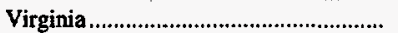 & 31.38 & 29.59 & 29.55 & 30.37 & 30.26 & 31.01 & 6.1 & .9 & .1 \\
\hline 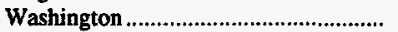 & $\mathbf{w}$ & $w$ & w & $\mathbf{w}$ & w & 28.36 & $\mathbf{w}$ & $\mathbf{w}$ & $w$ \\
\hline 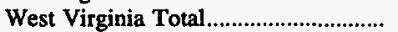 & 29.96 & 30.23 & 30.40 & 31.03 & 31.55 & 34.06 & -.9 & -1.3 & -1.4 \\
\hline Northern & 27.46 & 29.51 & 30.96 & 32.00 & 33.24 & 33.01 & -6.9 & -4.7 & -2.0 \\
\hline Southern & 30.94 & 30.54 & 30.20 & 30.60 & 30.78 & 34.69 & 1.3 & .1 & -1.3 \\
\hline Wyoming & 7.25 & 7.53 & 8.06 & 8.97 & 8.92 & 11.96 & -3.7 & -5.0 & -5.4 \\
\hline Appalachian Total ${ }^{1}$ & 30.25 & 30.16 & 30.47 & 30.81 & 31.63 & 33.12 & $\mathbf{3}$ & -1.1 & -1.0 \\
\hline Interior Total 1 & 20.73 & 21.90 & 22.08 & 23.82 & 24.10 & 25.85 & -5.3 & -3.7 & -2.4 \\
\hline Western Total ${ }^{1}$ & 11.19 & 11.65 & 12.28 & 12.78 & 12.91 & 15.47 & -4.0 & -3.5 & -3.5 \\
\hline East of Miss. River ................................ & 29.04 & 29.15 & 29.56 & 30.13 & 30.84 & 32.43 & -.4 & -1.5 & -1.2 \\
\hline West of Miss. River............................... & 11.56 & 12,03 & 12.68 & 13.21 & 13.29 & 15.56 & -3.9 & -3.4 & $-\mathbf{3 . 3}$ \\
\hline U.S. Total & 20.76 & 21.40 & 21.88 & 23.18 & 23.69 & 26.23 & -3.0 & -3.3 & -2.6 \\
\hline
\end{tabular}

1 For a definition of coal-producing regions, see Appendix $\mathbf{C}$.

Witheld to avoid disclosure of individual company data.

Notes: Average mine price is calculated by dividing the total free on board (f.o.b.) mine value of the coal produced by the total production. A measure of dispersion of these average prices at the State level (interquartile range) is given in Appendix D, Table D2. Excludes silt, culm, refuse bank, slurry dam, and dredge operations except for Pennsylvania anthracite. Excludes mines producing less than 10,000 short tons, which are not required to provide these data.

Sources: Energy Information Administration, Form EIA-7A, "Coal Production Report"; State Mining Agency Coal Production Reports; and/or U.S. Department of Labor, Mine Safety and Health Administration, Form 7000-2, "Quarterly Mine Employment and Coal Production Report." 
Table B11. Average Price of Coal Delivered to Electric Utilities by Census Division and State, 1986, 1991-1995

(Nominal Dollars per Metric Ton)

\begin{tabular}{|c|c|c|c|c|c|c|c|c|c|}
\hline \multirow{2}{*}{ Census Division and State } & \multirow{2}{*}{1995} & \multirow{2}{*}{1994} & \multirow{2}{*}{1993} & \multirow{2}{*}{1992} & \multirow{2}{*}{1991} & \multirow{2}{*}{1986} & \multirow{2}{*}{$\begin{array}{c}\text { Percent } \\
\text { Change } \\
\text { 1994-1995 }\end{array}$} & \multicolumn{2}{|c|}{ Average Annual Percent Change } \\
\hline & & & & & & & & 1991-1995 & $1986-1995$ \\
\hline New England Total ............................... & $\$ 47.78$ & $\$ 47.19$ & $\$ 47.78$ & $\$ 49.76$ & $\$ 51.95$ & $\$ 55.03$ & 1.2 & -2.1 & -1.5 \\
\hline 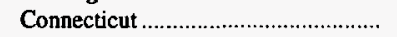 & 54.37 & 51.20 & 49.38 & 56.55 & 63.21 & 65.95 & 6.2 & -3.7 & -2.1 \\
\hline Massachusetts ..................................... & 47.00 & 47.40 & 47.83 & 48.62 & 49.97 & 51.81 & -.8 & -1.5 & -1.1 \\
\hline New Hampshire .................................. & 45.93 & 43.72 & 46.73 & 49.26 & 50.93 & 58.99 & 5.0 & -2.5 & -2.7 \\
\hline Middle Atlantic Total.......................... & 38.17 & 40.04 & $\mathbf{4 0 . 4 1}$ & 41.41 & 42.97 & 41.78 & -4.7 & -2.9 & -1.0 \\
\hline 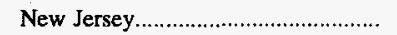 & 52.00 & 53.45 & 52.36 & 51.39 & 52.64 & 52.73 & -2.7 & -.3 & -.1 \\
\hline 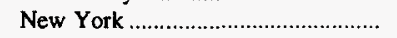 & 40.63 & 41.49 & 42.58 & 42.57 & 45.41 & 46.26 & -2.1 & -2.7 & -1.4 \\
\hline 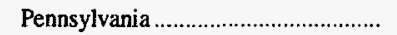 & 36.91 & 39.01 & 39.39 & 40.58 & 41.94 & 40.40 & -5.4 & -3.1 & -1.0 \\
\hline East North Central Total ................... & 32.70 & 33.69 & 34.15 & 35.33 & 35.97 & 42.65 & -2.9 & -2.3 & -2.9 \\
\hline 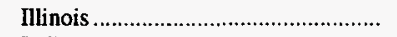 & 35.91 & 36.04 & 38.92 & 40.85 & 40.52 & 49.29 & -.3 & -3.0 & -3.4 \\
\hline Indiana & 28.59 & 29.54 & 29.47 & 30.75 & 31.31 & 37.15 & -3.2 & -2.2 & -2.9 \\
\hline Michigan & 34.12 & 36.26 & 36.57 & 37.73 & 38.80 & 46.83 & -5.9 & -3.2 & -3.4 \\
\hline 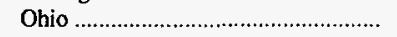 & 37.96 & 38.25 & 37.54 & 37.92 & 38.95 & 42.77 & -.8 & -.6 & -1.3 \\
\hline 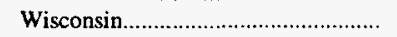 & 23.40 & 25.50 & 25.31 & 28.57 & 28.87 & 34.58 & -8.2 & -5.1 & -4.2 \\
\hline West North Central Total ................. & $\mathbf{1 7 . 7 5}$ & 18.47 & 18.60 & 20.86 & 21.43 & 24.71 & -3.9 & -4.6 & -3.6 \\
\hline Iowa & 18.88 & 19.17 & 19.32 & 21.58 & 21.63 & 27.78 & -1.5 & -3.3 & -4.2 \\
\hline 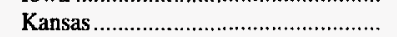 & 19.66 & 19.68 & 19.49 & 23.14 & 24.31 & 25.38 & -.1 & -5.2 & -2.8 \\
\hline 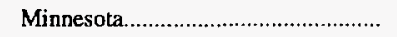 & 22.18 & 22.14 & 22.12 & 23.10 & 24.45 & 26.17 & .2 & -2.4 & -1.8 \\
\hline Missouri & 20.00 & 23.58 & 26.90 & 30.39 & 30.48 & 34.55 & -15.2 & -10.0 & -5.9 \\
\hline 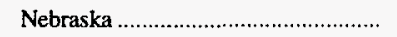 & 14.18 & 14.45 & 14.24 & 14.07 & 14.03 & 19.98 & -1.9 & .3 & -3.7 \\
\hline 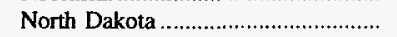 & 10.64 & 10.23 & 10.34 & 10.42 & 10.32 & 11.91 & 4.0 & .7 & -1.3 \\
\hline South Dakota & 15.82 & 14.44 & 14.66 & 15.07 & 15.04 & 16.64 & 9.6 & 1.3 & -.6 \\
\hline South Atlantic Total ............................... & 42.17 & 43.57 & 44.98 & 45.50 & 46.49 & 47.70 & -3.2 & -2.4 & -1.4 \\
\hline Delaware & 46.60 & 46.28 & 48.53 & 49.95 & 51.27 & 54.84 & .7 & -2.3 & -1.8 \\
\hline Florida & 48.42 & 48.18 & 48.04 & 49.63 & 50.56 & 52.20 & .5 & -1.1 & -8 \\
\hline 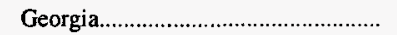 & 42.57 & 43.89 & 47.72 & 47.80 & 47.35 & 48.44 & -3.0 & -2.6 & -1.4 \\
\hline 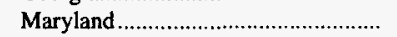 & 42.99 & 43.92 & 44.95 & 44.84 & 46.11 & 46.61 & -2.1 & -1.7 & -.9 \\
\hline North Carolina ...................................... & 44.72 & 46.04 & 46.69 & 47.40 & 49.04 & 51.14 & -2.9 & -2.3 & -1.5 \\
\hline South Carolina ....................................... & 42.83 & 43.92 & 44.28 & 43.14 & 45.60 & 50.95 & -2.5 & -1.5 & -1.9 \\
\hline Virginia & 40.68 & 40.84 & 41.41 & 41.67 & 42.85 & 48.35 & -.4 & -1.3 & -1.9 \\
\hline 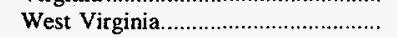 & 34.84 & 38.25 & 39.05 & 40.65 & 41.81 & 41.06 & -8.9 & -4.4 & -1.8 \\
\hline East South Central Total.................... & 33.16 & 35.75 & 36.71 & 36.43 & $\mathbf{3 7 . 4 0}$ & 41.43 & -7.2 & -3.0 & -2.4 \\
\hline Alabama & 40.78 & 44.55 & 46.91 & 45.93 & 48.31 & 51.26 & -8.4 & -4.1 & -2.5 \\
\hline 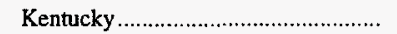 & 28.34 & 29.94 & 30.08 & 29.77 & 29.98 & 33.99 & -5.4 & -1.4 & -2.0 \\
\hline 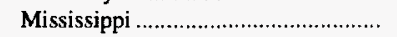 & 37.92 & 39.18 & 44.66 & 44.02 & 46.21 & 61.85 & -3.2 & -4.8 & -5.3 \\
\hline 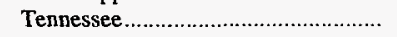 & 30.80 & 33.75 & 34.11 & 34.18 & 33.59 & 37.25 & -8.7 & -2.1 & -2.1 \\
\hline West South Central Total .................. & 22.78 & 22.91 & 24.40 & 24.86 & 25.33 & 26.87 & -.6 & -2.6 & -1.8 \\
\hline 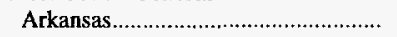 & 30.85 & 30.76 & 32.52 & 31.79 & 30.75 & 29.87 & .3 & .1 & .3 \\
\hline 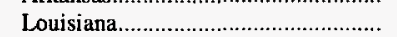 & 27.70 & 27.61 & 28.27 & 27.48 & 29.86 & 32.12 & .3 & -1.9 & -1.6 \\
\hline 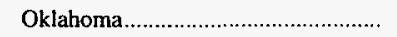 & 18.74 & 19.29 & 23.50 & 23.66 & 25.55 & 31.27 & -2.8 & -7.4 & -5.5 \\
\hline 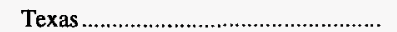 & 21.66 & 21.86 & 23.05 & 23.79 & 23.88 & 24.90 & -.9 & -2.4 & -1.5 \\
\hline 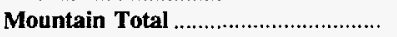 & 23.71 & 24.07 & 24.37 & 23.85 & 24.49 & 24.76 & -1.5 & -.8 & -.5 \\
\hline 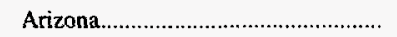 & 31.59 & 31.15 & 30.63 & 31.20 & 32.14 & 31.64 & 1.4 & -.4 & * \\
\hline 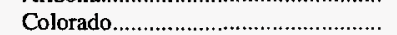 & 22.85 & 23.16 & 23.80 & 23.88 & 23.69 & 25.31 & -1.3 & -.9 & -1.1 \\
\hline 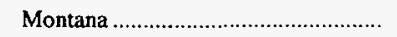 & 12.64 & 12.99 & 12.98 & 13.38 & 12.61 & 12.71 & -2.7 & $*$ & -.1 \\
\hline 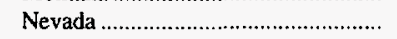 & 31.99 & 35.68 & 35.64 & 35.63 & 34.48 & 34.42 & -10.3 & -1.8 & -.8 \\
\hline New Mexico & 28.21 & 28.08 & 27.12 & 26.27 & 27.58 & 22.76 & .4 & .6 & 2.4 \\
\hline 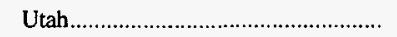 & 27.85 & 28.77 & 30.13 & 30.35 & 30.20 & 35.63 & -3.2 & -2.0 & -2.7 \\
\hline 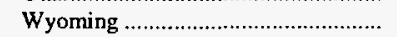 & 15.75 & 15.53 & 15.47 & 14.79 & 16.03 & 17.70 & 1.4 & -.4 & -1.3 \\
\hline 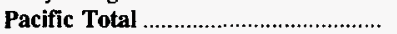 & 25.16 & 24.18 & 23.75 & 24.44 & 25.53 & 30.10 & 4.1 & -.3 & -2.0 \\
\hline 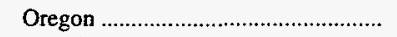 & 20.72 & 21.14 & 21.77 & 23.40 & 20.15 & - & -2.0 & .7 & - \\
\hline 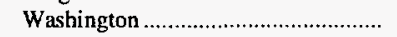 & 26.17 & 25.27 & 24.35 & 24.78 & 27.41 & 30.10 & 3.5 & -1.1 & -1.5 \\
\hline U.S. Total & 29.77 & 30.89 & 31.51 & 32.36 & 33.10 & 36.70 & -3.6 & -2.6 & -2.3 \\
\hline
\end{tabular}

* Data round to zero.

Note: Average prices are based on the cost including insurance and freight.

Source: Federal Energy Regulatory Commission, FERC Form 423, "Monthly Report of Cost and Quality of Fuels for Electric Plants." 
Table B12. Average Price of Coal Delivered to Other Industrial Plants by Census Division and State, 1986, 1991-1995

(Nominal Dollars per Metric Ton)

\begin{tabular}{|c|c|c|c|c|c|c|c|c|c|}
\hline \multirow{2}{*}{ Census Division and State } & \multirow{2}{*}{1995} & \multirow{2}{*}{1994} & \multirow{2}{*}{1993} & \multirow{2}{*}{1992} & \multirow{2}{*}{1991} & \multirow{2}{*}{1986} & \multirow{2}{*}{$\begin{array}{c}\text { Percent } \\
\text { Change } \\
1994-1995\end{array}$} & \multicolumn{2}{|c|}{ Average Annual Percent Change } \\
\hline & & & & & & & & 1991-1995 & 1986-1995 \\
\hline New England Total & $\$ 62.72$ & $\$ 61.44$ & $\$ 63.75$ & $\$ 72.43$ & $\$ 73.57$ & $\$ 70.51$ & 2.1 & -3.9 & -1.3 \\
\hline Connecticut & - & - & - & - & - & w & $\mathbf{w}$ & w & $w$ \\
\hline Maine & $w$ & $w$ & w & w & $\mathbf{w}$ & $\mathbf{w}$ & $w$ & $\mathbf{w}$ & w \\
\hline 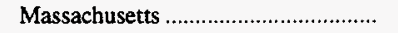 & $\mathbf{w}$ & $\mathbf{w}$ & w & w & $\mathbf{w}$ & $w$ & $w$ & $w$ & $\mathbf{w}$ \\
\hline 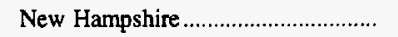 & - & - & - & - & - & $w$ & $\mathbf{w}$ & w & $w$ \\
\hline Middle Atlantic Total. ........................... & $\mathbf{w}$ & $\mathbf{w}$ & $\mathbf{w}$ & $\mathbf{w}$ & $\mathbf{w}$ & $\mathbf{w}$ & $\mathbf{w}$ & $\mathbf{w}$ & $\mathbf{w}$ \\
\hline New Jersey........................................... & w & $w$ & w & w & w & $w$ & w & $w$ & w \\
\hline 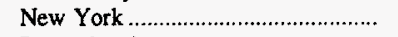 & 46.19 & 46.52 & 46.47 & 48.44 & 48.27 & 48.23 & -.7 & -1.1 & -.5 \\
\hline 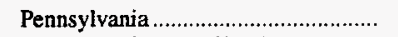 & 37.56 & 37.10 & 37.53 & 39.35 & 39.57 & 42.08 & 1.2 & -1.3 & -1.3 \\
\hline East North Central Total .................. & 38.45 & 38.27 & 38.07 & 38.64 & 39.66 & 43.09 & .5 & -.8 & -1.3 \\
\hline 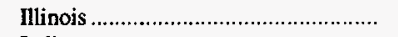 & 32.00 & 32.11 & 32.43 & 32.23 & 33.96 & 40.69 & -.3 & -1.5 & -2.6 \\
\hline Indiana & 36.53 & 34.56 & 34.07 & 34.81 & 36.38 & 37.80 & 5.7 & .1 & -.4 \\
\hline Michigan & 45.39 & 45.42 & 45.70 & 46.49 & 48.16 & 52.07 & -.1 & -1.5 & -1.5 \\
\hline Ohio & 38.78 & 39.40 & 38.38 & 38.84 & 38.41 & 39.12 & $-1,6$ & .2 & -.1 \\
\hline Wisconsin & 44.32 & 45.45 & 45.02 & 46.60 & 47.74 & 52.61 & -2.5 & -1.8 & -1.9 \\
\hline West North Central Total .................. & 20.86 & 20.52 & 19.84 & 19.57 & 20.21 & 21.30 & 1.7 & .8 & -.2 \\
\hline Iowa & 32.23 & 31.44 & 30.87 & 29.94 & 32.13 & 39.18 & 2.5 & .1 & -2.1 \\
\hline 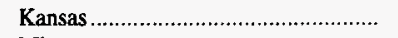 & 35.74 & 35.55 & 36.44 & 35.23 & 33.96 & 41.08 & .5 & 1.3 & -1.5 \\
\hline 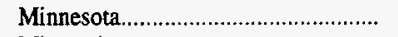 & 37.92 & 39.31 & 39.47 & 39.27 & 39.97 & 44.75 & -3.5 & -1.3 & -1.8 \\
\hline 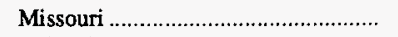 & 36.17 & 36.24 & 35.40 & 34.70 & 34,48 & 34.46 & -.2 & 1.2 & .5 \\
\hline 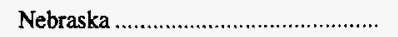 & w & w & w & w & w & 33.92 & w & w & $w$ \\
\hline 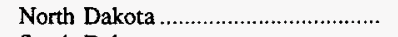 & w & $w$ & $w$ & $\mathbf{w}$ & w & w & $\mathbf{w}$ & w & $w$ \\
\hline 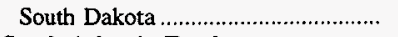 & $w$ & $w$ & w & $\mathbf{w}$ & w & w & w & $w$ & $\mathbf{w}$ \\
\hline South Atlantic Total & $\mathbf{w}$ & $\mathbf{w}$ & $\mathbf{w}$ & $\mathbf{w}$ & $\mathbf{w}$ & $\mathbf{w}$ & $\mathbf{w}$ & $\mathbf{w}$ & $\mathbf{w}$ \\
\hline 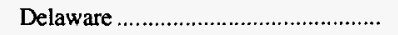 & w & w & w & w & w & w & w & $w$ & w \\
\hline Florida & 51.41 & 51.37 & 53.22 & 53.20 & 52.27 & 54.36 & .1 & -.4 & -.6 \\
\hline 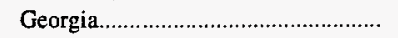 & 49.21 & 50.39 & 49.82 & 49.80 & 49.55 & 48.29 & -2.3 & -.2 & .2 \\
\hline 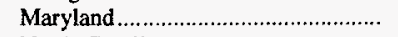 & 34.90 & 35.47 & 35.47 & 36.18 & 37.16 & 36.29 & -1.6 & -1.5 & -.4 \\
\hline 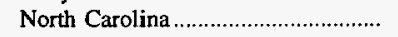 & 47.72 & 48.08 & 47.89 & 47.91 & 47.45 & 50.40 & -.7 & .1 & -.6 \\
\hline 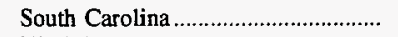 & 47.58 & 48.32 & 47.78 & 47.74 & 47.61 & 48.60 & -1.5 & * & -.2 \\
\hline 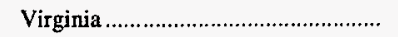 & 46.85 & 45.82 & 45.49 & 45.16 & 44.67 & 44.41 & 2.2 & 1.2 & .6 \\
\hline West Virginia & 37.05 & 36.07 & 36.28 & 35.20 & 35.37 & 36.78 & 2.7 & 1.2 & .1 \\
\hline East South Central Total....................... & $\mathbf{w}$ & $\mathbf{w}$ & $\mathbf{w}$ & $\mathbf{w}$ & $\mathbf{w}$ & $\mathbf{w}$ & $\mathbf{w}$ & $\mathbf{w}$ & $\mathbf{w}$ \\
\hline Alabama & 43.58 & 42.70 & 43.00 & 43.83 & 44.57 & 47.73 & 2.0 & -.6 & -1.0 \\
\hline 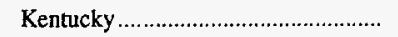 & 48.60 & 47.64 & 46.63 & 48.26 & 51.30 & 48.49 & 2.0 & -1.3 & * \\
\hline 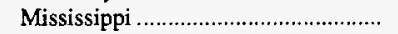 & $\mathbf{w}$ & $\mathbf{w}$ & $\mathbf{w}$ & $\mathbf{w}$ & $\mathbf{w}$ & $w$ & $w$ & $\mathbf{w}$ & $w$ \\
\hline 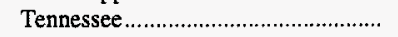 & 39.33 & 38.96 & 39.04 & 39.16 & 39.31 & 42.02 & 1.0 & $*$ & -.7 \\
\hline West South Central Total ..................... & 24.29 & 25.29 & 23.57 & 25.13 & 25.70 & $\mathbf{w}$ & -4.0 & -1.4 & $\mathbf{w}$ \\
\hline 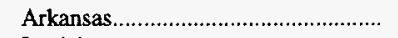 & 47.97 & 48.81 & 48.57 & 49.18 & 49.71 & 50.51 & -1.7 & -.9 & -.6 \\
\hline 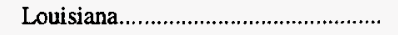 & w & w & w & w & w & w & $w$ & w & $\mathbf{w}$ \\
\hline 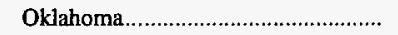 & w & w & $\mathbf{w}$ & w & w & 43.83 & $\mathbf{w}$ & $w$ & $w$ \\
\hline 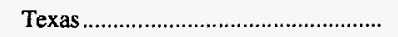 & 20.68 & 21.54 & 19.38 & 19.99 & 20.51 & 26.41 & -4.0 & .2 & -2.7 \\
\hline Mountain Total & 29.83 & 31.73 & 31.43 & 31.59 & 32.63 & 34.10 & -6.0 & -2.2 & -1.5 \\
\hline 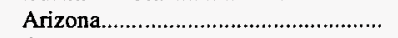 & 44.60 & 45.58 & 44.65 & 45.14 & 44.20 & w & -2.1 & .2 & w \\
\hline Colorado & 28.78 & 31.92 & 31.56 & 33.44 & 32.27 & 29.30 & -9.8 & -2.8 & -.2 \\
\hline Idaho & 37.60 & 36.76 & 36.13 & 36.70 & 37.38 & 40.26 & 2.3 & .1 & -.8 \\
\hline Montana & w & w & w & $\mathbf{w}$ & $w$ & w & w & w & w \\
\hline Nevada & w & w & w & w & $w$ & w & $w$ & $w$ & w \\
\hline 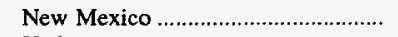 & w & w & $w$ & w & w & $w$ & $w$ & $w$ & w \\
\hline Utah & 21.76 & 29.29 & 29.23 & 27.94 & 29.13 & 30.41 & -25.7 & -7.0 & -3.6 \\
\hline Wyoming & 25.05 & 25.21 & 25.83 & 26.09 & 27.76 & w & -.6 & -2.5 & w \\
\hline 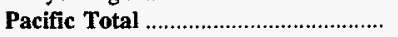 & 48.15 & 49.51 & 48.31 & 47.80 & 50.43 & 54.03 & -2.8 & -1.1 & -1.3 \\
\hline 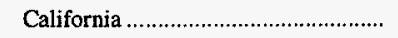 & 45.32 & 47.83 & 47.25 & 46.35 & 49.37 & 56.79 & -5.3 & -2.1 & -2.5 \\
\hline 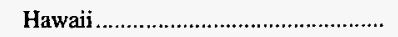 & $w$ & w & w & w & w & 64.32 & $\mathbf{w}$ & $w$ & w \\
\hline 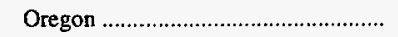 & w & $\mathbf{w}$ & w & w & w & $\mathbf{w}$ & $w$ & $w$ & $\mathbf{w}$ \\
\hline Washington & 65.20 & 64.89 & 58.54 & 62.00 & 65.22 & 45.73 & .5 & $*$ & 4.0 \\
\hline U.S. Total & 35.74 & 35.88 & 35.53 & 36.14 & 36.97 & 39.51 & -.4 & -.8 & -1.1 \\
\hline
\end{tabular}

* Data round to zero.

w Withheld to avoid disclosure of individual company data.

Notes: Price data are for manufacturing plants only. Average prices are based on the cost including insurance, freight, and taxes.

Source: Energy Information Administration. Form ELA-3, "Quarterly Coal Consumption - Manufacturing Plants." 
Table B13. Average Price of Coal Delivered to Coke Plants by Census Division and State, 1986, 1991-1995

(Nominal Dollars per Metric Ton)

\begin{tabular}{|c|c|c|c|c|c|c|c|c|c|}
\hline \multirow{2}{*}{ Census Division and State } & \multirow{2}{*}{1995} & \multirow{2}{*}{1994} & \multirow{2}{*}{1993} & \multirow{2}{*}{1992} & \multirow{2}{*}{1991} & \multirow{2}{*}{1986} & \multirow{2}{*}{$\begin{array}{c}\text { Percent } \\
\text { Change } \\
\text { 1994-1995 }\end{array}$} & \multicolumn{2}{|c|}{ Average Annual Percent Change } \\
\hline & & & & & & & & 1991-1995 & $1986-1995$ \\
\hline Middle Atlantic Total.......................... & $\mathbf{w}$ & $\mathbf{w}$ & $\mathbf{w}$ & $\mathbf{w}$ & $w$ & $\mathbf{w}$ & $\mathbf{w}$ & $\mathbf{w}$ & $\mathbf{w}$ \\
\hline New York & $w$ & $w$ & w & w & w & w & w & w & w \\
\hline 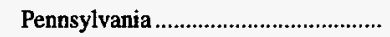 & $\$ 50.83$ & $\$ 50.98$ & $\$ 51.16$ & $\$ 51.25$ & $\$ 51.65$ & $\$ 52.06$ & -0.3 & -0.4 & -0.3 \\
\hline East North Central Total ................... & 54.12 & 52.06 & 54.58 & 55.53 & 55.89 & $\mathbf{5 9 . 4 6}$ & 3.9 & -.8 & -1.0 \\
\hline 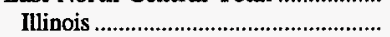 & w & $\mathbf{w}$ & w & w & w & 54.14 & $w$ & w & $\mathbf{w}$ \\
\hline 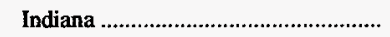 & 58.13 & 56.11 & 57.64 & 59.21 & 58.77 & 60.62 & 3.6 & -.3 & -.5 \\
\hline 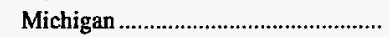 & w & w & - & - & w & w & $w$ & $w$ & $w$ \\
\hline Ohio & 46.50 & 46.32 & 49.68 & 51.45 & 50.87 & 60.17 & .4 & -2.2 & -2.8 \\
\hline West North Central Total ................ & - & - & - & - & - & w & - & $\mathbf{w}$ & $\mathbf{w}$ \\
\hline 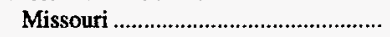 & - & - & - & - & - & $w$ & - & w & w \\
\hline South Atlantic Total............................. & $\mathbf{w}$ & $\mathbf{w}$ & $\mathbf{w}$ & $\mathbf{w}$ & $\mathbf{w}$ & $\mathbf{w}$ & $\mathbf{w}$ & $\mathbf{w}$ & $\mathbf{w}$ \\
\hline Maryland & - & - & - & - & $w$ & $w$ & $w$ & w & $w$ \\
\hline 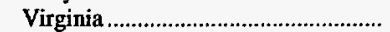 & w & $\mathbf{w}$ & w & $\mathbf{w}$ & $\mathbf{w}$ & $w$ & $\mathbf{w}$ & $w$ & $w$ \\
\hline 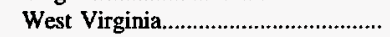 & $\mathrm{w}$ & w & $\mathrm{w}$ & $\mathbf{w}$ & $\mathbf{w}$ & $w$ & w & $w$ & $w$ \\
\hline 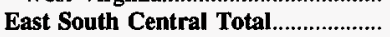 & $\mathbf{w}$ & $\mathbf{w}$ & $\mathbf{w}$ & $w$ & $\mathbf{w}$ & $\mathbf{w}$ & $\mathbf{w}$ & $\mathbf{w}$ & $\mathbf{w}$ \\
\hline Alabama & 53.38 & 52.31 & 52.36 & 52.70 & 53.29 & 53.37 & 2.0 & * & $*$ \\
\hline 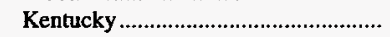 & w & w & w & $\mathbf{w}$ & $\mathbf{w}$ & w & $w$ & $w$ & $w$ \\
\hline 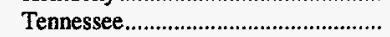 & - & - & - & - & $w$ & $w$ & - & $w$ & $\mathbf{w}$ \\
\hline West South Central Total .................. & - & - & - & - & - & $\mathbf{w}$ & - & $\mathbf{w}$ & $\mathbf{w}$ \\
\hline 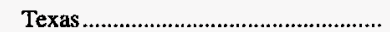 & - & - & - & - & - & $w$ & - & $w$ & $w$ \\
\hline 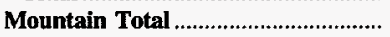 & $\mathbf{w}$ & $\mathbf{w}$ & $\mathbf{w}$ & $\mathbf{w}$ & $\mathbf{w}$ & $\mathbf{w}$ & $\mathbf{w}$ & $\mathbf{w}$ & $\mathbf{w}$ \\
\hline 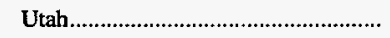 & $w$ & $\mathbf{w}$ & $\mathbf{w}$ & $\mathbf{w}$ & $\mathbf{w}$ & $w$ & $w$ & w & w \\
\hline U.S. Total & 52.18 & 51.32 & $\mathbf{5 2 . 3 0}$ & 52.82 & 53.88 & 56.05 & 1.7 & -.8 & -.8 \\
\hline
\end{tabular}

* Data round to zero.

withheld to avoid disclosure of individual company data.

Note: Average prices are based on the cost including insurance, freight, and taxes.

Source: Energy Information Administration, Form EIA-5, "Coke Plant Report - Quarterly." 
Table B14. Average Price of U.S. Coal Imports by Continent and Country of Origin, 1986, 1991-1995

(Nominal Dollars per Metric Ton)

\begin{tabular}{|c|c|c|c|c|c|c|c|c|c|}
\hline \multirow{2}{*}{$\begin{array}{l}\text { Continent and Country } \\
\text { of Origin }\end{array}$} & \multirow{2}{*}{1995} & \multirow{2}{*}{1994} & \multirow{2}{*}{1993} & \multirow{2}{*}{1992} & \multirow{2}{*}{1991} & \multirow{2}{*}{1986} & \multirow{2}{*}{$\begin{array}{c}\text { Percent } \\
\text { Change } \\
\text { 1994-1995 }\end{array}$} & \multicolumn{2}{|c|}{ Average Annual Percent Change } \\
\hline & & & & & & & & 1991-1995 & $1986-1995$ \\
\hline North America Total............................. & $\$ 35.92$ & $\$ 33.74$ & $\$ 31.97$ & $\$ 30.73$ & $\$ 27.67$ & $\mathbf{\$ 3 2 . 9 0}$ & 6.5 & 6.7 & 1.0 \\
\hline 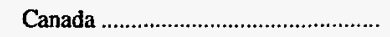 & 35.92 & 33.74 & 31.98 & 30.73 & 27.67 & 32.90 & 6.5 & 6.7 & 1.0 \\
\hline Mexico & - & - & 23.35 & - & - & - & - & - & - \\
\hline South America Total ............................ & 36.11 & 31.97 & 30.46 & 36.42 & 38.19 & 38.80 & 13.0 & -1.4 & -.8 \\
\hline 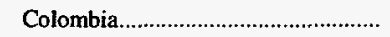 & 34.33 & 30.27 & 30.04 & 35.55 & 36.24 & 38.80 & 13.4 & -1.3 & -1.3 \\
\hline 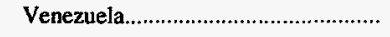 & 38.74 & 35.73 & 31.82 & 39.25 & 45.06 & - & 8.4 & -3.7 & - \\
\hline 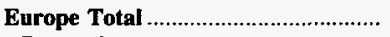 & 28.27 & - & 35.65 & 37.49 & - & - & - & - & - \\
\hline 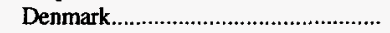 & - & - & 35.65 & - & - & - & - & - & - \\
\hline United Kingdom ............................... & 28.27 & - & - & 37.49 & - & - & - & - & - \\
\hline 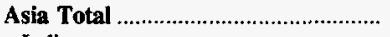 & 38.73 & $\mathbf{3 7 . 5 8}$ & 47.07 & 42.71 & - & - & 3.1 & - & - \\
\hline India & - & - & - & 26.07 & - & - & - & - & - \\
\hline 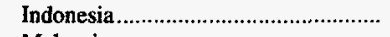 & 38.73 & 37.26 & 47.07 & 45.13 & - & - & 3.9 & - & - \\
\hline 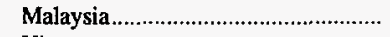 & - & - & - & 51.87 & - & - & - & - & - \\
\hline 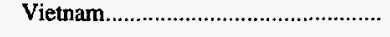 & - & 53.00 & - & - & - & - & -100.0 & - & - \\
\hline Oceania \& Australia Total .................. & 37.00 & 34.35 & 34.79 & 39.76 & 41.86 & 51.41 & 7.7 & $-\mathbf{3 . 0}$ & -3.6 \\
\hline 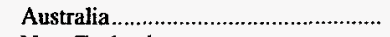 & 34.16 & 33.09 & 34.79 & 39.76 & 41.86 & 51.41 & 3.2 & -4.9 & -4.4 \\
\hline 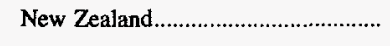 & 51.17 & 48.67 & - & - & - & - & 5.1 & - & - \\
\hline Africa Total & - & 27.92 & 30.66 & $\mathbf{5 4 . 3 3}$ & - & 41.24 & -100.0 & - & -100.0 \\
\hline South Africa, Rep of & - & 27.92 & - & 54.33 & - & 41.24 & -100.0 & - & -100.0 \\
\hline 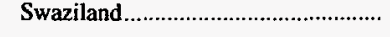 & - & - & 30.66 & - & - & - & - & - & - \\
\hline 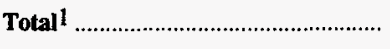 & 36.50 & 33.05 & 32.37 & 35.80 & 35.65 & 39.25 & 10.4 & .6 & -.8 \\
\hline U.S. Total ${ }^{2}$ & 37.62 & 33.30 & 32.94 & 36.88 & 36.51 & 39.70 & 13.0 & .7 & -.6 \\
\hline
\end{tabular}

1 The average prices presented in this table, with the exception of U.S. Total, are considered to be representative prices for coal imports and fall within the range of $\$ 20$ to $\$ 50$ per short ton $(\$ 18.14$ to $\$ 45.36$ per metric ton), inclusively.

2 U.S. Total is the average price of all coal imports.

Notes: Average price is based on the customs import value. Coal imports include coal to Puerto Rico and the Virgin Islands.

Source: U.S. Department of Commerce, Bureau of the Census, "Monthly Report IM 145." 
Table B15. Average Price of U.S. Coal Exports by Destination, 1986, 1991-1995 (Nominal Dollars per Metric Ton)

\begin{tabular}{|c|c|c|c|c|c|c|c|c|c|}
\hline \multirow{2}{*}{$\begin{array}{c}\text { Continent and Country } \\
\text { of Destination }\end{array}$} & \multirow{2}{*}{1995} & \multirow{2}{*}{1994} & \multirow{2}{*}{1993} & \multirow{2}{*}{1992} & \multirow{2}{*}{1991} & \multirow{2}{*}{1986} & \multirow{2}{*}{$\begin{array}{c}\text { Percent } \\
\text { Change } \\
\text { 1994-1995 }\end{array}$} & \multicolumn{2}{|c|}{ Average Annual Percent Change } \\
\hline & & & & & & & & 1991-1995 & 1986-1995 \\
\hline 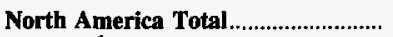 & $\mathbf{\$ 3 7 . 5 8}$ & $\$ 36.22$ & $\$ 37.65$ & $\$ 36.44$ & $\$ 37.05$ & $\$ 53.65$ & 3.7 & 0.3 & -3.9 \\
\hline 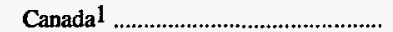 & 37.05 & 35.83 & 37.33 & 36.37 & 36.91 & 53.84 & 3.4 & .1 & -4.1 \\
\hline Mexico & 43.34 & 48.80 & 48.54 & 47.93 & 48.11 & 47.17 & -11.2 & -2.6 & -.9 \\
\hline Other ${ }^{2}$ & 37.47 & 38.99 & 38.69 & 40.11 & 39.36 & 41.17 & -3.9 & -1.2 & -1.0 \\
\hline South America Total ........................... & 47.87 & 46.61 & 48.25 & 50.20 & 50.97 & 52.64 & 2.7 & -1.5 & -1.0 \\
\hline Argentina & 47.09 & 46.85 & 47.63 & 50.09 & 50.26 & 52.20 & .5 & -1.6 & -1.1 \\
\hline 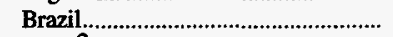 & 48.37 & 46.59 & 48.32 & 50.23 & 51.20 & 52.70 & 3.8 & -1.4 & -.9 \\
\hline Other 2 & 36.98 & 44.81 & 43.51 & 47.36 & 42.77 & 52.63 & -17.5 & -3.6 & -3.8 \\
\hline 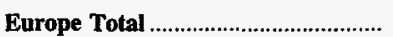 & 46.57 & 46.65 & 47.82 & 47.03 & 47.49 & 48.18 & -.2 & -.5 & -.4 \\
\hline Belgium \& Luxembourg ........................ & 47.91 & 46.55 & 47.55 & 48.51 & 49.40 & 49.48 & 2.9 & -.8 & -.3 \\
\hline 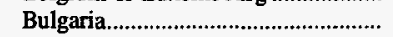 & 48.54 & 46.40 & 46.25 & 47.72 & 46.92 & 54.00 & 4.6 & .8 & -1.2 \\
\hline 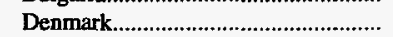 & 35.12 & 32.22 & 38.53 & 36.03 & 36.32 & 39.71 & 9.0 & -8 & -1.3 \\
\hline 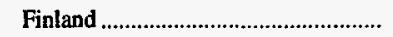 & 43.51 & 45.34 & 43.66 & 44.99 & 49.35 & 34.70 & -4.0 & -3.1 & 2.5 \\
\hline France. & 48.19 & 48.76 & 46.56 & 43.40 & 44.29 & 47.30 & -1.2 & 2.1 & .2 \\
\hline 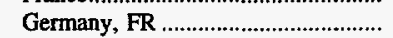 & 38.57 & 49.97 & 42.97 & 42.19 & 43.36 & 48.46 & -22.8 & -2.9 & -2.5 \\
\hline Ireland & 39.76 & 37.28 & 39.51 & 40.57 & 43.57 & 50.56 & 6.6 & -2.3 & -2.6 \\
\hline Italy & 48.66 & 47.40 & 48.86 & 49.97 & 50.02 & 47.52 & 2.7 & -.7 & .3 \\
\hline 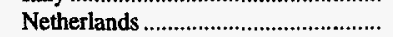 & 46.44 & 46.28 & 48.75 & 47.78 & 47.60 & 50.00 & .3 & -.6 & -.8 \\
\hline 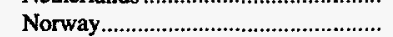 & - & 52.22 & - & 52.86 & 35.40 & 50.38 & -100.0 & -100.0 & -100.0 \\
\hline Portugal & 40.18 & 39.96 & 41.56 & 44.91 & 45.05 & 46.18 & .6 & -2.8 & -1.5 \\
\hline 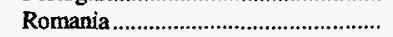 & 46.66 & 38.69 & 39.99 & 45.88 & 50.58 & 46.58 & 20.6 & -2.0 & * \\
\hline Spain & 52.24 & 51.09 & 51.84 & 51.61 & 49.85 & 49.51 & 2.2 & 1.2 & .6 \\
\hline Sweden & 52.44 & 50.22 & 50.66 & 51.44 & 52.60 & 50.58 & 4.4 & -1 & .4 \\
\hline Turkey & 47.16 & 45.51 & 46.94 & 50.20 & 51.63 & 50.05 & 3.6 & -2.2 & -.6 \\
\hline 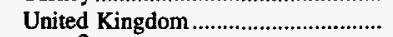 & 47.69 & 49.84 & 50.60 & 50.20 & 51.09 & 52.22 & -4.3 & -1.7 & -1.0 \\
\hline Other ${ }^{2}$ & 41.96 & 42.04 & 43.65 & 49.32 & 51.15 & 45.47 & -.2 & -4.8 & -.9 \\
\hline 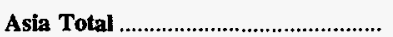 & 43.20 & 42.62 & 44.74 & 47.02 & 47.67 & 48.77 & 1.4 & -2.4 & -1.3 \\
\hline China (Taiwan) & 40.73 & 42.60 & 43.53 & 45.45 & 46.00 & 45.91 & -4.4 & -3.0 & -1.3 \\
\hline 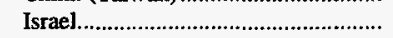 & 39.45 & 36.63 & 38.35 & 43.11 & 43.95 & 42.53 & 7.7 & -2.7 & -.8 \\
\hline Japan & 43.14 & 42.53 & 45.06 & 47.30 & 47.83 & 52.28 & 1.4 & -2.5 & -2.1 \\
\hline 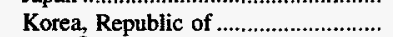 & 45.71 & 44.35 & 46.43 & 49.53 & 50.65 & 45.58 & 3.1 & -2.5 & $*$ \\
\hline Other 2 & 37.92 & 41.05 & 48.75 & 40.85 & 42.56 & 43.08 & -7.6 & -2.8 & -1.4 \\
\hline Oceania \& Australia Total .................. & 43.82 & 44.04 & 37.99 & 38.08 & - & 43.98 & -.5 & - & * \\
\hline Other $^{2}$ & 43.82 & 44.04 & 37.99 & 38.08 & - & 43.98 & -.5 & - & * \\
\hline Africa Total & $\mathbf{4 5 . 4 0}$ & 48.05 & 46.90 & 47.37 & 45.49 & 48.93 & -5.5 & * & -.8 \\
\hline Algeria & 52.69 & 47.66 & 48.85 & 51.10 & 51.26 & 52.53 & 10.5 & .7 & * \\
\hline Egypt. & 50.88 & 47.55 & 49.45 & 50.80 & 50.36 & 48.05 & 7.0 & .3 & .6 \\
\hline Morocco & 36.37 & 38.62 & 37.32 & 37.26 & 37.18 & 46.23 & -5.8 & -.5 & -2.6 \\
\hline 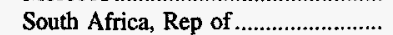 & 52.23 & 50.34 & 51.67 & 52.68 & 53.92 & - & 3.8 & -.8 & - \\
\hline Other ${ }^{2}$ & - & - & 44.68 & - & 44.99 & 47.57 & - & -100.0 & -100.0 \\
\hline Total 3 & 44.79 & 44.36 & 45.77 & 45.68 & 46.69 & 49.52 & 1.0 & -1.0 & -1.1 \\
\hline U.S. Total ${ }^{4}$ & 44.39 & 44.02 & 45.65 & 45.57 & 46.73 & 50.65 & .8 & -1.3 & -1.4 \\
\hline
\end{tabular}

1 Based on the U.S. - Canada Free Trade Agreement, as of January 1990, the U.S. Department of Commerce began reporting statistics on U.S. exports to Canada based on information on imports provided monthly by the Canadian government.

2 Includes countries with exports less than or equal to 50,000 short tons in 1994.

3 The average prices presented in this table, with the exception of U.S. Total, are considered to be representative prices for coal exports and fall within the range of $\$ 28$ to $\$ 50$ per short ton ( $\$ 25.40$ to $\$ 45.36$ per metric ton), inclusively.

4 U.S. Total is the average price of all coal exports.

* Data round to zero.

Note: Average price is based on the free alongside ship (f.a.s.) value.

Source: U.S. Department of Commerce, Bureau of the Census, "Monthly Report EM 545." 



\section{Appendix C}

\section{References}

Figure C1. Coal-Bearing Areas of the United States

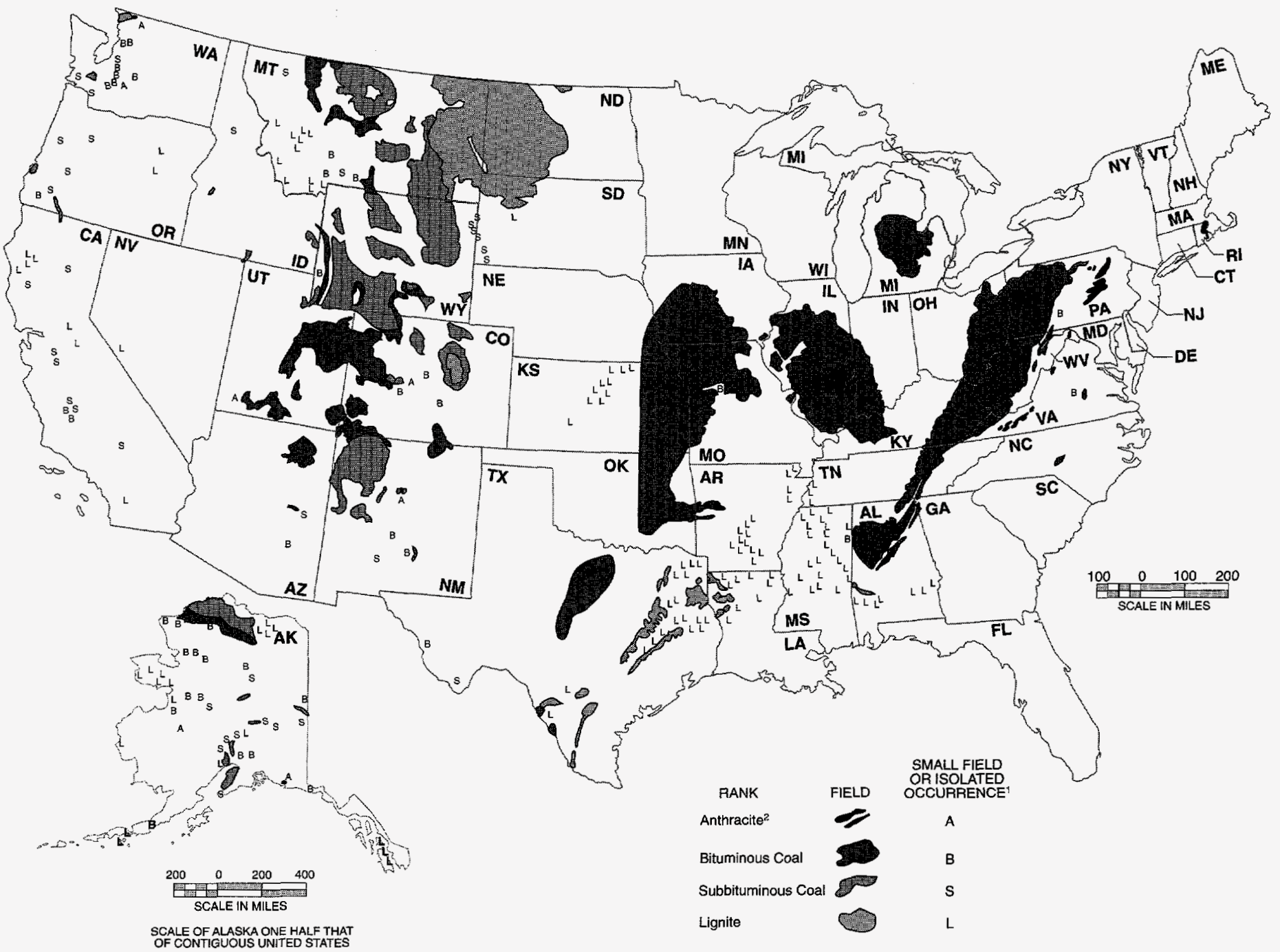




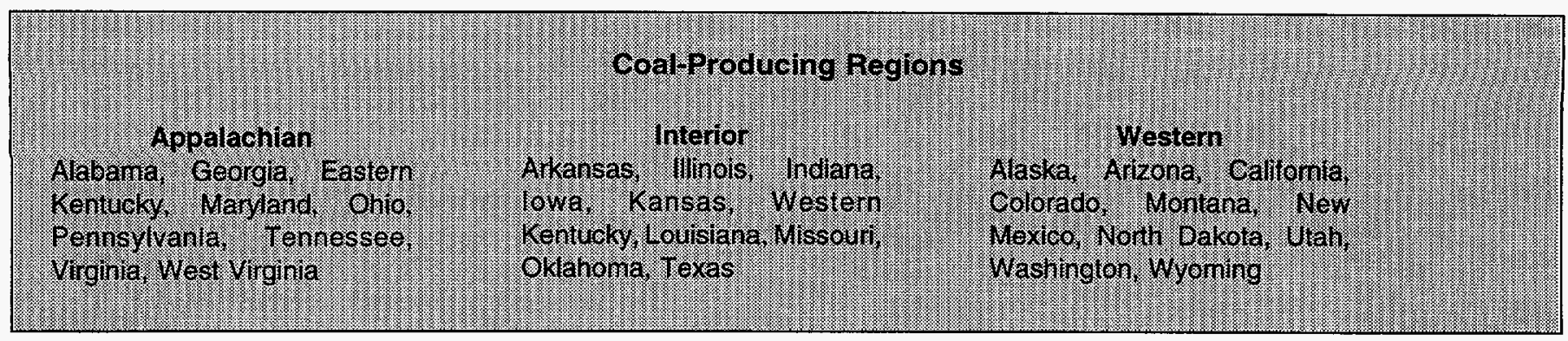

\section{Table C1. Classification of Coals by Rank}

\section{Principles}

The lower rank of coals can be classified based on heat content. The heat content of the higher rank coals is generally above 14,000 Btu per pound for each coal rank group (except for meta-anthracite, which trends slightly lower), and heat content ranges vary within a relatively narrow range. Since heat content is not a dependable criterion for these higher rank coals, their rank categories are instead described by degree of metamorphism, or "coalification," a property that is measured by fixed carbon content. Finally, the agglomerating character of bituminous coals is a critical attribute for certain coal consumers, and thus agglomerating character has come to define the distinctions between certain adjacent coal groups. Some high-volatile $\mathrm{C}$ bituminous and subbituminous $\mathrm{A}$ coals can be distinguished only on the basis of agglomerating character.

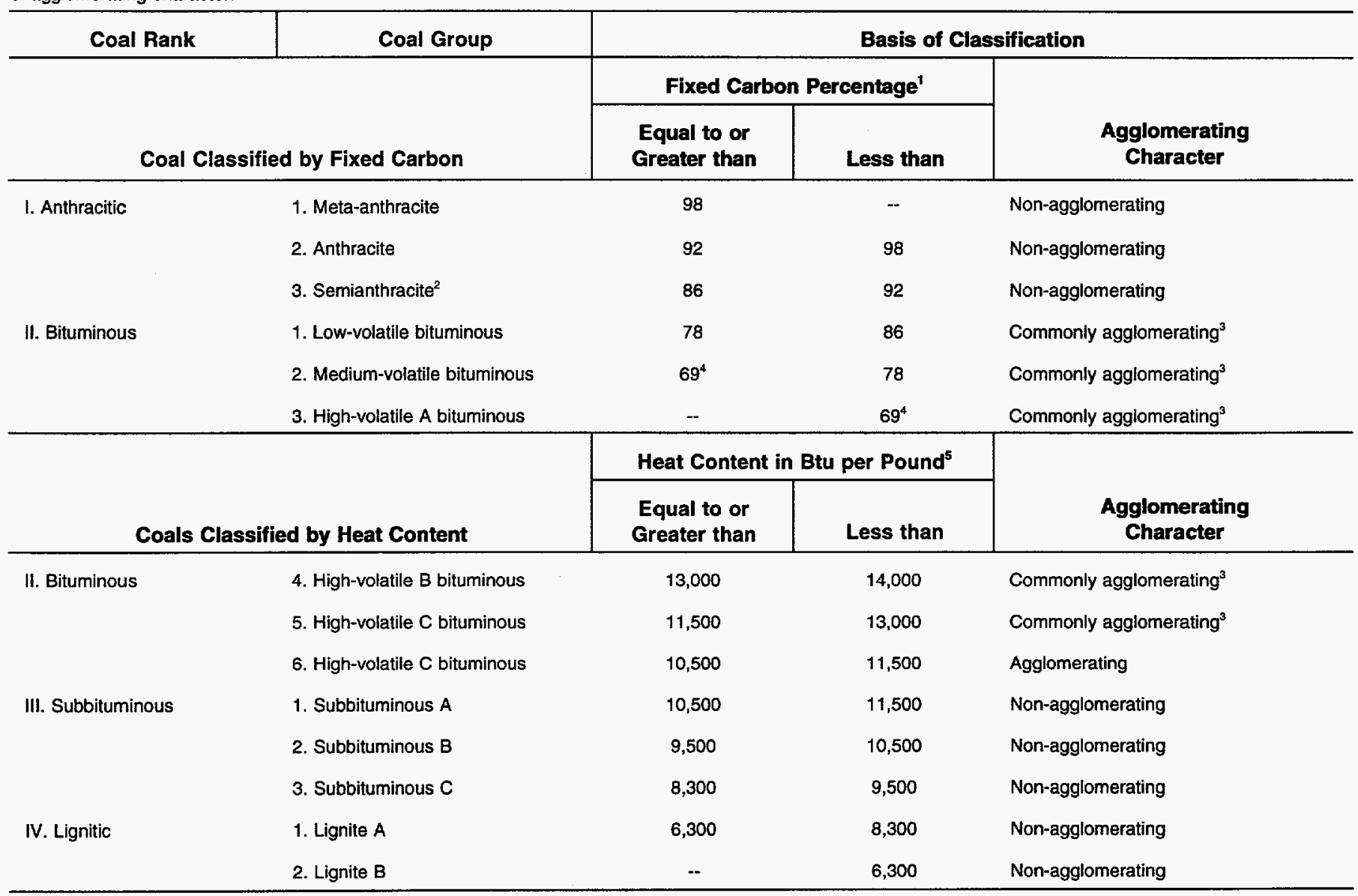

'Percentages are based on dry mineral-matter-free coal. Volatile matter (not shown) is the complement of fixed carbon; that is, the percentages of fixed carbon and volatile matter sum to 100 percent. As fixed carbon percentage decreases, therefore, volatile matter percentage increases by the same amount.

2If agglomerating, classify in low-volatile group of the bituminous class.

${ }^{3}$ There may be nonagglomerating varieties in the bituminous class, most notably in the high-volatile $\mathrm{C}$ bituminous group.

${ }^{4}$ Coals having 69 percent or more fixed carbon are classified according to fixed carbon, regardless of Btu value. Coals with less than 69 percent fixed carbon, but with 14,000 or more Btu per pound, are classified as high-volatile A bituminous.

${ }^{5}$ Calorific values in Btu per pound, on a moist mineral-matter-free basis.

Note: Terms in this table are defined in the Glossary.

Source: Adapted from American Society for Testing and Materials 1988, Standard Classification of Coal by Rank, ASTM Designation D 388-91a. 


\section{Coal Reports and Feature Articles}

\section{Coal Reports}

- Weekly Coal Production, DOE/EIA-0218 (96-33).

- Coal Data: A Reference, DOE/EIA-0064 (93), February 1995.

- State Coal Profiles, DOE/EIA-0576, January 1994.

- Quarterly Coal Report, DOE/EIA-0121(96/1Q).

- The Changing Structure of the U.S. Coal Industry: An Update, DOE/EIA-0513(93), July 1993.

- U.S. Coal Reserves: A Review and Update DOE/EIA-0529(95), August 1996.

- The U.S. Coal Industry, 1970-1990: Two Decades of Change, DOA/EIA-0559, November 1992.

- Trends in Contract Coal Transportation, 1979-1987, DOE/EIA-0549, September 1991.

- Electric Power Monthly, DOE/EIA-0226(96/08), August 1996.

- Electric Power Annual, DOE/EIA-0348(95), Vol. 1, July 1996.

- Longwall Mining, DOE/EIA-TR-0588 March 1995.

- Monthly Energy Review, DOE/EIA-0035(96/07) July 1996.

- Electric Utility Phase I Acid Rain Compliance Strategies for the Clean Air Act Amendments of 1990, DOE/EIA-0582, March 1994.
- Cost and Quality of Fuels for Electric Utility Plants 1994, DOE/EIA-0191(94), July 1995.

\section{Feature Articles}

- "Carbon Dioxide Emission Factors for Coal," Quarterly Coal Report, DOE/EIA-0121 (94/1Q), August 1994.

- "Federal and Indian Coal Lands: A Growing Source of Energy and Revenue," Coal Production 1992, DOE/EIA-0118(92), October 1993.

- "Coal Supply and Demand in 1993: A Review, 1993," Mining Engineering, May 1994, pp.433-436.

- "Wyoming Coal: An Overview," Coal Production 1991, DOE/EIA-0118(91), October 1992.

- "Profile of New Coal Mines in the 1980's," Coal Production 1990, DOE/EIA-0118), September 1991.

- "The Comparability of Resource and Reserve Data for Crude Oil, Natural Gas, Coal, and Uranium," Quarterly Coal Report OctoberDecember 1994, DOE/EIA-0121 (94/4Q) May 1995.

- "Annual Review 1995: Coal Overview," Mining Engineering, Vol. 48, No. 5, May 1996, pp. 41-46.

- "Coal Geology, Reserves and Production in Northern and Central Appalachia," Mining Engineering, Special Edition, December 1995. 
Table C2. Approximate Heat Content of Coal

(Million Btu per Short Ton)

\begin{tabular}{c|c|c|c|c|c|c|c}
\hline $\begin{array}{c}\text { Coal Rank } \\
\text { Sector }\end{array}$ & 1982 & 1983 & 1984 & 1985 & 1986 & 1987 & 1988 \\
\hline
\end{tabular}

Anthracite

\begin{tabular}{|c|c|c|c|c|c|c|c|}
\hline 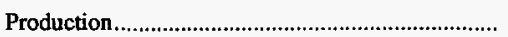 & 23.289 & 22.734 & 23.107 & 22.428 & 23.084 & 23.108 & 23.266 \\
\hline Consumption & 22.518 & 21.583 & 22.322 & 20.817 & 21.512 & 22.435 & 22.423 \\
\hline 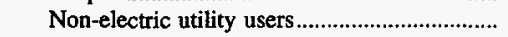 & 24.578 & 24.536 & 25.128 & 23.031 & 24.399 & 26.293 & 26.021 \\
\hline Electric utilities & 18.160 & 16.516 & 17.018 & 16.784 & 15.578 & 15.962 & 17.312 \\
\hline 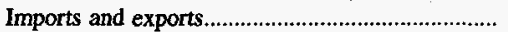 & 25.400 & 25.400 & 25.400 & 25.400 & 25.400 & 25.400 & 25.400 \\
\hline
\end{tabular}

Bituminous Coal and Lignite

\begin{tabular}{|c|c|c|c|c|c|c|c|}
\hline Production & 22.233 & 22.048 & 22.005 & 21.867 & 21.908 & 21.918 & 21.817 \\
\hline 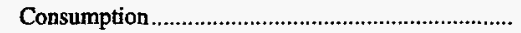 & 21.670 & 21.576 & 21.570 & 21.368 & 21.462 & 21.514 & 21.324 \\
\hline Residential and commercial ................................... & 22.226 & 22.438 & 22.406 & 22.568 & 22.669 & 22.800 & 23.135 \\
\hline $\begin{array}{l}\text { Coke plants.................................................... } \\
\text { Other industrial and }\end{array}$ & 26.800 & 26.800 & 26.800 & 26.800 & 26.800 & 26.800 & 26.800 \\
\hline 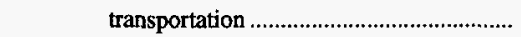 & 22.695 & 22.680 & 22.525 & 22.013 & 22.185 & 22.360 & 22.341 \\
\hline 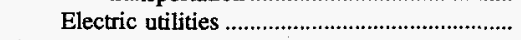 & 21.200 & 21.141 & 21.108 & 20.965 & 21.091 & 21.143 & 20.905 \\
\hline Imports & 25.000 & 25.000 & 25.000 & 25.000 & 25.000 & 25.000 & 25.000 \\
\hline Exports & 26.231 & 26.300 & 26.410 & 26.320 & 26.308 & 26.304 & 26.308 \\
\hline Coal Coke & 24.800 & 24.800 & 24.800 & 24.800 & 24.800 & 24.800 & 24.800 \\
\hline & 1989 & 1990 & 1991 & 1992 & 1993 & 1994 & 1995 \\
\hline
\end{tabular}

Anthracite

\begin{tabular}{|c|c|c|c|c|c|c|c|}
\hline Production & 23.385 & 23.574 & 22.573 & 22.572 & 22.573 & 22.572 & 22.573 \\
\hline Consumption & 22.623 & 21.668 & 21.410 & 21.423 & 21.262 & 21.828 & 20.860 \\
\hline 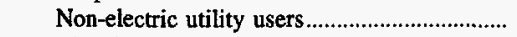 & 27.196 & 25.199 & 25.268 & 24.617 & 24.096 & 25.037 & 24.872 \\
\hline Electric utilities & 16.310 & 16.140 & 15.858 & 16.944 & 16.534 & 14.680 & 14.568 \\
\hline 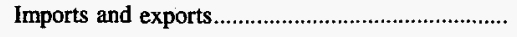 & 25.400 & 25.400 & 25.400 & 25.400 & 25.400 & 25.400 & 25.400 \\
\hline \multicolumn{8}{|l|}{ Bituminous Coal and Lignite } \\
\hline Production & 21.759 & 21.819 & 21.678 & 21.643 & 21.383 & 21.347 & 21.272 \\
\hline Consumption & 21.268 & 21.330 & 21.146 & 21.142 & 20.983 & 21.011 & 20.852 \\
\hline 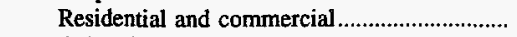 & 22.917 & 22.678 & 22.635 & 22.768 & 22.749 & 22.683 & 23.785 \\
\hline 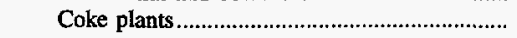 & 26.800 & 26.800 & 26.800 & 26.800 & 26.800 & 26.800 & 26.800 \\
\hline \multicolumn{8}{|l|}{ Other industrial and } \\
\hline transportation & 22.324 & 22.444 & 22.448 & 22.242 & 22.111 & 22.046 & 21.887 \\
\hline 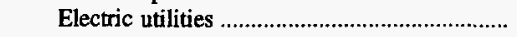 & 20.854 & 20.935 & 20.761 & 20.792 & 20.644 & 20.681 & 20.509 \\
\hline 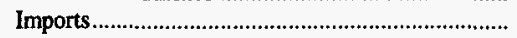 & 25.000 & 25.000 & 25.000 & 25.000 & 25.000 & 25.000 & 25.000 \\
\hline Exports & 26.166 & 26.207 & 26.192 & 26.165 & 26.341 & 26.335 & 26.212 \\
\hline Coal Coke & 24.800 & 24.800 & 24.800 & 24.800 & 24.800 & 24.800 & 24.800 \\
\hline
\end{tabular}

Note: Values for 1995 are preliminary.

Source: Calculated by Energy Information Administration. See Monthly Energy Review DOE/EIA-0035 Appendix A for detailed description. 


\section{Explanatory Notes}

\section{Data Sources}

All data in this report were collected by the Energy Information Administration (EIA), U.S. Department of Energy (DOE), except: import and export data, which were collected by the Bureau of the Census (Census Bureau), U.S. Department of Commerce; supplemental export data which were collected by King's Publishing Corporation, Knoxville, Tennessee; Federal and Indian land leasing data which were collected by the U.S. Department of the Interior (Bureau of Land Management and Minerals Management Service); and miner injury and fatality data which were collected by the U.S. Department of Labor (Mine Safety and Health Administration).

\section{Coal Surveys}

As early as the 1880 's, the U.S. Geological Survey began collecting coal data under a voluntary reporting system. The responsibility for gathering this information was transferred to the Bureau of Mines in the 1920 's, initially under the U.S. Department of Commerce and later under the U.S. Department of the Interior, which published the data in its Minerals Yearbook. Except for a brief period from 1937 to 1943, when bituminous coal data were collected under the mandatory authority of the Bituminous Coal Act, the Bureau of Mines continued to conduct voluntary coal surveys until the Department of Energy was created.

\section{Coal Production Report (Form EIA-7A)}

The Energy Information Administration (EIA) began collecting annual coal production data on October 1 , 1977. The 1995 coal production and identification data in this report were collected on Form EIA-7A, "Coal Production Report," from companies that produced, processed, or prepared coal in 1995. All other data collected on Form EIA-7A are reported for only those companies that owned a mining operation that produced, processed, or prepared 10,000 short tons or more of coal in 1995 and preparation plants with 5,000 or more employee hours.

So that the EIA may fulfill its data collection functions as specified in the Federal Energy Administration Act of 1974 (Public Law 93-275), response to this survey is mandatory. EIA compares respondents to this survey with lists of mining operations maintained by various State coal mining/licensing agencies and the Mine Safety and Health Administration (MSHA), U.S. Department of Labor, to identify new respondents. No sampling procedures are used. In 1995 , there were 2,278 mining operations that produced, processed, or prepared 10,000 or more short tons of coal. Of these mining operations, 77.6 percent $(1,767)$ responded to the EIA-7A survey. In 1995, there were 1,716 mines that produced 10,000 or more short tons of coal. Of these mines, 70.2 percent $(1,205)$ responded to the EIA-7A survey; they accounted for 987 million short tons, or 95.6 percent of the 1995 production total. All of the data were collected by mail and were edited to ensure that they were complete and accurate.

As in all surveys, data from Form EIA-7A, "Coal Production Report," are subject to various sources of error: (1) coverage (the list of respondents may not be complete or, on the other hand, there may be double counting), (2) nonresponse (all units that are surveyed may not respond or may not provide all the information requested), (3) respondents (respondents may commit errors in reporting the data), (4) processing (the data collection agency may lose or incorrectly transcribe the submissions), (5) concept (the data collection elements may not measure the items they were intended to measure), and (6) adjustment (errors may be made in estimating values for missing data).

Because the annual coal production survey (Form EIA-7A) is not a sample survey, the estimates shown 
Table D1. Sources of Data for Total U.S. Coal Production and Number of Mining Operations (Thousand Short Tons)

\begin{tabular}{|c|c|c|c|c|c|c|}
\hline \multirow{3}{*}{$\begin{array}{c}\text { Coal-Producing } \\
\text { State }\end{array}$} & \multicolumn{2}{|c|}{ Received } & \multicolumn{2}{|c|}{ Generated Data Sources } & \multicolumn{2}{|c|}{ Total } \\
\hline & \multicolumn{2}{|c|}{ Form EIA-7A } & \multicolumn{2}{|c|}{$\begin{array}{c}\text { Derived From } \\
\text { Mine Safety and } \\
\text { Health Administration Data }\end{array}$} & \multirow{2}{*}{$\begin{array}{l}\text { Number of } \\
\text { Operations }\end{array}$} & \multirow{2}{*}{ Production } \\
\hline & $\begin{array}{l}\text { Number of } \\
\text { Operations }\end{array}$ & Production & $\begin{array}{l}\text { Number of } \\
\text { Operations }\end{array}$ & Production & & \\
\hline 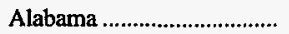 & 62 & 24,297 & 22 & 344 & 84 & 24,640 \\
\hline Alaska & 1 & 1,698 & - & - & 1 & 1,698 \\
\hline 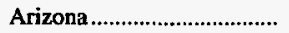 & 3 & 11,947 & - & - & 3 & 11,947 \\
\hline 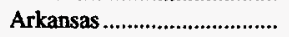 & 3 & 29 & - & - & 3 & 29 \\
\hline Colorado & 18 & 25,306 & 2 & 405 & 20 & 25,710 \\
\hline 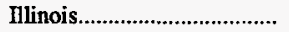 & 36 & 48,120 & 4 & 60 & 40 & 48,180 \\
\hline 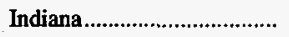 & 48 & 25,982 & 3 & 25 & 51 & 26,007 \\
\hline 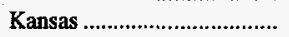 & 1 & 285 & - & - & 1 & 285 \\
\hline Kentucky Total.......................... & 544 & 134,743 & 289 & 18,996 & 833 & 153,739 \\
\hline Eastern & 479 & 100,028 & 272 & 18,514 & 751 & 118,541 \\
\hline 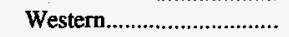 & 65 & 34,715 & 17 & 483 & 82 & 35,198 \\
\hline 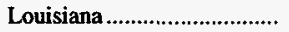 & 2 & 3,719 & - & - & 2 & 3,719 \\
\hline 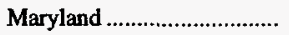 & 16 & 3,570 & 5 & 97 & 21 & 3,667 \\
\hline 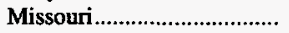 & 5 & 539 & 1 & 9 & 6 & 548 \\
\hline 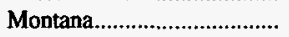 & 8 & 39,451 & - & - & 8 & 39,451 \\
\hline 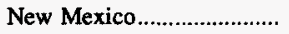 & 9 & 26,813 & - & - & 9 & 26,813 \\
\hline 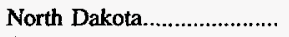 & 7 & 30,112 & - & - & 7 & 30,112 \\
\hline Ohio & 116 & 25,687 & 24 & 431 & 140 & 26,118 \\
\hline Oklahoma & 11 & 1,846 & 2 & 30 & 13 & 1,876 \\
\hline Pennsylvania Total............... & 453 & 59,872 & 168 & 1,703 & 621 & 61,576 \\
\hline 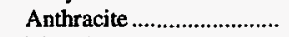 & 136 & 4,289 & 77 & 393 & 213 & 468 \\
\hline 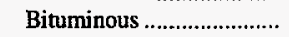 & 317 & 55,583 & 91 & 1,310 & 408 & 5,689 \\
\hline 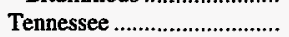 & 32 & 3,157 & 11 & 64 & 43 & 3,221 \\
\hline Texas & 14 & 52,684 & - & - & 14 & 52,684 \\
\hline Utah & 14 & 24,843 & 2 & 324 & 16 & 25,167 \\
\hline 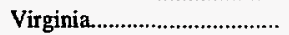 & 174 & 28,364 & 97 & 5,735 & 271 & 34,099 \\
\hline 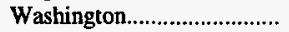 & 3 & 4,868 & - & - & 3 & 4,868 \\
\hline West Virginia Total ............ & 431 & 146,192 & 180 & 16,805 & 611 & 162,997 \\
\hline Northern............................. & 113 & 44,315 & 30 & 1,800 & 143 & 46,114 \\
\hline 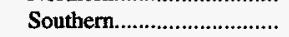 & 318 & 101,877 & 150 & 15,005 & 468 & 116,883 \\
\hline Wyoming & 28 & 263,805 & 1 & 18 & 29 & 263,822 \\
\hline 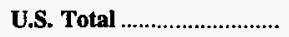 & 2,039 & 987,928 & 811 & 45,046 & 2,850 & $1,032,974$ \\
\hline
\end{tabular}

Notes: Coal production excludes silt, culm, refuse bank, slurry dam, and dredge production except for Pennsylvania anthracite. Number of mining operations includes preparation plants. All available State mining agency production data were reviewed, but none were included in this report because production data reported on Form EIA-7A to the Energy Information Administration and on Form 7000-1 to the Mine Safety and Health Administration were found to be complete. Totals may not equal sum of components because of independent rounding.

Sources: Energy Information Administration, Form EIA-7A, "Coal Production Report"; U.S. Department of Labor, Mine Safety and Health Administration, Form 7000-2, "Quarterly Mine Employment and Coal Production Repart."

in this report are not subject to sampling error. ${ }^{1}$ It is not possible to present estimates of nonsampling error, but precautionary steps were taken at each stage of the survey design to minimize the possible occurrence of these errors. These steps are described below.

The forms are logged within 24 hours of receipt and assigned to a team of data editors consisting of Coal Data Systems Branch personnel. The editors screen the forms for legibility, completeness, and consistency. Names and address changes are updated in the files. The reported data are compared with data from previous years and with secondary sources such as Mine Safety and Health Administration and State mining agency reports. Inconsistencies are identified and the respondents contacted for clarification. Computer edits are generated to identify keypunch errors, errors made by coders, out-of-range codes, and unlikely data combinations. Errors are corrected to conform to the data on the submissions or revised after telephone conversations with company representatives. All changes to reported data are documented. EIA maintains data from the Form EIA-7A on an automated database at its computing facility in Washington, D.C. The survey forms are filed by EIA identification number organized by State and county.

The survey forms were sent via regular mail in January with a due date of March 1, 1996. Nonresponse letters were mailed March 8 to those mining operations that had not submitted their forms. Subsequent to the letter, attempts were made to contact, by telephone, those nonrespondents whose reported 1994 production was 100,000 short tons or more.

1 Sampling error is a measure of the variation that occurs by chance because a sample rather than a complete enumeration of units is surveyed. 
Some forms could not be delivered. Where possible, address corrections were obtained. Some mining operations that had reported in earlier surveys or operated only in 1995 were no longer in business. Unobtainable data were derived from a secondary source: the Form 7000-2, "Quarterly Mine Employment and Coal Production Report," collected by the Mine Safety and Health Administration. Missing production quantity data were derived from the secondary source for 585 mines, which produced 45 million short tons, or 4.4 percent of total production. The received and generated data sources for total U.S. coal production are shown in Table D1. Of the mines whose production exceeded 10,000 or more short tons, missing production quantity data were derived from the secondary source for 511 mines, representing 4.3 percent (44.4 million short tons).

Since 1992, California has not reported coal production as the courts determined that the product mined in that State did not meet the standard classification for coal.

Missing coalbed classification, coalbed thickness, and coal rank/group data were estimated using State mining agency production reports, geological data, and previous years' reports for these mines.

When a mine had a missing production value, its production was multiplied by the county-level average mine price to estimate the value. County-level average mine prices were calculated by dividing the total value for the appropriate disposition (open/captive) and type of mining (underground or surface) by the corresponding total production. All missing production was classified as open market unless information was available to classify it as captive. Of those mines whose production was 10,000 or more short tons, value data were estimated for 562 mines, representing 5.2 percent ( 53.8 million short tons) of the production total.

When an underground mine had unreported mining method, it was assumed that the mining method was conventional.

Employment data include direct labor hours, number of production days worked, average length of a production shift, average number of miners per shift, and average number of shifts per day. Of those mines whose production exceeded 10,000 or more short tons, one or more of these data elements were derived from secondary sources or estimated for 532 mines representing 4.4 percent ( 45.2 million short tons) of their production total. Of the mining operations that produced, processed, or prepared 10,000 or more short tons, one or more of these data elements were derived from secondary sources or estimated for 868 mining operations, or 38.1 percent of the total operations in this category.

Missing direct labor hours were estimated using county-level or State-level productivity-per-hour averages. The averages were calculated by dividing the total production at the county or State level by total direct labor hours at the same level for the appropriate type of mining (underground or surface). The quantity of production for the mine with missing data was divided by this productivity average to derive direct labor hours.

Missing average number of production days worked and length of a production shift were derived by using the corresponding county-level or State-level average for the appropriate type of mining (underground or surface).

Missing average number of miners per shift was estimated as the average number of miners working daily. The average number of miners working daily was calculated by multiplying the average length of a production shift by the number of production days worked, and then dividing this number into the total direct labor hours.

Missing average number of shifts per day was estimated as one shift per day.

Missing recoverable coal reserves data were estimated by using the mine's 1994 recoverable reserves minus the mine's 1995 production. If this calculation could not be made, the mine's projected production for 1996 was used. If recoverable coal reserves for 1994 and 1995 and projected production for 1996 were all missing, no estimate was made. In 1995, recoverable reserves were reported by or estimated for 1,186 mines, representing 976.9 million short tons, or 69.1 percent of the mines whose production exceeded 10,000 or more short tons.

Missing recovery percentage data were estimated by using 1995 recovery percentage averages at the State level for the appropriate type of mining (underground or surface).

Missing productive capacity data were estimated by assuming productive capacity was equal to 1995 production. If productive capacity was reported as less than annual production, productive capacity was equated to 1995 production. There were 627 in-scope mines with production of $\mathbf{1 7 7 . 8}$ million short tons for which 1995 production was used as a proxy for productive capacity, resulting in 100 percent capacity utilization.

These mines included the MSHA generated mines, mines with productive capacity less than 1995 production, and mines that did not report productive capacity and could not be contacted. If these mines were excluded from the calculation of percent utilization, the U.S. total becomes 76.13 versus 79.40 , when those mines are included.

In 1995 , there were 26 mines that produced 1.2 million short tons of refuse bituminous and subbituminous coal. Those operations are not included in this report. In 1995, there were 2.3 million short tons of anthracite refuse produced and included in this report. An additional 4 million short tons of anthracite refuse was recovered and used by nonutility power producers in Pennsylvania is not included in this report. 
In order to protect the confidentiality of individual respondent's data, a policy was implemented to ensure that the reporting of survey data on mine prices and recoverable reserves in this publication would not associate those data with a particular company. The final phase in the data quality assurance and control procedures is determining which data must be suppressed (withheld) during publication to provide the necessary confidentiality for mines or companies that represent a significant portion of a reported data cell. All withholding analysis is done based upon production volumes. These procedures are performed as follows:

1. Primary Withholding Based on the Number of Respondents in a Cell -- All cells with three or fewer active coal mining operations are suppressed.

2. Residual Withholding Dominance Rule Phase 1 All cells containing between 4 and 10 active coal mining operations are examined. A cell is suppressed if any single respondent accounts for 75 percent or more of the volume for all respondents included in that cell.

3. Residual Withholding Dominance Rule Phase 2 All cells in which two active coal mining operations represent 90 percent or more of the volume for all respondents included in that cell are suppressed.

4. Complementary Suppression -- All tables are reviewed to identify cells which should have data withheld to prevent disclosure of already suppressed cells. An example of cells to be withheld during complementary suppression is underground price, if the surface price is withheld during primary or residual suppression. Because the total price is published, if the underground price is not withheld, the surface price could be calculated using the total price, the underground price, and the underground, surface, and total volumes.

5. Most complementary suppression involves type of mining considerations. Other complementary suppression is based on regional level data. A State or region must be withheld during complementary suppression because an already withheld State could be calculated using other States and the regional total. Cells are also selected for complementary suppression that represent the smallest volumes or that were withheld in prior years.

6. Inter-table effects are also examined regarding complementary suppression. For example, States that are withheld in one State table can influence the complementary suppression of an associated State table. This analysis is very similar to that done at the regional level, except that two tables are involved rather than one. Finally, similar tables are reviewed to ensure that all like suppressed cells are consistently withheld (suppressed) in all tables in which they appear.

The withholding/suppression of data is performed as an adjunct to the quality assurance (QA) procedures. The work is performed by survey editors, and the QA staff and is reviewed by the survey manager before being submitted to division-level QA review.
All sensitive cells identified in withholding analysis are denoted with the symbol/letter "w." The use of the symbol/letter applies to primary, complementary and inter-table suppressions as well as all withheld data. The symbol/letter " $w$ " is footnoted as follows: "w Withheld to avoid disclosure of individual company data."

The interquartile range is a measure of dispersion of State-level average mine prices. Two States may have the same average mine price, but the spread about this price may be totally different. For a fixed average mine price, a larger interquartile range suggests a broader distribution of coal prices than a smaller interquartile range. The summary statistics (Table D2) given in this publication are weighted by production. The interquartile range (weighted by production) is computed in the following manner:

- Each cell is sorted according to average mine price, from the lowest to the highest.

- For each cell, the corresponding mine's production according to increasing average mine price is divided by that particular cell's total production and multiplied by 100 . These percentages are then added as a cumulative percentage of production.

- The first quartile $(\mathrm{Q} 1)$ is the associated mine price for which the cumulative percentage of production first passes 25 percent. Thus, at least 25 percent of that cell's total production is identified with prices at or lower than Q1.

- The third quartile (Q3), is the associated mine price for which the cumulative percentage of production first passes 75 percent. Thus, at least 75 percent of that cell's total production is identified with prices at or lower than Q3.

The interquartile range is $\mathrm{Q} 3-\mathrm{Q} 1$.

\section{Quarterly Coal Consumption Report - Manufacturing Plants (Form EIA-3)}

Form EIA-3 is used to survey U.S. manufacturers that consume 1,000 tons or more of coal per year for all uses other than coke production. These data were collected on a monthly basis until 1980, when the reporting cycle was revised to a quarterly schedule. Data on manufacturers' coal stocks, receipts, prices, and consumption are reported.

Through the end of 1988 , all manufacturers that consumed coal were required to file Form EIA-3. Beginning with the first quarter of 1989 , only those manufacturers that consumed 1,000 or more tons in the past year were required to report. In 1995, 730 manufacturers responded to the EIA-3 survey. The response rate for the current year was 100 percent. In order to identify undercoverage problems, the data from this survey are compared with shipments to manufacturers reported on EIA's "Coal Distribution Report," Form EIA-6. At present, the coal receipts reported by manufacturers on Form EIA-3 cover approximately 97 percent of the coal shipments to 
Table D2. Interquartile Range and Average Mine Price by State and Mine Type, 1995 (Dollars per Short Ton)

\begin{tabular}{|c|c|c|c|c|c|c|}
\hline \multirow{2}{*}{$\begin{array}{l}\text { Coal-Producing } \\
\text { State and } \\
\text { Region }\end{array}$} & \multicolumn{2}{|c|}{ Underground } & \multicolumn{2}{|c|}{ Surface } & \multicolumn{2}{|c|}{ Total } \\
\hline & $\begin{array}{c}\text { Average } \\
\text { Mine Price }\end{array}$ & $\begin{array}{c}\text { Interquartile } \\
\text { Range }\end{array}$ & $\begin{array}{c}\text { Average } \\
\text { Mine Price }\end{array}$ & $\begin{array}{c}\text { Interquartile } \\
\text { Range }\end{array}$ & $\begin{array}{c}\text { Average } \\
\text { Mine Price }\end{array}$ & $\begin{array}{c}\text { Interquartile } \\
\text { Range }\end{array}$ \\
\hline 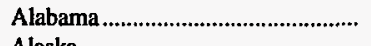 & 39.26 & 9.44 & 36.38 & 10.85 & 38.44 & 9.44 \\
\hline 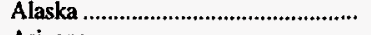 & - & - & $\mathbf{w}$ & w & w & $\mathbf{w}$ \\
\hline 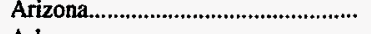 & - & - & $\mathbf{w}$ & $\mathbf{w}$ & $\mathbf{w}$ & w \\
\hline 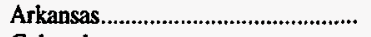 & - & - & w & $\mathbf{w}$ & $\mathbf{w}$ & $\mathbf{w}$ \\
\hline 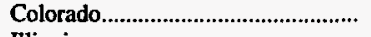 & 18.58 & .78 & 20.63 & 3.79 & 19.26 & 7.28 \\
\hline Illinois & 22.88 & 4.42 & 24.04 & 17.10 & 23.05 & 4.42 \\
\hline Indiana & $\mathbf{w}$ & $\mathbf{w}$ & $\mathbf{w}$ & $\mathbf{w}$ & 21.71 & 4.35 \\
\hline 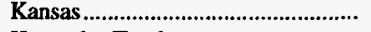 & - & - & $\mathbf{w}$ & $\mathbf{w}$ & $\mathbf{w}$ & w \\
\hline 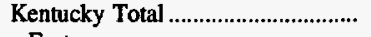 & 25.18 & 7.40 & 24.19 & 5.72 & 24.79 & 7.09 \\
\hline 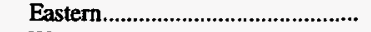 & 26.52 & 5.09 & 25.24 & 5.37 & 26.00 & 5.92 \\
\hline Western & 21.33 & 3.56 & 19.46 & 3.86 & 20.75 & 4.60 \\
\hline 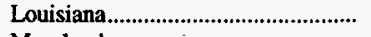 & - & - & w & $\mathbf{w}$ & $\mathbf{w}$ & $\mathbf{w}$ \\
\hline 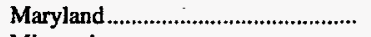 & w & $\mathbf{w}$ & $\mathbf{w}$ & $\mathbf{w}$ & 24.69 & - \\
\hline Missouri ............................................ & - & - & 18.91 & 9.50 & 18.91 & 9.50 \\
\hline 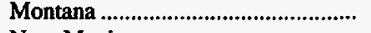 & - & - & 9.62 & 10.03 & 9.62 & 10.03 \\
\hline 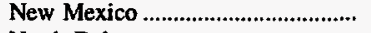 & w & $w$ & w & $\mathbf{w}$ & 23.80 & 3.36 \\
\hline North Dakota & - & - & 7.99 & .88 & 7.99 & .88 \\
\hline 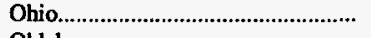 & 28.98 & 16.28 & 22.92 & 6.77 & 25.97 & 12.30 \\
\hline 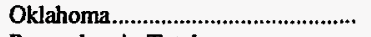 & $w$ & $\mathbf{w}$ & w & $\mathbf{w}$ & 24.13 & 4.31 \\
\hline Pennsylvania Total & 27.09 & 2.39 & 26.14 & 9.06 & 26.78 & 9.06 \\
\hline 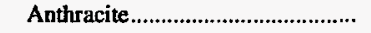 & 36.91 & 8.13 & 39.97 & 38.33 & 39.78 & 37.91 \\
\hline 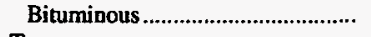 & 27.02 & 2.40 & 22.45 & 8.15 & 25.77 & 5.41 \\
\hline 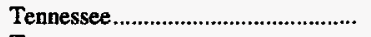 & w & $\mathbf{w}$ & $w$ & $\mathbf{w}$ & 26.94 & 1.81 \\
\hline Texas & - & - & 12,16 & 2.47 & 12.16 & 2.47 \\
\hline Utah & 19.10 & 2.91 & - & - & 19.10 & 2.91 \\
\hline Virginia & 29.20 & 3.92 & 26.34 & 4.51 & 28.47 & 7.01 \\
\hline 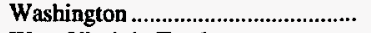 & - & - & w & w & $\mathbf{w}$ & $\mathbf{w}$ \\
\hline West Virginia Total ............................... & 27.77 & 5.88 & 25.95 & 3.65 & 27.18 & 5.68 \\
\hline Northern & 25.17 & 7.01 & 22.97 & 5.63 & 24.91 & 7.00 \\
\hline Southern ............................................. & 29.30 & 3.76 & 26.29 & 3.79 & 28.07 & 5.24 \\
\hline 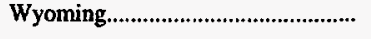 & w & w & w & $w$ & 6.58 & 2.06 \\
\hline 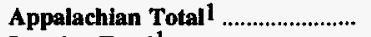 & 28.24 & 5.71 & 25.97 & $\mathbf{7 . 5 7}$ & 27.45 & 6.74 \\
\hline Interior Total 1 & 22.57 & 4.68 & 16.19 & 8.18 & 18.81 & 9.70 \\
\hline 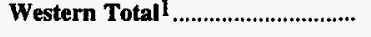 & 18.85 & 5.36 & 9.13 & 3.19 & 10.15 & 10.43 \\
\hline 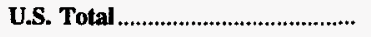 & 26.18 & 8.50 & 14.25 & 16.30 & 18.83 & 19.25 \\
\hline
\end{tabular}

1 For a definition of coal-producing regions, see Appendix C.

Withheld to avoid disclosure of individual company data.

Note: Excludes silt, culm, refuse bank, slurry dam, and dredge operations except for Pennsylvania anthracite. Excludes mines producing less than 10,000 short tons of coal during the year. Average Mine Price is calculated by dividing the total free on board (f.o.b) mine value of the coal produced by the total production.

Sources: Energy Information Administration, Form EIA-7A, “Coal Production Report”; State Mining Agency Coal Production Reports; and/or U.S. Department of Labor, Mine Safety and Health Administration, Form 7000-2, "Quarterly Mine Employment and Coal Production Report."

manufacturers on Form EIA-6. Consequently, the coal consumption data gathered on the Form EIA-3 do not represent the total consumption at manufac-turing plants. See Technical Note 5 for data adjustment procedures for coal consumption for the other industrial sector.

Current year data from this survey are preliminary and unrevised in the January - March, April - June, and July - September issues of the Quarterly Coal Report (DOE/EIA-0121). In the October - December issue, any revisions necessary for the entire year are applied and the data are considered final.

The respondent list of manufacturers for Form EIA-3 is compared with lists of coal-consuming manufacturing plants from State Air Quality and Energy Offices. When new respondents are found, they are added to the survey mailing list.

\section{Annual Coal Quality Report - Manufacturing Plants (Form EIA-3A)}

Form EIA-3A contains questions on the origin of coal (State or Country), the quantity of coal receipts, the Btu, sulfur and ash content of the coal receipts, and the basis used to determine the coal quality data. The threshold for the annual collection will be the same as for the EIA-3: manufacturing plants that consume in excess of 1,000 short tons of coal per per year. In 1995, 730 manufacturers responded to the EIA-3A survey. The response rate for the current year was 100 percent. 


\section{Coke Plant Report (Form ElA-5)}

Form EIA-5, a quarterly report of coal receipts, carbonization, and stocks, and of coke and breeze production, distribution, and stocks, is used to survey all U.S. coke plants.

Coke plants were surveyed monthly and a supplemental survey was taken annually until 1981 , when the reporting cycle was revised to a quarterly schedule with an annual supplemental survey. In 1985, collection of the annual supplement was ended.

In 1995, there were 28 respondents to the EIA-5 survey, and the response rate was 100 percent. The respondent list for this survey is updated by continuous monitoring of the industry literature.

Current year data from this survey are preliminary and unrevised in the January - March, April - June, and July - September issues of the Quarterly Coal Report (DOE/EIA-0121). In the October - December issue, any revisions necessary for the entire year are applied and the data are considered final.

\section{Annual Coal Quality Report - Coke Plants (Form EIA-5A)}

This form contains questions on the origin of coal (State or country), the quantity of coal receipts, the volatile matter, sulfur and ash content, and the basis used to determine the coal quality data. There is no threshold for this form. In 1995, there were 28 respondents to the EIA-5A survey, and the response rate was 100 percent.

\section{Coal Distribution Report (Form EIA-6)}

Form EIA-6 is used to survey all U.S. companies (producers and/or distributors) that own or purchase and distribute more than 50,000 short tons annually. ${ }^{2}$ Data on coal production and purchases, distribution by consumer category, and method of transportation are reported.

In 1995 , there were about 1,100 respondents to the EIA-6 survey. Until the end of 1988, coal distribution companies were required to report production on a Bureau of Mines district basis. For the year 1989, respondents were required to report on a BOM district/State basis. Beginning with the first quarter of 1990 , respondents were required to report on a State basis. The response rate for the current quarter was 100 percent. The annual production total reported on Form EIA-6 exceeds 99 percent of total production as reported by all mines on Form EIA-7A, "Coal Production Report," due to the difference in reporting thresholds. The data gathered on the Form EIA- 6 only represent the domestic coal distributed during the quarter. Therefore, imported coal distributed during the quarter is not included.

Current year data from this survey are preliminary and unrevised in the January - March, April - June, and July - September issued of the Quarterly Coal Report (DOE/EIA-0121). In the October - December issue, any revisions necessary for the entire year are applied and the data are considered final.

The respondent list for this survey is updated by comparing it with lists of coal producers from the Mine Safety and Health Administration (MSHA), U.S. Department of Labor, and from similar lists maintained by various State agencies. Also, new respondents are frequently identified on Form EIA- 6 itself when other companies are named as sources of coal purchases.

\section{Electric Utility Surveys}

Coal data appear in this report from three surveys of electric utilities --from all generating electric utilities and from fossil-fueled plants.

The Census Bureau collected and published the results of a census taken every 5 years from 1902 to 1937 on the electric light and power industries and some data on industrial production of electric energy. The U.S. Geological Survey collected data on capacity and generation of electric utilities from 1920 to 1936, when this activity was turned over to the Federal Power Commission (FPC).

All data are presented as reported on the surveys. No estimates or other adjustments are made for missing data. The data are maintained in a computer system and are edited to ensure that they are reasonable, consistent, and complete. For additional information from these surveys and for other electric utility data, see the EIA publication, Electric Power Monthly (DOE/EIA-0226).

\section{Monthly Power Plant Report (Form EIA-759)}

Form EIA-759 (which, until 1982, was called FPC Form 4) is used to survey all generating electric utilities. The Federal Power Act and FPC Order Number 141 define the legislative authority to collect power production data. Consumption and stocks of coal and other fuels at each plant are reported. The respondents to Form EIA-759, approximately 3,000 plants, account for 100 percent of total electric utility generation.

Current year data from this survey are considered final.

2 For the States of Arkansas, Maryland, Oklahoma, and the anthracite portion of Pennsylvania, the threshold is 10,000 tons. 


\section{Monthly Report of Cost and Quality of Fuels for Electric Plants (FERC Form 423)}

Federal Energy Regulatory Commission (FERC) Form 423 is used to survey all fossil-fueled plants with a total steam-generating capacity of 50 megawatts or more. It is submitted by approximately 230 electric utilities. (Before 1983, this form was called FPC Form 423 , and all fossil-fueled plants with a total generating capacity of 25 megawatts or more were surveyed.) In 1972, the FPC issued Order Number 453, which included the legislative authority to create FERC Form 423. Cost, quality, and source of fuels (by State or country of origin), including coal, are reported. Current year data from this survey are considered final.

\section{Steam-Electric Plant Operation and Design Report (Form EIA-767)}

The Form EIA-767 is a mandatory restricted-universe census of all electric power plants with a total existing or planned organic- or nuclear-fueled steamelectric generator nameplate rating of 10 or more megawatts. The entire form is filed by approximately 700 power plants with a nameplate capacity of 100 or more megawatts. An additional 200 power plants with a nameplate capacity between 10 and 100 megawatts submit information only on fuel consumption/quality, boiler/generator configuration, and flue-gas desulfurization equipment, if applicable. The Form EIA-767 is used to collect data annually on plant operations and equipment design (including boiler, generator, cooling system, flue gas desulfurization, flue gas particulate collectors, and stack data). Data from Form EIA-767 are used for economic, regulatory, and environmental analyses conducted by the DOE, the FERC, the Environmental Protection Agency, and the Department of Commerce.

Form EIA-767 data for 1995 are preliminary. Data for prior years are final.

\section{Annual Nonutility Power Producer Report (Form EIA-867)}

The Form EIA-867 is a restricted-universe census used to collect annual data from all existing and planned nonutility power producers in the United States. In 1992, the reporting threshold of the Form EIA-867 was lowered to include all facilities with a combined nameplate capacity of 1 or more megawatts. Previously data were collected every 3 years from facilities with a nameplate capacity between 1 and 5 megawatts. For the purpose of this data collection, a nonutility power producer is an enterprise that has electric generating capacity and is not an electric utility. They include qualifying cogenerators, qualifying small power producers, and other nonutility generators (including independent power producers) without a designated franchised service area. The form is used to collect data on the installed capacity, energy consumption, generation, and electric energy sales to electric utilities from approximately 2,000 facilities.

Form EIA-867, coal consumption data for 1989 through 1995 are: $876,1600,6000,10000,12344$, 15140 and 20800 thousand short tons.

\section{Export and Import Data}

Export and import data (except imports to electric utilities, manufacturing plants and coke plants, which are reported on the FERC Form 423, EIA-3A, and EIA-5A, respectively.) are obtained from the Census Bureau--export data from the monthly EM 545 (formerly EM 522) report, import data from the monthly IM 145 report. The Census Bureau compiles these data monthly from documents filed with the U.S. Customs Service as required by law. They include shippers' export declaration forms, import entry forms, and warehouse withdrawal forms. No sampling procedures are used. The Census Bureau publication Guide to Foreign Trade Statistics describes the foreign trade statistics program, including the EM 545 and IM 145 monthly reports. Data from these surveys are considered final at the time of publication.

Foreign distribution of U.S. coal, major exporting State, and destination, along with foreign distribution of metallurgical and steam coal (Tables 62, 63, and 64, respectively), was determined using EIA-6 distribution data by origin State, and coal export data from King's COALBASE (King Publishing Corporation, Knoxville, Tennessee) which gives the metallurgical and steam breakdown as well as the country destination data. The percentage of metallurgical and steam coal for each country of destination are applied to the EIA-6 export figures for each State of origin to derive coal distribution data that link State of origin to countries of destination by type of coal. The King's destination country data are considered to be more accurate than the Census country-of-destination data because it account for transhipments through intermediate countries to final destination countries, whereas the Census data would designate the destination as the intermediate country.

Copies of the survey forms and instructions used to collect data appearing in this publication can be obtained by calling EIA's National Energy Information Center at (202) 586-8800. 



\section{Technical Notes}

\section{Differences in Related Coal Data}

Coal Production versus Coal Distribution. Coal production represents newly mined coal. Coal distribution represents shipments of newly mined coal and coal from producer/distributor stockpiles (previously mined coal).

Coal Distribution versus Coal Receipts. Differences in coal distribution data and coal receipts data are due to the time lag between distribution and receipt of coal shipments, and due to the survey threshold differences. In addition, coal distributed includes only domestic coal, whereas receipts include imported coal.

Foreign Distribution of U.S. Coal versus U.S. Coal Exports. Foreign distribution of U.S. coal does not equal U.S. coal exports because there are differences in reporting time and survey thresholds.

Receipts of Imported Coal versus U.S. Coal Imports. Receipts of imported coal at electric utilities and manufacturing and coke plants does not equal U.S. coal imports due to reporting time differences. In addition, it does not include receipts at independent power producers.

\section{Other Industrial Plants and Manufacturing}

The other industrial plants end-use sector includes the manufacturing, agriculture, forestry and fishing, mining, and construction industries. Manufacturing accounts for approximately 97 percent of the coal receipts and consumption and 100 percent of the coal stocks in the other industrial plants sector as reported herein. Data sources for the other industrial plants sector and the manufacturing sector are Forms EIA-6 and EIA-3, respectively. The source statement in each table identifies the survey used to collect coal data for the other industrial plants sector, and the following technical notes describe the methodology used when data were derived.

\section{Residential and Commercial}

To reduce the reporting burden to coal users, the EIA does not conduct any survey of coal data from residential and commercial users of coal. Shipments of coal to this sector, reported by producers and distributors of coal on Form EIA-6, are equated to coal receipts and consumption by the residential and commercial sector, assuming no stock changes.

\section{Consumer Prices}

Prices are derived for each end-use sector as follows:

Electric Utilities. Prices are reported for each plant in cents-per-million Btu on FERC Form 423. The price per ton of coal is calculated at each plant using centsper-million Btu and the average Btu content per pound of coal for the appropriate rank of coal. The average prices appearing in the tables (e.g., across all States) are calculated by summing the dollar value at each plant (short tons of coal multiplied by price per short ton) and dividing by the corresponding total tons. For more information about prices of coal at electric utilities, see the EIA publication, Electric Power Monthly (DOE/EIA-0226).

Coke Plants. Respondents are asked to report the number of tons of coal received (or coke distributed) on Form EIA-5 and the total value of that coal (or coke) in dollars. Average prices are calculated by summing the reported values (e.g., across all States) and dividing by the corresponding total tons.

Other Industrial Plants. Respondents (manufacturing plants only) are asked to report the number of tons of coal received on Form EIA-3 and the total value of that coal in dollars. Average prices are calculated by summing the reported values across all States and dividing by the corresponding total tons.

Residential and Commercial. Data are not collected. See Technical Note 3. 


\section{Consumption}

\section{Annual Data}

Annual coal consumption data are sums of quarterly or monthly data described below except for nonutility power producers whose coal consumption is not included in this report. These data are however, reported on Form EIA-867 and published in the Electric Power Annual (DOE/EIA-0348).

Electric Utilities. Consumption is reported on Form EIA-759.

Nonutility Electric Generating Facilities. Consumption is reported on Form EIA-867.

Coke Plants. Consumption is reported on Form EIA-5.

Other Industrial Plants. In deriving a quarterly estimate for coal consumption for the other industrial plants sector, the first step is to equate consumption to beginning stocks plus receipts minus ending stocks. In terms of an equation, consumption can be expressed as $\mathrm{C}=\mathrm{Sb}+\mathrm{R}-\mathrm{Se}$, where $\mathrm{Sb}=$ beginning stocks, $\mathrm{R}=$ receipts, and $\mathrm{Se}=$ ending stocks.

Therefore, consumption is $\mathrm{C}=(\mathrm{Sb}-\mathrm{Se}$ (change in stocks)) $+\mathrm{R}$. Next, stock change at the State level is equated to the stock change for that State as reported on Form EIA-3, receipts at the State level are derived as described in Section 3, and a computed consumption is derived using the same equation for each State. Finally, the quarterly consumption $(C)$ at the State level is equated to the maximum of the computed consumption at the State level, as previously described, and the quarterly consumption for that State as reported on Form EIA-3. This process ensures that State-level consumption for the other industrial plants sector is always greater than or equal to the manufacturing sector consumption for that State. Total quarterly consumption for the other industrial plants sector is computed by summing the quarterly State-level consumption figures.

Residential and Commercial. Shipments to the residential and commercial sector as reported on Form EIA-6 are defined as consumption as well as receipts for this end-use sector.

EIA publishes monthly estimates of coal consumption in the Monthly Energy Review (DOE/EIA-0035).

Monthly coal consumption at electric utility plants is derived directly from Form EIA-759. Prior to 1980, monthly coal consumption at coke plants was derived directly from Form EIA-5. For 1981 through 1987, it was derived from the quarterly coal consumption reported on Form EIA-5, using the ratios of monthly to quarterly consumption in 1979 , the last year that coke plant data were collected monthly on Form EIA-5. These ratios by month (January - December) are $0.3377,0.3200,0.3423 ; 0.3529,0.3462,0.3009$; $0.3364,0.3347,0.3289$; and $0.3273,0.3301,0.3426$.
Starting with 1988 , monthly coal consumption at coke plants is derived from quarterly coal consumption reported on Form EIA-5, using ratios derived from monthly data on raw steel production published by the American Iron and Steel Institute (AISI) on Form AIS7. The ratio is the proportion of monthly raw steel production from open hearth and basic oxygen process furnaces to the quarterly raw steel production from those furnace types.

Prior to 1978, coal consumption for the other industrial plants sector (i.e., industrial users minus coke plants) was derived by using monthly data reported on Form EIA-3 to modify baseline coal consumption figures from the most recent Census of Manufactures or Annual Survey of Manufactures, Bureau of the Census, U.S. Department of Commerce. For 1978 through 1987, data from Forms EIA-3 and EIA-6 are used to compute monthly coal consumption for the other industrial plants sector.

Given the quarterly consumption for the other industrial plants sector (C), the monthly consumption for the sector $(\mathrm{Cm})$ is estimated for each month in the quarter as $\mathrm{Cm}=(\mathrm{Cm} 3 / \mathrm{C} 3) \times \mathrm{C}$ where $\mathrm{Cm} 3 / \mathrm{C} 3$ is the ratio of monthly to quarterly coal consumption as reported on Form EIA-3. For the 1978 coal consumption figures, the ratios used are based on 1978 EIA-3 data. For 1979 through 1987, the ratios used are based on the 1979 EIA-3 data. These 1979 ratios by month (January - December) are $0.3593,0.3264,0.3143$; $0.3485,0.3332,0.3183 ; 0.3317,0.3407,0.3276$; and $0.3045,0.3253,0.3702$.

Starting with 1988 , monthly coal consumption for the other industrial plants sector is derived from quarterly coal consumption reported on Form EIA-3 using monthly ratios derived from the industrial production indices published by the Board of Governors of the Federal Reserve System. Six major industry groups' indices are used as the basis for calculating the monthly ratios. These groups are foods (Standard Industrial Classification (SIC) 20), paper and products (SIC 26), chemicals and products (SIC 28), petroleum products (SIC 29), clay, glass, stone products (SIC 32 ), and primary metals (SIC 33).

The monthly ratios are computed as the monthly sum of weighted indices as a proportion of the quarterly sum of weighted indices, using the 1985 proportion as the weight.

Prior to 1980 , monthly coal consumption for the residential and commercial sector was derived by using monthly data reported on Form EIA-2, "Monthly Coal Report -- Retail Dealers and Upper Lake Docks," to modify baseline coal consumption figures developed by the Bureau of Mines, U.S. Department of the Interior.

For 1980 , the quarterly coal consumption figures in the residential and commercial sector are converted to monthly coal consumption figures using the ratios of monthly to quarterly coal deliveries to this sector in 1979 as reported on Form EIA-2. These 1979 ratios by month (January-December) are $0.4002,0.3502$, 
$0.2496 ; 0.4805,0.2901,0.2294 ; 0.3126,0.2952$, 0.3922 ; and $0.2931,0.3101,0.3968$. The 1981 and 1982 monthly coal consumption figures were derived using the 1979 ratios but were also modified according to heating/cooling degree-days. For 1983 through 1987, coal consumption figures are converted to monthly coal consumption figures using only the ratios of monthly to quarterly coal deliveries to this sector in 1979.

Starting with 1988 , monthly coal consumption figures are derived using the monthly national average population weighted heating/cooling degree-days obtained from the National Oceanic and Atmospheric Administration. The ratio is the proportion of the monthly national sum of heating and cooling degree-days to the quarterly sum.

\section{Stocks}

Annual stocks are calculated at the end of the year or the end of the fourth quarter. Coal stocks are derived for each end-use sector as follows:

Electric Utilities. Stocks are reported on Form EIA-759.

Coke Plants. Stocks are reported on Form EIA-5.

Other Industrial Plants. Stocks are reported on Form EIA-3, i.e., stocks at manufacturing plants only. Technical Note 2 discusses the difference between other industrial plants and manufacturing plants.

Residential and Commercial. Data are not available. See Technical Note 3.

Producer and Distributor. Stocks are reported on Form EIA-6.

\section{Methods of Transportation}

Rail: Shipments of coal moved to consumers by rail, either private or public/commercial. Included is coal hauled to or away from a railroad siding by truck.

Water Transportation: Shipments of coal moved by one of the three methods--river, Great Lakes, or tidewater piers and coastal ports. Included in these shipments is coal hauled to or from water loading facilities by other means of transportation.

River: Shipments of coal moved to consumers via river by barge, except shipments to Great Lakes coal loading docks or tidewater piers or coastal ports.

Great Lakes: Shipments of coal moved to consumers via the Great Lakes. These shipments are moved via the Great Lakes coal loading docks, which are iden- tified by name and locations as follows: Superior Midwest Energy Terminal, Superior, Wisconsin; Bessemer \& Lake Erie Coal Storage \& Transfer Facility, Conneaut, Ohio; B\&O Railroad Coal Loading Dock, Lorain, Ohio; C\&O Railroad Presque Isle Docks, Toledo, Ohio; Lakefront Dock \& Railroad Terminal Company Coal Loading Dock, Toledo, Ohio; N\&W Sandusky Coal Pier No. 3, Sandusky, Ohio; ConRail Coal Transfer Facilities, Ashtabula, Ohio; Rail to Water Transfer Corporation Dock, Chicago, Illinois.

Tidewater Piers and Coastal Ports: Shipments of coal moved to tidewater piers and coastal ports for further shipments to consumers via coastal water or ocean. The tidewater piers are identified by name and location as follows: B\&O Curtis Bay Coal Piers, Baltimore, Maryland; C\&O Coal Piers Nos. 14 \& 15 , Newport News, Virginia; N\&W Lamberts Point Coal Piers Nos. 5 \& 6, Norfolk, Virginia; Alabama State Docks Bulk Handling Plant, Mobile, Alabama; Alabama State Docks/McDuffie Terminals, Mobile, Alabama; Canton Coal Piers, Baltimore Harbor on the Chesapeake Bay; Greenwich Coal Pier, Greenwich Point, Philadelphia, Pennsylvania, on Delaware River; Port Richmond Pier, Pier 18 Port Richmond, Philadelphia, Pennsylvania, on the Delaware River; Galveston Regional Coal Distribution Center, Pelican Island, Galveston, Texas; International Marine Terminals/Plaquemines Parish Terminal, Mile 57 AHP-Mississippi River, approximately 30 miles south of New Orleans; Energy Terminals of Houston, Inc., a Subsidiary of Soros Associates, Houston, Texas. Coastal Ports are those located at Charleston, South Carolina; New York, New York; San Diego, California; Los Angeles, California; and Seattle, Washington.

Truck: Shipments of coal moved to consumers by truck.

Tramway, Conveyor, or Slurry Pipeline: Shipments of coal moved to consumers by tramway, conveyor, or slurry pipeline.

\section{Census Export and Import Data}

Export and import data are obtained from the Bureau of the Census, U.S. Department of Commerce, where they are compiled monthly from documents filed with the U.S. Customs Service, as required by law.

Each coal shipment is reported in short tons with corresponding total dollar values. EIA converts all value data obtained from the Census Bureau to average price data by dividing the dollar value by the quantity.

Based on an analysis and sample validation of the Census Bureau import and export data conducted by the EIA, it was determined that some of the coal and coke data collected from the Census Bureau may be misleading or incorrect (particularly those data associ- 
ated with very small quantities or very high prices). Because of this, a methodology was developed to edit the Census Bureau price data.

Prior to 1989 , certain data cells had been suppressed for publication purposes only: (1) average import coal prices of $\$ 50.00$ or more per short ton; (2) average export coal prices of $\$ 60.00$ or more per short ton; (3) average coke prices of $\$ 200.00$ or more per short ton; (4) all percent changes of 500 percent or more.

Beginning with 1989 , coal export data were categorized as metallurgical coal and steam coal, rather than as bituminous steam coal, lignite, anthracite, and bituminous metallurgical coal.

In addition, coal export tables were revised to present those countries to which the United States exported more than 50,000 short tons in the prior calendar year. The remaining countries in each continent were aggregated in an "other" category. This reduces the number of empty cells and highlights the major importers of U.S. coal. All coke export and import, and coal import countries and quantities are displayed.

The following methodology was used to derive the typical average prices as presented in the price tables. For all coal, a price distribution was derived from the prior calendar year export price data. Since extreme price variations in the Census Bureau data are the exception rather than the rule, the price distribution was used to identify a typical price range. The price distribution, from low to high, along with the frequency of each price (quantity) was analyzed to determine the representative prices. The extreme prices at both ends of the distribution were eliminated to arrive at a price range that covered at least 90 percent of the exports. This price range was considered to include typical or representative prices. Considering the records that fell within the typical price range, the weighted average price was calculated by country of destination and type of coal.

The same procedure was used to determine the typical average prices of coal imports. In addition to the average prices based on the above methodology, a U.S. total row is presented in the price tables, which represents the average price using all the Census Bureau data.

For reporting purposes, the month of exportation reflects the month in which the shipment leaves the United States. The month of importation generally is based on the month in which the U.S. Customs Service releases the merchandise to the importer. For both sets of data, however, there can exist a small carry-over from the actual month of exportation or importation to a subsequent month, usually the succeeding month. A number of factors in processing account for this, e.g., late receipt of a document for an end-of-month shipment, or rejection of a shipment by the computer due to failure to meet established edit criteria. These limitations should be considered when making comparisons.

Based on the U.S. - Canada Free Trade Agreement, as of January 1990, the U.S. Department of Commerce began reporting statistics on U.S. exports to Canada based on information on imports provided monthly by the Canadian government.

Comparing Census reported imported coal figures in Table 34 with EIA reported imported coal receipts at electric utilities, manufacturers, and coke plants for 1994 shows a difference of about 1.8 million short tons. The main reason for this is that the EIA receipts data do not cover imported coal received by nonutility power producers who are not in the manufacturing sector.

\section{Revisions}

All data published in this report are considered final. The Office of Coal, Nuclear, Electric and Alternate Fuels has adopted the following policy with respect to the revision and correction of recurrent data in energy publications:

1. Annual survey data collected by this office are published either as preliminary or final when first appearing in a data report. Data initially released as preliminary will be so noted in the report. These data will be revised, if necessary, and declared final in the next publication of the data.

2. All monthly and quarterly survey data collected by this office are published as preliminary. These data are revised only after the completion of the 12-month cycle of the data. No revisions are made to the published data before this.

3. After data are published as final, corrections will be made only in the event of a greater than one percent difference at the national level. Corrections for differences that are less than the 1-percent threshold are left to the discretion of the Office Director.

\section{Price Data and Taxes}

F.O.B. mine coal prices and prices of coal delivered to or received by end-use consumers (electric utility plants, manufacturing plants, and coke plants) as reported in this publication include relevant local, State and Federal excise and sales taxes. 
Table D3. Implicit Price Deflator, 1986-1995

\begin{tabular}{c|c}
\hline Year & $\begin{array}{c}\text { Implicit Price Deflator } \\
(\mathbf{1 9 9 2}=\mathbf{1 0 0 )}\end{array}$ \\
\hline $1986^{\mathrm{R}}$ & 80.6 \\
$1987^{\mathrm{R}}$ & 83.1 \\
$1988^{\mathrm{R}}$ & 86.1 \\
$1989^{\mathrm{R}}$ & 89.7 \\
$1990^{\mathrm{R}}$ & 93.6 \\
$1991^{\mathrm{R}}$ & 97.3 \\
$1992^{\mathrm{R}}$ & 100.0 \\
$1993^{\mathrm{R}}$ & 102.6 \\
$1994^{\mathrm{R}}$ & 105.0 \\
$1995^{\mathrm{R}}$ & 107.5 \\
\hline
\end{tabular}

$\mathbf{R}$ Revised data.

Source: Bureau of Economic Analysis, U.S. Department of Commerce, Survey of Current Business. 



\section{Glossary}

Agglomerating Character: Agglomeration describes the caking properties of coal. Agglomerating character is determined by examination and testing of the residue when a small powdered sample is heated to 950 degrees centigrade under specified conditions. If the sample is "agglomerating," the residue will be coherent, show swelling or cell structure, and be capable of supporting a 500-gram weight without pulverizing.

Anthracite: A hard, black lustrous coal, often referred to as hard coal, containing a high percentage of fixed carbon and a low percentage of volatile matter. Comprises three groups classified according to the following ASTM Specification D388-91a, on a dry mineral-matter-free (mmf) basis:

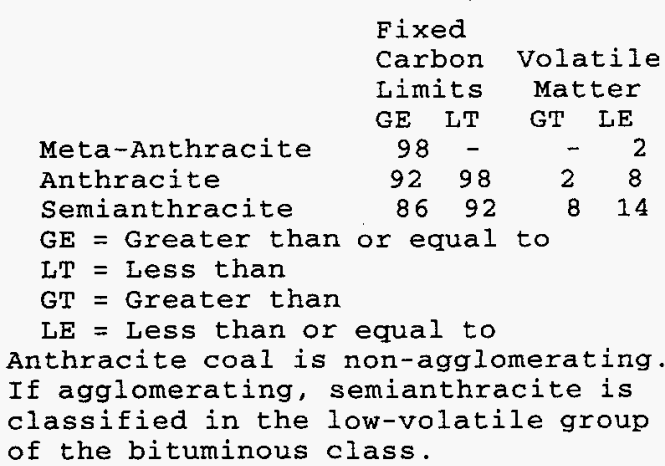

Ash: Impurities consisting of silica, iron, alumina, and other incombustible matter that are contained in coal. Ash increases the weight of coal, adds to the cost of handling, and can affect the burning characteristics. Ash content is measured as a percent by weight of coal on an "as received" or a "dry" (moisture-free, usually part of a laboratory analysis) basis.

Auger Mine: A surface mine where coal is recovered through the use of a large-diameter drill driven into a coalbed in a hillside. It usually follows contour surface mining, particularly when the overburden is too costly to excavate.

\section{Average Annual Percent Change:}

$$
\sqrt[n]{\frac{V_{n}}{V_{0}}-1}
$$

Where: $V_{0}=$ the value for the base period.

$V_{n}=$ the value for the $n^{\text {th }}$ period.

$\mathrm{n}=$ the number of periods.
Average Daily Production: The ratio of the total production at a mining operation to the total number of production days worked at the operation.

Average Length of a Shift: The arithmetic mean number of hours worked during a production shift. Overtime is included if usually worked during the year.

Average Mine Price: The ratio of the total value of the coal produced at the mine to the total production tonnage. (See F.O.B. mine price.)

Average Number of Employees per Shift: The arithmetic mean number of employees working during a production shift. Includes all employees except office workers. (See direct labor hours.)

Average Number of Miners Working Daily: The arithmetic mean number of miners working each day at a mining operation. Includes maintenance as well as production work performed.

Average Number of Shifts per Day: The arithmetic mean number of shifts each day at a mining operation. Includes maintenance as well as production shifts.

Average Production per Miner per Day: The product of the average production per miner per hour at a mining operation and the average length of a production shift at the operation.

Average Production per Miner per Hour: The ratio of the total production at a mining operation to the total direct labor hours worked at the operation.

Average Production per Miner per Shift: Calculated by multiplying average production per miner per hour by the average length of a miner shift.

Average Quality of Coal: Refers to individual measurements such as heat value, fixed cabon, moisture, ash, sulfur, phosphorus, major, minor, and trace elements, coking properties, petrologic properties, and particular organic constituents. The individual quality elements may be aggregated in various ways to classify coal for such special purposes as metallurgical, gas, petrochemical, and blending usages.

Average Recovery Percentage: Average recovery percentage represents the percentage of coal that can be recovered from coal reserves at reporting mines, averaged for all mines in the reported geographic area.

Bituminous Coal: The most common coal. It is dense and black (often with well-defined bands of 
bright and dull material). Its moisture content is usually less than 20 percent. It is used for generating electricity, making coke, and for space heating. Comprises five groups classified according to ASTM Specification D-388-91a, on a dry mineral-matter-free mmf basis for fixed-carbon and volatile matter and a moist mmf basis for calorific value. Coals having 69 percent or more fixed carbon on the dry, mineralmatter-free basis shall be classified according to fixed carbon, regardless of calorific (heating) value. Highvolatile $\mathrm{C}$ bituminous coal is agglomerating, but other bituminous coals are commonly agglomerating. However, it is recognized that there may be nonagglomerating varieties in these groups of the bituminous class, and there are notable exceptions in the high-volatile $\mathrm{C}$ bituminous group. Coals with less than 69 percent fixed carbon, but with 14,000 or more Btu per pound, are classified as high-volatile $A$ bituminous.

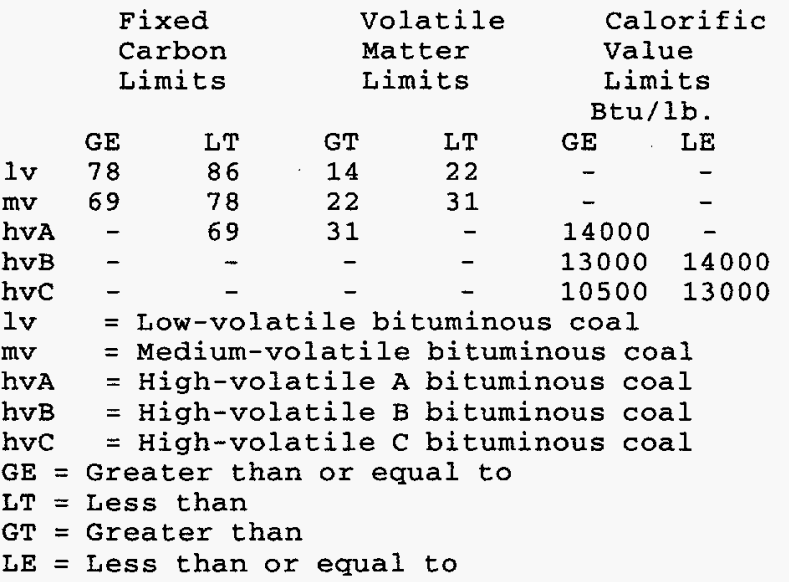

Btu (British thermal unit): The amount of heat needed to raise the temperature of 1 pound of water by 1 degree fahrenheit. The Btu is a convenient measure by which to compare the energy content of various fuels.

Cannel Coal: A variety of bituminous coal that is noncaking, contains a high percentage of volatile matter, ignites easily, and burns with a luminous smokey flame.

Capacity Utilization: Capacity utilization is computed by dividing production by productive capacity and multiplying by 100 .

Captive Coal: Coal produced and consumed by the mine operator, a subsidiary, or parent company (for example, steel companies and electric utilities).

Carbon Dioxide: $\mathrm{CO}_{2} \mathrm{~A}$ colorless, odorless, incombustible gas formed during combustion in fossil-fuel electric generation plants.

Census Divisions: The nine geographic divisions of the United States established by the Bureau of the Census, U.S. Department of Commerce for statistical analysis. The boundaries of Census divisions coincide with State boundaries. In some cases, the Pacific
Division is subdivided into the Pacific Contiguous and Pacific Noncontiguous areas.

CIF: See Cost, Insurance, Freight.

Coal Carbonized: The amount of coal decomposed into solid coke and gaseous products by heating in a coke oven in a limited air supply or in the absence of air.

\section{Coal (Coke): See Coke (coal).}

Coal Mining Productivity: Coal mining productivity is calculated by dividing total coal production by the total direct labor hours worked by all mine employees.

Coal Preparation: The process of sizing and cleaning coal to meet market specifications by removing impurities such as rock, sulfur, etc. May include crushing, screening, or mechanical cleaning.

Coal-Producing Regions: A geographic classification of coal-producing States. The States in the Appalachian Region are Alabama, Georgia, eastern Kentucky, Maryland, Ohio, Pennsylvania, Tennessee, Virginia, and West Virginia. The States in the Interior Region are Arkansas, Illinois, Indiana, Iowa, Kansas, western Kentucky, Louisiana, Missouri, Oklahoma, and Texas. The States in the Western Region are Alaska, Arizona, California, Colorado, Montana, New Mexico, North Dakota, Utah, Washington, and Wyoming.

Coal-Producing States: The States where mined and/or purchased coal originates are defined as follows: Alabama, Alaska, Arizona, Arkansas, California, Colorado, Illinois, Indiana, Iowa, Kansas, Kentucky Eastern, Kentucky Western, Louisiana, Maryland, Missouri, Montana, New Mexico, North Dakota, Ohio, Oklahoma, Pennsylvania anthracite, Pennsylvania bituminous, Tennessee, Texas, Utah, Virginia, Washington, West Virginia Northern, West Virginia Southern, and Wyoming. The following Coal-Producing States are split in origin of coal, as defined below:

- Kentucky, Eastern All mines located in counties other than the Western Kentucky counties.

- Kentucky, Western All mines in the following counties in Western Kentucky: Butler, Caldwell, Christian, Crittenden, Daviess, Edmonson, Grayson, Hancock, Henderson, Hopkins, Logan, McLean, Muhlenberg, Ohio, Simpson, Todd, Union, Warren, and Webster.

- Pennsylvania Anthracite All mines in the following counties: Carbon, Columbia, Dauphin, Lackawanna, Lebanon, Luzerne, Northumberland, Schuylkill, Sullivan, and Susquehanna. All anthracite mines in Bradford County.

- Pennslyvania Bituminous All mines located in counties other than the Pennsylvania anthracite counties and all bituminous mines in Bradford County.

- West Virginia, Northern All mines in the following counties (formerly defined as Coal- 
Producing Districts 1, 3, \& 6): Barbour, Brooke, Braxton, Calhoun, Doddridge, Gilmer, Grant, Hancock, Harrison, Jackson, Lewis, Marion, Marshall, Mineral, Monongalia, Ohio, Pleasants, Preston, Randolph, Ritchie, Roane, Taylor, Tucker, Upshur, Webster, Wetzel, Wirt, and Wood.

-West Virginia, Southern All mines in the following counties (formerly defined as CoalProducing Districts $7 \&$ 8): Boone, Cabell, Clay, Fayette, Greenbrier, Kanawha, Lincoln, Logan, Mason, McDowell, Mercer, Mingo, Monroe, Nicholas, Pocahontas, Putnam, Raleigh, Summers, Wayne, and Wyoming.

Coal Rank/Group: A classification of coal based on fixed carbon, volatile matter, calorific (heating) value, and agglomerating character. Coal is ranked progressively from lignite (least carbonaceous) to anthracite (most carbonaceous). The rank of coal can also determined by measuring the reflectance of vitrinite, one of several organic components of coal. The lower rank coal can be classified based on heat content. The heat content of the higher rank coals is generally above 14 thousand Btu per pound for each coal rank group (except for meta-anthracite, which trends slightly lower), and heat content ranges vary within a relatively narrow range. Since heat content is not a dependable criterion for these higher rank coals, their rank categories are instead described by degree of metamorphism, or "coalification," a property that is measured by fixed carbon content. Finally, the agglomerating character of bituminous coals is a critical attribute for certain coal consumers, and thus agglomerating character has come to define the distinctions between certain adjacent coal groups. Some high-volatile $\mathrm{C}$ bituminous and subbituminous $\mathrm{A}$ coals can be distinguished only on the basis of agglomerating character. Percentages are based on dry mineral-matter-free coal. Volatile matter (not shown) is the complement of fixed carbon; that is, the percentages of fixed carbon and volatile matter sum to 100 percent. As fixed carbon percentage decreases, therefore, volatile matter percentage increases by the same amount.

Coal Stocks: The supply of coal at a mine, plant, or utility at the end of the reporting period.

Coalbed: A bed or stratum of coal. Also called a coal seam.

Cogenerator: A generating facility that produces electricity and another form of useful thermal energy (such as heat or steam) used for industrial, commercial, heating, and cooling purposes. To receive status as a qualifying facility (QF) under the Public Utility Regulatory Policies Act (PURPA), the facility must produce electric energy and "another form of useful thermal energy through the sequencial use of energy," and meet certain ownership, operating, and efficiency criteria established by the Federal Energy Regulatory Commission (FERC). (See the Code of Federal Regulation, Title 18, Part 292.)

Coke (coal): In general, coke is made from bituminous coal (or blends of bituminous coal) from which the volatile constituents are driven off by baking in an oven at temperatures as high as 2,000 degrees Fahrenheit, so that the fixed carbon and ash are fused together. Coke is hard and porous, has a gray, submetallic luster, and is strong enough to support a load of iron ore in a blast furnace. It is used both as a fuel and a reducing agent in smelting iron ore in a blast furnace. Coke has a heating value of 24.8 million Btu per short ton.

Coke Plants: Plants where coal is carbonized in slot or beehive ovens for the manufacture of coke.

Continuous Mining: A form of room-and-pillar mining in which a continuous mining machine extracts and removes coal from the working face in one operation; no blasting is required.

Conventional Mining: The oldest form of room-andpillar mining which consists of a series of operations that involve cutting the coalbed so it breaks easily when blasted with explosives or high-pressure air, and then loading the broken coal.

Cost, Insurance, Freight (CIF): A type of sale in which the buyer of the product agrees to pay a unit price that includes the F.O.B. value of the product at the point of origin plus all costs of insurance and transportation. This type of transaction differs from a "delivered" purchase in that the buyer accepts the quantity as determined at the loading port (as certified by the Bill of Loading and Quality Report) rather than pay on the basis of the quantity and quality ascertained at the unloading port. It is similar to the terms of an F.O.B. sale, except that the seller, as a service for which he is compensated, arranges for transportation and insurance.

Crude Oil: A mixture of hydrocarbons that exists in liquid phase in underground reservoirs and remains liquid at amospheric pressure after passing through surface-separating facilities. Included are lease condensate and liquid hydrocarbons produced from tar sands, gilsonite and oil shale. Drip gases are also included, but topped crude (residual oil) and other unfinished oils are excluded. Liquids produced at natural gas processing plants and mixed with crude oil are likewise excluded where identifiable. Crude oil is considered as either domestic or foreign, according to the following: or from its "outer continental shelf" as defined in 43 U.S.C. 1331. States. Imported Athabasca hydrocarbons are included.

Culm: Waste from Pennsylvania anthracite preparation plants, consisting of coarse rock fragments containing as much as 30 percent small-sized coal; sometimes defined as including very fine coal particles called silt. Its heat value ranges from 8 to 17 million Btu per short ton.

Customs District: Customs districts, as defined by the Bureau of the Census, U.S. Department of Commerce, "Monthly Report EM 545," are as follows

- Eastern: Bridgeport, CT, Washington, DC, Boston, MA, Baltimore, MD, Portland, ME, Buffalo, NY, New York City, NY, Ogdensburg, 
NY, Philadelphia, PA, Providence, RI, Norfolk, VA, St. Albans, VT.

- Southern: Mobile, AL, Savannah, GA, Miami, FL, Tampa, FL, New Orleans, LA, Wilmington, NC, San Juan, PR, Charleston, SC, Dallas-Fort Worth, TX, El Paso, TX, Houston-Galveston, TX, Laredo, TX, Virgin Islands.

-Western: Anchorage, AK, Nogales, AZ, Los Angeles, CA, San Diego, CA, San Fracisco, CA, Honolulu, HI, Great Falls, MT, Portland, OR, Seattle, WA.

- Northern: Chicago, IL, Detroit, MI, Duluth, MN, Minneapolis, MN, St. Louis, MO, Pembina, ND, Cleveland, $\mathrm{OH}$, Milwaukee, WI.

Demonstrated Reserve Base: A collective term for the sum of coal in both measured and indicated resource categories of reliability which represents 100 percent of the coal in these categories in place as of a certain date. Includes beds of bituminous coal and anthracite 28 inches or more thick and beds of subbituminous coal 60 inches or more thick that occur at depths to 1 thousand feet. Includes beds of lignite 60 inches or more thick that can be surface mined. Includes also thinner and/or deeper beds that presently are being mined or for which there is evidence that they could be mined commercially at this time. Represents that portion of the identified coal resource from which reserves are calculated.

Depletion: The subtraction of both the tonnage produced and the tonnage lost to mining from identified resources to determine the remaining tonnage as of a certain time.

Depletion Factor: The multiplier applied to the tonnage produced to compute depletion. This multiplier takes into account both the tonnage recovered and the tonnage lost due to mining. The depletion factor is the reciprocal of the recovery factor in relation to a given quantity of production.

Direct Labor Hours: Direct labor hours worked by all mining employees at a mining operation during the year. Includes hours worked by those employees engaged in production, preparation, development, maintenance, repair, shop or yard work, management, and technical or engineering work. Excludes office workers. Excludes vacation and leave hours.

Distillate Fuel Oil: A general classification for one of the petroleum fractions produced in conventional distillation operations. Included are products known as No.1, No.2, and No.4 fuel oils and No.1, No.2, and No.4 diesel fuels. It is used primarily for space heating, on-and-off-highway diesel engine fuel (including railroad engine fuel and fuel for agricultural machinery), and electric power generation.

Dredge Mining: A method of recovering coal from rivers or streams.

Drift Mine: An underground mine that has a horizontal entry dug to a coalbed in a hillside.
Dry (Coal) Basis: Coal quality data calculated to a theoretical basis in which no moisture is associated with the sample. This basis is determined by measuring the weight loss of a sample when its inherent moisture is driven off under controlled conditions of low temperature air-drying followed by heating to just above the boiling point of water (104 to 110 degrees centigrade).

Electricity: A form of energy generated by friction, induction, or chemical change that is caused by the presence and motion of elementary charged particles of which matter consists.

Electricity Generation: The process of producing electric energy or transforming other forms of energy into electric energy. Also the amount of electric energy produced or expressed in watthours (Wh).

Electricity Generation, Gross: The total amount of electric energy produced by the generating station or stations, measured at the generator terminals.

Electricity Generation, Net: Gross generation less electricity consumed at the generating plant for station use. Electricity required for pumping at pumped-storage plants is regarded as plant use and is deducted from gross generation.

Electric Power Plant: A station containing prime movers, electric generators, and auxiliary equipment for converting mechanical, chemical, and/or fission energy into electric energy.

Electric Utility: A corporation, person, agency, authority, or other legal entity or instrumentality that owns and/or operates facilities within the United States, its territories, or Puerto Rico for the generation, transmission, distribution, or sale of electric energy primarily for use by the public and files forms listed in the Code of Federal Regulations, Title 18, Part 141. Facilities that qualify as cogenerators or small power producers under the Public Utility Regulatory Policies Act (PURPA) and exempt wholesale generators under Energy Policy Act of 1992 are not considered electric utilities. See definition of nonutility power producer.

Electric Utility Sector: The electric utility sector consists of privately and publicly owned establishments that generate, transmit, distribute, or sell electricity primarily for use by the public and that meet the definition of an electric utility. Nonutility power producers are not included in the electric utility sector.

Emissions: The pollutants dischaged into the atmosphere in exhaust gases. For coal-burning plants, these emissions are primarily Carbon Dioxide $\left(\mathrm{CO}_{2}\right)$, Nitrogen Oxide $\left(\mathrm{NO}_{\mathrm{x}}\right)$, and Sulfur Dioxide $\left(\mathrm{SO}_{2}\right)$.

Energy: The capacity for doing work as measured by the capability of doing work (potential energy) or the conversion of this capability to motion (kinetic energy). Energy has several forms, some of which are easily convertible and can be changed to another form useful for work. Most of the world's convertible 
energy comes from fossil fuels that are burned to produce heat that is then used as a transfer medium to mechanical or other means in order to accomplish tasks. Electrical energy is usually measured in kilowatthours, while heat energy is usually measured in British thermal units.

Energy Consumption: The use of energy as a source of heat or power or as an input in the manufacturing process.

Exports: Shipments of goods from the 50 States and the District of Columbia to foreign countries, Puerto Rico, the Virgin Islands, and other U.S. possessions and territories.

Fahrenheit: A temperature scale on which the boiling point of water is at 212 degrees above zero on the scale and the freezing point is at 32 degrees above zero at standard atmospheric pressure.

F.A.S. Value: Free alongside ship value. The value of a commodity at the port of exportation, generally including the purchase price plus all charges incurred in placing the commodity alongside the carrier at the port of exportation in the country of exportation.

Federal Energy Regulatory Commission (FERC): A quasi-independent regulatory agency within the Department of Energy having jurisdiction over interstate electricity sales, wholesale electric rates, hydroelectric licensing, natural gas pricing, oil pipeline rates, and gas pipeline certification.

Federal Coal Lease: A lease granted to a mining company to produce coal from land owned and administered by the Federal Government in exchange for royalties and other revenues.

Federal Power Act: Enacted in 1920, and amended in 1935, the Act consists of three parts. The first part incorporated the Federal Water Power Act administered by the former Federal Power Commission, whose activities were confined almost entirely to licensing non-Federal hydroelectric projects. Parts II and III were added with the passage of the Public Utility Act. These parts extended the Act's jurisdiction to include regulating the interstate transmission of electrical energy and rates for its sale as wholesale in interstate commerce. The Federal Energy Regulatory Commission is now charged with the administration of this law.

Federal Power Commission: The predecessor agency of the Federal Energy Regulatory Commission. The Federal Power Commission (FPC) was created by an Act of Congress under the Federal Water Power Act on June 10, 1920. It was charged originally with regulating the electric power and natural gas industries. The FPC was abolished on September 20,1977, when the Department of Energy was created. The functions of the FPC were divided between the Department of Energy and the Federal Energy Regulatory Commission.

FERC: The Federal Energy Regulatory Commission.
Fixed Carbon: The nonvolatile matter in coal minus the ash. Fixed carbon is the solid residue other than ash obtained by prescribed methods of destructive distillation of a coal. Fixed carbon is the part of the total carbon that remains when coal is heated in a closed vessel until all volatile matter is driven off.

Flue Gas Desulfurization Unit (Scrubber): Equipment used to remove sulfur oxides from the combustion gases of a boiler plant before discharge to the atmosphere. Chemicals, such as lime, are used as the scrubbing media.

Flue Gas Particulate Collectors: Equipment used to remove fly ash from the combustion gases of a boiler plant before discharge to the atmosphere. Particulate collectors include electrostatic precipitators, mechanical collectors (cyclones), fabric filters (baghouses), and wet scrubbers.

F.O.B. Mine Price: The free on board mine price. This is the price paid for coal at the mining operation site. It excludes freight or shipping and insurance costs.

Foreign-Controlled Firms: Foreign-controlled firms are U.S. coal producers with more than 50 percent of their stock or assets owned by a foreign firm.

Fossil-Fuel Electric Generation: Electric generation in which the prime mover is a turbine rotated by highpressure steam produced in a boiler by heat from burning fossil fuels.

Geothermal Energy: Energy from the internal heat of the earth, which may be residual heat, friction heat, or a result of radioactive decay. The heat is found in rocks and fluids at various depths and can be extracted by drilling and/or pumping.

Greenhouse Effect: The increasing mean global surface temperature of the earth caused by gases in the atmosphere (including carbon dioxide, methane, nitrous oxide, ozone, and chlorofluorocarbon). The greenhouse effect allows solar radiation to penetrate but absorbs the infrared radiation returning to space.

Gross Domestic Product (GDP): The total value of goods and services produced by labor and property in the United States. As long as the labor and property are located in the United States, the supplier (that is, the workers and, for property, the owners) may be either U.S. residents or residents of foreign countries.

Hand Loading: An underground loading method by which coal is removed from the working face by manual labor through the use of a shovel for conveyance to the surface. Though rapidly disappearing, it is still used in very small-tonnage mines.

Highwall: the unexcavated face of exposed overburden and coal in a surface mine.

High-Volatile A Bituminous Coal: See Bituminous coal. 
High-Volatile B Bituminous Coal: See Bituminous coal.

High-Volatile C Bituminous Coal: See Bituminous coal.

High-Volatile (specific sub-group unknown): See Bituminous coal.

Hydroelectric Power: The harnessing of flowing water to produce mechanical or electrical energy.

Implicit Price Deflator: The implicit price deflator, published by the U.S. Department of Commerce, Bureau of Economic Analysis, is used to convert nominal figures to real figures.

Imports: Receipts of goods into the 50 States and the District of Columbia from foreign countries and from Puerto Rico, the Virgin Islands, and other U.S. possessions and territories.

Indian Coal Lease: $A$ lease granted to a mining company to produce coal from Indian lands in exchange for royalties and other revenues; obtained by direct negotiatian with the Indians, but subject to approval and administration by the U.S. Department of the Interior.

Industrial Sector: The industrial sector comprises manufacturing industries which make up the largest part of the sector, along with mining, construction, agriculture, fisheries, and forestry. Establishments in the sector range from steel mills, to small farms, to companies assembling electronic components. The SIC codes used to classify establishments as industrial are 1 through 39.

Interquartile Range: The interquartile range is the range within which the middle 50 percent of observations are concentrated. See Appendix D, Section "Interquartile Range."

Jet Fuel: The term includes kerosene-type jet fuel and naphtha-type jet fuel. Kerosene-type jet fuel is a kerosene-quality product used primarily for commercial turbojet and turboprop aircraft engines. Naphthatype jet fuel is a fuel in the heavy naphthas range used primarily for military turbojet and turboprop aircraft engines.

Lease Condensate: A natural gas liquid recovered from gas well gas (associated and non-associated) in lease separators or natural gas field facilities. Lease condensate consists primarily of pentanes and heavier hydrocarbons.

Lignite: A brownish-black coal of low rank with high inherent moisture and volatile matter (used almost exclusively for electric power generation). Similar coal in Europe and Australia are also referred to as brown coal. Lignite comprises two groups classified according to the following ASTM Specifiaction D-388-91a for calorific values on a moist mineralmatter-free basis:

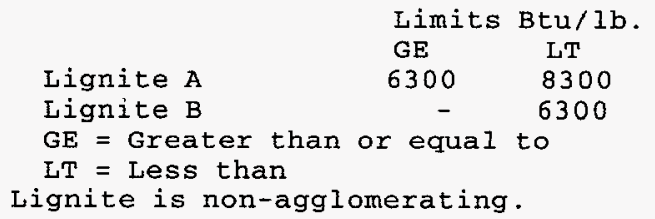

Lignite A: See Lignite.

Lignite B: See Lignite.

Longwall Mining: A form of underground coal mining which is gaining in importance in the United States and can be used at greater depths than roomand-pillar mining. In longwall mining, a cutting machine is pulled back and forth across a panel of coal 300 to 600 feet wide and as much as a mile long, with the broken coal moved by conveyor. Longwall mining is done under movable roof supports that are advanced as the bed is cut. The roof in the mined-out area is allowed to fall as the mining advances.

Low-Volatile Bituminous Coal: See Bituminous Coal.

Major Coal-Producing States: Any State that produces more than 12 million short tons of coal during the year.

Manufacturing (except coke plants): Those industrial users/plants, not including coke plants, that are engaged in the mechanical or chemical transformation of materials or substances into new (i.e., finished or semifinished) products. Includes coal used for gasification/liquifaction.

Medium-Volatile Bituminous Coal: See Bituminous Coal.

Merchant Coke Plant: A coke plant where coke is produced primarily for sale on the commercial (open) market.

Meta-Anthracite: See Anthracite.

Metallurgical Coal: Coal that meets the requirements for making coke. It must be low in ash and sulfur and form a coke that is capable of supporting the charge of iron ore and limestone in a blast furnace. A blend of two or more bituminous coals is usually required to make coke.

Metric Ton: A unit of weight equal to 2,204.6 pounds.

Mine Type: See Surface Mine and Underground Mine.

Mineral-Matter-Free Basis: Mineral matter in coal is the parent material in coal from which ash is derived, and which comes from minerals present in the original plant materials that formed the coal, or from extraneous sources such as sediments and precipitates from mineralized water is called the mineral matter. Mineral matter in coal cannot be analytically determined and is commonly calculated using data on 
ash and ash-forming constituents. Coal analyses are calculated to the mineral-matter-free basis by adjusting formulas used in calculations in order to deduct the weight of mineral matter from the total coal.

Moist (Coal) Basis: "Moist" coal contains its natural inherent or bed moisture, but does not include water adhering to the surface. Coal analyses expressed on a moist basis are performed or adjusted so as to describe the data when the coal contains only that moisture which exists in the bed in its natural state of deposition, and when the coal has not lost any moisture due to drying.

Naphtha: A genetic term applied to a petroleum fraction with an approximate boiling range between 122 and 400 degrees fahrenheit.

Natural Gas: A mixture of hydrocarbons and small quantities of various nonhydrocarbons existing in the gaseous phase or in solution with crude oil in underground reservoirs.

Natural Gas (Dry): The marketable portion of natural gas production, which is obtained by subtracting extraction losses, including natural gas liquids removed at natural gas processing plants, from total production.

Natural Gas Plant Liquids (NGPL): Natural gas liquids recovered from natural gas in processing plants and, in some situations, from natural gas field facilities, as well as those extracted by fractionators. Natural gas plant liquids are defined according to the published specifications of the Gas Processors Association and the American Society for Testing and Materials as follows: ethane, propane, normal butane, isobutane, pentanes plus, and other products from natural gas processing plants (i.e., products meeting the standards for finished petroleum products produced at natural gas processing plants, such as finished motor gasoline, finished avaiation gasoline, special naphthas, kerosene, distillate fuel oil, and miscellaneous products).

Nitrogen Oxide: $\quad N O_{x}$. A gas formed in hightemperature environments when nitrogen and oxygen are present together. This typically occurs in a combustion chamber such as those in fossil-fuel burning electric utilities. Nitrogen oxide emissions are a contributor to acid rain.

Nominal Price: The price paid for a product or service at the time of the transaction. The nominal price, which is expressed in current dollars, is not adjusted to remove the effect of changes in the purchasing power of the dollar.

Nonutility Power Producers: A corporation, person, agency, authority, or other legal entity or instrumentality that owns electric generating capacity and is not an electric utility. Nonutility power producers include qualifying cogenerators, qualifying small- power producers, and other nonutility generators (including independent power producers) without a designated franchised service area and which do not file forms listed in the Code of Federal Regulations, Title 18, Part 141. (See Electric Utility.)

Nuclear Electric Power: Electricity generated by an electric power plant whose turbines are driven by steam generated in a reactor by heat from the fissioning of nuclear fuel.

Number of Mines: The number of mines, or mines collocated with preparation plants or tipples, located in a particular geographic area (State or region). If a mine is mining coal across two counties within a State, or across two States, then it is counted as two operations. This is done so that EIA can separate production by State and county.

Number of Mining Operations: The number of mining operations includes preparation plants with greater than 5,000 total direct labor hours. Mining operations that consist of a mine and preparation plant or a preparation plant only will be counted as two operations, if the preparation plant processes both underground and surface coal. Exluded are silt, culm, refuse bank, slurry dam, and dredge operations except for Pennsylvania anthracite. Excludes mines producing less than 10,000 short tons of coal during the year, and preparation plants with less than 5,000 employee hours.

Open Market Coal: Coal sold in the open market, i.e., coal sold to companies other than the reporting company's parent company or an operating subsidiary of the parent company.

Operating Subsidiary: A company which is controlled through the ownership of voting stock, or a corporate joint venture in which a corporation is owned by a small group of businesses as a separate and specific business or project for the mutual benefit of the members of the group.

Other Industrial Plant: Industrial users, not including coke plants, engaged in the mechanical or chemical transformation of materials or substances into new products (manufacturing); and companies engaged in the agriculture, mining, or construction industries.

Other Unions: See Union Type.

Overburden: Any material, consolidated or unconsolidated, that overlies a coal deposit.

Parent Company: A company which solely or jointly owns the reporting company and which is not itself a subsidiary of, or owned by, another company.

Percent Utilization: The ratio of total production to productive capacity, times 100 .

Petroleum: Petroleum includes residential and distillate fuel oils, crude oil, and all other petroleum fuels, excluding petroleum coke.

Petroleum Coke: A residue that is the final product of the condensation process in cracking. The product 
is either marketable petroleum coke or catalyst petroleum coke.

Petroleum Products: Products obtained from the processing of crude oil (including lease condensate), natural gas, and other hydrocarbon compounds. Petroleum products include unfinished oils, liquefied petroleum gases, pentanes plus, aviation gasoline, motor gasoline, naphtha-type jet fuel, kerosene-type jet fuel, kerosene, distillate fuel oil, residual fuel oil, petrochemical feedstocks, special naphthas, lubricants, waxes, petroleum coke, asphalt, road oil, still gas, and miscellaneous products.

Photovoltiac and Solar Thermal Energy (as used at electric utilities): Energy radiated by the sun as electromagnetic waves (electromagnetic radiation) that is converted at electric utilities into electricity by means of solar (photovoltiac) cells or concentrating (focusing) collectors.

Preparation Plant: A mining facility at which coal is crushed, screened, and mechanically cleaned.

Producer and Distributor Coal Stocks: Producer and distributor coal stocks consist of coal held in stock by producers/distributors at the end of a reporting period.

Productive Capacity: The maximum amount of coal that a mining operation can produce or process during a period with the existing mining equipment and/or preparation plant in place, assuming that the labor and materials sufficient to utilize the plant and equipment are available, and that the market exists for the maximum production.

\section{Quadrillion Btu: $10^{15}$ Btu.}

Real Price: A price that has been adjusted to remove the effect of changes in the purchasing power of the dollar. Real prices, which are expressed in constant dollars, usually reflect buying power relative to a base year.

Recoverable Coal Reserves at Mines: The quantity of coal that can be recovered (i.e., mined) from existing coal reserves, as reported on Form EIA-7A.

Recoverable Reserves of Coal: An estimate of the amount of coal that can be recovered (mined) from the accessible reserves of the demonstrated reserve base.

Recovery Percentage: The percentage of coal that can be recovered from the coal deposits at existing mines.

Refuse Bank: A repository for waste material generated by the coal cleaning process.

Refuse Mine: A surface mine where coal is recovered from previously mined coal. It may also be known as a silt bank, culm bank, refuse bank, slurry dam, or dredge operation.

Report Year: The calendar year beginning at 12:00 a.m. January 1 and ending at 11:59 p.m. December 31 .
Residential and Commercial Sector: Housing units; wholesale and retail businesses (except coal wholesale dealers); health institutions (hospitals); social and educational institutions (schools and universities); and Federal, State, and local governments (military installations, prisons, office buildings).

Residual Fuel Oil: The heavier oils that remain after the distillate fuel oils and and lighter hydrocarbons are distilled away in refinery operations and that conform to ASTM Specifications D396 and 975 . Included are No. 5, a residual fuel oil of medium viscosity; Navy Special, for use in steam-powered vessels in government service and in shore power plants; and No. 6, which includes Bunker $C$ fuel oil and is used for commercial and industrial heating, electricity generation, and to power ships. Imports of residual fuel oil include imported crude oil burned as fuel.

Room-and-Pillar Mining: The most common method of underground mining in which the mine roof is supported mainly by coal pillars left at regular intervals. Rooms are places where the coal is mined; pillars are areas of coal left between the rooms. Room-and-pillar mining is done either by conventional or continuous mining.

Royalties: Payments, in money or kind, of a stated share of production from mineral deposits, by the lessee to the lessor. Royalties may be an established minimum, a sliding-scale, or a step-scale. A step-scale royalty rate increases by steps as the average production on the lease increases. A sliding-scale royalty rate is based on average production and applies to all production from the lease.

Sales Volume: The reported output from Federal and/or Indian lands, the basis of royalties. It is approximately equivalent to production, which includes coal sold, and coal added to stockpiles.

Scoop Loading: An underground loading method by which coal is removed from the working face by a tractor unit equipped with a hydraulically operated bucket attached to the front; also called a front-end loader.

Semianthracite: See Anthracite.

Shaft Mine: An underground mine that reaches the coalbed by means of a vertical shaft. In addition to the passages providing entry to the coalbed, a network of other passages are also dug, some to provide access to various parts of the mine and some for ventilation.

Short Ton: A unit of weight equal to 2,000 pounds.

Shortwall Mining: A form of underground mining that involves the use of a continuous mining machine and movable roof supports to shear coal panels 150 to 200 feet wide and more than half a mile long. Although similar to longwall mining, shortwall mining is generally more flexible because of the smaller working area. Productivity is lower than with 
longwall mining because the coal is hauled to the mine face by shuttle cars as opposed to conveyors.

\section{SIC: See Standard Industrial Classification.}

Silt: Waste from Pennsylvania anthracite preparation plants, consisting of coarse rock fragments containing as much as 30 percent small-sized coal; sometimes defined as including very fine coal particles called silt. Its heat value ranges from 8 to 17 million Btu per short ton. Synonymous with culm.

Silt, Culm Refuse Bank, or Slurry Dam Mining: A mining operation producing coal from these sources of coal. (See refuse mine.)

Slope Mine: An underground mine in which the entry is driven at an angle to reach the coal deposit.

Slurry Dam: A repository for the silt or culm from a preparation plant.

Solar Energy: The radiant energy of the sun, which can be converted into other forms of energy, such as heat or electricity.

Solar Thermal Collector: A devise designed to receive solar radiation and convert it into thermal energy. Normally, a solar thermal collector includes a frame, glazing, and an absorber, together with appropriate insulation. The heat collected by the solar thermal collector may be used immediately or stored for later use.

Standard Industrial Classification (SIC): A set of codes developed by the Office of Management and Budget which categorizes industries to groups with similar economic activities.

Steam Coal: All noncoking coal.

Stocks: The supply of coal or coke at a mine, plant, or utility at the end of the reporting period.

Strategic Petroleum Reserve (SPR): Petroleum stocks maintained by the Federal Government for use during periods of major supply interruption.

Strip (Surface) Mining: A method used on flat terrain to recover coal by mining long strips successively. The material excavated from the strip being mined is deposited in the strip previously mined.

Subbituminous Coal: A dull black coal of rank intermediate between lignite and bituminous, consisting of subbituminous $A$ coal, subbituminous $B$ coal, and subbituminous $C$ coal, classified according to the following ASTM Specification D-388-91a on a moist mineral-matter-free basis:

\begin{tabular}{lcc} 
& \multicolumn{2}{c}{ Calorific } \\
& Value \\
& Limits \\
& Btu/1b. \\
& GE & LT \\
Subbituminous A Coal & 10500 & 11500 \\
Subbituminous B Coal & 9500 & 10500 \\
Subbituminous C Coal & 8300 & 9500 \\
GE = Greater than or equal to & \\
LT = Less than & & \\
Subbituminous coal is non-agglomerating.
\end{tabular}

Subbituminous A Coal: See Subbituminous Coal.

Subbituminous B Coal: See Subbituminous Coal.

Subbituminous C Coal: See Subbituminous Coal.

Sulfur: One of the elements present in varying quantities in coal that contributes to environmental degradation when coal is burned. In terms of sulfur content by weight, coal is generally classified as low (less than or equal to 1 percent), medium (greater than 1 percent and less than or equal to 3 percent), and high (greater than 3 percent). Sulfur content is measured as a percent by weight of coal on an "as received" or a "dry" (moisture-free, usually part of a laboratory analysis) basis.

Sulfur Dioxide: $\mathrm{SO}_{2}$. A caustic, corrosive gas that is a by-product of combustion and emissions from fossil-fuel burning electric utility plants. The primary agent in the production of acid rain.

Supplemental Gaseous Fuels: Any gaseous substance that, introduced into or commingled with natural gas, increases the volume available for disposition. Such substances include, but are not limited to, propane-air, refinery gas, coke oven gas, still gas, manufactured gas, biomass gas, or air or inert gases added for Btu stabilization.

Surface Mine: A coal-producing mine that is usually within a few hundred feet of the surface. Earth and rock above or around the coal (overburden) is removed to expose the coalbed, which is then mined with surface excavation equipment such as draglines, power shovels, bulldozers, loaders, and augers. It may also be known as an area, contour, open-pit, strip, or auger mine.

Tipple: A central facility used in loading coal for transportation by rail or truck.

Transportation Sector: The transportation sector consists of private and public vehicles that move people and commodities. Included are automobiles, trucks. buses, motorcycles, railroads and railways (including streetcars), aircraft, ships, barges, and natural gas pipelines.

Underground Mine: A mine where coal is produced by tunneling into the earth to the coalbed, which is then mined with underground mining equipment such as cutting machines and continuous, longwall, and shortwall mining machines. Underground mines are classified according to the type of opening used to 
reach the coal, i.e., drift (level tunnel), slope (inclined tunnel), or shaft (vertical tunnel).

Unfinished Oils: All oils requiring further refinery processing, except those requiring only mechanical blending. Includes naphthas and lighter oils, kerosene and light gas oils, heavy gas oils, and residuum.

Union Type: Union type consists of United Mine Workers of America (UMWA), and the following "Other Union" types: Southern Labor Union (SLU), Appalachian Miners of America (AMA), Scotia Employees Association (SEA), International Union of Operation Engineers (IUOE), Utility Workers of America (UWA), Progressive Mine Workers Association (PMWA), International Brotherhood of Electrical Workers (IBEW), International Chemical Workers Union (ICWU), Redstone Workers Association (RWA), Chariton Valley Independent Union (CVIU), American Federation of Labor - Congress of Industrial Organization (AFL-CIO), Labors International (LABO), Crow Hollow Miners (CROW), Coal Strippers (COAL), United Steel Workers (USW), Independent Miners Association (IMA), Independent Union (INUN), Independence Miners, Brokers, and Truckers Association (IMBT), Council of Southern Mountains (CSM), International Brotherhood of Teamsters, Chauffeurs, Warehousemen and Helpers Union (TEAM), Thompson Creek Workers Association (TCWA), United Brotherhood of Clay Workers (UBCW), Wilmot Employees Independent Union (WEIU), Independent Strip Miners Union (ISMU), Independent Miners (IM), Independent Workers (IW), Coal Strippers Union (CSU), Independent Miners Union (IMU), Independent Coal Workers (ICW), Independent Strip Mining Workers (ISMW), Independent Strip Union (ISU), Association of Bituminous Contractors (ABC), Arch Minerals Employees Associ- ation (AMEA), United Paperworkers International Union (UPIU), Welch Miners Union (WMU), Falcon Coal Employees Association (FCEA), Justus Employees Association (JEA), International Construction Union (ICU), Brotherhood of Miners (BOM), Western Energy Workers (WEW), Carlin Independent Union (CIU), International Association of United Workers Union (IAWU), and Stove, Furnace and Allied Appliance Workers International Union of N. A. (SFAW).

U.S. Coal Exports: Amount of U.S. coal shipped to foreign destinations, as reported in the U.S. Department of Commerce, Bureau of Census, "Monthly Report EM 545."

U.S. Coal Imports: Amount of foreign coal shipped to the United States, as reported in the U.S. Department of Commerce, Bureau of the Census, "Monthly Report IM 145."

Wind Energy (as used at electric utilities): The kinetic energy of wind converted at electric utilities into mechanical energy by wind turbines (i.e., blades rotating from the hub) that drive generators to produce electricity for distribution.

Wood and Waste (as used at electric utilities): Wood energy, garbage, bagasse, sewerage gas, and other industrial, agricultural, and urban refuse used to generate electricity for distribution.

Volatile Matter: Those products, exclusive of moisture, given off by a material as gas or vapor. Volatile matter is determined by heating the coal to 950 degrees centigrade under carefully controlled conditions and measuring the weight loss, excluding weight of moisture driven off at 105 degrees centigrade. 


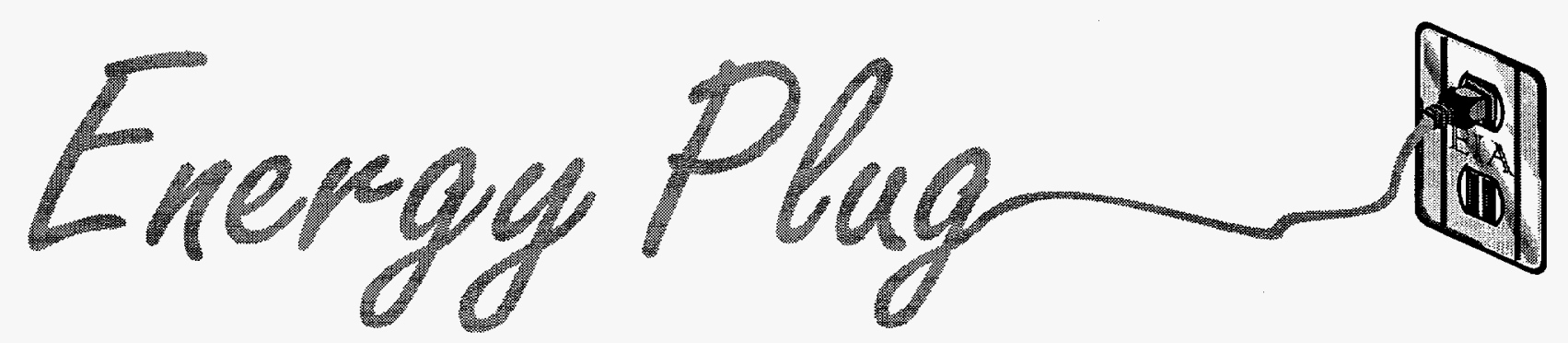

http://www.eia.doe.gov gopher://gopher.eia.doe.gov ftp://ftp.eia.doe.gov http: //www. eia.doe.gov gopher: //gopher.eia.doe.gov

\section{EIA Electronic Media Meet Customer Needs}

The Energy Information Administration (EIA) annually surveys its customers to learn how satisfied they are with EIA's service and information. In this year's February survey of 264 telephone customers, 91 percent said they were "satisfied" or "very satisfied" with EIA information quality. However, only 73 percent were satisfied with data timeliness and nearly four out of five said that earlier release of 95-percent accurate data would be satisfactory. There also seems to be a trend toward wider customer use of EIA electronic media. Such use increased from 49 percent in 1995 to 60 percent this year.

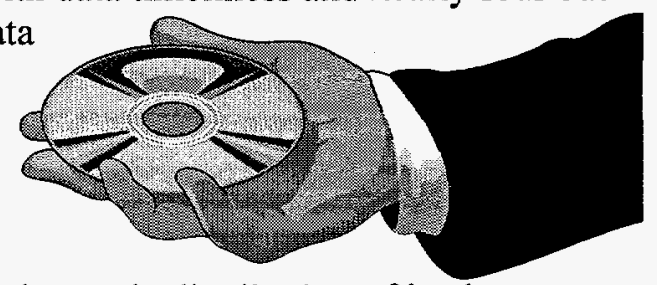

These results are driving EIA to strengthen its focus on electronic distribution of its data, most of which can now be accessed via one or more electronic media. The most important of these are described below. For more information, contact the National Energy Information Center at 202-586-8800 or via internet e-mail at infoctr@eia.doe.gov.

EIA World Wide Web Site-press releases, new publications and services, key EIA contacts, e-mail listserv sign-up, and energy events calendar, plus links to data stores on petroleum, natural gas, coal, nuclear power, renewables, electricity, multifuel summaries, international energy, enduse consumption, forecasts, and more. The URL is http://www.eia.doe.gov.

Energy InfoDisc CD-ROM-a quarterly compendium of more than 180 of EIA's data, analysis, and forecasting reports and directories published during the previous 12 months. Single issues or annual subscriptions are available from the U.S. Department of Commerce; call 202-482-1986 or 1-800-STAT-USA. More information can be found on the EIA web site at http://www.eia.doe.gov/infodisc.html.

Internet E-Mail-allows subscribers to receive press releases and selected EIA publications electronically, including Petroleum Market Report, Short-Term Energy Outlook Summary, Electric Power Monthly Summary, and more than a dozen others. Sign up via EIA's web site.

FTP Site-access to EIA's complete energy data stores, which are generally organized by fuel type. Files are often large and may require a reader to view. The URL is ftp://eia.doe.gov, or access via the EIA web site.

Data Files-a wide variety of EIA data files, ranging from the Annual Energy Review data series to the Oil and Gas Field Code Master List, is available on computer tape or diskette. For ordering information, contact the National Energy Information Center at 202-586-8800 or via internet e-mail at infoctr@eia.doe.gov. 